WHC-SP-1096

\title{
Planning Integration FY 1995 Multi-Year Program Plan (MYPP)/Fiscal Year Work Plan (FYWP)
}

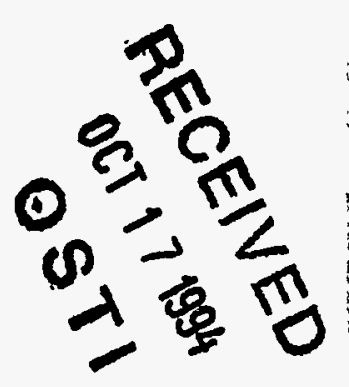

Date Published

September 1994

Prepared for the U.S. Department of Energy Office of Environmental Restoration and Waste Management

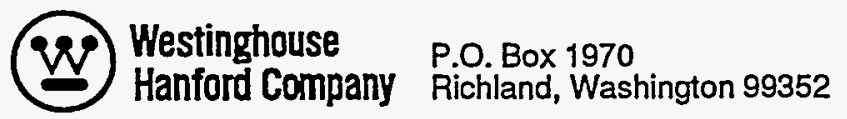

Hanford Operations and Engineering Contractor for the 
LEGAL DISCLAIMER

This report was prepared as an account of work sponsored by an agency of the United States Government. Neither the

United States Government nor any agency thereot, nor any of their employees, nor any of their contractors, subcontractors or their employees, makes any warranty, express or implied, or assumes any legal liability or responsibility for the accuracy, completeness, or any third party's use or the results of such use of any information, apparatus, product, or process disclosed, or represents that its use would not iniringe privately owned rights. Reference herein to any specific commercial product, process, or service by trade name, trademark, manufacturer, or otherwise, does not necessarily constitute or imply its endorsement, recommendation, or favoring by the United States Government or any agency thereof or its contractors or subcontractors. The views and opinions of authors expressed herein do not necessarily state or reflect those of the United States Government or any agency thereof.

This report has been reproduced from the best available copy. Available in paper copy and microfiche.

Available to the U.S. Department of Energy

and its contractors from

Office of Scientific and Technical Information

P.O. Box 62

Oak Ridge, TN 37831

(615) 576-8401

Printed in the United States of America

DISCLM-3.CHP (1.91) 


\section{DISCLAIMER}

Portions of this document may be illegible in electronic image products. Images are produced from the best available original document. 


\section{WESTINGHOUSE HANFORD COMPANY UNCLASSIFIED DOCUMENT RELEASE RECORD}

Document No::WHC-SP-1096

Copy No.:

Document Title: Planning Integ. FY 1995 Multi-Year Program Plan/Fiscal Year Work Plan

THIS IS A COPY CONTROLLED DOCUMENT. CUSTODIAN IS RESPONSIBLE FOR RECORDING

ALL INFORMATION UPON RECEIVING A NEW RELEASE

\begin{tabular}{|c|c|c|c|c|c|c|c|c|}
\hline $\begin{array}{l}\text { Rel. } \\
\text { No. }\end{array}$ & $\begin{array}{c}\text { Date } \\
\text { Inserted }\end{array}$ & $\begin{array}{c}\text { Revised By } \\
\text { Initials }\end{array}$ & $\begin{array}{l}\text { Rel. } \\
\text { No. }\end{array}$ & $\begin{array}{c}\text { Date } \\
\text { Inserted }\end{array}$ & $\begin{array}{l}\text { Revised By } \\
\text { Initials }\end{array}$ & $\begin{array}{l}\text { Rel. } \\
\text { No. }\end{array}$ & $\begin{array}{c}\text { Date } \\
\text { Inserted }\end{array}$ & $\begin{array}{l}\text { Revised By } \\
\text { Initials }\end{array}$ \\
\hline & & & & & & & & \\
\hline & & & & & & & & \\
\hline & & & & & & & 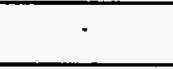 & \\
\hline & & & & & & & & \\
\hline & & & & & & & & \\
\hline & & & & & & & & \\
\hline & & & & & & & & \\
\hline & & & & & & & & \\
\hline & & & & & & & & \\
\hline & & & & & & & & \\
\hline & & & & & & & & \\
\hline & & & & & & & & \\
\hline & & & & & & & & . \\
\hline & & & & & & & & \\
\hline & & & & & & & & \\
\hline & & & & & & & & \\
\hline & & & & & & & & \\
\hline & & & & & & & & \\
\hline & & & & & & & & \\
\hline & & & & & & & & \\
\hline & & & & & & & & \\
\hline & & & & & & & & \\
\hline & & & & & & & & \\
\hline & & & & & & & & \\
\hline & & & & & & & & \\
\hline & & & & & & & & \\
\hline & & & & & & & & \\
\hline & & & & & & & & \\
\hline & & & & & & & & \\
\hline & & & & & & & & \\
\hline & & & & & & & & \\
\hline
\end{tabular}




\section{RELEASE AUTHORIZATION}

Document Number: $\quad$ WHC-SP-1096

Document Title: $\quad$ Planning Integration FY 1995 Multi-Year Program Plan (MYPP) Fiscal Year Work Plan (FYWP).

Release Date: $\quad 9 / 20 / 94$

This document was reviewed following the procedures described in WHC-CM-3-4 and is:

APPROVED FOR PUBLIC RELEASE

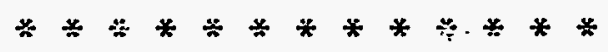

WHC Information Release Administration Specialist:

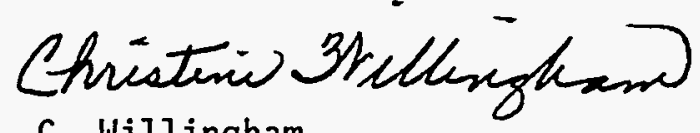

C. Willingham $9 / 20 / 94$

(Signature)

(Date) 


\section{MYPP Approval Sheet}

\subsubsection{Planning Integration Program}

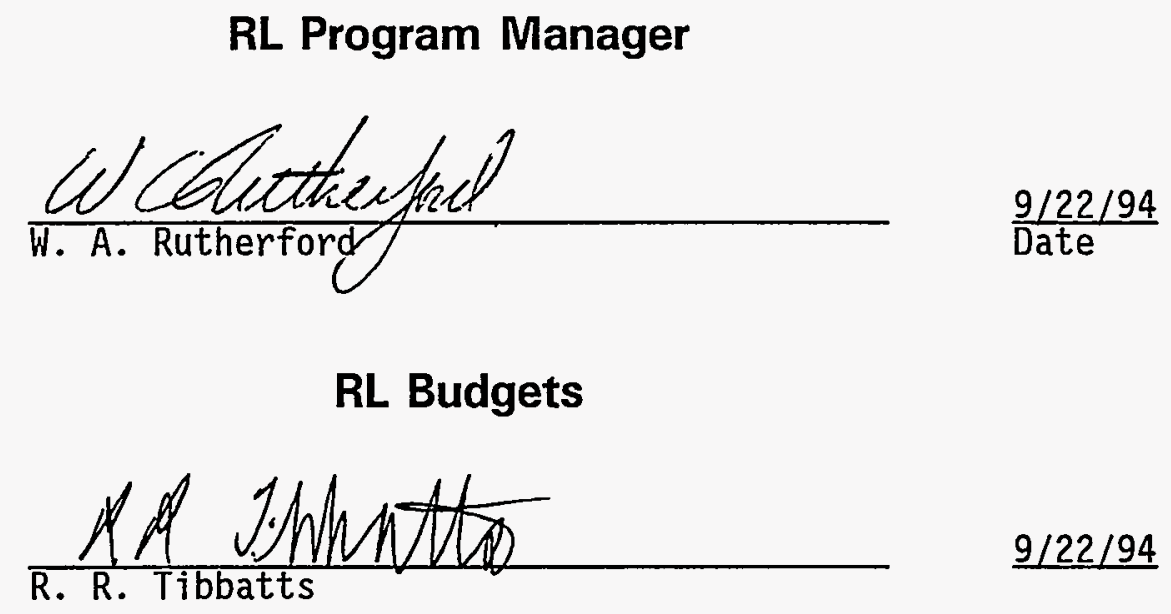

Assistant Manager-Contracting Officer's Representative
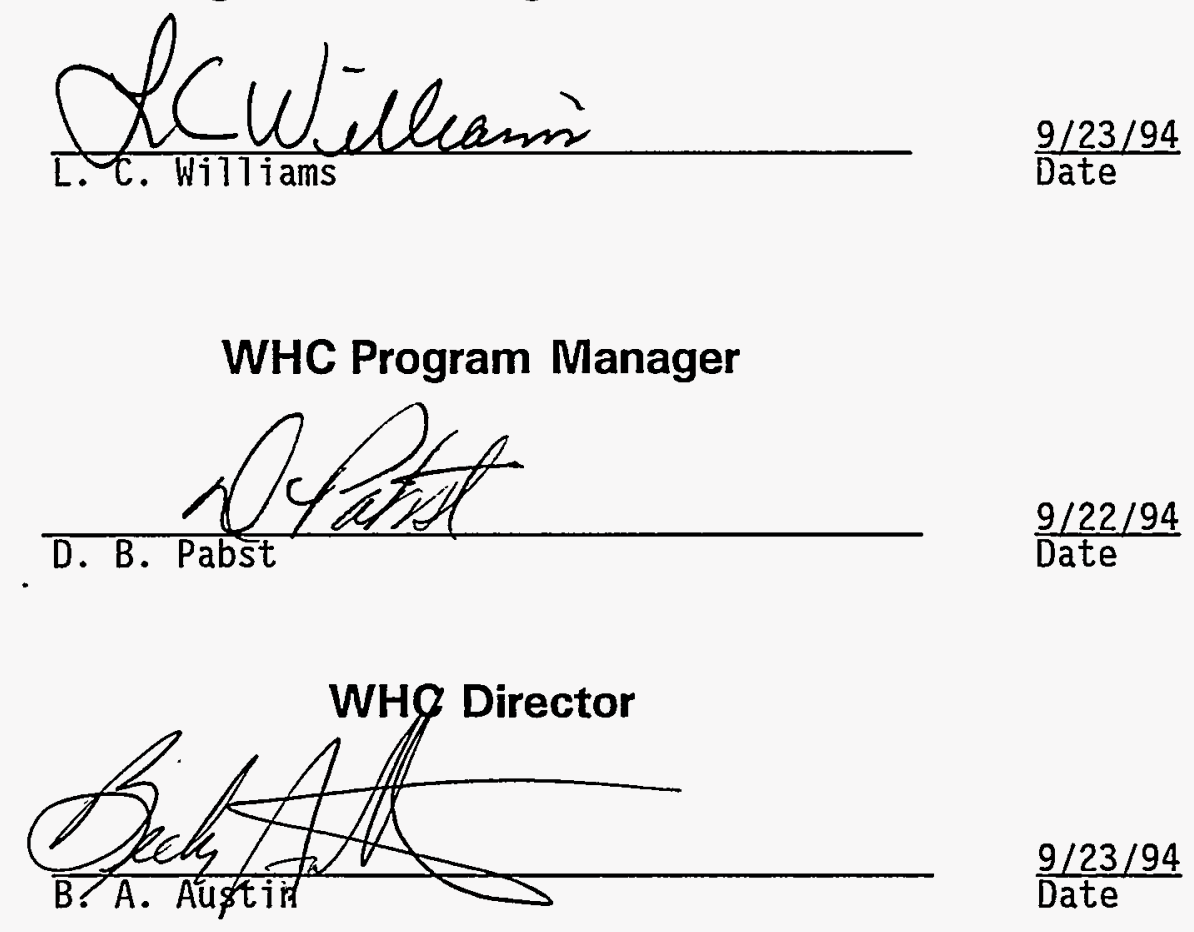


\section{MYPP Approval Sheet \\ WBS 1.8.2 \\ PLANNING INTEGRATION PROGRAM}

Program Element Managers

Page 1 of 2

WBS 1.8.2.1 - Work Identification

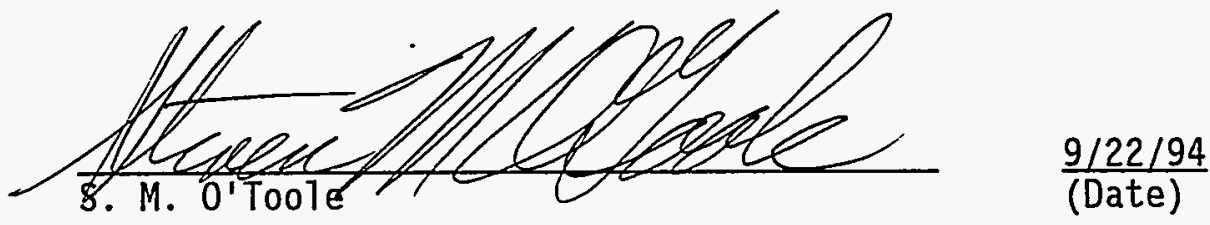

WBS 1.8.2.2 - Baseline Reporting and System Development

URIJ f

L. R. Hafer $\underline{9 / 22 / 94}$

(Date)

WBS 1.8.2.3 - Program Baseline Integration

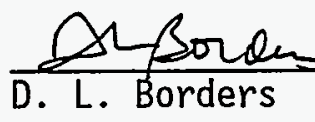

$\frac{9 / 22 / 94}{(\operatorname{Date})}$ (Date)

WBS 1.8.2.4 - Technical Baseline Development

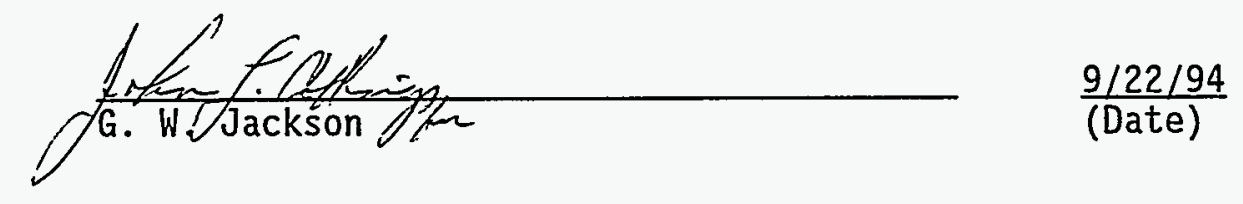




\section{MYPP Approval Sheet}

\section{WBS 1.8.2 \\ PLANNING INTEGRATION PROGRAM}

\section{Program Element Managers}

Page 2 of 2

WBS 1.8.2.5 - Planning Integration Program Management

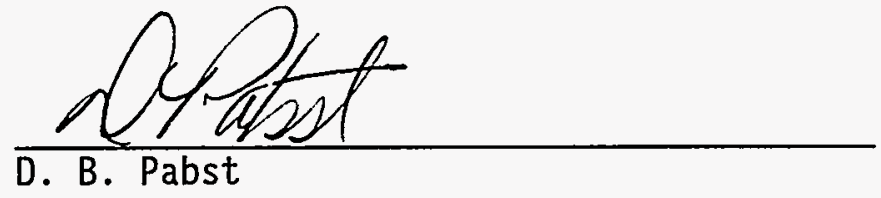

$\frac{9 / 22 / 94}{\text { (Date) }}$

WBS 1.8.2.6 - Integrated Risk Assessment

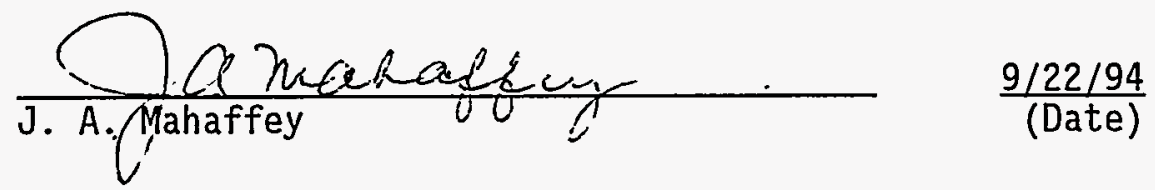

WBS 1.8.2.7 - Public Involvement

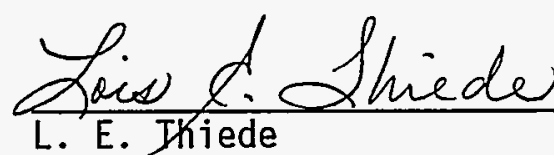

$\frac{9 / 22 / 94}{(\operatorname{Date})}$

(Date)

WBS 1.8.2.8 - ADS Preparation and Coordination

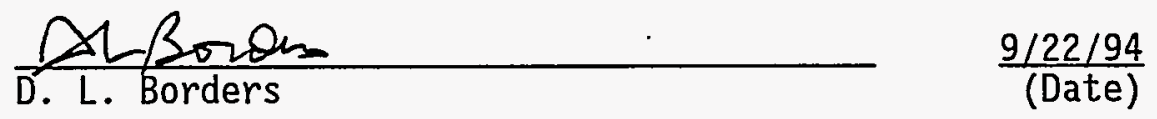




\section{Planning Integration Program}

Multi-Year Program Plan

\section{INTRODUCTION}

This Multi-Year Program P1an (MYPP) for the P1anning Integration Program, Work Breakdown Structure (WBS) Element 1.8.2, is the primary management tool to document the technical, schedule, and cost baseline for work directed by the U.S. Department of Energy (DOE), Richland Operations Office (RL). As an approved document, it establishes a binding agreement between $R L$ and the performing contractors for the work to be performed. It was prepared by the Westinghouse Hanford Company (WHC) and the Pacific Northwest Laboratory (PNL).

This MYPP provides a picture from fiscal year (FY) 1995 through FY 2001 for the Planning Integration Program. The MYPP provides a window of detailed information for the first three years. It also provides "execution year" work plans. The MYPP provides summary information for the next four years, documenting the same period as the Activity Data Sheets.

Guidance for the preparation of this MYPP was promulgated by RL in a June 3 , 1994, Tetter to contractors, "1995 Multi-Year Program Plan/Fiscal Year Work Plan - Program Plan Guidance." Because of the need to proceed with preparing the MYPP, WHC processed internal guidance in an Apri1 19, 1994, memo, "FY 1995 MYPP CaT1 Letter." The MYPP was generated based upon the integrated target case discussed in the current budget year's Activity Data Sheets.

Only that work scope identified, scheduled, and costed in this MYPP is authorized to be performed. Appropriate changes to the content of this MYPP document, excluding attachments, appendices, or other appurtenances, will be made through appropriate Class I change control. Levels for approving changes are defined in WHC-MCS-2-5, paragraph 4.1; WHC-CM-6-1, EP-2.2; RL-TPA-0900001 , TP-MG-02; and, RLP 5000.6. 
WHC-SP-1096

$09 / 23 / 94$

This page intentionally left blank. 
1.0 PROGRAM OVERVIEW ..................... . . . . . . .

1.A Program Mission and Vision .............. IA-1

1.A.1 Program Vision .............. . 1A-1

1.A.2 Program Mission ............... 1A-1

1.B Program Strategy and Mission ............ 1B-1

1.B.a Work Identification Strategy ........... . 1B-1

1.B.b Baseline Reporting and Systems Development Strategy . IB-2

1.B.c Program Baseline Integration Strategy ...... 1B-3

1.B.d Technical Baseline Development Strategy ....... . 1B-3

1.B.e PTanning Integration Program Management Strategy . . 1B-5

1.B.f Integrated Risk Assessment Strategy ........ 1B-5

1.B.g Public Involvement Strategy .......... . 1B-6

1.B.h Activity Data Sheet Preparation and Coordination

Strategy ............. . . 1B-6

1.B.1 Technical Functions and Requirements ...... 1B-9

1.B.2 Program Objectives .......... 1B-11

1.B.2.1 Work Identification Objectives .... 1B-12

1.B.2.2 Baseline Reporting and System Development

objectives .......... 1B-12

1.B.2.3 Program Baseline Integration objectives . . 1B-13

1.B.2.4 Technical Basel ine Development Objectives . 1B-13

1.B.2.5 Pianning Integration Program Management objectives ........... 1B-14

1.B.2.6 Integrated Risk Assessment Objectives . . 1B-14

1.B.2.7 Public Involvement Objectives ...... 1B-15

1.B.2.8 Activity Data Sheet Preparation and . . . . . 1B-15
Coordination Objectives ...

2.0 PROGRAM BASELINE ...................... 2-1

2.A Work Breakdown Structure Information ........ 2A-1

2.A.1 Work Breakdown Structure . . . . . . . . . 2A-2

2.A.2 Program Work Scope (WBS Dictionaries) . . . . . 2A-6

Planning Integration Program 1.8.2 ...... 2A-6

Work Identification $1.8 .2 .1 \ldots \ldots$ 2A-8

Strategic Quality PTanning 1.8.2.1.1 ..... 2A-10

Hanford Mission P7anning 1.8.2.1.2 . . . . . . 2A-12

Risk Management 1.8.2.1.3 . . . . . . . . 2A-14

Baseline Reporting and Systems Development 1.8.2.2 • 2A-16

Performance Reporting 1.8.2.2.1...... 2A-18

Business Systems Development 1.8.2.2.2 . . . . 2A-20

Management Direction 1.8.2.2.3 ... . . . 2A-22

Program Baseline Integration 1.8.2.3 .. . . . 2A-23

Program Baseline Development and Assessment 1.8.2.3.1 2A-24

Management Direction 1.8.2.3.2 . . . . . . . 2A-27

Technical Baseline Development 1.8.2.4 . . . . 2A-28

Systems Engineering Architecture 1.8.2.4.1 . . . 2A-30

Systems Engineering Management 1.8.2.4.2 ... 2A-32

Planning Integration Program Management 1.8.2.5 . . 2A-34

Management Direction 1.8.2.5.2 ....... 2A-37 
Integrated Risk Assessment Program 1.8.2.6 . . . 2A-38

Integrated Risk Assessment Program 1.8.2.6.1 . . . 2A-40

Public Involvement 1.8.2.7 ......... . 2A-42

Pub7 ic Involvement 1.8.2.7.1 .......... 2A-43

ADS Preparation and Coordination 1.8.2.8.... . 2A-44

ADS Preparation and Coordination 1.8.2.8.1 . . . 2A-46

Management Direction 1.8.2.8.2 . . . . . . . 2A-48

2.B Responsibility Assignments . . . . . . . . . . . $2 \mathrm{~B}-1$

2.C Program Logics . . . . . . . . . . . . . . . . . . . . 2C-1

2.D Program Master Basel ine Schedule ............ . 2D-1

2.E Program Performance Baseline Schedule . . . . . . . . . 2E-1

2.F Milestone List . . . . . . . . . . . . . . . . $2 \mathrm{~F}-1$

2.G Milestone Description Sheets . . . . . . . . . . . $2 \mathrm{G}-1$

2.H Program Cost Baseline . . . . . . . . . . . . . . . . $2 \mathrm{H}-1$

2.I Bases of Estimates . . . . . . . . . . . . . . . . 2I-1

2.I.1 Work Identification Basis of Estimate... . . . . . 2I-1

2.I.2 Baseline Reporting and System Development Basis of

Estimate ................ . 2I-1

2.I.3 Program Baseline Integration Basis of Estimate . . . 2I-1

2.I.4 Technical Baseline Development Basis of Estimate . . 2I-2

2.I.5 Planning Integration Program Management Basis of

Estimate .............. 2I-3

2.I.6 Integrated Risk Assessment Basis of Estimate . . . . 2I-3

2.I.7 Public Involvement Basis of Estimate . . . . . . . 2I-3

2.I.8 Activity Data Sheet Preparation and Coordination Basis of Estimate............. 2I-4

2.J Waste Type Data ... . . . . . . . . . . . . 2J-1

2.K Planned Staffing . . . . . . . . . . . . . . . . . $2 \mathrm{~K}-1$

2.L Building Blocks.................. $2 \mathrm{~L}-1$

3.0 FISCAL YEAR WORK PLAN . . . . . . . . . . . . . . . . . . . 3-1

3.A Cost Estimate Baseline by Month . . . . . . . . . . . . . 3A-1

3.B Program Funding ................. 3B-1

3.C Carry Over .................... $3 C-1$

3.D Cost Account Authorizations ............. 3D-1 


\section{List of Figures}

Figure 2.A.1-1. Figure 2.A.1-2. Figure 2.B-1. Figure 2.C-1.

Figure 2.C.1-1. Figure 2.C.2-1.

Figure 2.C.3-1. Figure 2.C.4-1. Figure 2.C.5-1. Figure 2.C.6-1. Figure 2.C.7-1. Figure 2.C.8-1.

Figure 2.D-1.

Figure 2.E-1.
Hanford Site Work Breakdown Structure ...... 2A-3 Planning Integration Work Breakdown Structure ... 2A-4 Responsibility Assignment Matrix . . . . . . 2B-2 Program Leve1 Logics, 1.8.2 . . . . . . . . 2C-2 Work Identification Logic Diagram, 1.8.2.1 . . . . 2C-3 Baseline Reporting and Systems Development Logic Diagram, 1.8.2.2 ........... 2C-5 Program Baseline Integration Logic Diagram, 1.8.2.3 . 2C-6 Technical Baseline Development Logic Diagram, 1.8.2.4 2C-7 Program Management Logic Diagram, 1.8.2.5 . . . . 2C-8 Integrated Risk Assessment Logic Diagram, 1.8.2.6 . . 2C-9 Pub7ic Involvement Logic Diagram, 1.8.2.7 .... . 2C-10 ADS Preparation and Coordination Logic Diagram, 1.8.2.8 ............. 2C-12 Program Master Baseline Schedule ........ 2D-2 Program Performance Baseline Schedule ...... 2E-3

List of Tables

Table 1.B.f-1. Table 2.F-1. Table 2.F-2. Table 2.F-3. Table 2. H-1. Table 2.K-1. Table 2.K-2. Table 3.A-1.
Overall Temporal Plan for Risk Assessment . . . . 1B-6 Program Element Designators . . . . . . . . . 2F-2 Milestone List by Due Date ... . . . . . . 2F-3 Milestone List by Milestone Control Number . . . . 2F-13 Cost Baseline Summary by Fiscal Year ....... 2H-2 Program Staffing Levels, WHC .......... 2K-1 Program Staffing Levels, PNL . . . . . . . . 2K-3 Cost Basel ine Summary by Month ......... 3A-1 
WHC-SP-1096

$09 / 23 / 94$

This page intentionally left blank.

$v i$ 
MULTI-YEAR PROGRAM PLAN

PLANNING INTEGRATION PROGRAM, WBS 1.8 .2

\section{$1.0 \quad$ PROGRAM OVERVIEW}

The Planning Integration Program provides sitewide $\mathrm{planning,} \mathrm{integration,}$ oversight, and analysis of the work scope being performed, or planned to be performed, across the Hanford Site. Part of the planning process is to develop and publish the site guidance to each of the performing programs across the site for developing planning documents such as the Activity Data Sheets and the Multi-Year Program Plans.

Planning Integration also provides oversight and primary customer interface for the Project Tracking System (PTS) and the Site Management System (SMS).

Planning Integration provides the resources necessary to conduct the sitewide strategic planning analyses and the systems engineering activities.

Risks are assessed and managed at a very high level across the site.

Planning Integration provides for its own public involvement required to support the above actions.

This activity provides the management interface with the DOE customer and with the contractor management for the program. Baseline development, management, and change control for this program are contained within the work scope.

The work scope contained within this program will continue at varying levels of resource requirements over the execution year plus six years.

The primary assumption is that funding will continue to be constrained. This will require a prioritization system be implemented to assign available resources to the work scope with the highest benefit in risk reduction, schedule optimization, and public acceptability. 
Planning Integration Multi-Year Program Plan

This page intentionally left blank. 


\section{A PROGRAM MISSION AND VISION}

\section{A.1 Program Vision}

- Activities on the Hanford Site are planned, analyzed, prioritized, organized, and effectively controlled and integrated according to system-engineered, risk-based program plans developed by the responsible line organizations.

\section{A.2 Program Mission}

The mission of the Planning Integration Program is to develop technically sound, publicly acceptable, and realistically achievable plans that when executed will enable all site programs to accomplish their missions. The P1anning Integration Program will prepare for and coordinate the preparation of integrated, system-engineered $\mathrm{plans}$ and efficient work-scope prioritization methods that consider health and environment risks and incorporate stakeholder values. This will result in the implementation of prioritized and measurable baselined $\mathrm{p} 7$ ans. 
WHC-SP-1096

$09 / 23 / 94$

Planning Integration Multi-Year Program Plan

This page intentionally left blank.

$1 A-2$ 
To accomplish the program mission, the Planning Integration Program will use the following overview strategies:

- The Hanford Site work scope will be completely defined using systems engineering and integrated $\mathrm{planning}$ techniques. Systems engineering principles will be used to completely define work scope assignment and interfaces. Program execution logic will be developed for site work.

- Baseline work scope and logic will be planned and scheduled over a multi-year period with structured estimating and resource-loaded schedules. Al1 Activity Data Sheet products will be produced from the programs MYPP/Fiscal Year Work PTans (FYWPS).

- Multi-year baselines will be developed and maintained. Execution of the plan will be reported so that progress against baseline, management corrective actions, and financial status can be assessed. Management of the program baselines will be maintained with rigorous change control processes.

- Analysis of the Hanford Site's program baselines will be conducted to validate that the site's limited resources are applied to the work with the highest value.

\section{B.a Work Identification Strategy}

The strategy for this program element includes providing support ( $p$ lanning, facilitation, and document compilation) to the Hanford Quality Council to prepare the Hanford Strategic P1an. Recommendations wil1 be made to ensure continuous process improvements. The strategy also includes the following.

Hanford's vision, mission, and goals will be developed by the Hanford Quality Council and pub7ished in the Hanford Strategic Plan. It will be the framework to align the technical baseline and the program baselines.

The Hanford Strategic Plan will be consistent with DOE-Headquarters (HQ) Environmental Management Strategic Plan.

The Hanford Mission Plan (HMP) Site Guidance will provide guidance to the programs preparing MYPPs. It will be driven by the Hanford Strategic Plan. It will contain the Site Planning Basis (decisions and assumptions) generated by Systems Engineering. The site Work Breakdown Structure (WBS) wil1 use the systems-engineered Cleanup Product Breakdown Structure as a part of the basis. Public values from the Future Site Uses Working Group and Tank Waste Task Force will be included in the Site Guidance. 
Planning Integration Multi-Year Program P1an

The HMP will be prepared by a multi-contractor team in conjunction with RL and programs.

A sitewide prioritization criteria will be published in the HMP Site Guidance to assure a consistent approach to resource allocation. The criteria will consider risk, stakeholder values, legal, and economic factors.

The Site Guidance will not only provide a summary of the systems-engineered, technical baseline, but it will direct program planning. to incorporate program systems-engineered, technical baselines into the MYPPS.

The Hanford Site program baselines and interfaces will be analyzed and assessed to identify key decision points, interface issues, inconsistencies, omissions, or overlaps.

Site budget priority 7 istings will be analyzed against risk factors, public values, and risk perceptions to provide input to decision makers. This will ensure a consistent approach and match resources to requirements.

The decision-making process wi17 be supported by introducing risk management techniques and facilitating compilation of risk data applicable to technical decisions (systems engineering) planning decisions (site and program baselines), the Hanford Federal Facility Agreement and Consent Order (Tri-Party Agreement) negotiations, etc.

The interfaces between site decision makers, program decision makers, and integrated risk assessment will be supported in order to provide a consistent framework for risk-based decision making and risk analysis.

The program baselines will be analyzed for opportunities to expedite the cleanup component of the mission.

A risk-based decision-making process will be implemented.

\section{B.b Baseline Reporting and Systems Development Strategy}

Performance towards the sitewide multi-year baselines will be reported so that progress against the baseline, management corrective actions, and financial status can be assessed. Reporting will be automated as appropriate and fed from a single source to maintain consistency.

Baseline management for the sitewide programs will be maintained through a rigorously documented change control process, which allows for tracking of change requests, maintains submittal and disposition statistics, and provides training as requested. 
Planning Integration MuTti-Year Program Plan

Planning systems will be developed which provide the capability to integrate schedule, cost, and report performance. Scope and schedules will drive budgets and performance, with status available to program managers as needed.

\section{B.c Program Baseline Integration Strategy}

Guidance will be issued to the direct and indirect funded programs providing site context and direction in the preparation of technical, schedule, and cost baselines. This strategy will provide a common direction (assumptions, interfaces, responsibilities, and strategic issues) for the programs to use in their baseline preparation and baseline maintenance.

The technical, schedule, and cost baselines will be independently reviewed on an ongoing basis. This review will assure the accuracy and consistency of the baselines to approved standards and criteria, and provide for sitewide baseline integration.

An additional strategy is the development and maintenance of the Hanford Site Integrated Schedule which provides a schedule, capable of identifying critical paths, integrated across the site's major work scope. This will assist management in the identification of adverse conditions in program interfaces and schedule conflicts.

\section{B.d Technical Baseline Development Strategy}

The technical baseline for the Hanford Cleanup mission will be established using formalized systems engineering methodology as required by RLPD 4900.1 Systems Engineering. The technical baseline developed in this element will be a primary input to program element·1.8.2.1.2 Hanford Mission Planning which provides technical content, priorities, and program scope to MYPP planning.

Systems engineering is a prescriptive process which defines the deliverables needed to complete the mission, as well as the facilities and infrastructure needed to produce the deliverables. The top level deliverables for the cleanup system include the identification of waste shipped offsite, waste disposed onsite, transferred resources, etc. In order to better define the top level deliverables, systems engineering also defines the sub-products which make up the top level deliverables. In turn, the sub-products are defined in terms of their component parts. The processes continue to develop a hierarchy of items which when delivered complete the system. Decomposition continues until each part of the deliverable set can be produced onsite, procured offsite, or provided by an onsite contractor.

The definition and development of the top level deliverables (products) is the responsibility of this program element. Definition of the Tower products is provided by the various programs/organizations involved in cleanup work. This 
Planning Integration Multi-Year Program Plan

program element is al so responsible for ensuring that the entire systems engineering process is conducted within the framework of a single integrated process which is completely continuous throughout the mission.

Development of a technical baseline using systems engineering is an iterative process. The first time through, rough definition of a deliverable hierarchy should be produced. After review by the system owners, technical staff, and stakeholders a second iteration should produce a significantly more detailed and better specified deliverable set. The improved set then should be optimized to ensure consistency, remove redundancies, eliminate omissions, and improve efficiencies. Because of the complexity of the cleanup system, optimization is a difficult process and can be perused only to a limited degree each year. The whole process needs to be repeated continuously to maintain a valid baseline in an evolving requirements environment.

The specific strategy to use systems engineering to develop the technical baseline is as follows:

- In the initial year (FY 1994), develop a systems engineering management plan (SEMP), Systems Engineering Manual (SEM), and associated implementing procedures, and conduct the first iteration of a 11 required systems engineering activities to produce an initial rough baseline.

- In the second Year (FY 1995) modify the SEM and SEMP to reflect field experience; revise the rough baseline to respond to reviewer comments and requirements changes; iterate (update) the entire SE process to refine baseline definition; and complete three optimization cases including $\mathrm{M}-33$, the 200 Area disposal complex, and a third case to be identified later. Upon the completion of the third case, a site specification will be developed which defines the baseline in better detail and reflects optimization of three high impact mission deliverables.

- In the third and fourth years the process will be repeated. It is expected that in this timeframe the baseline specification will achieve sufficient stability and quality that all components can be acquired.

- In subsequent years the system definition is expected to evolve slowly as new information or opportunities emerge, or as requirements change. During this period sitewide systems engineering activities are expected to decline to the level needed to validate lower systems work and to assure that the deliverables are integrated to complete the overall cleanup mission. 
Planning Integration Multi-Year Program P1an

\section{B.e Planning Integration Program Management Strategy}

The strategy for providing program management for the Planning Integration Program rests in the development and sustenance of a programmatic baseline. This baseline must specifically identify and document the scope, schedule, and budgets necessary to perform the assigned work. Once the baseline is emplaced, a rigorous management and change control process will be implemented. This strategy is founded on the principles that: a firm business relationship between the customer (RL) and the contractors (WHC and PNL) exists; that only the work identified in the MYPP is authorized; and, that only authorized work will be performed.

\section{B.f Integrated Risk Assessment Strategy}

The Integrated Risk Assessment Program (IRAP) develops and implements a methodology for producing Hanford sitewide risk assessments and associated uncertainties. These methods are applied to produce estimates of health and safety risks to the public, workers, and ecosystem for three time periods, each projected into the future: at the current time; during remediation; and, for the final site endstate. This risk analysis requires understanding temporal and spatial aspects of cleanup.

The approach for development of a broad understanding of Hanford Site risks, based on anticipated levels of funding, is given in Table 1. The initial development will require about five years. On a three- to five-year cycle, depending on needs, risk assessments will be updated to include new information. Results of risk assessments will be analyzed to understand areas in which additional information would reduce uncertainties in risk and clarify decision options. New results (when available) will be input to risk assessments to update them.

Specifically, risk results will be provided to various Hanford activities to allow Hanford sitewide planning and management to set priorities and allocate budgets across programs and to specify technology performance criteria in terms of risk reduction. Risk results will also be reformulated to meet other needs, such as making decisions about alternatives in developing a site-level technical baseline.

Risk results also support: communication to defend the site budget to Congress and to DOE-HQ; development of an integrated cleanup management and $\mathrm{planning}$ concept in collaboration with stakeholders; communication with the scientific community about knowledge gaps for cleanup planning and implementation (including for risk assessment); and, communication with DOE and regulators about a cleanup planning and management concept for Hanford, including communication about contaminants that are drivers of cleanup, risk reduction, and regulatory reform needed for safer and more cost effective cleanup. 


\begin{tabular}{|c|c|c|c|}
\hline Period & \multirow[t]{2}{*}{ Basel ine } & \multirow[t]{2}{*}{ Endstate } & \multirow[t]{2}{*}{ During Remediation } \\
\hline Work Accompl ished & & & \\
\hline FY 1995 & Public health (finish) & & \\
\hline FY 1996 & $\begin{array}{l}\text { Worker health and } \\
\text { safety }\end{array}$ & & \\
\hline FY 1997 & Ecological health & $\begin{array}{l}\text { Publ ic health (Tri - } \\
\text { Party Agreement) }\end{array}$ & $\begin{array}{l}\text { Worker heal th and } \\
\text { safety }\end{array}$ \\
\hline FY 1998 & & $\begin{array}{l}\text { Worker health and } \\
\text { safety (Tri-Party } \\
\text { Agreement) } \\
\text { Ecological health } \\
\text { (Tri-Party Agreement) }\end{array}$ & Publ ic heal th \\
\hline FY 1999 & $\begin{array}{l}\text { Update - public health } \\
\text { Update - worker heal th } \\
\text { and safety }\end{array}$ & & Ecological health \\
\hline FY 2000 & $\begin{array}{l}\text { Update - ecological } \\
\text { health }\end{array}$ & $\begin{array}{l}\text { Update - Public heal th } \\
\text { (Tri-Party Agreement) }\end{array}$ & \\
\hline
\end{tabular}

Table 1.B.f-1. Overall temporal plan for risk assessments to evaluate the public, worker, and ecological risks at the current time (baseline), during remediation, and in the final site endstate.

\section{B.g Public Involvement Strategy}

Public Involvement will compile the necessary public involvement needs from Program Elements. Activities to fulfill the public involvement requirements of the Hanford Federal Facility Agreement and Consent Order, Paragraphs 148 and 149 , and to fulfill the public involvement requirements associated with the recommendations of the Defense Nuclear Facility Safety Board will be incorporated into the program Public Involvement strategy. Public Involvement will facilitate an analysis of the sequential flow of the planning processes. Based on this flow, Public Involvement will help the program elements develop quarterly public involvement action plans for the planning processes. The implementation of an action plan will be followed by an evaluation to see how well action plan objectives were met. As the foundation processes are understood and accepted by the public, Indian nations, and interested parties, the subsequent action plans can focus on the next logical process(es).

\section{B.h Activity Data Sheet Preparation and Coordination Strategy}

Guidance wi11 be provided for the preparation and documentation of Activity Data Sheets to assure they are in accordance with approved criteria, have a 
Planning Integration Multi-Year Program Plan

clear and well defined basis, and are consistent across the site. The database (Resource Pricing Module) used for documenting the pricing of the cost estimates within the ADSs, will be maintained and validated. Ongoing interface with the programs and projects will provide a coordinated budget submittal for Hanford baseline planning and allocation of resources through a sitewide prioritization system.

Additionally, ongoing reviews will be performed and documented on ADS development to provide technical validation of the work scope and schedule as they relate to the identified funding estimates. 
Planning Integration Multi-Year Program Plan

This page intentionally left blank.

$1 B-8$ 


\section{B.I Technical Functions and Requirements}

To accomplish the vision and mission, the Planning Integration Program must meet these technical requirements:

- $\quad$ Requirement 1 - A disciplined process will be implemented that identifies and integrates the technical scope, schedule, and resource baselines of site programs in FY 1994 and incorporated in the FY 1995 MYPPs/FYWPs. (RL Site Management System Guidance, DOE-HQ Activity Data Sheets Guidance, Defense Authorization Act, OMB A-109, DOE Orders 4700.1 and 4700.5 ).

- $\quad$ Requirement 2 - Resources must be allocated and prioritized considering critical path schedules, risk, regulatory compliance, and performance. The initial application of critical path scheduling analysis will be during the first quarter of FY 1995 for the TWRS. (Tri-Party Agreement, paragraph 138.)

- Requirement 3 - Technical, schedule, risk, and resource baselines must consider input from stakeholders. Input will be received through various outreach initiatives and public interfaces. (DOE Activity Data Sheets DOE-HQ Guidance, DOE/EH 0231, Tri-Party Agreement, "Public Participation in Environmental Restoration Activities").

- $\quad$ Requirement 4 - A system must be implemented that measures performance against fully integrated baselines by the beginning of FY 1995 (RL Site Management System Guidance and Defense Authorization Act).

- Requirement 5 - Technical, schedule, and cost baselines must be independently reviewed to the approved standards and criteria. This must be an ongoing activity (EM-30 Cost and Schedule Estimating Guide).

- $\quad$ Requirement 6 - Budget requests must be based on approved technical, schedule, and cost baselines, and incorporate legal and regulatory compliance. This is an ongoing activity (Tri-Party Agreement, paragraph 149).

- $\quad$ Requirement 7 - Risks addressed by cleanup requirements must be estimated on the basis of best scientific evidence available and the risk placed within the context of other risks to which the public is exposed (H.R. 2445). 
WHC-SP-1096

$09 / 23 / 94$

Planning Integration Multi-Year Program Plan

This page intentionally left blank. 
Planning Integration MuTti-Year Program P1an

\section{B.2 Program Objectives}

To support the technical requirements of the Planning Integration Program, the key objectives that must be met are:

- Implement a structured planning process that builds from future site-use considerations, evaluates site-level alternatives using risk and systems engineering techniques, develops site-level decision criteria for alternative selection (including risk), selects a site-level planning baseline; and identifies and assigns total site work scope (including required technology), responsibilities, and interfaces for MYPP/FYWP development (Requirement 1).

- Implement a disciplined and structured systems engineering process for the site to completely identify work scope, interfaces, products, and performance measures as primary input to the site-level planning process and the development of technical scope for the program baselines reflected in the MYPP/FYWPS (Requirement 1).

- Coordinate the development of integrated program baselines, which will provide the ability to measure performance accurately and be maintained formally through change management (Requirement 1).

- Integrate the Hanford Site scheduling, critical path development, cost estimating, reporting, and financial data systems into a sitewide management systems network, to ensure that site resources are used effectively (Requirement 1).

- Develop the Activity Data Sheets as a byproduct of the MYPP/FYWP planning baseline (Requirement 1).

- Perform monthly analysis on the Site Integrated Schedule to determine areas requiring greater attention, to aid in prioritizing work and allocating resources (Requirements 1, 2, and 4).

- Develop risk information for the range of inventories on the Hanford Site in a form that can be used in the program prioritization and resource allocation process developed under WBS 1.8.2.1.3 and 1.8.2.6.1 (Requirements 2 and 7 ).

- Participation by the various stakeholder groups in defining the technical work scope to "clean up" the Hanford Site. Participation by the stakeholders in defining the end state, and developing alternatives, selection criteria, and the top-level alternative selection process. Understanding and acceptance by the stakeholders of the risks of the existing inventory and the risk implications of site-level alternatives (Requirements 3 and 7). 
Planning Integration Multi-Year Program Plan

- Implement a public-participation process that allows stakeholder input on Hanford planning and budgeting processes (Requirement 3 ).

- Review the Multi-Year Program Plans/Fiscal Year Work P1ans, and Activity Data Sheets to ensure that they reflect a 11 environmental compliance and safety activities. The Activity Data Sheets wi11 be used as the basis for the annual budget request (Requirement 5 \& 6 ).

- Develop a singTe database for the generation of both PTS and SMS reports (Requirement 4).

- Support RL and programs in: developing and validating cost baselines; enhancing Activity-Based Cost Estimating methods for validation of cost baselines; and, conducting independent reviews of technical, schedule and cost baselines (Requirements 1, 4, and 5).

\section{B.2.1 Work Identification Objectives}

The objectives for this program element are:

- Ensure the site contractors and programs share a common understanding of the Site mission, vision, goals, and planning basis (decisions and assumptions).

- Ensure continuity and traceability of Hanford work identification to the DOE-HQ Environmental Management Strategic Plan.

- Ensure site and programs plans are developed and implemented which are technically feasible, publicly acceptable, and realistically achievable.

- Support site-level decision makers to ensure that decisions are consistent, logical and defendable.

- Assure consideration of risk (health, safety, environmental, technical, and programmatic) by site decision makers.

1.B.2.2 Baseline Reporting and System Development Objectives

Baseline Reporting and System Development objectives are:

- Develop a single database for the generation of both PTS and SMS reports. 
- Submit an Executive Summary report which accurately depicts program consolidated performance at a level suitable for senior management, regulator, and stakeholder review.

- Maintain a change control process that provides the level of service appropriate to the needs of the programs, but one that can track each change package individually as well as maintain statistical performance trends for al1 programs.

- Integrate scheduling, cost estimating, performance reporting, and financial data systems into a sitewide management systems network.

\section{B.2.3 Program Baseline Integration Objectives}

Program Baseline Integration objectives will be met by:

- Providing specific guidance to the programs for preparation of technical, schedule, and cost baselines.

- Developing a Site Integrated Schedule which is supported by the Program Level I and II schedules.

- Performing technical reviews and analysis of Program Baseline Plans.

- Implementing supporting guidance (i.e., Activity-Based Cost estimating training, coordination and development workshops, procedure revision, training to updated procedures, etc.) to support this effort.

\section{B.2.4 Technical Baseline Development Objectives}

The key objectives that must be met to support this program element are:

- Implement systems engineering processes to develop the technical baseline which meets the requirements of RLPD 4900.1 , Systems Engineering.

- Develop the manuals, procedures, and training materials needed to conduct an effective systems engineering effort throughout the cleanup. system.

- Define the top level deliverables which, when complete, fulfill all cleanup systems requirements. This top level definition will provide the framework and coordination needed to integrate al1 lower level mission deliverables defined by the participating programs or organizations. 
Planning Integration

Multi-Year Program Plan

- Optimize the primary technical systems architectures selected to deliver the mission.

- Support technical review of al1 systems engineering outputs by the appropriate authority, including Defense Nuclear Facility Safety Board, National Academy of Sciences, stakeholder panels, RL and DOE-HQ.

\section{B.2.5 Planning Integration Program Management Objectives}

The objectives of $\mathrm{Planning}$ Integration Program Management are:

- Generate timely and accurate inputs for the ADS 7250 submittal.

- Develop the MYPP/FYWP to establish a credible technical, schedule, and cost baseline.

- Generate accurate and timely monthly inputs for the SMS and PTS reports.

- Conduct performance analyses of a11 program elements within the 1.8.2 program.

- Coordinate all the efforts within the 1.8 .2 program.

- Assure timely and disciplined control of the approved technical, schedule, and cost baseline through a rigorous change control process.

\section{B.2.6 Integrated Risk Assessment Objectives}

The goal of IRAP is to develop technically sound health and ecological risk assessment input to support making risk-based decisions about environmental restoration and waste management at Hanford. The following are IRAP objectives:

- Coordinate the risk management/risk-based decision making task with 1.8.2 program element activities including Systems Engineering, Stakeholder Involvement, and Strategic Planning.

- Coordinate with risk assessment activities being conducted in programs at Hanford to ensure (as much as possible) consistency in data, assumptions, and methods.

- Coordinate with various Hanford programs to assure consistency of results, or to understand reasons for a lack of consistency, and enable use of programmatic results by IRAP, as feasible. 
- Interface with DOE complex-wide risk assessment activities, both to use information produced by complex-wide programs and to provide risk results from the Hanford Site.

- Involve stakeholders, including the various publics, the Indian nations, regulators, funding organizations, scientific communities, contractors, $\mathrm{DOE}$, and workers.

- Develop a technical review panel to provide technical guidance and review on methods and priorities.

\section{B.2.7 Public Involvement Objectives}

The Public Involvement objectives are:

- Ensure that Hanford's planning process is open to the full view and input of those it serves, consistent with applicable laws, regulations, and contracts.

- Establish and maintain the credibility of Hanford's planning process with interested stakeholders.

\section{B.2.8 Activity Data Sheet Preparation and Coordination Objectives}

The objectives associated with ADS Preparation and Coordination include the technical assessment and analysis of ADSs to ensure compliance with standards and guidance provided by DOE. They are:

- Ensure consistency and integration of the budget submittal for Hanford baseline planning and allocation of resources.

- Assure consistency among programs, as well as between the ADSs and the Program Baseline Plans.

- Maintain the Resource Pricing Module to assure consistent and accurate pricing to support the budget submittal. 
WHC-SP-1096

$09 / 23 / 94$

Planning Integration Multi-Year Program Plan

This page intentionally left blank.

1B-16 
Planning Integration Multi-Year Program Plan

\section{$2.0 \quad$ PROGRAM BASELINE}

The program baseline contains the definition of the work scope to be accomplished, the schedule by which that work scope will be accomplished, and the costs for accomplishing that work scope within the established schedule. The baseline is maintained through a rigorous change control process.

The program baseline consists of work scope, schedule, and cost data necessary to establish a baseline for the program against which the program's performance can and will be measured. The data includes the program WBS, from the program's relationship to the site WBS, as well as details to the cost account leve1. The data also includes the responsibility assignment matrix to the cost account leve1; the WBS Dictionaries for the program to the activity leve1; program and program element logic diagrams; the program master baseline scheduTe; the program performance baseline schedule; cost summary data for the seven-year period (execution year plus six years); a summary of the bases of estimates for these costs; the planned staffing by job category; and, building blocks identifying work scope priorities.

This data forms the Planning Integration Program multi-year baseline through FY 2001, and will be used as the basis for the budget formulation process, as contained within the Activity Data Sheets. 
Planning Integration Multi-Year Program Plan

This page intentionally left blank. 
Planning Integration Multi-Year Program Plan

\section{A WORK BREAKDOWN STRUCTURE INFORMATION}

This section includes the following data:

1. The Hanford Site WBS

2. The Planning Integration Program WBS

3. The Planning Integration Program work scope definition as contained within the WBS Dictionaries

These data define the program's technical work scope through the use of the WBS Dictionaries, and decomposes and delegates this work scope to the accountable program element, which is further decomposed and delegated to the . activity and cost account. 
WHC-SP-1096

$09 / 23 / 94$

Planning Integration

Multi-Year Program Plan

This page intentionally left blank.

$2 A-1 A$ 


\section{A.1 Work Breakdown Structure}

All activities performed at the Hanford Site are described in the Hanford Site WBS. Refer to figure 2.A.1-1 for the Hanford Site WBS. Within the Hanford Site WBS is "EM-30, Waste Management," which is element 1.0 of the Hanford Site WBS. This element is further decomposed to elements such as: Tank Waste Remediation (1.1), Solid/Liquid Waste and Decontamination Services (1.2), and Program Management (1.8).

Program Management (1.8) contains two programs.

- RL Direction and Grant Management Program (1.8.1)

- Planning Integration Program (1.8.2)

The focus of this MYPP is on PIanning Integration Program (1.8.2). Figure 2.A.1-1 provides the relationship of the Planning Integration Program to other programs on the Site.

The Planning Integration Program WBS is depicted in figure 2.A.1-2. This figure identifies the decomposition of the work scope assigned to the Planning Integration .Program from the program level, through to the program elements, activities, and cost account levels. Details of those work scope assignments are found in the following section titled, "Program Work Scope," which contains al7 the WBS Dictionaries within the Planning Integration program. 


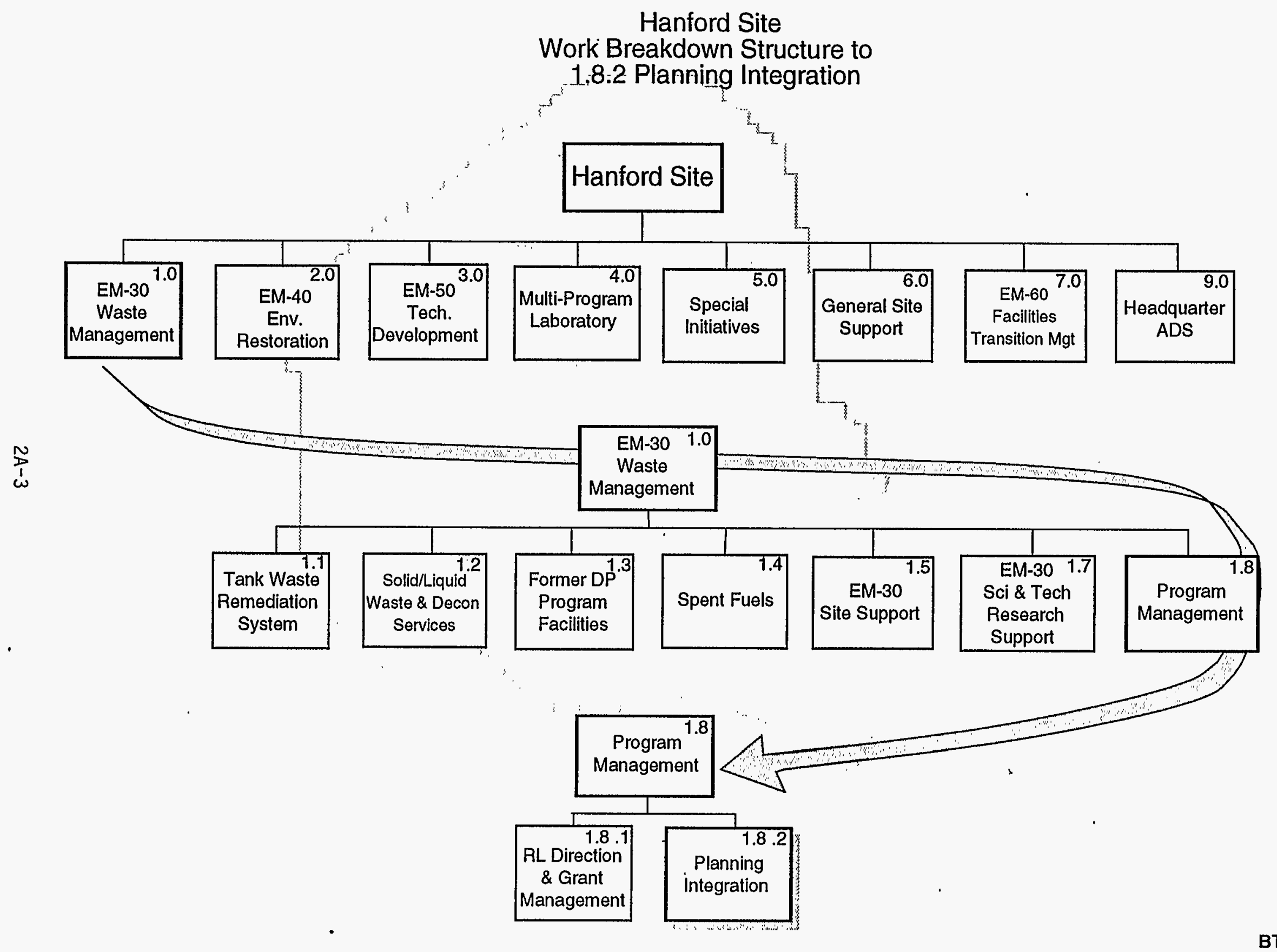




\section{FY 1995 MYPP/FYWP PLANNING INTEGRATION PROGRAM \\ 1.8.2}

2.A.1 Work Breakdown Structure

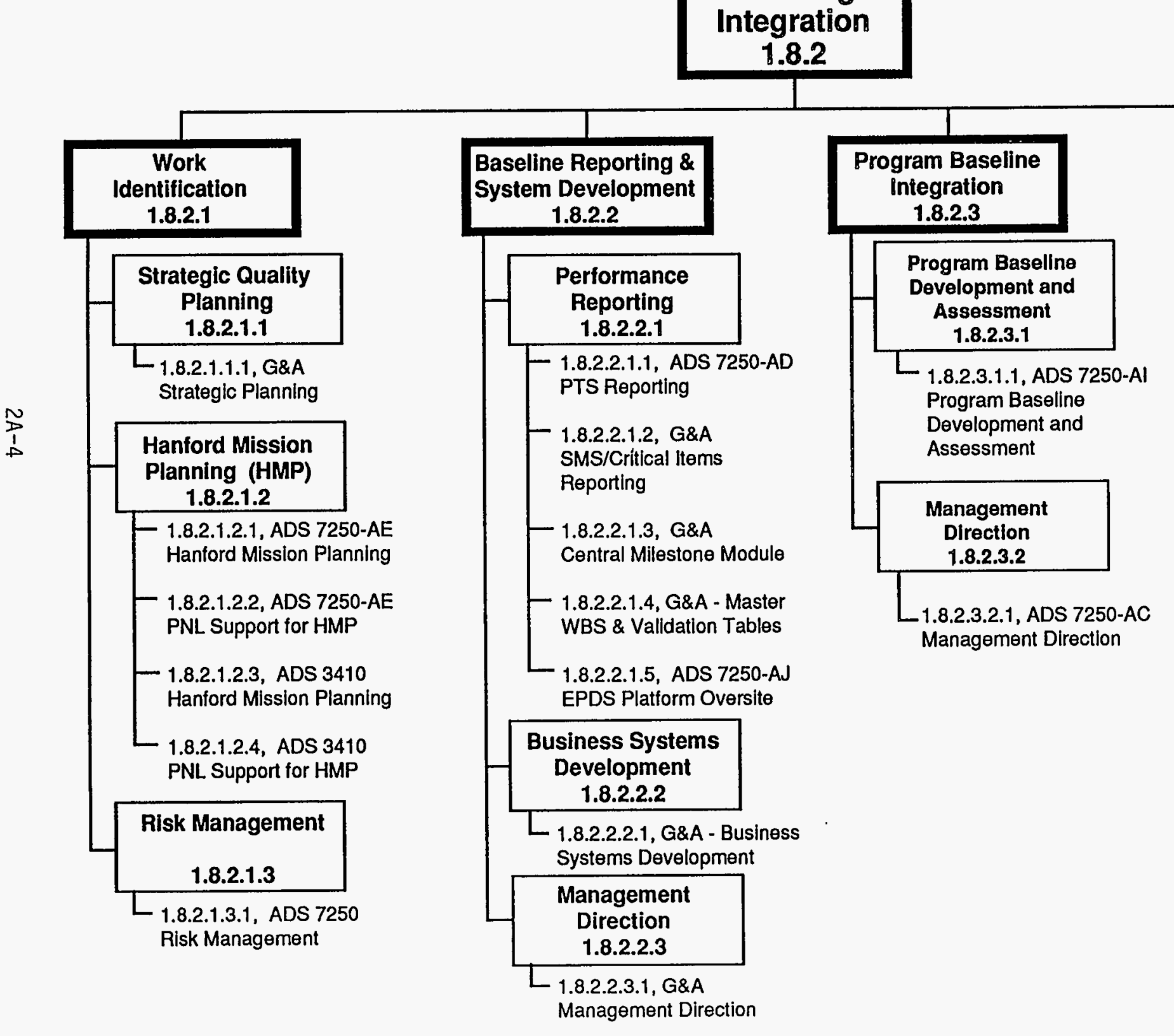

Technical Baseline

Development

1.8.2.4

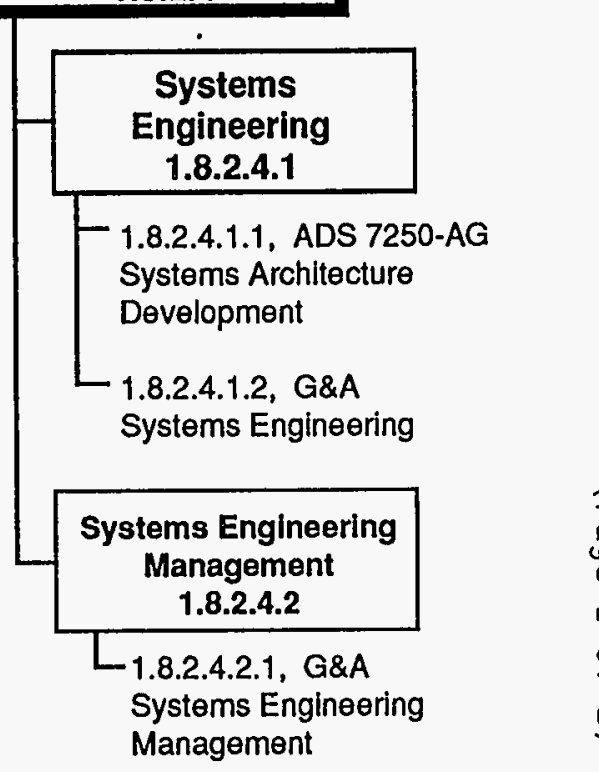

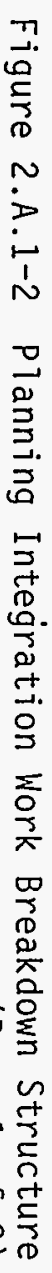

Business Systems

evelopment

1.82221 G\&A - Business Systems Development 1.8.2.2.3

Management Direction 


\section{FY 1995 MYPP/FYWP \\ PLANNING INTEGRATION PROGRAM}

\section{8 .2}
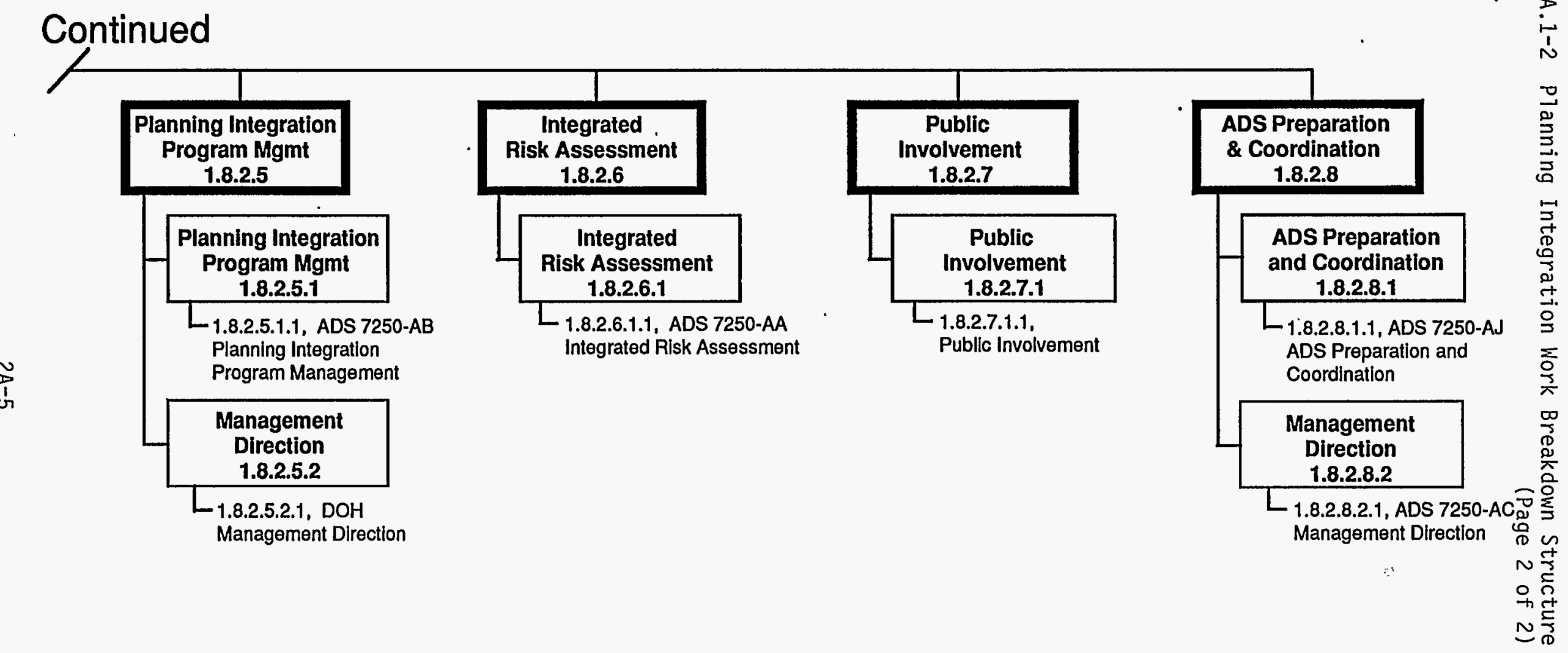
WHC-SP-1096

$09 / 23 / 94$

Planning Integration Multi-Year Program Plan

This page intentionally left blank. 


\title{
PLANNING INTEGRATION PROGRAM
}

\author{
1.8 .2
}

\section{A.2. WBS Dictionary.}

WORK BREAKDOWN STRUCTURE DICTIONARY

\begin{tabular}{|c|c|c|c|}
\hline $\begin{array}{l}1 \text { PROJECT TITLE/PARTICIPANT } \\
\text { Planning Integration Program }\end{array}$ & & $\begin{array}{l}2 \text { DATE } \\
08 / 31 / 94\end{array}$ & $\begin{array}{l}3 \text { IDENTIFICATION NO. } \\
\text { N/A }\end{array}$ \\
\hline $\begin{array}{l}4 \text { WBS ELEMENT CODE } \\
1.8 .2\end{array}$ & & $\begin{array}{l}5 \text { WBS ELEMENT TITLE } \\
\text { Planning Integratio }\end{array}$ & \\
\hline $\begin{array}{l}6 \text { INDEX LINE NO. } \\
\text { N/A }\end{array}$ & $\begin{array}{l}7 \text { REVISION N } \\
\text { ReV } 0\end{array}$ & AND AUTHORIZATION & $\begin{array}{l}8 \text { DATE } \\
08 / 31 / 94\end{array}$ \\
\hline
\end{tabular}

9 APPROVED CHANGES

None

\begin{tabular}{|l|l}
\hline $\begin{array}{l}10 \text { SYSTEM DESIGN DESCRIPTION } \\
\text { N/A }\end{array}$ & 11 BUDGET AND REPORTING NUMBER \\
EW3110010/EW2010700/G\&A
\end{tabular}

12 ELEMENT TASK DESCRIPTION

A. COST CONTENT

Labor

Materials

Purchased Services

Internal Services

IRM

\section{B. TECHNICAL CONTENT/WORK STATEMENT}

The Planning Integration Program provides sitewide planning, integration, oversite, and analysis of the work scope being performed, or planned to be performed, across the Hanford site. Part of the planning process is to develop and publish the site guidance to each of the performing programs across the site for developing planning documents such as the Activity Data Sheets and the Multi-Year Program Plans.

- continued- 


\section{B. TECHNICAL CONTENT/WORK STATEMENT (continued)}

Planning Integration also provides oversite and primary customer interface for the Project Tracking System, and the Site Management System.

Planning Integration provides the resources necessary to conduct the site wide strategic planning analyses and the systems engineering activities.

Risks are assessed and managed at a very high level across the site.

Planning Integration provides for $i$ ts own public involvement required to support the above actions.

This activity provides the management interface with the DOE customer and with the contractor management. Baseline development, management, and change control for this program is contained within the workscope.

The workscope contained within this activity is described in greater detail in the subordinate program elements:

1.8.2.1 Work Definition

1.8.2.2 Performance Reporting

1.8.2.3 Program Baseline Integration

1.8.2.4 Technical Baseline Development

1.8.2.5 Planning Integration Program Management

1.8.2.6 Integrated Risk Assessment

1.8.2.7 Public Involvement

1.8.2.8 ADS Preparation and Coordination

This workscope contained within this program will continue at varying levels of resource consumption over the Life of activity at the Hanford site. 


\section{A.2. WBS Dictionary}

WORK BREAKDOWN STRUCTURE DICTIONARY

\begin{tabular}{|l|l|l|}
\hline $\begin{array}{l}1 \text { PROJECT TITLE/PARTICIPANT } \\
\text { Planning Integration Program }\end{array}$ & $\begin{array}{l}2 \text { DATE } \\
08 / 31 / 94\end{array}$ & $\begin{array}{l}3 \text { IDENTIFICATION NO. } \\
\text { N/A }\end{array}$ \\
\hline 4 WBS ELEMENT CODE & $\begin{array}{l}5 \text { WBS ELEMENT TITLE } \\
\text { Work Identification }\end{array}$ \\
\hline 6 INDEX LINE NO. & $\begin{array}{l}7 \text { REVISION NO AND AUTHORIZATION } \\
\text { N/A }\end{array}$ & $\begin{array}{l}8 \text { DATE } \\
08 / 31 / 94\end{array}$ \\
\hline
\end{tabular}

9 APPROVED CHANGES

None

10 SYSTEM DESIGN DESCRIPTION

N/A
11 BUDGET AND REPORTING NUMBER

EW3110010/EW2010700/G\&A

12 ELEMENT TASK DESCRIPTION

A. COST CONTENT

Labor

Materials

Purchased Services

Internal Services

IRM

B. TECHNICAL CONTENT/WORK STATEMENT

Work Identification results in the broad definition of the site's long range work scope, framework and site-level risk management. It is the initial phase of the planning cycle defined in RLPD 5000.1,

RLID 5000.2 and RLID 5000.12. The annual cycle will continue until the mission cannot be changed,

long range planning decisions are complete, the framework is stable, strategic issues are resolved and only program level risk decisions remain as determined by the Hanford Quality Council (HQC).

The program elements associated with Strategic Planning are:

1.8.2.1.1 Strategic Quality Planning

1.8.2.1.2 Hanford Mission Planning

1.8.2.1.3 Risk Management

-continued- 


\section{WORK IDENTIFICATION}

\section{B. TECHNICAL CONTENT/WORK STATEMENT (continued)}

The primary products are the Hanford Strategic Plan (HSP), the Hanford Mission Plan (Site Guidance and Program Integration) and the development and implementation of site-level risk management (risk-based decision making). These primary products are supported by additional studies (material balances, cost, schedule) and analysis of program baselines.

The work identification interfaces include linkage of the HSP to the Environmental. Management Strategic Plan. Work identification uses the Systems Engineering technical baseline in the Hanford Mission Plan and formulation guidance to programs. Work identification assists systems engineering in the development of the site technical baseline (guidance, analysis and integration). Work identification analyzes individual program MYPPS for consistency and integration of the program basel ine assumptions, technical logic, waste data, regulatory drivers, decision points and priorities. The analys is will assist determination that the basel ine is realistic. Lastly, information requirements will be coordinated with Public Involvement to assure exchanges with the Hanford Advisory Board, public, and stakeholders on the mission, priorities, and risk.

Assumptions that guide this Program Element are:

The U.S. Department of Energy, Richland Operations Office (RL), Planning and Integration Division (PID) will direct WHC to continue support to the HQC and the preparation of the annual strategic plan.

RL-PID will direct Westinghouse Hanford Company (WHC) to continue preparation of the annual update of the Hanford Mission Plan "Si te Guidance."

Multi-contractor team effort will continue to be employed to prepare the Hanford Mission Plan products.

Preparation of the Hanford Mission Plan "Integration Analysis" is dependent on funding and resources. 


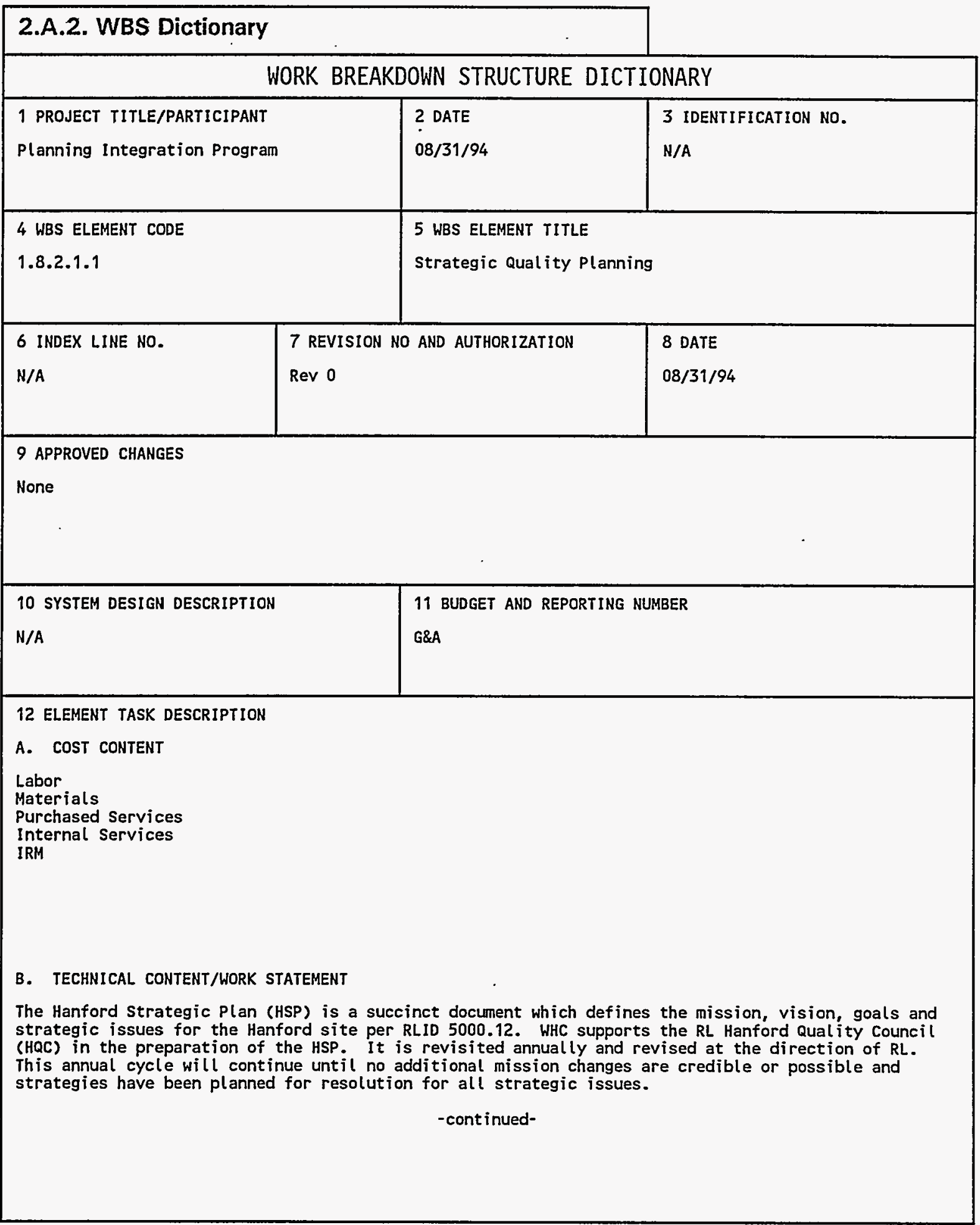




\section{STRATEGIC QUALITY PLANNING}

B. TECHNICAL CONTENT/WORK STATEMENT (continued)

The HSP is support to DOE-HQ strategic planning and drives the long range planning process at the Hanford Site. It is linked to the U.S. Department of Energy Strategic Plan and the Environmental Management

Strategic Plan. The primary product of this program activity is the annual completion of the HSP update.

The completed document will be distributed by this program element. The HSP is an input to the Hanford

Mission P(an Site Guidance (HMP) as well as the Systems Engineering Planning Basis for Multi-Year Program Plans (MYPPS). Another key part of this effort is the planning and facilitation of the ongoing Hanford Quality Council (HQC) meetings. This includes guidance and facilitation of sitewide strategy and goal teams.

Key assumptions for this activity are:

RL-PID will direct WHC to continue support the HQC and the annual preparation of the strategic plan. The HQC and its teams will develop the materials compiled by WHC in the Hanford Strategic Plan.

G\&A funding will be available for the Hanford Quality Council support. The HQC and its teams will be funded by other sources. 


\section{A.2. WBS Dictionary}

WORK BREAKDOWN STRUCTURE DICTIONARY

\begin{tabular}{|c|c|c|}
\hline $\begin{array}{l}1 \text { PROJECT TITLE/PARTICIPANT } \\
\text { Planning Integration Program }\end{array}$ & $\begin{array}{l}2 \text { DATE } \\
08 / 31 / 94\end{array}$ & $\begin{array}{l}3 \text { IDENTIFICATION NO. } \\
\text { N/A }\end{array}$ \\
\hline $\begin{array}{l}4 \text { WBS ELEMENT CODE } \\
1.8 .2 .1 .2\end{array}$ & $\begin{array}{l}5 \text { WBS ELEMENT TITLE } \\
\text { Hanford Mission Planning }\end{array}$ & \\
\hline $\begin{array}{l}6 \text { INDEX LINE NO. } \\
\text { N/A }\end{array}$ & $\begin{array}{l}7 \text { REVISION NO AND AUTHORIZATION } \\
\text { ReV } 0\end{array}$ & $\begin{array}{l}8 \text { DATE } \\
08 / 31 / 94\end{array}$ \\
\hline
\end{tabular}

9 APPROVED CHANGES

None

\begin{tabular}{|l|l|}
\hline $\begin{array}{l}10 \text { SYSTEM DESIGN DESCRIPTION } \\
\text { N/A }\end{array}$ & $\begin{array}{l}11 \text { BUDGET AND REPORTING NUMBER } \\
\text { EW3110010/EW2010700 }\end{array}$ \\
\hline
\end{tabular}

12 ELEMENT TASK DESCRIPTION

A. COST CONTENT

Labor

Materials

Purchased Services

Internal Services

B. TECHNICAL CONTENT/WORK STATEMENT

The Hanford Mission Plan (HMP) is a succinct document that provides sitewide program guidance integration and baseline documentation per RLID 5000.2 and RLID 5000.12. The HMP is revised annually according to input from site program elements, the DOE, stakeholders and the public. The HMP is driven by the Hanford Strategic Plan (HSP) and is developed using a Systems Engineering approach. It consists of two major pieces - site guidance and integration analysis. Site guidance provides a comprehensive definition of the total site mission as outlined in the HSP, including a WBS which defines the essential site program elements from which multi-year program planning evolves. The integration analysis (currently not funded for FY1995) is a repository for Multi-Year Program Plan (MYPP) generated data such as technical logic, waste data, technology, regulatory drivers, decision points and risk comparison. The annual cycle will continue until the last baseline planning cycle. 


\section{HANFORD MISSION PLANNING}

\section{TECHNICAL CONTENT/WORK STATEMENT (continued)}

Hanford mission planning provides support to RL in determining an integrated sitewide cleanup strategy which encompasses the disposition of material inventories, facilities and land. The HMP provides guidance to site programs that prepare their respective MYPPs. Hanford mission planning provides a link from the technical baseline to program planning. The site WBS is designed from a Systems Engineering (SE) Product Breakdown Structure (PBS). In parallel, the site level OBS is developed. Hanford Mission Planning supports the development of the SE technical basel ine update and optimization by providing the Hanford Strategic Analysis (HSA) material balances, ROM Schedule and cost estimates. The SE technical baseline and planning basis will be summarized in the FY1996 HMP site Guidance. Priority criteria and a high level priority list are maintained working with Risk Management and the Hanford Quality Council. Public values from the Future Site Use Working Group and the Tank Waste Task Force will be summarized in the HMP Site Guidance. Additional public values from formal committees, such as the Hanford Advisory Board, will be entered to update these two references.

Key assumptions for this activity are:

Initial Systems Engineering Technical Baseline will be issued in FY 1994.

The linkage of the Systems Engineering Technical Baseline with the program baselines will be the Hanford Mission Plan "Site Guidance."

Systems Engineering is the site technical decision process.

Hanford Mission Planning funding will be from both the EM-30 and EM-40. ADS 7250 will provide 80 percent of funding and ADS 3410 will provide the remaining 20 percent. 


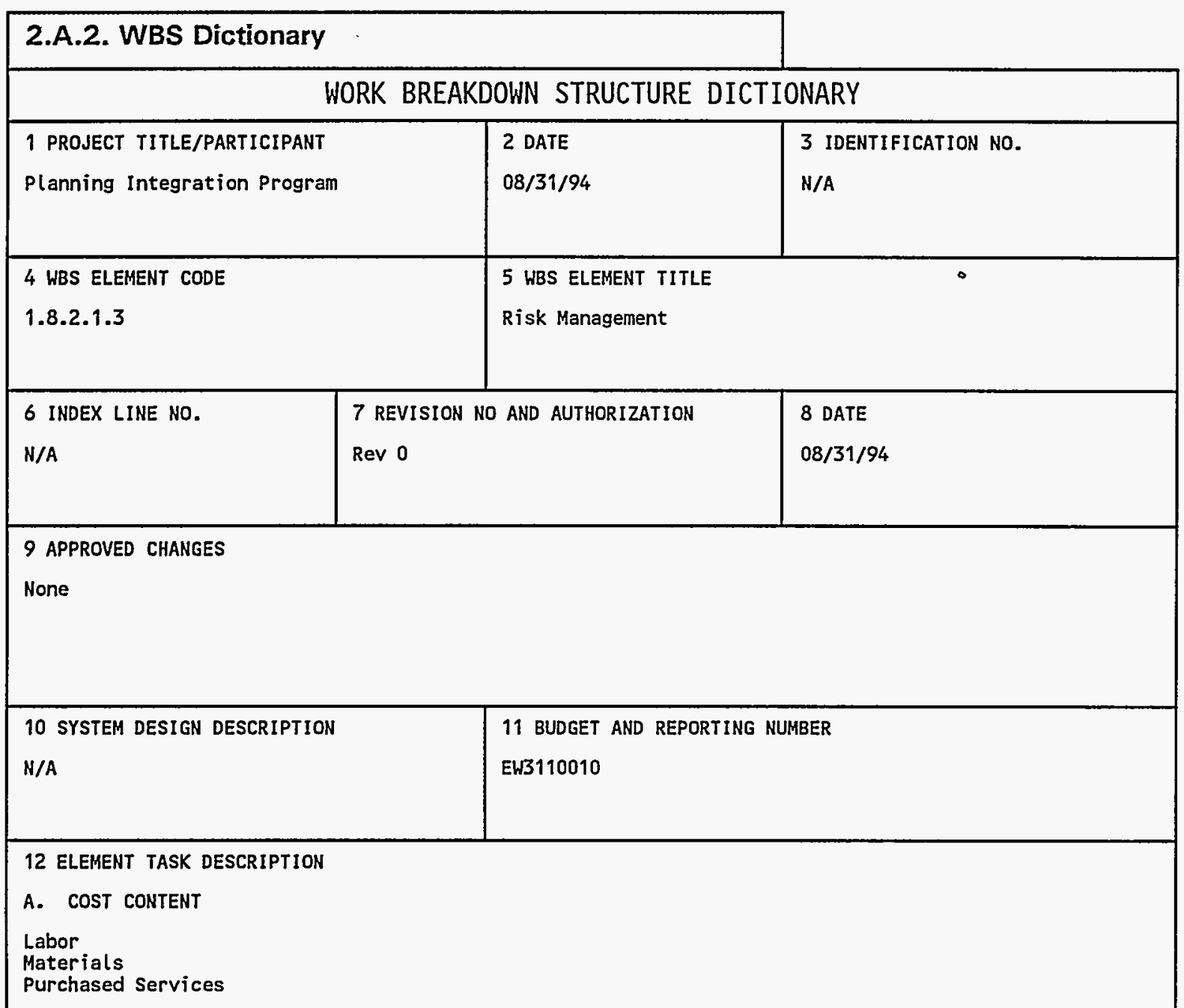

\section{B. TECHNICAL CONTENT/WORK STATEMENT}

The overall purpose of risk management at Hanford is to eliminate or reduce risks to the public, workers, and the environment associated with the storage, treatment, and disposal of hazardous and radiological waste. Hanford managers have been directed to allocate its resources to those activities which address the greatest risks and which provide the greatest risk reduction. To do so, Hanford will implement a Risk Management System at all levels of the Site. The overall goal of the risk management task is the design, implementation, and assessment of this System and the incorporation of risk-based decision making at all levels of management.

-cont inued- 
B. TECHNICAL CONTENT/WORK STATEMENT (continued)

It is assumed that this project will continue for the next several years to support resource allocation and decision making, although the form and content of the project will evolve over time. Initially, efforts will concentrate on determining, in cooperation with the Integrated Risk Assessment Program, a site-wide picture of risk, which can be made available to decision makers and in implementing risk-based decision processes. Risk-based decision making includes public values and concerns, so analysts will ensure perceived risk, as well as hazard, is included in decision making.

Annually, analysts will perform cost-risk-benefit analyses of the Hanford technical program and will advise managers on resource allocation in accordance with priority criteria established by those managers.

Prioritization methodology tools will be developed, implemented, and improved over the next several years as transition to the cleanup mission is completed. Other missions of science and technology and economic diversity will be included, as well.

Key interfaces of risk management are:

- Systems engineering--for technical alternatives and assumptions

- Mission Planning--for prioritization criteria and site guidance

Integrated Risk Assessment--for ES\&H risk information and focus

- Programs--for program risk information

- Public Involvement--for stakeholder involvement in decision making

Since resource allocation and decision making continue indefinitely, risk management is considered to be an ongoing task that diminishes over the next several years as risk-based decision making becomes the norm and prioritization tools are solidified.

Key deliverables are:

- In FY 1995: implementation of prioritization methods Cost-risk-benefit analys is

Public risk perceptions

RL Risk Management Procedure

Si tewide risk activities

- In FY 1996 and beyond, annual reports on:

Analysis of risks and costs at Hanford

Application of risk-based decision making at Hanford 


\section{A.2. WBS Dictionary}

WORK BREAKDOWN STRUCTURE DICTIONARY

\begin{tabular}{|c|c|c|c|}
\hline \multicolumn{2}{|l|}{$\begin{array}{l}1 \text { PROJECT TITLE/PARTICIPANT } \\
\text { Planning Integration Program }\end{array}$} & $\begin{array}{l}2 \text { DATE } \\
08 / 31 / 94\end{array}$ & $\begin{array}{l}3 \text { IDENTIFICATION NO. } \\
\text { N/A }\end{array}$ \\
\hline $\begin{array}{l}4 \text { WBS ELEMENT CODE } \\
1.8 .2 .2\end{array}$ & & \multicolumn{2}{|c|}{$\begin{array}{l}5 \text { WBS ELEMENT TITLE } \\
\text { Basel ine Reporting and Systems Development }\end{array}$} \\
\hline $\begin{array}{l}6 \text { INDEX LINE NO. } \\
\text { N/A }\end{array}$ & \multicolumn{2}{|c|}{$\begin{array}{l}7 \text { REVISION NO AND AUTHORIZATION } \\
\text { REV } 0\end{array}$} & $\begin{array}{l}8 \text { DATE } \\
08 / 31 / 94\end{array}$ \\
\hline \multicolumn{4}{|l|}{9 APPROVED CHANGES } \\
\hline $\begin{array}{l}10 \text { SYSTEM DESIGN DESCRIPTION } \\
\text { N/A }\end{array}$ & & $\begin{array}{l}11 \text { BUDGET AND REPOF } \\
\text { EW3110010/G\&A }\end{array}$ & MBER \\
\hline
\end{tabular}

12 ELEMENT TASK DESCRIPTION

A. COST CONTENT

Labor

Materials

Purchased Services

Internal Services

IRM

\section{B. TECHNICAL CONTENT/WORK STATEMENT}

The Baseline Reporting and Systems Development program element provides the infrastructure for performance reporting, including coordination (sitewide) of the Site Management System (SMS) and Progress Tracking System (PTS) reports; program systems development, implementation, and training; WHC centralized change control processing; and company project management procedure development, including training and surveillance activity to those procedures.

Requirements include integrating a standard scheduling system to allow the production of resourceloaded schedules by job category and provide integration of cost (the effort to produce this schedule and cost data is not included in the scope of this WBS). Ensure that the proper training in basic scheduling, logic/resource-loaded schedules, and standard scheduling software is available for the site.

The PTS activity involves integration and implementation of the DOE-HQ mandated reporting requirements required across the DOE complex.

The SMS activity involves the coordination and compilation of the individual SMS program reports and summarization of this data into a SMS Executive Summary report, as mandated by RL.

Also included in this program element is the Central Milestone Module, which is a database that houses all RL, DOE-HQ, Hanford Federal Facility Agreement and Consent Order, and Defense Nuclear Facility Safety Board milestones for the Hanford site. Additionally, oversight of the Site WBS, EPDS Platform, and client server development for 1.8 .2 activities is provided. Coordination of the Critical Items Report is performed within this program element. -cont inued- 


\section{BASELINE REPORTING AND SYSTEMS DEVELOPMENT}

B. TECHNICAL CONTENT/WORK STATEMENT (continued)

Key interfaces include all WHC and some PNL/BHI programs, the RL Planning Integration Division, and selected stakeholders as needed.

This program element continues as long as work is planned, performed, and reported on at Hanford.

C. KEY ASSUMPTIONS

It is assumed that SMS and PTS reporting continue with no significant development required to facilitate additional DOE-HQ or RL reporting. Additionally, the scope of work included in this program element assumes continued adherence to RL project management directives and related good management practices. Also, some level of scheduling support will be provided to the other programs. 


\section{A.2. WBS Dictionary}

WORK BREAKDOWN STRUCTURE DICTIONARY

\begin{tabular}{|c|c|c|c|}
\hline $\begin{array}{l}1 \text { PROJECT TITLE/PARTICIPANT } \\
\text { Planning Integration Program }\end{array}$ & & $\begin{array}{l}2 \text { DATE } \\
08 / 31 / 94\end{array}$ & $\begin{array}{l}3 \text { IDENTIFICATION NO. } \\
\text { N/A }\end{array}$ \\
\hline $\begin{array}{l}4 \text { WBS ELEMENT CODE } \\
1.8 .2 .2 .1\end{array}$ & & $\begin{array}{l}5 \text { WBS ELEMENT TITLE } \\
\text { Performance Reporting }\end{array}$ & \\
\hline $\begin{array}{l}6 \text { INDEX LINE NO. } \\
\text { N/A }\end{array}$ & $\begin{array}{l}7 \text { REVISION NO } \\
\text { ReV } 0\end{array}$ & AND AUTHORIZATION & $\begin{array}{l}8 \text { DATE } \\
08 / 31 / 94\end{array}$ \\
\hline \multicolumn{4}{|l|}{$\begin{array}{l}9 \text { APPROVED CHANGES } \\
\text { None }\end{array}$} \\
\hline $\begin{array}{l}10 \text { SYSTEM DESIGN DESCRIPTION } \\
\text { N/A }\end{array}$ & & $\begin{array}{l}11 \text { BUDGET AND REPORTIN } \\
\text { EW3110010/G\&A }\end{array}$ & MBER \\
\hline
\end{tabular}

12 ELEMENT TASK DESCRIPTION

A. COST CONTENT

Labor

Materials

Purchased Services

Internal Services

IRM

\section{B. TECHNICAL CONTENT/WORK STATEMENT}

Performance Reporting consists primarily of three reports, the Progress Tracking System (PTS), the Site Management System (SMS) and the Critical Items Report. Also included are the Central Milestone Module (CMM); the Master WBS Dictionary; oversight of the Environmental Planning Database System (EPDS); development and implementation of the client server platform; and participation in the computer steering group.

The PTS activity involves integration and implementation of the U.S. Department of Energy-Headquarters (DOE-HQ) mandated PTS reporting requirement. DOE-HQ established an Environmental Management (EM) program reporting system to track workscope progress at the activity data sheet (ADS) level. PTS serves as the primary EM management tool, which provides a consistent EM-wide reporting format and database for responding to internal and external requests for program information. This is an ongoing activity required to meet monthly reporting submittals to DOE-HQ from each site.

-cont inued- 


\section{B. TECHNICAL CONTENT/WORK STATEMENT (continuEd)}

PTS reports activity status for all EM ADSs and technical task plans (TTPS). Non-EM ADSs are not included in this activity. PTS integrates data from the SMS and the Financial Data System (FDS) and incorporates them into the PTS format via the EPDS.

The SMS activity involves the coordination and compilation of the individual sMS program reports and summarization of this data into an SMS executive summary and program summary report. This activity is the primary vehicle used by Hanford Site organizations to manage the work necessary to accomplish the goals and objectives of the RL mission. This is an ongoing activity to meet monthly reporting submittals to RL as required by RLID 5000.5, "Richland Field office-site Management System," and RLID 5000.7, "RL Site Management System Reporting."

The SMS Monthly Report statuses and measures the accomplishments, progress, and expenditures against an approved program baseline for all activities (direct and indirect). The Performance Reporting organization conducts monthly data quality checks of the information submitted by the programs.

in depth analysis is the responsibility of each individual program in conjunction with their RL program monitor prior to the monthly submittals to Performance Reporting. The SMS report is an.assimilation of data from the milestone database, Hanford Federal Facility Agreement and Consent Order (Tri-Party Agreement) TPA database, Level 0 schedule, Change Control, and FDS into a standardized format. Currently, incorporation of the SMS report is a manual process, which we are working toward automating by FY 1996.

The Critical Items Report is a biweekly contractors report to the RL Manager which provides essential information to RL - significant issues that require their action; issues submitted to DOE for action which have not yet been answered and which threaten progress; and, issues which Westinghouse Hanford Company (WHC) is taking management action on but do not require any DOE action. This report is coordinated by Performance Reporting and input is obtained from the WHC Level 1 managers.

With the concurrence of RL-PID, the CMM database was developed. CMM houses all RL, DOE-HQ, Tri-Party Agreement and DNFSB milestones for the Hanford Site in one central location. Milestones are statused within this database and provided to the Multi-Year Program Plan/Fiscal Year Work Plan; ADS preparation, PTS and SMS reports. Programs also have the ability to enter and track contractor milestones at their discretion.

The Master WBS Dictionary is a database which encompasses the DOE-HQ WBS number/titles, RL WBS numbers/titles, ADS numbers/titles, and DOE-HQ/RL Contractor Program Managers. This database provides input to all reports mentioned above to provide consistency and credibility between DOE-HQ and RL reports.

Oversight of EPDS is technical management of the computer platform on which all products mentioned above reside. Duties of the team lead include: chair weekly team meetings; interface with IRM leads: integration/prioritization of work for each component; approval of specifications; and test modifications for implementation of upgrades.

The client server platform is a project to enhance the existing Paradox 3.5 for Dos platform to a more efficient user-friendly computer environment. It will enable a greater number of concurrent users in each module. It will be windows compatible and able to interface directly with the DOE-HQ database currently under development for ADS, PTS and financial information.

Participation in the computer steering Group includes attendance at monthly meetings, reviewing company wide computer strategy documents, approving acquisitions of new systems, and developing the organizational microcomputer statement of strategy.

\section{KEY ASSUMPTIONS}

It is assumed that the PTS and SMS Reports will continue to be a requirement for submission to RL and DOE-HQ on a monthly basis. It is assumed that the Critical I tems Report will continue to be a biweekly requirement for submission to RL. Also assumed is that the 4700.1 Project Management Report will continue to be a quarterly requirement to DOE-HQ.

It is also assumed that the Environmental Restoration contractor will fund its participation in the activities associated with this WBS element. 


\section{A.2. WBS Dictionary}

WORK BREAKDOWN STRUCTURE DICTIONARY

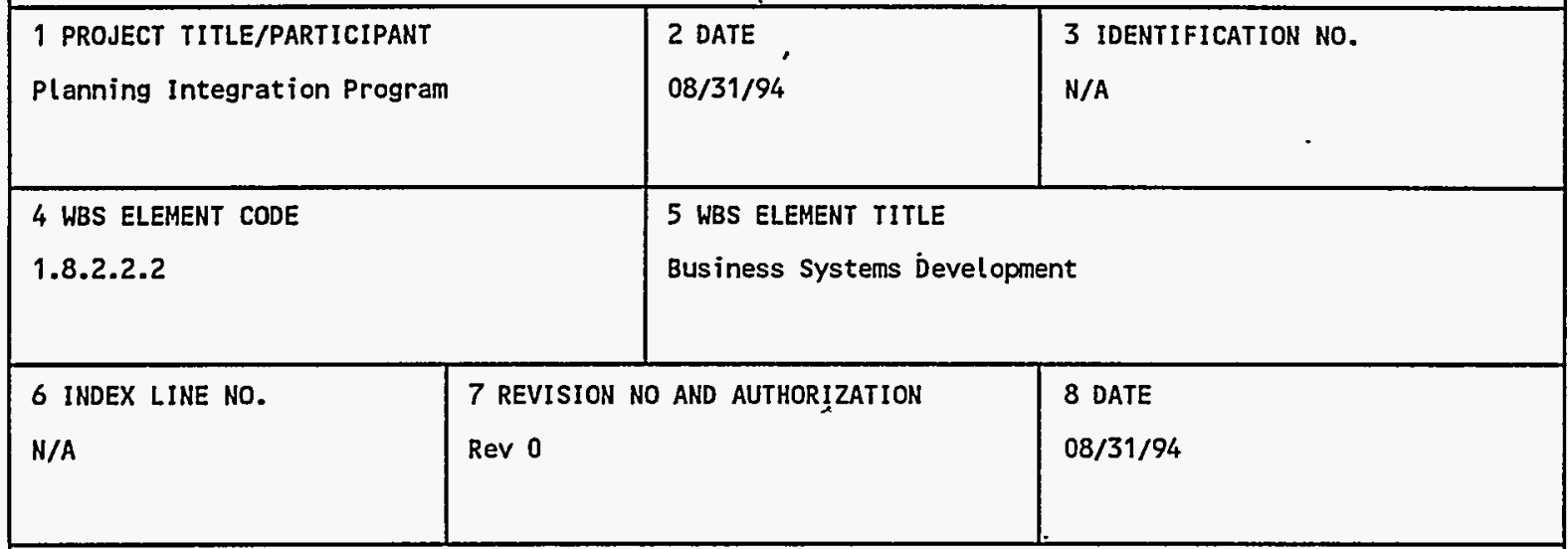

9 APPROVED CHANGES

None

\begin{tabular}{l|l}
\hline 10 SYSTEM DESIGN DESCRIPTION & 11 BUDGET AND REPORTING NUMBER \\
N/A & G\&A
\end{tabular}

12 ELEMENT TASK DESCRIPTION

A. COST CONTENT

Labor

Materials

Purchased Services

Internal Services

IRM

\section{B. TECHNICAL CONTENT/WORK STATEMENT}

The technical content and workscope associated with this effort includes the development and maintenance of company level procedures that support the Department of Energy, Richland Office's directives in the site Management System area. This includes the Management Control System manual that comprises over twenty procedures used to organize, plan, monitor and control work by Westinghouse Hanford Company personnel and other support organizations. A WHC System Description is also maintained which overviews the MCS process for all Programs/Projects under the jurisdiction of Hestinghouse Hanford. Surveillance activities are also conducted during the year and are based on a developed schedule. Programs, projects or processes are scrutinized and checked against released procedures. Records are kept and reports are provided with recommendations. As part of the MCS processes, this workscope also administers the company's change control system for all Class 1 Change 


\section{BUSINESS SYSTEMS DEVELOPMENT}

\section{FY 1995 MYPP}

\subsubsection{2}

B. TECHNICAL CONTENT/WORK STATEMENT (continued)

Requests. This includes providing assistance to the program/project control administrators, interfacing with RL's Planning integration Division, and the development of the EPDS change control module. Being the subject matter experts in the MCS area for the company, this function develops, maintains and assures that site personnel are adequately trained utilizing the procedures and forms required to manage and control all programs and projects assigned to WHC.

This workscope is also responsible for providing the standard scheduling system implementation across all WHC programs. This includes directing the BCSR P/X programming and configuration control personnel, and directing site program control personnel and the site support personnel supporting the core implementation team. Product testing, modification, installation and upgrade of the P/X system are all a part of the scope of work. This technical team conducts monthly meetings with site personnel and acts as the technical advisor for the product. Being the subject experts on the product, training is provided for all site personnel utilizing $P / X$. This includes training on the use of the product as a tool for scheduling, interface training between $P / X$ and the Financial Data System (FDS) in the development of Cost Account Plans (CAPS) and integrating the efforts of the program data administrators. Advanced training will also be provided for report \& graphic standardization and customization. Management training is also provided for those managers using the product for statusing their customers.

A prototype project will also be initiated in FY 1995 to explore the possibility of moving the budget and performance functions from FDS to $P / X$. This project will explore the feasibility of using a "warehouse" methodology of storing and retrieving data.

The management and administration effort supports the workscope associated with the above activities and also includes company requirements for WHC personnel. These areas include personnel and safety matters and other responsibilities of providing the organization with the tools to complete their workscope.

\section{KEY ASSUMPTIONS}

It is assumed that continued adherence to RL project management directives and related good management practices will be required. Also, some level of scheduling support will be provided to the other programs. 
FY 1995 MYPP

1.8.2.2.3

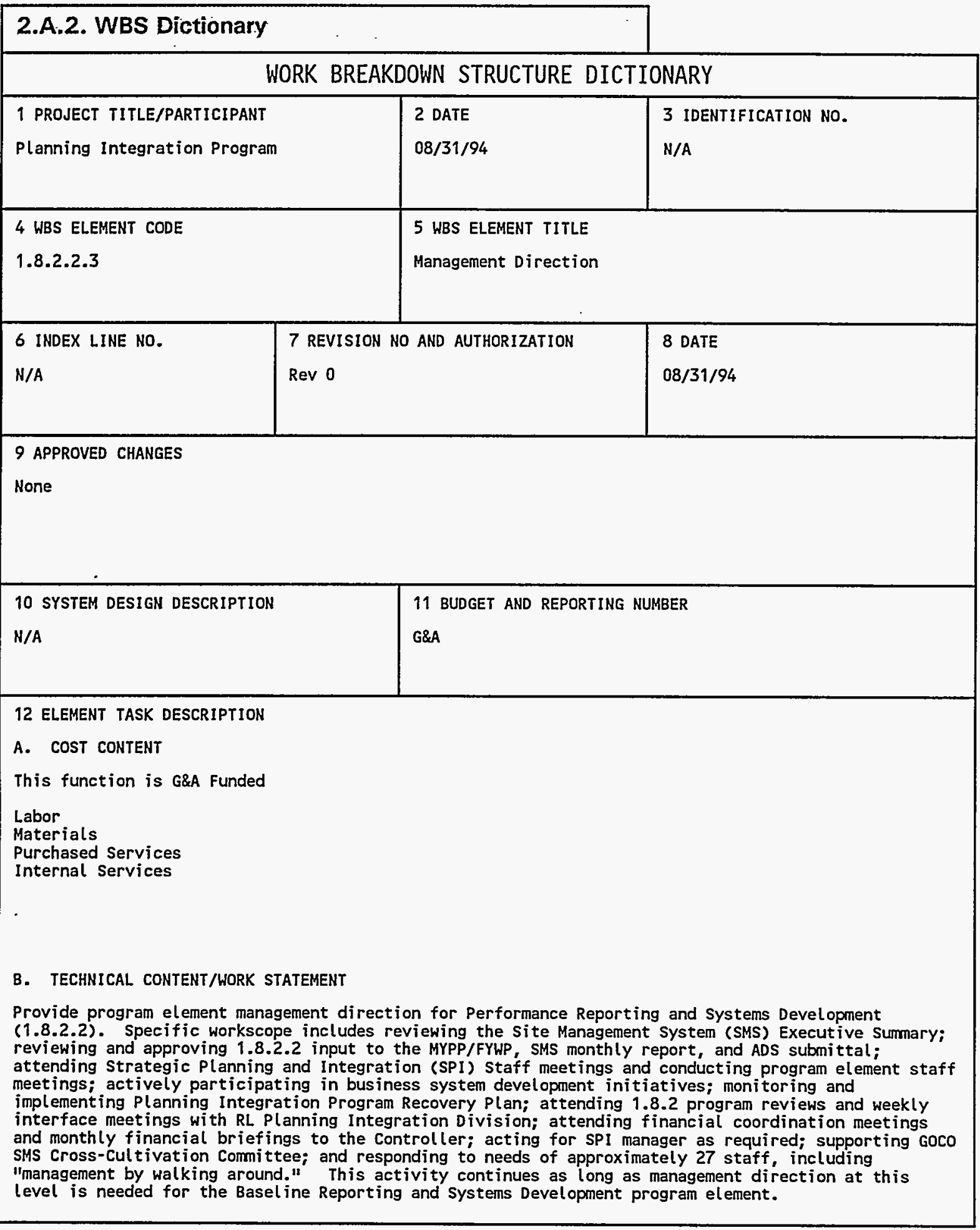


1.8.2.3

\begin{tabular}{|c|c|c|}
\hline \multicolumn{3}{|c|}{ WORK BREAKDOWN STRUCTURE DICTIONARY } \\
\hline $\begin{array}{l}1 \text { PROJECT TITLE/PARTICIPANT } \\
\text { Planning Integration Program }\end{array}$ & $\begin{array}{l}2 \text { DATE } \\
08 / 31 / 94\end{array}$ & $\begin{array}{l}3 \text { IDENTIFICATION NO. } \\
\text { N/A }\end{array}$ \\
\hline $\begin{array}{l}4 \text { WBS ELEMENT CODE } \\
1.8 .2 .3\end{array}$ & \multicolumn{2}{|c|}{$\begin{array}{l}5 \text { WBS ELEMENT TITLE } \\
\text { Program Basel ine Integration }\end{array}$} \\
\hline \begin{tabular}{l|l}
6 INDEX LINE NO. \\
N/A
\end{tabular} & $\begin{array}{l}7 \text { REVISION NO AND AUTHORIZATION } \\
\text { REV } 0\end{array}$ & $\begin{array}{l}8 \text { DATE } \\
08 / 31 / 94 \\
\end{array}$ \\
\hline \multicolumn{3}{|l|}{$\begin{array}{l}9 \text { APPROVED CHANGES } \\
\text { None }\end{array}$} \\
\hline $\begin{array}{l}10 \text { SYSTEM DESIGN DESCRIPTION } \\
\text { N/A }\end{array}$ & \multicolumn{2}{|c|}{$\begin{array}{l}11 \text { BUDGET AND REPORTING NUMBER } \\
\text { EW3110010 }\end{array}$} \\
\hline \multicolumn{3}{|l|}{$\begin{array}{l}\text { A. COST CONTENT } \\
\text { Labor } \\
\text { Materials } \\
\text { Purchased Services } \\
\text { Internal Services }\end{array}$} \\
\hline \multicolumn{3}{|c|}{$\begin{array}{l}\text { B. TECHNICAL CONTENT/WORK STATEMENT } \\
\text { This program element directs and integrates the sitewide program basel ine consistent with the } \\
\text { objectives of the Hanford site and RL guidance as provided through the Hanford Mission Plan. This } \\
\text { includes overall guidance and direction for the site MYPP/FYPs, Site Integrated Schedule, and } \\
\text { allocation of funds to the programs. This workscope provides the lead and support to responsible line } \\
\text { organizations in the preparation of a multi-year integrated site basel ine. }\end{array}$} \\
\hline $\begin{array}{l}\text { This Program element consists } \\
\text { (1.8.2.3.1) and Management Di } \\
\text { the Activity dictionaries. }\end{array}$ & $\begin{array}{l}\text { S of two Activities: Program Base } \\
\text { irection (1.8.2.3.2). Details of }\end{array}$ & $\begin{array}{l}\text { evelopment and Assessment } \\
\text { f these Activities can be found in }\end{array}$ \\
\hline
\end{tabular}


PROGRAM BASELINE DEVELOPMENT AND ASSESSMENT

FY 1995 MYPP

1.8.2.3.1

\section{A.2. WBS Dictionary}

WORK BREAKDOWN STRUCTURE DICTIONARY

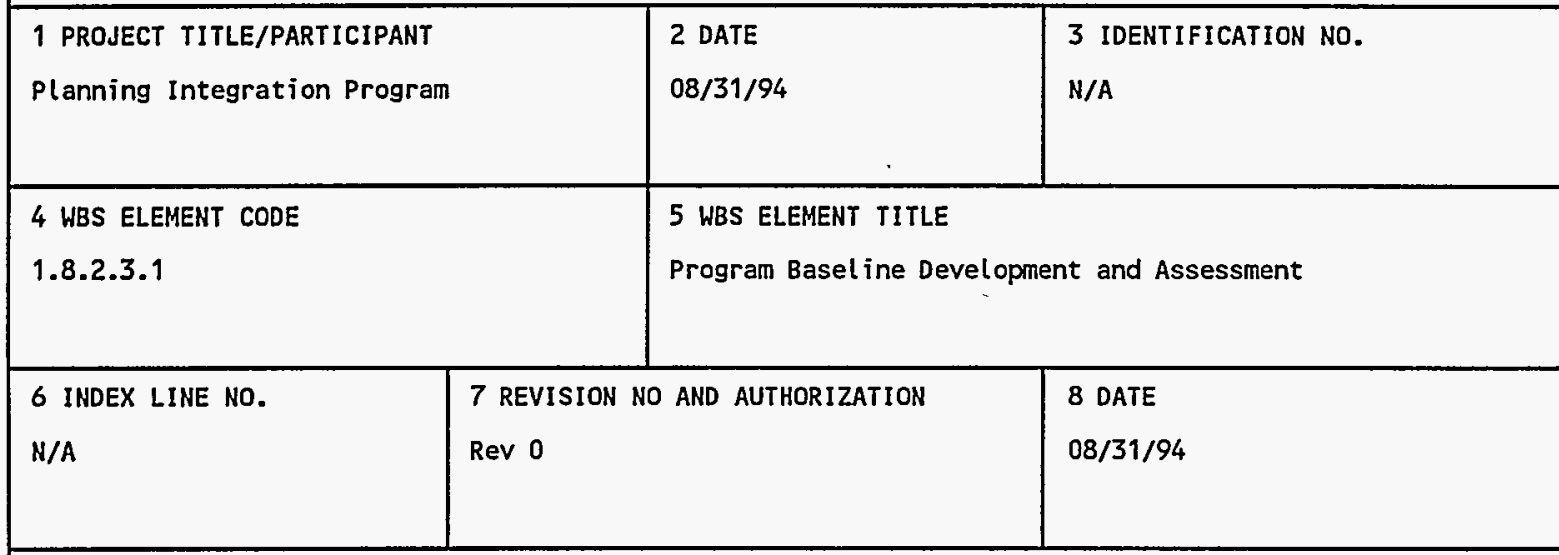

9 APPROVED CHANGES

None

10 SYSTEM DESIGN DESCRIPTION

11 BUDGET AND REPORTING NUMBER

N/A

EW3110010

12 ELEMENT TASK DESCRIPTION

A. COST CONTENT

Labor

Materials

Purchased Services

Internal Services

\section{B. TECHNICAL CONTENT/WORK STATEMENT}

This activity is the direction, training, and coordination of the Hanford Site's Multi-Year Program Plan (MYPP)/Fiscal Year Work Plans (FYWP) (the Hanford site programatic basel ine documents), and is required by RL as part of the Hanford Site Management System (SHS). Using the technical basel ine guidance provided by the Hanford Mission Plan, these documents define the scope/technical, schedule, and cost baselines for each direct program and support (indirect-funded) program across the Hanford site. Contained within the documents will be program-level objectives and technical requirements, basel ines assumptions, Work Breakdown Structure (WBS) and dictionaries, program logics and schedules at the activity (1.8.2.3.2. X) level. Also included are crosscut data on staffing levels and information by waste type. In addition, this activity will include; integration to produce a site schedule and logic; and assisting in the development of a sitewide workscope prioritization system to guide the budget allocation process.

This activity also provides the review of MYPP/FYWPS for quality and compliance with DOE guidance as specified in the call letter, RLID 5700.4 "Cost Estimating," RLID 5700.9 "Cost Estimating," EM-30 guidance, and WHC-CM-2-17 "Budget Formulation Manual." Reports of findings and corrective actions will be per WHC-CM-2-17. The purpose is to ensure that budget requests are based on validated program planning.

-continued- 


\section{FY 1995 MYPP}

\section{PROGRAM BASELINE DEVELOPMENT AND ASSESSMENT}

\subsubsection{1}

\section{B. TECHNICAL CONTENT/WORK STATEMENT (continued)}

Following are specific workscopes in this WBS:

1.8.2.3.1.1 - DIRECT FUNDED PROGRAM - The Multi-Year Program Plan (MYPP) and Fiscal Year Work Plan (FYWP), were consolidated in 1994 and form the Hanford programatic baseline. This workscope describes the activities required to manage the Program Baseline Plans process for the Hanford site which includes preparing call letters and providing direction and guidance to programs to prepare baseline documentation. It also includes routinely interfacing with programs and RL to maximize customer satisfaction and minimize problems in document preparation.

Details are:

- Coordinate workshops with RL and contractors necessary to issue the annual site guidance letter for preparation of the Program Basel ine Plan.

- Work with programs to promote training of new procedures and methods for better preparation of Program Baseline Plans, (ie., system Engineering, Risk Management, and Activity Based Cost Estimating)

- Assist programs in their recovery plan development during preparation to support milestone completion target

- Work with RL and programs to resolve issues that arise during preparation of Program Baseline Plans

- Interface with system Engineering and programs to resolve integration issues that arise from the integration analysis

- Issue production support guidance for the release and approval of Program Basel ine Plans

- Issue status reports to management during the development of Program Basel ine Plans

- Continue to support process improvement to make the baseline tool that the programs use to record changes to the program and to support performance measurement

- Assist in the preparation of 1.8.2.3.1 Program Baseline Plan and ADS submittal

- Support development of the Hanford Site Integrated schedule

- Support administration of activities for Site Management System (SMS) reporting, weekly report, and request for special assignments

1.8.2.3.1.2 - INDIRECT FUNDED PROGRAMS - The key focus during the preparation of the site Support Program Plans (SSPP) is to have support programs perform internal and external assessments to document their customers' requirements and identify ways to improve their products and services through process improvements or outsourcing. The SSPP preparation support will:

- Coordinate workshops with RL. and contractors to develop the annual site guidance letter for preparation of the SSPP

- Work with RL and programs to solve SSPP preparation issues

- Assist support programs to coordinate workshops to identify and document customer requirements for the support organization

- Work with the programs and their RL monitors to enhance the SSPP process

- Provide the necessary training to understand and use new procedures and methods for preparing better SSPPS, (i.e., Developing and writing business plans; and Using Systems Engineering input, Risk Management Guidance, and Activity Based Cost estimating tools)

- Issue guidance for production support for release and approval of SSPP

- Issue status reports to management during development of SSPP

- Assist programs with additional support and recovery plan development during sspP preparation to help ensure milestone completion on target

- Support the development of SSPP process improvements aimed at making the SSPPS useful multiyear planning documents with an execution year performance baseline containing realistic performance measurements and objectives

- Assist in the preparation of 1.8.2.3.1 Program Baseline Plans and ADS submittals

- Support administrative activities such as SMS reporting, weekly report and completion of Special Assignments

- Issue production support guidance for the release and approval of SSPP

- Issue status reports to management during the development of the SSPP

- Assist programs with additional support and recovery plan development during the preparation of the SSPP supporting milestone completion an target

- Continue to support the SSPP improvement development process making the SSPP the baseline tool the at the programs can record changes to the program and support performance measurement

- Support administration of activities for SMS reporting, weekly report and request for Special Assignment. 


\section{PROGRAM BASELINE DEVELOPMENT AND ASSESSMENT FY 1995 MYPP \\ 1.8.2.3.1}

B. TECHNICAL CONTENT/WORK STATEMENT (continuEd)

1.8.2.3.1.3 - SCHEDULING - The Hanford Site Integrated Schedule (HSIS) is a tool providing visibility to management of those activities having the greatest potential to impact the completion of the cleanup mission. The HSIS is a merger of Program Master Schedules and as such, identifies impacts from a sitewide perspective.

- Construct and issue HSIS related specifications and guidance for HSIS development to the programs

- Provide coordination and central point of contact for the HSIS development team

- Consol idate individual Program Master Basel ine Schedules (PMBS) into the HSIS

- Identify and resolve integration issues with support from the programs and strategic planning

- Ensure implementation of System Engineering technical baseline into the HSIS baseline

- Support implementation of phased HSIS features to upgrade the HSIS fully meeting the requirements of RLID 5000.11, "Scheduling" in accordance with the HSIS Implementation Action Plan

- Consolidate monthly update status from the programs into HSIS

- Develop methodologies to perform schedule analysis that focuses management attention on important and high priority work as well as performance vs. baseline to support development of site priorities

- Provide RL and site programs with an analysis of the longest duration critical path leading to completion of the site mission. The analysis will be used by management to identify adverse conditions, schedule impacts, and determine program status.

- Provide coordination with Systems Engineering and Program Integration Team to identify HSIS problems that will support completion of impacted milestones on their approved baseline dates

- Support the 1.8.2 program schedule development and ADS submittal

LEVEL 0 SCHEDULE - Graphically portrays the RL/DOE-HQ and Tri-Party Agreement milestones through the year 2000. It is structured to the RL WBS and includes all Hanford contractor's workscope with a summarization of milestone progress. This schedule will be statused monthly.

- Monthly coordination and statusing of the schedule

- Maintain schedule baseline utilizing the change control process

- Obtain forecasted completion dates

- Acquire RL and contractor approval signatures

- Produce status of Level 0 schedule to SMS reporting.

1.8.2.3.1.4 - PLANNING SERVICES - Provide program assessment accomplished by independent support and reviews of each direct program for the Hanford Site. Baseline reviews provide an independent review and validation of workscope, cost and schedule data as they relate to formulation of the Program Baseline Plans. Planning Services additionally will:

- Continue to provide training to site personnel in Activity Based Cost Estimating. Outscored $A B C$ training will be given to Program personnel on a monthly basis.

- Update the Budget Formulation Manual to reflect changing requirements/procedures regarding the cost estimating process

- Training development activities associated with cost, schedule and technical baselines

- Provide Integration Analysis activities data to management and Hanford Mission

Planning (1.8.2.1.2) on integration issues found as a result of a review of the MYPP/FYWPS. The total effort is being shared by WBS 1.8.2.1.2 and 1.8.2.3.1. The total analysis will include:

1.8.2.1.2 (Please refer to 1.8.2.1.2 to determine if funded)

Waste Data Analysis

Regulatory Analysis

Technology Analysis

Assumptions, Issues, and Interfaces (joint effort with 1.8.2.3.1)

1.8.2.3.1 (All items funded for FY 1995)

Full time Equivalent Analysis

Hanford Site Integrated Schedule Critical Path

Budget Profile

Assumptions, Issues, and Interfaces (joint effort with 1.8.2.1.2)

1.8.2.3.1.5 - DEPARTMENT ADMINISTRATION - Provides departmental tasks associated with preparation of Activity Data Sheets, the Planning Integration MYPP, and the planning and management of the group. Department Administration additionally will provide for Personnel Administration, Special Projects and Contracted Services Procurement. Essentially these activities provide for the administration of Program Baseline Development and Assessment.

This element will continue until the Hanford mission is completed.

It is assumed that the Environmental Restoration contractor will fund its participation in the activities associated with this WBS element. 


\begin{tabular}{|c|c|c|c|}
\hline \multicolumn{3}{|c|}{ 2.A.2. WBS Dictionary } & \\
\hline \multicolumn{4}{|c|}{ WORK BREAKDOWN STRUCTURE DICTIONARY } \\
\hline $\begin{array}{l}1 \text { PROJECT TITLE/PARTICIPANT } \\
\text { Planning Integration Program }\end{array}$ & & $\begin{array}{l}2 \text { DATE } \\
08 / 31 / 94\end{array}$ & $\begin{array}{l}3 \text { IDENTIFICATION NO. } \\
\text { N/A }\end{array}$ \\
\hline $\begin{array}{l}4 \text { WBS ELEMENT CODE } \\
1.8 .2 .3 .2\end{array}$ & & $\begin{array}{l}5 \text { WBS ELEMENT TITLE } \\
\text { Management Direction }\end{array}$ & \\
\hline $\begin{array}{l}6 \text { INDEX LINE NO. } \\
\text { N/A }\end{array}$ & $\begin{array}{l}7 \text { REVISION NC } \\
\text { ReV } 0\end{array}$ & AND AUTHORIZATION & $\begin{array}{l}8 \text { DATE } \\
08 / 31 / 94\end{array}$ \\
\hline \multicolumn{4}{|c|}{$\begin{array}{l}9 \text { APPROVED CHANGES } \\
\text { None }\end{array}$} \\
\hline $\begin{array}{l}10 \text { SYSTEM DESIGN DESCRIPTION } \\
\text { N/A }\end{array}$ & & $\begin{array}{l}11 \text { BUDGET AND REPORTING NUM } \\
\text { EW3110010 }\end{array}$ & MBER \\
\hline
\end{tabular}

12 ELEMENT TASK DESCRIPTION

A. COST CONTENT

Labor

Materials

Purchased Services

Internal Services

\section{B. TECHNICAL CONTENT/WORK STATEMENT}

Program Baseline Integration (PBI) management direction is the management function associated with oversight of the Multi-Year Planning effort. Oversight is most significantly focused on the direction and integration of program basel ine plans and integrated logics/schedules. PBI management direction also shares with WBS element 1-8.2.8.2 direct responsibility for direction and coordination of the Program Integration Team (PIT), a team composed of program managers across the site which is directly responsible for integration and prioritization of site workscope and allocation of associated budget resources. This activity is associated with ADS 7250 , subactivities $A I$ and $A C$.

KEY ASSUMPTION

The key assumption which directs the workscope associated with this activity include acknowledgement that the mission of the PBI organization is to direct, coordinate, and support effective planning by responsible line organizations. This includes the development of the planning required to guide site programs in the preparation of their multi-year baselines using integrated planning techniques and which can be validated through independent reviews and audits. The products will be driven by approved, integrated, logic driven program plans. This mission will be accomplished through teaming effort of all Hanford programs and contractor planning organizations, in partnership with the U.S. Department of Energy, Richland Operations Office.

This activity will continue until the Hanford mission is completed.

It is also assumed that the Environmental Restoration contractor will fund its participation in the activities associated with this WBS element. 


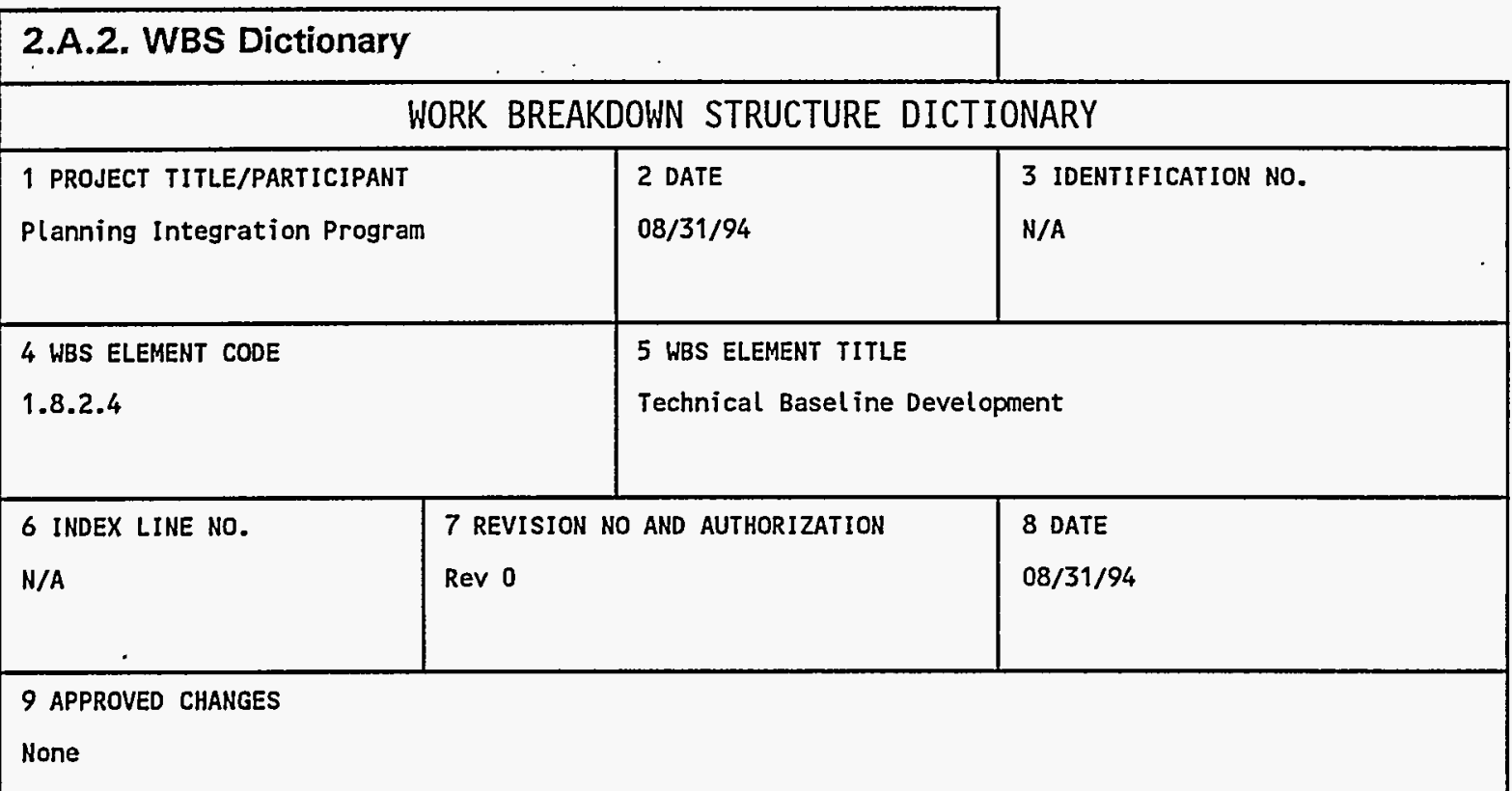

10 SYSTEM DESIGN DESCRIPTION

N/A

11 BUDGET AND REPORTING NUMBER

EW3110010/G\&A

12 ELEMENT TASK DESCRIPTION

A. COST CONTENT

Labor

Materials

Purchased Services

Internal Services

\section{B. TECHNICAL CONTENT/WORK STATEMENT}

This element will define the sitewide technical baseline for all Hanford Cleanup work using Systems Engineering (SE) methodology. Systems Engineering for the Hanford Cleanup system is a requirement of RL Policy Directive 4900.1. All work in this element will be performed to meet the requirements of RLPD 4900.1. The technical baseline will cover the top levels of the entire cleanup system. The top levels of the system will be developed within this effort. Lower levels will be developed by, and using the funds of, the various participating programs and organizations. This element will provide the integrating structure and overall system optimization.

The SE work conducted in this element consists of establishment of procedures, instructions, training methods, configuration management, associated information systems, as well as utilization of SE to establish the technical baseline. The effort develops a baseline which is technically sound and independent of program structure. 


\section{TECHNICAL BASELINE DEVELOPMENT}

\section{B. TECHNICAL CONTENT/WORK STATEMENT (continued)}

The primary deliverable is a technical specification for the cleanup mission which (1) includes all mission components down to the project/program level; (2) is optimized by elimination of overlaps, utilization of common resources, and selection of methods which minimize expenditures while minimizing risk and schedule duration; (3) is acceptable to the public, regulators and appropriate scientific/engineering assessment authority; and (4) is endorsed by DOE. The specification will be based on sound risk assessment/management principles, and include rough order of magnitude cost and schedule estimates. Its development process will be reviewed with the Hanford Advisory Board, stakeholders, and other interested parties.

The site specification will be developed as a result of mission analysis, functional analysis, requirements definition and allocation, measures of success or parametrics, alternative development, and alternative evaluation and selection. In its mature state SE will transition to verification of completed $i$ tems with respect to systems requirements, planning the integration of completed components, and evaluation of the completed system with respect to mission requirements.

This element is complete when the top level cleanup system is fully specified with respect to end state and mission delivery system. Since the cleanup system is expected to continually evolve over mission life, the systems engineering work will continue until the cleanup mission is complete.

Key milestones include completion of the development of systems engineering procedures and processes by the end of FY 1996, initial product structure in FY 1994, initial site specification in FY 1995. The basel ine technical base produced by this element and the associated projects and programs will be used to plan all Cleanup activities.

This WBS element is subdivided into two successors as follows:

\subsection{System Engineering,}

1.8.2.4.2 Systems Engineering Management. 


\subsubsection{1}

\section{A.2. WBS Dictionary}

\begin{tabular}{|l|l|l|}
\hline \multicolumn{3}{|c|}{ WORK BREAKDOWN STRUCTURE DICTIONARY } \\
\hline $\begin{array}{l}1 \text { PROJECT TITLE/PARTICIPANT } \\
\text { Planning Integration Program }\end{array}$ & $\begin{array}{l}2 \text { DATE } \\
08 / 31 / 94\end{array}$ & N/A IDENTIFICATION NO. \\
\hline $\begin{array}{l}\text { W WBS ELEMENT CODE } \\
1.8 .2 .4 .1\end{array}$ & $\begin{array}{l}5 \text { WBS ELEMENT TITLE } \\
\text { Systems Engineering Architecture }\end{array}$ \\
\hline 6 INDEX LINE NO. & 7 REVISION NO AND AUTHORIZATION & $\begin{array}{l}8 \text { DATE } \\
\text { N/A }\end{array}$ \\
& Rev 0 & \\
\hline
\end{tabular}

9 APPROVED CHANGES

None

\begin{tabular}{|l|l}
\hline 10 SYSTEM DESIGN DESCRIPTION & $\begin{array}{l}11 \text { BUDGET AND REPORTING NUMBER } \\
\text { E/A }\end{array}$ \\
EW3110010/G\&A
\end{tabular}

12 ELEMENT TASK OESCRIPTION

A. COST CONTENT

Labor

Materials

Purchased Services

Internal Services

\section{B. TECHNICAL CONTENT/WORK STATEMENT}

This element conducts the top level systems engineering which leads to definition of the top level deliverables and lays the foundation for integration and optimization of program/organizational level systems engineering results. Input from the Hanford Mission plan, the Hanford Strategic plan, TPA, and various external requirements is translated (using SE methods) to specific interim and final deliverables which complete the mission. This element also integrates the program level SE work with the top level results to produce an integrated site-cleanup technical specification. Integration activities include optimization of both inter-related components and total system performance. 
B. TECHNICAL CONTENT/HORK STATEMENT (continued)

The site specification becomes the technical basis used to guide the Multi-Year planning process. All Programmatic MYPPs will be based on assigned portions of the site specification. This task will require considerable investment in the early years $(2-3)$. As the cleanup system definition reaches maturity, the investment will decline to a level consistent with maintaining system integrity and consistency with mission direction changes.

This element is subdivided into two lower tiers, namely:

1.8.2.4.1.1 Systems Architecture Development which is the direct funded portion of the Architecture work and

1 8.2.4.1.2 G\&A Systems Engineering which covers the indirect work. 
FY 1995 MYPP

1.8.2.4.2

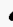

\section{A.2. WBS Dictionary}

WORK BREAKDOWN STRUCTURE DICTIONARY

\begin{tabular}{|c|c|c|c|}
\hline $\begin{array}{l}1 \text { PROJECT TITLE/PARTICIPANT } \\
\text { Planning Integration Program }\end{array}$ & & $\begin{array}{l}2 \text { DATE } \\
08 / 31 / 94\end{array}$ & $\begin{array}{l}3 \text { IDENTIFICATION NO. } \\
\text { N/A }\end{array}$ \\
\hline $\begin{array}{l}4 \text { WBS ELEMENT CODE } \\
1.8 .2 .4 .2\end{array}$ & & $\begin{array}{l}5 \text { WBS ELEMENT TITLE } \\
\text { Systems Engineering }\end{array}$ & ment \\
\hline $\begin{array}{l}6 \text { INDEX LINE NO. } \\
\text { N/A }\end{array}$ & $\begin{array}{l}7 \text { REVISION NO } \\
\text { ReV } 0\end{array}$ & AND AUTHORIZATION & $\begin{array}{l}8 \text { DATE } \\
08 / 31 / 94\end{array}$ \\
\hline $\begin{array}{l}9 \text { APPROVED CHANGES } \\
\text { None }\end{array}$ & & & \\
\hline \multicolumn{4}{|c|}{. } \\
\hline $\begin{array}{l}10 \text { SYSTEM DESIGN DESCRIPTION } \\
\text { N/A }\end{array}$ & & $\begin{array}{l}11 \text { BUDGET AND REPOR } \\
\text { G\&A }\end{array}$ & MBER \\
\hline
\end{tabular}

12 ELEMENT TASK DESCRIPTION

A. COST CONTENT

Labor

Materials

Purchased Services

Internal Services

\section{B. TECHNICAL CONTENT/WORK STATEMENT}

This element will provide the coordination needed to manage and control formal site systems engineering baselines. The output from this element translates the general systems engineering requirements of RLPD 4900.1 into procedures and instructions that can be implemented uniformly in the field. Development of the training materials needed to qualify performing staff is also included. Coordination will be provided for mission/functional definition, requirements, alternatives, technical performance, and design configuration. This element will also provide development and integration of Program/Project SEMPs, SEMs, and other implementation standards and procedures. Policies, processes and specifications necessary to support systems engineering performance at Hanford will be provided and maintained. Information management procedures for maintaining the systems engineering basel ines will be established to support effective configuration management program and to provide linkage to the site Management System (SMS). 


\section{SYSTEMS ENGINEERING MANAGEMENT}

B. TECHNICAL CONTENT/WORK STATEMENT (continued)

This task will require considerable investment in the early years $(2-3)$. As the cleanup system definition reaches maturity, the investment will decline to a level consistent with maintaining system integrity and consistency with mission direction changes. This element will be active throughout mission life. All work in this element is conducted in element 1.8.2.4.2. 


\section{A.2. WBS Dictionary}

WORK BREAKDOWN STRUCTURE DICTIONARY

\begin{tabular}{|c|c|c|c|}
\hline $\begin{array}{l}1 \text { PROJECT TITLE/PARTICIPANT } \\
\text { Planning Integration Program }\end{array}$ & & $\begin{array}{l}2 \text { DATE } \\
08 / 31 / 94\end{array}$ & $\begin{array}{l}3 \text { IDENTIFICATION NO. } \\
\text { N/A }\end{array}$ \\
\hline $\begin{array}{l}4 \text { WBS ELEMENT CODE } \\
1.8 .2 .5\end{array}$ & & $\begin{array}{l}5 \text { WBS ELEMENT TITLE } \\
\text { Planning Integratio }\end{array}$ & am Management \\
\hline $\begin{array}{l}6 \text { INDEX LINE NO. } \\
\text { N/A }\end{array}$ & $\begin{array}{l}7 \text { REVISION NO } \\
\operatorname{ReV} 0\end{array}$ & AND AUTHORIZATION & $\begin{array}{l}8 \text { DATE } \\
08 / 31 / 94\end{array}$ \\
\hline \multicolumn{4}{|l|}{$\begin{array}{l}9 \text { APPROVED CHANGES } \\
\text { None }\end{array}$} \\
\hline $\begin{array}{l}10 \text { SYSTEM DESIGN DESCRIPTION } \\
\text { N/A }\end{array}$ & & $\begin{array}{l}11 \text { BUDGET AND REPOR } \\
\text { EW3110010 }\end{array}$ & MBER \\
\hline
\end{tabular}

12 ELEMENT TASK DESCRIPTION

A. COST CONTENT

Labor

Materials

Purchased Services

Internal Services

B. TECHNICAL CONTENT/WORK STATEMENT

Planning Integration Program Management coordinates the yearly generation of ADS 7250 inputs, the MYPP/FYWP and the monthly SMS and PTS reports for the 1.8.2 program and provides management oversight for all elements of the 1.8.2 Planning Integration program. The program activities are:

\subsection{Planning Integration Program Management \\ 1.8.2.5.2 Management Direction}

The primary products are the 1.8.2 Planning Integration ADS 7250 inputs, MYPP/FYWP (technical, cost and schedule performance baseline), and the monthly SMS and PTS reports.

The other functions contained within this program element are control of the technical, cost and schedule basel ine through a rigorous change control process, performance analysis against this baseline, the coordination of all the 1.8.2 program elements efforts and the management of the total 1.8.2 program efforts. Planning Integration Program Management is required for the life of the program. 


\section{A.2. WBS Dictionary}

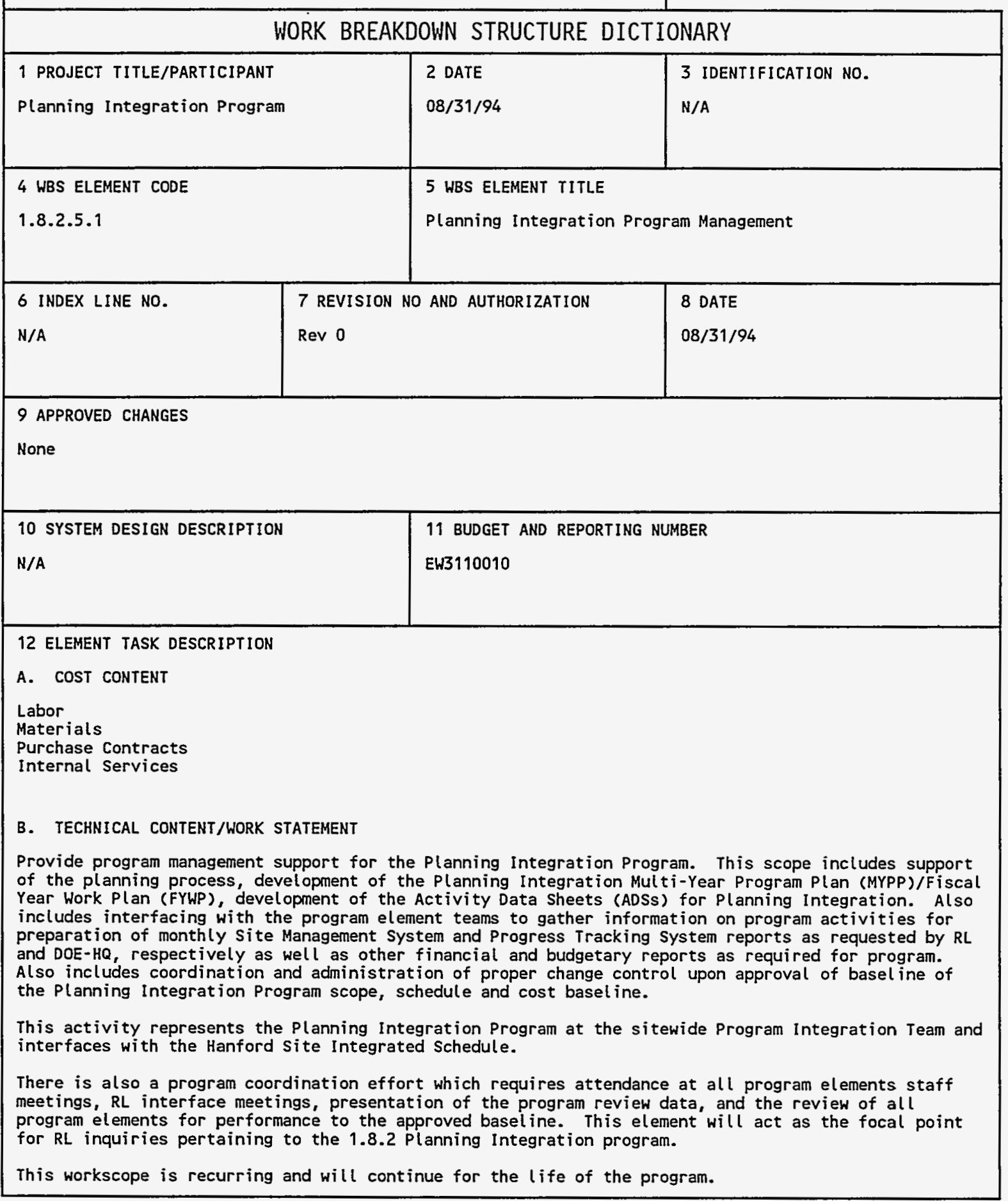




\section{B. TECHNICAL CONTENT/WORK STATEMENT (continued)}

This program management function is only capable of supporting limited budget-related exercises with its internal resources. The limited list of exercises which are supported are listed below:

- Review and prioritization of EM key unfunded workscope for year of execution,

- Review of OMB Budget Passback (for year following execution),

- Budget Amendment Package - action to change President's Budget before Congress reviews it. If Budget Amendment does not happen, we resort to a Reprograming package,

- Budget Reprograming Actions - preparation of a reprograming package to change the

Appropriation allocation. Requires RL, DOE-HQ and congressional approval, preparation,

- Integration of EM workscope priorities - Integrated Priority List to drive ADS preparation,

- Mid-year review with RL and DOE-HQ,

- DOE HQ Internal Review of Budget (IRB) - DOE-HQ review of the field office Budget Request (ADSS revīewed from all Field offices for $E M)$,

- Ecology and EPA review of ADS 7250,

- DOE-HQ Independent Review of ADSs,

- ADS reviews with RL,

- 1.8.2 Priority List reviews with RL. Needed for year of execution, planning year and budget request,

- Baseline Validation Reviews - internal assessment with RL,

- Public involvement for ADS review. 


\section{A.2. WBS Dictionary}

WORK BREAKDOWN STRUCTURE DICTIONARY

\begin{tabular}{|l|l|l|}
\hline $\begin{array}{l}\text { 1 PROJECT TITLE/PARTICIPANT } \\
\text { Planning Integration Program }\end{array}$ & $\begin{array}{l}2 \text { DATE } \\
08 / 31 / 94\end{array}$ & $\begin{array}{l}3 \text { IDENTIFICATION NO. } \\
\text { N/A }\end{array}$ \\
\hline $\begin{array}{l}\text { WBS ELEMENT CODE } \\
1.8 .2 .5 .2\end{array}$ & $\begin{array}{l}5 \text { WBS ELEMENT TITLE } \\
\text { Management Direction }\end{array}$ \\
\hline $\begin{array}{l}\text { N INDEX LINE NO. } \\
\text { N/A }\end{array}$ & $\begin{array}{l}7 \text { REVISION NO AND AUTHORIZATION } \\
\text { ReV } 0\end{array}$ & $\begin{array}{l}8 \text { DATE } \\
08 / 31 / 94\end{array}$ \\
\hline
\end{tabular}

9 APPROVED CHANGES

None

\begin{tabular}{|l|l|}
\hline $\begin{array}{l}10 \text { SYSTEM DESIGN DESCRIPTION } \\
\text { N/A }\end{array}$ & $\begin{array}{l}11 \text { BUDGET AND REPORTING NUMBER } \\
\text { Department Overhead }\end{array}$ \\
\hline
\end{tabular}

12 ELEMENT TASK DESCRIPTION

A. COST CONTENT

Labor

Materials

Purchased Services

Internal Services

B. TECHNICAL CONTENT/WORK STATEMENT

The workscope contained within this activity includes the management oversight of the following 1.8 .2 program el ements:

1.8.2.1 Work Definition

1.8.2.2 Performance Reporting

1.8.2.3 Program Basel ine Integration

1.8.2.4 Technical Basel ine Development

1.8.2.5 Planning Integration Program Management

1.8.2.6 Integrated Risk Assessment

1.8.2.7 Publ ic Involvement

1.8.2.8 ADS Preparation and Coordination

The activity also includes interfacing with WHC and RL senior management concerning program direction and policy.

This activity is funded from department overheads. It has no direct funding. It is purely level of effort work. This activity is required for the life of the program. 


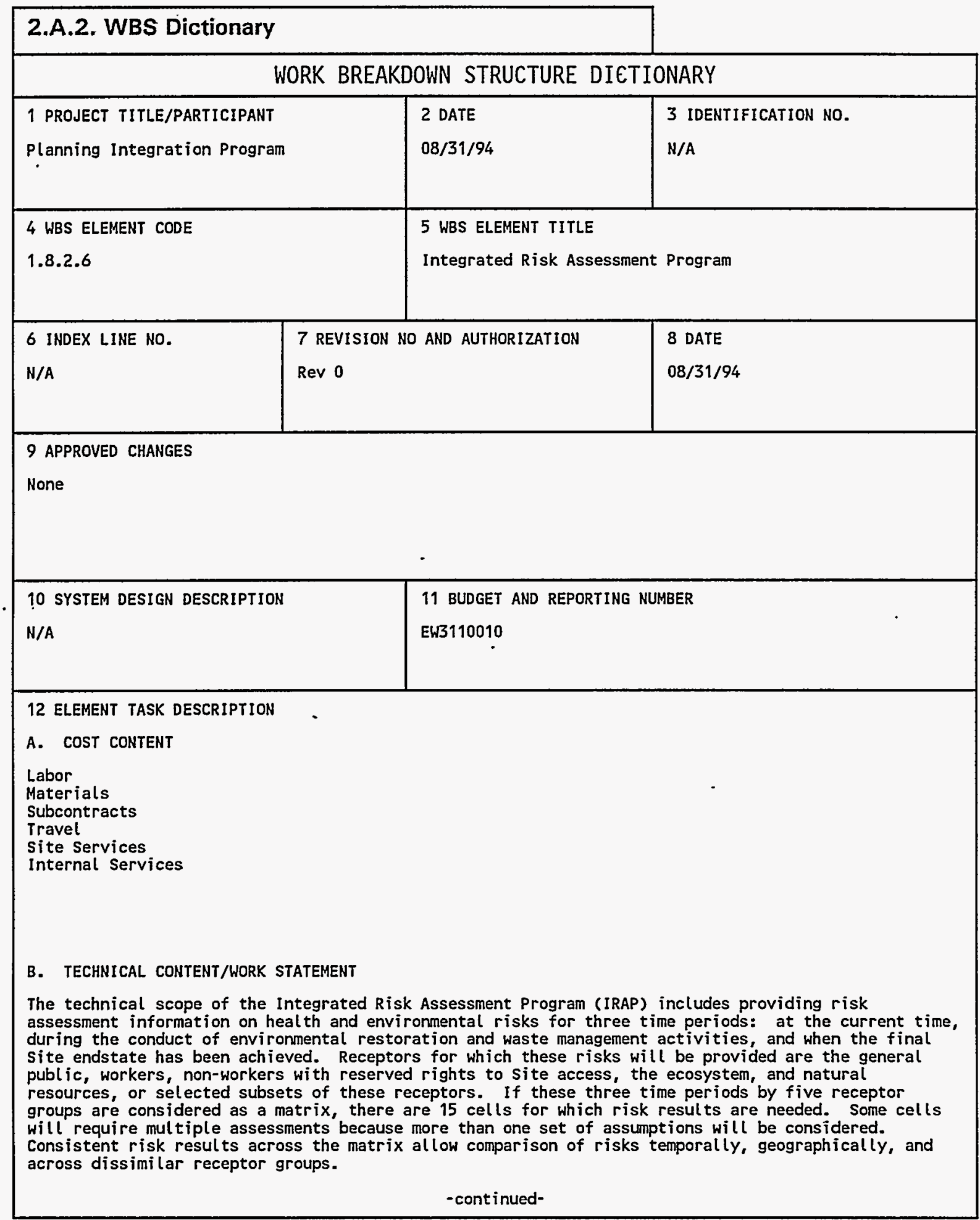




\section{B. TECHNICAL CONTENT/WORK STATEMENT (continued)}

Accomplishment of the entire matrix of IRAP risk assessments will take several years to complete. In each cell of the matrix, an exhaustive assessment will not be done, but assessments will be chosen so that exhaustive inferences are possible (e.g., some activities will have only upper and/or lower bounds). In addition, updates of aspects of completed assessments will be required as additional inventory information and characterization data become available, and as the site endstate specified in the TriParty Agreement changes.

IRAP products are risk assessment results and their reformulation in various ways to provide needed information for decision making by other Hanford and DOE activities. As noted below, IRAP products also include input to the MYPP guidance document to Hanford programs $(1.1,1.2,1.3,2.1,7.1)$. Thus, products will be reports and other written materials.

Users of IRAP risk results include those staff developing the technical basel ine (1.8.2.4), implementing risk-based decision making for the purposes of site-level planning (1.8.2.1), and conducting risk assessment activities for complex-level planning under the auspices of EM-6, Integrated Risk Evaluation. Results will allow users to set risk-based priorities across Hanford site programs. Priorities can be set based on baseline risk results, on potential for risk reduction (current minus endstate, such as the endstate specified in the Tri-Party Agreement), or on potential for net risk reduction (current plus during minus endstate), or on other metrics.

Inputs to IRAP planning include information on stakeholder values, both information obtained from stakeholder involvement at the 1.8 .2 program level (by 1.8.2.7) and information developed at a technical level by IRAP (including technical stakeholder inputs and guidance provided by IRAP's Technical Review Board). Input also includes descriptions of risk information needs from the technical baseline (1.8.2.4) and from strategic planning (1.8.2.1). This information will be used to guide IRAP planning activities, including to develop the IRAP portion of the 1.8.2 ADS and MYPP.

Based on planning needs, MYPP guidance to Hanford programs will be developed. This guidance will specify inputs required, the form of inputs, and technical methodologies for developing inputs. Guidance to Hanford programs on IRAP methodologies will be developed to reflect stakeholder values and input from the IRAP Technical Review Board. Methodologies will be reviewed both by technical stakeholders and by internal/external peers, including Hanford site programs (both DOE and WHC), safety and emergency preparedness organizations, and others. Once comments are resolved, methodology documents will be provided to Hanford programs as guidance for providing IRAP inputs, and IRAP staff will be available to help in their implementation.

Inputs to IRAP from Hanford programs will at a minimum also include materials prescribed in the MYPP guidance. Inputs from Hanford programs are expected to include inventory information and release scenarios used to translate inventory information to source-term information. Where programmatic capabilities exist, inputs may include results of applying specific methodologies on environmental transport of released contaminants for public and ecological health risks to provide concentration at the receptor, risk results, and associated uncertainty/sensitivity information. Exact assumptions to be used about exposure pathways, time durations of exposure, levels of exposure, receptor characteristics, and heal th and ecological endpoints will be developed with stakeholder participation and provided as part of programmatic guidance.

Once IRAP obtains input from Hanford programs, results will be reconciled. Gaps will be filled by IRAP staff, including risk results and associated uncertainty/sensitivity information for Hanford programs without risk assessment capabilities. Finally, results will be integrated by IRAP staff and provided to users. Internal and external review will be conducted of integrated risk assessment results, including verification of all computations. When review comments are resolved, results will be provided to users. 
INTEGRATED RISK ASSESSMENT PROGRAM

FY 1995 MYPP

1.8.2.6.1

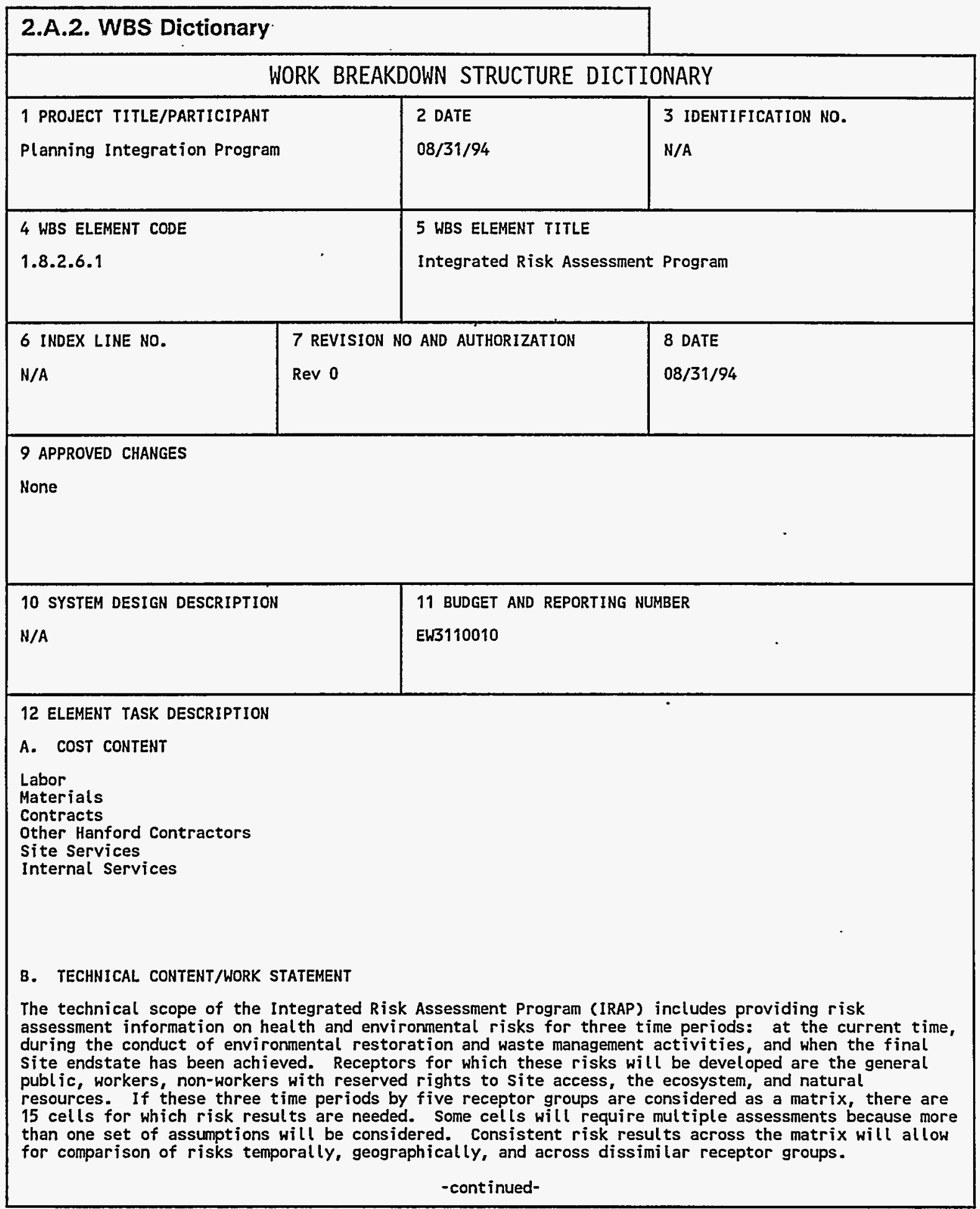




\section{B. TECHNICAL CONTENT/WORK STATEMENT (continued)}

Accomplishment of the entire matrix of IRAP risk assessments will take five years to complete. Because an exhaustive assessment will not be done as each cell of the matrix is "completed" (even though assessments will be chosen so that exhaustive inferences are possible), some assessments may have to be evaluated for previously considered time periods or receptors in the course of completion of specific cells of the matrix. In addition, updates of aspects of completed assessments will be required as additional inventory information and characterization data become available, continuing the IRAP beyond the initial five years.

IRAP products are risk assessment results and their combination in various ways to provide requested information to other Hanford and DOE activities. Products will be documented in the form of reports. Results will be provided to various users, including those developing the technical baseline (1.8.2.4), those implementing risk-based decision making for the purposes of site-level planning (1.8.2.1), programs $(1.1,1.2,1.3,2.1,7.1)$, and those conducting risk assessment activities for complex-level planning under the auspices of EM-6, Integrated Risk Evaluation. Some risk results will be provided to programs; purposes vary from information only to use in some aspect of program planning or in filling legal requi rements.

Inputs to IRAP planning include information on stakeholder values, both those collected from stakeholder involvement (at the 1.8.2 program level) by 1.8 .2 .7 and those from programmatic activities $(1.1,1.2,1.3$, $2.1,7.1)$. Input also includes descriptions of risk information needs from the technical basel ine (1.8.2.4) and from strategic planning (1.8.2.1). This information will be used to guide IRAP planning activities, including developing ADSs and producing MYPPS.

IRAP methodology will be developed to reflect stakeholder values and input of a technical review panel. IRAP methods will be reviewed both by stakeholders (at the technical level) and by internal/external peers.

Development of inventory information requires participation of programs $(1.1,1.2,1.3,2.1,7.1)$, as does the translation of inventory data to source term information by development of release scenarios (e.g., assumptions). Similar(y, environmental transport of released contaminants requires programmatic review for acceptability of assumptions. Transport results will provide concentration at the receptor. Exact assumptions on exposure pathways, time durations of exposure, and levels of exposure will be developed with stakeholder participation. Finally, inputs from various stakeholders on critical receptors, on health and ecological endpoints of interest, and on their relative importance will be developed. Taken together, this information (inventory data and assumptions regarding source term, environmental transport, exposure assessment, effect assessment) constitutes scenarios for which risks will be obtained or assessed by IRAP.

Reviews of scenarios will be conducted by management at Hanford, by programs (1.1, 1.2, 1.3, 2.1, 7.1), by safety and emergency preparedness organizations, and by other stakeholders. Once comments are resolved, risks associated with scenarios will be evaluated using computerized tools that are an implementation of methods and scenarios defined previously. Internal and external review will be conducted of risk assessment results, including verification of all computations. When review comnents are resolved, results will be provided to users.

This effort assumes that funding requirements identified in the FY 1996 ADS Integrated Target Case are maintained. 


\section{A.2. WBS Dictionary}

\begin{tabular}{|c|c|c|c|}
\hline \multicolumn{4}{|c|}{ WORK BREAKDOWN STRUCTURE DICTIONARY } \\
\hline $\begin{array}{l}1 \text { PROJECT TITLE/PARTICIPANT } \\
\text { Planning Integration Program }\end{array}$ & & $\begin{array}{l}2 \text { DATE } \\
08 / 31 / 94\end{array}$ & $\begin{array}{l}3 \text { IDENTIFICATION NO. } \\
\text { N/A }\end{array}$ \\
\hline $\begin{array}{l}4 \text { WBS ELEMENT CODE } \\
1.8 .2 .7\end{array}$ & & $\begin{array}{l}5 \text { WBS ELEMENT TITLE } \\
\text { Public Involvement }\end{array}$ & \\
\hline $\begin{array}{l}6 \text { INDEX LINE NO. } \\
\text { N/A }\end{array}$ & $\begin{array}{l}7 \text { REVISION NO } \\
\text { ReV } 0\end{array}$ & AND AUTHORIZATION & $\begin{array}{l}8 \text { DATE } \\
08 / 31 / 94\end{array}$ \\
\hline $\begin{array}{l}9 \text { APPROVED CHANGES } \\
\text { None }\end{array}$ & & & \\
\hline $\begin{array}{l}10 \text { SYSTEM DESIGN DESCRIPTION } \\
\text { N/A }\end{array}$ & & $\begin{array}{l}11 \text { BUDGET AND REPORT } \\
\text { G\&A }\end{array}$ & MBER \\
\hline
\end{tabular}

12 ELEMENT TASK DÉSCRIPTION

A. COST CONTENT

Labor

Materials

Purchased Services

Internal Services

\section{B. TECHNICAL CONTENT/WORK STATEMENT}

The Public Involvement Element provides integrated public involvement support to 1.8 .2 to establish and maintain the legitimacy of Hanford's planning process with interested stakeholders and the interested Indian nations. Public Involvement will develop mechanisms to help the Hanford Advisory Board, public, and interested parties understand Hanford's planning process and how different Program Elements and planning components are related.

A public involvement plan was developed in FY 1994. It established the basic framework around which the Planning Integration Program will provide to the Hanford Advisory Board, the public, and interested parties insight and involvement in the Hanford planning process.

Public Involvement will facilitate an analysis with 1.8.2 Program Elements to determine what planning components need public involvement. This element will then help develop and implement appropriate Action Plans to involve members of the public in Hanford's formulation, planning, execution and reporting planning processes. Activities to fulfill the public involvement requirements of the Hanford Federal Facility Agreement and Consent Order, Paragraphs 148 and 149 , and to fulfill the public involvement requirements associated with the recommendations of the Defense Nuclear Facility Safety Board will be incorporated into the program Action Plans. Public Involvement will also help evaluate the effectiveness of the actions to establish understanding and credibility of the planning process. 


\section{PUBLIC INVOLVEIMENT}

\subsubsection{1}

\section{A.2. WBS Dictionary}

WORK BREAKDOWN STRUCTURE DICTIONARY

\begin{tabular}{|l|l|l|}
\hline $\begin{array}{l}\text { 1 PROJECT TITLE/PARTICIPANT } \\
\text { Planning Integration Program }\end{array}$ & $\begin{array}{l}2 \text { DATE } \\
08 / 31 / 94\end{array}$ & $\begin{array}{l}\text { N/A } \\
\text { IDENTIFICATION NO. }\end{array}$ \\
\hline $\begin{array}{l}1.8 .2 .7 .1 \\
\text { WBS ELEMENT CODE }\end{array}$ & $\begin{array}{l}5 \text { WBS ELEMENT TITLE } \\
\text { Public Involvement }\end{array}$ \\
\hline N/A & $\begin{array}{l}7 \text { REVISION NO AND AUTHORIZATION } \\
\text { Rev O LINE NO. }\end{array}$ & $\begin{array}{l}8 \text { DATE } \\
08 / 31 / 94\end{array}$ \\
\hline
\end{tabular}

9 APPROVED CHANGES

None

\begin{tabular}{|l|l}
\hline 10 SYSTEM DESIGN DESCRIPTION & 11 BUDGET AND REPORTING NUMBER \\
N/A & G\&A
\end{tabular}

12 ELEMENT TASK DESCRIPTION

A. COST CONTENT

Labor

Materials

Purchased Services

Internal Services

B. TECHNICAL CONTENT/WORK STATEMENT

The Public Involvement Element provides integrated public involvement support to 1.8 .2 to establish and maintain the Legitimacy of Hanford's planning process with interested stakeholders and the interested Indian nations. Public Involvement will develop mechanisms to help the Hanford Advisory Board, public, and interested parties understand Hanford's planning process and how different Program Elements and planning components are related.

A public involvement plan was developed in FY 1994. It established the basic framework around which the Planning Integration Program will provide to the Hanford Advisory Board, the public, and interested parties insight and involvement in the Hanford planning process.

Public Involvement will facilitate an analysis with 1.8.2 Program Elements to determine what planning components need public involvement. This element wili then help develop and implement appropriate Action Plans to involve members of the public in Hanford's formulation, planning, execution and reporting planning processes. Public Involvement will also help evaluate the effectiveness of the actions to establish understanding and credibility of the planning process. 


\section{ADS PREPARATION AND COORDINATION}

\section{A.2. WBS Dictionary}

WORK BREAKDOWN STRUCTURE DICTIONARY

\begin{tabular}{|c|c|c|c|}
\hline $\begin{array}{l}1 \text { PROJECT TITLE/PARTICIPANT } \\
\text { Planning Integration Program }\end{array}$ & & $\begin{array}{l}2 \text { DATE } \\
08 / 31 / 94\end{array}$ & $\begin{array}{l}3 \text { IDENTIFICATION NO. } \\
\text { N/A }\end{array}$ \\
\hline $\begin{array}{l}4 \text { WBS ELEMENT CODE } \\
1.8 .2 .8\end{array}$ & & $\begin{array}{l}5 \text { WBS ELEMENT TITLE } \\
\text { ADS Preparation and }\end{array}$ & nation \\
\hline $\begin{array}{l}6 \text { INDEX LINE NO. } \\
\text { N/A }\end{array}$ & $\begin{array}{l}7 \text { REVISION N } \\
\text { ReV } 0\end{array}$ & AND AUTHORIZATION & $\begin{array}{l}8 \text { DATE } \\
08 / 31 / 94\end{array}$ \\
\hline \multicolumn{4}{|l|}{$\begin{array}{l}9 \text { APPROVED CHANGES } \\
\text { None }\end{array}$} \\
\hline $\begin{array}{l}10 \text { SYSTEM DESIGN DESCRIPTION } \\
\text { N/A }\end{array}$ & & $\begin{array}{l}11 \text { BUDGET AND REPOR } \\
\text { EW3110010 }\end{array}$ & MBER \\
\hline
\end{tabular}

12 ELEMENT TASK DESCRIPTION

A. COST CONTENT

Labor

Materials

Purchased Services

Internal Services

\section{TECHNICAL CONTENT/WORK STATEMENT}

This Program Element provides for the development of site preparation guidance, training, coordination, submittal, and distribution of the Hanford Site Environmental Restoration and Waste Management Activity Data Sheet (ADS) and supporting documentation. It also includes Program Integration Team activities and budget exercises.

This program element consists of two Activities: ADS Preparation Coordination (1.8.2.8.1) and Management Direction (1.8.2.8.2). Details of each of these Activities can be found in the Activity dictionaries.

Selected special activities, budget exercises, and planning actions that occur throughout a given fiscal year are contained in this program element. These items are hard to schedule and difficult to quantify resource requirements for these items. A listing of these $i$ tems are: -continued- 


\section{ADS PREPARATION AND COORDINATION}

B. TECHNICAL CONTENT/WORK STATEMENT (continued)

- Review and prioritization of EM key unfunded workscope for year of execution,

- Review of OMB Budget Passback (for year following execution).

- Budget Amendment Package - action to change President's Budget before Congress reviews it. If Budget Amendment does not happen, we resort to a Reprograming package,

- Budget Reprograming Actions - preparation of a reprogramming package to change the Appropriation allocation. Requires RL, DOE-HQ and congressional approval, preparation,

- Integration of EM workscope priorities - Integrated Priority List to drive ADS preparation,

- Mid-year review with RL and DOE-HQ,

- DOE-HQ Internal Review of Budget (IRB) - DOE-HQ review of the field office Budget Request (ADSs reviewed from all Field offices for EM),

- Ecology and EPA review of site ADSs,

- DOE-HQ Independent Review of ADSs,

- ADS reviews with RL,

- 1.8.2 Priority List reviews with RL. Needed for year of execution, planning year and budget request,

- Baseline Validation Reviews - internal assessment with RL,

- Public Involvement for ADS review.

This element will continue until the Hanford mission is completed.

It is assumed that the Environmental Restoration contractor will fund its participation in the activities associated with this WBS element. 
1.8.2.8.1

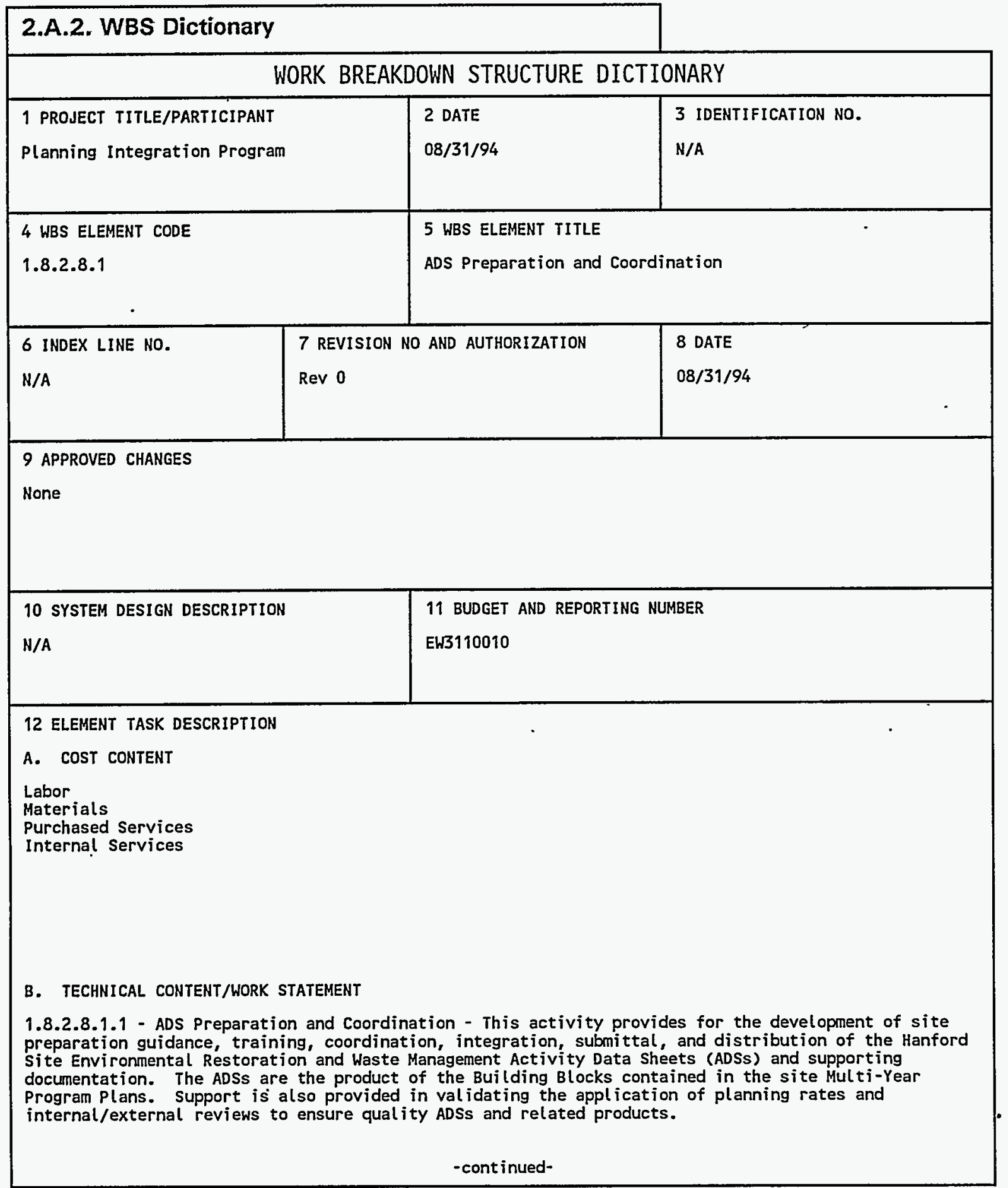




\section{ADS PREPARATION AND COORDINATION}

\section{FY 1995 MYPP}

\subsubsection{1}

\section{B. TECHNICAL CONTENT/WORK STATEMENT (continued)}

1.8.2.8.1.2 - Public Involvement - This activity is also responsible for the development and implementation of public involvement activities as mandated by the Tri-Party Agreement Requirements Paragraph 148/149 and Keystone Report. This encompasses the development of cost data and related written materials designed to inform the public, and solicit input to be used in the decision-making process. It will interface with WBS element 1.8.2.7 in these actions.

1.8.2.8.1.3 - System Developrent and Support - This activity also includes supporting analysis of the annual EM and Safety and Health budget submittal, coordinating site-wide workscope prioritization and integration exercises, and coordinating and summarizing budgetary information and related briefing materials as requested by DOE-HQ and RL.

This activity is also responsible for supporting various site activities by providing EPDS generated data.

This activity will continue until the Hanford mission is completed.

It is assumed that the Environmental Restoration contractor will fund its participation in the activities associated with this WBS element. 
FY 1995 MYPP

MANAGEMENT DIRECTION

\subsubsection{2}

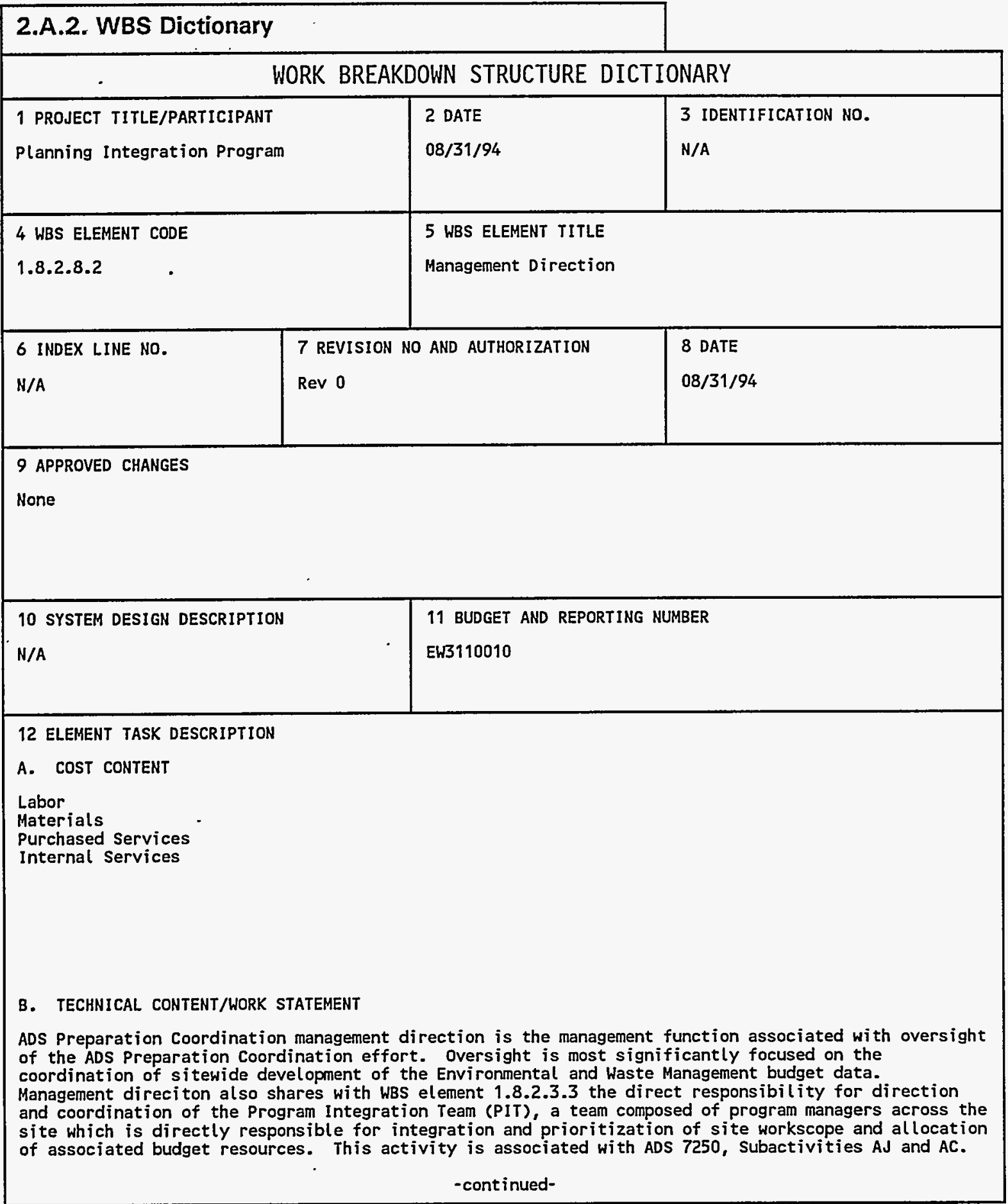




\section{FY 1995 MYPP}

1.8.2.8.2

\section{KEY ASSUMPTION}

The key assumption which directs activities associated with this activity include acknowledgement that the mission of this activity is to lead and support effective planning and budgeting by responsible line organizations. The mission will be accompl ished through teaming effort of all Hanford programs and contractor planning organizations, in partnership with the U.S. Department of Energy, Richland Operations. Office.

This workscope includes the assistance in defining of the Hanford public participation process for stakeholder review and input into the site ADSs and the EM budget and planning activities.

This activity will continue until the Hanford mission is completed. 
Planning Integration Multi-Year Program Plan

This page intentionally left blank. 
Planning Integration Multi-Year Program P1an

\section{B RESPONSIBILITY ASSIGNMENTS}

The Responsibility Assignment Matrix (RAM) reflects the established Planning Integration Program work breakdown structure from the program level to the cost account level. The RAM assigns responsibilities to individuals, organizations, and companies at each level. The RAM will be updated annualiy through the MYPP/FYWP update process, and as required outside of this MYPP/FYWP. The RAM is not a controlled portion of the program baseline, and updates to the RAM do not require change control processes. 


\section{Planning Integration Program \\ WBS 1.8.2}

\section{FY 1995 MYPP/FYWP}

\section{B. RESPONSIBILITY ASSIGNMENT MATRIX (RAM)}

\begin{tabular}{|l|l|l|l|l|l||}
\hline \hline $\begin{array}{l}\text { Program } \\
\text { Element }\end{array}$ & Activity & \multicolumn{1}{|c|}{$\begin{array}{c}\text { Cost } \\
\text { Account }\end{array}$} & \multicolumn{1}{|c|}{ Title } & \multicolumn{1}{|c|}{$\begin{array}{c}\text { Responsible } \\
\text { Manager }\end{array}$} & \multicolumn{1}{|c|}{$\begin{array}{c}\text { Resp/ } \\
\text { Org. }\end{array}$} \\
\hline \hline 1.8 .2 & & & Planning Integration & D.B. Pabst & WHC/SPI \\
\hline 1.8 .2 .1 & & & Work Identification & H.H. Yoshikawa & WHC/SPI \\
\hline & 1.8 .2 .1 .1 & 1.8 .2 .1 .1 .1 & Strategic Quality Planning & H.H. Yoshikawa & WHC/SPI \\
\hline & 1.8 .2 .1 .2 & 1.8 .2 .1 .2 .1 & Hanford Mission Planning (HMP) & S.M. O'Toole & WHC/SPI \\
\hline & & 1.8 .2 .1 .2 .2 & PNL Support for HMP & W.A. Hesser & PNL \\
\hline & & 1.8 .2 .1 .2 .3 & Hanford Mission Planning & S.M. O'Toole & WHC/SPI \\
\hline & & 1.8 .2 .1 .2 .4 & PNL Support for HMP & W.A. Hesser & PNL \\
\hline & 1.8 .2 .1 .3 & 1.8 .2 .1 .3 .1 & Risk Management & W.A. Hesser & PNL \\
\hline & & & $\begin{array}{l}\text { Baseline Reporting \& System } \\
\text { Development }\end{array}$ & L.R. Hafer & WHC/SPI \\
\hline & 1.8 .2 .2 .1 & & Performance Reporting & E.A. Schultz & WHC/SPI \\
\hline & & 1.8 .2 .2 .1 .1 & PTS Reporting & E.A. Schultz & WHC/SPI \\
\hline & & 1.8 .2 .2 .1 .2 & SMS/Critical Items Reporting & E.A. Schultz & WHC/SPI \\
\hline
\end{tabular}




\section{Planning Integration Program \\ WBS 1.8 .2}

\section{FY 1995 MYPP/FYWP}

\begin{tabular}{|c|c|c|c|c|c|}
\hline \multicolumn{6}{|c|}{ 2B. RESPONSIBILITY ASSIGNMENT MATRIX (RAM) } \\
\hline $\begin{array}{l}\text { Program } \\
\text { Element }\end{array}$ & Activity & $\begin{array}{l}\text { Cost } \\
\text { Account }\end{array}$ & Title & $\begin{array}{c}\text { Responsible } \\
\text { Manager }\end{array}$ & $\begin{array}{l}\text { Resp/ } \\
\text { Org. }\end{array}$ \\
\hline & & 1.8 .2 .2 .1 .3 & Central Milestone Module & E.A. Schultz & WHC/SPI \\
\hline & & 1.8 .2 .2 .1 .4 & Master WBS and Validation Tables & E.A. Schultz & WHC/SPI \\
\hline & & 1.8.2.2.1.5 & EPDS Platform Oversite & E.A. Schultz & WHC/SPI \\
\hline & 1.8.2.2.2 & 1.8.2.2.2.1 & Business Systems Development & P.J. Bence & WHC/SPI \\
\hline & 1.8.2.2.3 & 1.8.2.2.3.1 & Management Direction & L.R. Hafer & WHC/SPI \\
\hline 1.8 .2 .3 & & & Program Baseline Integration & D.L. Borders & WHC/SPI \\
\hline & 1.8.2.3.1 & 1.8.2.3.1.1 & $\begin{array}{l}\text { Program Baseline Development and } \\
\text { Assessment }\end{array}$ & J.K. Fish & WHC/SPI \\
\hline & 1.8.2.3.2 & 1.8 .2 .3 .2 .1 & Management Direction & D.L. Borders & WHC/SPI \\
\hline 1.8.2.4 & & & Technical Baseline Development & G.W. Jackson & WHC/SE \\
\hline & 1.8.2.4.1 & 1.8.2.4.1.1 & Systems Architecture Development & J.C. Collings & WHC/SE \\
\hline & & 1.8.2.4.1.2 & Systems Engineering & J.C. Collings & WHC/SE \\
\hline & 1.8.2.4.2 & 1.8.2.4.2.1 & Systems Engineering Management & P.K. Shen & WHC/SE \\
\hline
\end{tabular}




\section{Planning Integration Program \\ WBS 1.8.2}

\section{FY 1995 MYPP/FYWP}

\begin{tabular}{|c|c|c|c|c|c|}
\hline \multicolumn{6}{|c|}{ 2B. RESPONSIBILITY ASSIGNMENT MATRIX (RAM) } \\
\hline $\begin{array}{l}\text { Program } \\
\text { Element } \\
\end{array}$ & Activity & $\begin{array}{c}\text { Cost } \\
\text { Account } \\
\end{array}$ & Title & $\begin{array}{c}\text { Responsibie } \\
\text { Manager }\end{array}$ & $\begin{array}{l}\text { Resp/ } \\
\text { Org. } \\
\end{array}$ \\
\hline 1.8.2.5 & 1.8.2.5.1 & 1.8.2.5.1.1 & $\begin{array}{l}\text { Planning Integration Program } \\
\text { Management }\end{array}$ & D.B. Pabst & WHC/SPI \\
\hline & 1.8 .2 .5 .2 & 1.8 .2 .5 .2 .1 & Management Direction & D.B. Pabst & WHC/SPI \\
\hline 1.8 .2 .6 & 1.8.2.6.1 & & Integrated Risk Assessment & J.A. Mahaffey & PNL \\
\hline 1.8 .2 .7 & 1.8.2.7.1 & & Public Involvement & L.E. Thiede & $\mathrm{WHC} / \mathrm{COM}^{-}$ \\
\hline 1.8.2.8 & & & ADS Preparation and Coordination & D.L. Borders & WHC/SPI \\
\hline & 1.8.2.8.1 & 1.8.2.8.1.1 & ADS Preparation and Coordination & C. Edwards & WHC/SPI \\
\hline & 1.8.2.8.2 & 1.8.2.8.2.1 & Management Direction & D.L. Borders & WHC/SPI \\
\hline
\end{tabular}




\author{
Planning Integration \\ Multi-Year Program Plan
}

\title{
2.C PROGRAM LOGICS
}

Once the scope of the effort is defined in the Work Breakdown Structure, the next step in the planning process is to develop the flows for performing that work scope. This is defined as the logics associated with the work scope. The logics describe the flow, the "how", and identify where the interfaces to and from other activities associate with the work scope. The logics are not time phased, nor are they resource loaded. They represent work concepts, not discreet work packages. They identify predecessor and successor activities that dictate when in the flow a certain action must occur.

The conventions used in the Planning Integration Program logics are:

- A box or square connotes an activity being performed by the program or program element.

- An oval or a circle connotes an interface to or from an outside program, program element, customer, or stakeholder. Wherever possible, the WBS element identifier for the interface is listed.

- A diamond connotes a "yes/no" decision point.

The logic diagrams for the Planning Integration Program and its subordinate program elements are reflected in the following. 
WHC-SP-1096

$09 / 23 / 94$

Planning Integration Multi-Year Program Plan

This page intentionally left blank. 


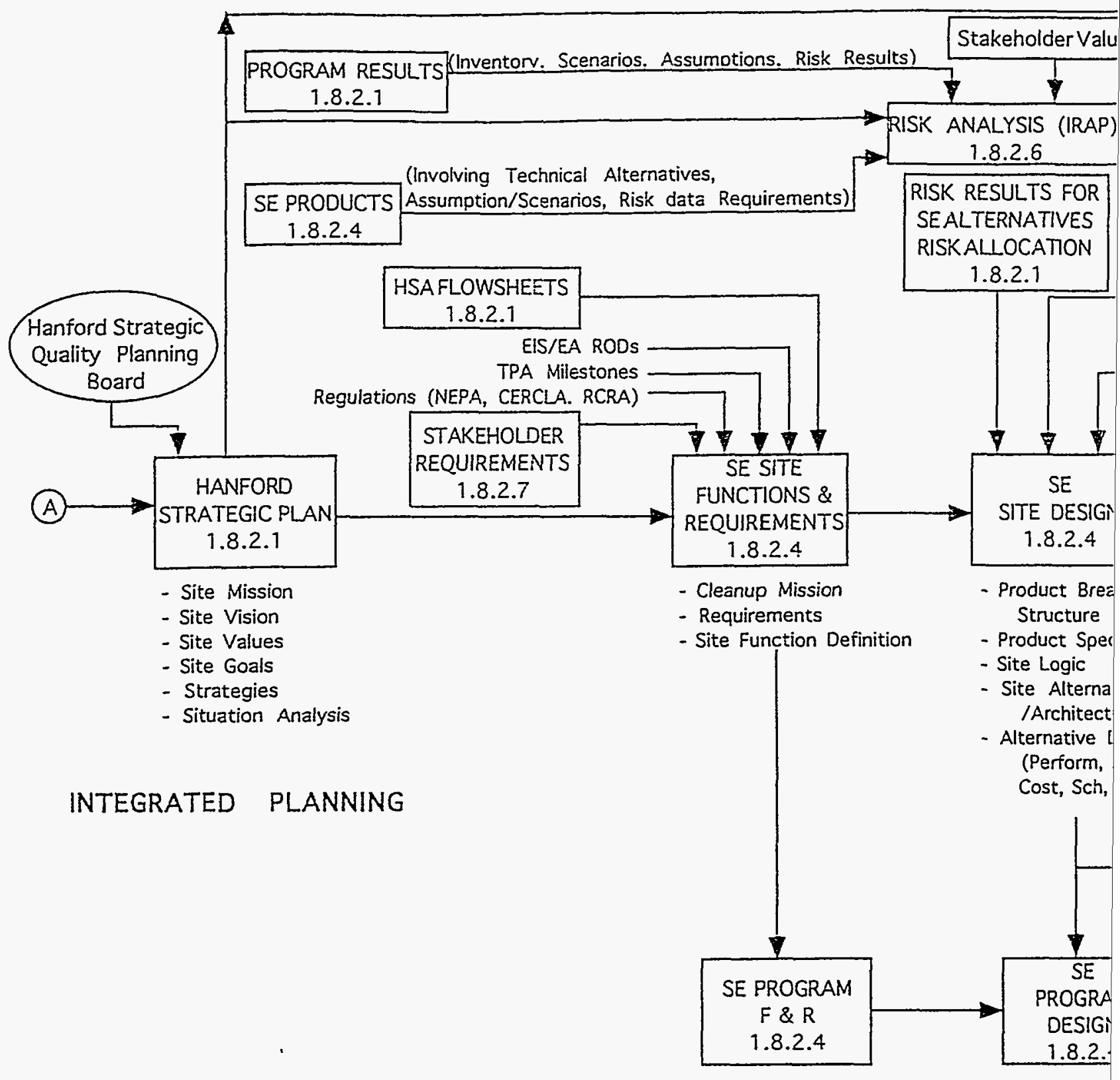

SYSTEMS ENGINEERING 


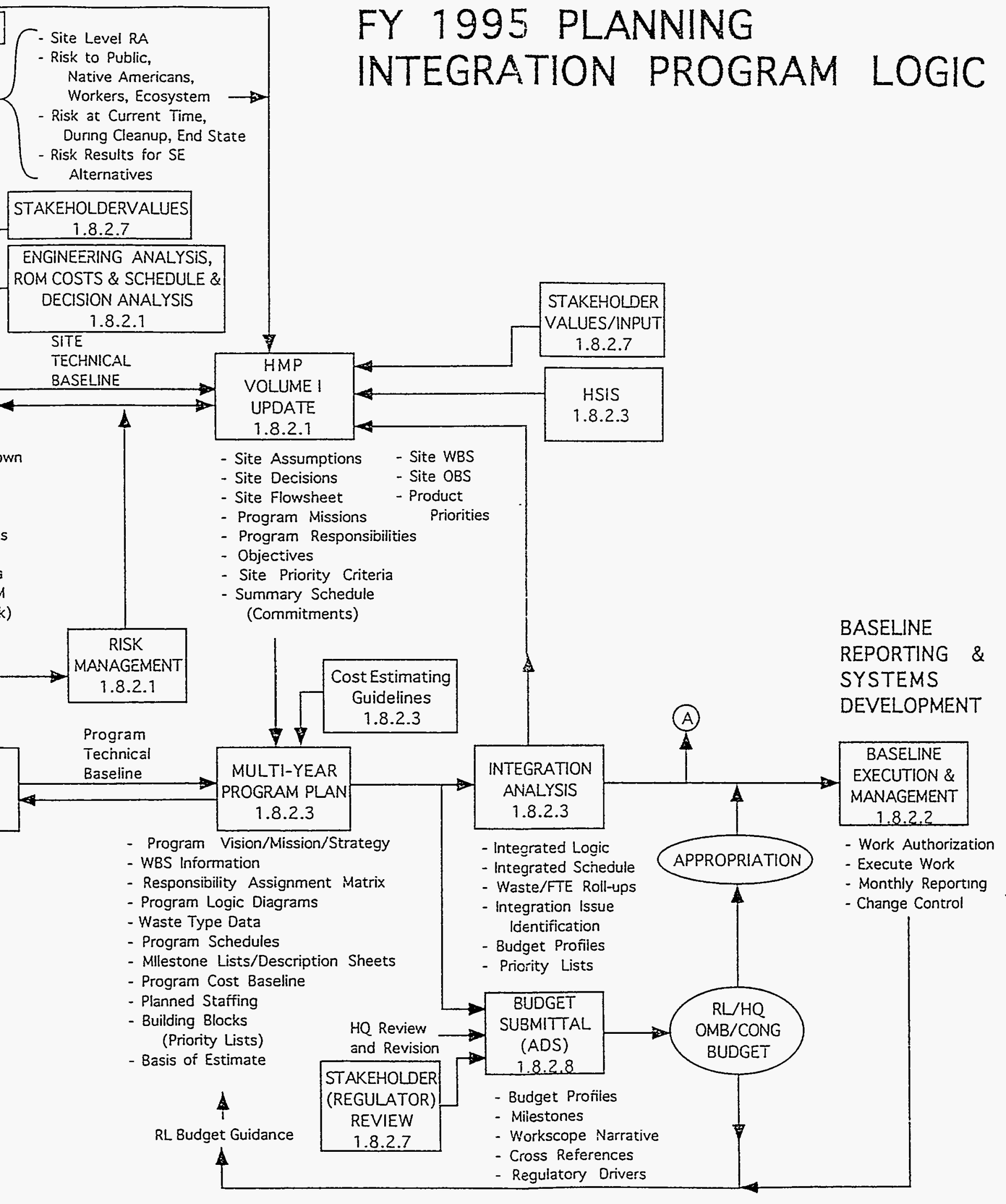

PROGRAM BASELINE INTEGRATION/ADC PREPARATION \& COORDINATION 
Fig. 2.C.1-1 Logic Diagrain '

(Page 2 of 2 )

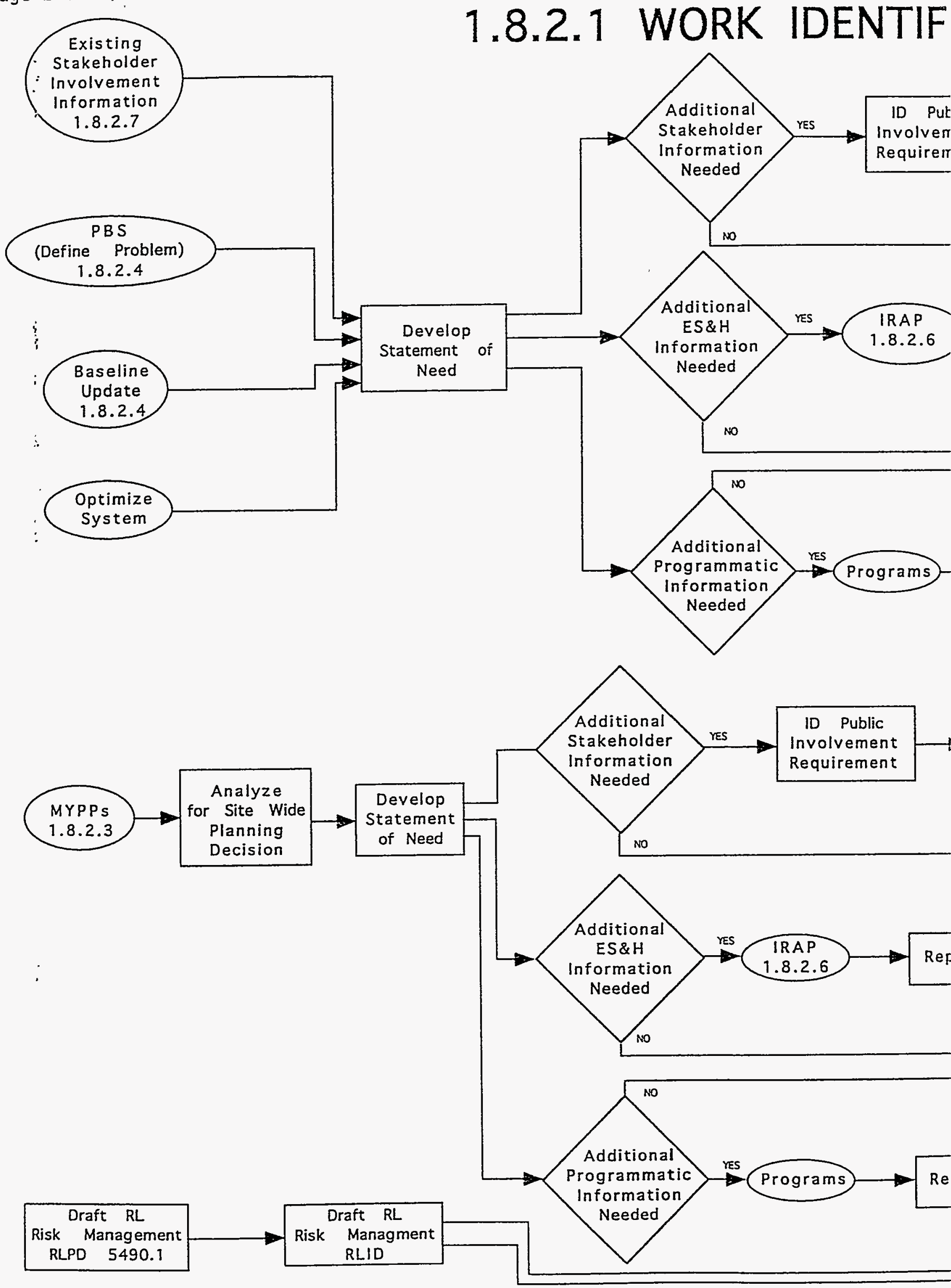


Fig. 2.C.1-1 Logic Diagram

(Page 1 of 2)

\subsubsection{WORK IDENTIFICATIOI}

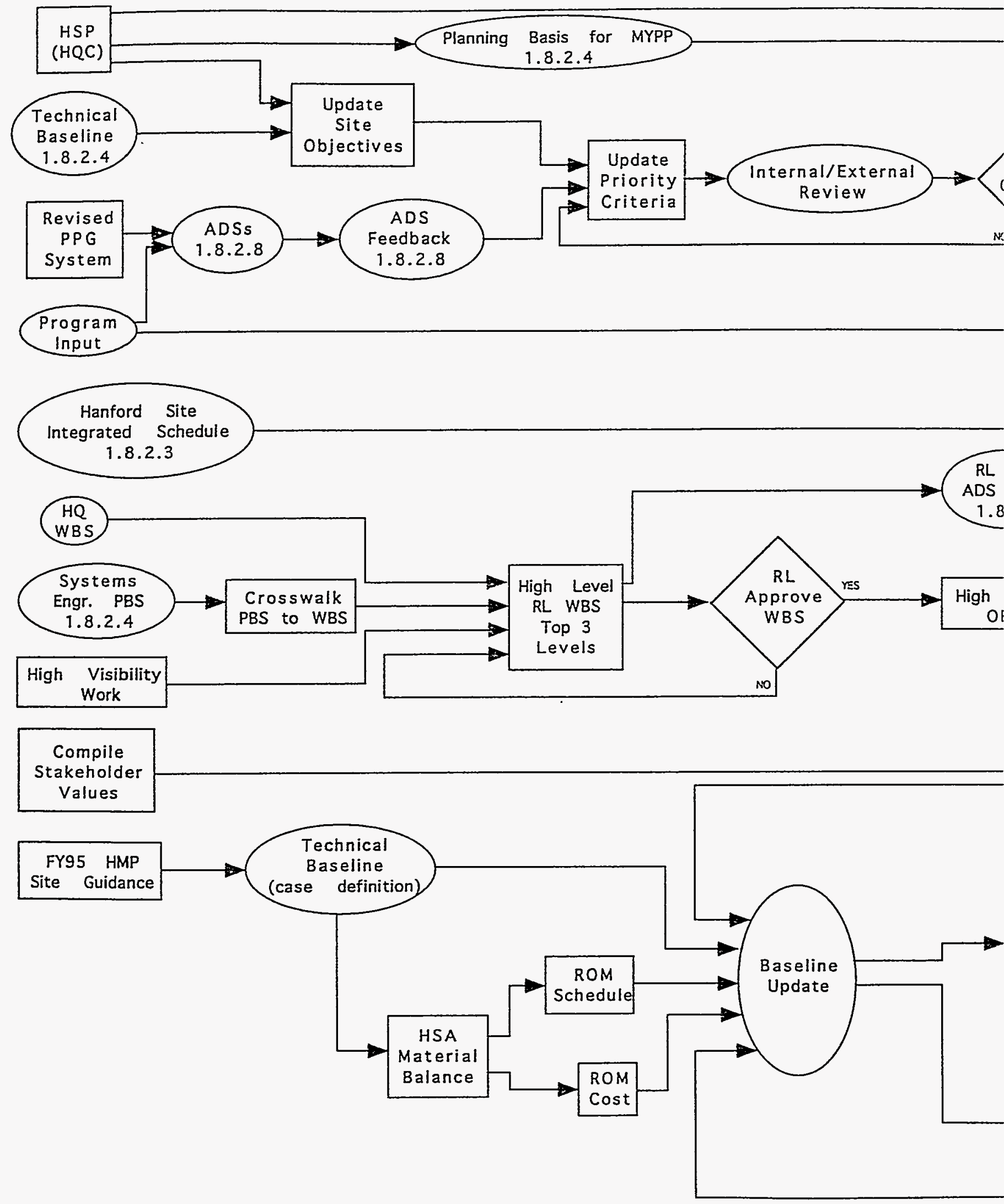




\section{7/26/94 (Page 1 of 2)}

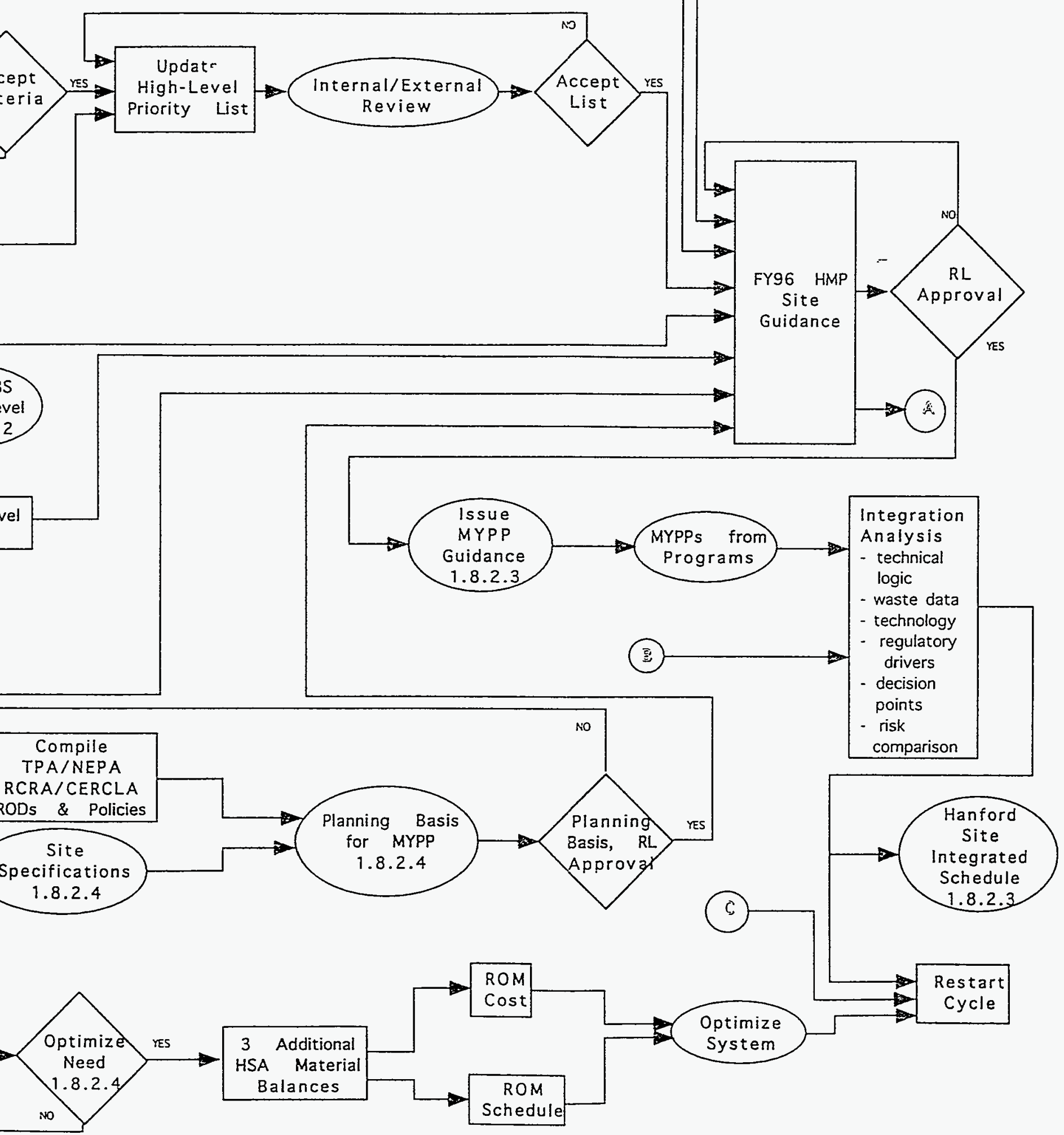




\section{BASELINE REPORTING AND SYSTEMS DEVELOPMENT 1.8.2.2}

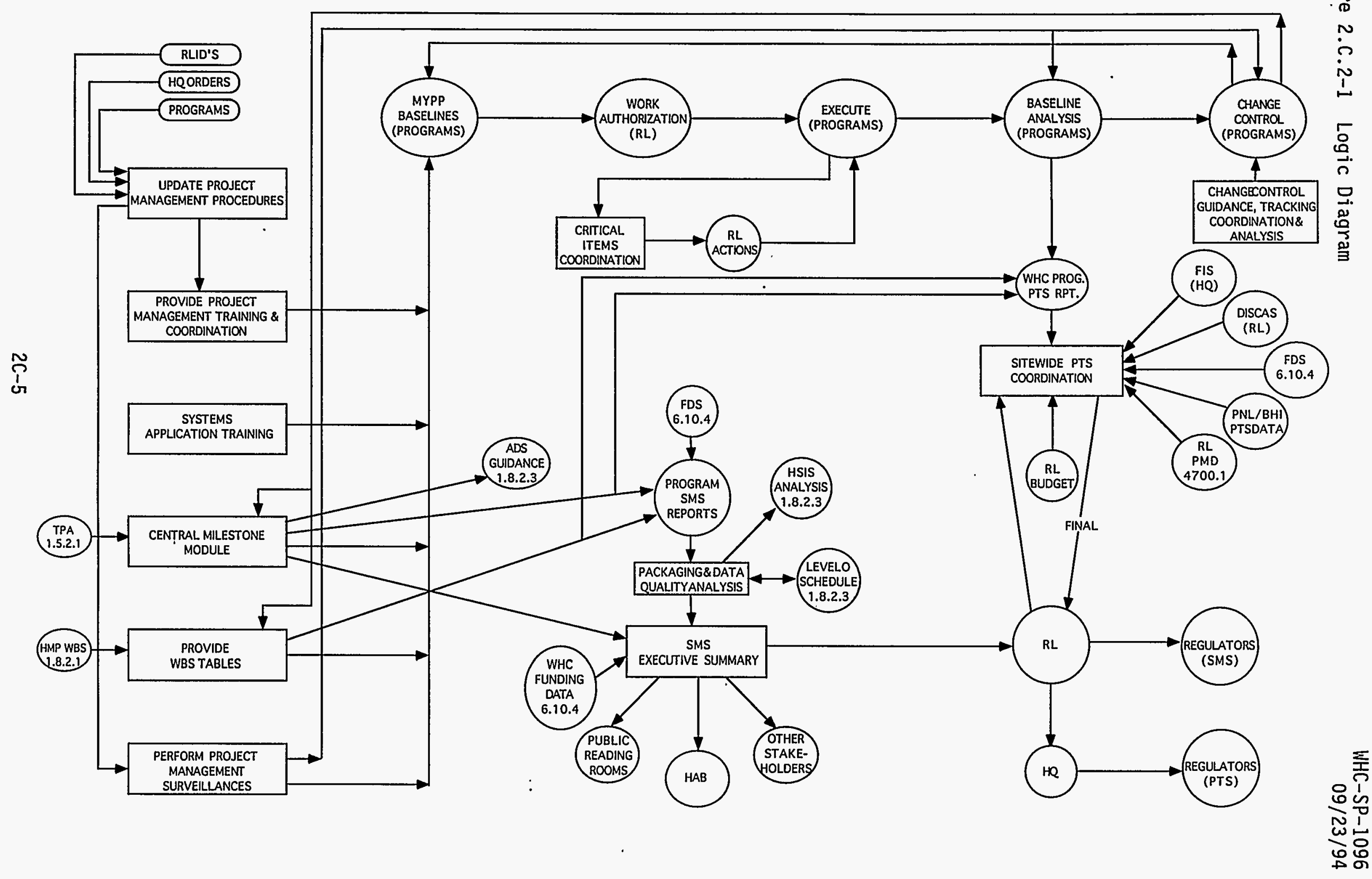




\section{PROGRAM BASELINE INTEGRATION 1.8.2.3}

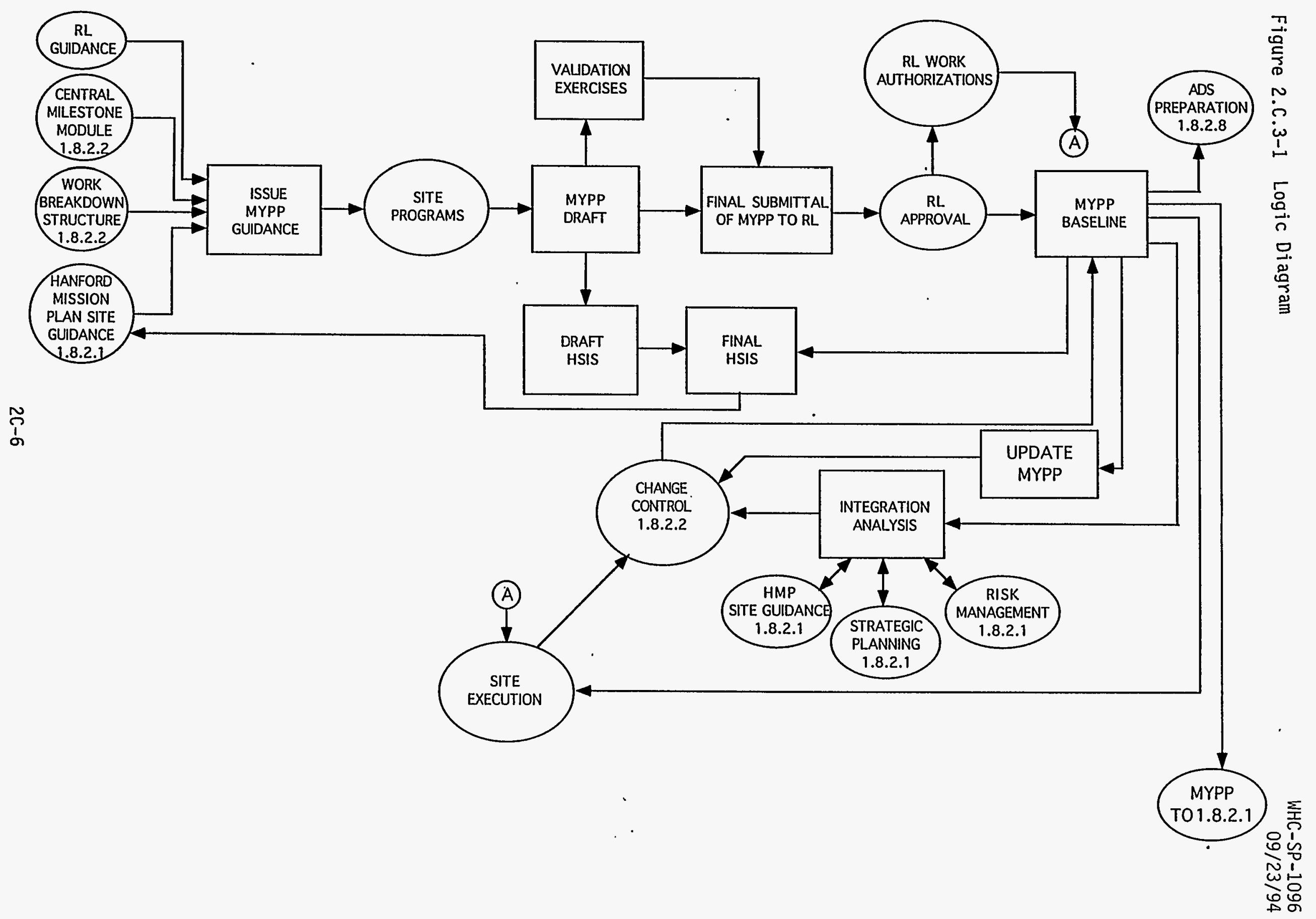




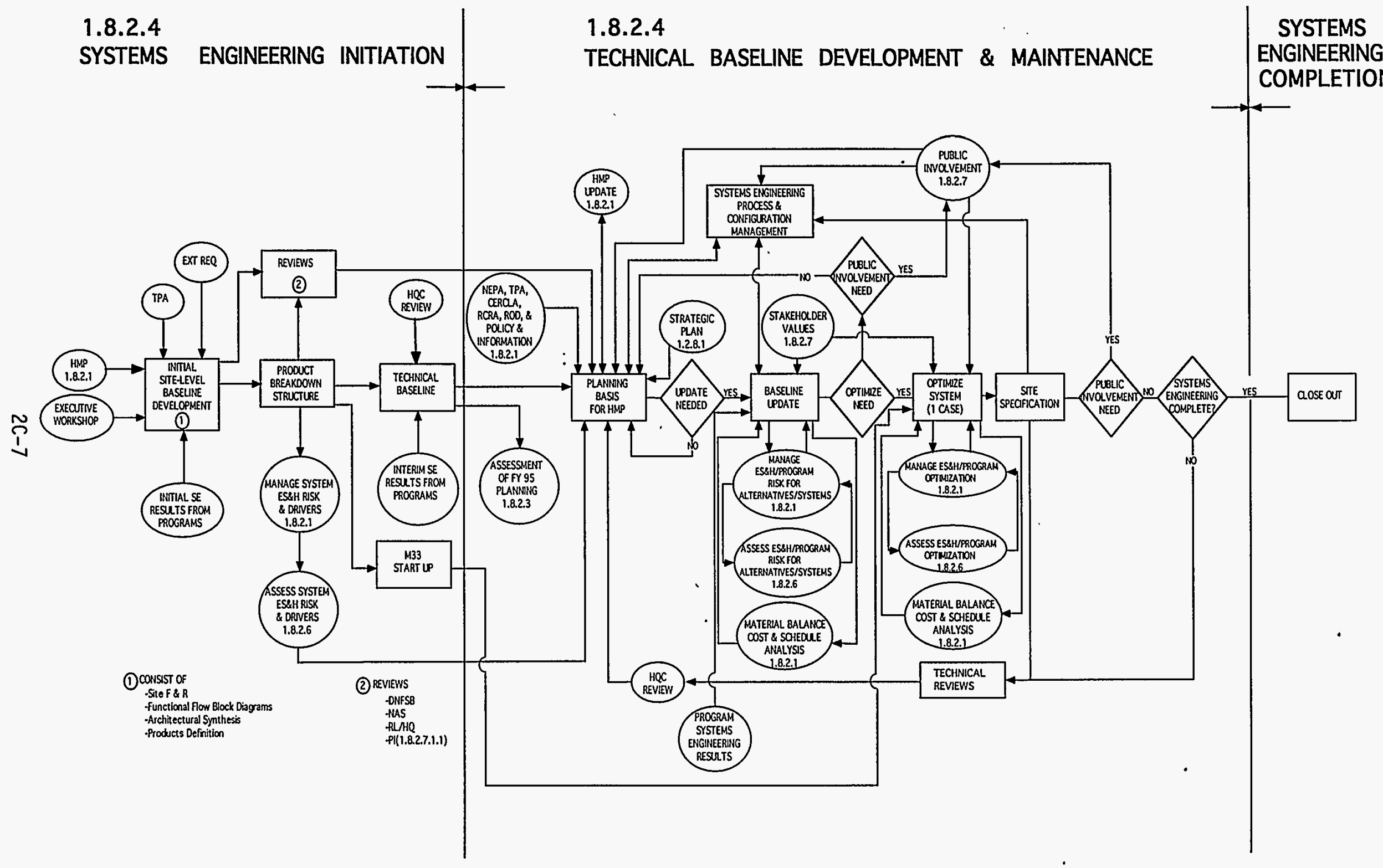

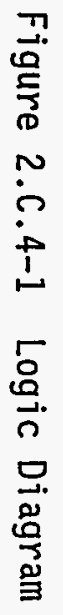

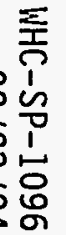




\section{PROGRAM MANAGEMENT $\quad \mathbf{1} \mathbf{8 . 2 . 5}$}

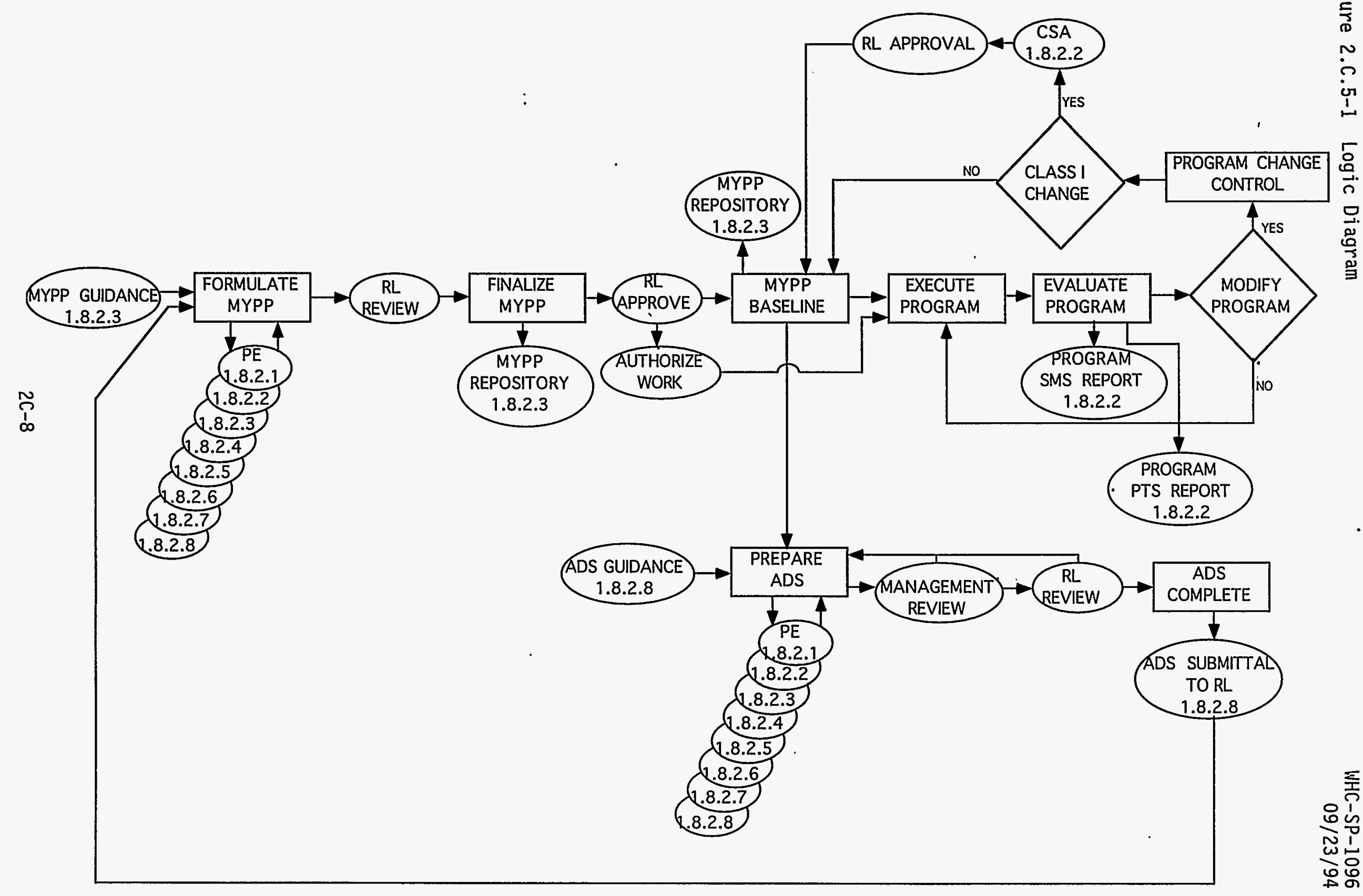


Fig. 2.C.6-1 Logic Diagram

RISK ASSESS

1.8.2.6

$7 / 27 / 94$

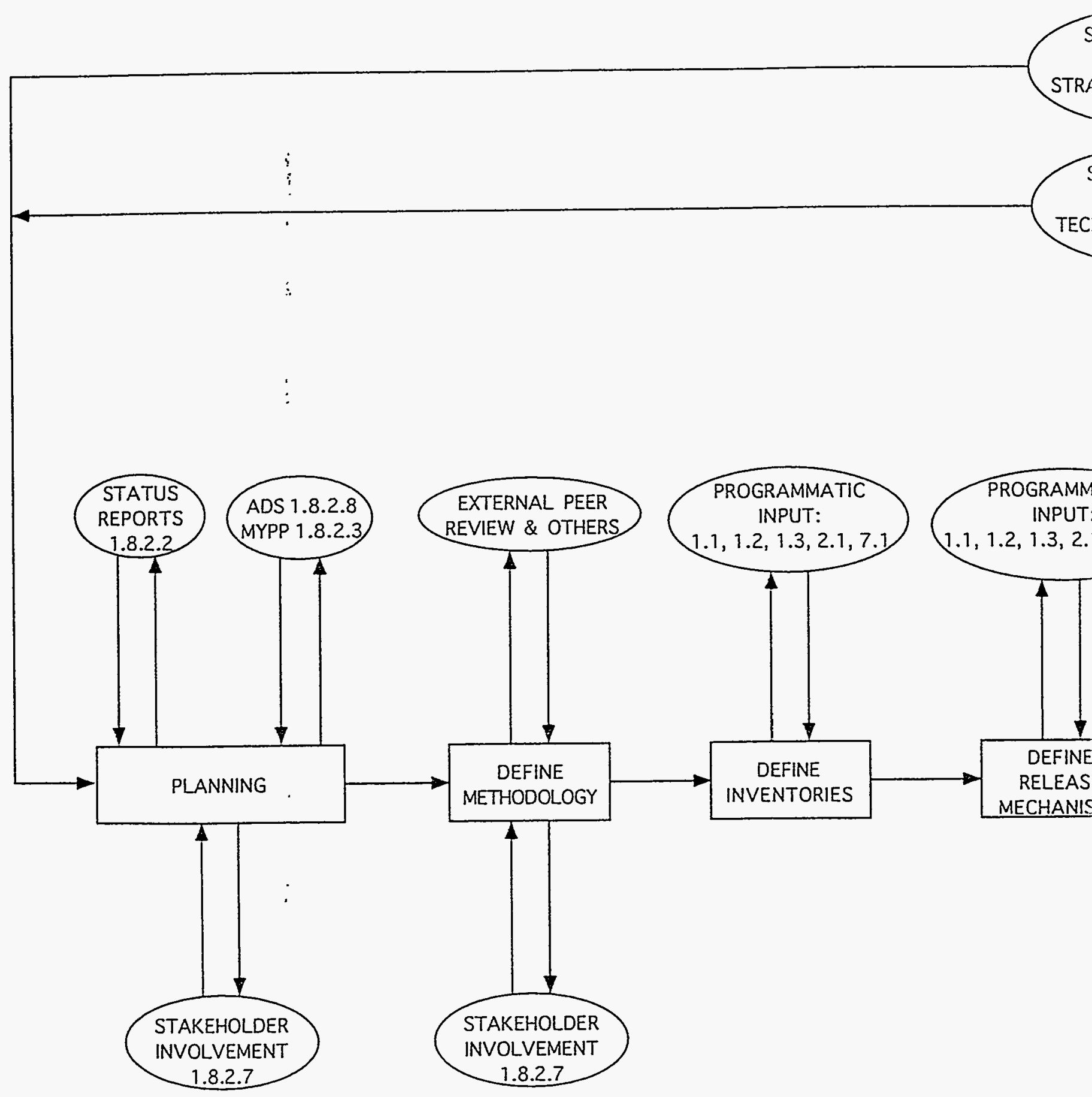




\section{ENT LOGIC/INTERFACES}

\section{EMENT OF OBLEM: \\ IC PLANNING \\ 1.8.2.1 \\ TEMENT OF \\ ROBLEM: \\ CAL BASELINE}

1.8.2.4

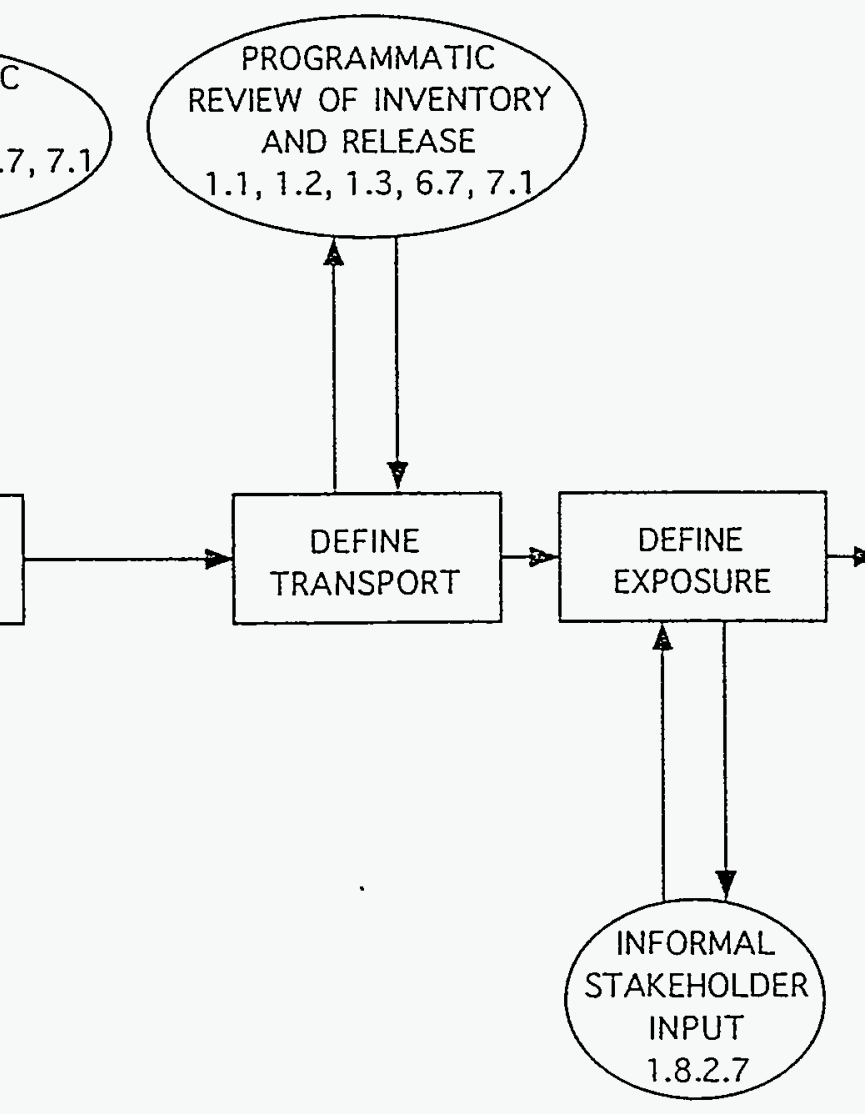

PEVIOGRAMMATIC

AND RELEASE

$1.1,1.2,1.3,6.7,7.1$
MANAGEMENT

EXTERNAL PEER

REVIEW

1.0, 2.0, 6.0, 7.0.

OTHERS

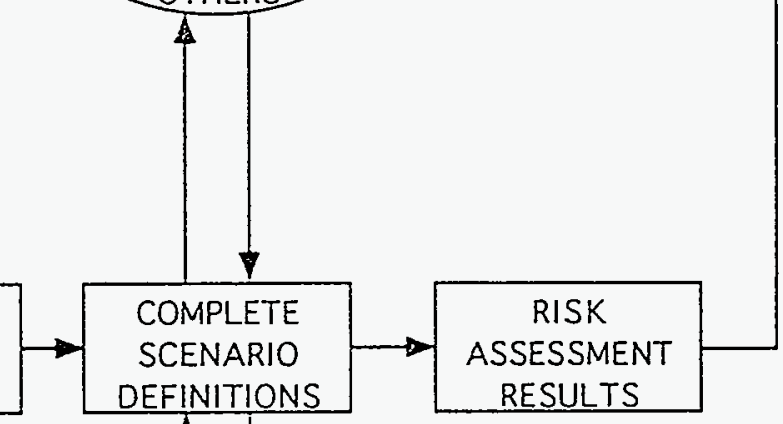




\section{PUBLIC INVOLVEMENT 1.8.2.7 (PAGE 1 OF 2) $8 / 3 / 94$}

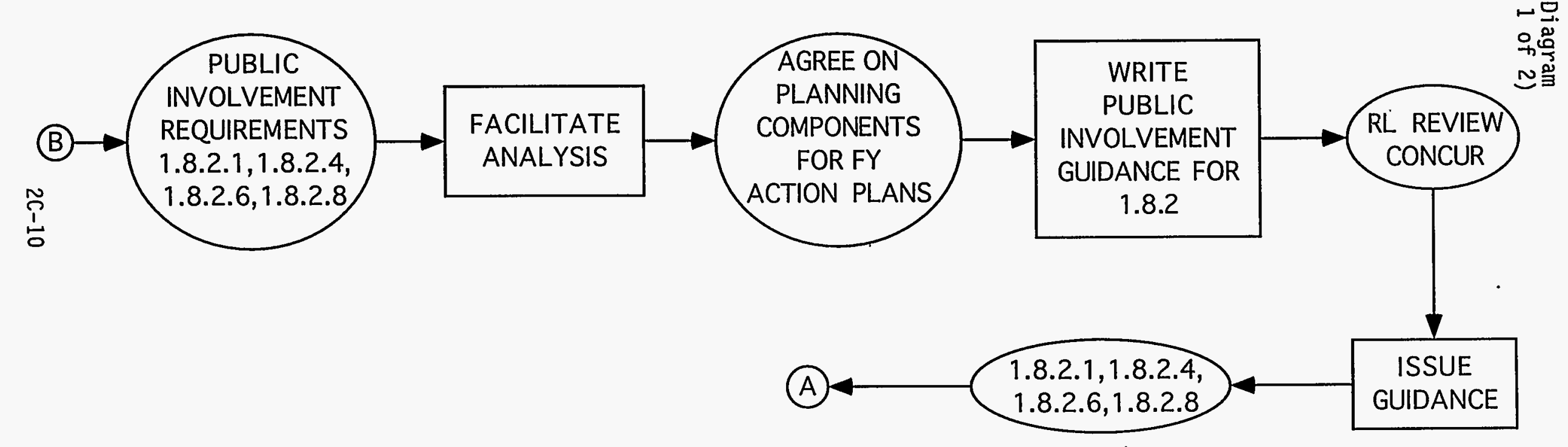


PUBLIC INVOLVEMENT 1.8.2.7 (PAGE 2 OF 2) 8/4/94

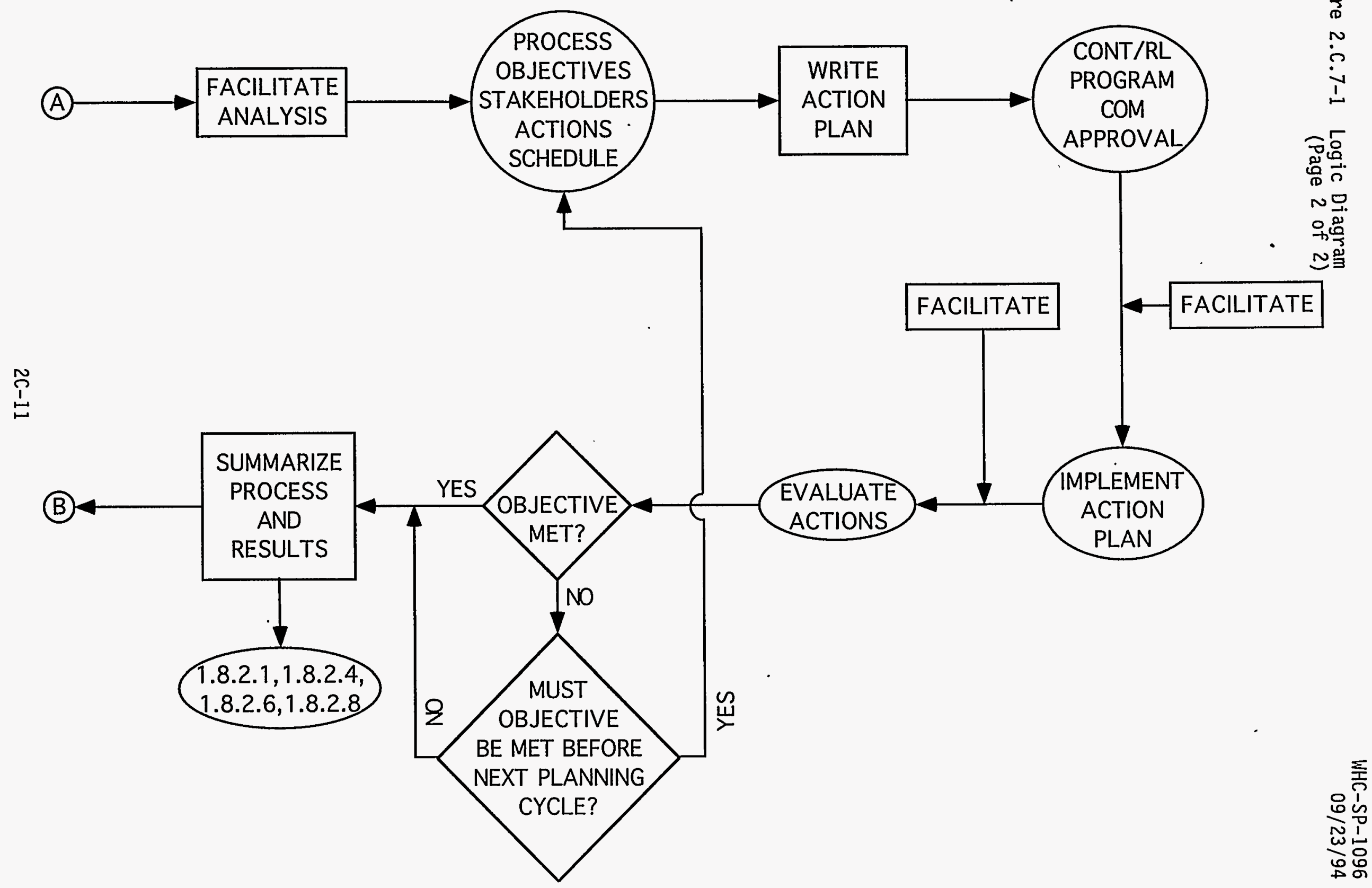




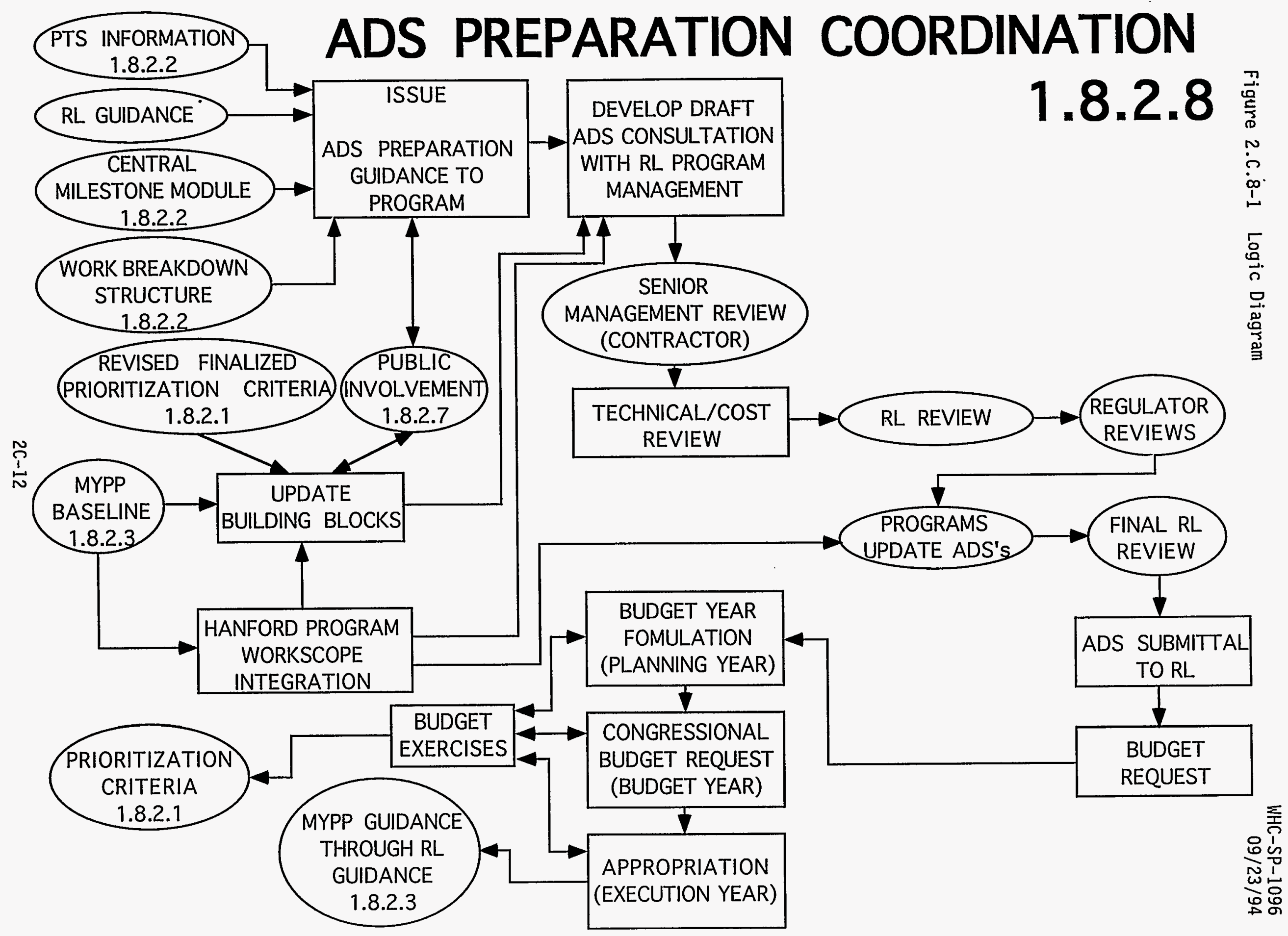




\section{D PROGRAM MASTER BASELINE SCHEDULE}

The approved Program Master Baseline Schedule is the RL program basel ine schedule, developed for and incorporated into the program's FYWP, to become the FY performance schedule baseline. The Program Master Baseline Schedule (PMBS) is developed at the Program level, with activities defined at the Program element leve1, and is based upon the MYPP. A71 Program DOE-HQ and $\mathrm{RL}$ milestones shall be included in this schedule.

The PMBS for the Planning Integration Program is a summarization of the detailed program element schedules for the first three years of the plan, followed by four additional years at a much greater level of summarization. Over the first three years, a11 RL and DOE-HQ milestones are displayed. In the following summarized years, only significant RL and DOE-HQ milestones are depicted.

The PMBS for the Planning Integration Program is a reflection of the integration of the program elements into a network of logicaliy interfaced schedule activities. Due to the summarization, not all interfaces are shown on the PMBS. 


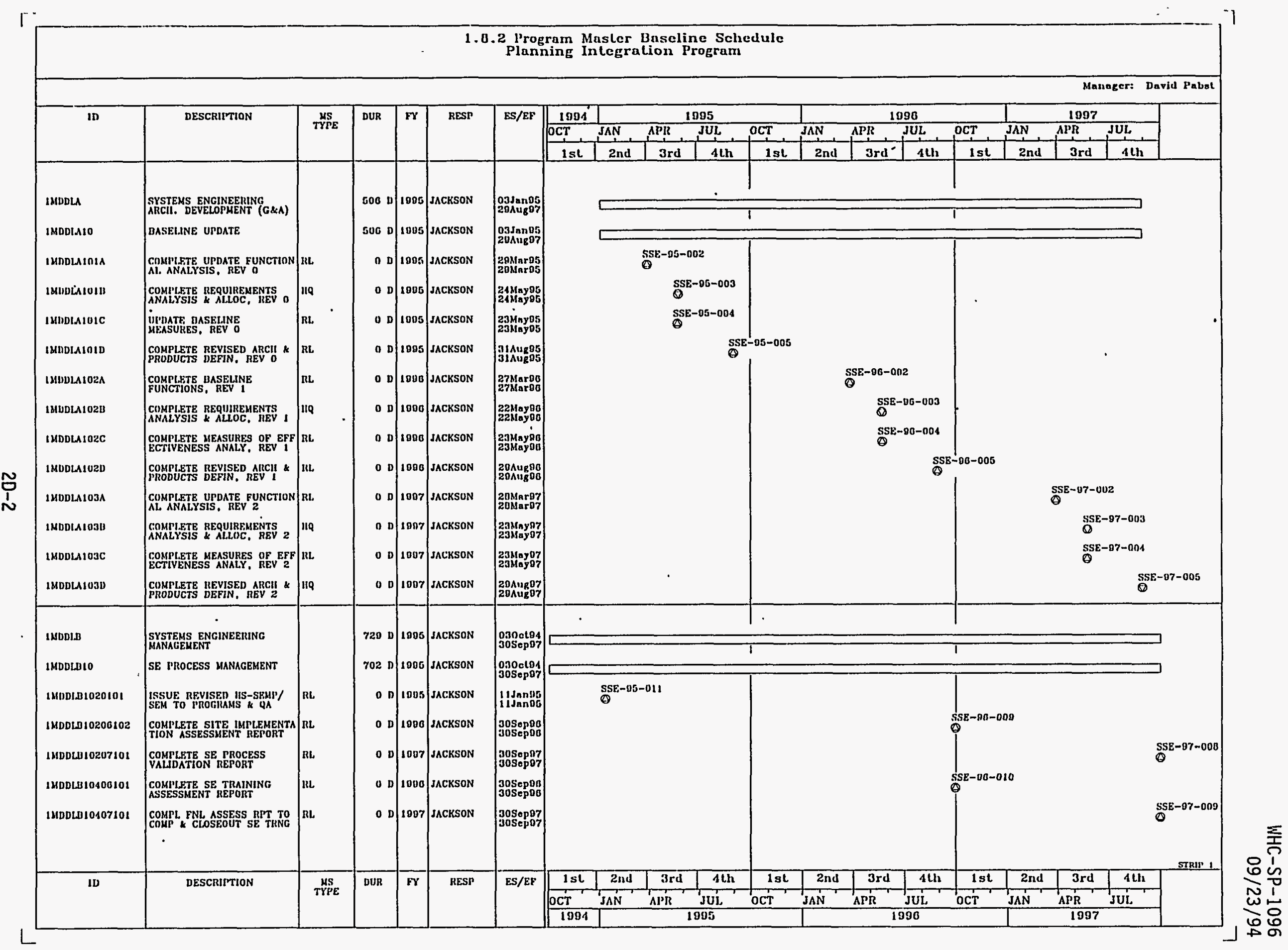




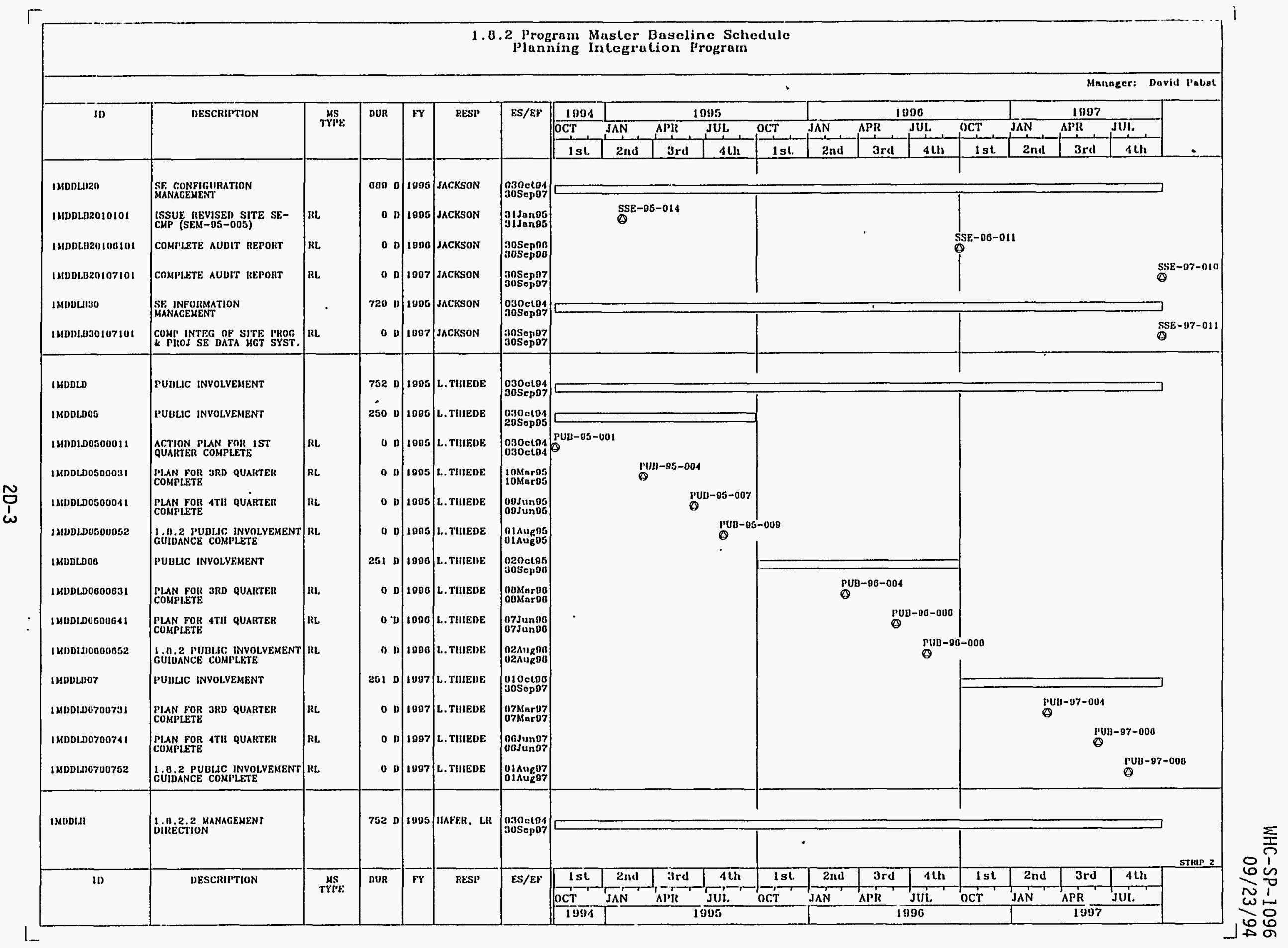




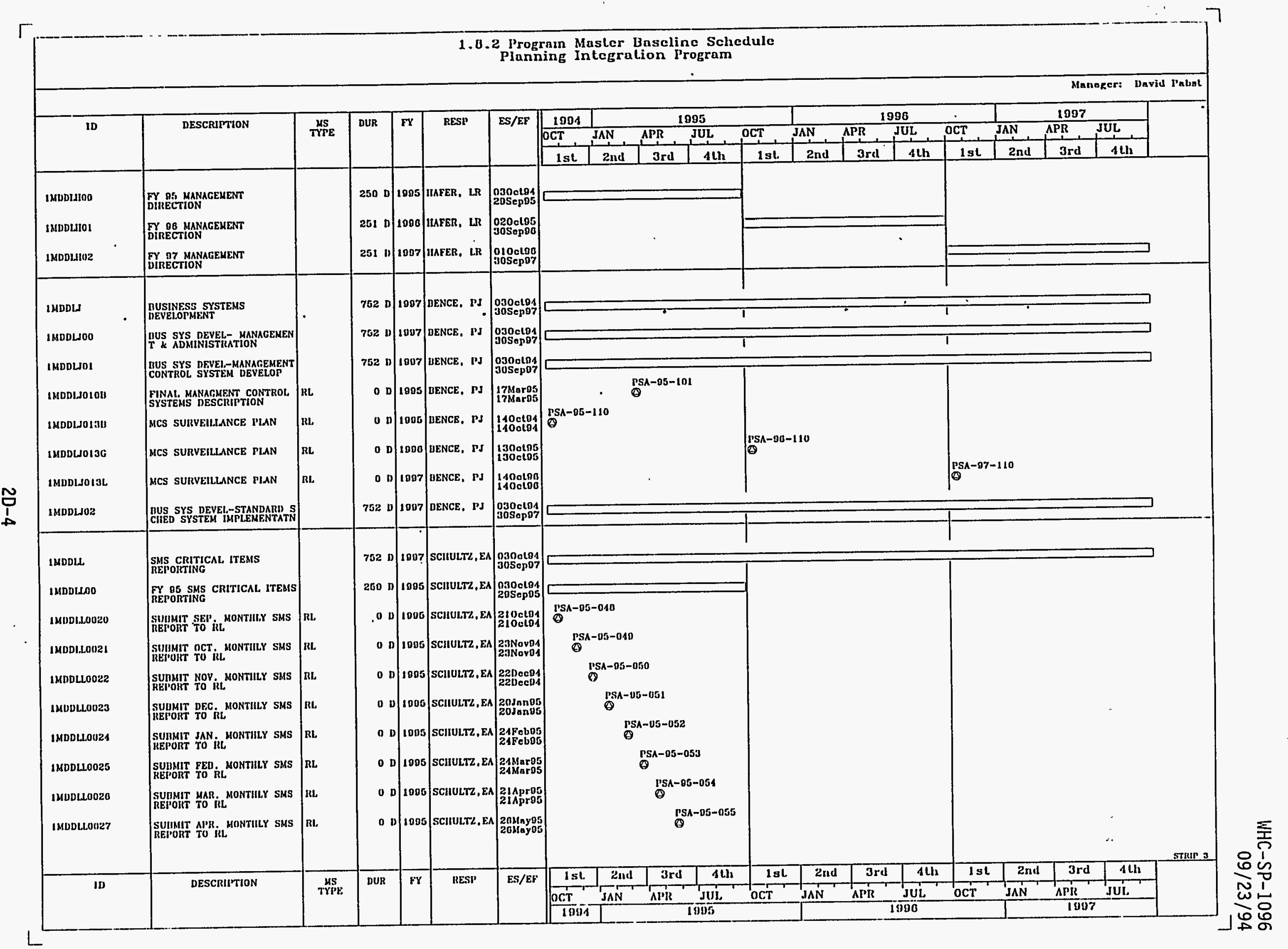




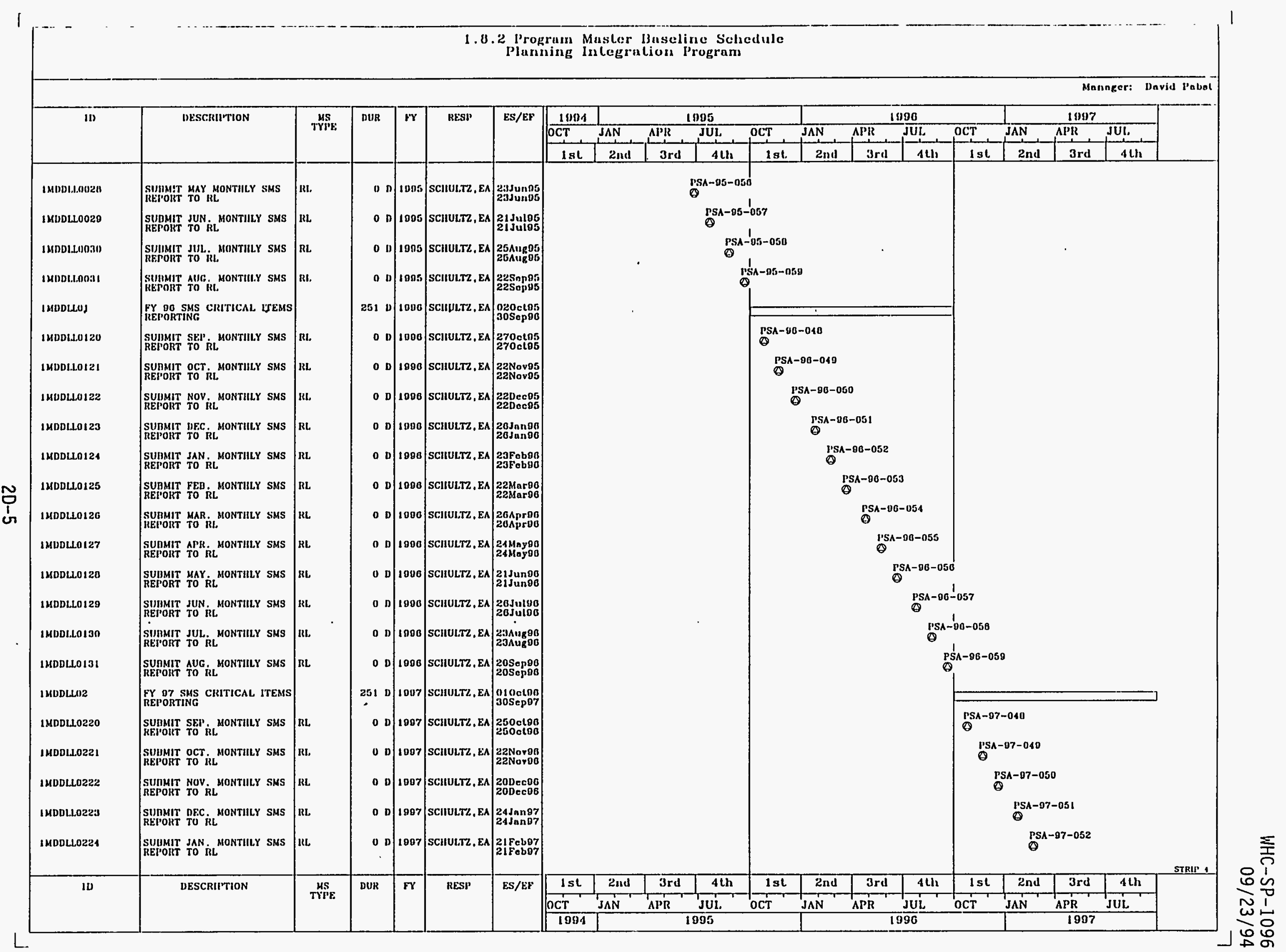




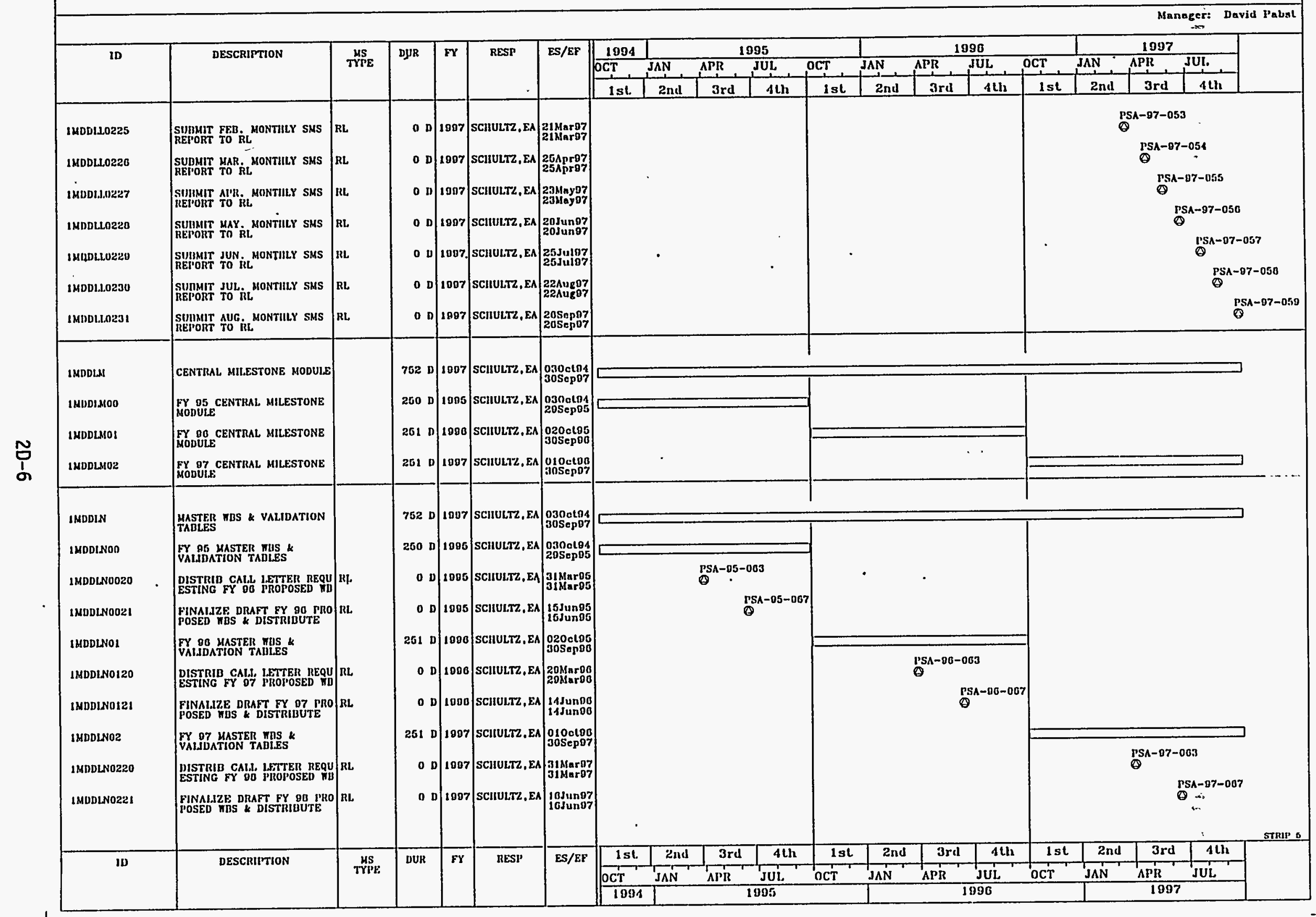

L- 


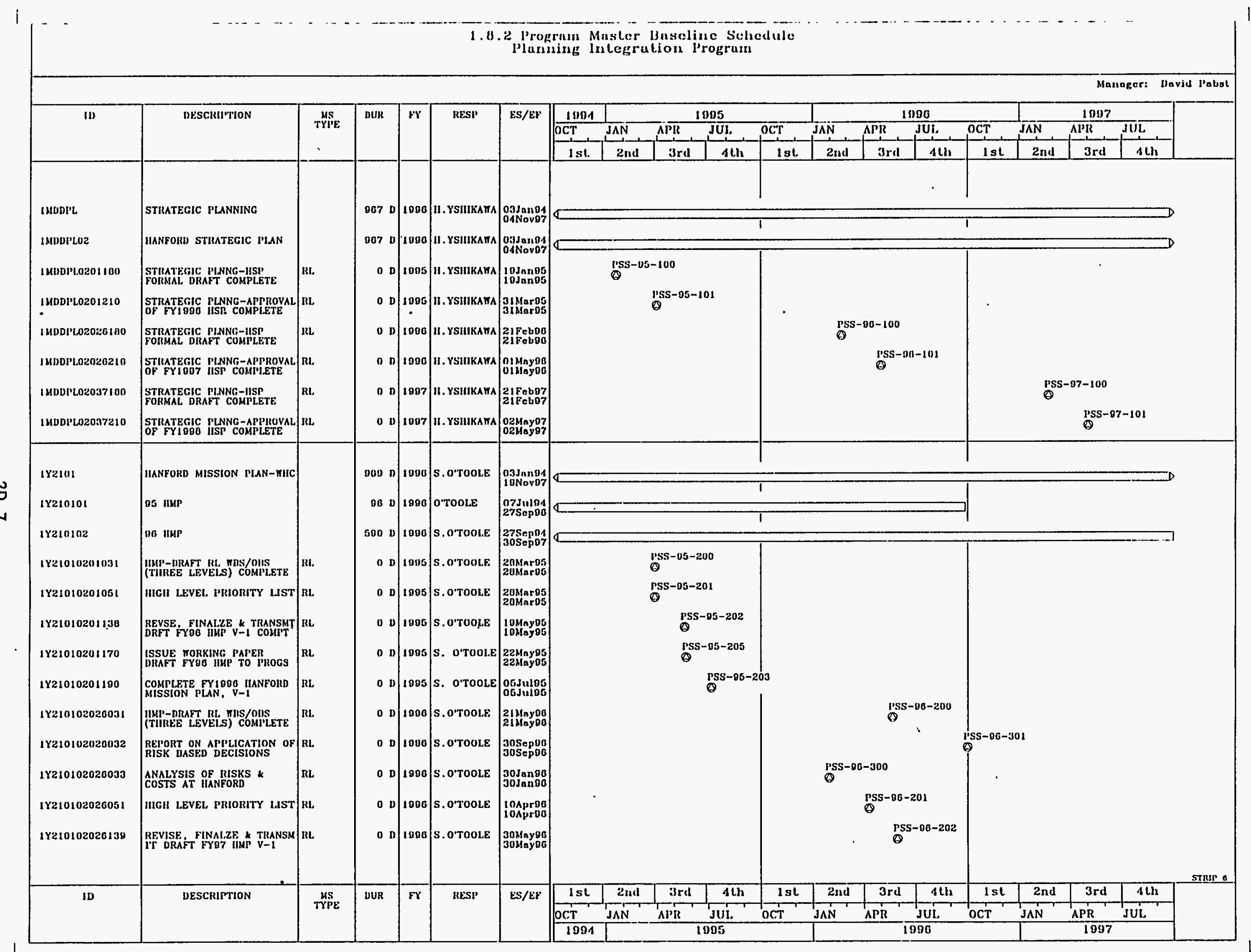

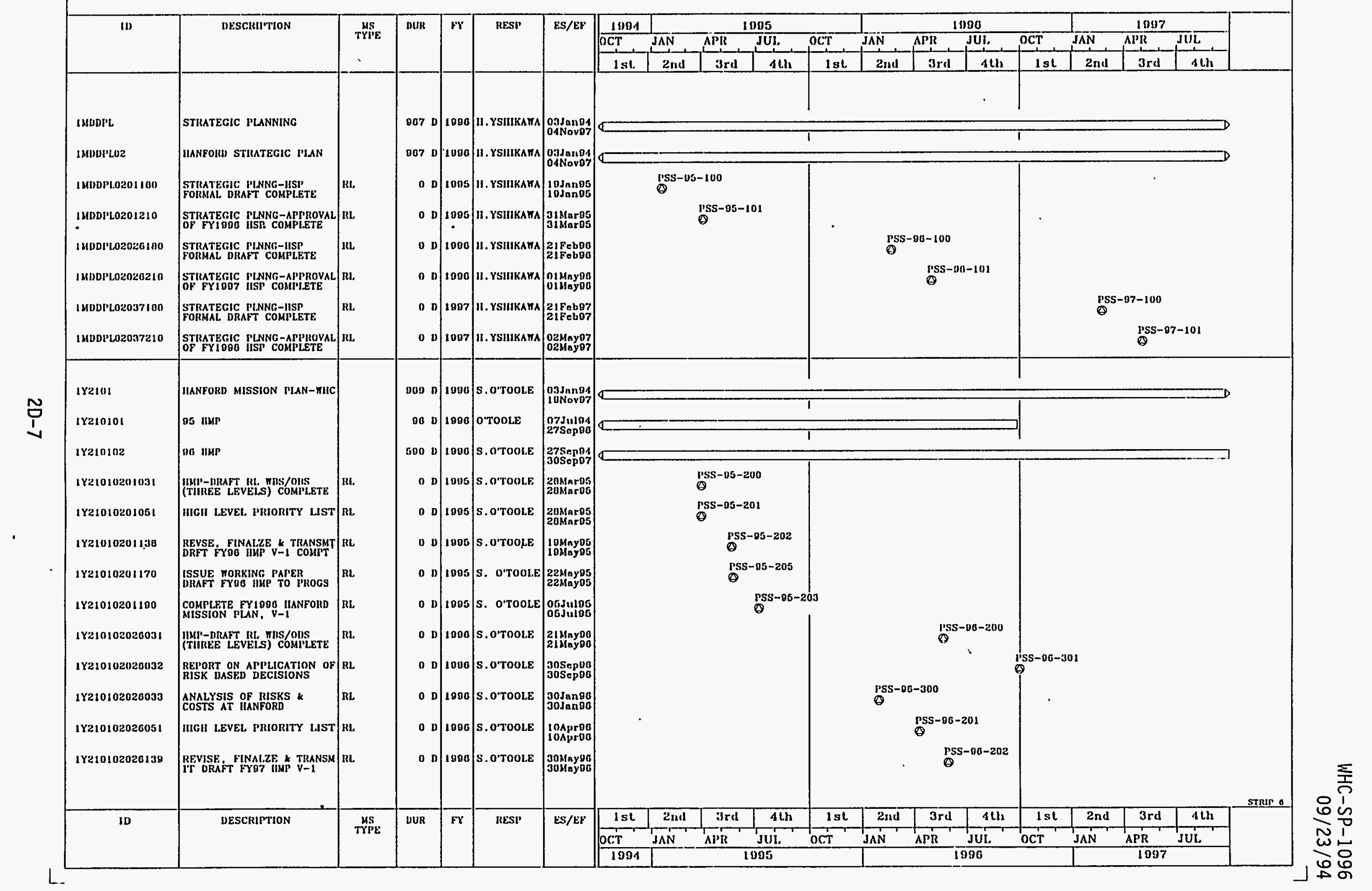




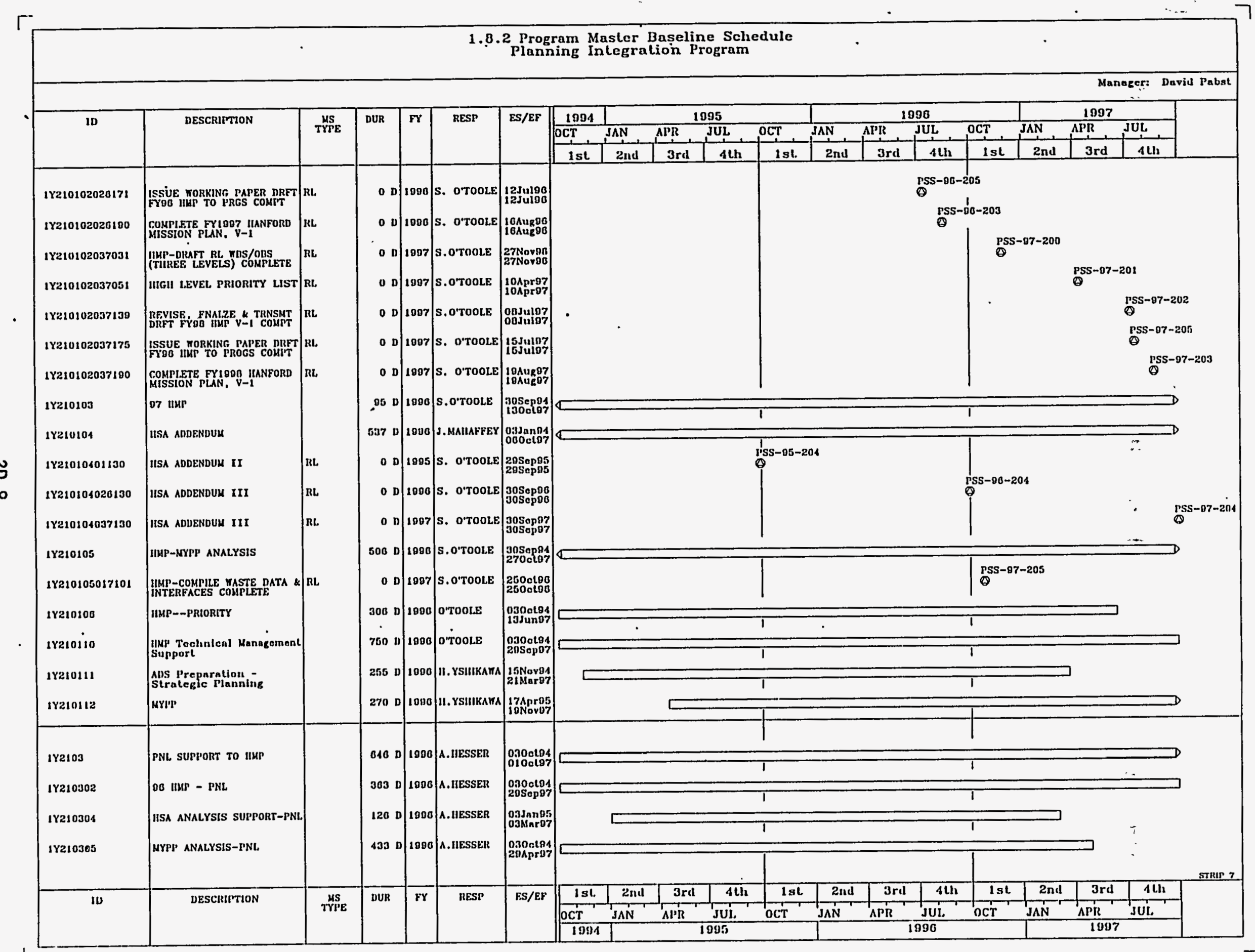




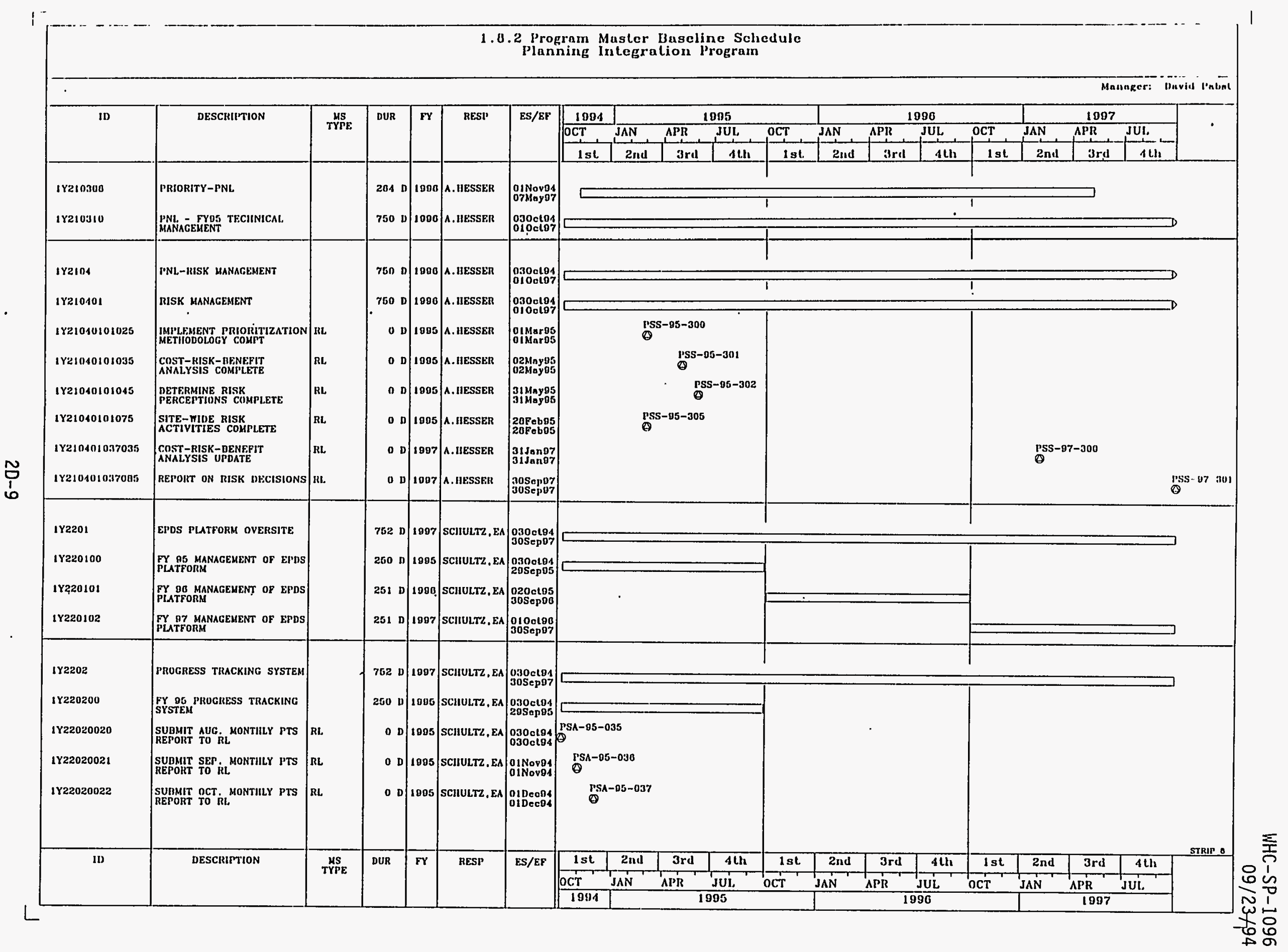




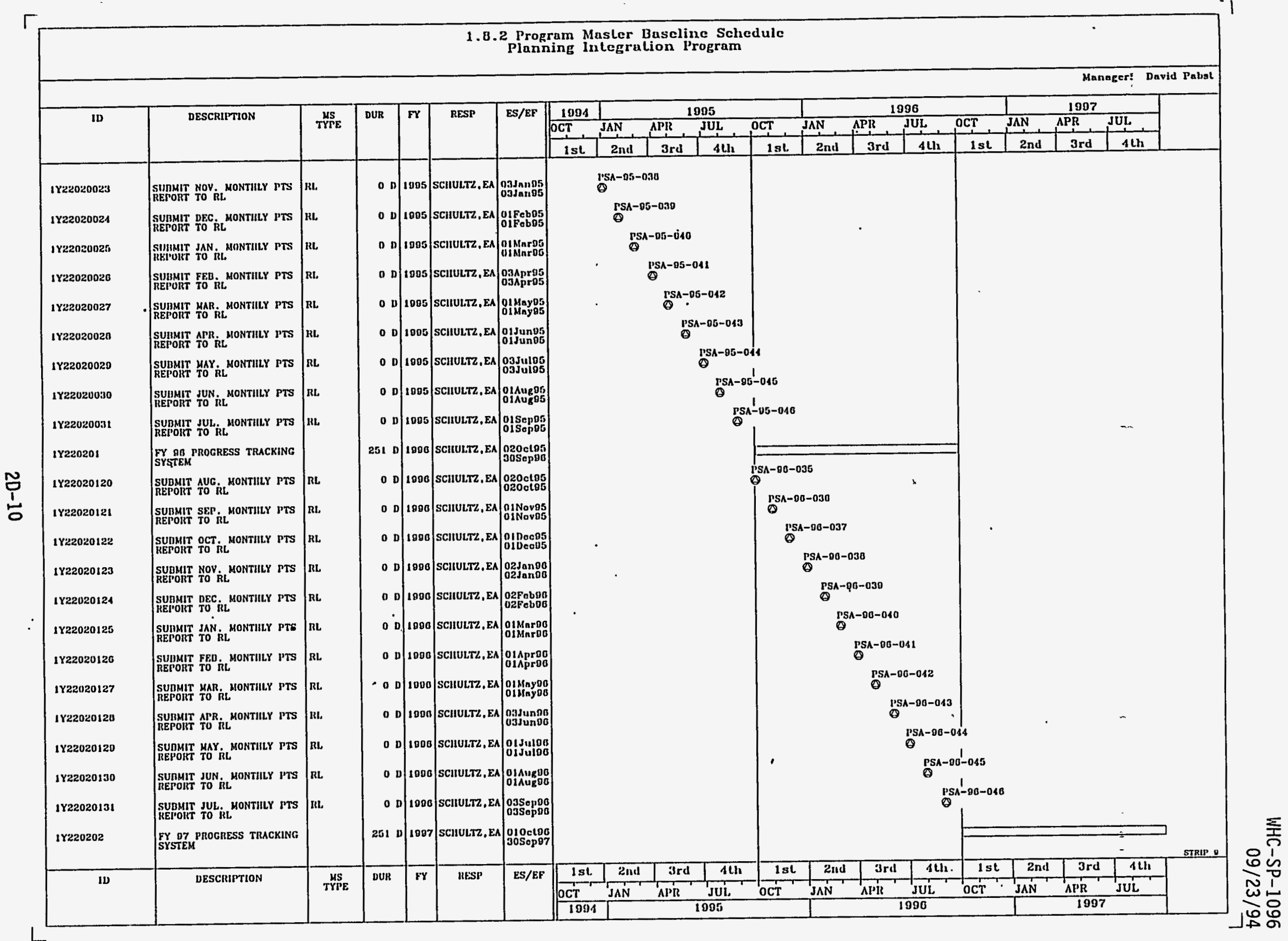




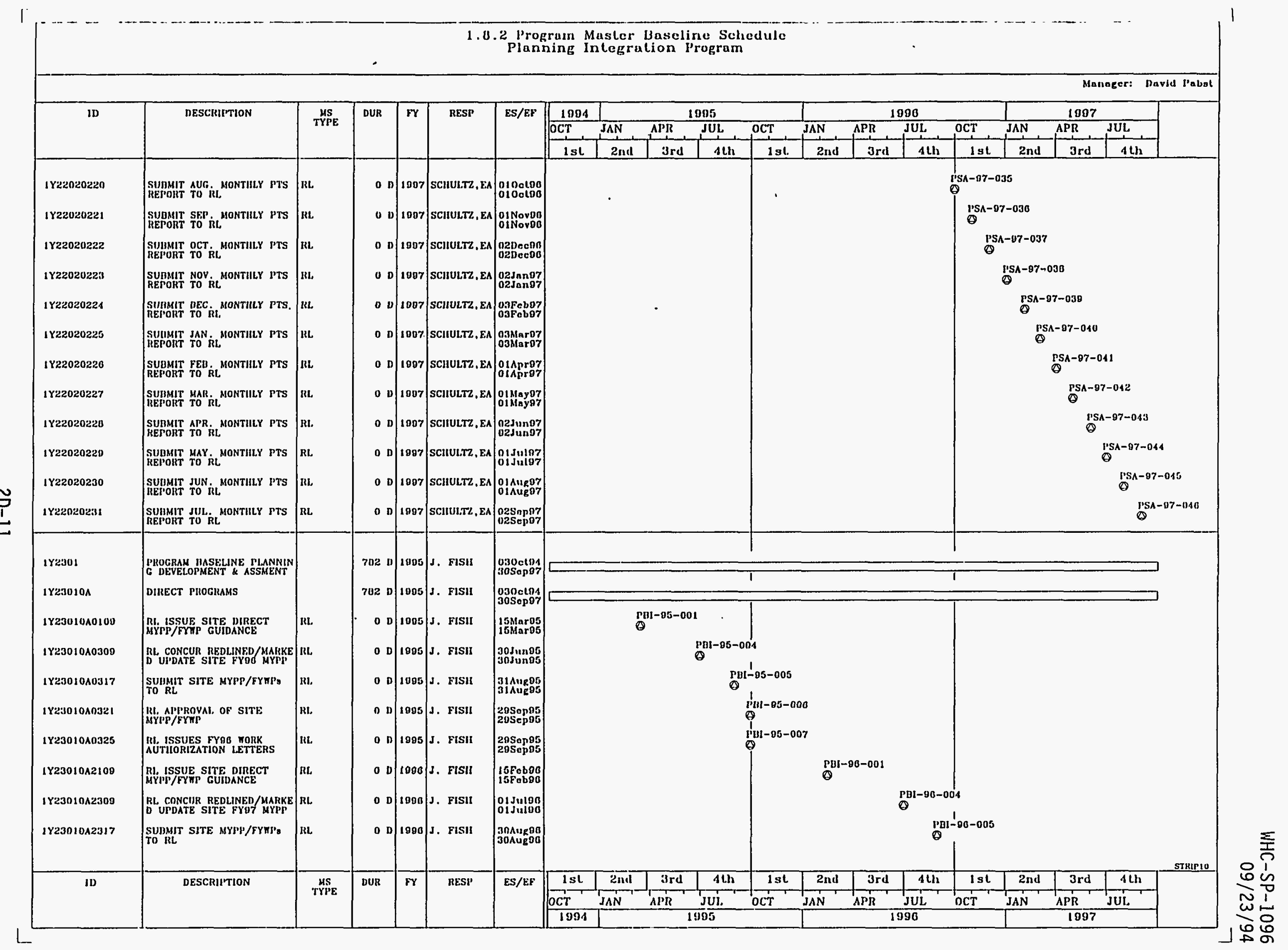




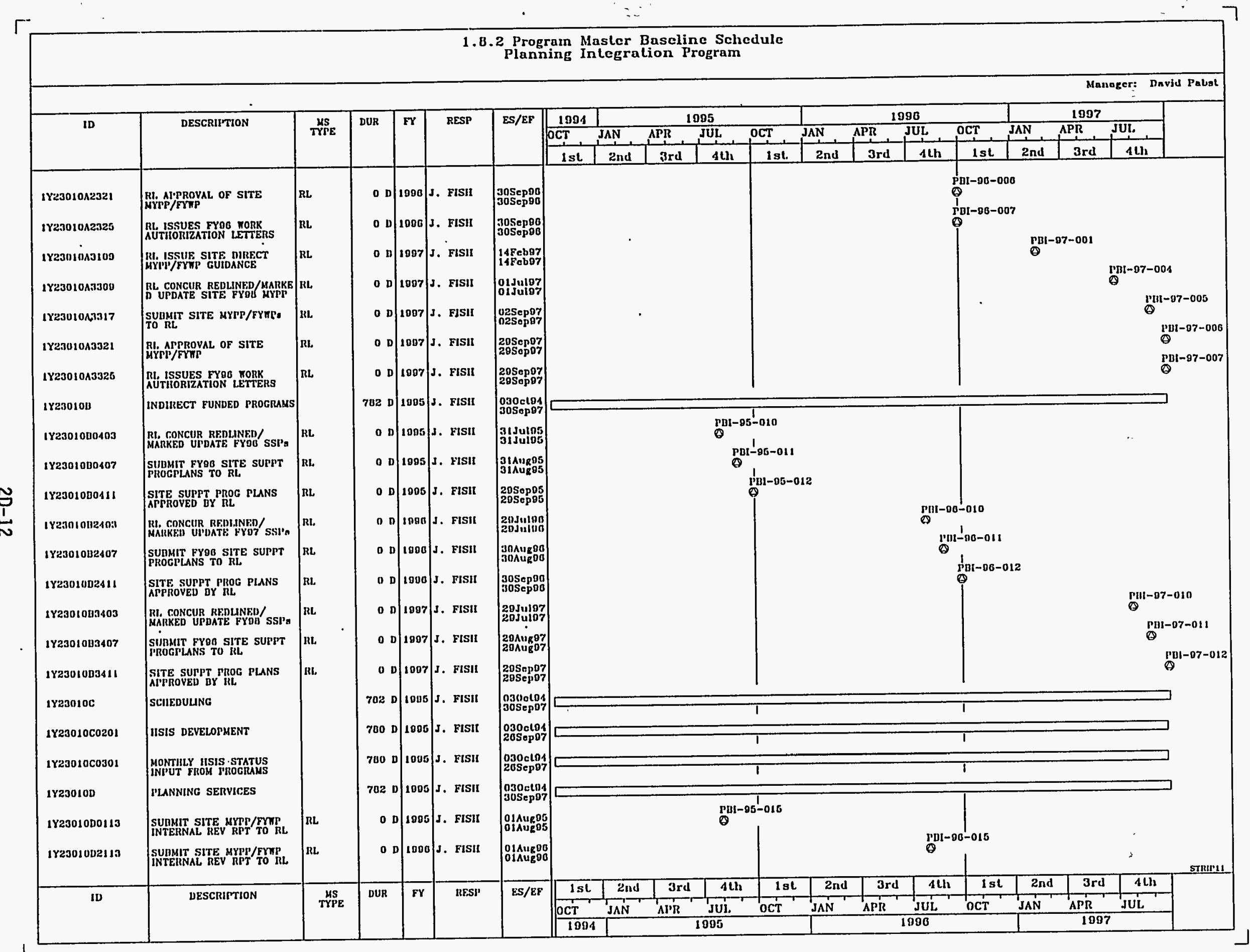




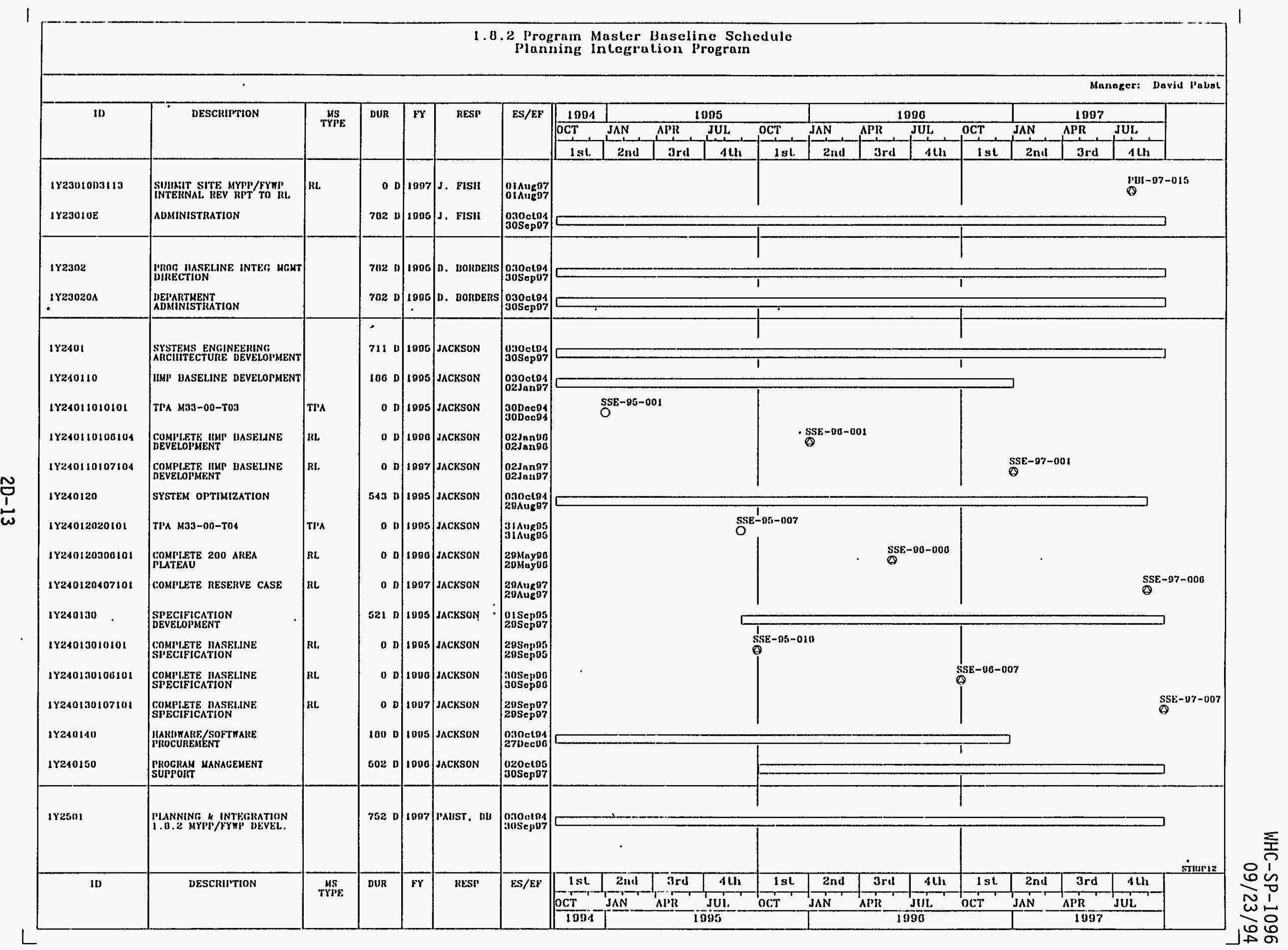




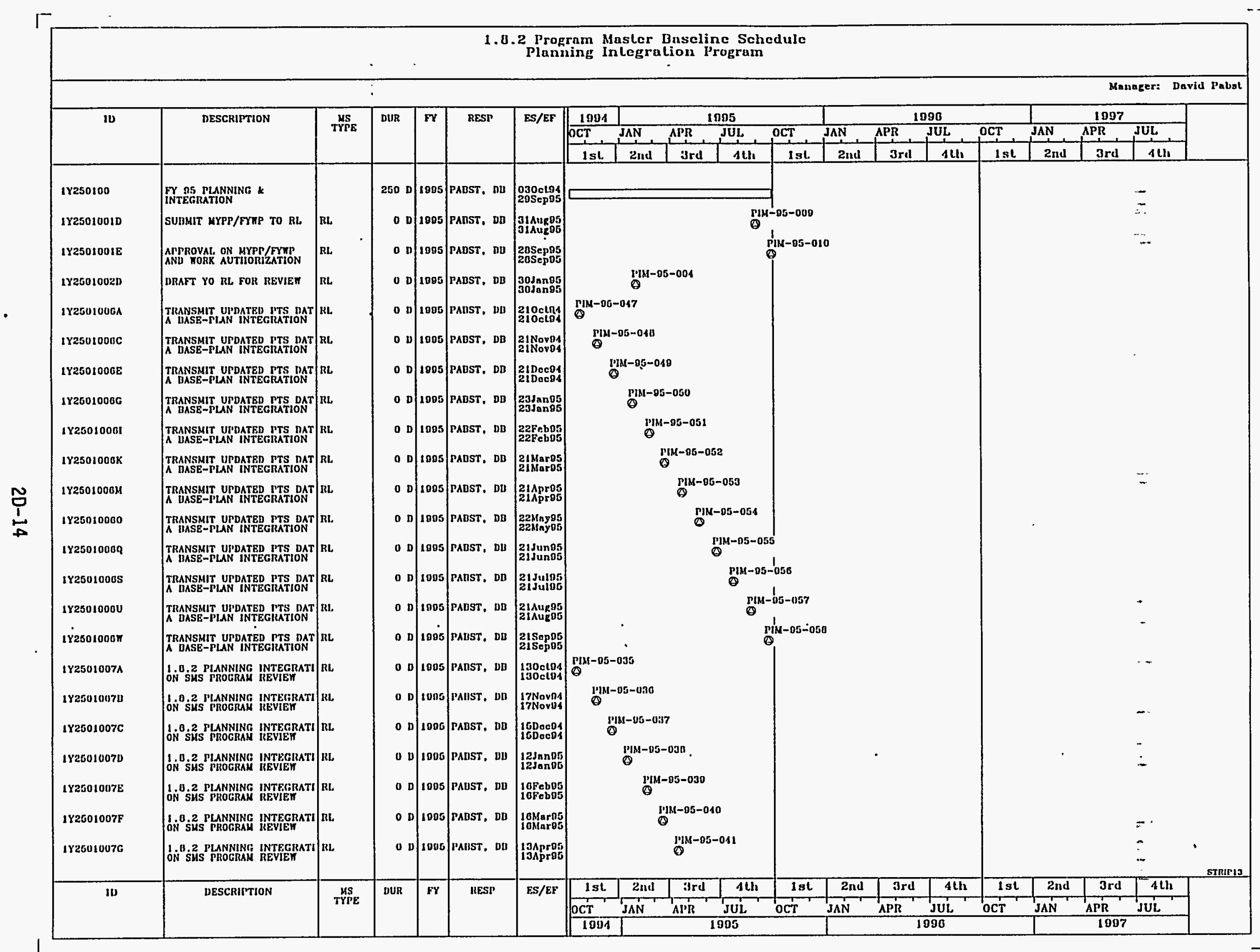

Program Master Baseline Schedule 


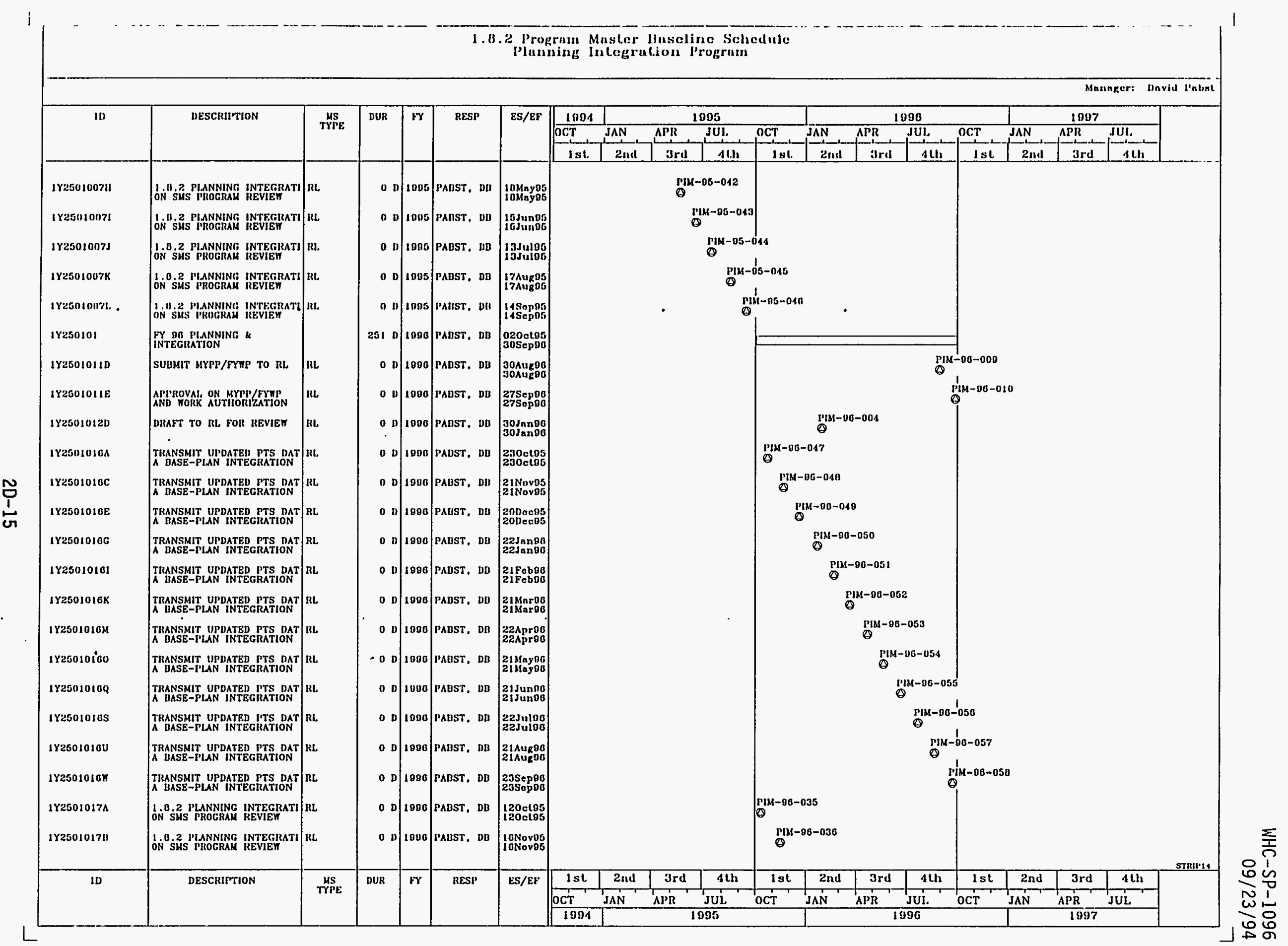




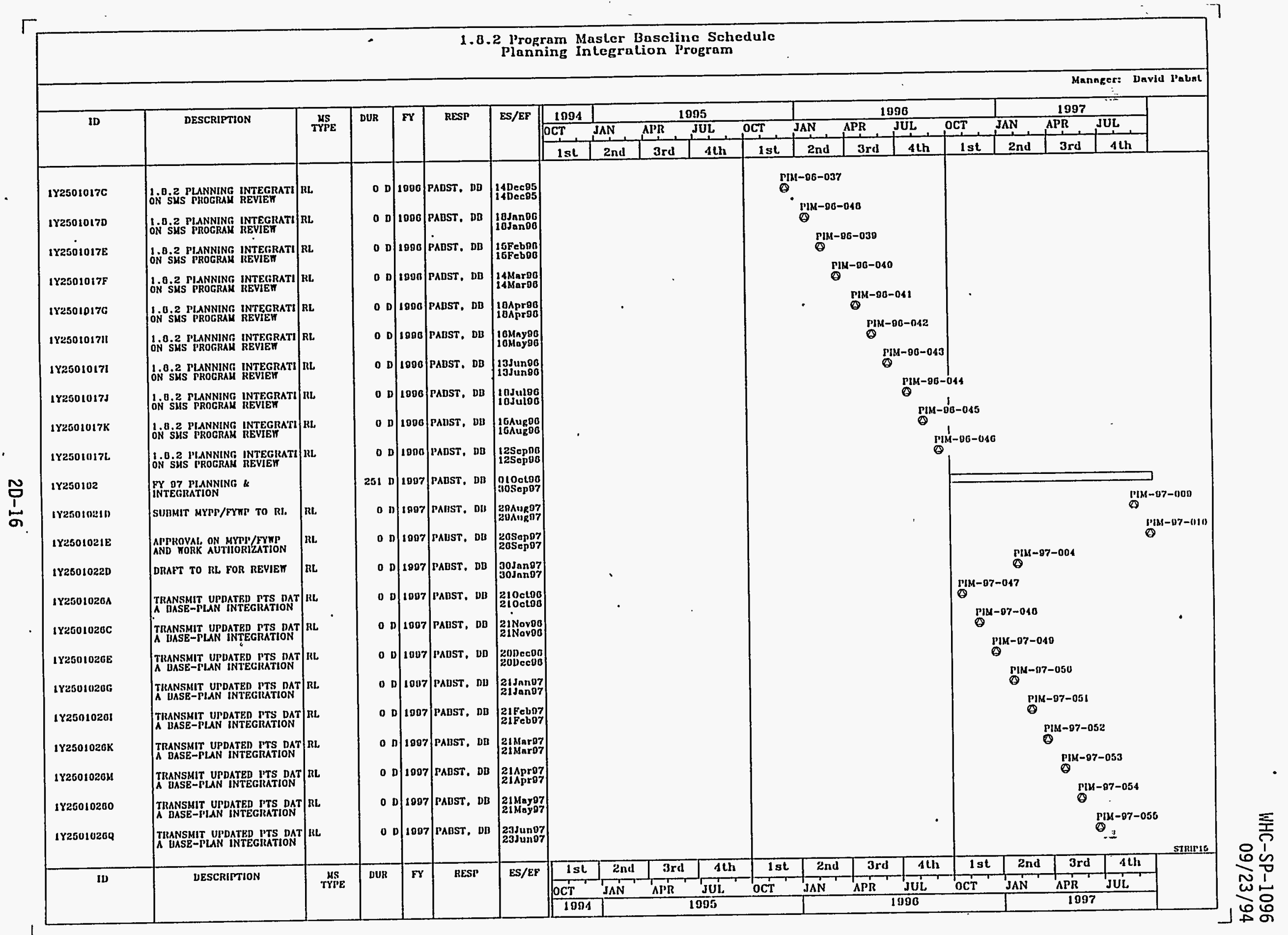




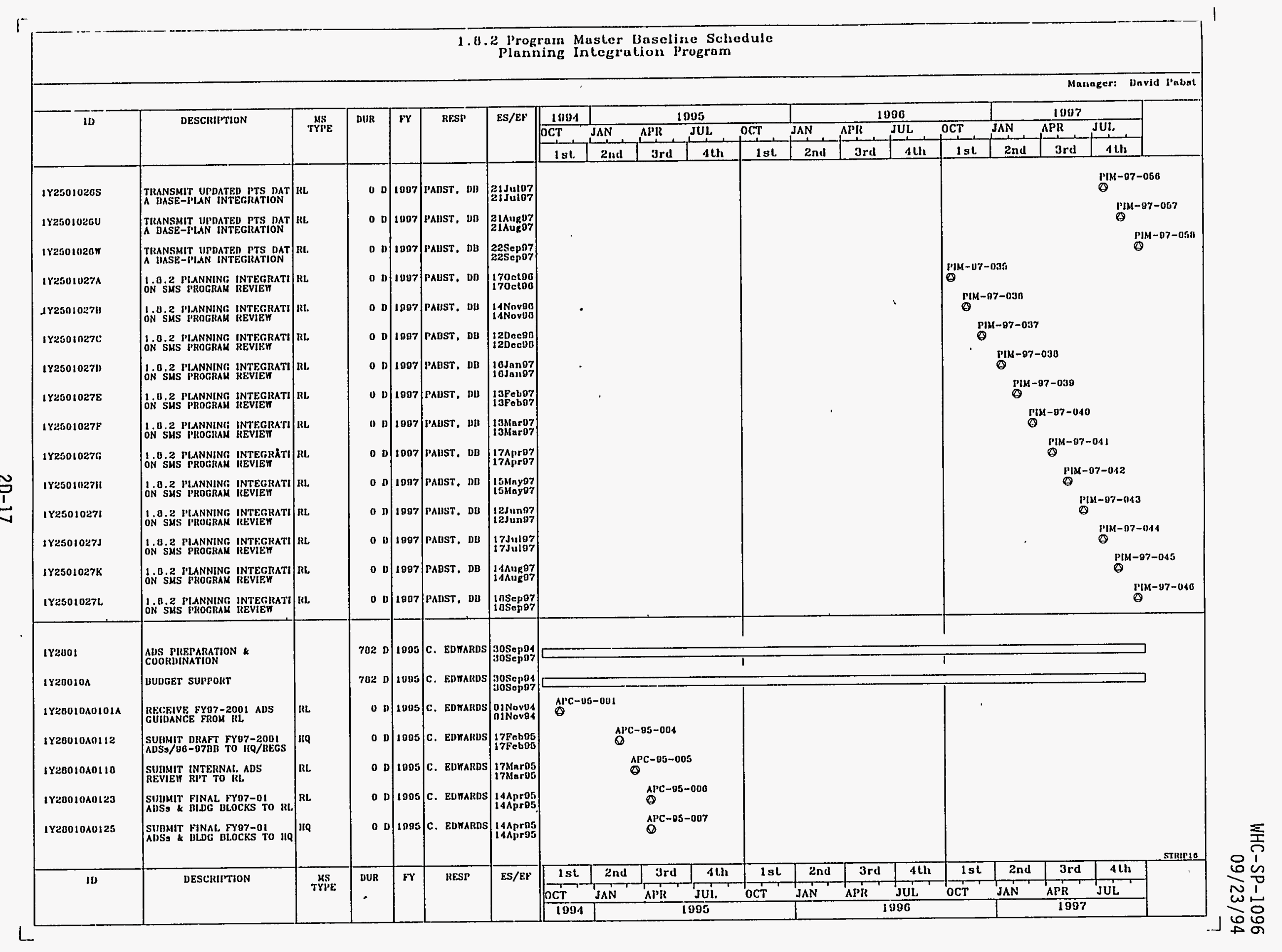




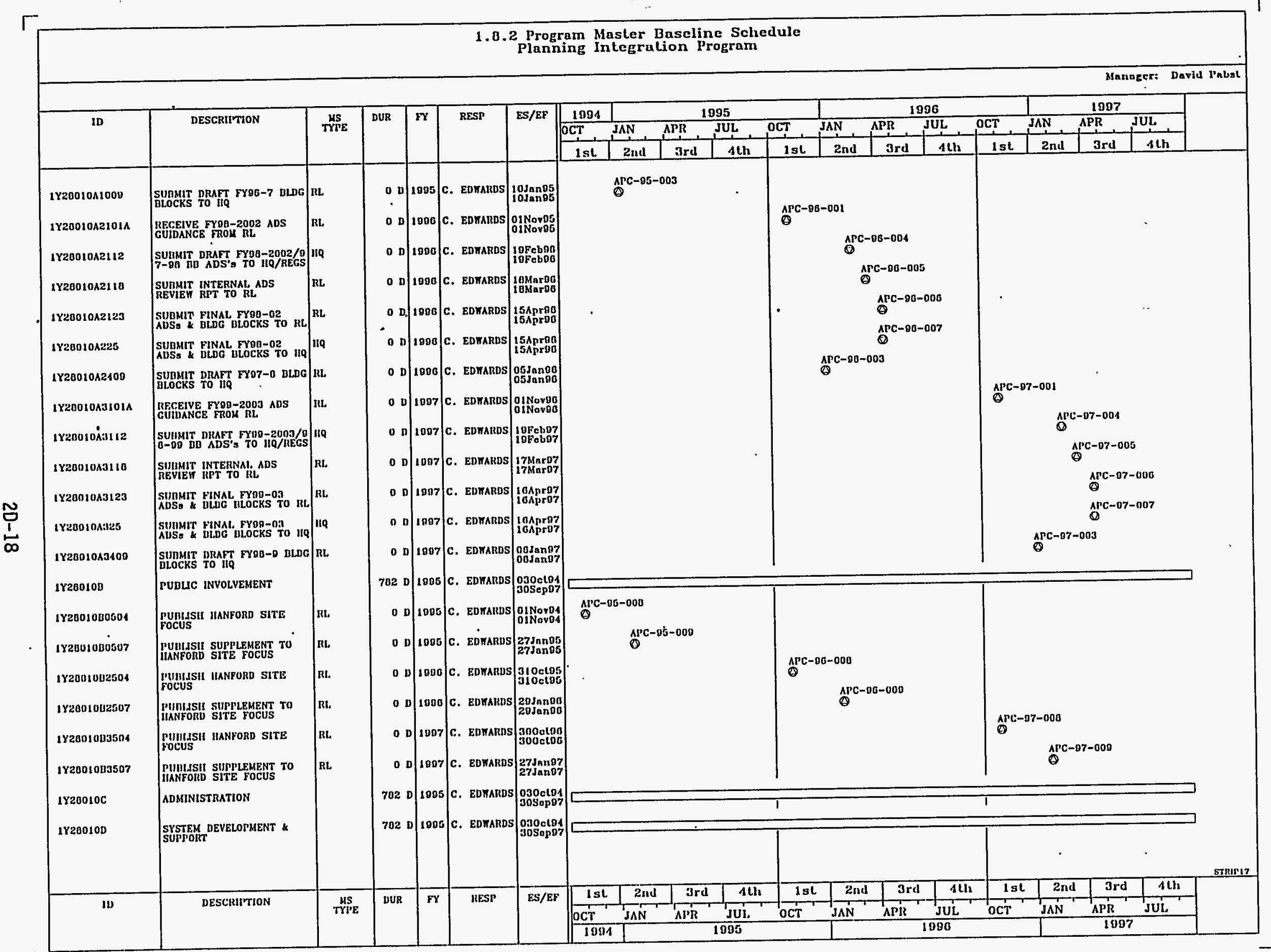




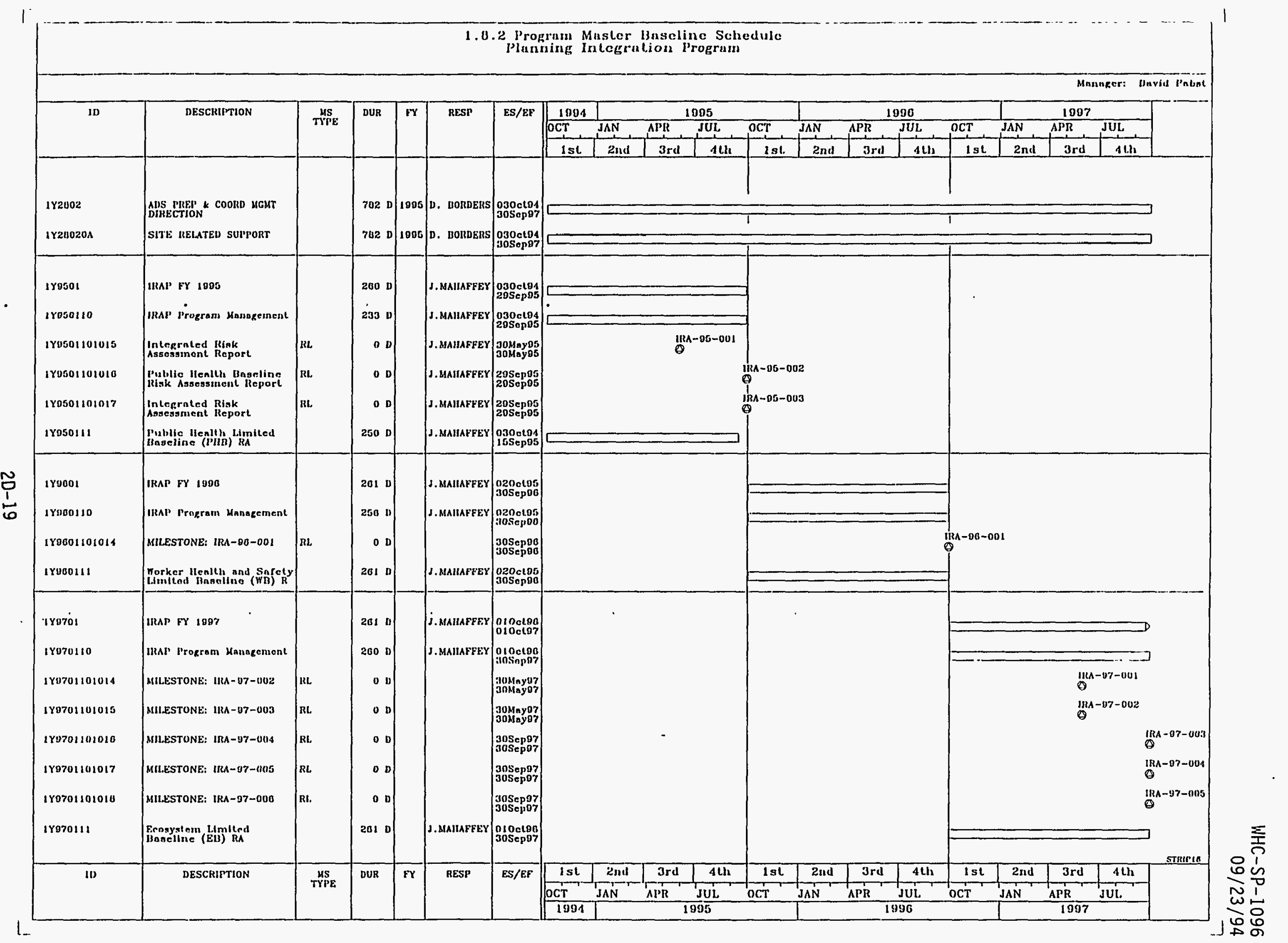




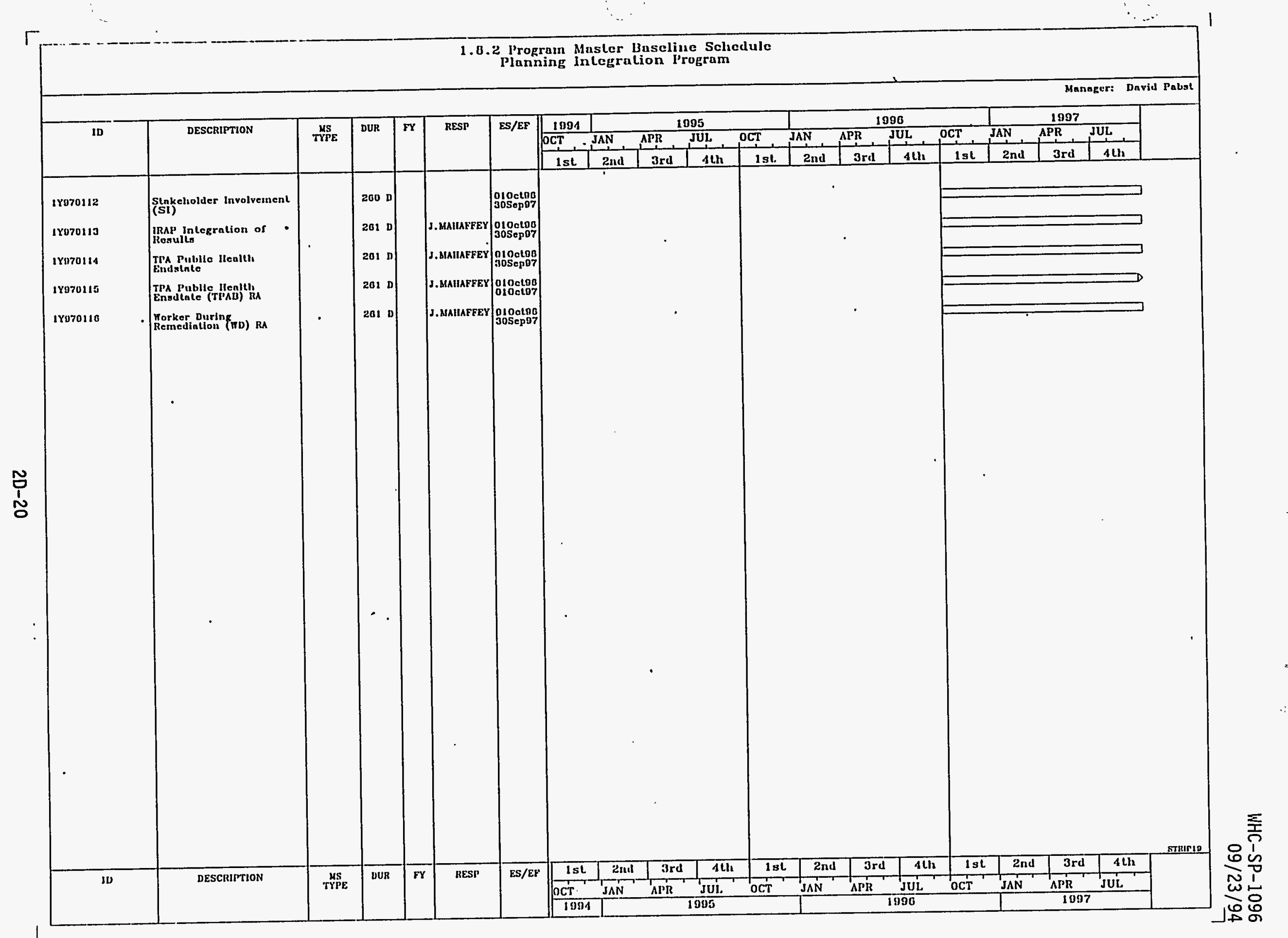




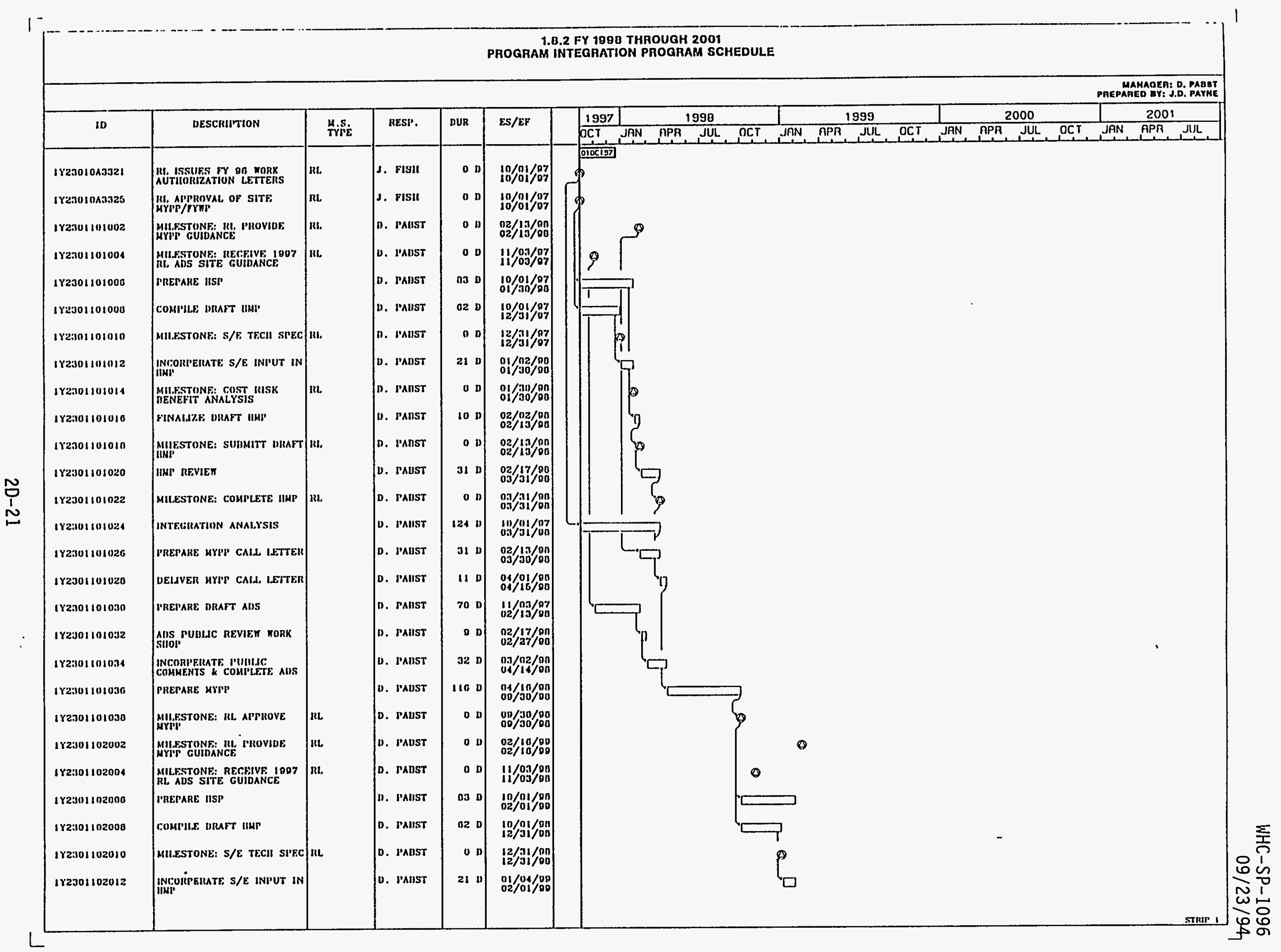




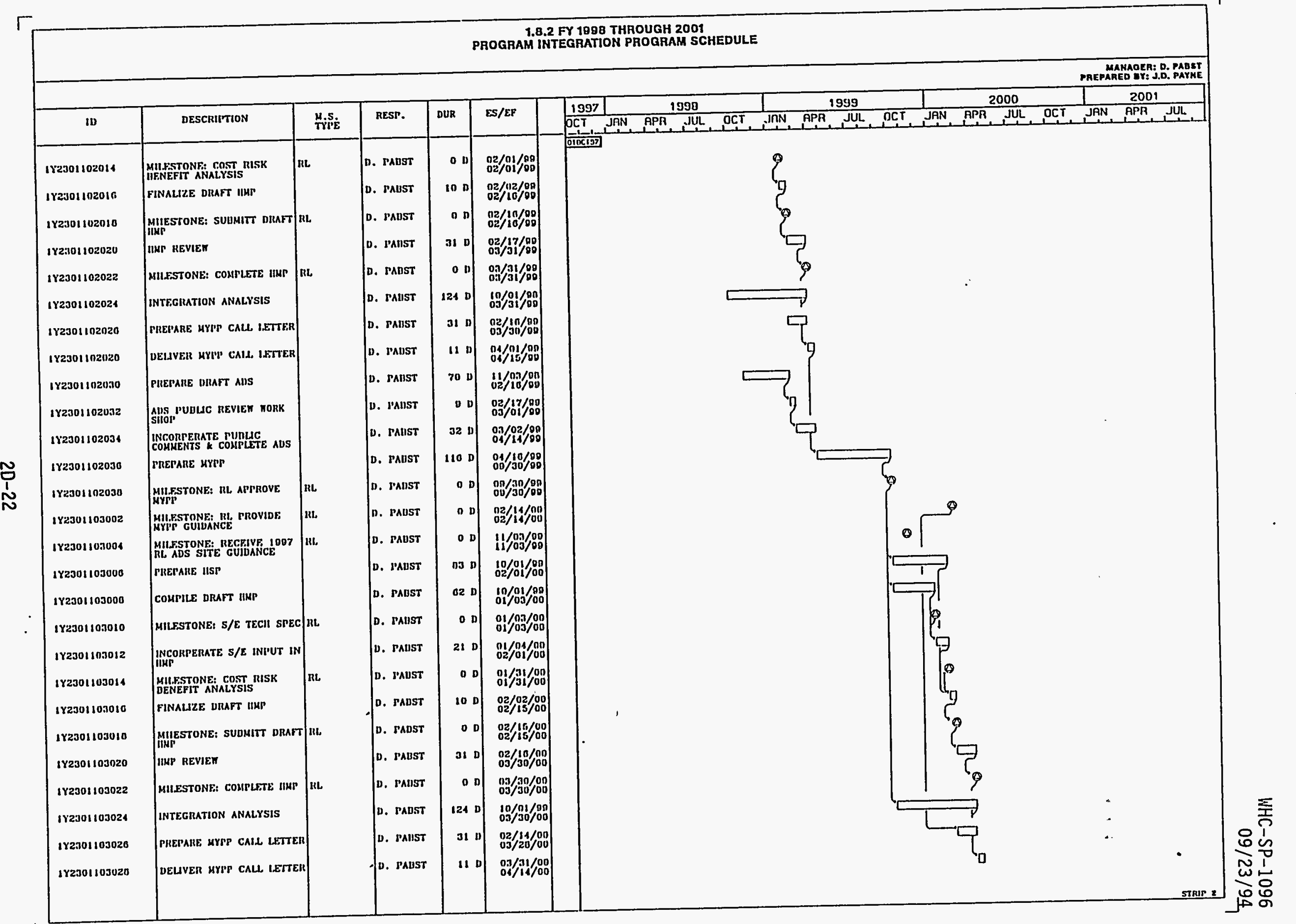




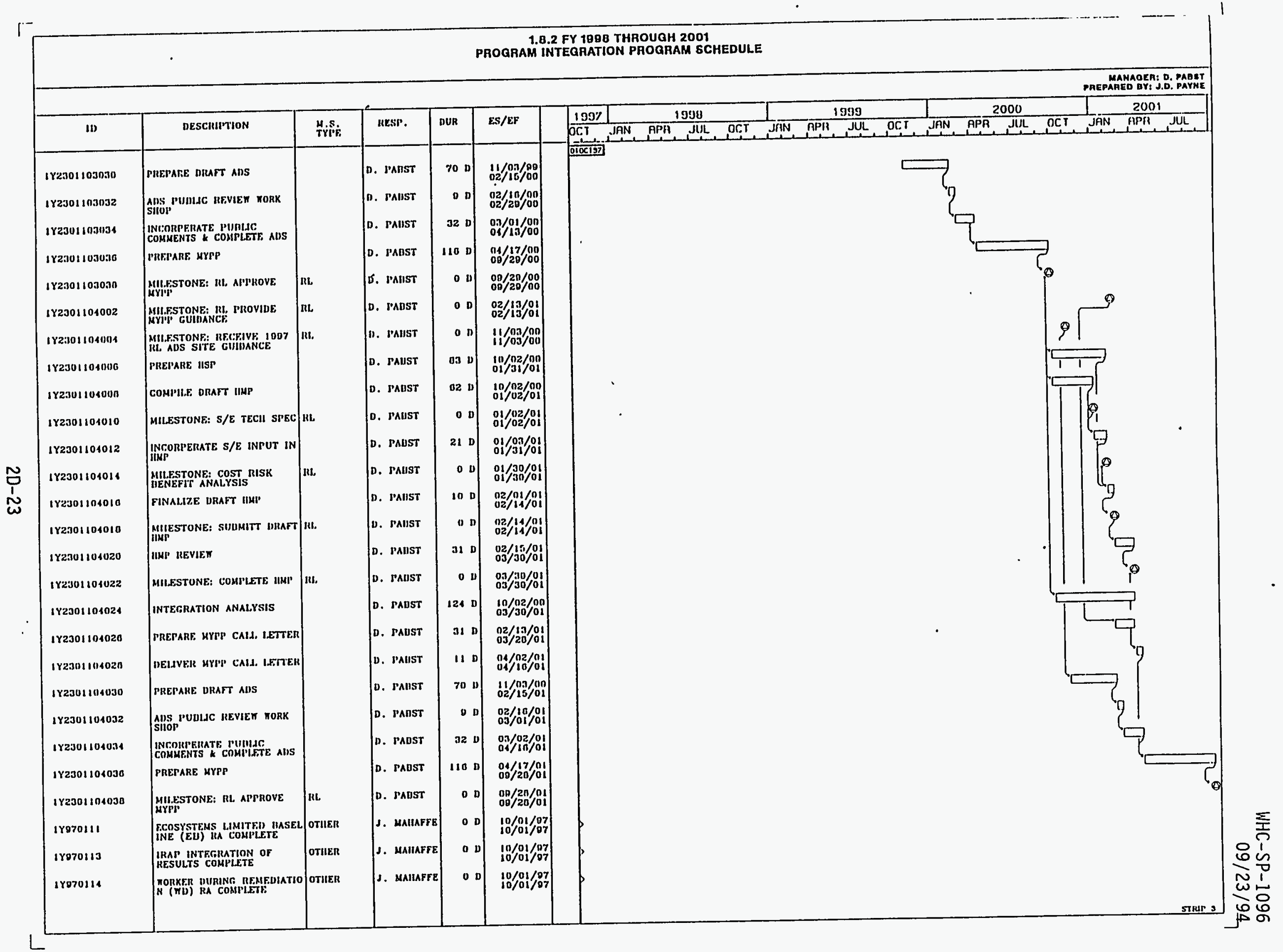




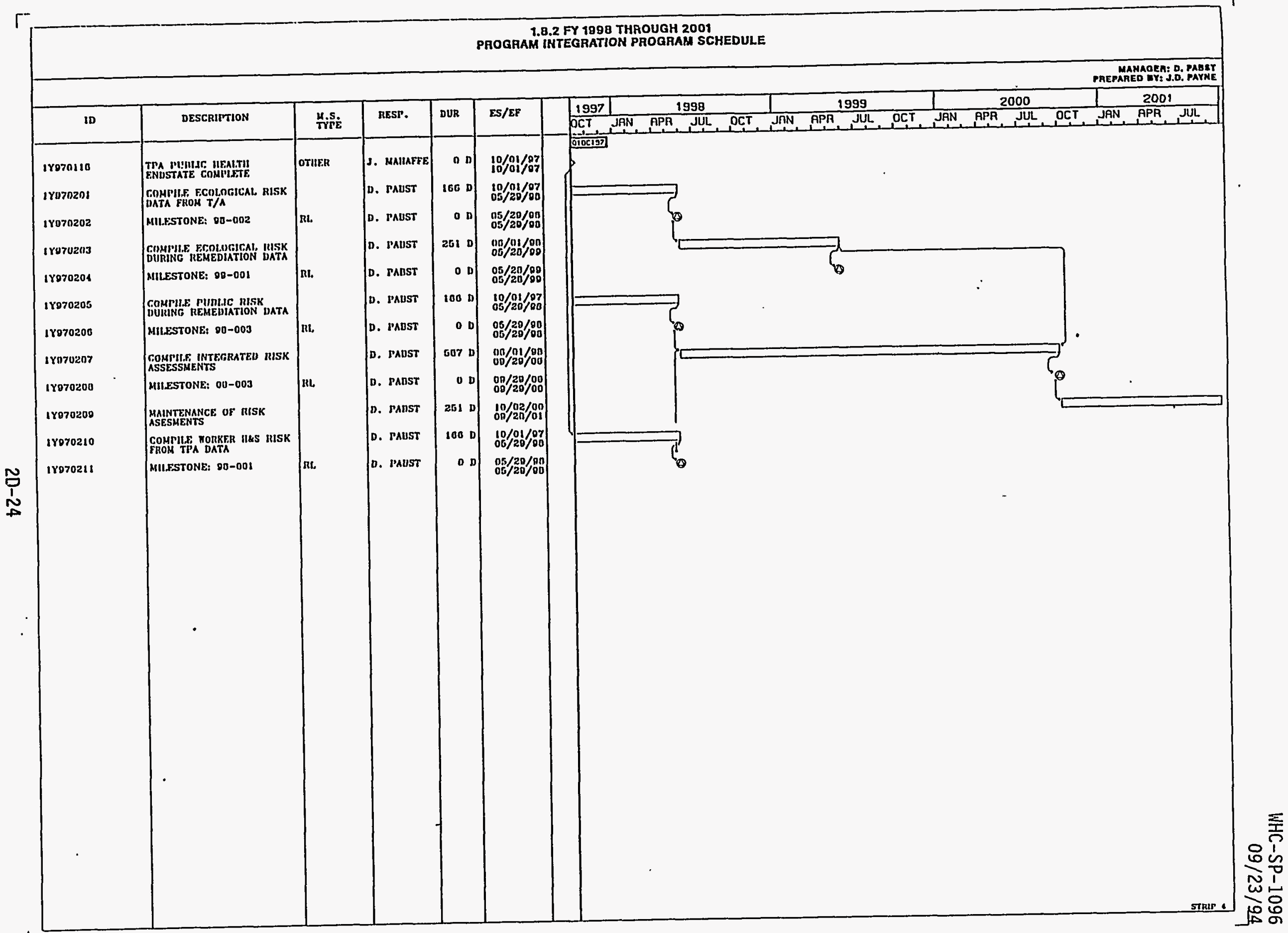




\section{E PROGRAM PERFORMANCE BASELINE SCHEDULE}

The approved Program Performance Baseline ScheduTe is the FYWP performance execution, management, and reporting schedule, developed at the cost Account level, with activities defined at the Work Package or Task Package Level, and based upon a detailed breakdown of the PMBS activities. Actual cost data shall be accumulated and reported at the Program Performance Baseline Schedule (PPBS) level for performance evaluation.

The PPBS for the PIanning Integration Program provides the detailed scheduling tool to be used in managing the schedule and reporting schedule progress and performance over the fiscal year. It provides the greatest detail and insight into the program's time-phased plans. Interfaces within program elements, and to external entities are identified. 
WHC-SP-1096

$09 / 23 / 94$

Planning Integration MuTti-Year Program Plan

Figure 2.E-1. Program Performance Baseline Schedule 


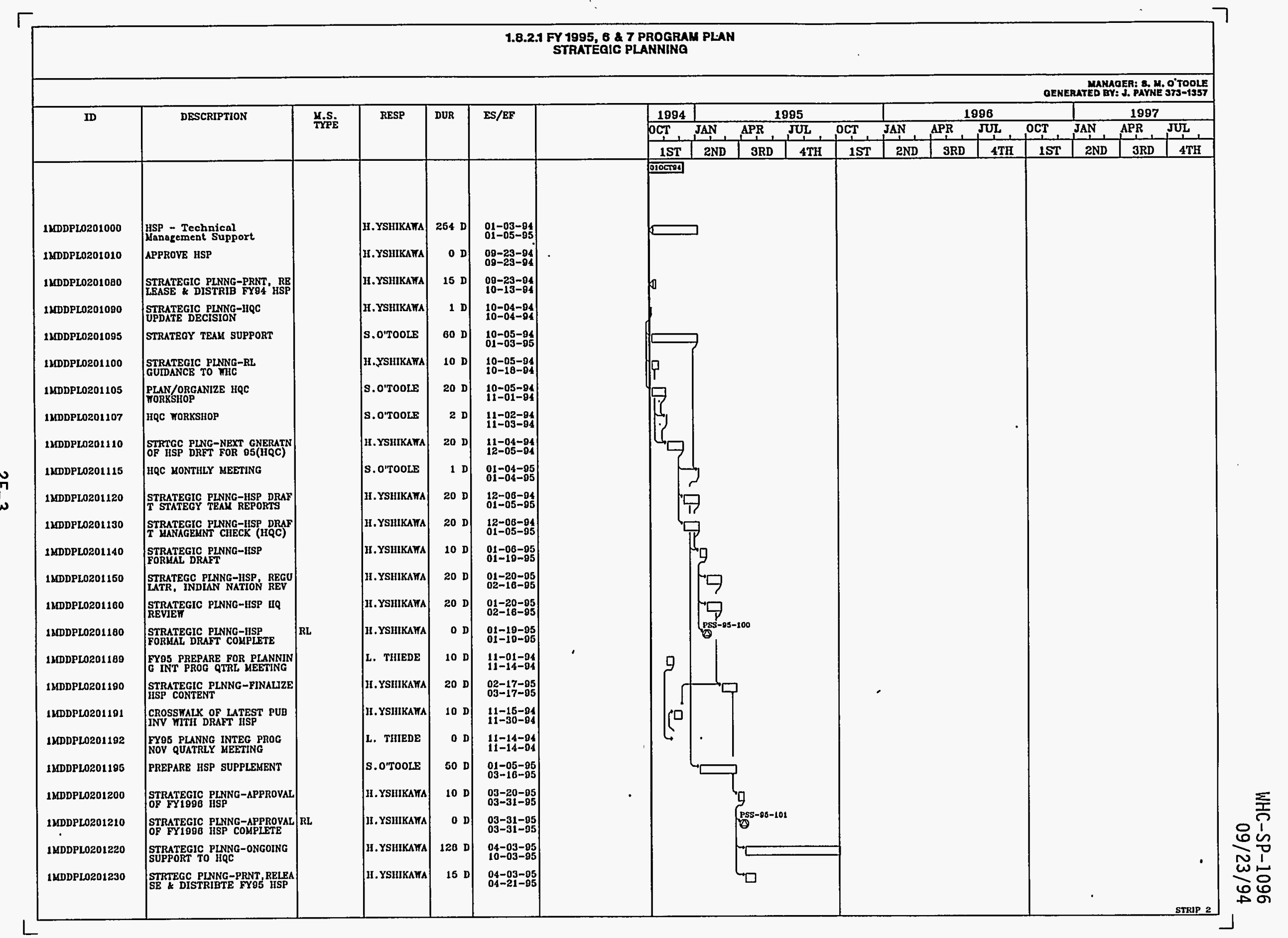




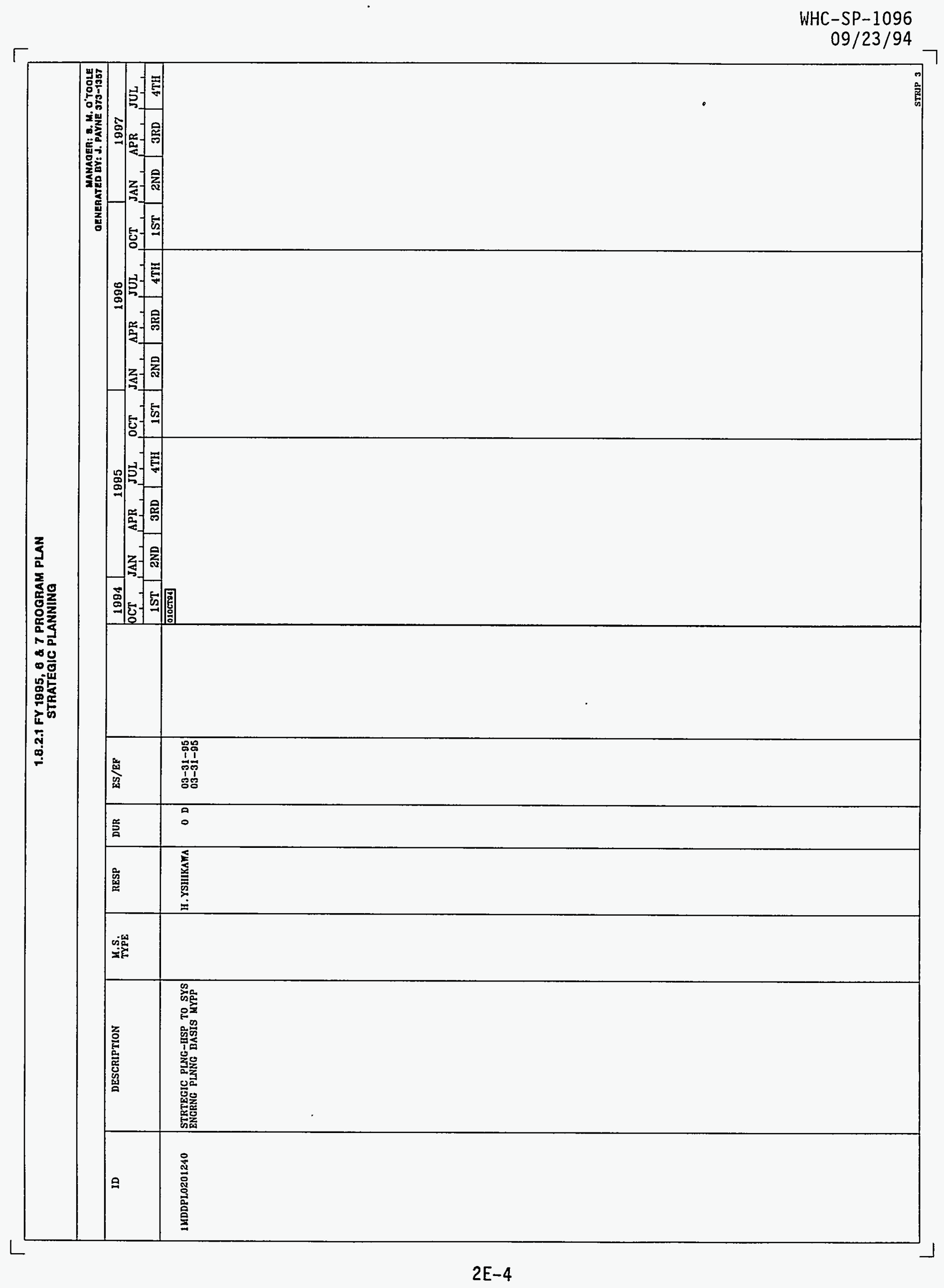




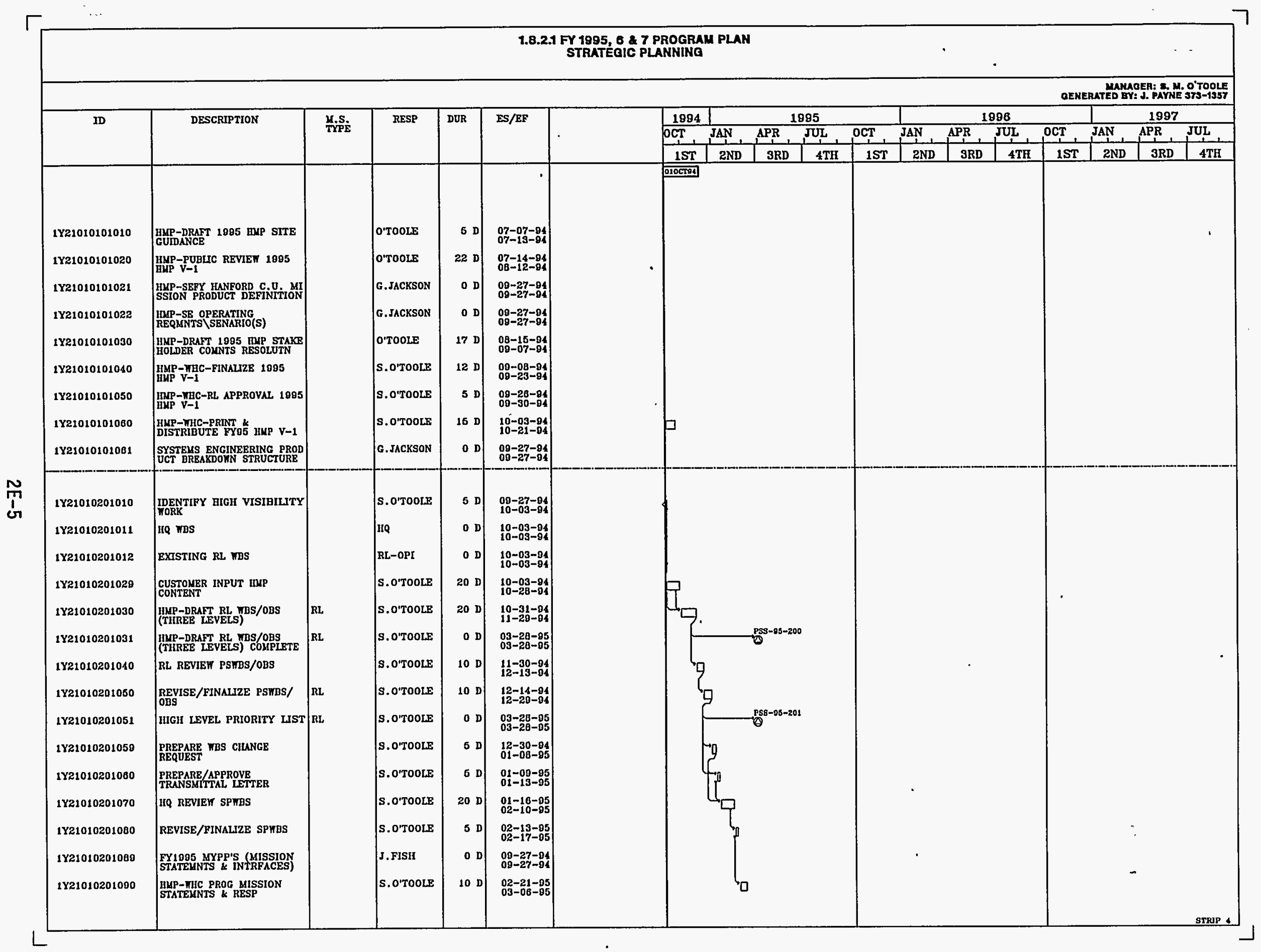

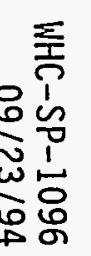




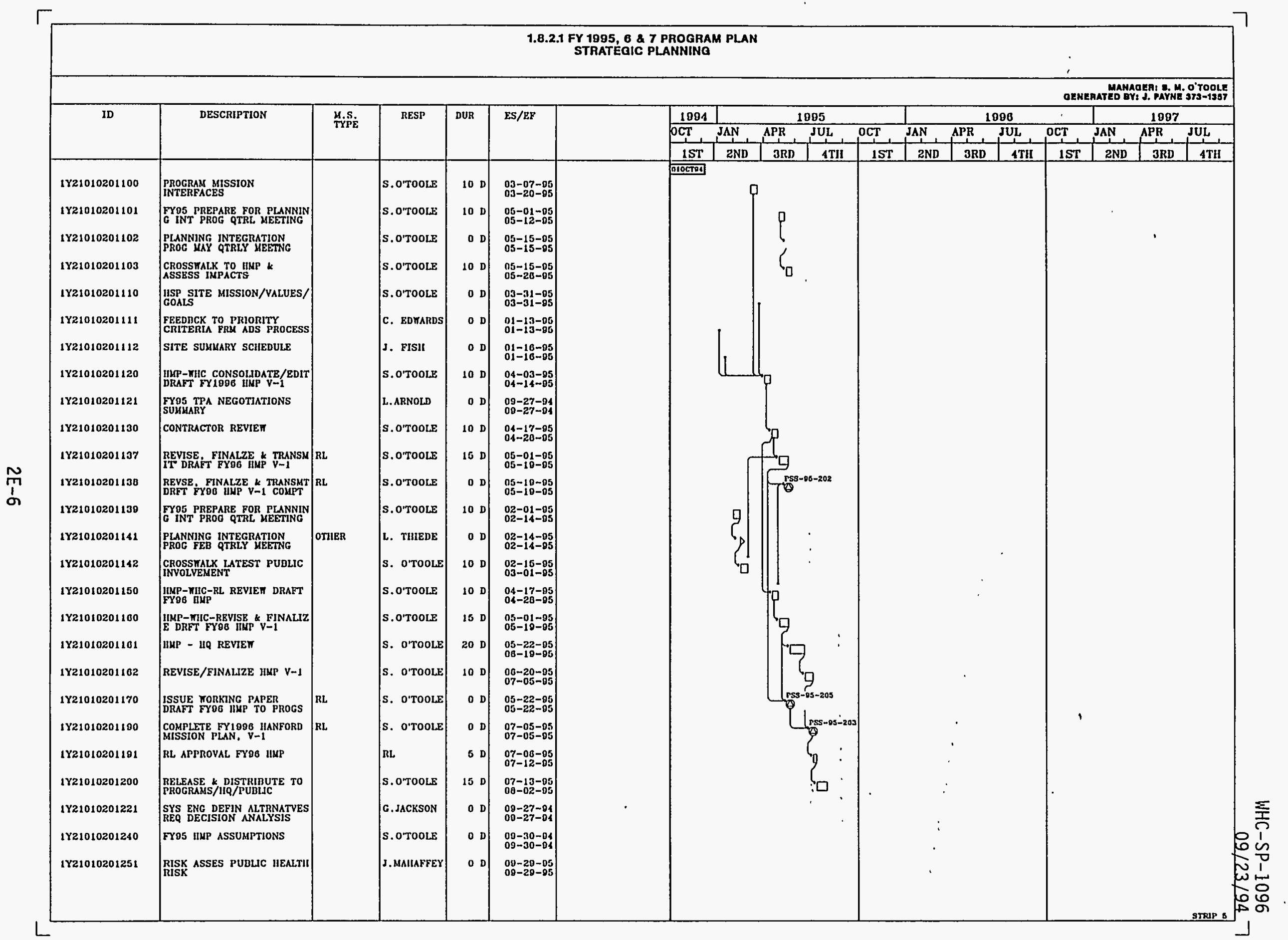




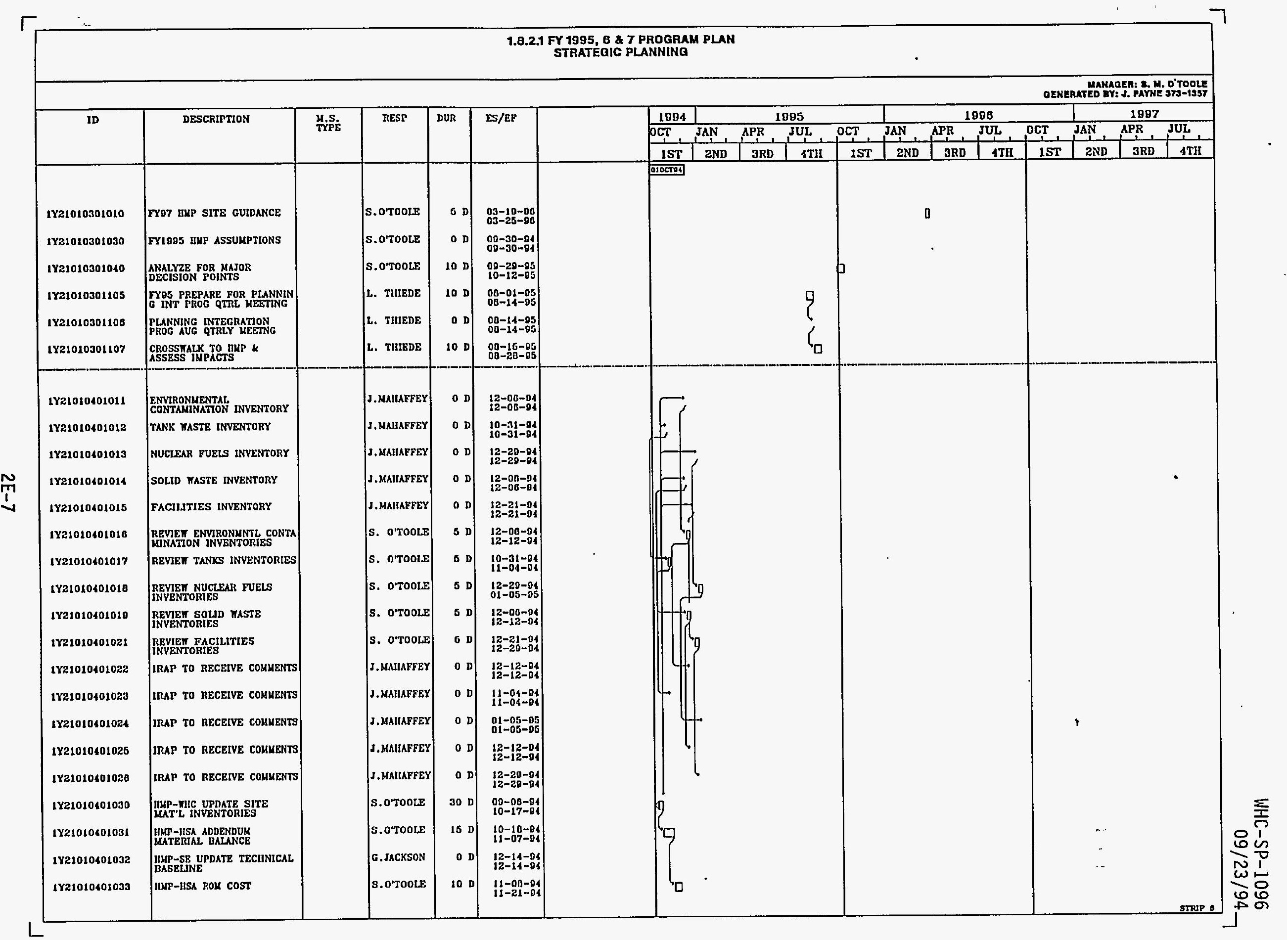




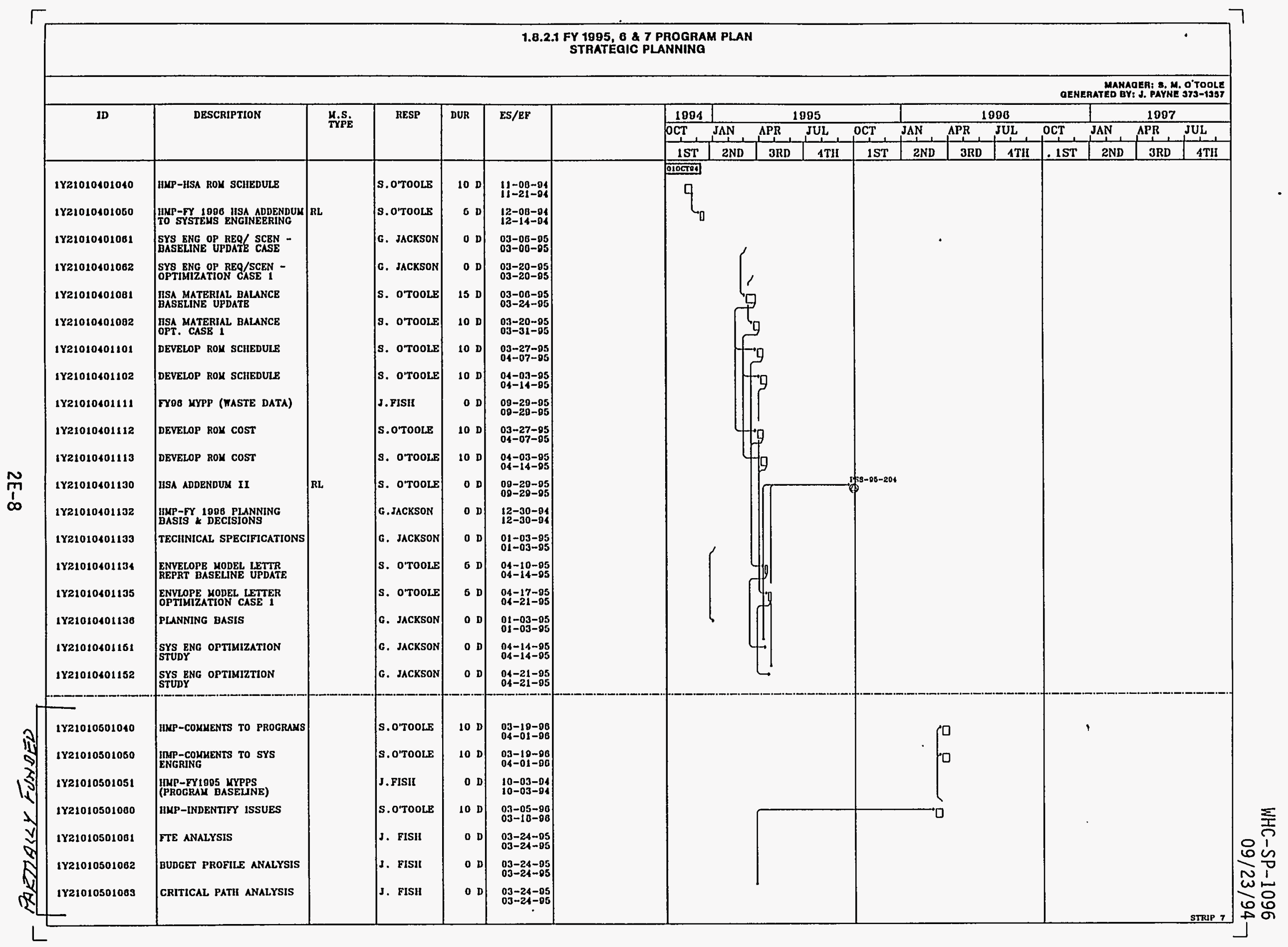




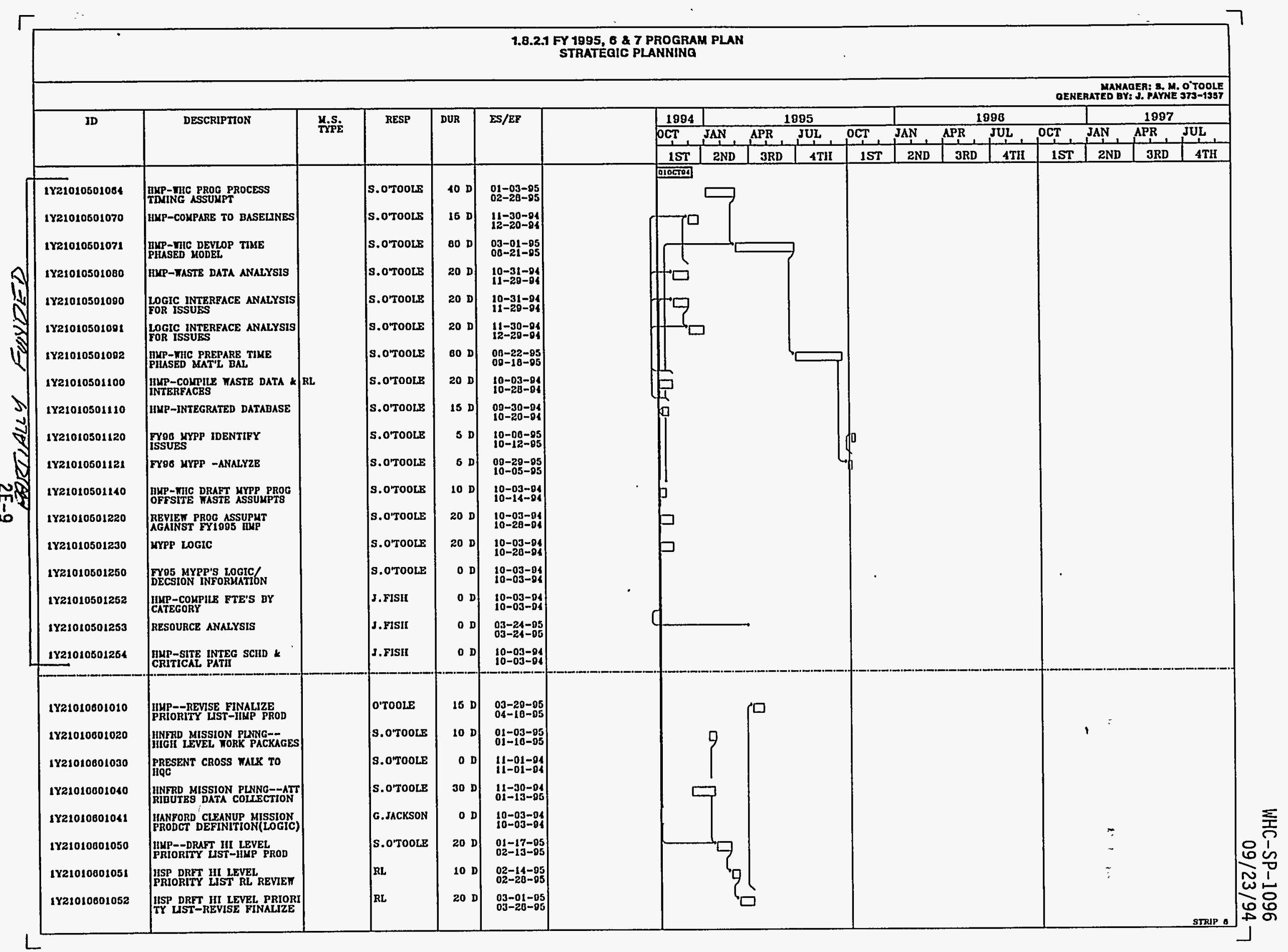




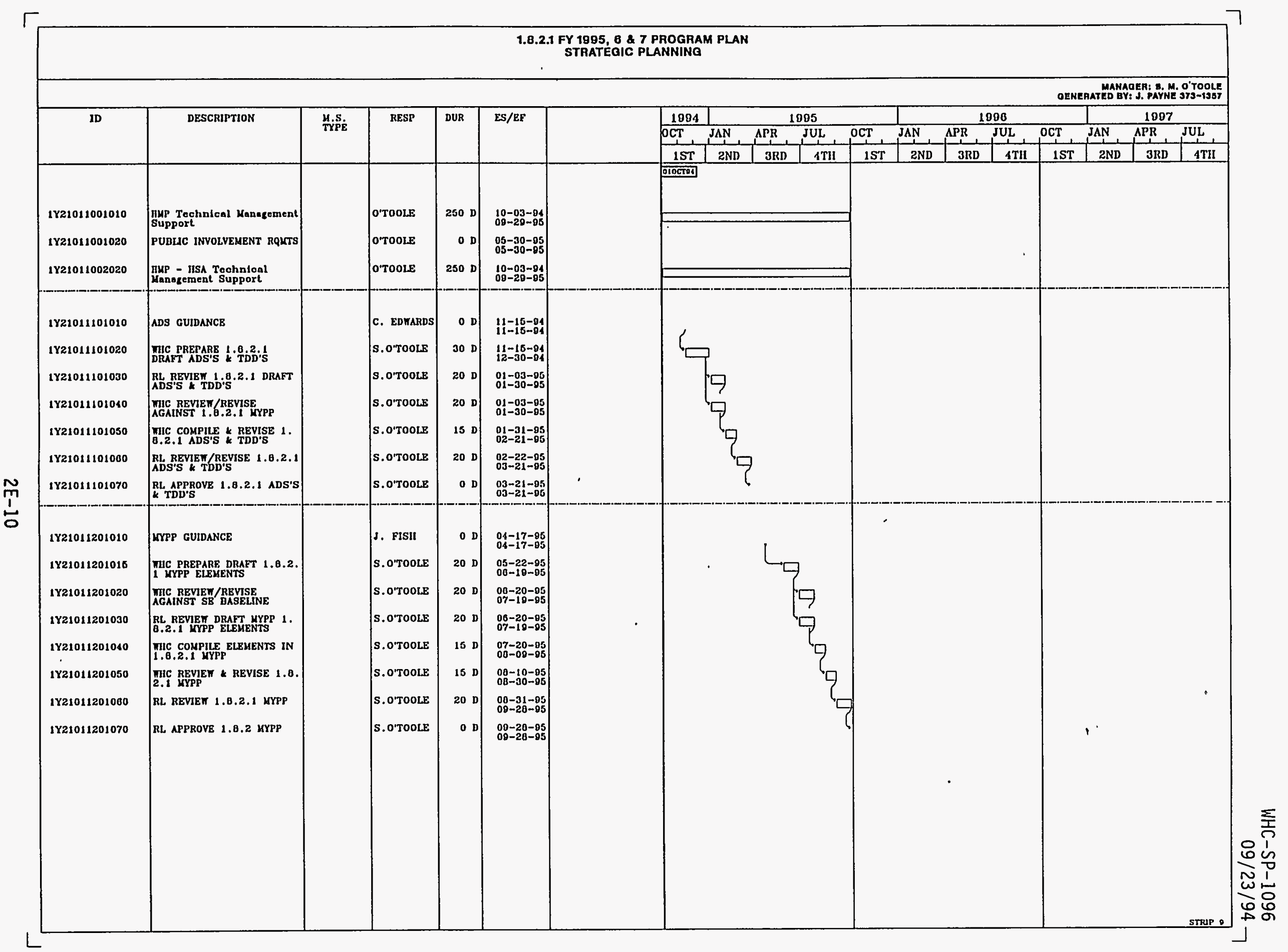




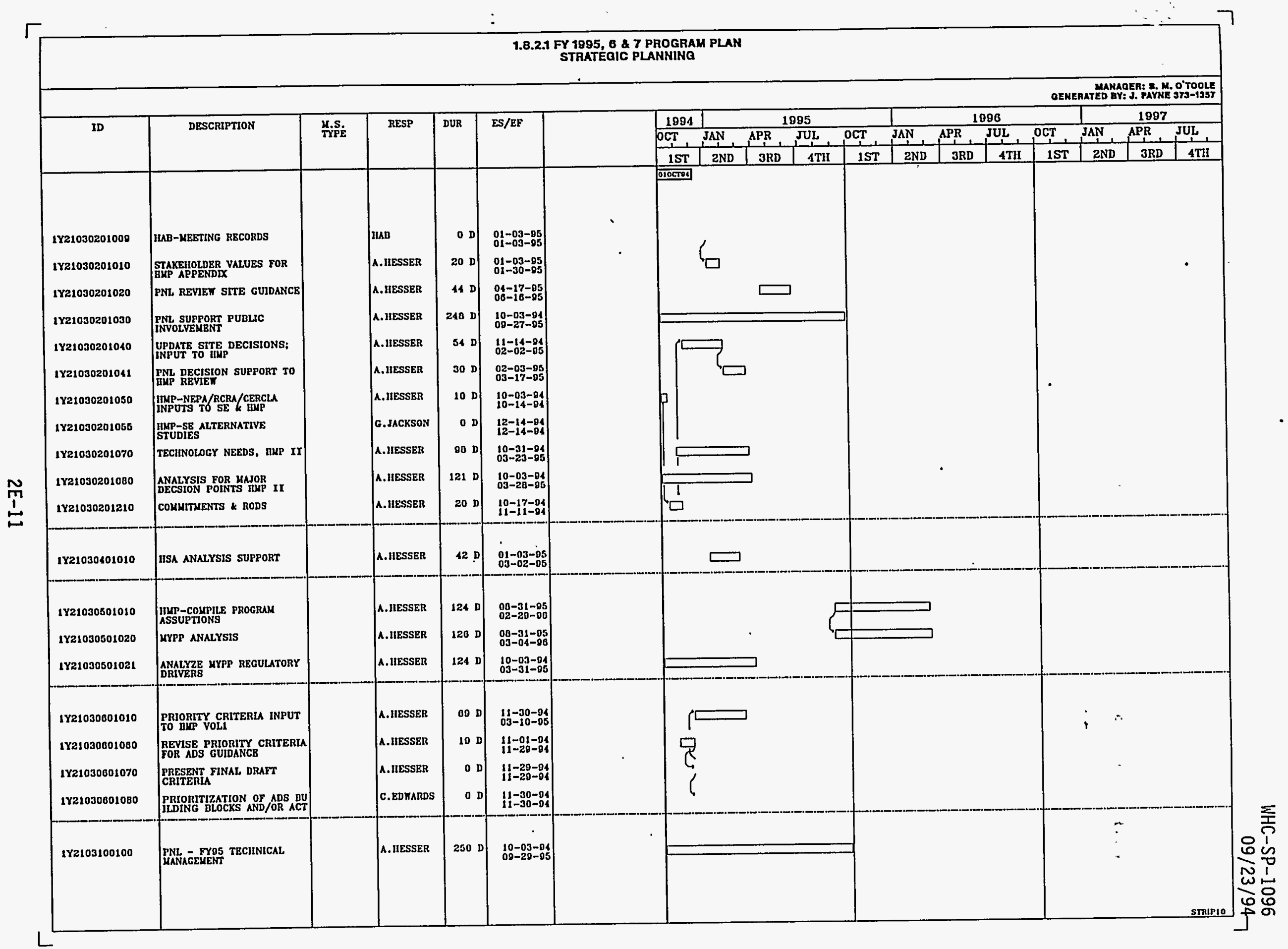




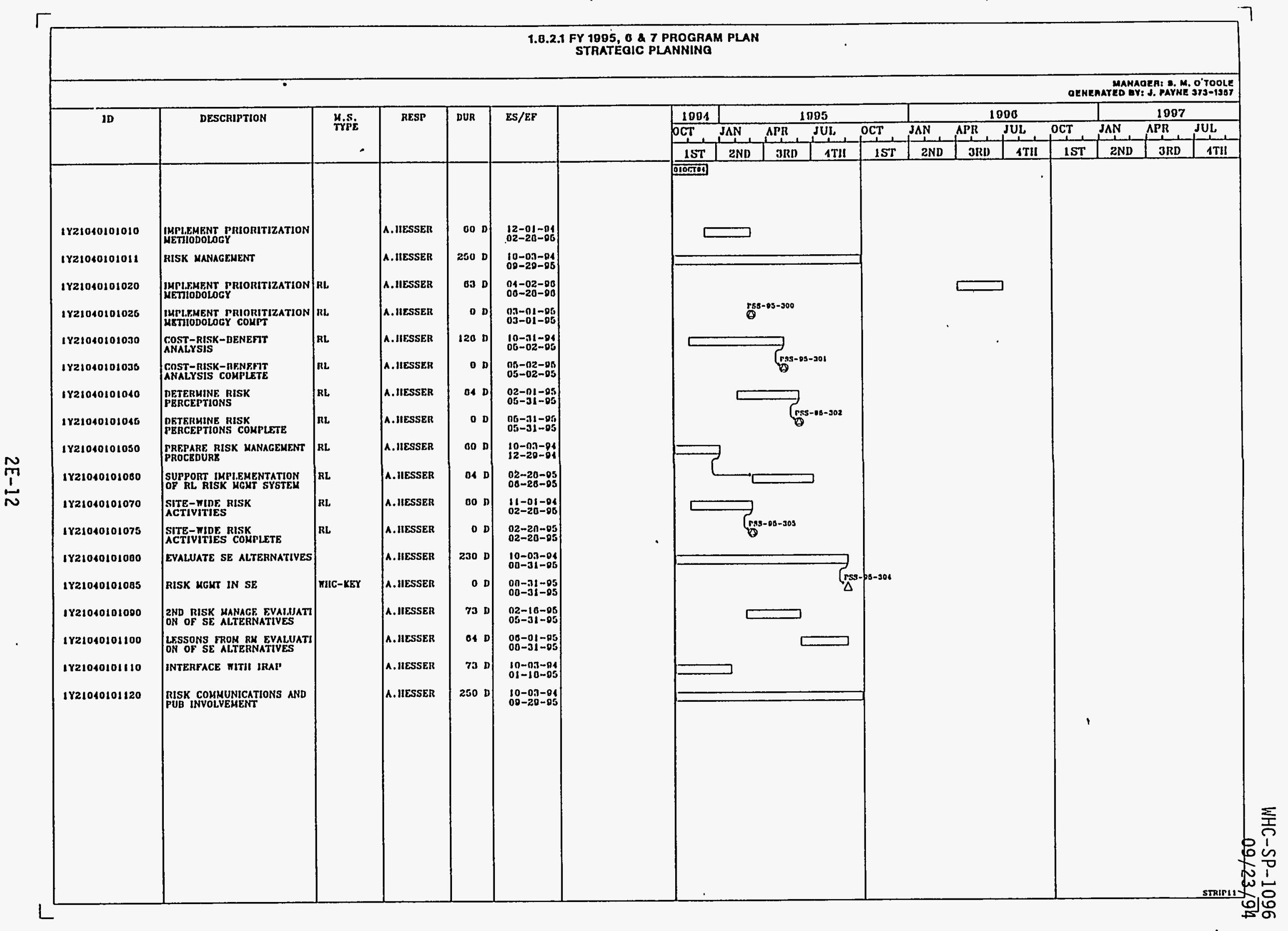




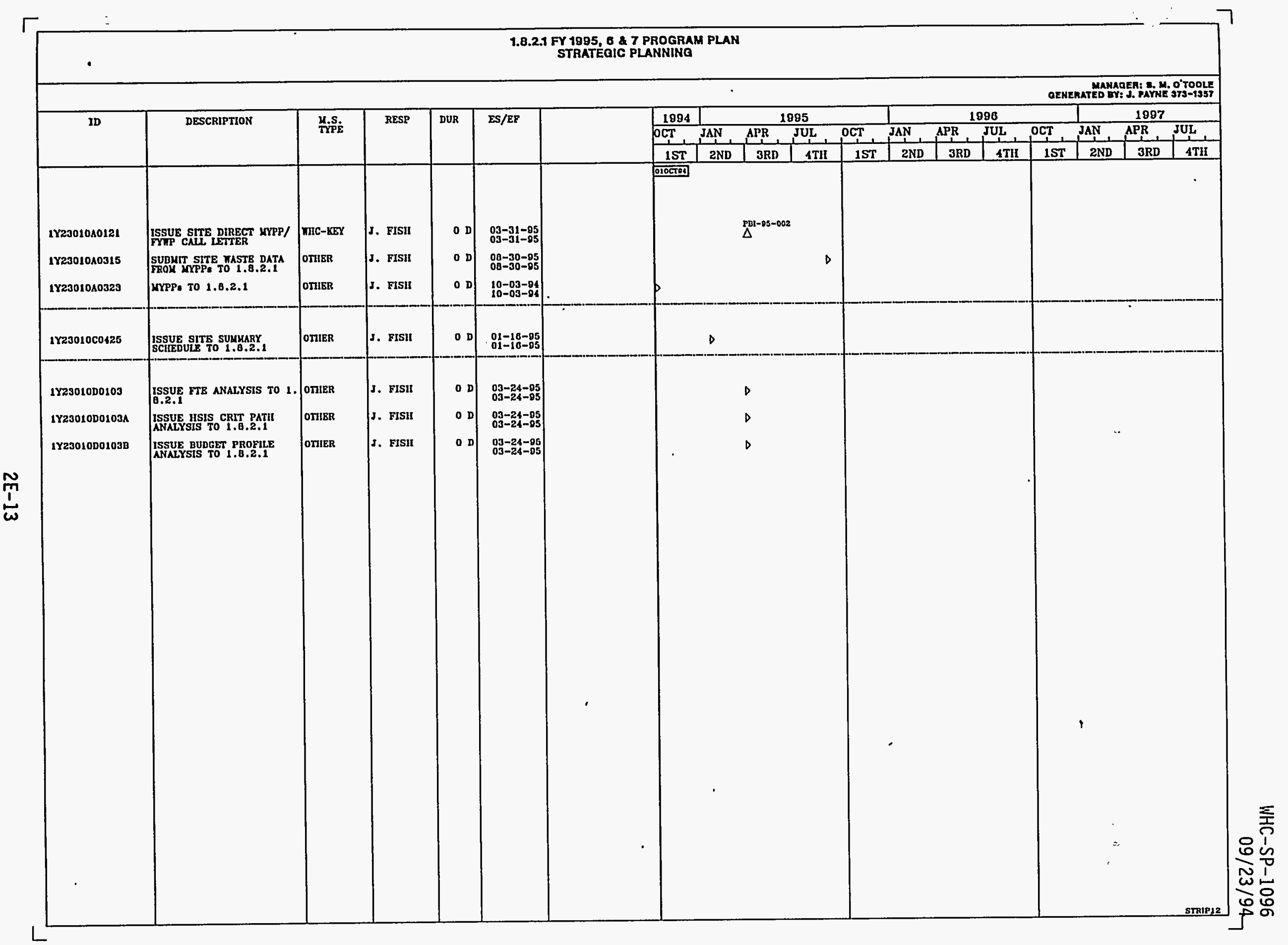




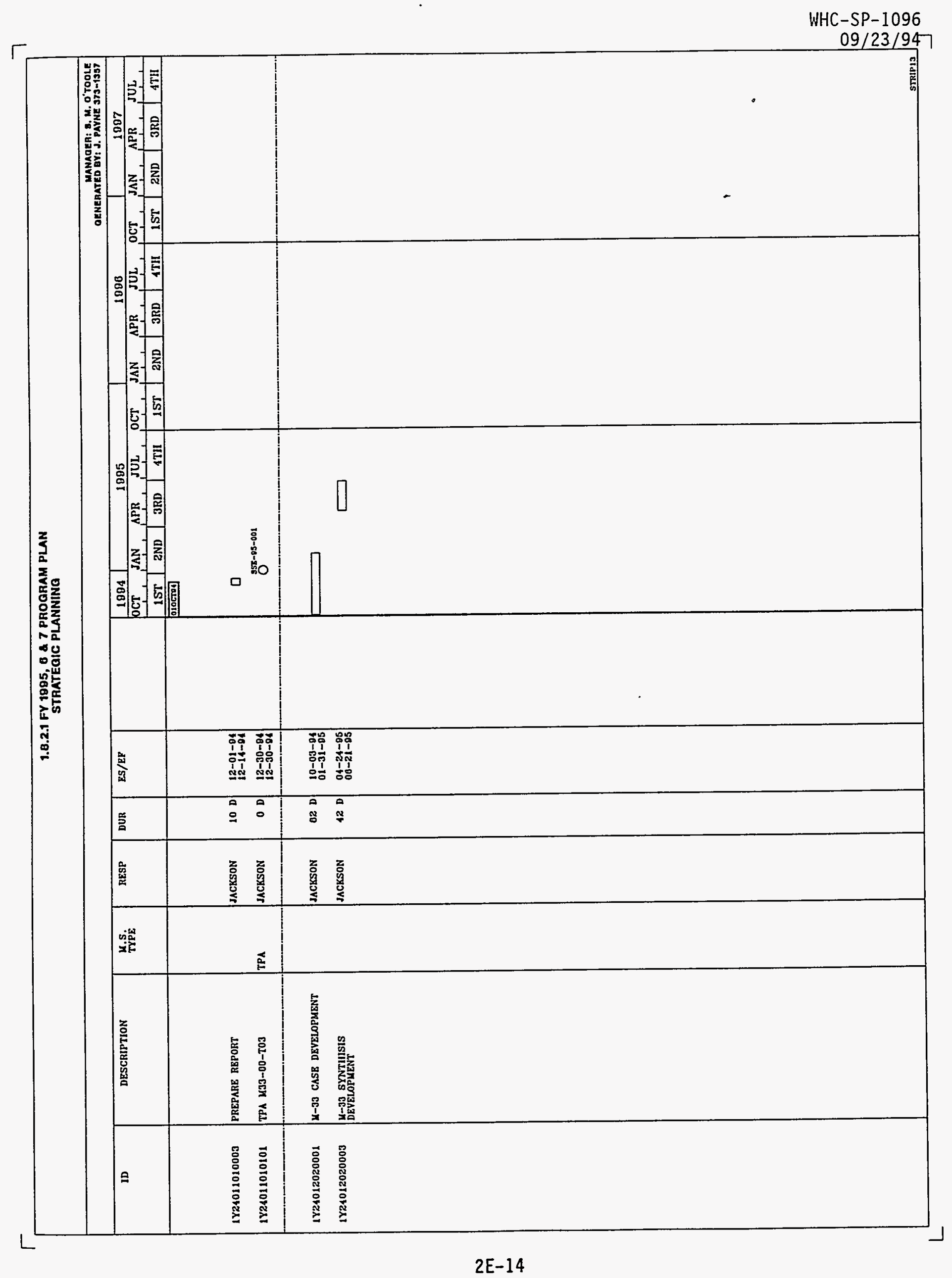




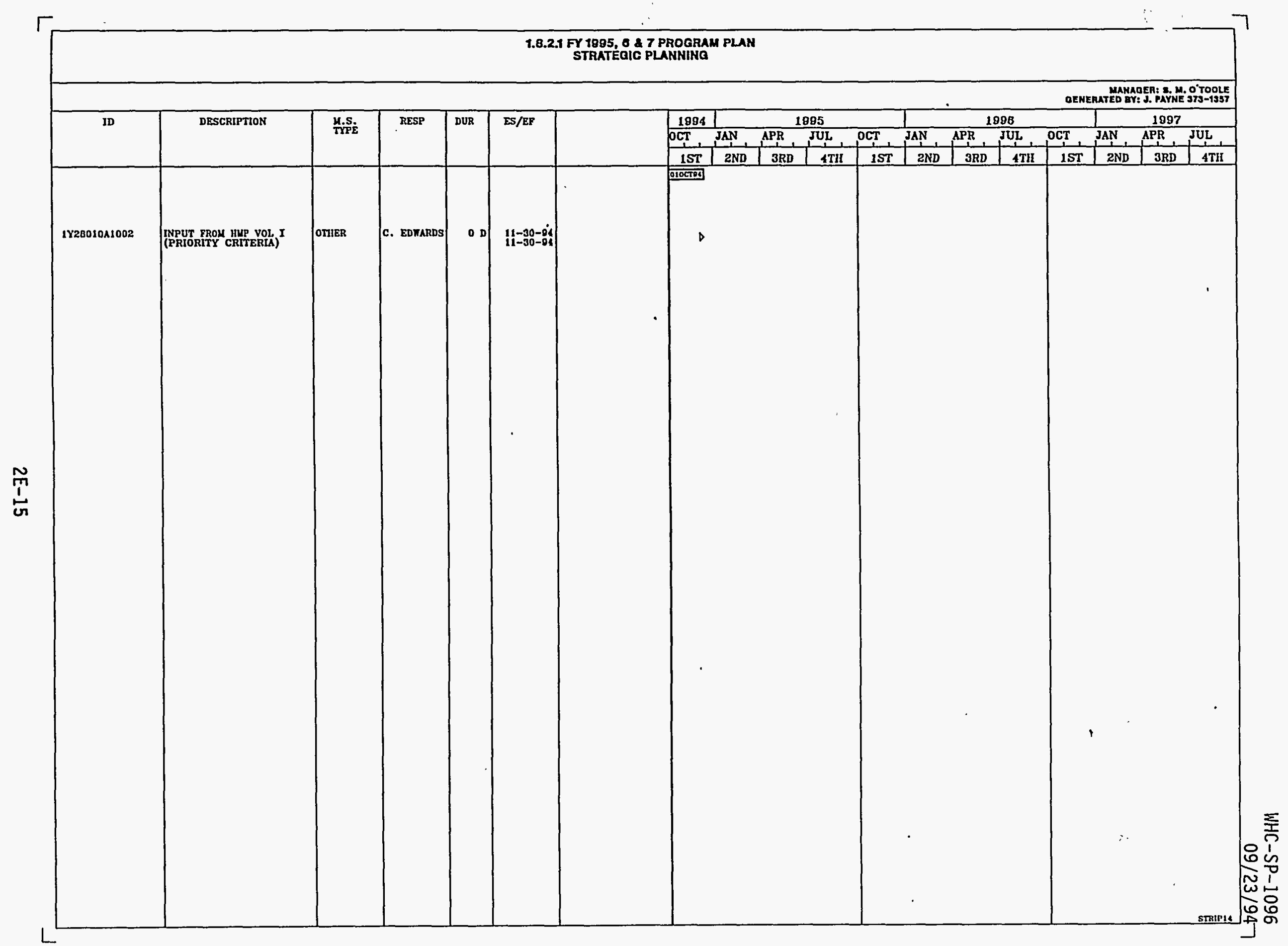




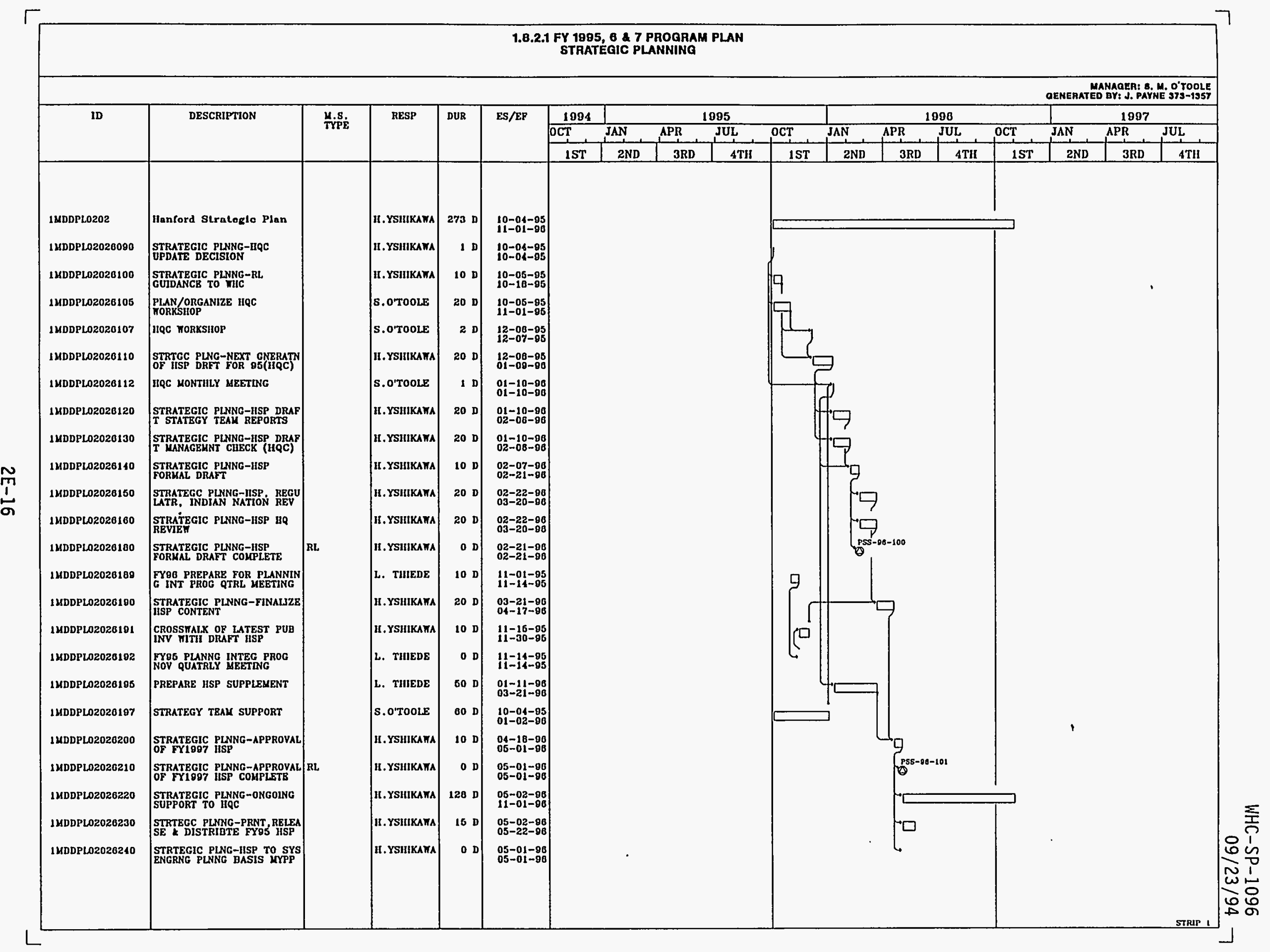




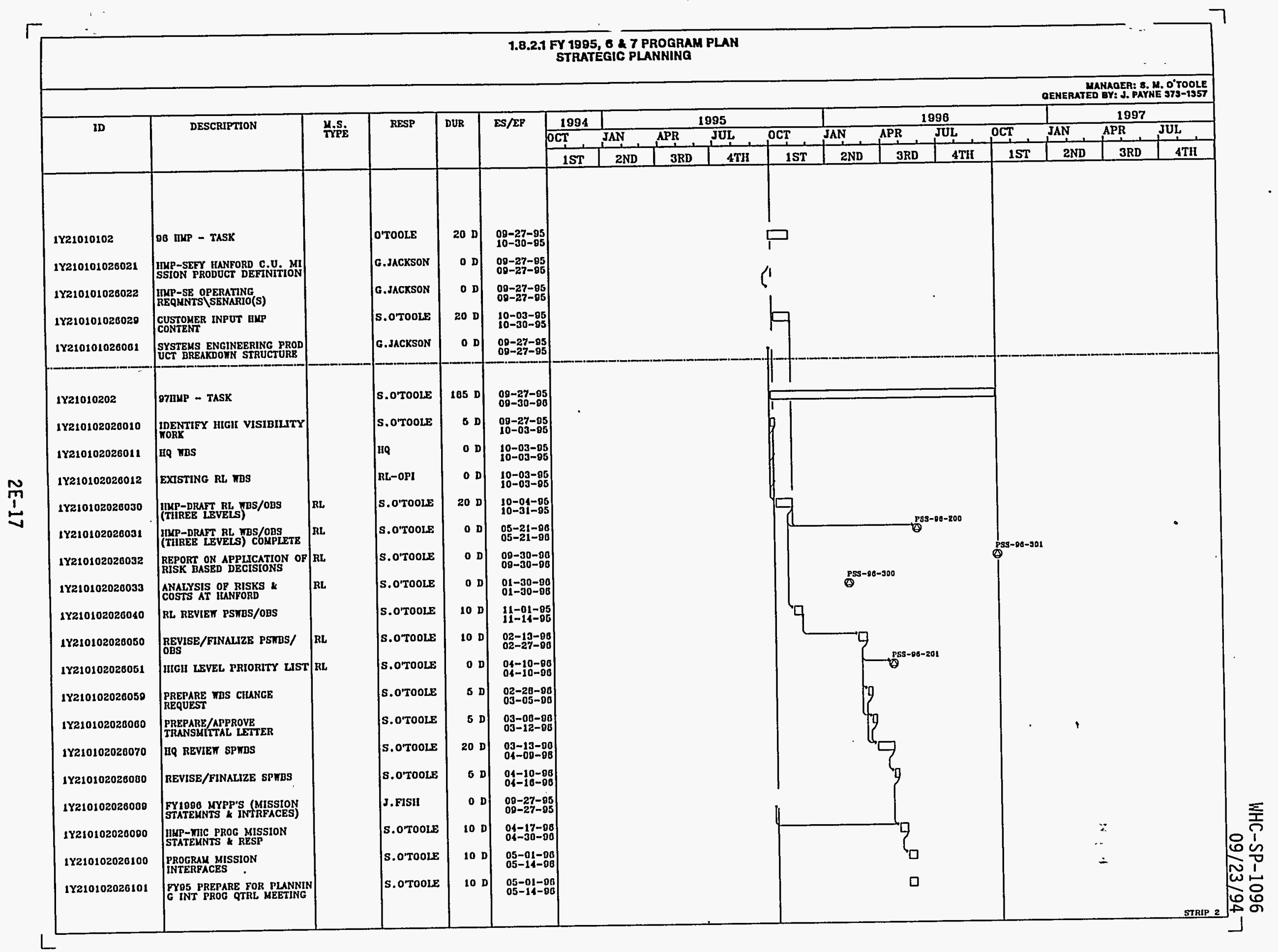




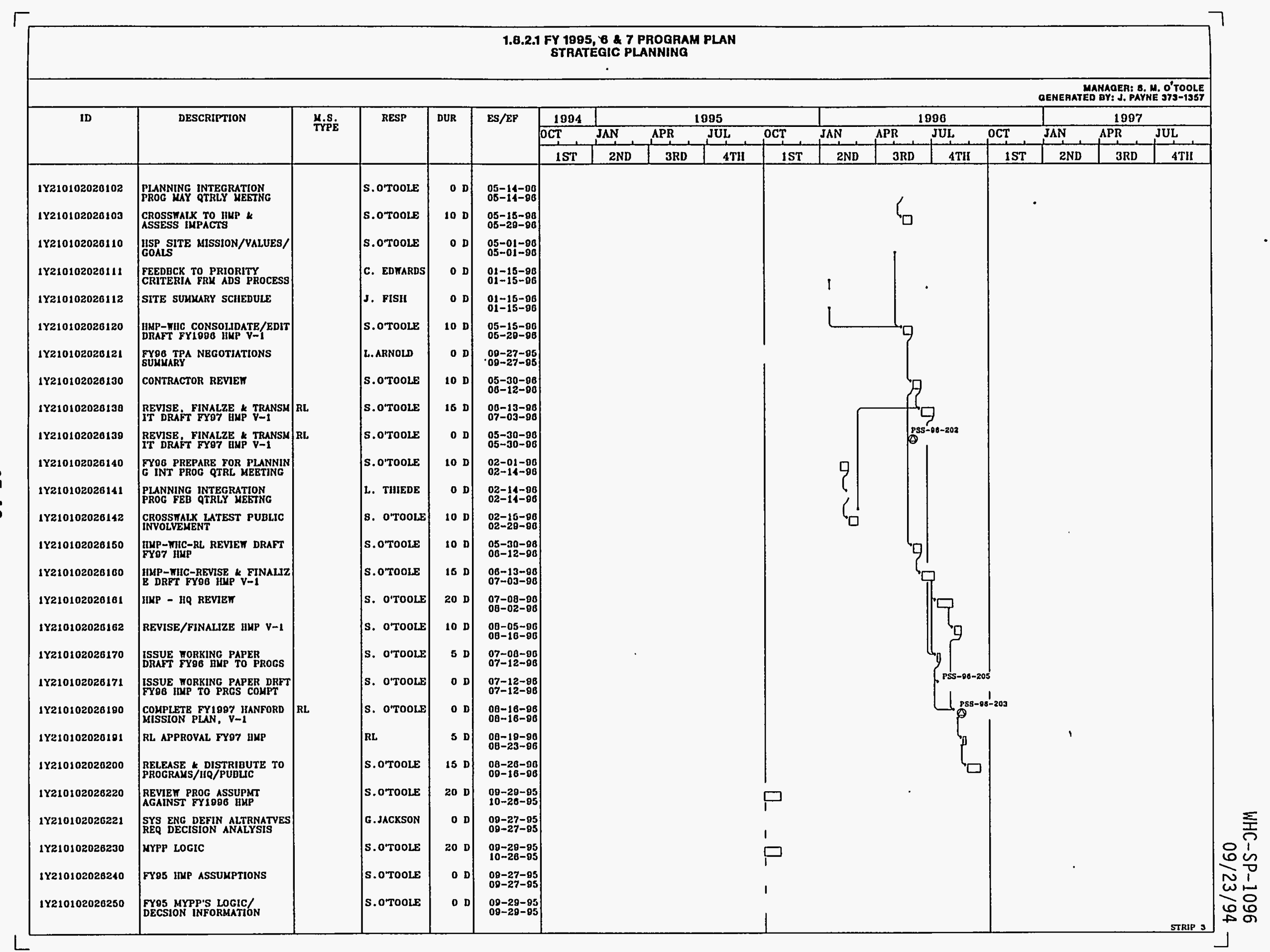




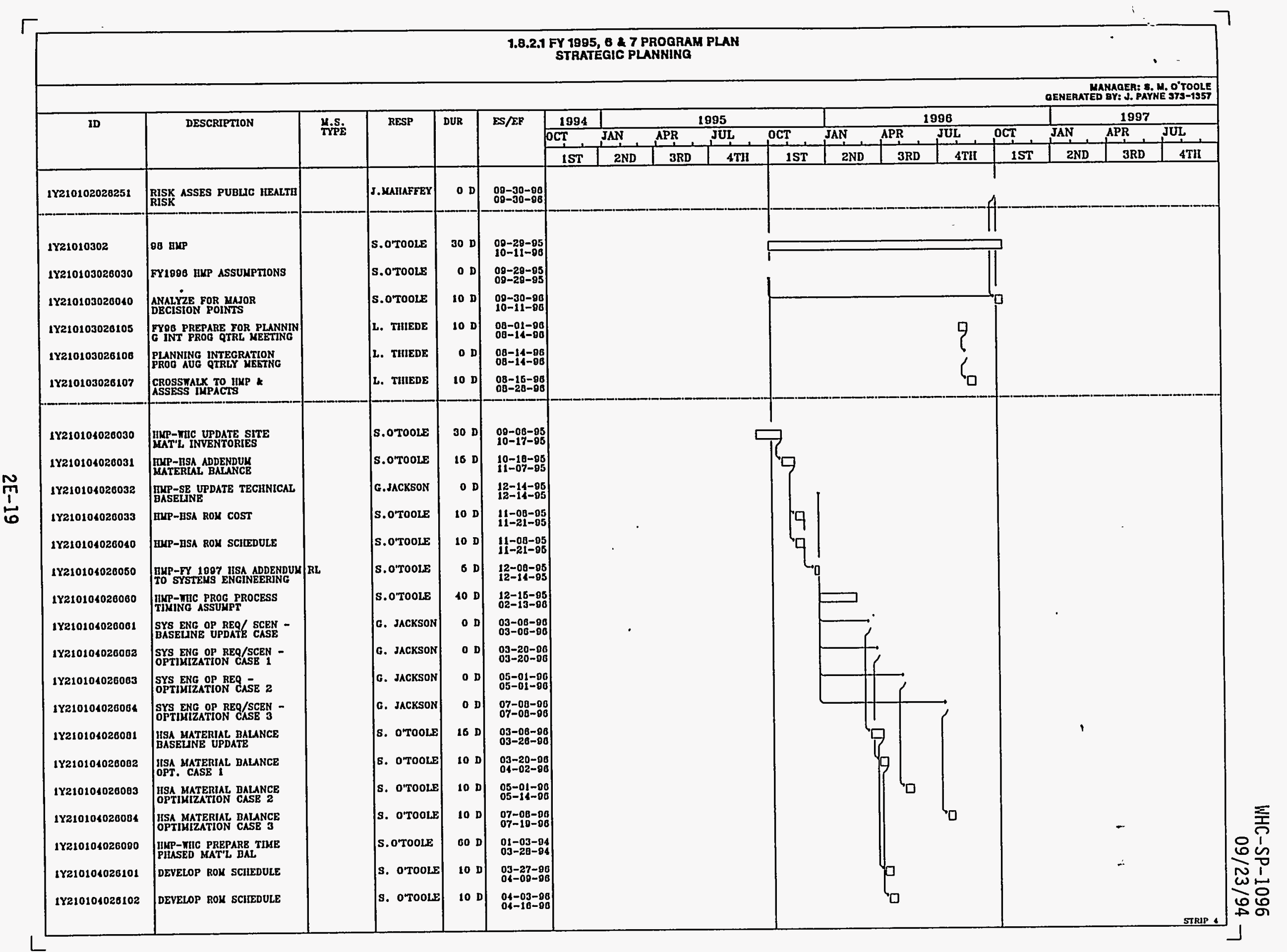




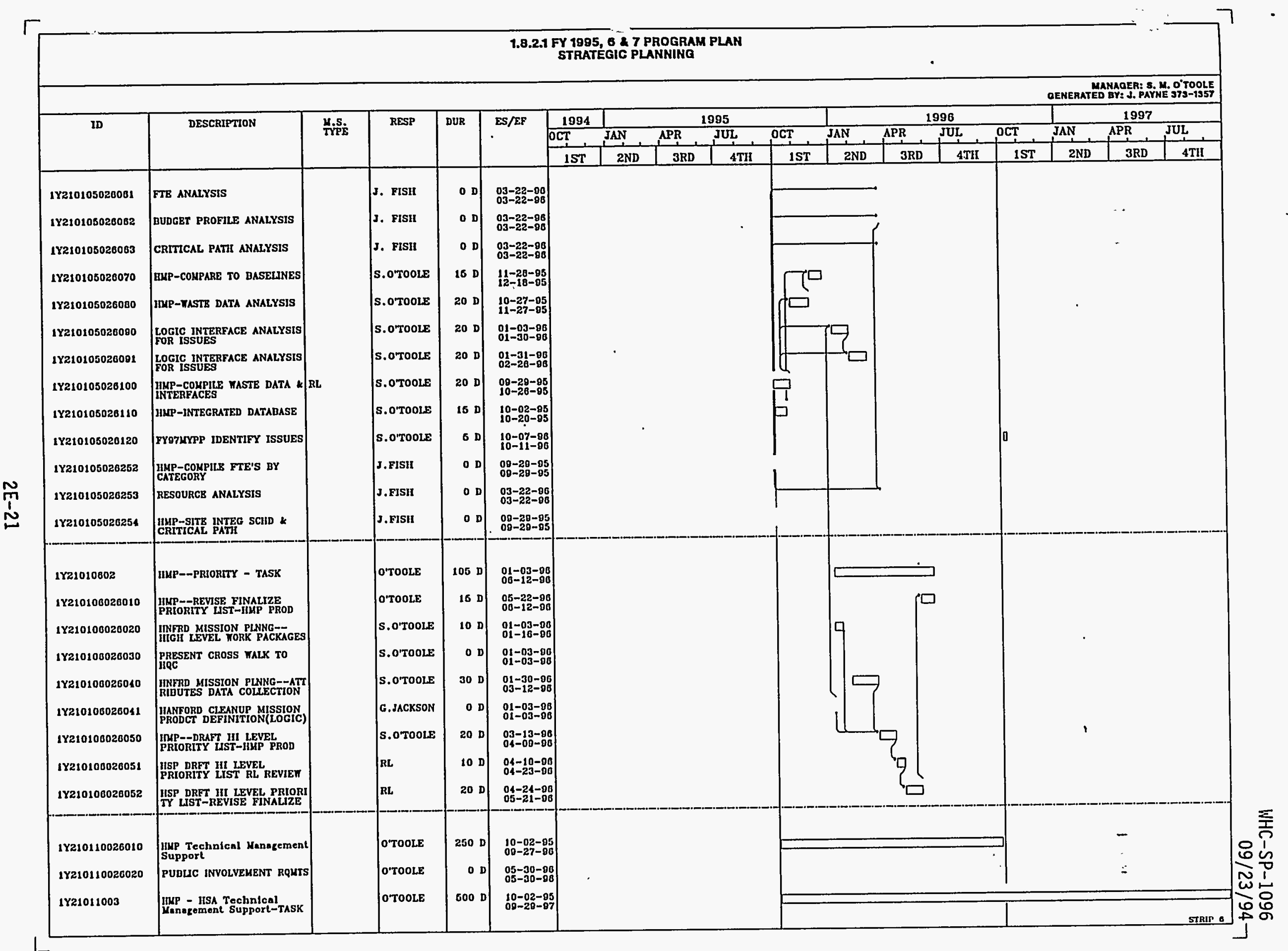




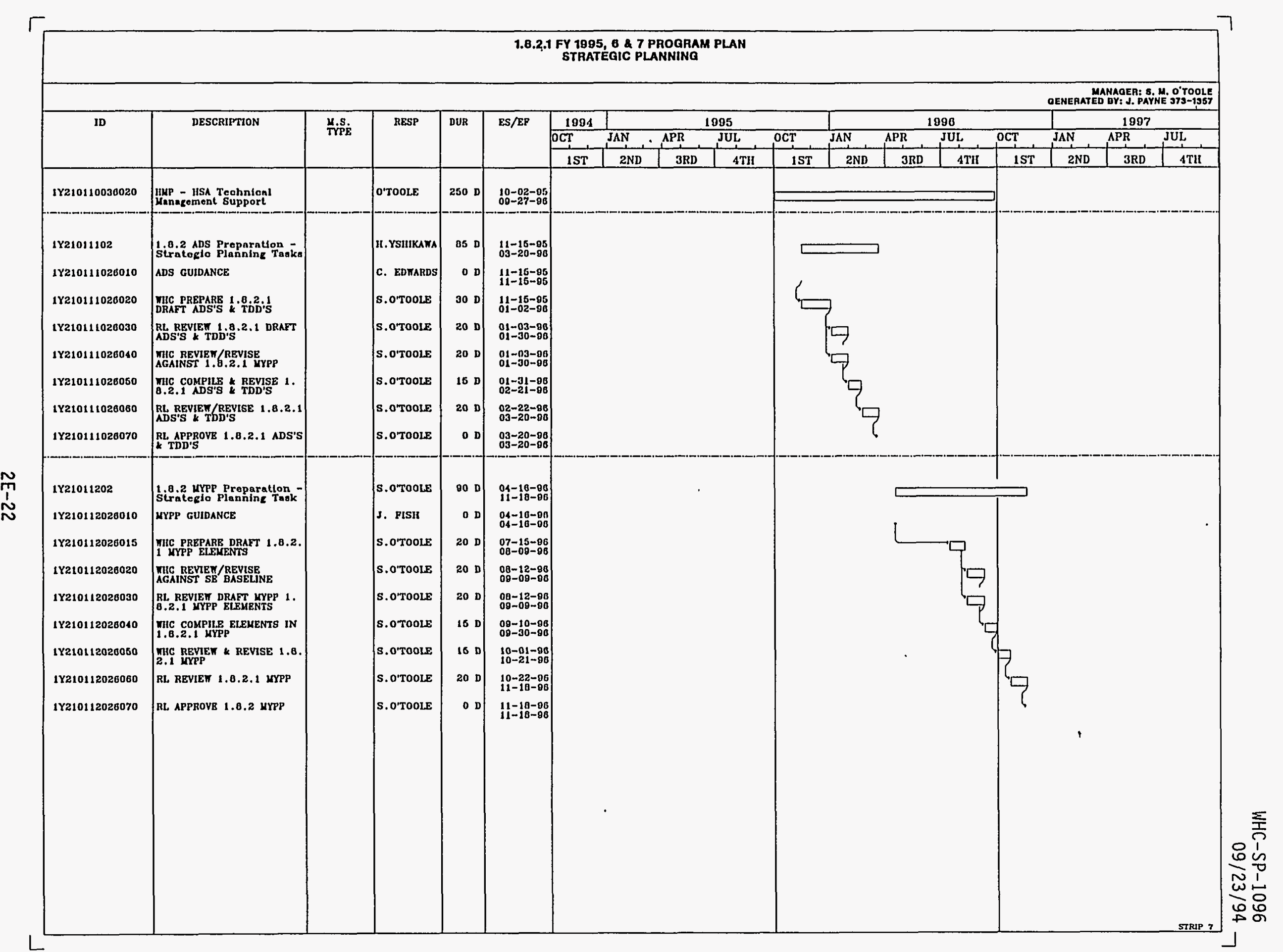




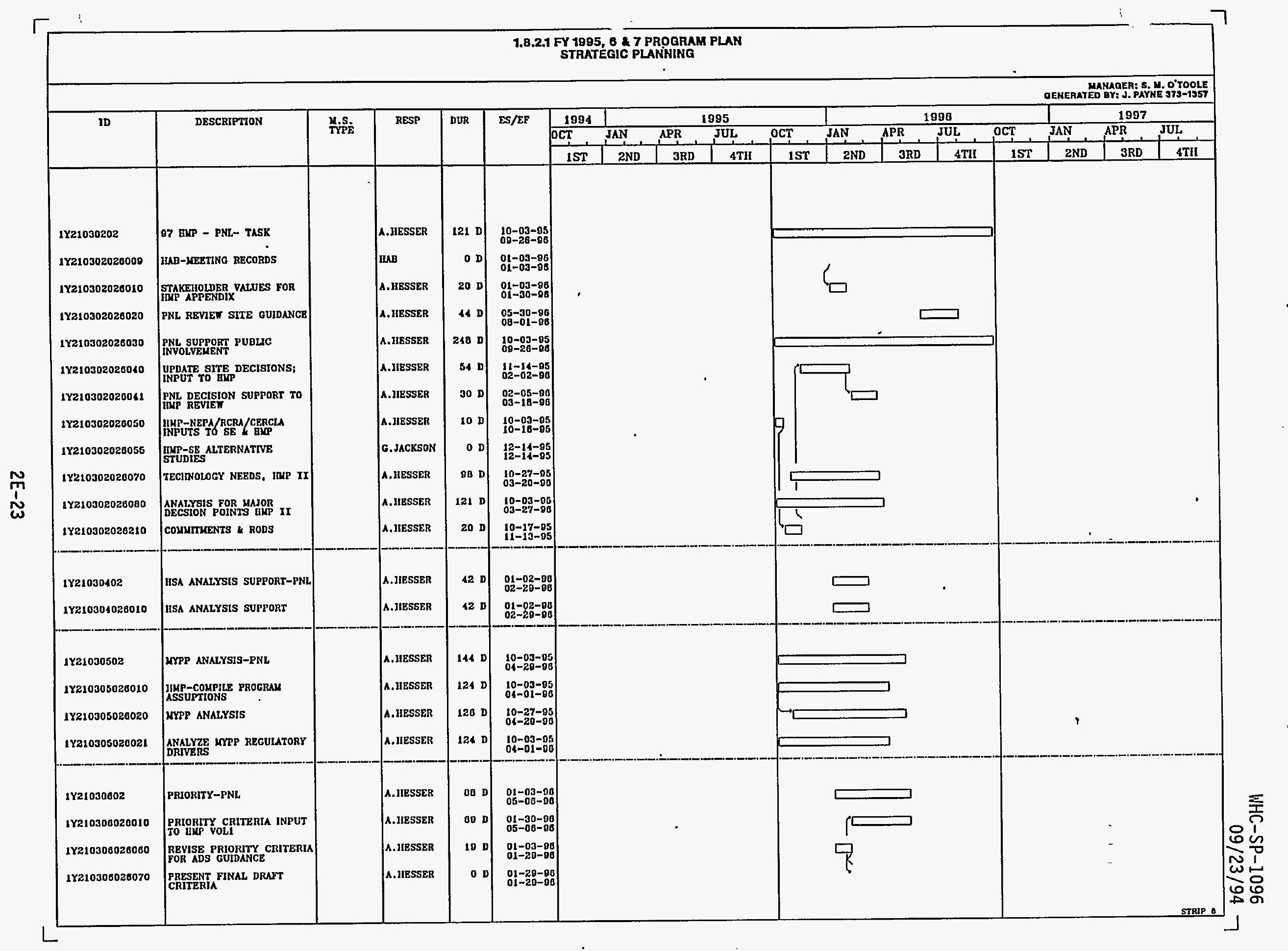




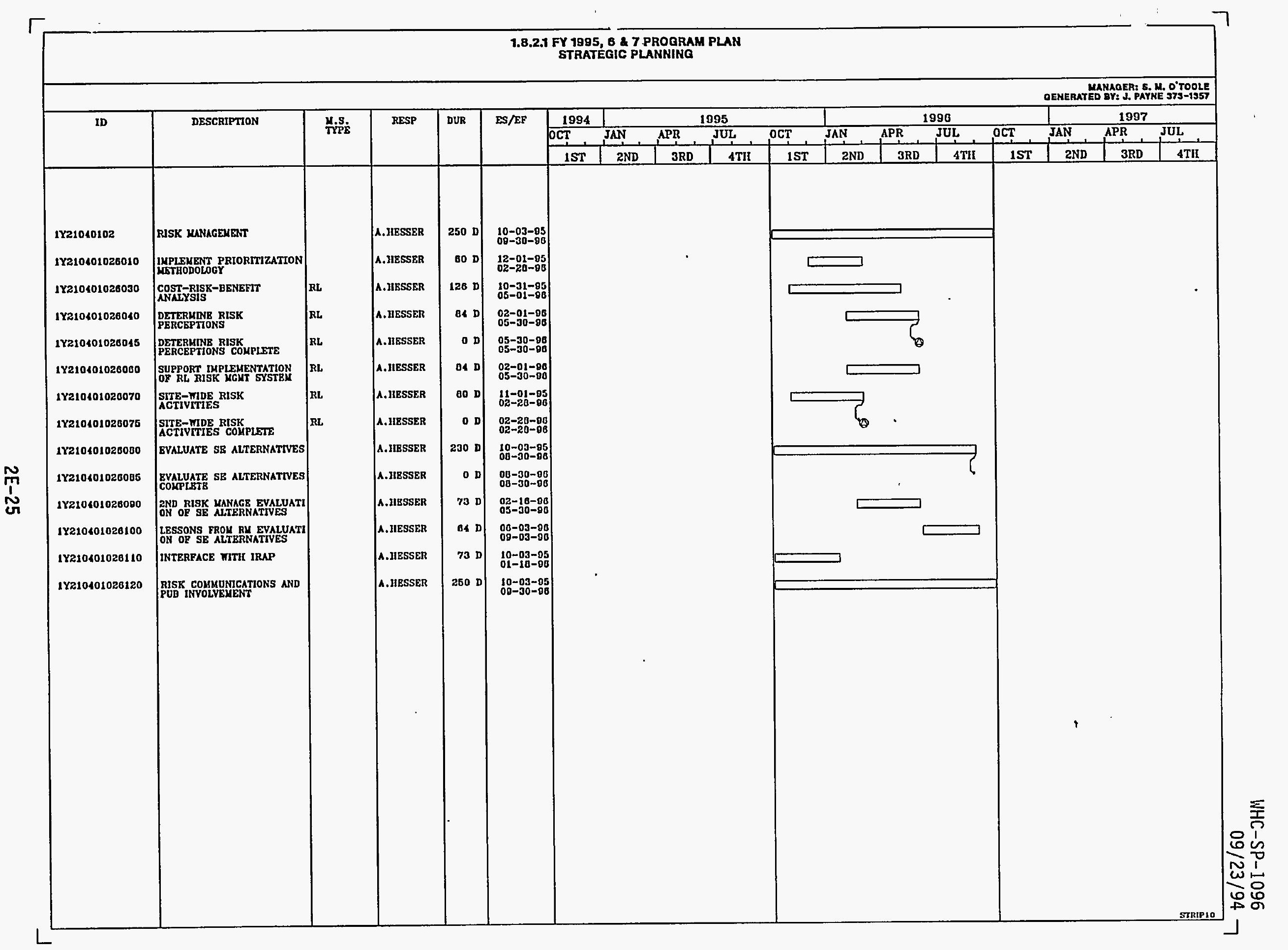




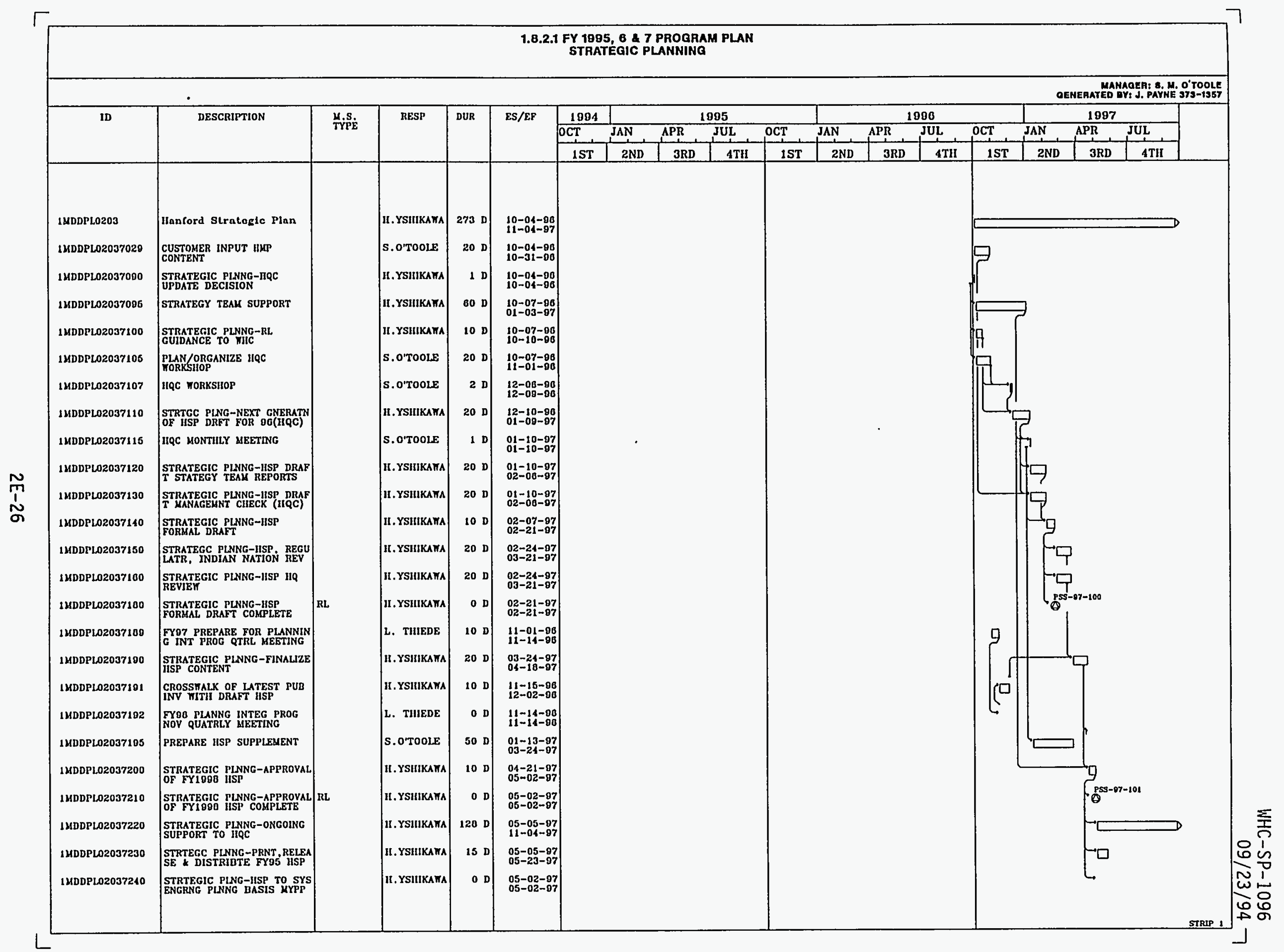




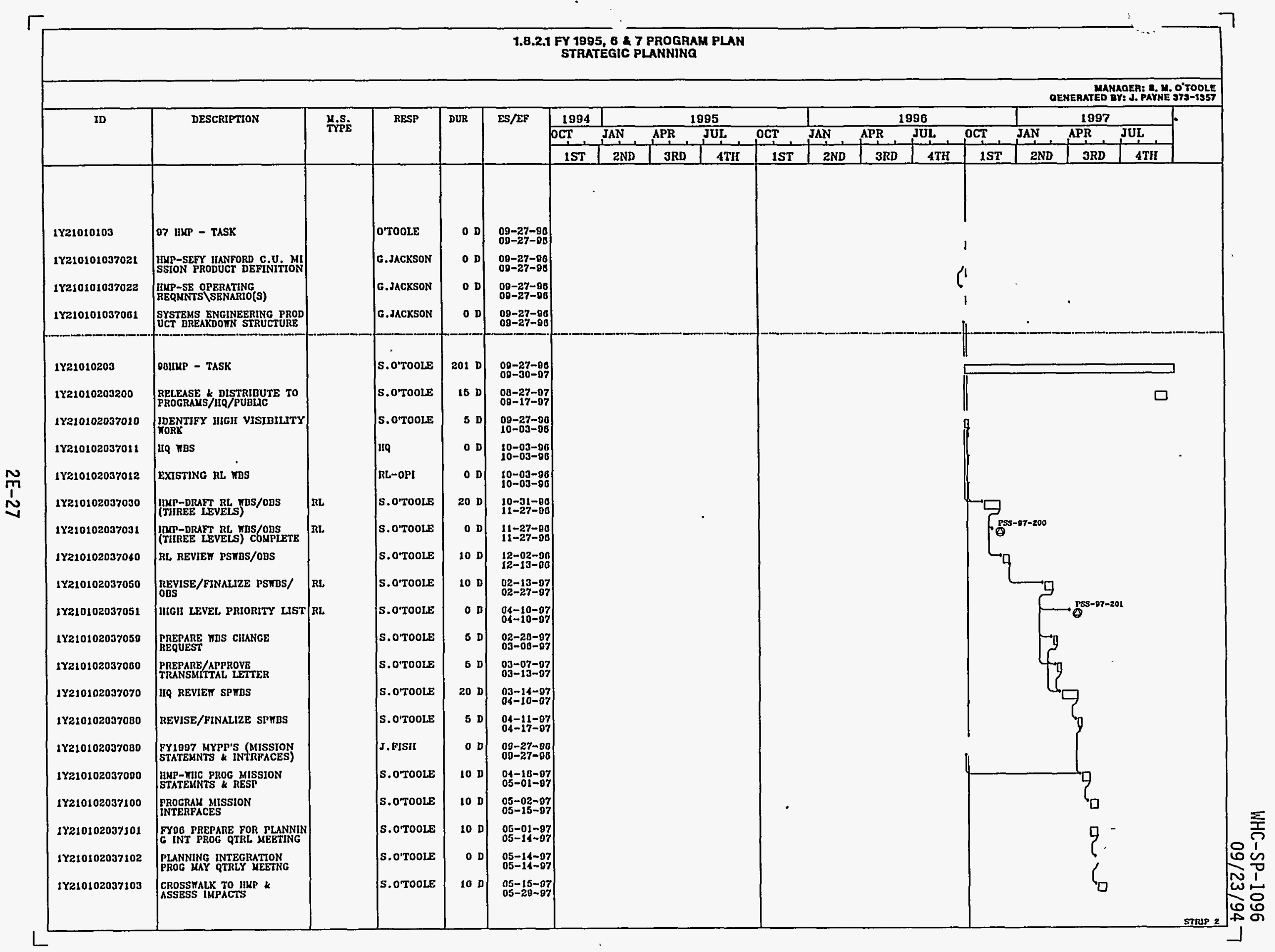




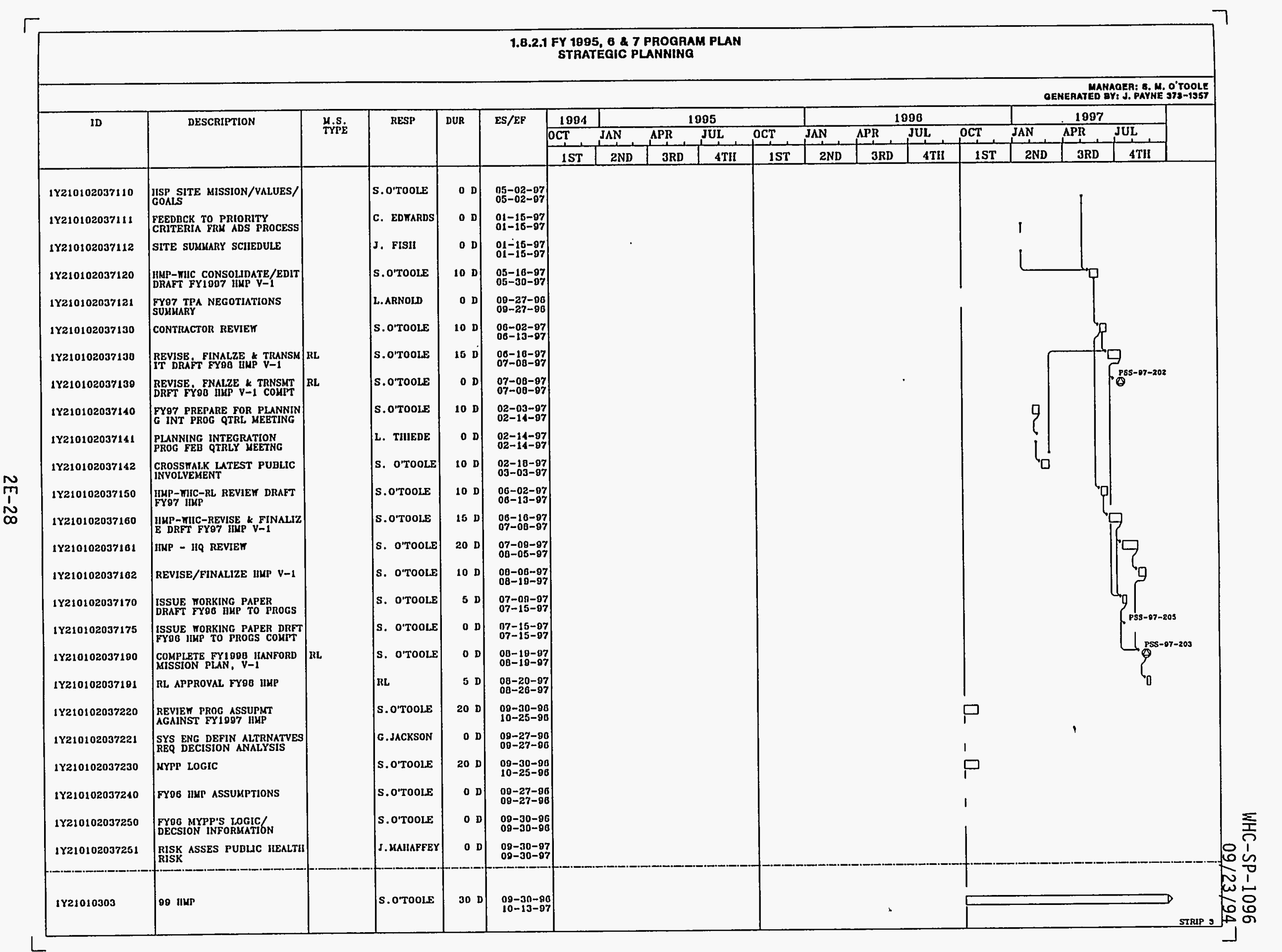




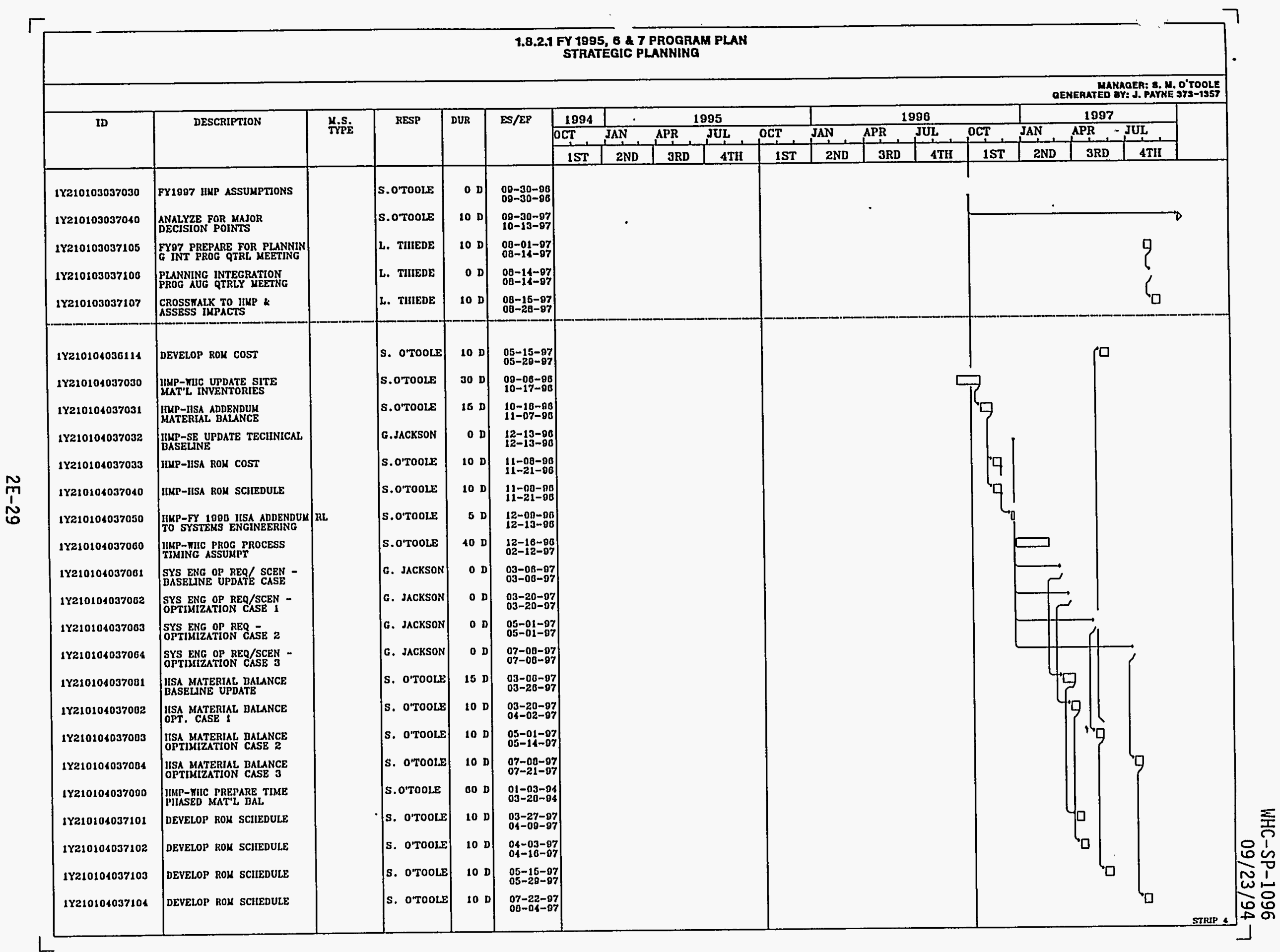




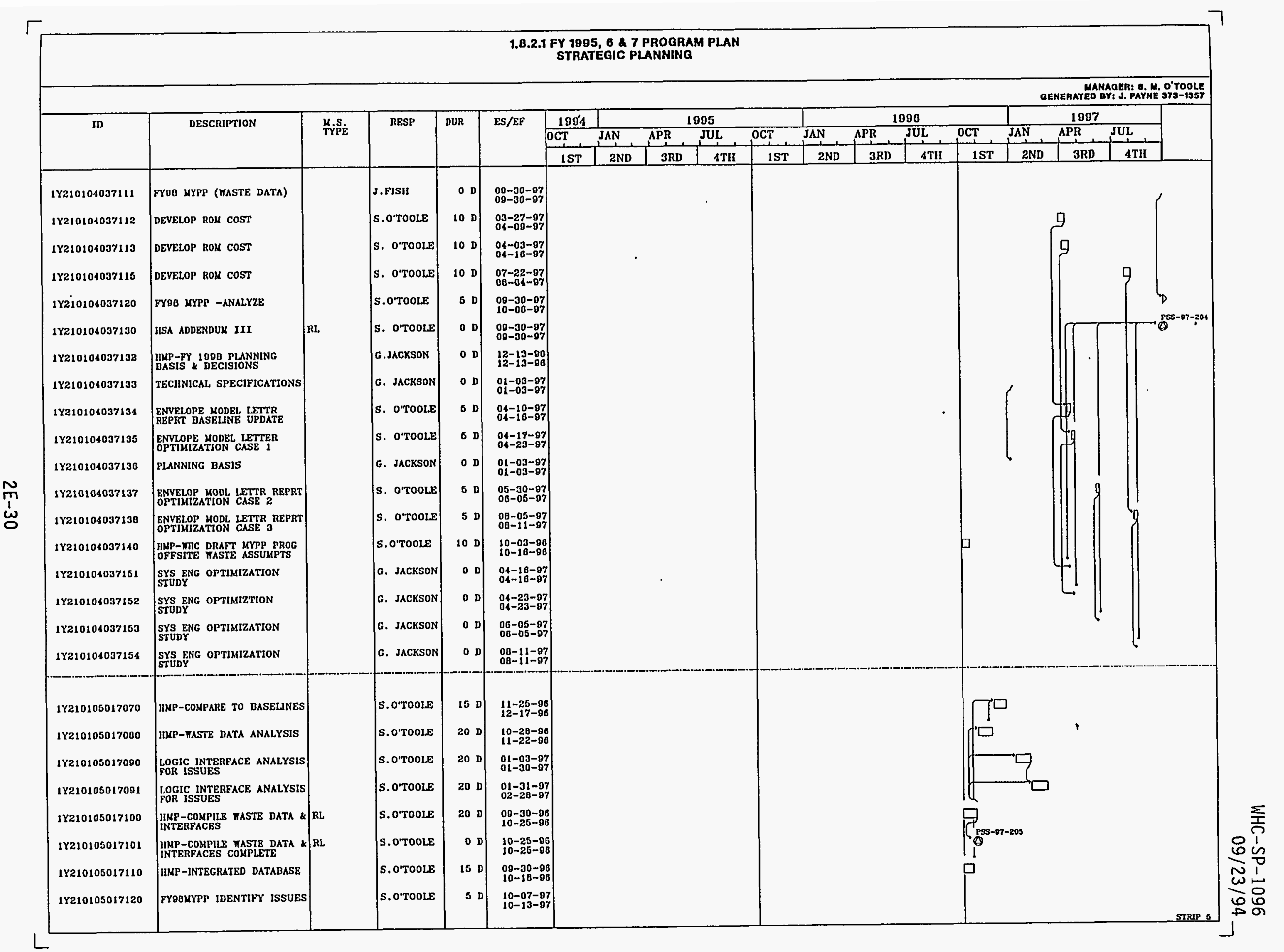




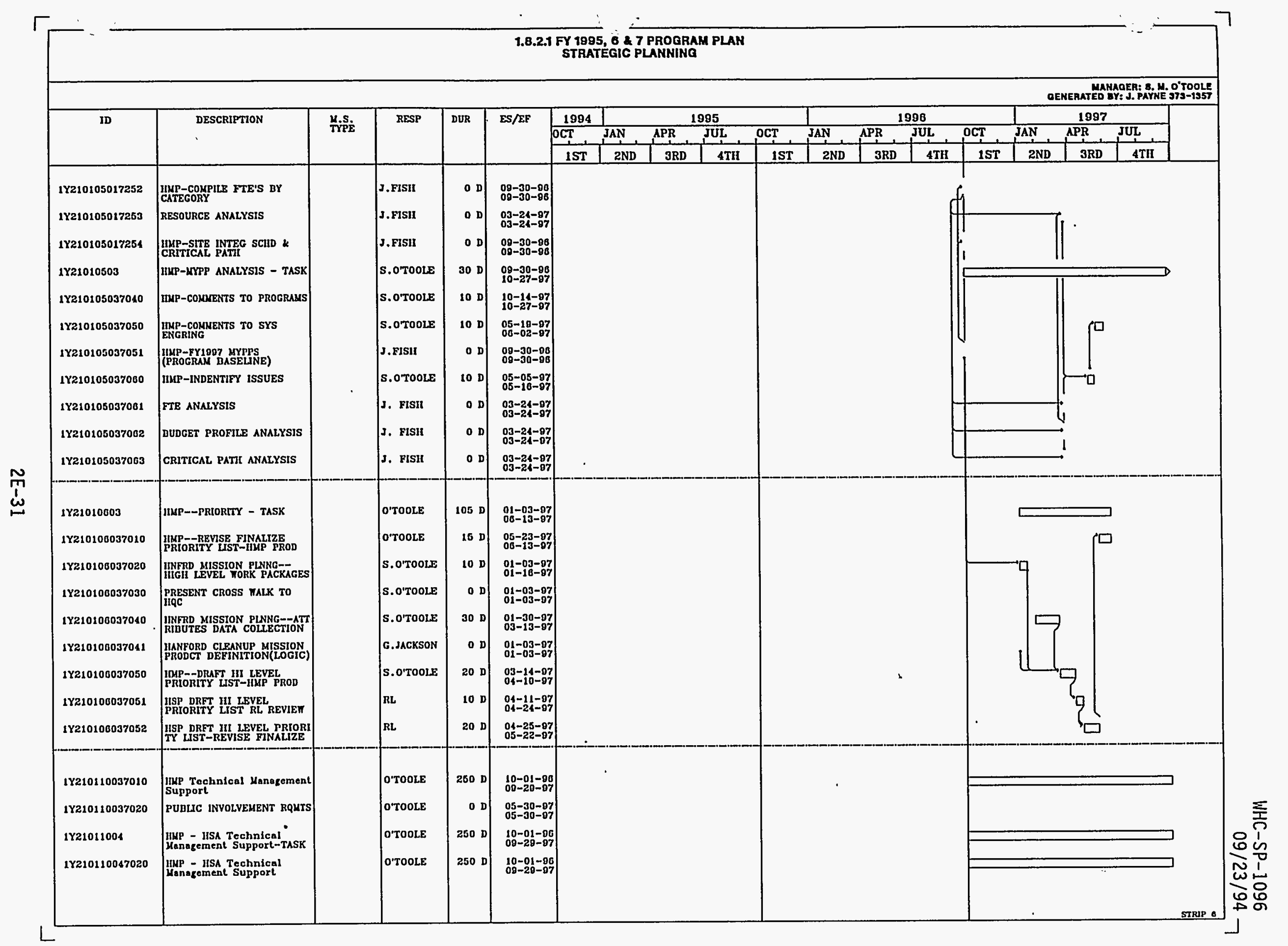




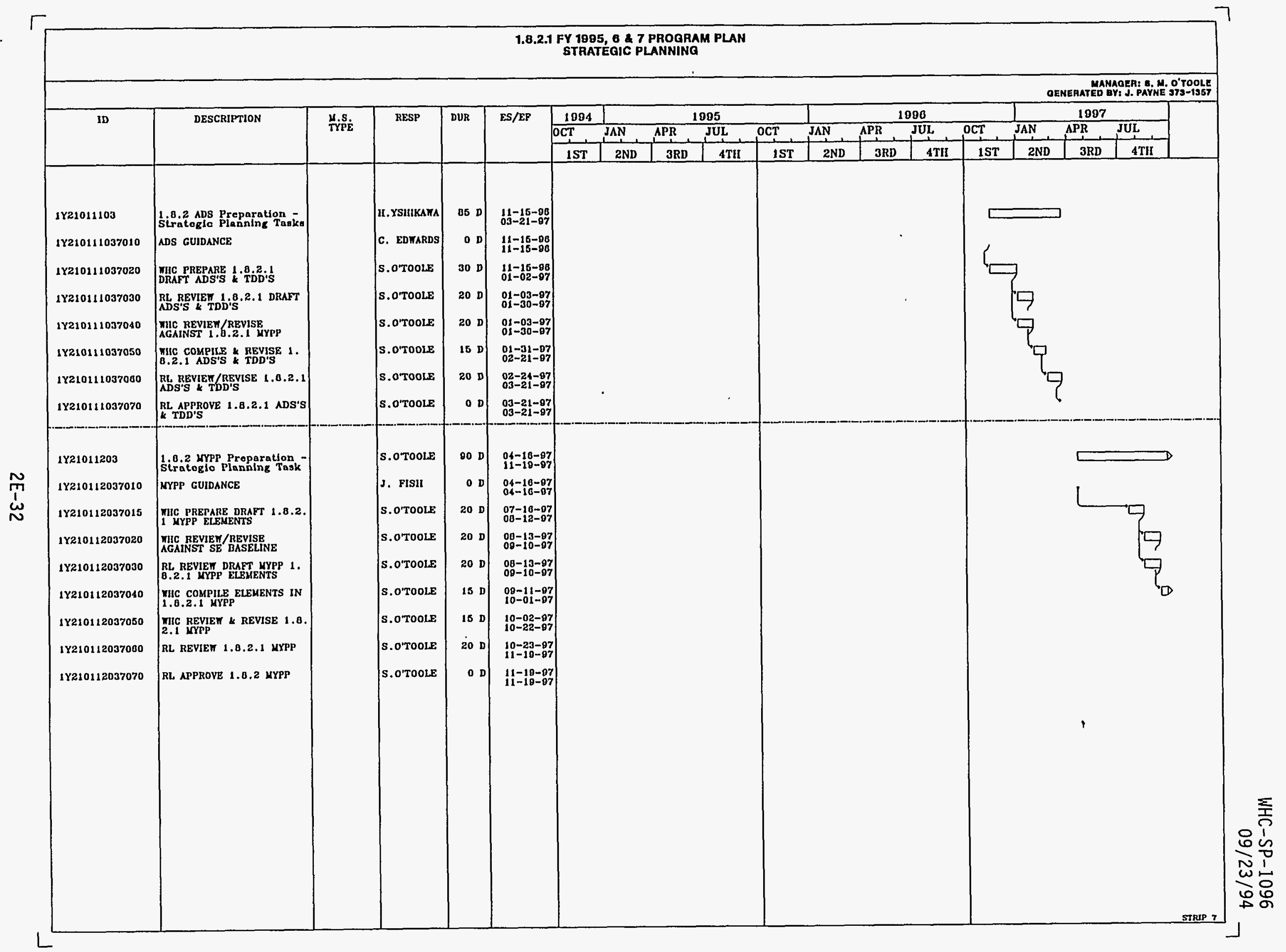




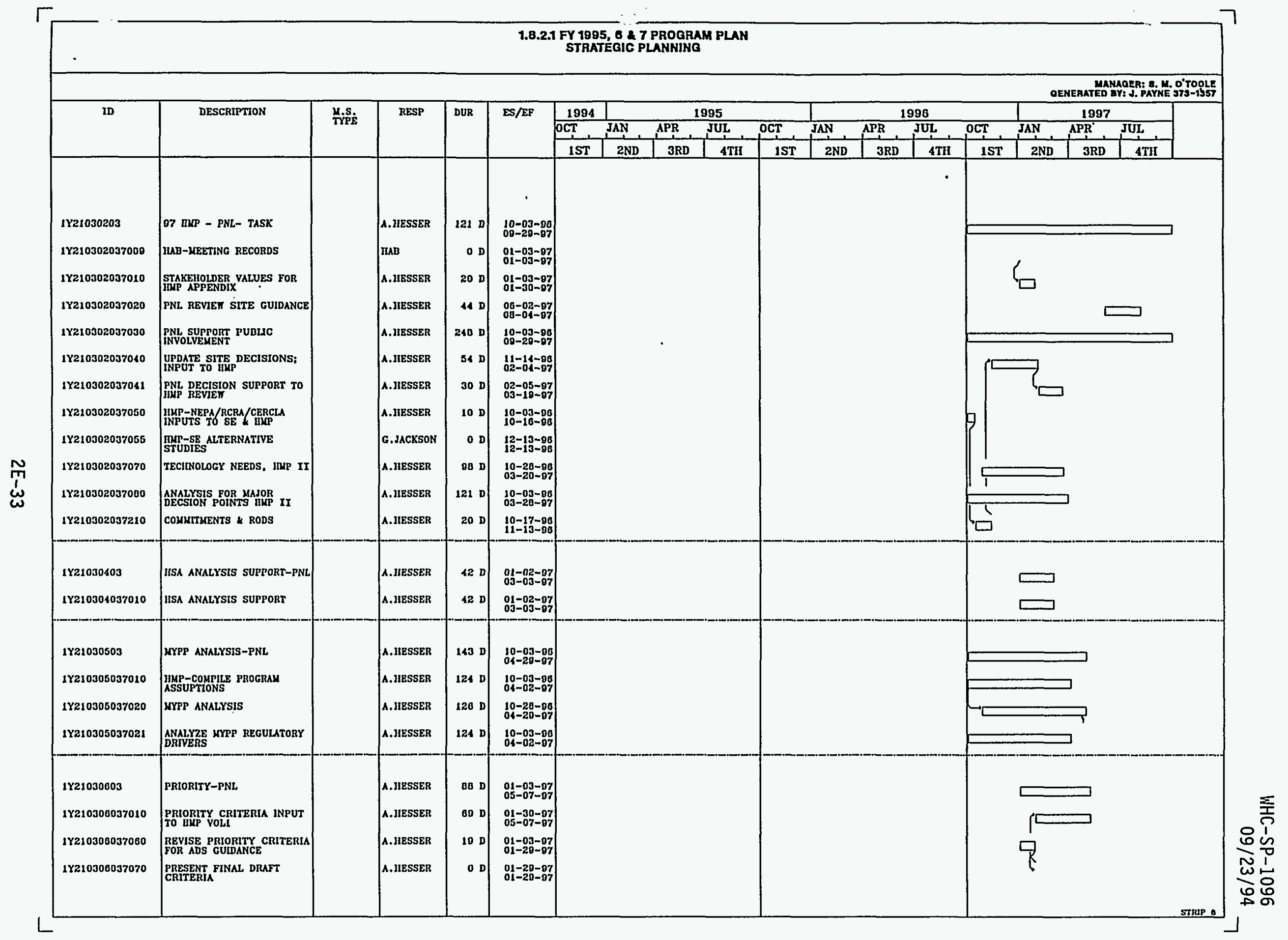




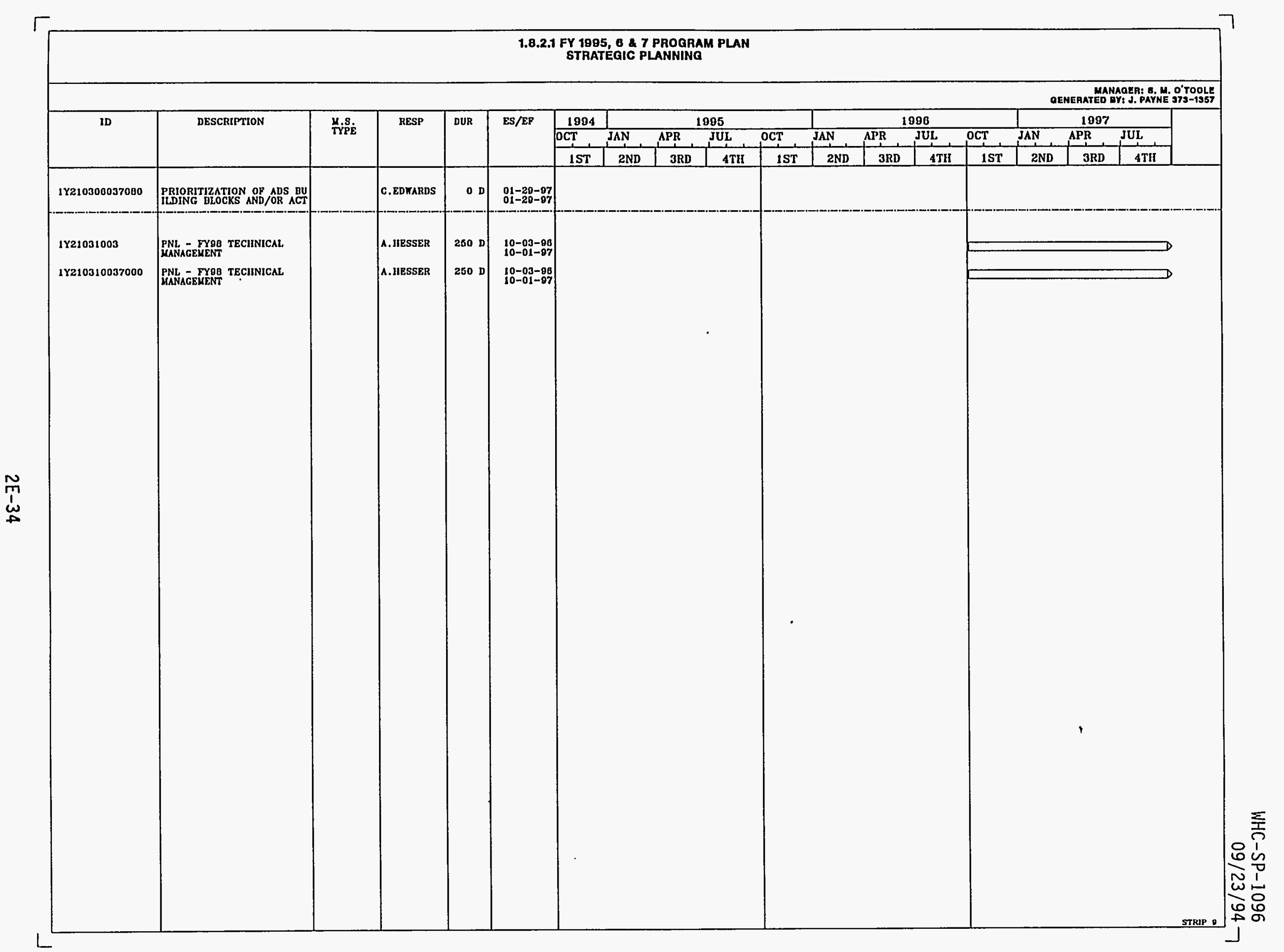




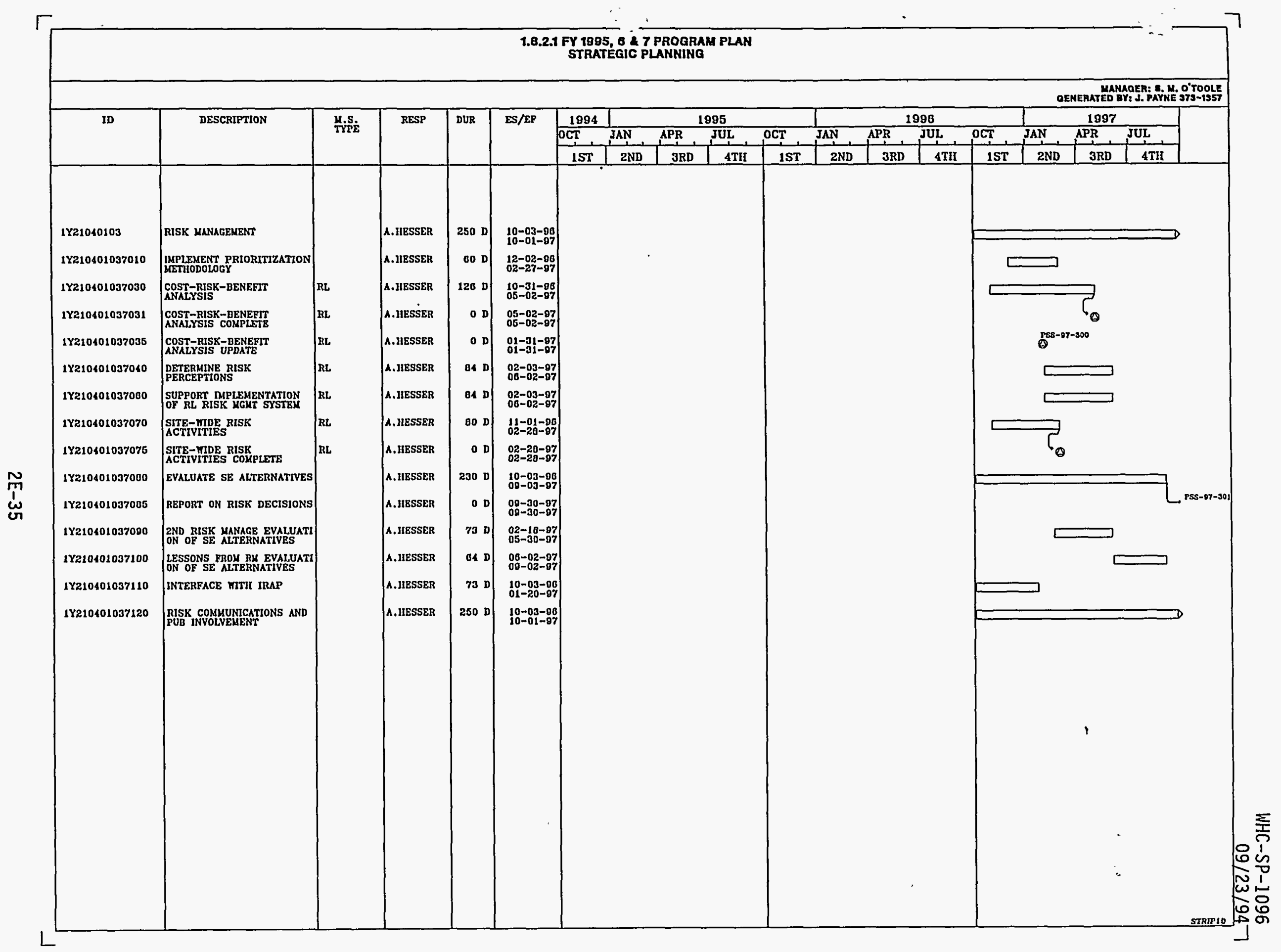




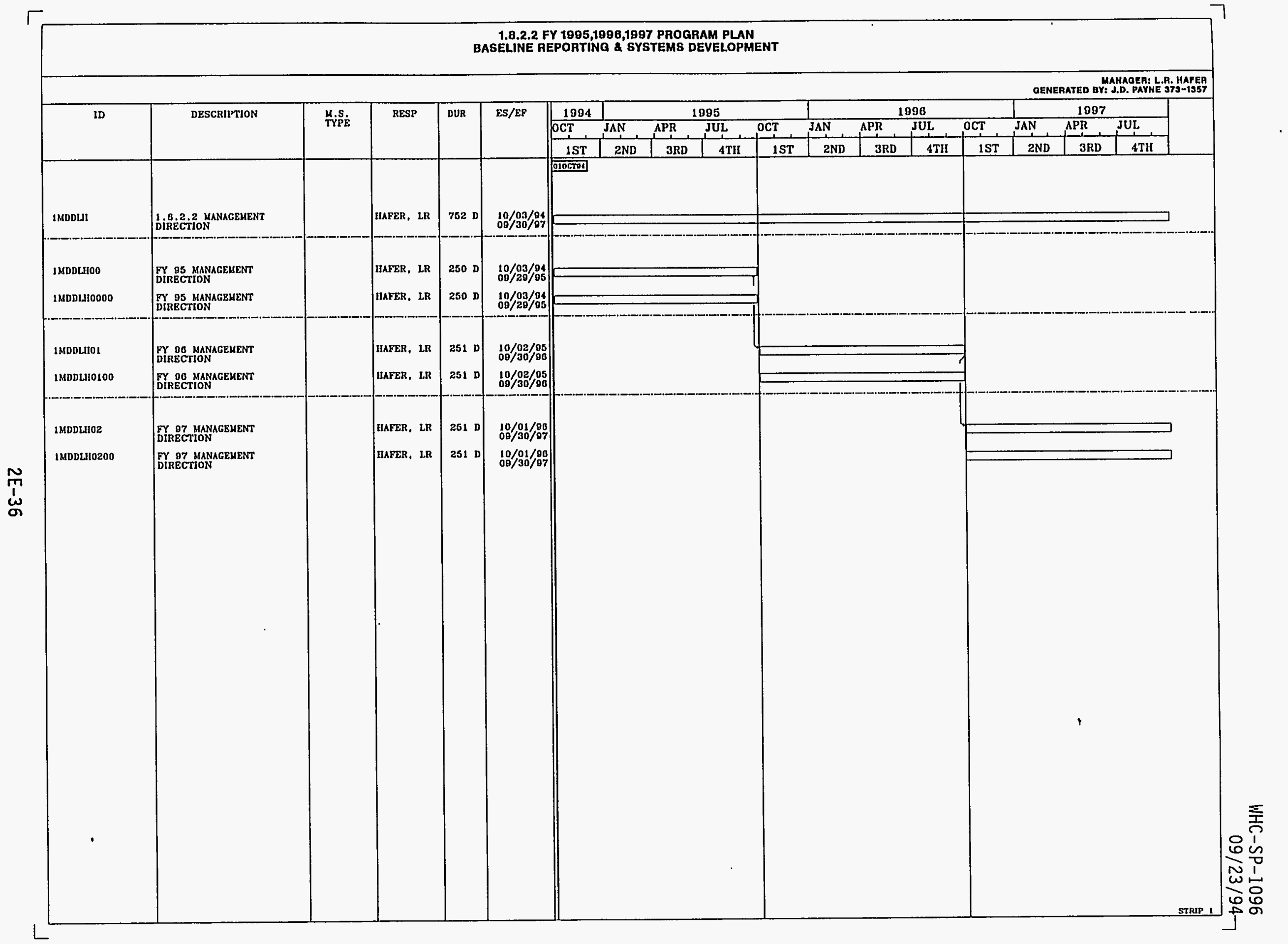




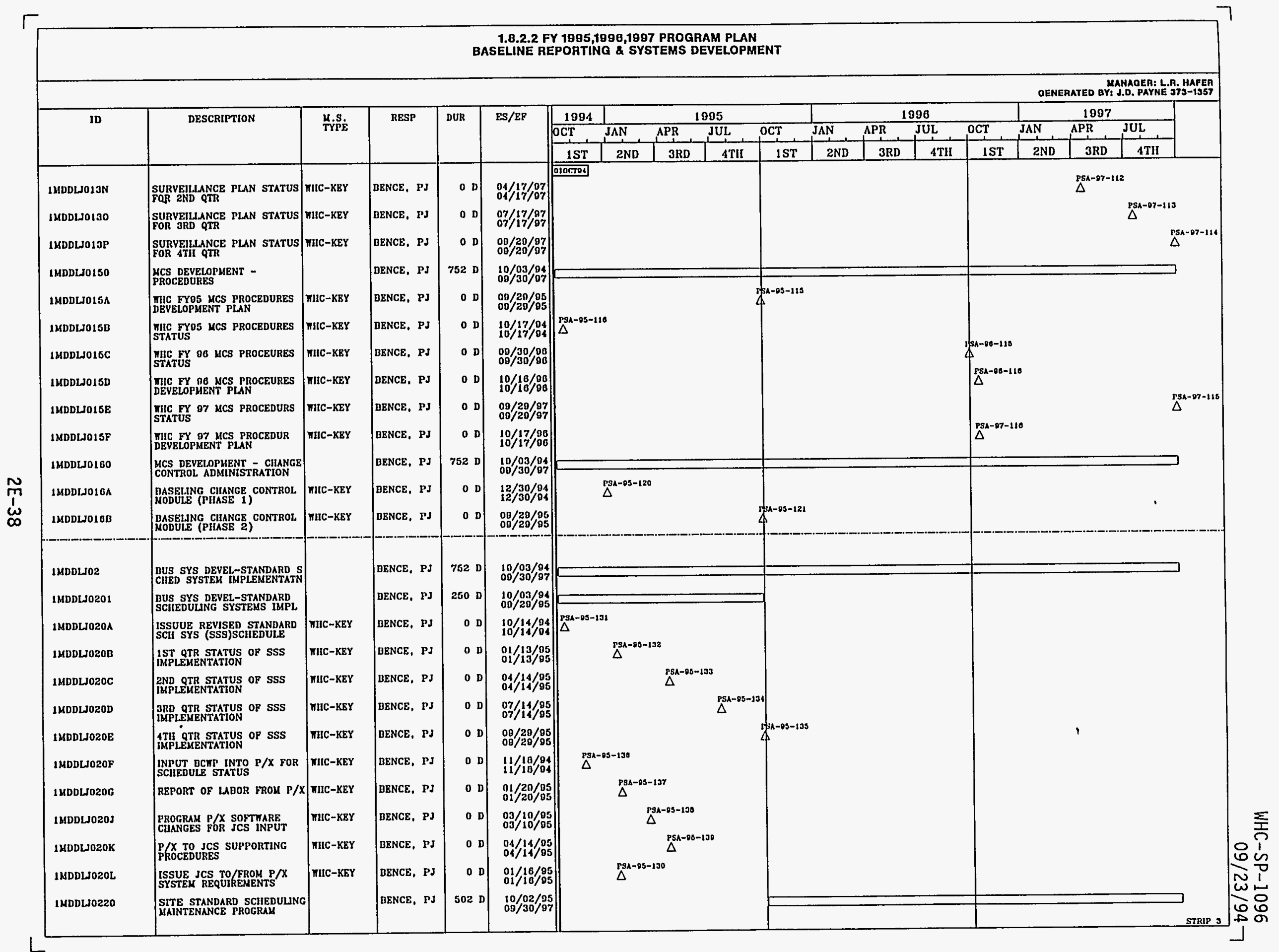




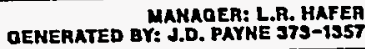

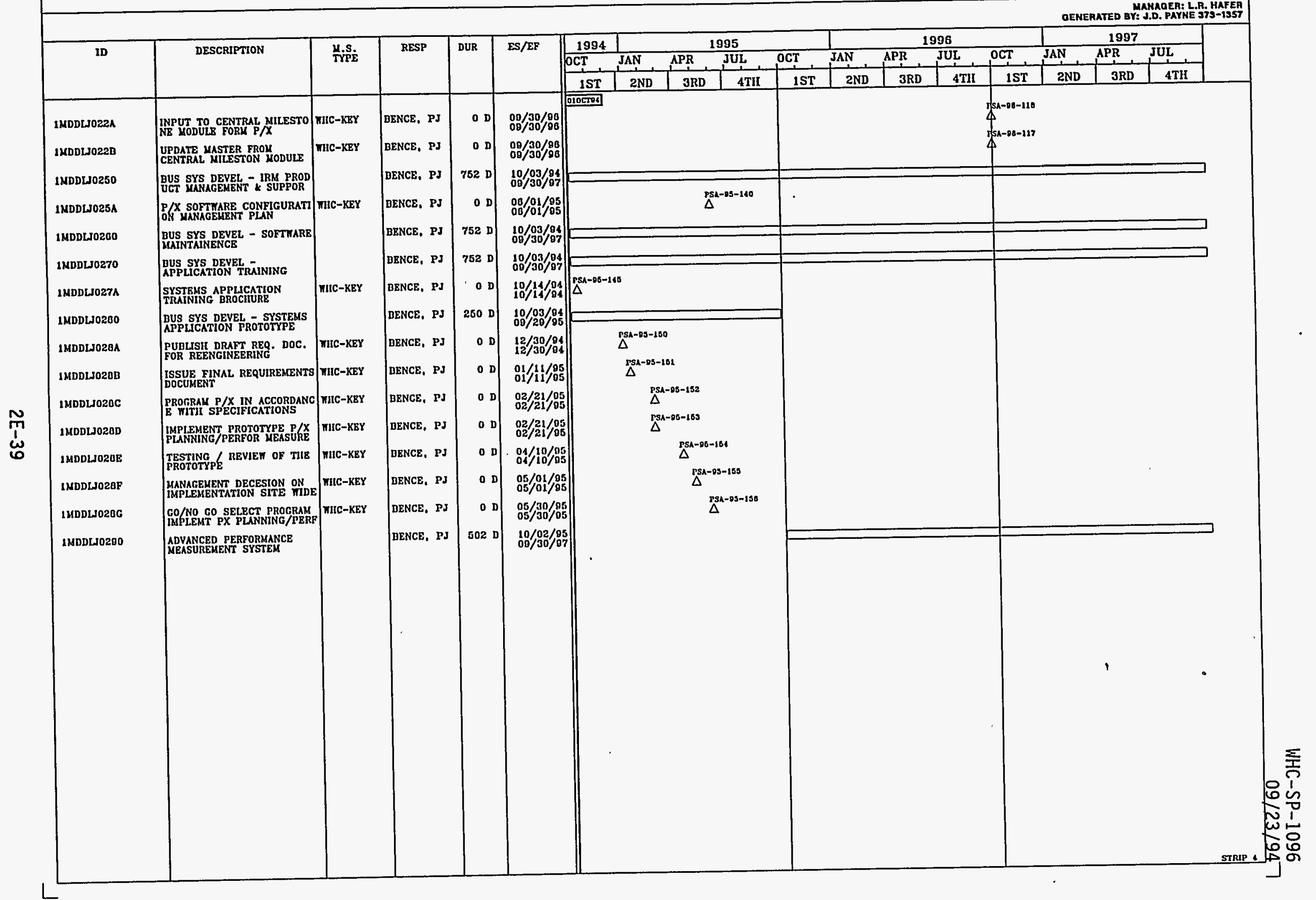




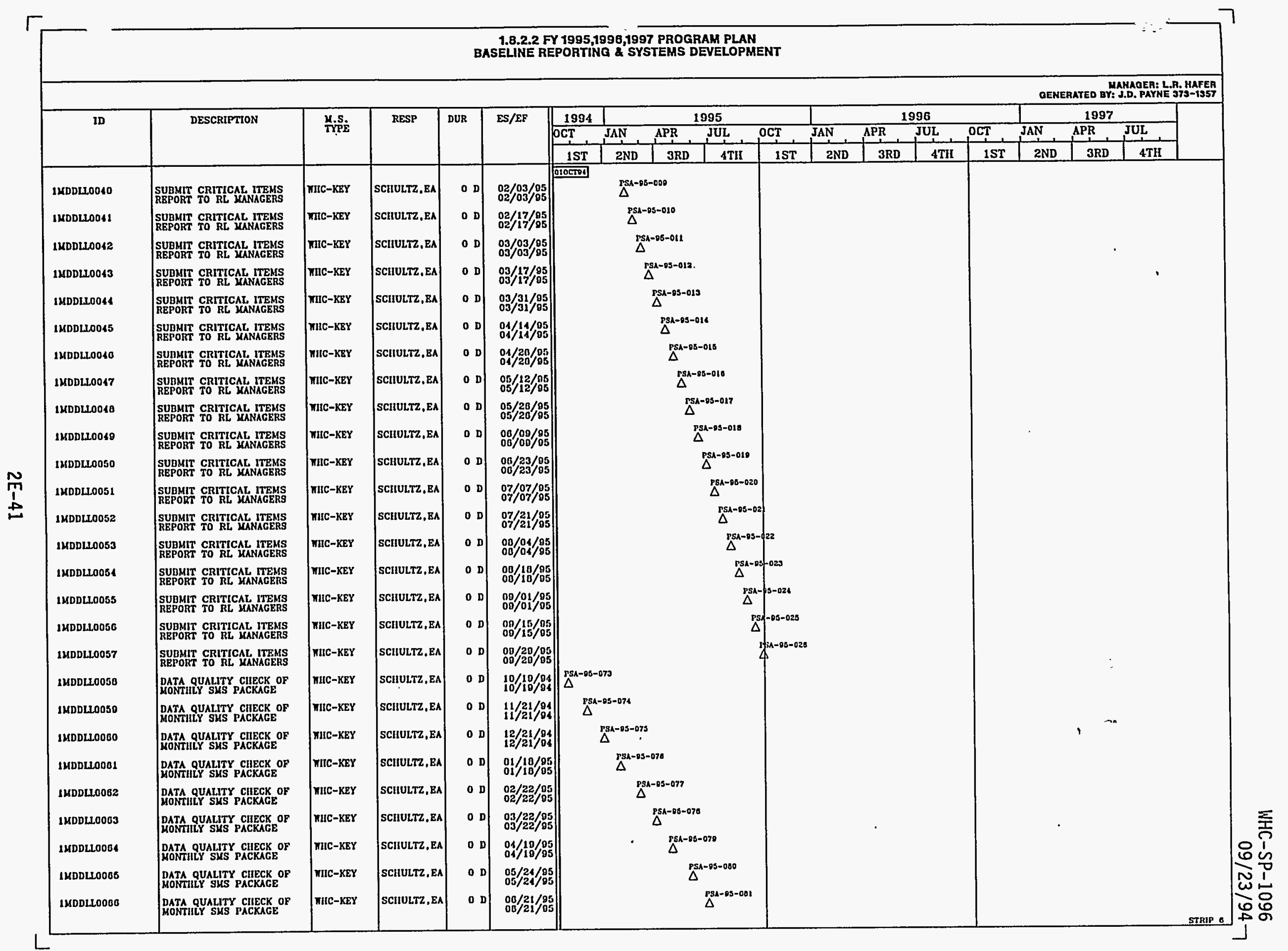




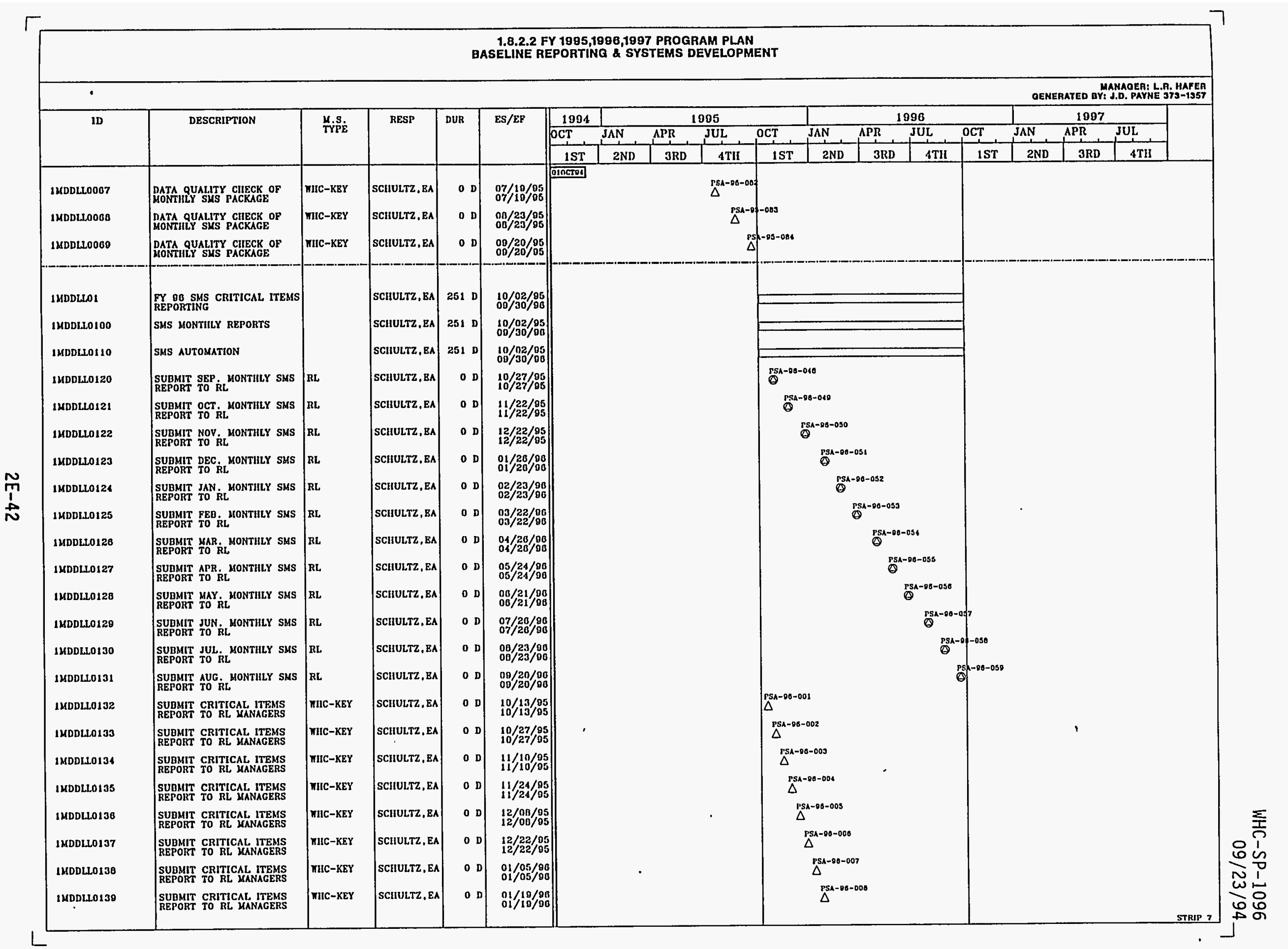




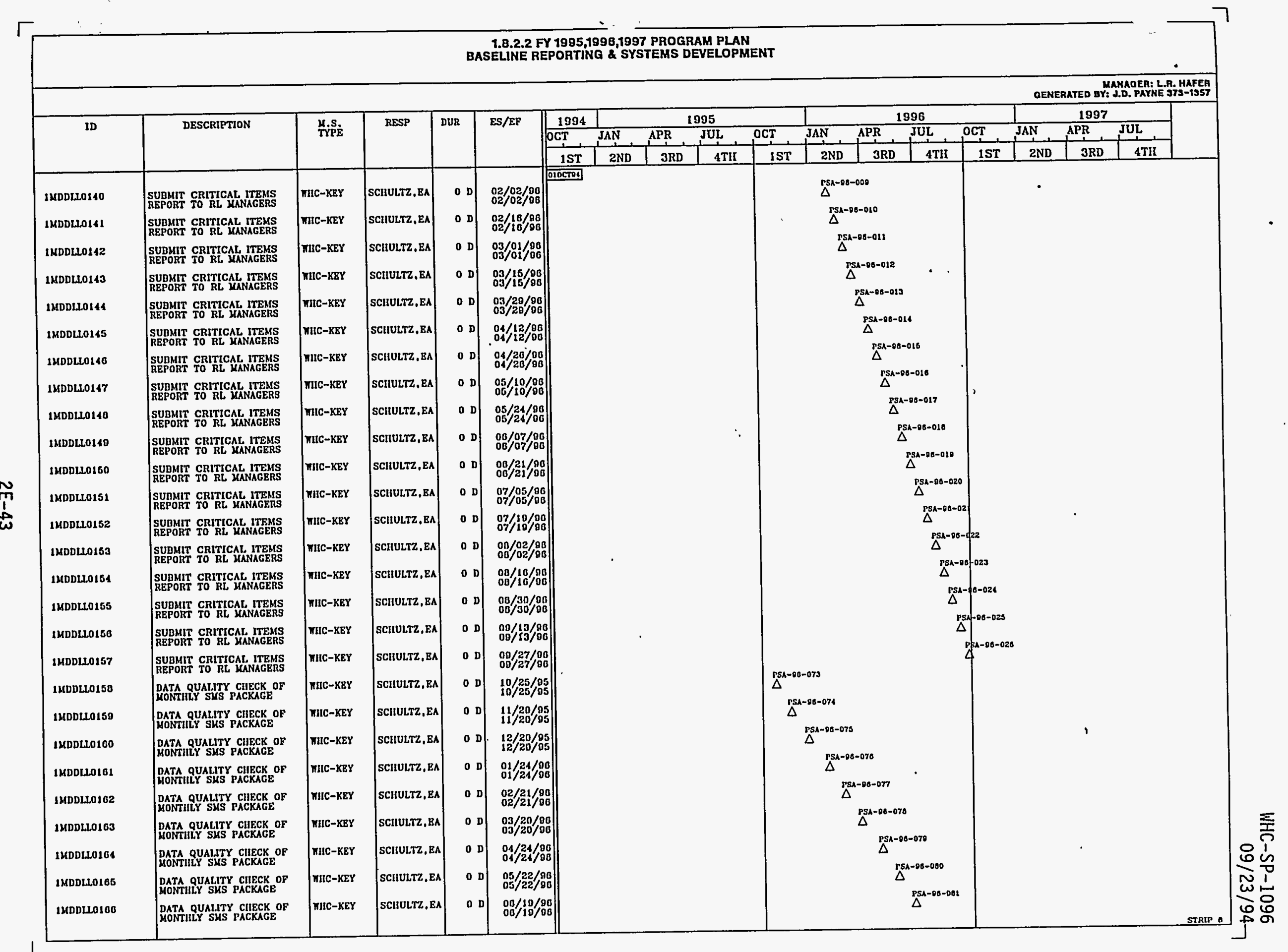




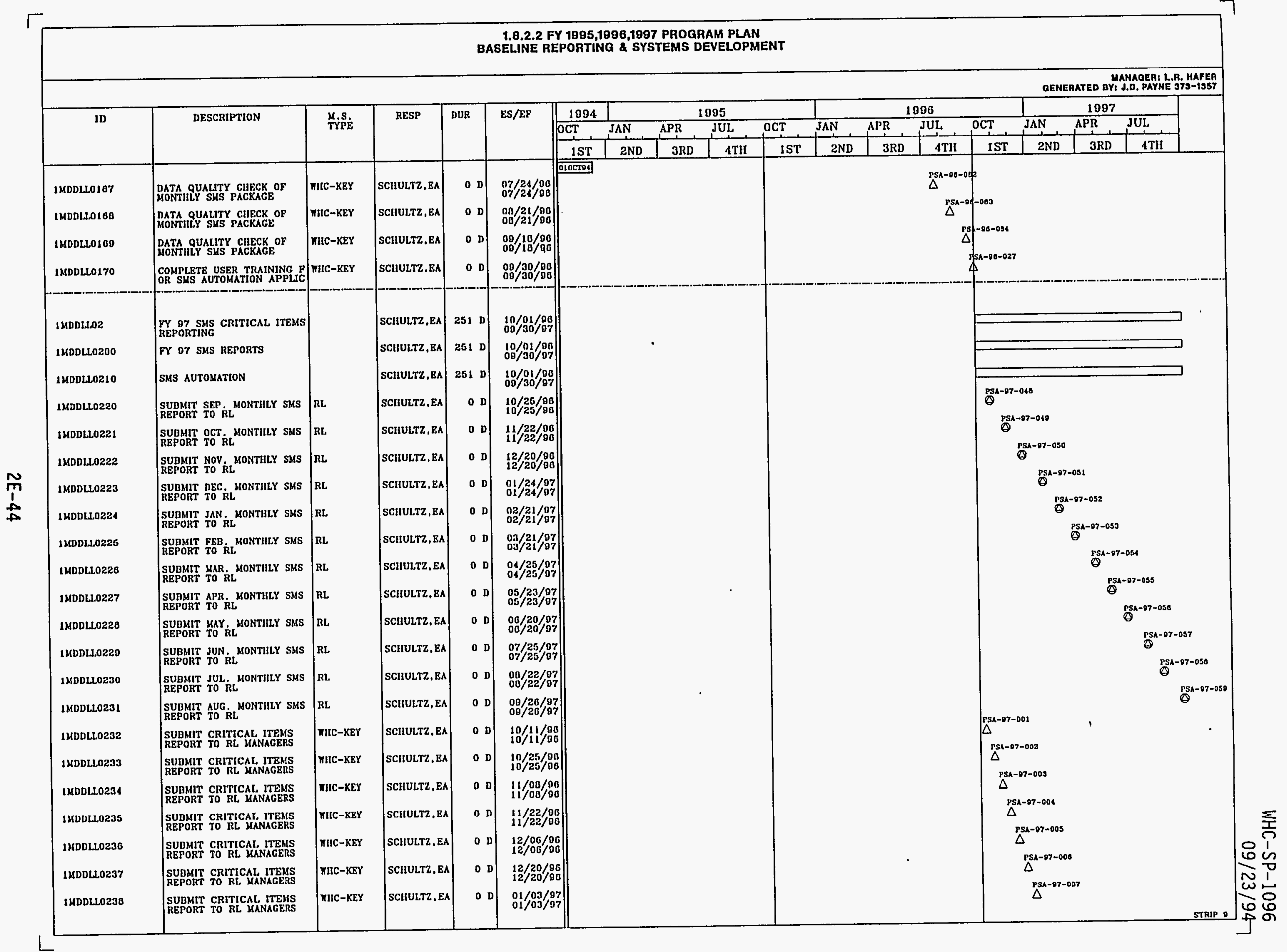




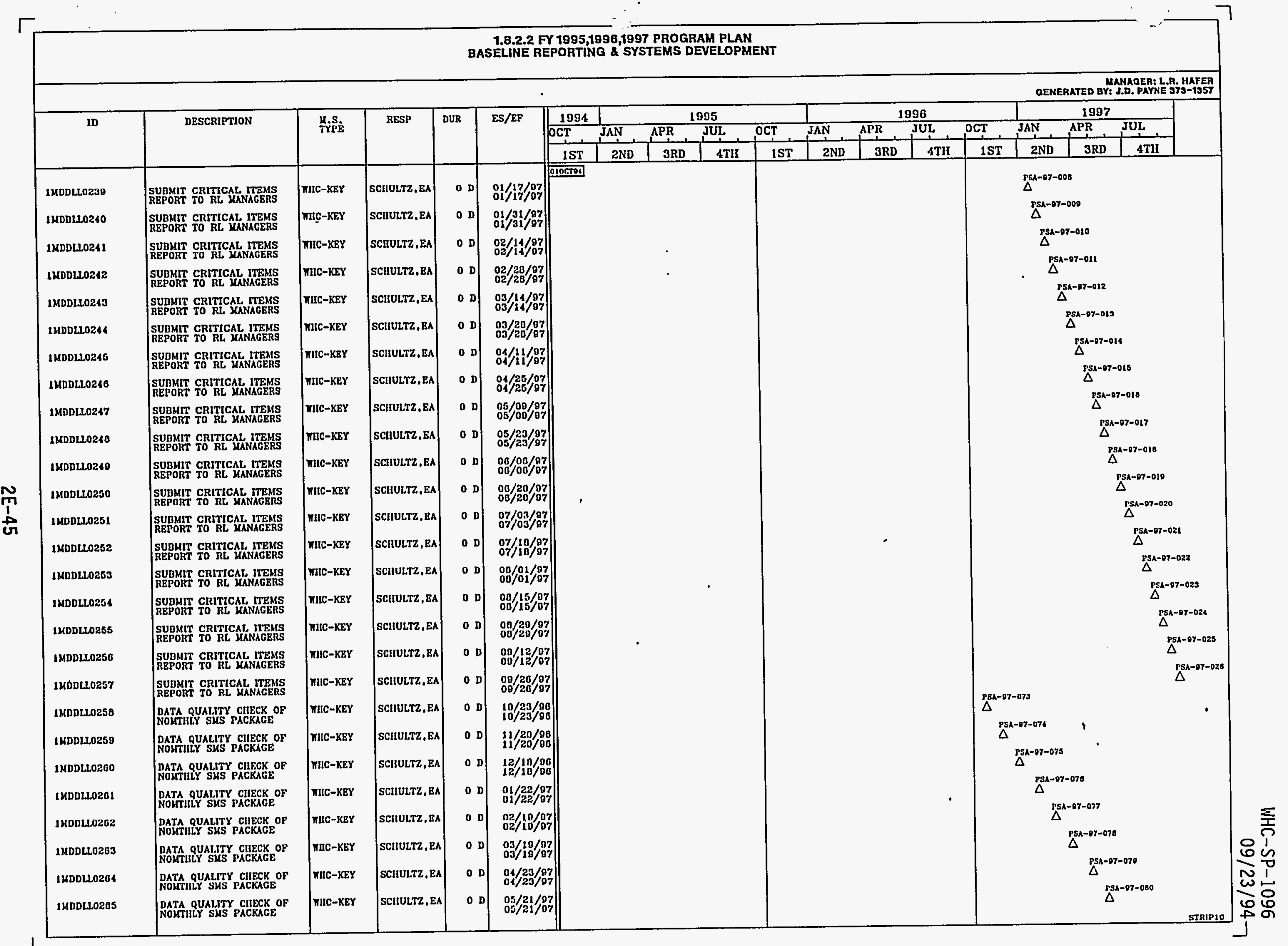




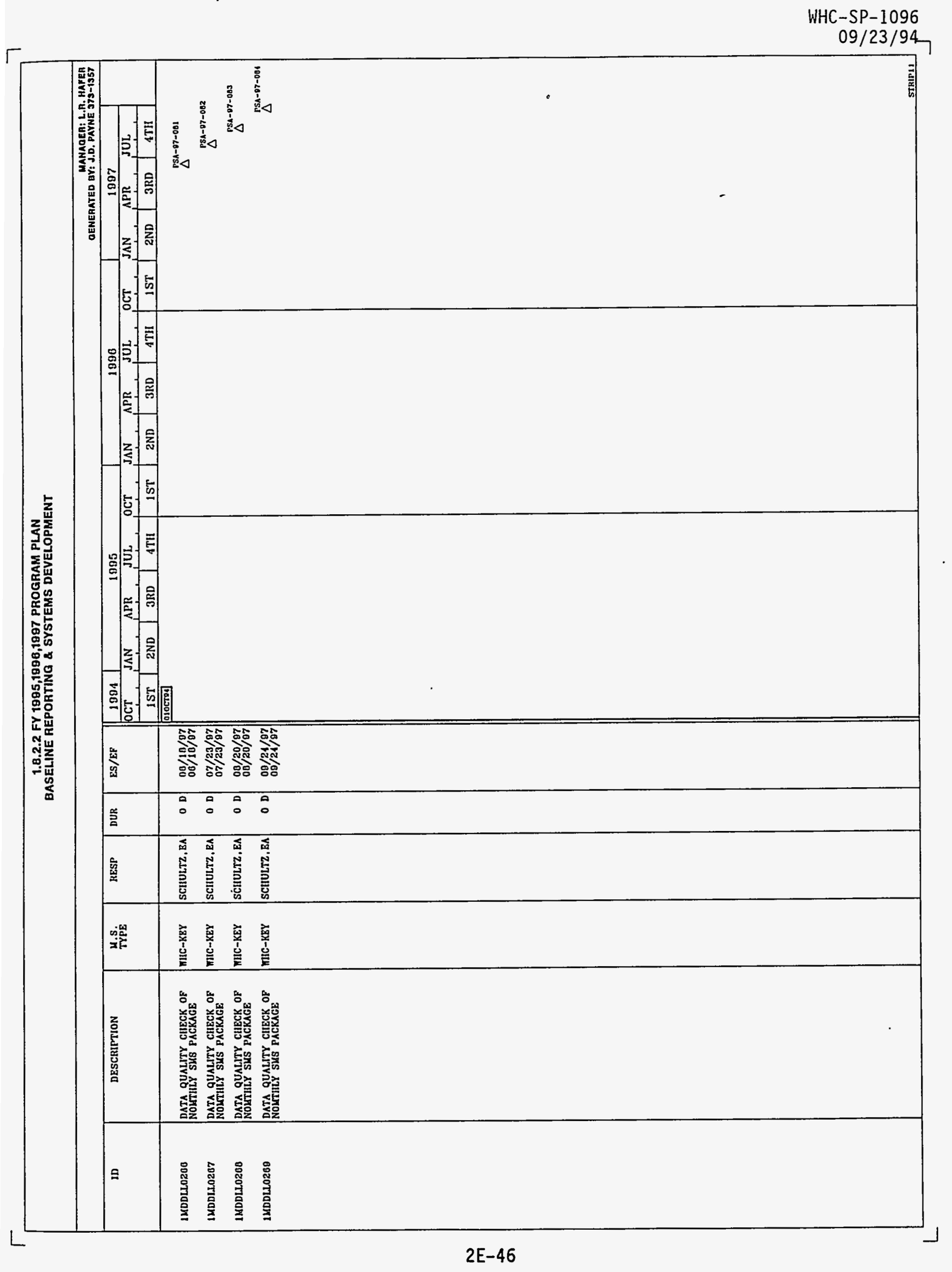




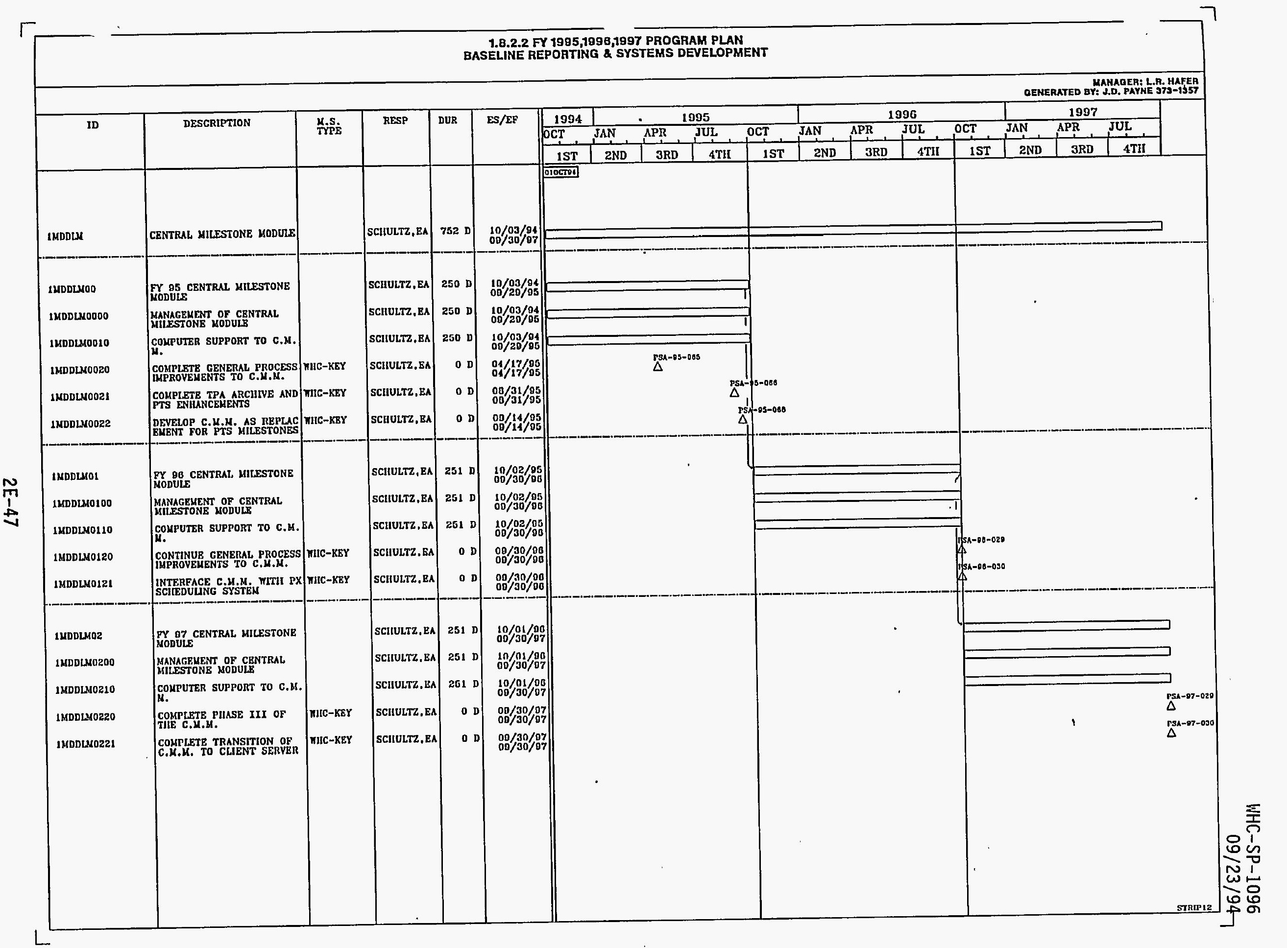




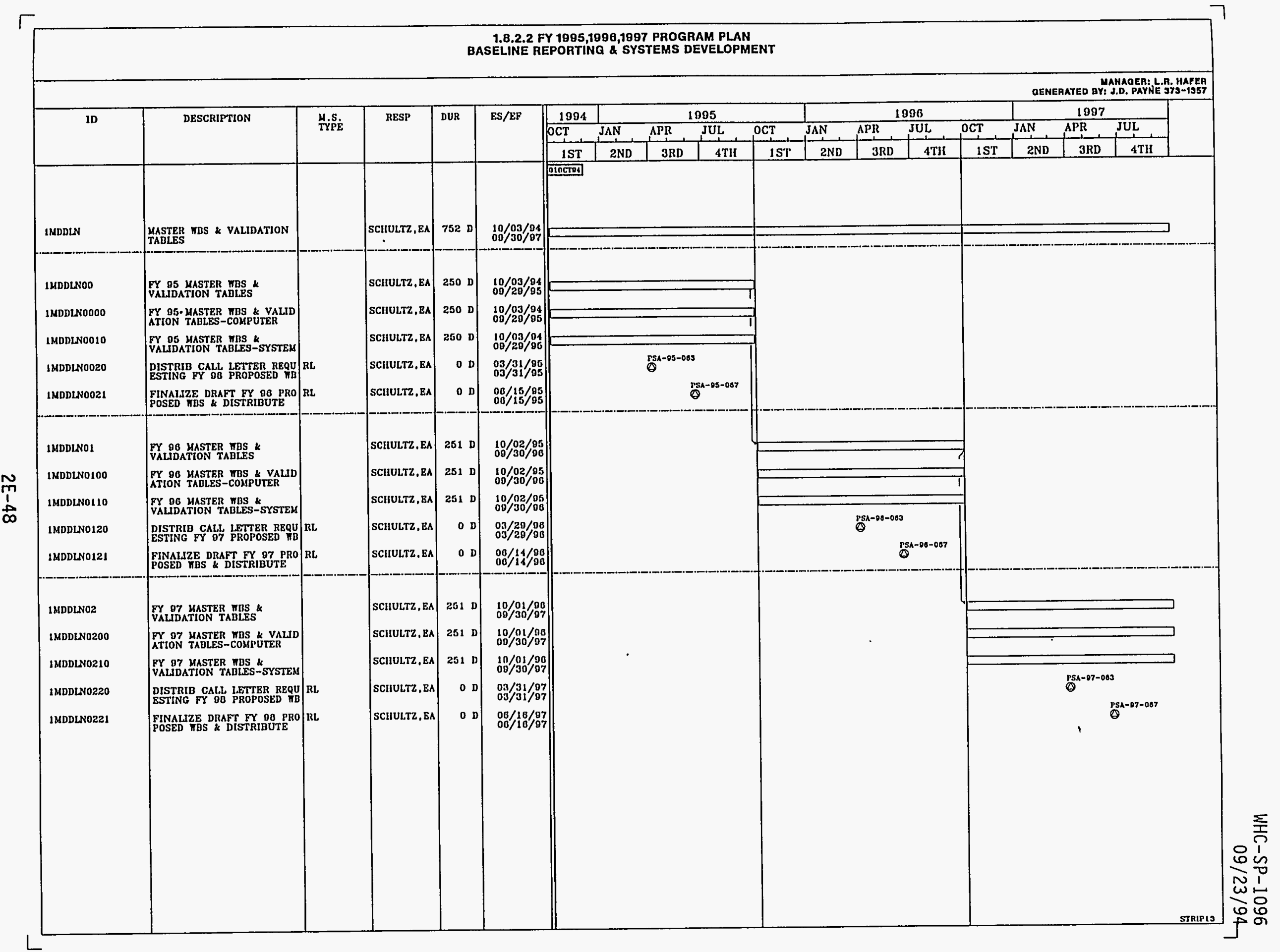




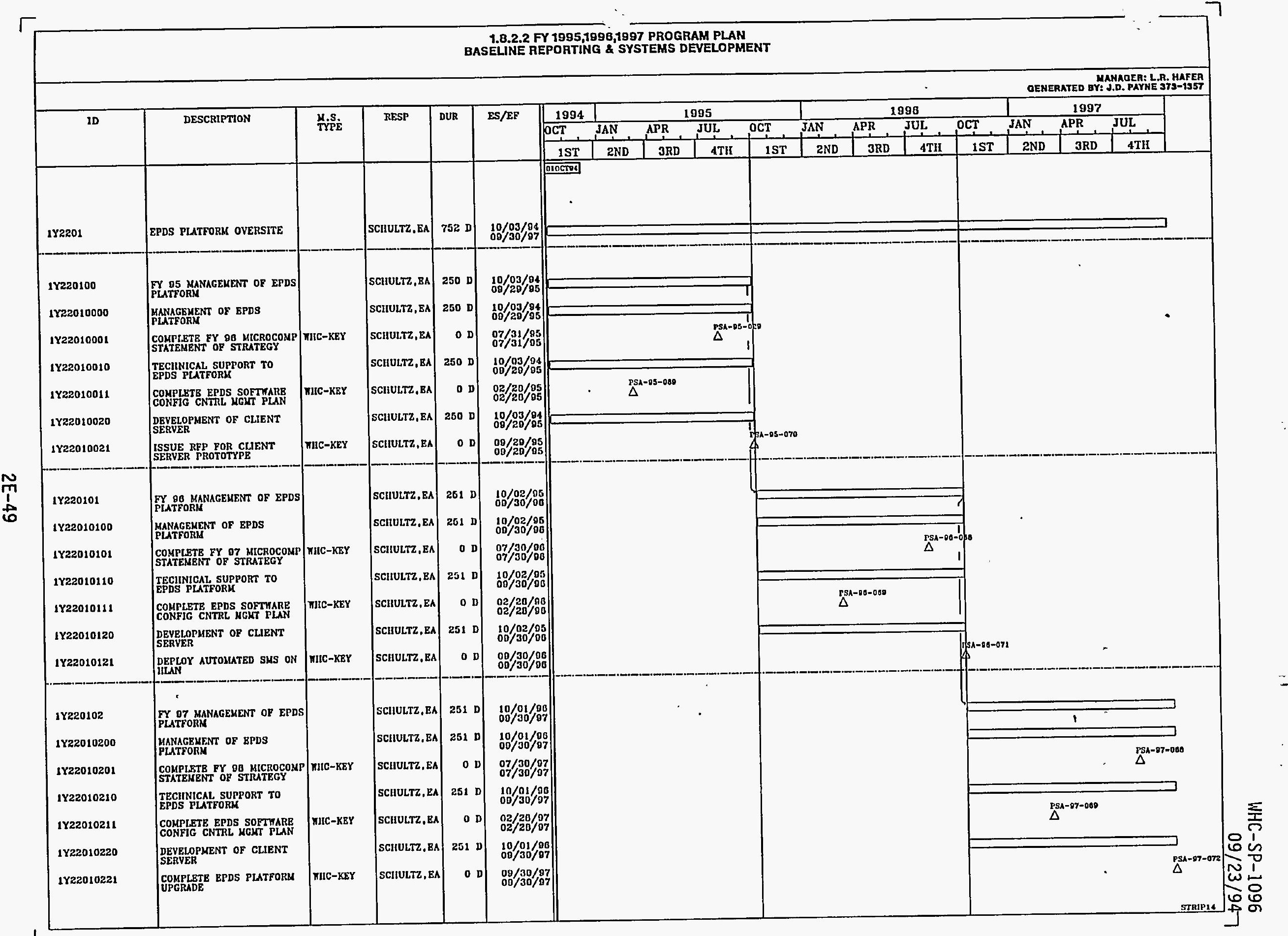




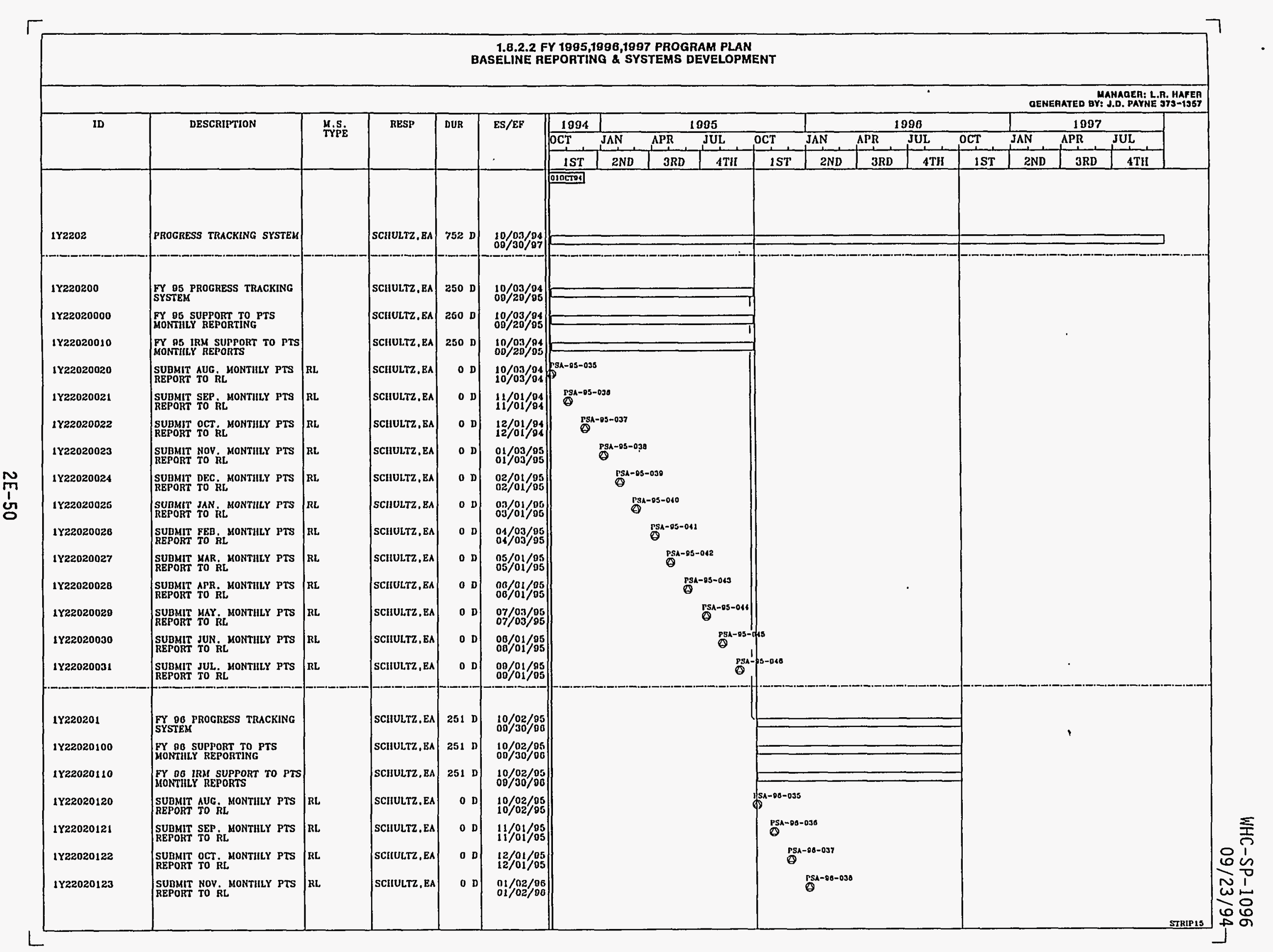




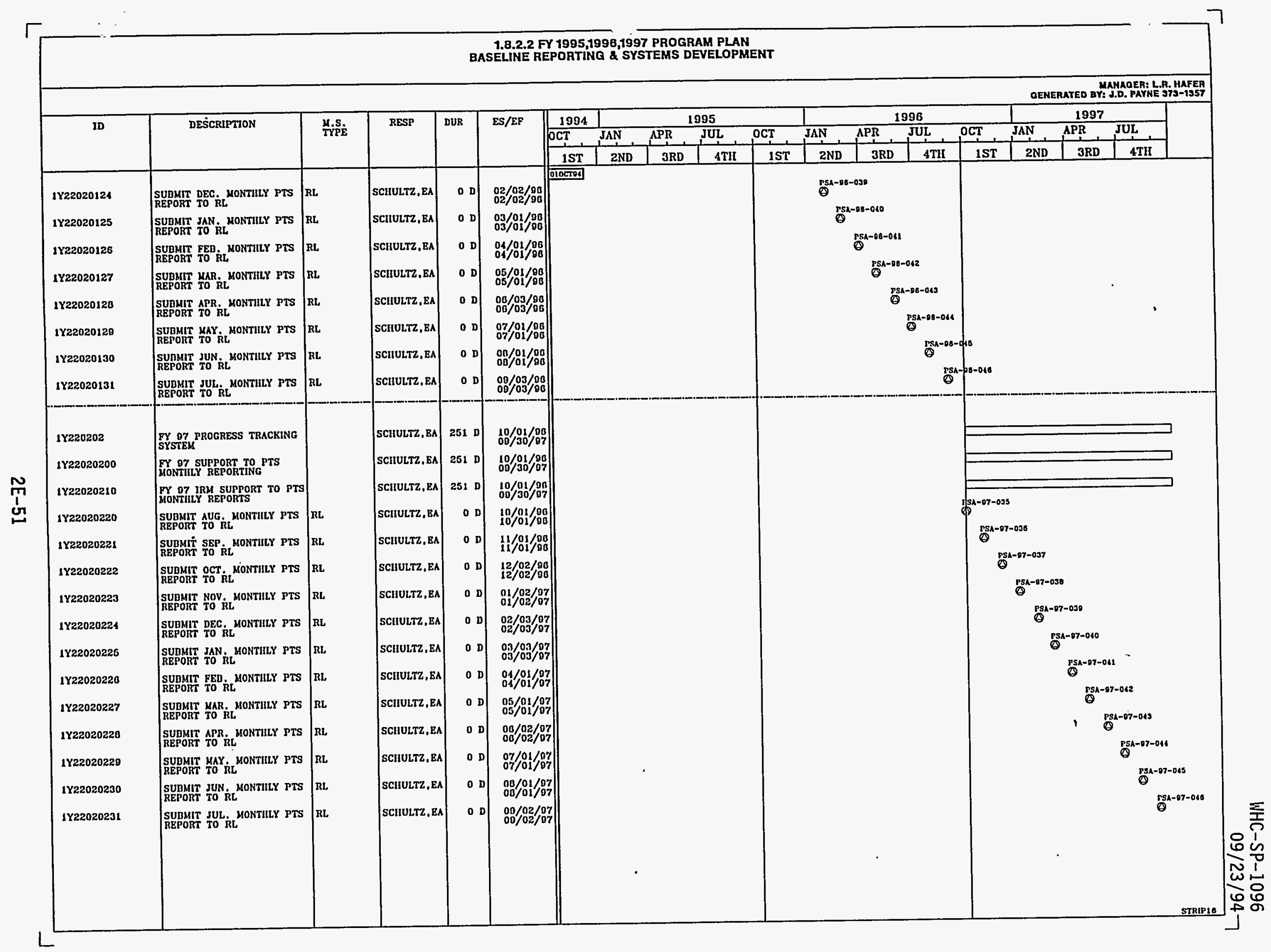




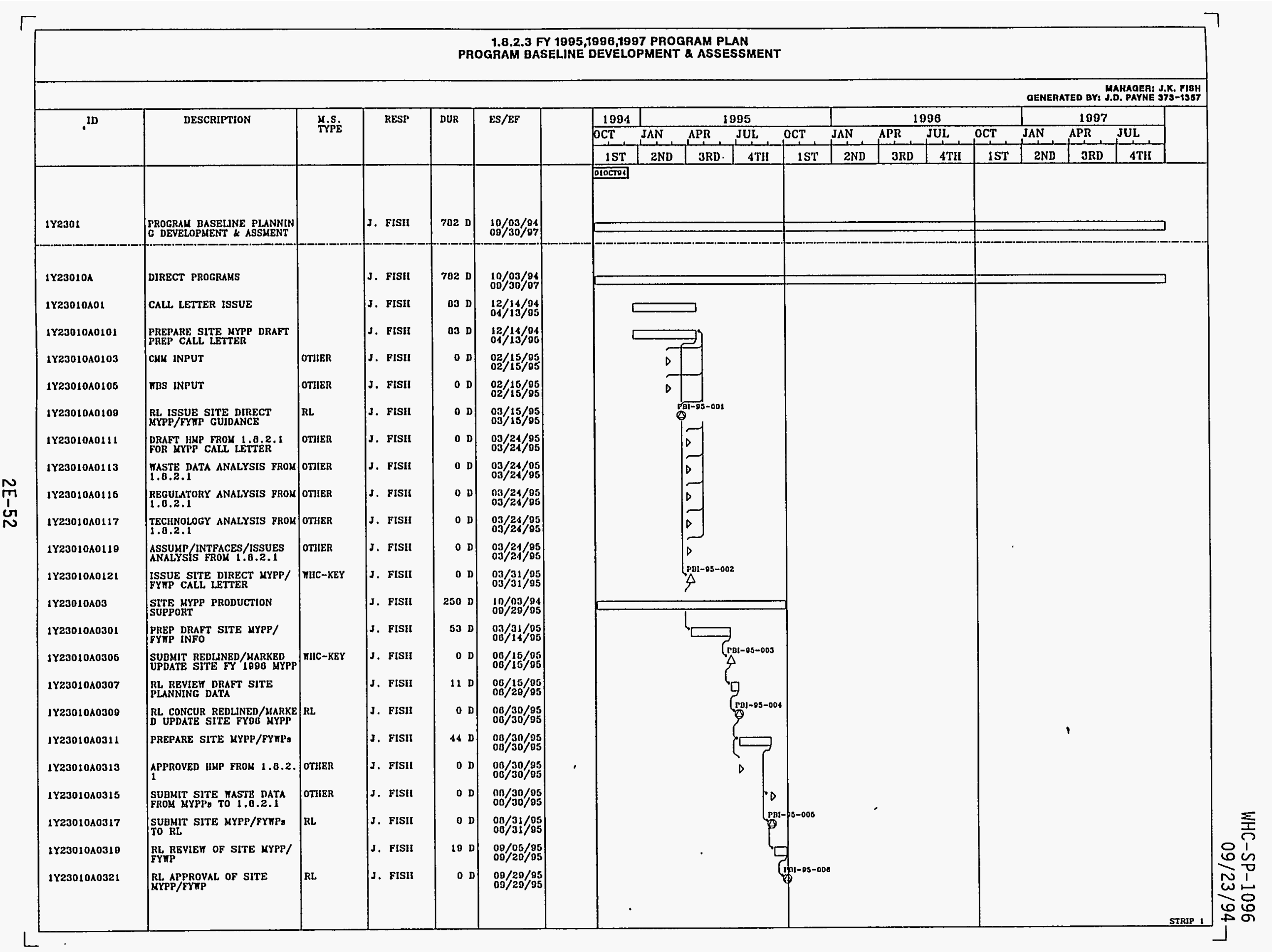




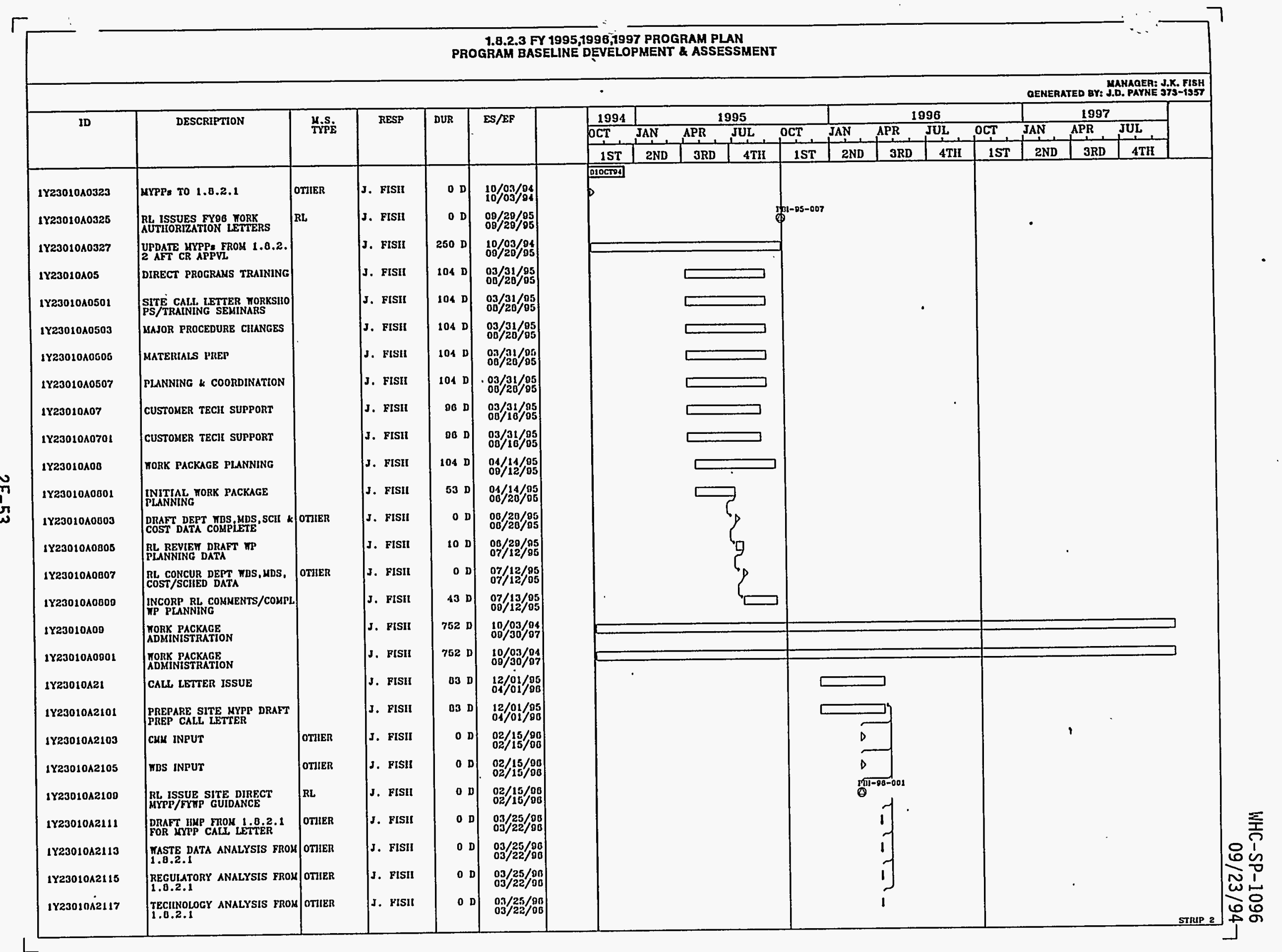




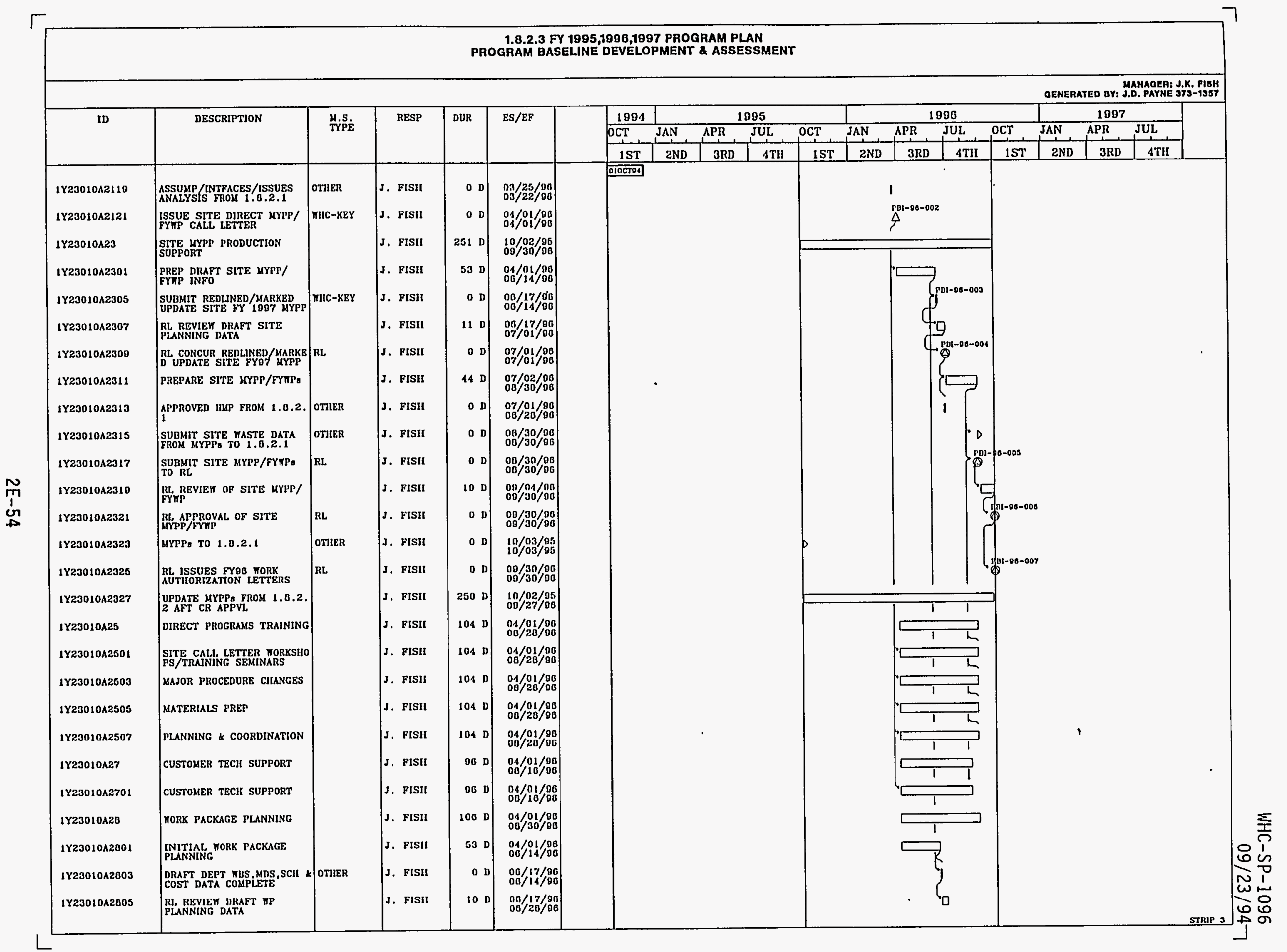




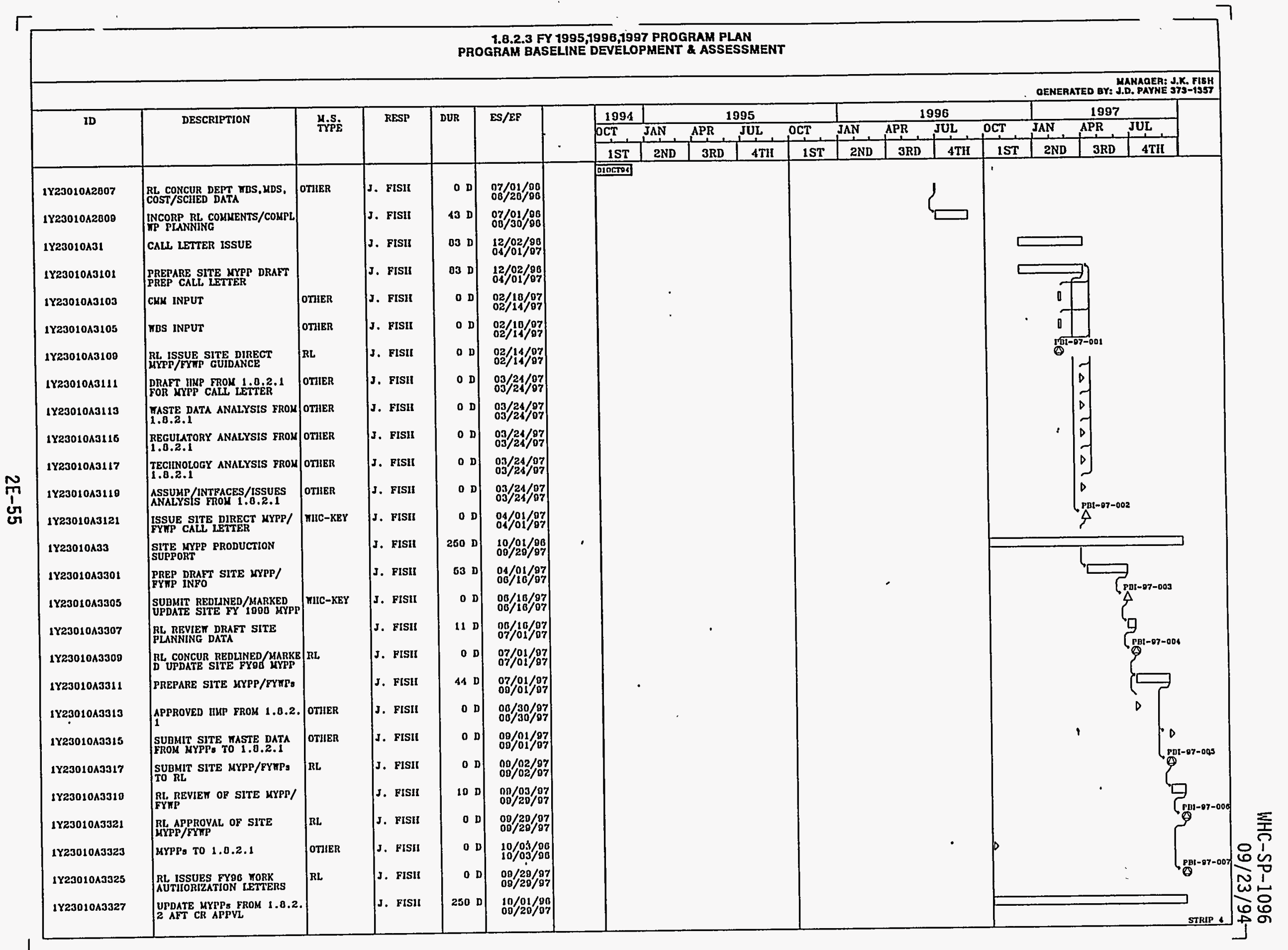




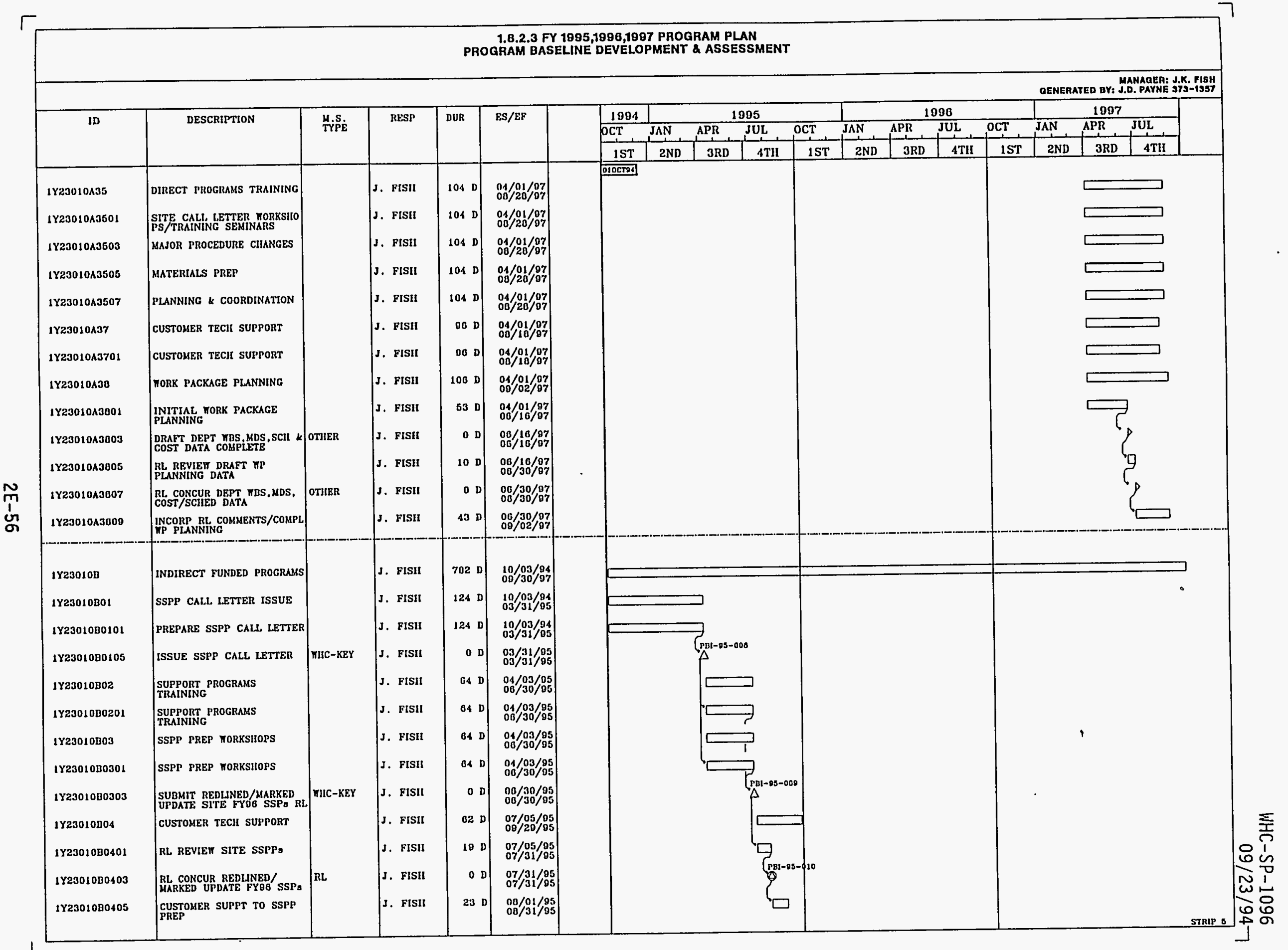




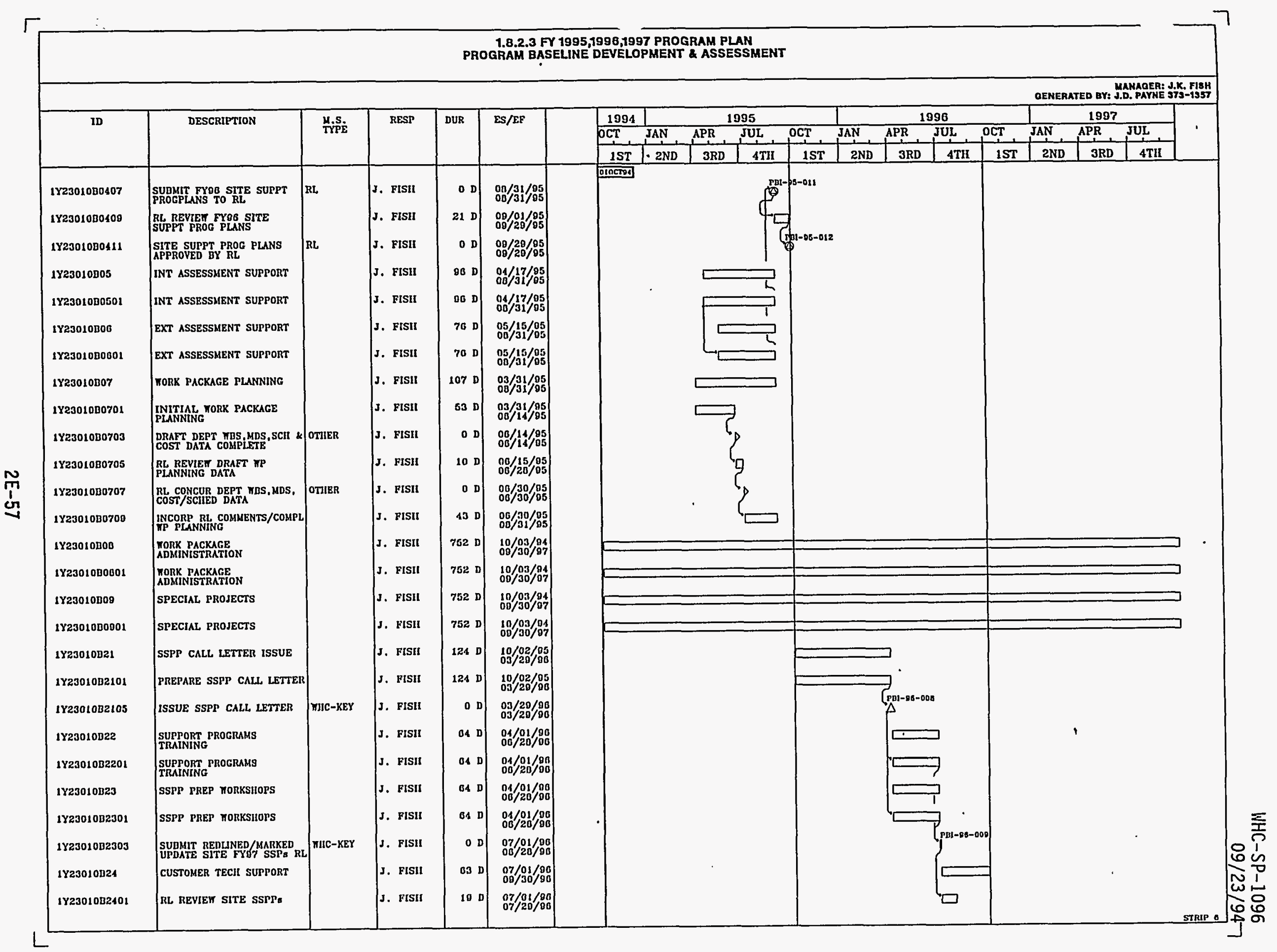




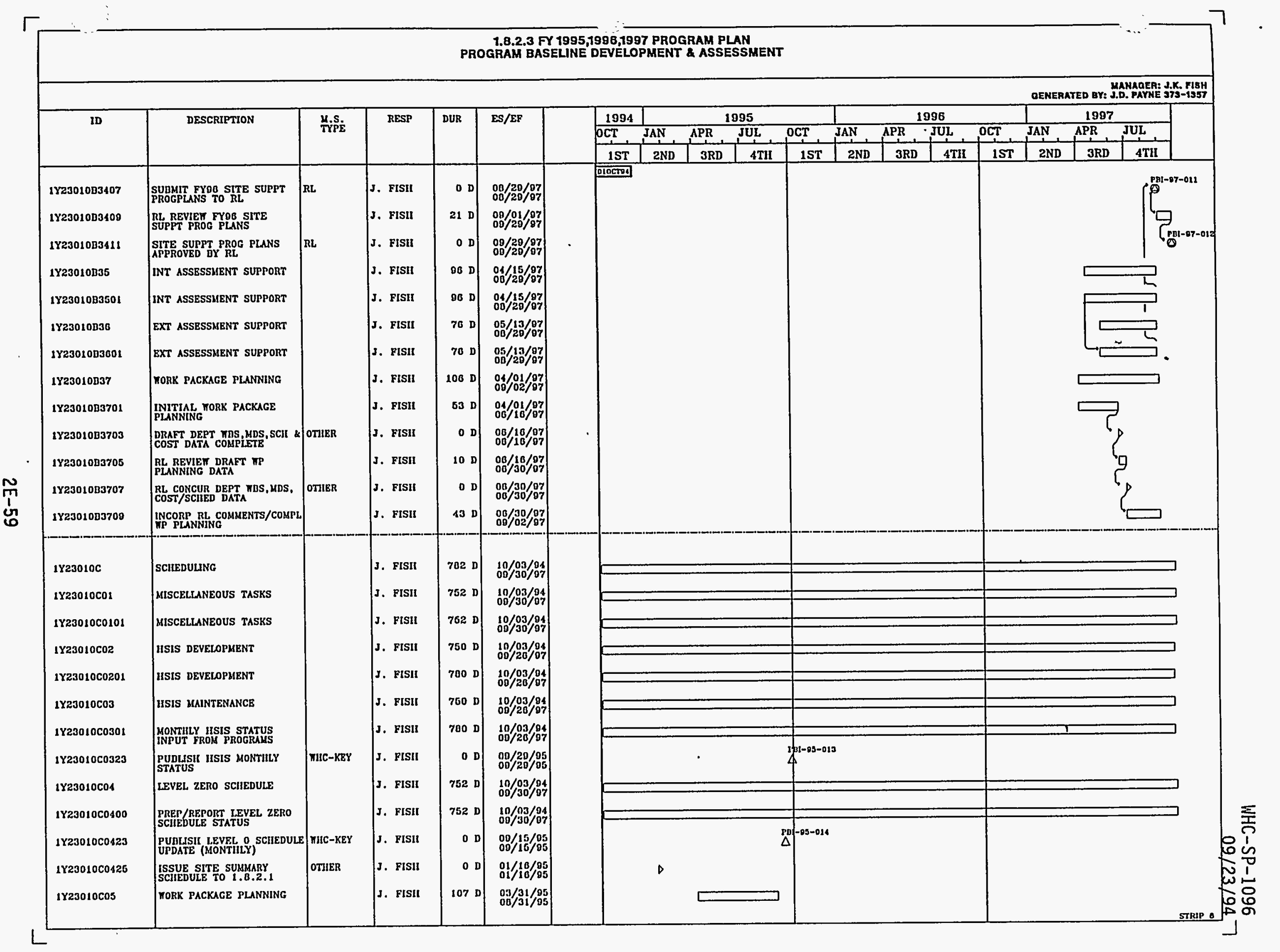




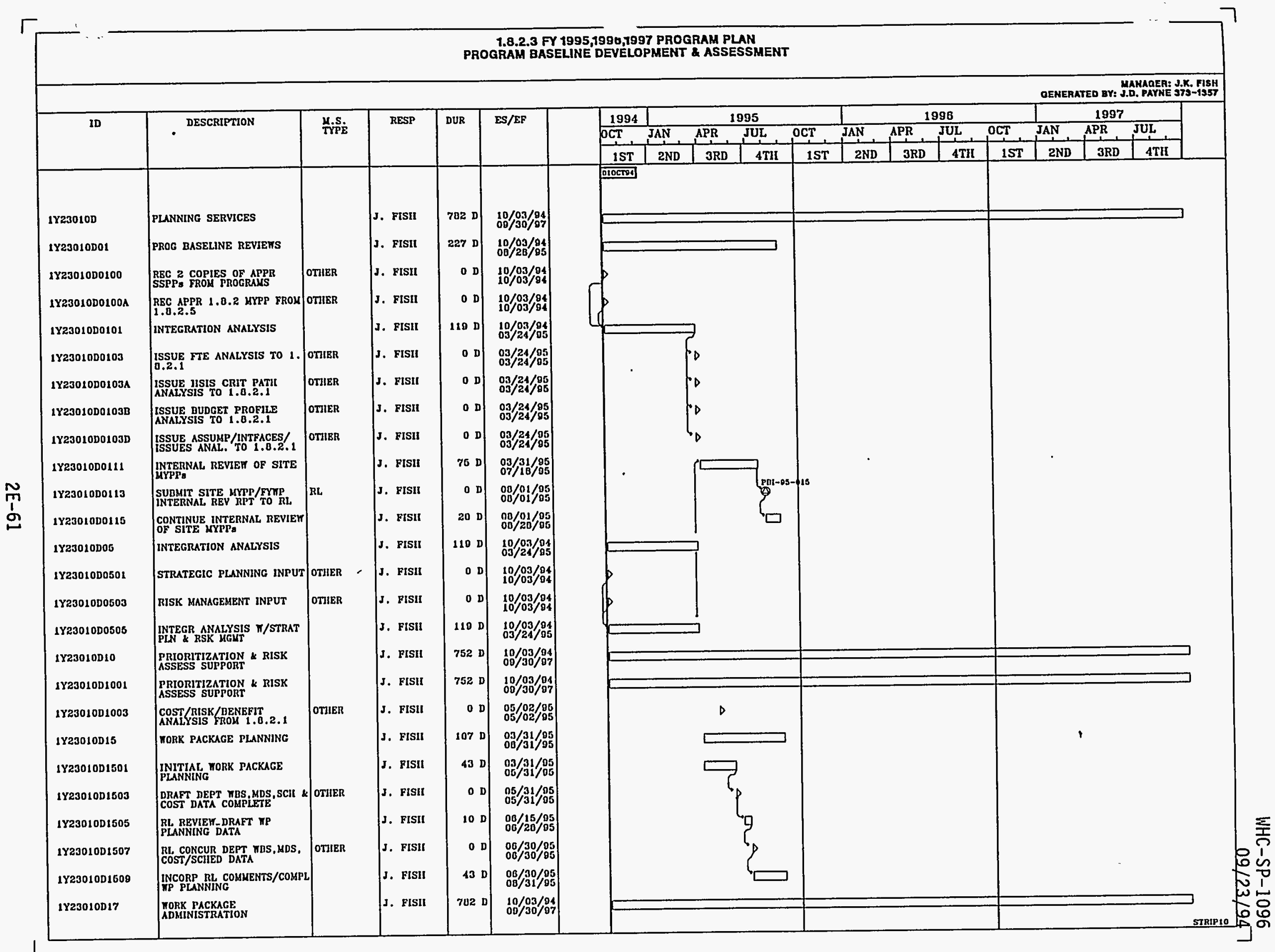




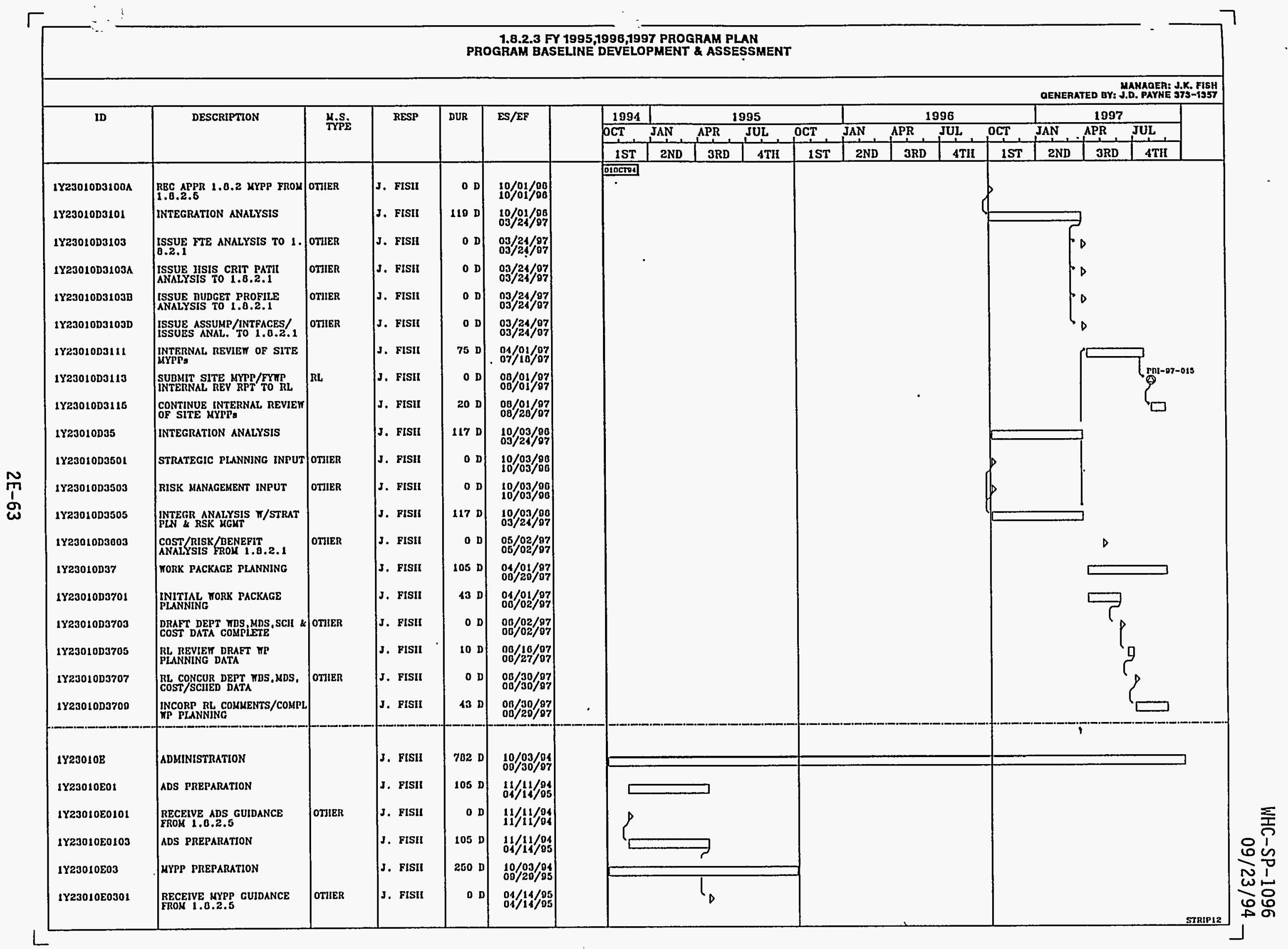




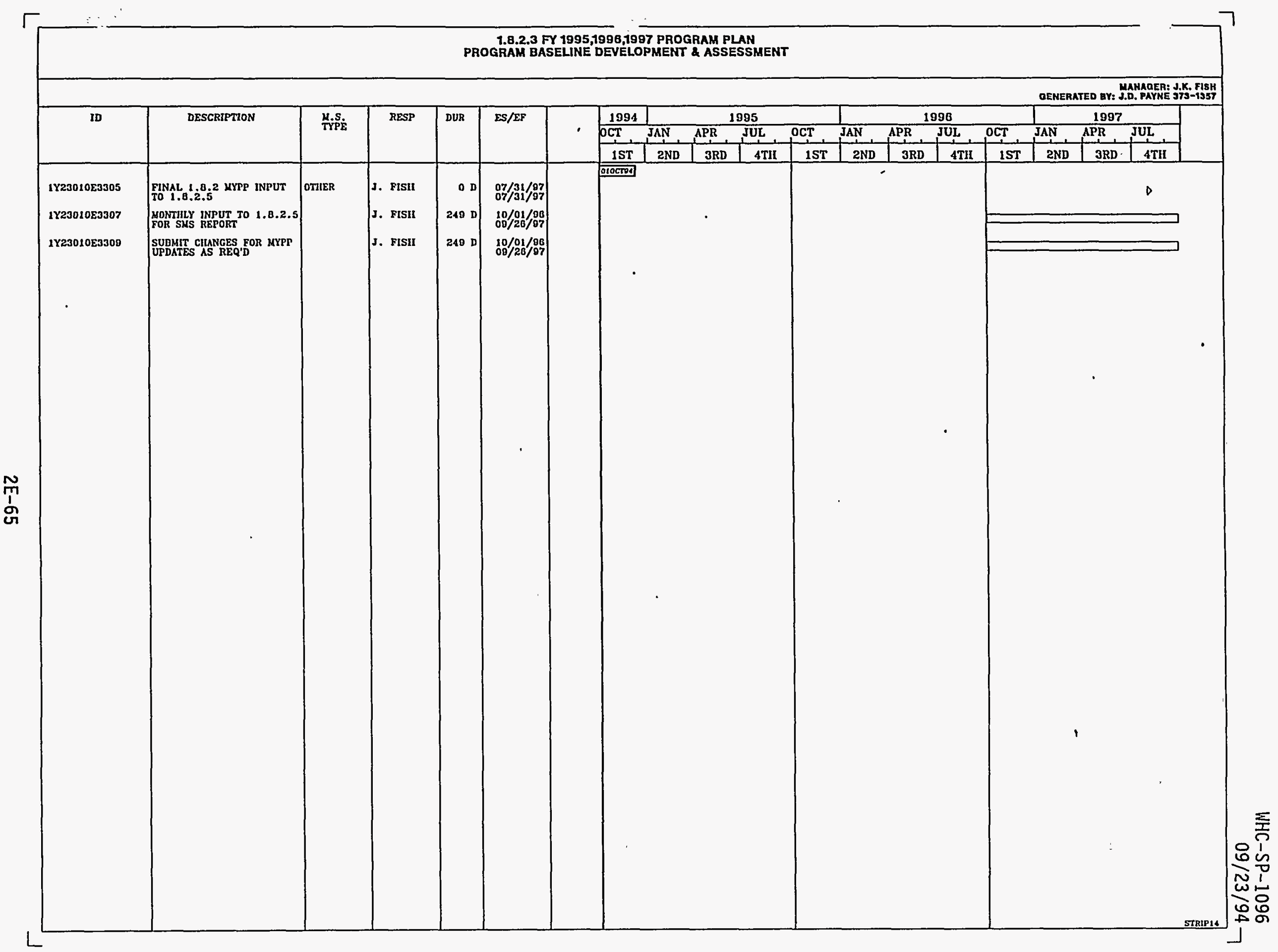




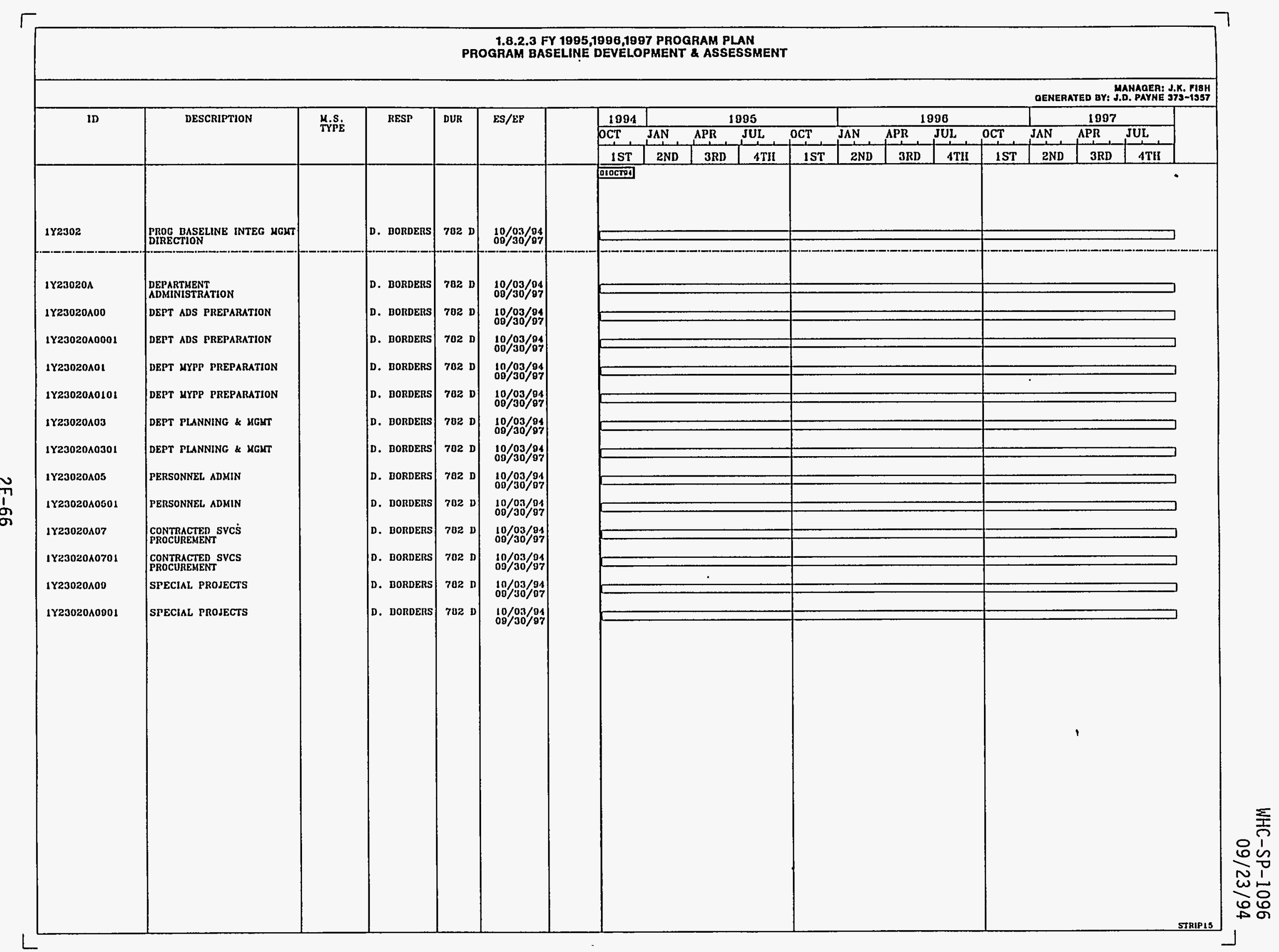




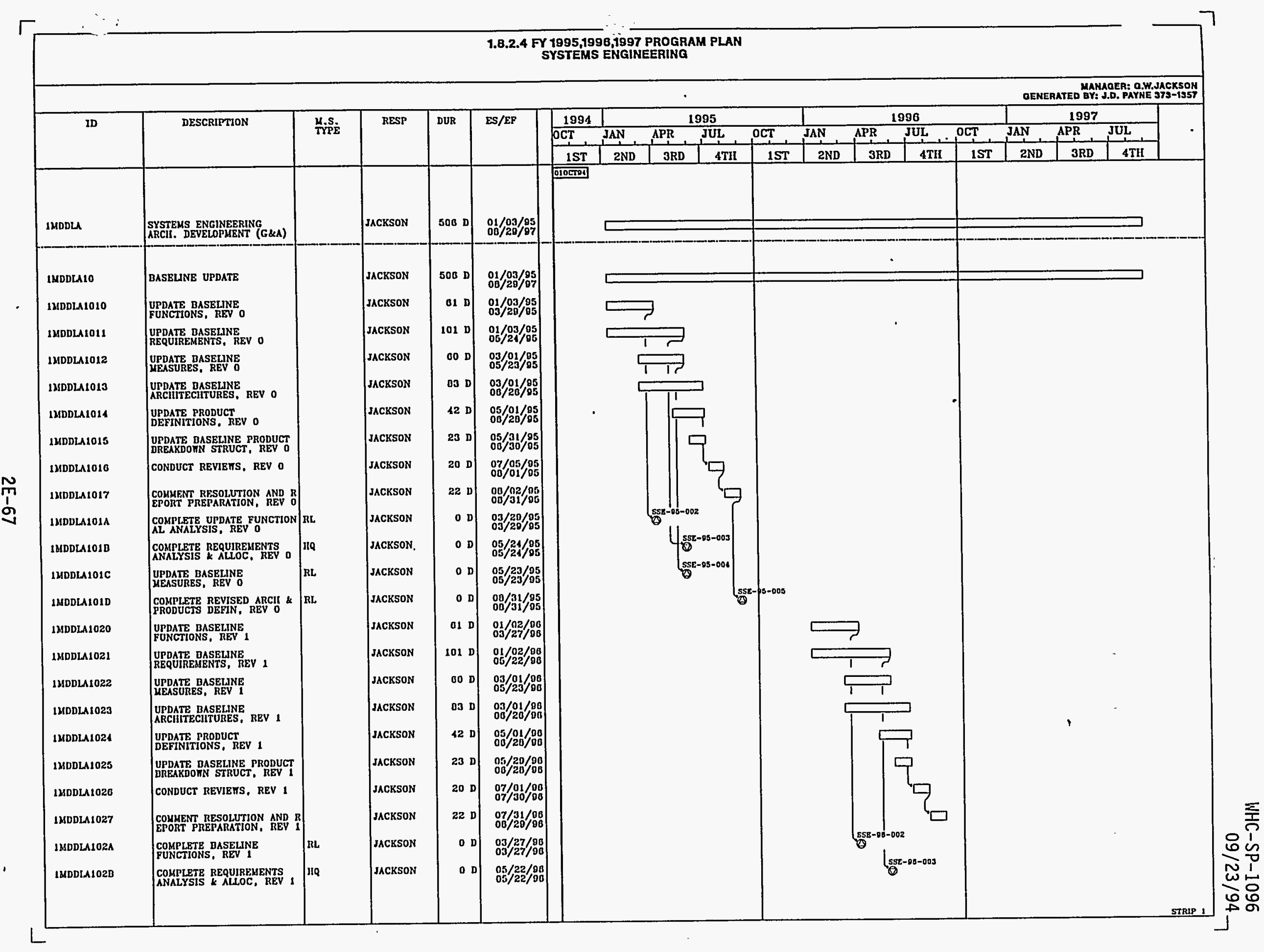




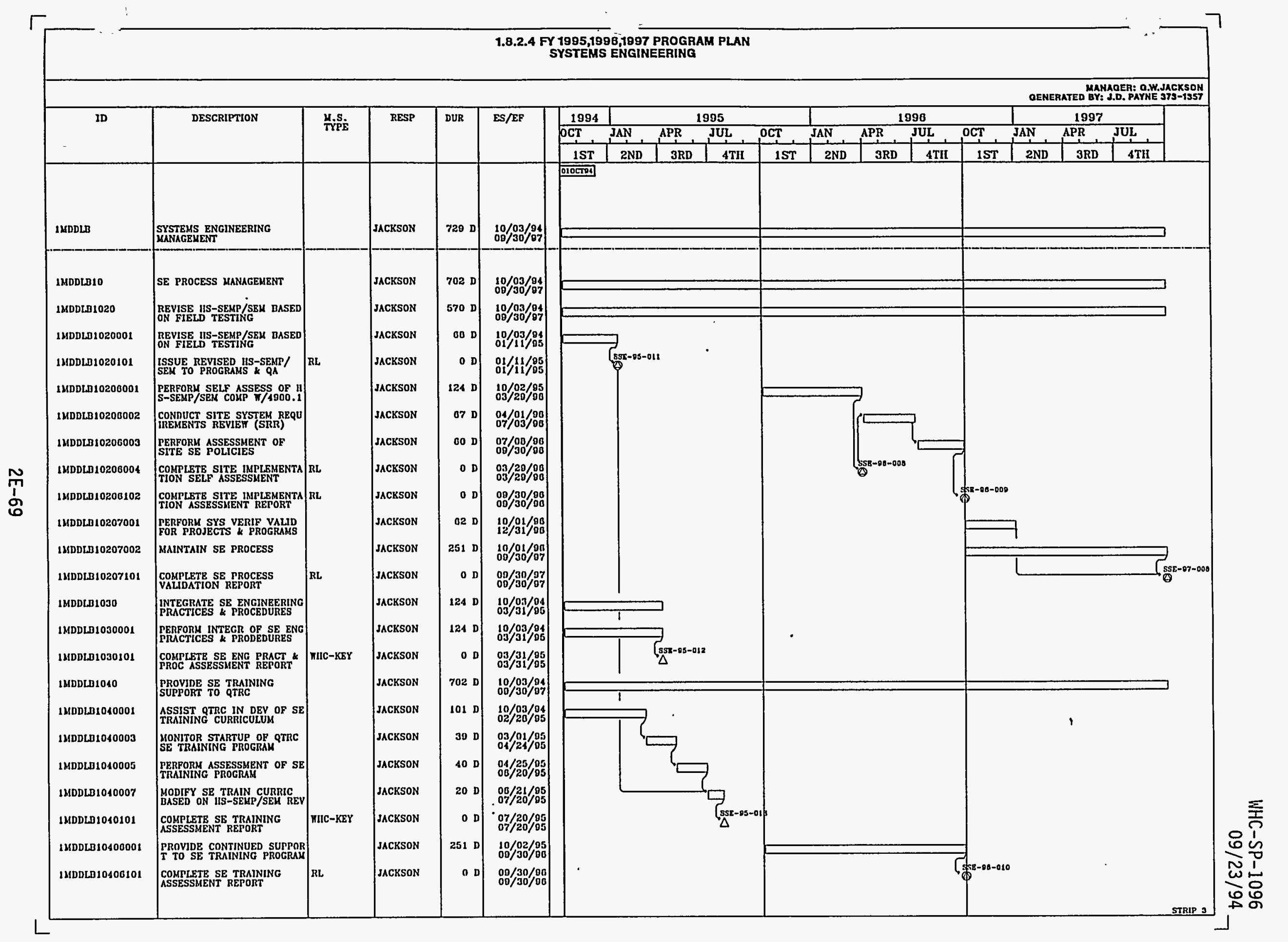




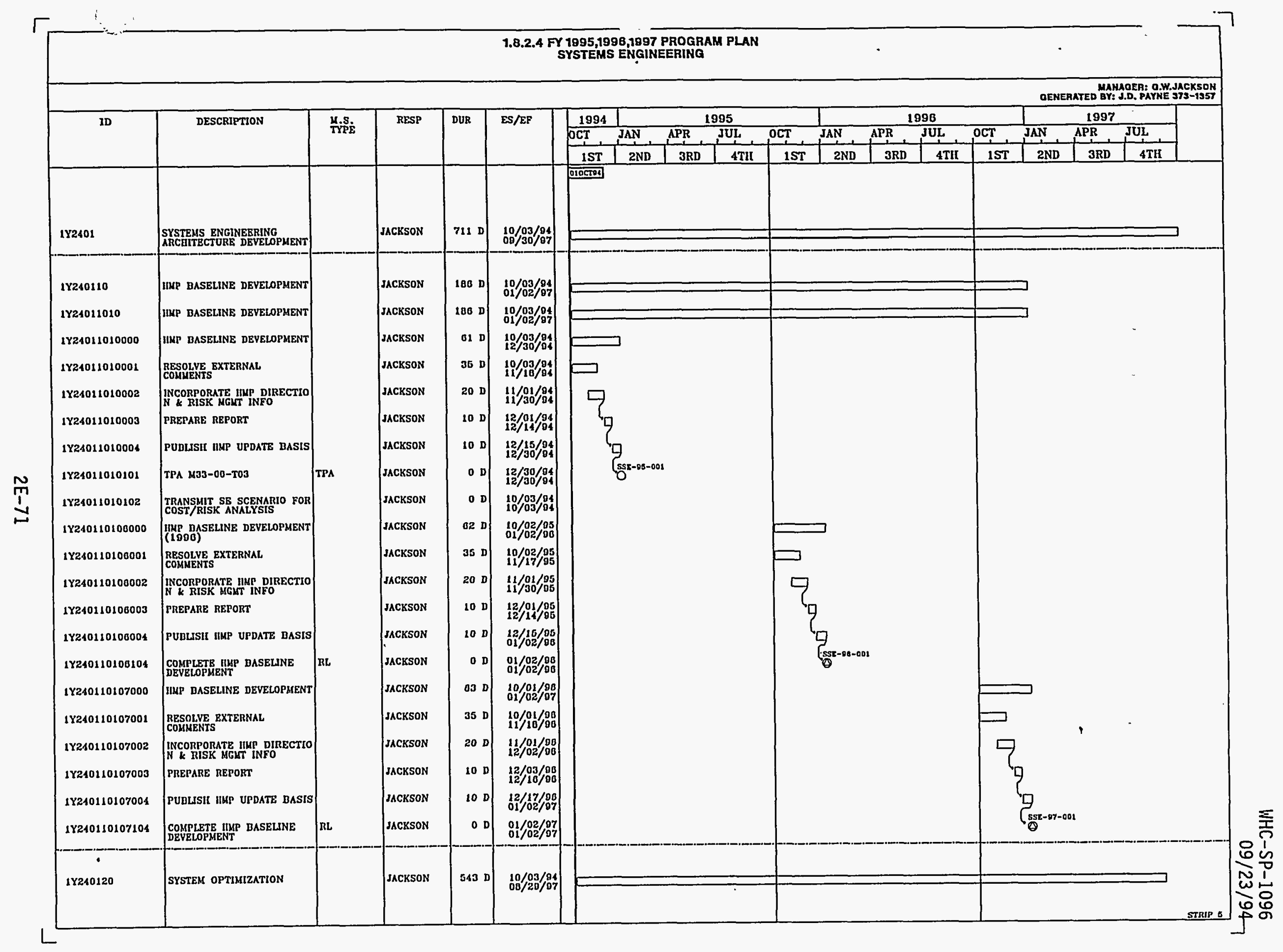




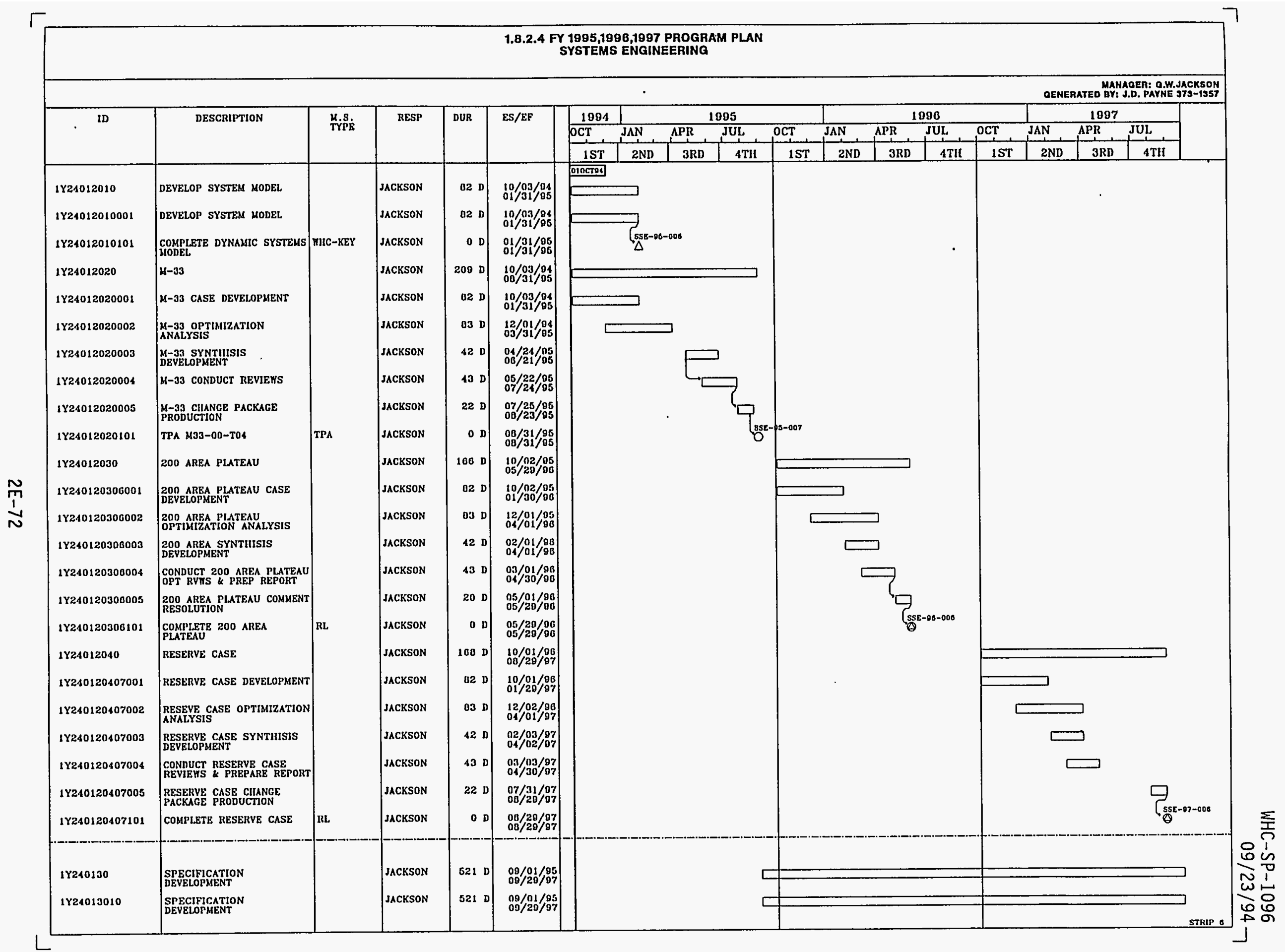




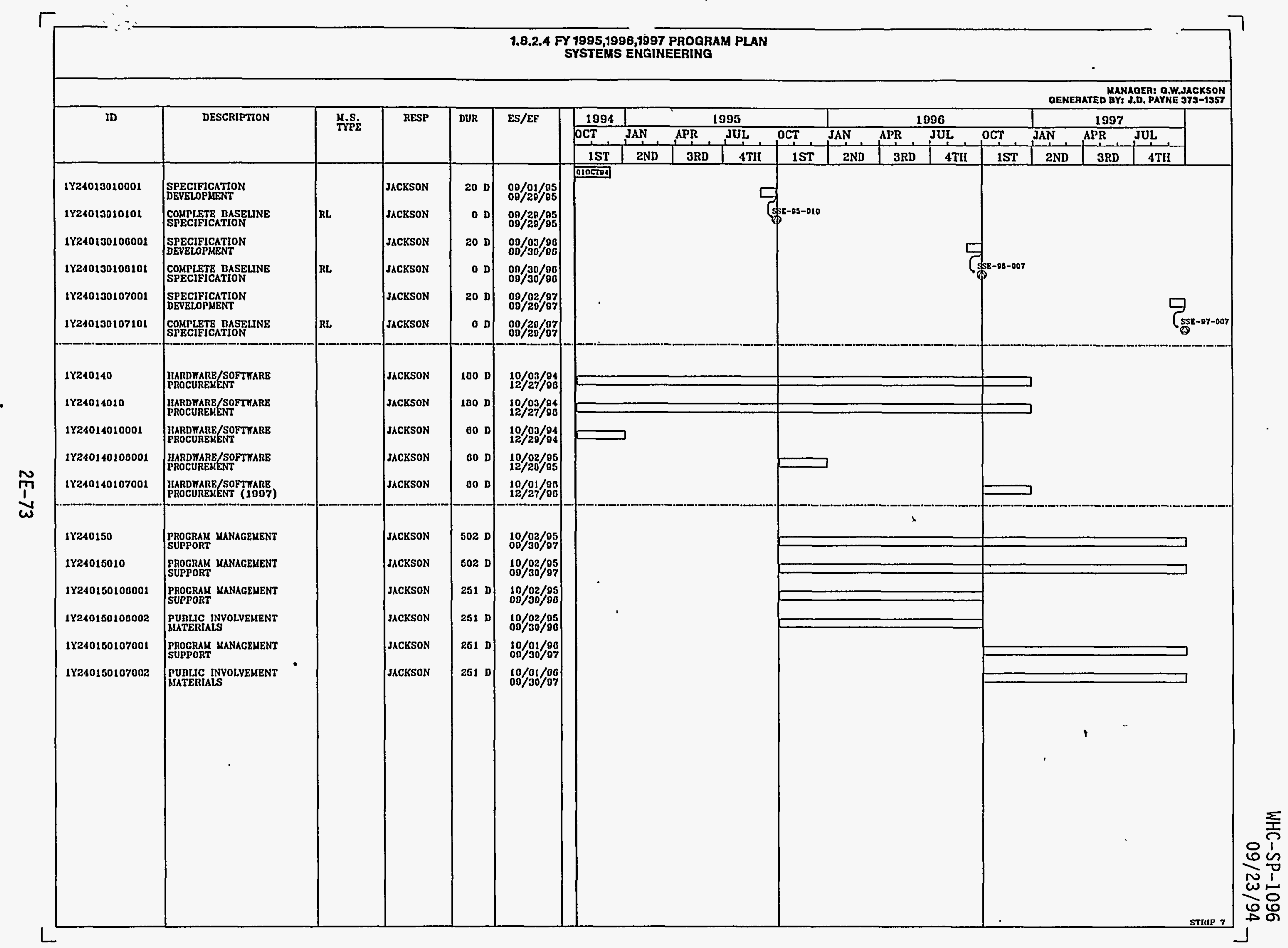




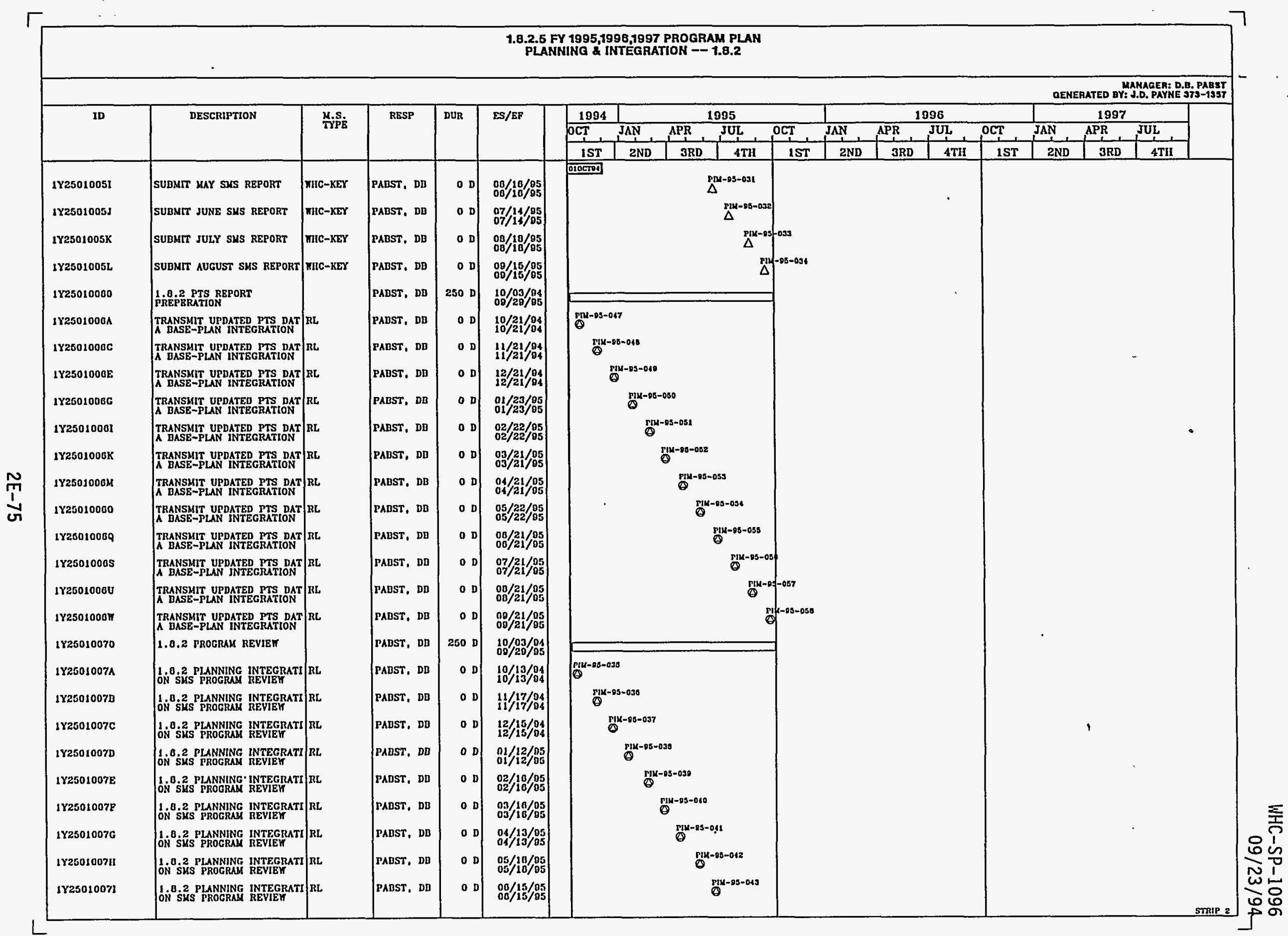




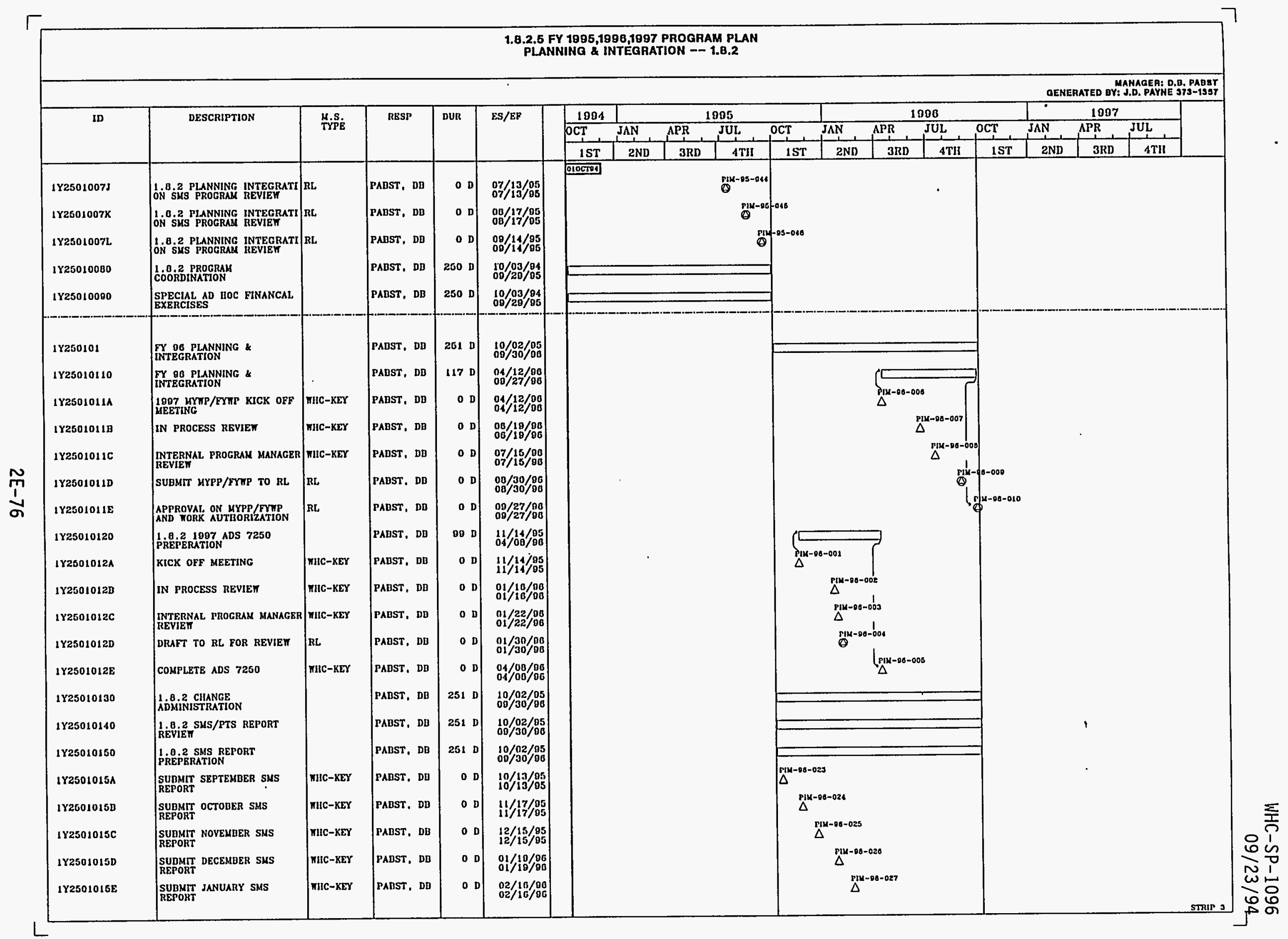




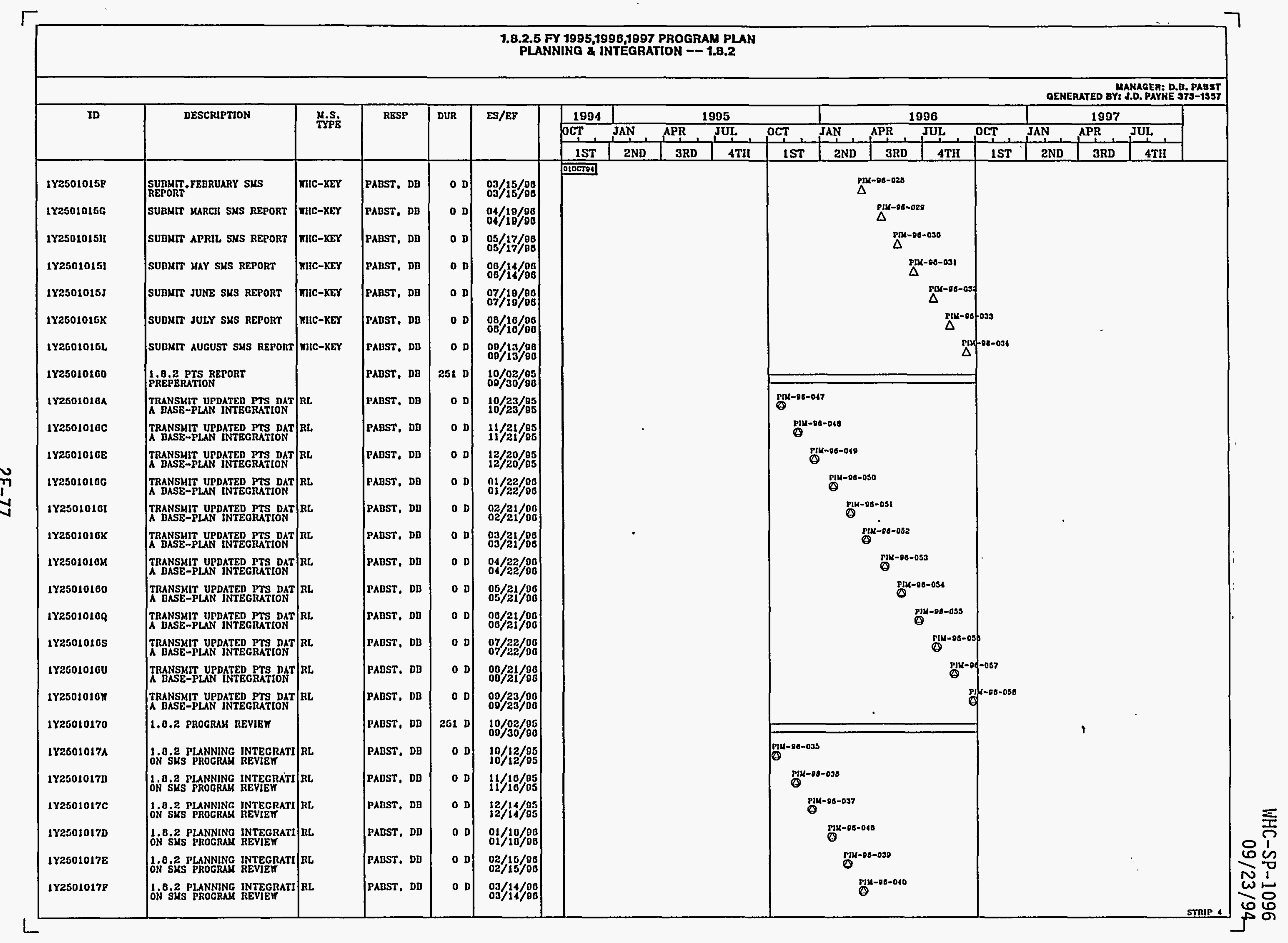




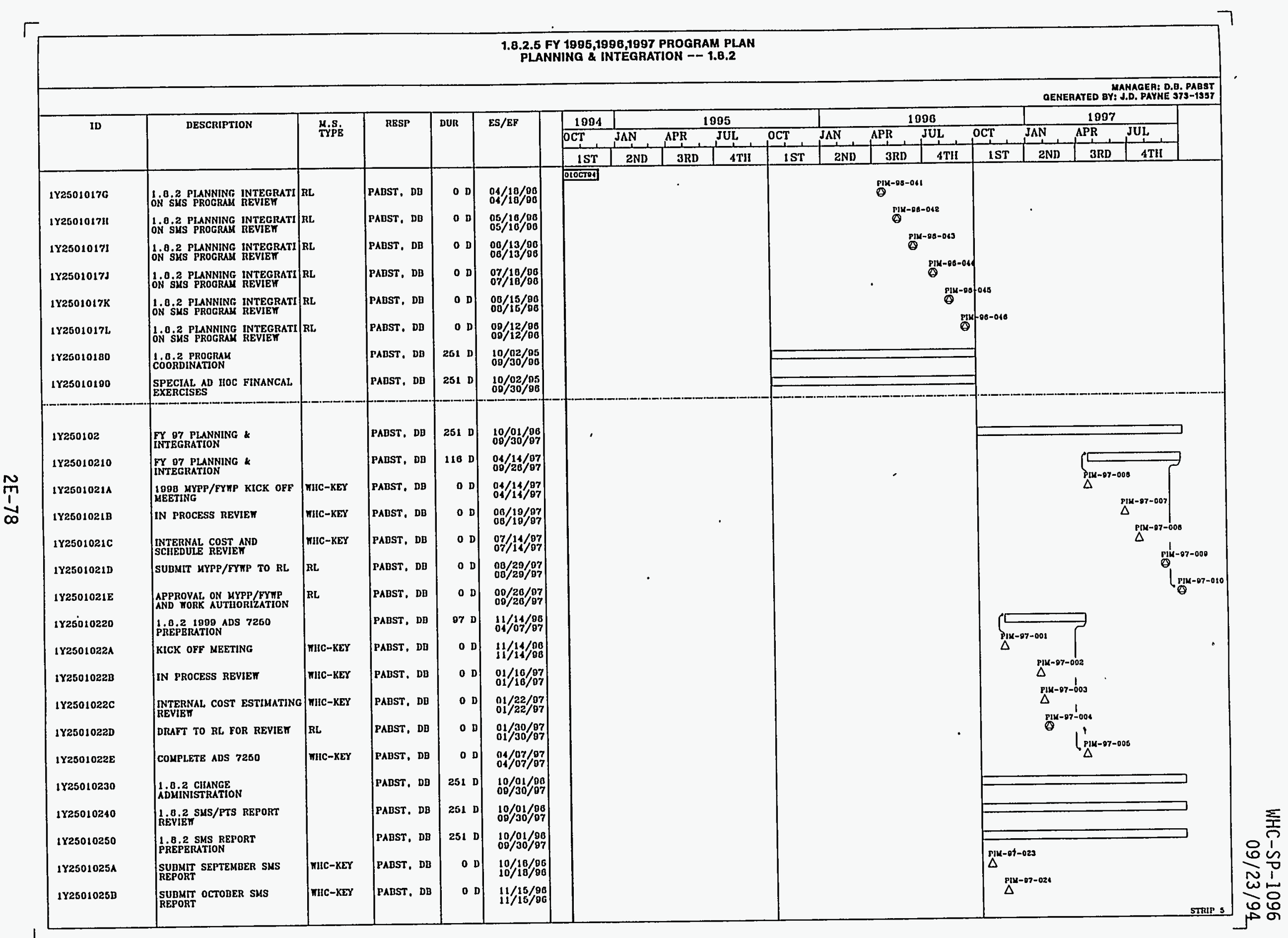




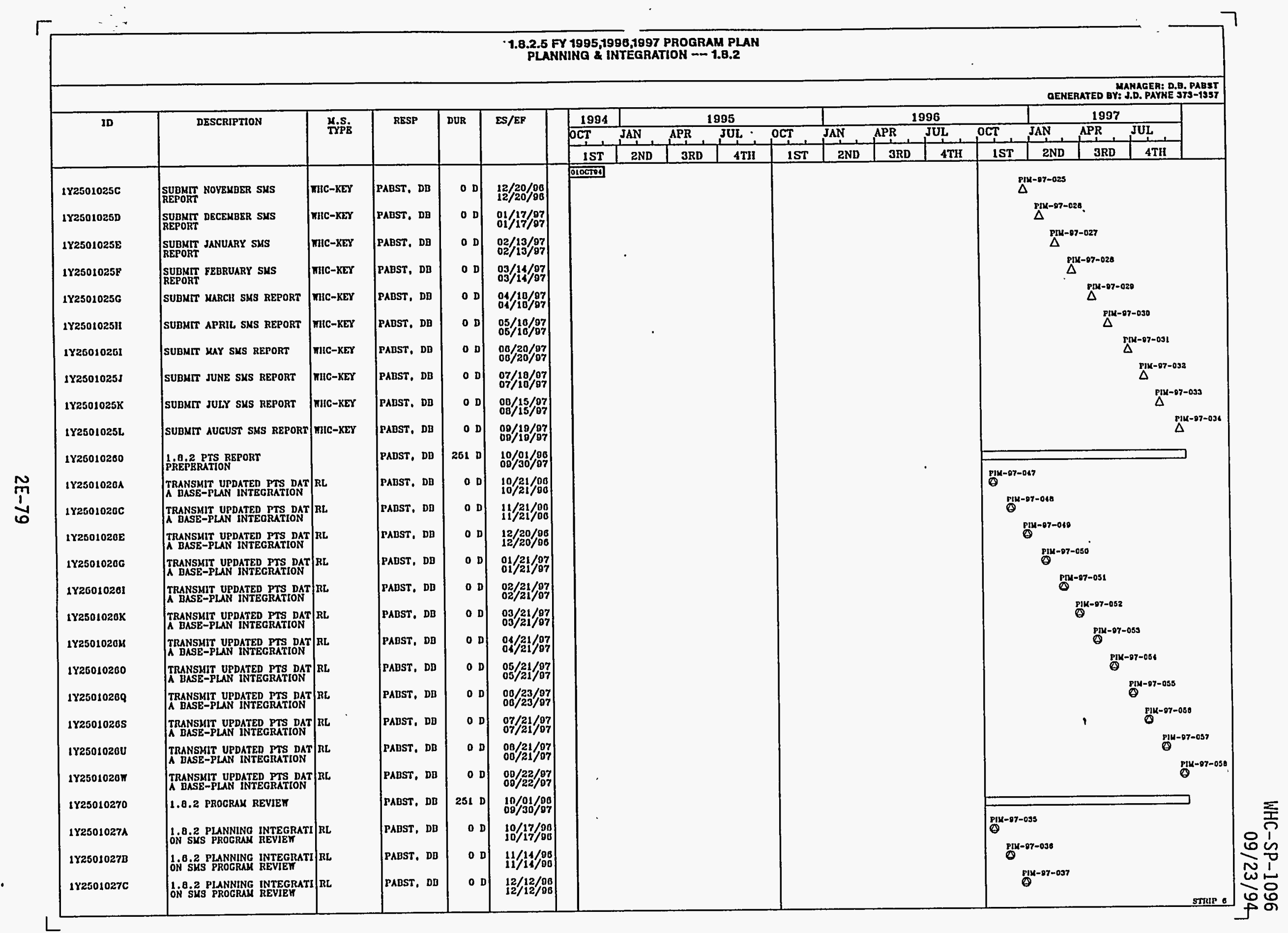




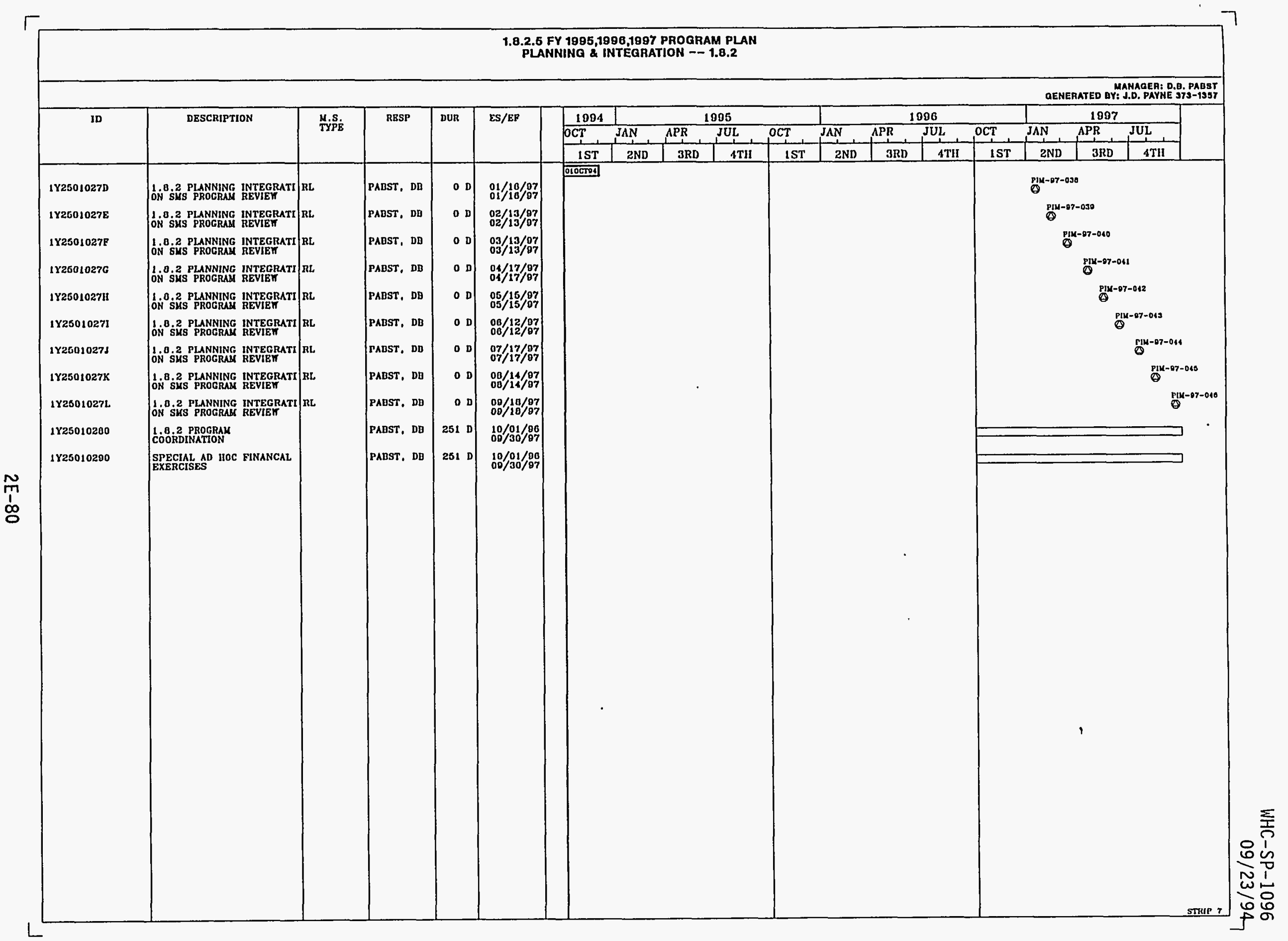




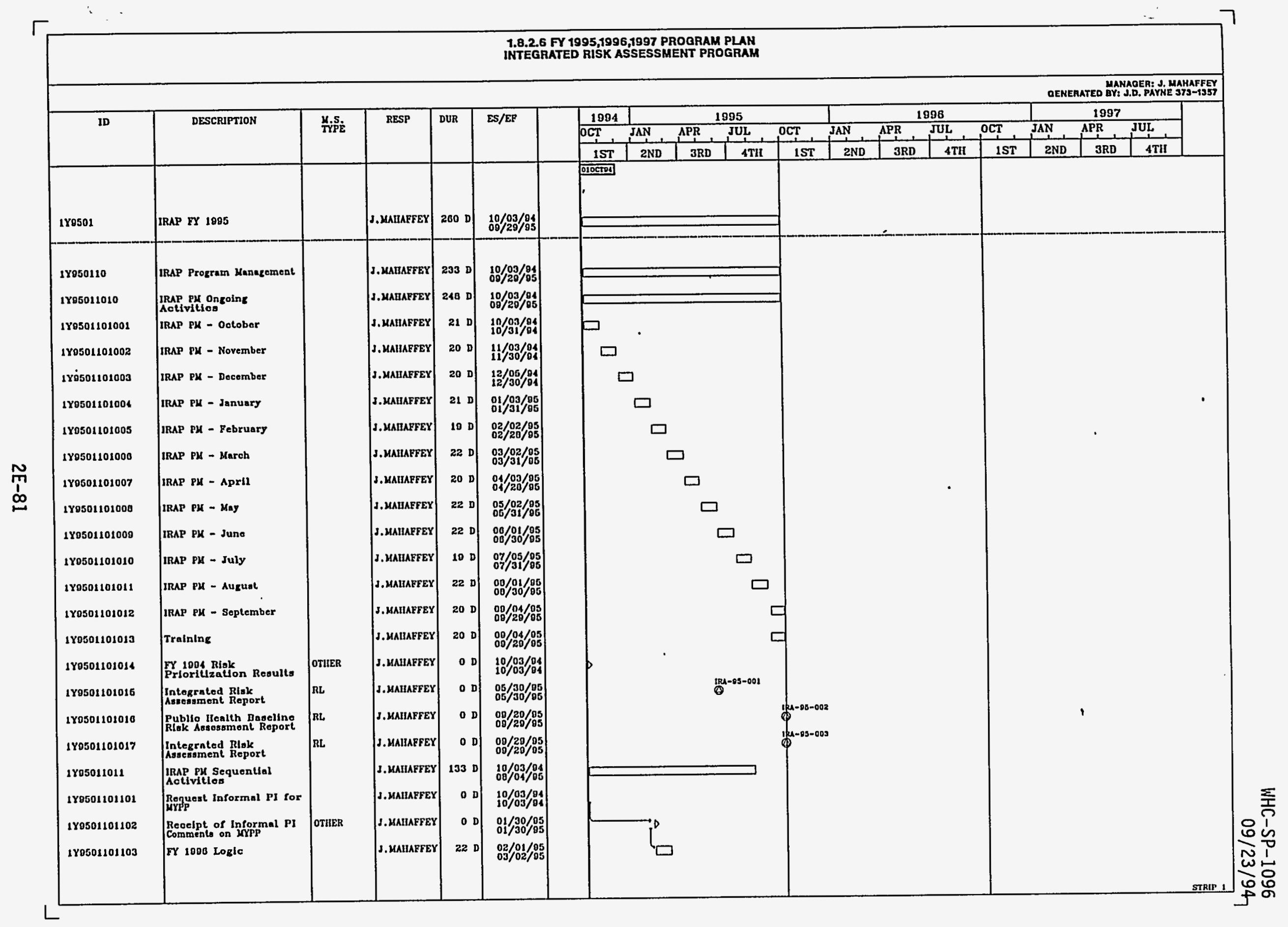




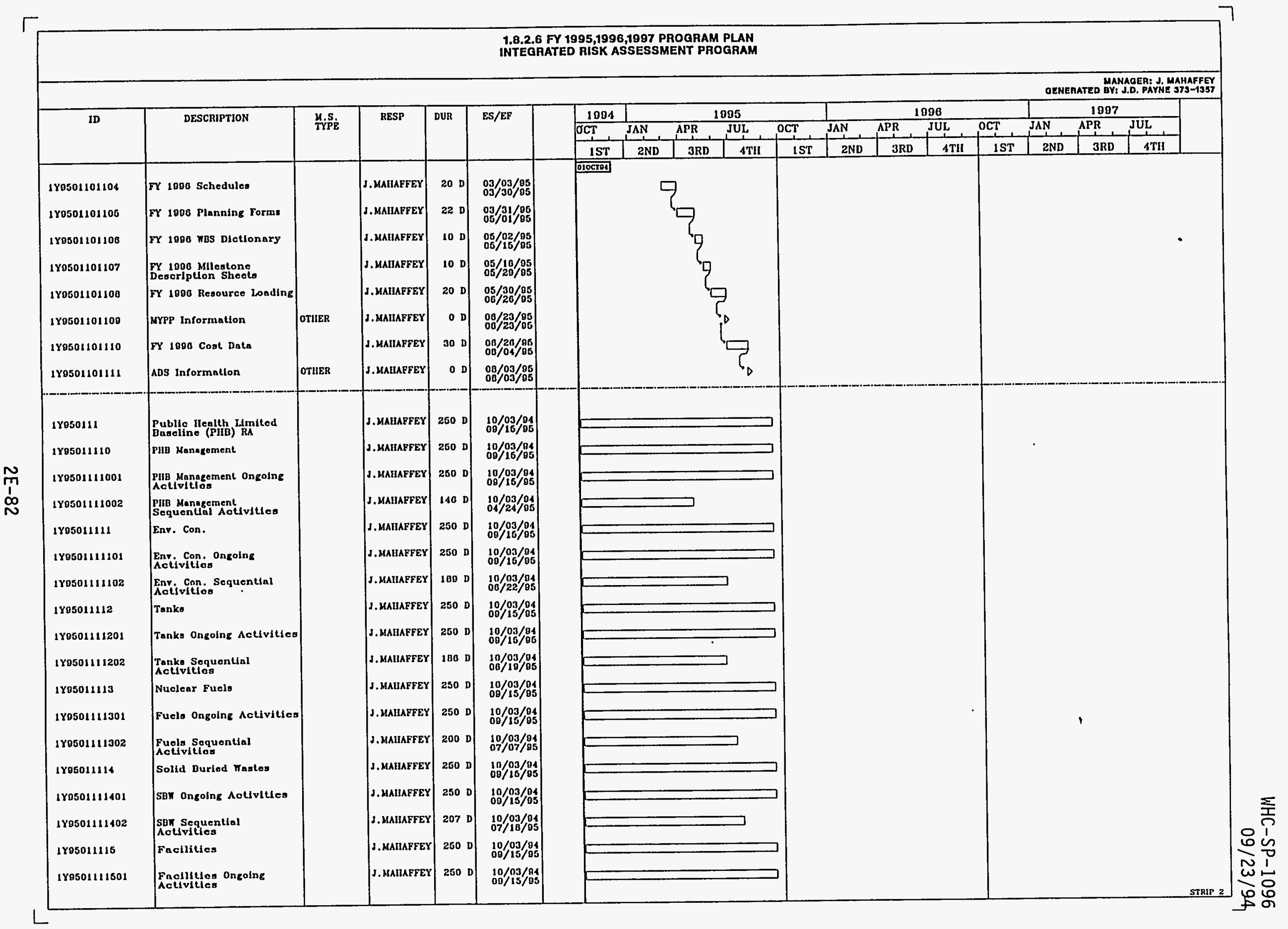




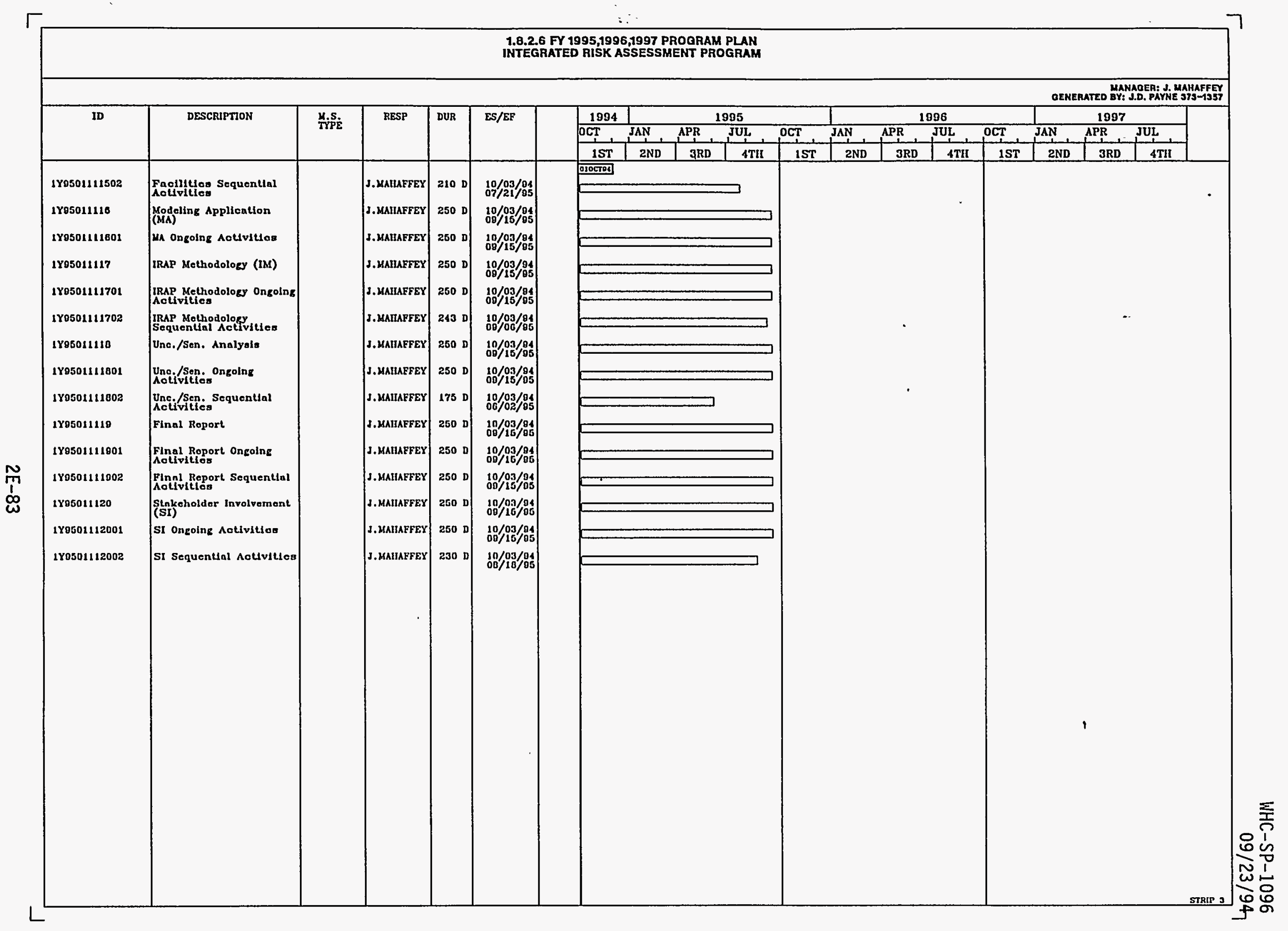




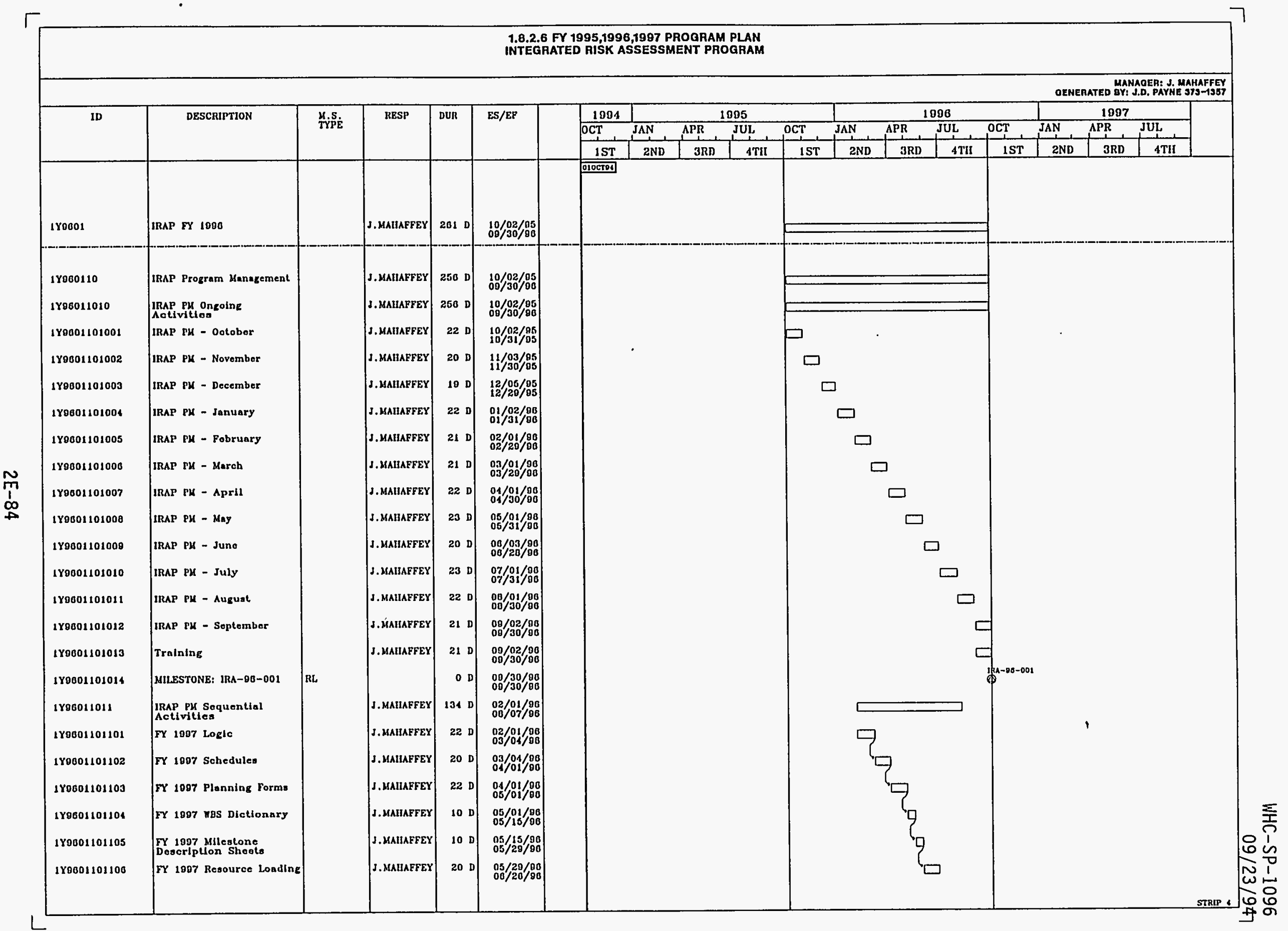




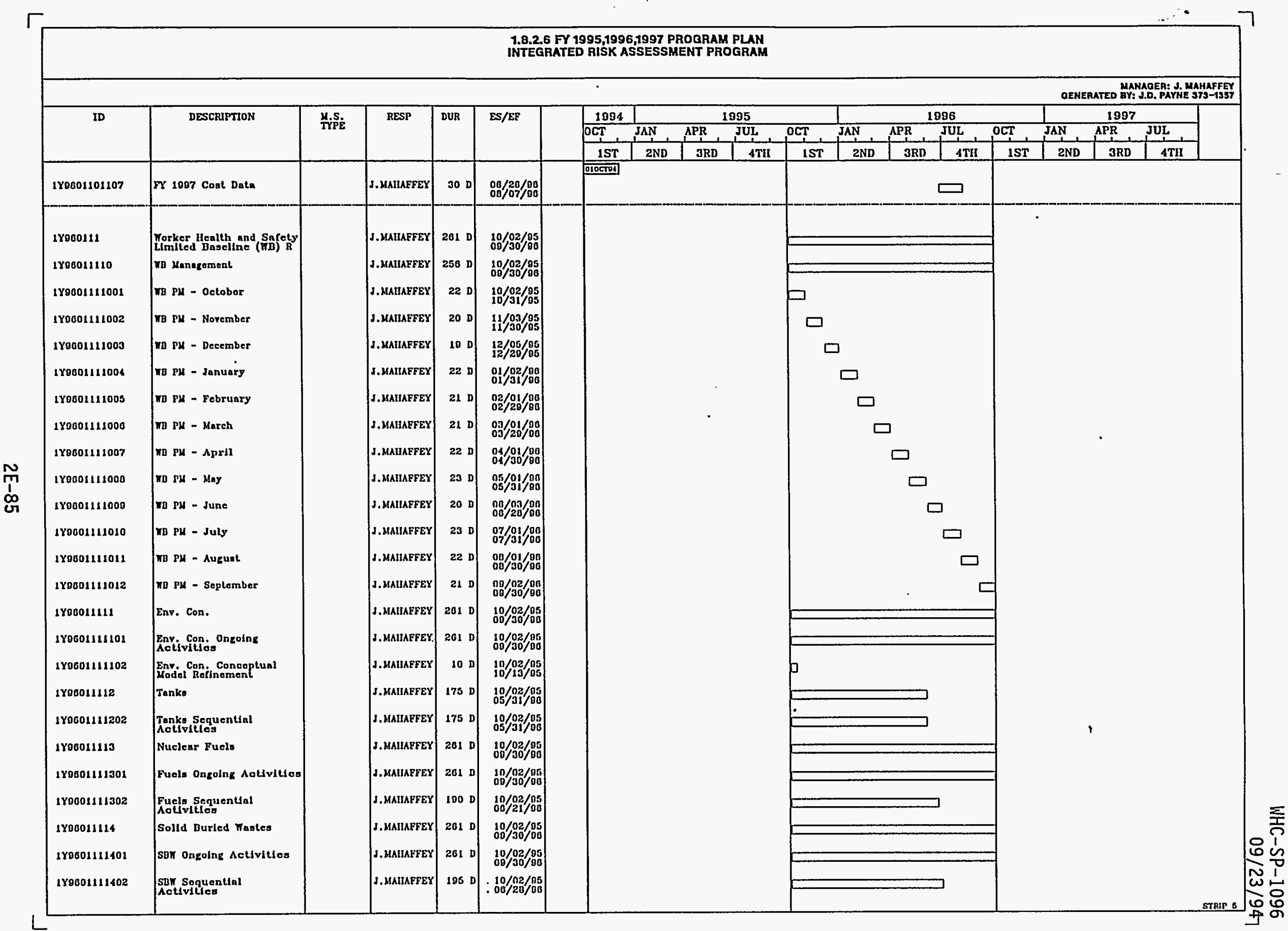




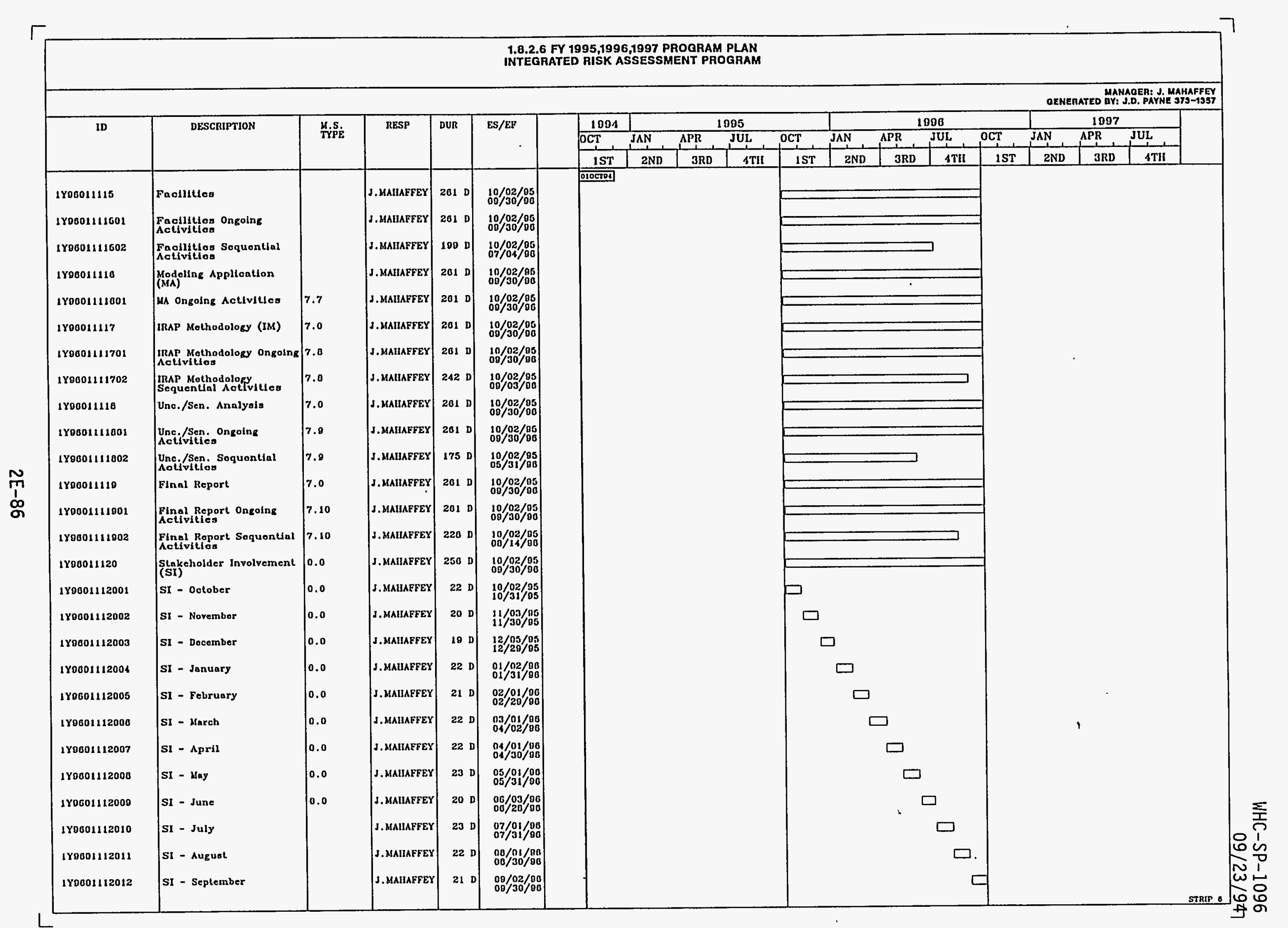




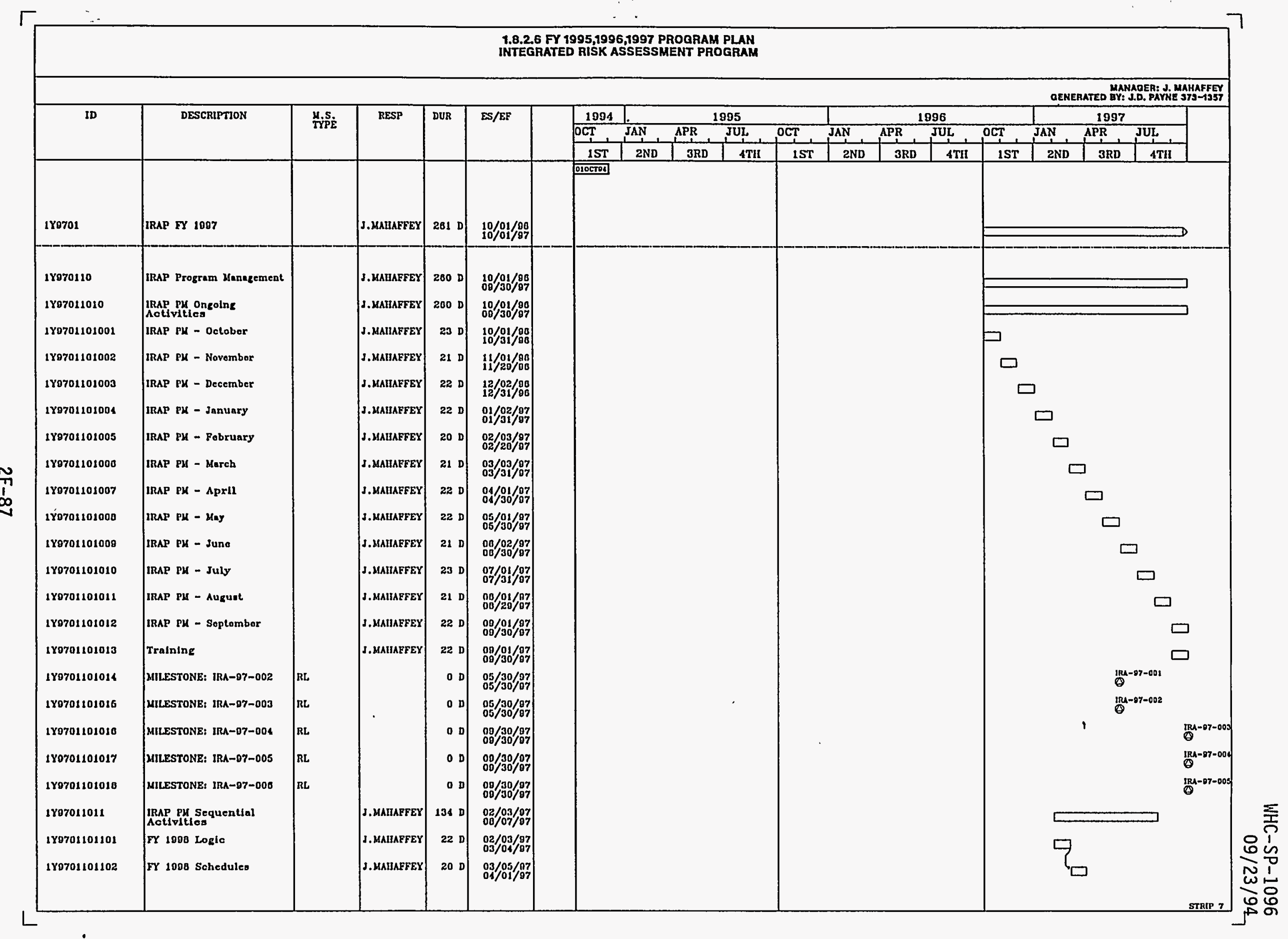




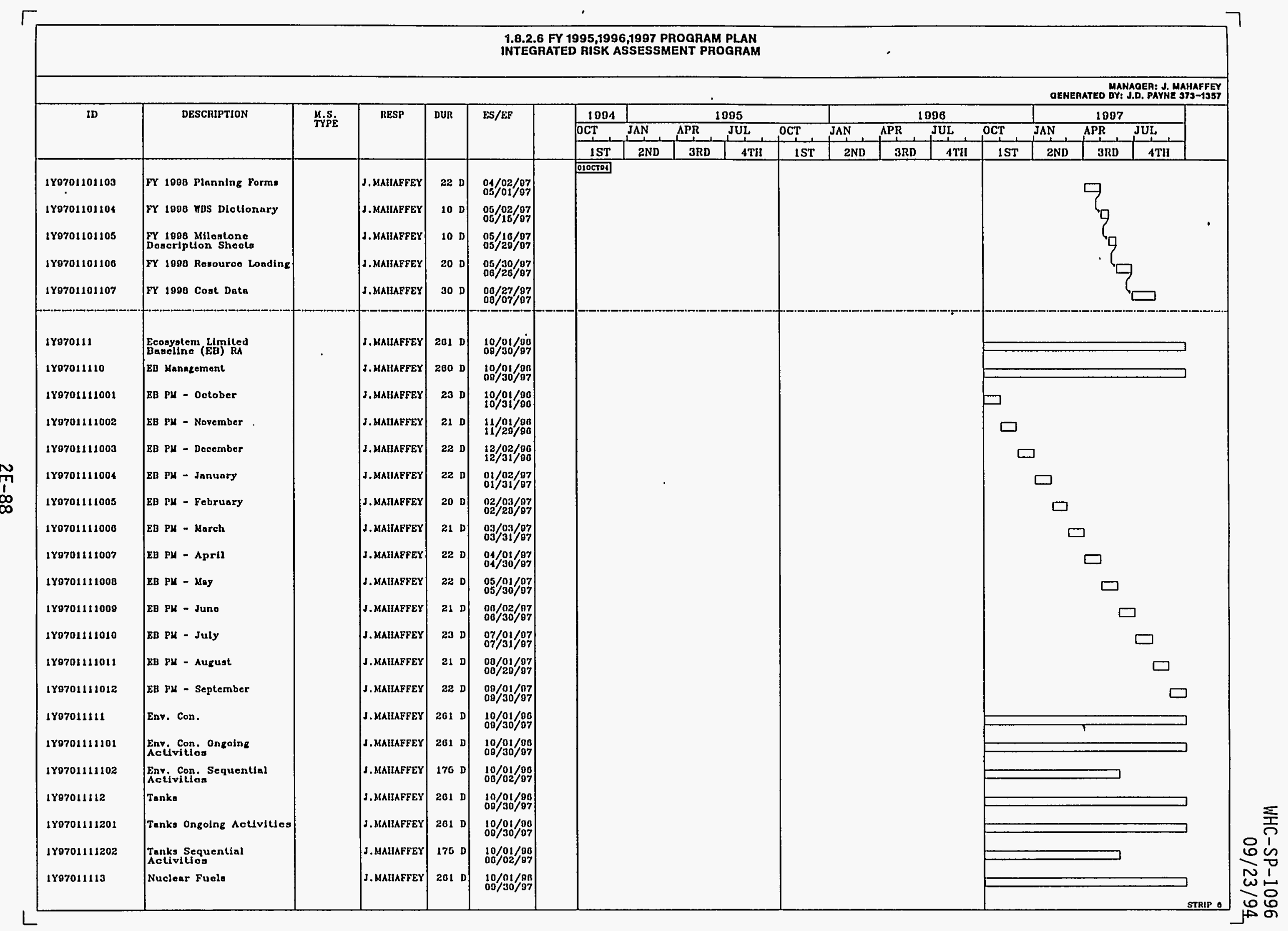




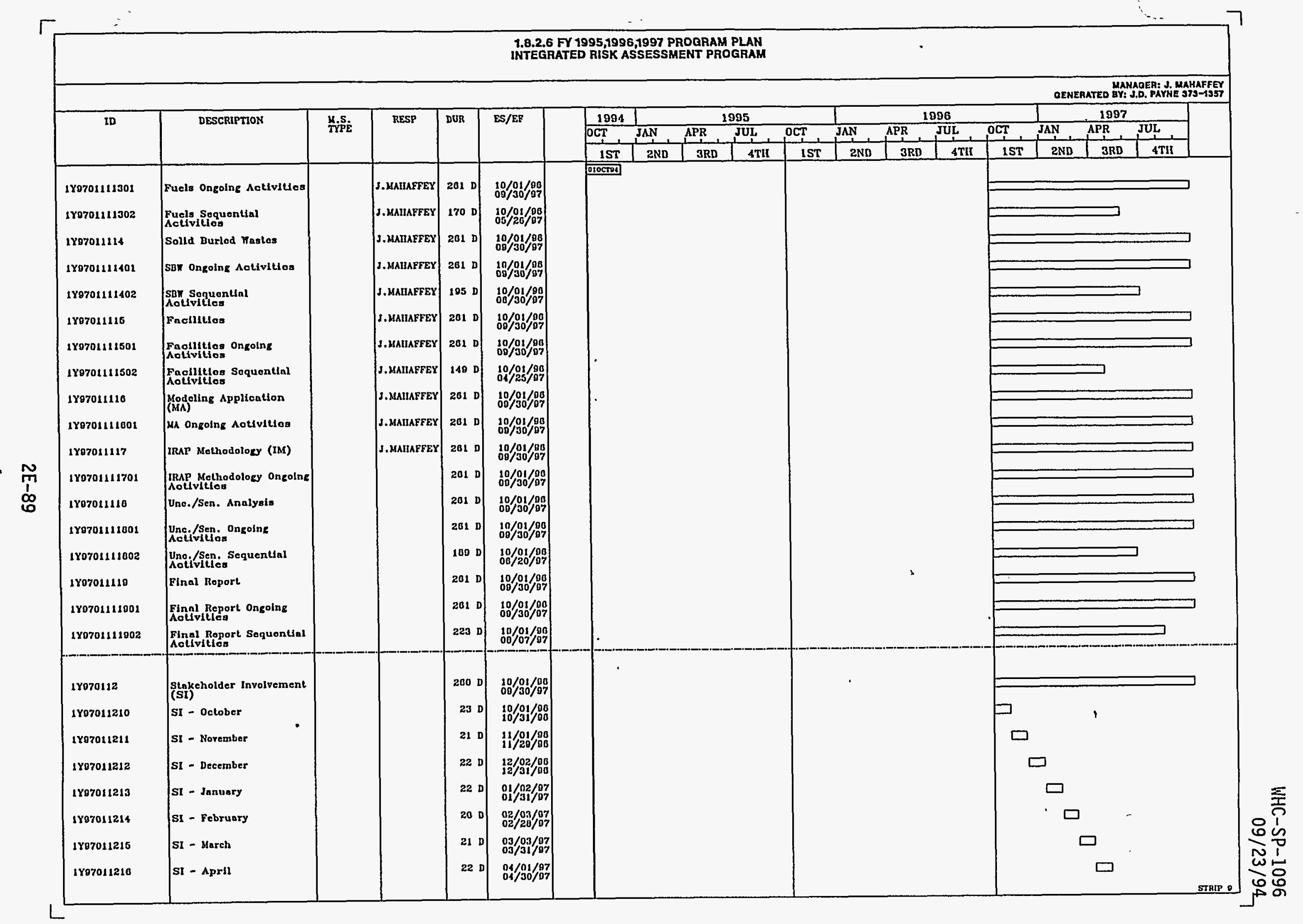




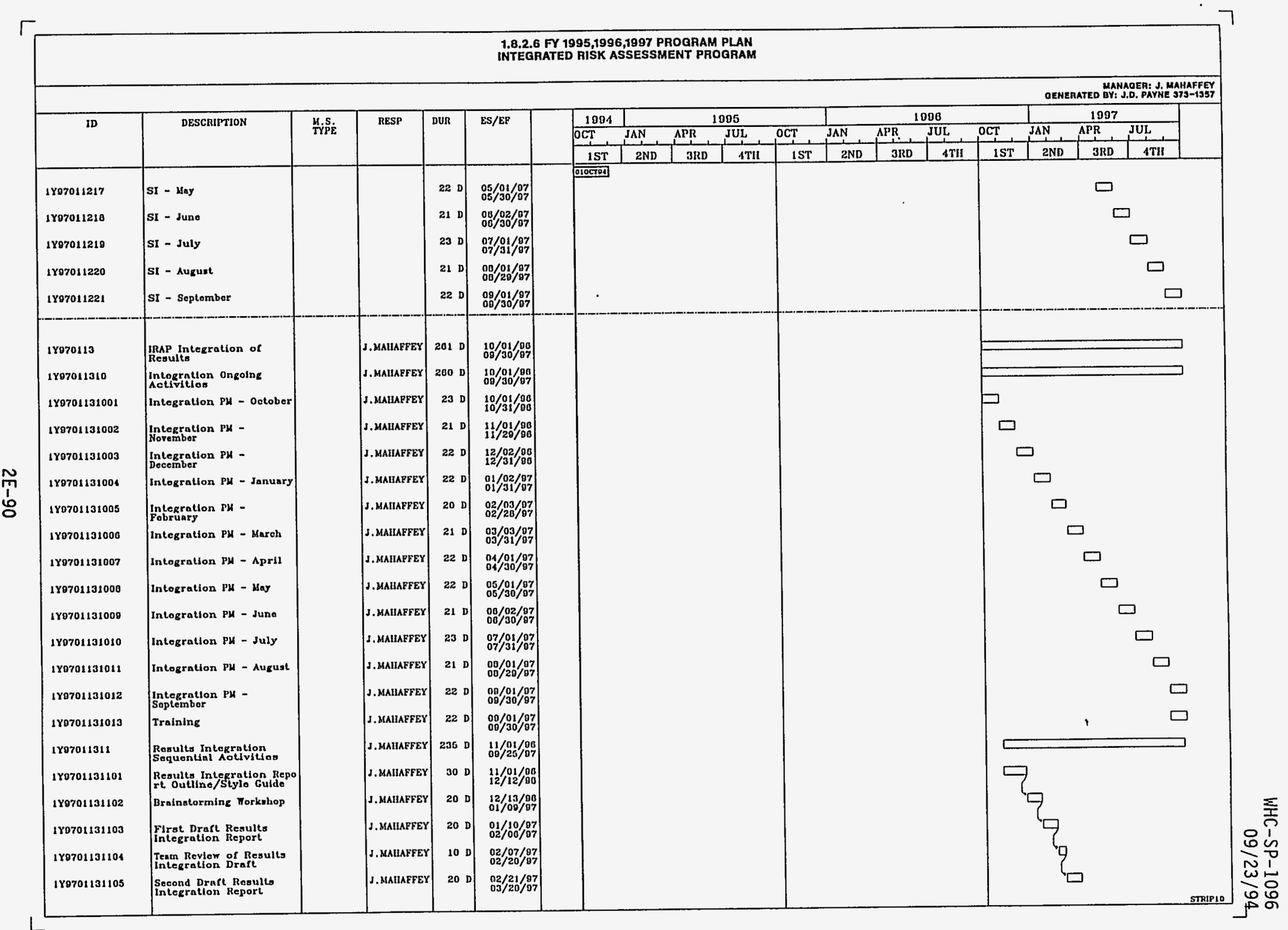




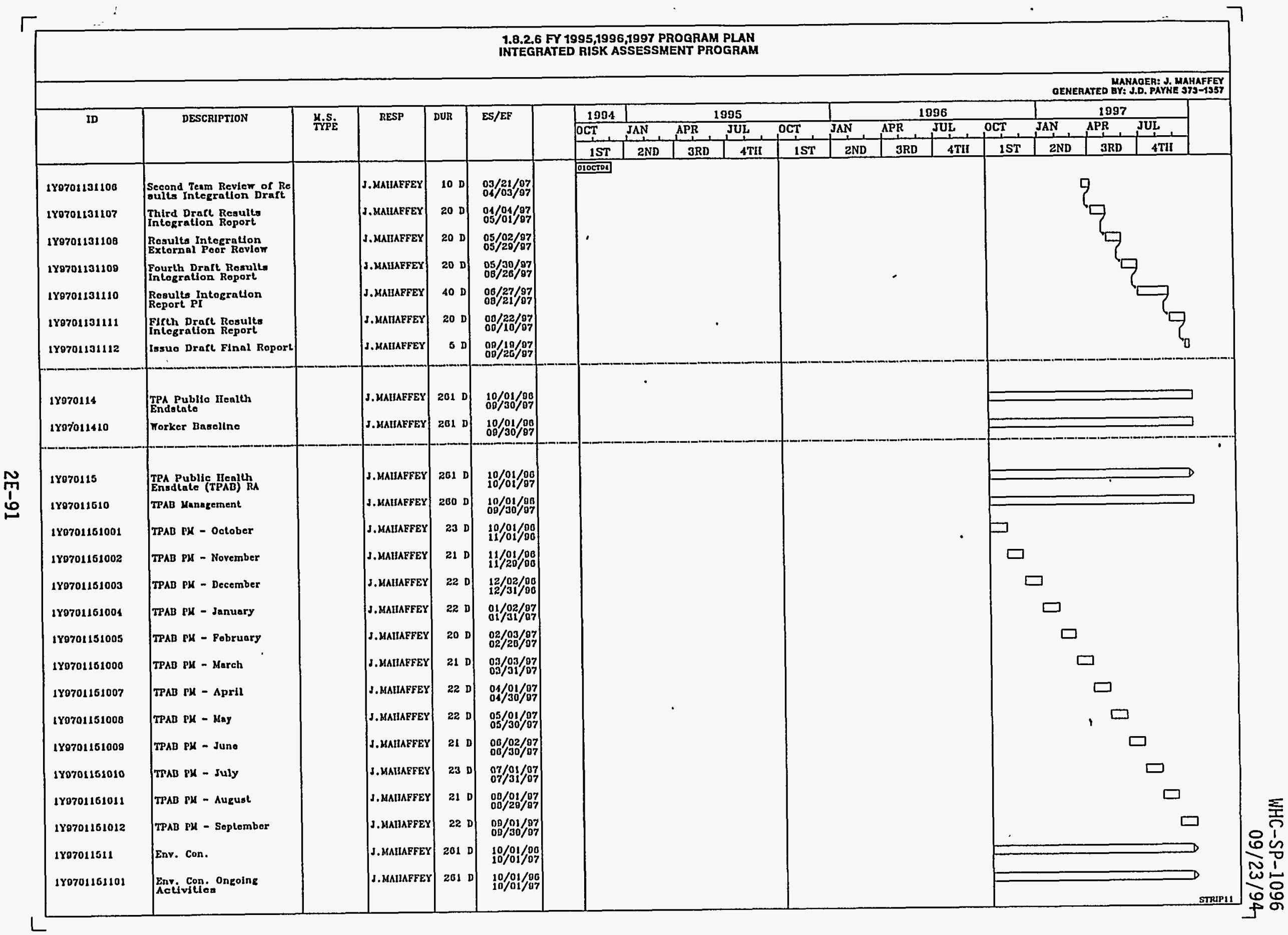




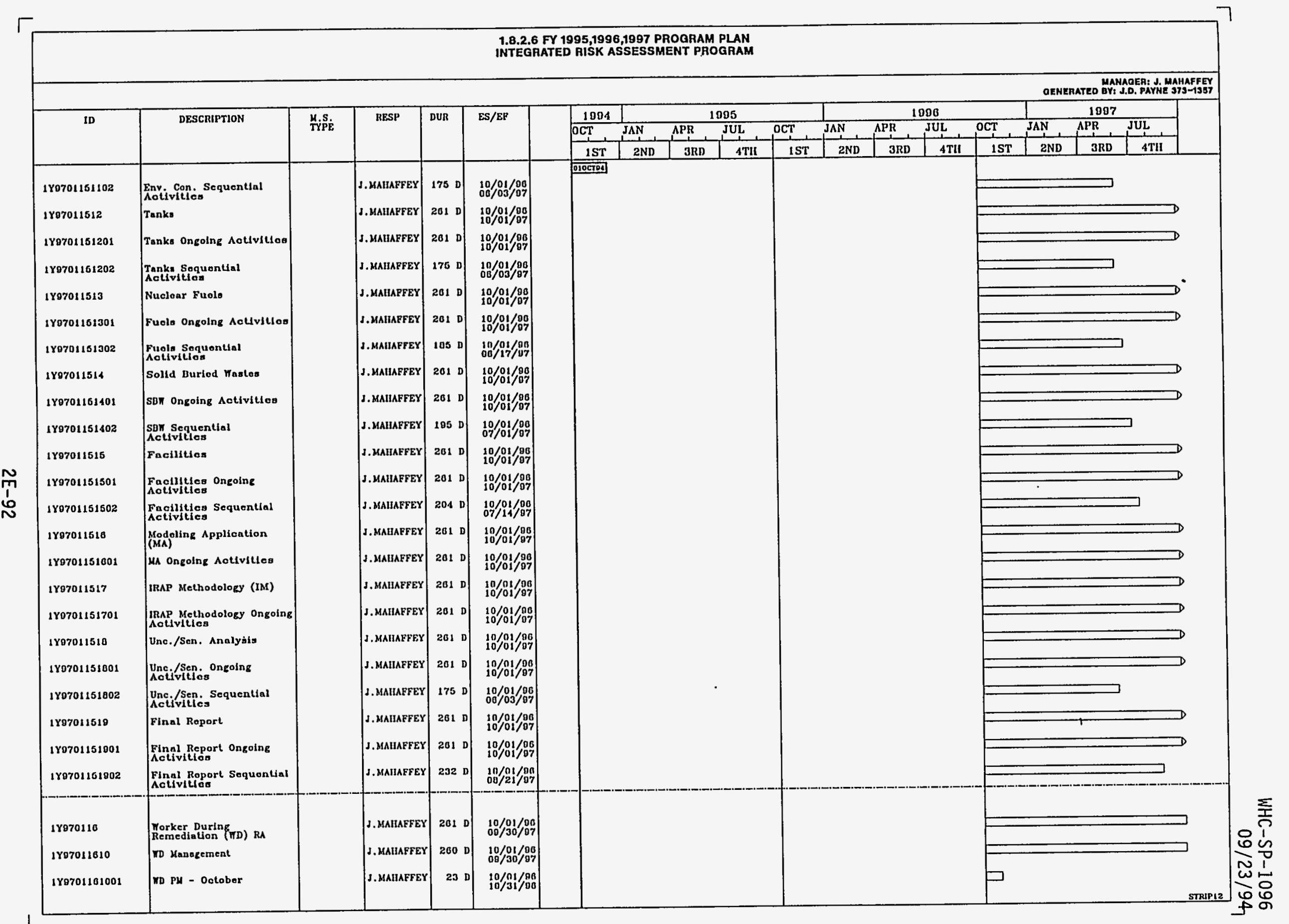




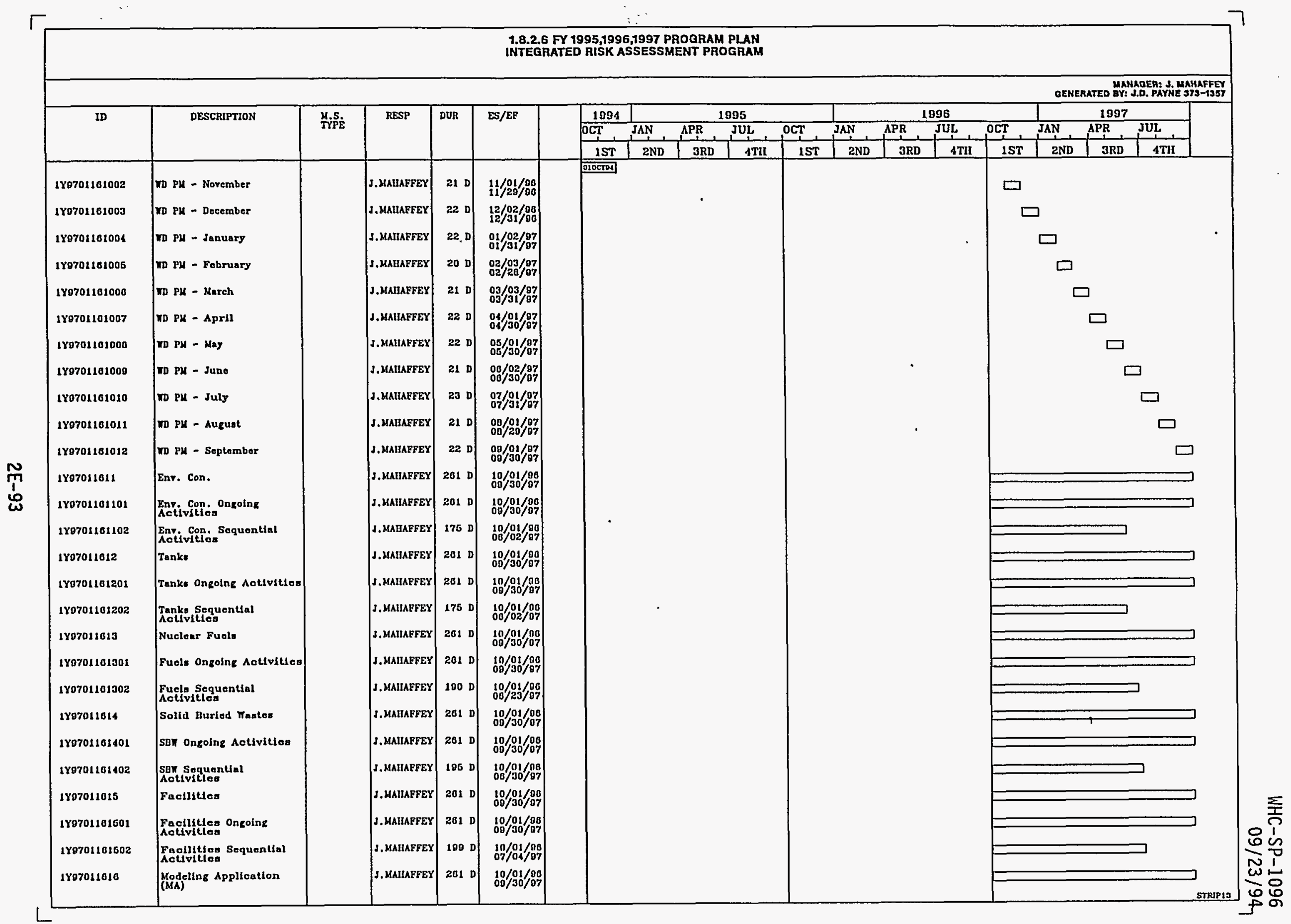




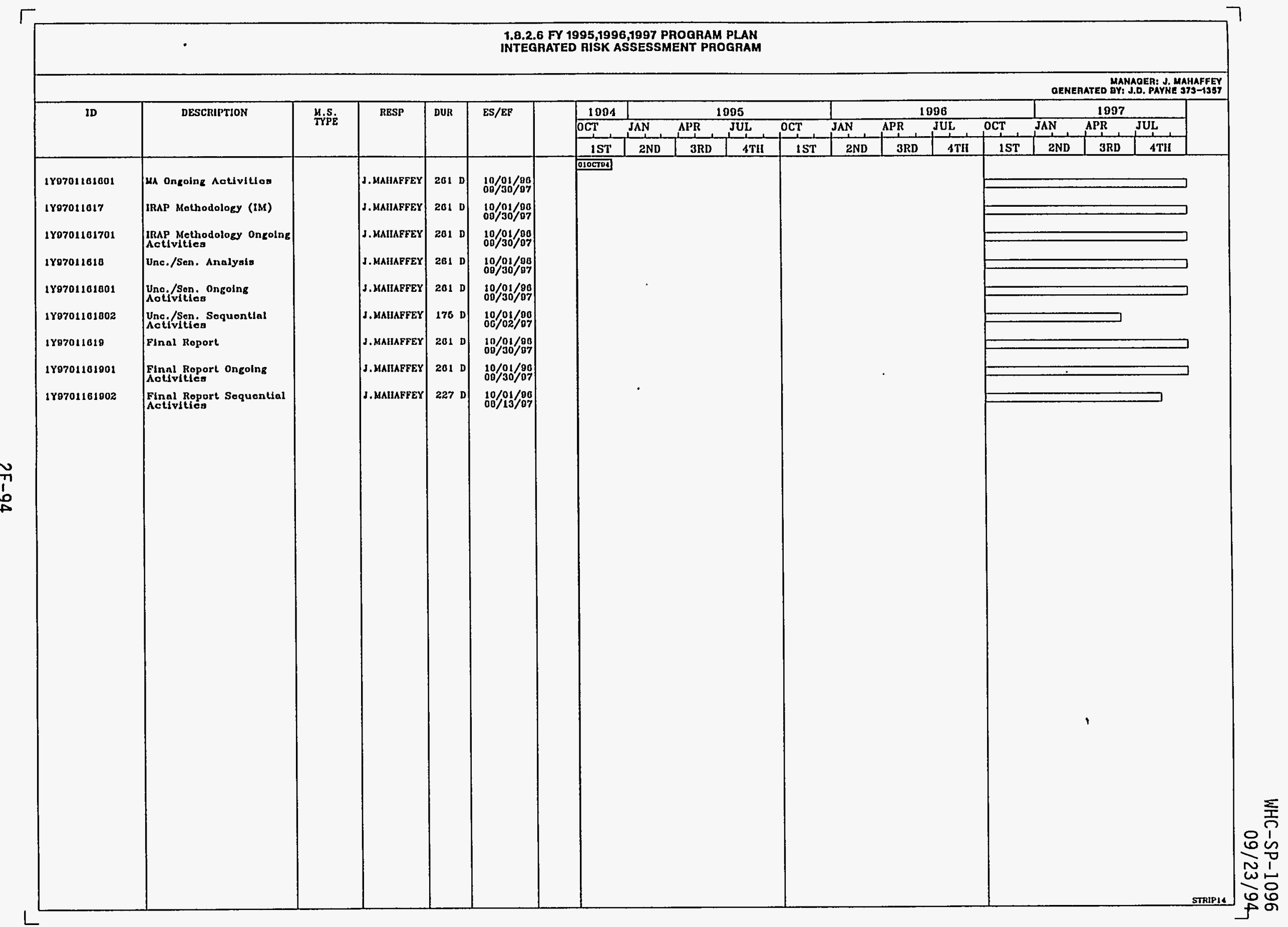




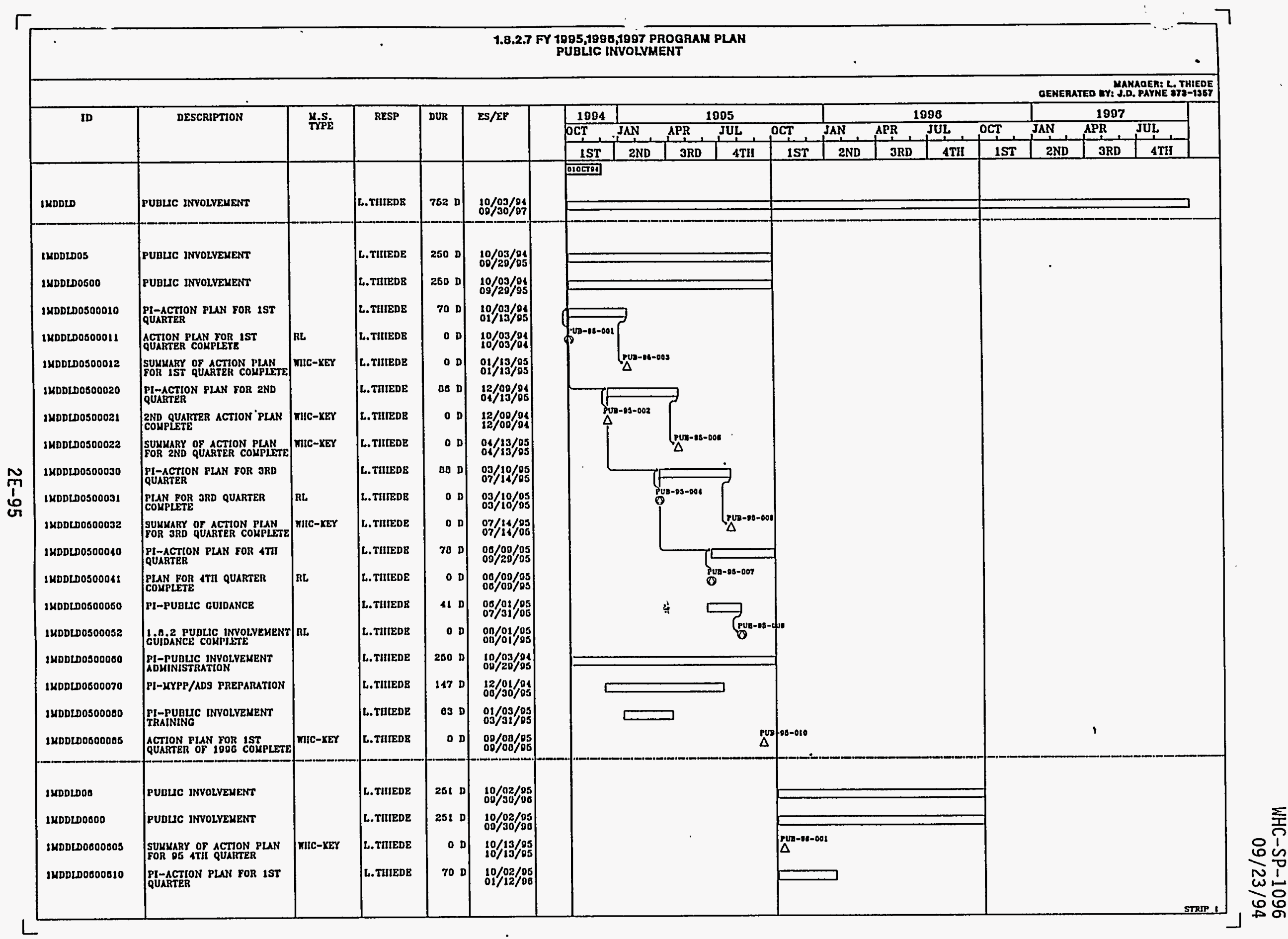




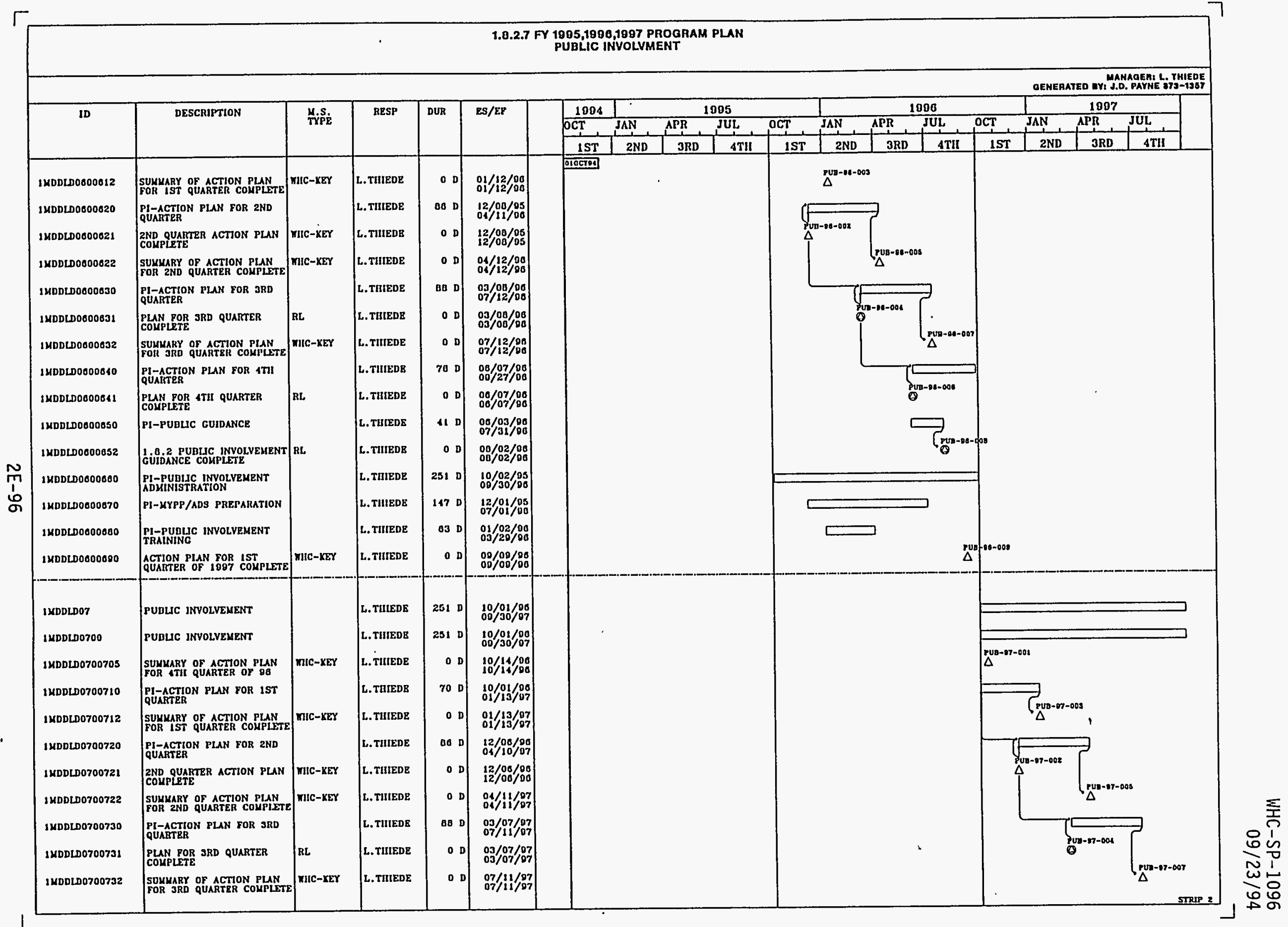




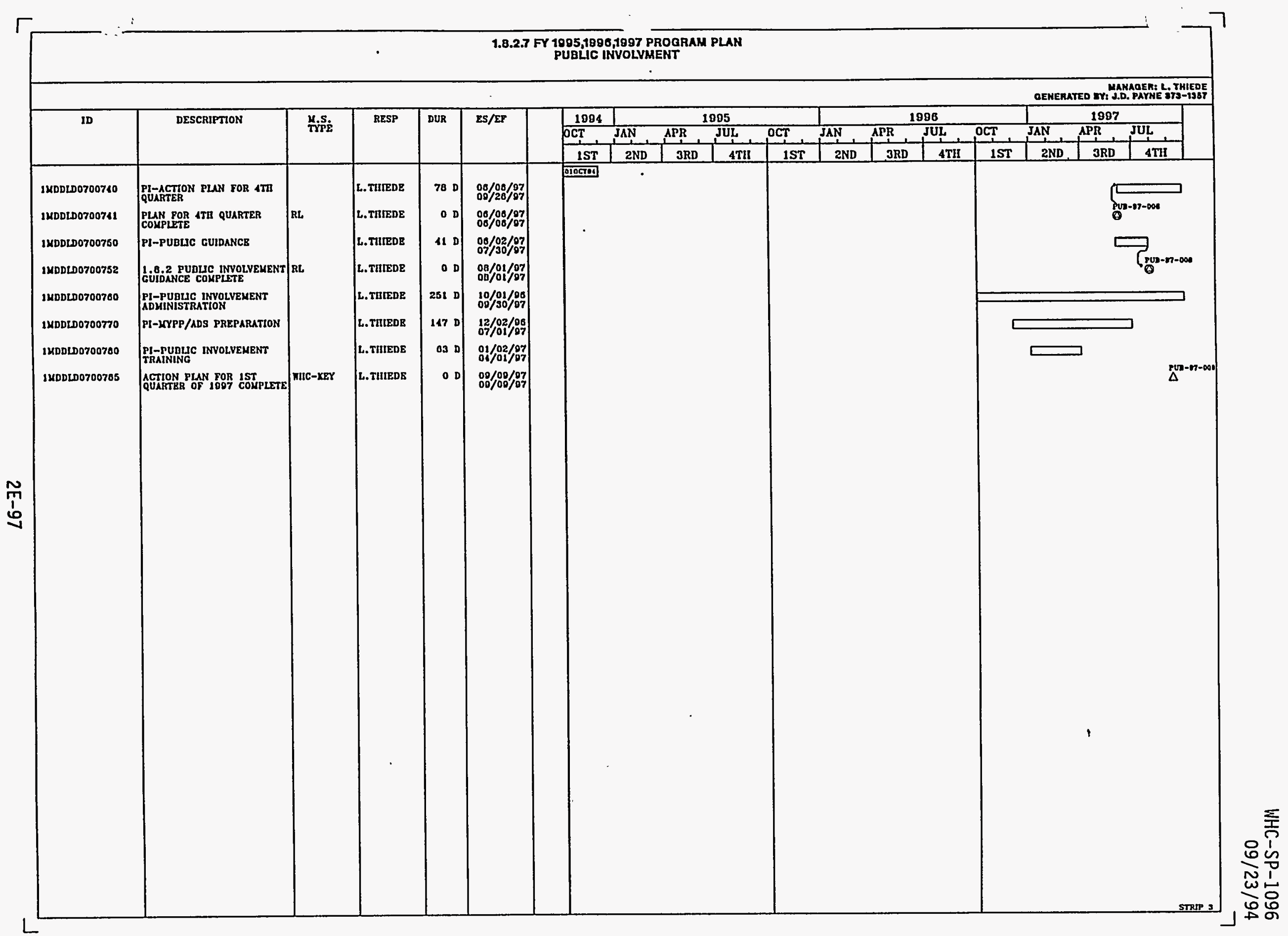




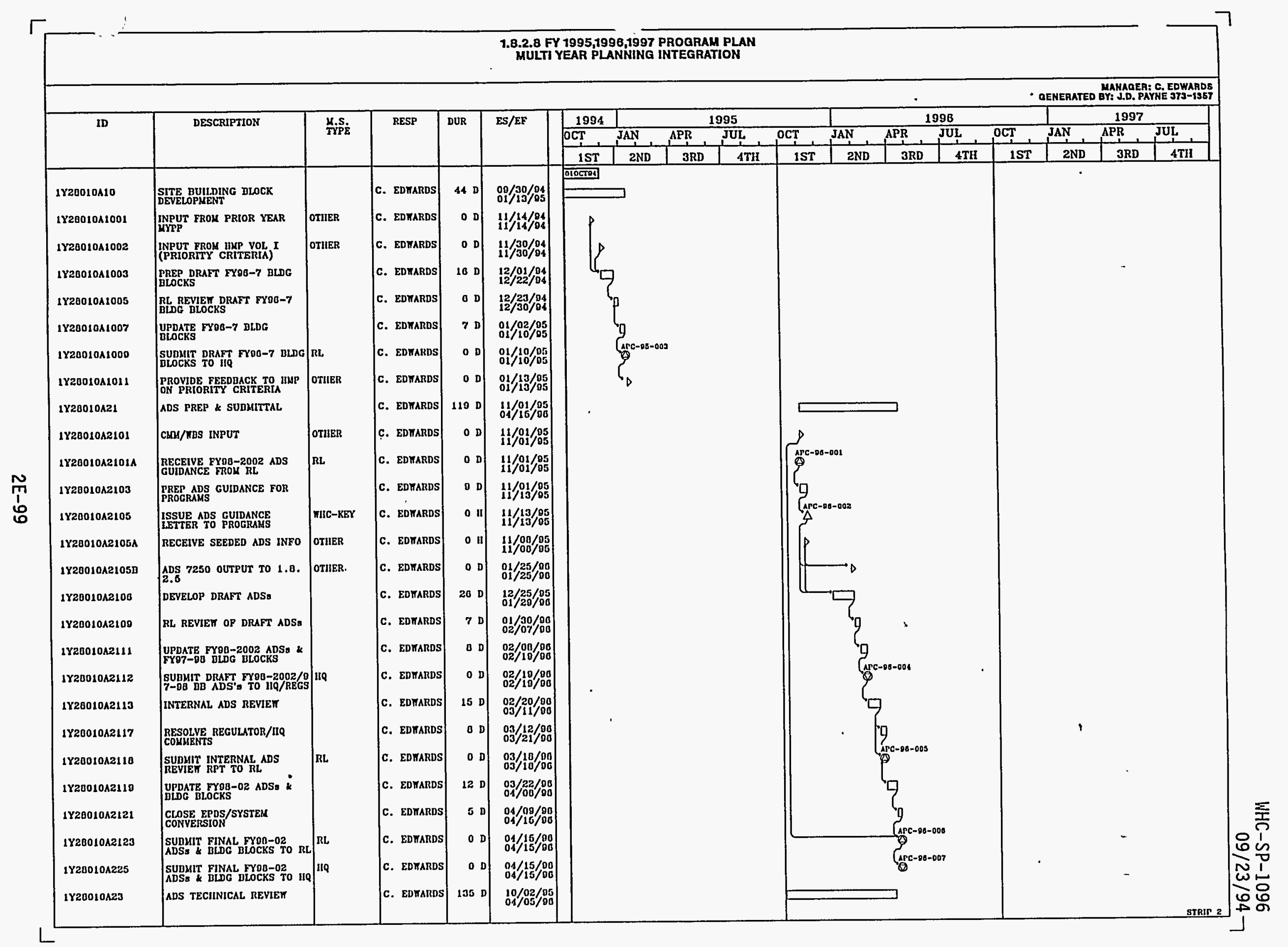




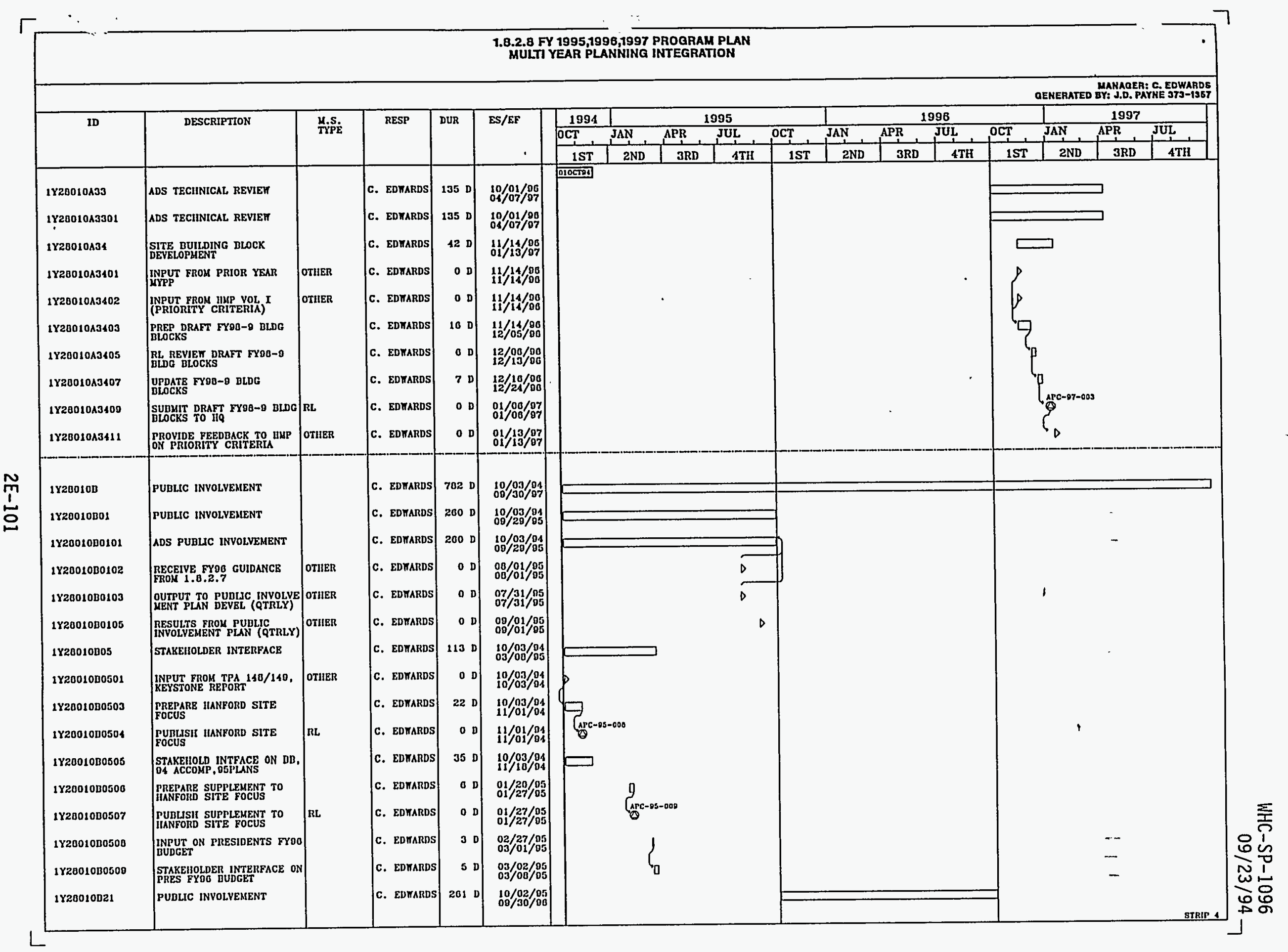




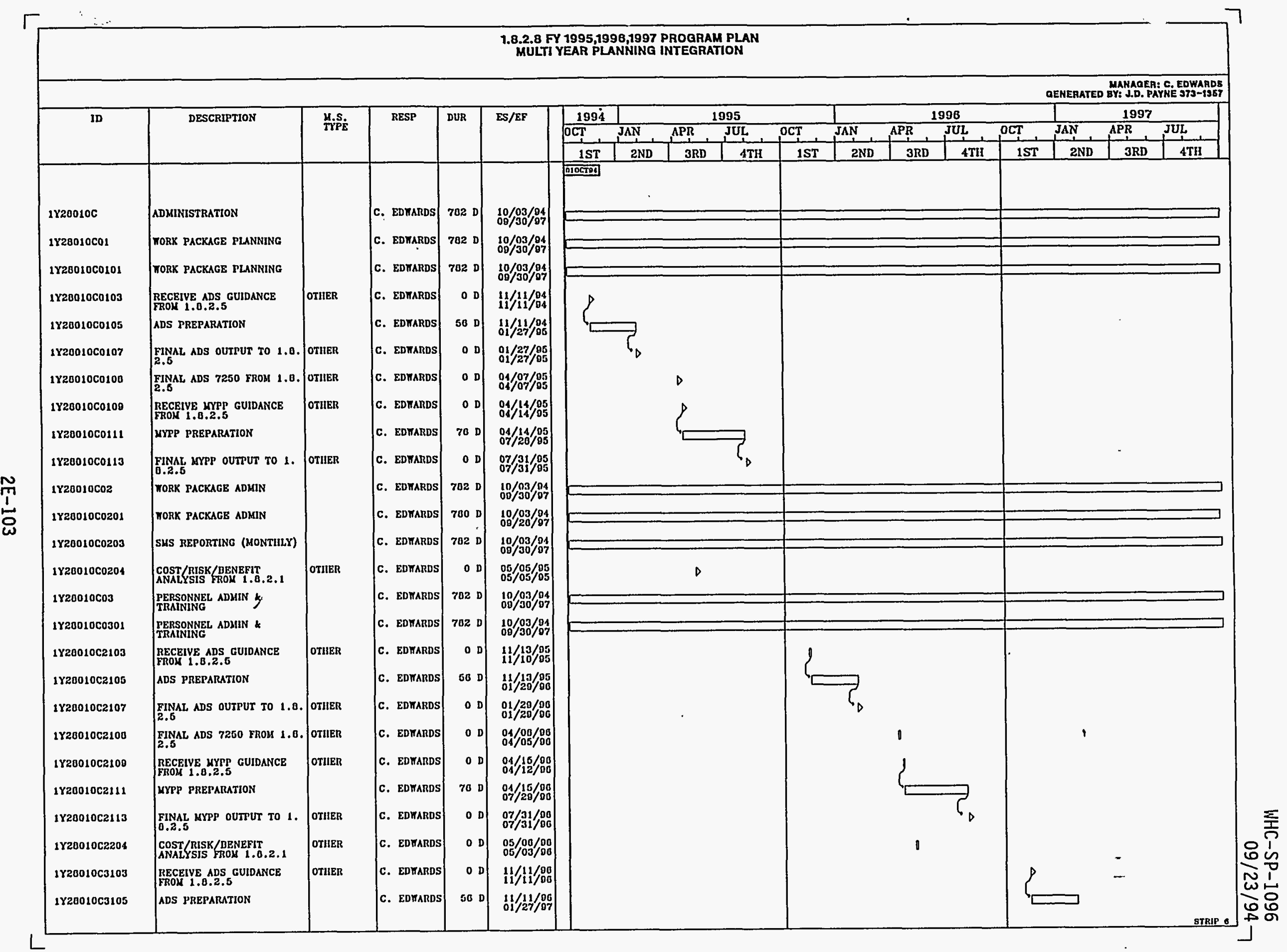




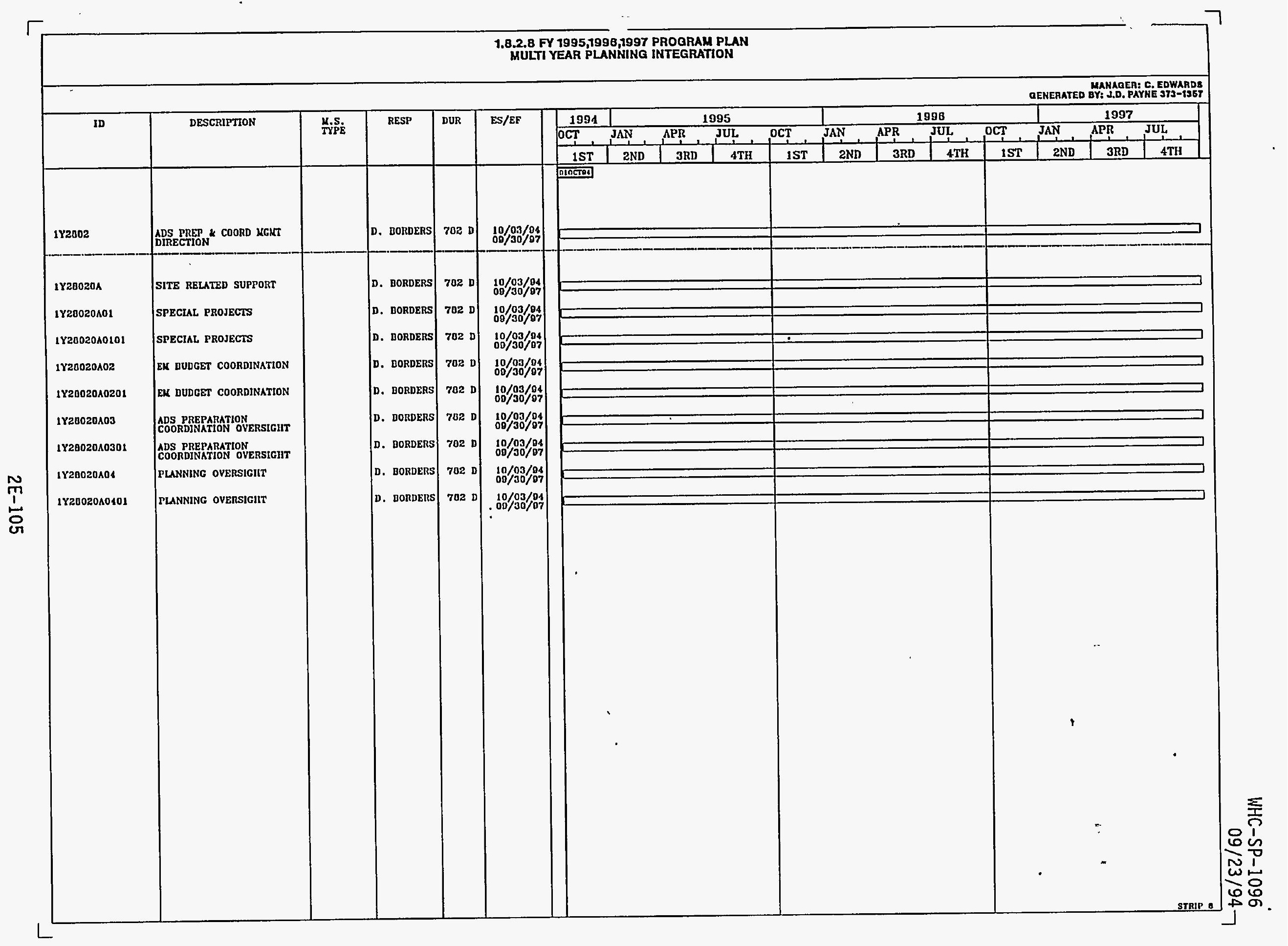


Planning Integration Multi-Year Program Plan

This page intentionally left blank. 
Planning Integration Multi-Year Program Plan

\section{F MILESTONE LIST}

The Milestone Control Log (MCL) is a listing of al1 DOE-HQ, RL, and contractor key milestones. The MCL wi11 be maintained as an ancil7ary to the change control process. As approved changes to the milestones occur, the MCL will be updated.

A1l milestones are controlled through a change control process. Changes to DOE-HQ and RL milestones are processed through a Class I baseline change request. Changes to contractor key milestones are analyzed for impacts to senior milestones ( $R L$ or DOE-HQ). If a modification to a contractor key milestone affects a senior milestone, a Class I baseline change request will be necessary. Otherwise, changes to contractor key milestones are processed through a $\mathrm{Class}$ II baseline change request.

Each milestone is identified by a unique milestone control number. Each milestone can be located in the schedule by this unique number. The number sequence is assigned by the program element. Each program element is assigned a block of milestone control numbers, utilizing the following convention.

Three character designator for the

Program Element
Two character designator for the fiscal year in which the milestone is due

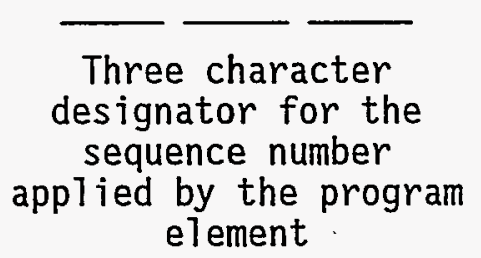

Three character designator for the sequence number element

Example: SSE-95-001, is a milestone that is the responsibility of Systems Engineering (WBS 1.8.2.4), will be completed in FY 1995 , and has been assigned sequence number 001 . 


\section{Planning Integration \\ MuTti-Year Program Plan}

The program element designators are contained in table 2.F-1.

\begin{tabular}{|c|c|c|}
\hline Identivitier & 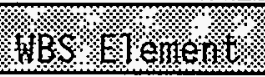 & 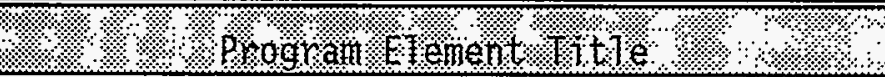 \\
\hline PSS & 1.8 .2 .1 & Work Identification \\
\hline PSA & 1.8 .2 .2 & Baseline Reporting and System Development \\
\hline PBI & 1.8 .2 .3 & Program Baseline Integration \\
\hline SSE & 1.8 .2 .4 & Technical Baseline Development \\
\hline PIM & 1.8 .2 .5 & Program Management \\
\hline IRA & 1.8 .2 .6 & Integrated Risk Assessment \\
\hline PUB & 1.8 .2 .7 & Public Involvement \\
\hline APC & 1.8 .2 .8 & $\begin{array}{c}\text { Activity Data Sheet Preparation and } \\
\text { Coordination }\end{array}$ \\
\hline
\end{tabular}

Table 2.F-1. Program Element Designators

There are two data sorts of the MCL contained within the MYPP/FYWP. The first sort, table 2.F-2, is by due date, irrespective of program element responsible for the work. The second sort, table 2.F-3, is sorted by milestone control number, irrespective of due date. 
Planning Integration Multi-Year Program Plan

1

Table 2.F-2. Milestone List by Due Date (9 pages)

$2 F-3$ 
Run Date 21Sep94 19:50

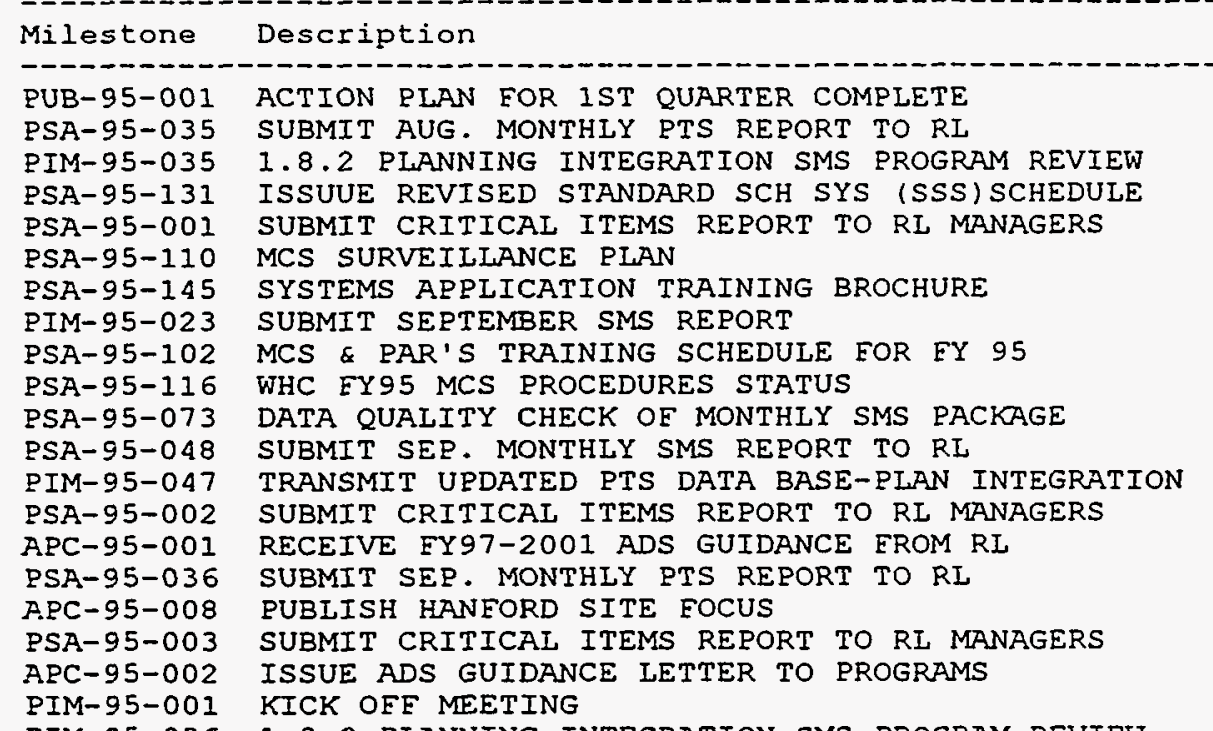

PIM-95-001 PIM-95-036 PIM-95-024 PSA-95-136 PSA-95-074 PIM-95-048 PSA-95-049 PSA-95-004 PSA-95-037 PUB-95-002 PSA-95-005 PIM-95-037 PIM-95-025 PSA-95-100 PSA-95-075 PIM-95-049 PSA-95-050 PSA-95-006 PSA-95-150 PSA-95-120 SSE-95-001 PSA-95-038 PSA-95-007 APC-95-003 PSA-95-151 SSE-95-011 PIM-95-038 PIM-95-026 PSA-95-132 PUB-95-003 PSA-95-111 PIM-95-002 PSA-95-130 PSA-95-076 PSS-95-100 PSA-95-008 PSA-95-137 PIM-95-003 PSA-95-051 PIM-95-050 APC-95-009 PIM-95-004 SSE-95-006 SSE-95-014 PSA-95-039 PSA-95-009 PIM-95-039 PSA-95-010 PIM-95-027 APC-95-004 - PSA-25-153
1.8 .2 PLANNING INTEGRATION SMS PROGRAM REVIEW SUBMIT OCTOBER SMS REPORT

DATA QUALITY CHECK OF MONTHLY SMS PACKAGE

TRANSMIT UPDATED PTS DATA BASE-PLAN INTEGRATION

SUBMIT OCT. MONTHLY SMS REPORT TO RL

SUBMIT CRITICAL ITEMS REPORT TO RL MANAGERS

SUBMIT OCT. MONTHLY PTS REPORT TO RL

2ND QUARTER ACTION PLAN COMPIETE

SUBMIT CRITICAL ITEMS REPORT TO RL MANAGERS

1.8.2 PLANNING INTEGRATION SMS PROGRAM REVIEW

SUBMIT NOVEMBER SMS REPORT

DRAET MANAGMENT CONTROL SYSTEMS DESCRIPTION

DATA QUAIITY CHECK OF MONTHLY SMS PACKAGE

TRANSMIT UPDATED PTS DATA BASE-PLAN INTEGRATION

SUBMIT NOV. MONTHLY SMS REPORT TO RI

SUBMIT CRITICAI ITEMS REPORT TO RL MANAGERS

PUBLISH DRAFT REQ. DOC. FOR REENGINEERING

BASEIING CHANGE CONTROL MODUIE (PHASE 1)

TPA M33-00-T03

SUBMIT NOV. MONTHLY PTS REPORT TO RL

SUBMIT CRITICAI ITEMS REPORT TO RL MANAGERS

SUBMIT DRAET FY96-7 BLDG BLOCKS TO HQ

ISSUE FINAI REQUIREMENTS DOCUMENT

ISSUE REVISED HS-SEMP/SEM TO PROGRAMS \& $Q A$

1.8 .2 PIANNING INTEGRATION SMS PROGRAM REVIEW SUBMIT DECEMBER SMS REPORT

IST QTR STATUS OF SSS IMPLEMENTATION

SUMMARY OE ACTION PLAN FOR 1ST QUARTER COMPLETE

SURVEILIANCE PIAN STATUS REPORT FOR IST QTR

IN PROCESS REVIEW

ISSUE JCS TO/EROM $P / X$ SYSTEM REQUIREMENTS

DATA QUALITY CHECK OF MONTHLY SMS PACKAGE

STRATEGIC PINNG-HSP EORMAL DRAFT COMPLETE

SUBMIT CRITICAL ITEMS REPORT TO RL MANAGERS

REPORT OF I.ABOR EROM $P / X$

INTERNAL PROGRAM MANAGER REVIEW

SUBMIT DEC. MONTHIY SMS REPORT TO RL

TRANSMIT UPDATED PTS DATA BASE-PLAN INTEGRATION

PUBLISH SUPPIEMENT TO HANFORD SITE FOCUS

DRAFT YO RI EOR REVIEW

COMPIETE DYNAMIC SYSTEMS MODEL

ISSUE REVISED SITE SE-CMP (SEM-95-005)

SUBMIT DEC. MONTHLY PTS REPORT TO RI

SUBMIT CRITICAL ITEMS REPORT TO RL MANAGERS 1.8.2 PIANNING INTEGRATION SMS PROGRAM REVIEW SUBMIT CRITICAL ITEMS REPORT TO RL MANAGERS

SUBMIT JANUARY SMS REPORT

SUBMIT DRAFT FY97-2001 ADSS/96-97BB TO HO/REGS

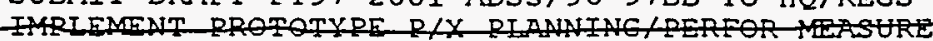

INPUT BCWP INTO $\mathrm{P} / \mathrm{X}$ FOR SCHEDULE STATUS
Type

Finish

$K$ RI Reportable

K RI Reportable

$K$ RI Reportable

$R$ Westinghouse Reportable

$R$ Westinghouse Reportable

K RI Reportable

$R$ Westinghouse Reportable

$R$ Westinghouse Reportable

$R$ Westinghouse Reportable

$R$ Westinghouse Reportable

$R$ Westinghouse Reportable

$K$ RI Reportable

$K$ RI Reportable

$R$ Westinghouse Reportable

K RL Reportable

$K$ RI Reportable

$K$ RI Reportable

$R$ Westinghouse Reportable

$R$ Westinghouse Reportable

$R$ Westinghouse Reportable

$K$ RI Reportable

$R$ Westinghouse Reportable

$R$ Westinghouse Reportable

$R$ Westinghouse Reportable

$K$ RI Reportable

$K$ RI Reportable

$R$ Westinghouse Reportable

K RL Reportable

$R$ Westinghouse Reportable

$R$ Westinghouse Reportable

$K$ RI Reportable

$R$ Westinghouse Reportable

$R$ Westinghouse Reportable

$R$ Westinghouse Reportable

K RL Reportable

K RL Reportable

$R$ Westinghouse Reportable

$R$ Westinghouse Reportable

$R$ Westinghouse Reportable

T Tri-Party Agreement

K RL Reportable

R Westinghouse Reportable

K RI Reportable

$R$ Westinghouse Reportable

K RL Reportable

K RI Reportable

$R$ Westinghouse Reportable

$R$ Westinghouse Reportable

$R$ Westinghouse Reportable

$R$ Westinghouse Reportable

$R$ Westinghouse Reportable

$R$ Westinghouse Reportable

$R$ Westinghouse Reportable

K RI Reportable

$R$ Westinghouse Reportable

$R$ Westinghouse Reportable

$R$ Westinghouse Reportable

$K$ RI Reportable

K RI Reportable

K RL Reportable

K RI Reportable

$R$ Westinghouse Reportable

K RI Reportable

$K$ RI Reportable

$R$ Westinghouse Reportable

K RI Reportable

$R$ Westinghouse Reportable

$R$ Westinghouse Reportable

M Major-HQ Reportable

R westinghouse Reportabie
$030 c t 94$

o30ct 94

130 ct 94

$140 \operatorname{ct} 94$

140 ct 94

140 ct 94

140 oct 94

$140 \operatorname{ct} 94$

140 ot 94

$170 c t 94$

$190 \operatorname{ct} 94$

$210 \operatorname{cts} 4$

$210 \operatorname{ct} 94$

280 ct 94

01Nov94

OINov94

01Nov94

11 Nov 94

11 Nov 94

14 Nov 94

17 Nov 94

18 Nov 94

18 Nov 94

21 Nov 94

21 Nov 94

23 Nov 94

25 Nov 94

01 Dec 94

09Dec 94

O9Dec 94

15Dec 94

16 Dec 94

16 Dec 94

21 Dec 94

21Dec 94

22Dec94

23 Dec 94

30Dec 94

30Dec 94

30Dec 94

$03 \mathrm{Jan} 95$

$06 J a n 95$

$10 \mathrm{Jan} 95$

$11 \mathrm{Jan} 95$

11 Jan95

$12 \mathrm{Jan} 95$

13 Jan95

$13 \mathrm{Jan} 95$

$13 \mathrm{Jan} 95$

$16 \mathrm{Jan} 95$

$16 \mathrm{Jan} 95$

$16 \mathrm{Jan} 95$

$18 \mathrm{Jan} 95$

$19 \mathrm{Jan} 95$

20Jan 95

$20 \mathrm{Jan} 95$

$20 J a n 95$

$20 J a n 95$

23Jan95

$27 \mathrm{Jan} 95$

$30 \mathrm{Jan} 95$

31 Jan 95

31 Jan 95

01 Feb95

03 Eeb95

$16 \mathrm{Feb} 95$

$17 \mathrm{Feb} 95$

$17 \mathrm{Feb} 95$

17 Eeb95

21 Feb95 
Run Date 21Sep94 19:50

Milestone Description

Type

Finish

Á-95-152

ESA-95-077

PIM-95-051

PSA-95-052

PSA-95-069

PSS-95-305

PSA $-95-040$

PSS-95-300

PSA-95-011

PUB-95-004

PSA-95-138

PBI-95-001

PIM-95-040

APC-95-005

PIM-95-028

PSA-95-012

RSA-95-101

PIM-95-052

PSA-95-078

PSA-95-053

RSS-95-201

PSS-95-200

SSE-95-002

PBI-95-002

PSA-95-013

PSA-95-063

PSS-95-10I

PBI-95-008

SSE-95-012

RSA-95-041

PIM-95-005

*) $29 \pi-95-154$

-M-95-041

B-95-006

ESA-95-014

RSA-95-139

PSA $-95-133$

PIM-95-029

PIM-95-006

APC-95-006

APC-95-007

PSA-95-065

RSA-95-112

PSA-95-079

PSA-95-054

PIM-95-053

PSA-95-015

PSA-95-042

大 PSA-95-155

PSS-95-301

PSA-95-016

PIM-95-042

PIM-95-030

PSS-95-202

PSS-95-205

PIM-95-054

SSE-95-004

PSA-95-080

SSE-95-003

PSA-95-055

PSA-95-017

IRA-95-001

PSS-95-302

PSA $-95-043$

जSA-95-140

B-95-007

$\triangle A-95-018$

PBI-95-003

PIM-95-043

PSA-95-067
PROGRAM $\mathrm{P} / \mathrm{X}$ IN ACCORDANCE WITH SPECIEICATIONS DATA QUALITY CHECK OE MONTHLY SMS PACKAGE TRANSMIT UPDATED PTS DATA BASE-PLAN-INTEGRATION SUBMIT JAN. MONTHLY SMS REPORT TO RL COMPLETE EPDS SOFTWARE CONEIG CNTRL MGMT PLAN SITE-WIDE RISK ACTIVITIES COMPLETE

SUBMIT JAN. MONTHLY PTS REPORT TO RL IMPLEMENT RRIORITIZATION METHODOLOGY COMPT SUBMIT CRITICAL ITEMS REPORT TO RI MANAGERS PLAN EOR 3RD OUARTER COMPLETE

PROGRAM P/X SOETWARE CHANGES FOR JCS INPUT RL ISSUE SITE DIRECT MYPP/FYWP GUIDANCE

1.8.2 PLANNING INTEGRATION SMS PROGRAM REVIEW SUBMIT INTERNAL ADS REVIEW RPT TO RL

SUBMIT EEBURARY SMS REPORT

SUBMIT CRITICAL ITEMS REPORT TO RL MANAGERS EINAL MANAGMENT CONTROL SYSTEMS DESCRIPTION TRANSMIT UPDATED PTS DATA BASE-PLAN INTEGRATION DATA QUALITY CHECK OE MONTHLY SMS PACKAGE SUBMIT FEB. MONTHLY SMS REPORT TO RL HIGH LEVEL PRIORITY IIST

HMP-DRAFT RI WBS/OBS (THREE LEVELS) COMPLETE COMPLETE UPDATE FUNCTIONAL ANALYSIS, REV 0 ISSUE SITE DIRECT MYPP/FYWP CALL LETTER SUBMIT CRITICAI ITEMS REPORT TO RL MANAGERS DISTRIB CAIL IETTER REQUESTING FY 96 PROPOSED WB STRATEGIC PLNNG-APPROVAL OF EY1996 HSP COMPLETE ISSUE SSPP CAIL IETTER

COMPLETE SE ENG PRACT \& PROC ASSESSMENT REPORT SUBMIT EEB. MONTHLY PTS REPORT TO RI

COMPIETE ADS 7250

TESTING, REVIENOETIE-RROTOTYE

1.8.2 PIANNING INTEGRATION SMS PROGRAM REVIEW

SUMMARY OF ACTION PLAN FOR 2ND QUARTER COMPLETE

SUBMIT CRITICAL ITEMS REPORT TO RL MANAGERS

P/X TO JCS SUPPORTING PROCEDURES

2ND QTR STATUS OF SSS IMPLEMENTATION

SUBMIT MARCH SMS REPORT

1996 MYPP/FYWP KICK OFF MEETING

SUBMIT FINAL EY97-01 ADSS \& BLDG BLOCKS TO RL SUBMIT EINAL EY97-01 ADSS \& BLDG BLOCKS TO HQ COMPLETE GENERAL PROCESS IMPROVEMENTS TO C.M.M. SURVEILLANCE PIAN STATUS REPORT FOR 2ND QTR DATA QUALITY CHECK OF MONTHLY SMS PACKAGE SUBMIT MAR. MONTHLY SMS REPORT TO RL TRANSMIT UPDATED PTS DATA BASE-PLAN INTEGRATION SUBMIT CRITICAJ ITEMS REPORT TO RL MANAGERS SUBMIT MAR. MONTHLY PTS REPORT TO RL

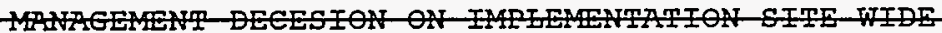
COST-RISK-BENEEIT ANALYSIS COMPLETE SUBMIT CRITICAI ITEMS REPORT TO RI MANAGERS 1.8.2 PLANNING INTEGRATION SMS PROGRAM REVIEW SUBMIT APRIL SMS REPORT

REVSE, EINALZE \& TRANSMT DRFT FY96 HMP V-1 COMPT ISSUE WORKING PAPER DRAET FY96 HMP TO PROGS TRANSMIT URDATED PTS DATA BASE-PIAN INTEGRATION UPDATE BASELINE MEASURES, REV O

DATA QUALITY CHECK OF MONTHLY SMS PACKAGE COMPLETE REQUIREMENTS ANAIYSIS \& ALLOC, REV 0 SUBMIT APR. MONTHLY SMS REPORT TO RL

SUBMIT CRITICAI ITEMS REPORT TO RL MANAGERS

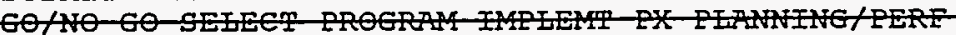

Integrated Risk Assessment Report

DETERMINE RISK PERCEPTIONS COMPIETE

SUBMIT APR. MONTHLY PTS REPORT TO RL

$\mathrm{P} / \mathrm{X}$ SOFTWARE CONEIGURATION MANAGEMENT PLAN

PLAN FOR 4 TH QUARTER COMPLETE

SUBMIT CRITICAL ITEMS REPORT TO RL MANAGERS

SUBMIT REDLINED/MARKED UPDATE SITE FY 1996 MYPP 1.8.2 PLANNING INTEGRATION SMS PROGRAM REVIEW FINAIIZE DRAET FY 96 PROPOSED WBS \& DISTRIBUTE
R Westinghouse Reportable R Westinghouse Reportable

K RL Reportable

K RL Reportable

R Westinghouse Reportable

$\therefore$ K RI Reportable

K RL Reportable

K RL Reportable

$R$ Westinghouse Reportable

K RL Reportable

$R$ Westinghouse Reportable

K RL Reportable

K RL Reportable

K RL Reportable

$R$ Westinghouse Reportable

$R$ Westinghouse Reportable

K RI Reportable

K RL Reportable

$R$ Westinghouse Reportable

K RL Reportable

K RL Reportable

K RI Reportable

K RL Reportable

$R$ Westinghouse Reportable

$R$ Westinghouse Reportable

K RI Reportable

K RL Reportable

$R$ Westinghouse Reportable

$R$ Westinghouse Reportable

K RI Reportable

$R$ Westinghouse Reportable

R Wegting Reportable

K RL Reportable

$\mathrm{R}$ Westinghouse Reportable

$R$ Westinghouse Reportable

$R$ Westinghouse Reportable

$R$ Westinghouse Reportable

$R$ Westinghouse Reportable

R Westinghouse Reportable

K RL Reportable

M Major-HQ Reportable

$R$ Westinghouse Reportable

$R$ Westinghouse Reportable

$R$ Westinghouse Reportable

K RL Reportable

K RL Reportable

$R$ Westinghouse Reportable K RI Reportable

R Wostinghousa Reportable

K RL Reportable

$R$ Westinghouse Reportable

K RL Reportable

$R$ Westinghouse Reportable

K RI Reportable

K RI Reportable

K RI Reportable

K RI Reportable

R Westinghouse Reportable

K RL Reportable

K RL Reportable

$R$ Westinghouse Reportable festimgirouse-Reportable

$K$ RI Reportable

$K$ RL Reportable

K RL Reportable

$R$ Westinghouse Reportable

K RI Reportable

$R$ Westinghouse Reportable

$R$ Westinghouse Reportable

K RI Reportable

K RL Reportable
21 Feb95

22 Eeb95

22 Eeb95

24 Eeb95

28 Eeb 95

28 Eeb95

01 Mar 95

01 Mar 95

03 Mar 95

10 Mar 95

$10 \operatorname{Mar} 95$

15 Mar 95

16 Mar 95

17 Mar 95

17 Mar 95

17 Mar95

17 Mar95

21 Mar 95

22Mar 95

$24 \operatorname{Mar} 95$

28Mar 95

28Mar 95

$29 \operatorname{Mar} 95$

31 Mar95

31 Mar 95

31 Mar 95

$31 \mathrm{Mar} 95$

31 Mar 95

31 Mar 95

03Apr95

07 Apr 95

10Apres

13Apr 95

13Apr95

14 Apr 95

14Apr 95

14Apr95

14Apr 95

14Apr 95

14Apr 95

14Apr 95

17Apr95

17Apr95

19 Apr 95

21Apr95

21Apr95

28Apr95

01 May 95

01Mays

02 May 95

12 May 95

18 May 95

19 May95

19 May95

22 May 95

22 May 95

23 May 95

24 May 95

24 May 95

26 May 95

26 May 95

30 May 5

30 May 95

31 May 95

01 Jun95

01 Jun95

09Jun95

09 Jun95

15Jun95

15Jun95

$15 \mathrm{Jun} 95$ 
Milestone

PIM-95-031

PIM-95-007

PIM-95-055

PSA-95-08I

PSA-95-019

PSA-95-056

PBI-95-004

PBI-95-009

PSA-95-044

PSS-95-203

PSA-95-020

PIM-95-044

PIM-95-032

PIM-95-008

PSA-95-134

PUB-95-008

PSA-95-113

PSA-95-082

SSE-95-013

PSA-95-057

PIM-95-056

PSA-95-021

PSA-95-029

PBI-95-010

PBI-95-015

PSA-95-045

PUB-95-009

PSA-95-022

PIM-95-045

PIM-95-033

PSA-95-023

PIM-95-057

PSA-95-083

PSA-95-058

SSE-95-015

PSA-95-066

SSE-95-007

PIM-95-009

PSS-95-304

PBI-95-011

SSE-95-005

PBI-95-005

PSA-95-046

PSA-95-024

PUB-95-010

PSA-95-068

PIM-95-046

PIM-95-034

PBI-95-014

PSA-95-025

PSA-95-084

PIM-95-058

PSA-95-059

PIM-95-010

PSA-95-114

PBI-95-013

PSA-95-135

PSA-95-026

PSA-95-070

PSS-95-204

PSA-95-115

PSA-95-121

PBI-95-006

IRA-95-003

IRA-95-002

PBI-95-012

PBI-95-007

SSE-95-010

PSA-96-035

PIM-96-035

PIM-96-023

Description

Type

Einish

SUBMIT MAY SMS REPORT

IN PROCESS REVIEW

TRANSMIT UPDATED PTS DATA BASE-PLAN INTEGRATION

DATA QUALITY CHECK OF MONTHLY SMS PACKAGE

SUBMIT CRITICAL ITEMS REPORT TO RI MANAGERS

SUBMIT MAY MONTHLY SMS REPORT TO RL

RL CONCUR REDIINED/MARKED UPDATE SITE FY96 MYPP SUBMIT REDLINED/MARKED UPDATE SITE FY96 SSPS RL SUBMIT MAY. MONTHIY PTS REPORT TO RL

COMPLETE FY1996 HANFORD MISSION PLAN, V-1

SUBMIT CRITICAL ITEMS REPORT TO RL MANAGERS

1.8.2 PLANNING INTEGRATION SMS PROGRAM REVIEW

SUBMIT JUNE SMS REPORT

INTERNAL PROGRAM MANAGER REVIEW

3RD QTR STATUS OF SSS IMPLEMENTATION

SUMMARY OF ACTION PLAN FOR 3RD QUARTER COMPLETE

SURVEILLANCE PLAN STATUS REPORT FOR 3RD QTR

DATA QUALITY CHECK OF MONTHLY SMS PACKAGE

COMPLETE SE TRAINING ASSESSMENT REPORT

SUBMIT JUN. MONTHLY SMS REPORT TO RL

TRANSMIT UPDATED PTS DATA BASE-PLAN INTEGRATION

SUBMIT CRITICAL ITEMS REPORT TO RL MANAGERS

COMPLETE FY 96 MICROCOMP STATEMENT OF STRATEGY

RL CONCUR REDLINED/MARKED UPDATE FY96 SSPS

SUBMIT SITE MYPP/EYWP INTERNAL REV RPT TO RL

SUBMIT JUN. MONTHLY PTS REPORT TO RL

1.8 .2 PUBLIC INVOLVEMENT GUIDANCE COMPLETE

SUBMIT CRITICAL ITEMS REPORT TO RL MANAGERS

1.8.2 PLANNING INTEGRATION SMS PROGRAM REVIEW

SUBMIT JULY SMS REPORT

SUBMIT CRITICAL ITEMS REPORT TO RL MANAGERS

TRANSMIT UPDATED PTS DATA BASE-PLAN INTEGRATION

DATA QUALITY CHECK OE MONTHLY SMS PACKAGE

SUBMIT JUL. MONTHLY SMS REPORT TO RI

COMPLETE DEF. \& IMPL. INFOR STRUCT \& CNTRL' SYS

COMPLETE TPA ARCHIVE AND PTS ENHANCEMENTS

TPA M33-00-TO4

SUBMIT MYPP/FYWP TO RL

RISK MGMT IN SE

SUBMIT FY96 SITE SUPPT PROGPLANS TO RL

COMPLETE REVISED ARCH \& PRODUCTS DEFIN, REV 0 SUBMIT SITE MYPP/FYWPS TO RL

SUBMIT JUL. MONTHLY PTS REPORT TO RL

SUBMIT CRITICAL ITEMS REPORT TO RL MANAGERS

ACTION PLAN EOR IST QUARTER OF 1996 COMPLETE

DEVELOP C.M.M. AS REPLACEMENT FOR PTS MILESTONES

1.8.2 PLANNING INTEGRATION SMS PROGRAM REVIEW

SUBMIT AUGUST SMS REPORT

PUBLISH LEVEL O SCHEDULE UPDATE (MONTHLY)

SUBMIT CRITICAL ITEMS REPORT TO RL MANAGERS

DATA QUALITY CHECK OF MONTHLY SMS PACKAGE

TRANSMIT URDATED PTS DATA BASE-PLAN INTEGRATION

SUBMIT AUG. MONTHLY SMS REPORT TO RL

APPROVAL ON MYPP/FYWP AND WORK AUTHORIZATION

SURVEILLANCE PLAN STATUS REPORT FOR 4TH QTR

PUBLISH HSIS MONTHLY STATUS

$4 T H$ QTR STATUS OE SSS IMPIEMENTATION

SUBMIT CRITICAI ITEMS REPORT TO RL MANAGERS

ISSUE RFP FOR CLIENT SERVER PROTOTYPE

HSA ADDENDUM II

WHC FY95 MCS PROCEDURES DEVELOPMENT PLAN

BASELING CHANGE CONTROL MODULE (PHASE 2)

RI APPROVAL OF SITE MYPP/FYWP

Integrated Risk Assessment Report

Public Health Baseline Risk Assessment Report

SITE SUPPT PROG PLANS APPROVED BY RL

RI ISSUES FY96 WORK AUTHORIZATION LETTERS

COMPIETE BASELINE SPECIFICATION

SUBMIT AUG. MONTHLY PTS REPORT TO RL

1.8.2 PIANNING INTEGRATION SMS PROGRAM REVIEW

SUBMIT SEPTEMBER SMS REPORT
$R$ Westinghouse Reportable

$R$ Westinghouse Reportable

K RL Reportable

$R$ Westinghouse Reportable

$R$ Westinghouse Reportable

K RL Reportable

K RL Reportable

R Westinghouse Reportable

K RL Reportable

K RL Reportable

$R$ Westinghouse Reportable

K RL Reportable

$R$ Westinghouse Reportable

$R$ Westinghouse Reportable

$R$ Westinghouse Reportable

$R$ Westinghouse Reportable

$R$ Westinghouse Reportable

$R$ Westinghouse Reportable

$R$ Westinghouse Reportable

K RL Reportable

K RL Reportable

$R$ Westinghouse Reportable

$R$ Westinghouse Reportable

K RL Reportable

K RL Reportable

K RL Reportable

K RL Reportable

$R$ Westinghouse Reportable

K RL Reportable

$R$ Westinghouse Reportable

$R$ Westinghouse Reportable

K RL Reportable

$R$ Westinghouse Reportable

K RL Reportable

O Other Mgt Reportable

$R$ Westinghouse Reportable

T Tri-Party Agreement

K RL Reportable

$R$ Westinghouse Reportable

K RL Reportable

K RL Reportable

K RI Reportable

K RL Reportable

$R$ Westinghouse Reportable

$R$ Westinghouse Reportable

$R$ Westinghouse Reportable

K RL Reportable

$R$ Westinghouse Reportable

$R$ Westinghouse Reportable

$R$ Westinghouse Reportable

$R$ Westinghouse Reportable

K RL Reportable

K RL Reportable

K RL Reportable

$R$ Westinghouse Reportable

$R$ Westinghouse Reportable

$R$ Westinghouse Reportable

$R$ Westinghouse Reportable

$R$ Westinghouse Reportable

K RL Reportable

$R$ Westinghouse Reportable

$R$ Westinghouse Reportable

K RL Reportable

K RL Reportable

K RL Reportable

K RL Reportable

K RL Reportable

K RI Reportable

K RI Reportable

K RL Reportable

$\mathrm{R}$ Westinghouse Reportable

16 Jun 95

19 Jun 95

21 Jun95

21Jun95

23Jun95

23Jun 95

30 Jun 95

30 Jun 95

03Jul 95

05Jul95

$07 J u 195$

13 Jul 95

14 Jul 95

14 Jul 95

14 Jul 95

$14 J u 195$

17 Jul 95

19Jul 95

20Jul 95

21Jul 95

21 Jul 95

$21 J u 195$

31Ju195

31Jul95

01Aug 95

01 Aug 95

01 Aug 95

04 Aug 95

17 Aug 95

18 Aug 95

18 Aug 95

21Aug 95

23 Aug 95

25Aug95

28Aug 95

31 Aug 95

31 Aug 95

31 Aug 95

31 Aug 95

31 Aug 95

31 Aug95

31 Aug 95

01 Sep95

01 Sep 95

08 Sep 95

14 Sep 95

14 Sep 95

15 Sep 95

15 Sep 95

$15 \operatorname{sep} 95$

20 Sep 95

21 Sep 95

22 sep 95

28 sep95

29Sep95

29 sep 95

29Sep95

29 Sep 95

29 sep95

29 Sep 95

29Sep95

29Sep95

29Sep95

29Sep95

29 sep95

29Sep95

29 Sep95

29Sep95

020 ct 95

120 ct 95 
Run Date 21Sep94 19:50

\begin{tabular}{|c|c|c|c|c|}
\hline Milestone & Description & Type & & Finish \\
\hline$A-96$ & SUBMIT CRITICAL ITEMS REPORT TO RL MANAGERS & se & rtable & \\
\hline $5 A-96$ & MCS SURVEILIANCE PLAN & & & \\
\hline PUB-9 & SUMMARY OF ACTION PLAN EOR 95 & $\mathrm{R}$ Westinghouse & & $130 c t 95$ \\
\hline PSA- & MCS \& RAR'S TRAINING SCHEDULE FOR FY 96 & $\mathrm{R}$ Westinghouse & & $160 \mathrm{ct} 95$ \\
\hline PIM- & TRANSMIT URDATED PTS DATA BASE-PLAN INTEGRATION & K RI Reportable & & \\
\hline PSA- & DATA QUALITY CHECK OF MONTHLY SMS PACKAGE & R Westin & ble & \\
\hline PSA $\rightarrow$ & SUBMIT CRITICAI ITEMS REPORT TO RL MANAGERS & R Westinghouse & & \\
\hline PSA- & SUBMIT SEP. MONTHLY SMS REPORT TO RL & K RL Reportable & & \\
\hline APC & PUBLISH HANEORD SITE FOCUS & K RL Rep & & $=95$ \\
\hline 01 & RECEIVE FY98-2002 ADS GUIDANCE EROM RL & K RL Rep & & 011 \\
\hline ESA- & SUBMIT SEP. MONTHLY PTS REPORT TO RL & K RL Reportable & & $01 \mathrm{~N}$ \\
\hline$P S A-S$ & SUBMIT CRITICAI ITEMS REPORT TO RI MANAGERS & R Westinghouse & ortable & v95 \\
\hline$A P C-S$ & ISSUE ADS GUIDANCE LETTER TO PROGRAMS & R Westin & le & v95 \\
\hline PIM-96 & KICK OEF MEETING & $R$ Westin & le & 795 \\
\hline PIM-S & 1.8.2 PLANNING INTEGRATION SMS PROGRAM REVIEW & K RL Rep & & \\
\hline PIM- & SUBMIT OCTOBER SMS REPORT & $\mathrm{R}$ Westin & able & 95 \\
\hline PSA & DATA QUALITY CHECK OF MONTHLY SMS PACKAGE & R Westin & le & $20 \mathrm{~N}$ \\
\hline PIM- & TRANSMIT UPDATED PTS DATA BASE-PIAN INTEGRATION & K RI Rep & & $21 \mathrm{~N}$ \\
\hline esA- & SUBMIT OCT. MONTHLY SMS REPORT TO RL & K RI Rep & & 221 \\
\hline 04 & SUBMIT CRITICAI ITEMS REPORT TO RL MANAGERS & $\mathrm{R}$ Westin & table & 895 \\
\hline SA-S & SUBMIT OCT. MONTHLY PTS REPORT TO RI & K RI Rep & & $=95$ \\
\hline$S A-9$ & SUBMIT CRITICAI ITEMS REPORT TO RI MANAGERS & R Westin & portable & 081 \\
\hline PUB -9 & 2ND QUARTER ACTION PLAN COMPLETE & $\mathrm{R} W$ & le & \\
\hline PIM-S & 1.8.2 PLANNING INTEGRATION SMS PROGRAM REVIEW & K RI ReI & & $14 \mathrm{D}$ \\
\hline IM & SUBMIT NOVEMBER SMS REPORT & R Westin & le & 95 \\
\hline 75 & DATA QUALITY CHECK OF MONTHLY SMS PACKAGE & R Westil & & $: 95$ \\
\hline$I M$ & TRANSMIT UPDATED PTS DATA BASE-PLAN INTEGRATION & K RL ReE & & $=95$ \\
\hline SA- & SUBMIT NOV. MONTHLY SMS REPORT TO RL & K RL Rep & & 95 \\
\hline$S A-$ & SUBMIT CRITICAI ITEMS REPORT TO RI MANAGERS & R Westin & portable & 22 \\
\hline & SUBMIT NOV. MONTHLY PTS REPORT TO RI & K RI ReE & & 025 \\
\hline E & COMPLETE HMP BASELINE DEVELOPMENT & K RI Rep & & 02 \\
\hline PC-9 & SUBMIT DRAFT FY97-8 BLDG BLOCKS TO HQ & K RL Re & & 196 \\
\hline & SUBMIT CRITICAI ITEMS REPORT TO RL MANAGERS & $\mathbf{R} \mathbf{W}$ & & 196 \\
\hline & SUMMARY OE ACTION RLAN FOR 1ST QUARTER COMPLETE & 1se & $1 e$ & \\
\hline SSA-9 & SURVEILLANCE PLAN STATUS 1ST QTR & use & le & $15 i$ \\
\hline PIM-9 & IN PROCESS REVIEW & R Westinghouse & Reportable & $16 \mathrm{~J}$ \\
\hline PIM & 1.8 .2 PLANNING INTEGRATION SMS PROGRAM REVIEW & K RL Rep & & 296 \\
\hline PIM & SUBMIT DECEMBER SMS REPORT & R Westi & & \\
\hline PSA- & SUBMIT CRITICAI ITEMS REPORT TO RI MANAGERS & $\mathrm{R}$ & & n96 \\
\hline 03 & INTERNAI PROGRAM MANAGER REVIEW & $\mathrm{R} W \epsilon$ & portable & 296 \\
\hline & TRANSMIT UPDATED PTS DATA BASE-PLAN INTEGRATION & K RI & & 22 \\
\hline PSA & DATA QUALITY CHECK OE MONTHLY SMS PACKAGE & R Westin & portable & $24 i$ \\
\hline 1 & JBMIT DEC. MONTHLY SMS REPORT TO RI & K RL Rep & & 996 \\
\hline $\mathrm{PC}-9$ & JBLISH SUPPLEMENT TO HANEORD SITE FOCUS & K RL Rep & & 96 \\
\hline PIM-96 & DRAFT TO RL FOR REVIEW & K RL Rep & & 296 \\
\hline PSS-9 & ANALYSIS OF RISKS \& COSTS AT HANFORD & K RI Rep & & 196 \\
\hline 09 & SUBMIT CRITICAL ITEMS REPORT TO RI MANAGERS & R Westil & Dle & 02. \\
\hline & BMIT DEC. MONTHLY RTS REPORT TO RL & K RL Rep & & 696 \\
\hline$B I-96$ & RL ISSUE SITE DIRECT MYPP/FYWP GUIDANCE · & K RL Rep & & 96 \\
\hline$T M$ & 1.8.2 PLANNING INTEGRATION SMS PROGRAM REVIEW & K RI Rep & & \\
\hline PIM-9 & SUBMIT JANUARY SMS REPORT & R Westin & & 96 \\
\hline & SUBMIT CRITICAL ITEMS REPORT TO RI MANAGERS & $\mathrm{R}$ & & \\
\hline & SUBMIT DRAFT FY98-2002/97-98 BB ADS'S TO HQ/REGS & Repo & & 19 \\
\hline & DATA QUALITY CHECK OE MONTHLY SMS PACKAGE & R Westin & $\mathrm{R}$ & \\
\hline & RANSMIT URDATED PTS DATA BASE-PLAN INTEGRATION & K RI ReF & & 296 \\
\hline 00 & RATEGIC PLNNG-HSP EORMAL DRAFT COMPLETE & K RL Rep & & \\
\hline 52 & SUBMIT JAN. MONTHLY SMS REPORT TO RI & K RL Rep & & \\
\hline & COMPLETE ERDS SOFTWARE CONEIG CNTRI MGMT PLAN & R Westi & & \\
\hline & SUBMIT JAN. MONTHLY PTS REPORT TO RL & $\mathrm{K} R$ & & 011 \\
\hline & SUBMIT CRITICAL ITEMS REPORT TO RI MANAGERS & $\mathrm{R}$ Westin & & $=96$ \\
\hline & RLAN FOR 3RD QUARTER COMPLETE & K RL Rel & & $\mathbf{r 9 6}$ \\
\hline & 1.8.2 PLANNING INTEGRATION SMS PROGRAM REVIEW & K RI Rep & & 596 \\
\hline 028 & SUBMIT EEBRUARY SMS REPORT & $\mathrm{R}$ Westin & & \\
\hline 012 & SUBMIT CRITICAU ITEMS REPORT TO RL MANAGERS & R Westi & & \\
\hline & SUBMIT INTERNAL ADS REVIEW RPT TO RL & K RL. Rer & & 596 \\
\hline & DATA QUALITY CHECK OF MONTHLY SMS PACKAGE & R Westin & Reportable & $\operatorname{arg6}$ \\
\hline & RANSMIT UPDATED PTS DATA BASE-PLAN INTEGF & K RI Rep & & $\operatorname{arg6}$ \\
\hline & SUBMIT FEB. MONTHLY SMS REPORT TO RL & K RL Rep & & r96 \\
\hline & COMPLETE BASELINE EUNCTIONS, REV 1 & K RL Repox & & 196 \\
\hline & ISTRIB CALL LETTER REQUESTING FY 97 & & & \\
\hline & DMTT CDTTTCAT TTFMS RFPORT TO RT, MA & & & \\
\hline
\end{tabular}


Run Date 21Sep94 19:50

Milestone

SSE-96-008

PBI-96-008

PSA-96-041

P.BI $-96^{\circ}-002$

PIM-96-005

PSS $-96-201$

PUB-96-005

PSA-96-014

PIM-96-006

PSA-96-112

APC-96-007

APC-96-006

PIM-96-041

PIM-96-029

PIM-96-053

PSA-96-079

PSA-96-054

PSA-96-015

PSA-96-042

PSS-96-10I

PSA-96-016

PIM-96-042

PIM-96-030

PIM-96-054

P.SS-96-200

PSA-96-080

SSE-96-003

SSE-96-004

PSA-96-017

PSA-96-055

SSE-96-006

PSS-96-202

PSA-96-043

PUB-96-006

PSA-96-018

PIM-96-043

PSA-96-067

PIM-96-031

PBI-96-003

PIM-96-007

P.SA-96-081

PSA-96-056

PSA-96-019

PIM-96-055

PBI-96-009

PSA-96-044

PBI-96-004

PSA-96-020

PUB-96-007

PSS-96-205

PSA-96-113

PIM-96-008

PIM-96-044

PSA-96-021

PIM-96-032

PIM-96-056

PSA-96-082

PSA-96-057

PIBI-96-010

IPSA-96-068

IPBI-96-015

TPSA-96-045

RUB-96-008

PSA-96-022

PIM-96-045

PSA-96-023

PIM-96-033

PSS-96-203

PSA-96-083

PIM-96-057

PSA-96-058
Description

Type

Einish

COMPLETE SITE IMPLEMENTATION SELF ASSESSMENT

ISSUE SSPP CALL LETTER

SUBMIT EEB. MONTHLY PTS REPORT TO RI

ISSUE SITE DIRECT MYPP/FYWP CALL LETTER

COMPLETE ADS 7250

HIGH LEVEL PRIORITY LIST

SUMMARY OF ACTION PLAN FOR 2ND QUARTER COMPLETE

SUBMIT CRITICAL ITEMS REPORT TO RL MANAGERS

1997 MYWP/FYWP KICK OEF MEETING

SURVEILLANCE PLAN STATUS 2ND QTR

SUBMIT FINAL FY98-02 ADSS \& BLDG BLOCKS TO HQ

SUBMIT EINAL EY98-02 ADSS \& BLDG BLOCKS TO RI

1.8 .2 PLANNING INTEGRATION SMS PROGRAM REVIEW

SUBMIT MARCH SMS REPORT

TRANSMIT UPDATED PTS DATA BASE-PIAN INTEGRATION

DATA QUALITY CHECK OF MONTHLY SMS PACKAGE

SUBMIT MAR. MONTHLY SMS REPORT TO RI

SUBMIT CRITICAL ITEMS REPORT TO RL MANAGERS

SUBMIT MAR. MONTHLY PTS REPORT TO RL

STRATEGIC PLNNG-APPROVAL OF FY1997 HSP COMPIETE

SUBMIT CRITICAL ITEMS REPORT TO RI MANAGERS

1.8.2 PLANNING INTEGRATION SMS PROGRAM REVIEW

SUBMIT APRIL SMS REPORT

TRANSMIT UPDATED PTS DATA BASE-PIAN INTEGRATION

HMP-DRAFT RL WBS/OBS (THREE LEVELS) COMPLETE

DATA OUALITY CHECK OF MONTHLY SMS PACKAGE

COMPLETE REQUIREMENTS ANALYSIS \& ALLOC, REV 1

COMPLETE MEASURES OF EFEECTIVENESS ANALY, REV 1

SUBMIT CRITICAL ITEMS REPORT TO RL MANAGERS

SUBMIT APR. MONTHLY SMS REPORT TO RI

COMPLETE 200 AREA PLATEAU

REVISE, FINALZE \& TRANSMIT DRAFT FY97 HMP V-1

SUBMIT APR. MONTHLY PTS REPORT TO RL

PLAN FOR 4TH QUARTER COMPLETE

SUBMIT CRITICAL ITEMS REPORT TO RL MANAGERS

1.8.2 PLANNING INTEGRATION SMS PROGRAM REVIEW

FINALIZE DRAFT FY 97 PROPOSED WBS \& DISTRIBUTE

SUBMIT MAY SMS REPORT

SUBMIT REDLINED/MARKED UPDATE SITE FY 1997 MYPP

IN PROCESS REVIEW

DATA QUALITY CHECK OF MONTHLY SMS PACKAGE

SUBMIT MAY. MONTHLY SMS REPORT TO RL

SUBMIT CRITICAL ITEMS REPORT TO RL MANAGERS

TRANSMIT UPDATED PTS DATA BASE-PIAN INTEGRATION

SUBMIT REDIINED/MARKED UPDATE SITE FY97 SSPS RI

SUBMIT MAY. MONTHLY PTS REPORT TO RL

RL CONCUR REDLINED/MARKED UPDATE SITE FY97 MYPP SUBMIT CRITICAL ITEMS REPORT TO RL MANAGERS

SUMMARY OF ACTION PLAN FOR 3RD QUARTER COMPLETE

ISSUE WORKING PAPER DRFT FY96 HMP TO PRGS COMPT

SURVEILLANCE PIAN STATUS 3RD QTR

INTERNAI PROGRAM MANAGER REVIEW

1.8.2 PI.ANNING INTEGRATION SMS PROGRAM REVIEW

SUBMIT CRITICAL ITEMS REPORT TO RL MANAGERS

SUBMIT JUNE SMS REPORT

TRANSMIT UPDATED PTS DATA BASE-PLAN INTEGRATION

DATA QUALITY CHECK OE MONTHLY SMS PACKAGE

SUBMIT JUN. MONTHLY SMS REPORT TO RI

RL CONCUR REDLINED/MARKED UPDATE FY97 SSPS

COMPLETE FY 97 MICROCOMP STATEMENT OF STRATEGY

SUBMIT SITE MYPP/FYWP INTERNAL REV RPT TO RL

SUBMIT JUN. MONTHLY PTS REPORT TO RL

1.8.2 PUBLIC INVOLVEMENT GUIDANCE COMPLETE

SUBMIT CRITICAL ITEMS REPORT TO RL MANAGERS

1.8.2 PLANNING INTEGRATION SMS PROGRAM REVIEW

SUBMIT CRITICAI ITEMS REPORT TO RL MANAGERS

SUBMIT JULY SMS REPORT

COMPLETE FY1997 HANEORD MISSION PLAN, V-1

DATA QUALITY CHECK OF MONTHLY SMS PACKAGE

TRANSMIT UPDATED PTS DATA BASE-PLAN INTEGRATION

SUBMIT JUL. MONTHLY SMS REPORT TO RL
$R$ Westinghouse Reportable $R$ Westinghouse Reportable

K RL Reportable

$R$ Westinghouse Reportable

$R$ Westinghouse Reportable

K RL Reportable

$R$ Westinghouse Reportable

$R$ Westinghouse Reportable

$R$ Westinghouse Reportable

$R$ Westinghouse Reportable

M Major-HQ Reportable

K RL Reportable

$K$ RI Reportable

$R$ Westinghouse Reportable

K RL Reportable

$R$ Westinghouse Reportable

$K$ RL Reportable

R Westinghouse Reportable

K RL Reportable

K RL Reportable

$R$ Westinghouse Reportable

K RL Reportable

$R$ Westinghouse Reportable

K RL Reportable

K RL Reportable

$R$ Westinghouse Reportable

K RL Reportable

$K$ RL Reportable

$R$ Westinghouse Reportable

K RL Reportable

K RI Reportable

K RL Reportable

K RL Reportable

K RL Reportable

$R$ Westinghouse Reportable

K RL Reportable

K RL Reportable

$R$ Westinghouse Reportable

$R$ Westinghouse Reportable

$R$ Westinghouse Reportable

$R$ Westinghouse Reportable

$K$ RI Reportable

$R$ Westinghouse Reportable

K RI Reportable

K RL Reportable

K RI Reportable

$R$ Westinghouse Reportable

$R$ Westinghouse Reportable

K RI Reportable

$R$ Westinghouse Reportable

$R$ Westinghouse Reportable

K RL Reportable

$R$ Westinghouse Reportable

$R$ Westinghouse Reportable

K RL Reportable

$R$ Westinghouse Reportable

K RL Reportable

K RL Reportable

$R$ Westinghouse Reportable

K RL Reportable

K RI Reportable

K RL Reportable

$R$ Westinghouse Reportable

K RL Reportable

$R$ Westinghouse Reportable

$R$ Westinghouse Reportable

$K$ RI Reportable

$R$ Westinghouse Reportable

K RL Reportable

K RI Reportable
$R$ Westinghouse Reportable

$29 \operatorname{Mar} 96$

$29 \operatorname{Mar} 96$

01 Apr 96

01 Apr 96

08 Apr 96

10 Apr 96

12Apr 96

12 Apr 96

$12 \operatorname{Apr} 96$

15 Apr 96

15 Apr 96

15 Apr 96

18Apr 96

19Apr 96

22Apr 96

24 Apr 96

26 Apr 96

26 Apr 96

01 May 96

01 May 96

10 May 96

16 May 96

17 May 96

21 May 96

21 May 96

22 May 96

22 May 96

23 May 96

24 May 96

24 May 96

29 May 96

30 May 96

03Jun96

07Jun96

07Jun96

13Jun96

$14 J$ un 96

14 Jun 96

14 Jun96

19 Jun 96

19 Jun 96

21 Jun 96

21Jun96

21Jun96

28 Jun96

01Jul96

01Jul96

05Jul96

$12 \mathrm{Jul} 96$

12Jul96

15Jul 96

15Jul96

$18 J u I 96$

19JuI96

$19 J u 196$

22Jul96

24 Jul 96

26Jul96

29Jul96

$30 \mathrm{Jul} 96$

01 Aug 96

01Aug 96

02Aug 96

02 Aug 96

15 Aug 96

16 Aug 96

16 Aug 96

16 Aug 96

21 Aug 96

21 Aug 96

23Aug 96 
Run Date 21Sep94 19:50

\begin{tabular}{|c|c|c|c|c|}
\hline Milestone & Description & Type & & Einish \\
\hline $\begin{array}{r}J E-96-005 \\
P S A-96-024\end{array}$ & $\begin{array}{l}\text { COMPLETE REVISED ARCH \& PRODUCTS DEFIN, REV } 1 \\
\text { SUBMIT CRITICAL ITEMS REPORT TO RL MANAGERS }\end{array}$ & $\begin{array}{l}\text { K RL Reportable } \\
\text { R Westinghouse }\end{array}$ & Reportable & $\begin{array}{l}29 \text { Aug } 96 \\
30 \text { Aug96 }\end{array}$ \\
\hline $\begin{array}{l}\text { PSA-96-024 } \\
\text { PIM-96-009 }\end{array}$ & SUBMIT MYPP/FYWP TO /RL ، « & K RL Reportable & a & $\begin{array}{l}30 \text { Aug } 96 \\
30 \text { Aug } 96\end{array}$ \\
\hline PBI-96-011 & SUBMIT FY96 SITE SUPPT PROGPLANS TO RL & K RI Reportable & & 30 Aug 96 \\
\hline PBI-96-005 & SUBMIT SITE MYPP/FYWPS TO RL & K RL Reportable & & 30 Aug 96 \\
\hline PSA-96-046 & SUBMIT JUL. MONTHLY PTS REPORT TO RL & K RL Reportable & & 03 Sep96 \\
\hline PUB-96-009 & ACTION PLAN FOR IST QUARTER OE 1997 COMPLETE & R Westinghouse & Reportable & $09 \operatorname{sep} 96$ \\
\hline PIM-96-046 & 1.8 .2 PIANNING INTEGRATION SMS PROGRAM REVIEW & $K \cdot R L$ Reportable & & 12 Sep 96 \\
\hline RIM-96-034 & SUBMIT AUGUST SMS REPORT & R Westinghouse & Reportable & 13 Sep 96 \\
\hline PSA-96-025 & SUBMIT CRITICAL ITEMS REPORT TO RL MANAGERS & R Westinghouse & Reportable & $135 e p 96$ \\
\hline PBI-96-014 & PUBLISH LEVEL 0 SCHEDULE UPDATE. (MONTHLY) & $\mathrm{R}$ Westinghouse & Reportable & 13 Sep96 \\
\hline PSA-96-084 & DATA QUAIITY CHECK OF MONTHLY SMS RACKAGE & $\mathrm{R}$ Westinghouse & Reportable & 18 Sep 96 \\
\hline PSA-96-059 & SUBMIT AUG. MONTHLY SMS REPORT TO RL & K RL Reportable & & 20 Sep 96 \\
\hline PIM-96-058 & TRANSMIT UPDATED PTS DATA BASE-PLAN INTEGRATION & K RL Reportabile & & 23sep96 \\
\hline PSA-96-026 & SUBMIT CRITICAI ITEMS REPORT TO RI MANAGERS & R Westinghouse & Reportable & 27Sep96 \\
\hline PIM-96-010 & APPROVAI ON MYPP/FYWR AND WORK AUTHORIZATION & K RL Reportable & & 27Sep96 \\
\hline PBI-96-013 & PUBLISH HSIS MONTHLY STATUS & $\mathrm{R}$ Westinghouse & Reportable & 27Sep96 \\
\hline $96-117$ & UPDATE MASTER EROM CENTRAI MILESTON MODULE & R Westinghouse & Reportable & 30 sep96 \\
\hline PSA-96-118 & INPUT TO CENTRAL MILESTONE MODULE FORM $\mathrm{P} / \mathrm{X}$ & $\mathrm{R}$ Westinghouse & Reportable & 30 Sep 96 \\
\hline PSA-96-114 & SURVEILIAANCE PLAN STATUS 4TH QTR & $\mathrm{R}$ Westinghouse & Reportable & 30 Sep96 \\
\hline PSA-96-027 & COMPLETE USER TRAINING EOR SMS AUTOMATION APPLIC & $\mathrm{R}$ Westinghouse & Reportable & 30 Sep96 \\
\hline PSA-96-071 & DEPLOY AUTOMATED SMS ON HLAN & $\mathrm{R}$ Westinghouse & Repor & 30 Sep 96 \\
\hline PSA-96-030 & INTEREACE C.M.M. WITH PX SCHEDULING SYSTEM & R Westinghouse & Repos & 30 Sep 96 \\
\hline PSA-96-115 & WHC FY 96 MCS PROCEURES STATUS & $\mathrm{R}$ Westinghouse & Reportable & 30 sep 96 \\
\hline PSA-96-029 & CONTINUE GENERAI PROCESS IMPROVEMENTS TO C.M.M. & R Westinghouse & Reportable & 30 Sep96 \\
\hline IRA-96-001 & MILESTONE: IRA-96-001 & K RI Reportable & & 30 Sep 96 \\
\hline PSS-96-204 & HSA ADDENDUM III & K RL Reportable & & 30 Sep 96 \\
\hline PSS-96-301 & REPORT ON APPLICATION OE RISK BASED DECISIONS & K RI Reportable & & 30 sep 96 \\
\hline SSE-96-0II & COMPLETE AUDIT REPORT & K RI Reportable & & 30 Sep 96 \\
\hline PBI-96-007 & RL ISSUES EY96 WORK AUTHORIZATION LETTERS & K RL Reportable & & 30 Sep96 \\
\hline PBI-96-006 & RL APRROVAI OF SITE MYPP/EYWP & K RI Reportable & & 30 sep 96 \\
\hline SSE-96 & COMPLETE BASELINE SPECIFICATION & K RI Reportable & & 30 Sep 96 \\
\hline รE-96 & COMPLETE SITE IMPLEMENTATION ASSESSMENT REPORT & K RI Reportable & & 30 Sep 96 \\
\hline$I-96-012$ & SITE SUPPT PROG PLANS APPROVED BY RI & K RL Reportable & & 30 sep 96 \\
\hline SE-96-010 & COMPLETE SE TRAINING ASSESSMENT REPORT & K RL Reportable & & 30 Sep 96 \\
\hline PSA-97-035 & SUBMIT AUG. MONTHLY PTS REPORT TO.RL & K RI Reportable & & $010 \operatorname{ct} 96$ \\
\hline PSA-97-001 & SUBMIT CRITICAI ITEMS REPORT TO RL MANAGERS & $\mathrm{R}$ Westinghouse & Reportable & $110 c t 96$ \\
\hline PSA-97-110 & MCS SURVEILIANCE PLIAN & K RL Reportable & & $140 c t 96$ \\
\hline PUB-97-001 & SUMMARY OF ACTION PLAN FOR 4TH QUARTER OF 96 & R Westinghouse & ble & $140 c t 96$ \\
\hline $97-102$ & MCS \& PAR'S TRAINING SCHEDULE EOR EY 97 & use & Reportable & $150 c t 96$ \\
\hline PSA-96-116 & WHC EY 96 MCS PROCEURES DEVELOPMENT PLAN & stinghouse & Reportable & $160 c t 96$ \\
\hline PSA-97-116 & WHC EY 97 MCS PROCEDUR DEVELOPMENT PLAN & R Westinghouse & Reportable & $170 c t 96$ \\
\hline $97-035$ & 1.8.2 PLANNING INTEGRATION SMS PROGRAM REVIEW & K RL Reportable & & $170 c t 96$ \\
\hline $7-023$ & SUBMIT SEPTEMBER SMS REPORT & R Westinghouse & Reportable & $180 \operatorname{ctg} 6$ \\
\hline PIM-97-047 & TRANSMIT URDATED PTS DATA BASE-PLAN INTEGRATION & K RL Reportable & & $210 c t 96$ \\
\hline PSA-97-073 & DATA QUALITY CHECK OF NOMTHLY SMS PACKAGE & R Westin & Reporta & $230 c t 96$ \\
\hline PSA-97-048 & SUBMIT SEP. MONTHLY SMS REPORT TO RI & K RL Reportable & & $250 \operatorname{ct} 96$ \\
\hline PSA-97-002 & SUBMIT CRITICAI ITEMS REPORT TO RL MANAGERS & R Westinghouse & Reportable & $250 \operatorname{ct} 96$ \\
\hline $97-205$ & HMP-COMPILE WASTE DATA \& INTEREACES COMPLETE & K RL Reportable & & $250 c t 96$ \\
\hline $97-008$ & PUBLISH HANEORD SITE FOCUS & K RL Reportable & & $300 c t 96$ \\
\hline $97-036$ & SUBMIT SEP. MONTHLY PTS REPORT TO RI & K RI Reportable & & 01 Nov 96 \\
\hline $97-001$ & RECEIVE FY99-2003 ADS GUIDANCE FROM RI & K RI Repor & & 01 Nov9 6 \\
\hline PSA-97-003 & SUBMIT CRITICAL ITEMS REPORT TO RL MANAGERS & R Westing & Reportable & 08Nov96 \\
\hline APC-97-002 & ISSUE ADS GUIDANCE LETTER TO PROGRAMS & $\mathrm{R}$ Westinghouse & Rep & 13 Nov9 6 \\
\hline-001 & KICK OFE MEETING & R Westinghouse & Reportable & 14 Nov 96 \\
\hline $97-036$ & 1.8.2 PLANNING INTEGRATION SMS PROGRAM REVIEW & K RI Reportable & & 14 Nov 96 \\
\hline $97-024$ & SUBMIT OCTOBER SMS REPORT & $\mathrm{R}$ Westinghouse & Report & 15 Nov 96 \\
\hline $97-074$ & DATA QUAIITY CHECK OF NOMTHLY SMS PACKAGE & R Westinghouse & Reportable & 20Nov96 \\
\hline $97-048$ & TRANSMIT URDATED PTS DATA BASE-PIAN INTEGRATION & K RL Rep & & 21 Nov96 \\
\hline $97-004$ & SUBMIT CRITICAL ITEMS REPORT TO RI MANAGERS & R Westinghouse & Reportable & 22Nov96 \\
\hline $97-049$ & SUBMIT OCT. MONTHLY SMS REPORT TO RL & K RI Reportable & & 22 Nov 96 \\
\hline $97-200$ & HMP-DRAFT RI WBS/OBS (THREE LEVELS) COMPLETE & K RI Reportable & & 27 Nov 96 \\
\hline $97-037$ & SUBMIT OCT. MONTHLY PTS REPORT TO RL & K RI Reportable & & 02Dec 96 \\
\hline PUB-97-002 & 2ND QUARTER ACTION PLAN COMPLETE & R Westinghouse & & 06 Dec 96 \\
\hline PSA-97-005 & SUBMIT CRITICAI ITEMS REPORT TO RL MANAGERS & R Westinghouse & rtable & 06 Dec 96 \\
\hline IM-97-037 & 1.8 .2 PLANNING INTEGRATION SMS PROGRAM REVIEW & K RI Reportable & & 12Dec 96 \\
\hline$-97-075$ & DATA QUALITY CHECK OF NOMTHLY SMS PACKAGE & R Westinghouse & Reportable & 18Dec 96 \\
\hline & SUBMIT NOVEMBER SMS RERORT & R Westinghouse & Reportable & 20Dec 96 \\
\hline SA-97-050 & SUBMIT NOV. MONTHLY SMS REPORT TO RL & K RL Reportable & & 20Dec 96 \\
\hline PSA-97-006 & SUBMIT CRITICAL ITEMS REPORT TO RI MANAGERS & R Westingho & & 20Dec 96 \\
\hline $8-047$ & TRANSMIT URDATED PTS DATA BASE-PIAN INTEGRATION & K RL Report & & 20Dec 96 \\
\hline
\end{tabular}


Run Date 21Sep94 19:50

\section{Milestone Description}

PSA-97-038

SSE-97-001

PSA-97-007

APC $-97-003$

PUB-97-003

PIM-97-038

PSA-97-111

PIM-97-002

PSA-97-008

PIM-97-026

PIM-97-050

PIM-97-003

PSA-97-076

PSA-97-051

APC-97-009

PIM-97-004

PSS $-97-300$

PSA-97-009

PSA $-97-039$

PIM-97-039

PIM-97-027

PSA-97-010

PBI-97-001

PSA-97-077

APC-97-004

PSA-97-052

PIM-97-051

PSS-97-100

PSA-97-01I

PSA-97-069

PSA-97-040

PUB-97-004

PIM-97-040

PIM-97-028

PSA-97-012

APC-97-005

PSA-97-078

PSA-97-053

PIM-97-052

PSA-97-013

SSE-97-002

PSA-97-063

PBI-97-008

PSA-97-041

PBI-97-002

PIM-97-005

PSS-97-201

PUB-97-005

PSA-97-014

PIM-97-006

APC-97-007

APC-97-006

PIM-97-041

PSA-97-112

PIM-97-029

PIM-97-053

PSA-97-079

PSA-97-054

PSA-97-015

PSA-97-042

PSS-97-101

PSA-97-016

PIM-97-042

PIM-97-030

PIM-97-054

PSA-97-080

PSA-97-055

PSA-97-017

SSE-97-003

SSE-97-004

IRA-97-002
SUBMIT NOV. MONTHLY PTS REPORT TO RL

COMPLETE HMP BASELINE DEVELOPMENT

SUBMIT CRITICAI ITEMS REPORT TO RL MANAGERS

SUBMIT DRAET EY98-9 BLDG BLOCKS TO HQ

SUMMARY OF ACTION PLAN FOR IST QUARTER COMPLETE

1.8.2 PLANNING INTEGRATION SMS PROGRAM REVIEW

SURVEILIANCE PLAN STATUS FOR IST QTR

IN PROCESS REVIEW

SUBMIT CRITICAL ITEMS REPORT TO RL MANAGERS

SUBMIT DECEMBER SMS REPORT

TRANSMIT UPDATED PTS DATA BASE-PLAN INTEGRATION

INTERNAL COST ESTIMATING REVIEW

DATA QUAIITY CHECK OF NOMTHLY SMS PACKAGE

SUBMIT DEC. MONTHLY SMS REPORT TO RL

PUBLISH SUPPLEMENT TO HANFORD SITE FOCUS

DRAFT TO RL FOR REVIEW

COST-RISK-BENEFIT ANALYSIS UPDATE

SUBMIT CRITICAL ITEMS REPORT TO RL MANAGERS

SUBMIT DEC. MONTHLY PTS REPORT TO RL

1.8.2 PLANNING INTEGRATION SMS PROGRAM REVIEW

SUBMIT JANUARY SMS REPORT

SUBMIT CRITICAL ITEMS REPORT TO RL MANAGERS

RL ISSUE SITE DIRECT MYPP/EYWP GUIDANCE

DATA QUALITY CHECK OF NOMTHLY SMS PACKAGE

SUBMIT DRAFT FY99-2003/98-99 BB ADS's TO HQ/REGS

SUBMIT JAN. MONTHLY SMS REPORT TO RL

TRANSMIT UPDATED PTS DATA BASE-PLAN INTEGRATION

STRATEGIC PLNNG-HSP EORMAL DRAET COMPLETE

SUBMIT CRITICAL ITEMS REPORT TO RL MANAGERS

COMPLETE EPDS SOFTWARE CONFIG CNTRL MGMT PLAN

SUBMIT JAN. MONTHLY PTS REPORT TO RL

PLAN FOR 3RD QUARTER COMPLETE

1.8.2 PLANNING INTEGRATION SMS PROGRAM REVIEW

SUBMIT FEBRUARY SMS REPORT

SUBMIT CRITICAL ITEMS REPORT TO RL MANAGERS

SUBMIT INTERNAL ADS REVIEW RPT TO RI

DATA QUALITY CHECK OF NOMTHLY SMS PACKAGE

SUBMIT FEB. MONTHLY SMS REPORT TO RL

TRANSMIT UPDATED PTS DATA BASE-PLAN INTEGRATION

SUBMIT CRITICAL ITEMS REPORT TO RL MANAGERS

COMPLETE UPDATE FUNCTIONAL ANALYSIS, REV 2

DISTRIB CALL LETTER REQUESTING FY 98 PROPOSED WB

ISSUE SSPP CALI LETTER

SUBMIT FEB. MONTHLY PTS REPORT TO RL

ISSUE SITE DIRECT MYPP/EYWR CALI LETTER

COMPLETE ADS 7250

HIGH LEVEL PRIORITY LIST

SUMMARY OF ACTION PLAN FOR 2ND QUARTER COMPLETE

SUBMIT CRITICAL ITEMS REPORT TO RL MANAGERS

1998 MYPP/EYWP KICK OFF MEETING

SUBMIT FINAI FY99-03 ADSS \& BLDG BLOCKS TO HO

SUBMIT EINAL FY99-03 ADSS \& BLDG BLOCKS TO RL

1.8 .2 PLANNING INTEGRATION SMS PROGRAM REVIEW

SURVEILLANCE PLAN STATUS FOR 2ND QTR

SUBMIT MARCH SMS REPORT

TRANSMIT UPDATED PTS DATA BASE-PLAN INTEGRATION

DATA QUALITY CHECK OF NOMTHLY SMS PACKAGE

SUBMIT MAR. MONTHLY SMS REPORT TO RL

SUBMIT CRITICAI ITEMS REPORT TO RL MANAGERS

SUBMIT MAR. MONTHLY PTS REPORT TO RI

STRATEGIC PLNNG-APPROVAL OF FY1998 HSP COMPLETE

SUBMIT CRITICAI ITEMS REPORT TO RL MANAGERS

1.8 .2 PLANNING INTEGRATION SMS PROGRAM REVIEW

SUBMIT APRIL SMS REPORT

TRANSMIT UPDATED PTS DATA BASE-PLAN INTEGRATION

DATA QUALITY CHECK OE NOMTHLY SMS PACKAGE

SUBMIT APR. MONTHLY SMS REPORT TO RL

SUBMIT CRITICAL ITEMS REPORT TO RL MANAGERS

COMPLETE REQUIREMENTS ANALYSIS \& ALLOC, REV 2

COMPLETE MEASURES OF EFEECTIVENESS ANALY, REV 2

MILESTONE : IRA-97-003
Type

Finish

K RI Reportable

K RL Reportable

$R$ Westinghouse Reportable

K RL Reportable

$R$ Westinghouse Reportable

$K$ RL Reportable

$R$ Westinghouse Reportable

$R$ Westinghouse Reportable

$R$ Westinghouse Reportable

$R$ Westinghouse Reportable

K RI Reportable

$R$ Westinghouse Reportable

$R$ Westinghouse Reportable

K RI Reportable

K RL Reportable

K RI Reportable

K RI Reportable

$R$ Westinghouse Reportable

K RL Reportable

K RL Reportable

$R$ Westinghouse Reportable

$R$ Westinghouse Reportable

K RL Reportable

R Westinghouse Reportable

M Major-HQ Reportable

K RL Reportable

K RL Reportable

K RL Reportable

$R$ Westinghouse Reportable

$R$ Westinghouse Reportable

K RI Reportable

K RL Reportable

K RL Reportable

$R$ Westinghouse Reportable

R Westinghouse Reportable

K RL Reportable

R Westinghouse Reportable

K RL Reportable

K RL Reportable

$R$ Westinghouse Reportable

K RL Reportable

K RL Reportable

$R$ Westinghouse Reportable

K RL Reportable

$R$ Westinghouse Reportable

$R$ Westinghouse Reportable

$K$ RL Reportable

$R$ Westinghouse Reportable

$R$ Westinghouse Reportable

$R$ Westinghouse Reportable

M Major-HQ Reportable

K RL Reportable

K RL Reportable

$R$ Westinghouse Reportable

$R$ Westinghouse Reportable

K RL Reportable

$R$ Westinghouse Reportable

$K$ RI Reportable

$R$ Westinghouse Reportable

K RI Reportable

K RI Reportable

$R$ Westinghouse Reportable

K RL Reportable

$R$ Westinghouse Reportable

K RI Reportable

$R$ Westinghouse Reportable

K RI Reportable

$\mathrm{R}$ Westinghouse Reportable

K RI Reportable

K RI Reportable

K RL Reportable

$02 \operatorname{Jan} 97$

$02 \operatorname{Jan} 97$

$03 \mathrm{Jan} 97$

$06 \mathrm{Jan} 97$

$13 \mathrm{Jan} 97$

$16 \mathrm{Jan} 97$

$16 \operatorname{Jan} 97$

$16 \mathrm{Jan} 97$

$17 \operatorname{Jan} 97$

17Jan 97

21 Jan 97

$22 \operatorname{Jan} 97$

22 Jan 97

$24 \mathrm{Jan} 97$

$27 \operatorname{Jan} 97$

30Jan 97

$31 \operatorname{Jan} 97$

31 Jan 97

03 Feb97

13 Eeb 97

13 Feb 97

14 Feb97

14 Feb 97

19 Eeb97

19 Feb 97

21 Feb97

21 Feb97

21 Feb97

28 Feb 97

28 Feb 97

$03 \operatorname{Mar} 97$

07 Mar 97

$13 \operatorname{Mar} 97$

$14 \operatorname{Mar} 97$

$14 \operatorname{Mar} 97$

$17 \operatorname{Mar} 97$

19 Mar 97

21Mar97

$21 \operatorname{Mar} 97$

28 Mar 97

$28 \operatorname{Mar} 97$

31 Mar 97

31 Mar 97

01Apr 97

01Apr 97

07Apr 97

10Apr 97

11Apr 97

11Apr 97

14Apr 97

16Apr 97

16Apr 97

17 Apr 97

17Apr 97

18Apr97

21Apr 97

23Apr 97

25Apr 97

25Apr 97

01 May 97

02 May 97

09 May 97

15 May 97

16 May 97

21 May 97

21 May 97

23 May 97

23 May 97

23 May 97

23 May 97

30 May 97 
Run Date 21Sep94 19:50

Dilestone
RA-97-001 MILESTONE: IRA-97-002
PSA-97-043 SUBMIT ARR. MONTHLY PTS REPORT TO RI
PSA-97-018 SUBMIT CRITICAL ITEMS REPORT TO RI MANAGERS
PUB-97-006

PIM-97-043 1.8.2 PLANNING INTEGRATION SMS PROGRAM REVIEW

PSA-97-067 FINALIZE DRAET FY 98 PROPOSED WBS \& DISTRIBUTE

PBI-97-003 SUBMIT REDLINED/MARKED UPDATE SITE FY 1998 MYPP

PSA-97-081 DATA QUAIITY CHECK OE NOMTHLY SMS PACKAGE

PIM-97-007 IN PROCESS REVIEW

PIM-97-031 SUBMIT MAY SMS REPORT

PSA-97-056 SUBMIT MAY. MONTHLY SMS REPORT TO RL

PSA-97-019 SUBMIT CRITICAL ITEMS REPORT TO RL MANAGERS

PIM-97-055

EBI-97-009

PSA $-97-044$

PBI-97-004

PSA-97-020

PSS $-97-202$

RUB-97-007

PIM-97-008

RSS-97-205

PSA-97-113

RIM-97-044

PSA-97-021

PIM-97-032

PIM-97-056

PSA-97-082

PSA-97-057

PBI-97-010

PSA-97-068

PSA-9.7-045

PBI-97-015

IB-97-008

5A-97-022

PIM-97-045

PIM-97-033

PSA-97-023

PSS-97-203

PSA-97-083

PIM-97-057

ESA-97-058

PSA-97-024

PIM-97-009

SSE-97-006

RBI-97-011

SSE-97-005

PSA-97-046

PBI-97-005

PUB-97-009

PSA-97-025

PBI-97-014

PIM-97-046

PIM-97-034

PIM-97-058

PSA-97-084

PSA-97-026

PSA-97-059

PIM-97-010

RBI-97-013

PSA-97-115

PSA-97-114

SSE-97-007

PBI-97-007

PBI-97-012

PBI-97-006

'SA-97-030

(A-97-004

LRA-97-003

PSA-97-029

IRA-97-005

PSS-97-204
TRANSMIT UPDATED PTS DATA BASE-PLAN INTEGRATION

SUBMIT REDLINED/MARKED UPDATE SITE FY98 SSPS RL SUBMIT MAY. MONTHLY PTS REPORT TO RI

RL CONCUR REDLINED/MARKED UPDATE SITE FY98 MYPP SUBMIT CRITICAI ITEMS REPORT TO RL MANAGERS

REVISE, FNAIZE \& TRNSMT DRFT FY98 HMP V-1 COMPT SUMMARY OF ACTION PLAN EOR 3RD QUARTER COMPLETE INTERNAI COST AND SCHEDULE REVIEW

ISSUE WORKING PAPER DRET FY96 HMP TO PROGS COMPT SURVEILLANCE PLAN STATUS FOR 3RD QTR SUBMIT CRITICAI ITEMS REPORT TO RL MANAGERS SUBMIT JUNE SMS REPORT

TRANSMIT UPDATED PTS DATA BASE-PIAN INTEGRATION DATA QUALITY CHECK OE NOMTHLY SMS PACKAGE SUBMIT JUN. MONTHLY SMS REPORT TO RL

RI CONCUR REDLINED/MARKED UPDATE FY98 SSPS COMPLETE EY 98 MICROCOMP STATEMENT OF STRATEGY SUBMIT JUN. MONTHLY PTS REPORT TO RL SUBMIT SITE MYPP/FYWP INTERNAL REV RPT TO RL 1.8.2 PUBLIC INVOLVEMENT GUIDANCE COMPLETE SUBMIT CRITICAL ITEMS REPORT TO RL MANAGERS 1.8.2 PLANNING INTEGRATION SMS PROGRAM REVIEW SUBMIT JULY SMS REPORT

SUBMIT CRITICAL ITEMS REPORT TO RL MANAGERS COMPLETE EY1998 HANEORD MISSION PLAN, V-1 DATA QUALITY CHECK OF NOMTHLY SMS PACKAGE TRANSMIT UPDATED PTS DATA BASE-PLAN INTEGRATION SUBMIT JUL. MONTHLY SMS REPORT TO RL

SUBMIT CRITICAI ITEMS REPORT TO RL MANAGERS SUBMIT MYPP/EYWP TO RL

COMPLETE RESERVE CASE

SUBMIT FY96 SITE SUPPT PROGPLANS TO RL

COMPLETE REVISED ARCH \& PRODUCTS DEEIN, REV 2

SUBMIT JUL. MONTHLY PTS REPORT TO RL

SUBMIT SITE MYPR/FYWPS TO RI

ACTION RIAN FOR IST QUARTER OF 1997 COMPLETE

SUBMIT CRITICAI ITEMS REPORT TO RI MANAGERS

PUBIISH LEVEL O SCHEDULE UPDATE (MONTHLY)

1.8 .2 PLANNING INTEGRATION SMS PROGRAM REVIEW

SUBMIT AUGUST SMS REPORT

TRANSMIT UPDATED PTS DATA BASE-PLAN INTEGRATION

DATA QUALITY CHECK OF NOMTHLY SMS PACKAGE

SUBMIT CRITICAL ITEMS REPORT TO RI MANAGERS

SUBMIT AUG. MONTHLY SMS REPORT TO RI

APPROVAL ON MYPP/FYWR AND WORK AUTHORIZATION

PUBIISH HSIS MONTHLY STATUS

WHC FY 97 MCS PROCEDURS STATUS

SURVEILIANCE PLAN STATUS FOR 4TH QTR

COMPLETE BASELINE SPECIEICATION

RI ISSUES EY96 WORK AUTHORIZATION LETTERS

SITE SUPPT PROG PIANS APRROVED BY RL

RL APPROVAL OE SITE MYPP/FYWP

COMPLETE TRANSITION OE C.M.M. TO CLIENT SERVER

MILESTONE : IRA-97-005

COMPLETE PHASE III OE THE C.M.M.

MILESTONE: IRA-97-006

HSA ADDENDUM III

$2 F-11$
1.8.2 PLANNING INTEGRATION SMS PROGRAM REVIEW

MILESTONE : IRA-97-004
Type

Finish

K RL Reportable

K RI Reportable

$R$ Westinghouse Reportable:

K RI Reportable

K RL Reportable

K RL Reportable

$R$ Westinghouse Reportable

$R$ Westinghouse Reportable

$R$ Westinghouse Reportable

$R$ Westinghouse Reportable

K RI Reportable

$R$ Westinghouse Reportable

K RL Reportable

$R$ Westinghouse Reportable

K RI Reportable

K RL Reportable

$R$ Westinghouse Reportable

K RL Reportable

$R$ Westinghouse Reportable

$R$ Westinghouse Reportable

K RL Reportable

$R$ Westinghouse Reportable

K RI Reportable

$R$ Westinghouse Reportable

$R$ Westinghouse Reportable

K RL Reportable

$R$ Westinghouse Reportable

$K$ RL Reportable

K RL Reportable

R Westinghouse Reportable

K RI Reportable

K RI Reportable

K RL Reportable

$R$ Westinghouse Reportable

$K$ RL Reportable

$R$ Westinghouse Reportable

$R$ Westinghouse Reportable

K RL Reportable

$R$ Westinghouse Reportable

K RI Reportable

K RI Reportable

$R$ Westinghouse Reportable

K RL Reportable

K RL Reportable

K RI Reportable

K RL Reportable

K RI Reportable

K RI Reportable

$R$ Westinghouse Reportable

$R$ Westinghouse Reportable

$R$ Westinghouse Reportable

K RI Reportable

$R$ Westinghouse Reportable

K RI Reportable

$R$ Westinghouse Reportable

$R$ Westinghouse Reportable

$K$ RI Reportable

K RI Reportable

R Westinghouse Reportable

$R$ Westinghouse Reportable

$R$ Westinghouse Reportable

K RL Reportable

K RI Reportable

K RL Reportable

K RL Reportable

$R$ Westinghouse Reportable

K RI Reportable

K RI Reportable

$\mathrm{R}$ Westinghouse Reportable

K RL Reportable

K RI Reportable
30 May 97

$02 J u n 97$

$06 J u n 97$

$06 J u n 97$

12Jun97

16Jun97

16Jun 97

18Jun97

19Jun97

20Jun97

20Jun97

20Jun 97

23Jun97

30Jun97

01Jul97

01Jul97

03Jul97

08Jul 97

11Jul97

$14 \mathrm{Ju} 197$

$15 J u 197$

17Jul97

17Jul97

$18 J u 197$

$18 J u 197$

21Jul97

23Jul97

25Jul97

29Jul97

$30 \mathrm{Jul} 97$

01Aug 97

01Aug 97

01Aug 97

01 Aug 97

14 Aug 97

15Aug 97

15 Aug 97

19 Aug 97

20 Aug 97

21 Aug 97

22Aug 97

29Aug 97

29Aug 97

29Aug 97

29Aug 97

29Aug 97

02 Sep 97

02 Sep 97

09 Sep97

12 Sep97

15 Sep 97

18 Sep 97

19Sep97

22Sep 97

24 Sep 97

26 Sep 97

26Sep 97

26 Sep 97

29Sep97

29 Sep 97

29Sep 97

29 Sep 97

29Sep97

29Sep 97

29Sep97

30 Sep 97

30 sep 97

30 Sep 97

30 sep 97

30 Sep 97

30Sep 97 
Run Date 21Sep94 19:50

\begin{tabular}{|c|c|c|c|c|}
\hline Milestone & Description & & Type & Einish \\
\hline PSS $-97-301$ & REPORT ON RISK DECISIONS & & K RL Reportable & $30 \mathrm{~s}$ \\
\hline PSA $-97-072$ & COMPLETE EPDS PLATEORM UPGRADE & & R Westinghouse Reportable & 30 Sep 97 \\
\hline SSE-97-009 & COMPL FNL ASSESS RPT TO COMP \& & CLOSEOUT SE TRNG & K RL Reportable & 30 Sep 97 \\
\hline SSE-97-010 & COMPLETE AUDIT REPORT & & K RL Reportable & 30 sep 97 \\
\hline SSE-97-008 & COMPLETE SE PROCESS VALIDATION & REPORT & K RL Reportable & 30 Sep 97 \\
\hline$S S E-97-011$ & COMP INTEG OF SITE PROG \& PROJ & SE DATA MGT SYST. & K RL Reportable & 30 Sep97 \\
\hline
\end{tabular}


Planning Integration Multi-Year -Program Plan

Table 2.F-3. Milestone List by Milestone Control Number (9 pages) 
Run Date 21Sep94 19:52

Milestone

APC $-95-001$

APC-95-002

APC $-95-003$

APC-95-004

APC-95-005

APC-95-006

APC-95-007

APC-95-008

APC-95-009

APC-96-001

APC-96-002

APC $-96-003$

APC-96-004

APC-96-005

APC-96-006

APC-96-007

APC $-96-008$

APC $-96-009$

APC-97-001

APC $-97-002$

APC-97-003

APC-97-004

APC-97-005

APC $-97-006$

APC-97-007

APC-97-008

APC-97-009

IRA-95-001

IRA-95-002

IRA-95-003

IRA-96-001

IRA-97-001

IRA-97-002

IRA-97-003

IRA-97-004

IRA-97-005

PBI-95-001

PBI-95-002

PBI-95-003

PBI-95-004

PBI-95-005

PBI-95-006

PBI-95-007

PBI-95-008

PBI-95-009

PBI-95-010

PBI-95-011

PBI-95-012

PBI-95-013

PBI-95-014

PBI-95-015

PBI-96-001

PBI-96-002

PBI-96-003

PBI-96-004

PBI-96-005

PBI-96-006

IPBI-96-007

IPBI-96-008

IFBI-96-009

PBI-96-010

PBII-96-011

PBI-96-012

PBI-96-013

PBI-96-014

PBI-96-015

PBI-97-001

PBI-97-002

PBI-97-003

PBI-97-004

PBI-97-005
Description

RECEIVE FY97-2001 ADS GUIDANCE FROM RI

ISSUE ADS GUIDANCE LETTER TO PROGRAMS

SUBMIT DRAFT FY96-7 BLDG BLOCKS TO HQ

SUBMIT DRAFT FY97-2001 ADSs/96-97BB TO HQ/REGS

SUBMIT INTERNAL ADS REVIEW RPT TO RL

SUBMIT FINAL FY97-01 ADSs \& BLDG BLOCKS TO RL

SUBMIT EINAL EY97-01 ADSS \& BLDG BLOCKS TO HQ PUBLISH HANFORD SITE FOCUS

PUBLISH SUPPLEMENT TO HANFORD SITE FOCUS

RECEIVE FY98-2002 ADS GUIDANCE EROM RL

ISSUE ADS GUIDANCE LETTER TO PROGRAMS

SUBMIT DRAET FY97-8 BLDG BLOCKS TO HQ

SUBMIT DRAET FY98-2002/97-98 BB ADS'S TO HQ/REGS

SUBMIT INTERNAI ADS REVIEW RPT TO RL

SUBMIT FINAL FY98-02 ADSS \& BLDG BLOCKS TO RL

SUBMIT FINAL FY98-02 ADSS \& BLDG BLOCKS TO HQ

PUBLISH HANEORD SITE FOCUS

PUBLISH SUPRLEMENT TO HANFORD SITE FOCUS

RECEIVE FY99-2003 ADS GUIDANCE EROM RL

ISSUE ADS GUIDANCE LETTER TO PROGRAMS

SUBMIT DRAFT FY98-9 BLDG BLOCKS TO HQ

SUBMIT DRAET FY99-2003/98-99 BB ADS'S TO HQ/REGS

SUBMIT INTERNAL ADS REVIEW RPT TO RL

SUBMIT FINAL FY99-03 ADSS \& BLDG BLOCKS TO RL

SUBMIT FINAL FY99-03 ADSS \& BLDG BLOCKS TO HQ

PUBLISH HANEORD SITE FOCUS

PUBLISH SUPPLEMENT TO HANFORD SITE FOCUS

Integrated Risk Assessment Report

Public Health Baseline Risk Assessment Report

Integrated Risk Assessment Report

MILESTONE : IRA-96-001

MILESTONE : IRA-97-002

MILESTONE : IRA-97-003

MIIESTONE : IRA-97-004

MILESTONE : IRA-97-005

MIIESTONE : IRA-97-006

RI ISSUE SITE DIRECT MYPP/FYWP GUIDANCE

ISSUE SITE DIRECT MYPP/EYWP CALL LETTER

SUBMIT REDLINED/MARKED UPDATE SITE FY 1996 MYPP RL CONCUR REDLINED/MARKED UPDATE SITE FY96 MYPP SUBMIT SITE MYPP/EYWPS TO RL

RI APPROVAI OE SITE MYPP/EYWP

RL ISSUES FY96 WORK AUTHORIZATION LETTERS

ISSUE SSPP CALL LETTER

SUBMIT REDLINED/MARKED UPDATE SITE FY96 SSPS RL RL CONCUR REDLINED/MARKED UPDATE FY96 SSPS

SUBMIT FY96 SITE SUPPT PROGPIANS TO RL

SITE SUPPT PROG PLANS APPROVED BY RL

PUBLISH HSIS MONTHLY STATUS

PUBLISH LEVEL O SCHEDULE UPDATE (MONTHLY)

SUBMIT SITE MYPP/EYWP INTERNAL REV RPT TO RL

RL ISSUE SITE DIRECT MYPP/FYWP GUIDANCE

ISSUE SITE DIRECT MYPP/EYWP CALL LETTER

SUBMIT REDLINED/MARKED UPDATE SITE FY 1997 MYPP

RL CONCUR REDLINED/MARKED UPDATE SITE FY97 MYPP

SUBMIT SITE MYPP/FYWPS TO RL

RL APPROVAL OF SITE MYPP/EYWP

RL ISSUES FY96 WORK AUTHORIZATION LETTERS

ISSUE SSPP CAIL LETTER

SUBMIT REDLINED/MARKED UPDATE SITE FY97 SSPS RL RL CONCUR REDLINED/MARKED UPDATE FY97 SSPS

SUBMIT FY96 SITE SUPPT PROGPIANS TO RL

SITE SUPPT PROG PLANS ARPROVED BY RL

PUBLISH HSIS MONTHLY STATUS

PUBLISH LEVEL 0 SCHEDULE UPDATE (MONTHLY)

SUBMIT SITE MYPP/EYWP INTERNAI REV RPT TO RL

RL ISSUE SITE DIRECT MYPP/FYWP GUIDANCE

ISSUE SITE DIRECT MYPP/EYWP CALI LETTER

SUBMIT REDLINED/MARKED UPDATE SITE FY 1998 MYPP

RI CONCUR REDLINED/MARKED UPDATE SITE FY98 MYPP

SUBMIT SITE MYPP/EYWPS TO RL
Type

Finish

K RL Reportable

R Westinghouse Reportable

K RL Reportable

M Major-HQ Reportable

K RL Reportable

$K$ RL Reportable

M Major-HQ Reportable

K RL Reportable

K RL Reportable

K RI Reportable

R Westinghouse Reportable

K RL Reportable

M Major-HQ Reportable

K RL Reportable

K RL Reportable

M Major-HQ Reportable

K RL Reportable

K RL Reportable

$K$ RL Reportable

$R$ Westinghouse Reportable

K RL Reportable

M Major-HQ Reportable

K RL Reportable

K RL Reportable

M Major-HQ Reportable

K RL Reportable

K RL Reportable

K RI Reportable

K RL Reportable

K RL Reportable

K RL Reportable

K RL Reportable

K RI Reportable

K RL Reportable

K RL Reportable

K RL Reportable

K RL Reportable

$R$ Westinghouse Reportable

$R$ Westinghouse Reportable

K RL Reportable

K RI Reportable

K RI Reportable

K RL Reportable

$R$ Westinghouse Reportable

$R$ Westinghouse Reportable

K RI Reportable

K RI Reportable

K RL Reportable

$R$ Westinghouse Reportable

$R$ Westinghouse Reportable

$K$ RL Reportable

$K$ RI Reportable

$R$ Westinghouse Reportable

$R$ Westinghouse Reportable

K RL Reportable

K RL Reportable

K RL Reportable

K RL Reportable

$R$ Westinghouse Reportable

$R$ Westinghouse Reportable

K RL Reportable

K RI Reportable

$K$ RL Reportable

$R$ Westinghouse Reportable

$R$ Westinghouse Reportable

K RL Reportable

K RL Reportable

$R$ Westinghouse Reportable

$R$ Westinghouse Reportable

K RL Reportable

K RL Reportable

01 Nov 94

11 Nov94

10Jan95

17 Eeb95

17 Mar95

14 Apr 95

14Apr95

01 Nov94

27 Jan 95

01 Nov95

13 Nov 95

$05 J a n 96$

19 Feb96

$18 \operatorname{Mar} 96$

15Apr 96

15Apr 96

$310 c t 95$

29Jan96

01Nov96

13 Nov96

$06 \mathrm{Jan} 97$

$19 \mathrm{Feb} 97$

$17 \mathrm{Mar} 97$

16 Apr 97

16Apr97

300 ct 96

$27 \mathrm{Jan} 97$

30 May 95

29Sep95

29Sep95

30 Sep 96

उ0May 97

30 May 97

30sep9:

30 sep 97

30 Sep 97

15 Mar 95

31 Mar 95

15Jun 95

30 Jun 95

31 Aug 95

29Sep95

29Sep 95

31 Mar 95

30 Jun 95

31 Jul95

31 Aug 95

29 Sep95

29Sep95

15 Sep 95

01Aug 95

$15 \mathrm{Feb} 96$

01Apr 96

14 Jun96

01JuI96

30 Aug 96

30 sep96

30 Sep96

29 Mar 96

28 Jun96

29Jul96

30 Aug 96

30 Sep9 6

27Sep96

13 Sep 96

01Aug 96

$14 \mathrm{Eeb}^{\circ}$

01 Apr 97

16 Jun97

01Jul97

02 Sep 97 
Run Date 21Sep94 19:52

Milestone

I-97-006

PBI-97-007

PBI-97-008

PBI-97-009

PBI-97-010

PBI-97-011

PBI-97-012

PBI-97-013

PBI-97-014

PBI-97-015

PIM-95-001

PIM-95-002

PIM-95-003

PIM-95-004

PIM-95-005

PIM-95-006

PIM-95-007

PIM-95-008

RIM-95-009

PIM-95-010

PIM-95-023

PIM-95-024

PIM-95-025

PIM-95-026

PIM-95-027

PIM-95-028

PIM-95-029

PIM-95-030

PIM-95-031

PIM-95-032

PIM-95-033

PIM-95-034

-M-95-035

.M-95-036 rIM-95-037

RIM-95-038

PIM-95-039

PIM-95-040

PIM-95-041

PIM-95-042

PIM-95-043

PIM-95-044

PIM-95-045

PIM-95-046

PIM-95-047

PIM-95-048

PIM-95-049

PIM-95-050

PIM-95-051

PIM-95-052

PIM-95-053

PIM-95-054

PIM-95-055

PIM-95-056

PIM-95-057

PIM-95-058

PIM-96-001

PIM-96-002

PIM-96-003

PIM-96-004

PIM-96-005

PIM-96-006

PIM-96-007

PIM-96-008

PIM-96-009

SIM-96-010

M-96-023

M-96-024

PIM-96-025

PIM-96-026

PIM-96-027
Description

RL APPROVAL OF SITE MYPP/EYWP

RL ISSUES EY96 WORK AUTHORIZATION LETTERS

ISSUE SSPP CALL LETTER

SUBMIT REDLINED/MARKED UPDATE SITE FY98 SSPS RL RL CONCUR REDIINED/MARKED UPDATE FY98 SSPS

SUBMIT FY96 SITE SUPPT PROGPLANS TO RL

SITE SUPPT RROG PLANS APPROVED BY RI

PUBLISH HSIS MONTHLY STATUS

PUBLISH LEVEI 0 SCHEDULE UPDATE (MONTHLY)

SUBMIT SITE MYPP/EYWP INTERNAL REV RPT TO RL

KICK OEE MEETING

IN PROCESS REVIEW

INTERNAI PROGRAM MANAGER REVIEW

DRAET YO RL FOR REVIEW

COMPLETE ADS 7250

1996 MYPP/EYWP KICK OFE MEETING

IN PROCESS REVIEW

INTERNAI PROGRAM MANAGER REVIEW

SUBMIT MYPP/FYWP TO RL

APPROVAL ON MYPP/FYWP AND WORK AUTHORIZATION

SUBMIT SEPTEMBER SMS REPORI

SUBMIT OCTOBER SMS REPORT

SUBMIT NOVEMBER SMS REPORT

SUBMIT DECEMBER SMS REPORT

SUBMIT JANUARY SMS REPORT

SUBMIT FEBURARY SMS REPORT

SUBMIT MARCH SMS REPORT

SUBMIT APRIL SMS REPORT

SUBMIT MAY SMS REPORT

SUBMIT JUNE SMS REPORT

SUBMIT JULY SMS REPORT

SUBMIT AUGUST SMS REPORT

1.8.2 PLANNING INTEGRATION SMS PROGRAM REVIEW

1.8.2 PLANNING INTEGRATION SMS PROGRAM REVIEW

1.8.2 PLANNING INTEGRATION SMS PROGRAM REVIEW

1.8.2 PLANNING INTEGRATION SMS PROGRAM REVIEW

1.8.2 PLANNING INTEGRATION SMS PROGRAM REVIEW

1.8.2 PLANNING INTEGRATION SMS PROGRAM REVIEW

1.8 .2 PLANNING INTEGRATION SMS PROGRAM REVIEW

1.8.2 PLANNING INTEGRATION SMS PROGRAM REVIEW

1.8.2 RLANNING INTEGRATION SMS PROGRAM REVIEW

1.8.2 RLANNING INTEGRATION SMS PROGRAM REVIEW

1.8 .2 PLANNING INTEGRATION SMS PROGRAM REVIEW 1.8 .2 PLANNING INTEGRATION SMS PROGRAM REVIEW TRANSMIT UPDATED PTS DATA BASE-PIAN INTEGRATION TRANSMIT UPDATED PTS DATA BASE-PIAN INTEGRATION TRANSMIT UPDATED RTS DATA BASE-PLAN INTEGRATION TRANSMIT UPDATED PTS DATA BASE-PLAN INTEGRATION TRANSMIT UPDATED PTS DATA BASE-PLAN INTEGRATION TRANSMIT UPDATED PTS DATA BASE-PIAN INTEGRATION TRANSMIT UPDATED PTS DATA BASE-PIAN INTEGRATION TRANSMIT UPDATED PTS DATA BASE-PLAN INTEGRATION TRANSMIT UPDATED PTS DATA BASE-PILAN INTEGRATION TRANSMIT UPDATED PTS DATA BASE-PLAN INTEGRATION TRANSMTT UPDATED PTS DATA BASE-PIAN INTEGRATION TRANSMIT UPDATED PTS DATA BASE-PLAN INTEGRATION KICK OEF MEETING

IN PROCESS REVIEW

INTERNAL PROGRAM MANAGER REVIEW

DRAET TO RL FOR REVIEW

COMPLETE ADS 7250

1997 MYWP/EYWP KICK OFF MEETING

IN PROCESS REVIEW

INTERNAL PROGRAM MANAGER REVIEW

SUBMIT MYPE/EYWP TO RL

APPROVAL ON MYPP/EYWP AND WORK AUTHORIZATION

SUBMIT SEPTEMBER SMS REPORT

SUBMIT OCTOBER SMS REPORT

SUBMIT NOVEMBER SMS REPORT

SUBMIT DECEMBER SMS REPORT

SUBMIT JANUARY SMS REPORT
Type

Finish

K RL Reportable

K RL Reportable

$R$ Westinghouse Reportable

$R$ Westinghouse Reportable

K RL Reportable.

K RL Reportable

$K$ RI Reportable

$R$ Westinghouse Reportable

$R$ Westinghouse Reportable

K RL Reportable

$R$ Westinghouse Reportable

$R$ Westinghouse Reportable

$R$ Westinghouse Reportable

K RL Reportable

$R$ Westinghouse Reportable

$R$ Westinghouse Reportable

$R$ Westinghouse Reportable

$R$ Westinghouse Reportable

K RI Reportable

K RL Reportable

$R$ Westinghouse Reportable

$R$ Westinghouse Reportable

$R$ Westinghouse Reportable

$R$ Westinghouse Reportable

$R$ Westinghouse Reportable

$R$ Westinghouse Reportable

$R$ Westinghouse Reportable

$R$ Westinghouse Reportable

$R$ Westinghouse Reportable

$R$ Westinghouse Reportable

$R$ Westinghouse Reportable

$R$ Westinghouse Reportable

K RL Reportable

K RL Reportable

K RL Repartable

K RL Reportable

K RL Reportable

K RL Reportable

K RI Reportable

K RI Reportable

K RL Reportable

K RI Reportable

$K$ RI Reportable

K RL Reportable

K RI Reportable

K RL Reportable

K RL Reportable

$K$ RI Reportable

$K$ RI Reportable

K RL Reportable

K RL Reportable

$K$ RI Reportable

$K$ RI Reportable

K RL Reportable

K RI Reportable

K RI Reportable

$R$ Westinghouse Reportable

$R$ Westinghouse Reportable

$R$ Westinghouse Reportable

K RI Reportable

$R$ Westinghouse Reportable

$R$ Westinghouse Reportable

$R$ Westinghouse Reportable

$R$ Westinghouse Reportable

K RL Reportable

K RL Reportable

$R$ Westinghouse Reportable

$R$ Westinghouse Reportable

$R$ Westinghouse Reportable

$R$ Westinghouse Reportable

$R$ Westinghouse Reportable

$29 \operatorname{Sep} 97$

29Sep97

$31 \operatorname{Mar} 97$

30 Jun 97

29Jul.97

29Aug 97

$29 \operatorname{Sep} 97$

29Sep 97

15 Sep 97

01 Aug 97

14 Nov 94

16 Jan95

20 Jan95

30 Jan 95

07 Apr 95

14 Apr 95

19 Jun 95

$14 \mathrm{Jul} 95$

31 Aug 95

28Sep95

140 ct 94

18 Nov 94

16Dec 94

13 Jan95

17 Eeb95

17Mar95

14Apr95

19 May 95

16 Jun95

14 Jul 95

18 Aug 95

15 Sep95

130 ct 94

17 Nov94

15Dec 94

12Jan95

$16 \mathrm{Feb} 95$

$16 \mathrm{Mar} 95$

13 Apr 95

$18 \mathrm{May} 95$

15Jun95

$13 \mathrm{Ju} 195$

17 Aug 95

14 Sep95

210 ct 94

21Nov94

21Dec 94

23Jan 95

$22 \mathrm{Feb} 95$

$21 \mathrm{Mar} 95$

21 Apr 95

22 May 95

21Jun95

21Jul95

21 Aug 95

21Sep95

14 Nov 95

$16 \mathrm{Jan} 96$

22Jan96

$30 \mathrm{Jan} 96$

08Apr 96

12Apr96

19 Jun96

15Jul96

30 Aug 96

27Sep96

130 ct95

17 Nov 95

15Dec 95 
Run Date 21Sep94 19:52

\section{Milestone Description}

PIM-96-028 PIM-96-029

PIM-96-030

PIM-96-031

PIM-96-032

PIM-96-033

PIM-96-034

PIM-96-035

PIM-96-036

PIM-96-037

PIM-96-039

PIM-96-040

PIM-96-041

PIM-96-042

PIM-96-043

PIM-96-044

PIM-96-045

PIM-96-046

PIM-96-047

PIM-96-048

PIM-96-048

PIM-96-049

PIM-96-050

PIM-96-05I

PIM-96-052

PIM-96-053

PIM-96-054

PIM-96-055

PIM-96-056

PIM-96-057

PIM-96-058

PIM-97-001

PIM-97-002

PIM-97-003

PIM-97-004

PIM-97-005

PIM-97-006

PIM-97-007

PIM-97-008

PIM-97-009

PIM-97-010

PIM-97-023

PIM-97-024

PIM-97-025

PIM-97-026

PIM-97-027

PIM-97-028

PIM-97-029

PIM-97-030

PIM-97-031

PIM-97-032

PIM-97-033

PIM-97-034

PIM-97-035

PIM-97-036

PIM-97-037

PIM-97-038

PIM-97-039

PIM-97-040

PIM-97-041

PIM-97-042

PIM-97-043

PIM-97-044

PIM-97-045

PIM-97-046

PIM-97-047

PIM-97-048

PIM-97-049

PIM-97-050

PIM-97-051

PIM-97-052

\section{SUBMIT FEBRUARY SMS REPORT}

SUBMIT MARCH SMS REPORT

SUBMIT APRIL SMS REPORT

SUBMIT MAY SMS REPORT

SUBMIT JUNE SMS REPORT

SUBMIT JULY SMS REPORT

SUBMIT AUGUST SMS REPORT

1.8.2 PLANNING INTEGRATION SMS PROGRAM REVIEW

1.8.2 PLANNING INTEGRATION SMS PROGRAM REVIEW

1.8.2 PLANNING INTEGRATION SMS PROGRAM REVIEW

1.8.2 PLANNING INTEGRATION SMS PROGRAM REVIEW

1.8 .2 PLANNING INTEGRATION SMS PROGRAM REVIEW

1.8.2 PLANNING INTEGRATION SMS PROGRAM REVIEW

1.8.2 PLANNING INTEGRATION SMS PROGRAM REVIEW

1.8 .2 PLANNING INTEGRATION SMS PROGRAM REVIEW

1.8 .2 PLANNING INTEGRATION SMS PROGRAM REVIEW

1.8 .2 PLANNING INTEGRATION SMS PROGRAM REVIEW

1.8.2 PIANNING INTEGRATION SMS PROGRAM REVIEW

TRANSMIT UPDATED PTS DATA BASE-PIAN INTEGRATION

1.8.2 PLANNING INTEGRATION SMS PROGRAM REVIEW

TRANSMIT UPDATED PTS DATA BASE-PIAN INTEGRATION

TRANSMIT UPDATED PTS DATA BASE-PLAN INTEGRATION

TRANSMIT UPDATED PTS DATA BASE-PLAN INTEGRATION

TRANSMIT UPDATED PTS DATA BASE-PLAN INTEGRATION

TRANSMIT UPDATED PTS DATA BASE-PI.AN INTEGRATION

TRANSMIT UPDATED PTS DATA BASE-PLAN INTEGRATION TRANSMIT UPDATED PTS DATA BASE-PLAN INTEGRATION TRANSMIT UPDATED PTS DATA BASE-PLAN INTEGRATION TRANSMIT UPDATED PTS DATA BASE-PLAN INTEGRATION TRANSMIT UPDATED PTS DATA BASE-PLAN INTEGRATION TRANSMIT UPDATED PTS DATA BASE-PLAN INTEGRATION KICK OEF MEETING

IN PROCESS REVIEW

INTERNAL COST ESTIMATING REVIEW

DRAFT TO RL FOR REVIEW

COMPLETE ADS 72.50

1998 MYPP/FYWP KICK OFE MEETING

IN PROCESS REVIEW

INTERNAL COST AND SCHEDULE REVIEW

SUBMIT MYPP/EYWP TO RL

APPROVAL ON MYPP/FYWP AND WORK AUTHORIZATION

SUBMIT SEPTEMBER SMS REPORT

SUBMIT OCTOBER SMS REPORT

SUBMIT NOVEMBER SMS REPORT

SUBMIT DECEMBER SMS REPORT

SUBMIT JANUARY SMS REPORT

SUBMIT FEBRUARY SMS RERORT

SUBMIT MARCH SMS REPORT

SUBMIT APRIL SMS REPORT

SUBMIT MAY SMS REPORT

SUBMIT JUNE SMS REPORT

SUBMIT JULY SMS REPORT

SUBMIT AUGUST SMS REPORT

1.8.2 PLANNING INTEGRATION SMS PROGRAM REVIEW

1.8 .2 PIANNING INTEGRATION SMS PROGRAM REVIEW

1.8.2 PLANNING INTEGRATION SMS PROGRAM REVIEW

1.8.2 PLANNING INTEGRATION SMS PROGRAM RE்VIEW

1.8.2 PLANNING INTEGRATION SMS PROGRAM REVIEW

1.8.2 PLANNING INTEGRATION SMS PROGRAM REVIEW

1.8.2 PLANNING INTEGRATION SMS PROGRAM REVIEW

1.8.2 PIANNING INTEGRATION SMS PROGRAM REVIEW

1.8.2 PLANNING INTEGRATION SMS PROGRAM REVIEW

1.8 .2 PLANNING INTEGRATION SMS PROGRAM REVIEW

1.8.2 PLANNING INTEGRATION SMS PROGRAM REVIEW

1.8.2 PLANNING INTEGRATION SMS PROGRAM REVIEW

TRANSMIT UPDATED PTS DATA BASE-PILAN INTEGRATION

TRANSMIT UPDATED PTS DATA BASE-PLAN INTEGRATION TRANSMIT UPDATED PTS DATA BASE-PLAN INTEGRATION TRANSMIT UPDATED PTS DATA BASE-PLAN INTEGRATION TRANSMIT UPDATED PTS DATA BASE-PLAN INTEGRATION TRANSMIT UPDATED PTS DATA BASE-PLAN INTEGRATION
Type

Finish

$R$ Westinghouse Reportable $R$ Westinghouse Reportable $R$ Westinghouse Reportable $R$ Westinghouse Reportable $R$ Westinghouse Reportable $R$ Westinghouse Reportable R Westinghouse Reportable K RL Reportable

K RL Reportable

K RI Reportable

K RL Reportable

K RL Reportable

K RU Reportable

K RL Reportable

K RL Reportable

K RL Reportable

K RL Reportable

K RL Reportable

K RL Reportable

K RL Reportable

K RL Reportable

K RL Reportable

K RL Reportable

K RL Reportable

K RL Reportable

K RI Reportable

K RL Reportable

K RL Reportable

K RL Reportable

K RL Reportable

K RL Reportable

$R$ Westinghouse Reportable

$R$ Westinghouse Reportable

$R$ Westinghouse Reportable

$K$ RL Reportable

$R$ Westinghouse Reportable

$R$ Westinghouse Reportable

$R$ Westinghouse Reportable

$R$ Westinghouse Reportable

K RL Reportable

K RI Reportable

$R$ Westinghouse Reportable

$R$ Westinghouse Reportable

$R$ Westinghouse Reportable

$R$ Westinghouse Reportable

$R$ Westinghouse Reportable

$R$ Westinghouse Reportable

$R$ Westinghouse Reportable

$R$ Westinghouse Reportable

$R$ Westinghouse Reportable

$R$ Westinghouse Reportable

$R$ Westinghouse Reportable

$R$ Westinghouse Reportable

K RL Reportable

K RL Reportable

K RL Reportable

K RL Reportable

K RL Reportable

K RL Reportable

K RI Reportable

K RI Reportable

K RL Reportable

K RI Reportable

K RL Reportable

K RL Reportable

K RI Reportable

K RI Reportable

K RI Reportable

K RI Reportable

K RI Reportable

K RI Reportable

$15 \operatorname{Mar} 96$

19 Apr 96

17 May 96

14 Jun 96

19 Jul96

16 Aug 96

13 Sep96

$120 \mathrm{ct} 95$

16 Nov 95

14 Dec 95

15 Feb9 6

14 Mar 96

18 Apr 96

16 May 96

13 Jun 96

18 Jul96

15Aug 96

$12 \operatorname{Sep} 96$

230 ct 95

18 Jan 96

21 Nov 95

20Dec 95

22 Jan96

21 Feb96

21 Mar 96

22Apr 96

21 May 96

21Jun96

22Jul 96

21Aug 96

23 Sep 96

14 Nov9 6

$16 \mathrm{Jan} 97$

22Jan 97

$30 \mathrm{Jan} 97$

07 Apr 97

14 Apr 97

19Jun97

14 Jul 97

29 Aug 97

26 Sep 97

180 ct 96

15 Nov 96

20Dec 96

17 Jan 97

13 Feb 97

14 Mar 97

18Apr 97

16 May 97

20Jun97

18 Ju197

15Aug 97

19 Sep 97

$170 c t 96$

14 Nov 96

12Dec 96

$16 \mathrm{Jan} 97$

$13 \mathrm{Feb} 97$

$13 \mathrm{Mar} 97$

17 Apr 97

15 May 97

$12 J u n 97$

17Jul97

14 Aug 97

18 Sep97

210 ct 96

21 Nov9 6

20Dec 96

21Jan97

21 Feb 97 
Run Date 21Sep94 19:52

- $i$ itestome

Description

Type

Finish

$-1 \mathrm{M}-97-053$

PIM-97-054

PIM-97-055

PIM-97-056

PIM-97-057

PIM-97-058

PSA-95-001

PSA-95-002

PSA-95-003

PSA-95-004

PSA-95-005

PSA-95-006

PSA-95-007

PSA-95-008

PSA-95-009

PSA-95-010

PSA-95-011

PSA-95-012

PSA-95-013

PSA-95-014

PSA-95-015

PSA-95-016

PSA-95-017

PSA-95-018

PSA-95-019

PSA-95-020

PSA-95-021

RSA-95-022

PSA-95-023

PSA-95-024

PSA-95-025

PSA-95-026

A-95-029

A-95-035

PSA-95-036

PSA-95-037

PSA-95-038

PSA-95-039

PSA-95-040

PSA-95-041

PSA-95-042

PSA-95-043

RSA-95-044

PSA-95-045

PSA-95-046

RSA-95-048

PSA-95-049

PSA-95-050

PSA-95-051.

PSA-95-052

PSA-95-053

PSA-95-054

PSA-95-055

PSA-95-056

RSA-95-057

PSA-95-058

PSA-95-059

PSA-95-063

PSA-95-065

PSA-95-066

PSA-95-067

RSA-95-068

RSA-95-069

PSA-95-070

PSA-95-073

SA-95-074

A-95-075

-SA-95-076

PSA-95-077

PSA-95-078

RSA-95-079
TRANSMIT UPDATED PTS DATA BASE-PLAN INTEGRATION TRANSMIT UPDATED PTS DATA BASE-PLAN INTEGRATION TRANSMIT UPDATED PTS DATA BASE-PLAN INTEGRATION TRANSMIT URDATED PTS DATA BASE-PLAN INTEGRATION TRANSMIT UPDATED PTS DATA BASE-PLAN INTEGRATION TRANSMIT UPDATED PTS DATA BASE-PLAN INTEGRATION SUBMIT CRITICAL ITEMS REPORT TO RL MANAGERS SUBMIT CRITICAL ITEMS REPORT TO RL MANAGERS SUBMIT CRITICAL ITEMS REPORT TO RL MANAGERS SUBMIT CRITICAL ITEMS REPORT TO RL MANAGERS SUBMIT CRITICAI ITEMS REPORT TO RL MANAGERS SUBMIT CRITICAI ITEMS REPORT TO RL MANAGERS SUBMIT CRITICAL ITEMS REPORT TO RL MANAGERS SUBMIT CRITICAI ITEMS REPORT TO RI MANAGERS SUBMIT CRITICAL ITEMS REPORT TO RI MANAGERS SUBMIT CRITICAL ITEMS REPORT TO RL MANAGERS SUBMIT CRITICAI ITEMS REPORT TO RL MANAGERS SUBMIT CRITICAL ITEMS REPORT TO RL MANAGERS SUBMIT CRITICAL ITEMS REPORT TO RI MANAGERS SUBMIT CRITICAI ITEMS REPORT TO RL MANAGERS SUBMIT CRITICAI ITEMS REPORT TO RL MANAGERS SUBMIT CRITICAI ITEMS REPORT TO RL MANAGERS SUBMIT CRITICAL ITEMS REPORT TO RL MANAGERS SUBMIT CRITICAL ITEMS REPORT TO RL MANAGERS SUBMIT CRITICAL ITEMS REPORT TO RL MANAGERS SUBMIT CRITICAL ITEMS REPORT TO RL MANAGERS SUBMIT CRITICAI ITEMS REPORT TO RI MANAGERS SUBMIT CRITICAI ITEMS REPORT TO RL MANAGERS SUBMIT CRITICAI ITEMS REPORT TO RI MANAGERS SUBMIT CRITICAL ITEMS REPORT TO RI MANAGERS SUBMIT CRITICAL ITEMS REPORT TO RL MANAGERS SUBMIT CRITICAL ITEMS REPORT TO RI MANAGERS COMPLETE FY 96 MICROCOMP STATEMENT OF STRATEGY SUBMIT AUG. MONTHLY PTS REPORT TO RI SUBMIT SEP. MONTHLY PTS REPORT TO RI SUBMIT OCT. MONTHLY PTS REPORT TO RI SUBMIT NOV. MONTHLY PTS REPORT TO RI SUBMIT DEC. MONTHLY PTS REPORT TO RL SUBMIT JAN. MONTHLY RTS REPORT TO RL SUBMIT EEB. MONTHLY PTS REPORT TO RI SUBMIT MAR. MONTHLY PTS REPORT TO RI, SUBMIT ARR. MONTHLY PTS REPORT TO RI SUBMIT MAY. MONTHLY PTS REPORT TO RL SUBMIT JUN. MONTHLY PTS REPORT TO RI SUBMIT JUL. MONTHLY PTS REPORT TO RI SUBMIT SEP. MONTHLY SMS REPORT TO RI SUBMIT OCT. MONTHLY SMS REPORT TO RI SUBMIT NOV. MONTHLY SMS REPORT TO RL SUBMIT DEC. MONTHLY SMS REPORT TO RI SUBMIT JAN. MONTHLY SMS REPORT TO RL SUBMIT EEB. MONTHLY SMS REPORT TO RI SUBMIT MAR. MONTHLY SMS REPORT TO RL SUBMIT APR. MONTHLY SMS REPORT TO RI SUBMIT MAY MONTHLY SMS REPORT TO RL SUBMIT JUN. MONTHLY SMS REPORT TO RL SUBMIT JUL. MONTHLY SMS REPORT TO RL SUBMIT AUG. MONTHLY SMS REPORT TO RI DISTRIB CALI IETTER REQUESTING FY 96 PROPOSED WB COMPLETE GENERAL PROCESS IMPROVEMENTS TO C.M.M. COMPLETE TPA ARCHIVE AND PTS ENHANCEMENTS

FINALIZE DRAFT FY 96 PROPOSED WBS \& DISTRIBUTE DEVELOP C.M.M. AS REPIACEMENT FOR PTS MILESTONES COMPLETE ERDS SOETWARE CONEIG CNTRL MGMT PLAN ISSUE REP EOR CLIENT SERVER PROTOTYPE DATA QUALITY CHECK OF MONTHLY SMS PACKAGE DATA QUALITY CHECK OE MONTHLY SMS PACKAGE DATA QUALITY CHECK OE MONTHIY SMS PACKAGE DATA QUALITY CHECK OE MONTHLY SMS PACKAGE DATA QUALITY CHECK OF MONTHLY SMS PACKAGE DATA QUALITY CHECK OE MONTHLY SMS PACKAGE DATA QUALITY CHECK OF MONTHLY SMS PACKAGE
K RL Reportable

K RL Reportable

K RI'"Reportáble :"

K 'RI Reportiable

K RI Reportable

$K$ RI Reportable

$R$ Westinghouse Reportable

$R$ Westinghouse Reportable

$R$ Westinghouse Reportable

$R$ Westinghouse Reportable

$R$ Westinghouse Reportable

$R$ Westinghouse Reportable

$R$ Westinghouse Reportable

$R$ Westinghouse Reportable

$R$ Westinghouse Reportable

$R$ Westinghouse Reportable

$R$ Westinghouse Reportable

$R$ Westinghouse Reportable

$R$ Westinghouse Reportable

$R$ Westinghouse Reportable

$R$ Westinghouse Reportable

$R$ Westinghouse Reportable

$R$ Westinghouse Reportable

$R$ Westinghouse Reportable

$R$ Westinghouse Reportable

$R$ Westinghouse Reportable

$R$ Westinghouse Reportable

$R$ Westinghouse Reportable

$R$ Westinghouse Reportable

$R$ Westinghouse Reportable

$R$ Westinghouse Reportable

$R$ Westinghouse Reportable

$R$ Westinghouse Reportable

K RL Reportable

$K$ RL Reportable

K RL Reportable

K RI Reportable

K RI Reportable

K RI Reportable

K RI Reportable

K RI Reportable

K RL Reportable

K RL Reportable

K RI Reportable

K RI Reportable

K RL Reportable

K RL Reportable

K RI Reportable

K RI Reportable

K RL Reportable

K RI Reportable

K RI, Reportable

K RI Reportable

K RI Reportable

K RI Reportable

K RI, Reportable

K RI Reportable

K RI Reportable

$R$ Westinghouse Reportable

$R$ Westinghouse Reportable

K RI Reportable

$R$ Westinghouse Reportable $R$ Westinghouse Reportable $R$ Westinghouse Reportable $R$ Westinghouse Reportable $R$ Westinghouse Reportable $R$ Westinghouse Reportable $R$ Westinghouse Reportable $R$ Westinghouse Reportable $R$ Westinghouse Reportable $R$ Westinghouse Reportable

21Apr97

21 May 97

$23 J$ un 97

21Jul97

21Aug 97

22Sep97

140 ct 94

280 ct 94

11 Nov 94

25 Nov9 4

09Dec94

23Dec 94

06Jan95

20Jan95

03 Eeb95

17 Feb95

03Mar 95

17 Mar95

31 Mar 95

14 Apr 95

28Apr 95

$12 \mathrm{May} 95$

26 May 95

09Jun95

23Jun95

07JuI 95

21Ju195

04Aug 95

18Aug95

01 Sep95

15 Sep95

29 Sep 95

31Jul95

030ct 94

01Nov 94

01 Dec 94.

03Jan95

01 Eeb95

01Mar95

03 Apr 95

01 May 95

01 Jun95

03Jul95

01Aug 95

01 Sep95

210 ct 94

23 Nov 94

22Dec 94

20Jan 95

24 Feb95

24 Mar 95

21 Apr 95

26 May 95

23 Jun 95

21Jul95

25Aug 95

22Sep95

31 Mar95

17Apr 95

31 Aug 95

15 Jun95

14 Sep 95

28 Eeb95

2 9Sep 95

190 ct94

21 Nov9 4

21Dec 94

$18 \mathrm{Jan} 95$

22 Eeb 95

22Mar 95

19Apr95 
Run Date 21Sep94 19:52

Milestone Description

PSA-95-080 DATA QUALITY CHECK OE MONTHLY SMS PACKAGE PSA-95-081 DATA QUALITY CHECK OF MONTHLY SMS PACKAGE PSA-95-082 DATA QUALITY CHECK OF MONTHLY SMS PACKAGE PSA-95-083 DATA QUALITY CHECK OE MONTHLY SMS PACKAGE PSA-95-084 DATA QUALITY CHECK OF MONTHLY SMS PACKAGE PSA-95-100 DRAFT MANAGMENT CONTROL SYSTEMS DESCRIPTION PSA-95-101 EINAL MANAGMENT CONTROL SYSTEMS DESCRIPTION PSA-95-102 PSA-95-110 PSA-95-111 PSA-95-112 PSA-95-113 PSA-95-114 PSA-95-115 PSA-95-116 PSA-95-120 PSA-95-121 PSA-95-130 PSA-95-131 PSA-95-132 PSA-95-133 PSA-95-134 PSA-95-135 PSA-95-136 ESA-95-137 PSA-95-138 ISA-95-139 PSA-95-140 PSA-95-145 PSA-95-150 PSA-95-151 PSA-95-152 $+5 A-95-153$ ISA- 153 $15-95-155$ DSA-25-156 PSA-96-001 PSA-96-002 PSA-96-003 P.SA-96-004 BSA-96-005 PSA-96-006 PSA-96-007 P.SA-96-008 PSA-96-009 PSA-96-010 PSA-96-011 PSA-96-012 R.SA-96-013 PSA-96-014 ESA-96-015 PSA-96-016 PSA-96-017 PSA-96-018 RSA-96-019 ESA-96-020 PSA-96-021 PSA-96-022 IPSA-96-023 IPSA-96-024 IPSA-96-025 IPSA-96-026 PSA-96-027 P.SA-96-029 PSA-96-030 PSA-96-035 IRSA-96-036 IPSA-96-037 P.SA-96-038 IESA-96-039 P.SA-96-040
MCS \& PAR'S TRAINING SCHEDULE FOR EY 95 MCS SURVEILLANCE PLAN

SURVEILLANCE PLAN STATUS REPORT FOR IST QTR SURVEILIANCE PLAN STATUS REPORT FOR 2ND QTR SURVEILIANCE PLAN STATUS REPORT FOR 3RD QTR SURVEILIANCE PLAN STATUS REPORT FOR $4 T H$ QTR WHC FY95 MCS PROCEDURES DEVELOPMENT PLAN WHC FY95 MCS PROCEDURES STATUS BASEIING CHANGE CONTROL MODULE (PHASE 1) BASELING CHANGE CONTROL MODULE (PHASE 2) ISSUE JCS TO/EROM P/X SYSTEM REQUIREMENTS ISSUUE REVISED STANDARD SCH SYS (SSS) SCHEDULE IST QTR STATUS OE SSS IMPLEMENTATION 2ND OTR STATUS OF SSS IMPLEMENTATION 3RD QTR STATUS OF SSS IMPLEMENTATION $4 T H$ QTR STATUS OE SSS IMPLEMENTATION INPUT BCWP INTO $\mathrm{P} / \mathrm{X}$ FOR SCHEDULE STATUS REPORT OF IAABOR FROM $P / X$ PROGRAM $\mathrm{P} / \mathrm{X}$ SOETWARE CHANGES FOR JCS INPUT $\mathrm{P} / \mathrm{X}$ TO JCS SUPPORTING PROCEDURES

$P / X$ SOFTWARE CONFIGURATION MANAGEMENT PLAN SYSTEMS APPLICATION TRAINING BROCHURE PUBLISH DRAET REQ. DOC. FOR REENGINEERING ISSUE EINAL REQUIREMENTS DOCUMENT PROGRAM $P / X$ IN ACCORDANCE WITH SPECIEICATIONS

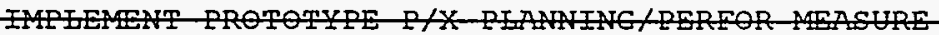

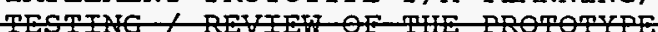
A A GENENT DEEESION ON IMPIEATETATION-SITE WIDE GO/NO GO SEIEGT PROGRAM IMPI ENT PY PIANAING/PERT SUBMIT CRITICAL ITEMS REPORT TO RL MANAGERS SUBMIT CRITICAL ITEMS REPORT TO RL MANAGERS SUBMIT CRITICAL ITEMS REPORT TO RL MANAGERS SUBMIT CRITICAL ITEMS REPORT TO RL MANAGERS SUBMIT CRITICAL ITEMS REPORT TO RL MANAGERS SUBMIT CRITICAL ITEMS REPORT TO RI, MANAGERS SUBMIT CRITICAL ITEMS REPORT TO RL MANAGERS SUBMIT CRITICAL ITEMS REPORT TO RL MANAGERS SUBMIT CRITICAL ITEMS REPORT TO RL MANAGERS SUBMIT CRITICAL ITEMS REPORT TO RL MANAGERS SUBMIT CRITICAL ITEMS REPORT TO RL MANAGERS SUBMIT CRITICAL ITEMS REPORT TO RL MANAGERS SUBMIT CRITICAL ITEMS REPORT TO RL MANAGERS SUBMIT CRITICAI ITEMS REPORT TO RL MANAGERS SUBMIT CRITICAL ITEMS REPORT TO RL MANAGERS SUBMIT CRITICAL ITEMS REPORT TO RL MANAGERS SUBMIT CRITICAL ITEMS REPORT TO RL MANAGERS SUBMIT CRITICAL ITEMS REPORT TO RL MANAGERS SUBMIT CRITICAL ITEMS REPORT TO RL MANAGERS SUBMIT CRITICAL ITEMS REPORT TO RL MANAGERS SUBMIT CRITICAL ITEMS REPORT TO RL MANAGERS SUBMIT CRITICAL ITEMS REPORT TO RL MANAGERS SUBMIT CRITICAL ITEMS REPORT TO RL MANAGERS SUBMIT CRITICAL ITEMS REPORT TO RL MANAGERS SUBMIT CRITICAL ITEMS REPORT TO RL MANAGERS SUBMIT CRITICAL ITEMS REPORT TO RL MANAGERS COMPLETE USER TRAINING FOR SMS AUTOMATION APPLIC CONTINUE GENERAI PROCESS IMPROVEMENTS TO C.M.M. INTEREACE C.M.M. WITH PX SCHEDULING SYSTEM SUBMIT AUG. MONTHLY PTS REPORT TO RL SUBMIT SEP. MONTHLY PTS REPORT TO RL SUBMIT OCT. MONTHLY PTS REPORT TO RL SUBMIT NOV. MONTHLY PTS REPORT TO RI SUBMIT DEC. MONTHLY PTS REPORT TO RI SUBMIT JAN. MONTHLY PTS REPORT TO RL
$R$ Westinghouse Reportable

$R$ Westinghouse Reportable

$R$ Westinghouse Reportable

$R$ Westinghouse Reportable

$R$ Westinghouse Reportable

$R$ Westinghouse Reportable

$K$ RL Reportable

$R$ Westinghouse Reportable

K RL Reportable

$R$ Westinghouse Reportable

$R$ Westinghouse Reportable

$R$ Westinghouse Reportable

$R$ Westinghouse Reportable

$R$ Westinghouse Reportable

$R$ Westinghouse Reportable

$R$ Westinghouse Reportable

$R$ Westinghouse Reportable

$R$ Westinghouse Reportable

$R$ Westinghouse Reportable

$R$ Westinghouse Reportable

$R$ Westinghouse Reportable

$R$ Westinghouse Reportable

$R$ Westinghouse Reportable

$R$ Westinghouse Reportable

$R$ Westinghouse Reportable

$R$ Westinghouse Reportable

$R$ Westinghouse Reportable

$R$ Westinghouse Reportable

$R$ Westinghouse Reportable

$R$ Westinghouse Reportable

$R$ Westinghouse Reportable

$R$ Westinghouse Reportable

$R$ West in Reportable

R-Wegtingouse-Reportable $R$ - Hestinghouse- Reportable $R$ Westinghouse Reportable

$R$ Westinghouse Reportable

$R$ Westinghouse Reportable

$R$ Westinghouse Reportable

$R$ Westinghouse Reportable

$R$ Westinghouse Reportable

$R$ Westinghouse Reportable

$R$ Westinghouse Reportable

$R$ Westinghouse Reportable

$R$ Westinghouse Reportable

$R$ Westinghouse Reportable

$R$ Westinghouse Reportable

$R$ Westinghouse Reportable

$R$ Westinghouse Reportable

$R$ Westinghouse Reportable

$R$ Westinghouse Reportable

$R$ Westinghouse Reportable

$R$ Westinghouse Reportable

$R$ Westinghouse Reportable

$R$ Westinghouse Reportable

$R$ Westinghouse Reportable

$R$ Westinghouse Reportable

$R$ Westinghouse Reportable

$R$ Westinghouse Reportable

$R$ Westinghouse Reportable

$R$ Westinghouse Reportable

$R$ Westinghouse Reportable

$R$ Westinghouse Reportable

$R$ Westinghouse Reportable

K RL Reportable

K RL Reportable

$K$ RL Reportable

K RL Reportable

K RL Reportable

K RL Reportable
Finish

24 May 95

21 Jun 95

19 Jul95

23 Aug 95

20Sep 95

16 Dec 94

$17 \mathrm{Mar} 95$

140 ct 94

140 ct 94

$16 \mathrm{Jan} 95$

17 Apr 95

17 Jul95

29Sep95

29Sep95

$170 c t 94$

30Dec 94

29Sep95

16 Jan 95

$140 c t 94$

$13 \mathrm{Jan} 95$

14 Apr 95

14 Jul 95

29 Sep 95

18 Nov 94

20Jan 95

10 Mar 95

14Apr 95

01 Jun 95

$140 \operatorname{ct} 94$

30Dec 94

11 Jan95

$21 \mathrm{Feb} 95$

21 Felog 5

10 10

$01140 y$

$304 a y 95$

130 ct 95

$270 c t 95$

10 Nov 95

24 Nov 95

08 Dec 95

22 Dec 95

05Jan96

19 Jan96

02 Feb96

16 Feb9 6

01 Mar 96

$15 \operatorname{Mar} 96$

29 Mar 96

12Apr 96

26Apr96

10 May 96

24 May 96

07 Jun96

21 Jun 96

05Jul96

19Jul96

02Aug 96

16 Aug 96

30 Aug 96

13 Sep 96

27 Sep 96

30 Sep 96

30 Sep 96

30 sep 96

02 Oct 95

01Nov95

01 Dec 95

02Jan96

02 Feb96

01 Mar 96 
Run Date 21Sep94 19:52

Milestone Description Type

Finish

ذA-96-041

BSA-96-042

PSA $-96-043$

PSA-96-044

PSA-96-045

RSA-96-046

PSA-96-048

PSA-96-049

RSA-96-050

PSA-96-051

PSA-96-052

PSA-96-053

PSA-96-054

RSA-96-055

PSA-96-056

PSA-96-057

PSA-96-058

PSA-96-059

PSA-96-063

PSA-96-0.67

BSA-96-068

PSA-96-069

PSA-96-071

PSA-96-073

PSA-96-074

PSA-96-075

PSA-96-076

PSA-96-077

PSA-96-078

PSA-96-079

PSA-96-080

PSA-96-081

:A-96-082

.A-96-083

PSA-96-084

PSA-96-102

PSA-96-110

PSA-96-111

PSA-96-112

PSA-96-113

PSA-96-114

PSA-96-115

PSA-96-116

PSA-96-117

BSA-96-118

PSA-97-001

PSA-97-002

PSA-97-003

PSA-97-004

PSA-97-005

PSA-97-006

PSA-97-007

PSA-97-008

PSA-97-009

PSA-97-010

PSA-97-011

PSA-97-012

PSA-97-013

PSA-97-014

PSA-97-015

PSA-97-016

PSA-97-017

PSA-97-018

PSA-97-019

PSA-97-020

7 SA-97-021

'A-97-022

-SA-97-023

PSA-97-024

PSA-97-025

PSA-97-026
SUBMIT FEB. MONTHLY PTS REPORT TO RI SUBMIT MAR. MONTHLY PTS REPORT TO RL SUBMIT ARR. MONTHLY, PTS REPORT TO RL SUBMIT MAY: MONTHLY PTS REPORT TO RL SUBMIT JUN. MONTHLY PTS REPORT TO RL SUBMIT JUL. MONTHLY PTS REPORT TO RL SUBMIT SEP. MONTHLY SMS REPORT TO RI SUBMIT OCT. MONTHLY SMS REPORT TO RL SUBMIT NOV. MONTHLY SMS REPORT TO RL SUBMIT DEC. MONTHLY SMS REPORT TO RL SUBMIT JAN. MONTHLY SMS REPORT TO RL SUBMIT FEB. MONTHLY SMS REPORT TO RL SUBMIT MAR. MONTHLY SMS REPORT TO RL SUBMIT ARR. MONTHLY SMS REPORT TO RL SUBMIT MAY. MONTHLY SMS REPORT TO RL SUBMIT JUN. MONTHLY SMS REPORT TO RL SUBMIT JUL. MONTHLY SMS REPORT TO RL SUBMIT AUG. MONTHLY SMS REPORT TO RL DISTRIB CAIL LETTER REQUESTING FY 97 RROPOSED WB FINALIZE DRAET FY 97 PROPOSED WBS \& DISTRIBUTE COMPLETE FY 97 MICROCOMP STATEMENT OF STRATEGY COMPLETE EPDS SOETWARE CONEIG CNTRL MGMT PLAN DERLOY AUTOMATED SMS ON HLAN

DATA QUALITY CHECK OF MONTHLY SMS PACKAGE DATA QUALITY CHECK OF MONTHLY SMS PACKAGE DATA QUALITY CHECK OF MONTHLY SMS PACKAGE DATA QUALITY CHECK OF MONTHLY SMS PACKAGE DATA QUALITY CHECK OF MONTHLY SMS PACKAGE DATA QUAIITY CHECK OF MONTHLY SMS PACKAGE DATA QUAIITY CHECK OF MONTHLY SMS PACKAGE DATA QUALITY CHECK OF MONTHLY SMS PACKAGE DATA QUALITY CHECK OF MONTHLY SMS PACKAGE DATA QUALITY CHECK OF MONTHLY SMS PACKAGE DATA QUALITY CHECK OF MONTHLY SMS PACKAGE DATA QUALITY CHECK OE MONTHLY SMS PACKAGE MCS \& PAR'S TRAINING SCHEDULE FOR FY 96 MCS SURVEILI.ANCE PI.AN

SURVEILLANCE PLAN STATUS 1ST QTR SURVEILLANCE PLAN STATUS 2ND QTR SURVEILIANCE PIAN STATUS 3RD QTR SURVEILLANCE PLAN STATUS 4TH QTR WHC FY 96 MCS PROCEURES STATUS

WHC FY 96 MCS PROCEURES DEVELOPMENT PIAN URDATE MASTER EROM CENTRAL MILESTON MODULE INPUT TO CENTRAI MILESTONE MODULE FORM $\mathrm{P} / \mathrm{X}$ SUBMIT CRITICAL ITEMS REPORT TO RL MANAGERS SUBMIT CRITICAL ITEMS REPORT TO RL MANAGERS SUBMIT CRITICAI ITEMS REPORT TO RL MANAGERS SUBMIT CRITICAI ITEMS REPORT TO RL MANAGERS SUBMIT CRITICAI ITEMS REPORT TO RI MANAGERS SUBMIT CRITICAL ITEMS REPORT TO RL MANAGERS SUBMIT CRITICAI ITEMS REPORT TO RL MANAGERS SUBMIT CRITICAI ITEMS REPORT TO RL MANAGERS SUBMIT CRITICAL ITEMS REPORT TO RL MANAGERS SUBMIT CRITICAL ITEMS REPORT TO RI MANAGERS SUBMIT CRITICAI ITEMS REPORT TO RI MANAGERS SUBMI'T CRITICAL ITEMS REPORT TO RL MANAGERS SUBMIT CRITICAI ITEMS REPORT TO RL MANAGERS SUBMIT CRITICAL ITEMS REPORT TO RI MANAGERS SUBMIT CRITICAL ITEMS REPORT TO RL MANAGERS SUBMIT CRITICAI ITEMS REPORT TO RL MANAGERS SUBMIT CRITICAI ITEMS REPORT TO RL MANAGERS SUBMIT CRITICAL ITEMS REPORT TO RL MANAGERS SUBMIT CRITICAL ITEMS REPORT TO RL MANAGERS SUBMIT CRITICAI ITEMS REPORT TO RL MANAGERS SUBMIT CRITICAL ITEMS REPORT TO RI MANAGERS SUBMIT CRITICAL ITEMS REPORT TO RL MANAGERS SUBMIT CRITICAI ITEMS REPORT TO RI MANAGERS SUBMIT CRITICAL ITEMS REPORT TO RL MANAGERS SUBMIT CRITICAL ITEMS REPORT TO RI MANAGERS SUBMIT CRITICAL ITEMS REPORT TO RL MANAGERS
K RL Reportable

K RL Reportable

K RL Reportable

K RL Reportảbile

K RL Reportable

K RL Reportable

K RL Reportable

K RI Reportable

K RI Reportable

K RL Reportable

K RL Reportable

K RL Reportable

K RL Reportable

K RL Reportable

K RL Reportable

K RL Reportable

$K$ RI Reportable

K RL Reportable

K RL Reportable

K RL Reportable

$R$ Westinghouse Reportable

$R$ Westinghouse Reportable

$R$ Westinghouse Reportable

$R$ Westinghouse Reportable

$R$ Westinghouse Reportable

$R$ Westinghouse Reportable

$R$ Westinghouse Reportable

$R$ Westinghouse Reportable

$R$ Westinghouse Reportable

$R$ Westinghouse Reportable

$R$ Westinghouse Reportable

$R$ Westinghouse Reportable

$R$ Westinghouse Reportable

$R$ Westinghouse Reportable

$R$ Westinghouse Reportable

$R$ Westinghouse Reportable

K RI Reportable

$R$ Westinghouse Reportable

$R$ Westinghouse Reportable

$R$ Westinghouse Reportable

$R$ Westinghouse Reportable

$R$ Westinghouse Reportable

$R$ Westinghouse Reportable

$R$ Westinghouse Reportable

$R$ Westinghouse Reportable

$R$ Westinghouse Reportable

$R$ Westinghouse Reportable

$R$ Westinghouse Reportable

$R$ Westinghouse Reportable

$R$ Westinghouse Reportable

$R$ Westinghouse Reportable

$R$ Westinghouse Reportable

$R$ Westinghouse Reportable

$R$ Westinghouse Reportable

$R$ Westinghouse Reportable

$R$ Westinghouse Reportable

$R$ Westinghouse Reportable

$R$ Westinghouse Reportable

$R$ Westinghouse Reportable

$R$ Westinghouse Reportable

$R$ Westinghouse Reportable

$R$ Westinghouse Reportable

$R$ Westinghouse Reportable

$R$ Westinghouse Reportable

$R$ Westinghouse Reportable

$R$ Westinghouse Reportable

$R$ Westinghouse Reportable

$R$ Westinghouse Reportable

$R$ Westinghouse Reportable

$R$ Westinghouse Reportable

$R$ Westinghouse Reportable

01 Apr 96

01 May 96

03Jun96

01Jul96

01 Aug 96

03 Sep96

270 ct95

22 Nov95

22Dec 95

$26 \operatorname{Jan} 96$

23 Feb96

$22 \operatorname{Mar} 96$

26 Apr 96

24 May 96

21Jun 96

26Jul96

23 Aug 96

20 Sep96

$29 \operatorname{Mar} 96$

$14 J u n 96$

$30 J u 196$

28 Feb 96

30 Sep 96

$250 c t 95$

20 Nov 95

20Dec 95

24 Jan96

21 Feb 96

20 Mar 96

24 Apr 96

22May96

$19 J u n 96$

24 Jul 96

21Aug 96

18 Sep96

160 ct 95

130 ct 95

15Jan96

15Apr 96

15Jul96

30 Sep 96

30 Sep 96

160 ct 96

30 Sep 96

30 Sep96

110 ct 96

250ct 96

08Nov96

22Nov96

06Dec 96

20Dec 96

$03 \mathrm{Jan} 97$

$17 \mathrm{Jan} 97$

31 Jan 97

14 Feb 97

28 Feb 97

$14 \mathrm{Mar} 97$

28Mar 97

11 Apr 97

25Apr 97

09 May 97

23 May 97

$06 J u n 97$

20Jun97

03Jul97

18 Jul97

01 Aug 97

15 Aug 97

29Aug 97

12 Sep 97

$265 e p 97$ 


\section{Milestone}

PSA-97-029 PSA-97-030 PSA-97-035 PSA $-97-036$ PSA $-97-037$ PSA-97-038 PSA-97-039 PSA-97-040 PSA-97-041 PSA-97-042 PSA-97-043 PSA-97-044 PSA-97-045 PSA-97-046 PSA $-97-048$ PSA-97-049 PSA-97-050 PSA-97-051 PSA-97-052 PSA-97-053 PSA-97-054 PSA-97-055 PSA-97-056 PSA-97-057 PSA-97-058 PSA-97-059 PSA-97-063 PSA-97-067 PSA-97-068 PSA-97-069 PSA-97-072 PSA-97-073 PSA-97-074 PSA-97-075 PSA-97-076 PSA-97-077 PSA-97-078 PSA-97-079 PSA-97-080 PSA-97-081 PSA-97-082 PSA-97-083 PSA-97-084 PSA-97-102 PSA-97-110 PSA-97-111 PSA-97-112 PSA-97-113 PSA-97-114 PSA-97-115 PSA-97-116 PSS-95-100 PSS-95-101 PSS-95-200 PSS-95-201 PSS-95-202 PSS-95-203 PSS-95-204 PSS- $95-205$ PSS-95-300 PSS-95-301 PSS-95-302 PSS-95-304 PSS-95-305 PSS-96-100 PSS-96-101 PSS-96-200 PSS-96-201 PSS-96-202 PSS-96-203
Description

COMPLETE PHASE III OF THE C.M.M. COMPIETE TRANSITION OF C.M.M. TO CLIENT SERVER SUBMIT AUG. MONTHLY PTS REPORT TO RL SUBMIT SEP. MONTHLY PTS REPORT TO RL SUBMIT OCT. MONTHLY PTS REPORT TO RL SUBMIT NOV. MONTHLY PTS REPORT TO RL SUBMIT DEC. MONTHLY PTS REPORT TO RL SUBMIT JAN. MONTHLY PTS REPORT TO RL SUBMIT FEB. MONTHLY PTS REPORT TO RL SUBMIT MAR. MONTHLY PTS REPORT TO RL SUBMIT APR. MONTHLY PTS REPORT TO RL SUBMIT MAY. MONTHLY PTS REPORT TO RL SUBMIT JUN, MONTHLY PTS REPORT TO RL SUBMIT JUL. MONTHLY PTS REPORT TO RI SUBMIT SEP. MONTHLY SMS REPORT TO RL SUBMIT OCT. MONTHLY SMS REPORT TO RL SUBMIT NOV. MONTHLY SMS REPORT TO RL SUBMIT DEC. MONTHLY SMS REPORT TO RL SUBMIT JAN. MONTHLY SMS REPORT TO RL SUBMIT FEB. MONTHLY SMS REPORT TO RL SUBMIT MAR. MONTHLY SMS REPORT TO RL SUBMIT ARR. MONTHLY SMS REPORT TO RI SUBMIT MAY. MONTHLY SMS REPORT TO RL SUBMIT JUN. MONTHLY SMS REPORT TO RI SUBMIT JUL. MONTHLY SMS REPORT TO RL SUBMIT AUG. MONTHLY SMS REPORT TO RL DISTRIB CALL IETTER REQUESTING FY 98 PROPOSED WB FINAIIZE DRAFT FY 98 PROPOSED WBS \& DISTRIBUTE COMPLETE FY 98 MICROCOMP STATEMENT OF STRATEGY COMPLETE EPDS SOETWARE CONFIG CNTRL MGMT PLAN COMPLETE EPDS PLATFORM UPGRADE

DATA QUALITY CHECK OF NOMTHLY SMS PACKAGE DATA QUALITY CHECK OE NOMTHLY SMS PACKAGE DATA QUALITY CHECK OE NOMTHLY SMS PACKAGE DATA QUALITY CHECK OE NOMTHLY SMS PACKAGE DATA QUAIITY CHECK OE NOMTHLY SMS PACKAGE DATA QUAIITY CHECK OE NOMTHLY SMS PACKAGE DATA QUALITY CHECK OF NOMTHLY SMS PACKAGE DATA QUALITY CHECK OE NOMTHLY SMS PACKAGE DATA QUALITY CHECK OE NOMTHLY SMS PACKAGE DATA QUALITY CHECK OF NOMTHLY SMS PACKAGE DATA QUALITY CHECK OE NOMTHLY SMS PACKAGE DATA QUALITY CHECK OF NOMTHLY SMS PACKAGE MCS \& PAR'S TRAINING SCHEDULE FOR FY 97

MCS SURVEILLANCE PLAN

SURVEILLANCE PLAN STATUS FOR IST QTR SURVEILIANCE PIAN STATUS FOR 2ND QTR SURVEILIANCE PIAN STATUS FOR 3RD QTR SURVEILIANCE PLAN STATUS FOR $4 T H$ QTR WHC FY 97 MCS PROCEDURS STATUS

WHC FY 97 MCS PROCEDUR DEVELOPMENT PLAN

STRATEGIC PLNNG-HSP FORMAL DRAFT COMPLETE STRATEGIC PLNNG-APPROVAL OE FYI996 HSP COMPLETE HMP-DRAET RL WBS/OBS (THREE LEVELS) COMPLETE HIGH LEVEI PRIORITY IIST

REVSE, FINALZE \& TRANSMT DRFT FY96 HMP V-1 COMPT COMPLETE FY1996 HANEORD MISSION PLAN, V-I

HSA ADDENDUM II

ISSUE WORKING PAPER DRAFT FY96 HMP TO PROGS IMPLEMENT PRIORITIZATION METHODOLOGY COMPT COST-RISK-BENEEIT ANALYSIS COMPLETE

DETERMINE RISK PERCEPTIONS COMPLETE

RISK MGMT IN SE

SITE-WIDE RISK ACTIVITIES COMPLETE

STRATEGIC PLNNG-HSP FORMAI DRAFT COMPLETE

STRATEGIC PLNNG-APRROVAI OF FY1997 HSP COMPLETE HMP-DRAFT RL WBS/OBS (THREE LEVELS) COMPIETE HIGH LEVEL PRIORITY LIST

REVISE, EINALZE \& TRANSMIT DRAET FY97 HMP V-1 COMPLETE FY1997 HANEORD MISSION PLAN, V-1
Type

Einist

$R$ Westinghouse Reportable

$R$ Westinghouse Reportable

K RL Reportable

$K$ RI Reportable

K RL Reportable

K RL Reportable

K RI Reportable

K RI Reportable

K RL Reportable

K RL Reportable

K RL Reportable

K RL Reportable

K RI Reportable

K RL Reportable

K RL Reportable

K RL Reportabie

K RI Reportable

K RL Reportable

K RI Reportable

K RI Reportable

K RL Reportable

K RL Reportable

K RI Reportable

K RI Reportable

K RL Reportable

K RI Reportable

K RL Reportable

K RI Reportable

$R$ Westinghouse Reportable

$R$ Westinghouse Reportable

$R$ Westinghouse Reportable

$R$ Westinghouse Reportable

$R$ Westinghouse Reportable

$R$ Westinghouse Reportable

$R$ Westinghouse Reportable

$R$ Westinghouse Reportable

$R$ Westinghouse Reportable

$R$ Westinghouse Reportable

$R$ Westinghouse Reportable

$R$ Westinghouse Reportable

$R$ Westinghouse Reportable

$R$ Westinghouse Reportable

$R$ Westinghouse Reportable

$R$ Westinghouse Reportable

K RL Reportable

$R$ Westinghouse Reportable

$R$ Westinghouse Reportable

$R$ Westinghouse Reportable

$R$ Westinghouse Reportable

$R$ Westinghouse Reportable

$R$ Westinghouse Reportable

K RI Reportable

K RI Reportable

K RL Reportable

K RI Reportable

K RI Reportable

K RL Reportable

K RL Reportable

$K$ RL Reportable

K RL Reportable

K RL Reportable

K RL Reportable

$R$ Westinghouse Reportable

K RI Reportable

K RI Reportable

$K$ RI Reportable

K RI Reportable

K RL Reportable

K RL Reportable

K RL Reportable
30 sep 97

30 Sep 97

010 ct 96

01Nov96

02Dec 96

$02 \operatorname{Jan} 97$

$03 \mathrm{Feb} 97$

03 Mar 97

01 Apr 97

01 May 97

$02 J u n 97$

01Ju197

01 Aug 97

02 sep 97

250 ct 96

22 Nov 96

20Dec 96

24 Jan 97

21 Feb97

$21 \mathrm{Mar} 97$

25Apr 97

23 May 97

20Jun97

25Jul97

22Aug 97

26 Sep 97

$31 \mathrm{Mar} 97$

$16 J u n 97$

$30 \mathrm{Jul} 97$

28 Eeb97

30 sep 97

230 ct 96

2 Nov96

18 Dec 96

22Jan97

$19 \mathrm{Eeb} 97$

19 Mar97

23 Apr 97

21 May 97

18 Jun 97

23Jul97

20 Aug 97

24 Sep 97

$150 \mathrm{ct} 96$

140 ct 96

16 Jan 97

17 Apr 97

17Jul97

29 Sep 97

29 Sep 97

170 ct 96

19 Jang 5

31 Mar 95

28 Mar 95

28 Mar 95

19 May 95

05Jul 95

29 Sep95

22 May 95

01Mar 95

02 May 95

31 May 95

31 Aug 95

$28 \mathrm{Feb} 95$

21 Eeb96

01May 96

21May 96

10 Apr 96

30 May 96

16 Aug 96 
Run Date 21sep94 19:52

\begin{tabular}{|c|c|c|c|}
\hline ilestone & Description & Type & Finish \\
\hline $\begin{array}{l}\text { SS }-96-204 \\
S S-96-205\end{array}$ & $\begin{array}{l}\text { HSA ADDENDUM III } \\
\text { ISSUE WORKING PAPER DRET FY96 HMP TO PRGS COMPT }\end{array}$ & $\begin{array}{l}\text { K RL Reportable } \\
\text { K RL Reportable }\end{array}$ & \\
\hline PSS $-96-300$ & ANALYSIS OE RISKS \& COSTS AT HANEORD & K RI :Reportábles & 30 Jan 96 \\
\hline PSS $-96-301$ & REPORT ON APPLICATION OE RISK BASED DECISIONS & K RL Reportable & 30 Sep 96 \\
\hline PSS $-97-100$ & STRATEGIC PLNNG-HSP FORMAL DRAET COMPLETE & K RL Reportable & $21 \mathrm{Eeb} 97$ \\
\hline$-97-101$ & STRATEGIC RLNNG-ARPROVAL OF EY1998 HSP COMPLETE & K RI Reportable & $02 \mathrm{May} 97$ \\
\hline$-97-200$ & HMP-DRAFT RL WBS/OBS (THREE LEVELS) COMPLETE & K RI Reportable & 27 Nover 96 \\
\hline$-97-201$ & HIGH IEVEL PRIORITY IIST & K RI Reportable & 10Apr97 \\
\hline$-97-202$ & REVISE, FNAIZE \& TRNSMT DRET FY98 HMP V-1 COMPT & K RL Reportable & $08 J u 197$ \\
\hline PSS $-97-203$ & COMPLETE EY1998 HANFORD MISSION PLAN, V-1 & K RL Reportable & 19Aug97 \\
\hline PSS-97-204 & HSA ADDENDUM III & K RL Reportable & 30 sep 97 \\
\hline$-97-205$ & ISSUE WORKING PAPER DRET FY96 HMP TO PROGS COMPT & K RI Reportable & $15 J u 197$ \\
\hline RSS $-97-205$ & HMP-COMPILE WASTE DATA \& INTEREACES COMPLETE & K RI Reportable & $250 \operatorname{ct} 96$ \\
\hline$-97-300$ & COST-RISK-BENEFIT ANALYSIS UPDATE & K RI Reportable & $31 \mathrm{Jan} 97$ \\
\hline$-97-301$ & REPORT ON RISK DECISIONS & K RI Reportable & 30 Sep97 \\
\hline$-95-001$ & ACTION PLAN FOR IST QUARTER COMPLETE & K RI Reportable & $030 \operatorname{ctg} 4$ \\
\hline $.95-002$ & 2ND QUARTER ACTION PLAN COMPLETE & R Westinghouse Reportable & 09Dec94 \\
\hline$-95-003$ & SUMMARY OE ACTION PLAN FOR IST QUARTER COMPLETE & R Westinghouse Reportable & $13 \mathrm{Jan} 95$ \\
\hline PUB-95-004 & PIAN FOR 3RD QUARTER COMPLETE & K RI Reportable & $10 \mathrm{Mar} 95$ \\
\hline PUB-95-006 & SUMMARY OF ACTION PLAN FOR 2ND QUARTER COMPLETE & R Westinghouse Reportable & 13Apr95 \\
\hline$-95-007$ & PLAN EOR 4 TH QUARTER COMPLETE & K RI Reportable & $09 J u n 95$ \\
\hline $95-008$ & SUMMARY OE ACTION PLAN FOR 3RD QUARTER COMPLETE & R Westinghouse Reportable & 14 Jul95 \\
\hline PUB $-95-009$ & 1.8.2 PUBLIC INVOLVEMENT GUIDANCE COMPLETE & K RI Reportable & 01Aug 95 \\
\hline PUB $-95-010$ & ACTION PIAN EOR IST QUARTER OE 1996 COMPLETE & R Westinghouse Reportable & 08 Sep95 \\
\hline EUB-96-001 & SUMMARY OE ACTION PLAN FOR $954 \mathrm{TH}$ QUARTER & R Westinghouse Reportable & $130 c t 95$ \\
\hline$-96-002$ & 2ND QUARTER ACTION PLAN COMPLETE & $\mathrm{R}$ Westinghouse & $08 D e c 95$ \\
\hline ?UB-96-003 & SUMMARY OE ACTION PIAN FOR IST QUARTER COMPLETE & R Westinghouse & 12 Jan 96 \\
\hline PUB $-96-004$ & PLAN FOR 3RD QUARTER COMPLETE & K RI Reportable & $08 \operatorname{Mar} 96$ \\
\hline PUB-96-005 & SUMMARY OF ACTION PLAN FOR 2ND QUARTER COMPLETE & R Westinghouse Reportable & 12Apr96 \\
\hline PUB-96-006 & PLAN FOR $4 T H$ QUARTER COMPLETE & K RI Reportable & 07Jun96 \\
\hline PUB-96-007 & SUMMARY OE ACTION PLAN FOR 3RD QUARTER COMPLETE & R Westinghouse Reportable & 12Jul96 \\
\hline UUB-96-008 & 1.8 .2 PUBLIC INVOLVEMENT GUIDANCE COMPLETE & K RI Reportable & 02 Aug 96 \\
\hline iB-96-009 & ACTION PLAN EOR IST QUARTER OF 1997 COMPLETE & stinghouse Reportable & 09 Sep96 \\
\hline$J B-97-001$ & SUMMARY OE ACTION PLAN FOR 4 TH QUARTER OF 96 & $\mathrm{R}$ Westinghouse & $140 c t 96$ \\
\hline PUB-97-002 & 2ND QUARTER ACTION PIAN COMPLETE & $\mathrm{R}$ Westinghouse & 06 Dec 96 \\
\hline$-97-003$ & SUMMARY OE ACTION PLAN EOR IST QUARTER COMPLETE & R Westinghouse Reportable & $13 \operatorname{Jan} 97$ \\
\hline $97-004$ & PLAN FOR 3RD QUARTER COMPLETE & K RI Reportable & $07 \mathrm{Mar} 97$ \\
\hline PUB-97-005 & SUMMARY OE ACTION PLAN FOR 2ND QUARTER COMPLETE & $R$ Westinghouse Reportable & 11 Apr 97 \\
\hline $37-006$ & PLAN EOR 4TH QUARTER COMPLETE & K RI Reportable & 06 Jun97 \\
\hline$-97-007$ & SUMMARY OF ACTION PLAN FOR 3RD QUARTER COMPLETE & R Westinghouse Reportable & 11 Jul97 \\
\hline UB-97-008 & 1.8 .2 PUBLIC INVOLVEMENT GUIDANCE COMPLETE & K RL Reportable & 01Aug 97 \\
\hline PUB-97-009 & ACTION PLAN FOR 1ST QUARTER OE 1997 COMPLETE & $R$ Westinghouse Rep & $09 \operatorname{Sep} 97$ \\
\hline SSE-95-001 & TRA M33-00-T03 & T Tri-Party Agreem & 30Dec 94 \\
\hline SSE-95-002 & COMPLETE UPDATE FUNCTIONAL ANALYSIS, REV 0 & K RL Reportable & $29 \operatorname{Mar} 95$ \\
\hline $5-003$ & COMPLETE REQUIREMENTS ANALYSIS \& ALLOC, REV 0 & K RI Reportable & 24 May 95 \\
\hline$-95-004$ & UPDATE BASELINE MEASURES, REV 0 & K RI Reportable & 23 May 95 \\
\hline$-95-005$ & COMPLETE REVISED ARCH \& PRODUCTS DEFIN, REV 0 & K RL Reportable & 31 Aug 95 \\
\hline$-95-006$ & COMPLETE DYNAMIC SYSTEMS MODEL & R Westinghouse Reportable & 31 Jangs \\
\hline $.95-007$ & TPA M33-00-T04 & T Tri-Party Agreement & 31 Aug 95 \\
\hline$-95-010$ & COMPLETE BASELINE SPECIFICATION & K RL Reportable & $295 e p 95$ \\
\hline $95-011$ & ISSUE REVISED HS-SEMP/SEM TO PROGRAMS \& $Q A$ & K RI Rep & $11 \mathrm{Jan} 95$ \\
\hline$-95-012$ & COMPLETE SE ENG PRACT \& PROC ASSESSMENT REPORT & $\mathrm{R}$ Westingh & $31 \operatorname{Mar} 95$ \\
\hline 5-013 & COMPLETE SE TRAINING ASSESSMENT REPORT & R Westinghouse Reportable & $20 J u 195$ \\
\hline $95-014$ & ISSUE REVISED SITE SE-CMP (SEM-95-005) & K RL Reportable & 31 Jan95 \\
\hline$-95-015$ & COMPLETE DEF. \& IMPL. INFOR STRUCT \& CNTRL SYS & o Other Mgt Reportable & 28 Aug 95 \\
\hline 001 & COMPLETE HMP BASELINE DEVELOPMENT & K RL Reportable & $02 J a n 96$ \\
\hline-002 & COMPLETE BASELINE EUNCTIONS, REV 1 & K RL Reportable & 27Mar96 \\
\hline-003 & COMPLETE REQUIREMENTS ANALYSIS \& ALLOC, REV 1 & K RL Reportable & 22 May 96 \\
\hline $96-004$ & COMPLETE MEASURES OF EEEECTIVENESS ANALY, REV 1 & K RL Reportable & 23 May 96 \\
\hline-005 & COMPLETE REVISED ARCH \& PRODUCTS DEEIN, REV 1 & K RL Reportable & 29Aug 96 \\
\hline & COMPLETE 200 AREA PLATEAU & K RL Reportable & 29 May 96 \\
\hline-007 & COMPLETE BASELINE SPECIEICATION & K RL Reportable & 30 Sep 96 \\
\hline $6-008$ & COMPLETE SITE IMPIEMENTATION SELE ASSESSMENT & $R$ Westinghouse $\mathrm{Re}$ & $29 \operatorname{Mar} 96$ \\
\hline$-96-009$ & COMPLETE SITE IMPLEMENTATION ASSESSMENT REPORT & K RL Reportable & 30 Sep 96 \\
\hline$-96-010$ & COMPLETE SE TRAINING ASSESSMENT REPORT & K RI Reportable & $30 \operatorname{sep} 96$ \\
\hline $5 E-96-011$ & COMPLETE AUDIT REPORT & K RI Reportable & Sep96 \\
\hline $7-001$ & COMPLETE HMP BASELINE DEVELOPMENT & K RI Reportable & $02 J a n 97$ \\
\hline SSE-97-002 & COMPLETE URDATE EUNCTIONAL ANALYSIS, REV 2 & K RL Reportable & $28 \operatorname{Mar} 97$ \\
\hline SSE-97-003 & COMPLETE REQUIREMENTS ANALYSIS \& ALLOC, REV 2 & K RL Reportable & 23 Мау 97 \\
\hline$-97-004$ & COMPLETE MEASURES OF EEEECTIVENESS ANALY, REV 2 & K RI Reportable & 23 May 97 \\
\hline 7-005 & COMPLETE REVISED ARCH \& PRODUCTS DEFIN, REV 2 & K RL Reportable & 29Aug97 \\
\hline
\end{tabular}


Run Date 21Sep94 19:52

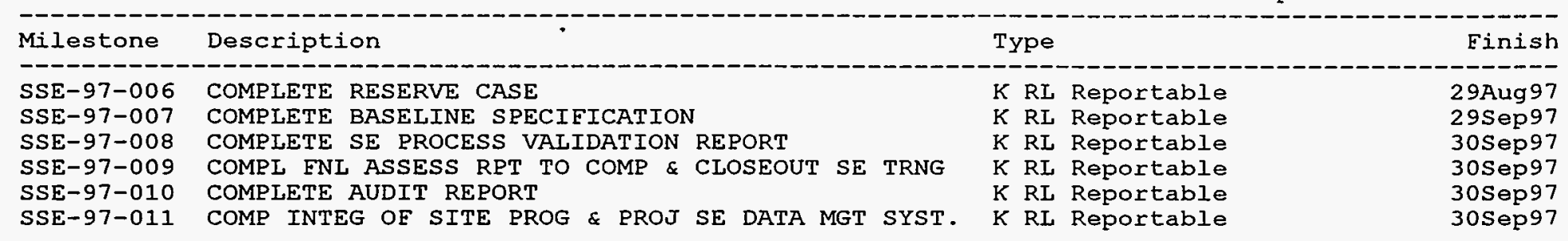




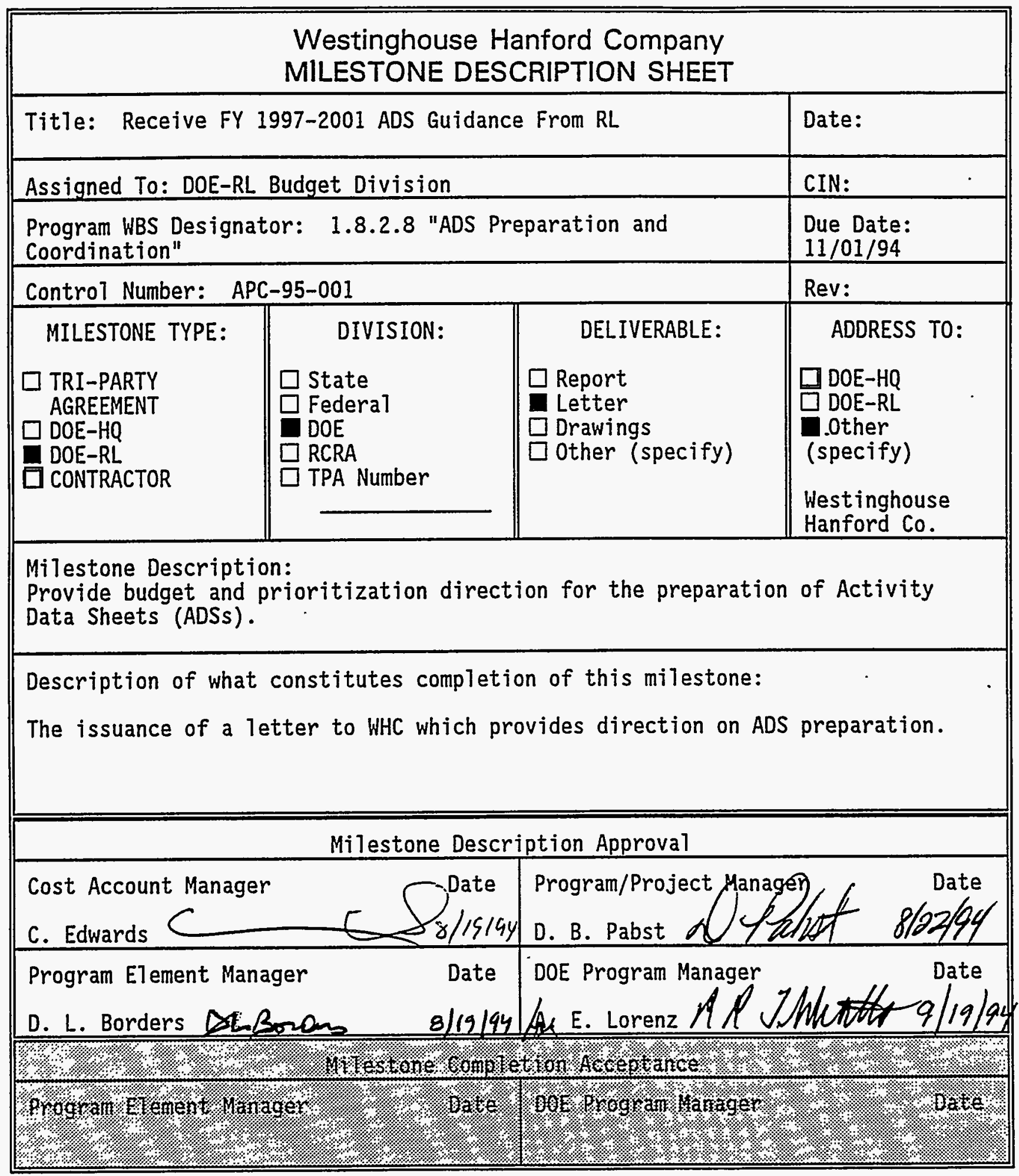




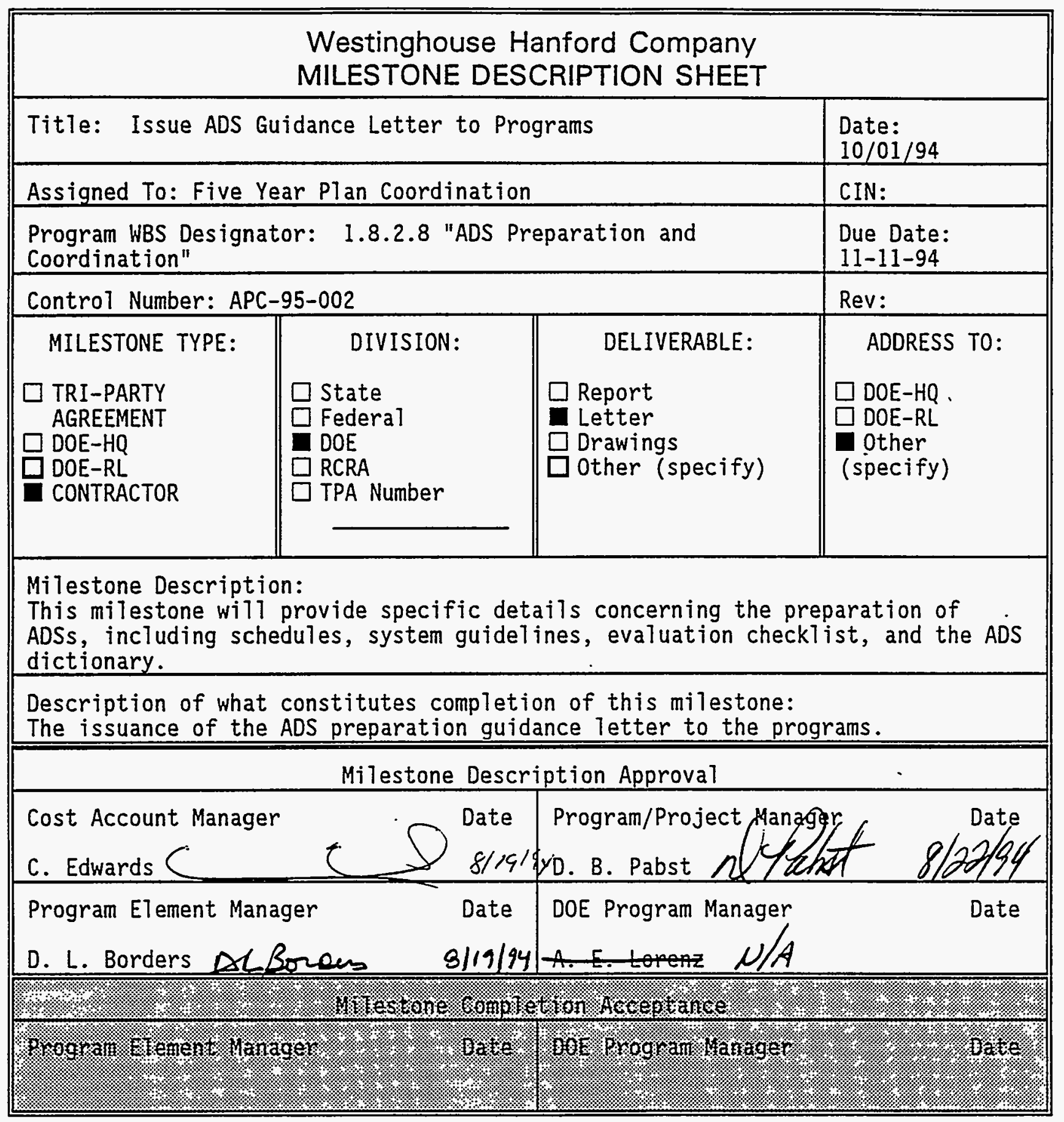




\section{Westinghouse Hanford Company MILESTONE DESCRIPTION SHEET}

\begin{tabular}{|c|c|c|c|}
\hline \multicolumn{3}{|c|}{$\begin{array}{l}\text { Title: } \\
\text { Submit Draft FY 1996-1997 Building BTocks to HQ }\end{array}$} & $\begin{array}{l}\text { Date: } \\
\quad 7 / 25 / 94\end{array}$ \\
\hline \multicolumn{3}{|c|}{$\begin{array}{l}\text { Assigned To: } \\
\text { Five Year Planning Coordination }\end{array}$} & CIN: \\
\hline \multicolumn{3}{|c|}{$\begin{array}{l}\text { Program WBS Designator: } \\
\text { 1.8.2.8 "ADS Preparation and Coordination" }\end{array}$} & $\begin{array}{c}\text { Due Date: } \\
01 / 06 / 95 \\
\end{array}$ \\
\hline \multicolumn{3}{|c|}{ Control Number: APC-95-003 } & Rev: \\
\hline MILESTONE TYPE: & DIVISION: & DELIVERABLE: & ADDRESS TO: \\
\hline $\begin{array}{l}\square \text { DOE-HQ } \\
\text { DOE-RL } \\
\text { CONTRACTOR }\end{array}$ & $\begin{array}{l}\square \text { State } \\
\square \text { Federat } \\
\square \text { DOE } \\
\square \text { RCRA } \\
\square \text { TPA Number }\end{array}$ & $\begin{array}{l}\square \text { Report } \\
\square \text { Letter } \\
\square \text { Drawings } \\
\square \text { Other (specify) }\end{array}$ & $\begin{array}{l}\square \text { DOE-HQ } \\
\text { DOE-RL } \\
\square \text { Other (specify) }\end{array}$ \\
\hline
\end{tabular}

Milestone Description:

Submittal of Building blocks for FY 1996 and FY 1997 to support FY 1997 ADS planning to DOE-RL programs for Waste Management, Facility Transition, and Environmental Restoration. A17 program's building blocks will be subject to full review, integration and approvaT by WHC and RL Senior management. The building biocks will be developed utilizing the RL priority system.

Description of what constitutes completion of this milestone:

Submittal of each program's Building Blocks to RL.

\begin{tabular}{|c|c|}
\hline \multicolumn{2}{|c|}{ Milestone Description Approval } \\
\hline C. Edwards & Program/Project Mapagey of \\
\hline $\begin{array}{l}\text { Program Element Manager } \\
\text { D.L. Borders } 8 / 19 / 92\end{array}$ & DOE Monitor I whatto \\
\hline \multicolumn{2}{|c|}{ Milestone Completion Acceptance } \\
\hline $\begin{array}{c}\text { Program ETement Manager } \\
\end{array}$ & DOE Program Manager $\quad \square$ \\
\hline
\end{tabular}




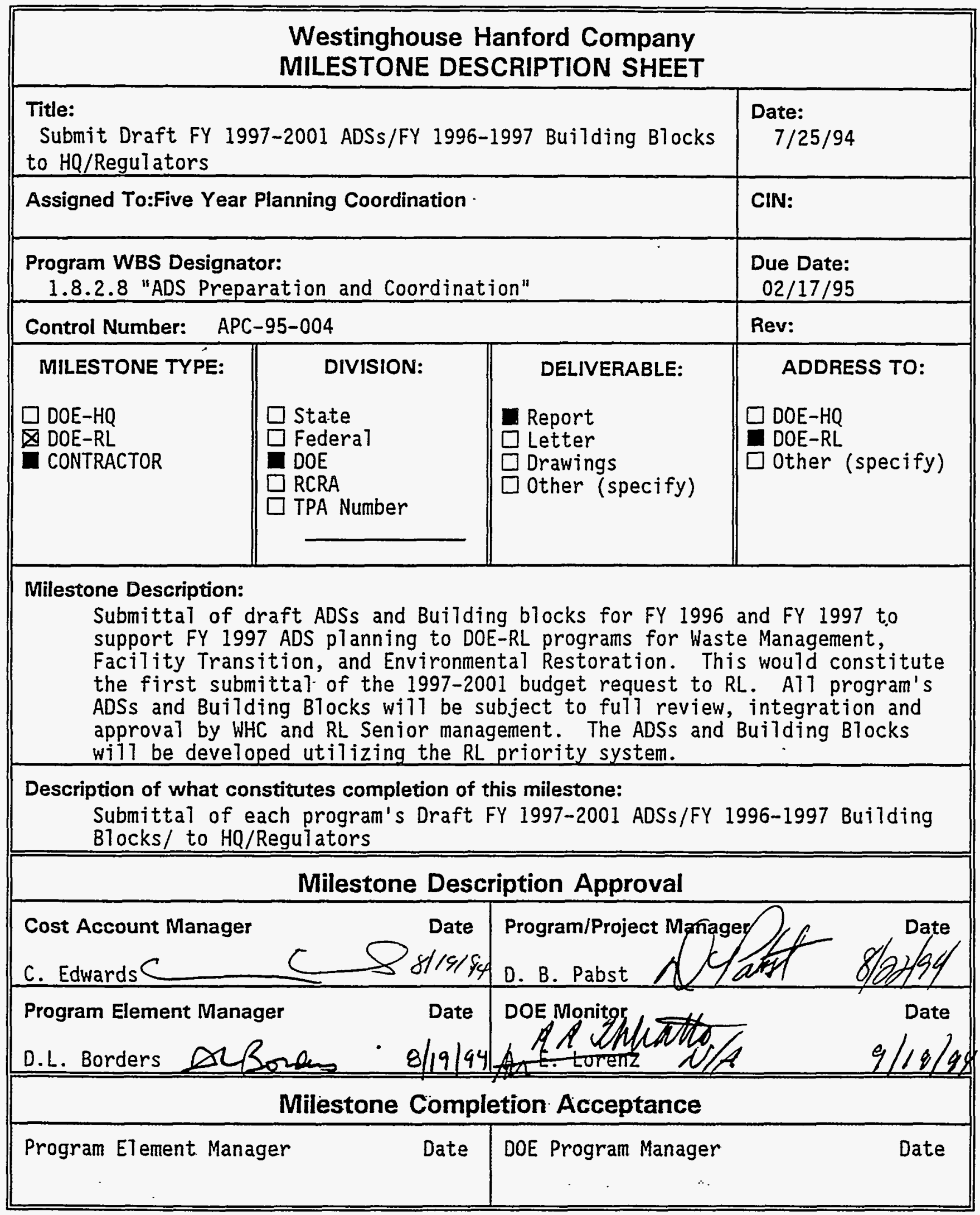




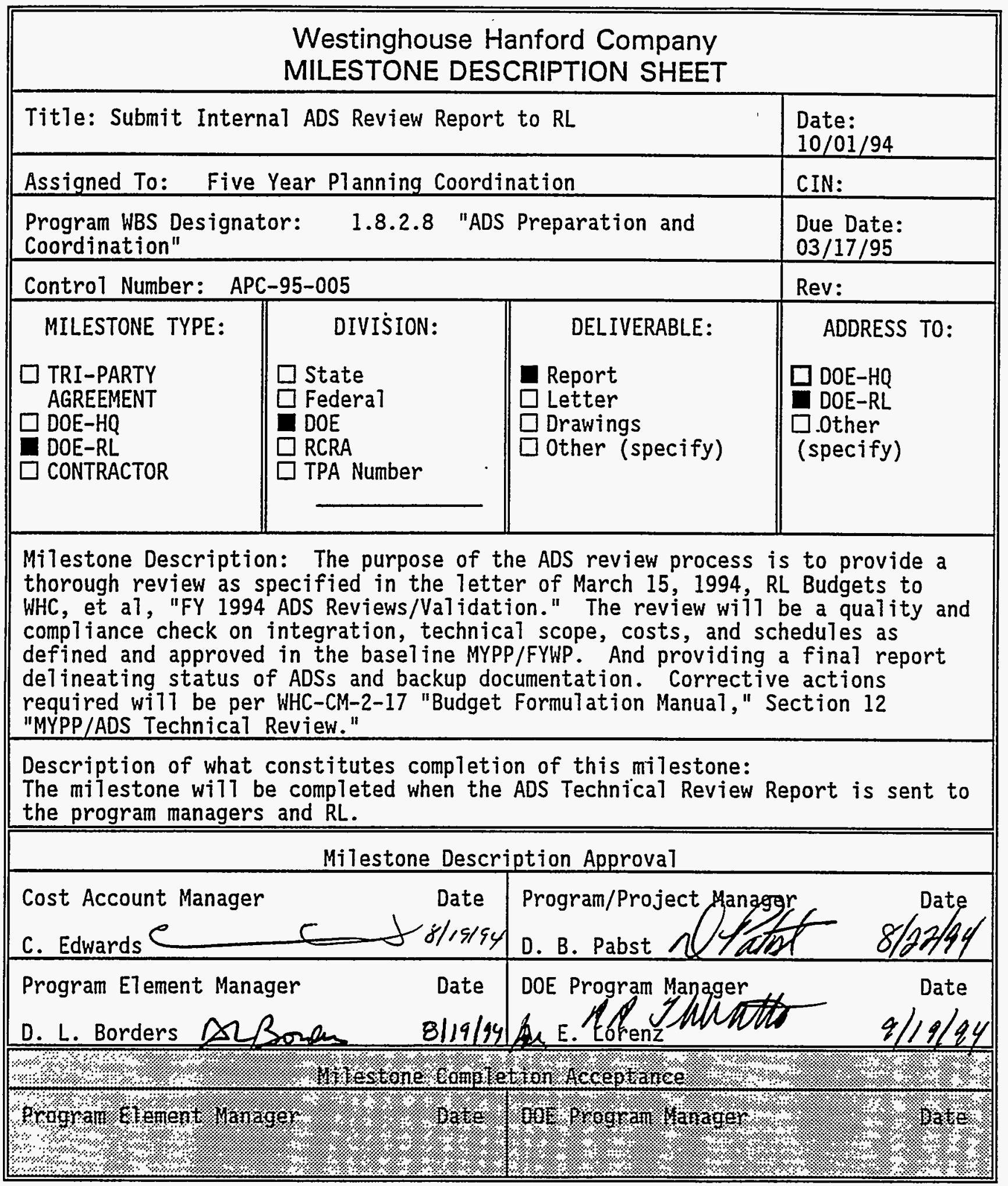




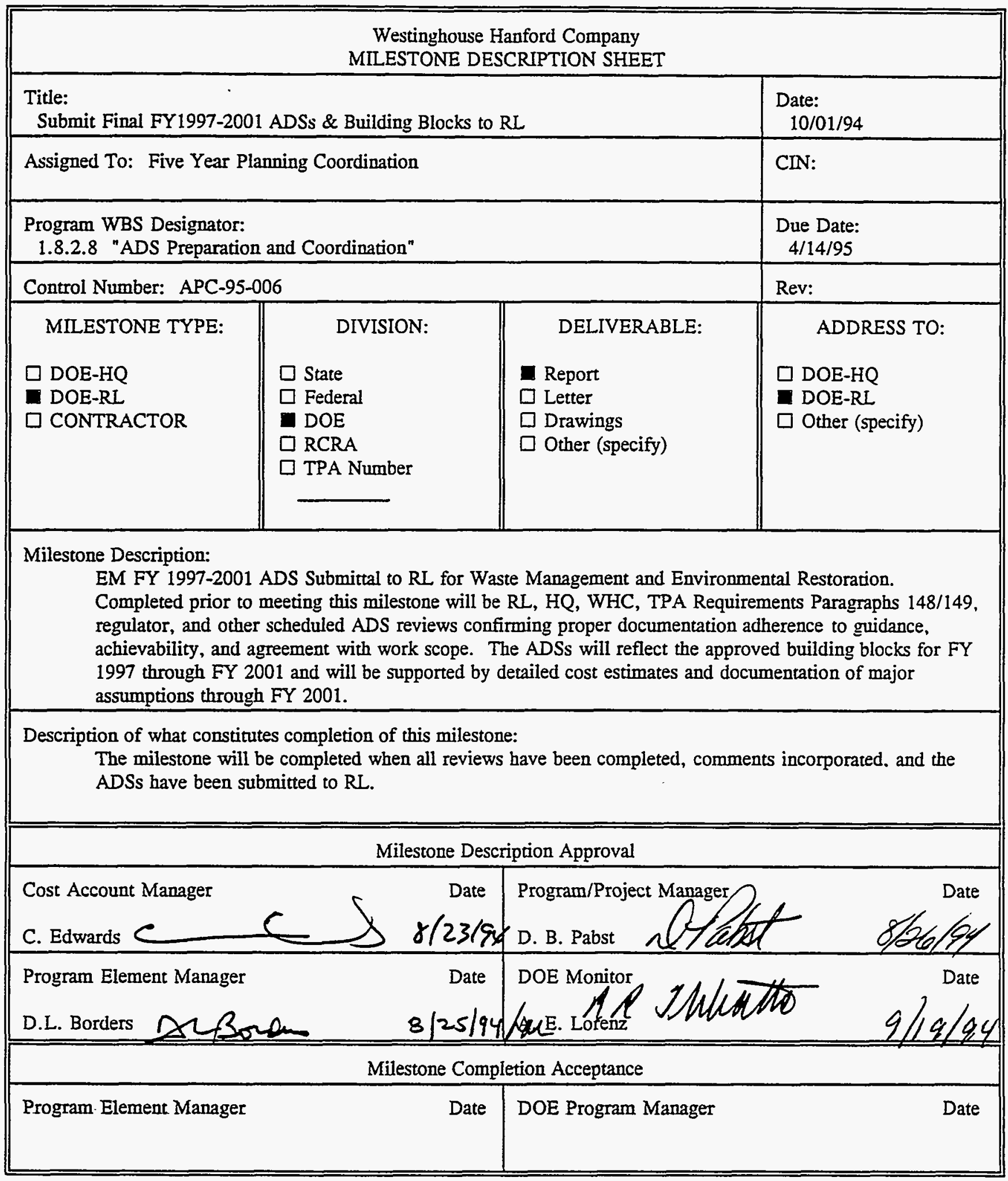




\begin{tabular}{|c|c|c|c|}
\hline \multicolumn{4}{|c|}{$\begin{array}{l}\text { Westinghouse Hanford Company } \\
\text { MILESTONE DESCRIPTION SHEET }\end{array}$} \\
\hline \multicolumn{3}{|c|}{$\begin{array}{l}\text { Title: } \\
\text { Submit Final FY1997-2001 ADSs \& Building Blocks to HQ }\end{array}$} & $\begin{array}{l}\text { Date: } \\
7 / 25 / 94 \\
\end{array}$ \\
\hline \multicolumn{3}{|c|}{$\begin{array}{l}\text { Assigned To: } \\
\text { Five Year Planning Coordination }\end{array}$} & CIN: \\
\hline \multicolumn{3}{|c|}{$\begin{array}{l}\text { Program WBS Designator: } \\
\text { 1.8.2.8 "ADS Preparation and Coordination" }\end{array}$} & $\begin{array}{c}\text { Due Date: } \\
4 / 14 / 95\end{array}$ \\
\hline \multicolumn{3}{|c|}{ Control Number: APC-95-007 } & Rev: \\
\hline $\begin{array}{l}\text { MILESTONE TYPE: } \\
\square \text { DOE-HQ } \\
\square \text { DOE-RL } \\
\square \text { CONTRACTOR }\end{array}$ & $\begin{array}{l}\quad \text { DIVISION: } \\
\square \text { State } \\
\square \text { Federal } \\
\square \text { DOE } \\
\square \text { RCRA } \\
\square \text { TPA Number }\end{array}$ & $\begin{aligned} & \text { DELIVERABLE: } \\
& \\
& \text { Report } \\
& \square \text { Letter } \\
& \square \text { Drawings } \\
& \square \text { Other (specify) }\end{aligned}$ & $\begin{aligned} & \text { ADDRESS TO: } \\
& \text { DOE-HQ } \\
& \text { DOE-RI } \\
& \text { Other (specify) }\end{aligned}$ \\
\hline \multicolumn{4}{|c|}{$\begin{array}{l}\text { Milestone Description: } \\
\text { EM FY 1997-2001 ADS Submittal to DOE-HQ for Waste Management and Environmental Restoration. } \\
\text { Completed prior to meeting this milestone will be RL, HQ, WHC, TPA Requirements Paragraphs 148/149, } \\
\text { regulator, and other scheduled ADS reviews confirming proper documentation adherence to guidance, and } \\
\text { achievability. The ADSs will reflect the RL approved building blocks for FY } 1997 \text { through FY } 2001 \text { and } \\
\text { will be supported by detailed cost estimates and documentation of major assumptions through FY } 2001 \text {. }\end{array}$} \\
\hline \multicolumn{4}{|c|}{$\begin{array}{l}\text { Description of what constitutes completion of this milestone: } \\
\text { The milestone will be completed when all reviews have been completed, comments incorporated, and the } \\
\text { ADSs have been submitted to HQ by RL. }\end{array}$} \\
\hline \multicolumn{4}{|c|}{ Milestone Description Approval } \\
\hline \multicolumn{2}{|l|}{$\begin{array}{l}\text { Cost Account Manager } \\
\text { C. EdwardS }\end{array}$} & Program/Project Mag & Date \\
\hline \multicolumn{2}{|c|}{ Program Element Manager } & DOE Monitor & $q / 19 / q$ \\
\hline$\because$ & \multicolumn{2}{|c|}{ Milestone Completion Acceptance } & \\
\hline DOE Program & ger & Date & \\
\hline
\end{tabular}




\begin{tabular}{|c|c|c|c|}
\hline \multicolumn{4}{|c|}{$\begin{array}{l}\text { Westinghouse Hanford Company } \\
\text { MILESTONE DESCRIPTION SHEET }\end{array}$} \\
\hline \multicolumn{3}{|c|}{$\begin{array}{l}\text { Title: } \\
\text { Publish Hanford Site Focus }\end{array}$} & $\begin{array}{l}\text { Date: } \\
08 / 22 / 94\end{array}$ \\
\hline \multicolumn{3}{|c|}{$\begin{array}{l}\text { Assigned To: } \\
\text { ADS Preparation and Coordination }\end{array}$} & CIN: \\
\hline \multicolumn{3}{|c|}{$\begin{array}{l}\text { Program WBS Designator: } \\
\text { 1.8.2.8 "ADS Preparation and Coordination" }\end{array}$} & $\begin{array}{l}\text { Due Date: } \\
11 / 01 / 94\end{array}$ \\
\hline \multicolumn{3}{|c|}{ Control Number: APC-95-008 } & Rev: \\
\hline $\begin{array}{l}\text { MILESTONE TYPE: } \\
\square \text { DOE-HQ } \\
\text { DOE-RL } \\
\square \text { CONTRACTOR }\end{array}$ & $\begin{aligned} & \text { DIVISION: } \\
& \square \text { State } \\
& \text { Federal } \\
& \text { DOE } \\
& \square \text { RCRA } \\
& \square \text { TPA Number }\end{aligned}$ & $\begin{array}{l}\text { DELIVERABLE: } \\
\square \text { Report } \\
\square \text { Letter } \\
\square \text { Drawings } \\
\square \text { Other (specify) } \\
\text { Hanford Site Focus }\end{array}$ & $\begin{array}{l}\text { ADDRESS TO: } \\
\square \text { DOE-HQ } \\
\square \text { DOE-RL } \\
\text { Other (specify) } \\
\text { Public }\end{array}$ \\
\hline \multicolumn{4}{|c|}{$\begin{array}{l}\text { Milestone Description: } \\
\text { Prepare and publish the Hanford Site Focus. The Hanford Site Focus will report on: The just completed fiscal year; } \\
\text { Plans for the execurion year based on actual budget received; Priorities, planning processes, and values. } \\
\text { The level of detail will be sufficient to be understood by, but not overwhelm, the public. After review by DOE-RL, } \\
\text { publish the document. }\end{array}$} \\
\hline \multicolumn{4}{|c|}{$\begin{array}{l}\text { Description of what constitutes completion of this milestone: } \\
\text { The milestone will be completed when the Hanford Site Focus is published. }\end{array}$} \\
\hline \multicolumn{4}{|c|}{ Milestone Description Approval } \\
\hline \multicolumn{2}{|c|}{ Cost Account Manager } & \multicolumn{2}{|c|}{ D. B. Pabst } \\
\hline \multicolumn{2}{|c|}{$\begin{array}{lc}\text { Program Element Manager } & \text { Date } \\
\text { D.L. Borders } & 8 / 25 / 94\end{array}$} & \multicolumn{2}{|c|}{ DOE Monitor } \\
\hline \multicolumn{4}{|c|}{ Milestone Completion:Acceptance } \\
\hline Program Element Manag & & DOE Program Manager & Date \\
\hline
\end{tabular}




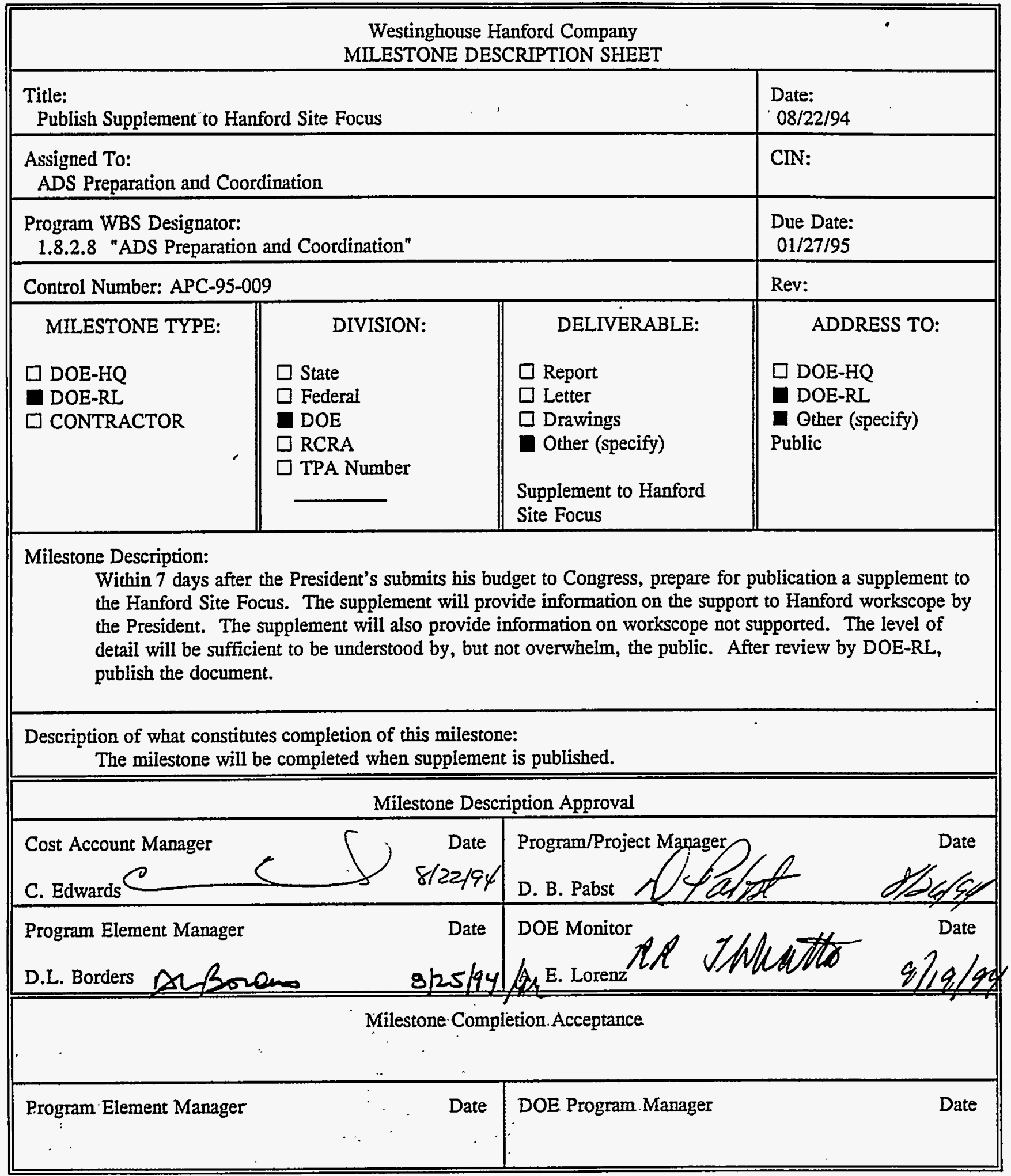




\begin{tabular}{|c|c|c|}
\hline \multicolumn{3}{|c|}{$\begin{array}{l}\text { Westinghouse Hanford Company } \\
\text { MILESTONE DESCRIPTION SHEET }\end{array}$} \\
\hline \multicolumn{2}{|c|}{ Title: Receive FY 1998-2002 ADS Guidance From RL } & Date: \\
\hline \multicolumn{2}{|l|}{ Assigned To: DOE-RL Budget Division } & CIN: \\
\hline \multicolumn{2}{|c|}{$\begin{array}{l}\text { Program WBS Designator: } 1.8 .2 .8 \text { "ADS Preparation and } \\
\text { Coordination" }\end{array}$} & $\begin{array}{l}\text { Due Date: } \\
11 / 01 / 95\end{array}$ \\
\hline \multicolumn{2}{|l|}{ Control Number: APC-96-001 } & Rev: \\
\hline 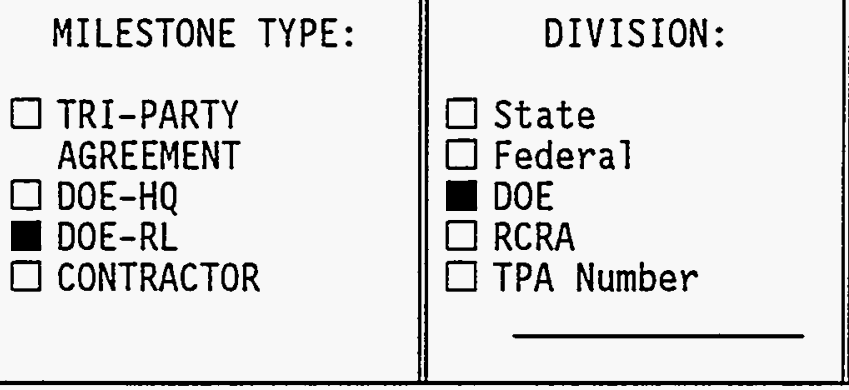 & $\begin{array}{l}\text { DELIVERABLE: } \\
\square \text { Report } \\
\text { Letter } \\
\square \text { Drawings } \\
\square \text { Other (specify) }\end{array}$ & $\begin{array}{l}\text { ADDRESS TO: } \\
\square \text { DOE-HQ } \\
\square \text { DOE-RL } \\
\text {. Other } \\
\text { (specify) } \\
\text { Westinghouse } \\
\text { Hanford Co. }\end{array}$ \\
\hline \multicolumn{3}{|c|}{$\begin{array}{l}\text { Milestone Description: } \\
\text { Provide budget and prioritization direction for the preparation of Activity } \\
\text { Data Sheets (ADSs). }\end{array}$} \\
\hline \multicolumn{3}{|c|}{$\begin{array}{l}\text { Description of what constitutes completion of this milestone: } \\
\text { The issuance of a letter to WHC which provides direction on AD }\end{array}$} \\
\hline \multicolumn{3}{|c|}{ Milestone Description Approval } \\
\hline \multicolumn{2}{|l|}{ 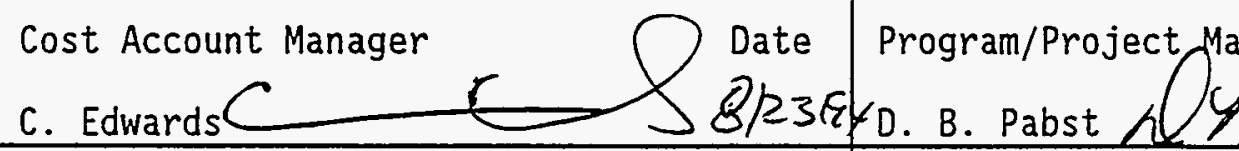 } & $8 / 26 / 94$ \\
\hline \multicolumn{2}{|c|}{ 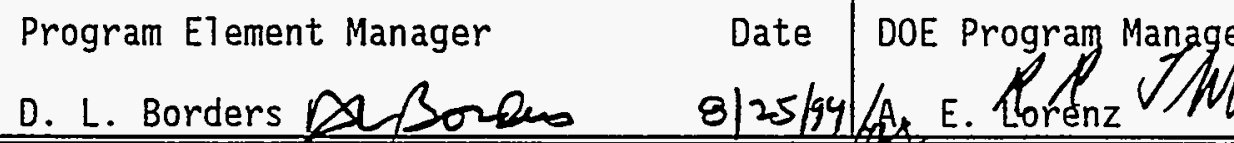 } & Hol $\quad$ Date \\
\hline \multicolumn{3}{|c|}{ 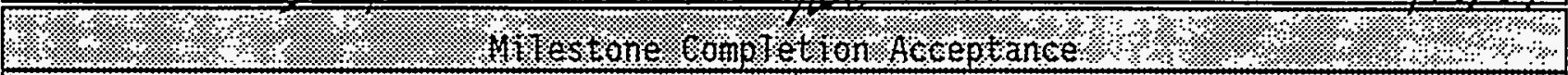 } \\
\hline \multicolumn{3}{|c|}{ 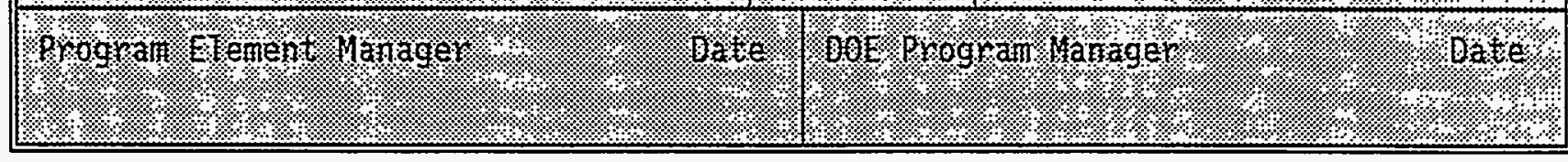 } \\
\hline
\end{tabular}




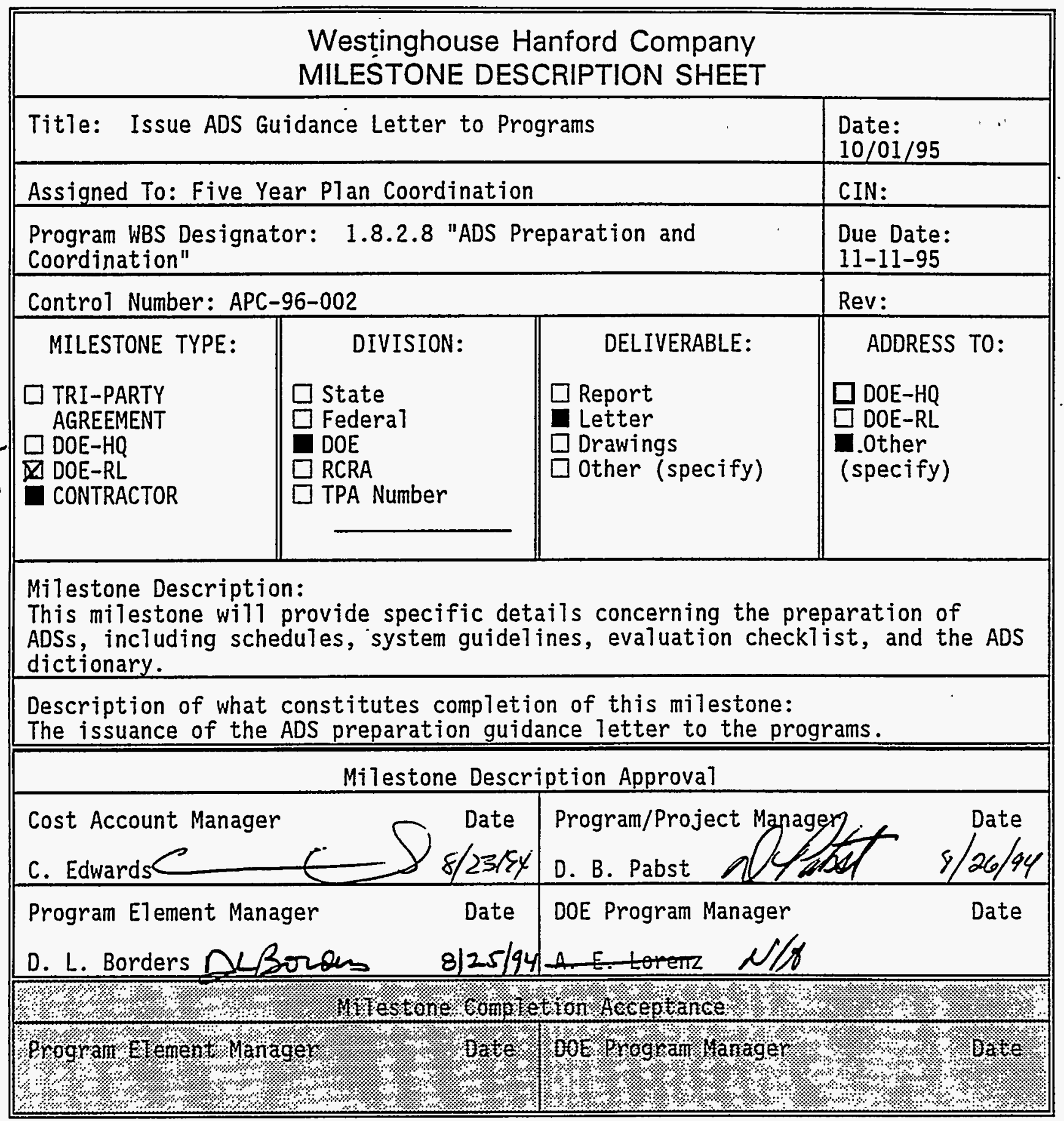




\section{Westinghouse Hanford Company MILESTONE DESCRIPTION SHEET}

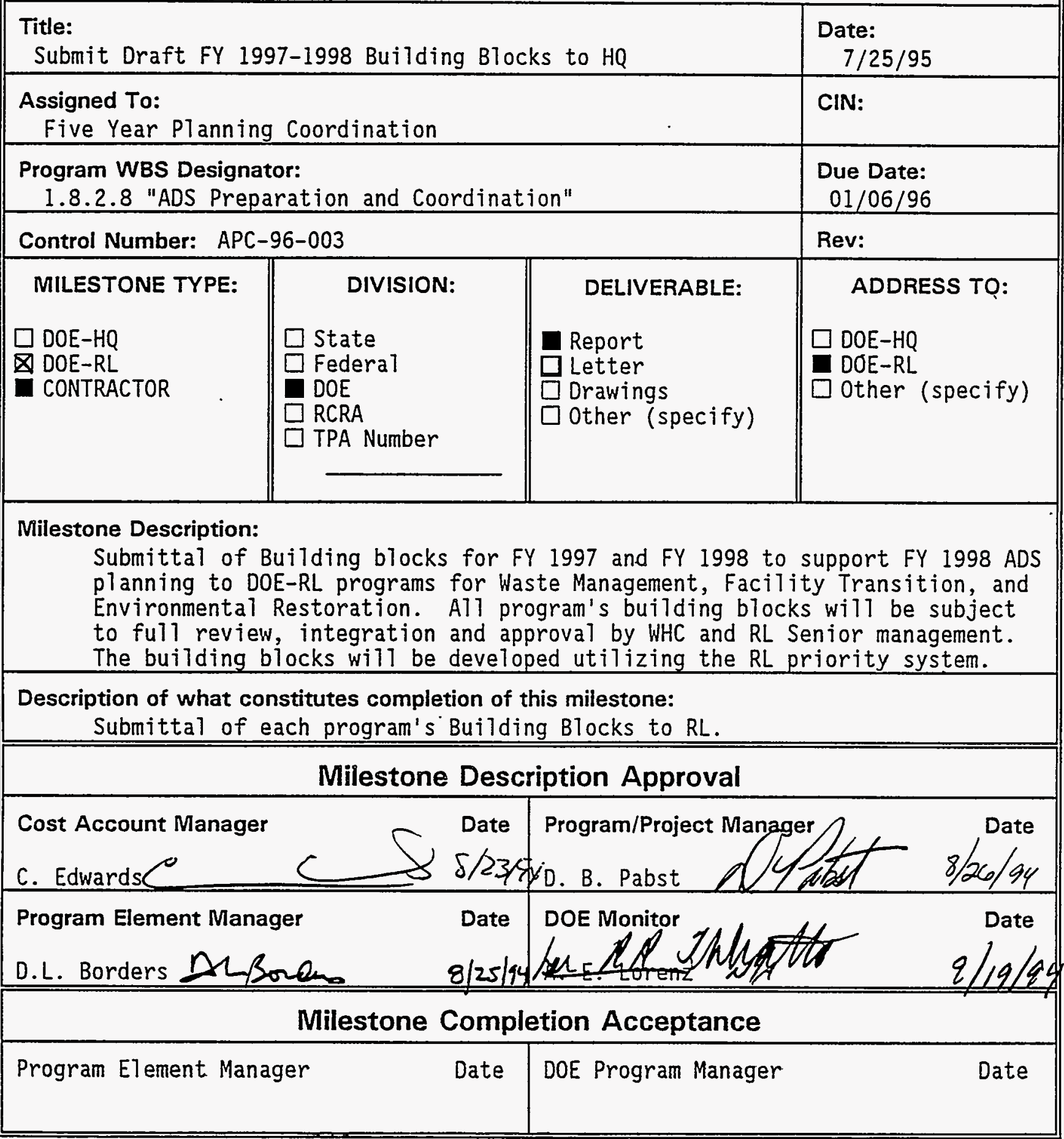




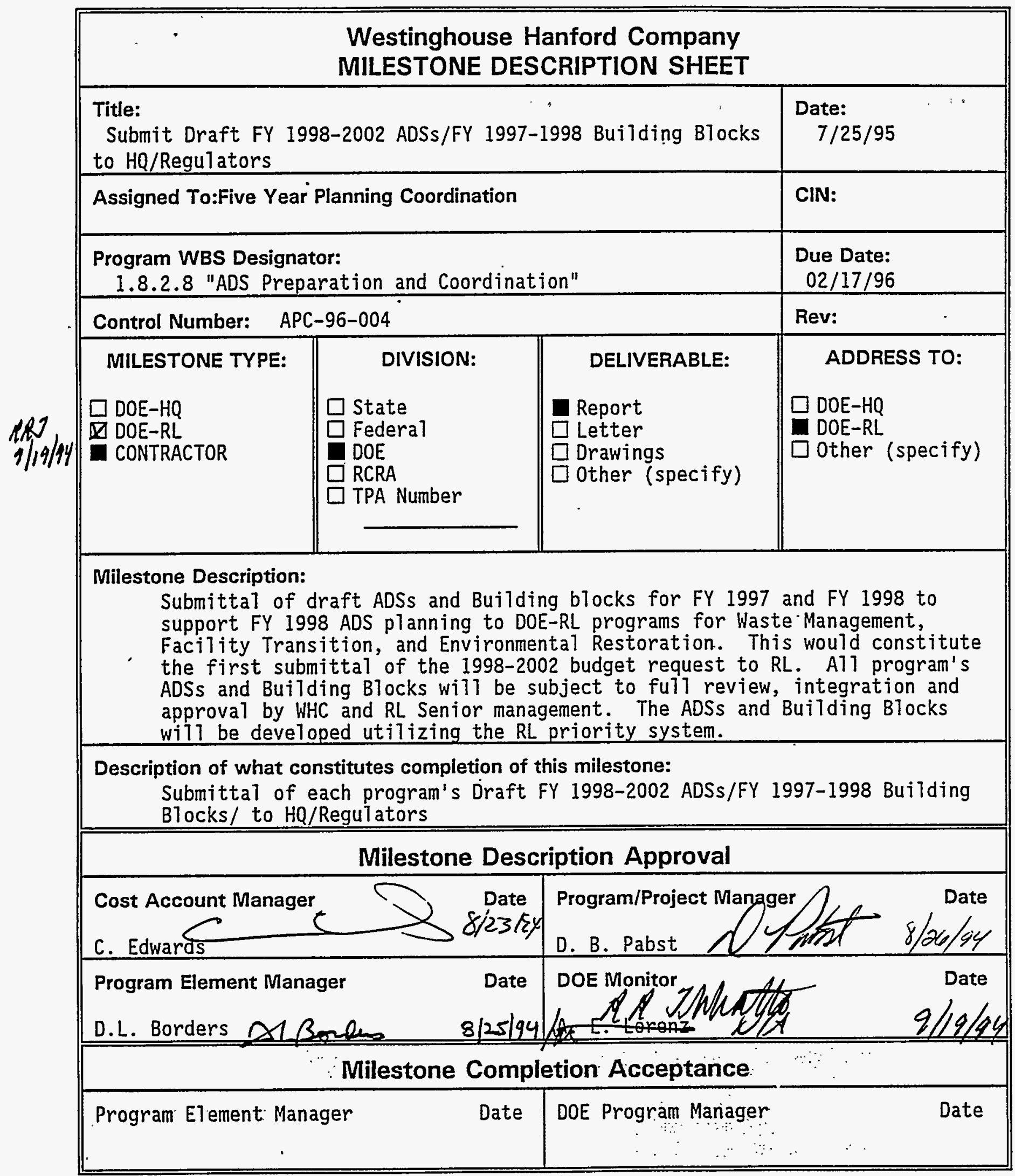




\begin{tabular}{|l||l|l||}
\hline \multicolumn{3}{|c||}{ Westinghouse Hanford Company } \\
MILESTONE DESCRIPTION SHEET
\end{tabular}




\begin{tabular}{|c|c|c|c|}
\hline \multicolumn{4}{|c|}{$\begin{array}{l}\text { Westinghouse Hanford Company } \\
\text { MILESTONE DESCRIPTION SHEET }\end{array}$} \\
\hline \multicolumn{3}{|c|}{$\begin{array}{l}\text { Title: } \\
\text { Submit Final FY1998-2002 ADSs \& Building Blocks to RL }\end{array}$} & $\begin{array}{l}\text { Date: } \\
\text { 10/01/95 }\end{array}$ \\
\hline \multicolumn{3}{|c|}{ Assigned To: Five Year Planning Coordination } & CIN: \\
\hline \multicolumn{3}{|c|}{$\begin{array}{l}\text { Program WBS Designator: } \\
\text { 1.8.2.8 "ADS Preparation and Coordination" }\end{array}$} & $\begin{array}{c}\text { Due Date: } \\
4 / 14 / 96\end{array}$ \\
\hline \multicolumn{3}{|c|}{ Control Number: APC-96-006 } & Rev: \\
\hline $\begin{array}{l}\text { MILESTONE TYPE: } \\
\square \text { DOE-HQ } \\
\square \text { DOE-RL } \\
\square \text { CONTRACTOR }\end{array}$ & $\begin{array}{l}\quad \text { DIVISION: } \\
\quad \square \text { State } \\
\square \text { Federal } \\
\square \text { DOE } \\
\square \text { RCRA } \\
\square \text { TPA Number }\end{array}$ & $\begin{aligned} & \text { DELIVERABLE: } \\
& \text { Report } \\
& \text { Retter } \\
& \square \text { Drawings } \\
& \square \text { Other (specify) }\end{aligned}$ & $\begin{aligned} & \text { ADDRESS TO: } \\
& \\
& \text { DOE-HQ } \\
& \text { DOE-RL } \\
& \square \text { Other (specify) }\end{aligned}$ \\
\hline \multicolumn{4}{|c|}{$\begin{array}{l}\text { Milestone Description: } \\
\text { EM FY 1998-2002 ADS Submittal to RL for Waste Management and Environmental Restoration. } \\
\text { Completed prior to meeting this milestone will be RL, HQ, WHC, TPA Requirements Paragraphs 148/149, } \\
\text { regulator, and other scheduled ADS reviews confirming proper documentation adherence to guidance, } \\
\text { achievability, and agreement with work scope. The ADSs will reflect the approved building blocks for FY } \\
1998 \text { through FY } 2002 \text { and will be supported by detailed cost estimates and documentation of major } \\
\text { assumptions through FY } 2002 \text {. }\end{array}$} \\
\hline \multicolumn{4}{|c|}{$\begin{array}{l}\text { Description of. what constintes completion of this milestone: } \\
\text { The milestone will be completed when all reviews have been completed, comments incorporated, and the } \\
\text { ADSs have been submitted to RL. }\end{array}$} \\
\hline \multicolumn{4}{|c|}{ Milestone Description Approval } \\
\hline $\begin{array}{l}\text { Cost Account Manager } \\
\text { C. Edwards } 0\end{array}$ & $\oiint^{\prime} \delta / 23 / \varepsilon y$ & $\begin{array}{l}\text { Program/Project Manage } \\
\text { D. B. Pabst }\end{array}$ & $8 / 26 / 91$ \\
\hline \multicolumn{2}{|c|}{$\begin{array}{lr}\text { Program Element Manager } & \text { Date } \\
\text { D.L. Borders } & 8 / 25 / 94\end{array}$} & \multicolumn{2}{|c|}{ DOE Monitor } \\
\hline \multicolumn{4}{|c|}{ Milestone Completion Acceptance } \\
\hline Program:Element Manag & Date & DOE Program Manager & Date \\
\hline
\end{tabular}




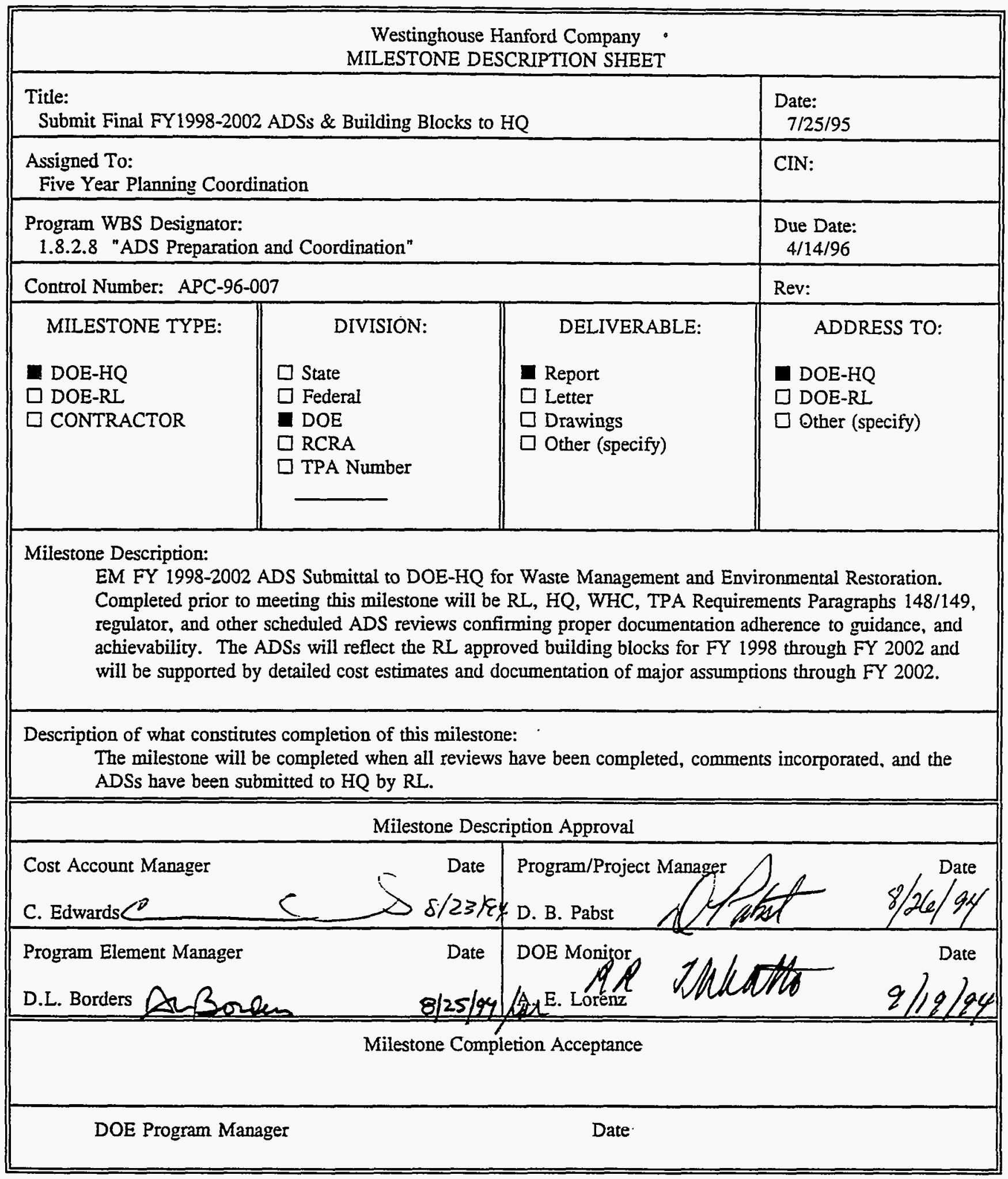




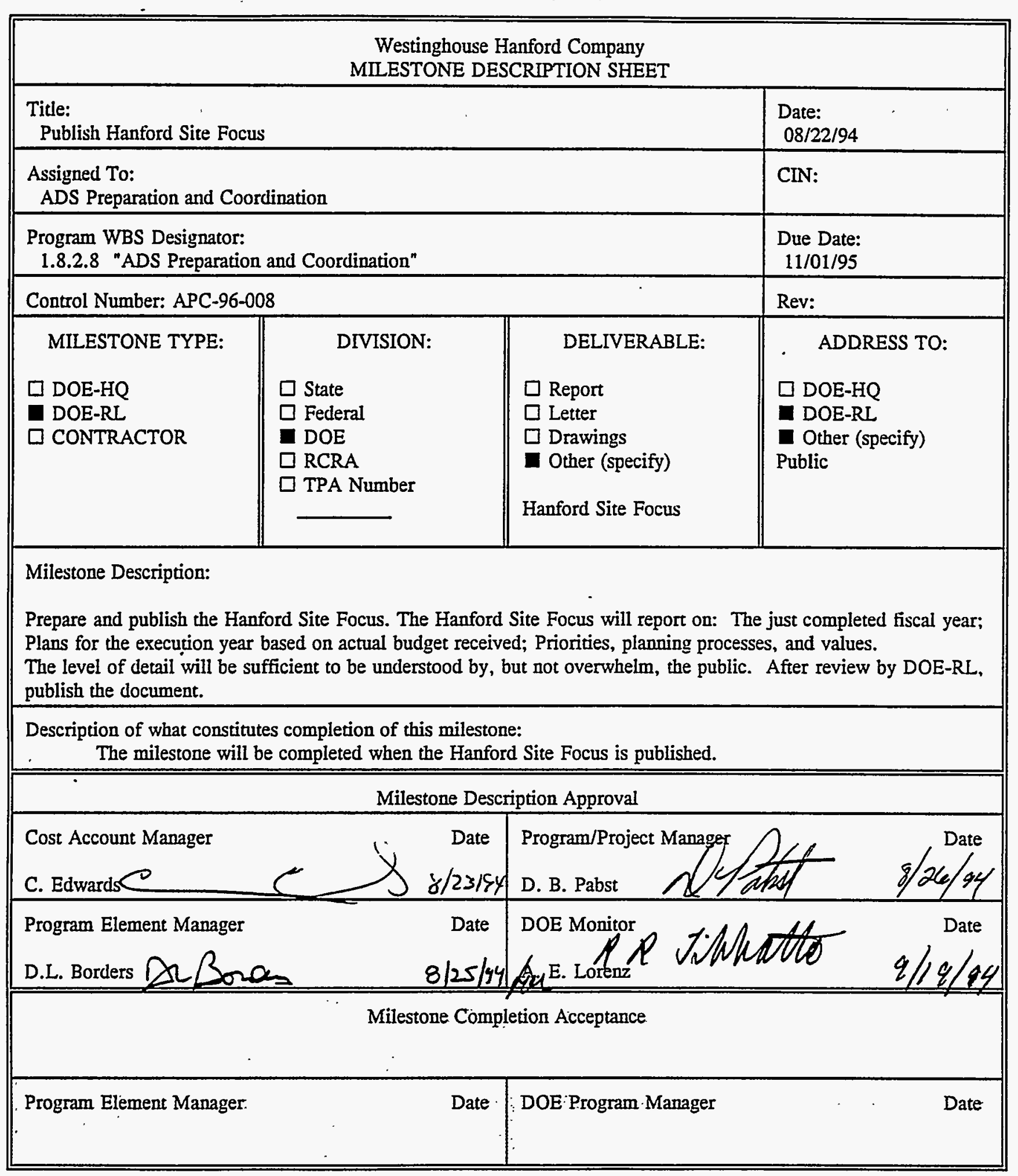




\begin{tabular}{|c|c|c|c|}
\hline \multicolumn{4}{|c|}{$\begin{array}{l}\text { Westinghouse Hanford Company } \\
\text { MILESTONE DESCRIPTION SHEET }\end{array}$} \\
\hline \multicolumn{3}{|c|}{$\begin{array}{l}\text { Title: } \\
\text { Publish Supplement to Hanford Site Focus }\end{array}$} & $\begin{array}{l}\text { Date: } \\
08 / 22 / 94\end{array}$ \\
\hline \multicolumn{3}{|c|}{$\begin{array}{l}\text { Assigned To: } \\
\text { ADS Preparation and Coordination }\end{array}$} & CIN: \\
\hline \multicolumn{3}{|c|}{$\begin{array}{l}\text { Program WBS Designator: } \\
\text { 1.8.2.8 "ADS Preparation and Coordination" }\end{array}$} & $\begin{array}{l}\text { Due Date: } \\
01 / 27 / 96\end{array}$ \\
\hline \multicolumn{3}{|c|}{ Control Number: APC-96-009 } & Rev: \\
\hline $\begin{array}{l}\text { MILESTONE TYPE: } \\
\square \text { DOE-HQ } \\
\text { DOE-RL } \\
\square \text { CONTRACTOR }\end{array}$ & $\begin{array}{l}\quad \text { DIVISION: } \\
\square \text { State } \\
\square \text { Federal } \\
\square \text { DOE } \\
\square \text { RCRA } \\
\square \text { TPA Number }\end{array}$ & $\begin{array}{l}\quad \text { DELIVERABLE: } \\
\square \text { Report } \\
\square \text { Letter } \\
\square \text { Drawings } \\
\square \text { Other (specify) } \\
\text { Supplement to Hanford } \\
\text { Site Focus }\end{array}$ & $\begin{array}{l}\text { ADDRESS TO: } \\
\square \text { DOE-HQ } \\
\text { DOE-RL } \\
\text { Qther (specify) } \\
\text { Public }\end{array}$ \\
\hline \multicolumn{4}{|c|}{$\begin{array}{l}\text { Milestone Description: } \\
\text { Within } 7 \text { days after the President's submits his budget to Congress, prepare for publication a supplement to } \\
\text { the Hanford Site Focus. The supplement will provide information on the support to Hanford workscope by } \\
\text { the President. The supplement will also provide information on workscope not supported. The level of } \\
\text { detail will be sufficient to be understood by, but not overwhelm, the public. After review by DOE-RL, } \\
\text { publish the document. }\end{array}$} \\
\hline \multicolumn{4}{|c|}{$\begin{array}{l}\text { Description of what constitutes completion of this milestone: } \\
\text { The milestone will be completed when supplement is published. }\end{array}$} \\
\hline \multicolumn{4}{|c|}{ Milestone Description Approval } \\
\hline \multicolumn{2}{|l|}{$\begin{array}{l}\text { Cost Account Manager } \\
\text { C. Edwards }\end{array}$} & P. B. Pabst & $8 / 26 / 94$ \\
\hline \multicolumn{2}{|c|}{$\begin{array}{lr}\text { Program Element Manager } & \text { Date } \\
\text { D.L. Borders } & 8 / 25 / 94\end{array}$} & \multicolumn{2}{|c|}{ DOE Monitor } \\
\hline \multicolumn{4}{|c|}{ Milestone Completion Acceptance } \\
\hline Program Element Manag & & DOE Program Manager & Date \\
\hline
\end{tabular}




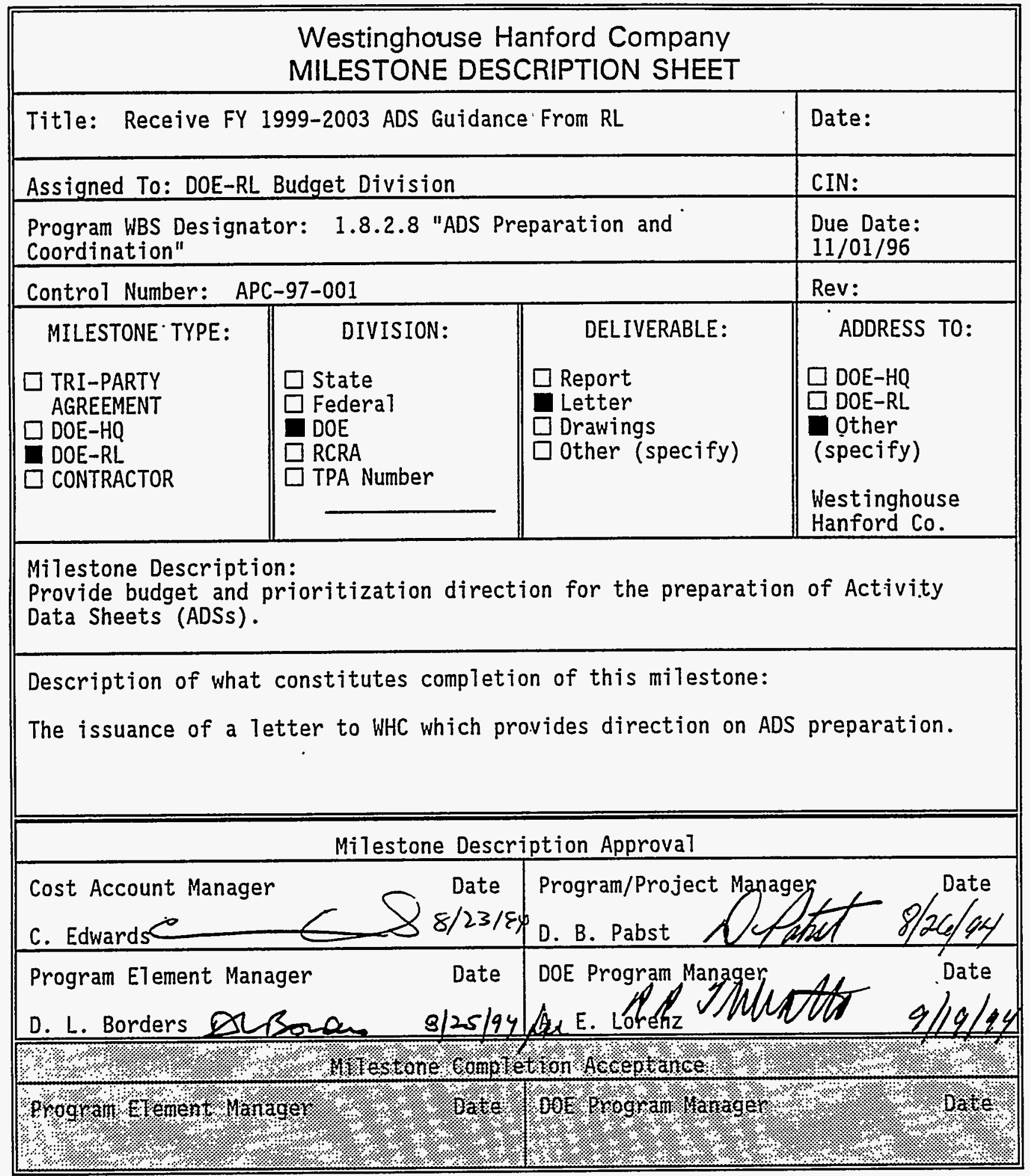




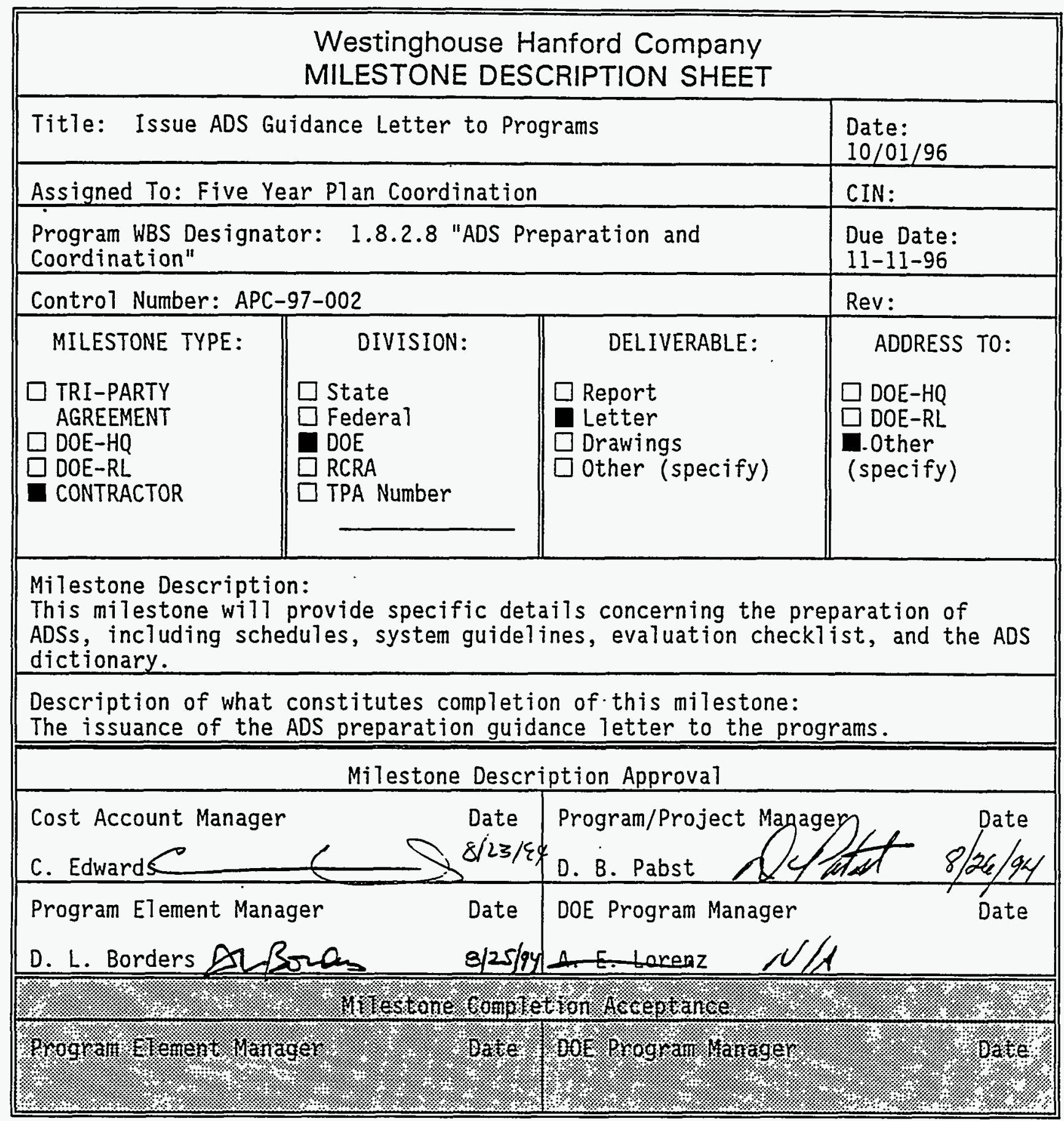




\begin{tabular}{|c|c|c|}
\hline \multicolumn{3}{|c|}{$\begin{array}{l}\text { Westinghouse Hanford Company } \\
\text { MILESTONE DESCRIPTION SHEET }\end{array}$} \\
\hline \multicolumn{2}{|c|}{$\begin{array}{l}\text { Title: } \\
\text { Submit Draft FY 1998-1999 Building Blocks to HQ }\end{array}$} & $\begin{array}{l}\text { Date: } \\
\quad 7 / 25 / 96\end{array}$ \\
\hline \multicolumn{2}{|l|}{$\begin{array}{l}\text { Assigned To: } \\
\quad \text { Five Year Planning Coordination }\end{array}$} & CIN: \\
\hline \multicolumn{2}{|c|}{$\begin{array}{l}\text { Program WBS Designator: } \\
\text { 1.8.2.8 "ADS Preparation and Coordination" }\end{array}$} & $\begin{array}{l}\text { Due Date: } \\
01 / 06 / 97 \\
\end{array}$ \\
\hline \multicolumn{2}{|l|}{ Control Number: APC-97-003 } & Rev: \\
\hline 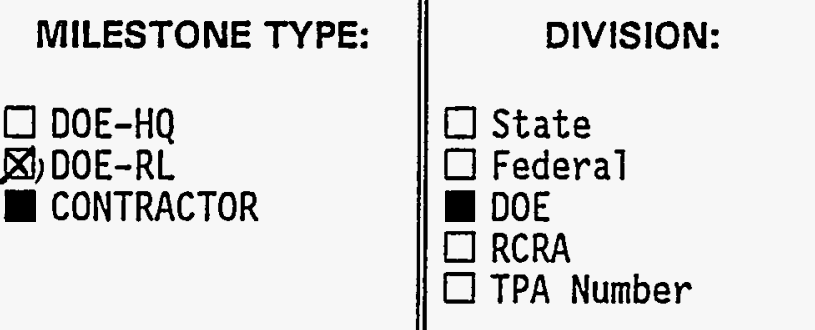 & $\begin{array}{l}\text { DELIVERABLE: } \\
\text { Report } \\
\square \text { Letter } \\
\square \text { Drawings } \\
\square \text { Other (specify) }\end{array}$ & $\begin{array}{c}\text { ADDRESS TO: } \\
\square \text { DOE-HQ } \\
\text { DOE-RL } \\
\square \text { Other (specify) }\end{array}$ \\
\hline \multicolumn{3}{|c|}{$\begin{array}{l}\text { Milestone Description: } \\
\text { Submittal of Building blocks for FY } 1998 \text { and FY } 1999 \text { to support FY } 1999 \text { ADS } \\
\text { planning to DOE-RL programs for Waste Management, Facility Transition, and } \\
\text { Environmental Restoration. All program's building blocks will be subject } \\
\text { to full review, integration and approval by WHC and RL Senior management. } \\
\text { The building blocks will be developed utilizing the RL priority system. }\end{array}$} \\
\hline \multicolumn{3}{|c|}{$\begin{array}{l}\text { Description of what constitutes completion of this milestone: } \\
\text { Submittal of each program's Building Blocks to RL. } \\
\end{array}$} \\
\hline \multicolumn{3}{|c|}{ Milestone Description Approval } \\
\hline Cost Account Manager & \multicolumn{2}{|c|}{ Program/Project Manager } \\
\hline $\begin{array}{l}\text { Program Element Manager } \\
\text { D.L. Borders Date }\end{array}$ & \multicolumn{2}{|c|}{ DOE Monitor } \\
\hline \multicolumn{3}{|c|}{ Milestone Completion Acceptance } \\
\hline \begin{tabular}{c} 
Program El ement Manager \\
\hdashline$\quad \therefore \quad$ \\
\hdashline$\quad:$ \\
\end{tabular} & DOE Program Manager & Date \\
\hline
\end{tabular}




\begin{tabular}{|c|c|c|c|}
\hline \multicolumn{4}{|c|}{$\begin{array}{l}\text { Westinghouse Hanford Company } \\
\text { MILESTONE DESCRIPTION SHEET }\end{array}$} \\
\hline \multicolumn{3}{|c|}{$\begin{array}{l}\text { Title: } \\
\text { Submit Draft FY 1999-2003 ADSs/FY 1997-1998 Building Blocks } \\
\text { to HQ/Regulators }\end{array}$} & $\begin{array}{l}\text { Date: } \\
\quad 7 / 25 / 96\end{array}$ \\
\hline \multicolumn{3}{|c|}{ Assigned To:Five Year Planning Coordination } & CIN: \\
\hline \multicolumn{3}{|c|}{$\begin{array}{l}\text { Program WBS Designator: } \\
\text { 1.8.2.8 "ADS Preparation and Coordination" }\end{array}$} & $\begin{array}{l}\text { Due Date: } \\
02 / 17 / 97 \\
\end{array}$ \\
\hline \multicolumn{3}{|c|}{ Control Number: APC-97-004 } & Rev: \\
\hline $\begin{array}{l}\text { MILESTONE TYPE: } \\
\qquad \text { DOE-HQ } \\
\text { D DOE-RL } \\
\text { CONTRACTOR }\end{array}$ & 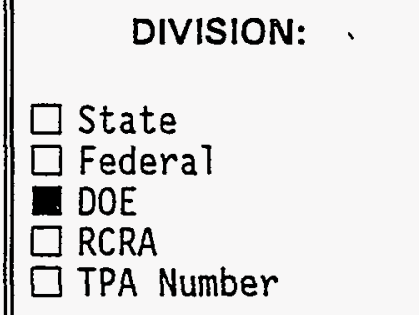 & $\begin{aligned} & \text { DELIVERABLE: } \\
& \text { Report } \\
& \text { Letter } \\
& \text { Drawings } \\
\square & \text { Other (specify) }\end{aligned}$ & $\begin{array}{c}\text { ADDRESS TO: } \\
\square \text { DOE-HQ } \\
\text { DOE-RL } \\
\square \text { other (specify) }\end{array}$ \\
\hline \multicolumn{4}{|c|}{$\begin{array}{l}\text { Milestone Description: } \\
\text { Submittal of draft ADSs and Building blocks for FY } 1998 \text { and FY } 1999 \text { to } \\
\text { support FY } 1999 \text { ADS planning to DOE-RL programs for Waste Management, } \\
\text { Facility Transition, and Environmental Restoration. This would constitute } \\
\text { the first submittal of the } 1999-2003 \text { budget request to RL. All program's } \\
\text { ADSs and Building Blocks will be subject to full review, integration and } \\
\text { approval by WHC and RL Senior management. The ADSs and Building Blocks } \\
\text { will be developed utilizing the RL priority system. }\end{array}$} \\
\hline \multicolumn{4}{|c|}{$\begin{array}{l}\text { Description of what constitutes completion of this milestone: } \\
\text { Submittal of each program's Draft FY 1999-2003 ADSS/FY 1998-1999 Building } \\
\text { Blocks/ to HQ/Regulators }\end{array}$} \\
\hline \multicolumn{4}{|c|}{ Milestone Description Approval } \\
\hline \multicolumn{2}{|c|}{ Cost Account Manager } & \multicolumn{2}{|c|}{ Program/Project Mangger $8 / 26 / 94$} \\
\hline \multicolumn{2}{|c|}{ 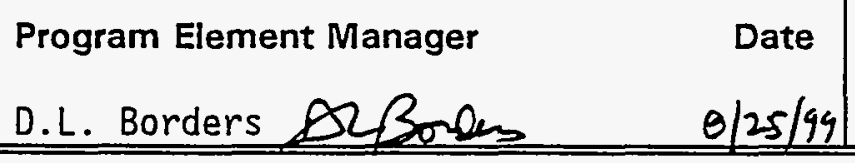 } & \multicolumn{2}{|c|}{ 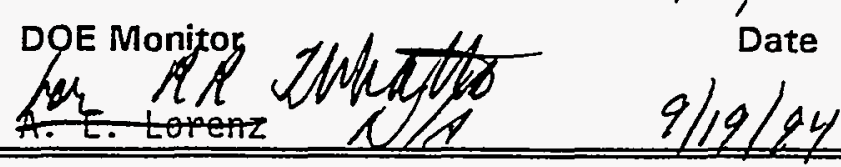 } \\
\hline \multicolumn{4}{|c|}{ Milestone Completion Acceptance } \\
\hline \multicolumn{2}{|c|}{ Program Element Manager Date } & DOE Program Manager & Date \\
\hline
\end{tabular}




\begin{tabular}{|c|c|c|}
\hline \multicolumn{3}{|c|}{$\begin{array}{l}\text { Westinghouse Hanford Company } \\
\text { MILESTONE DESCRIPTION SHEET }\end{array}$} \\
\hline \multicolumn{2}{|c|}{ Title: Submit Internal ADS Review Report to RL } & $\begin{array}{l}\text { Date: } \\
10 / 01 / 96\end{array}$ \\
\hline \multicolumn{2}{|c|}{ Assigned To: Five Year Planning Coordination } & CIN: \\
\hline \multicolumn{2}{|c|}{$\begin{array}{l}\text { Program WBS Designator: } 1.8 .2 .8 \text { "ADS Preparation and } \\
\text { Coordination" }\end{array}$} & $\begin{array}{l}\text { Due Date: } \\
03 / 17 / 97\end{array}$ \\
\hline \multicolumn{2}{|c|}{ Control Number: APC-97-005 } & Rev: \\
\hline 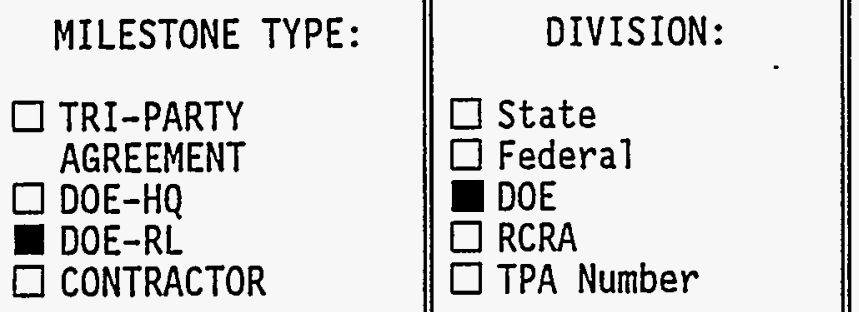 & $\begin{aligned} & \text { DELIVERABLE: } \\
& \text { Report } \\
& \text { Letter } \\
& \text { Drawings } \\
& \\
& \text { Other (specify) }\end{aligned}$ & $\begin{aligned} & \text { ADDRESS TO: } \\
& \text { DOE-HQ } \\
& \text { DOE-RL } \\
& \square \text {.Other } \\
& \text { (specify) }\end{aligned}$ \\
\hline \multicolumn{3}{|c|}{$\begin{array}{l}\text { Milestone Description: The purpose of the ADS review process is to provide a } \\
\text { thorough review as specified in the letter of March } 15,1996, \text { RL Budgets to } \\
\text { WHC, et a7, "FY } 1996 \text { ADS Reviews/Validation." The review wi11 be a quality and } \\
\text { compliance check on integration, technical scope, costs, and schedules as } \\
\text { defined and approved in the basel ine MYPP/FYWP. And providing a final report } \\
\text { delineating status of ADSs and backup documentation. Corrective actions } \\
\text { required will be per WHC-CM-2-17 "Budget Formulation Manual, "Section } 12 \\
\text { "MYPP/ADS Technical Review." }\end{array}$} \\
\hline \multicolumn{3}{|c|}{$\begin{array}{l}\text { Description of what constitutes completion of this milestone: } \\
\text { The milestone will be completed when the ADS Technical Review Report is sent to } \\
\text { the program managers and RL. }\end{array}$} \\
\hline \multicolumn{3}{|c|}{ Milestone Description Approval } \\
\hline Cost Account Manager & \multicolumn{2}{|c|}{ Program/Project Managgr $8 / 26 / 94$} \\
\hline $\begin{array}{lc}\text { Program Element Manager } & \text { Date } \\
\text { D. L. Borders D Bonas } & 8 / 25 / 94\end{array}$ & \multicolumn{2}{|c|}{ DOE Program Mangger $r$ Date } \\
\hline \multicolumn{3}{|c|}{ 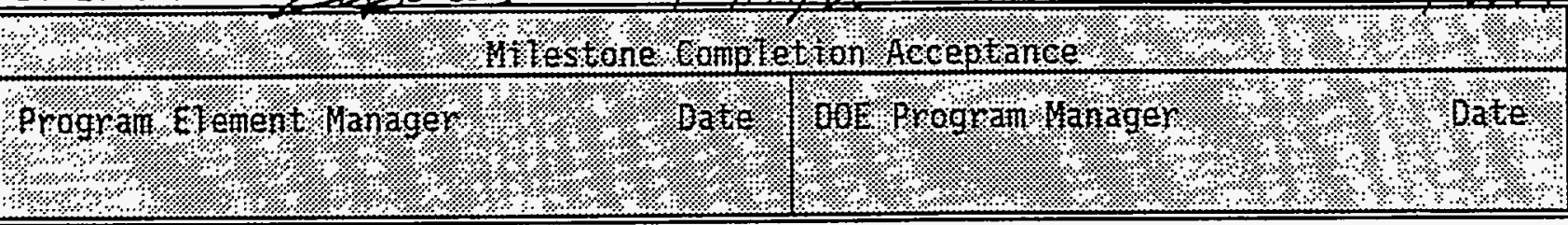 } \\
\hline
\end{tabular}




\begin{tabular}{|c|c|c|c|}
\hline \multicolumn{4}{|c|}{$\begin{array}{l}\text { Westinghouse Hanford Company } \\
\text { MILESTONE DESCRIPTION SHEET }\end{array}$} \\
\hline \multicolumn{3}{|c|}{$\begin{array}{l}\text { Title: } \\
\text { Submit Final FY1999-2003 ADSs \& Building Blocks to RL }\end{array}$} & $\begin{array}{l}\text { Date: } \\
\text { 10/01/96 }\end{array}$ \\
\hline \multicolumn{3}{|c|}{ Assigned To: Five Year Planning Coordination } & CIN: \\
\hline \multicolumn{3}{|c|}{$\begin{array}{l}\text { Program WBS Designator: } \\
\text { 1.8.2.8 "ADS Preparation and Coordination" }\end{array}$} & $\begin{array}{l}\text { Due Date: } \\
4 / 14 / 97\end{array}$ \\
\hline \multicolumn{3}{|c|}{ Control Number: APC-97-006 } & Rev: \\
\hline $\begin{array}{l}\text { MILESTONE TYPE: } \\
\square \text { DOE-HQ } \\
\square \text { DOE-RL } \\
\square \text { CONTRACTOR }\end{array}$ & $\begin{aligned} \quad \text { DIVISION: } \\
\\
\square \text { State } \\
\square \text { Federal } \\
\square \text { DOE } \\
\square \text { RCRA } \\
\square \text { TPA Number }\end{aligned}$ & $\begin{array}{l}\text { DELIVERABLE: } \\
\square \text { Report } \\
\square \text { Letter } \\
\square \text { Drawings } \\
\square \text { Other (specify) }\end{array}$ & $\begin{aligned} & \text { ADDRESS TO: } \\
& \square \text { DOE-HQ } \\
& \text { DOE-RL } \\
& \square \text { Other (specify) }\end{aligned}$ \\
\hline \multicolumn{4}{|c|}{$\begin{array}{l}\text { Milestone Description: } \\
\text { EM FY 1999-2003 ADS Submitral to RL for Waste Management and Environmental Restoration. } \\
\text { Completed prior to meeting this milestone will be RL, HQ, WHC, TPA Requirements Paragraphs 148/149, } \\
\text { regulator, and other scheduled ADS reviews confirming proper documentation adherence to guidance, } \\
\text { achievability, and agreement with work scope. The ADSs will reflect the approved building blocks for FY } \\
1999 \text { through FY } 2003 \text { and will be supported by detailed cost estimates and documentation of major } \\
\text { assumptions through FY } 2003 \text {. }\end{array}$} \\
\hline \multicolumn{4}{|c|}{$\begin{array}{l}\text { Description of what constitutes completion of this milestone: } \\
\text { The milestone will be completed when all reviews have been completed, comments incorporated, and the } \\
\text { ADSs have been submitted to RL. }\end{array}$} \\
\hline \multicolumn{4}{|c|}{ Milestone Description Approval } \\
\hline \multicolumn{2}{|c|}{ C. Edwards } & \multicolumn{2}{|c|}{ Drogram/Project Manager Babst $8 / 26 / 94$} \\
\hline \multicolumn{2}{|c|}{$\begin{array}{lr}\text { Program Element Manager } & \text { Date } \\
\text { D.L. Borders AuBorQuer } & 8 / 25 / 49 \\
\end{array}$} & \multicolumn{2}{|c|}{ 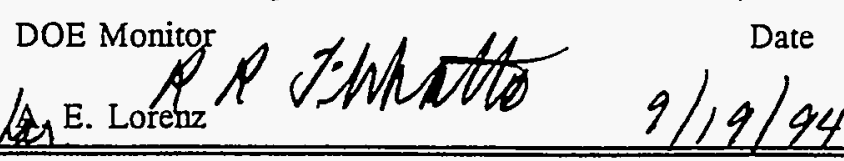 } \\
\hline \multicolumn{4}{|c|}{ Milestone Completion Acceptance } \\
\hline \multicolumn{2}{|l|}{ Program Element Manager } & DOE Program Manager & Date \\
\hline
\end{tabular}




\begin{tabular}{|c|c|c|c|}
\hline \multicolumn{4}{|c|}{$\begin{array}{l}\text { Westinghouse Hanford Company } \\
\text { MILESTONE DESCRIPTION SHEET }\end{array}$} \\
\hline \multicolumn{3}{|c|}{$\begin{array}{l}\text { Title: } \\
\text { Submit Final FY1999-2003 ADSs \& Building Blocks to HQ }\end{array}$} & $\begin{array}{l}\text { Date: } \\
\quad 7 / 25 / 96\end{array}$ \\
\hline \multicolumn{3}{|c|}{$\begin{array}{l}\text { Assigned To: } \\
\text { Five Year Planning Coordination }\end{array}$} & CIN: \\
\hline \multicolumn{3}{|c|}{$\begin{array}{l}\text { Program WBS Designator: } \\
\text { 1.8.2.8 "ADS Preparation and Coordination" }\end{array}$} & $\begin{array}{c}\text { Due Date: } \\
\text { 4/14/97 }\end{array}$ \\
\hline \multicolumn{3}{|c|}{ Control Number: APC-97-007 } & Rev: \\
\hline $\begin{array}{l}\text { MILESTONE TYPE: } \\
\text { DOE-HQ } \\
\square \text { DOE-RL } \\
\square \text { CONTRACTOR }\end{array}$ & $\begin{array}{l}\quad \text { DIVISION: } \\
\square \text { State } \\
\square \text { Federal } \\
\square \text { DOE } \\
\square \text { RCRA } \\
\square \text { TPA Number }\end{array}$ & $\begin{aligned} & \text { DELIVERABLE: } \\
& \text { Report } \\
& \square \text { Letter } \\
& \text { Drawings } \\
& \square \text { Other (specify) }\end{aligned}$ & $\begin{aligned} & \text { ADDRESS TO: } \\
& \text { DOE-HQ } \\
& \square \text { DOE-RL } \\
& \square \text { Other (specify) }\end{aligned}$ \\
\hline \multicolumn{4}{|c|}{$\begin{array}{l}\text { Milestone Description: } \\
\text { EM FY 1999-2003 ADS Submittal to DOE-HQ for Waste Management and Environmental Restoration. } \\
\text { Completed prior to meeting this milestone will be RL, HQ, WHC, TPA Requirements Paragraphs 148/149, } \\
\text { regulator, and other scheduled ADS reviews confirming proper documentation adherence to guidance, and } \\
\text { achievability. The ADSs will reflect the RL approved building blocks for FY } 1999 \text { through FY } 2003 \text { and } \\
\text { will be supported by detailed cost estimates and documentation of major assumptions through FY } 2003 \text {. }\end{array}$} \\
\hline \multicolumn{4}{|c|}{$\begin{array}{l}\text { Description of what constitutes completion of this milestone: } \\
\text { The milestone will be completed when all reviews have been completed, comments incorporated, and the } \\
\text { ADSs have been submitted to HQ by RL. }\end{array}$} \\
\hline \multicolumn{4}{|c|}{ Milestone Description Approval } \\
\hline \multicolumn{2}{|l|}{$\begin{array}{l}\text { Cost Account Manager } \\
\text { C. Edwards }\end{array}$} & $\begin{array}{l}\text { Program/Project Mang } \\
\text { D. B. Pabst }\end{array}$ & $8 / 26 / 94$ \\
\hline \multicolumn{2}{|c|}{ Program Element Manager } & DOE Monitor Lorenz $A / A$ & UTtor $9 / 19 / 94$ \\
\hline \multicolumn{4}{|c|}{ Milestone Completion Acceptance } \\
\hline DOE Program N & iger & Date & \\
\hline
\end{tabular}




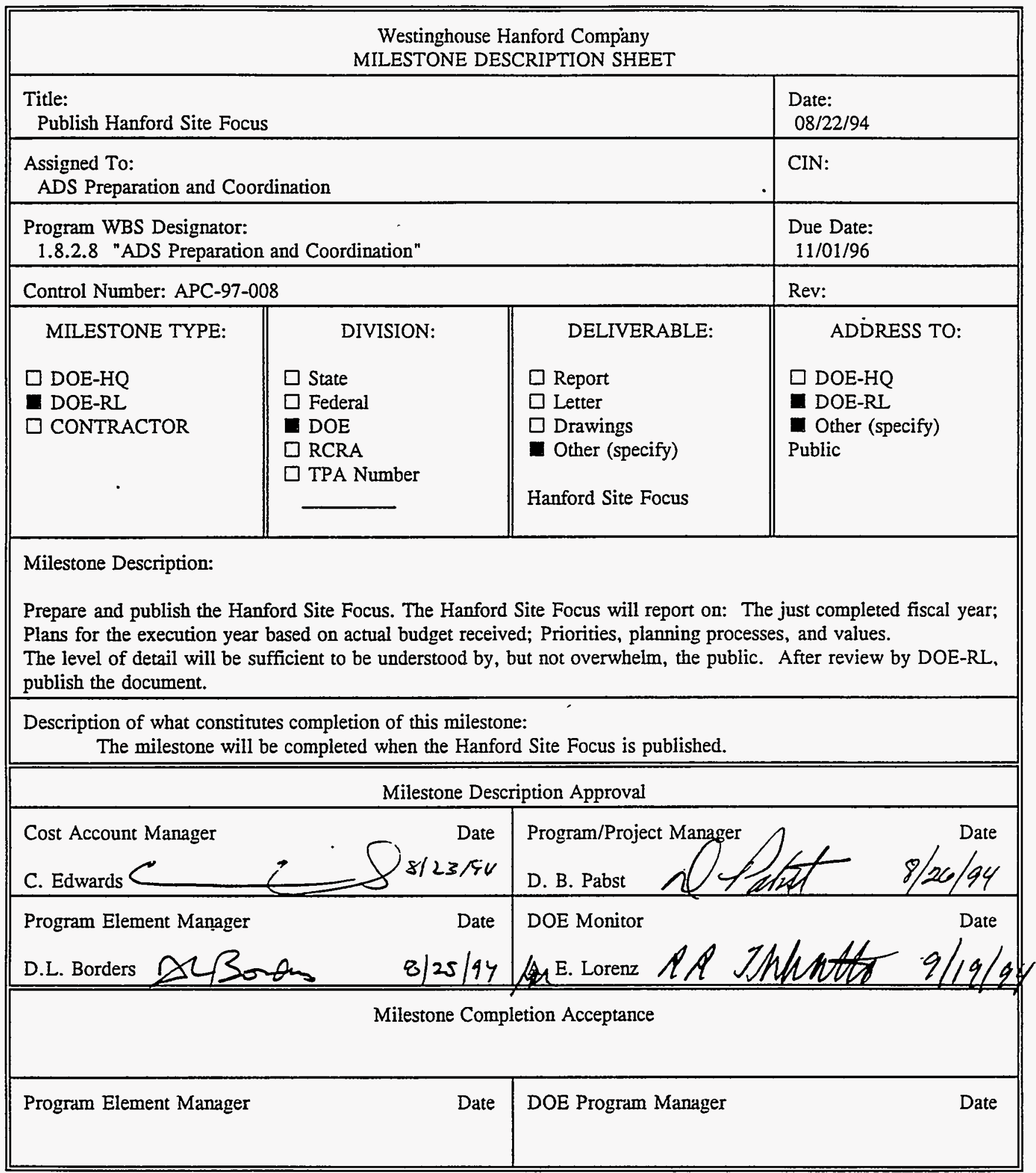




\begin{tabular}{|c|c|c|c|}
\hline \multicolumn{4}{|c|}{$\begin{array}{l}\text { Westinghouse Hanford Company } \\
\text { MILESTONE DESCRIPTION SHEET }\end{array}$} \\
\hline \multicolumn{3}{|c|}{$\begin{array}{l}\text { Title: } \\
\text { Publish Supplement to Hanford Site Focus }\end{array}$} & $\begin{array}{l}\text { Date: } \\
08 / 22 / 94\end{array}$ \\
\hline \multicolumn{3}{|c|}{$\begin{array}{l}\text { Assigned To: } \\
\text { ADS Preparation and Coordination }\end{array}$} & CIN: \\
\hline \multicolumn{3}{|c|}{$\begin{array}{l}\text { Program WBS Designator: } \\
\text { 1.8.2.8 "ADS Preparation and Coordination" }\end{array}$} & $\begin{array}{l}\text { Due Date: } \\
01 / 27 / 97\end{array}$ \\
\hline \multicolumn{3}{|c|}{ Control Number: APC-97-009 } & Rev: \\
\hline $\begin{array}{l}\text { MILESTONE TYPE: } \\
\square \text { DOE-HQ } \\
\square \text { DOE-RL } \\
\square \text { CONTRACTOR }\end{array}$ & $\begin{aligned} & \text { DIVISION: } \\
& \square \text { State } \\
& \square \text { Federal } \\
& \text { DOE } \\
& \square \text { RCRA } \\
& \square \text { TPA Number }\end{aligned}$ & $\begin{array}{l}\quad \text { DELIVERABLE: } \\
\square \text { Report } \\
\square \text { Letter } \\
\square \text { Drawings } \\
\square \text { Other (specify) } \\
\text { Supplement to Hanford } \\
\text { Site Focus }\end{array}$ & $\begin{array}{l}\text { ADDRESS TO: } \\
\square \text { DOE-HQ } \\
\square \text { DOE-RL } \\
\text { Other (specify) } \\
\text { Public }\end{array}$ \\
\hline \multicolumn{4}{|c|}{$\begin{array}{l}\text { Milestone Description: } \\
\text { Within } 7 \text { days after the President's submits his budget to Congress, prepare for publication a supplement to } \\
\text { the Hanford Site Focus. The supplement will provide information on the support to Hanford workscope by } \\
\text { the President. The supplement will also provide information on workscope not supported. The level of } \\
\text { detail will be sufficient to be understood by, but not overwhelm, the public. After review by DOE-RL, } \\
\text { publish the document. }\end{array}$} \\
\hline \multicolumn{4}{|c|}{$\begin{array}{l}\text { Description of what constitutes completion of this milestone: } \\
\text { The milestone will be completed when supplement is published. }\end{array}$} \\
\hline \multicolumn{4}{|c|}{ Milestone Description Approval } \\
\hline $\begin{array}{l}\text { Cost Account Manager } \\
\text { C. Edwards }<\end{array}$ & S. & Program/Project Manage & $8 / 26 / 9 y$ \\
\hline \multicolumn{3}{|c|}{ 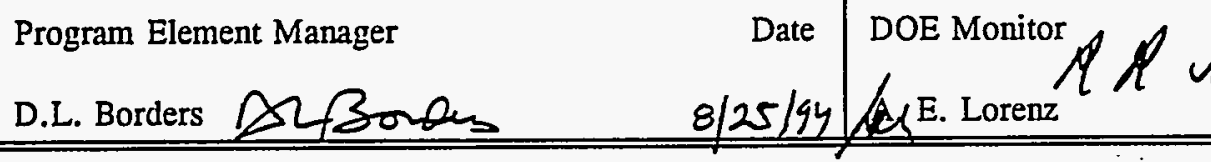 } & NTW $9 / 19 / 94$ \\
\hline \multicolumn{4}{|c|}{ Milestone Completion Acceptance } \\
\hline Program Element Manag & : & DOE Program Manager & Date \\
\hline
\end{tabular}


Planning Integration Multi-Year Program Plan

This page

intentionally left blank. 


\begin{tabular}{|c|c|c|c|}
\hline \multicolumn{4}{|c|}{$\begin{array}{l}\text { Westinghouse Hanford Company } \\
\text { MILESTONE DESCRIPTION SHEET }\end{array}$} \\
\hline \multicolumn{3}{|c|}{$\begin{array}{r}\text { Title: Integrated Risk Assessment Program - Fy } 1986 \text { Mypp } \\
\text { Pisik Guidamen the }\end{array}$} & Date: $9 / 17 / 94$ \\
\hline \multicolumn{3}{|c|}{ Assigned To: Judith A. Mahaffey N W J JAM } & \multirow{2}{*}{$\begin{array}{l}\text { CIN: } \\
\text { Due Date: } 5 / 30 / 95\end{array}$} \\
\hline \multicolumn{3}{|c|}{ Program WBS Designator: 1.8.2.6 } & \\
\hline \multicolumn{3}{|c|}{ Control Number: IRA-95-001 } & Rev: 0 \\
\hline $\begin{array}{l}\text { MILESTONE TYPE: } \\
\square \text { DOE-HQ } \\
\text { X DOE-RL } \\
\square \text { CONTRACTOR }\end{array}$ & $\begin{array}{l}\quad \text { DIVISION: } \\
\square \text { State } \\
\square \text { Federal } \\
\text { X DOE } \\
\square \text { RCRA } \\
\square \text { TPA Number }\end{array}$ & $\begin{array}{l}\text { DELIVERABLE: } \\
\times \text { Report } \\
\square \text { Letter } \\
\square \text { Drawings } \\
\square \text { Other (specify) }\end{array}$ & $\begin{array}{l}\text { ADDRESS TO: } \\
\square \text { DOE-HQ } \\
\text { X DOE-RL } \\
\square \text { Other (specify) }\end{array}$ \\
\hline \multirow{2}{*}{\multicolumn{4}{|c|}{$\begin{array}{l}\text { Milestone Description: One objective of Hanford cleanup is to reduce health and } \\
\text { ecological risks, but these risks have not yet been estimated consistently or compared } \\
\text { across Site programs. IRAP is comparing these risks consistently and comprehensively } \\
\text { at a Site level to understand why, when, and how much remediation is needed and to } \\
\text { assist systems engineering in evaluating remediation options from a risk perspective. } \\
\text { However, much required information is unavailable or unusable, and to expedite } \\
\text { establishing a risk baseline, information needs to be produced by programs so it can be } \\
\text { integrated across programs by IRAP. } \\
\text { To compare risks across programs, IRAP requires program-specific information, including } \\
\text { inventory information, release scenarios, and risk results. Guidance will be provided to } \\
\text { programs on what information is needed, when, and in what format. This deliverable will } \\
\text { direct that a document describing methods for qualitative and quantitative risk } \\
\text { assessments will be provided at the end of FY } 1995 \text { and early in FY 1996, respectively. } \\
\text { The FY } 1995 \text { document will provide guidance on methods and assumptions for } \\
\text { developing this information. Methods will include approaches both for assessing risks } \\
\text { and their uncertainties. }\end{array}$}} \\
\hline & & & \\
\hline \multicolumn{4}{|c|}{$\begin{array}{l}\text { Description of what constitutes completion of this milestone: Delivery of guidance to } \\
\text { programs as part of the FY } 1996 \text { MYPP guidance issues by the } 1.8 .2 \text { program. The } \\
\text { guidance will describe requirement for provision of risk related information to IRAP for } \\
\text { Site-wide integration. }\end{array}$} \\
\hline \multicolumn{4}{|c|}{ Milestone Description Approval } \\
\hline \multicolumn{2}{|c|}{$\begin{array}{l}\text { Cost Account Manager } \\
\text { Judith A. Mahaffey }\end{array}$} & \multicolumn{2}{|c|}{ Program/Project Manager 2 Date } \\
\hline
\end{tabular}




\begin{tabular}{|c|c|}
\hline \multicolumn{2}{|c|}{$\begin{array}{l}\text { Westinghouse Hanford Company } \\
\text { MILESTONE DESCRIPTION SHEET }\end{array}$} \\
\hline Title: Integrated Risk Assessment Program & Date: $9 / 17 / 94$ \\
\hline Assigned To: Judith A. Mahaffey & CIN: \\
\hline Program WBS Designator: 1.8.2.6 & Due Date: $5 / 30 / 95$ \\
\hline Control Number: IRA-95-001 & \begin{tabular}{l|l} 
Rev: 0 \\
\end{tabular} \\
\hline $\begin{array}{l}\text { Program Element Manager } \\
\text { Judith A. Mahaffey }\end{array}$ & 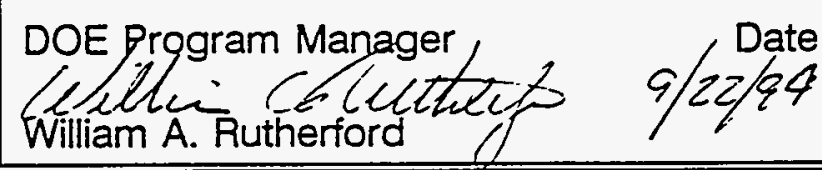 \\
\hline 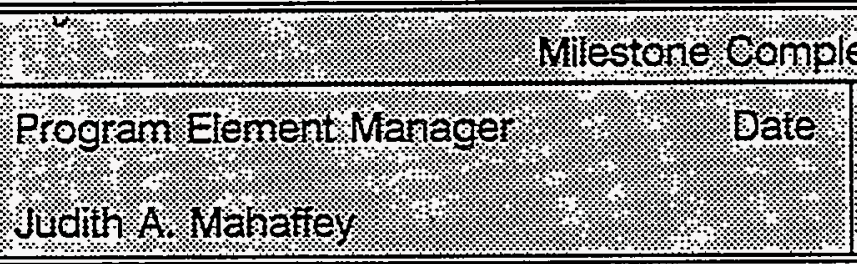 & $\begin{array}{l}\text { etion acceptance } \\
\text { DeE Progranthanager }\end{array}$ \\
\hline
\end{tabular}




\begin{tabular}{|c|c|c|c|}
\hline \multicolumn{4}{|c|}{$\begin{array}{l}\text { Westinghouse Hanford Company } \\
\text { MILESTONE DESCRIPTION SHEET }\end{array}$} \\
\hline \multicolumn{3}{|c|}{ 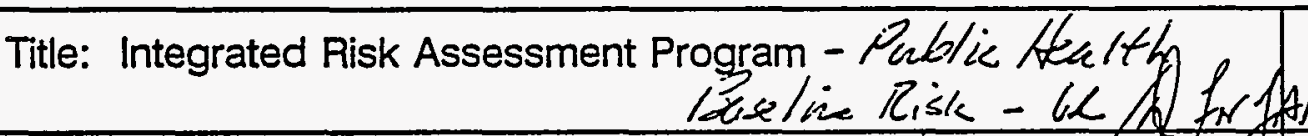 } & $\begin{array}{l}\text { Date: } 9 / 17 / 94 \\
M M\end{array}$ \\
\hline \multicolumn{3}{|c|}{ Assigned To: Judith A. Mahaffey } & CIN: \\
\hline \multicolumn{3}{|c|}{ Program WBS Designator: 1.8.2.6 } & Due Date: $9 / 30 / 95$ \\
\hline \multicolumn{3}{|c|}{ Control Number: IRA-95-002 } & Rev: 0 \\
\hline $\begin{array}{l}\text { MILESTONE TYPE: } \\
\square \text { DOE-HQ } \\
\text { X DOE-RL } \\
\square \text { CONTRACTOR }\end{array}$ & $\begin{array}{l}\quad \text { DIVISION: } \\
\square \text { State } \\
\square \text { Federal } \\
\text { X DOE } \\
\square \text { RCRA } \\
\square \text { TPA Number }\end{array}$ & $\begin{array}{l}\text { DELIVERABLE: } \\
X \text { Report } \\
\square \text { Letter } \\
\square \text { Drawings } \\
\square \text { Other (specify) }\end{array}$ & $\begin{array}{l}\text { ADDRESS TO: } \\
\square \text { DOE-HQ } \\
\text { X DOE-RL } \\
\square \text { Other (specify) }\end{array}$ \\
\hline \multirow{2}{*}{\multicolumn{4}{|c|}{$\begin{array}{l}\text { Milestone Description: One objective of Hanford cleanup is to reduce health and } \\
\text { ecological risks, but these risks have not yet been estimated consistently or compared } \\
\text { across Site programs. IRAP is comparing these risks consistently and comprehensively } \\
\text { at a Site level to understand why, when, and how much remediation is needed and to } \\
\text { assist systems engineering in evaluating remediation options from a risk perspective. } \\
\text { The first step of this work was initiated in mid-FY 1994. In FY 1994, scored and ranked } \\
\text { tables of components by exposure scenario for chemicals and radionuclides were } \\
\text { produced using qualitative methods. These were developed for individual or grouped } \\
\text { components in five program areas: tanks, buried solid wastes, contaminated soil and } \\
\text { groundwater from past practices, facilities, and nuclear materials. A document describing } \\
\text { methods and results was produced. In FY 1995, IRAP will conduct and document the } \\
\text { second step of this work for evaluating current public health risks from Hanford wastes } \\
\text { and materials (called a public health baseline) across programs at Hanford. The } \\
\text { purposes of this second step are to validate scores obtained in FY } 1994 \text { and to anchor } \\
\text { results to particular risk values. }\end{array}$}} \\
\hline & & & \\
\hline \multicolumn{4}{|c|}{$\begin{array}{l}\text { In FY 1995, public health baseline risks will be assessed and documented for selected } \\
\text { components so that all components either have an associated quantitative risk or } \\
\text { quantitative bounds. This will provide information both that validates the qualitative } \\
\text { ranking within and across program areas and that anchors ranking results to levels of risk } \\
\text { that guide decisions by regulators. Results will be provided to support Site-level decision } \\
\text { making about priorities and also will be capable of reconfiguration to support other } \\
\text { decisions. Risks associated with components may have to be reassessed in the future, } \\
\text { however, because (1) the FY } 1995 \text { assessment will not be exhaustive and (2) new } \\
\text { inventory or characterization data may become available. }\end{array}$} \\
\hline
\end{tabular}




\section{Westinghouse Hanford Company MILESTONE DESCRIPTION SHEET}

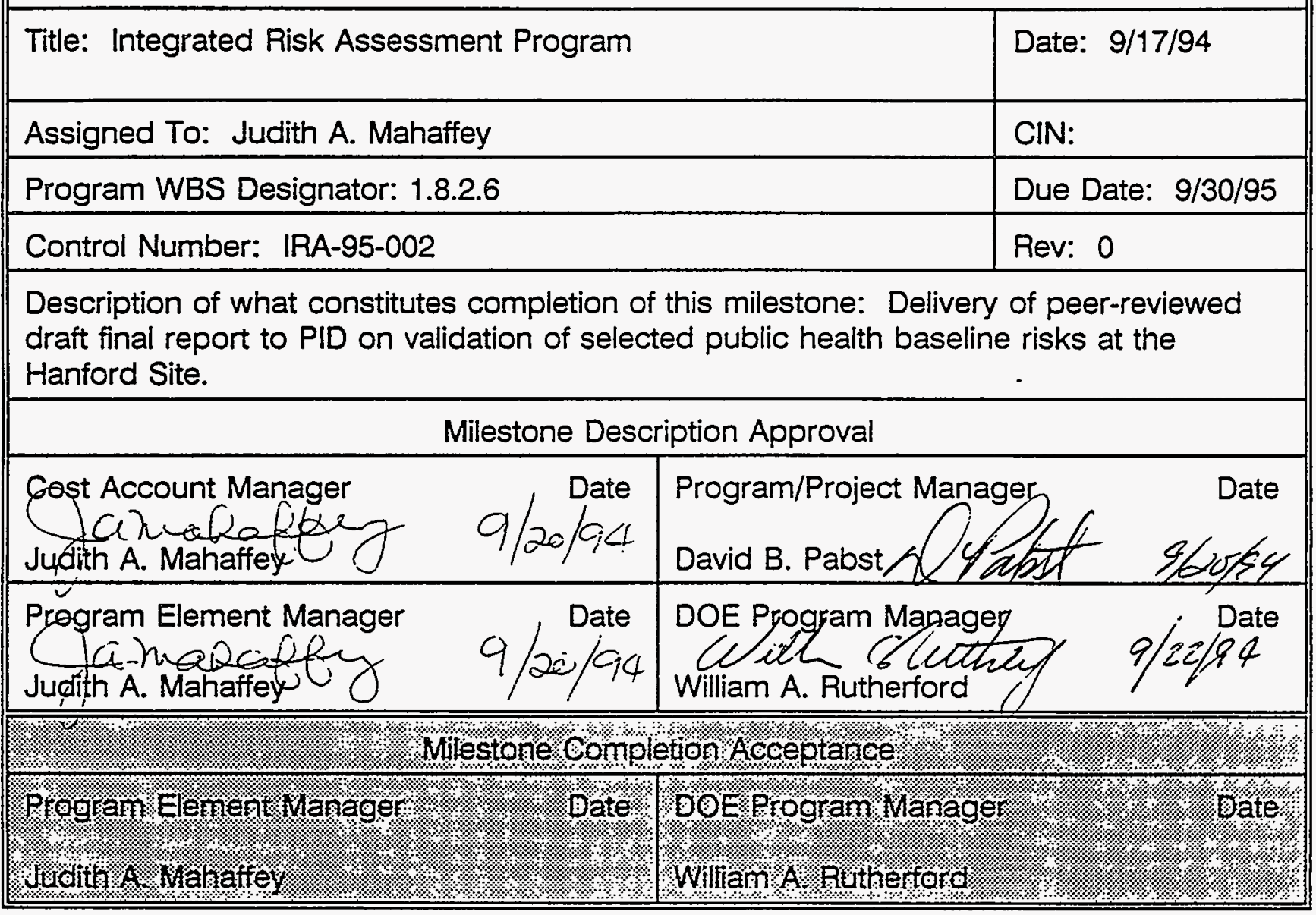




\begin{tabular}{|c|c|c|c|}
\hline \multicolumn{4}{|c|}{$\begin{array}{l}\text { Westinghouse Hanford Company } \\
\text { MILESTONE DESCRIPTION SHEET }\end{array}$} \\
\hline \multicolumn{4}{|c|}{ Title: Integrated Risk Assessment Program - Rauking of Risic gate: 9/17/94 } \\
\hline \multicolumn{4}{|c|}{ Assigned To: Judith A. Mahaffey } \\
\hline \multicolumn{3}{|c|}{ Program WBS Designator: 1.8.2.6 } & Due Date: $9 / 30 / 95$ \\
\hline \multicolumn{3}{|c|}{ Control Number: IRA-95-003 } & Rev: 0 \\
\hline $\begin{array}{l}\text { MILESTONE TYPE: } \\
\text { प DOE-HQ } \\
\text { X DOE-RL } \\
\square \text { CONTRACTOR }\end{array}$ & $\begin{array}{l}\quad \text { DIVISION: } \\
\square \text { State } \\
\square \text { Federal } \\
\text { X DOE } \\
\square \text { RCRA } \\
\square \text { TPA Number }\end{array}$ & $\begin{array}{l}\text { DELIVERABLE: } \\
X \text { Report } \\
\square \text { Letter } \\
\square \text { Drawings } \\
\square \text { Other (specify) }\end{array}$ & $\begin{array}{l}\text { ADDRESS TO: } \\
\square \text { DOE-HQ } \\
\text { X DOE-RL } \\
\square \text { Other (specify) }\end{array}$ \\
\hline \multicolumn{4}{|c|}{$\begin{array}{l}\text { Milestone Description: One objective of Hanford cleanup is to reduce health and } \\
\text { ecological risks, but these risks have not yet been estimated consistently or compared } \\
\text { across Site programs. IRAP is comparing these risks consistently and comprehensively } \\
\text { at a Site level to understand why, when, and how much remediation is needed and to } \\
\text { assist systems engineering in evaluating remediation options from a risk perspective. } \\
\text { In FY 1995, IRAP will conduct the first step of the process for evaluating current worker } \\
\text { health and safety risks and for evaluating current ecological health risks (called baseline } \\
\text { risks) from Hanford wastes and materials across programs at Hanford. This work will } \\
\text { build on the public health baseline scoring methods and application developed in FY } \\
1994 \text {. The purpose of this step is to develop and document comprehensive qualitative } \\
\text { scoring methods for worker and ecological risks, to be provided to Hanford Site } \\
\text { programs for application in early FY 1996. A report will be developed that describes } \\
\text { qualitative methods for scoring and ranking public, worker, and ecological baseline risks. }\end{array}$} \\
\hline \multicolumn{4}{|c|}{$\begin{array}{l}\text { Description of what constitutes completion of this milestone: Delivery of peer-reviewed } \\
\text { draft final report to PID on scoring and ranking of public health, worker health and safety, } \\
\text { and ecological health baseline risks at the Hanford Site. }\end{array}$} \\
\hline \multicolumn{4}{|c|}{ Milestone Description Approval } \\
\hline \multicolumn{2}{|c|}{$\begin{array}{l}\text { Cest Account Manager } \\
\text { a hua Rad Q y } \\
\text { Judith A. Mahaffey }\end{array}$} & $\begin{array}{l}\text { Program/Project } M \\
\text { David B. Pabst }\end{array}$ & Date \\
\hline \multicolumn{2}{|c|}{$\begin{array}{l}\text { Program Element Manager } \\
\text { Juedith A. Mahaffey }\end{array}$} & $\begin{array}{l}\text { DOE Program Mar } \\
\text { William A. Rutherfo }\end{array}$ & athey a/zifate \\
\hline
\end{tabular}




\begin{tabular}{|c|c|}
\hline \multicolumn{2}{|c|}{$\begin{array}{l}\text { Westinghouse Hanford Company } \\
\text { MILESTONE DESCRIPTION SHEET }\end{array}$} \\
\hline Title: Integrated Risk Assessment Program & Date: $9 / 17 / 94$ \\
\hline Assigned To: Judith A. Mahaffey & CIN: \\
\hline Program WBS Designator: 1.8.2.6 & Due Date: $9 / 30 / 95$ \\
\hline Control Number: IRA-95-003 & Rev: 0 \\
\hline 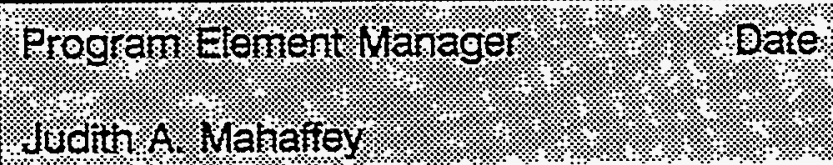 & 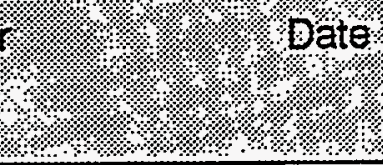 \\
\hline
\end{tabular}




\begin{tabular}{|c|c|c|c|}
\hline \multicolumn{4}{|c|}{$\begin{array}{l}\text { Westinghouse Hanford Company } \\
\text { MILESTONE DESCRIPTION SHEET }\end{array}$} \\
\hline \multicolumn{3}{|c|}{ Title: Integrated Risk Assessment Program } & Date: $6 / 15 / 94$ \\
\hline \multicolumn{3}{|c|}{ Assigned To: Judith A. Mahaffey } & CIN: \\
\hline \multicolumn{3}{|c|}{ Program WBS Designator: 1.8.2.6 } & Due Date: $9 / 30 / 96$ \\
\hline \multicolumn{3}{|c|}{ Control Number: IRA-96-001 } & Rev: 0 \\
\hline $\begin{array}{l}\text { MILESTONE TYPE: } \\
\square \text { DOE-HQ } \\
\text { X DOE-RL } \\
\square \text { CONTRACTOR }\end{array}$ & $\begin{aligned} & \text { DIVISION: } \\
& \square \text { State } \\
& \square \text { Federal } \\
\text { X DOE } & \\
& \text { RCRA } \\
& \square \text { TPA Number }\end{aligned}$ & $\begin{array}{l}\text { DELIVERABLE: } \\
\text { X Report } \\
\square \text { Letter } \\
\square \text { Drawings } \\
\square \text { Other (specify) }\end{array}$ & $\begin{array}{l}\text { ADDRESS TO: } \\
\square \text { DOE-HQ } \\
\text { X DOE-RL } \\
\square \text { Other (specify) }\end{array}$ \\
\hline \multirow{2}{*}{\multicolumn{4}{|c|}{$\begin{array}{l}\text { Milestone Description: One objective of Hanford cleanup is to reduce health and ecological } \\
\text { risks. IRAP is quantifying these risks consistently and comprehensively at a sitewide level to } \\
\text { understand if and when remediation is needed and to evaluate options for remediation from a } \\
\text { risk perspective. In FY 1996, IRAP will provide sitewide information on current worker health } \\
\text { and safety risks from Hanford wastes and materials (called a worker health and safety baseline). } \\
\text { This work was initiated in mid-FY 1994. Prioritized lists of elements (e.g., each facility or } \\
\text { complex of facilities is an element) were produced for tanks, buried wastes, currently } \\
\text { contaminated soil and groundwater, facilities, and nuclear materials (called mission areas). } \\
\text { Priorities were based on collected inventory, release scenario, and toxicity information that will } \\
\text { be updated and used to conduct risk assessments. Lists of elements are in order of expected risk } \\
\text { and mission areas can be cross linked. } \\
\text { In FY 1996, worker health and safety baseline risks will be assessed for selected elements from } \\
\text { lists so that all elements either have an associated quantitative risk or quantitative bounds. } \\
\text { Results will be provided to support site-level decision making about priorities and also will be } \\
\text { capable of reconfiguration to support other decisions. }\end{array}$}} \\
\hline & & & \\
\hline \multicolumn{4}{|c|}{$\begin{array}{l}\text { Risks associated with other elements may have to be assessed again in the future, however, } \\
\text { because (1) the FY } 1996 \text { assessment will not be exhaustive and (2) new inventory or } \\
\text { characterization data may become available. }\end{array}$} \\
\hline $\begin{array}{l}\text { Description of what co } \\
\text { final report to PID on }\end{array}$ & $\begin{array}{l}\text { stitutes completion } \\
\text { orker health and sa }\end{array}$ & $\begin{array}{l}\text { milestone: Delivery } \\
\text { aseline risks at the } \mathrm{Ha}\end{array}$ & $\begin{array}{l}\text { peer-reviewed draft } \\
\text { rd Site. }\end{array}$ \\
\hline
\end{tabular}




\begin{tabular}{||l|l||}
\hline \multicolumn{3}{|c||}{ Westinghouse Hanford Company } \\
MILESTONE DESCRIPTION SHEET
\end{tabular}




\begin{tabular}{|c|c|c|c|}
\hline \multicolumn{4}{|c|}{$\begin{array}{l}\text { Westinghouse Hanford Company } \\
\text { MILESTONE DESCRIPTION SHEET }\end{array}$} \\
\hline \multicolumn{3}{|c|}{ Title: Integrated Risk Assessment Program } & Date: $6 / 15 / 94$ \\
\hline \multicolumn{3}{|c|}{ Assigned To: Judith A. Mahaffey } & CIN: \\
\hline \multicolumn{3}{|c|}{ Program WBS Designator: 1.8.2.6 } & Due Date: $5 / 31 / 97$ \\
\hline \multicolumn{3}{|c|}{ Control Number: IRA-97-001 } & Rev: 0 \\
\hline $\begin{array}{l}\text { MILESTONE TYPE: } \\
\square \text { DOE-HQ } \\
\text { X DOE-RL } \\
\square \text { CONTRACTOR }\end{array}$ & $\begin{aligned} & \text { DIVISION: } \\
& \square \text { State } \\
& \text { Federal } \\
& \text { X DOE } \\
& \square \text { RCRA } \\
& \square \text { TPA Number }\end{aligned}$ & $\begin{array}{l}\text { DELIVERABLE: } \\
\text { X Report } \\
\square \text { Letter } \\
\square \text { Drawings } \\
\square \text { Other (specify) }\end{array}$ & $\begin{array}{l}\text { ADDRESS TO: } \\
\square \text { DOE-HQ } \\
\text { X DỌE-RL } \\
\square \text { Other (specify) }\end{array}$ \\
\hline \multirow{2}{*}{\multicolumn{4}{|c|}{$\begin{array}{l}\text { Milestone Description: One objective of Hanford cleanup is to reduce health and ecological } \\
\text { risks. IRAP is quantifying these risks consistently and comprehensively at a sitewide level to } \\
\text { understand if and when remediation is needed and to evaluate options for remediation from a } \\
\text { risk perspective. In FY 1997, IRAP will provide sitewide information on ecosystem risks from } \\
\text { Hanford wastes and materials (called an ecological baseline). } \\
\text { This work was initiated in mid-FY 1994. Prioritized lists of elements (e.g., each facility or } \\
\text { complex of facilities) were produced for tanks, buried wastes, currently contaminated soil and } \\
\text { groundwater, facilities, and nuclear materials (called mission areas). Priorities were based on } \\
\text { collected inventory, release scenario, and toxicity information that will be updated and used to } \\
\text { conduct risk assessments. Lists of elements are in order of expected risk and mission areas are } \\
\text { cross linked. }\end{array}$}} \\
\hline & & & \\
\hline \multicolumn{4}{|c|}{$\begin{array}{l}\text { In FY 1997, ecosystem baseline risks will be assessed for selected items from lists so all } \\
\text { elements either have an associated quantitative risk or quantitative bounds. Results will be } \\
\text { provided to support site-level decision making about priorities and also will be capable of } \\
\text { reconfiguration to support other decisions. }\end{array}$} \\
\hline \multicolumn{4}{|c|}{$\begin{array}{l}\text { Risks associated with other elements may have to be assessed again in the future, however, } \\
\text { because (1) this FY } 1997 \text { assessment will not be exhaustive and (2) new inventory or } \\
\text { characterization data may become available. }\end{array}$} \\
\hline \multicolumn{4}{|c|}{$\begin{array}{l}\text { Description of what constitutes completion of this milestone: Delivery of peer-reviewed draft } \\
\text { final report to PID on ecosystem baseline risks at the Hanford Site. }\end{array}$} \\
\hline
\end{tabular}




\begin{tabular}{|c|c|c|}
\hline \multicolumn{3}{|c|}{$\begin{array}{l}\text { Westinghouse Hanford Company } \\
\text { MILESTONE DESCRIPTION SHEET }\end{array}$} \\
\hline \multicolumn{2}{|l|}{ Title: Integrated Risk Assessment Program } & Date: $6 / 15 / 94$ \\
\hline \multicolumn{2}{|l|}{ Assigned To: Judith A. Mahaffey } & CIN: \\
\hline \multicolumn{2}{|l|}{ Program WBS Designator: 1.8.2.6 } & Due Date: $5 / 31 / 97$ \\
\hline \multicolumn{2}{|l|}{ Control Number: IRA-97-001 } & Rev: 0 \\
\hline \multicolumn{3}{|c|}{ Milestone Description Approval } \\
\hline $\begin{array}{l}\text { Cost Account Manager } \\
\text { Judicala. Wued offey } 8 / 26 / 94 \\
\text { Jufith A. Mahaffey }\end{array}$ & \multicolumn{2}{|c|}{ Pavid B. Pabst } \\
\hline $\begin{array}{l}\text { Program Element Manager } \\
\text { Jueduna. Muak offoy } 8 / 26 / 94 \\
\text { Judith A. Mahaffey }\end{array}$ & \multicolumn{2}{|c|}{$\begin{array}{l}\text { DOE Program Manager Date } \\
\text { William A. Rutherford } \\
\end{array}$} \\
\hline \multicolumn{3}{|c|}{ Milestone Completion Acceptance } \\
\hline $\begin{array}{l}\text { Program Element Manager } \quad \text { Date } \\
\text { Judith A. Mahaffey }\end{array}$ & \multicolumn{2}{|l|}{$\begin{array}{l}\text { DOE Program Manager } \\
\text { William A. Rutherford }\end{array}$} \\
\hline
\end{tabular}




\begin{tabular}{|c|c|c|c|}
\hline \multicolumn{4}{|c|}{$\begin{array}{l}\text { Westinghouse Hanford Company } \\
\text { MILESTONE DESCRIPTION SHEET }\end{array}$} \\
\hline \multicolumn{3}{|c|}{ Title: Integrated Risk Assessment Program } & Date: $6 / 15 / 94$ \\
\hline \multicolumn{3}{|c|}{ Assigned To: Judith A. Mahaffey } & CIN: \\
\hline \multicolumn{3}{|c|}{ Program WBS Designator: 1.8 .2 .6} & Due Date: $5 / 31 / 97$ \\
\hline \multicolumn{3}{|c|}{ Control Number: IRA-97-002 } & Rev: 0 \\
\hline $\begin{array}{l}\text { MILESTONE TYPE: } \\
\square \text { DOE-HQ } \\
\text { X DOE-RL } \\
\square \text { CONTRACTOR }\end{array}$ & $\begin{array}{l}\quad \text { DIVISION: } \\
\square \text { State } \\
\square \text { Federal } \\
\text { X DOE } \\
\square \text { RCRA } \\
\square \text { TPA Number }\end{array}$ & $\begin{array}{l}\text { DELIVERABLE: } \\
\text { X Report } \\
\square \text { Letter } \\
\square \text { Drawings } \\
\square \text { Other (specify) }\end{array}$ & $\begin{array}{l}\text { ADDRESS TO: } \\
\square \text { DOE-HQ } \\
\text { X DOE-RL } \\
\square \text { Other (specify) }\end{array}$ \\
\hline \multirow{2}{*}{\multicolumn{4}{|c|}{$\begin{array}{l}\text { Milestone Description: One objective of Hanford cleanup is to reduce health and ecological } \\
\text { risks. IRAP is quantifying these risks consistently and comprehensively at a sitewide level to } \\
\text { enable an evaluation of risk reduction achieved by cleanup activities and its value. In FY } 1997 \\
\text { IRAP will provide sitewide information on public health risks from residual Hanford wastes and } \\
\text { materials assuming that milestones associated with the Tri-Party Agreement (TPA) have been } \\
\text { accomplished. } \\
\text { Results will be compared to baseline public health risks to understand risk reduction } \\
\text { accomplished in another FY } 1997 \text { milestone; risk results will be provided to others to analyze } \\
\text { risk reduction value provided per dollar and to understand activities that need to be further } \\
\text { accomplished for DOE Hanford Site closure. }\end{array}$}} \\
\hline & & & \\
\hline \multicolumn{4}{|c|}{$\begin{array}{l}\text { In FY 1997, public health endstate risks will be assessed for selected elements from updates to } \\
\text { lists produced in FY } 1994 \text { so all elements either have an associated quantitative risk or } \\
\text { quantitative bounds. Results will be provided to support Site-level decision making about } \\
\text { priorities and also will be capable of reconfiguration to support other decisions. }\end{array}$} \\
\hline \multicolumn{4}{|c|}{$\begin{array}{l}\text { Other elements may have to be assessed again in the future, however, because (1) the FY } 1997 \\
\text { assessment will not be exhaustive, ( } 2 \text { ) new inventory and characterization data may become } \\
\text { available, and (3) TPA milestones may change. }\end{array}$} \\
\hline \multicolumn{4}{|c|}{$\begin{array}{l}\text { Description of what constitutes completion of this milestone: Delivery of peer-reviewed draft } \\
\text { final report to PID on public health risks after accomplishing remediation at the Hanford Site } \\
\text { according to TPA milestones. }\end{array}$} \\
\hline
\end{tabular}




\begin{tabular}{|c|c|c|}
\hline \multicolumn{3}{|c|}{$\begin{array}{l}\text { Westinghouse Hanford Company } \\
\text { MILESTONE DESCRIPTION SHEET }\end{array}$} \\
\hline \multicolumn{2}{|l|}{ Title: Integrated Risk Assessment Program } & Date: $6 / 15 / 94$ \\
\hline \multicolumn{2}{|l|}{ Assigned To: Judith A. Mahaffey } & CIN: \\
\hline \multicolumn{2}{|l|}{ Program WBS Designator: 1.8.2.6 } & Due Date: $5 / 31 / 97$ \\
\hline \multicolumn{2}{|l|}{ Control Number: IRA-97-002 } & Rev: 0 \\
\hline \multicolumn{3}{|c|}{ Milestone Description Approval } \\
\hline $\begin{array}{l}\text { Cost Account Manager } \\
\text { Date } \\
\text { Judith A. Mahaffey }\end{array}$ & \multicolumn{2}{|c|}{ Program/Project Manager B. Pabst } \\
\hline 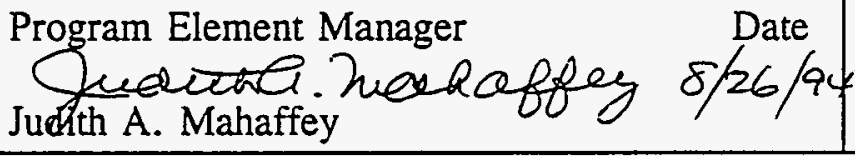 & \multicolumn{2}{|c|}{$\begin{array}{l}\text { DOE Program Manager Date } \\
\text { William A. Rutherford } \\
\end{array}$} \\
\hline \multicolumn{3}{|c|}{ Milestone Completion Acceptance } \\
\hline $\begin{array}{l}\text { Program Element Manager } \quad \text { Date } \\
\text { Judith A. Mahaffey }\end{array}$ & $\begin{array}{l}\text { DOE Program Manager } \\
\text { William A. Rutherford }\end{array}$ & \\
\hline
\end{tabular}




\begin{tabular}{|c|c|c|c|}
\hline \multicolumn{4}{|c|}{$\begin{array}{l}\text { Westinghouse Hanford Company } \\
\text { MILESTONE DESCRIPTION SHEET }\end{array}$} \\
\hline \multicolumn{3}{|c|}{ Title: Integrated Risk Assessment Program } & Date: $6 / 15 / 94$ \\
\hline \multicolumn{3}{|c|}{ Assigned To: Judith A. Mahaffey } & CIN: \\
\hline \multicolumn{3}{|c|}{ Program·WBS Designator: 1.8 .2 .6} & Due Date: $9 / 30 / 97$ \\
\hline \multicolumn{3}{|c|}{ Control Number: IRA-97-003 } & Rev: 0 \\
\hline $\begin{array}{l}\text { MILESTONE TYPE: } \\
\square \text { DOE-HQ } \\
\text { X DOE-RL } \\
\square \text { CONTRACTOR }\end{array}$ & $\begin{array}{l}\quad \text { DIVISION: } \\
\square \text { State } \\
\square \text { Federal } \\
\text { X DOE } \\
\square \text { RCRA } \\
\square \text { TPA Number }\end{array}$ & $\begin{array}{l}\text { DELIVERABLE: } \\
\text { X Report } \\
\square \text { Letter } \\
\square \text { Drawings } \\
\square \text { Other (specify) }\end{array}$ & $\begin{array}{l}\text { ADDRESS TO: } \\
\square \text { DOE-HQ } \\
\text { X DOE-RL } \\
\square \text { Other (specify) }\end{array}$ \\
\hline \multicolumn{4}{|c|}{$\begin{array}{l}\text { Milestone Description: One objective of Hanford cleanup is to reduce health and ecological } \\
\text { risks. IRAP is quantifying these risks consistently and comprehensively at a sitewide level. } \\
\text { Such quantification will enable an evaluation from a risk perspective of when public health risks } \\
\text { should dominate risk considerations in decision making, when worker health and safety risks are } \\
\text { of greatest concern, when ecological risk is a primary consideration, or (more likely) when } \\
\text { decisions on cleanup involve multiple risk considerations. } \\
\text { In FY 1997, IRAP will compare the three baseline risk assessments to understand how they are } \\
\text { related and what composite results mean in terms of drivers for and dependency among cleanup } \\
\text { decisions. Analyses will be based on IRAP baseline risk assessment reports delivered in } \\
\text { FY 1995, in FY 1996, and in FY } 1997 \text {. }\end{array}$} \\
\hline \multicolumn{4}{|c|}{$\begin{array}{l}\text { Description of what constitutes completion of this milestone: Delivery of peer-reviewed draft } \\
\text { final report to PID on integrated baseline risks (public health, worker health and safety, and } \\
\text { ecosystem health) at the Hanford Site. }\end{array}$} \\
\hline
\end{tabular}




\begin{tabular}{|c|c|c|}
\hline \multicolumn{3}{|c|}{$\begin{array}{l}\text { Westinghouse Hanford Company } \\
\text { MILESTONE DESCRIPTION SHEET }\end{array}$} \\
\hline \multicolumn{2}{|l|}{ Title: Integrated Risk Assessment Program } & Date: $6 / 15 / 94$ \\
\hline \multicolumn{2}{|l|}{ Assigned To: Judith A. Mahaffey } & CIN: \\
\hline \multicolumn{2}{|l|}{ Program WBS Designator: 1.8.2.6 } & Due Date: $9 / 30 / 97$ \\
\hline \multicolumn{2}{|l|}{ Control Number: IRA-97-003 } & Rev: 0 \\
\hline \multicolumn{3}{|c|}{ Milestone Description Approval } \\
\hline $\begin{array}{l}\text { Cost Account Manager } \\
\text { Date } \\
\text { Jutheth A. Mahaffey }\end{array}$ & \multicolumn{2}{|c|}{ Program/Project Manager B. Pabst } \\
\hline $\begin{array}{lc}\text { Program Element Manager } & \text { Date } \\
\text { Jududth A. Mahaffey } & \delta / 26 / 94\end{array}$ & \multicolumn{2}{|l|}{$\begin{array}{l}\text { DOE Program Manager } \\
\text { William A. Rutherford } \\
\end{array}$} \\
\hline \multicolumn{3}{|c|}{ Milestone Completion Acceptance } \\
\hline $\begin{array}{l}\text { Program Element Manager } \\
\text { Judith A. Mahaffey }\end{array}$ & \multicolumn{2}{|l|}{$\begin{array}{l}\text { DOE Program Manager } \\
\text { William A. Rutherford }\end{array}$} \\
\hline
\end{tabular}




\begin{tabular}{|c|c|c|c|}
\hline \multicolumn{4}{|c|}{$\begin{array}{l}\text { Westinghouse Hanford Company } \\
\text { MILESTONE DESCRIPTION SHEET }\end{array}$} \\
\hline \multicolumn{3}{|c|}{ Title: Integrated Risk Assessment Program } & Date: $6 / 15 / 94$ \\
\hline \multicolumn{3}{|c|}{ Assigned To: Judith A. Mahaffey } & CIN: \\
\hline \multicolumn{3}{|c|}{ Program WBS Designator: 1.8.2.6 } & Due Date: $9 / 30 / 97$ \\
\hline \multicolumn{3}{|c|}{ Control Number: IRA-97-004 } & Rev: 0 \\
\hline $\begin{array}{l}\text { MILESTONE TYPE: } \\
\square \text { DOE-HQ } \\
\text { X DOE-RL } \\
\square \text { CONTRACTOR }\end{array}$ & $\begin{array}{l}\quad \text { DIVISION: } \\
\quad \square \text { State } \\
\square \text { Federal } \\
\text { X DOE } \\
\square \text { RCRA } \\
\square \text { TPA Number }\end{array}$ & $\begin{array}{l}\text { DELIVERABLE: } \\
\text { X Report } \\
\square \text { Letter } \\
\square \text { Drawings } \\
\square \text { Other (specify) }\end{array}$ & $\begin{array}{l}\text { ADDRESS TO: } \\
\square \text { DOE-HQ } \\
\text { X DOE-RL } \\
\square \text { Other (specify) }\end{array}$ \\
\hline \multicolumn{4}{|c|}{$\begin{array}{l}\text { Milestone Description: One objective of Hanford cleanup is to reduce health and ecological } \\
\text { risks. IRAP is quantifying these risks consistently and comprehensively at a sitewide level to } \\
\text { enable an evaluation of risk reduction. } \\
\text { In FY 1997, IRAP will compare baseline and Tri-Party Agreement (TPA) endstate public health } \\
\text { risks to understand risk reduction accomplished, and to support other in evaluating risk reduction } \\
\text { value provided per dollar and activities that need to be further accomplished for DOE Hanford } \\
\text { site closure that are not included in the TPA. } \\
\text { Analyses will be based on IRAP reports on baseline and endstate public health risks delivered in } \\
\text { FY } 1995 \text { and in FY } 1997 \text {. }\end{array}$} \\
\hline \multicolumn{4}{|c|}{$\begin{array}{l}\text { Description of what constitutes completion of this milestone: Delivery of peer-reviewed draft } \\
\text { final report to PID on public health risks (comparing baseline and TPA endstate) at the Hanford } \\
\text { site. }\end{array}$} \\
\hline
\end{tabular}




\begin{tabular}{|c|c|c|}
\hline \multicolumn{3}{|c|}{$\begin{array}{l}\text { Westinghouse Hanford Company } \\
\text { MILESTONE DESCRIPTION SHEET }\end{array}$} \\
\hline Title: Integrated Risk Assessment Program & & Date: $6 / 15 / 94$ \\
\hline Assigned To: Judith A. Mahaffey & & CIN: \\
\hline Program WBS Designator: 1.8.2.6 & & Due Date: $9 / 30 / 97$ \\
\hline Control Number: IRA-97-004 & & Rev: 0 \\
\hline \multicolumn{3}{|c|}{ Milestone Description Approval } \\
\hline $\begin{array}{l}\text { Cost Account Manager } \\
\text { Date } \\
\text { Judith A. Mahaffey }\end{array}$ & $\begin{array}{l}\text { Program/Project Manag } \\
\text { David B. Pabst }\end{array}$ & Def 8/26/94 \\
\hline 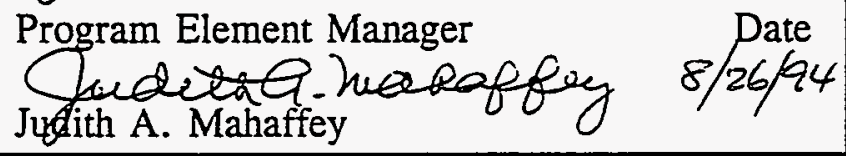 & $\begin{array}{l}\text { DOE Program Manager } \\
\text { William A. Rutherford }\end{array}$ & Date \\
\hline \multicolumn{3}{|c|}{ Milestone Completion Acceptance } \\
\hline $\begin{array}{l}\text { Program Element Manager } \\
\text { Judith A. Mahaffey }\end{array}$ & $\begin{array}{l}\text { DOE Program Manager } \\
\text { William A. Rutherford }\end{array}$ & Date \\
\hline
\end{tabular}




\begin{tabular}{|c|c|c|c|}
\hline \multicolumn{4}{|c|}{$\begin{array}{l}\text { Westinghouse Hanford Company } \\
\text { MILESTONE DESCRIPTION SHEET }\end{array}$} \\
\hline \multicolumn{3}{|c|}{ Title: Integrated Risk Assessment Program } & Dạte: $6 / 15 / 94$ \\
\hline \multicolumn{3}{|c|}{ Asssigned To: Judith A. Mahaffey } & CIN: \\
\hline \multicolumn{3}{|c|}{ Program WBS Designator: 1.8.2.6 } & Due Date: $9 / 30 / 97$ \\
\hline \multicolumn{3}{|c|}{ Control Number: IRA-97-005 } & Rev: 0 \\
\hline $\begin{array}{l}\text { MILESTONE TYPE: } \\
\square \text { DOE-HQ } \\
\text { X DOE-RL } \\
\square \text { CONTRACTOR }\end{array}$ & $\begin{array}{l}\quad \text { DIVISION: } \\
\square \text { State } \\
\square \text { Federal } \\
\text { X DOE } \\
\square \text { RCRA } \\
\square \text { TPA Number }\end{array}$ & $\begin{array}{l}\text { DELIVERABLE: } \\
\text { X Report } \\
\square \text { Letter } \\
\square \text { Drawings } \\
\square \text { Other (specify) }\end{array}$ & $\begin{array}{l}\text { ADDRESS TO: } \\
\square \text { DOE-HQ } \\
\text { X DOE-RL } \\
\square \text { Other (specify) }\end{array}$ \\
\hline \multicolumn{4}{|c|}{$\begin{array}{l}\text { Milestone Description: It is possible that Hanford Site cleanup is trading longer-term risks to the } \\
\text { public (from exposures) for shorter-term risks to workers (both from industrial accidents and } \\
\text { from exposures). Thus, one purpose of IRAP in FY } 1997 \text { is to provide sitewide information on } \\
\text { worker health and safety risks during remediation at Hanford. } \\
\text { This work will build on work initiated in mid-FY 1994, during which prioritized lists of } \\
\text { elements (e.g., each facility or complex of facilities is an element) were produced for tanks, } \\
\text { buried wastes, currently contaminated soil and groundwater, facilities, and nuclear materials } \\
\text { (called mission areas). Priorities were based on collection of information that will be updated } \\
\text { and used to conduct assessments or worker risks during remediation. } \\
\text { In FY } 1997 \text {, worker health and safety risks during remedial activities will be assessed for } \\
\text { selected items from lists so all elements either have an associated quantitative risk or quantitative } \\
\text { bounds. Results will be provided to support site-level decision making about potential tradeoffs } \\
\text { between public and worker risks, and to support decisions on other worker health and safety } \\
\text { issues, such as technology requirements for monitoring worker exposures or especially high-risk } \\
\text { remedial alternatives. Information will also will be capable of reconfiguration to support other } \\
\text { decisions. }\end{array}$} \\
\hline \multicolumn{4}{|c|}{$\begin{array}{l}\text { Description of what constitutes completion of this milestone: Delivery of peer-reviewed draft } \\
\text { final report to PID on worker health and safety risks during remediation at the Hanford Site. }\end{array}$} \\
\hline
\end{tabular}




\begin{tabular}{|c|c|c|}
\hline \multicolumn{3}{|c|}{$\begin{array}{l}\text { Westinghouse Hanford Company } \\
\text { MILESTONE DESCRIPTION SHEET }\end{array}$} \\
\hline Title: Integrated Risk Assessment Program & & Date: $6 / 15 / 94$ \\
\hline Assigned To: Judith A. Mahaffey & & CIN: \\
\hline Program WBS Designator: 1.8.2.6 & & Due Date: $9 / 30 / 97$ \\
\hline Control Number: IRA-97-005 & & Rev: 0 \\
\hline \multicolumn{3}{|c|}{ Milestone Description Approval } \\
\hline $\begin{array}{l}\text { Cost Account Manager } \\
\text { Oudota. Weebaffy } \\
\text { Judith A. Mahaffey }\end{array}$ & Program/Project Manage & Ditist Blovilut \\
\hline $\begin{array}{l}\text { Program Element Manager } \\
\text { Ous dietce, thateofiez } 8 / 26 / 94 \\
\text { Jughth A. Mahaffey }\end{array}$ & $\begin{array}{l}\text { DOE Program Manager } \\
\text { William A. Rutherford }\end{array}$ & Date \\
\hline \multicolumn{3}{|c|}{ Milestone Completion Acceptance } \\
\hline $\begin{array}{l}\text { Program Element Manager } \quad \text { Date } \\
\text { Judith A. Mahaffey }\end{array}$ & $\begin{array}{l}\text { DOE Program Manager } \\
\text { William A. Rutherford }\end{array}$ & Date \\
\hline
\end{tabular}


Planning Integration Multi-Year Progran Plan

\section{This page}

intentionally left blank. 


\begin{tabular}{|c|c|c|c|}
\hline \multicolumn{4}{|c|}{$\begin{array}{l}\text { Westinghouse Hanford Company } \\
\text { MILESTONE DESCRIPTION SHEET }\end{array}$} \\
\hline \multicolumn{3}{|c|}{$\begin{array}{l}\text { Title: } \\
\text { RL Issue Site FY } 1996 \text { MYPP/FYWP Guidance }\end{array}$} & $\begin{array}{l}\text { Date: } \\
10 / 01 / 94\end{array}$ \\
\hline \multicolumn{3}{|c|}{$\begin{array}{l}\text { Assigned To: } \\
\text { DOE-RL Planning Integration Division }\end{array}$} & CIN: \\
\hline \multicolumn{3}{|c|}{$\begin{array}{l}\text { Program WBS Designator: } \\
\text { 1.8.2.3 }\end{array}$} & $\begin{array}{l}\text { Due Date: } \\
03 / 15 / 95\end{array}$ \\
\hline \multicolumn{3}{|c|}{ Control Number: PBI-95-001 } & Rev: \\
\hline $\begin{array}{l}\text { MILESTONE TYPE: } \\
\square \text { DOE-HQ } \\
\text { DOE-RL } \\
\square \text { CONTRACTOR }\end{array}$ & $\begin{aligned} & \text { DIVISION: } \\
& \square \text { State } \\
& \text { Federal } \\
& \text { DOE } \\
& \square \text { RCRA } \\
& \square \text { TPA Number }\end{aligned}$ & $\begin{array}{l}\text { DELIVERABLE: } \\
\square \text { Report } \\
\mathbf{\square} \text { Letter } \\
\square \text { Drawings } \\
\square \text { Other (specify) }\end{array}$ & $\begin{array}{l}\text { ADDRESS TO: } \\
\text { D DOE-HQ } \\
\square \text { DOE-RL } \\
\text { Other (specify) } \\
\text { WHC Direct } \\
\text { Programs }\end{array}$ \\
\hline \multicolumn{4}{|c|}{$\begin{array}{l}\text { Milestone Description: } \\
\text { RL provide planning guidance to prepare the call letter for the annual site MYPP update. }\end{array}$} \\
\hline \multicolumn{4}{|c|}{$\begin{array}{l}\text { Description of what constitutes completion of this milestone: } \\
\text { The milestone will be completed when the letter identifying the RL requirements is issued } \\
\text { to site contractors. }\end{array}$} \\
\hline \multicolumn{4}{|c|}{ Milestone Description Approval } \\
\hline \multicolumn{2}{|c|}{ 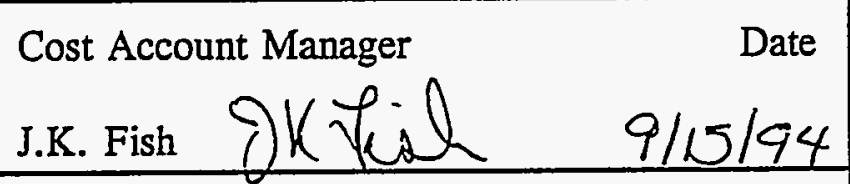 } & \multicolumn{2}{|c|}{ Drogram/Project Manages Dabst 1 Date } \\
\hline \multicolumn{2}{|c|}{$\begin{array}{rr}\text { Program Element Manager } & \text { Date } \\
\text { D.L. Borders } & \text { Sorans }\end{array}$} & \multicolumn{2}{|c|}{$\begin{array}{l}\text { DOE Monitor } \\
\text { W.A. Rutherford Ute }\end{array}$} \\
\hline \multicolumn{4}{|c|}{ 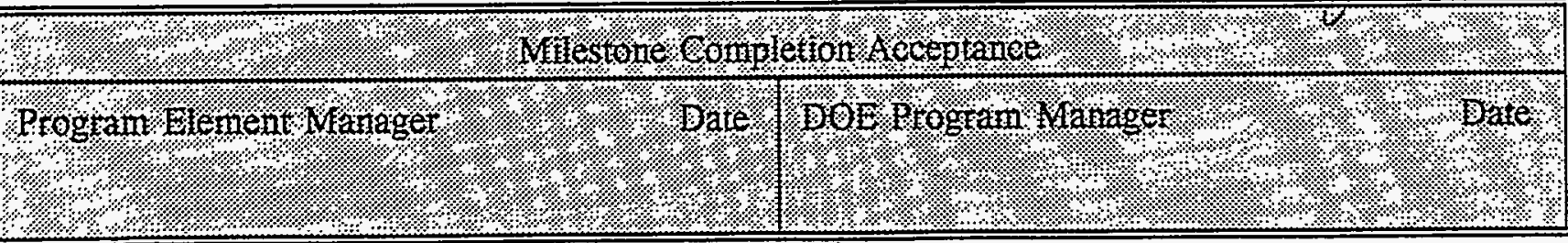 } \\
\hline
\end{tabular}




\begin{tabular}{|l||l|l||}
\hline \multicolumn{3}{|c|}{ Westinghouse Hanford Company } \\
MILESTONE DESCRIPTION SHEET
\end{tabular}




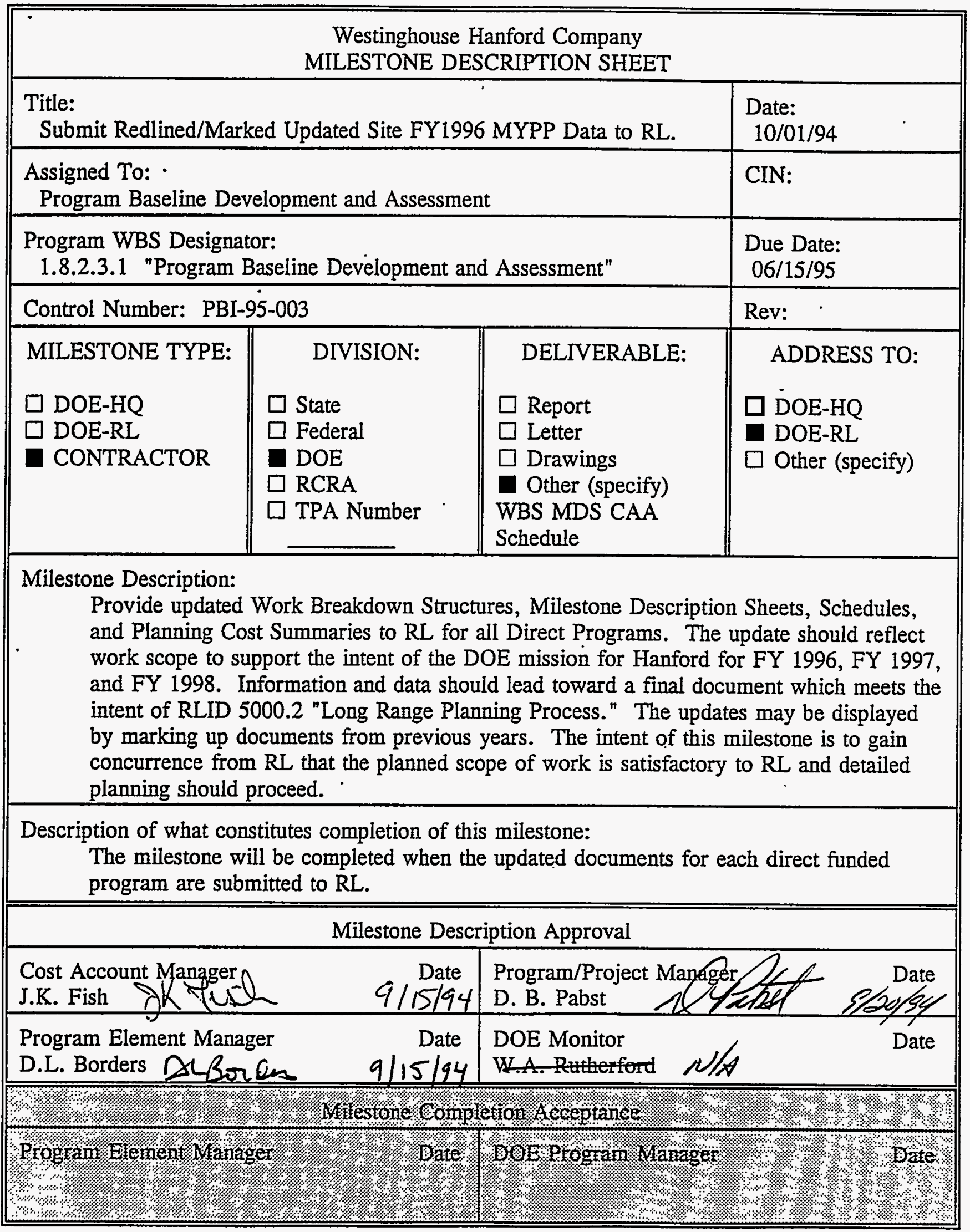




\begin{tabular}{|l||l|l||}
\hline \multicolumn{3}{|c||}{ Westinghouse Hanford Company } \\
MILESTONE DESCRIPTION SHEET
\end{tabular}




\begin{tabular}{|c|c|c|c|}
\hline \multicolumn{4}{|c|}{$\begin{array}{l}\text { Westinghouse Hanford Company } \\
\text { MILESTONE DESCRIPTION SHEET }\end{array}$} \\
\hline \multicolumn{3}{|c|}{$\begin{array}{l}\text { Title: } \\
\text { Submit Site FY } 1996 \text { MYPP/FYWPs to RL. }\end{array}$} & $\begin{array}{l}\text { Date: } \\
10 / 01 / 94\end{array}$ \\
\hline \multicolumn{3}{|c|}{$\begin{array}{l}\text { Assigned To: } \\
\text { Program Baseline Development and Assessment }\end{array}$} & CIN: \\
\hline \multicolumn{3}{|c|}{$\begin{array}{l}\text { Program WBS Designator: } \\
\text { 1.8.2.3.1 "Program Baseline Development and Assessment" }\end{array}$} & $\begin{array}{l}\text { Due Date: } \\
08 / 31 / 95\end{array}$ \\
\hline \multicolumn{3}{|c|}{ Control Number: PBI-95-005 } & Rev: \\
\hline $\begin{array}{l}\text { MILESTONE TYPE: } \\
\square \text { DOE-HQ } \\
\square \text { DOE-RL } \\
\square \text { CONTRACTOR }\end{array}$ & $\begin{aligned} & \text { DIVISION: } \\
& \square \text { State } \\
\square & \text { Federal } \\
& \text { DOE } \\
& \text { RCRA } \\
& \square \text { TPA Number }\end{aligned}$ & $\begin{array}{l}\text { DELIVERABLE: } \\
\text {. } \\
\square \text { Report } \\
\square \text { Letter } \\
\square \text { Drawings } \\
\square \text { Other (specify) } \\
\text { MYPP/FYWP } \\
\text { document . }\end{array}$ & $\begin{array}{l}\text { ADDRESS TO: } \\
\square \text { DOE-HQ } \\
\text { DOE-RL } \\
\square \text { Other (specify) }\end{array}$ \\
\hline \multicolumn{4}{|c|}{$\begin{array}{l}\text { Milestone Description: . } \\
\text { The FY } 1996 \text { MYPP's/FYWP's are the baseline documents with which WHC documents } \\
\text { scope, schedules, and resource requirements to RL. This includes all the items set forth } \\
\text { in the call letter issued to the direct programs. The final document must meet the intent } \\
\text { of RLID } 5000.2 \text { "Long Range Planning Process." FY 1996, FY 1997, and FY } 1998 \\
\text {. must contain data which is of validatable quality. }\end{array}$} \\
\hline \multicolumn{4}{|c|}{$\begin{array}{l}\text { Description of what constitutes completion of this milestone: } \\
\text { This milestone is completed when all of the direct-funded programs have submitted their } \\
\text { FY1996 MYPP's/FYWP's to RL. }\end{array}$} \\
\hline \multicolumn{4}{|c|}{ Milestone Description Approval } \\
\hline \multicolumn{2}{|c|}{ 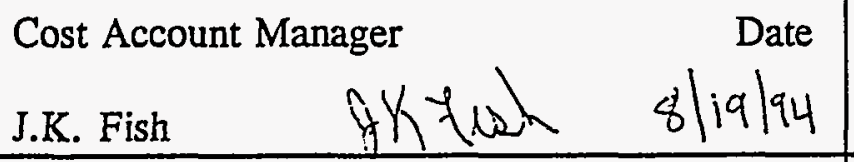 } & \multicolumn{2}{|c|}{ Drogram/Project Manager } \\
\hline \multicolumn{2}{|c|}{$\begin{array}{lr}\text { Program Element Manager } & \text { Date } \\
\text { D.L. Borders DLBonsas } 8 / 19 / 94\end{array}$} & \multicolumn{2}{|c|}{ DOE Monitor } \\
\hline \multicolumn{4}{|c|}{ 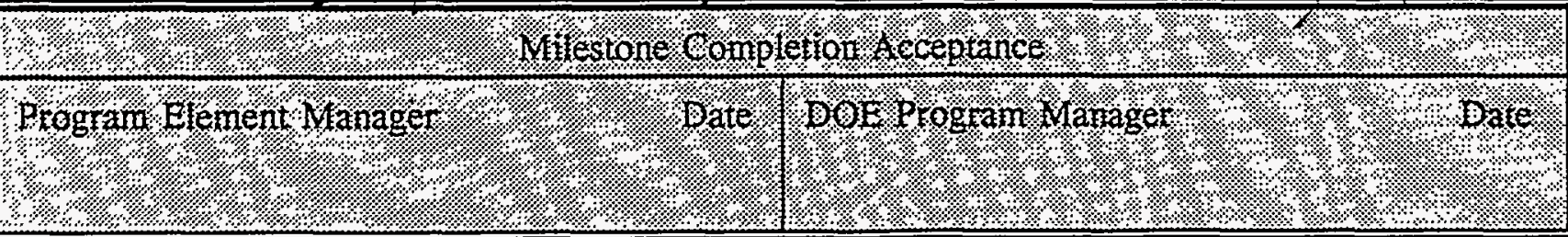 } \\
\hline
\end{tabular}




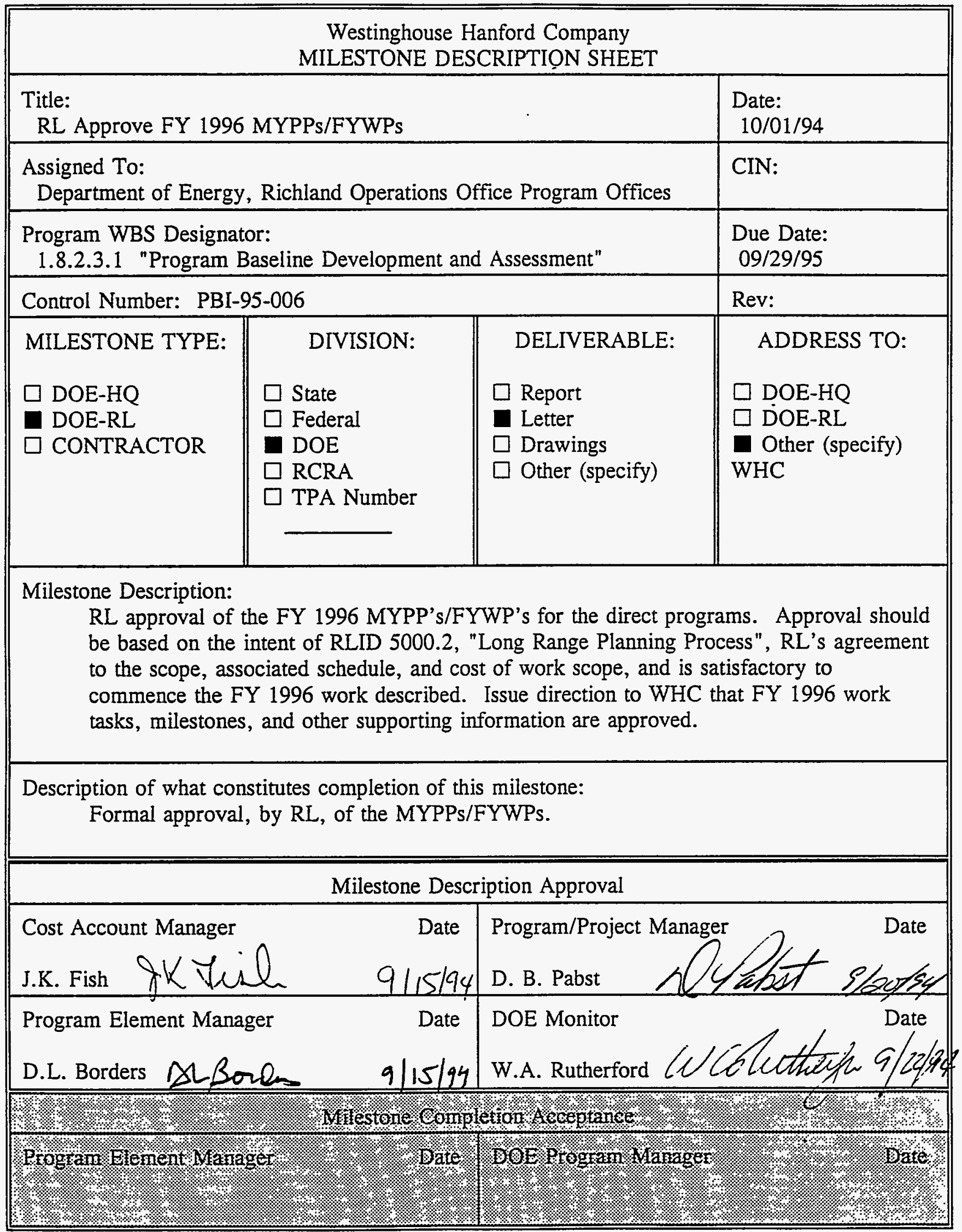




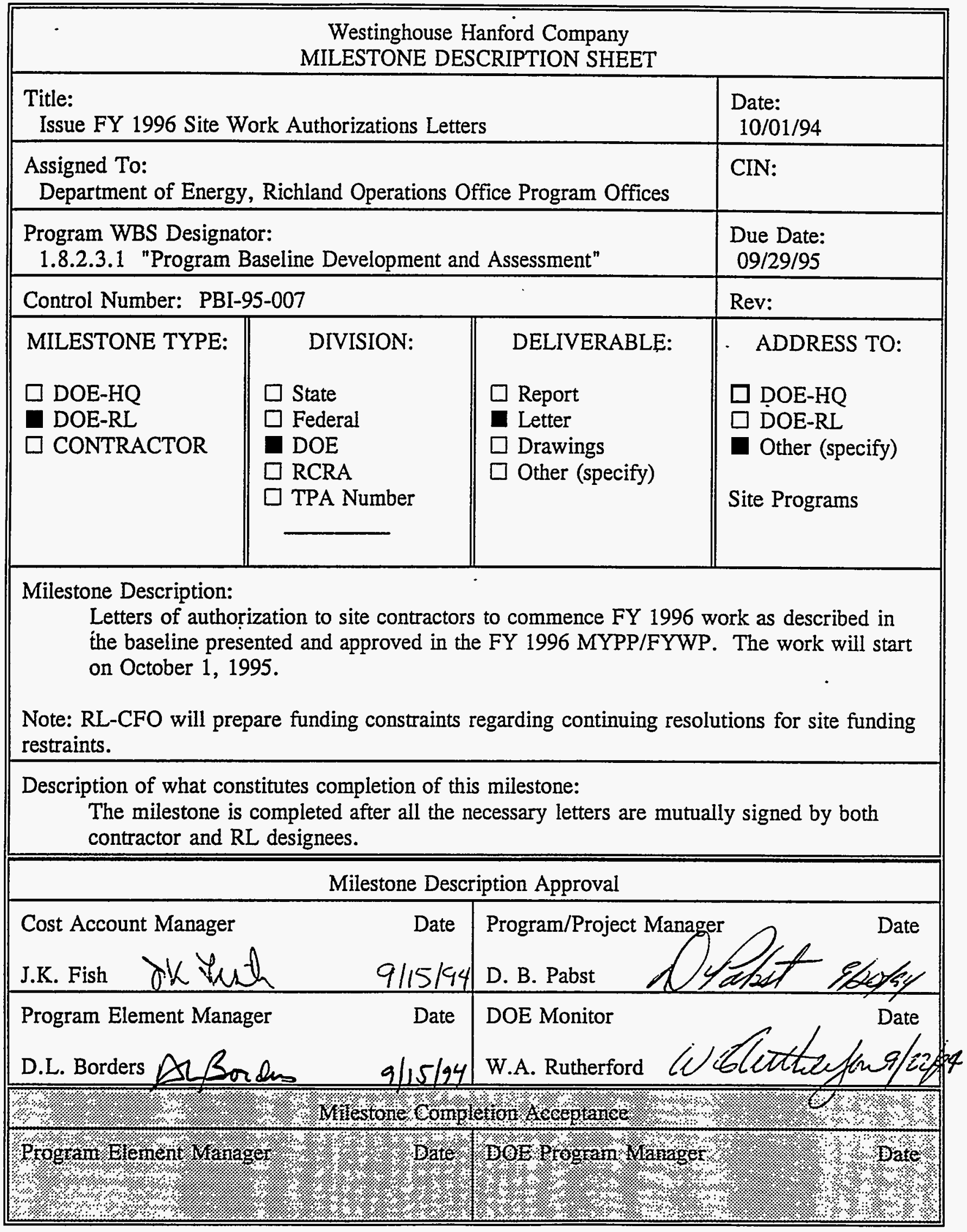




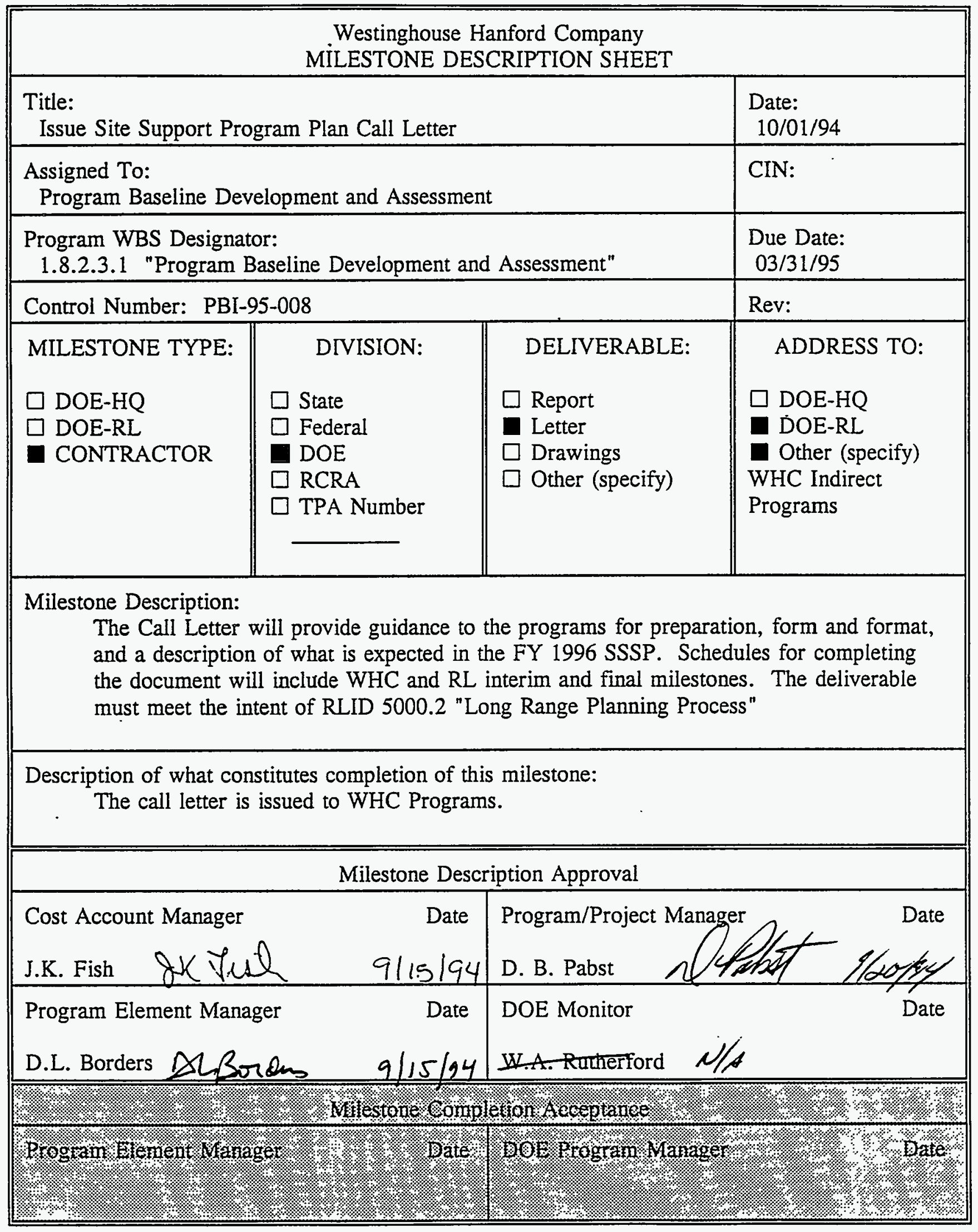




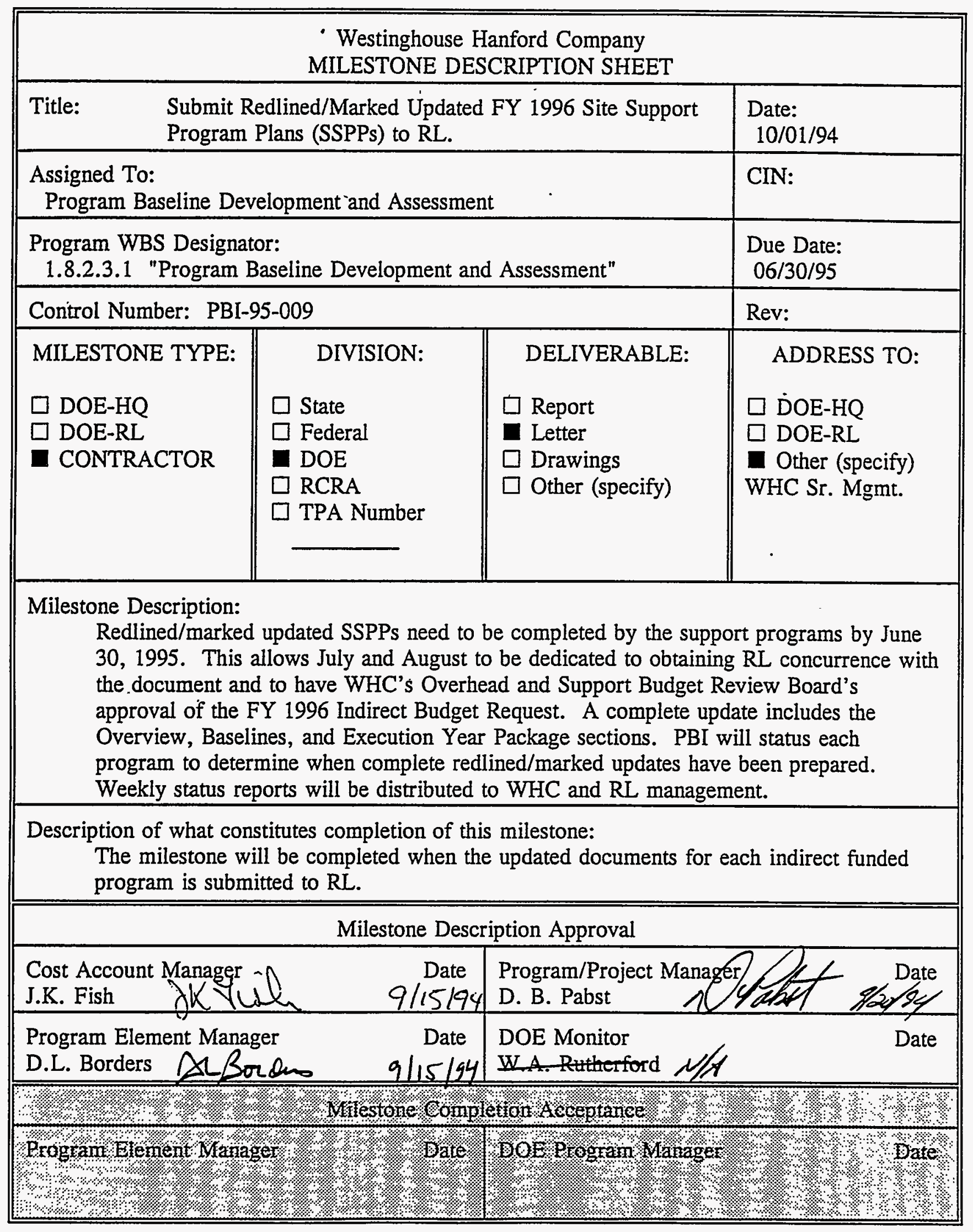




\begin{tabular}{|l|l||}
\hline \multicolumn{3}{|c|}{ Westinghouse Hanford Company } \\
MILESTONE DESCRIPTION SHEET
\end{tabular}




\begin{tabular}{|c|c|c|c|}
\hline \multicolumn{4}{|c|}{$\begin{array}{l}\text { Westinghouse Hanford Company } \\
\text { MILESTONE DESCRIPTION SHEET }\end{array}$} \\
\hline \multicolumn{3}{|c|}{$\begin{array}{l}\text { Title: } \\
\text { Submit FY } 1996 \text { Site Support Program Plans to RL. }\end{array}$} & $\begin{array}{l}\text { Date: } \\
10 / 01 / 94\end{array}$ \\
\hline \multicolumn{3}{|c|}{$\begin{array}{l}\text { Assigned To: } \\
\text { Program Baseline Development and Assessment }\end{array}$} & CIN: \\
\hline \multicolumn{3}{|c|}{$\begin{array}{l}\text { Program WBS Designator: } \\
\text { 1.8.2.3.1 "Program Baseline Development and Assessment" }\end{array}$} & $\begin{array}{l}\text { Due Date: } \\
08 / 31 / 95\end{array}$ \\
\hline \multicolumn{3}{|c|}{ Control Number: PBI-95-011 } & Rev: \\
\hline $\begin{array}{l}\text { MILESTONE TYPE: } \\
\square \text { DOE-HQ } \\
\square \text { DOE-RL } \\
\square \text { CONTRACTOR }\end{array}$ & $\begin{aligned} & \text { DIVISION: } \\
& \square \text { State } \\
\square & \text { Federal } \\
& \text { DOE } \\
& \text { RCRA } \\
& \square \text { TPA Number }\end{aligned}$ & $\begin{array}{l}\text { DELIVERABLE: } \\
\square \text { Report } \\
\square \text { Letter } \\
\square \text { Drawings } \\
\square \text { Other (specify) } \\
\text { SSPP documents }\end{array}$ & $\begin{array}{l}\text { ADDRESS TO: } \\
\square \text { DOE-HQ } \\
\square \text { DOE-RL } \\
\square \text { Other (specify) }\end{array}$ \\
\hline \multicolumn{4}{|c|}{$\begin{array}{l}\text { Milestone Description: } \\
\text { The FY } 1996 \text { Site Support Program Plans are the baseline documents with which WHC } \\
\text { documents scope, schedules, and resource requirements to RL. This includes all the } \\
\text { items set forth in the call letter. The final document must meet the intent of RLID } \\
5000.2 \text { "Long Range Planning Process." FY 1996, FY 1997, and FY } 1998 \text { must contain } \\
\text { data which is of validatable quality. }\end{array}$} \\
\hline \multicolumn{4}{|c|}{$\begin{array}{l}\text { Description of what constitutes completion of this milestone: } \\
\text { This milestone is completed when all of the WHC SSPPs have been submitted to RL. }\end{array}$} \\
\hline \multicolumn{4}{|c|}{ Milestone Description Approval } \\
\hline \multicolumn{2}{|c|}{$\begin{array}{l}\text { Cost Account Manager } \\
\text { J.K. Fish YKFich } 8-76-94\end{array}$} & \multicolumn{2}{|c|}{ Program/Project Majager B. Pabst } \\
\hline \multicolumn{2}{|c|}{$\begin{array}{l}\text { Program Element Manager } \\
\text { D.L. Borders } \triangle 1 \text { Date } \\
\text { Bors }\end{array}$} & \multicolumn{2}{|c|}{ DOE Monitor } \\
\hline \multicolumn{4}{|c|}{ 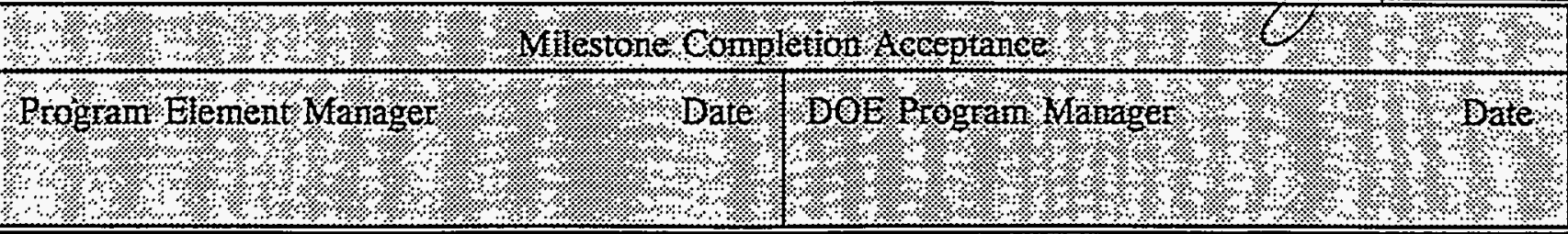 } \\
\hline
\end{tabular}




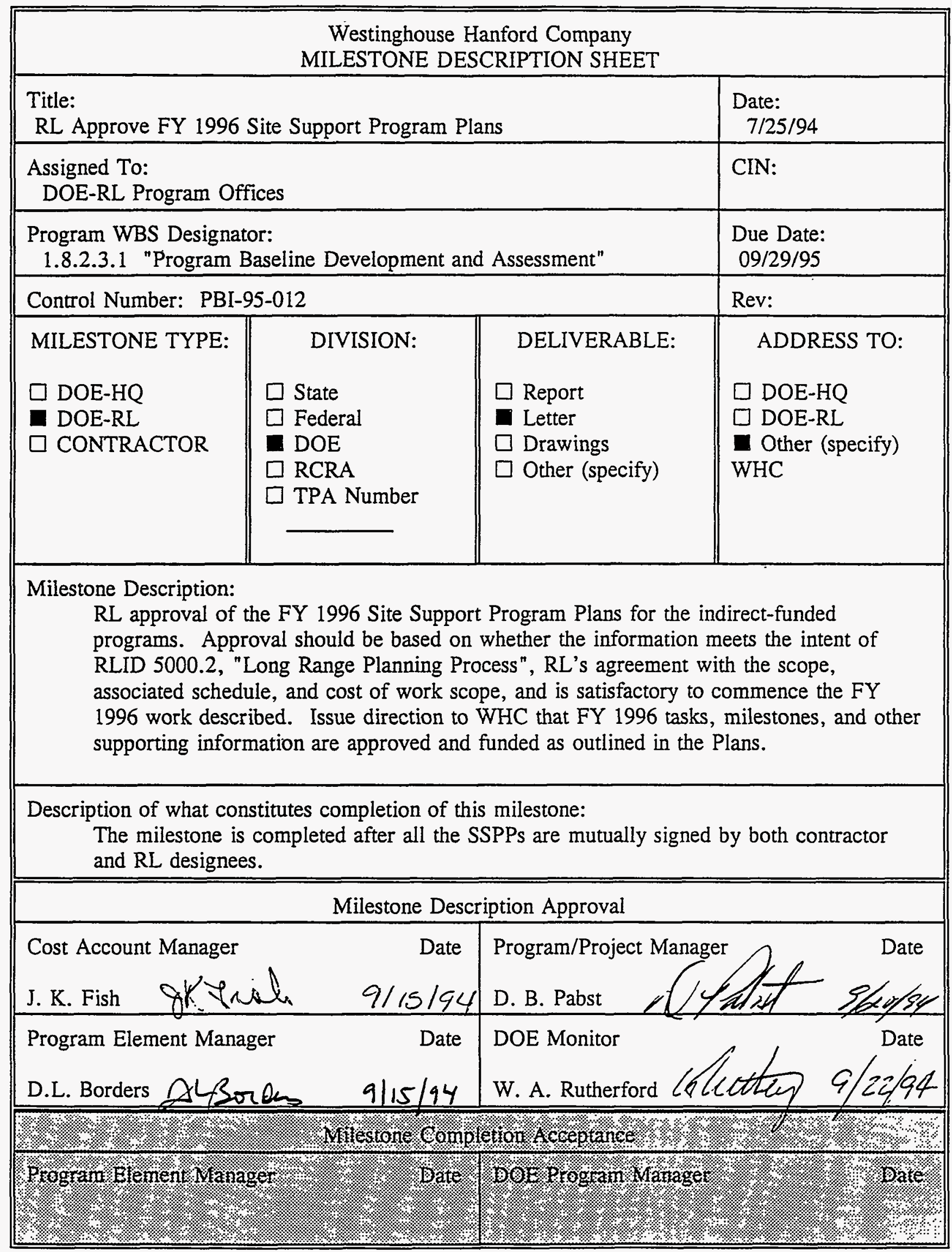




\begin{tabular}{|c|c|c|c|}
\hline \multicolumn{4}{|c|}{$\begin{array}{l}\text { Westinghouse Hanford Company } \\
\text { MILESTONE DESCRIPTION SHEET }\end{array}$} \\
\hline \multicolumn{3}{|c|}{$\begin{array}{l}\text { Title: } \\
\text { Publish Site-wide Integrated Logic and Schedule Reports }\end{array}$} & $\begin{array}{l}\text { Date: } \\
10 / 01 / 94\end{array}$ \\
\hline \multicolumn{3}{|c|}{$\begin{array}{l}\text { Assigned To: } \\
\text { Program Baseline Development and Assessment }\end{array}$} & CIN: \\
\hline \multicolumn{3}{|c|}{$\begin{array}{l}\text { Program WBS Designator: } \\
\text { 1.8.2.3.1 "Program Baseline Development and Assessment" }\end{array}$} & $\begin{array}{r}\text { Due Date: } \\
\text { Monthly }\end{array}$ \\
\hline \multicolumn{3}{|c|}{ Control Number: PBI-95-013 } & Rev: 0 \\
\hline $\begin{array}{l}\text { MILESTONE TYPE: } \\
\square \text { DOE-HQ } \\
\square \text { DOE-RL } \\
\square \text { CONTRACTOR }\end{array}$ & $\begin{aligned} & \text { DIVISION: } \\
& \square \text { State } \\
& \square \text { Federal } \\
& \text { DOE } \\
& \square \text { RCRA } \\
& \square \text { TPA Number }\end{aligned}$ & $\begin{array}{l}\text { DELIVERABLE: } \\
\text { 口 Report } \\
\square \text { Letter } \\
\square \text { Drawings } \\
\square \text { Other (specify) }\end{array}$ & $\begin{array}{l}\text { ADDRESS TO: } \\
\square \text { DOE-HQ } \\
\square \text { DOE-RL } \\
\square \text { Other (specify) }\end{array}$ \\
\hline \multicolumn{4}{|c|}{$\begin{array}{l}\text { Milestone Description: } \\
\text { A critical path tabular report generated from the Hanford Site Integrated Schedule (HSIS) } \\
\text { database reflecting the schedule data from the programmatic MYPPs/FYWPs. The } \\
\text { critical path tabular report will show activity titles, current status, scheduled and forecast } \\
\text { finish dates, and the total float related to each activity. The purpose of the HSIS is to aid } \\
\text { in the analysis of critical path activities which may impact completion of Hanford Site } \\
\text { cleanup. This information will be used to show program interfaces, work progress, and } \\
\text { used to develop plans of actions to mitigate potential impacts. In FY 1995, this will be } \\
\text { statused monthly via a critical path analysis with recommended corrective actions. }\end{array}$} \\
\hline \multicolumn{4}{|c|}{$\begin{array}{l}\text { Description of what constitutes completion of this milestone: } \\
\text { The critical path report will be transmitted via cover letter to RL each month. }\end{array}$} \\
\hline \multicolumn{4}{|c|}{ Milestone Description Approval } \\
\hline $\begin{array}{l}\text { Cost Account Manager } \\
\partial K=\text { Tish } \\
\text { J.K. Fish }\end{array}$ & $\begin{array}{r}\text { Date } \\
08 \cdot 22-94\end{array}$ & $\begin{array}{l}\text { Program/Project } \\
\text { D. B. Pabst }\end{array}$ & $8 / 29 / 94$ \\
\hline $\begin{array}{l}\text { Program Element Man } \\
\text { gk fish fo } \\
\text { D.L. Borders }\end{array}$ & $\begin{array}{r}\text { Date } \\
\text { Yoras }\end{array}$ & $\begin{array}{l}\text { DOE Monitor } \\
\text { H.A.R Rtherfort. }\end{array}$ & Date \\
\hline \multicolumn{4}{|c|}{ 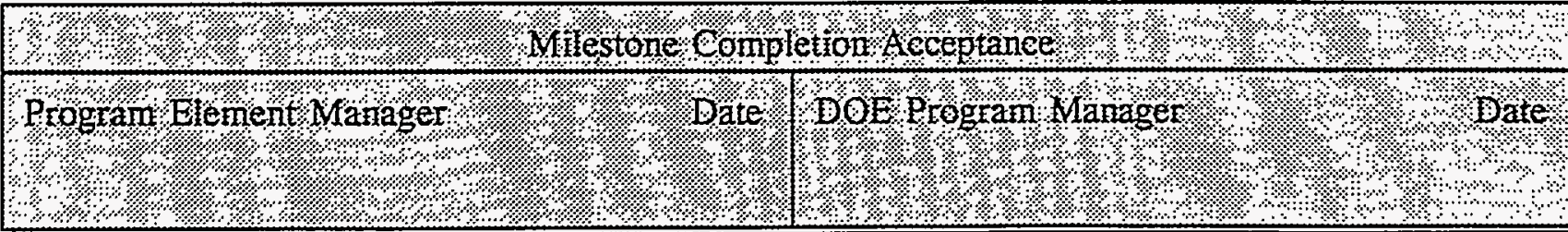 } \\
\hline
\end{tabular}




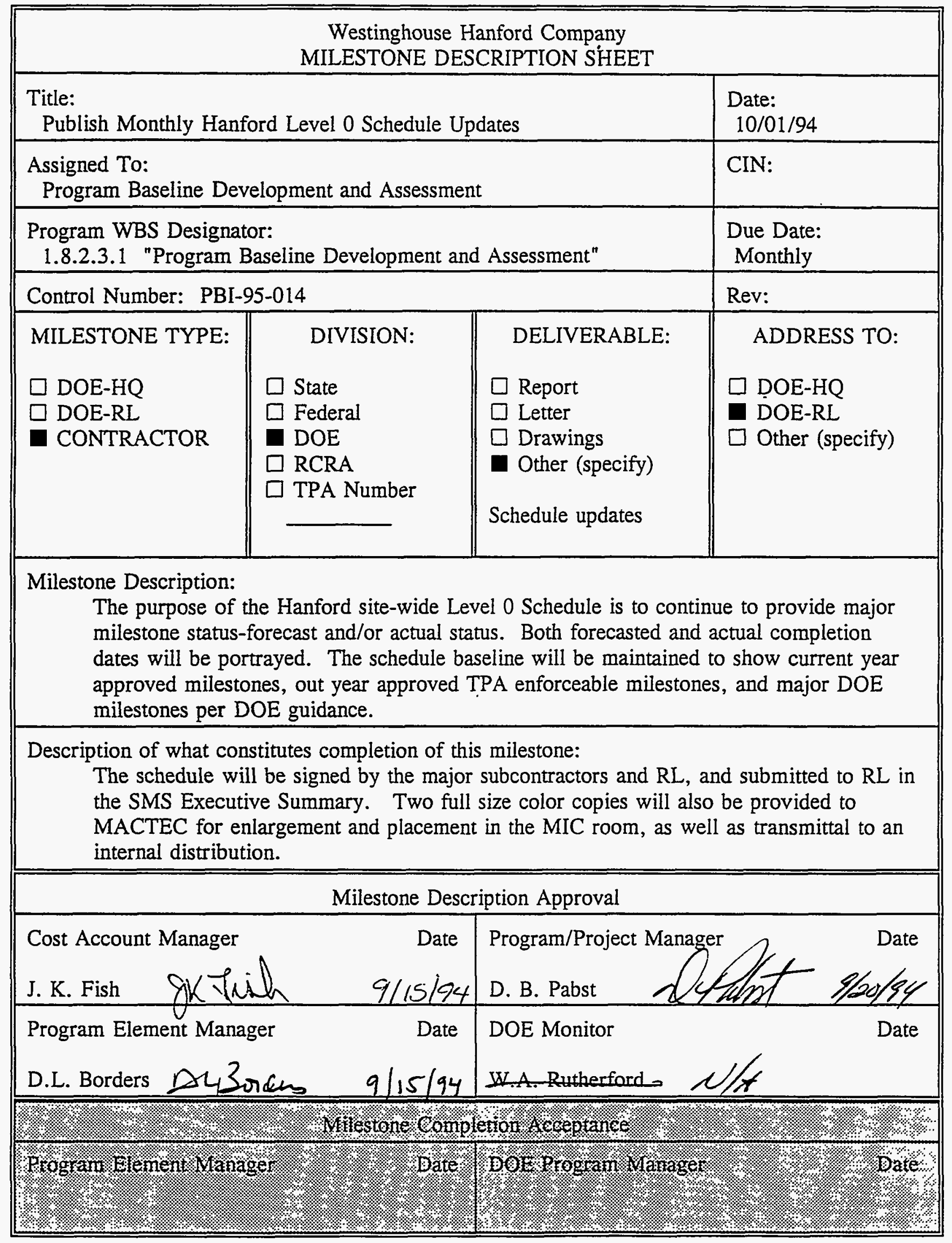




\begin{tabular}{||l|l||}
\hline \multicolumn{3}{|c|}{ Westinghouse Hanford Company } \\
MILESTONE DESCRIPTION SHEET
\end{tabular}




\begin{tabular}{||l|l|l||}
\hline \multicolumn{3}{|c||}{ Westinghouse Hanford Company } \\
MILESTONE DESCRIPTION SHEET
\end{tabular}




\begin{tabular}{|l||l|l||}
\hline \multicolumn{3}{|c|}{ Westinghouse Hanford Company } \\
MILESTONE DESCRIPTION SHEET
\end{tabular}




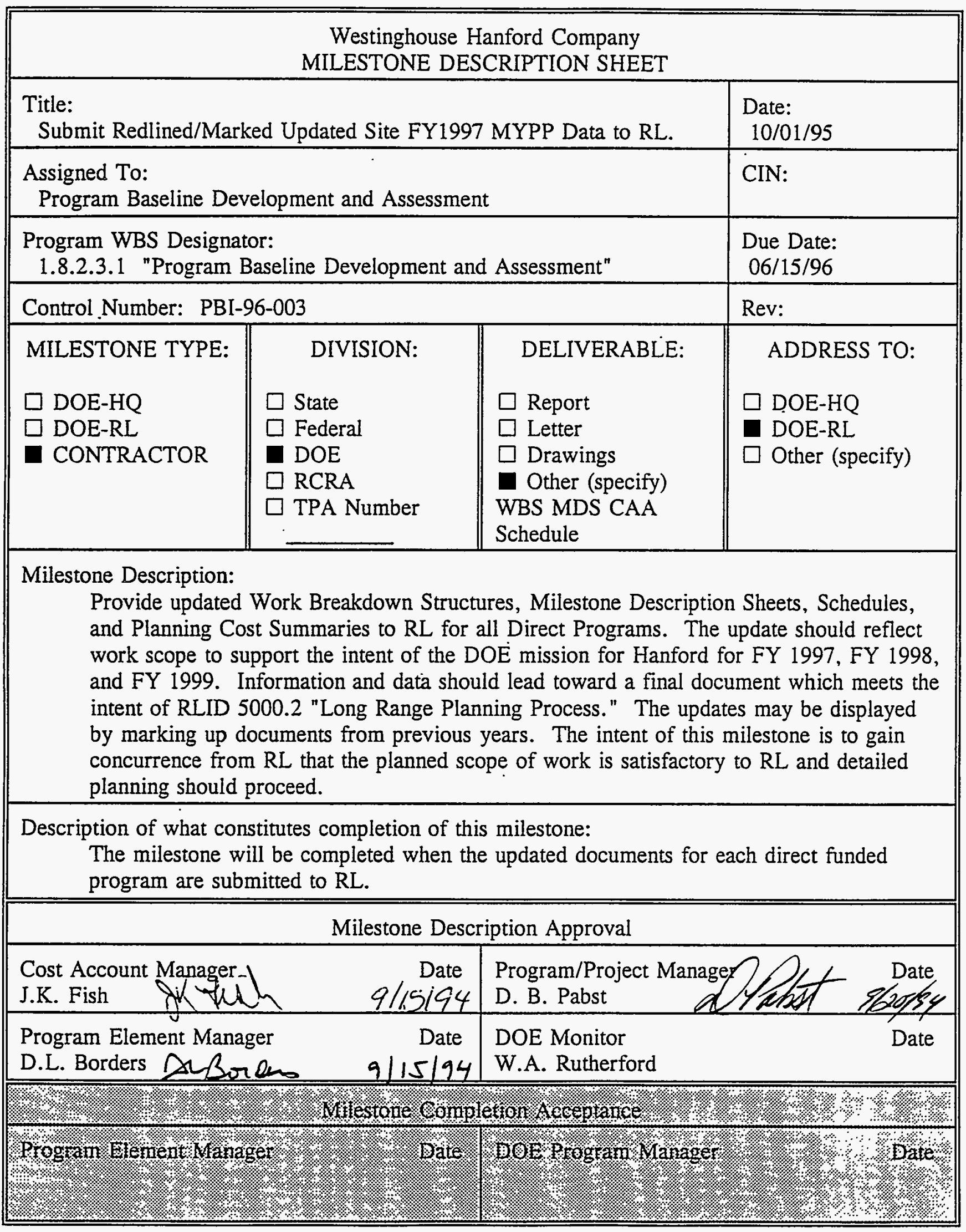




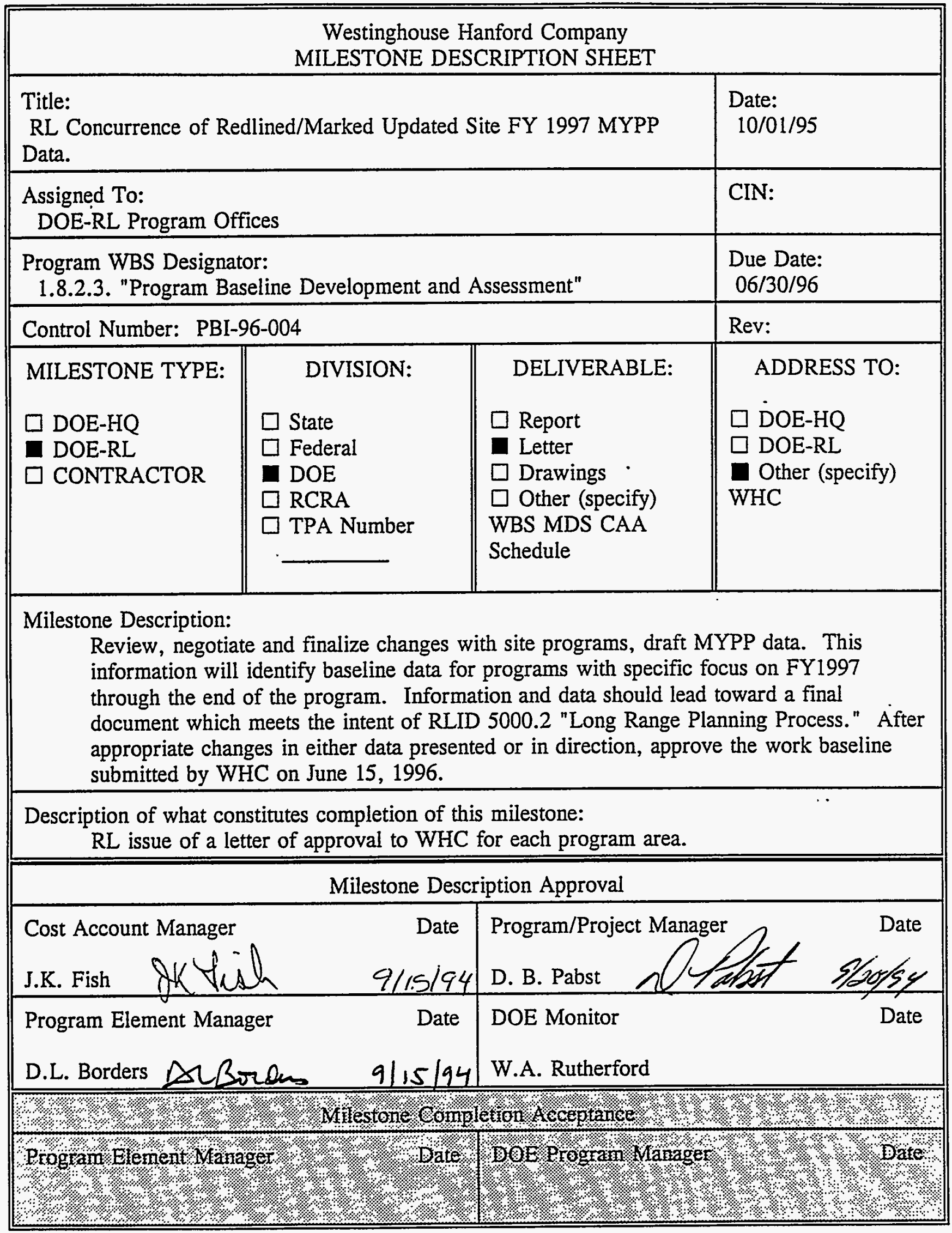




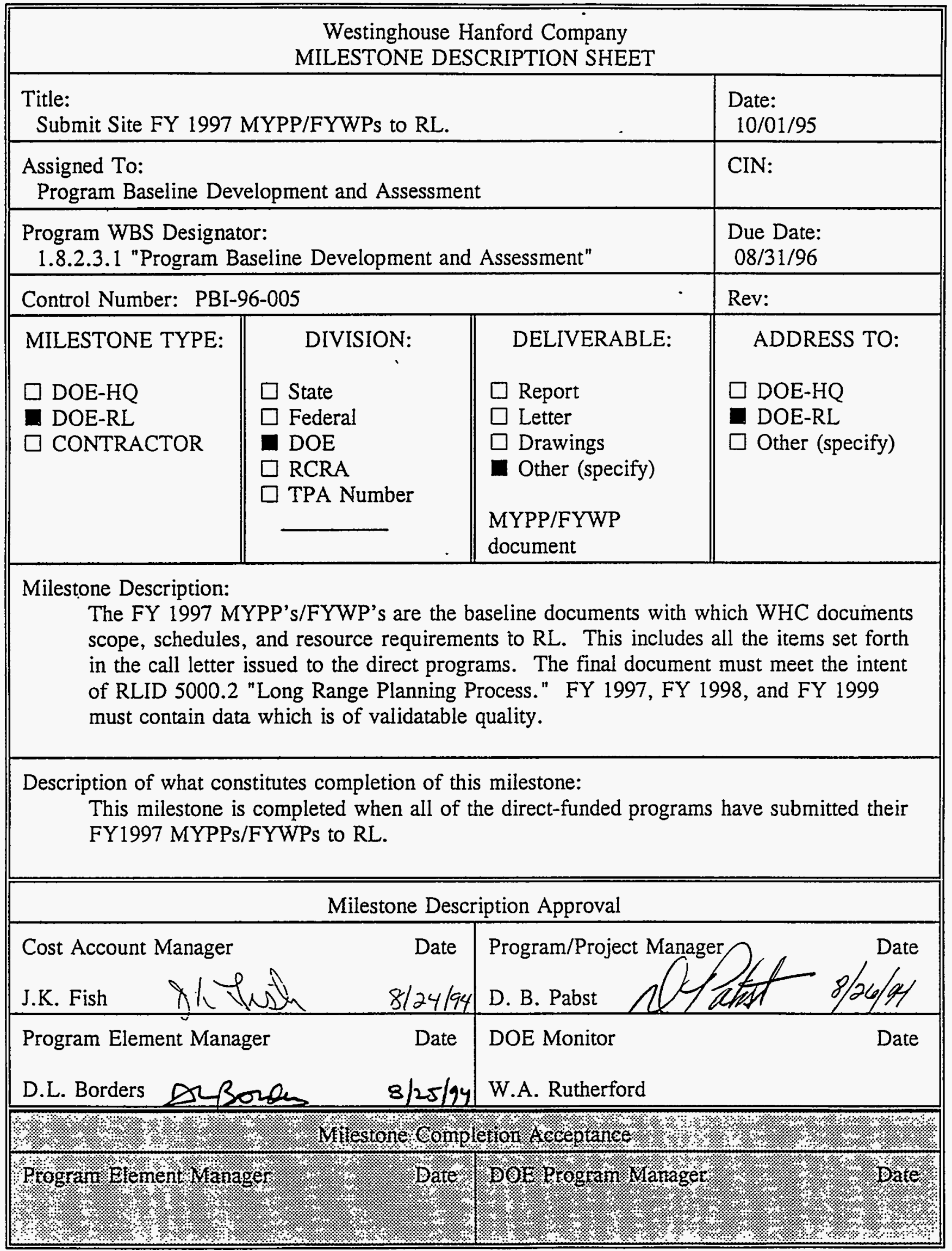




\begin{tabular}{|c|c|c|c|}
\hline \multicolumn{4}{|c|}{$\begin{array}{l}\text { Westinghouse Hanford Company } \\
\text { MILESTONE DESCRIPTION SHEET }\end{array}$} \\
\hline \multicolumn{3}{|c|}{$\begin{array}{l}\text { Title: } \\
\text { RL Approve FY } 1997 \text { MYPPs/FYWPs }\end{array}$} & $\begin{array}{l}\text { Date: } \\
10 / 01 / 95\end{array}$ \\
\hline \multicolumn{3}{|c|}{$\begin{array}{l}\text { Assigned To: } \\
\text { Department of Energy, Richland Operations Office Program Offices }\end{array}$} & CIN: \\
\hline \multicolumn{3}{|c|}{$\begin{array}{l}\text { Program WBS Designator: } \\
\text { 1.8.2.3.1 "Program Baseline Development and Assessment" }\end{array}$} & $\begin{array}{l}\text { Due Date: } \\
09 / 29 / 96\end{array}$ \\
\hline \multicolumn{3}{|c|}{ Control Number: PBI-96-006 } & Rev: \\
\hline $\begin{array}{l}\text { MILESTONE TYPE: } \\
\square \text { DOE-HQ } \\
\square \text { DOE-RL } \\
\square \text { CONTRACTOR }\end{array}$ & $\begin{array}{l}\quad \text { DIVISION: } \\
\square \text { State } \\
\square \text { Federal } \\
\text { DOE } \\
\square \text { RCRA } \\
\square \text { TPA Number }\end{array}$ & $\begin{array}{l}\text { DELIVERABLE: } \\
\square \text { Report } \\
\square \text { Letter } \\
\square \text { Drawings } \\
\square \text { Other (specify) }\end{array}$ & $\begin{array}{l}\text { ADDRESS TO: } \\
\square \text { DOE-HQ } \\
\square \text { DOE-RL } \\
\text { O Other (specify) } \\
\text { WHC }\end{array}$ \\
\hline \multicolumn{4}{|c|}{$\begin{array}{l}\text { Milestone Description: } \\
\text { RL approval of the FY } 1997 \text { MYPP's/FYWP's for the direct programs. Approval should } \\
\text { be based on the intent of RLID 5000.2, "Long Range Planning Process", RL's agreement } \\
\text { to the scope, associated schedule, and cost of work scope, and is satisfactory to } \\
\text { commence the FY } 1997 \text { work described. Issue direction to WHC that FY } 1997 \text { work } \\
\text { tasks, milestones, and other supporting information are approved. }\end{array}$} \\
\hline \multicolumn{4}{|c|}{$\begin{array}{l}\text { Description of what constitutes completion of this milestone: } \\
\text { Formal approval, by RL, of the MYPPs/FYWPs. }\end{array}$} \\
\hline \multicolumn{4}{|c|}{ Milestone Description Approval } \\
\hline \multicolumn{2}{|c|}{$\begin{array}{lr}\text { Cost Account Manager } & \text { Date } \\
\text { J.K. Fish } & 9 \% 15 / 94 \\
\end{array}$} & \multicolumn{2}{|c|}{ Program/Project Manager } \\
\hline \multicolumn{2}{|c|}{$\begin{array}{lr}\text { Program Element Manager } & \text { Date } \\
\text { D.L. Borders } A \text { Boran } & 9 / 15 / 94 \mid \\
\end{array}$} & \multicolumn{2}{|c|}{$\begin{array}{l}\text { DUE Monitor } \\
\text { W.A. Rutherford }\end{array}$} \\
\hline \multicolumn{4}{|c|}{ 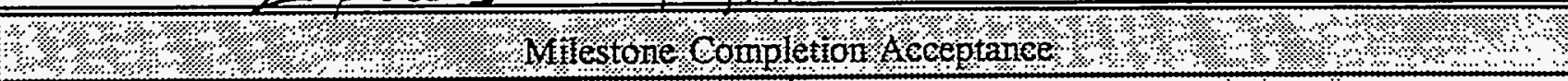 } \\
\hline \multicolumn{4}{|c|}{ 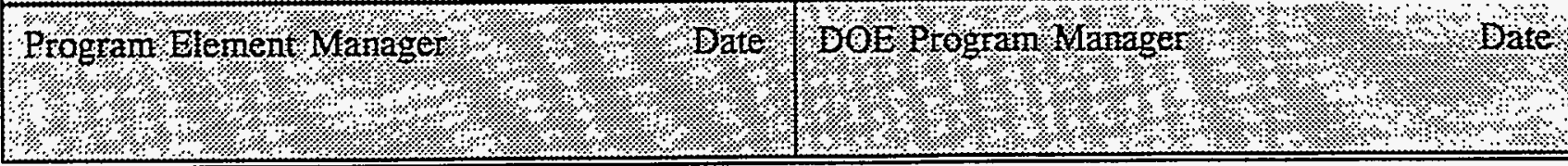 } \\
\hline
\end{tabular}




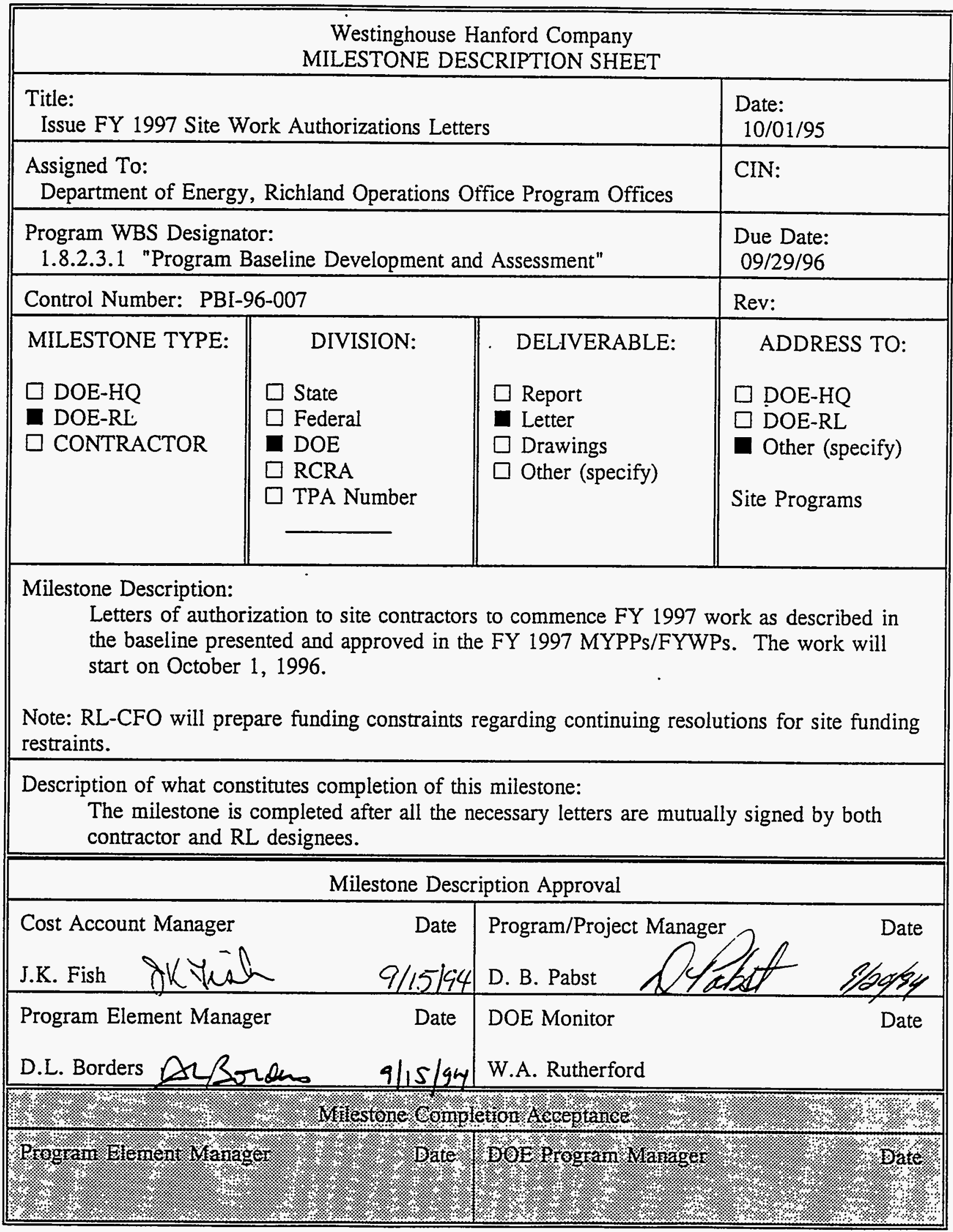




\begin{tabular}{|l||l||}
\hline \multicolumn{3}{|c|}{ Westinghouse Hanford Company } \\
MILESTONE DESCRIPTION SHEET
\end{tabular}




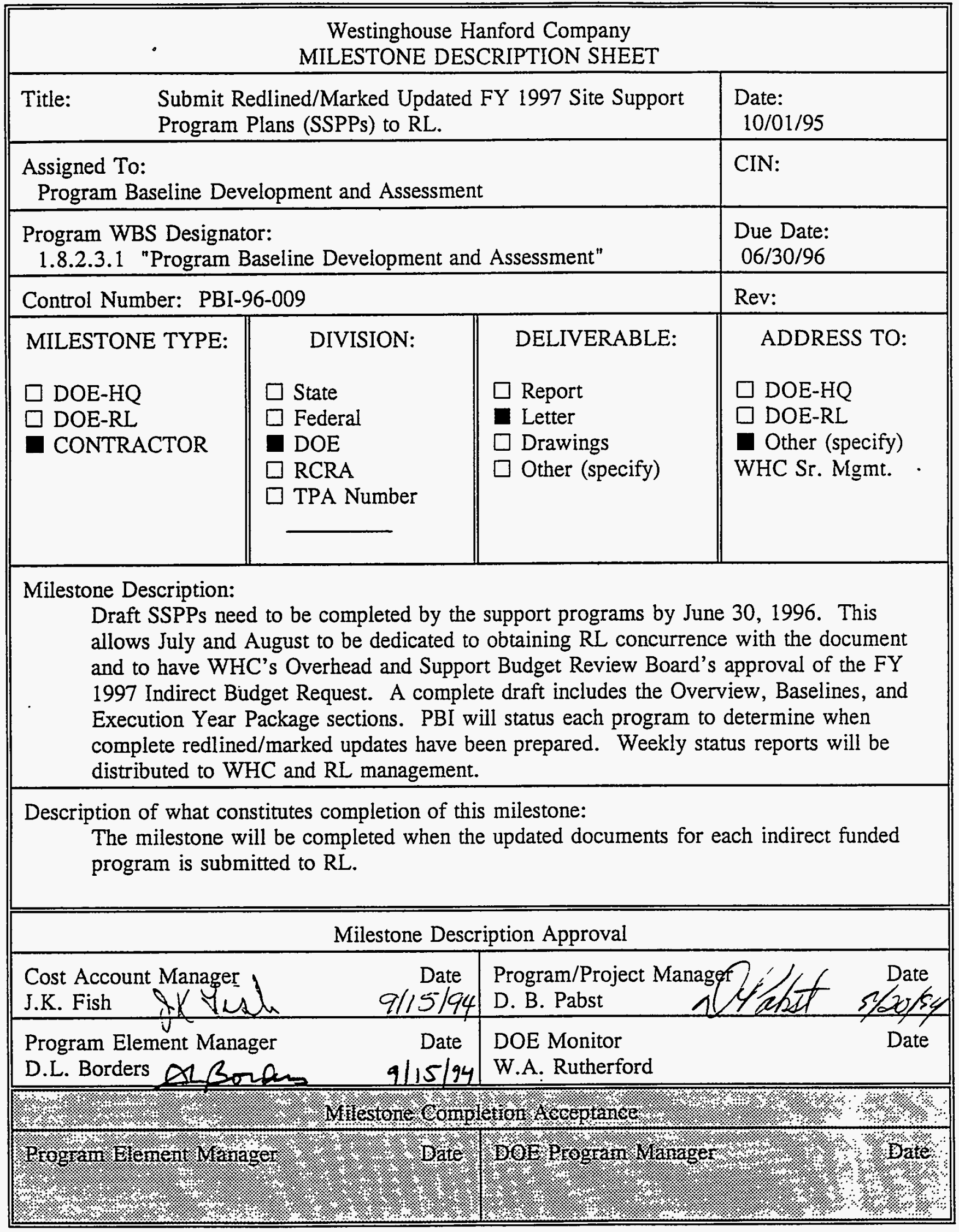




\begin{tabular}{|c|c|c|c|}
\hline \multicolumn{4}{|c|}{$\begin{array}{l}\text { Westinghouse Hanford Company } \\
\text { MILESTONE DESCRIPTION SHEET }\end{array}$} \\
\hline \multicolumn{3}{|c|}{$\begin{array}{l}\text { Title: } \\
\text { RL Concurrence of Redlined/Marked Updated FY } 1997 \text { SSPPs }\end{array}$} & $\begin{array}{l}\text { Date: } \\
\quad 10 / 01 / 95\end{array}$ \\
\hline \multicolumn{3}{|c|}{ Assigned To: DOE-RL Program Offices } & CIN: \\
\hline \multicolumn{3}{|c|}{$\begin{array}{l}\text { Program WBS Designator: } \\
\text { 1.8.2.3.1 "Program Baseline Development and Assessment" }\end{array}$} & $\begin{array}{l}\text { Due Date: } \\
07 / 31 / 96\end{array}$ \\
\hline \multicolumn{3}{|c|}{ Control Number: PBI-96-010 } & Rev: \\
\hline MILESTONE TYPE: & DIVISION: & DELIVERABLE: & ADDRESS TO: \\
\hline $\begin{array}{l}\square \text { DOE-HQ } \\
\square \text { DOE-RL } \\
\square \text { CONTRACTOR }\end{array}$ & $\begin{array}{l}\square \text { State } \\
\square \text { Federal } \\
\square \text { DOE } \\
\square \text { RCRA } \\
\square \text { TPA Number }\end{array}$ & $\begin{array}{l}\square \text { Report } \\
\square \text { Letter } \\
\square \text { Drawings } \\
\square \text { Other (specify) }\end{array}$ & $\begin{array}{l}\square \text { DOE-HQ } \\
\square \text { DOE-RL } \\
\text { Other (specify) } \\
\text { Letters from RL } \\
\text { Monitors to Site } \\
\text { Support Programs }\end{array}$ \\
\hline \multicolumn{4}{|c|}{$\begin{array}{l}\text { Milestone Description: } \\
\text { RL Program Offices will review redlined/marked updated Site Support Program Plans } \\
\text { (SSPPs) submitted to them on June 30, } 1996 \text { and provide comments needed for improving } \\
\text { the SSPPs. The comments should be of such a nature that once resolved by WHC } \\
\text { support programs, the SSPPs will be ready for RL approval after the August } 31 \text { deadline } \\
\text { for submitting final versions of the SSPPs to RL. }\end{array}$} \\
\hline \multicolumn{4}{|c|}{$\begin{array}{l}\text { Description of what constitutes completion of this milestone: } \\
\text { This milestone is completed once all updated SSPPs have been reviewed and returned to } \\
\text { the appropriate WHC program managers. The updates should be returned with a letter } \\
\text { that either concurs with the contents of the draft SSPPs or lists comments that need to be } \\
\text { resolved so that the final SSPPs can be rapidly approved by RL. WHC PBI will status } \\
\text { the support programs to provide periodic status of the review and comment process and } \\
\text { to determine when all updates have been reviewed. }\end{array}$} \\
\hline \multicolumn{4}{|c|}{ Milestone Description Approval } \\
\hline \multicolumn{2}{|c|}{ 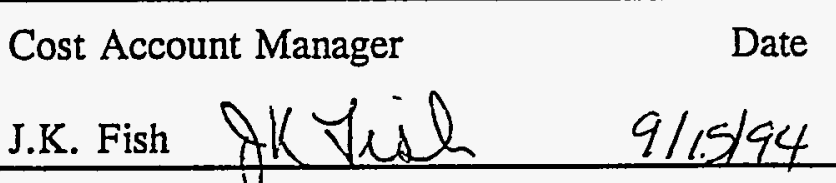 } & \multicolumn{2}{|c|}{ Program/Project Manager/Pabst Date } \\
\hline \multicolumn{2}{|c|}{$\begin{array}{lr}\text { Program Element Manager } & \text { Date } \\
\text { D.L. Borders } \triangle \text { Gonon } & 9 / 15 / 94 \\
\end{array}$} & $\begin{array}{l}\text { DOE Monitor } \\
\text { W.A. Rutherford } \\
\end{array}$ & Date \\
\hline \multicolumn{4}{|c|}{ 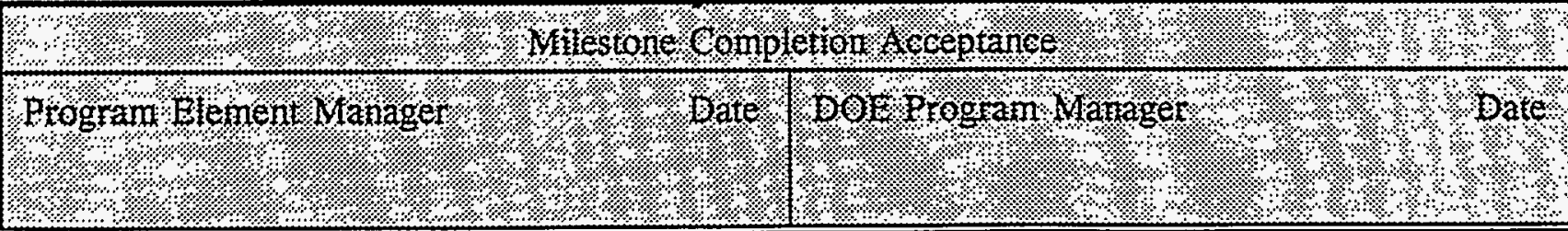 } \\
\hline
\end{tabular}




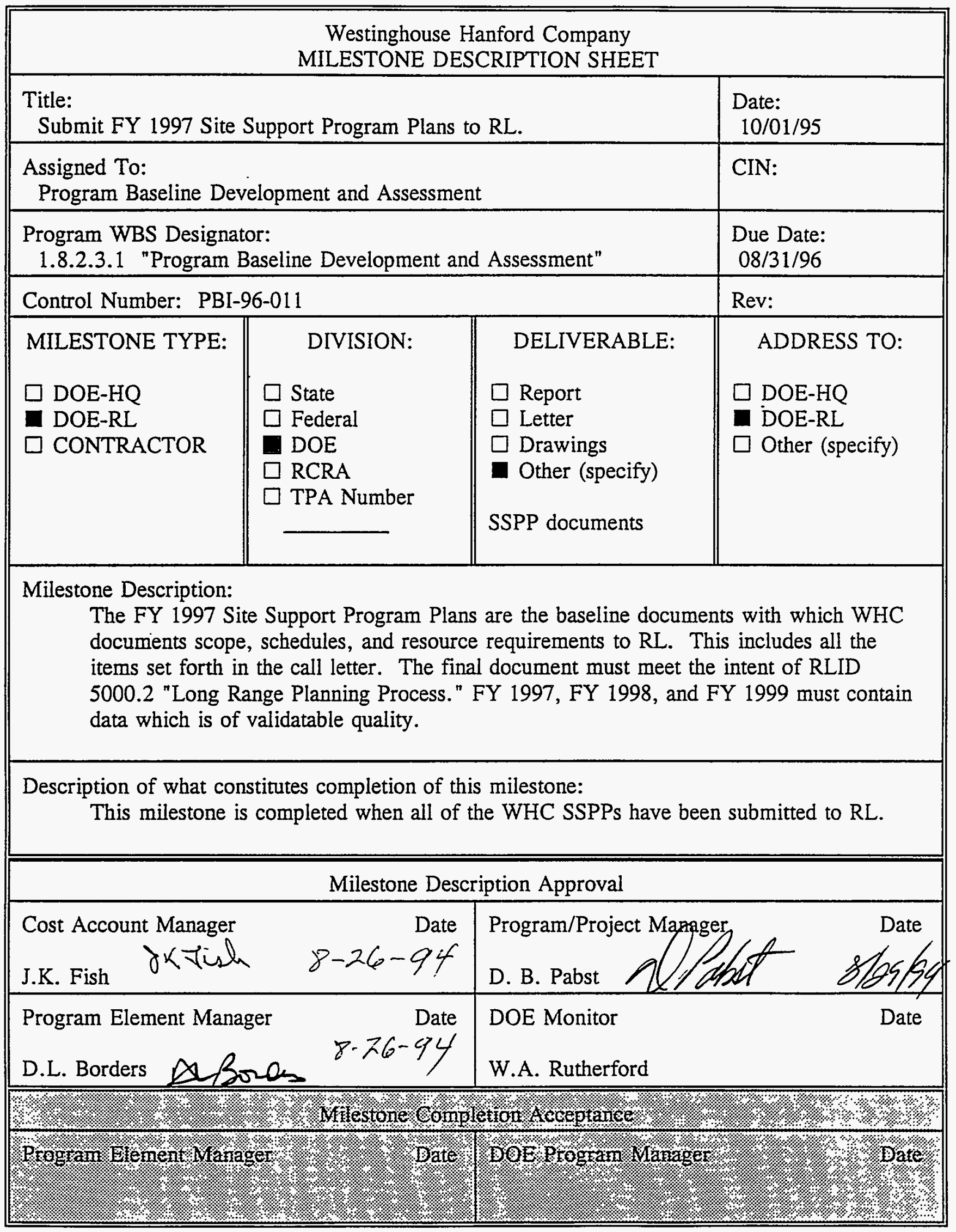




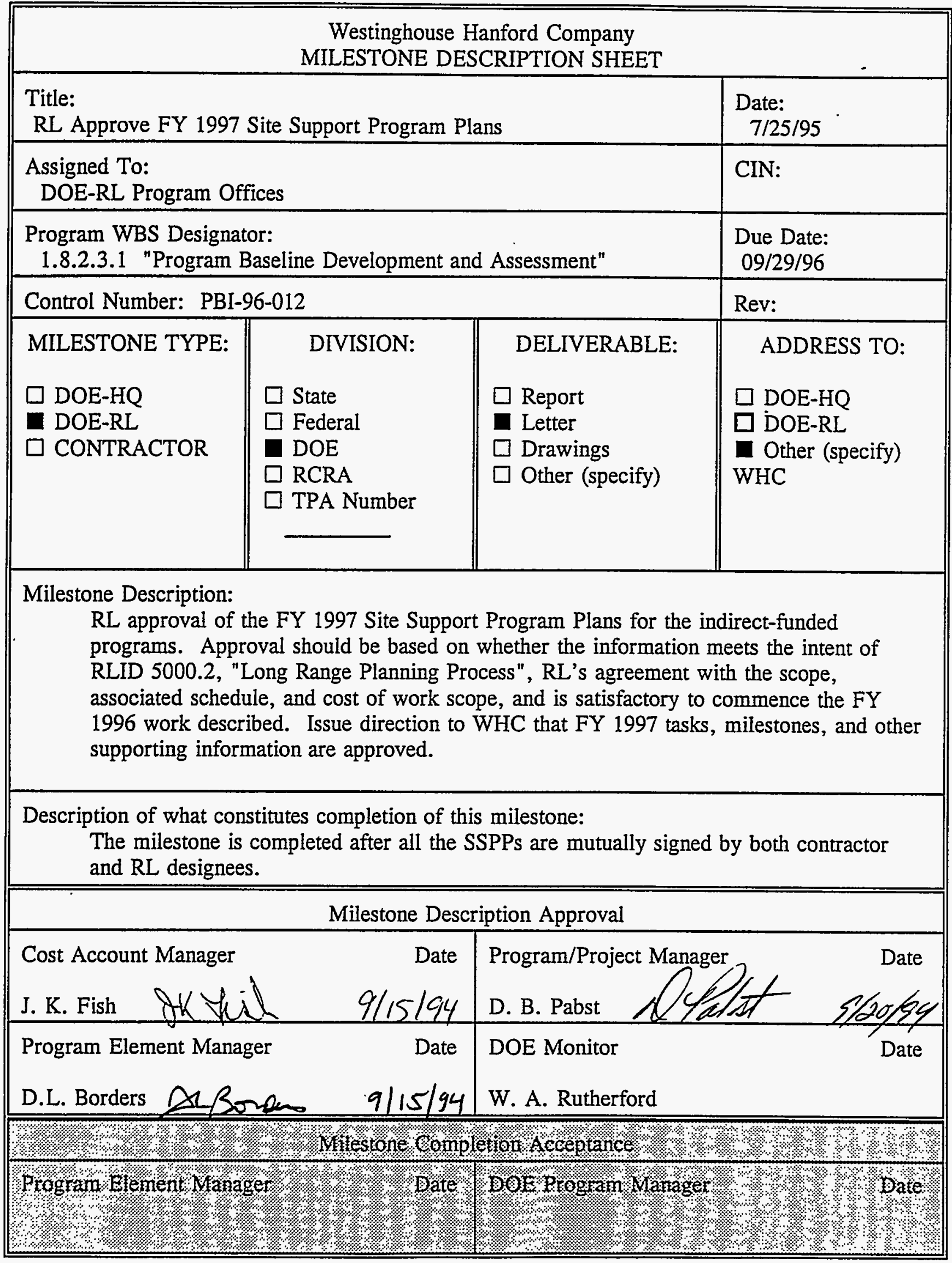




\begin{tabular}{||l|l||}
\hline \multicolumn{3}{|c|}{ Westinghouse Hanford Company } \\
MILESTONE DESCRIPTION SHEET
\end{tabular}




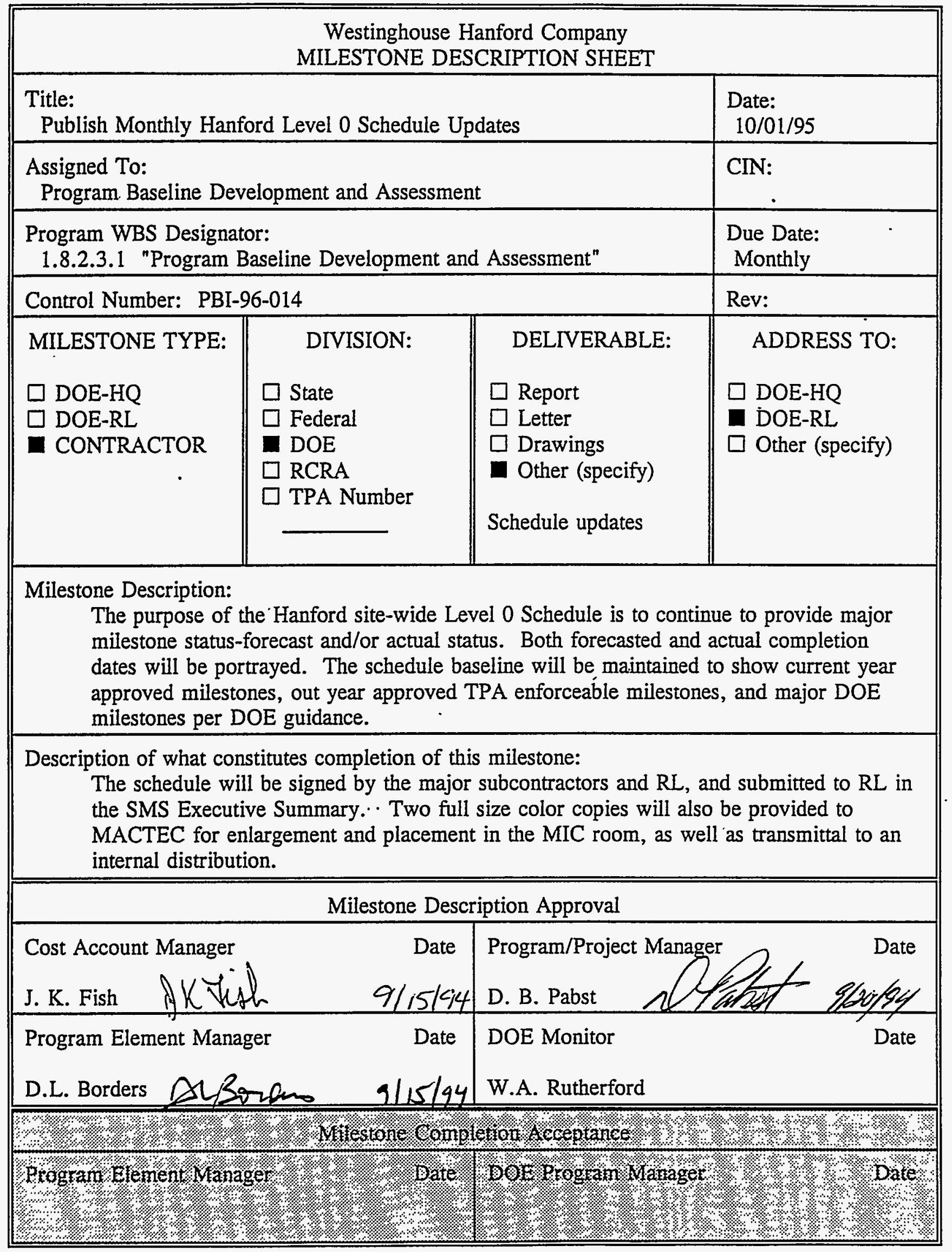




\begin{tabular}{|l|l|l||}
\hline \multicolumn{3}{|c|}{ Westinghouse Hanford Company } \\
MILESTONE DESCRIPTION SHEET
\end{tabular}




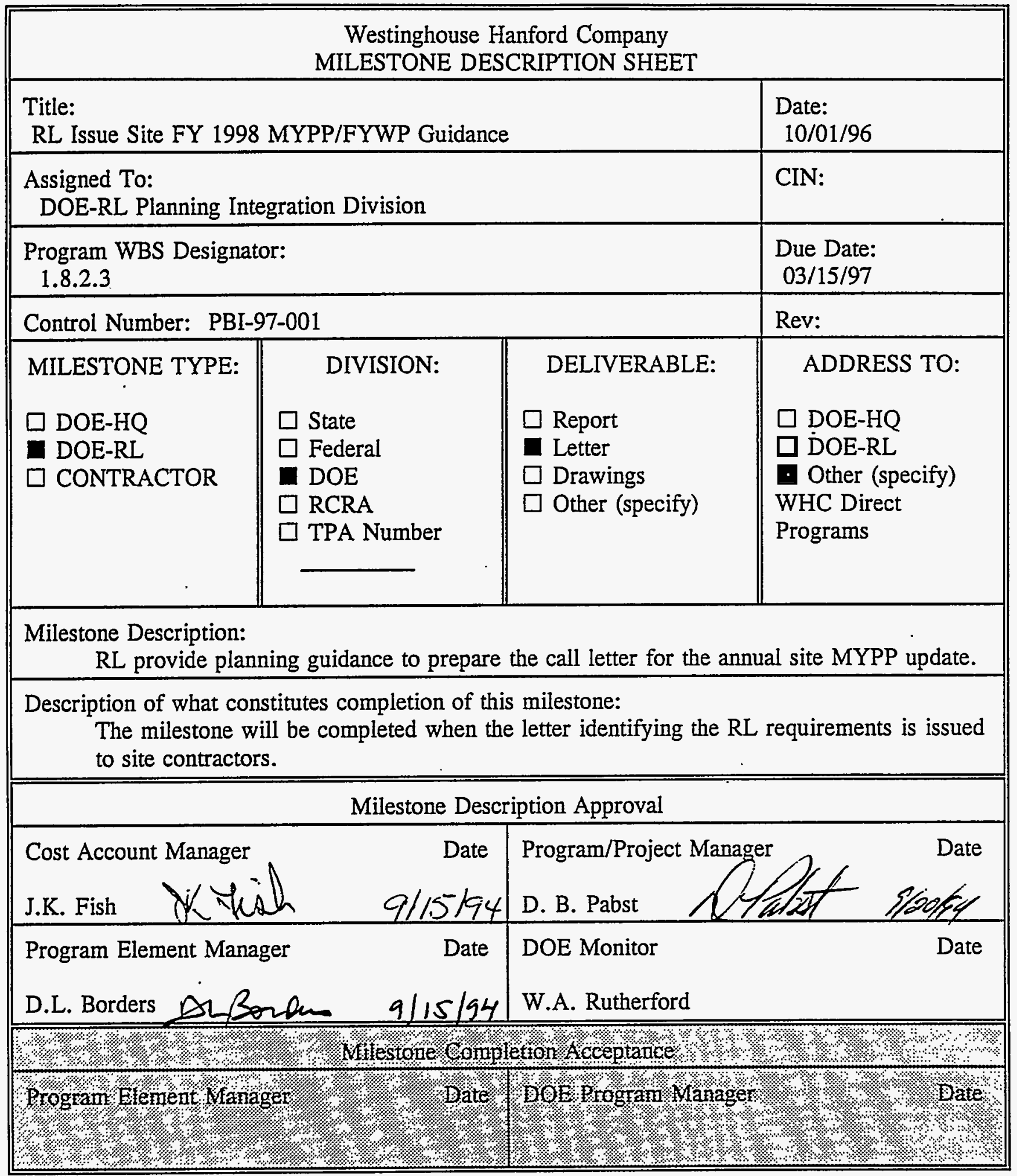




\begin{tabular}{||l|l||}
\hline \multicolumn{3}{|c||}{ Westinghouse Hanford Company } \\
MILESTONE DESCRIPTION SHEET
\end{tabular}




\begin{tabular}{||l|l||}
\hline \multicolumn{3}{|c|}{ Westinghouse Hanford Company } \\
MILESTONE DESCRIPTION SHEET
\end{tabular}




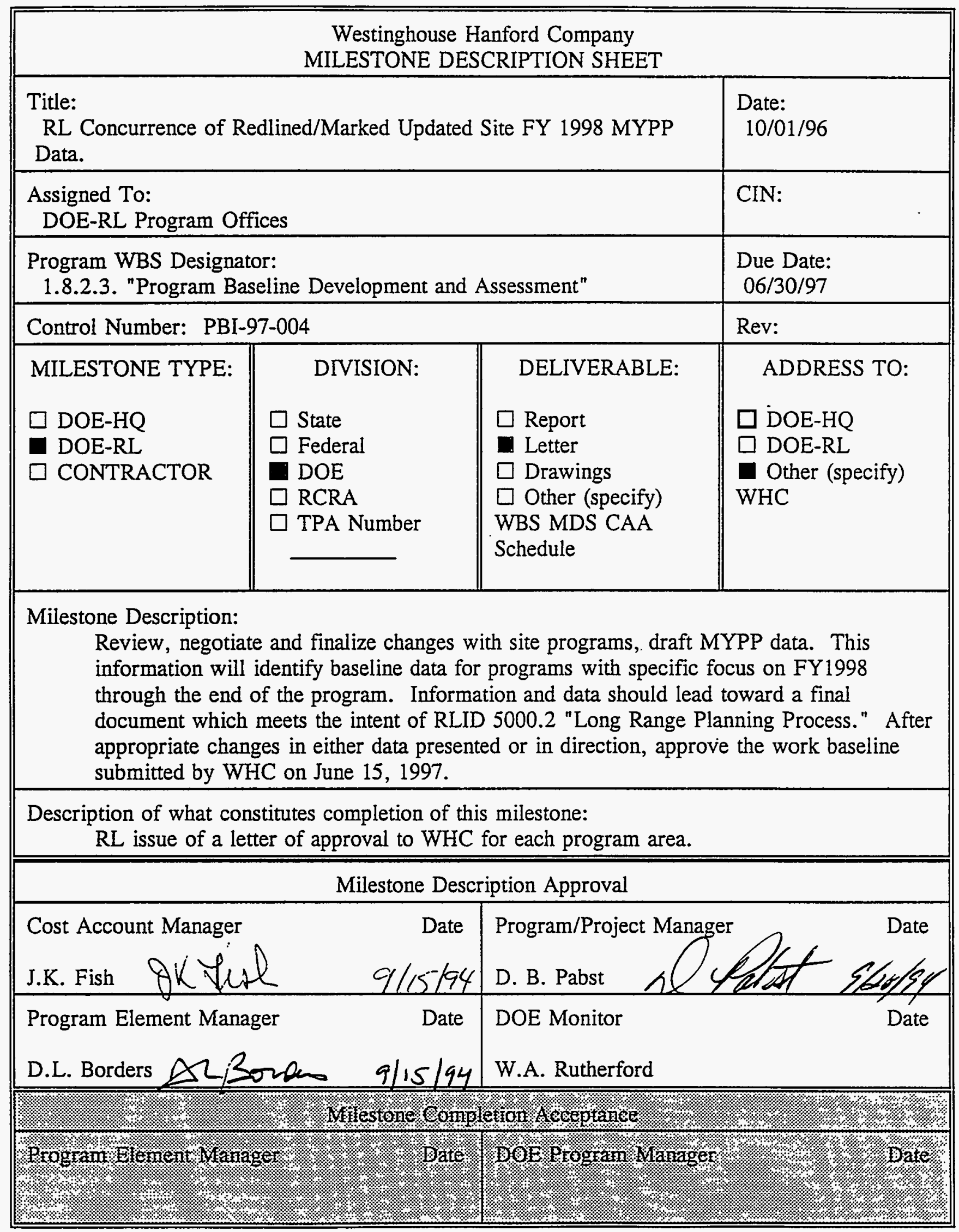




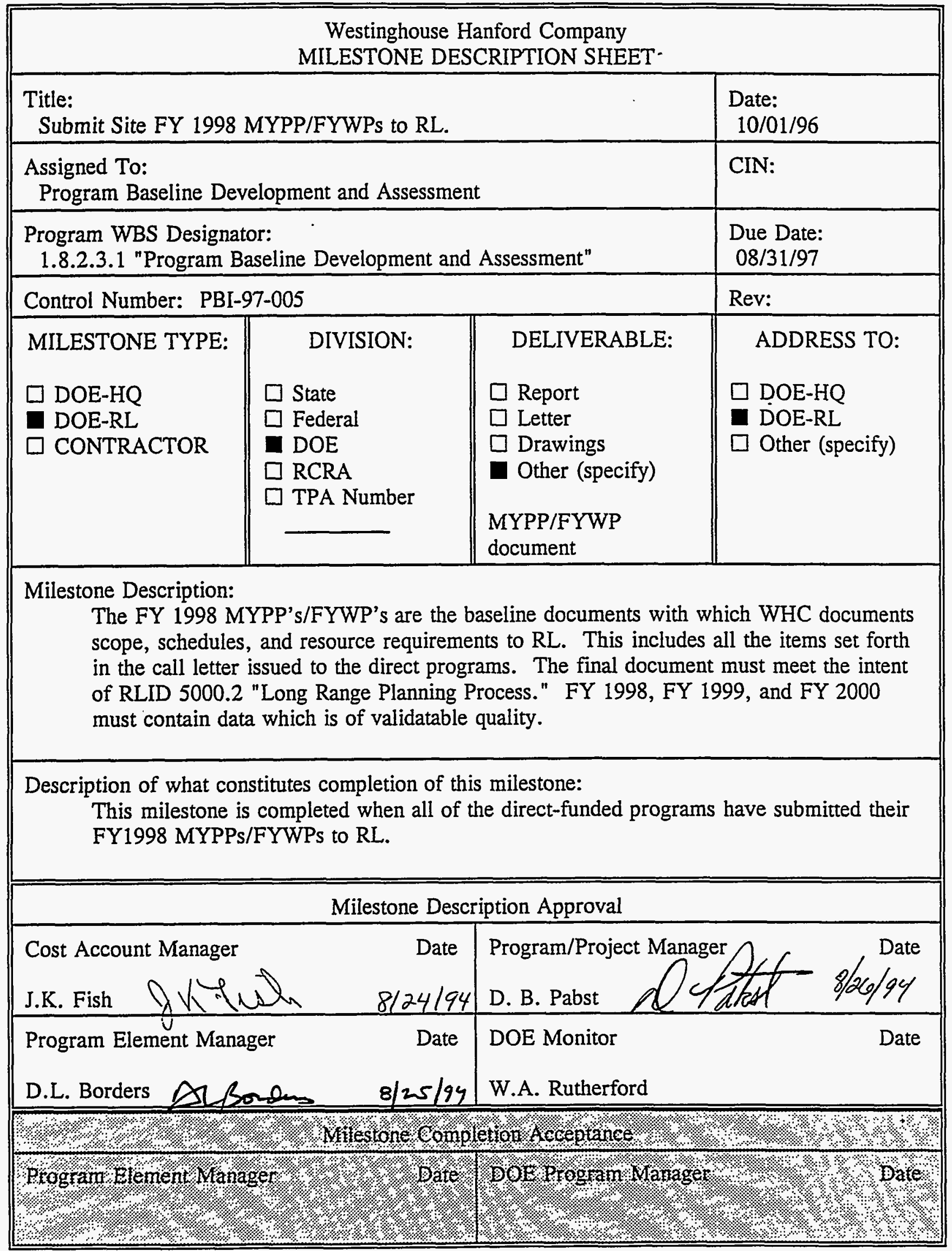




\begin{tabular}{|l|l||}
\hline \multicolumn{3}{|c||}{ Westinghouse Hanford Company } \\
MILESTONE DESCRIPTION SHEET
\end{tabular}




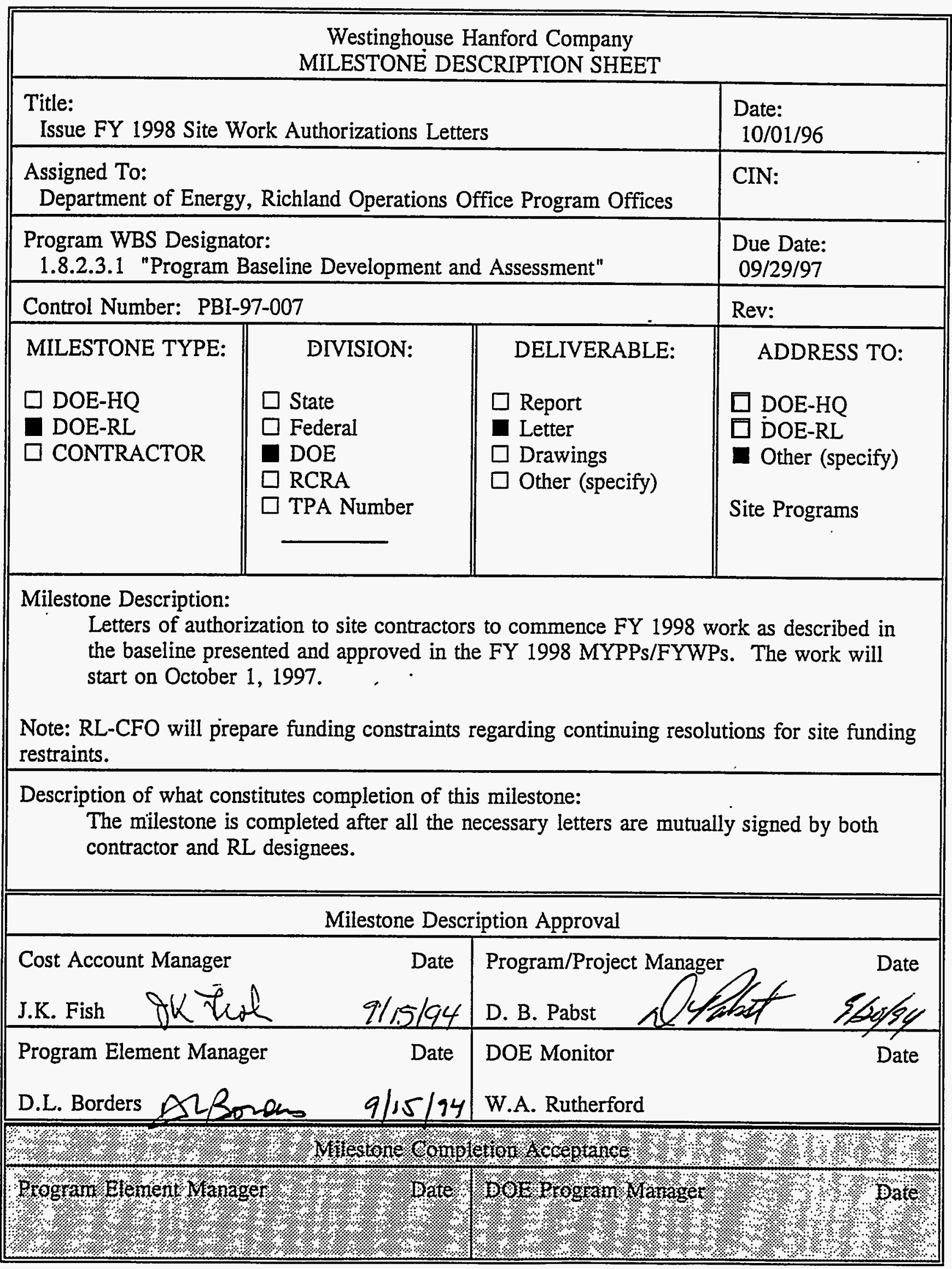




\begin{tabular}{|l||l|l||}
\hline \multicolumn{3}{|c|}{ Westinghouse Hanford Company } \\
MILESTONE DESCRIPTION SHEET
\end{tabular}




\begin{tabular}{||l|l||}
\hline \multicolumn{3}{|c|}{ Westinghouse Hanford Company } \\
MILESTONE DESCRIPTION SHEET
\end{tabular}




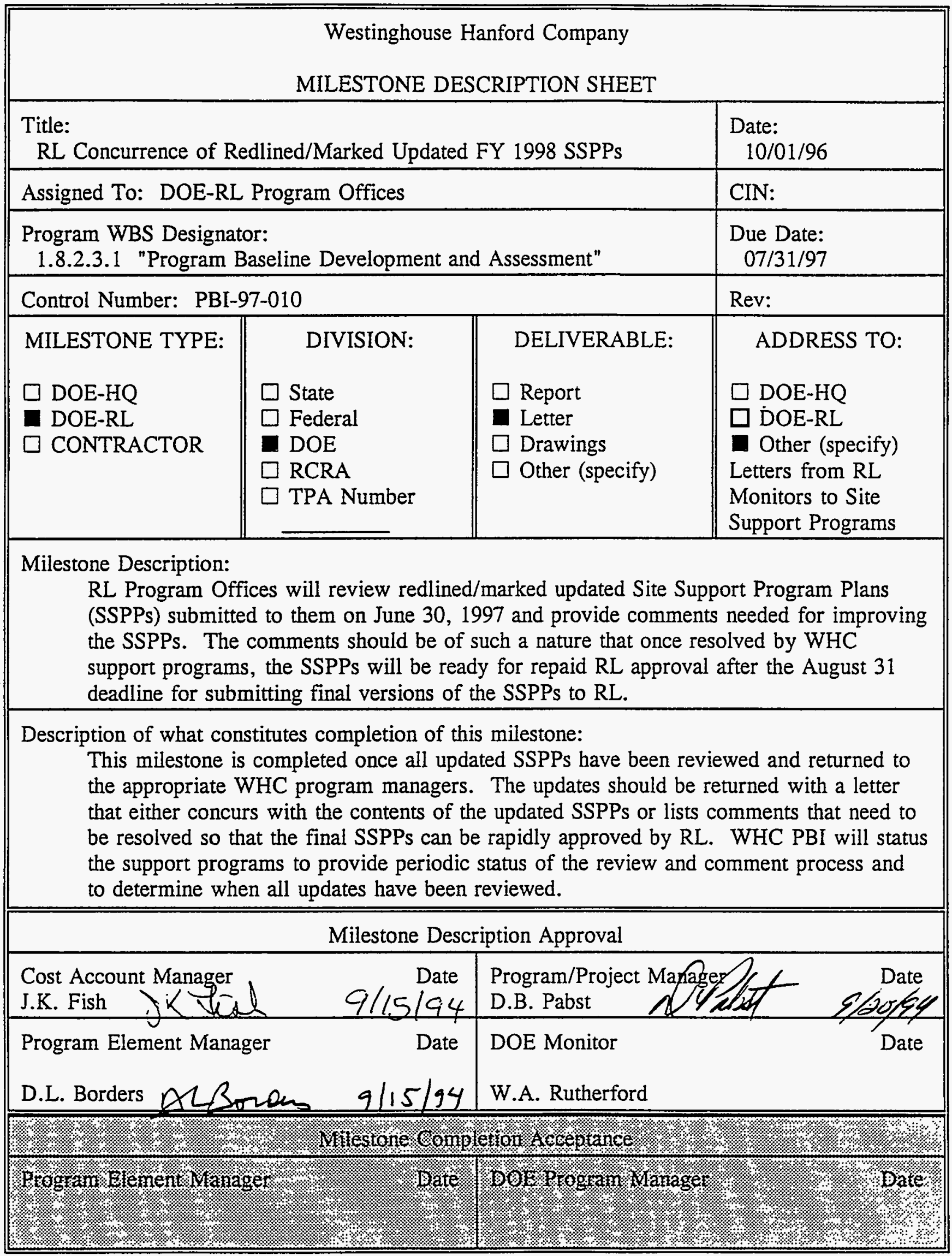




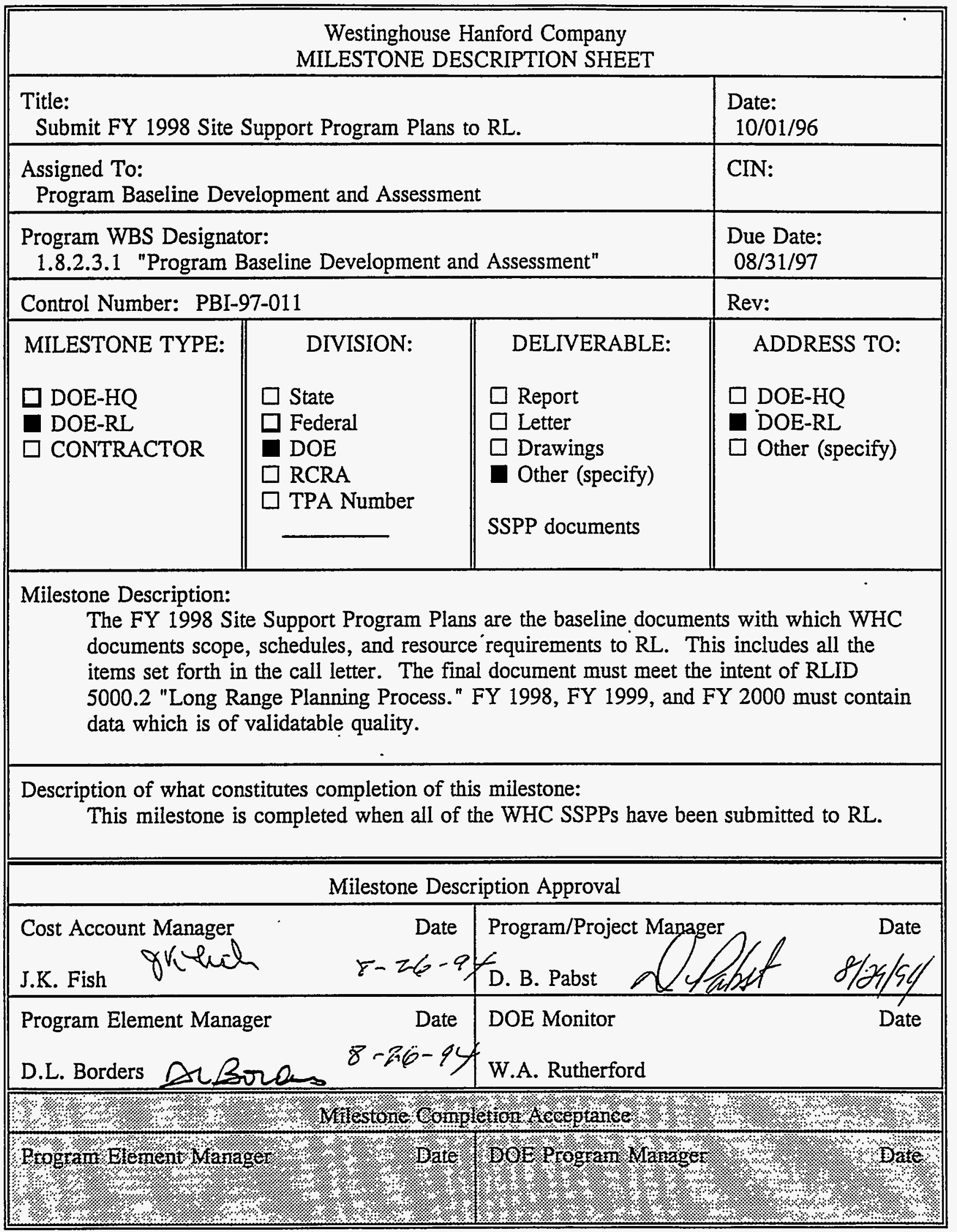




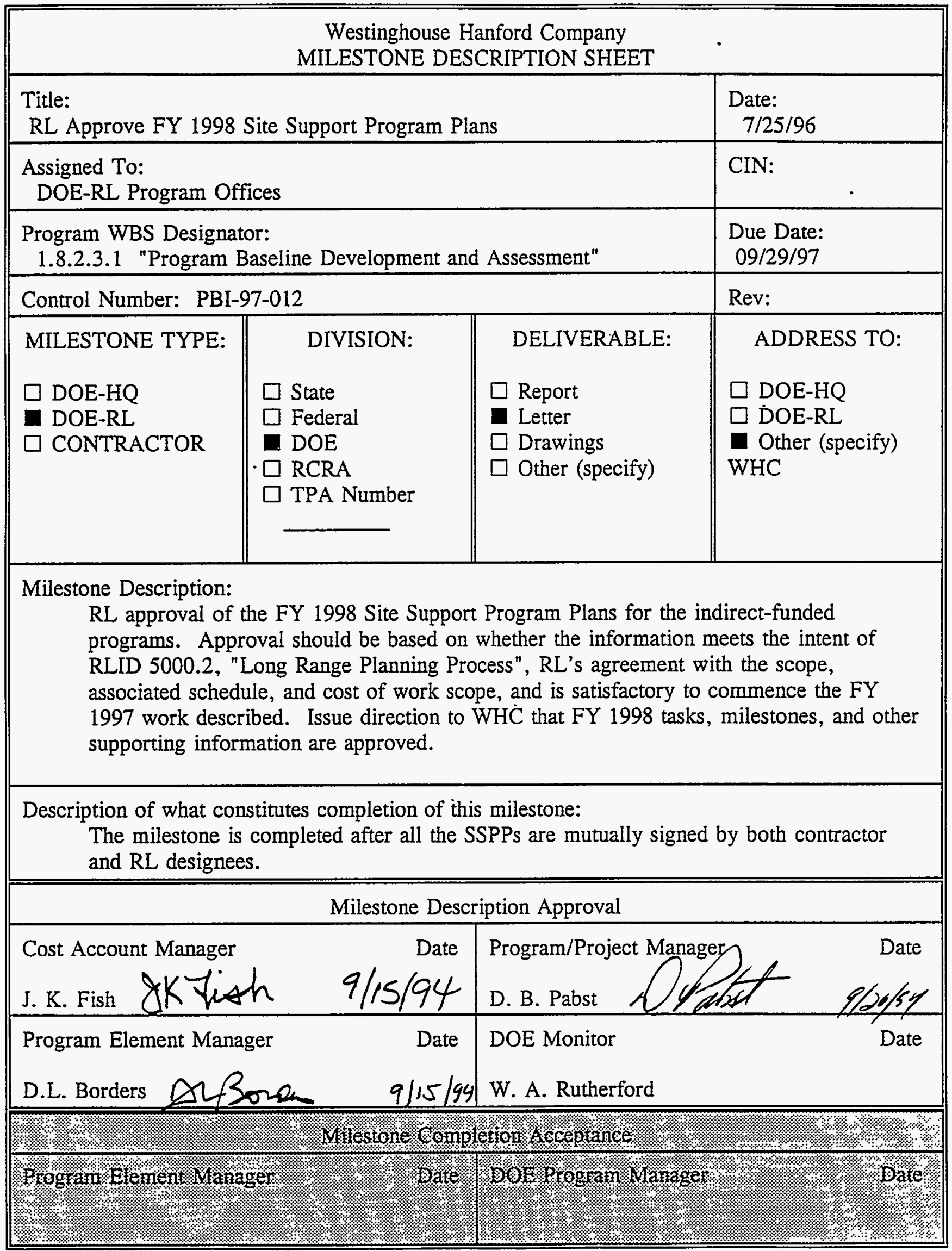




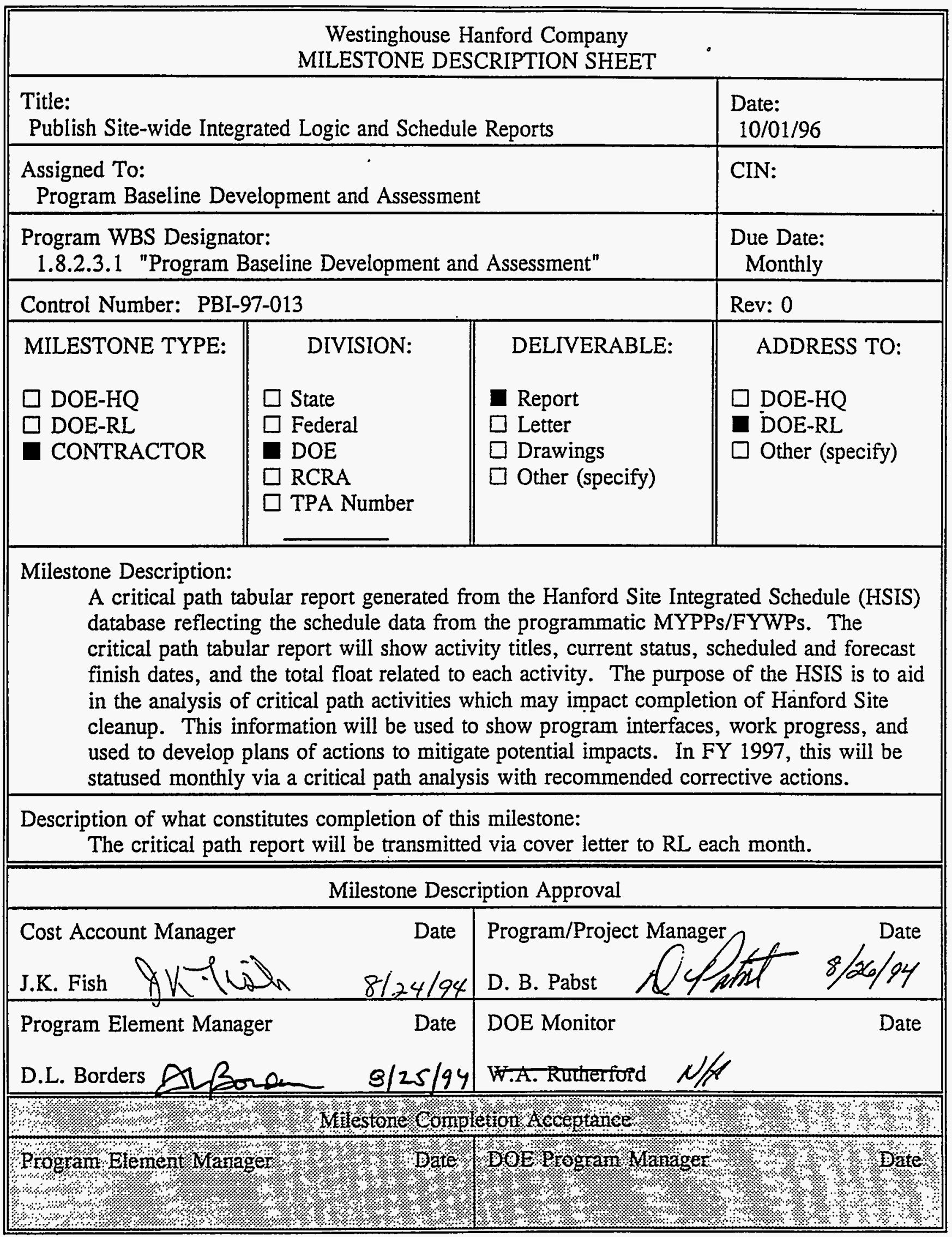




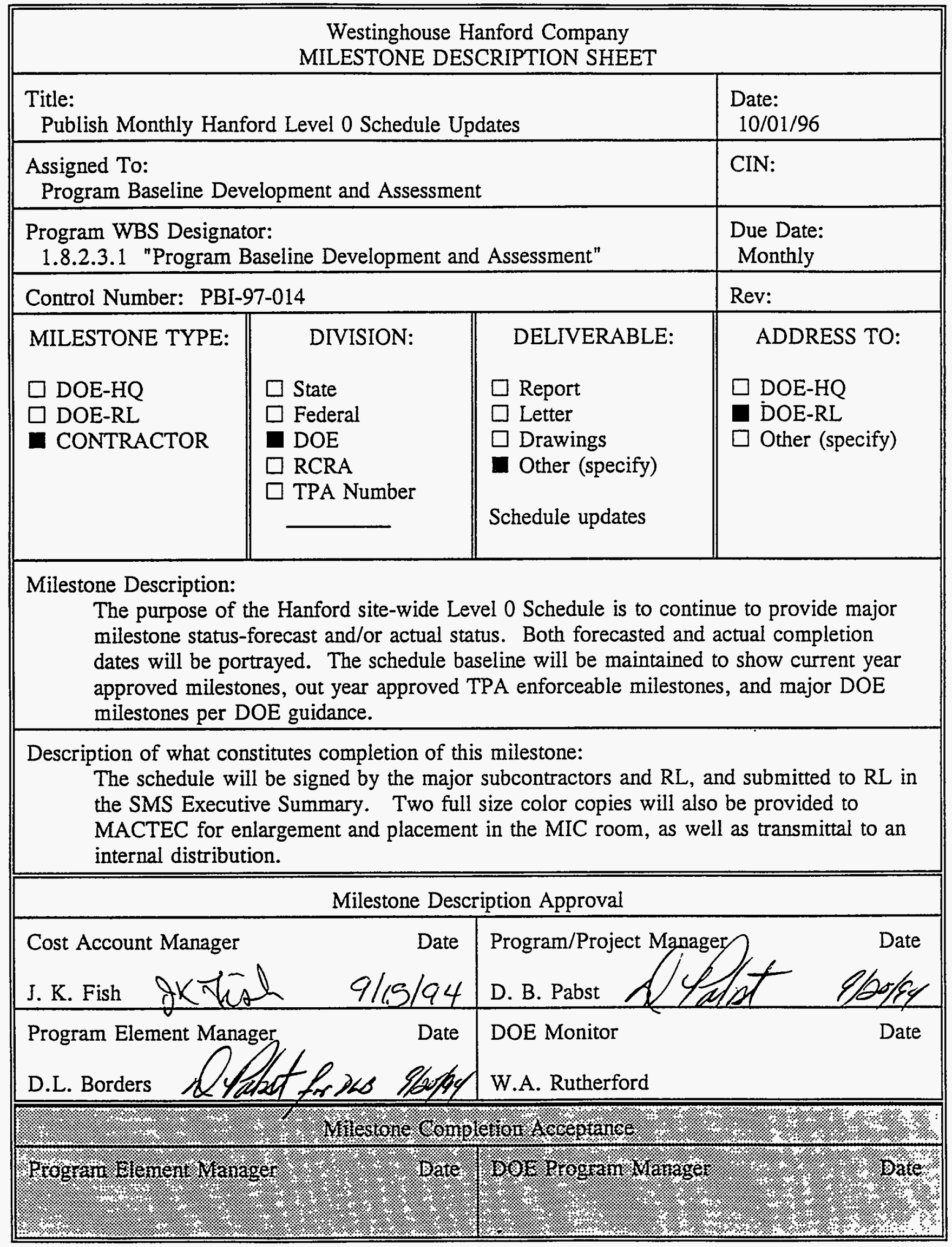




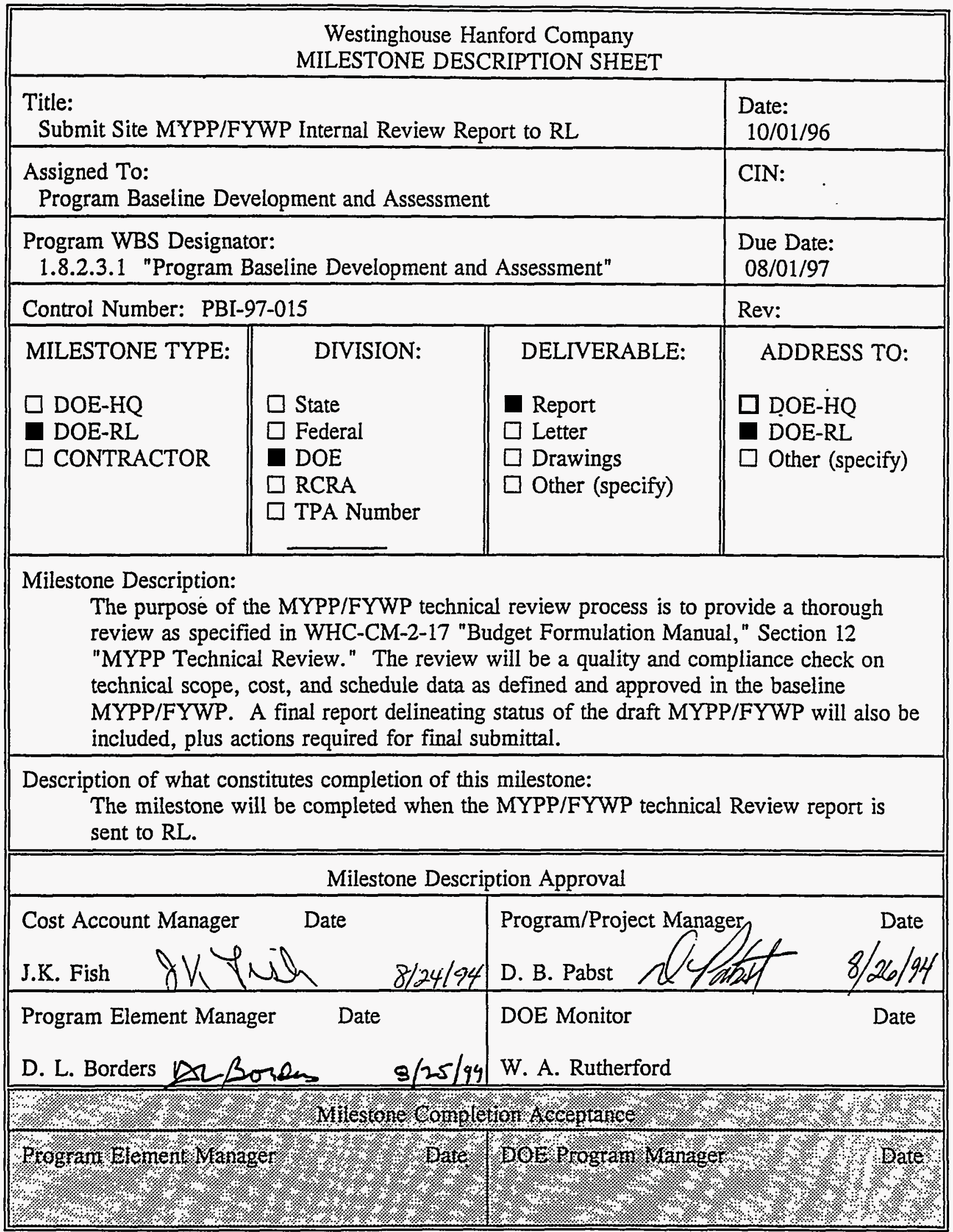


Planning Integration Multi-Year Progran Plan

\section{This page}

intentionally left blank. 


\begin{tabular}{|c|c|c|c|}
\hline \multicolumn{4}{|c|}{$\begin{array}{l}\text { Westinghouse Hanford Company } \\
\text { MILESTONE DESCRIPTION SHEET }\end{array}$} \\
\hline \multicolumn{3}{|c|}{ Title: ADS 7250 Preparation Kick-Off Meeting } & Date: 6-1-94 \\
\hline \multicolumn{3}{|c|}{ Assigned To: D. B. Pabst } & CIN: \\
\hline \multicolumn{3}{|c|}{ Program WBS Designator: 1 8.2.5.1 } & Due Date: $11-14-94$ \\
\hline \multicolumn{3}{|c|}{ Control Number: PIM-95-001 } & Rev: 0 \\
\hline $\begin{array}{l}\text { MILESTONE TYPE: } \\
\square \text { DOE-HQ } \\
\square \text { DOE-RL } \\
\text { X CONTRACTOR }\end{array}$ & $\begin{aligned} & \text { DIVISION: } \\
& \cdot \\
& \square \text { State } \\
& \square \text { Federal } \\
& \text { X DOE } \\
& \square \text { RCRA } \\
& \square \text { TPA Number }\end{aligned}$ & $\begin{array}{l}\quad \text { DELIVERABLE: } \\
\square \text { Report } \\
\square \text { X Letter } \\
\square \text { Drawings } \\
\square \text { Other (specify) } \\
\text { Instructions for ADS } \\
7250 \text { preparation }\end{array}$ & $\begin{array}{l}\quad \text { ADDRESS TO: } \\
\square \text { DOE-HQ } \\
\square \text { DOE-RL } \\
\text { X Other (specify) } \\
\text { All 1.8.2 Planning } \\
\text { Integration } \\
\text { organizations }\end{array}$ \\
\hline \multicolumn{4}{|c|}{$\begin{array}{l}\text { On receipt of the budget data from program element 1.8.2.8 ADS preparation and coordination, } \\
\text { a call letter will be prepared. }\end{array}$} \\
\hline \multicolumn{4}{|c|}{$\begin{array}{l}\text { This deliverable will contain instructions on preparation, detail required, RL site guidance and } \\
\text { preparation schedule. This data will be generated in the form of a call letter and distributed to } \\
\text { all 1.8.2 Planning Integration program elements and RL PID and PUB prior to the kick-off } \\
\text { meeting. }\end{array}$} \\
\hline \multicolumn{4}{|c|}{$\begin{array}{l}\text { Description of what constitutes completion of this milestone: } \\
\text { This milestone will be complete when the above data is distributed, RL comments dispositioned, } \\
\text { and the 1.8.2 Planning Integration kick-off meeting held to clarify any questions by the } \\
\text { participants. }\end{array}$} \\
\hline \multicolumn{4}{|c|}{ Milestone Description Approval } \\
\hline \multicolumn{2}{|c|}{$\begin{array}{l}\text { Cost Aceount Mangoger } \\
\sum \sum_{\text {R. Aflectul }} \text {. Beitrich }\end{array}$} & \multicolumn{2}{|c|}{ Drogram/Project Manager B. Pabst } \\
\hline \multicolumn{2}{|c|}{ Program Element Manager B. Pabst } & $\begin{array}{l}\text { DOE Monitor } \\
\text { N/A }\end{array}$ & Date \\
\hline \multicolumn{3}{|c|}{ 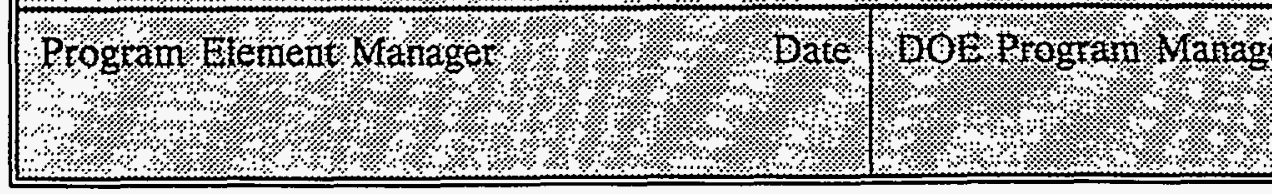 } & 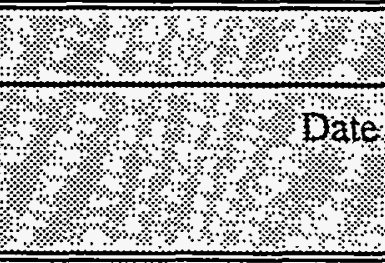 \\
\hline
\end{tabular}




\begin{tabular}{|l||l||}
\hline \multicolumn{3}{|c|}{ Westinghouse Hanford Company } \\
MILESTONE DESCRIPTION SHEET
\end{tabular}




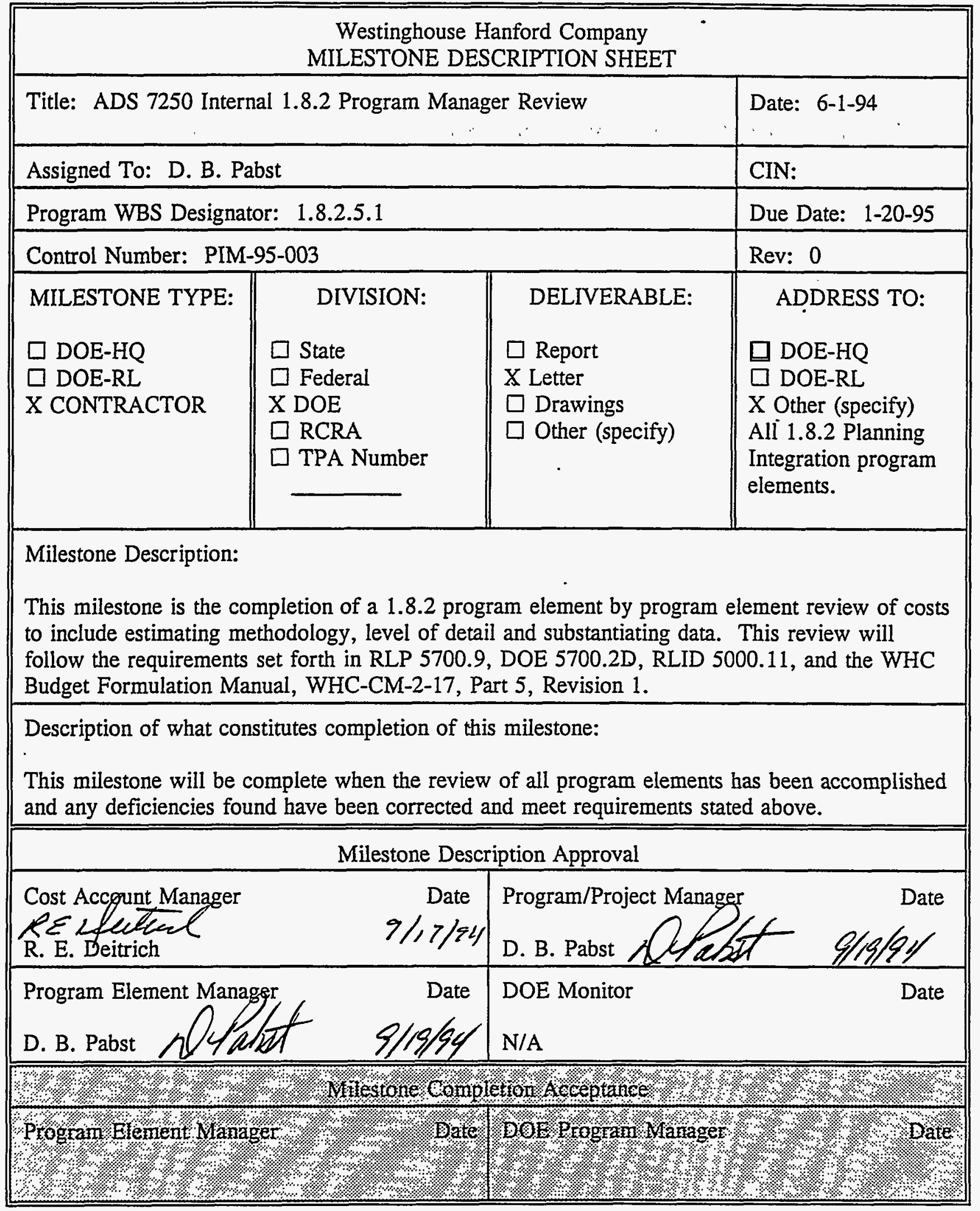




\begin{tabular}{||l||l||}
\hline \multicolumn{3}{|c|}{ Westinghouse Hanford Company } \\
MILESTONE DESCRIPTION SHEET
\end{tabular}




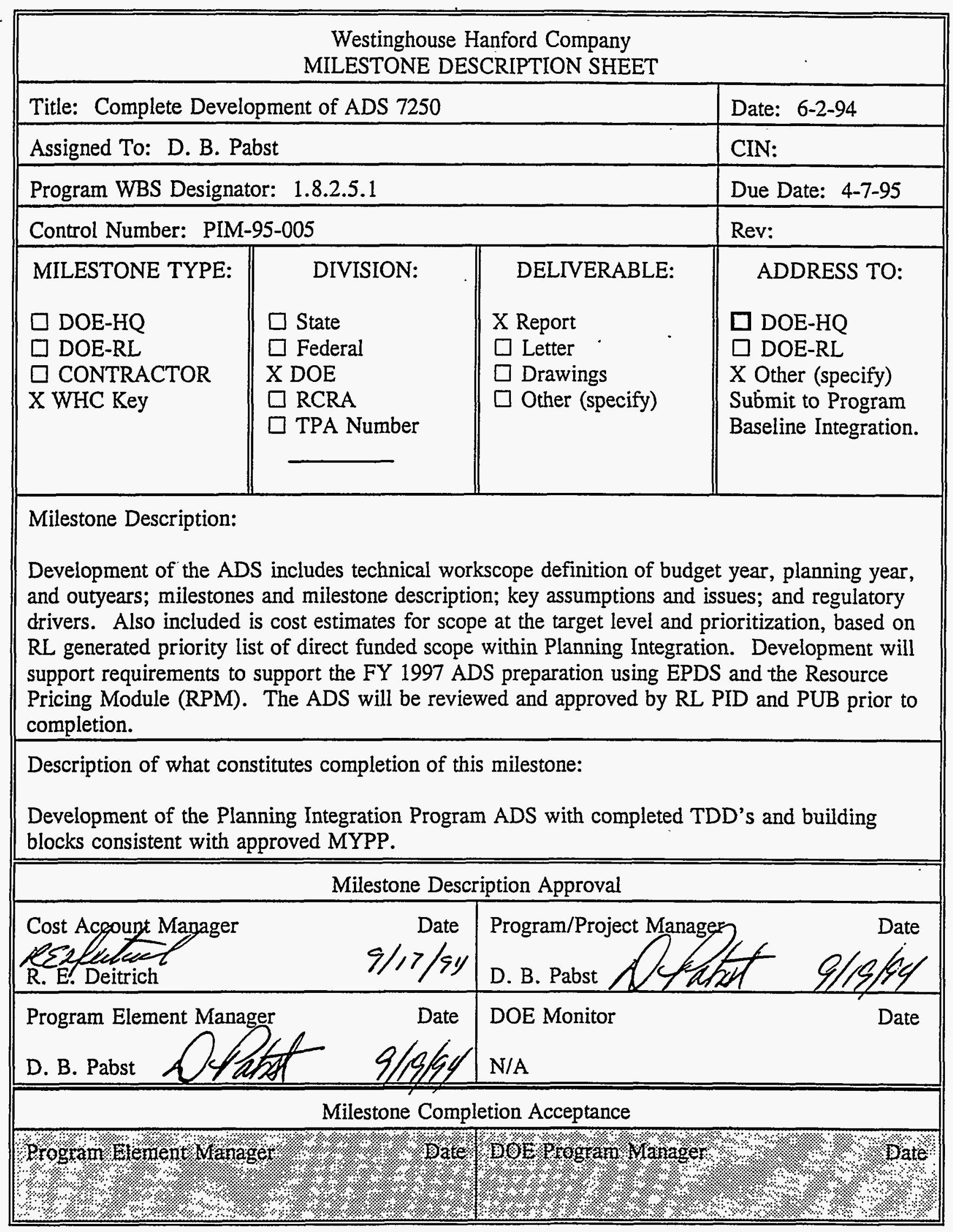




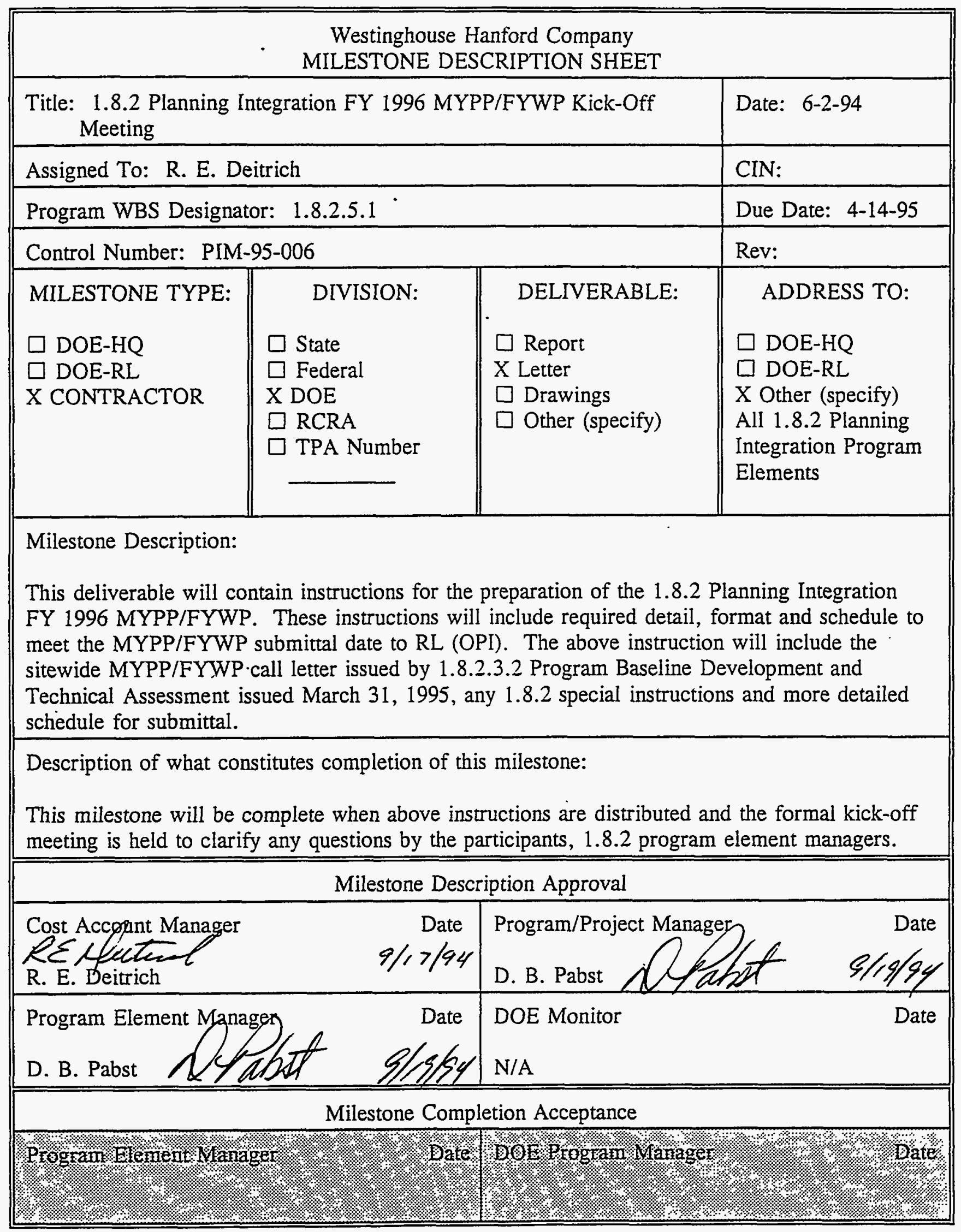




\begin{tabular}{|l||l|l||}
\hline \multicolumn{3}{|c|}{ Westinghouse Hanford Company } \\
MILESTONE DESCRIPTION SHEET
\end{tabular}




\begin{tabular}{|l||l|l||}
\hline \multicolumn{3}{|c|}{ Westinghouse Hantord Company } \\
MILESTONE DESCRIPTION SHEET
\end{tabular}




\begin{tabular}{|l||l|l||}
\hline \multicolumn{3}{|c|}{ Westinghouse Hanford Company } \\
MILESTNE DESCRIPTION SHEET
\end{tabular}




\begin{tabular}{|l||l|||}
\hline \multicolumn{3}{|c||}{ Westinghouse Hanford Company } \\
MILESTONE DESCRIPTION SHEET
\end{tabular}




\begin{tabular}{|c|c|c|c|}
\hline \multicolumn{4}{|c|}{$\begin{array}{l}\text { Westinghouse Hanford Company } \\
\text { MILESTONE DESCRIPTION SHEET }\end{array}$} \\
\hline \multicolumn{3}{|c|}{ Title: Submit Monthly SMS Report for Planning and Integration } & Date: $07 / 18 / 94$ \\
\hline \multicolumn{3}{|c|}{ Assigned To: David Pabst } & CIN: \\
\hline \multicolumn{3}{|c|}{ Program WBS Designator: 1.8.2.5 Planning and Integration Mgmt. } & Due Date: $10 / 14 / 94$ \\
\hline \multicolumn{3}{|c|}{ Control Number: PIM-95-023 } & Rev: \\
\hline $\begin{array}{l}\text { MILESTONE TYPE: } \\
\square \text { DOE-HQ } \\
\square \text { DOE-RL } \\
\text { x CONTRACTOR }\end{array}$ & $\begin{aligned} & \text { DIVISION: } \\
& \square \text { State } \\
& \square \text { Federal } \\
& \text { x DOE } \\
& \square \text { RCRA } \\
& \square \text { TPA Number }\end{aligned}$ & $\begin{array}{l}\text { DELIVERABLE: } \\
\text { x Report } \\
\square \text { Letter } \\
\square \text { Drawings } \\
\square \text { Other (specify) }\end{array}$ & $\begin{array}{l}\quad \text { ADDRESS TO: } \\
\square \text { DOE-HQ } \\
\square \text { DOE-RL } \\
\text { x Other (specify) } \\
\text { Deliver to } \\
\text { Performance } \\
\text { Reporting 1.8.2.2 }\end{array}$ \\
\hline \multicolumn{4}{|c|}{$\begin{array}{l}\text { Milestone Description: } \\
\text { This milestone supports RLID 5000.5, "Richland Field Office - Site Management System" and } \\
\text { RLID } 5000.7 \text {, "RL Site Management System Reporting" and consists of coordinating the } \\
\text { monthly Planning and Integration program's Site Management Systems (SMS) report for final } \\
\text { submission to the Performance Reporting Organization. The SMS report will status and measure } \\
\text { Planning and Integration's accomplishments, progress, and expenditures against the approved } \\
\text { baseline. Also included will be a status of any change to the approved program baseline. } \\
\text { Following the monthly program review with the Office of Planning Integration (OPI) RL, RL } \\
\text { comments will be incorporated into the monthly sitewide SMS report delivered to RL each } \\
\text { month in FY'1995. }\end{array}$} \\
\hline \multicolumn{4}{|c|}{$\begin{array}{l}\text { Description of what constitutes completion of this milestone: } \\
\text { The completion of this milestone is constituted by the final submission of the Planning and } \\
\text { Integration's SMS report. to the Performance Reporting Organization. }\end{array}$} \\
\hline \multicolumn{4}{|c|}{ Milestone Description Approval } \\
\hline \multicolumn{2}{|l|}{$\begin{array}{l}\text { Cost Accoxnt Manager } \\
\text { RE Sflecece } \\
\text { RE Deitrich }\end{array}$} & \multicolumn{2}{|c|}{ PBogram/Project Manager } \\
\hline \multicolumn{2}{|c|}{ Program Element Manager } & \multicolumn{2}{|c|}{$\begin{array}{l}\text { DOE Monitor Date } \\
\text { N/A }\end{array}$} \\
\hline \multicolumn{4}{|c|}{ 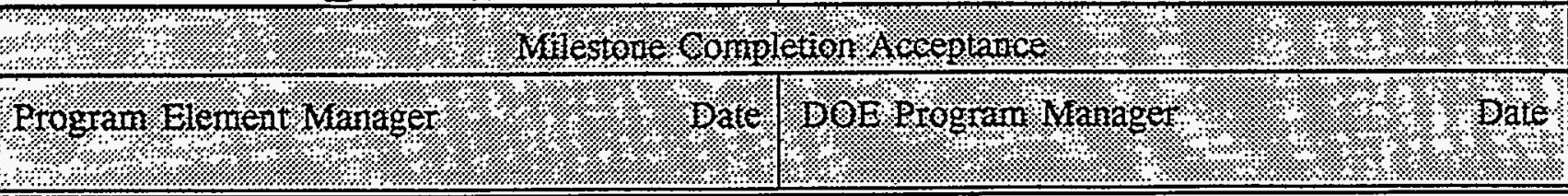 } \\
\hline
\end{tabular}




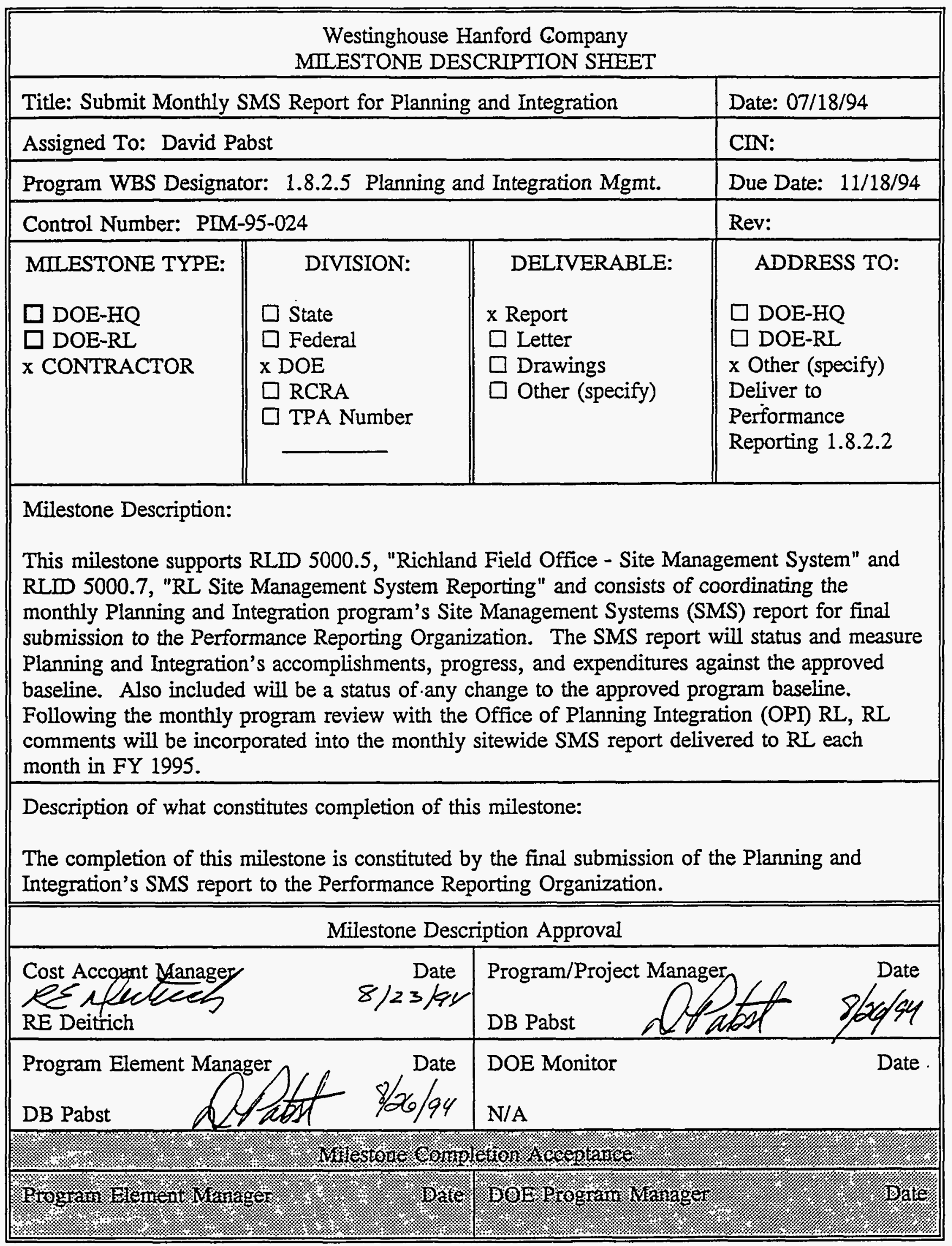




\begin{tabular}{|c|c|c|c|}
\hline \multicolumn{4}{|c|}{$\begin{array}{l}\text { Westinghouse Hanford Company } \\
\text { MILESTONE DESCRIPTION SHEET }\end{array}$} \\
\hline \multicolumn{3}{|c|}{ Title: Submit Monthly SMS Report for Planning and Integration } & Date: $07 / 18 / 94$ \\
\hline \multicolumn{3}{|c|}{ Assigned To: David Pabst } & CIN: \\
\hline \multicolumn{3}{|c|}{ Program WBS Designator: 1-8.2.5 Planning and Integration Mgmt. } & Due Date: $12 / 16 / 94$ \\
\hline \multicolumn{3}{|c|}{ Control Number: PIM-95-025 } & Rev: \\
\hline $\begin{array}{l}\text { MILESTONE TYPE: } \\
\square \text { DOE-HQ } \\
\square \text { DOE-RL } \\
x \text { CONTRACTOR }\end{array}$ & $\begin{array}{l}\quad \text { DIVISION: } \\
\quad \square \text { State } \\
\square \text { Federal } \\
\text { x DOE } \\
\square \text { RCRA } \\
\square \text { TPA Number }\end{array}$ & $\begin{array}{l}\text { DELIVERABLE: } \\
\text { x Report } \\
\square \text { Letter } \\
\square \text { Drawings } \\
\square \text { Other (specify) }\end{array}$ & $\begin{array}{l}\quad \text { ADDRESS TO: } \\
\square \text { DOE-HQ } \\
\square \text { DOE-RL } \\
\text { x Other (specify) } \\
\text { Deliver to } \\
\text { Performance } \\
\text { Reporting 1.8.2.2 }\end{array}$ \\
\hline \multicolumn{4}{|c|}{$\begin{array}{l}\text { This milestone supports RLID 5000.5, "Richland Field Office - Site Management System" and } \\
\text { RLID 5000.7, "RL Site Management System Reporting" and consists of coordinating the } \\
\text { monthly Planning and Integration program's Site Management Systems (SMS) report for final } \\
\text { submission to the Performance Reporting Organization. The SMS report will status and measure } \\
\text { Planning and Integration's accomplishments, progress, and expenditures against the approved } \\
\text { baseline. Also included will be a status of any change to the approved program baseline. } \\
\text { Following the monthly program review with the Office of Planning Integration (OPI) RL, RL } \\
\text { comments will be incorporated into the monthly sitewide SMS report delivered to RL each } \\
\text { month in FY } 1995 \text {. }\end{array}$} \\
\hline \multicolumn{4}{|c|}{$\begin{array}{l}\text { Description of what constitutes completion of this milestone: } \\
\text { The completion of this milestone is constituted by the final submission of the Planning and } \\
\text { Integration's SMS report to the Performance Reporting Organization. }\end{array}$} \\
\hline \multicolumn{4}{|c|}{ Milestone Description Approval } \\
\hline \multicolumn{2}{|l|}{$\begin{array}{l}\text { Cost Account Manager } \\
\text { RE Hecteal } \\
\text { RE Deifrich }\end{array}$} & \multicolumn{2}{|c|}{ PBogram/Project Manager } \\
\hline \multicolumn{2}{|c|}{ Program Element Manager } & \multicolumn{2}{|c|}{ DOE Monitor Date } \\
\hline \multicolumn{4}{|c|}{ 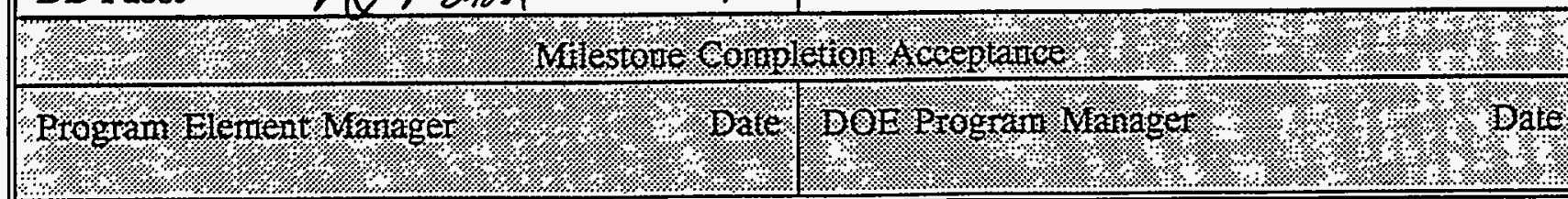 } \\
\hline
\end{tabular}




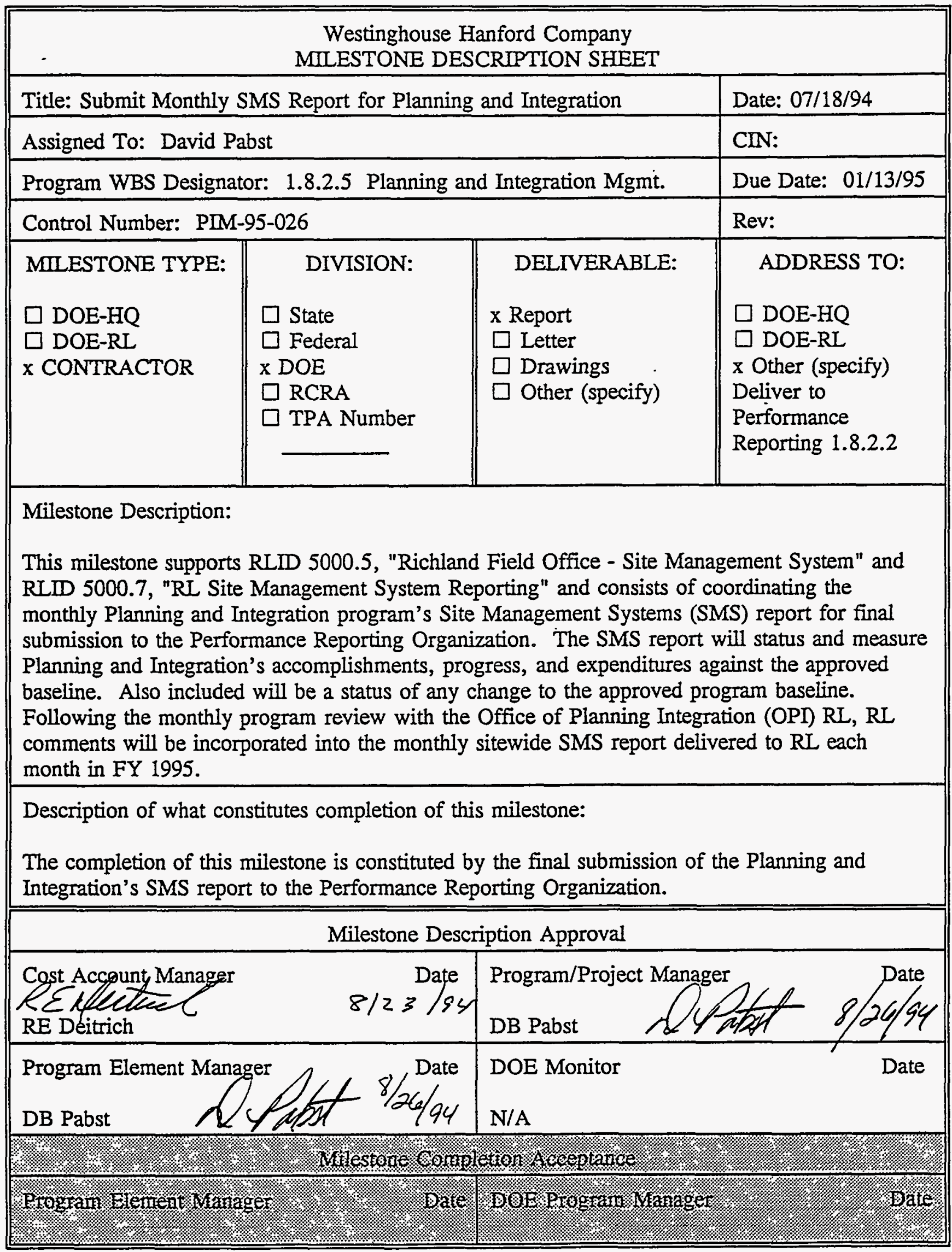




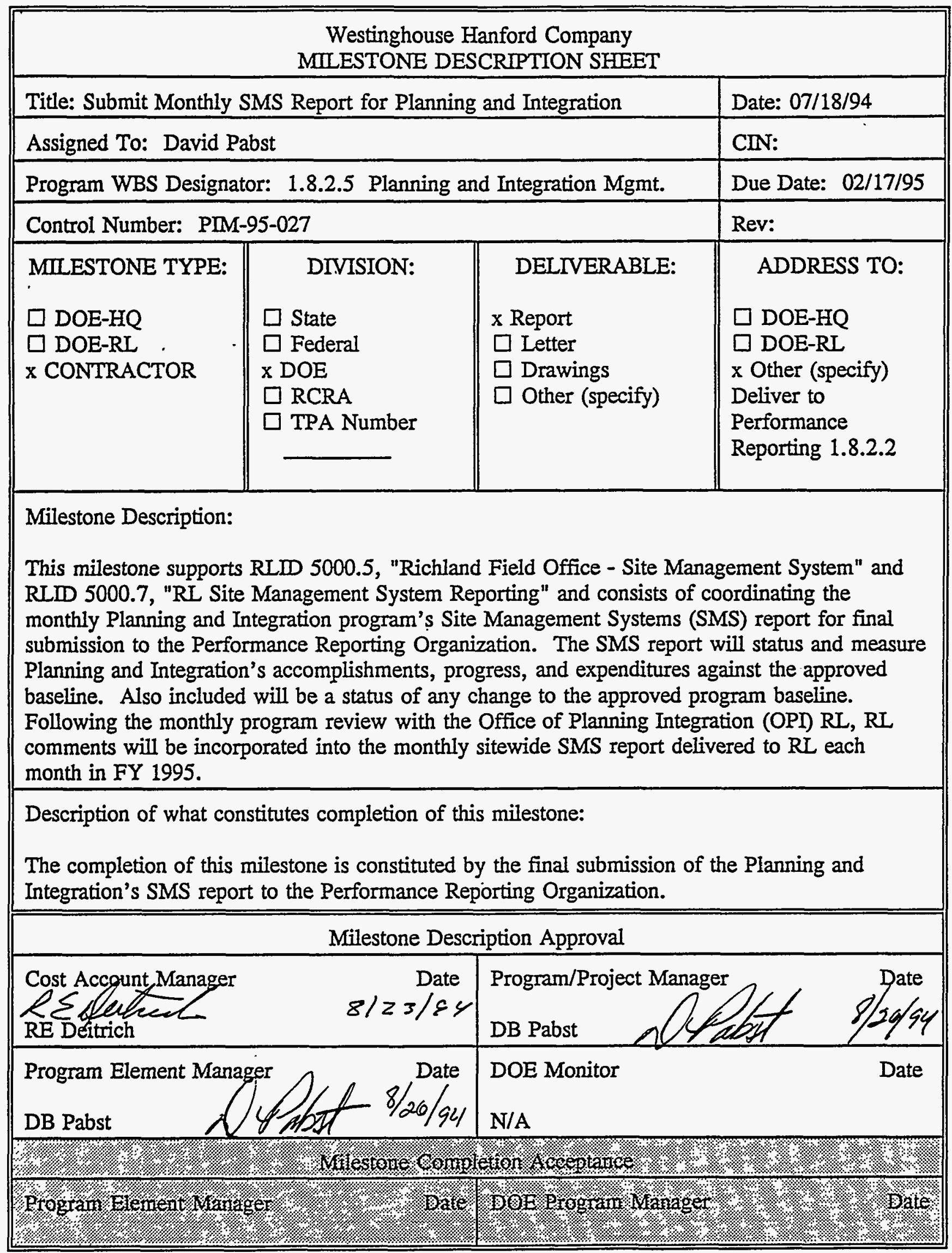




\begin{tabular}{|c|c|c|c|}
\hline \multicolumn{4}{|c|}{$\begin{array}{l}\text { Westinghouse Hanford Company } \\
\text { MILESTONE DESCRIPTION SHEET }\end{array}$} \\
\hline \multicolumn{3}{|c|}{ Title: Submit Monthly SMS Report for Planning and Integration } & Date: $07 / 18 / 94$ \\
\hline \multicolumn{3}{|c|}{ Assigned To: David Pabst } & CIN: \\
\hline \multicolumn{3}{|c|}{ Program WBS Designator: 1 8.2.5 Planning and Integration Mgmt. } & Due Date: $03 / 17 / 95$ \\
\hline \multicolumn{3}{|c|}{ Control Number: PIM-95-028 } & Rev: \\
\hline $\begin{array}{l}\text { MILESTONE TYPE: } \\
\square \text { DOE-HQ } \\
\square \text { DOE-RL } \\
\times \text { CONTRACTOR }\end{array}$ & $\begin{array}{l}\quad \text { DIVISION: } \\
\\
\square \text { State } \\
\square \text { Federal } \\
\text { x DOE } \\
\square \text { RCRA } \\
\square \text { TPA Number }\end{array}$ & $\begin{array}{l}\text { DELIVERABLE: } \\
\text { x Report } \\
\square \text { Letter } \\
\square \text { Drawings } \\
\square \text { Other (specify) }\end{array}$ & $\begin{array}{l}\text { ADDRESS TO: } \\
\square \text { DOE-HQ } \\
\square \text { DOE-RL } \\
\text { x Other (specify) } \\
\text { Deliver to } \\
\text { Performance } \\
\text { Reporting 1.8.2.2 }\end{array}$ \\
\hline \multicolumn{4}{|c|}{$\begin{array}{l}\text { This milestone supports RLID 5000.5, "Richland Field Office - Site Management System" and } \\
\text { RLID 5000.7, "RL Site Management System Reporting" and consists of coordinating the } \\
\text { monthly Planning and Integration program's Site Management Systems (SMS) report for final } \\
\text { submission to the Performance Reporting Organization. The SMS report will status and measure } \\
\text { Planning and Integration's accomplishments, progress, and expenditures against the approved } \\
\text { baseline. Also included will be a status of any change to the approved program baseline. } \\
\text { Following the monthly program review with the Office of Planning Integration (OPI) RL, RL } \\
\text { comments will be incorporated into the monthly sitewide SMS report delivered to RL each } \\
\text { month in FY } 1995 \text {. }\end{array}$} \\
\hline \multicolumn{4}{|c|}{$\begin{array}{l}\text { Description of what constitutes completion of this milestone: } \\
\text { The completion of this milestone is constituted by the final submission of the Planning and } \\
\text { Integration's SMS report to the Performance Reporting Organization. }\end{array}$} \\
\hline \multicolumn{4}{|c|}{ Milestone Description Approval } \\
\hline \multicolumn{2}{|l|}{ 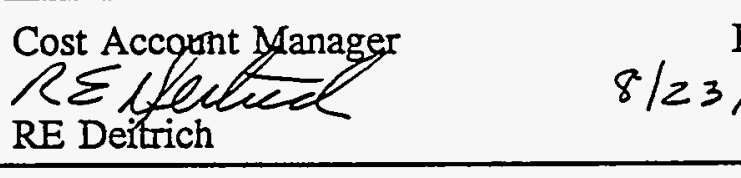 } & \multicolumn{2}{|c|}{ Program/Project Manager } \\
\hline \multicolumn{2}{|c|}{ Program Element Manager } & \multicolumn{2}{|c|}{ N/A } \\
\hline \multicolumn{4}{|c|}{ 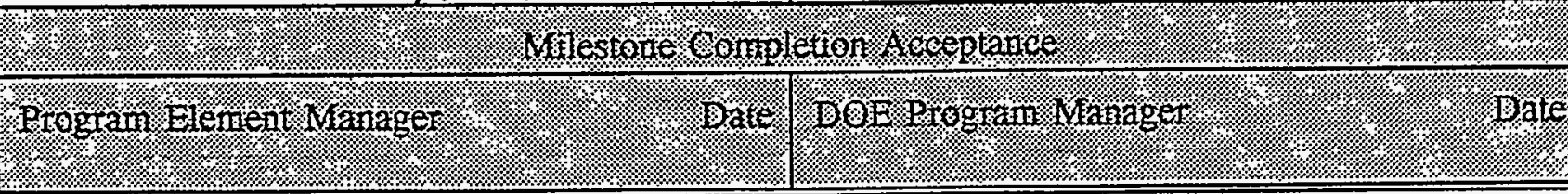 } \\
\hline
\end{tabular}




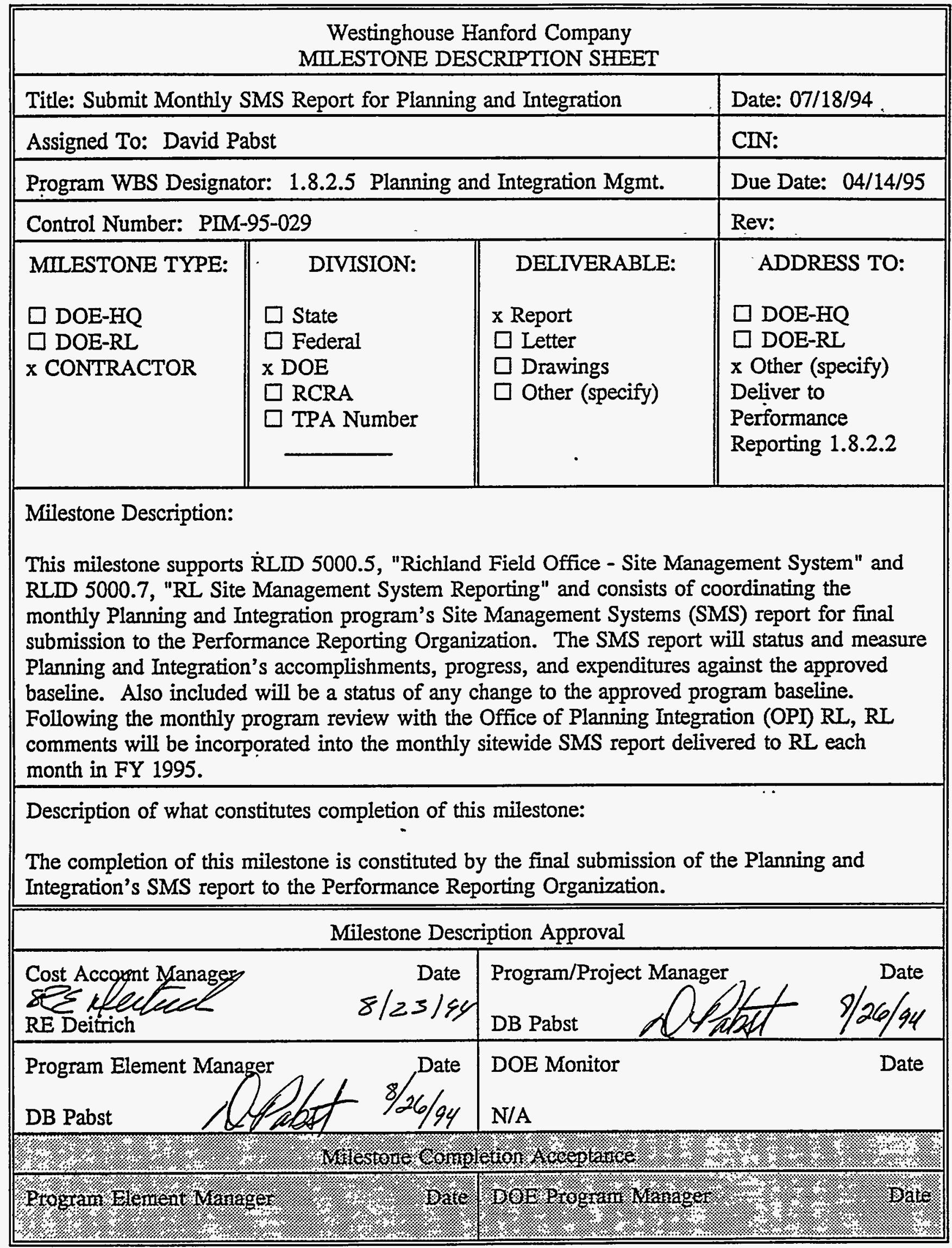




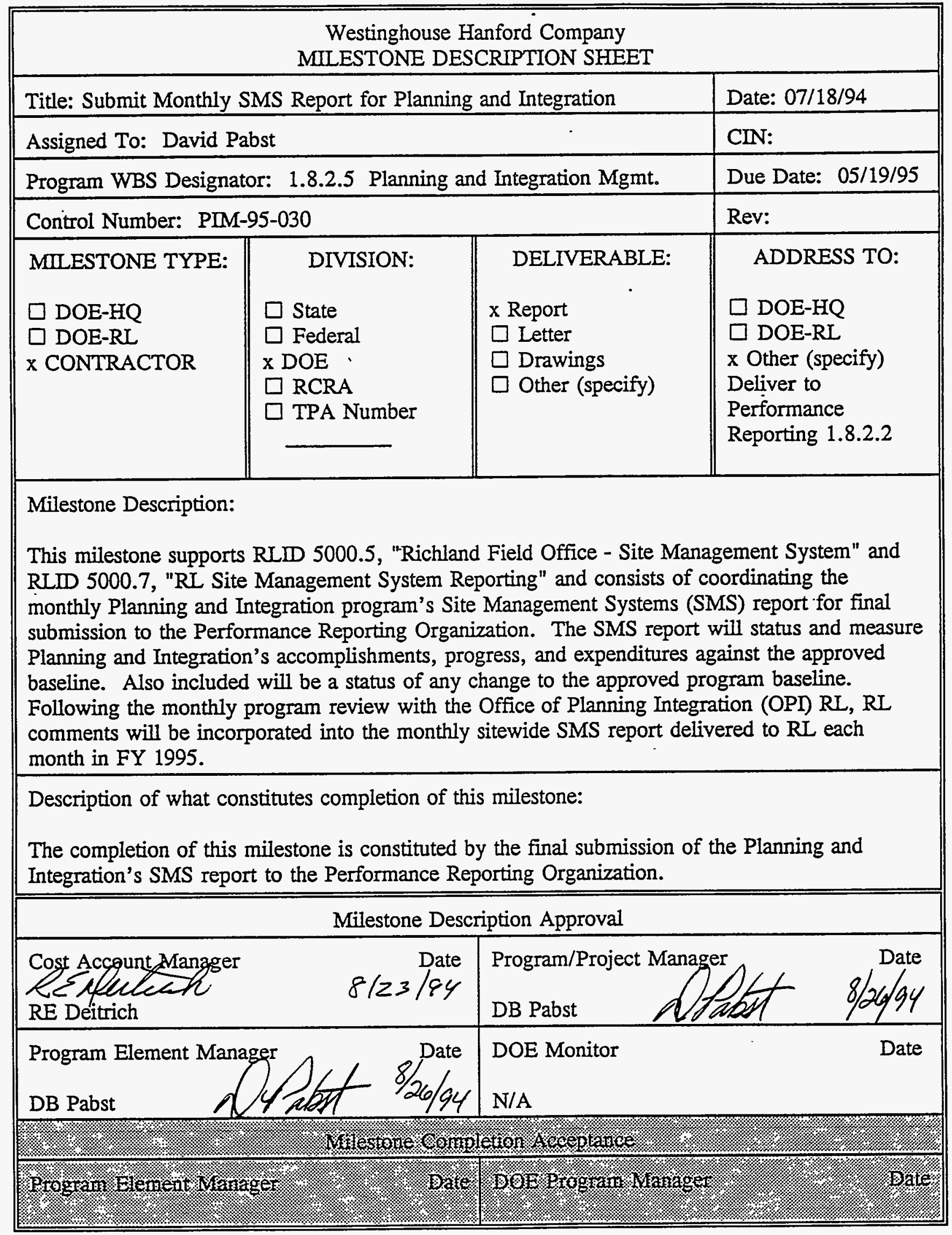




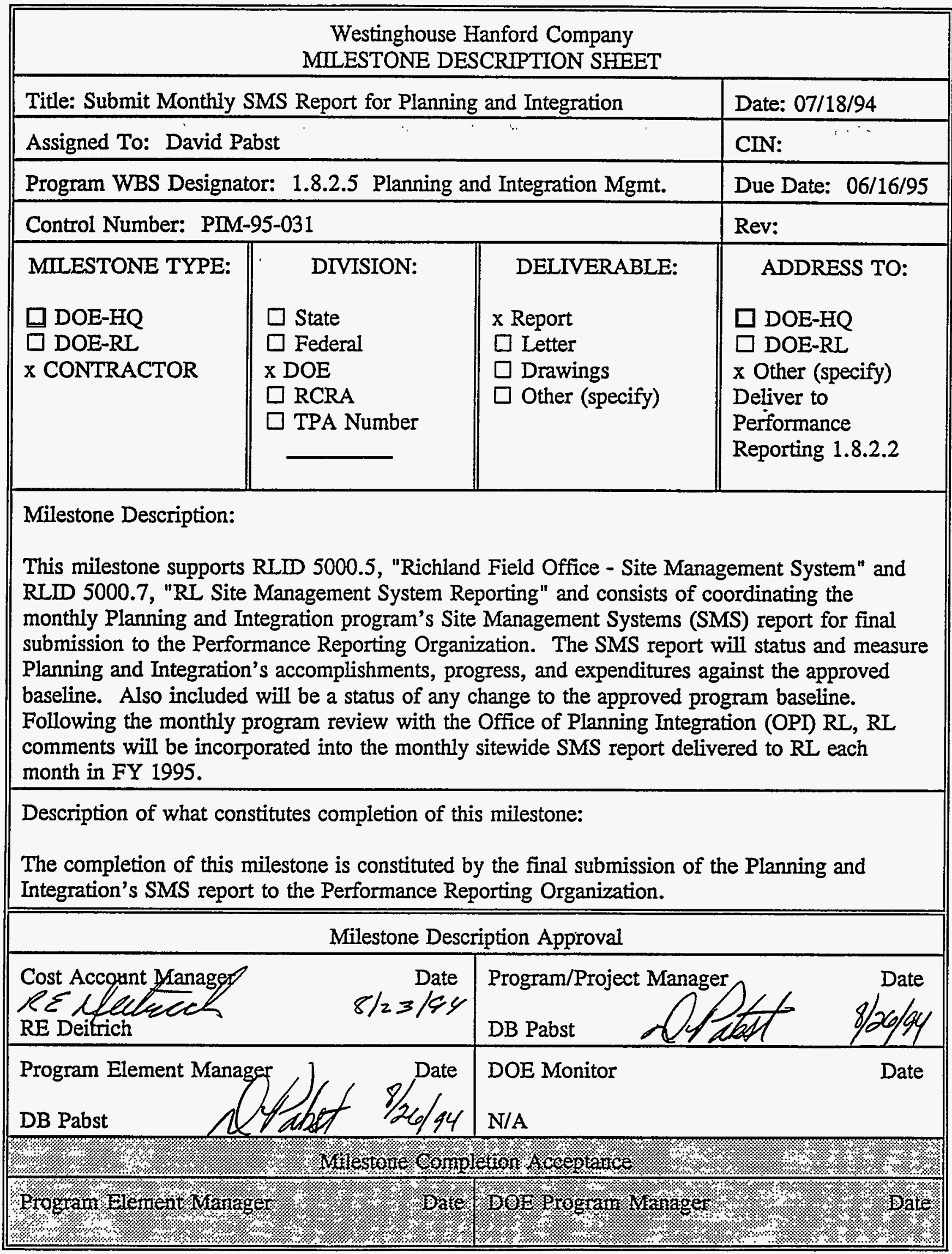




\begin{tabular}{|c|c|c|c|}
\hline \multicolumn{4}{|c|}{$\begin{array}{l}\text { Westinghouse Hanford Company } \\
\text { MILESTONE DESCRIPTION SHEET }\end{array}$} \\
\hline \multicolumn{3}{|c|}{ Title: Submit Monthly SMS Report for Planning and Integration } & Date: $07 / 18 / 94$ \\
\hline \multicolumn{3}{|c|}{ Assigned To: David Pabst } & CIN: \\
\hline \multicolumn{3}{|c|}{ Program WBS Designator: 1 8.2.5 Planning and Integration Mgmt. } & Due Date: $07 / 14 / 95$ \\
\hline \multicolumn{3}{|c|}{ Control Number: PIM-95-032 } & Rev: \\
\hline $\begin{array}{l}\text { MIIESTONE TYPE: } \\
\square \text { DOE-HQ } \\
\square \text { DOE-RL } \\
\text { x CONTRACTOR }\end{array}$ & $\begin{array}{l}\quad \text { DIVISION: } \\
\square \text { State } \\
\square \text { Federal } \\
\times \text { DOE } \\
\square \text { RCRA } \\
\square \text { TPA Number }\end{array}$ & $\begin{array}{l}\text { DELIVERABLE: } \\
\text { x Report } \\
\square \text {. Letter } \\
\square \text { Drawings } \\
\square \text { Other (specify) }\end{array}$ & $\begin{array}{l}\quad \text { ADDRESS TO: } \\
\square \text { DOE-HQ } \\
\square \text { DOE-RI } \\
\text { x Other (specify) } \\
\text { Deliver to } \\
\text { Performance } \\
\text { Reporting } 1.8 .2 .2\end{array}$ \\
\hline \multicolumn{4}{|c|}{$\begin{array}{l}\text { This milestone supports RLID } 5000.5 \text {, "Richland Field Office - Site Management System" and } \\
\text { RLID 5000.7, "RL Site Management System Reporting" and consists of coordinating the } \\
\text { monthly Planning and Integration program's Site Management Systems (SMS) report for final } \\
\text { submission to the Performance Reporting Organization. The SMS report will status and measure } \\
\text { Planning and Integration's accomplishments, progress, and expenditures against the approved } \\
\text { baseline. Also included will be a status of any change to the approved program baseline. } \\
\text { Following the monthly program review with the Office of Planning Integration (OPI) RL, RL } \\
\text { comments will be incorporated into the monthly sitewide SMS report delivered to RL each } \\
\text { month in FY 1995. }\end{array}$} \\
\hline \multicolumn{4}{|c|}{$\begin{array}{l}\text { Description of what constitutes completion of this milestone: } \\
\text { The completion of this milestone is constituted by the final submission of the Planning and } \\
\text { Integration's SMS report to the Performance Reporting Organization. }\end{array}$} \\
\hline \multicolumn{4}{|c|}{ Milestone Description Approval } \\
\hline \multicolumn{2}{|l|}{$\begin{array}{l}\text { Cost Accountianzger } \\
\text { RE fllecect } \\
\text { RE Deitrich }\end{array}$} & \multicolumn{2}{|c|}{ PB Pabst Date } \\
\hline \multicolumn{2}{|c|}{ Program Element Manager } & \multicolumn{2}{|l|}{$\begin{array}{l}\text { DOE Monitor } \\
\text { N/A }\end{array}$} \\
\hline \multicolumn{4}{|c|}{ 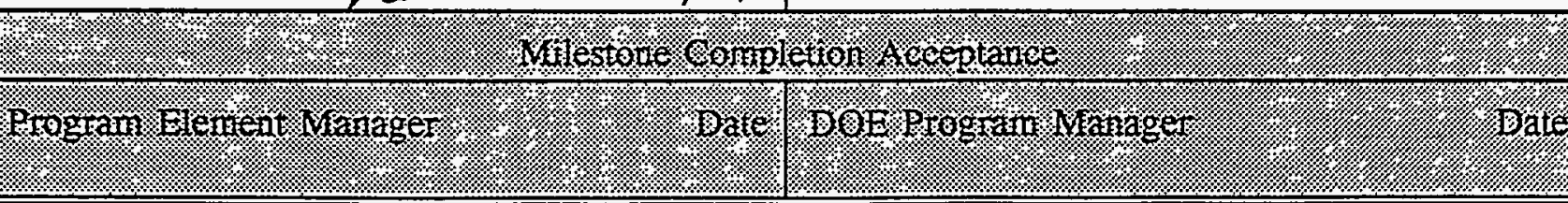 } \\
\hline
\end{tabular}




\begin{tabular}{|c|c|c|c|}
\hline \multicolumn{4}{|c|}{$\begin{array}{l}\text { Westinghouse Hanford Company } \\
\text { MILESTONE DESCRIPTION SHEET }\end{array}$} \\
\hline \multicolumn{3}{|c|}{ Title: Submit Monthly SMS Report for Planning and Integration } & Date: $07 / 18 / 94$ \\
\hline \multicolumn{3}{|c|}{ Assigned To: David Pabst } & CIN: \\
\hline \multicolumn{3}{|c|}{ Program WBS Designator: 1-8.2.5 Planning and Integration Mgmt. } & Due Date: $08 / 18 / 95$ \\
\hline \multicolumn{3}{|c|}{ Control Number: PIM-95-033 } & Rev: \\
\hline $\begin{array}{l}\text { MILESTONE TYPE: } \\
\square \text { DOE-HQ } \\
\square \text { DOE-RL } \\
\text { x CONTRACTOR }\end{array}$ & $\begin{array}{l}\quad \text { DIVISION: } \\
\square \text { State } \\
\square \text { Federal } \\
\text { x DOE } \\
\square \text { RCRA } \\
\square \text { TPA Number }\end{array}$ & $\begin{array}{l}\text { DELIVERABLE: } \\
\text { x Report } \\
\square \text { Letter } \\
\square \text { Drawings } \\
\square \text { Other (specify) }\end{array}$ & $\begin{array}{l}\quad \text { ADDRESS TO: } \\
\\
\square \text { DOE-HQ } \\
\square \text { DOE-RL } \\
\text { x Other (specify) } \\
\text { Deliver to } \\
\text { Performance } \\
\text { Reporting } 1.8 .2 .2\end{array}$ \\
\hline \multicolumn{4}{|c|}{$\begin{array}{l}\text { This milestone supports RLID 5000.5, "Richland Field Office - Site Management System" and } \\
\text { RLID 5000.7, "RL Site Management System Reporting" and consists of coordinating the } \\
\text { monthly Planning and Integration program's Site Management Systems (SMS) report for final } \\
\text { submission to the Performance Reporting Organization. The SMS report will status and measure } \\
\text { Planning and Integration's accomplishments, progress, and expenditures against the approved } \\
\text { baseline. Also included will be a status of any change to the approved program baseline. } \\
\text { Following the monthly program review with the Office of Planning Integration (OPI) RL, RL } \\
\text { comments will be incorporated into the monthly sitewide SMS report delivered to RL each } \\
\text { month in FY 1995. }\end{array}$} \\
\hline \multicolumn{4}{|c|}{$\begin{array}{l}\text { Description of what constitutes completion of this milestone: } \\
\text { The completion of this milestone is constituted by the final submission of the Planning and } \\
\text { Integration's SMS report to the Performance Reporting Organization. }\end{array}$} \\
\hline \multicolumn{4}{|c|}{ Milestone Description Approval } \\
\hline \multicolumn{2}{|l|}{$\begin{array}{l}\text { Cost Accolnt wanager } \\
\text { RE Afleelecel } \\
\text { RE Defrich }\end{array}$} & \multicolumn{2}{|c|}{ Program/Project Manager } \\
\hline \multirow{2}{*}{\multicolumn{2}{|c|}{ Program Element Manager }} & \multicolumn{2}{|c|}{ DOE Monitor Date } \\
\hline & & 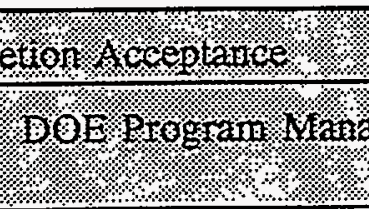 & 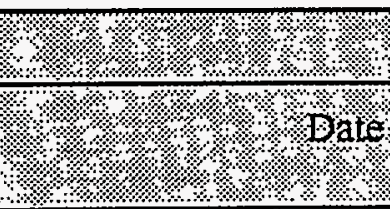 \\
\hline
\end{tabular}




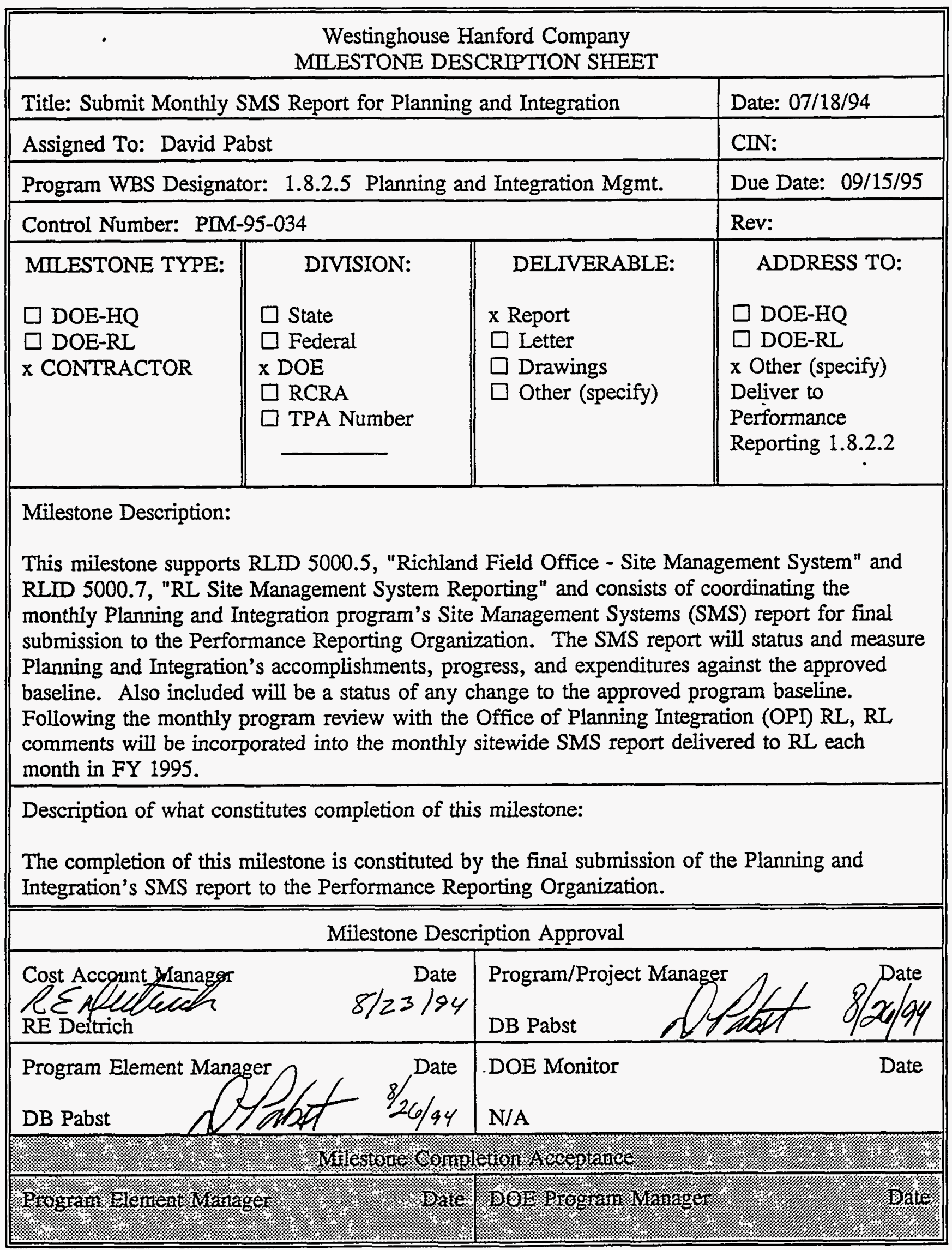




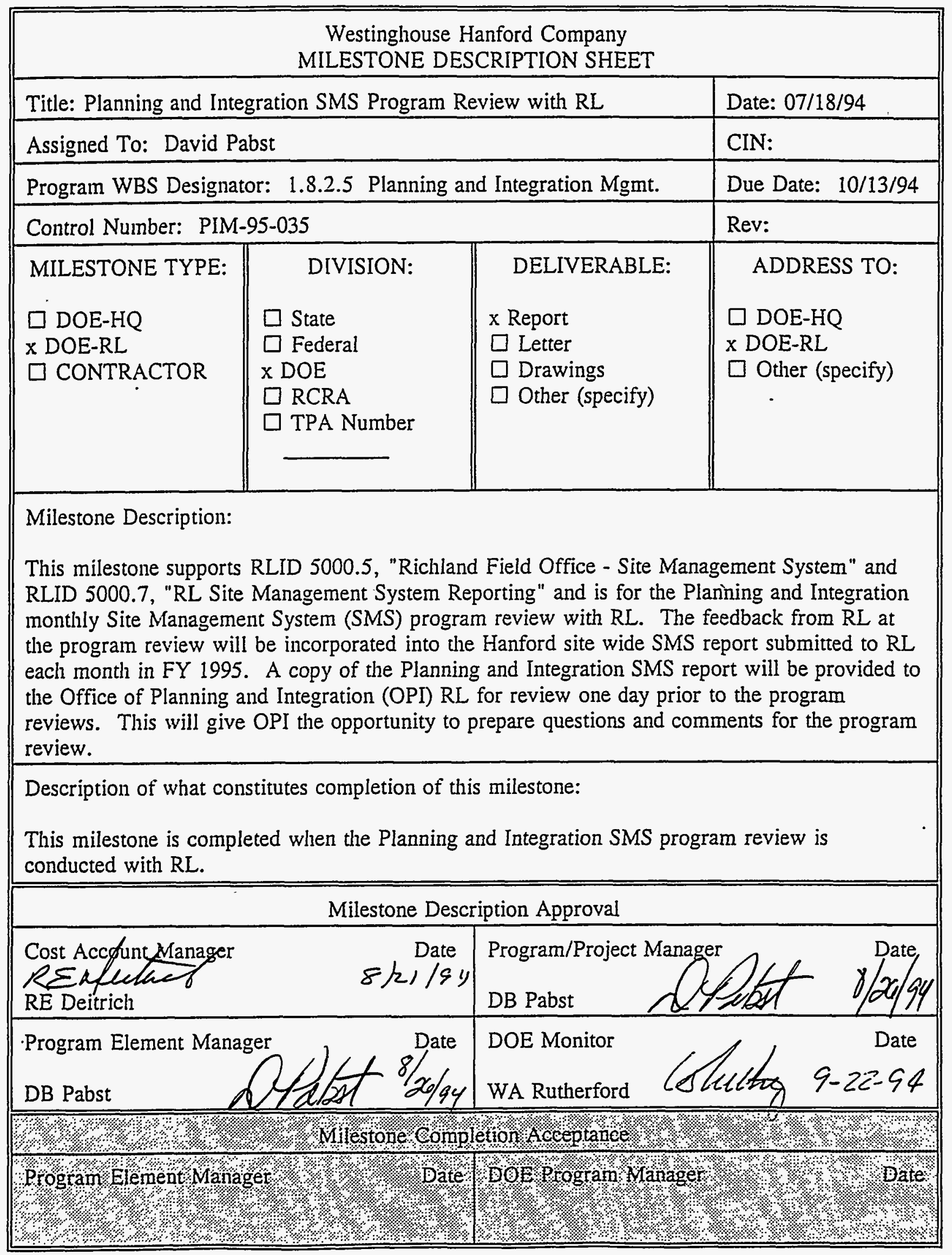




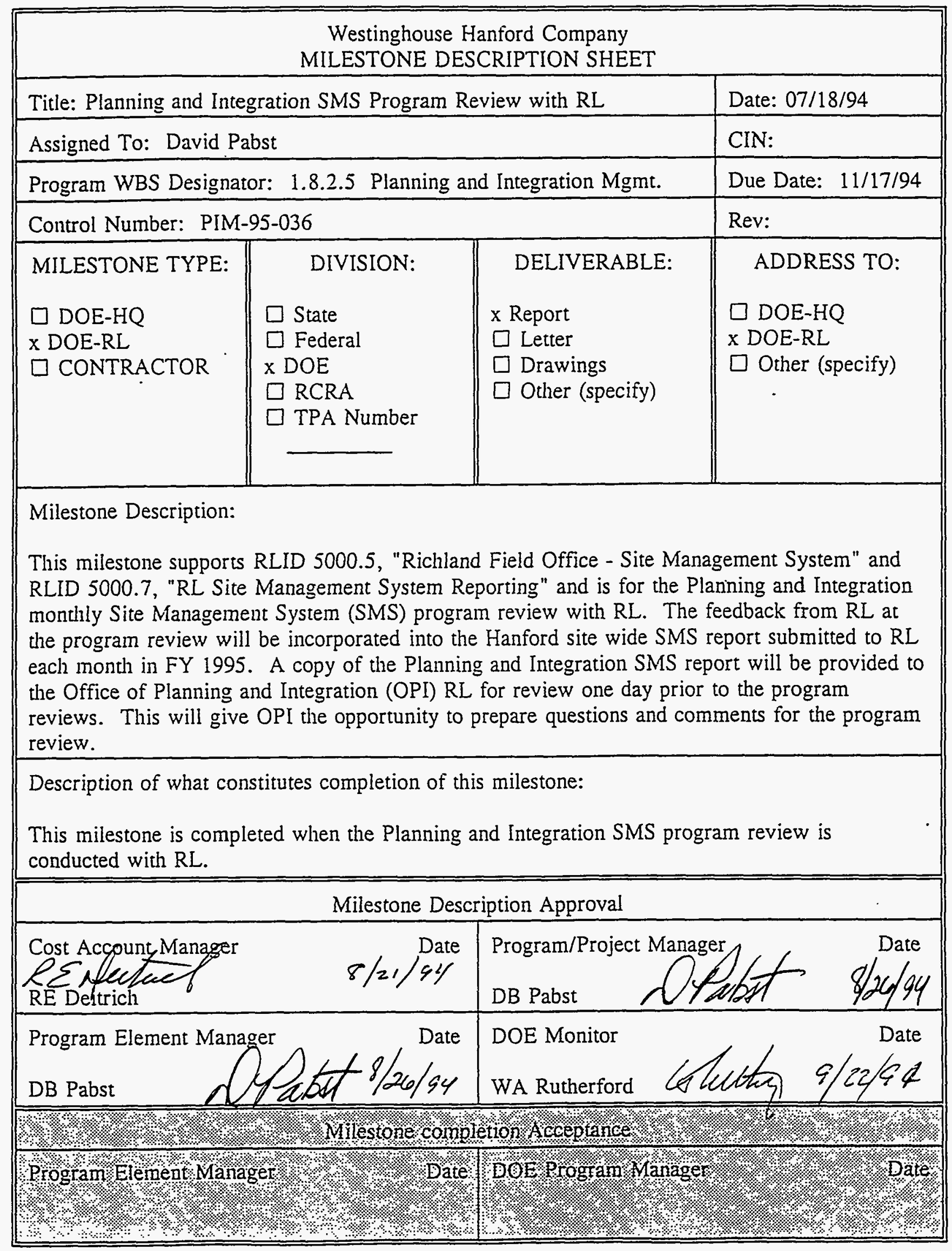




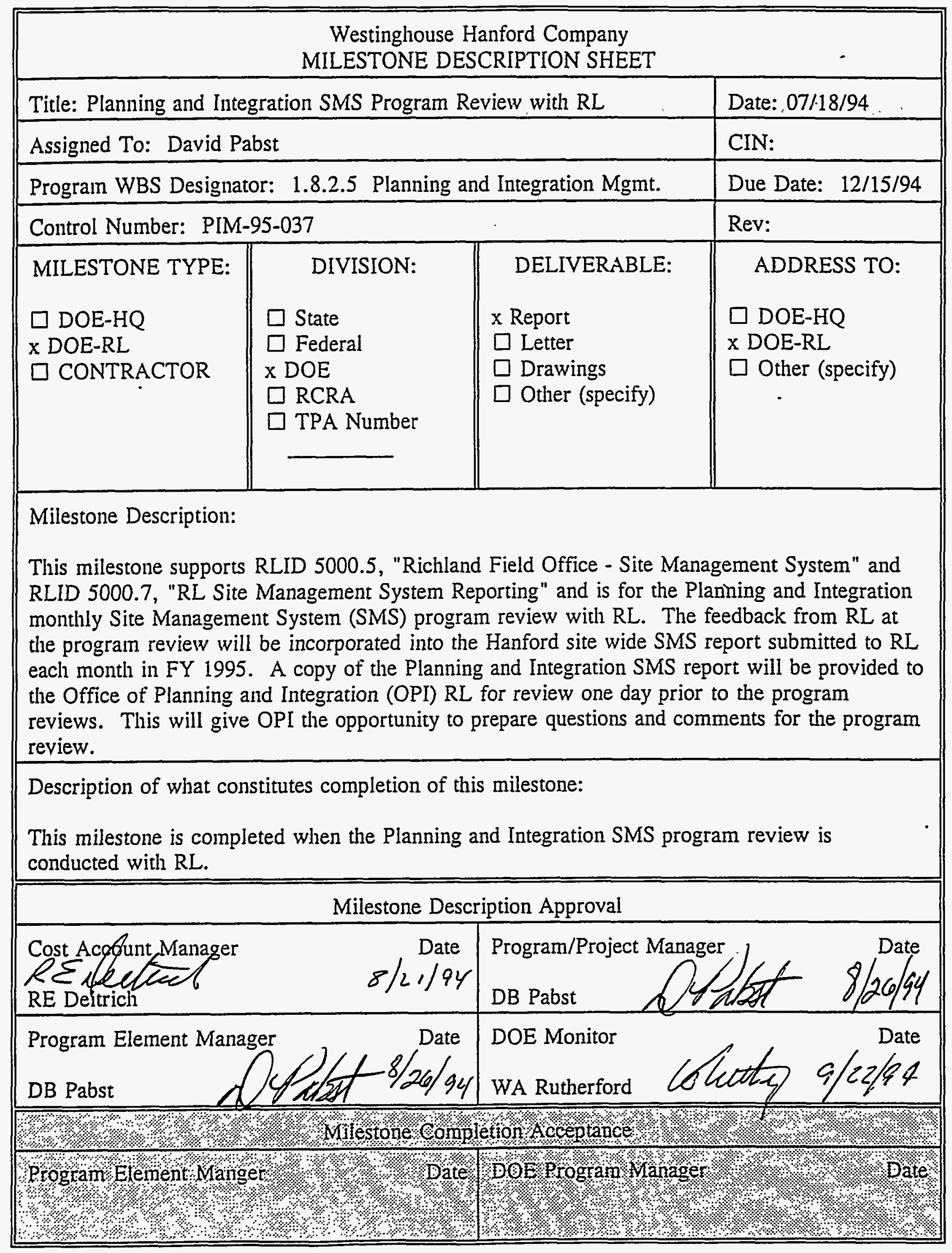




\begin{tabular}{|l||l||}
\hline \multicolumn{3}{|c|}{ Westinghouse Hanford Company } \\
MILESTONE DESCRIPTION SHEET
\end{tabular}




\begin{tabular}{|l|l||}
\hline \multicolumn{3}{|c|}{ Westinghouse Hanford Company } \\
MILESTONE DESCRIPTION SHEET
\end{tabular}




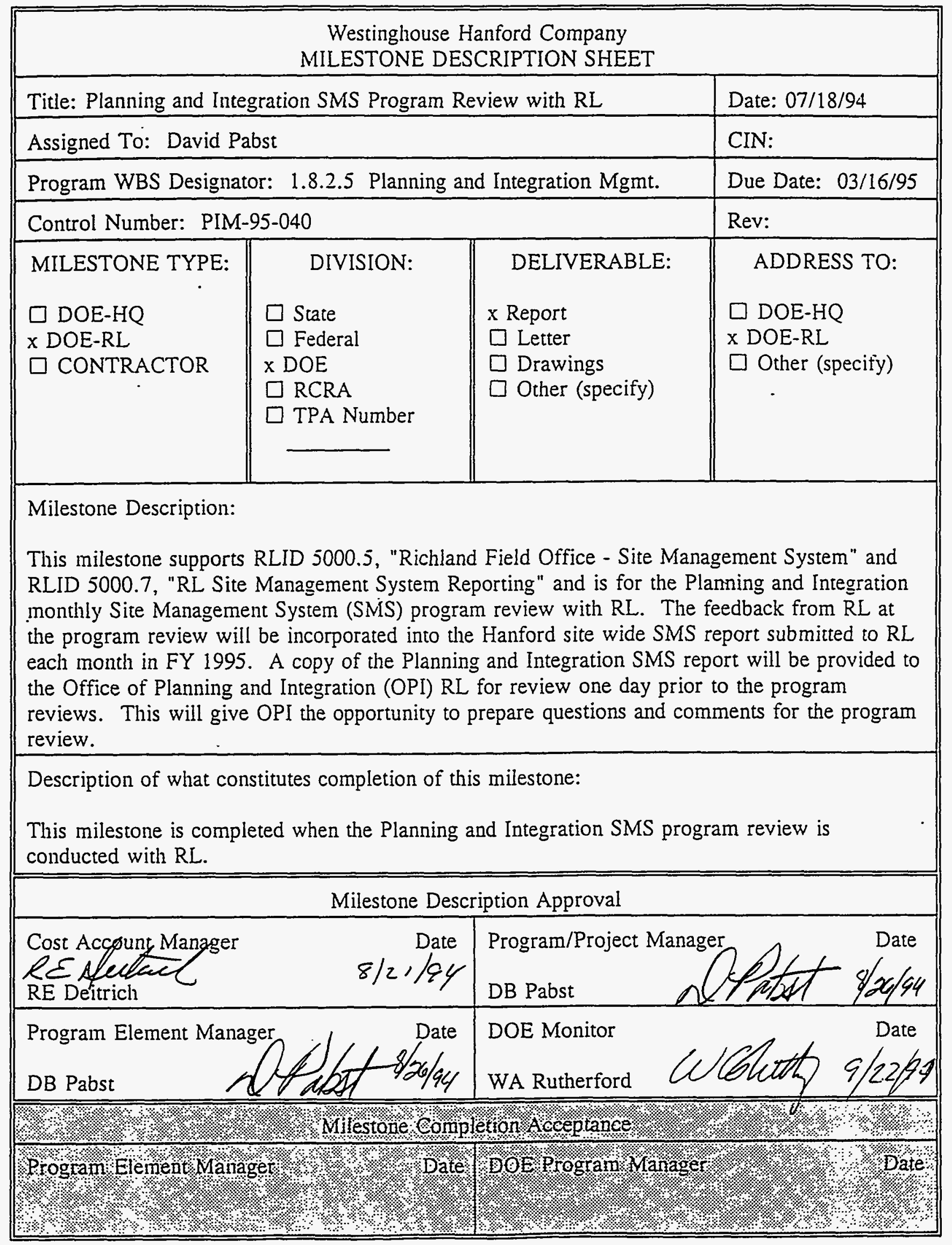




\begin{tabular}{|c|c|c|c|}
\hline \multicolumn{4}{|c|}{$\begin{array}{l}\text { Westinghouse Hanford Company } \\
\text { MILESTONE DESCRIPTION SHEET }\end{array}$} \\
\hline \multicolumn{3}{|c|}{ Title: Planning and Integration SMS Program Review with RL } & Date: $07 / 18 / 94$ \\
\hline \multicolumn{3}{|c|}{ Assigned To: David Pabst } & CIN: \\
\hline \multicolumn{3}{|c|}{ Program WBS Designator: 1.8.2.5 Planning and Integration Mgmt. } & Due Date: $04 / 13 / 95$ \\
\hline \multicolumn{3}{|c|}{ Control Number: PIM-95-041 } & Rev: \\
\hline $\begin{array}{l}\text { MILESTONE TYPE: } \\
\square \text { DOE-HQ } \\
\text { X DOE-RL } \\
\square \text { CONTRACTOR }\end{array}$ & $\begin{array}{l}\quad \text { DIVISION: } \\
\square \text { State } \\
\square \text { Federal } \\
\text { x DOE } \\
\square \text { RCRA } \\
\square \text { TPA Number }\end{array}$ & $\begin{array}{l}\text { DELIVERABLE: } \\
\times \text { Report } \\
\square \text { Letter } \\
\square \text { Drawings } \\
\square \text { Other (specify) }\end{array}$ & $\begin{array}{l}\text { ADDRESS TO: } \\
\square \text { DOE-HQ } \\
\text { x DOE-RL } \\
\square \text { Other (specify) }\end{array}$ \\
\hline \multicolumn{4}{|c|}{$\begin{array}{l}\text { This milestone supports RLID 5000.5, "Richland Field Office - Site Management System" and } \\
\text { RLID } 5000.7 \text {, "RL Site Management System Reporting" and is for the Plamning and Integration } \\
\text { monthly Site Management System (SMS) program review with RL. The feedback from RL at } \\
\text { the program review will be incorporated into the Hanford site wide SMS report submitted to RL } \\
\text { each month in FY } 1995 \text {. A copy of the Planning and Integration SMS report will be provided to } \\
\text { the Office of Planning and Integration (OPI) RL for review one day prior to the program } \\
\text { reviews. This will give OPI the opportunity to prepare questions and comments for the program } \\
\text { review. }\end{array}$} \\
\hline \multicolumn{4}{|c|}{$\begin{array}{l}\text { Description of what constitutes completion of this milestone: } \\
\text { This milestone is completed when the Planning and Integration SMS program review is } \\
\text { conducted with RL. }\end{array}$} \\
\hline \multicolumn{4}{|c|}{ Milestone Description Approval } \\
\hline \multicolumn{2}{|l|}{$\begin{array}{l}\text { Cost AccounyManzger } \\
\text { RE qeetew } \\
\text { RE Deitrich }\end{array}$} & \multicolumn{2}{|c|}{ Program/Project Manager Pabst Date } \\
\hline \multicolumn{2}{|c|}{ Program Element Manager Pabst } & \multicolumn{2}{|c|}{ DOE Monitor } \\
\hline \multicolumn{4}{|c|}{ 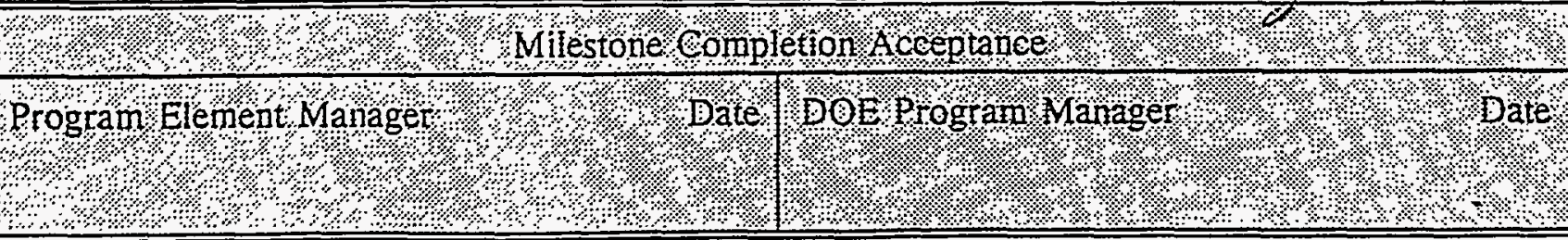 } \\
\hline
\end{tabular}




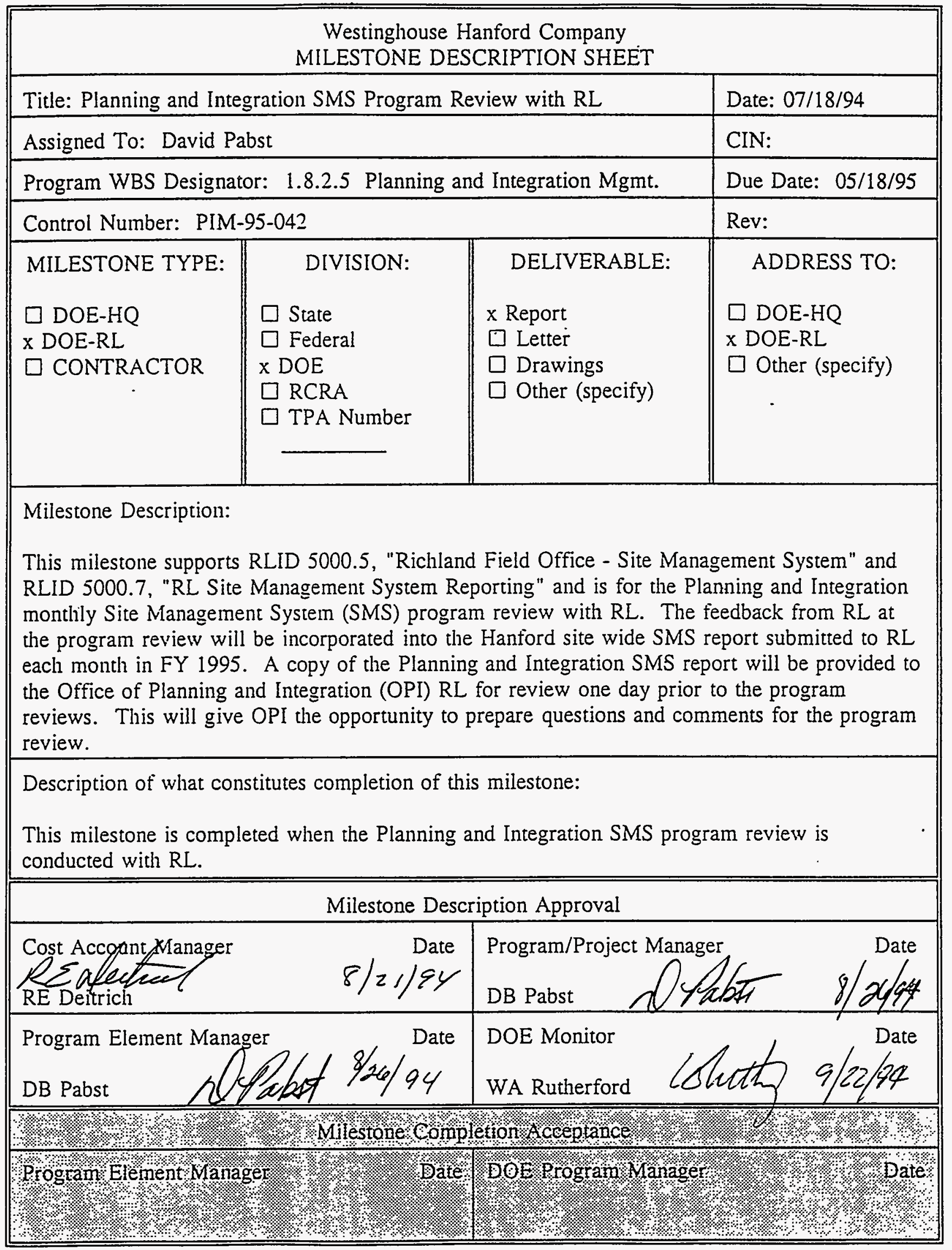




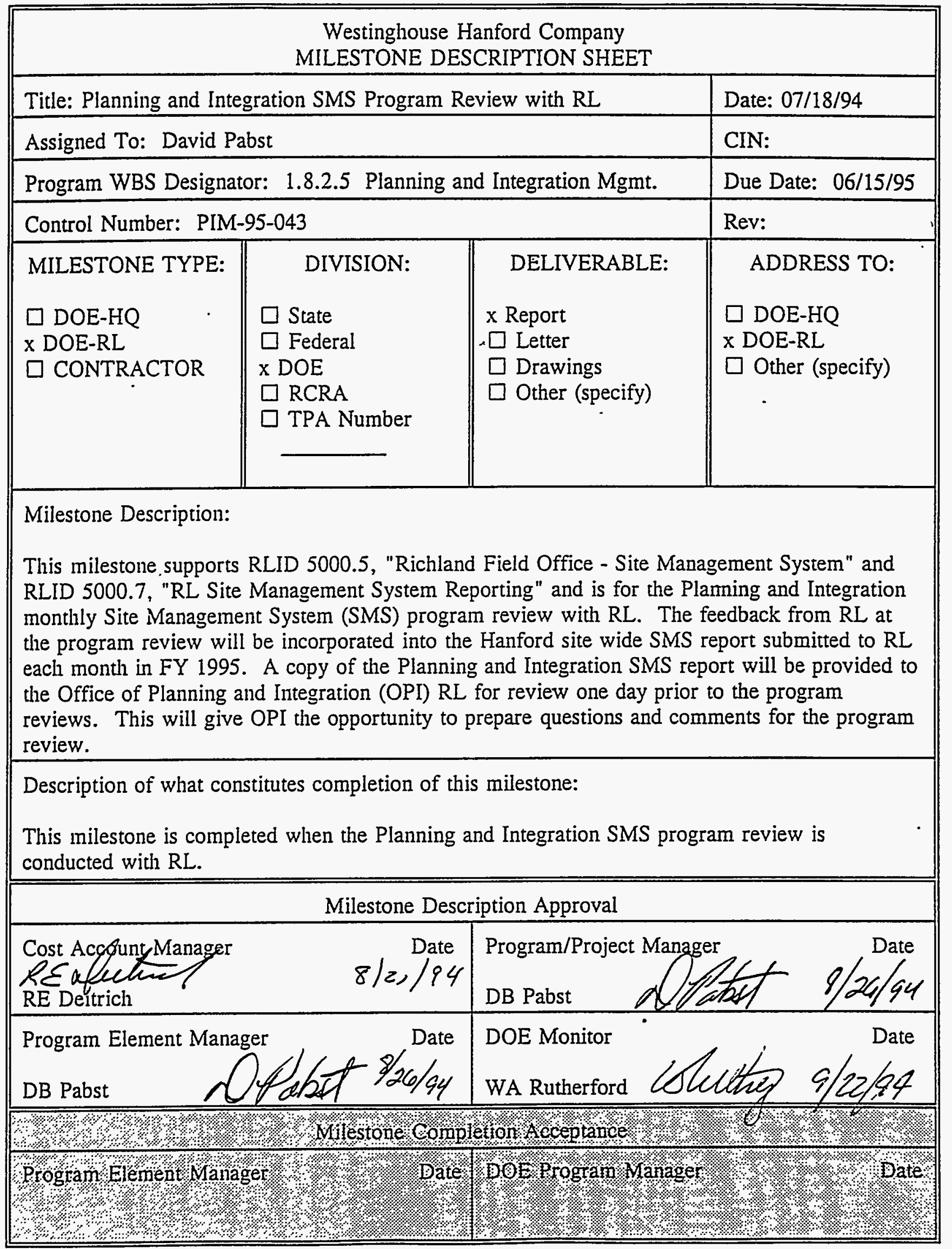




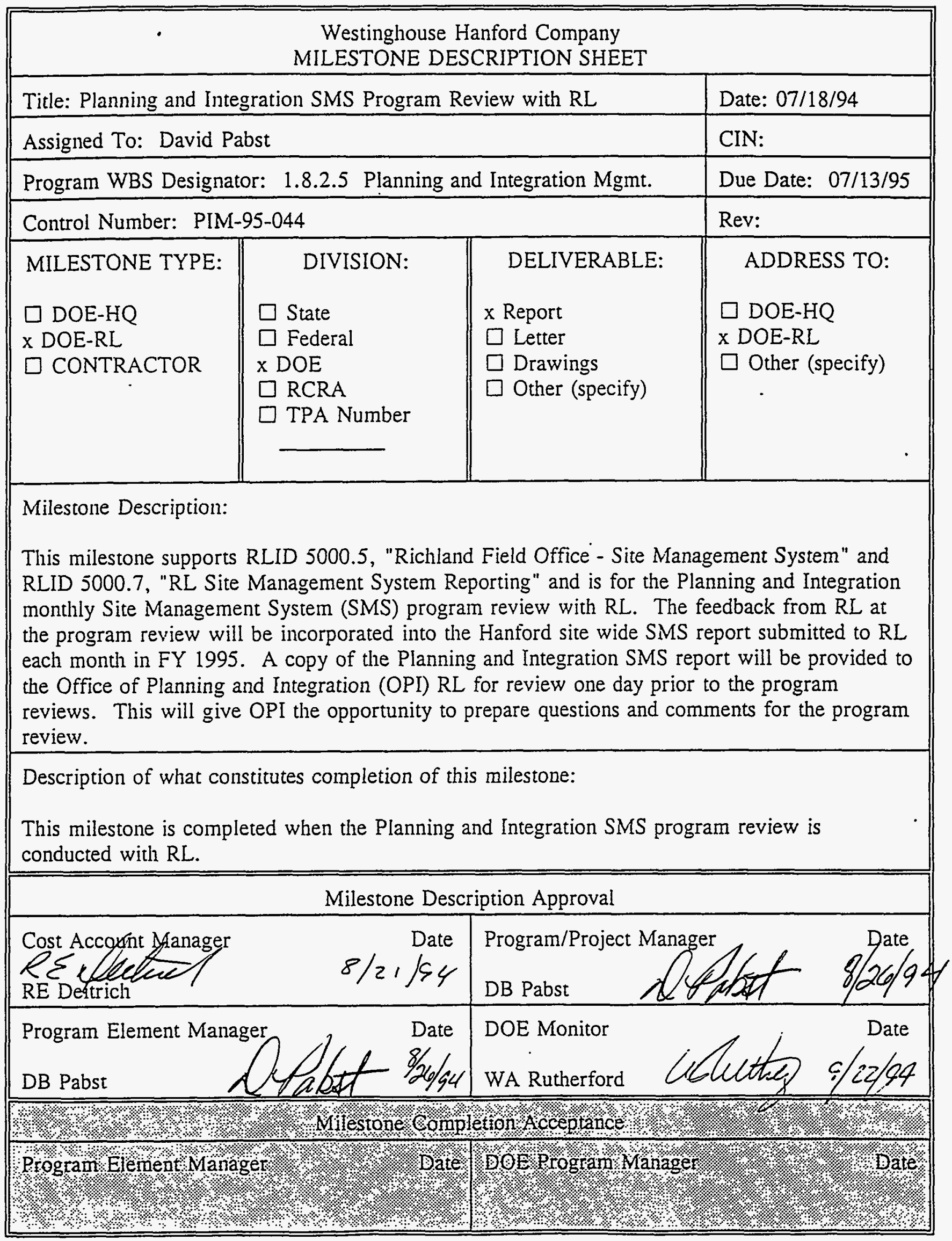




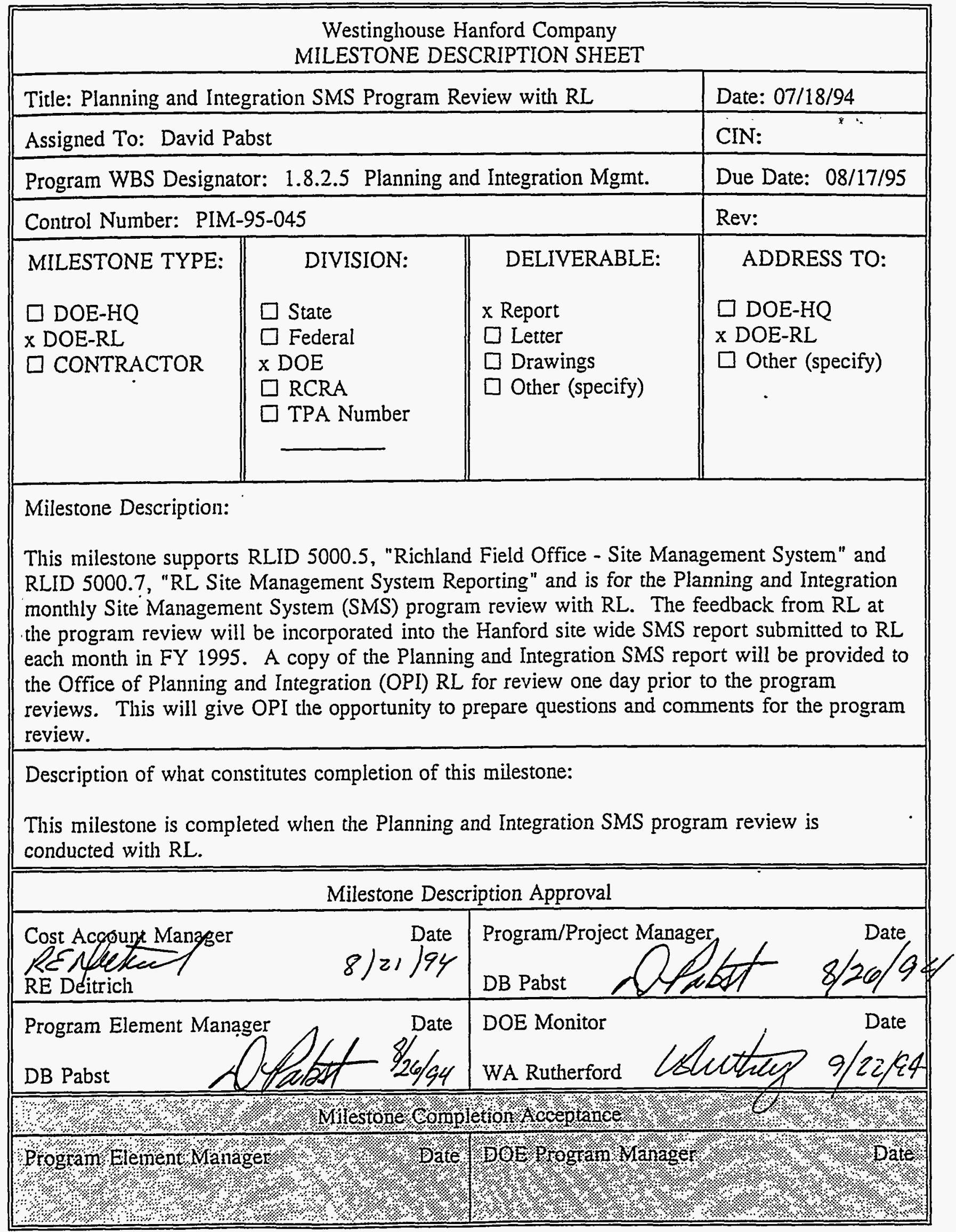




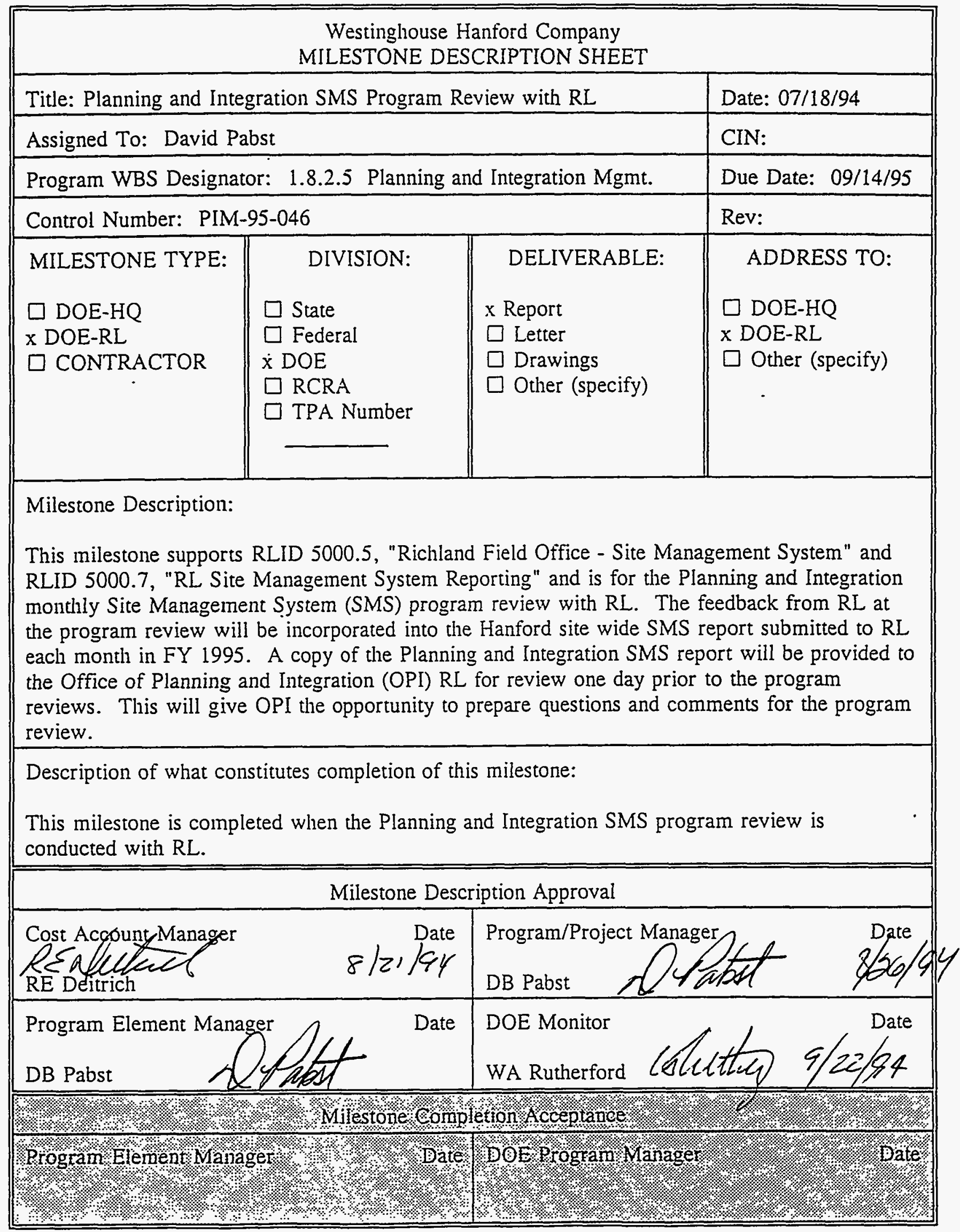




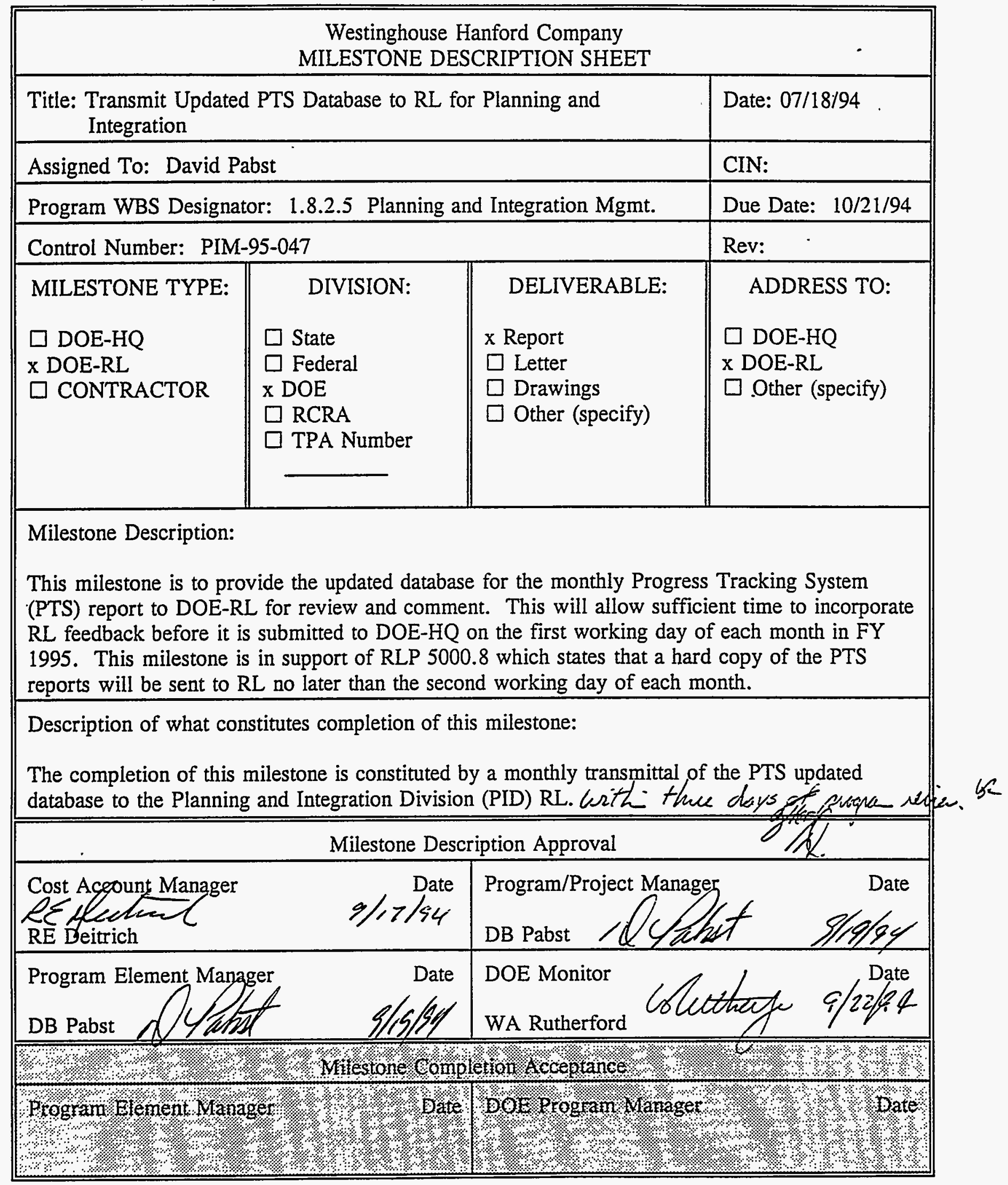




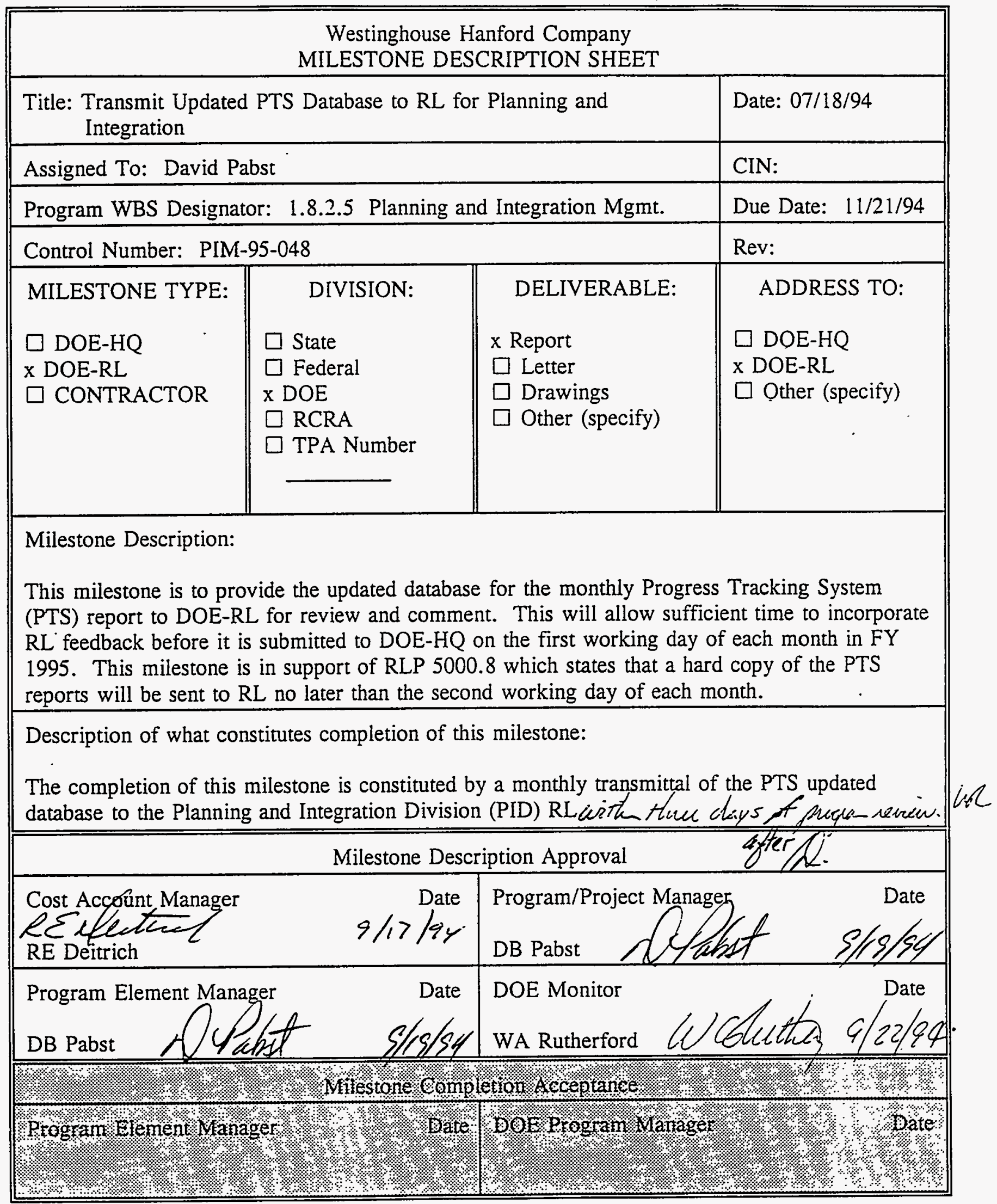




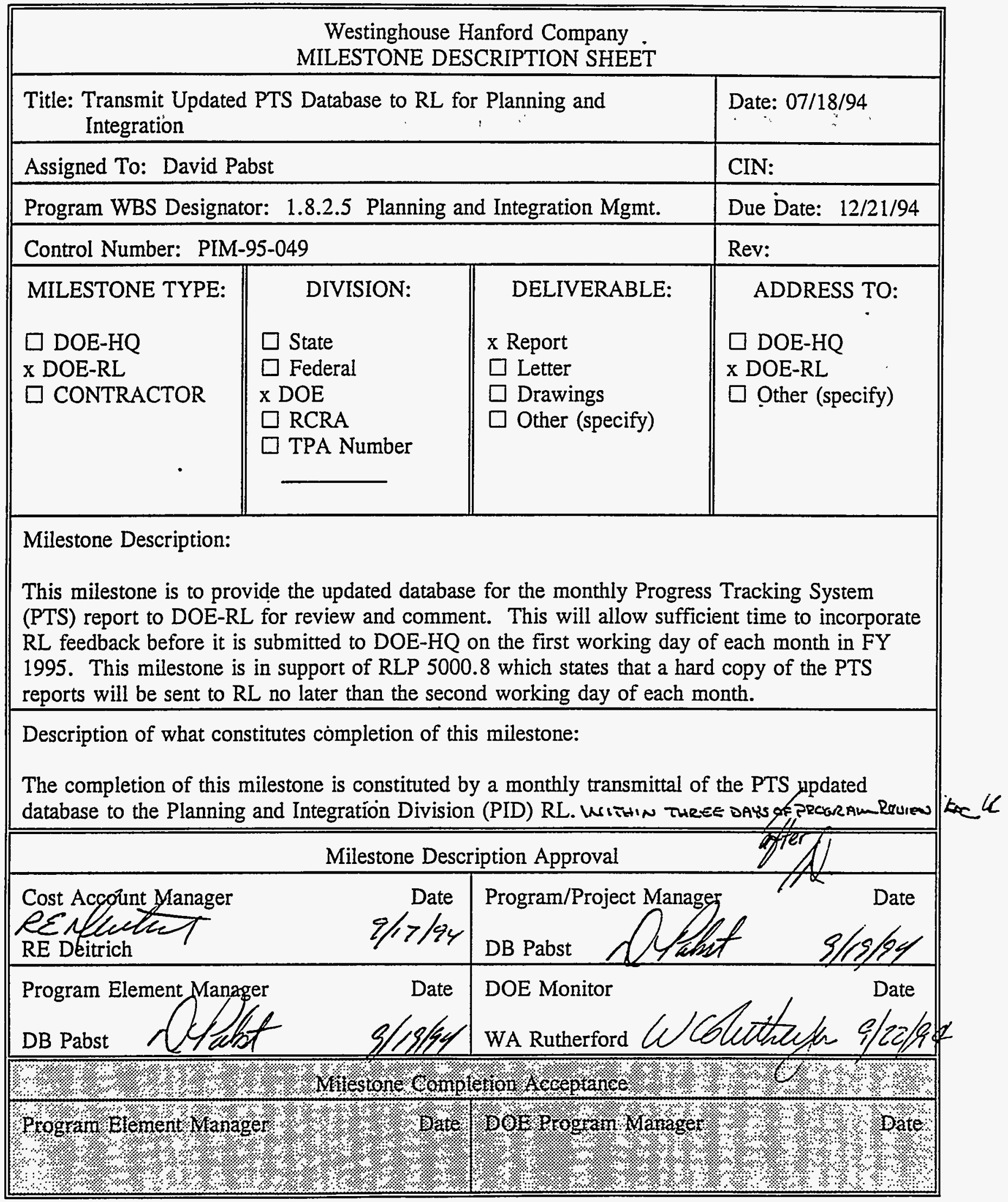




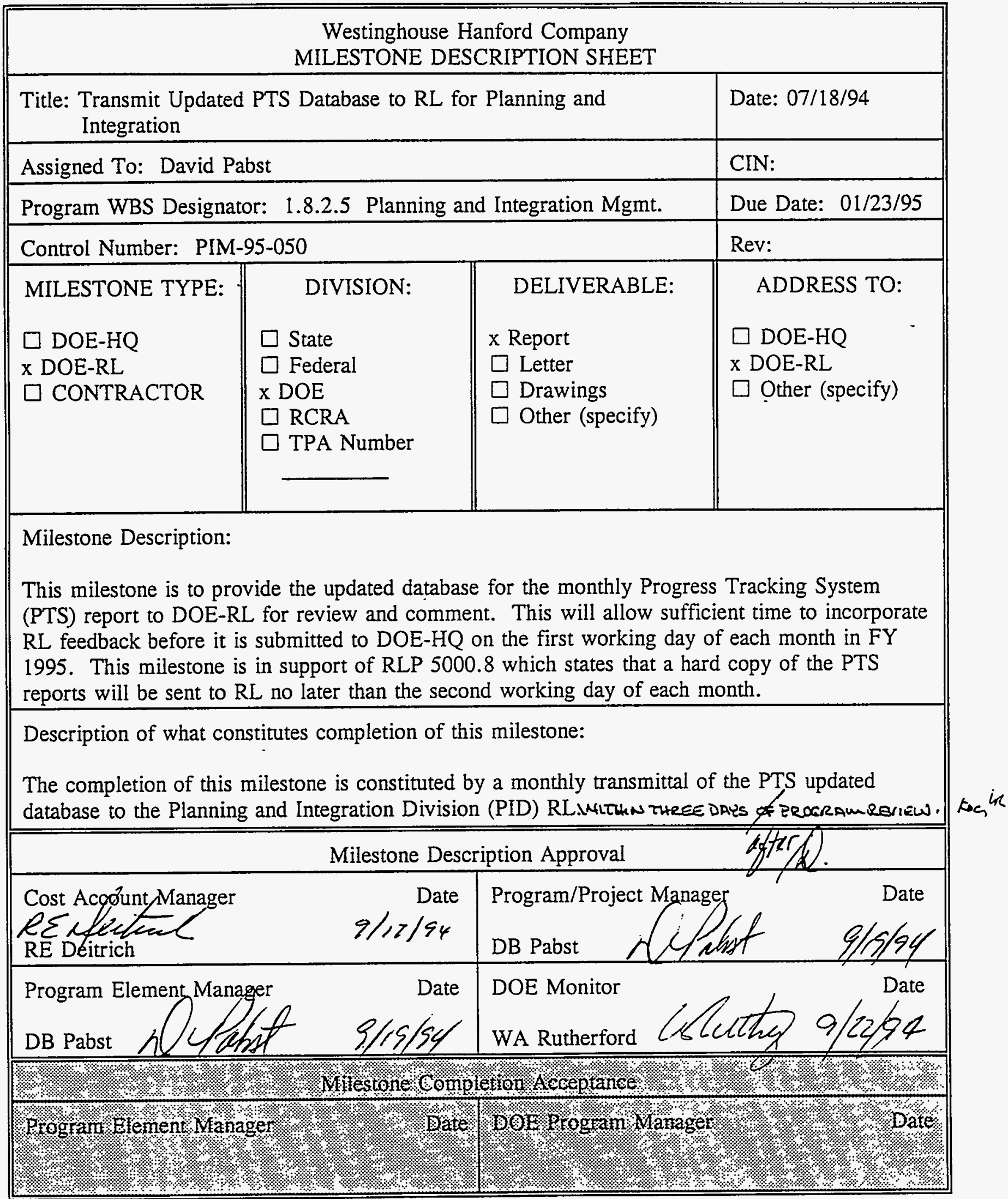




\begin{tabular}{|l||l|||}
\hline \multicolumn{3}{|c||}{ Westinghouse Hanford Company } \\
MILSTONE DESCRIPTION SHEET
\end{tabular}




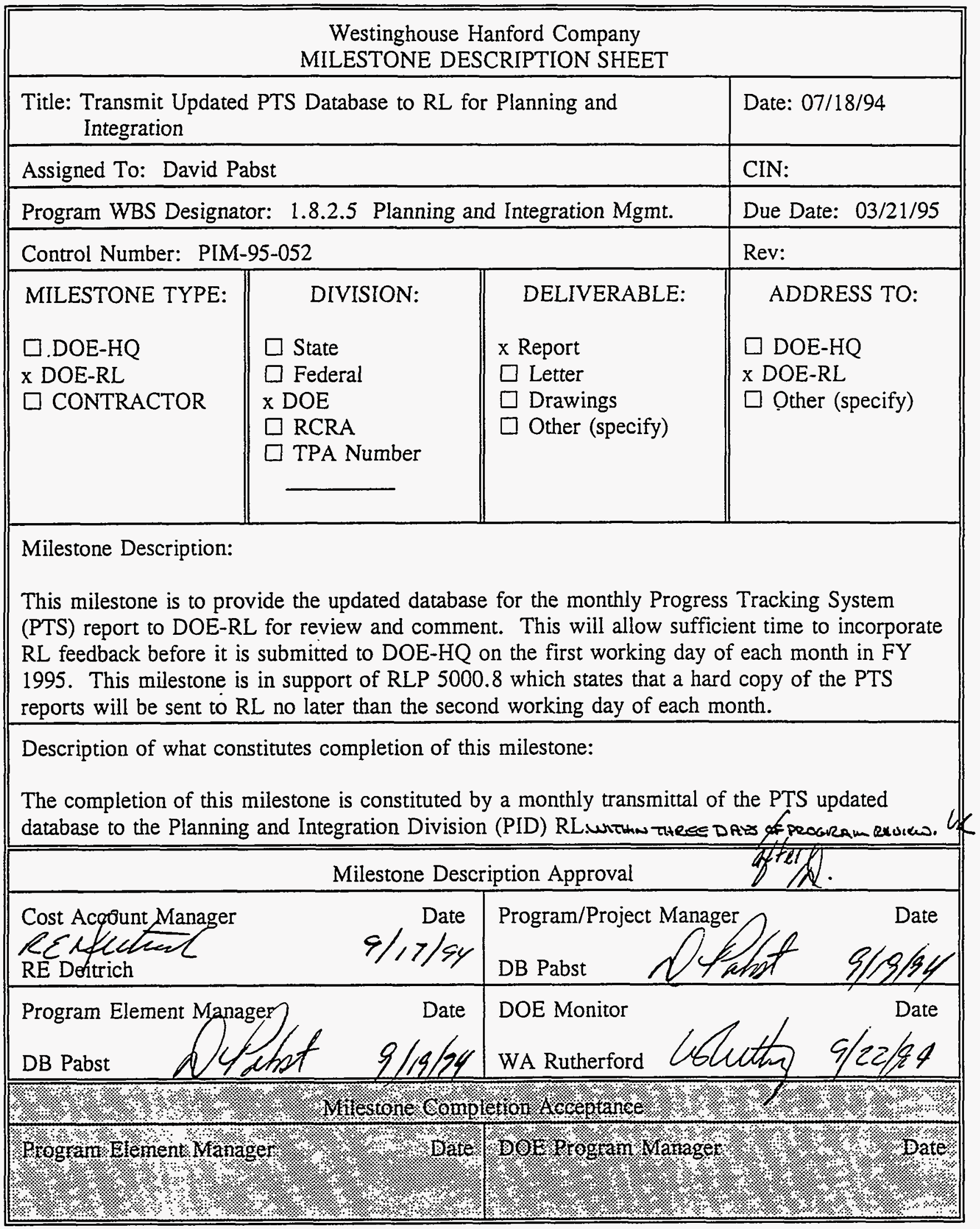




\begin{tabular}{|l||l|l||}
\hline \multicolumn{3}{|c|}{ Westinghouse Hanford Company } \\
MILESTONE DESCRIPTION SHEET
\end{tabular}




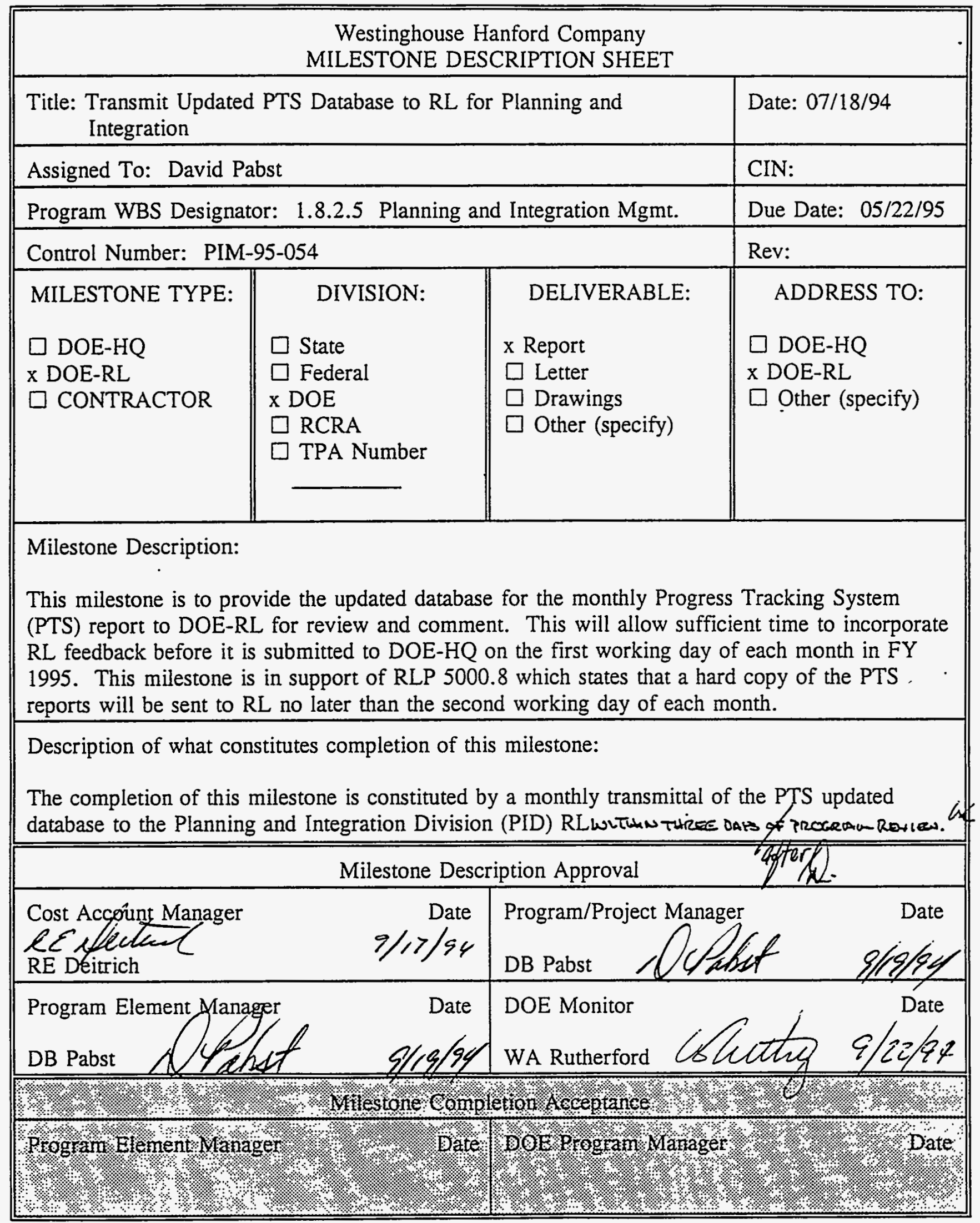




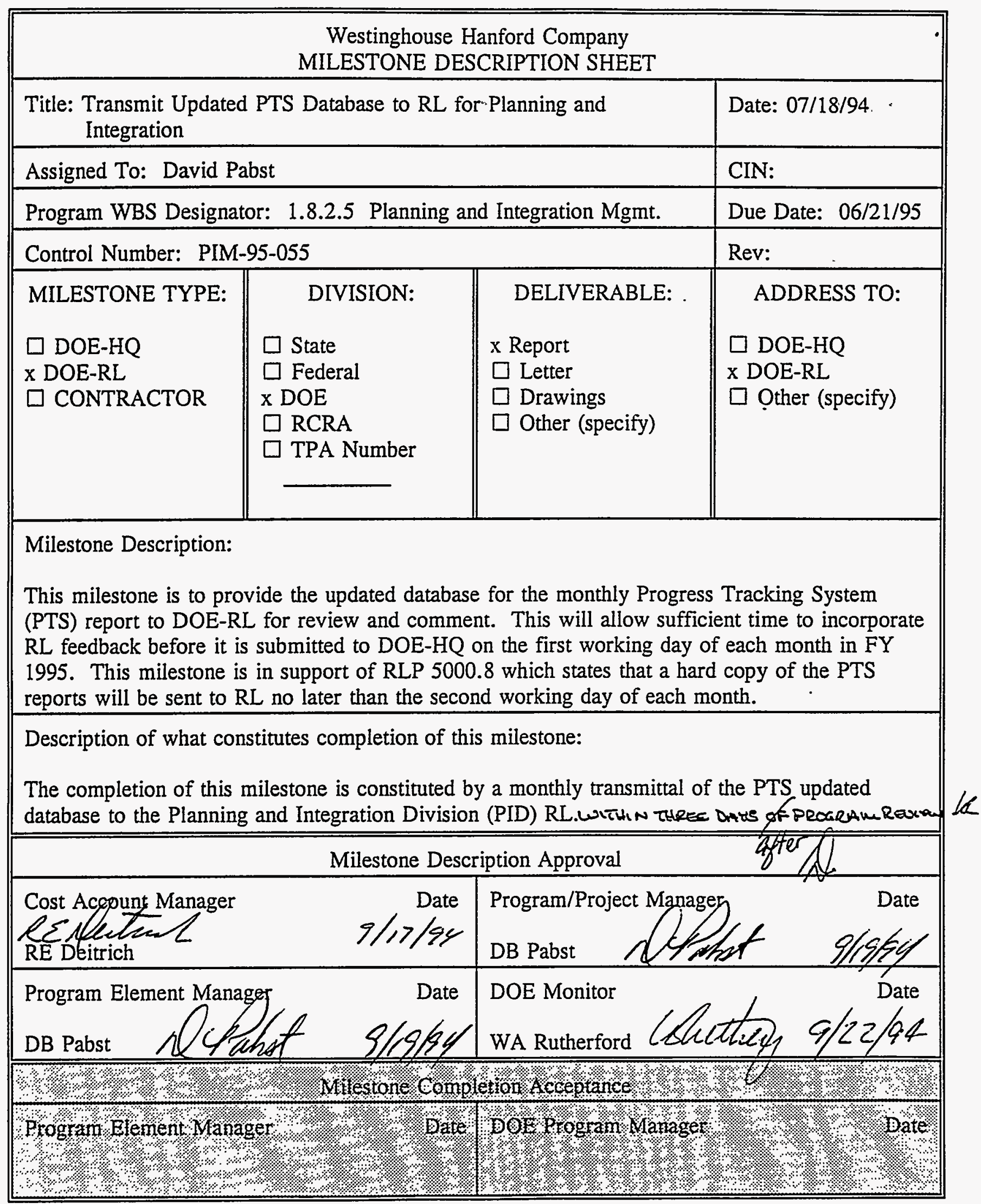




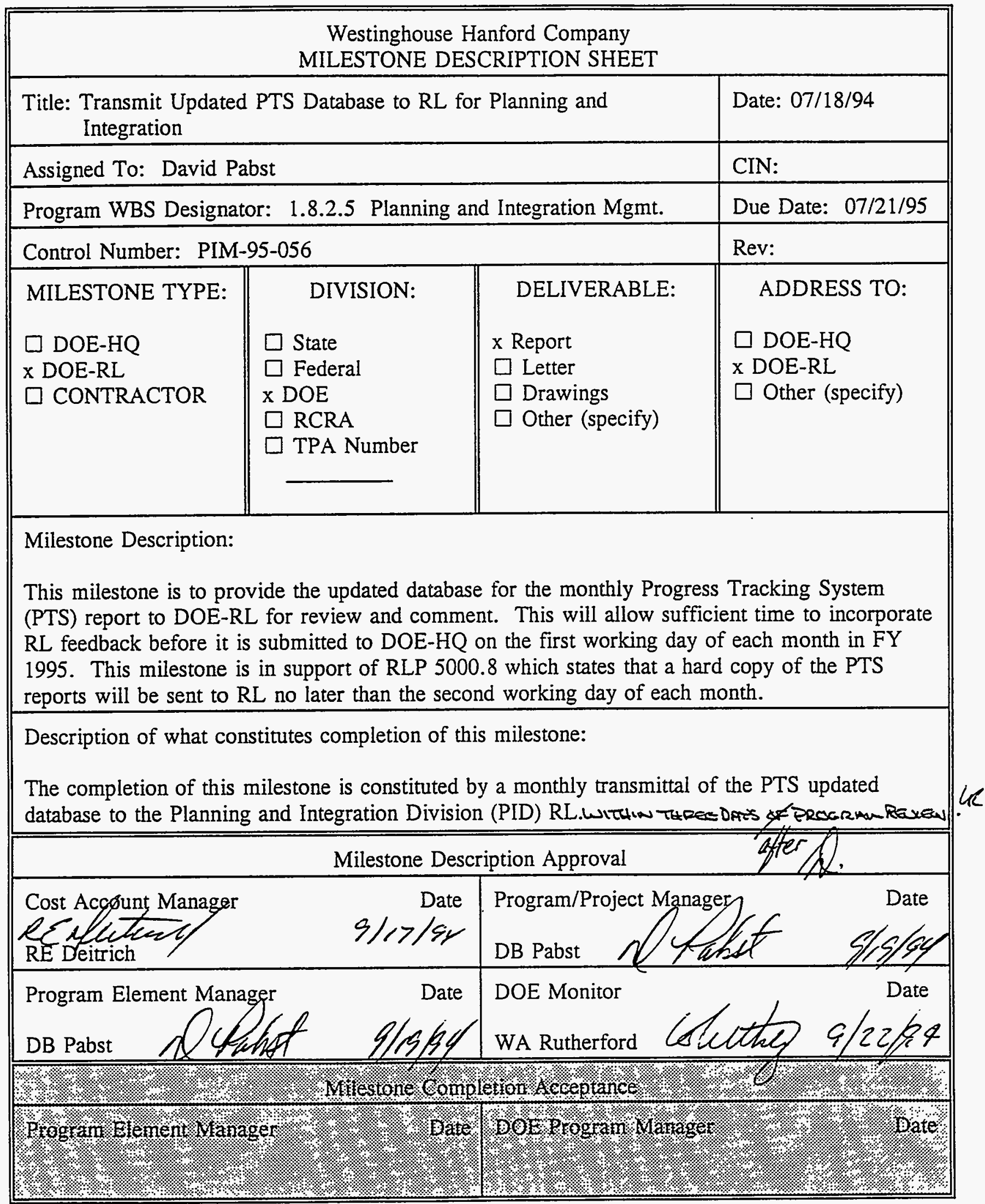




\begin{tabular}{|l||l||}
\hline \multicolumn{3}{|c|}{ Westinghouse Hanford Company } \\
MILESTONE DESCRIPTION SHEET
\end{tabular}




\begin{tabular}{|l||l||}
\hline \multicolumn{3}{|c|}{ Westinghouse Hanford Company } \\
MILESTONE DESCRIPTION SHEET
\end{tabular}




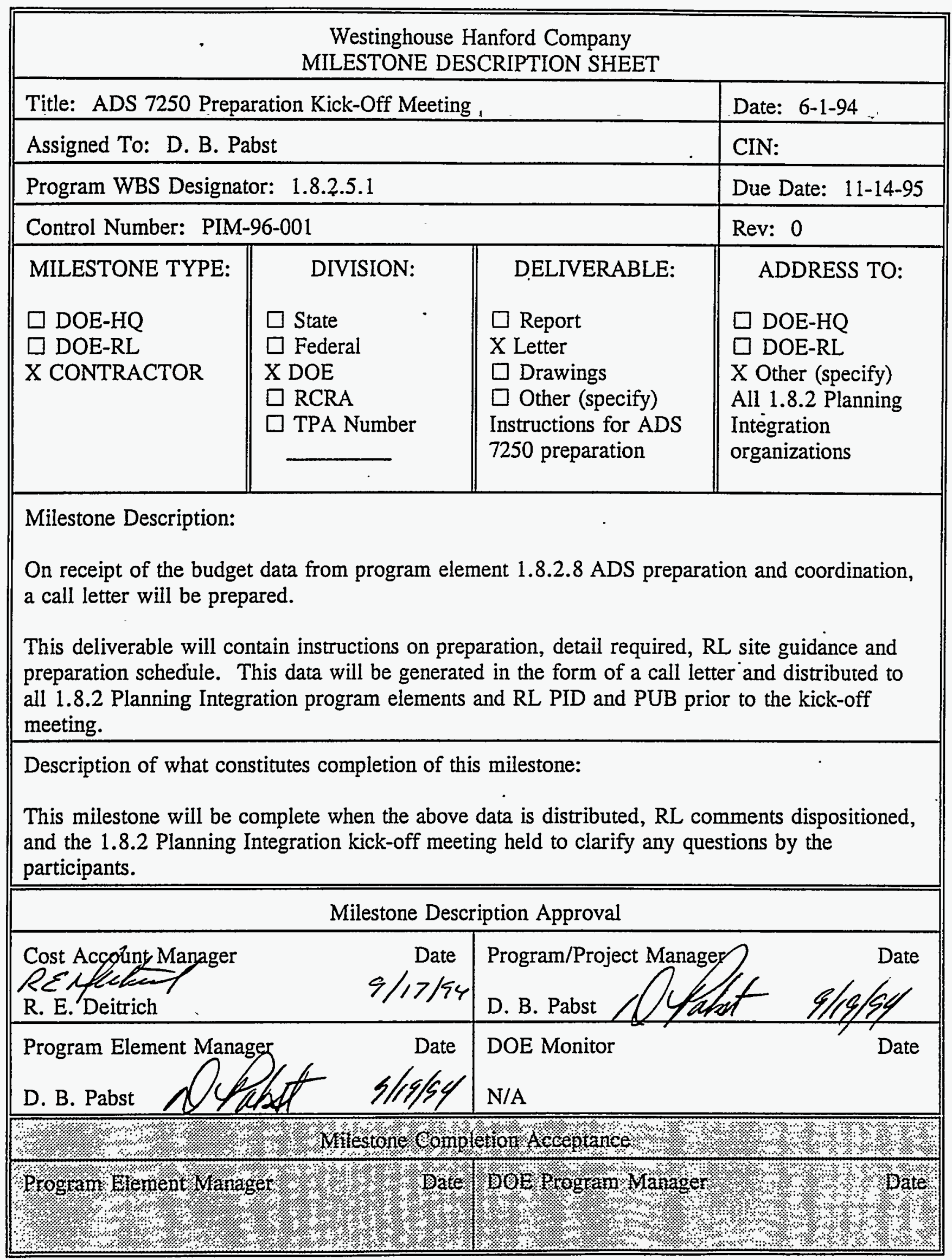




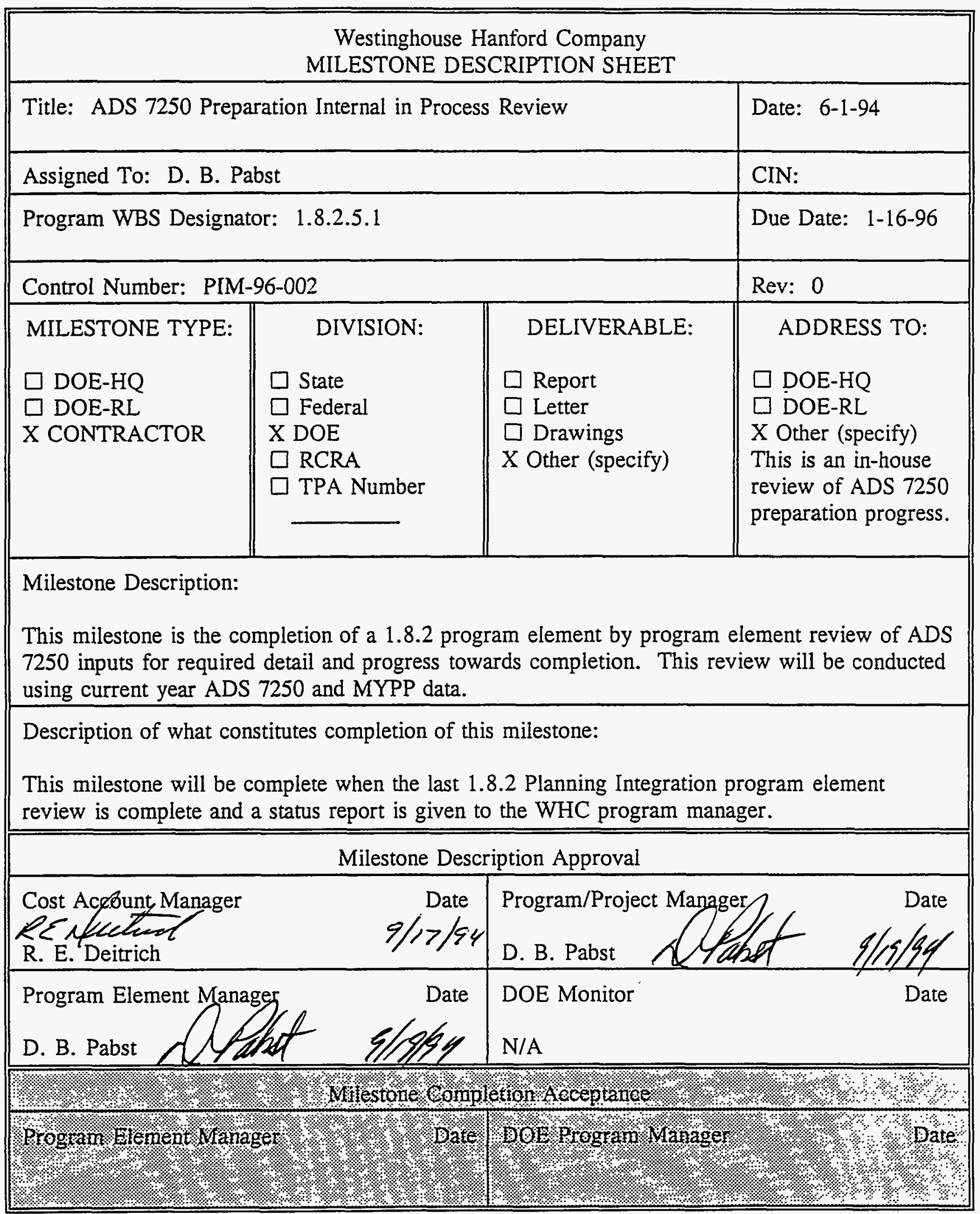




\begin{tabular}{||l||l||}
\hline \multicolumn{3}{|c|}{ Westinghouse Hanford Company } \\
MILESTONE DESCRIPTION SHEET
\end{tabular}




\begin{tabular}{||l||l||}
\hline \multicolumn{3}{|c|}{ Westinghouse Hanford Company } \\
MILESTONE DESCRIPTION SHEET
\end{tabular}




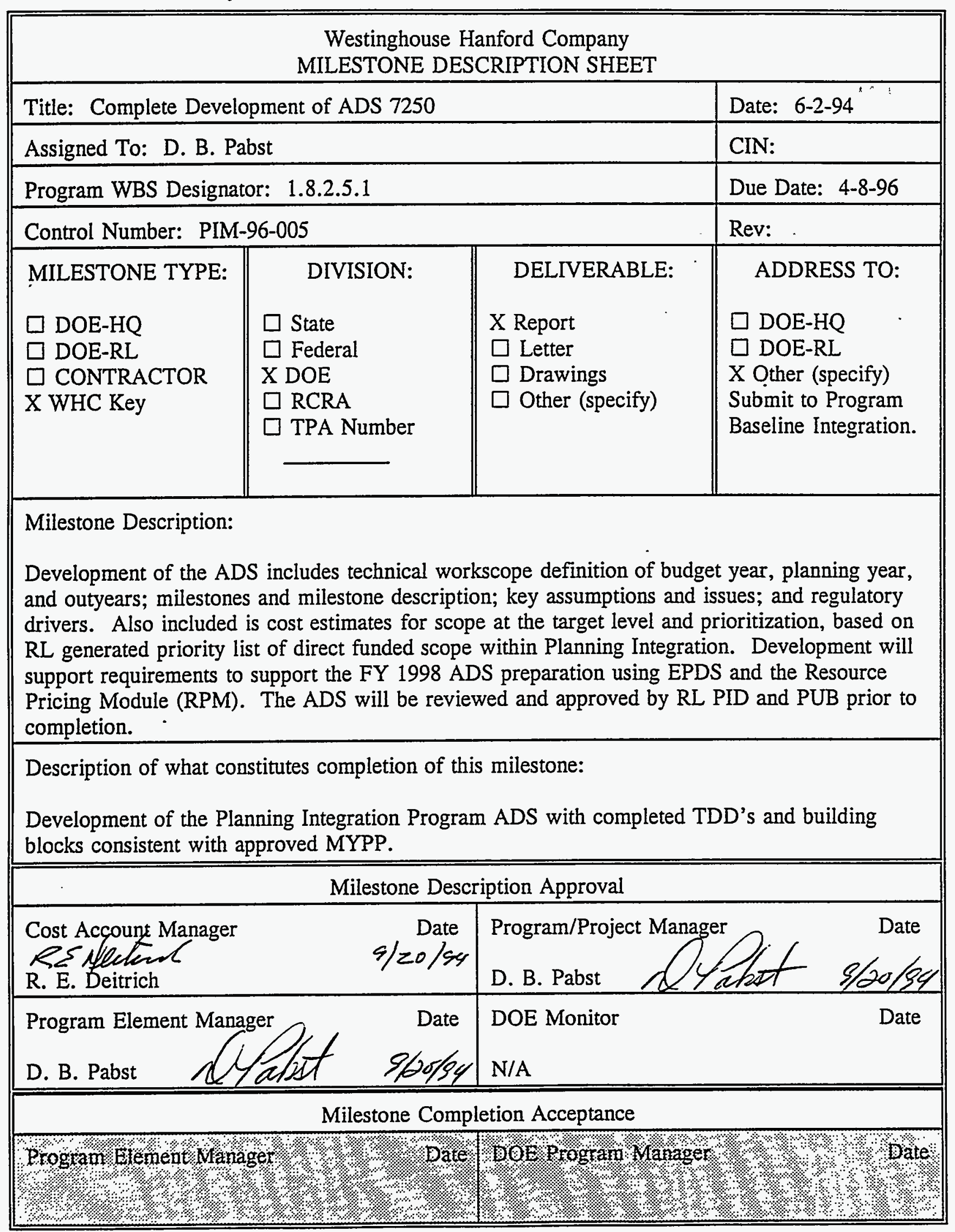




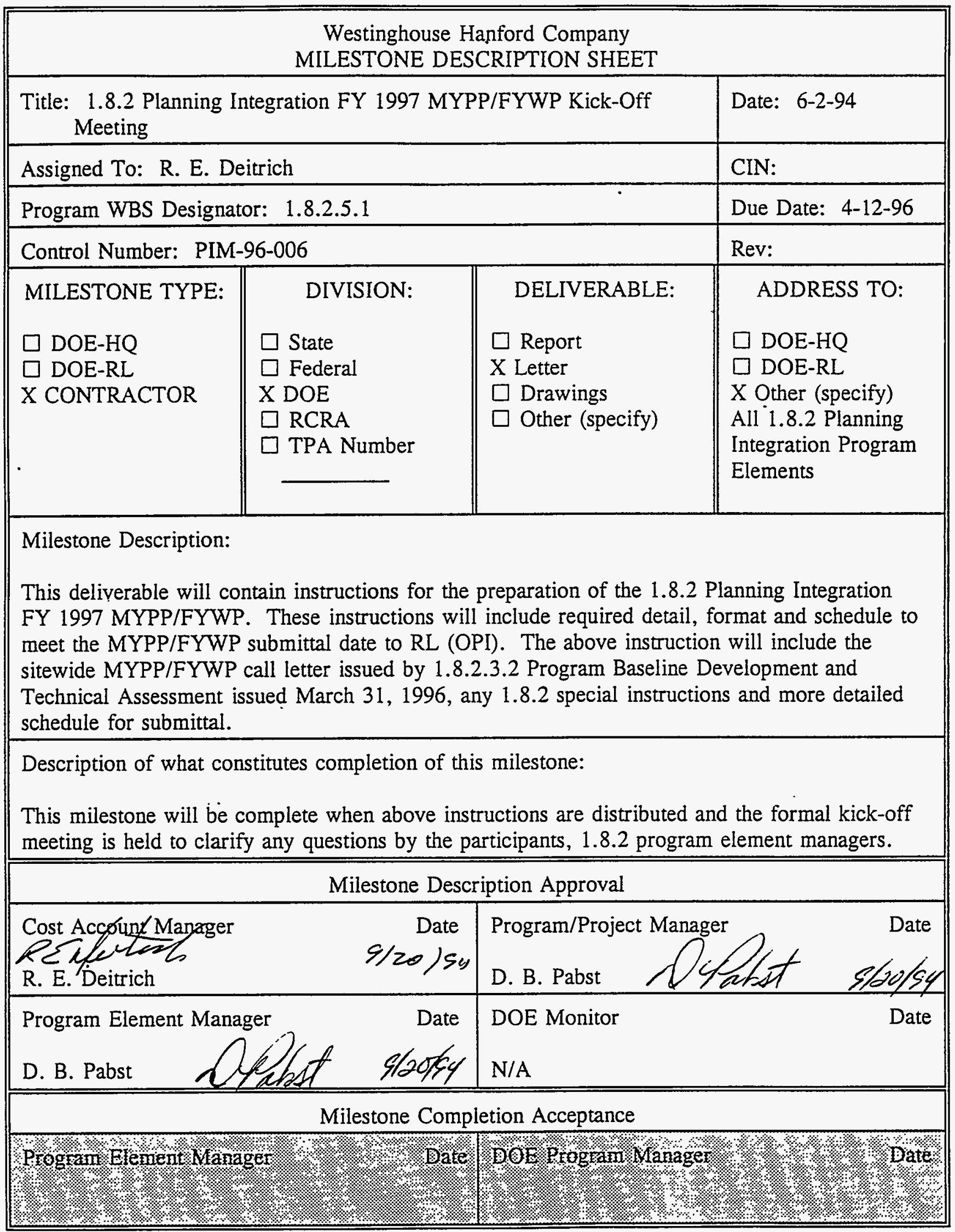




\begin{tabular}{|c|c|c|c|}
\hline \multicolumn{4}{|c|}{$\begin{array}{l}\text { Westinghouse Hanford Company } \\
\text { MILESTONE DESCRIPTION SHEET }\end{array}$} \\
\hline \multicolumn{3}{|c|}{ Title: 1 8.2 Planning Integration MYPP/FYWP in Process Reviews } & Date: $6-2-94$ \\
\hline \multicolumn{3}{|c|}{ Assigned To: D. B. Pabst } & CIN: \\
\hline \multicolumn{3}{|c|}{ Program WBS Designator: 1 8.2.5.1 } & Due Date: $6-19-96$ \\
\hline \multicolumn{3}{|c|}{ Control Number: PIM-96-007 } & Rev: \\
\hline $\begin{array}{l}\text { MILESTONE TYPE: } \\
\square \text { DOE-HQ } \\
\square \text { DOE-RL } \\
\text { X CONTRACTOR }\end{array}$ & $\begin{array}{l}\quad \text { DIVISION: } \\
\\
\square \text { State } \\
\square \text { Federal } \\
\text { X DOE } \\
\square \text { RCRA } \\
\square \text { TPA Number }\end{array}$ & $\begin{array}{l}\text { DELIVERABLE: } \\
\square \text { Report } \\
\square \text { Letter } \\
\square \text { Drawings } \\
\text { X Other (specify) } \\
\text { Review of } \\
\text { MYPP/FYWP } \\
\text { Progress }\end{array}$ & $\begin{array}{l}\quad \text { ADDRESS TO: } \\
\square \text { DOE-HQ } \\
\square \text { DOE-RL } \\
\text { X Other (specify) } \\
\text { Internal Review of } \\
\text { MYPP/FYWP } \\
\text { Progress. }\end{array}$ \\
\hline \multicolumn{4}{|c|}{$\begin{array}{l}\text { Milestone Description: } \\
\text { This milestone is a program element by program element review of } 1.8 .2 \text { Planning Integration } \\
\text { MYPP/FYWP inputs for required detail and progress towards RL submittal date. This review } \\
\text { will be performed by WHC and contractor personnel not involved with the generation of this } \\
\text { program plan. }\end{array}$} \\
\hline \multicolumn{4}{|c|}{$\begin{array}{l}\text { Description of what constitutes completion of this milestone: } \\
\text { This milestone will be complete when all the } 1.8 .2 \text { program elements have been reviewed. }\end{array}$} \\
\hline \multicolumn{4}{|c|}{ Milestone Description Approval } \\
\hline \multicolumn{2}{|c|}{$\begin{array}{l}\text { Cost Accojint Manager } \\
\text { Kéfectter } \\
\text { R.E. Deitrich }\end{array}$} & \multicolumn{2}{|c|}{ Program/Project Manager B. Pabst Date } \\
\hline \multicolumn{2}{|c|}{ Drogram Element Manager } & $\begin{array}{l}\text { DOE Monitor } \\
\text { N/A }\end{array}$ & Date \\
\hline \multicolumn{4}{|c|}{ 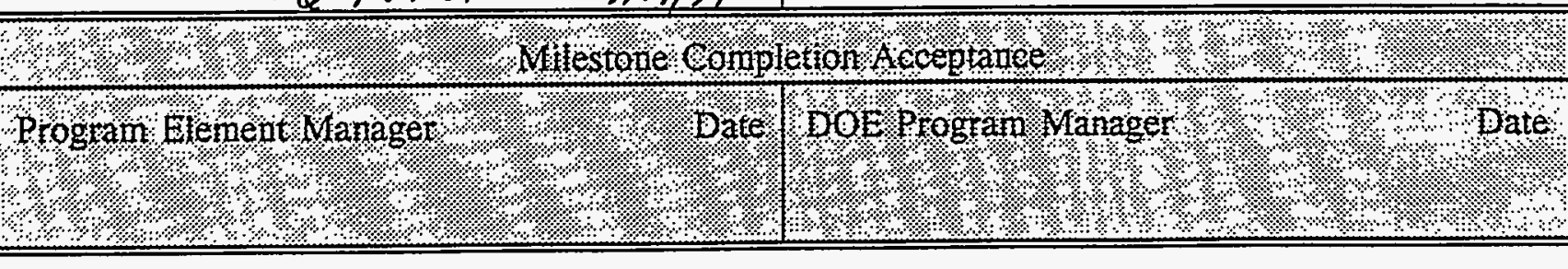 } \\
\hline
\end{tabular}




\begin{tabular}{|l||l|l||}
\hline \multicolumn{3}{|c|}{ Westinghouse Hanford Company } \\
MILESTONE DESCRIPTION SHEET
\end{tabular}




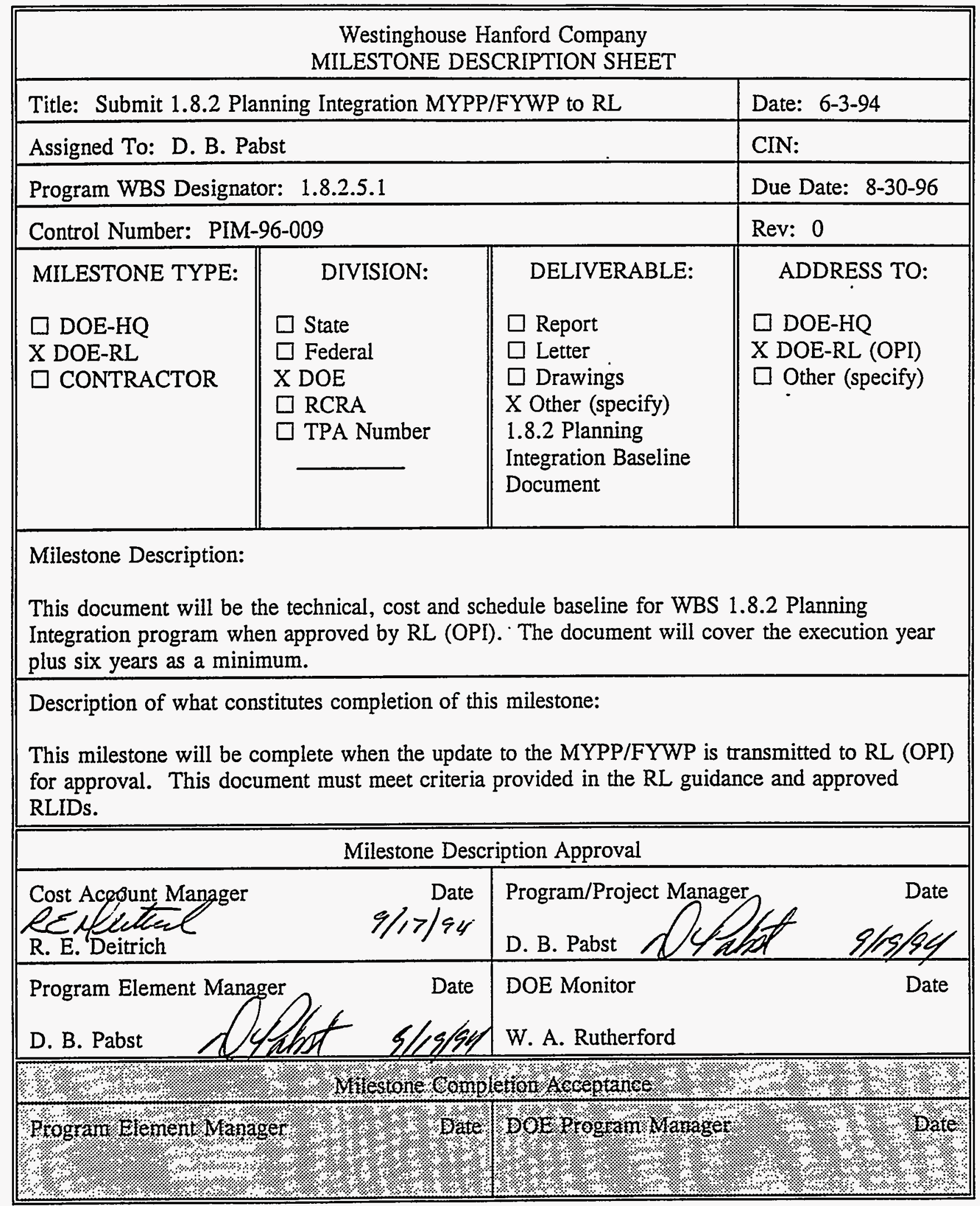




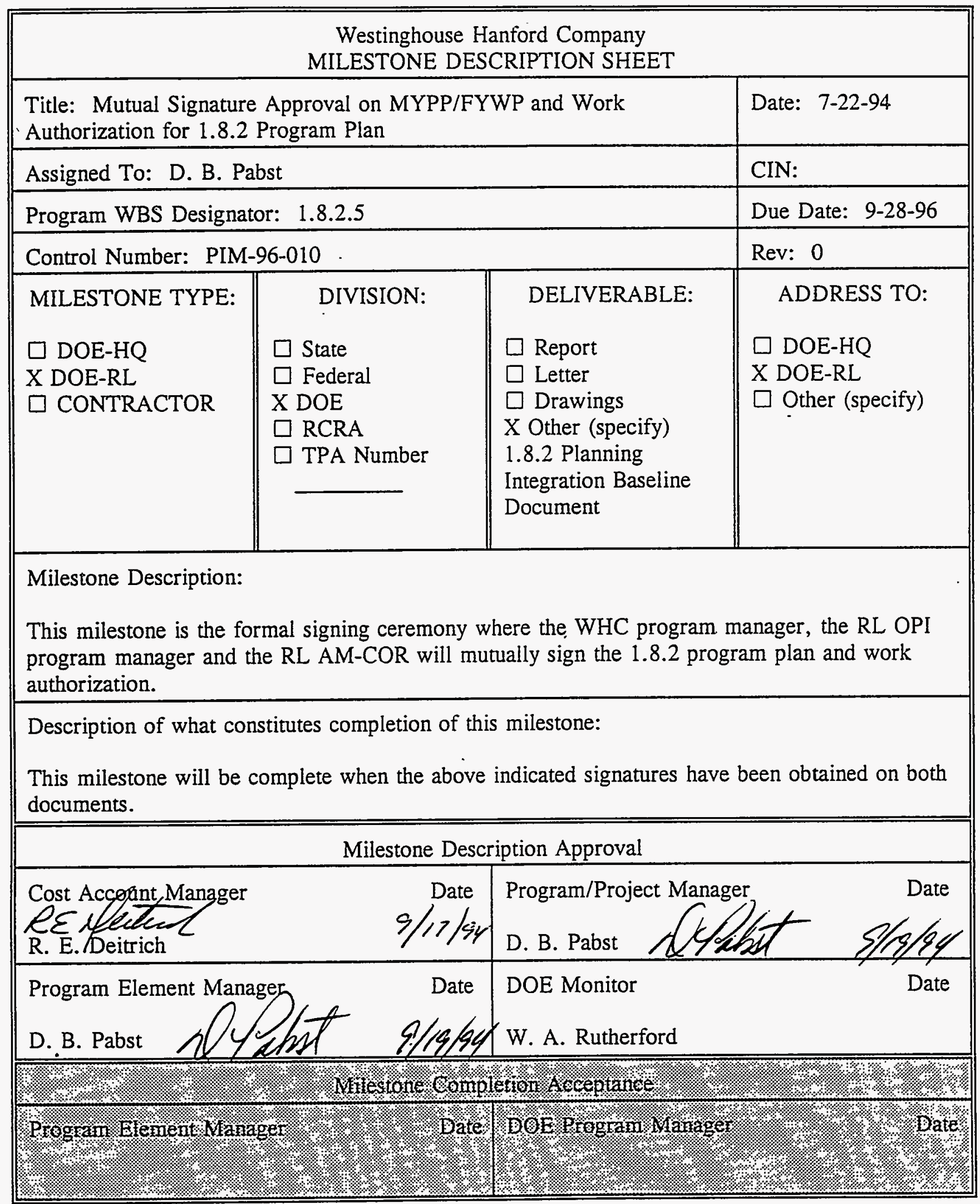




\begin{tabular}{|c|c|c|c|}
\hline \multicolumn{4}{|c|}{$\begin{array}{l}\text { Westinghouse Hanford Company } \\
\text { MILESTONE DESCRIPTION SHEET }\end{array}$} \\
\hline \multicolumn{3}{|c|}{ Title: Submit Monthly SMS Report for Planning and Integration } & Date: $07 / 18 / 94$ \\
\hline \multicolumn{3}{|c|}{ Assigned To: David Pabst } & CIN: \\
\hline \multicolumn{3}{|c|}{ Program WBS Designator: 1 8.2.5 Planning and Integration Mgmt. } & Due Date: $10 / 13 / 95$ \\
\hline \multicolumn{3}{|c|}{ Control Number: PIM-96-023 } & Rev: \\
\hline $\begin{array}{l}\text { MILESTONE TYPE: } \\
\square \text { DOE-HQ } \\
\square \text { DOE-RL } \\
\text { x CONTRACTOR }\end{array}$ & $\begin{array}{l}\quad \text { DIVISION: } \\
\quad \square \text { State } \\
\square \text { Federal } \\
\text { x DOE } \\
\square \text { RCRA } \\
\square \text { TPA Number }\end{array}$ & $\begin{array}{l}\text { DELIVERABLE: } \\
\text { x Report } \\
\square \text { Ĺtter } \\
\square \text { Drawings } \\
\square \text { Other (specify) }\end{array}$ & $\begin{array}{l}\quad \text { ADDRESS TO: } \\
\square \text { DOE-HQ } \\
\square \text { DOE-RL } \\
\square \text { Other (specify) } \\
\text { Deliver to } \\
\text { Performance } \\
\text { Reporting } 1.8 .2 .2\end{array}$ \\
\hline \multicolumn{4}{|c|}{$\begin{array}{l}\text { This milestone supports RLID } 5000.5 \text {, "Richland Field Office - Site Management System" and } \\
\text { RLID 5000.7, "RL Site Management System Reporting" and consists of coordinating the } \\
\text { monthly Planning and Integration program's Site Management Systems (SMS) report for final } \\
\text { submission to the Performance Reporting Organization. The SMS report will status and measure } \\
\text { Planning and Integration's accomplishments, progress, and expenditures against the approved } \\
\text { baseline. Also included will be a status of any change to the approved program baseline. } \\
\text { Following the monthly program review with the Office of Planning Integration (OPI) RL, RL } \\
\text { comments will be incorporated into the monthly sitewide SMS report delivered to RL each } \\
\text { month in FY 1995. }\end{array}$} \\
\hline \multicolumn{4}{|c|}{$\begin{array}{l}\text { Description of what constitutes completion of this milestone: } \\
\text { The completion of this milestone is constituted by the final submission of the Planning and } \\
\text { Integration's SMS report to the Performance Reporting Organization. }\end{array}$} \\
\hline \multicolumn{4}{|c|}{ Milestone Description Approval } \\
\hline \multicolumn{2}{|l|}{$\begin{array}{l}\text { Cost Accoynt Manager } \\
\text { RE Elfeetece } \\
\text { RE Deirich }\end{array}$} & \multicolumn{2}{|c|}{ Program/Project Manager } \\
\hline \multicolumn{2}{|c|}{ Program Element Manager } & \multicolumn{2}{|c|}{$\begin{array}{l}\text { DOE Monitor } \quad \text { Date } \\
\text { N/A }\end{array}$} \\
\hline \multicolumn{4}{|c|}{ 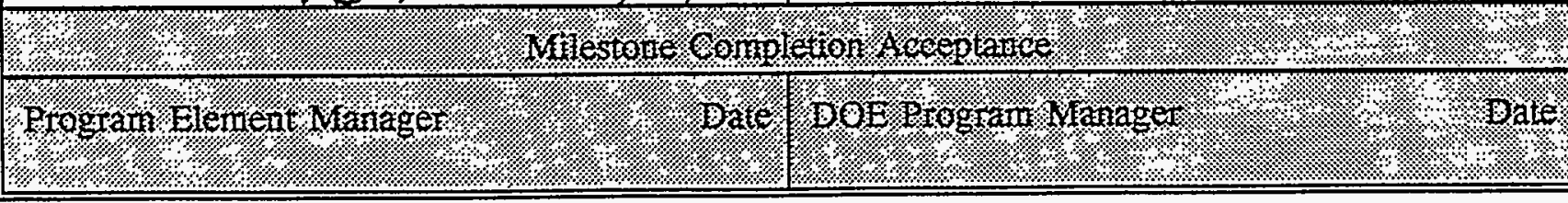 } \\
\hline
\end{tabular}




\begin{tabular}{|c|c|c|c|}
\hline \multicolumn{4}{|c|}{$\begin{array}{l}\text { Westinghouse Hanford Company } \\
\text { MILESTONE DESCRIPTION SHEET }\end{array}$} \\
\hline \multicolumn{3}{|c|}{ Title: Submit Monthly SMS Report for Planning and Integration } & Date: $07 / 18 / 94$ \\
\hline \multicolumn{3}{|c|}{ Assigned To: David Pabst } & CIN: \\
\hline \multicolumn{3}{|c|}{ Program WBS Designator: 188.2.5 Planning and Integration Mgmt. } & Due Date: $11 / 17 / 95$ \\
\hline \multicolumn{3}{|c|}{ Control Number: PIM-96-024 } & Rev: \\
\hline $\begin{array}{l}\text { MILESTONE TYPE: } \\
\square \text { DOE-HQ } \\
\square \text { DOE-RL } \\
\text { x CONTRACTOR }\end{array}$ & $\begin{aligned} & \text { DIVISION: } \\
& \square \text { State } \\
& \square \text { Federal } \\
& \text { x DOE } \\
& \square \text { RCRA } \\
& \square \text { TPA Number }\end{aligned}$ & $\begin{array}{l}\text { DELIVERABLE: } \\
x \text { Report } \\
\square \text { Letter } \\
\square \text { Drawings } \\
\square \text { Other (specify) }\end{array}$ & $\begin{array}{l}\quad \text { ADDRESS TO: } \\
\square \text { DOE-HQ } \\
\square \text { DOE-RL } \\
\text { x Other (specify) } \\
\text { Deliver to } \\
\text { Performance } \\
\text { Reporting 1.8.2.2 }\end{array}$ \\
\hline \multicolumn{4}{|c|}{$\begin{array}{l}\text { Milestone Description: } \\
\text { This milestone supports RLID } 5000.5 \text {, "Richland Field Office - Site Management System" and } \\
\text { RLDD } 5000.7 \text {, "RL Site Management System Reporting" and consists of coordinating the } \\
\text { monthly Planning and Integration program's Site Management Systems (SMS) report for final } \\
\text { submission to the Performance Reporting Organization. The SMS report will status and measure } \\
\text { Planning and Integration's accomplishments, progress, and expenditures against the approved } \\
\text { baseline. Also included will be a status of any change to the approved program baseline. } \\
\text { Following the monthly program review with the Office of Planning Integration (OPI) RL, RL } \\
\text { comments will be incorporated into the monthly sitewide SMS report delivered to RL each } \\
\text { month in FY 1995. }\end{array}$} \\
\hline \multicolumn{4}{|c|}{$\begin{array}{l}\text { Description of what constitutes completion of this milestone: } \\
\text { The completion of this milestone is constituted by the final submission of the Planning and } \\
\text { Integration's SMS report to the Performance Reporting Organization. }\end{array}$} \\
\hline \multicolumn{4}{|c|}{ Milestone Description Approval } \\
\hline \multicolumn{2}{|l|}{$\begin{array}{l}\text { Cost Account Manager } \\
\text { RE H whech } \\
\text { RE Deitrich }\end{array}$} & \multicolumn{2}{|c|}{ Program/Project Manager Pabst } \\
\hline \multicolumn{2}{|c|}{ Program Element Managey } & \multicolumn{2}{|c|}{$\begin{array}{ll}\text { DOE Monitor } & \text { Date } \\
\text { N/A } & \\
\end{array}$} \\
\hline \multicolumn{4}{|c|}{ 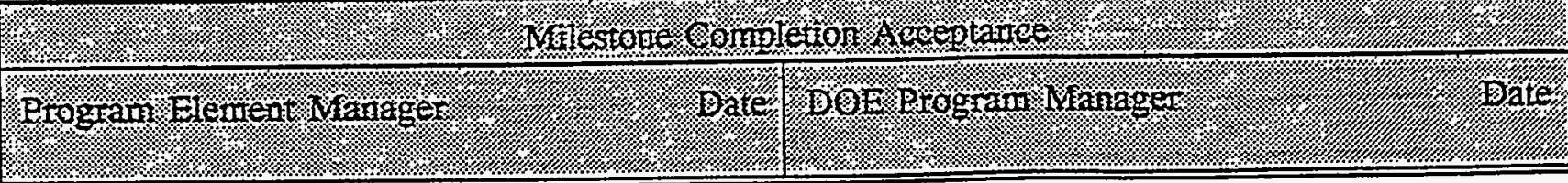 } \\
\hline
\end{tabular}




\begin{tabular}{|c|c|c|c|}
\hline \multicolumn{4}{|c|}{$\begin{array}{l}\text { Westinghouse Hanford Company } \\
\text { MILESTONE DESCRIPTION SHEET }\end{array}$} \\
\hline \multicolumn{3}{|c|}{ Title: Submit Monthly SMS Report for Planning and Integration } & Date: $07 / 18 / 94$ \\
\hline \multicolumn{3}{|c|}{ Assigned To: David Pabst } & CIN: \\
\hline \multicolumn{3}{|c|}{ Program WBS Designator: 1-8.2.5 Planning and Integration Mgmt. } & Due Date: $12 / 15 / 95$ \\
\hline \multicolumn{3}{|c|}{ Control Number: PIM-96-025 } & Rev: \\
\hline $\begin{array}{l}\text { MULESTONE TYPE: } \\
\square \text { DOE-HQ } \\
\square \text { DOE-RL } \\
\text { X CONTRACTOR }\end{array}$ & $\begin{array}{l}\quad \text { DIVISION: } \\
\square \text { State } \\
\square \text { Federal } \\
\times \text { DOE } \\
\square \text { RCRA } \\
\square \text { TPA Number }\end{array}$ & $\begin{array}{l}\text { DELIVERABLE: } \\
\quad \cdot \\
\text { x Report } \\
\square \text { Letter } \\
\square \text { Drawings } \\
\square \text { Other (specify) }\end{array}$ & $\begin{array}{l}\quad \text { ADDRESS TO: } \\
\square \text { DOE-HQ } \\
\square \text { DOE-RL } \\
\text { x Other (specify) } \\
\text { Deliver to } \\
\text { Performance } \\
\text { Reporting } 1.8 .2 .2\end{array}$ \\
\hline \multicolumn{4}{|c|}{$\begin{array}{l}\text { Milestone Description: } \\
\text { This milestone supports RLID 5000.5, "Richland Field Office - Site Management System" and } \\
\text { RLID 5000.7, "RL Site Management System Reporting" and consists of coordinating the } \\
\text { monthly Planning and Integration program's Site Management Systems (SMS) report for final } \\
\text { submission to the Performance Reporting Organization. The SMS report will status and measure } \\
\text { Planning and Integration's accomplishments, progress, and expenditures against the approved } \\
\text { baseline. Also included will be a status of any change to the approved program baseline. } \\
\text { Following the monthly program review with the Office of Planning Integration (OPI RL, RL } \\
\text { comments will be incorporated into the monthly sitewide SMS report delivered to RL each } \\
\text { month in FY 1995. }\end{array}$} \\
\hline \multicolumn{4}{|c|}{$\begin{array}{l}\text { Description of what constitutes completion of this milestone: } \\
\text { The completion of this milestone is constituted by the final submission of the Planning and } \\
\text { Integration's SMS report to the Performance Reporting Organization. }\end{array}$} \\
\hline \multicolumn{4}{|c|}{ Milestone Description Approval } \\
\hline \multicolumn{2}{|l|}{$\begin{array}{l}\text { Cost Acceuntyanager } \\
\text { RE Yfleleel } \\
\text { RE DEitrich }\end{array}$} & \multicolumn{2}{|c|}{ Program/Project Manager } \\
\hline \multicolumn{2}{|c|}{ Program Element Manager } & \multicolumn{2}{|l|}{$\begin{array}{l}\text { DOE Monitor } \\
\text { N/A }\end{array}$} \\
\hline \multicolumn{4}{|c|}{ 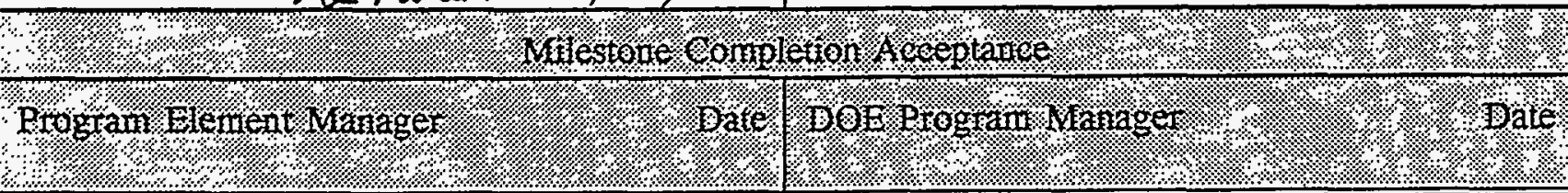 } \\
\hline
\end{tabular}




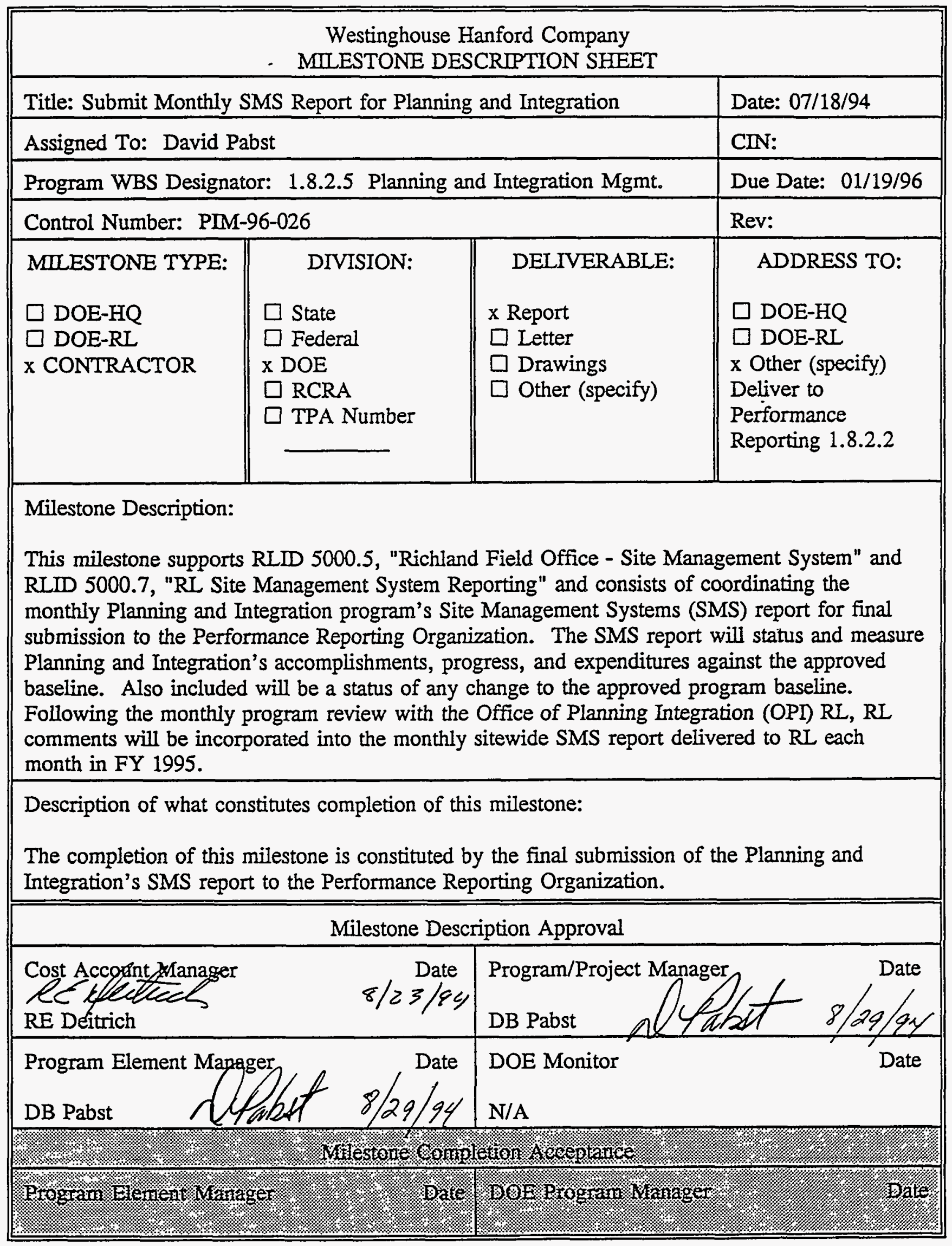




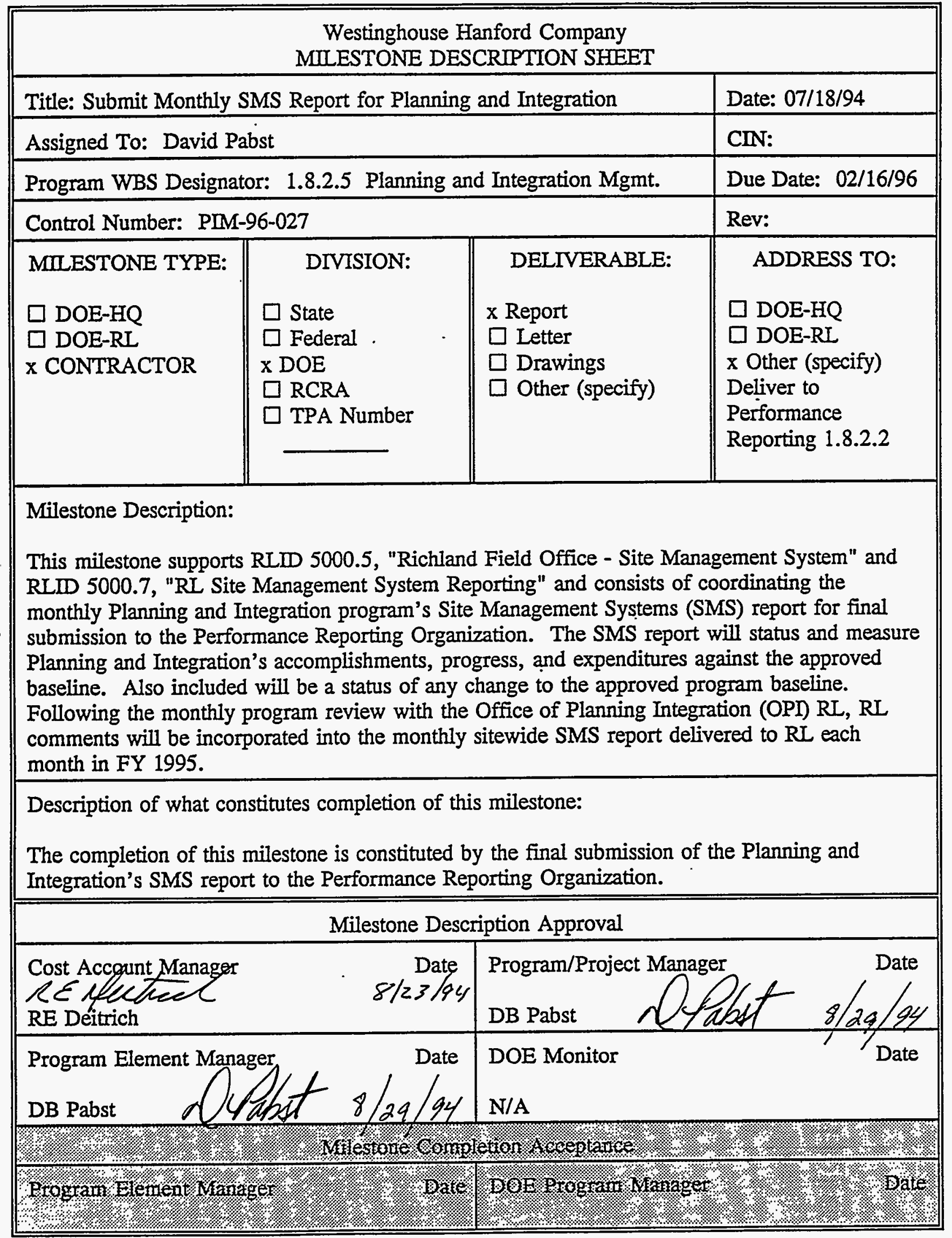




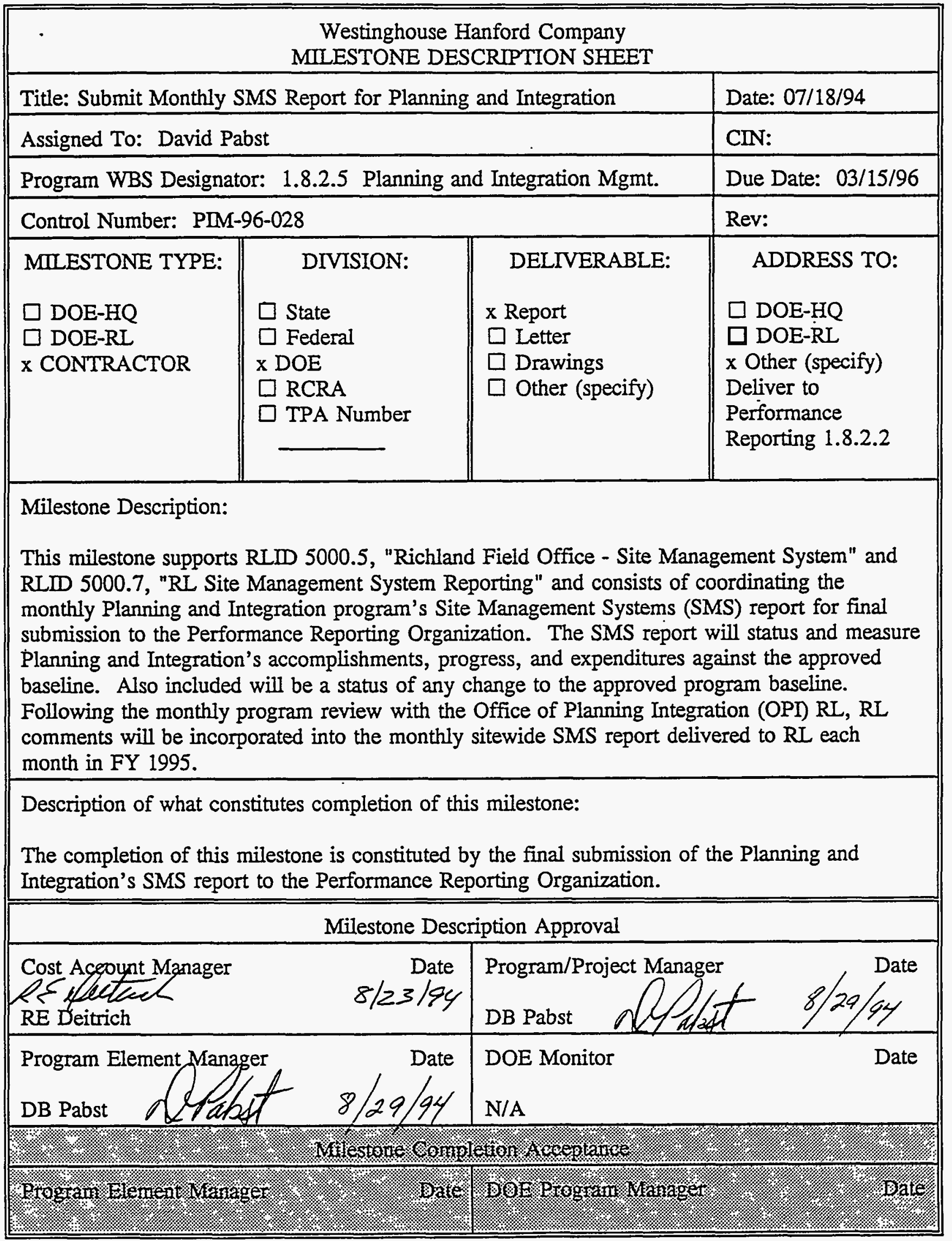




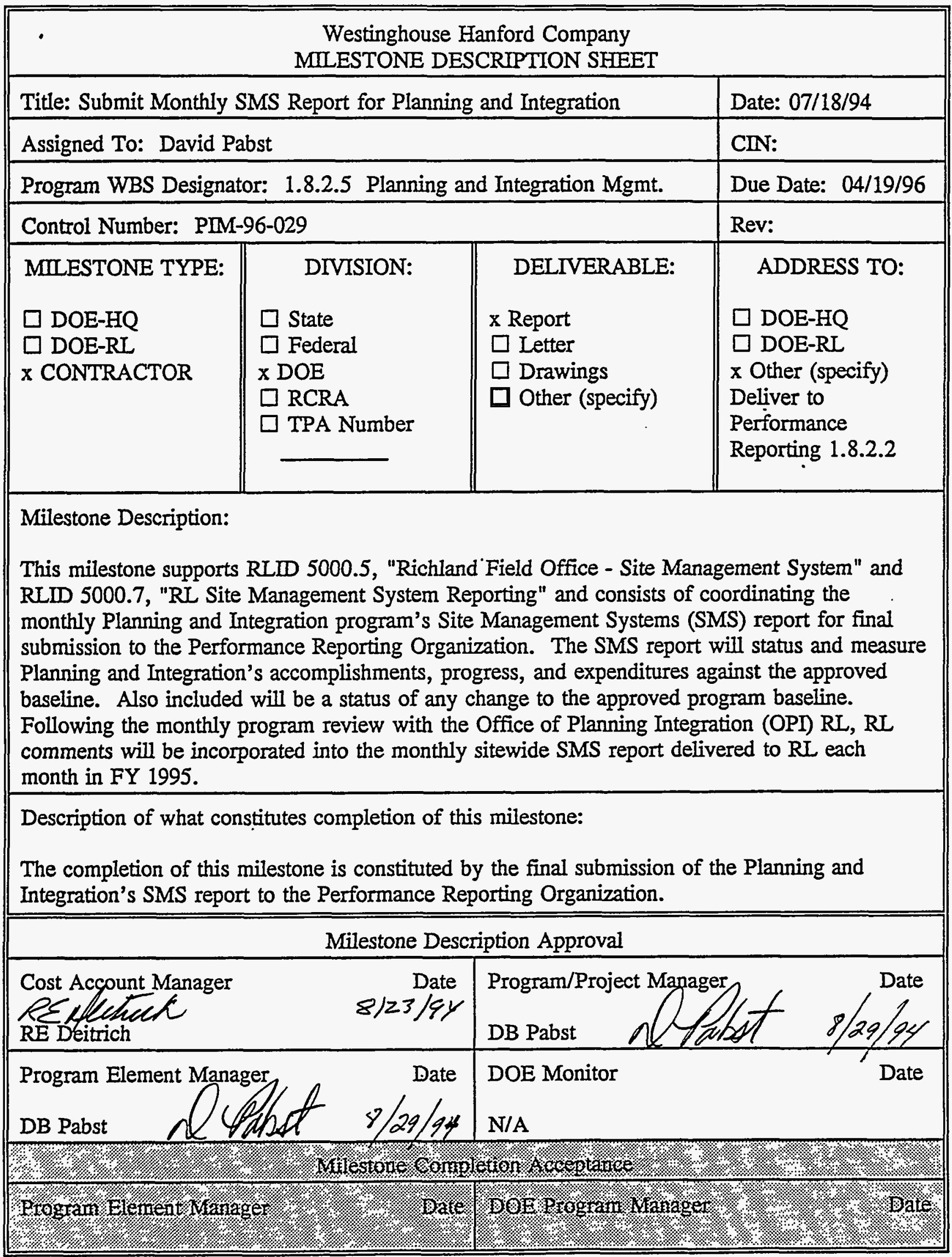




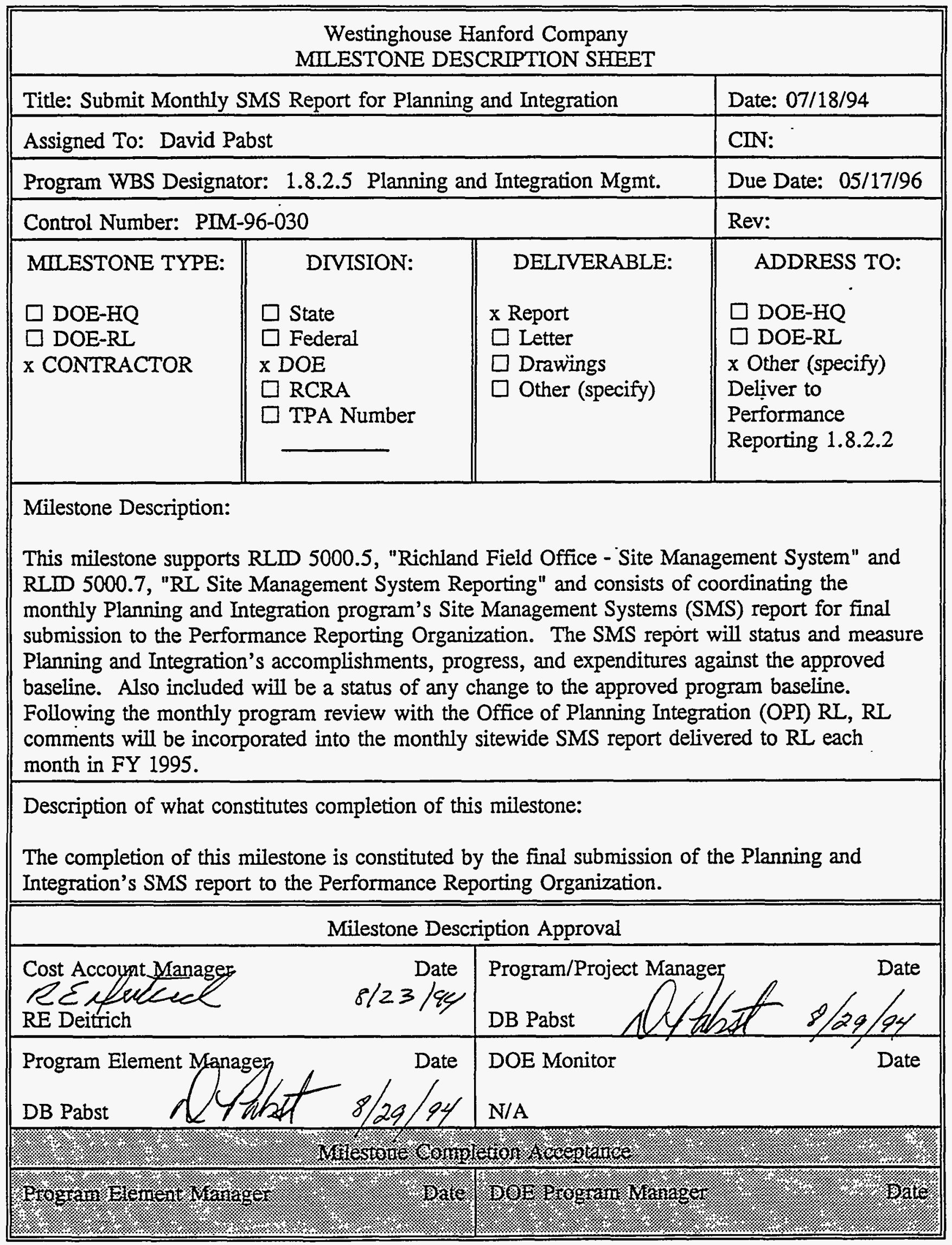




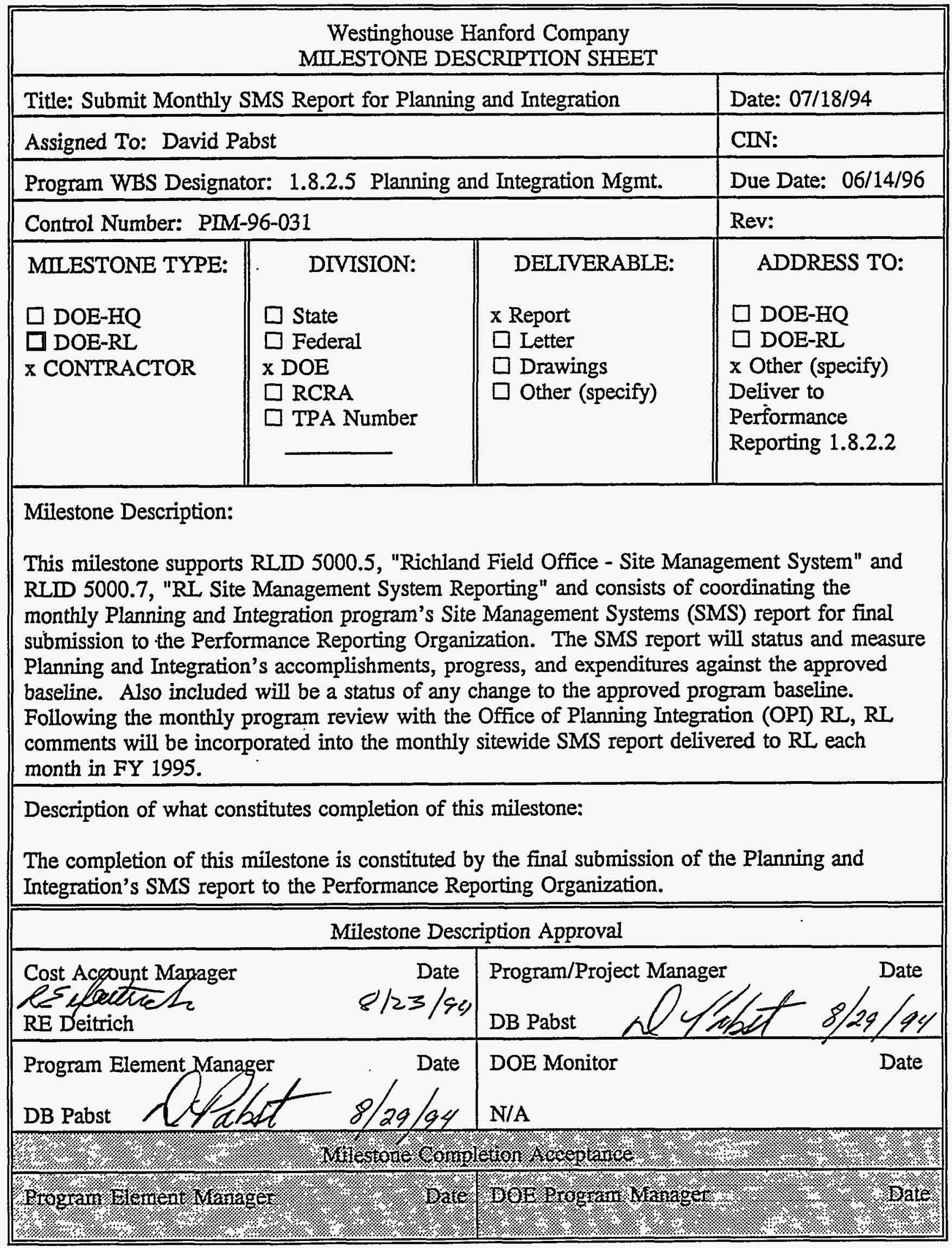




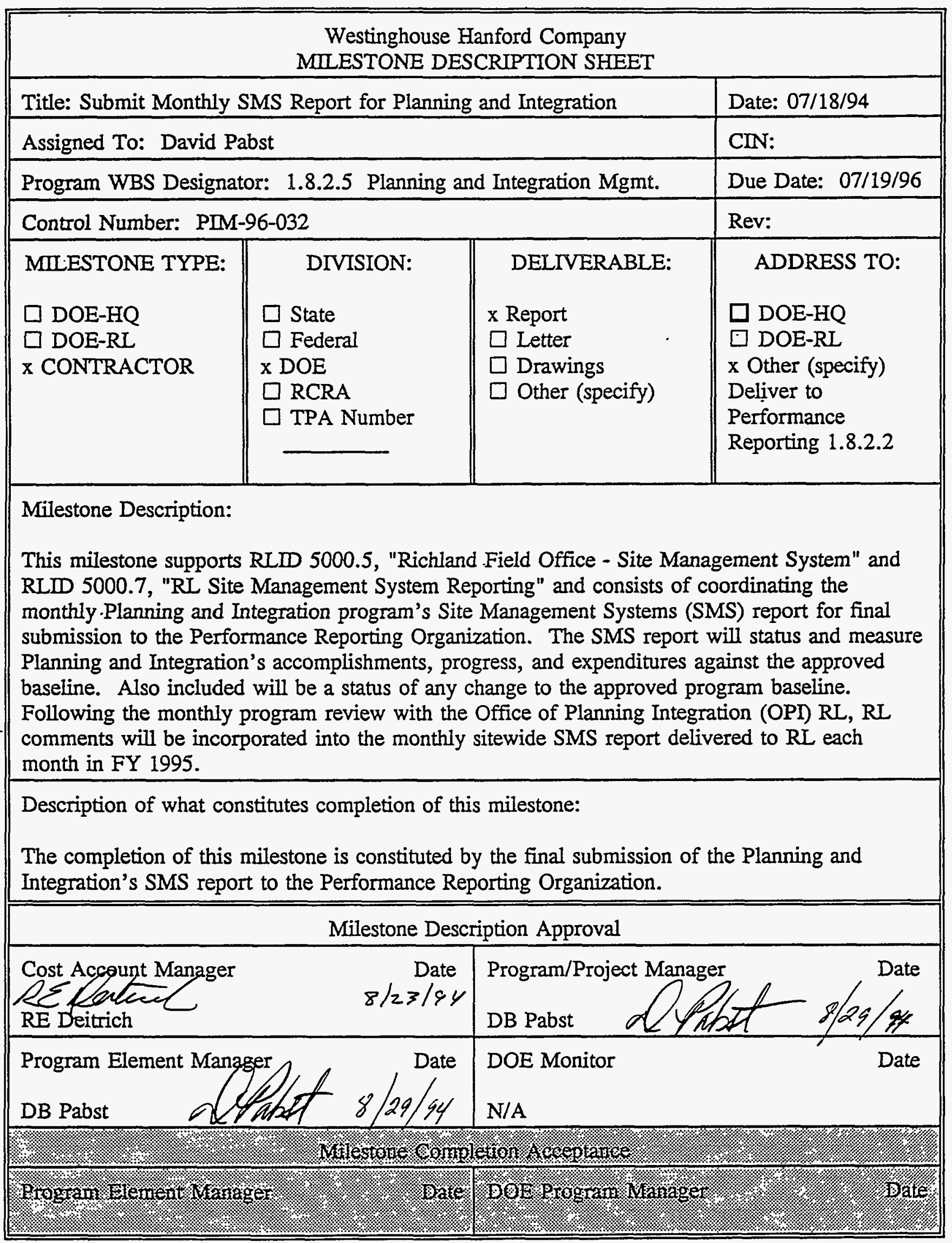




\begin{tabular}{|c|c|c|c|}
\hline \multicolumn{4}{|c|}{$\begin{array}{l}\text { Westinghouse Hanford Company } \\
\text { MILESTONE DESCRIPTION SHEET }\end{array}$} \\
\hline \multicolumn{3}{|c|}{ Title: Submit Monthly SMS Report for Planning and Integration } & Date: $07 / 18 / 94$ \\
\hline \multicolumn{3}{|c|}{ Assigned To: David Pabst } & CIN: \\
\hline \multicolumn{3}{|c|}{ Program WBS Designator: 1.8.2.5 Planning and Integration Mgmt. } & Due Date: $08 / 16 / 96$ \\
\hline \multicolumn{3}{|c|}{ Control Number: PIM-96-033 } & Rev: \\
\hline $\begin{array}{l}\text { MILESTONE TYPE: } \\
\square \text { DOE-HQ } \\
\square \text { DOE-RL } \\
\text { x CONTRACTOR }\end{array}$ & $\begin{array}{l}\quad \text { DIVISION: } \\
\\
\square \text { State } \\
\square \text { Federal } \\
\text { x DOE } \\
\square \text { RCRA } \\
\square \text { TPA Number }\end{array}$ & $\begin{array}{l}\text { DELIVERABLE: } \\
\text { x Report } \\
\square \text { Letter } \\
\square \text { Drawings } \\
\square \text { Other (specify) }\end{array}$ & $\begin{array}{l}\quad \text { ADDRESS TO: } \\
\square \text { DOE-HQ } \\
\square \text { DOE-RL } \\
\text { x Other (specify) } \\
\text { Deliver to } \\
\text { Performance } \\
\text { Reporting } 1.8 .2 .2\end{array}$ \\
\hline \multicolumn{4}{|c|}{$\begin{array}{l}\text { Milestone Description: } \\
\text { This milestone supports RLID 5000.5, "Richland Field Office - Site Management System" and } \\
\text { RLID 5000.7, "RL Site Management System Reporting" and consists of coordinating the } \\
\text { monthly Planning and Integration program's Site Management Systems (SMS) report for final } \\
\text { submission to the Performance Reporting Organization. The SMS report will status and measure } \\
\text { Planning and Integration's accomplishments, progress, and expenditures against the approved } \\
\text { baseline. Also included will be a status of any change to the approved program baseline. } \\
\text { Following the monthly program review with the Office of Planning Integration (OPI) RL, RL } \\
\text { comments will be incorporated into the monthly sitewide SMS report delivered to RL each } \\
\text { month in FY } 1995 \text {. }\end{array}$} \\
\hline \multicolumn{4}{|c|}{$\begin{array}{l}\text { Description of what constitutes completion of this milestone: } \\
\text { The completion of this milestone is constituted by the final submission of the Planning and } \\
\text { Integration's SMS report to the Performance Reporting Organization. }\end{array}$} \\
\hline \multicolumn{4}{|c|}{ Milestone Description Approval } \\
\hline \multicolumn{2}{|c|}{$\begin{array}{l}\text { Cost Account Manager } \\
\text { Re iflectede } \\
\text { RE Deitrich }\end{array}$} & \multicolumn{2}{|c|}{ DB Pabst } \\
\hline \multirow{2}{*}{\multicolumn{2}{|c|}{ Program Element Managef }} & \multicolumn{2}{|c|}{$\begin{array}{l}\text { DOE Monitor Date } \\
\text { N/A }\end{array}$} \\
\hline & & 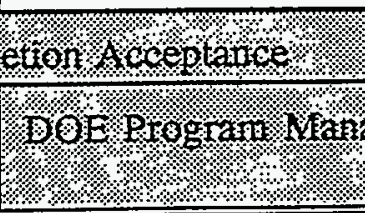 & 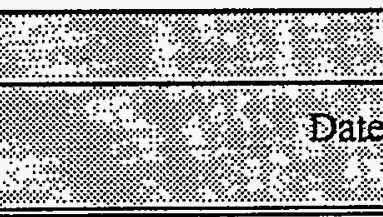 \\
\hline
\end{tabular}




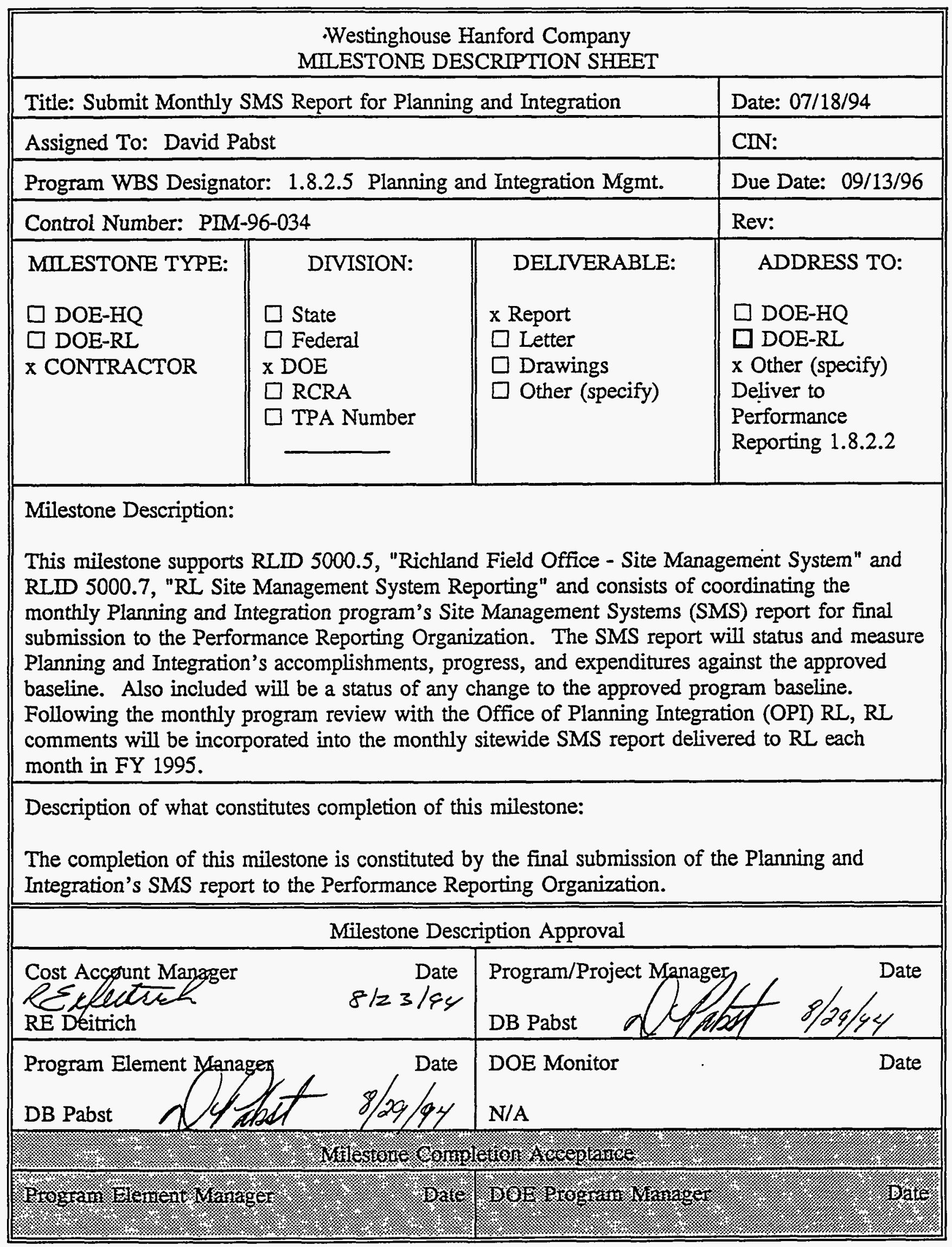




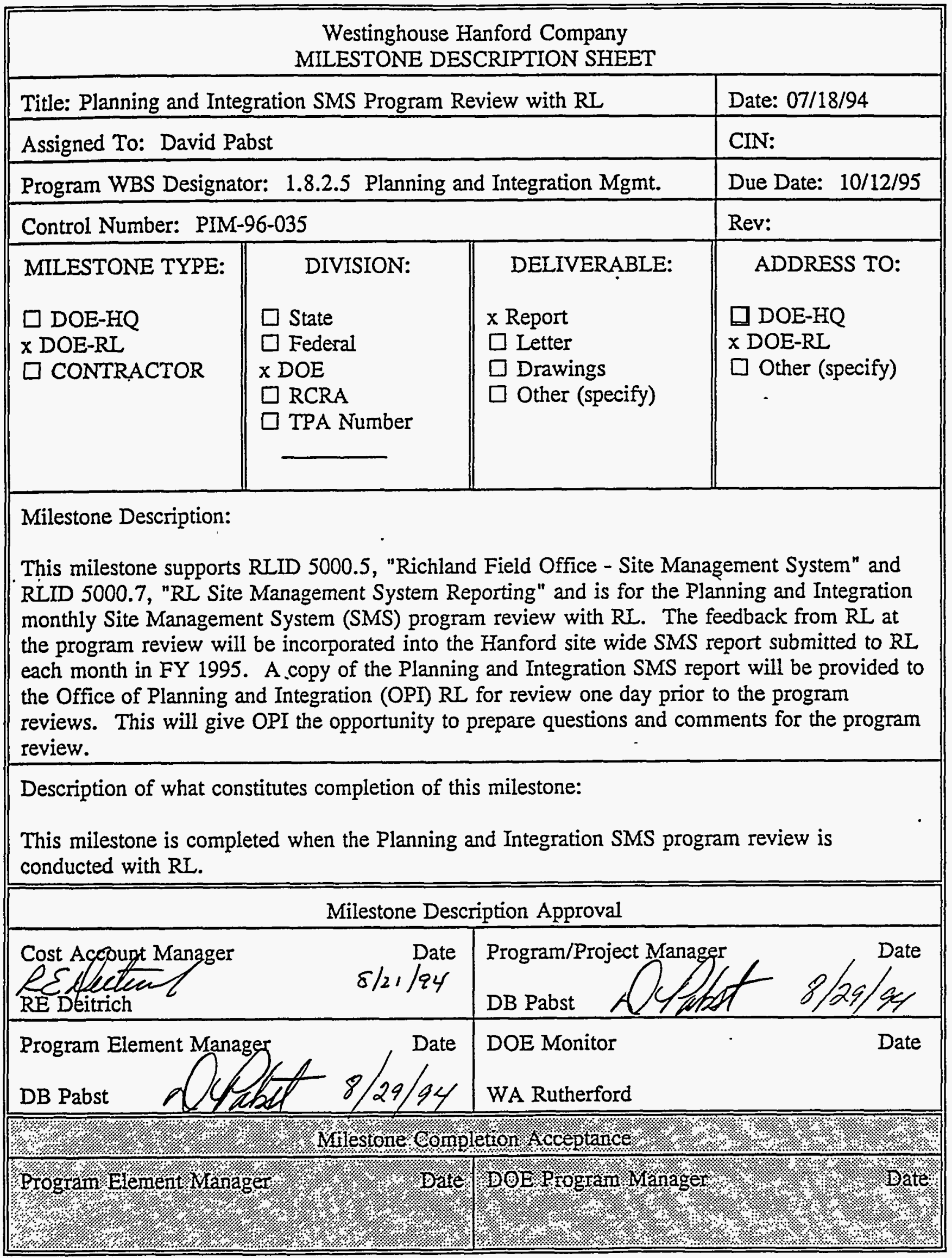




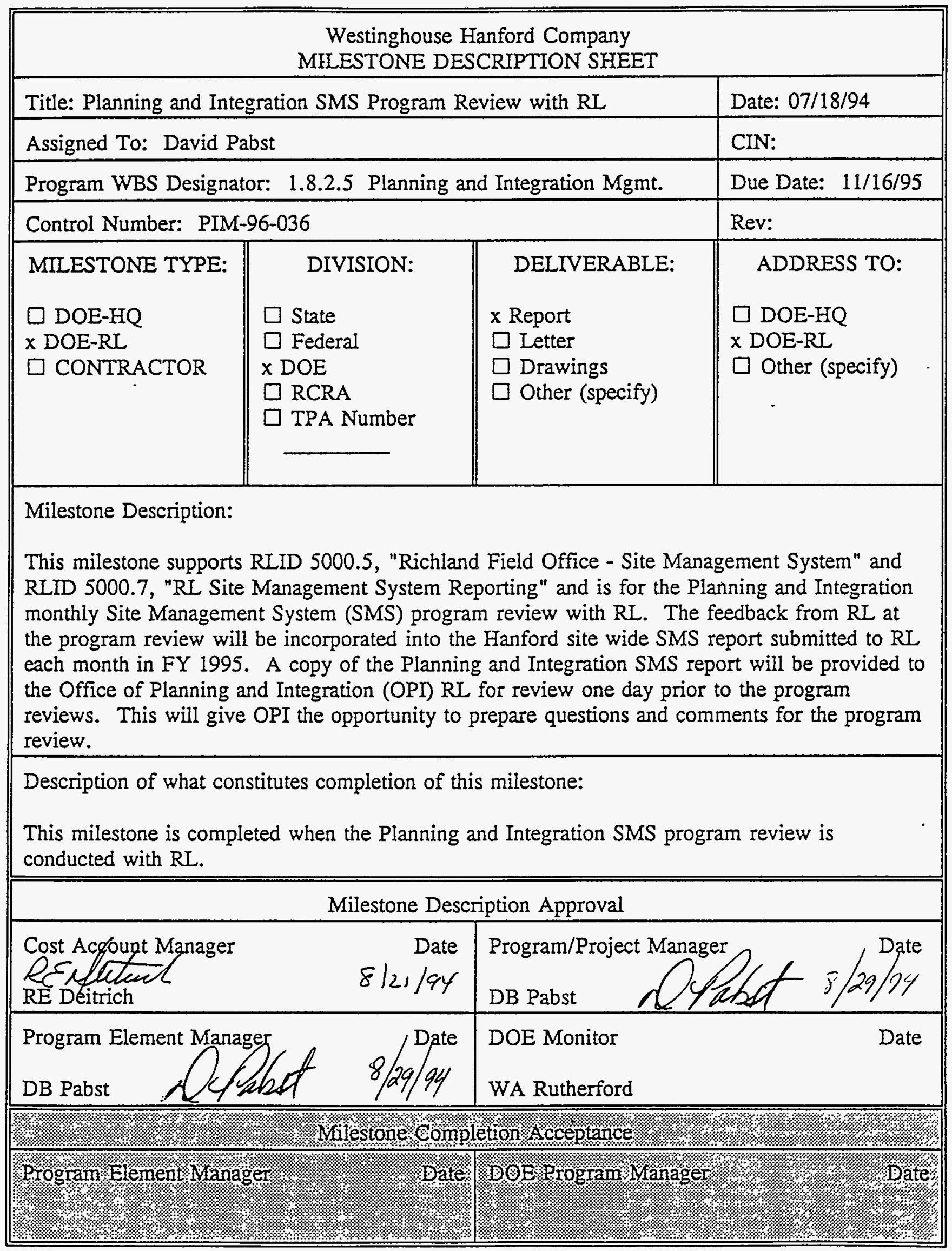




\begin{tabular}{|c|c|c|c|}
\hline \multicolumn{4}{|c|}{$\begin{array}{l}\text { Westinghouse Hanford Company } \\
\text { MILESTONE DESCRIPTION SHEET }\end{array}$} \\
\hline \multicolumn{3}{|c|}{ Title: Planning and Integration SMS Program Review with RL } & Date: $07 / 18 / 94$ \\
\hline \multicolumn{3}{|c|}{ Assigned To: David Pabst } & CIN: \\
\hline \multicolumn{3}{|c|}{ Program WBS Designator: 1 18.2.5 Planning and Integration Mgmt. } & Due Date: $12 / 14 / 95$ \\
\hline \multicolumn{3}{|c|}{ Control Number: PIM-96-037 } & Rev: \\
\hline $\begin{array}{l}\text { MILESTONE TYPE: } \\
\square \text { DOE-HQ } \\
\text { X DOE-RL } \\
\square \text { CONTRACTOR }\end{array}$ & $\begin{array}{l}\text { DIVISION: } \\
\square \text { State } \\
\square \text { Federal } \\
\times \text { DOE } \\
\square \text { RCRA } \\
\square \text { TPA Number }\end{array}$ & $\begin{array}{l}\text { DELIVERABLE: } \\
\times \text { Report } \\
\square \text { Letter } \\
\square \text { Drawings } \\
\square \text { Other (specify) }\end{array}$ & $\begin{array}{l}\text { ADDRESS TO: } \\
\square \text { DOE-HQ } \\
\text { x DOE-RL } \\
\square \text { Other (specify) }\end{array}$ \\
\hline \multicolumn{4}{|c|}{$\begin{array}{l}\text { This milestone supports RIID } 5000.5 \text {, "Richland Field Office - Site Management System" and } \\
\text { RLID } 5000.7 \text {, "RL Site Management System Reporting" and is for the Plañning and Integration } \\
\text { monthly Site Management System (SMS) program review with RL. The feedback from RL at } \\
\text { the program review will be incorporated into the Hanford site wide SMS report submitted to RL } \\
\text { each month in FY } 1995 \text {. A copy of the Planning and Integration SMS report will be provided to } \\
\text { the Office of Planning and Integration (OPI) RL for review one day prior to the program } \\
\text { reviews. This will give OPI the opportunity to prepare questions and comments for the program } \\
\text { review. }\end{array}$} \\
\hline \multicolumn{4}{|c|}{$\begin{array}{l}\text { Description of what constitutes completion of this milestone: } \\
\text { This milestone is completed when the Planning and Integration SMS program review is } \\
\text { conducted with RL. }\end{array}$} \\
\hline \multicolumn{4}{|c|}{ Milestone Description Approval } \\
\hline \multicolumn{2}{|l|}{$\begin{array}{l}\text { Cost Acesunt Magager } \\
\text { RE Afeleed } \\
\text { RE Deitrich }\end{array}$} & \multicolumn{2}{|c|}{ Program/Project Manager } \\
\hline \multicolumn{2}{|c|}{ Program Element Manzger } & \multicolumn{2}{|c|}{$\begin{array}{l}\text { DOE Monitor } \\
\text { WA Rutherford }\end{array}$} \\
\hline \multicolumn{4}{|c|}{ 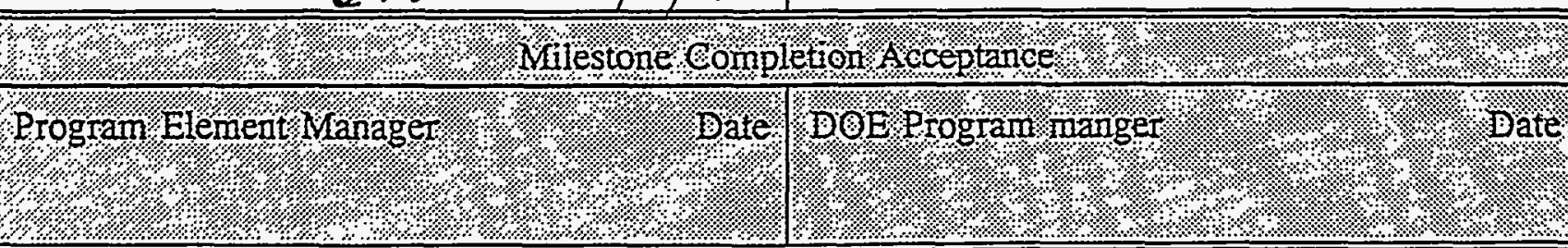 } \\
\hline
\end{tabular}




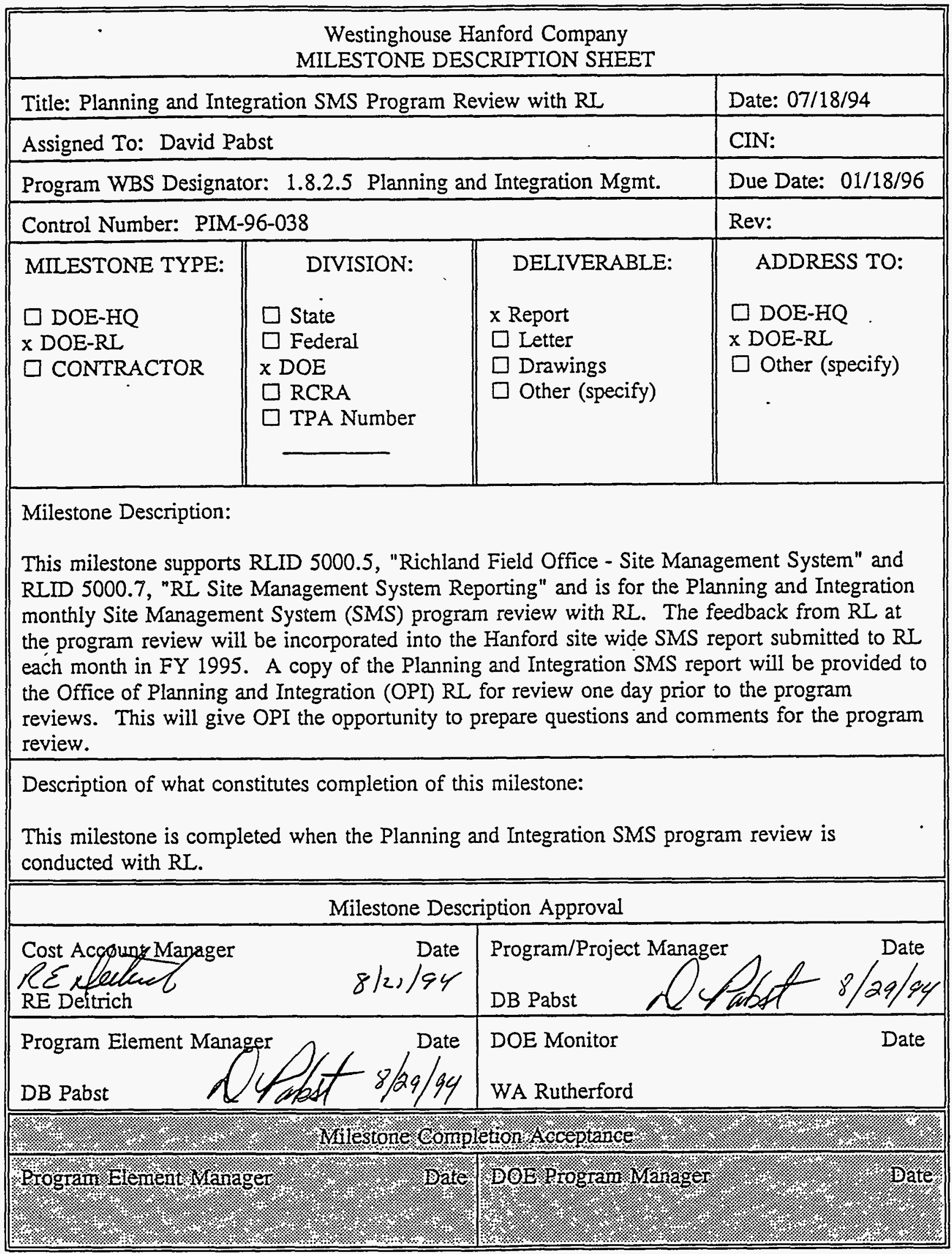




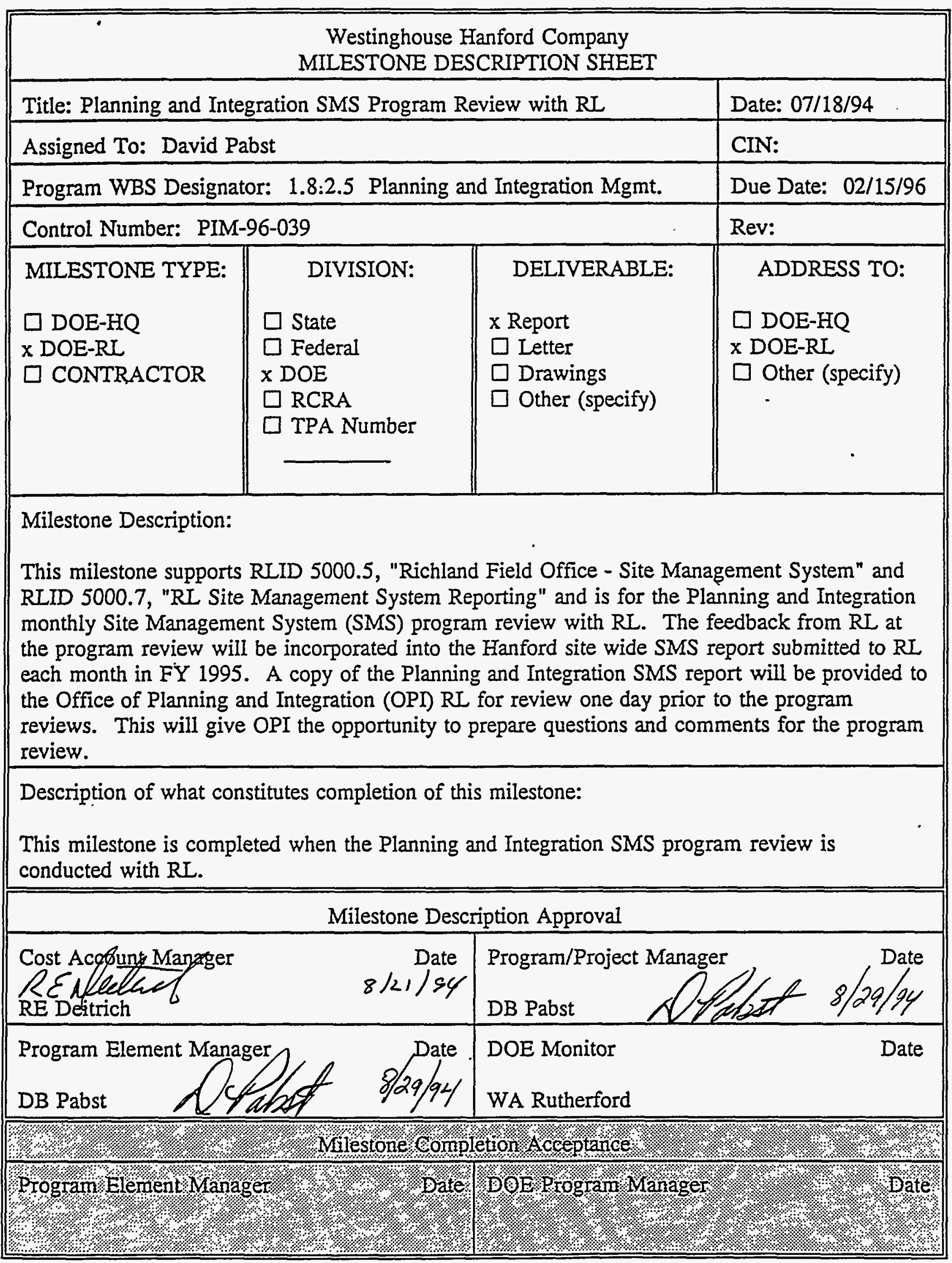




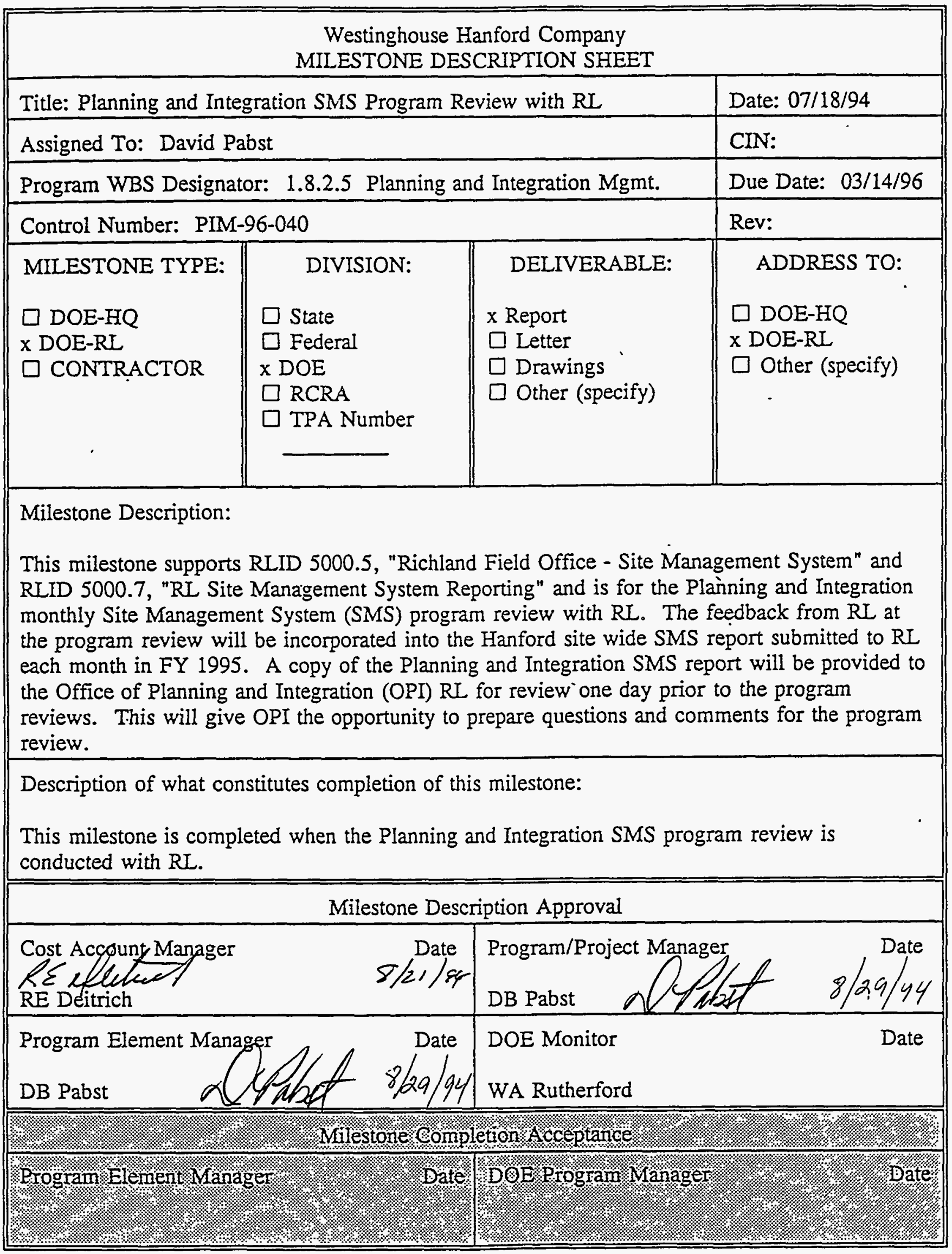




\begin{tabular}{|l||l||}
\hline \multicolumn{3}{|c|}{ Westinghouse Hanford Company } \\
MILESTONE DESCRIPTION SHEET
\end{tabular}




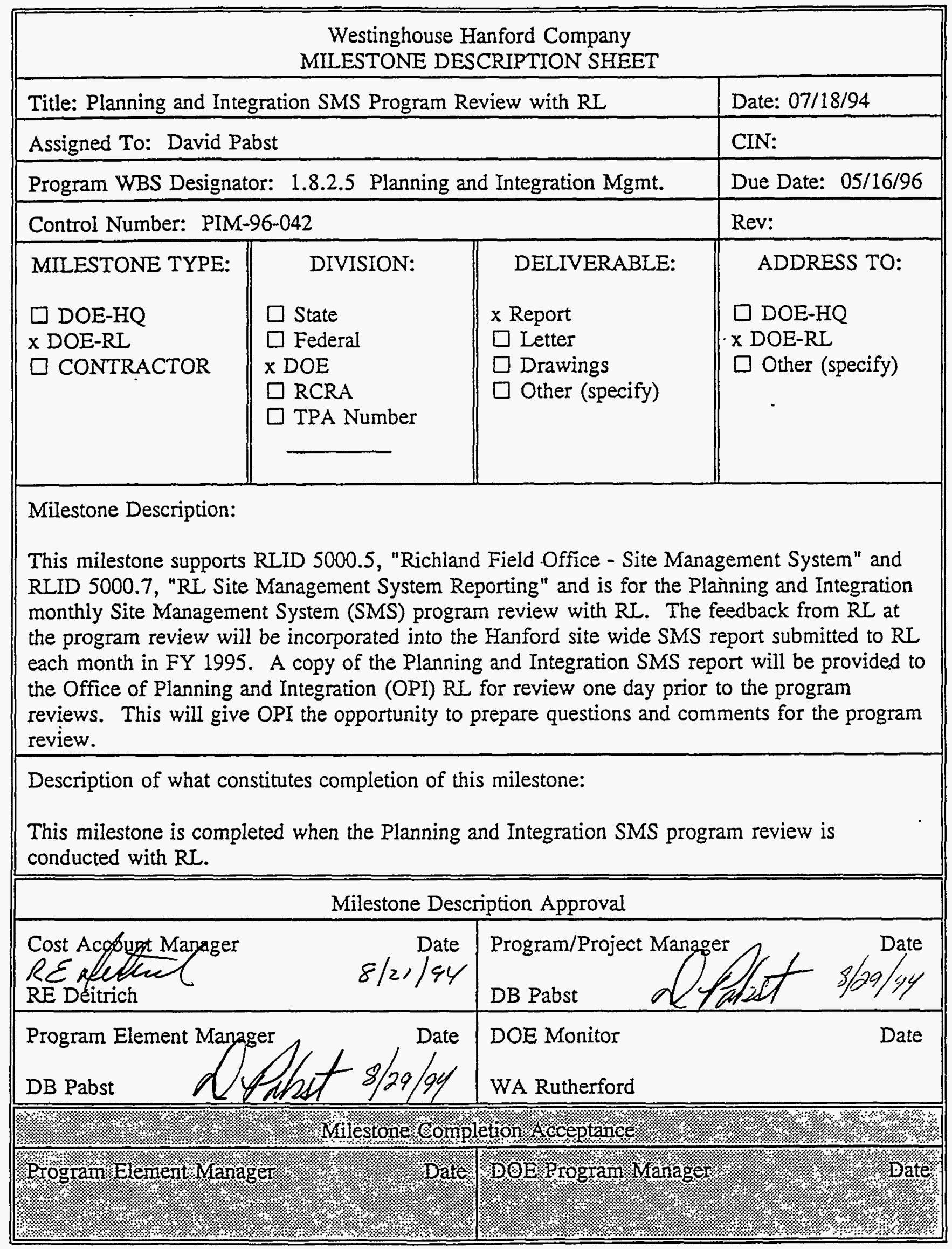




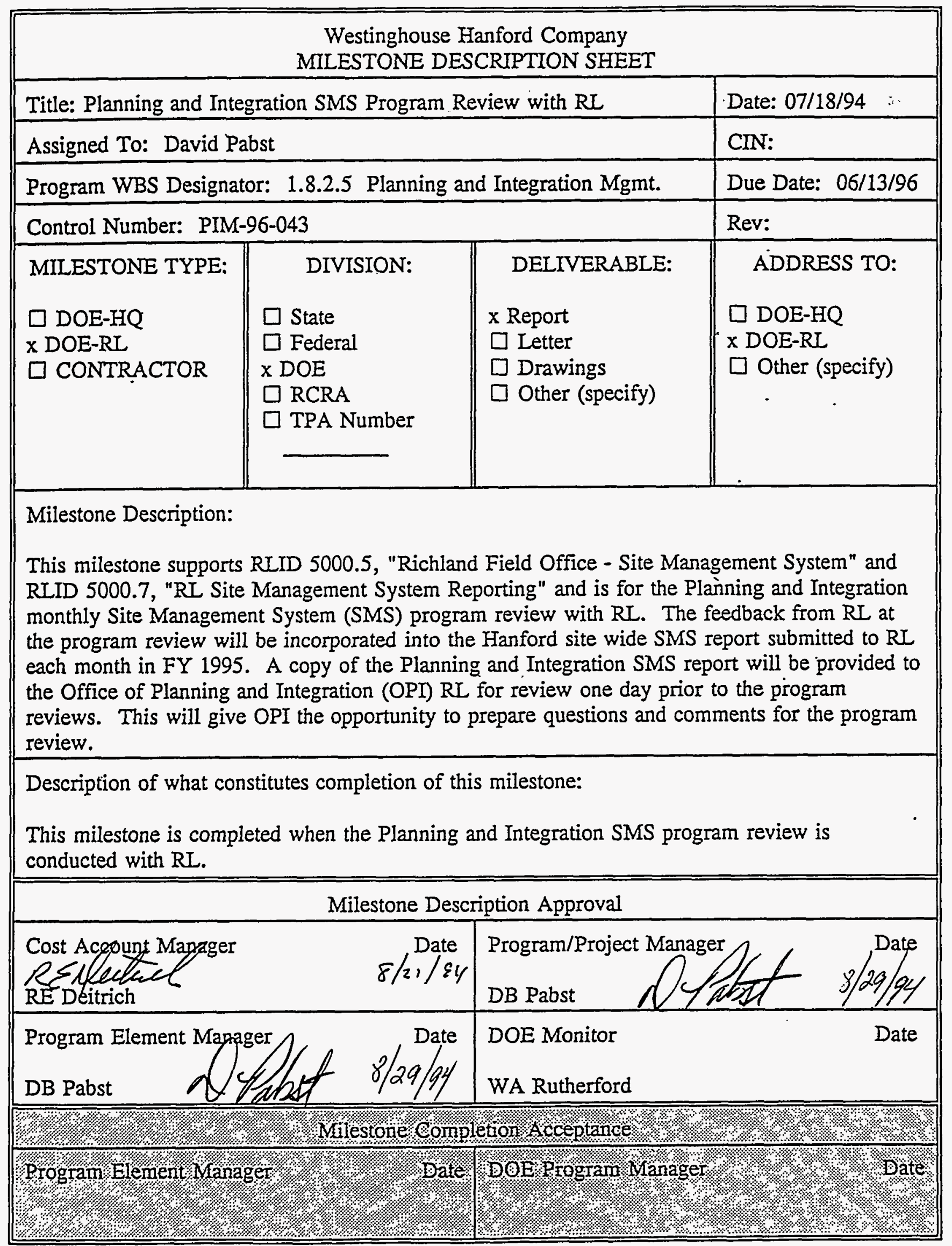




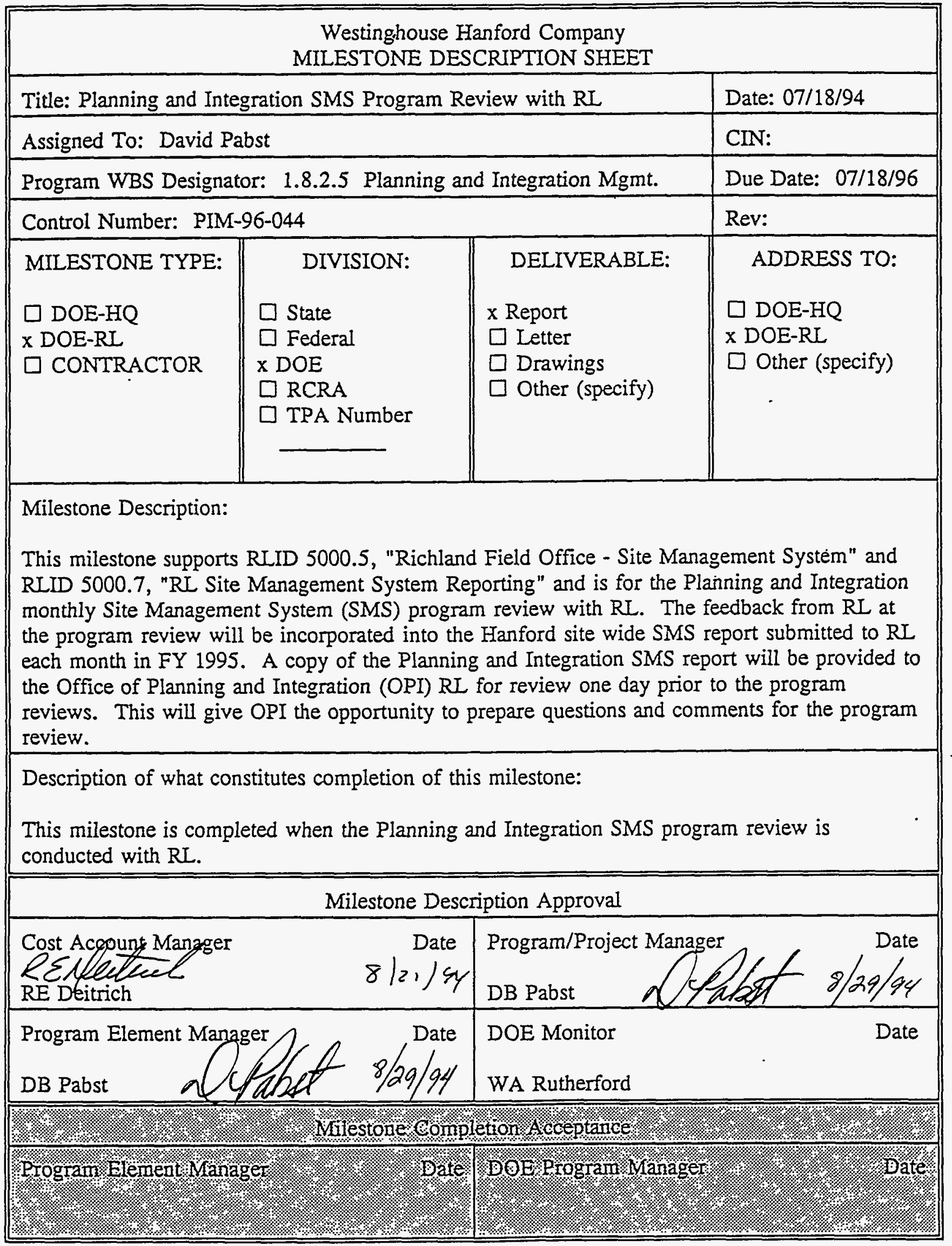




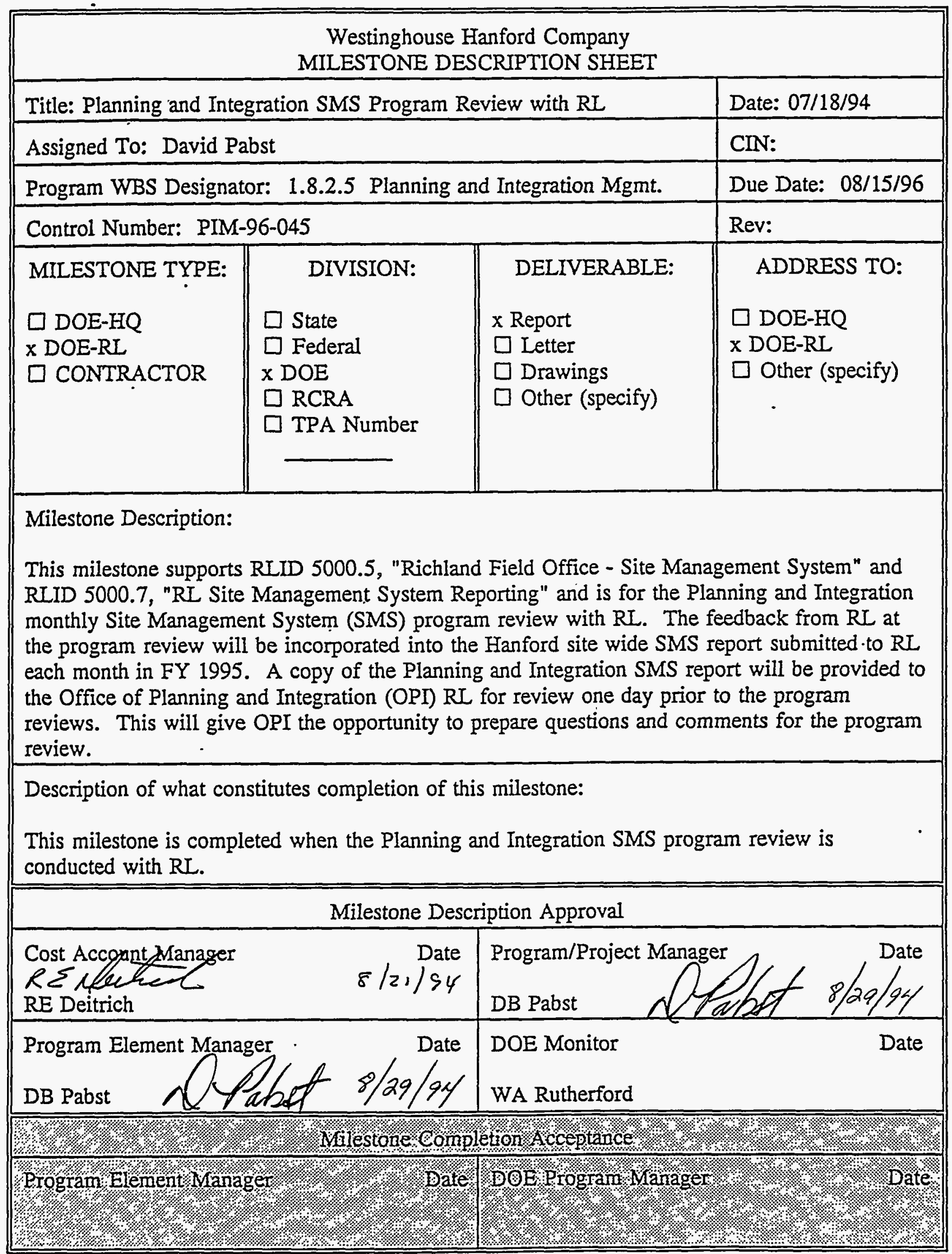




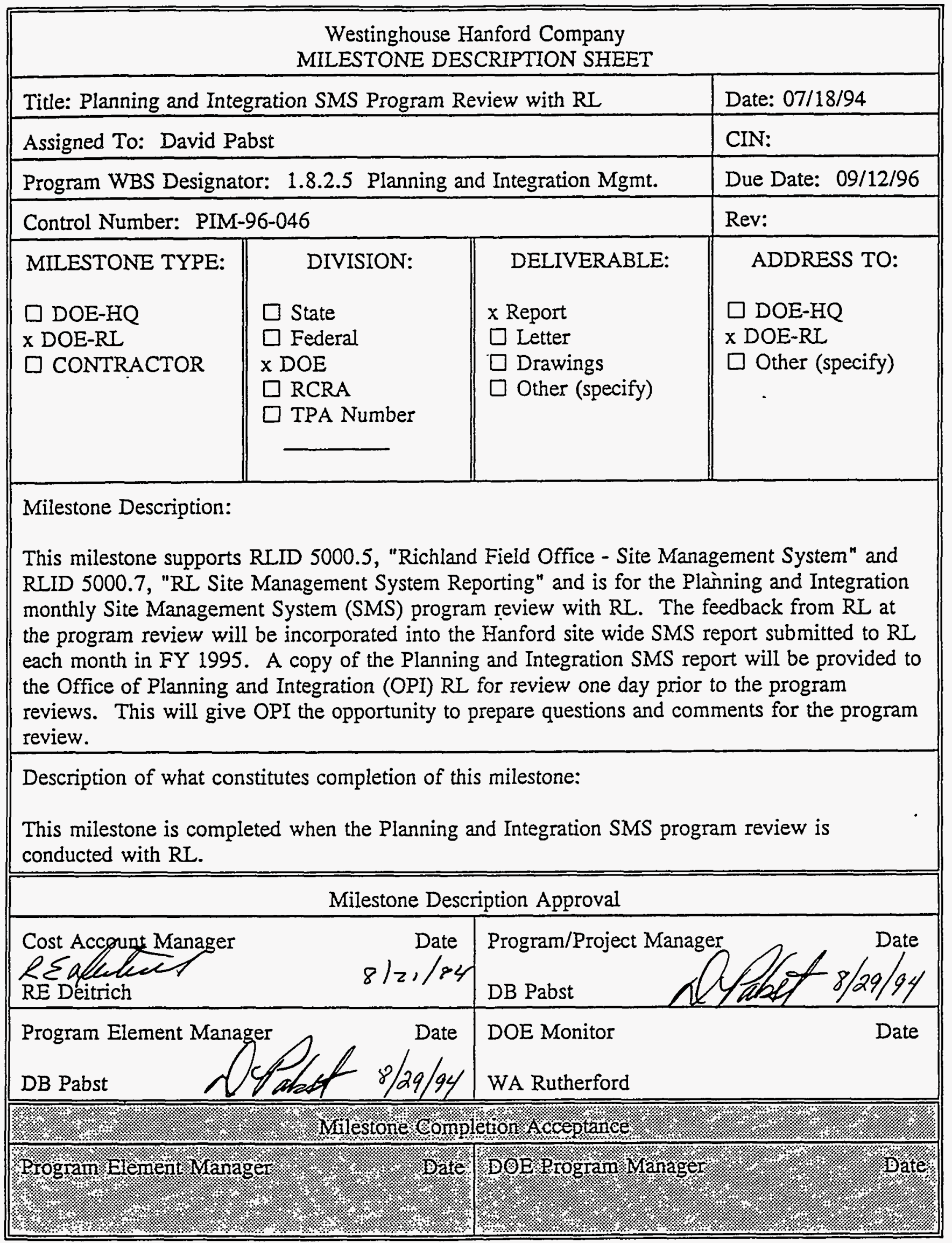




\begin{tabular}{|l||l|l||}
\hline \multicolumn{3}{|c|}{ Westinghouse Hanford Company } \\
MILESTONE DESCRIPTION SHEET
\end{tabular}




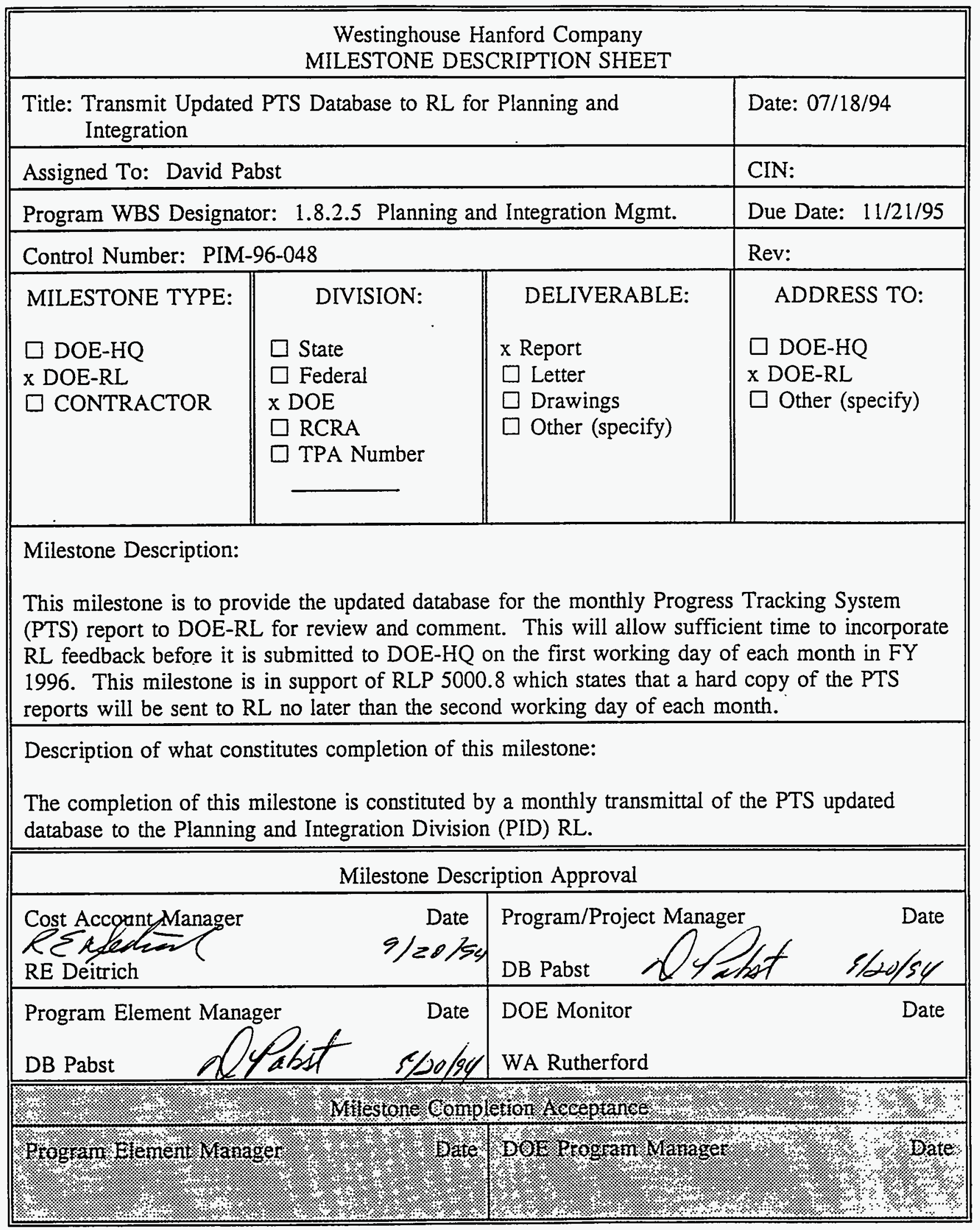




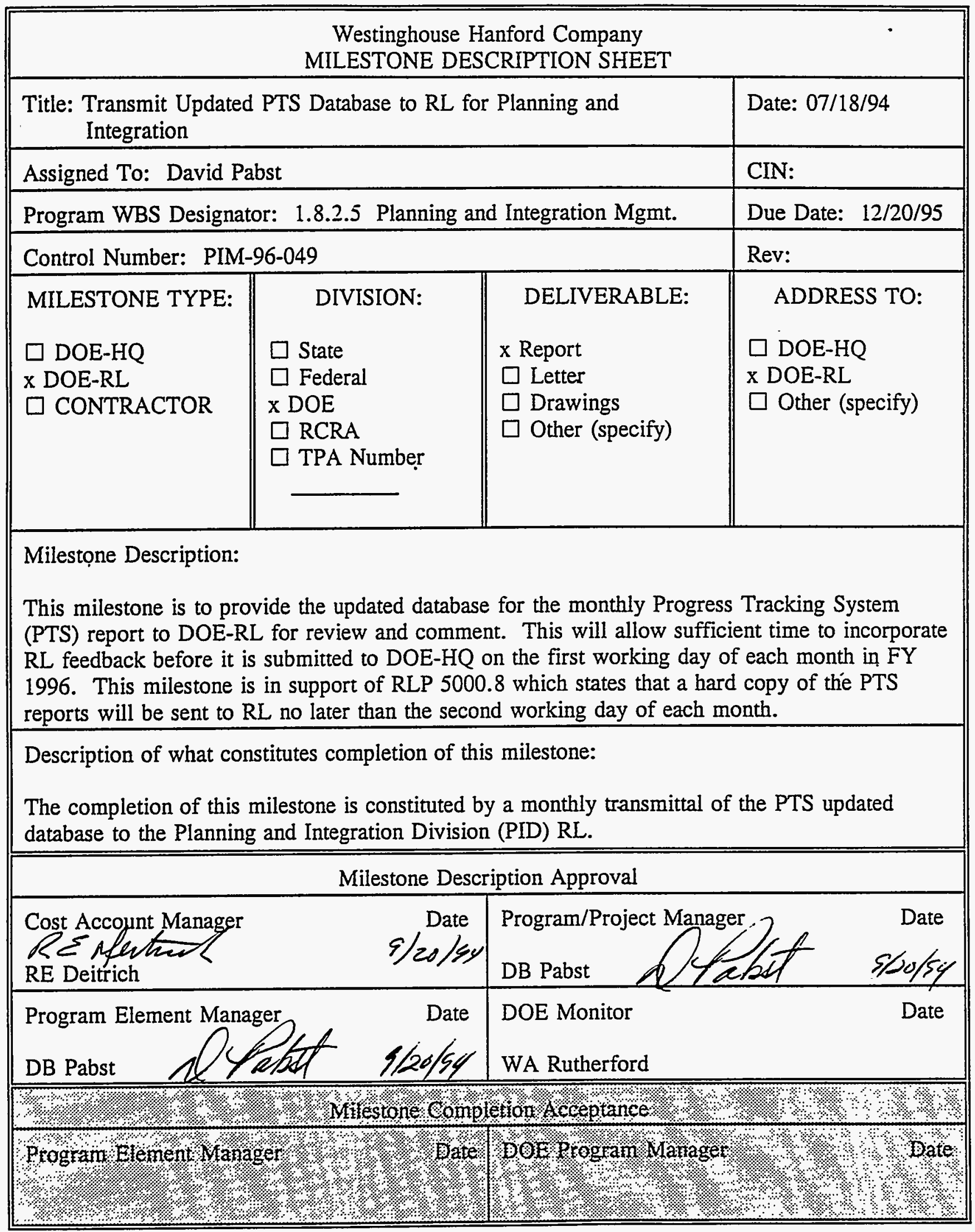




\begin{tabular}{|l|l|l||}
\hline \multicolumn{3}{|c|}{ Westinghouse Hanford Company } \\
MILESTONE DESCRIPTION SHEET
\end{tabular}




\begin{tabular}{|l||l|l||}
\hline \multicolumn{3}{|c|}{ Westinghouse Hanford Company } \\
MILESTONE DESCRIPTION SHEET
\end{tabular}




\begin{tabular}{|c|c|c|c|}
\hline \multicolumn{4}{|c|}{$\begin{array}{l}\text { Westinghouse Hanford Company } \\
\text { MILESTONE DESCRIPTION SHEET }\end{array}$} \\
\hline \multicolumn{3}{|c|}{$\begin{array}{l}\text { Title: Transmit Updated PTS Database to RL for Planning and } \\
\text { Integration }\end{array}$} & Date: $07 / 18 / 94$ \\
\hline \multicolumn{3}{|c|}{ Assigned To: David Pabst } & CIN: \\
\hline \multicolumn{3}{|c|}{ Program WBS Designator: 1.8.2.5 Planning and Integration Mgmt. } & Due Date: $03 / 21 / 96$ \\
\hline \multicolumn{3}{|c|}{ Control Number: PIM-96-052 } & Rev: \\
\hline $\begin{array}{l}\text { MILESTONE TYPE: } \\
\square \text { DOE-HQ } \\
\text { X DOE-RL } \\
\square \text { CONTRACTOR }\end{array}$ & $\begin{array}{l}\quad \text { DIVISION: } \\
\square \text { State } \\
\square \text { Federal } \\
\text { x DOE } \\
\square \text { RCRA } \\
\square \text { TPA Number }\end{array}$ & $\begin{array}{l}\text { DELIVERABLE: } \\
\times \text { Report } \\
\square \text { Letter } \\
\square \text { Drawings } \\
\square \text { Other (specify) }\end{array}$ & $\begin{array}{l}\text { ADDRESS TO: } \\
\square \text { DOE-HQ } \\
\text { x DOE-RL } \\
\square \text { Other (specify) }\end{array}$ \\
\hline \multicolumn{4}{|c|}{$\begin{array}{l}\text { This milestone is to provide the updated database for the monthly Progress Tracking System } \\
\text { (PTS) report to DOE-RL for review and comment. This will allow sufficient time to incorporate } \\
\text { RL feedback before it is submitted to DOE-HQ on the first working day of each month in FY } \\
\text { 1996. This milestone is in support of RLP } 5000.8 \text { which states that a hard copy of the PTS } \\
\text { reports will be sent to RL no later than the second working day of each month. }\end{array}$} \\
\hline \multicolumn{4}{|c|}{$\begin{array}{l}\text { Description of what constitutes completion of this milestone: } \\
\text { The completion of this milestone is constituted by a monthly transmittal of the PTS updated } \\
\text { database to the Planning and Integration Division (PID) RL. }\end{array}$} \\
\hline \multicolumn{4}{|c|}{ Milestone Description Approval } \\
\hline \multicolumn{2}{|c|}{$\begin{array}{l}\text { Cost Accbunt Manager } \\
\text { RE Deitrich } \\
\text { R/20/44 }\end{array}$} & \multicolumn{2}{|c|}{ Program/Project Manager Pabst Bate } \\
\hline \multicolumn{2}{|c|}{ 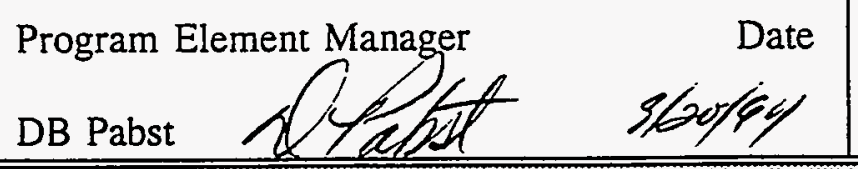 } & \multicolumn{2}{|l|}{$\begin{array}{l}\text { DOE Monitor } \\
\text { WA Rutherford }\end{array}$} \\
\hline \multicolumn{4}{|c|}{ 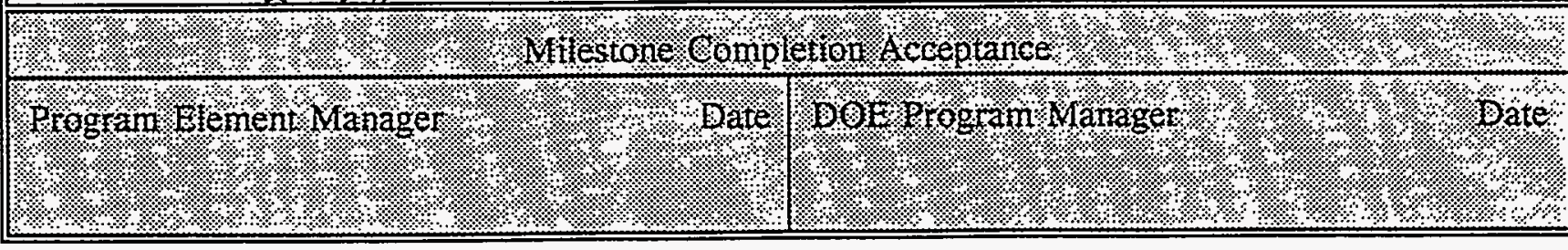 } \\
\hline
\end{tabular}




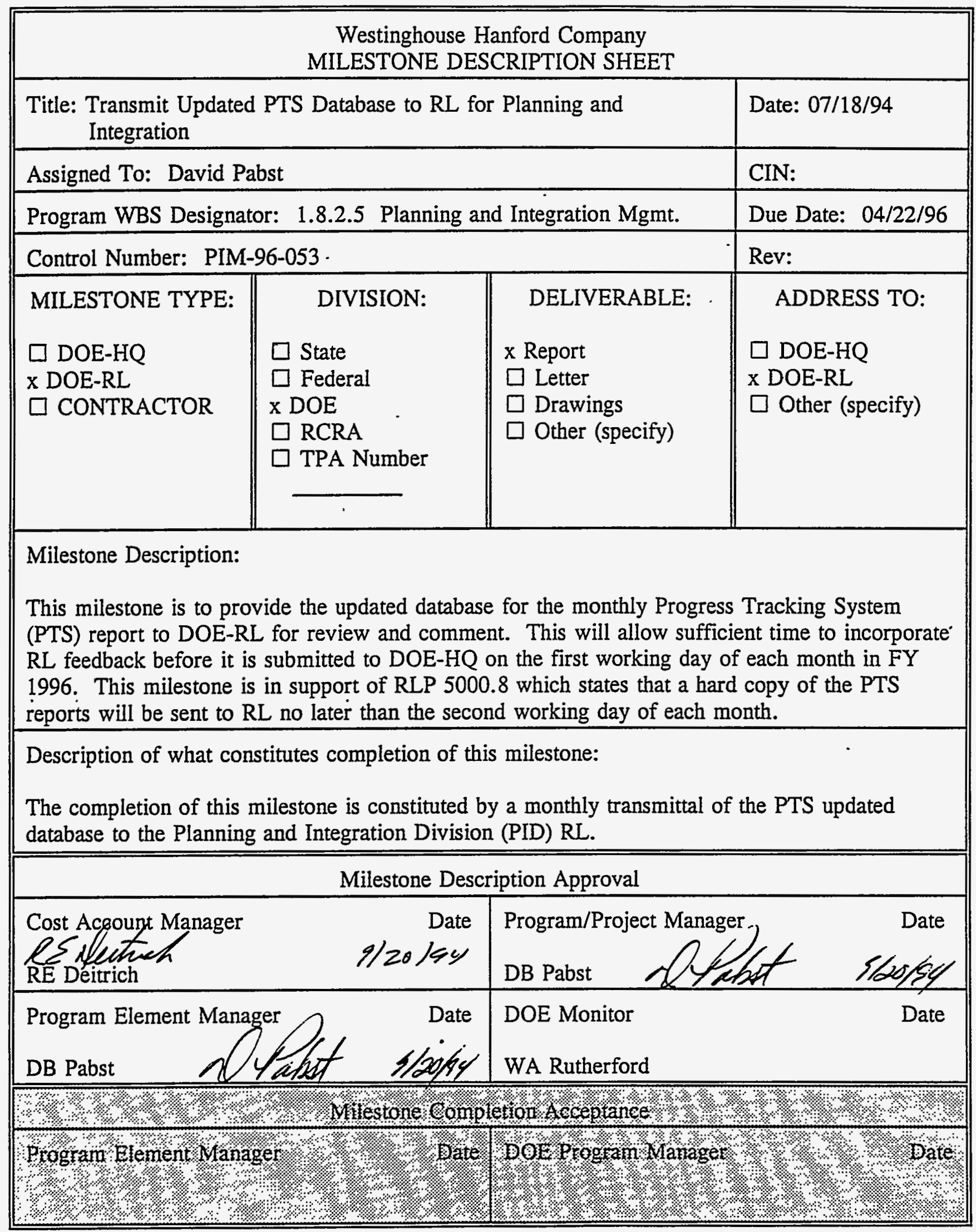




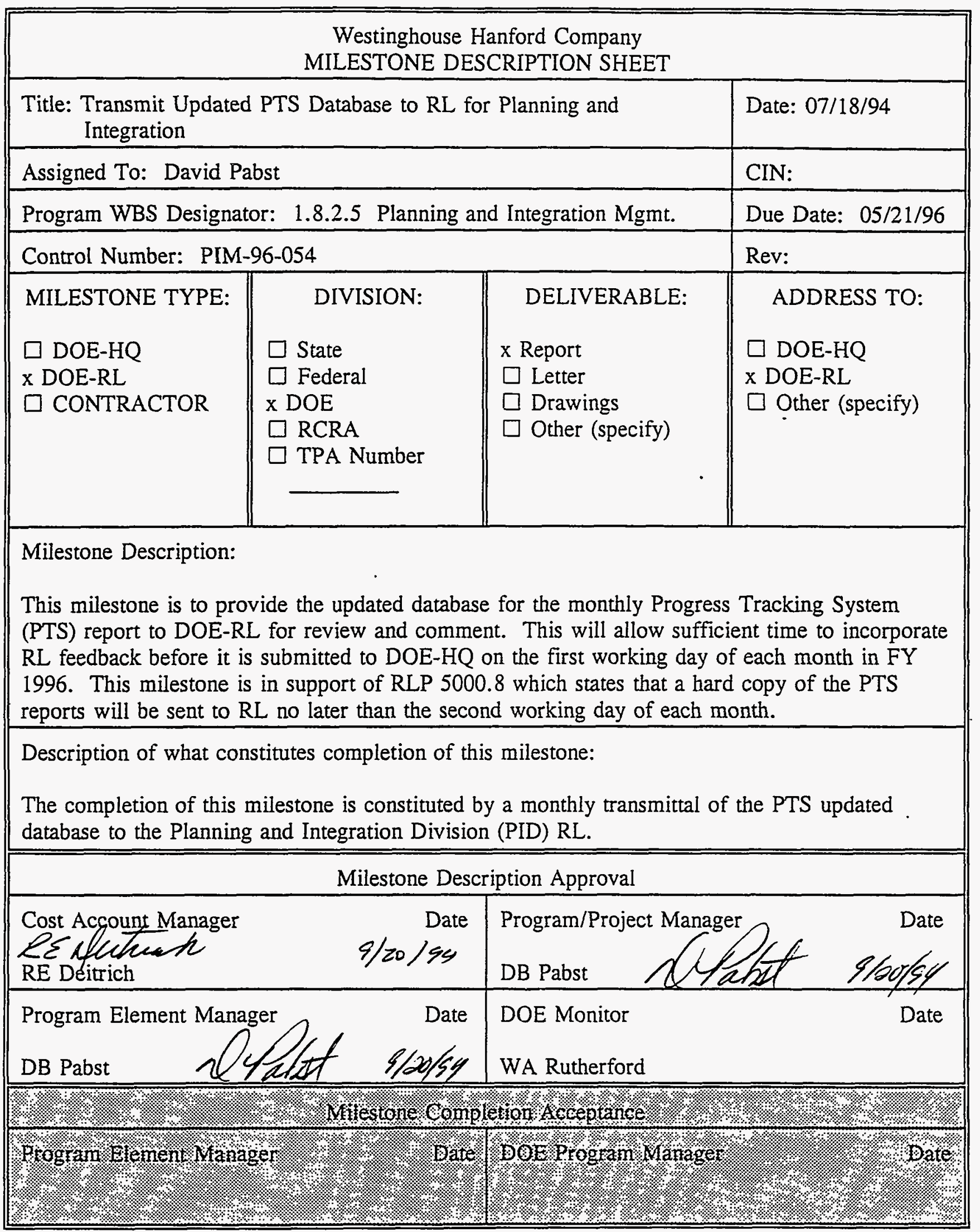




\begin{tabular}{|l|l|l||}
\hline \multicolumn{3}{|c|}{ Westinghouse Hanford Company } \\
MILESTONE DESCRIPTION SHEET
\end{tabular}




\begin{tabular}{|l||l||}
\hline \multicolumn{3}{|c|}{ Westinghouse Hanford Company } \\
MILESTONE DESCRIPTION SHEET
\end{tabular}




\begin{tabular}{|l||l||}
\hline \multicolumn{3}{|c|}{ Westinghouse Hanford Company } \\
MILESTONE DESCRIPTION SHEET
\end{tabular}




\begin{tabular}{|l|l|l||}
\hline \multicolumn{3}{|c|}{ Westinghouse Hanford Company } \\
MILESTONE DESCRIPTION SHEET
\end{tabular}




\begin{tabular}{|c|c|c|c|}
\hline \multicolumn{4}{|c|}{$\begin{array}{l}\text { Westinghouse Hanford Company } \\
\text { MILESTONE DESCRIPTION SHEET }\end{array}$} \\
\hline \multicolumn{3}{|c|}{ Title: ADS 7250 Preparation Kick-Off Meeting } & Date: $6-1-94$ \\
\hline \multicolumn{3}{|c|}{ Assigned To: D. B. Pabst } & CIN: \\
\hline \multicolumn{3}{|c|}{ Program WBS Designator: 1 .8.2.5.1 } & Due Date: $11-14-96$ \\
\hline \multicolumn{3}{|c|}{ Control Number: PIM-97-001 } & Rev: 0 \\
\hline $\begin{array}{l}\text { MILESTONE TYPE: } \\
\square \text { DOE-HQ } \\
\square \text { DOE-RL } \\
\text { X CONTRACTOR }\end{array}$ & $\begin{array}{l}\quad \text { DIVISION: } \\
\quad \square \text { State } \\
\square \text { Federal } \\
\text { X DOE } \\
\square \text { RCRA } \\
\square \text { TPA Number }\end{array}$ & $\begin{array}{l}\quad \text { DELIVERABLE: } \\
\square \text { Report } \\
\text { X Letter } \\
\square \text { Drawings } \\
\square \text { Other (specify) } \\
\text { Instructions for ADS } \\
7250 \text { preparation }\end{array}$ & $\begin{array}{l}\quad \text { ADDRESS TO: } \\
\square \text { DOE-HQ } \\
\square \text { DOE-RL } \\
\text { X Other (specify) } \\
\text { All 1.8.2 Planning } \\
\text { Integration } \\
\text { organizations }\end{array}$ \\
\hline \multicolumn{4}{|c|}{$\begin{array}{l}\text { Milestone Description: } \\
\text { On receipt of the budget data from program element 1.8.2.8 ADS preparation and coordination, } \\
\text { a call letter will be prepared. } \\
\text { This deliverable will contain instructions on preparation, detail required, RL site guidance and } \\
\text { preparation schedule. This data will be generated in the form of a call letter and distributed to } \\
\text { all 1.8.2 Planning Integration program elements and RL PID and PUB prior to the kick-off } \\
\text { meeting. }\end{array}$} \\
\hline \multicolumn{4}{|c|}{$\begin{array}{l}\text { Description of what constitutes completion of this milestone: } \\
\text { This milestone will be complete when the above data is distributed, RL comments dispositioned, } \\
\text { and the } 1.8 .2 \text { Planning Integration kick-off meeting held to clarify any questions by the } \\
\text { participants. }\end{array}$} \\
\hline \multicolumn{4}{|c|}{ Milestone Description Approval } \\
\hline \multicolumn{2}{|l|}{$\begin{array}{l}\text { Cost Acebunt Manager } \\
\text { Re Heeleal } \\
\text { R. E. Deitrich }\end{array}$} & \multicolumn{2}{|c|}{ Program/Project Mangeger Babst Plesfef } \\
\hline \multicolumn{2}{|c|}{ Program Element Manager } & \multicolumn{2}{|c|}{$\begin{array}{l}\text { DOE Monitor } \\
\text { N/A } \\
\end{array}$} \\
\hline \multicolumn{4}{|c|}{ 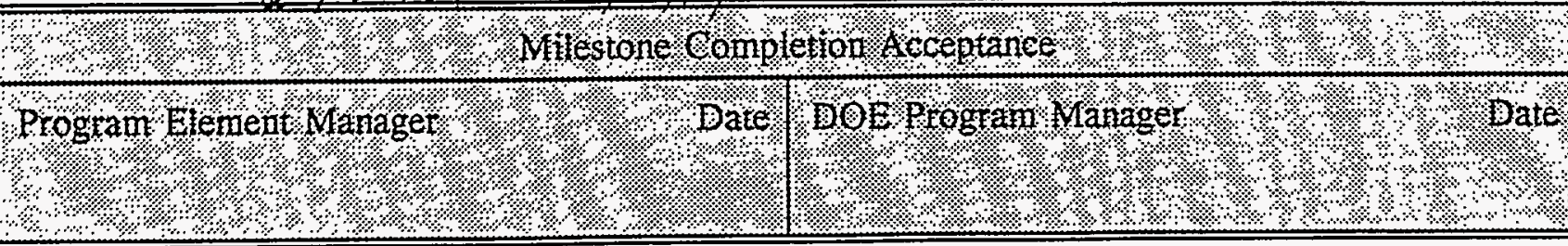 } \\
\hline
\end{tabular}




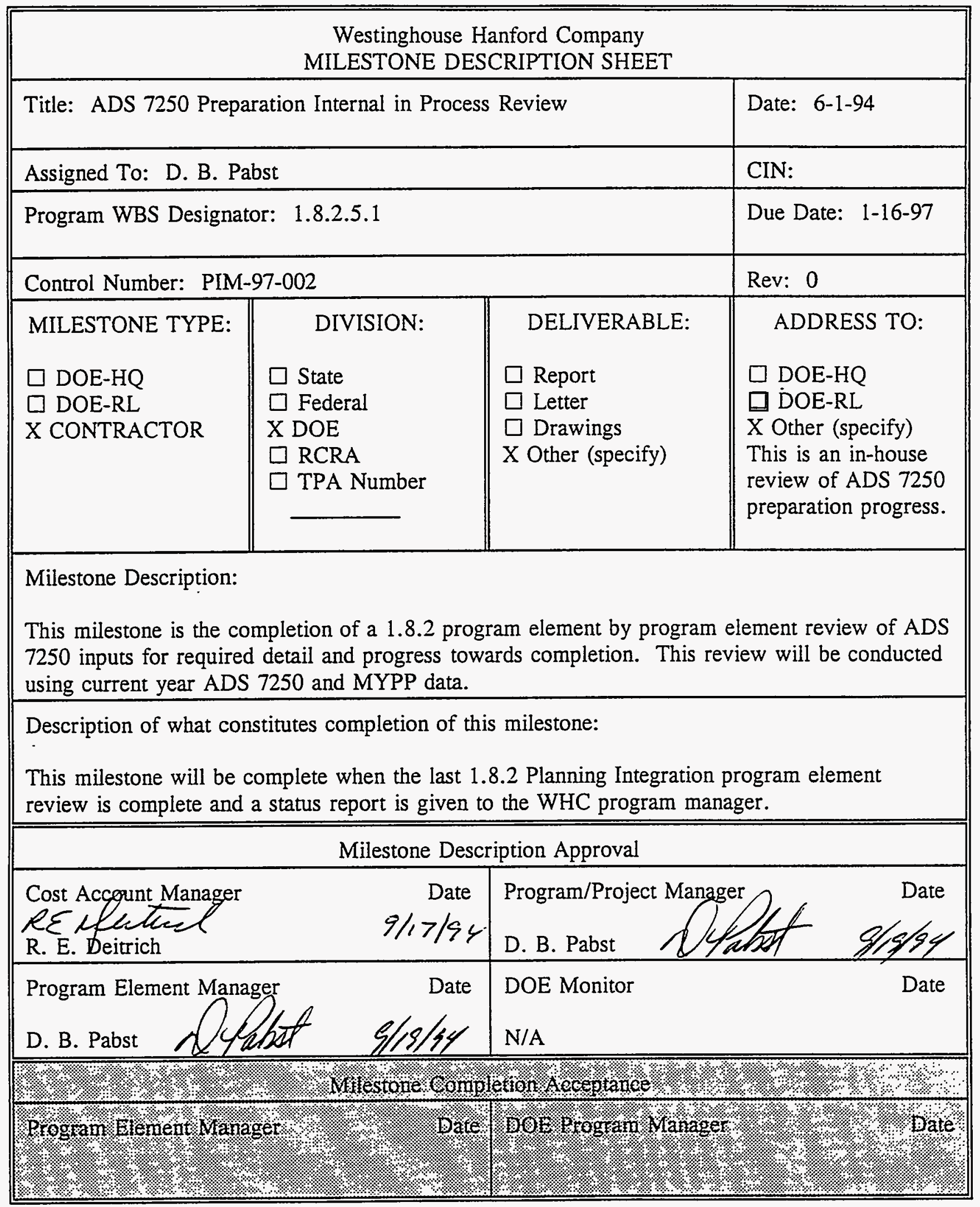




\begin{tabular}{|l||l||}
\hline \multicolumn{3}{|c|}{ Westinghouse Hanford Company } \\
MILESTONE DESCRIPTION SHEET
\end{tabular}




\begin{tabular}{|l||l|||}
\hline \multicolumn{3}{|c||}{ Westinghouse Hanford Company } \\
MILESTONE DESCRIPTION SHEET
\end{tabular}




\begin{tabular}{|c|c|c|c|}
\hline \multicolumn{4}{|c|}{$\begin{array}{l}\text { Westinghouse Hanford Company } \\
\text { MILESTONE DESCRIPTION SHEET }\end{array}$} \\
\hline \multicolumn{3}{|c|}{ Title: Complete Development of ADS 7250} & Date: $6-2-94$ \\
\hline \multicolumn{3}{|c|}{ Assigned To: D. B. Pabst } & CIN: \\
\hline \multicolumn{3}{|c|}{ Program WBS Designator: 1 8.2.5.1 } & Due Date: $4-7-97$ \\
\hline \multicolumn{3}{|c|}{ Control Number: PIM-97-005 } & Rev: \\
\hline $\begin{array}{l}\text { MILESTONE TYPE: } \\
\square \text { DOE-HQ } \\
\square \text { DOE-RL } \\
\square \text { CONTRACTOR } \\
\text { X WHC Key }\end{array}$ & $\begin{array}{l}\quad \text { DIVISION: } \\
\quad \square \text { State } \\
\square \text { Federal } \\
\text { X DOE } \\
\square \text { RCRA } \\
\square \text { TPA Number }\end{array}$ & $\begin{array}{l}\text { DELIVERABLE: } \\
\text { X Report } \\
\square \text { Letter } \\
\square \text { Drawings } \\
\square \text { Other (specify) }\end{array}$ & $\begin{array}{l}\quad \text { ADDRESS TO: } \\
\square \text { DOE-HQ } \\
\square \text { DOE-RL } \\
\square \text { D Other (specify) } \\
\text { Submit to Program } \\
\text { Baseline Integration. }\end{array}$ \\
\hline \multicolumn{4}{|c|}{$\begin{array}{l}\text { Milestone Description: } \\
\text { Development of the ADS includes technical workscope definition of budget year, planning year, } \\
\text { and outyears; milestones and milestone description; key assumptions and issues; and regulatory } \\
\text { drivers. Also included is cost estimates for scope at the target level and prioritization, based on } \\
\text { RL generated priority list of direct funded scope within Planning Integration. Development will } \\
\text { support requirements to support the FY } 1999 \text { ADS preparation using EPDS and the Resource } \\
\text { Pricing Module (RPM). The ADS will be reviewed and approved by RL PID and PUB prior to } \\
\text { completion. }\end{array}$} \\
\hline \multicolumn{4}{|c|}{$\begin{array}{l}\text { Description of what constitutes completion of this milestone: } \\
\text { Development of the Planning Integration Program ADS with completed TDD's and building } \\
\text { blocks consistent with approved MYPP. }\end{array}$} \\
\hline \multicolumn{4}{|c|}{ Milestone Description Approval } \\
\hline \multicolumn{2}{|l|}{$\begin{array}{l}\text { Cost Acgount Manager } \\
\text { RSE fectech } \\
\text { R. E. Deitrich }\end{array}$} & \multicolumn{2}{|c|}{ Program/Project Managen Pabst 14 Date } \\
\hline \multicolumn{2}{|c|}{ Drogram Element Manager } & $\begin{array}{l}\text { DOE Monitor } \\
\text { N/A }\end{array}$ & Date \\
\hline \multicolumn{4}{|c|}{ Milestone Completion Acceptance } \\
\hline Progtam Elenent Mran & (1) & 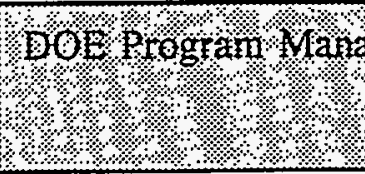 & 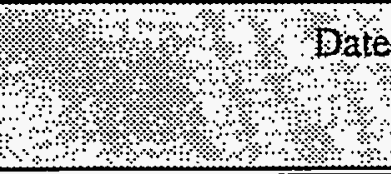 \\
\hline
\end{tabular}




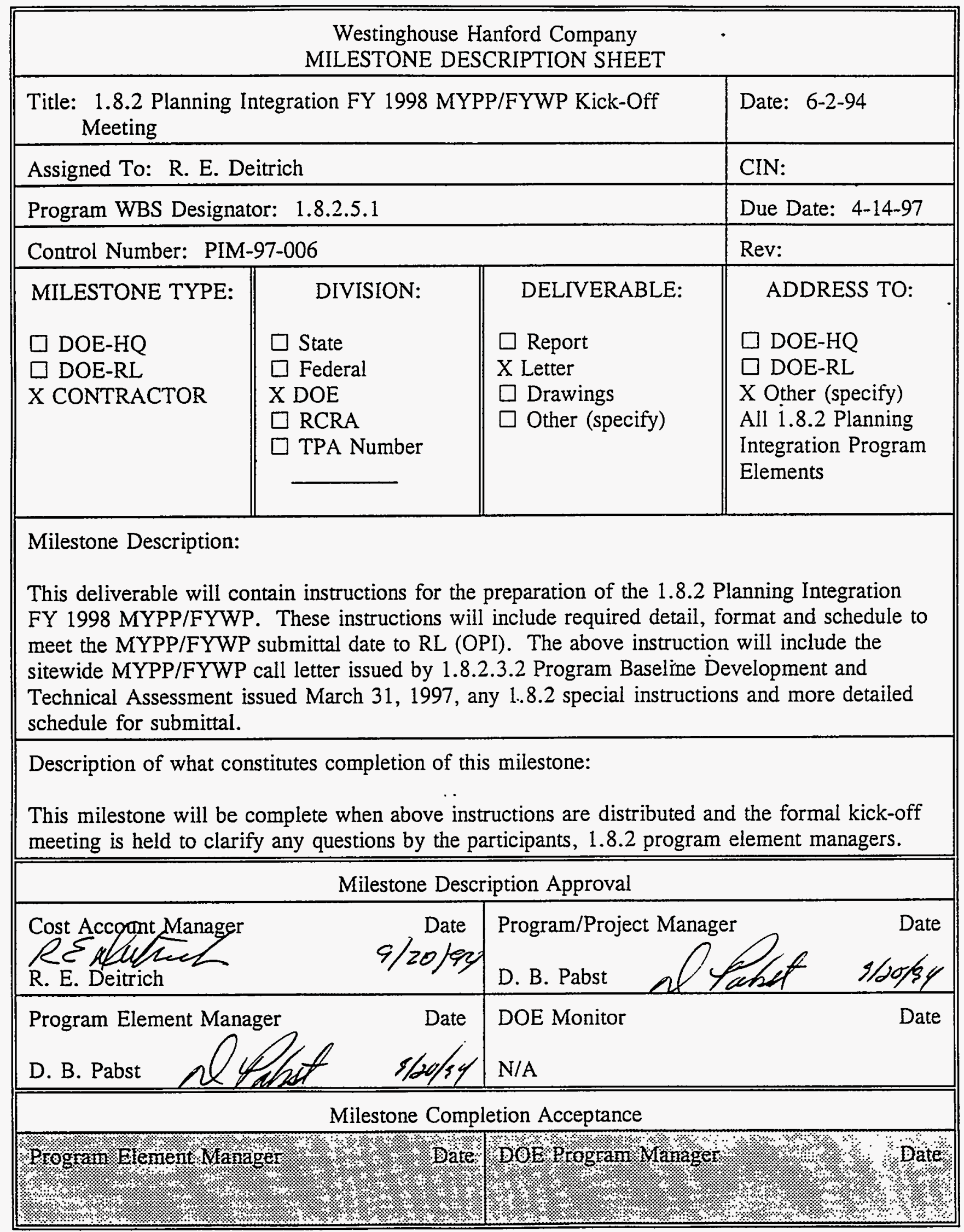




\begin{tabular}{|c|c|c|c|}
\hline \multicolumn{4}{|c|}{$\begin{array}{l}\text { Westinghouse Hanford Company } \\
\text { MILESTONE DESCRIPTION SHEET }\end{array}$} \\
\hline \multicolumn{3}{|c|}{ Title: 1.8.2 Planning Integration MYPP/FYWP in Process Reviews } & Date: $6-2-94$ \\
\hline \multicolumn{3}{|c|}{ Assigned To: D. B. Pabst } & CIN: \\
\hline \multicolumn{3}{|c|}{ Program WBS Designator: 1.8 .2 .5 .1} & Due Date: $6-19-97$ \\
\hline \multicolumn{3}{|c|}{ Control Number: PIM-97-007 } & Rev: \\
\hline $\begin{array}{l}\text { MILESTONE TYPE: } \\
\square \text { DOE-HQ } \\
\square \text { DOE-RL } \\
\text { X CONTRACTOR }\end{array}$ & $\begin{array}{l}\quad \text { DIVISION: } \\
\square \text { State } \\
\square \text { Federal } \\
\text { X DOE } \\
\square \text { RCRA } \\
\square \text { TPA Number }\end{array}$ & $\begin{array}{l}\text { DELIVERABLE: } \\
\square \text { Report } \\
\square \text { Letter } \\
\square \text { Drawings } \\
\text { X Other (specify) } \\
\text { Review of } \\
\text { MYPP/FYWP } \\
\text { Progress }\end{array}$ & $\begin{array}{l}\quad \text { ADDRESS TO: } \\
\square \text { DOE-HQ } \\
\square \text { DOE-RL } \\
\text { X Other (specify) } \\
\text { Internal Review of } \\
\text { MYPP/FYWP } \\
\text { Progress. }\end{array}$ \\
\hline \multicolumn{4}{|c|}{$\begin{array}{l}\text { Milestone Description: } \\
\text { This milestone is a program element by program element review of } 1.8 .2 \text { Planning Integration } \\
\text { MYPP/FYWP inputs for required detail and progress towards RL submittal date. This review } \\
\text { will be performed by WHC and contractor personnel not involved with the generation of this } \\
\text { program plan. }\end{array}$} \\
\hline \multicolumn{4}{|c|}{$\begin{array}{l}\text { Description of what constitutes completion of this milestone: } \\
\text { This milestone will be complete when all the } 1.8 .2 \text { program elements have been reviewed. }\end{array}$} \\
\hline \multicolumn{4}{|c|}{ Milestone Description Approval } \\
\hline \multicolumn{2}{|c|}{ 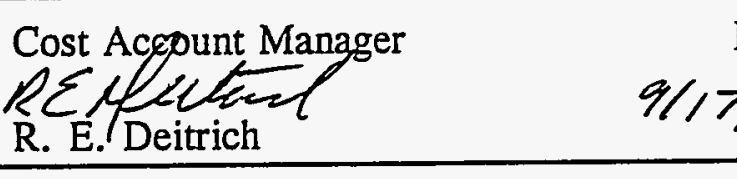 } & Program/Project Manager & Ohyt $\quad$ Date \\
\hline \multicolumn{2}{|c|}{ Program Element Manager } & \multicolumn{2}{|c|}{$\begin{array}{l}\text { DOE Monitor Date } \\
\text { N/A }\end{array}$} \\
\hline \multicolumn{4}{|c|}{ 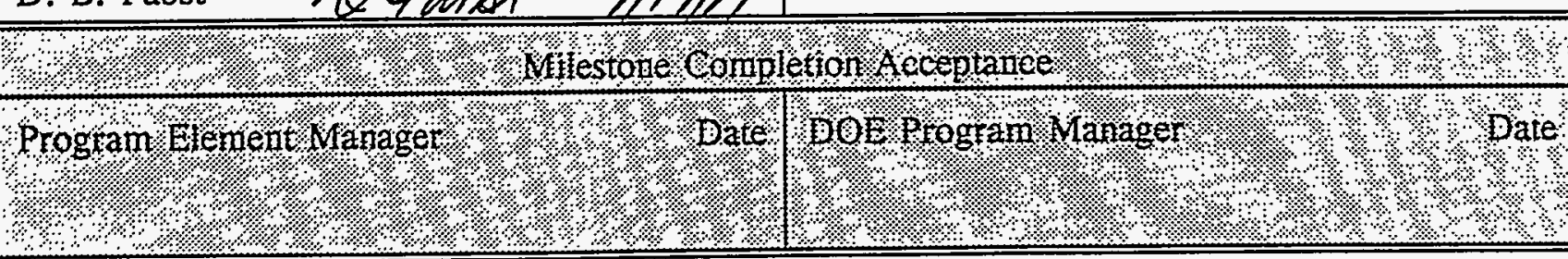 } \\
\hline
\end{tabular}




\begin{tabular}{|c|c|c|c|}
\hline \multicolumn{4}{|c|}{$\begin{array}{l}\text { Westinghouse Hanford Company } \\
\text { MILESTONE DESCRIPTION SHEET }\end{array}$} \\
\hline \multicolumn{3}{|c|}{$\begin{array}{l}\text { Title: } 1.8 .2 \text { Planning Integration Internal MYPP/FYWP Program } \\
\text { Manager Review }\end{array}$} & Date: $6-2-94$ \\
\hline \multicolumn{3}{|c|}{ Assigned To: R. E. Deitrich } & CIN: \\
\hline \multicolumn{3}{|c|}{ Program WBS Designator: 1 8.2.5.1 } & Due Date: 7-14-97 \\
\hline \multicolumn{3}{|c|}{ Control Number: PIM-97-008 } & Rev: 0 \\
\hline $\begin{array}{l}\text { MILESTONE TYPE: } \\
\square \text { DOE-HQ } \\
\square \text { DOE-RL } \\
\text { X CONTRACTOR }\end{array}$ & $\begin{aligned} & \text { DIVISION: } \\
& \square \text { State } \\
& \text { Federal } \\
& \text { X DOE } \\
& \square \text { RCRA } \\
& \square \text { TPA Number }\end{aligned}$ & $\begin{array}{l}\quad \text { DELIVERABLE: } \\
\qquad \text { Report } \\
\square \text { Letter } \\
\square \text { Drawings } \\
\text { X Other (specify) } \\
\text { Cost Estimating Data } \\
\text { Including Backup } \\
\text { Data }\end{array}$ & $\begin{array}{l}\quad \text { ADDRESS TO: } \\
\square \text { DOE-HQ } \\
\square \text { DOE-RL } \\
\text { X Other (specify) } \\
\text { A Planning } \\
\text { Integration Program } \\
\text { Elements }\end{array}$ \\
\hline \multicolumn{4}{|c|}{$\begin{array}{l}\text { This milestone is the completion of a } 1.8 .2 \text { program element by program element review of costs } \\
\text { and schedule to include estimating methodology, level of detail and substantiating data. This } \\
\text { review will follow the requirements set forth in RLID 5000.11, RLID 5700.4, and RLP 5000.9. }\end{array}$} \\
\hline \multicolumn{4}{|c|}{$\begin{array}{l}\text { Description of what constitutes completion of this milestone: } \\
\text { This milestone will be complete when the cost and schedule review of all program elements have } \\
\text { been accomplished and any deficiencies corrected to meet above stated requirements. }\end{array}$} \\
\hline \multicolumn{4}{|c|}{ Milestone Description Approval } \\
\hline \multicolumn{2}{|c|}{$\begin{array}{l}\text { Cost Account Manager } \\
\text { RE.ecectert } \\
\text { R.E. Deitrich }\end{array}$} & \multicolumn{2}{|c|}{ Program/Project Manager } \\
\hline \multicolumn{2}{|c|}{ Program Element Managey } & $\begin{array}{l}\text { DOE Monitor } \\
\text { N/A }\end{array}$ & Date \\
\hline \multicolumn{4}{|c|}{ 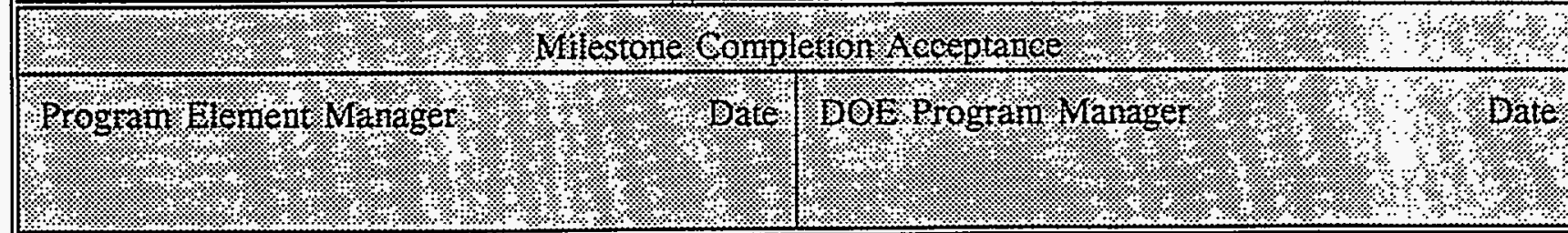 } \\
\hline
\end{tabular}




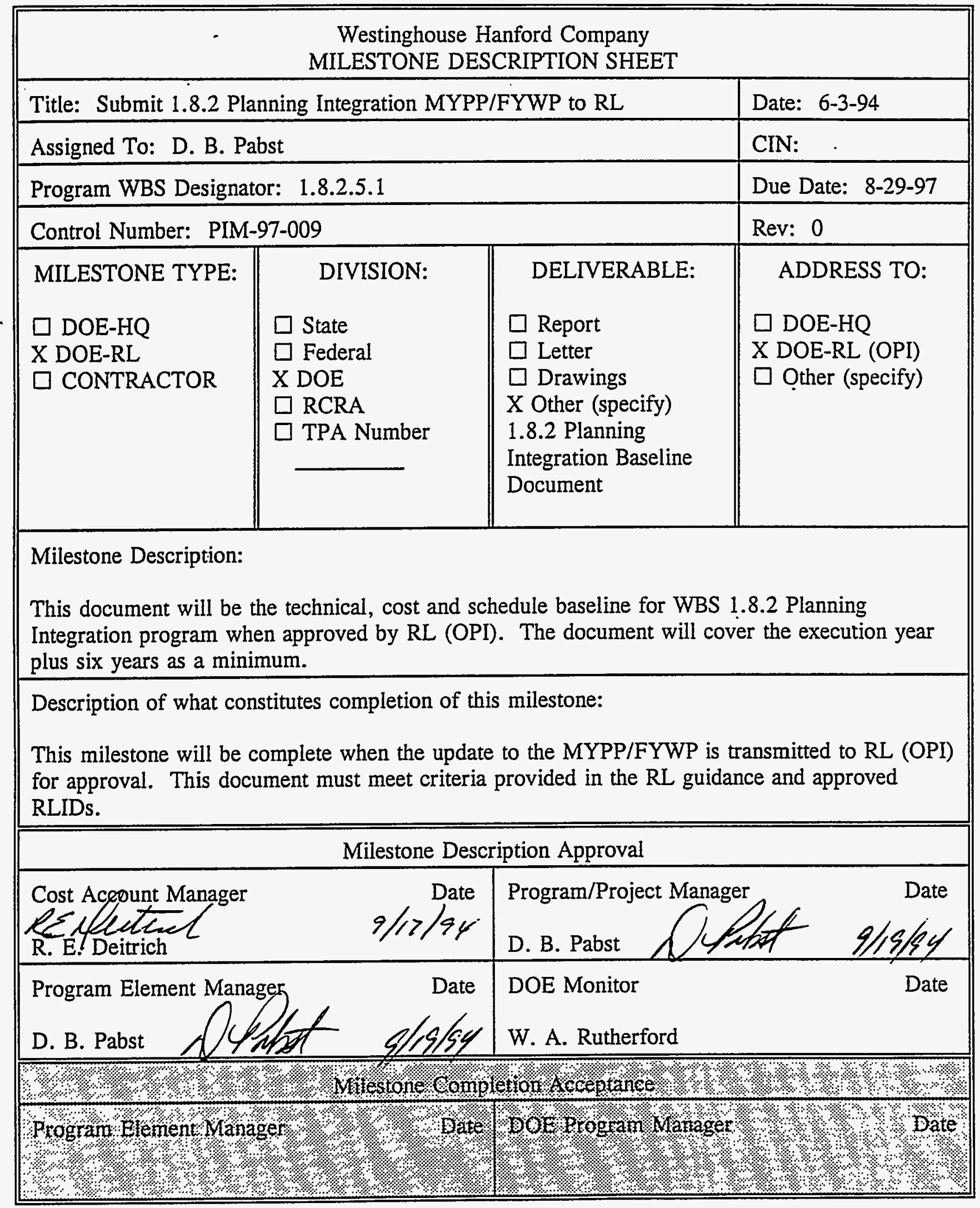




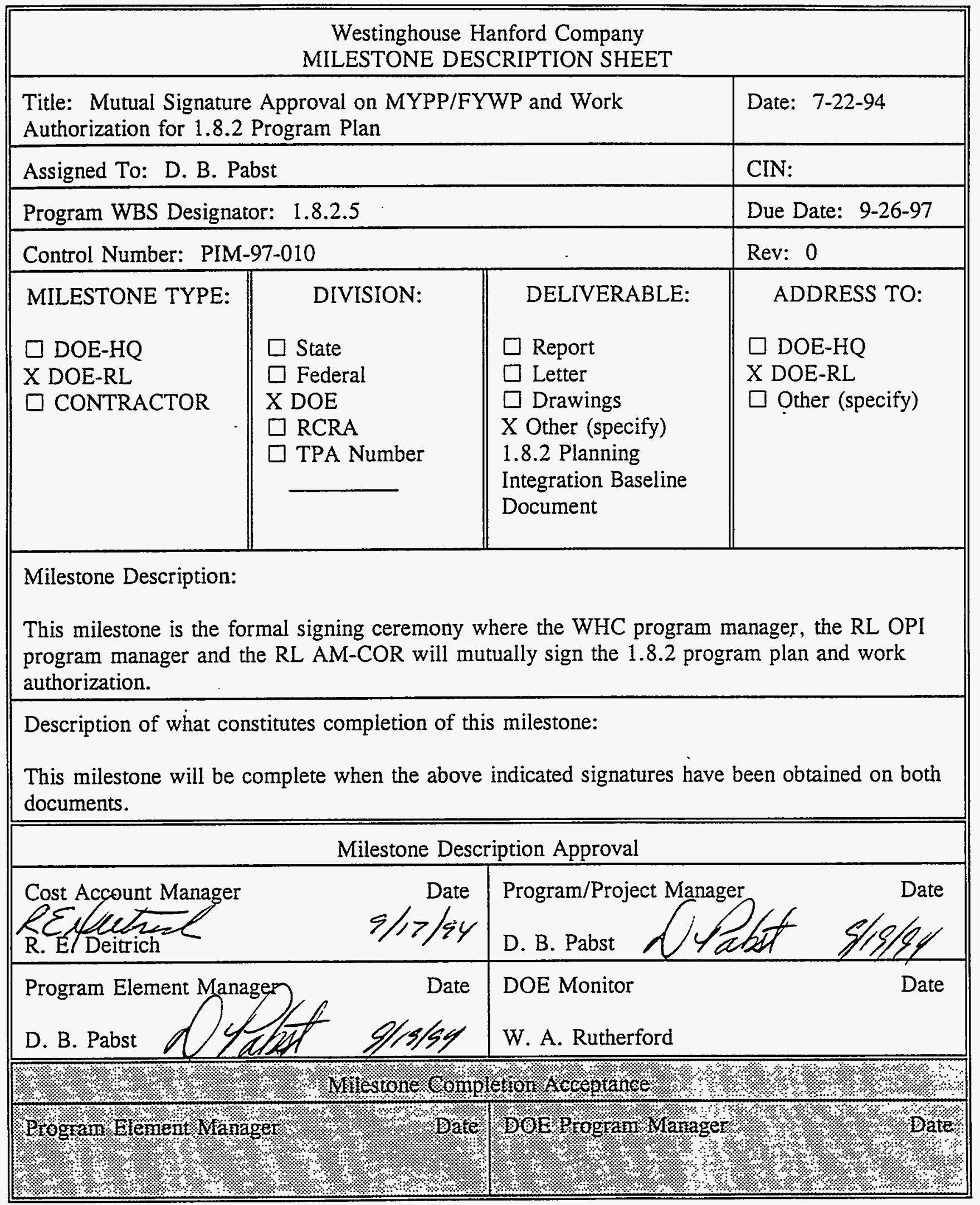




\begin{tabular}{|c|c|c|c|}
\hline \multicolumn{4}{|c|}{$\begin{array}{l}\text { Westinghouse Hanford Company } \\
\text { MILESTONE DESCRIPTION SHEET }\end{array}$} \\
\hline \multicolumn{3}{|c|}{ Title: Submit Monthly SMS Report for Planning and Integration } & Date: $07 / 18 / 94$ \\
\hline \multicolumn{3}{|c|}{ Assigned To: David Pabst } & CIN: \\
\hline \multicolumn{3}{|c|}{ Program .WBS Designator: 1 8.2.5 Planning and Integration Mgmt. } & Due Date: $10 / 18 / 96$ \\
\hline \multicolumn{3}{|c|}{ Control Number: PIM-97-023 } & Rev: \\
\hline $\begin{array}{l}\text { MILESTONE TYPE: } \\
\square \text { DOE-HQ } \\
\square \text { DOE-RL } \\
\text { X CONTRACTOR }\end{array}$ & $\begin{array}{l}\quad \text { DIVISION: } \\
\quad \square \text { State } \\
\square \text { Federal } \\
\text { x DOE } \\
\square \text { RCRA } \\
\square \text { TPA Number }\end{array}$ & $\begin{array}{l}\text { DELIVERABLE: } \\
\text { x Report } \\
\square \text { Letter } \\
\square \text { Drawings } \\
\square \text { Other (specify) }\end{array}$ & $\begin{array}{l}\quad \text { ADDRESS TO: } \\
\\
\square \text { DOE-HQ } \\
\square \text { DOE-RL } \\
\text { x Other (specify) } \\
\text { Deliver to } \\
\text { Performance } \\
\text { Reporting 1.8.2.2 }\end{array}$ \\
\hline \multicolumn{4}{|c|}{$\begin{array}{l}\text { Milestone Description: } \\
\text { This milestone supports RLID } 5000.5 \text {, "Richland Field Office - Site Management System" and } \\
\text { RLID 5000.7, "RL Site Management System Reporting" and consists of coordinating the } \\
\text { monthly Planning and Integration program's Site Management Systems (SMS) report for final } \\
\text { submission to the Performance Reporting Organization: The SMS report will status and measure } \\
\text { Planning and Integration's accomplishments, progress, and expenditures against the approved } \\
\text { baseline. Also included will be a status of any change to the approved program baseline. } \\
\text { Following the monthly program review with the Office of Planning Integration (OPI) RL, RL } \\
\text { comments will be incorporated into the monthly sitewide SMS report delivered to RL each } \\
\text { month in FY 1995. }\end{array}$} \\
\hline \multicolumn{4}{|c|}{$\begin{array}{l}\text { Description of what constitutes completion of this milestone: } \\
\text { The completion of this milestone is constituted by the final submission of the Planning and } \\
\text { Integration's SMS report to the Performance Reporting Organization. }\end{array}$} \\
\hline \multicolumn{4}{|c|}{ Milestone Description Approval } \\
\hline \multicolumn{2}{|l|}{$\begin{array}{l}\text { Cost Accognt, Manager } \\
\text { RE ffelece } \\
\text { RE Deitrich }\end{array}$} & \multicolumn{2}{|c|}{ PB Pabst } \\
\hline \multicolumn{2}{|c|}{ Program Element Managet } & \multicolumn{2}{|c|}{ DOE Monitor Date } \\
\hline \multicolumn{4}{|c|}{ 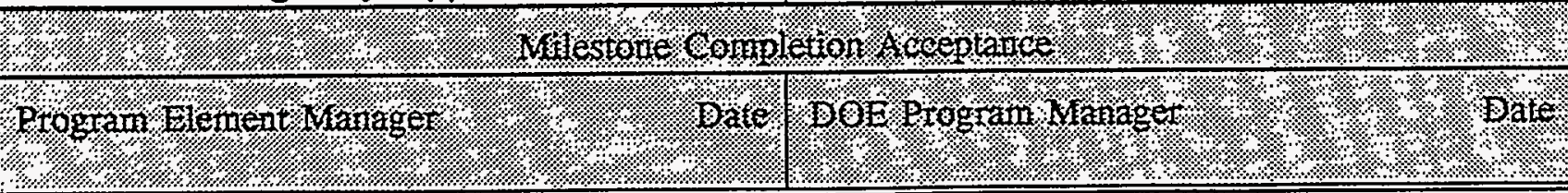 } \\
\hline
\end{tabular}




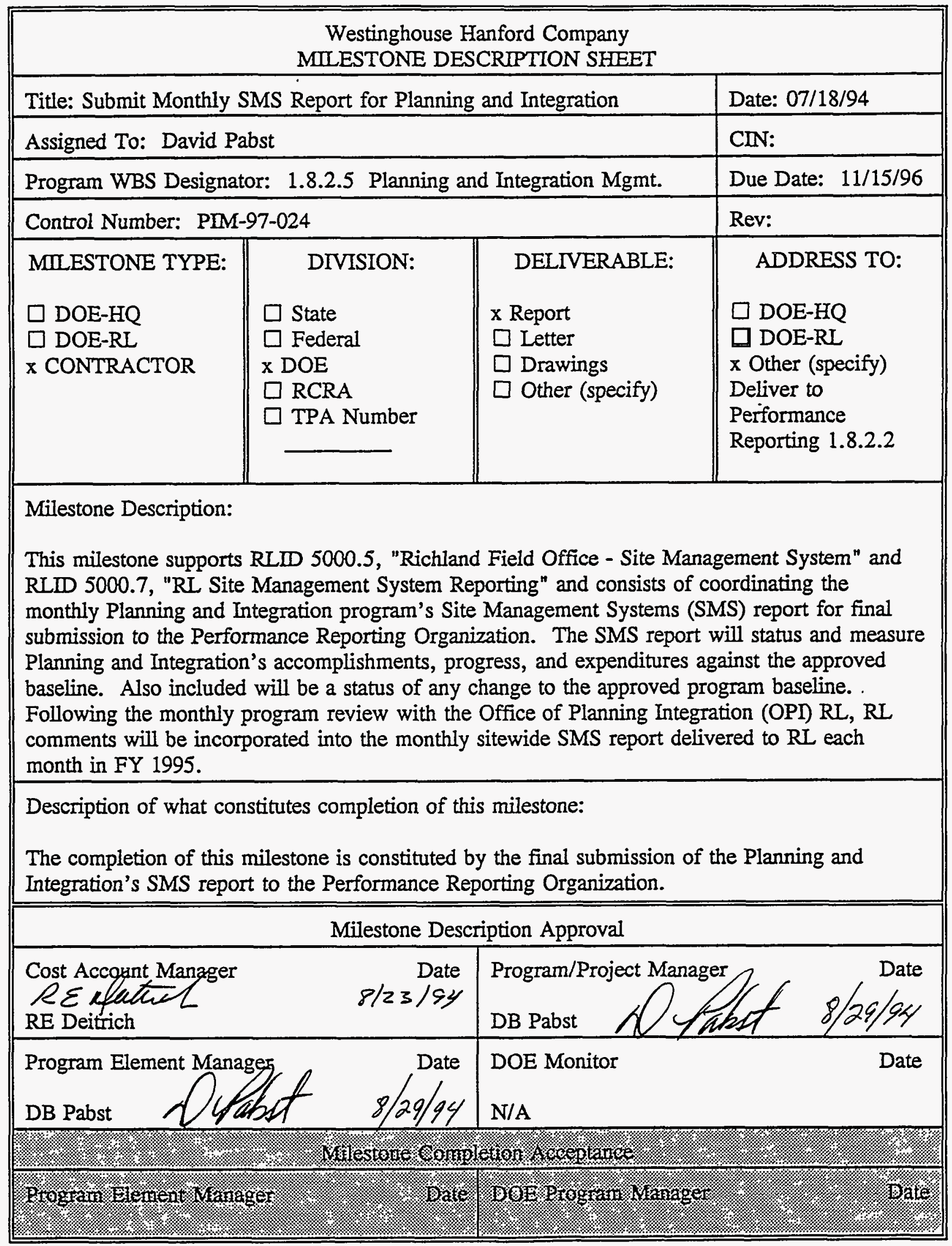




\begin{tabular}{|c|c|c|c|}
\hline \multicolumn{4}{|c|}{$\begin{array}{l}\text { Westinghouse Hanford Company } \\
\text { MILESTONE DESCRIPTION SHEET }\end{array}$} \\
\hline \multicolumn{3}{|c|}{ Title: Submit Monthly SMS Report for Planning and Integration } & Date: $07 / 18 / 94$ \\
\hline \multicolumn{3}{|c|}{ Assigned To: David Pabst } & CIN: \\
\hline \multicolumn{3}{|c|}{ Program WBS Designator: 1-8.2.5 Planning and Integration Mgmt. } & Due Date: $12 / 20 / 96$ \\
\hline \multicolumn{3}{|c|}{ Control Number: PIM-97-025 } & Rev: \\
\hline $\begin{array}{l}\text { MILESTONE TYPE: } \\
\square \text { DOE-HQ } \\
\square \text { DOE-RL } \\
\text { X CONTRACTOR }\end{array}$ & $\begin{array}{l}\quad \text { DIVISION: } \\
\quad \square \text { State } \\
\square \text { Federal } \\
\text { x DOE } \\
\square \text { RCRA } \\
\square \text { TPA Number }\end{array}$ & $\begin{array}{l}\text { DELIVERABLE: } \\
\text { x Report } \\
\square \text { Letter } \\
\square \text { Drawings } \\
\square \text { Other (specify) }\end{array}$ & $\begin{array}{l}\text { ADDRESS TO: } \\
\quad \text { · } \\
\square \text { DOE-HQ } \\
\square \text { DOE-RL } \\
\text { x Other (specify) } \\
\text { Deliver to } \\
\text { Performance } \\
\text { Reporting } 1.8 .2 .2\end{array}$ \\
\hline \multicolumn{4}{|c|}{$\begin{array}{l}\text { This milestone supports RLID 5000.5, "Richland Field Office - Site Management System" and } \\
\text { RLID 5000.7, "RL Site Management System Reporting" and consists of coordinating the } \\
\text { monthly Planning and Integration program's Site Management Systems (SMS) report for final } \\
\text { submission to the Performance Reporting Organization. The SMS report will status and measure } \\
\text { Planning and Integration's accomplishments, progress, and expenditures against the approved } \\
\text { baseline. Also included will be a status of any change to the approved program baseline. } \\
\text { Following the monthly program review with the Office of Planning Integration (OPI RL, RL } \\
\text { comments will be incorporated into the monthly sitewide SMS report delivered to RL each } \\
\text { month in FY 1995. }\end{array}$} \\
\hline \multicolumn{4}{|c|}{$\begin{array}{l}\text { Description of what constitutes completion of this milestone: } \\
\text { The completion of this milestone is constituted by the final submission of the Planning and } \\
\text { Integration's SMS report to the Performance Reporting Organization. }\end{array}$} \\
\hline \multicolumn{4}{|c|}{ Milestone Description Approval } \\
\hline \multicolumn{2}{|l|}{$\begin{array}{l}\text { Cost Account Manager } \\
\text { RE Efeitecen } \\
\text { RE Deitrich }\end{array}$} & \multicolumn{2}{|c|}{ Program/Project Manager } \\
\hline \multicolumn{2}{|c|}{ PBogram Element Manager } & \multicolumn{2}{|c|}{ DOE Monitor Date } \\
\hline \multicolumn{4}{|c|}{ 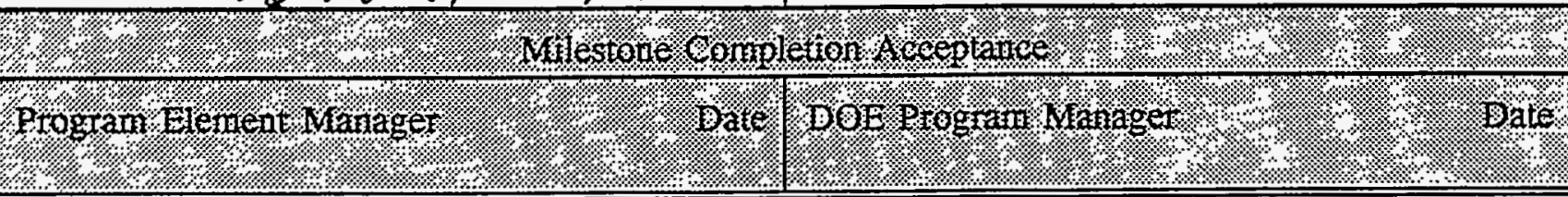 } \\
\hline
\end{tabular}




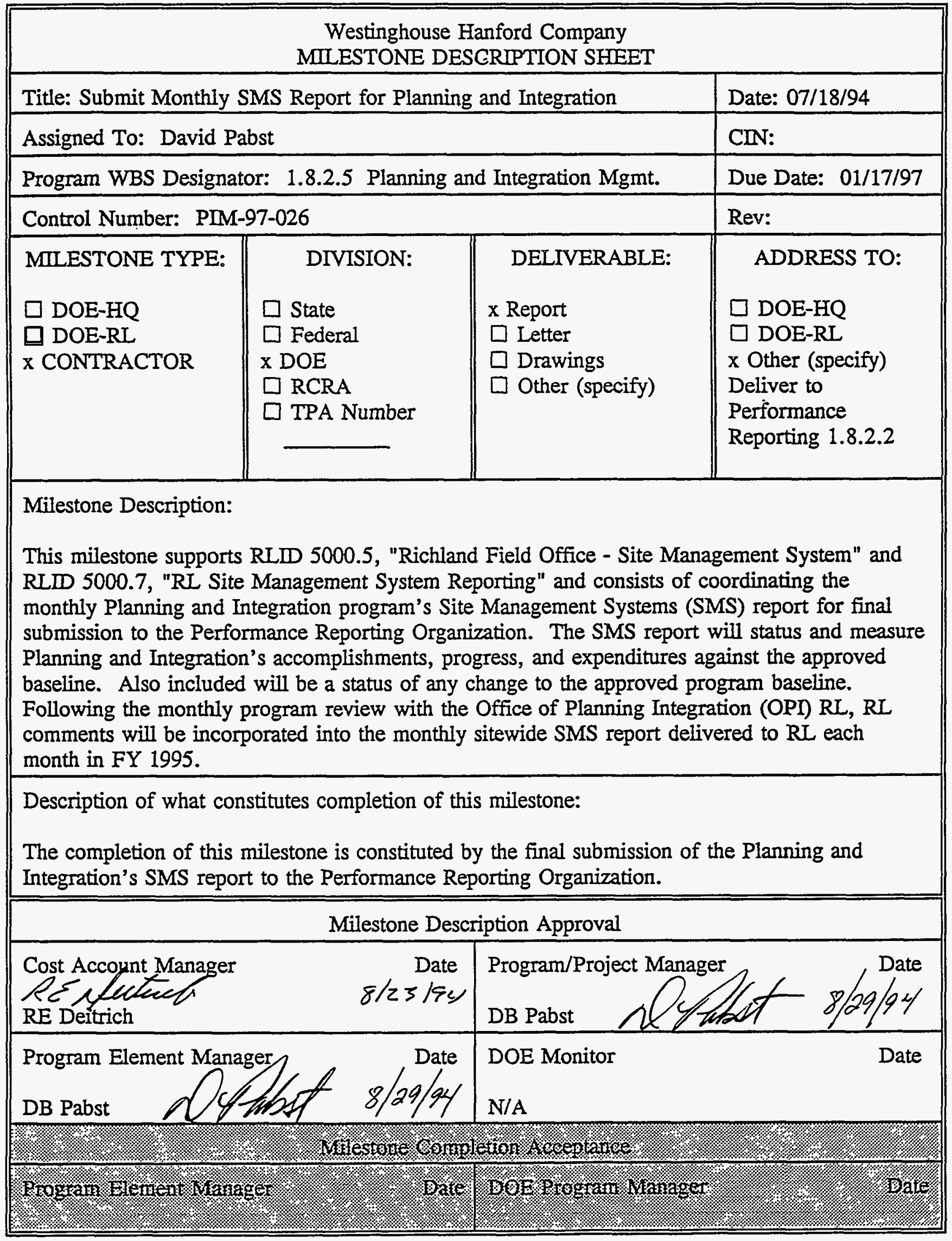




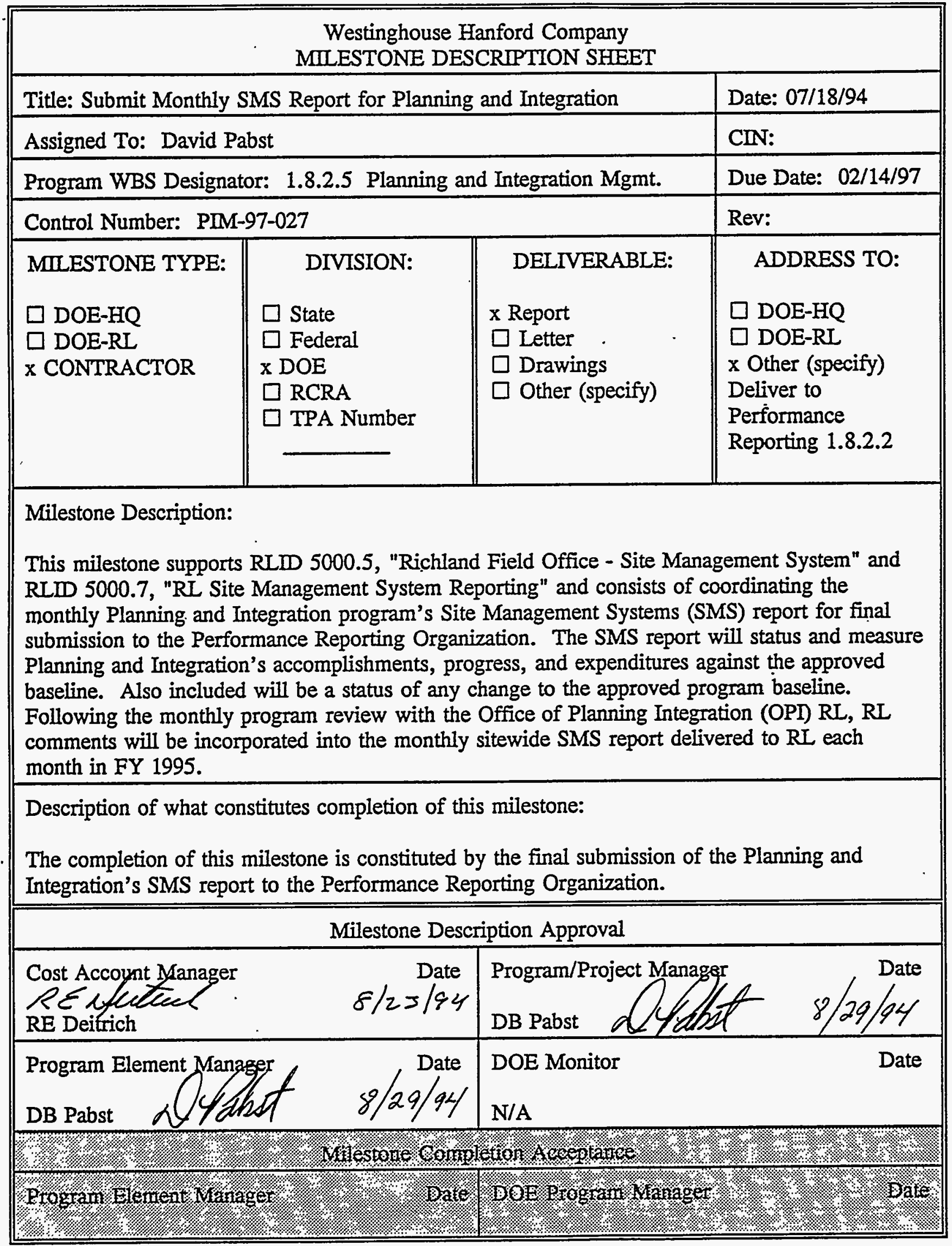




\begin{tabular}{|c|c|c|c|}
\hline \multicolumn{4}{|c|}{$\begin{array}{l}\text { Westinghouse Hanford Company } \\
\text { MULESTONE DESCRIPTION SHEET }\end{array}$} \\
\hline \multicolumn{3}{|c|}{ Title: Submit Monthly SMS Report for Planning and Integration } & Date: $07 / 18 / 94$ \\
\hline \multicolumn{3}{|c|}{ Assigned To: David Pabst } & CIN: \\
\hline \multicolumn{3}{|c|}{ Program WBS Designator: 1.8.2.5 Planning and Integration Mgmt. } & Due Date: $03 / 14 / 97$ \\
\hline \multicolumn{3}{|c|}{ Control Number: PIM-97-028 } & Rev: \\
\hline $\begin{array}{l}\text { MILESTONE TYPE: } \\
\square \text { DOE-HQ } \\
\square \text { DOE-RL } \\
\text { x CONTRACTOR }\end{array}$ & $\begin{array}{l}\quad \text { DIVISION: } \\
\quad \square \text { State } \\
\square \text { Federal } \\
\text { x DOE } \\
\square \text { RCRA } \\
\square \text { TPA Number }\end{array}$ & $\begin{array}{l}\text { DELIVERABLE: } \\
\text { x Report } \\
\square \text { Letter } \\
\square \text { Drawings } \\
\square \text { Other (specify) }\end{array}$ & $\begin{array}{l}\quad \text { ADDRESS TO: } \\
\\
\square \text { DOE-HQ } \\
\square \text { DOE-RL } \\
\text { x Other (specify) } \\
\text { Deliver to } \\
\text { Performance } \\
\text { Reporting 1.8.2.2 }\end{array}$ \\
\hline \multicolumn{4}{|c|}{$\begin{array}{l}\text { Milestone Description: } \\
\text { This milestone supports RLID 5000.5, "Richland Field Office - Site Management System" and } \\
\text { RLID } 5000.7 \text {, "RL Site Management System Reporting" and consists of coordinating the } \\
\text { monthly Planning and Integration program's Site Management Systems (SMS) report for final } \\
\text { submission to the Performance Reporting Organization. The SMS report will status and measure } \\
\text { Planning and Integration's accomplishments, progress, and expenditures against the approved } \\
\text { baseline. Also included will be a status of any change to the approved program baseline. } \\
\text { Following the monthly program review with the Office of Planning Integration (OPI) RL, RL } \\
\text { comments will be incorporated into the monthly sitewide SMS report delivered to RL each } \\
\text { month in FY } 1995 \text {. }\end{array}$} \\
\hline \multicolumn{4}{|c|}{$\begin{array}{l}\text { Description of what constitutes completion of this milestone: } \\
\text { The completion of this milestone is constituted by the final submission of the Planning and } \\
\text { Integration's SMS report to the Performance Reporting Organization. }\end{array}$} \\
\hline \multicolumn{4}{|c|}{ Milestone Description Approval } \\
\hline \multicolumn{2}{|l|}{$\begin{array}{l}\text { Cost Account Manager } \\
\text { RE Deifrich }\end{array}$} & \multicolumn{2}{|c|}{ Drogram/Project Manager } \\
\hline \multicolumn{2}{|c|}{ DBogram Element Manager } & \multicolumn{2}{|c|}{$\begin{array}{ll}\text { DOE Monitor } & \text { Date } \\
\text { N/A } & \\
\end{array}$} \\
\hline \multicolumn{4}{|c|}{ 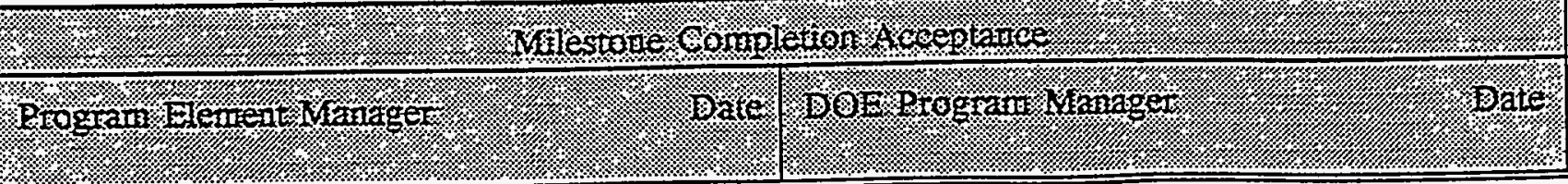 } \\
\hline
\end{tabular}




\begin{tabular}{|c|c|c|c|}
\hline \multicolumn{4}{|c|}{$\begin{array}{l}\text { Westinghouse Hanford Company } \\
\text { MUESTONE DESCRIPTION SHEET }\end{array}$} \\
\hline \multicolumn{3}{|c|}{ Title: Submit Monthly SMS Report for Planning and Integration } & Date: 07/18/94 \\
\hline \multicolumn{3}{|c|}{ Assigned To: David Pabst } & CIN: \\
\hline \multicolumn{3}{|c|}{ Program WBS Designator: 1.8.2.5 Planning and Integration Mgmt. } & Due Date: $04 / 18 / 97$ \\
\hline \multicolumn{3}{|c|}{ Control Number: PIM-97-0.29 } & Rev: \\
\hline $\begin{array}{l}\text { MOLESTONE TYPE: } \\
\square \text { DOE-HQ } \\
\square \text { DOE-RI } \\
\times \text { CONTRACTOR }\end{array}$ & $\begin{array}{l}\quad \text { DIVISION: } \\
\square \text { State } \\
\square \text { Federal } \\
\text { x DOE } \\
\square \text { RCRA } \\
\square \text { TPA Number }\end{array}$ & $\begin{array}{l}\text { DELTVERABLE: } \\
\text { x Report } \\
\square \text { Letter } \\
\square \text { Drawings } \\
\square \text { Other (specify) }\end{array}$ & $\begin{array}{l}\text { ADDRESS TO: } \\
\square \text { DOE-HQ } \\
\square \text { DOE-RL } \\
\text { X Other (specify) } \\
\text { Deliver to } \\
\text { Performance } \\
\text { Reporting } 1.8 .2 .2\end{array}$ \\
\hline \multicolumn{4}{|c|}{$\begin{array}{l}\text { Milestone Description: } \\
\text { This milestone supports RLID 5000.5, "Richland Field Office - Site Management System" and } \\
\text { RLID } 5000.7 \text {, "RI Site Management System Reporting" and consists of coordinating the } \\
\text { monthly Planning and Integration program's Site Management Systems (SMS) report for final } \\
\text { submission to the Performance Reporting Organization. The SMS report will status and measure } \\
\text { Planning and Integration's accomplishments, progress, and expenditures against the approved } \\
\text { baseline. Also included will be a status of any change to the approved program baseline. } \\
\text { Following the monthly program review with the Office of Planning Integration (OPI) RL, RL } \\
\text { comments will be incorporated into the monthly sitewide SMS repoit delivered to RL each } \\
\text { month in FY } 1995 \text {. }\end{array}$} \\
\hline \multicolumn{4}{|c|}{$\begin{array}{l}\text { Description of what constitutes completion of this milestone: } \\
\text { The completion of this milestone is constituted by the final submission of the Planning and } \\
\text { Integration's SMS report to the Performance Reporting Organization. }\end{array}$} \\
\hline \multicolumn{4}{|c|}{ 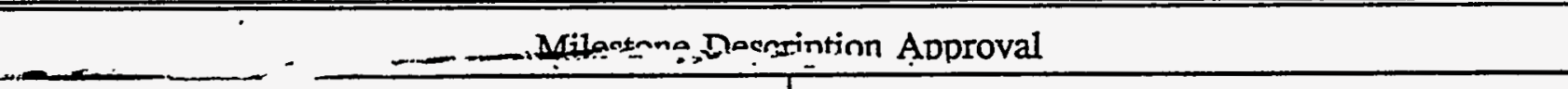 } \\
\hline \multicolumn{2}{|c|}{$\begin{array}{l}\text { Cost Accosant Manager } \\
\text { KE Plectece } \\
\text { RE Deitrich }\end{array}$} & \multicolumn{2}{|c|}{ PB Pabst } \\
\hline \multicolumn{2}{|c|}{ Program Element Managern Pabst $8 / 29 / 94$} & $\begin{array}{l}\text { DOE Monitor } \\
\text { N/A }\end{array}$ & Date \\
\hline \multicolumn{4}{|c|}{ 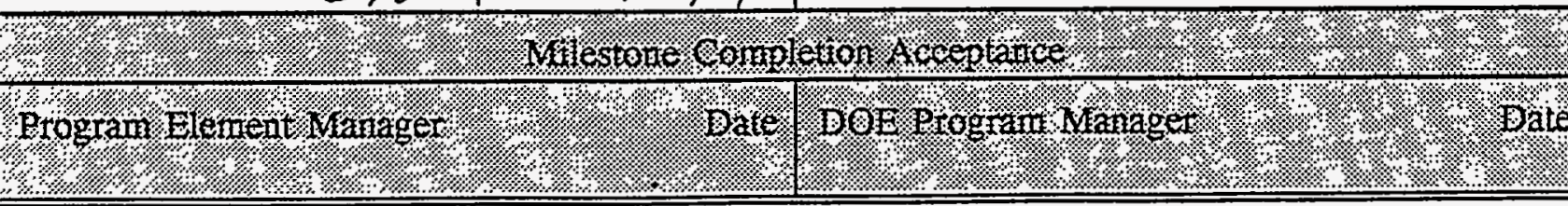 } \\
\hline
\end{tabular}




\begin{tabular}{|c|c|c|c|}
\hline \multicolumn{4}{|c|}{$\begin{array}{l}\text { Westinghouse Hanford Company } \\
\text { MILESTONE DESCRIPTION SHEET }\end{array}$} \\
\hline \multicolumn{3}{|c|}{ Title: Submit Monthly SMS Report for Planning and Integration } & Date: $07 / 18 / 94$ \\
\hline \multicolumn{3}{|c|}{ Assigned To: David Pabst } & CIN: \\
\hline \multicolumn{3}{|c|}{ Program WBS Designator: 1 1 8.2.5 Planning and Integration Mgmt. } & Due Date: $05 / 16 / 97$ \\
\hline \multicolumn{3}{|c|}{ Control Number: PIM-97-030 } & Rev: \\
\hline $\begin{array}{l}\text { MILESTONE TYPE: } \\
\square \text { DOE-HQ } \\
\square \text { DOE-RL } \\
x \text { CONTRACTOR }\end{array}$ & $\begin{array}{l}\quad \text { DIVISION: } \\
\quad \square \text { State } \\
\square \text { Federal } \\
\text { x DOE } \\
\square \text { RCRA } \\
\square \text { TPA Number }\end{array}$ & $\begin{array}{l}\text { DELIVERABLE: } \\
\text { x Report } \\
\square \text { Letter } \\
\square \text { Drawings } \\
\square \text { Other (specify) }\end{array}$ & $\begin{array}{l}\quad \text { ADDRESS TO: } \\
\square \text { DOE-HQ } \\
\square \text { DOE-RL } \\
\text { x Other (specify) } \\
\text { Deliver to } \\
\text { Performance } \\
\text { Reporting 1.8.2.2 }\end{array}$ \\
\hline \multicolumn{4}{|c|}{$\begin{array}{l}\text { Milestone Description: } \\
\text { This milestone supports RLID 5000.5, "Richland Field Office - Site Management System" and } \\
\text { RLID 5000.7, "RL Site Management System Reporting" and consists of coordinating the } \\
\text { monthly Planning and Integration program's Site Management Systems (SMS) report for final } \\
\text { submission to the Performance Reporting Organization. The SMS report will status and measure } \\
\text { Planning and Integration's accomplishments, progress, and expenditures against the approved } \\
\text { baseline. Also included will be a status of any change to the approved program baseline. } \\
\text { Following the monthly program review with the Office of Planning Integration (OPI) RL, RL } \\
\text { comments will be incorporated into the monthly sitewide SMS report delivered to RL each } \\
\text { month in FY 1995. }\end{array}$} \\
\hline \multicolumn{4}{|c|}{$\begin{array}{l}\text { Description of what constitutes completion of this milestone: } \\
\text { The completion of this milestone is constituted by the final submission of the Planning and } \\
\text { Integration's SMS report to the Performance Reporting Organization. }\end{array}$} \\
\hline \multicolumn{4}{|c|}{ Milestone Description Approval } \\
\hline \multicolumn{2}{|l|}{$\begin{array}{l}\text { Cost Accoant Manager } \\
\text { RE Deitrich }\end{array}$} & \multicolumn{2}{|c|}{ PB Pabst } \\
\hline \multicolumn{2}{|c|}{ Program Element Manager } & \multicolumn{2}{|c|}{$\begin{array}{l}\text { DOE Monitor Date } \\
\text { N/A }\end{array}$} \\
\hline \multicolumn{4}{|c|}{ 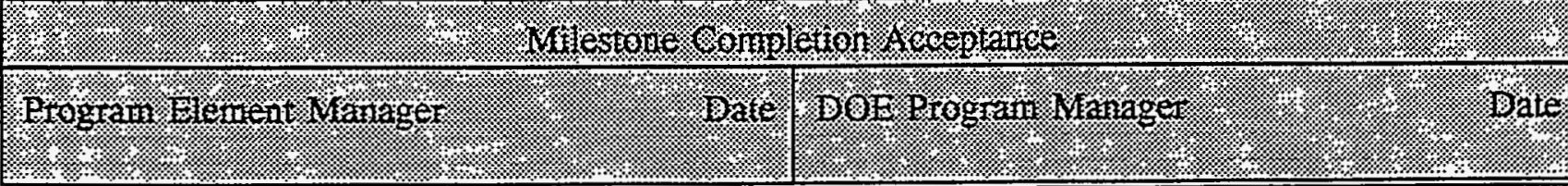 } \\
\hline
\end{tabular}




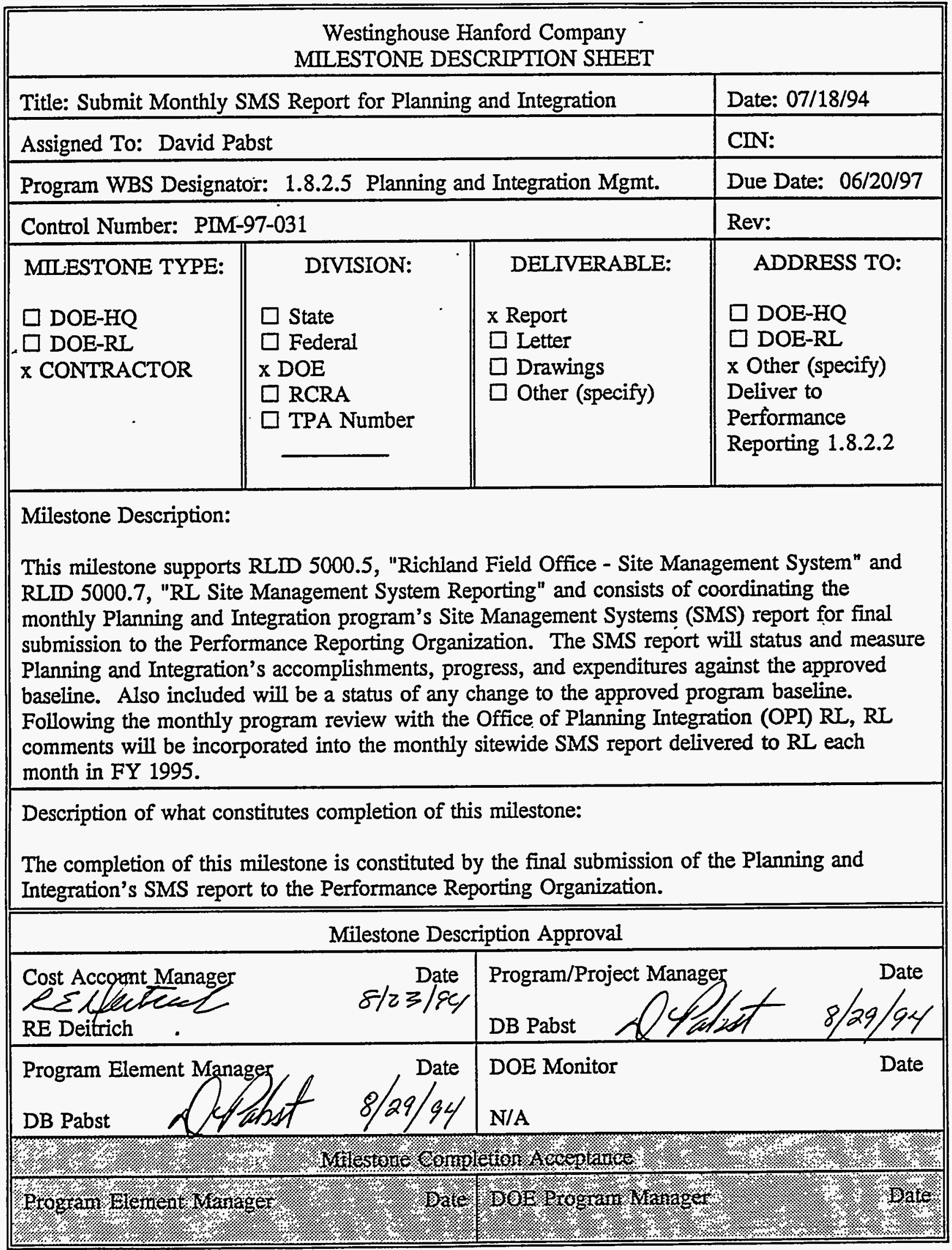




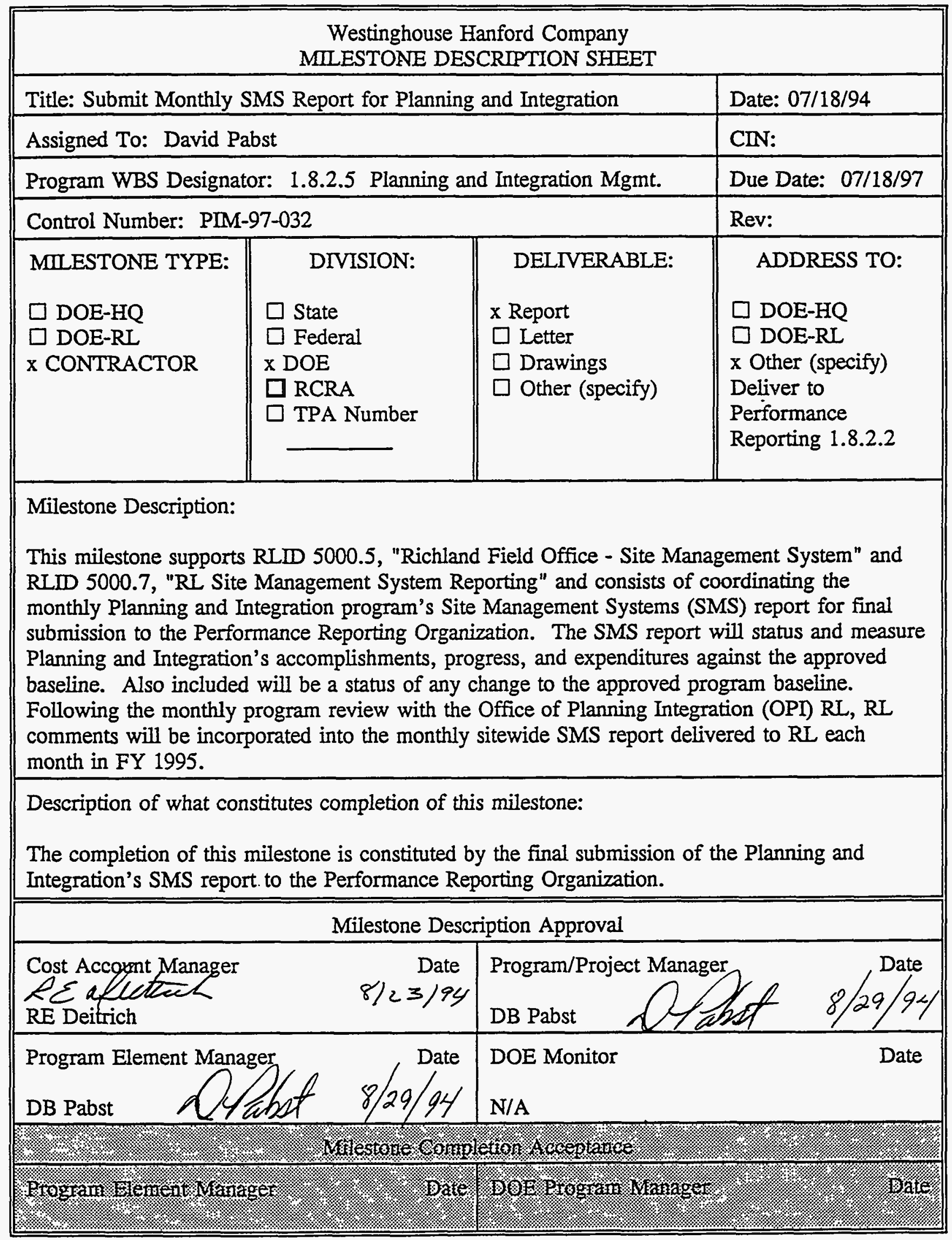




\begin{tabular}{|c|c|c|c|}
\hline \multicolumn{4}{|c|}{$\begin{array}{l}\text { Westinghouse Hanford Company } \\
\text { MILESTONE DESCRIPTION SHEET }\end{array}$} \\
\hline \multicolumn{3}{|c|}{ Title: Submit Monthly SMS Report for Planning and Integration } & Date: $07 / 18 / 94$ \\
\hline \multicolumn{3}{|c|}{ Assigned To: David Pabst } & CIN: \\
\hline \multicolumn{3}{|c|}{ Program WBS Designator: 1 1 8.2.5 Planning and Integration Mgmt. } & Due Date: $08 / 15 / 97$ \\
\hline \multicolumn{3}{|c|}{ Control Number: PIM-97-033 } & Rev: \\
\hline $\begin{array}{l}\text { MILESTONE TYPE: } \\
\square \text { DOE-HQ } \\
\square \text { DOE-RL } \\
x \text { CONTRACTOR }\end{array}$ & $\begin{array}{l}\quad \text { DIVISION: } \\
\square \text { State } \\
\square \text { Federal } \\
\text { x DOE } \\
\square \text { RCRA } \\
\square \text { TPA Number }\end{array}$ & $\begin{array}{l}\text { DELIVERABLE: } \\
\text { x Report } \\
\square \text { Letter } \\
\square \text { Drawings } \\
\square \text { Other (specify) }\end{array}$ & $\begin{array}{l}\quad \text { ADDRESS TO: } \\
\square \text { DOE-HQ } \\
\square \text { DOE-RL } \\
\text { x Other (specify) } \\
\text { Deliver to } \\
\text { Performance } \\
\text { Reporting } 1.8 .2 .2\end{array}$ \\
\hline \multicolumn{4}{|c|}{$\begin{array}{l}\text { Milestone Description: } \\
\text { This milestone supports RLID } 5000.5 \text {, "Richland Field Office - Site Management System" and } \\
\text { RLID 5000.7, "RL Site Management System Reporting" and consists of coordinating the } \\
\text { monthly Planning and Integration program's Site Management Systems (SMS) report for final } \\
\text { submission to the Performance Reporting Organization. The SMS report will status and measure } \\
\text { Planning and Integration's accomplishments, progress, and expenditures against the approved } \\
\text { baseline. Also included will be a status of any change to the approved program.baseline. } \\
\text { Following the monthly program review with the Office of Planning Integration (OPI) RL, RL } \\
\text { comments will be incorporated into the monthly sitewide SMS report delivered to RL each } \\
\text { month in FY } 1995 \text {. }\end{array}$} \\
\hline \multicolumn{4}{|c|}{$\begin{array}{l}\text { Description of what constitutes completion of this milestone: } \\
\text { The completion of this milestone is constituted by the final submission of the Planning and } \\
\text { Integration's SMS report to the Performance Reporting Organization. }\end{array}$} \\
\hline \multicolumn{4}{|c|}{ Milestone Description Approval } \\
\hline \multicolumn{2}{|l|}{$\begin{array}{l}\text { Cost Account Manager } \\
\text { RE Nefeitoech } \\
\text { RE Deirich }\end{array}$} & \multicolumn{2}{|c|}{ Program/Project Manager } \\
\hline \multicolumn{2}{|c|}{ Program Element Manager } & \multicolumn{2}{|c|}{ DOE Monitor Date } \\
\hline \multicolumn{4}{|c|}{ 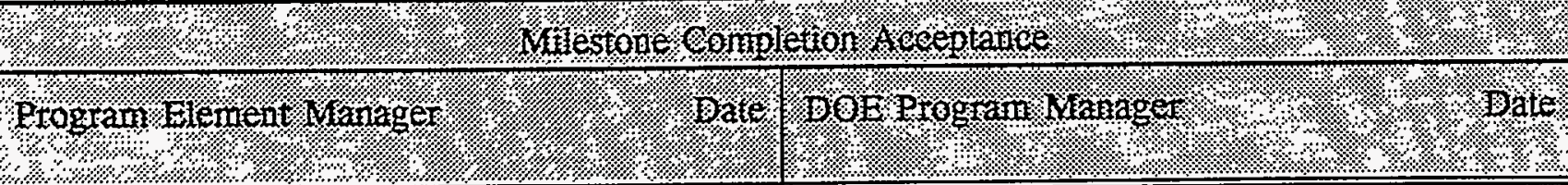 } \\
\hline
\end{tabular}




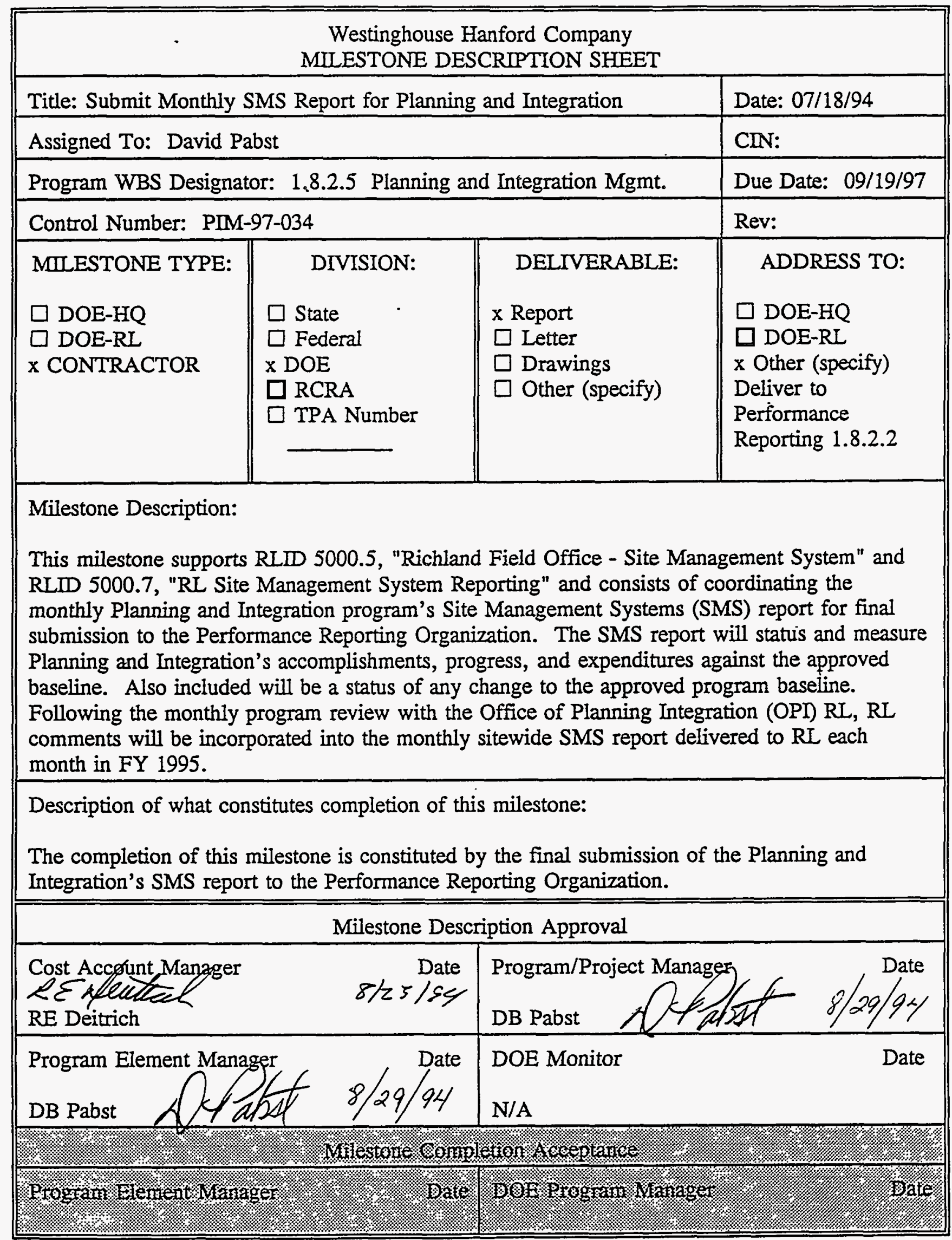




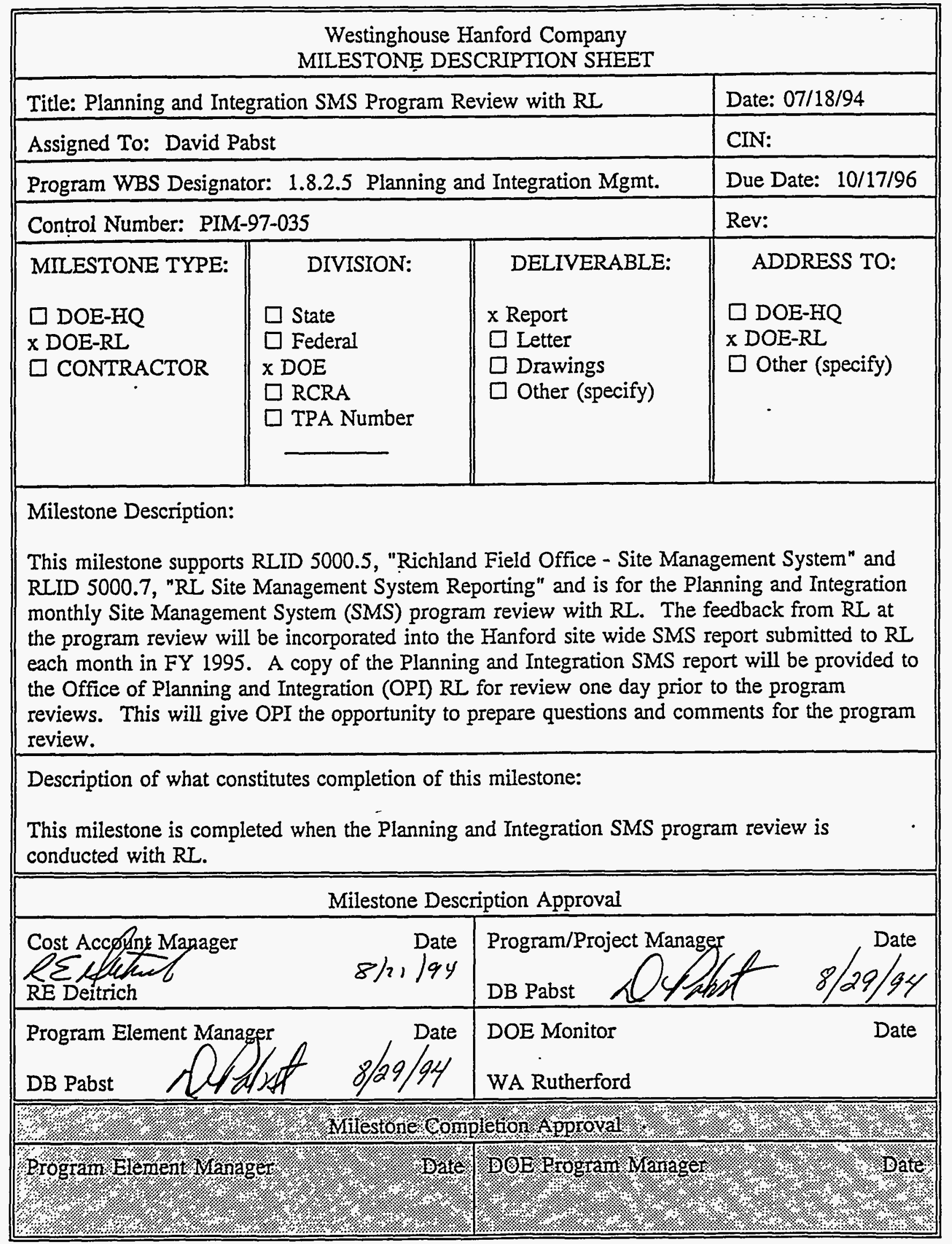




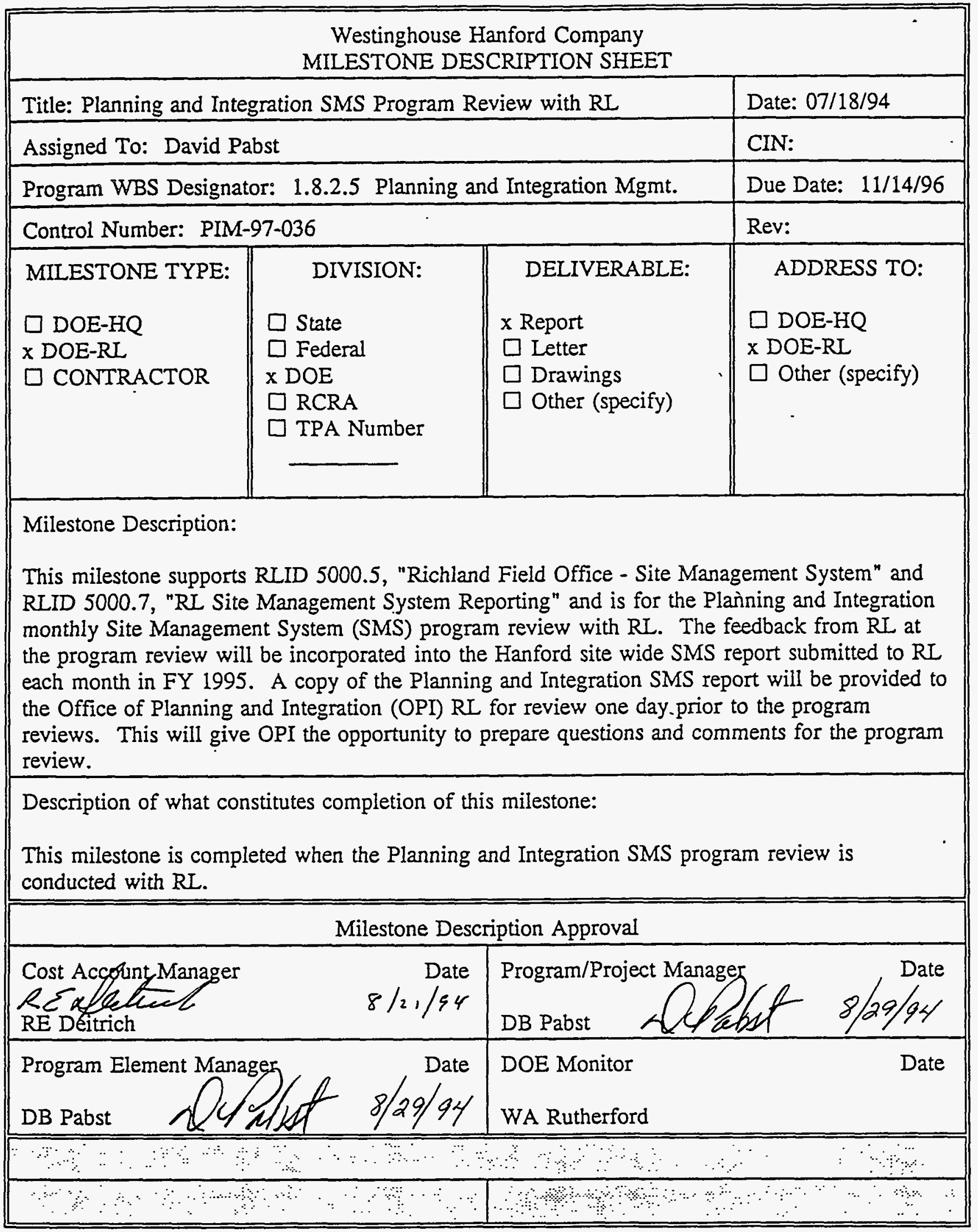




\begin{tabular}{|c|c|c|c|}
\hline \multicolumn{4}{|c|}{$\begin{array}{l}\text { Westinghouse Hanford Company } \\
\text { MILESTONE DESCRIPTION SHEET }\end{array}$} \\
\hline \multicolumn{3}{|c|}{ Title: Planning and Integration SMS Program Review with RL } & Date: $07 / 18 / 94$ \\
\hline \multicolumn{3}{|c|}{ Assigned To: David Pabst } & CIN: \\
\hline \multicolumn{3}{|c|}{ Program WBS Designator: 1.8.2.5 Planning and Integration Mgmt. } & Due Date: $12 / 12 / 96$ \\
\hline \multicolumn{3}{|c|}{ Control Number: PIM-97-037 } & Rev: \\
\hline $\begin{array}{l}\text { MULESTONE TYPE: } \\
\square \text { DOE-HQ } \\
\text { X DOE-RL } \\
\square \text { CONTRACTOR }\end{array}$ & $\begin{aligned} & \text { DIVISION: } \\
& \square \text { State } \\
& \square \text { Federal } \\
& \text { x DOE } \\
& \square \text { RCRA } \\
& \square \text { TPA Number }\end{aligned}$ & $\begin{array}{l}\text { DELIVERABLE: } \\
x \text { Report } \\
\square \text { Letter } \\
\square \text { Drawings } \\
\square \text { Other (specify) }\end{array}$ & $\begin{array}{l}\text { ADDRESS TO: } \\
\square \text { DOE-HQ } \\
\text { x DOE-RL } \\
\square \text { Other (specify) }\end{array}$ \\
\hline \multicolumn{4}{|c|}{$\begin{array}{l}\text { Milestone Description: } \\
\text { This milestone supports RLID 5000.5, "Richland Field Office - Site Management System" and } \\
\text { RLID 5000.7, "RI Site Management System Reporting" and is for the Planning and Integration } \\
\text { monthly Site Management System (SMS) program review with RL. The feedback from RI at } \\
\text { the program review will be incorporated into the Hanford site wide SMS report submitted to RL } \\
\text { each month in FY } 1995 \text {. A copy of the Planning and Integration SMS report will be provided to } \\
\text { the Office of Planning and Integration (OPD RI for review one day prior to the program } \\
\text { reviews. This will give OPI the opportunity to prepare questions and comments for the program } \\
\text { review. }\end{array}$} \\
\hline \multicolumn{4}{|c|}{$\begin{array}{l}\text { Description of what constitutes completion of this milestone: } \\
\text { This milestone is completed when the Planning and Integration SMS program review is } \\
\text { conducted with RL. }\end{array}$} \\
\hline \multicolumn{4}{|c|}{ Milestone Description Approval } \\
\hline \multicolumn{2}{|l|}{$\begin{array}{l}\text { Cost Accopint Manager } \\
\text { Ré fectued } \\
\text { RE Deftrich }\end{array}$} & \multicolumn{2}{|c|}{ Program/Project Manager } \\
\hline \multicolumn{2}{|c|}{ Drogram Element Manager } & \multicolumn{2}{|c|}{$\begin{array}{l}\text { DOE Monitor } \\
\text { WA Rutherford }\end{array}$} \\
\hline \multicolumn{4}{|c|}{ 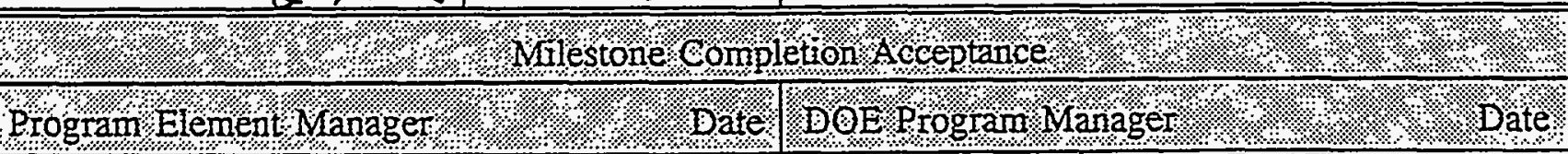 } \\
\hline
\end{tabular}




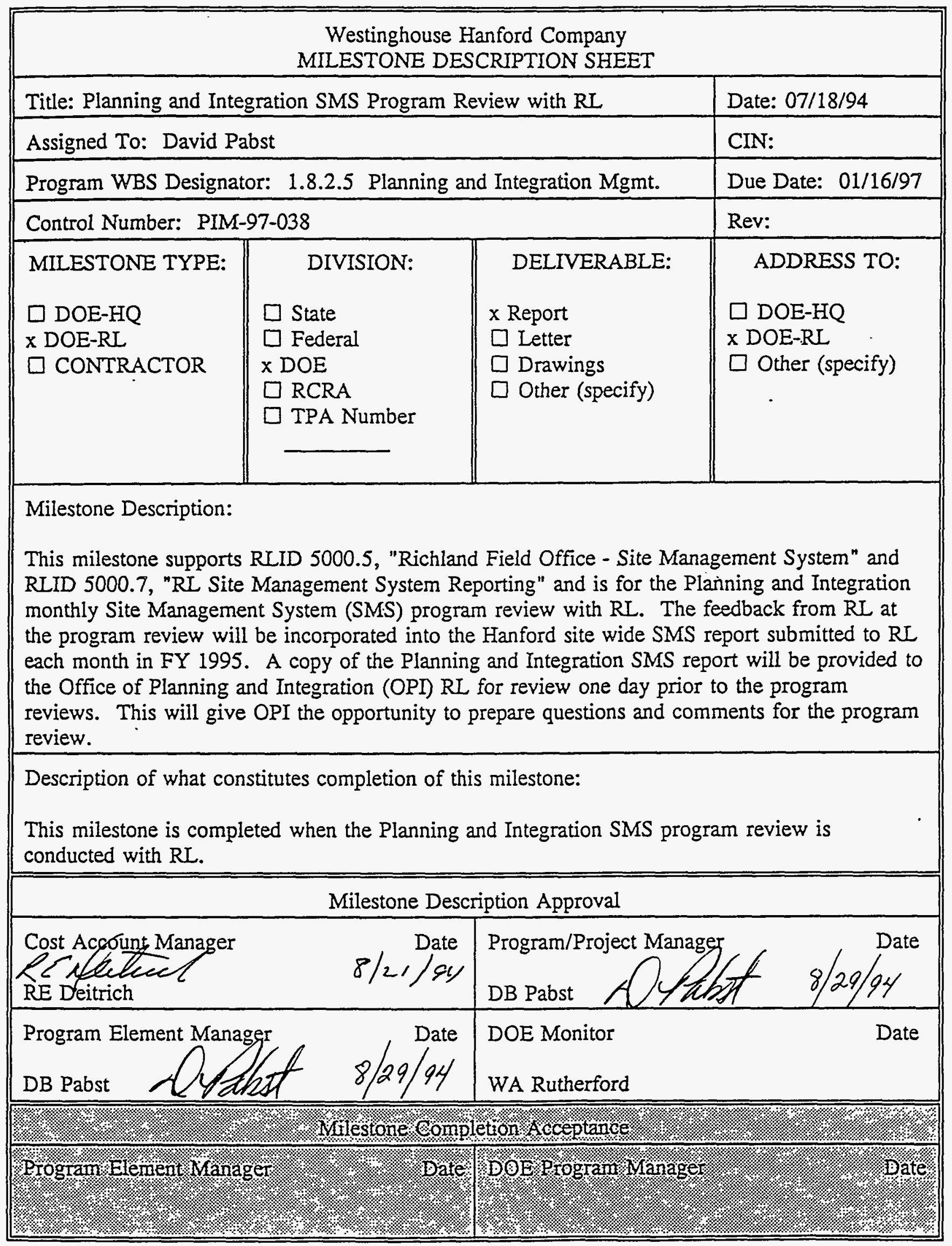




\begin{tabular}{|c|c|c|c|}
\hline \multicolumn{4}{|c|}{$\begin{array}{l}\text { Westinghouse Hanford Company } \\
\text { MILESTONE DESCRIPTION SHEET }\end{array}$} \\
\hline \multicolumn{3}{|c|}{ Title: Planning and Integration SMS Program Review with RL } & Date: 07 \\
\hline \multicolumn{3}{|c|}{ Assigned To: David Pabst } & CIN: \\
\hline \multicolumn{3}{|c|}{ Program WBS Designator: 1-8.2.5 Planning and Integration Mgmt. } & Due Date: $02 / 13 / 97$ \\
\hline \multicolumn{3}{|c|}{ Control Number: PIM-97-039 } & Rev: \\
\hline $\begin{array}{l}\text { MILESTONE TYPE: } \\
\square \text { DOE-HQ } \\
\times \text { DOE-RL } \\
\square \text { CONTRACTOR }\end{array}$ & $\begin{aligned} & \text { DIVISION: } \\
& \square \text { State } \\
& \square \text { Federal } \\
& \text { X DOE } \\
& \square \text { RCRA } \\
& \square \text { TPA Number }\end{aligned}$ & $\begin{array}{l}\text { DELIVERABLE: } \\
\text { x Report } \\
\square \text { Letter } \\
\square \text { Drawings } \\
\square \text { Other (specify) }\end{array}$ & $\begin{array}{l}\text { ADDRESS TO: } \\
\square \text { DOE-HQ } \\
\text { x DOE-RL } \\
\square \text { Other (specify) }\end{array}$ \\
\hline \multicolumn{4}{|c|}{$\begin{array}{l}\text { This milestone supports RLID 5000.5, "Richland Field Office - Site Management System" and } \\
\text { RLID 5000.7, "RL Site Management System Reporting" and is for the Plañning and Integration } \\
\text { monthly Site Management System (SMS) program review with RL. The feedback from RL at } \\
\text { the program review will be incorporated into the Hanford site wide SMS report submitted to RL } \\
\text { each month in FY } 1995 \text {. A copy of the Planning and Integration SMS report will be provided to } \\
\text { the Office of Planning and Integration (OPI) RL for review one day-prior to the program } \\
\text { reviews. This will give OPI the opportunity to prepare questions and comments for the program } \\
\text { review. }\end{array}$} \\
\hline \multicolumn{4}{|c|}{$\begin{array}{l}\text { This milestone is completed when the Planning and Integration SMS program review is } \\
\text { conducted with RL. }\end{array}$} \\
\hline \multicolumn{4}{|c|}{ Milestone Description Approval } \\
\hline \multicolumn{2}{|l|}{$\begin{array}{l}\text { Cost Account Manager } \\
\text { RE ifleitted } \\
\text { RE Deitrich }\end{array}$} & \multicolumn{2}{|c|}{ Diogram/Project Manager } \\
\hline \multicolumn{2}{|c|}{ Program Element Manager } & \multicolumn{2}{|c|}{$\begin{array}{l}\text { DOE Monitor } \\
\text { WA Rutherford }\end{array}$} \\
\hline
\end{tabular}




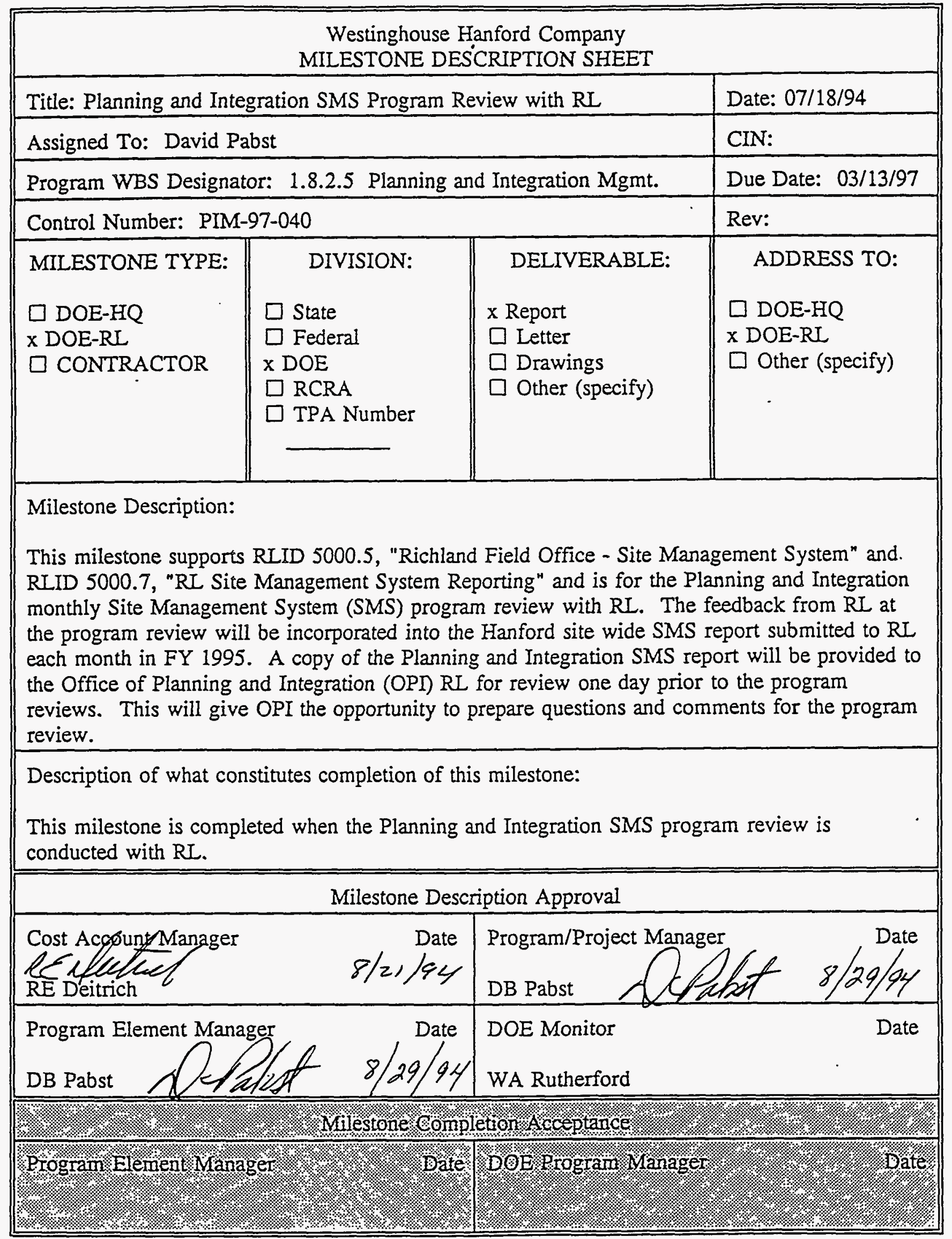




\begin{tabular}{|l|l|l||}
\hline \multicolumn{3}{|c|}{ Westinghouse Hanford Company } \\
MILESTONE DESCRIPTION SHEET
\end{tabular}




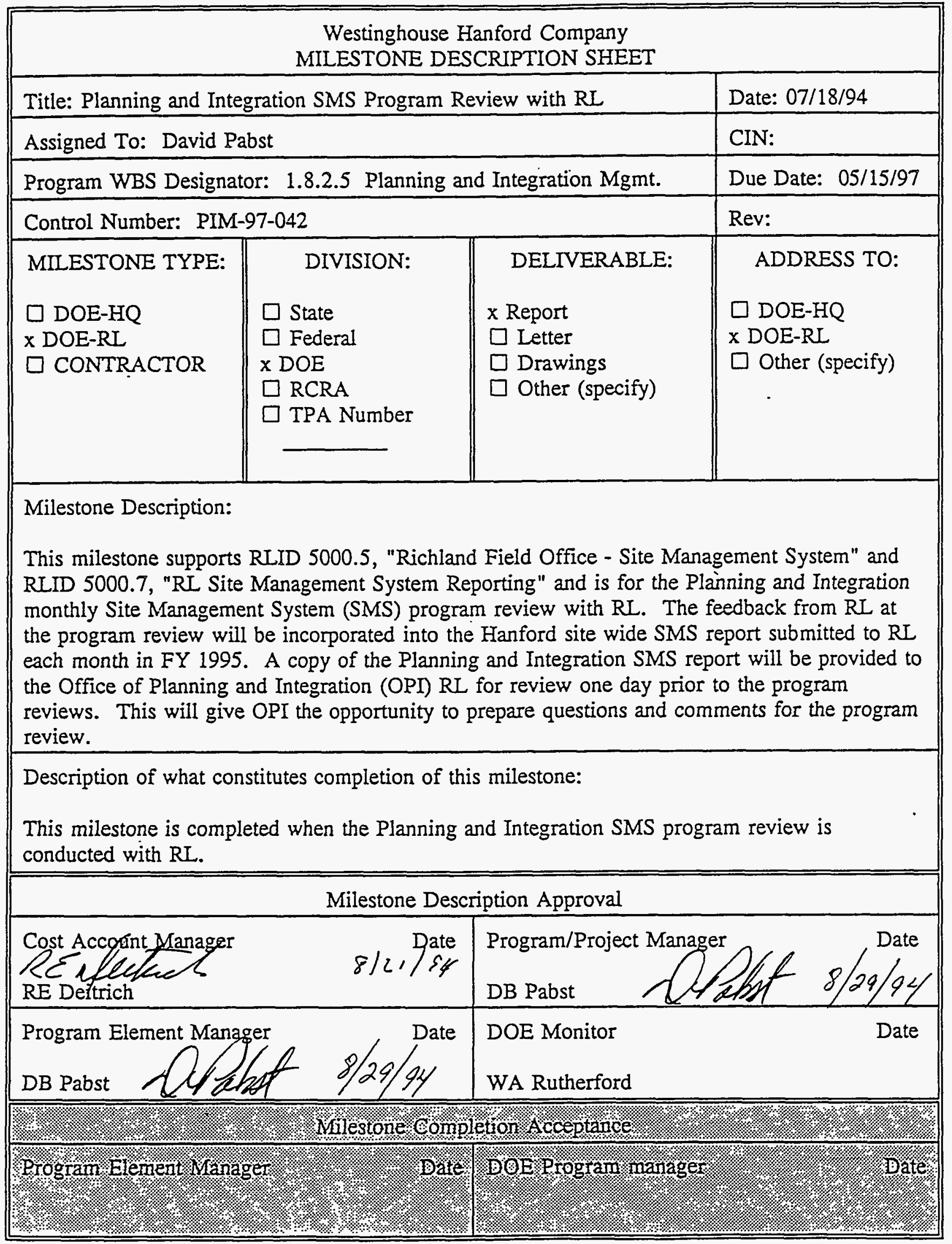




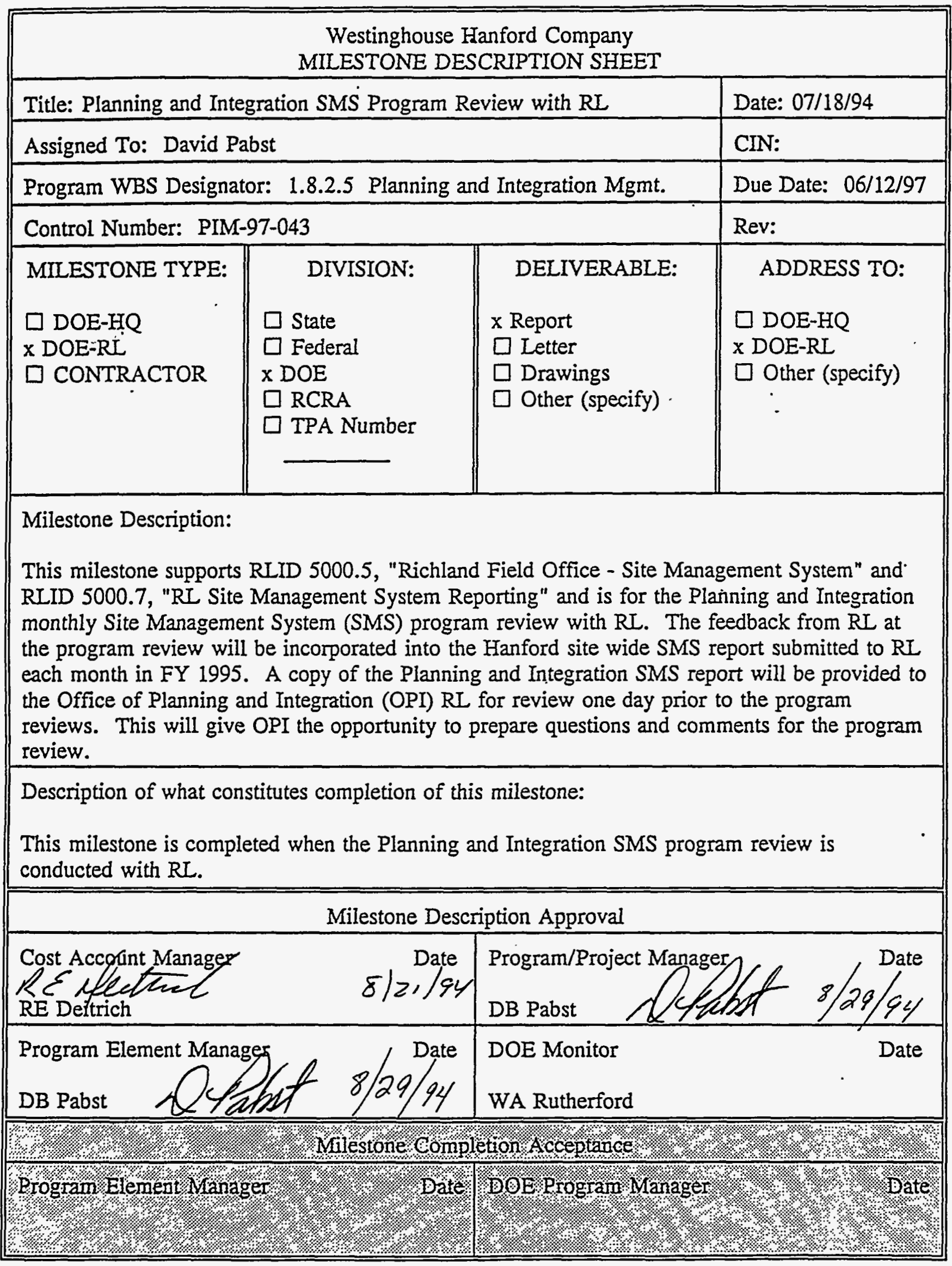




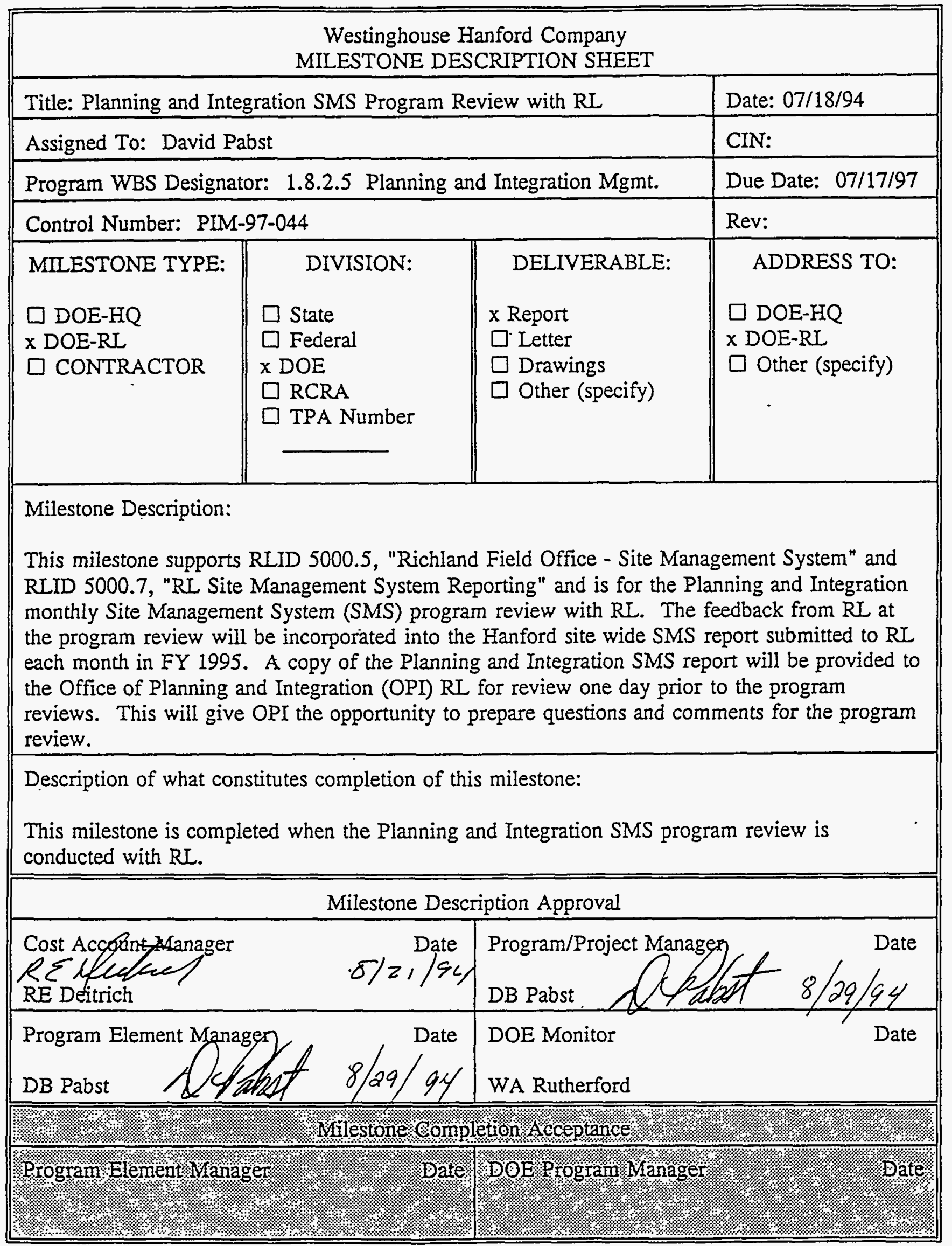


i)

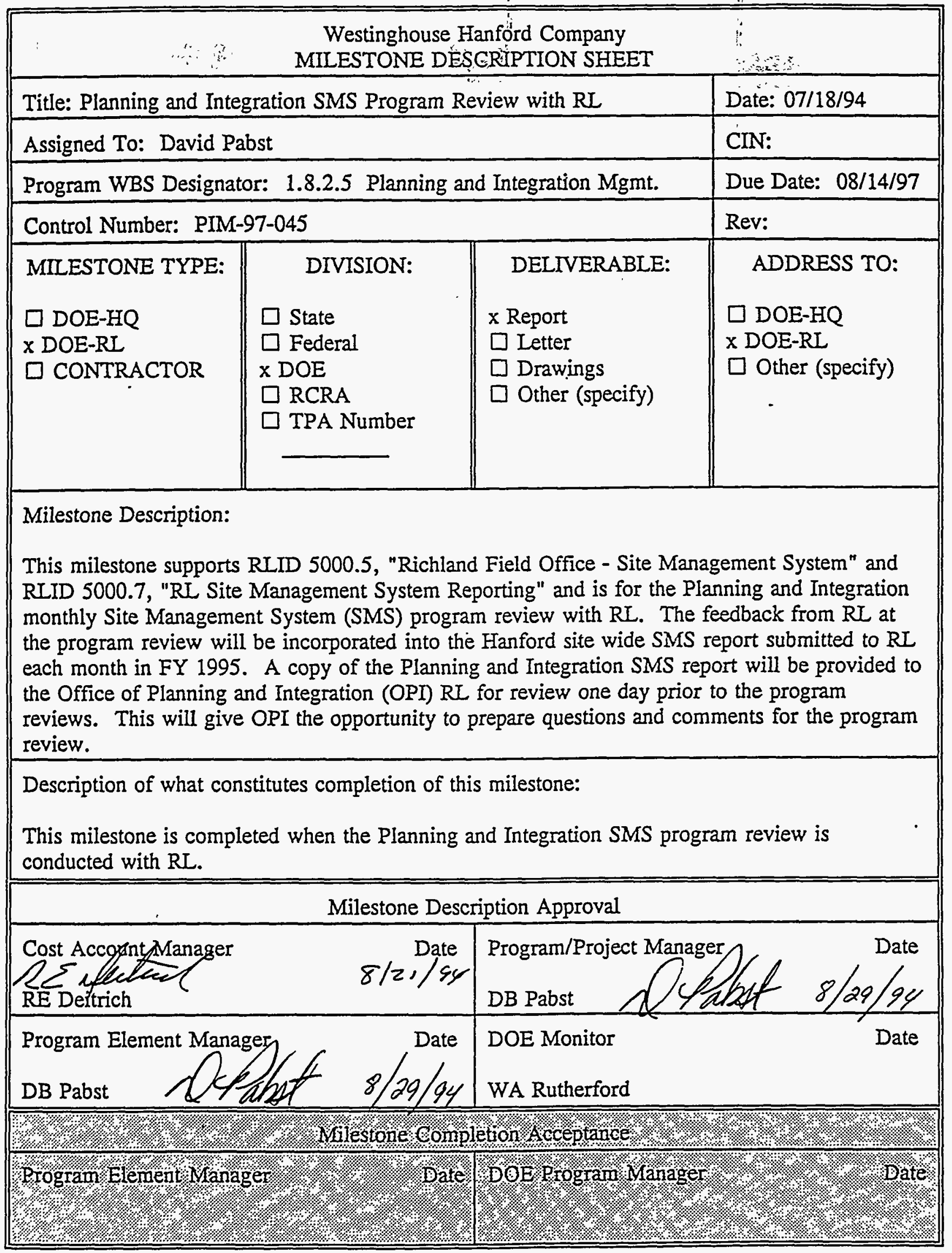




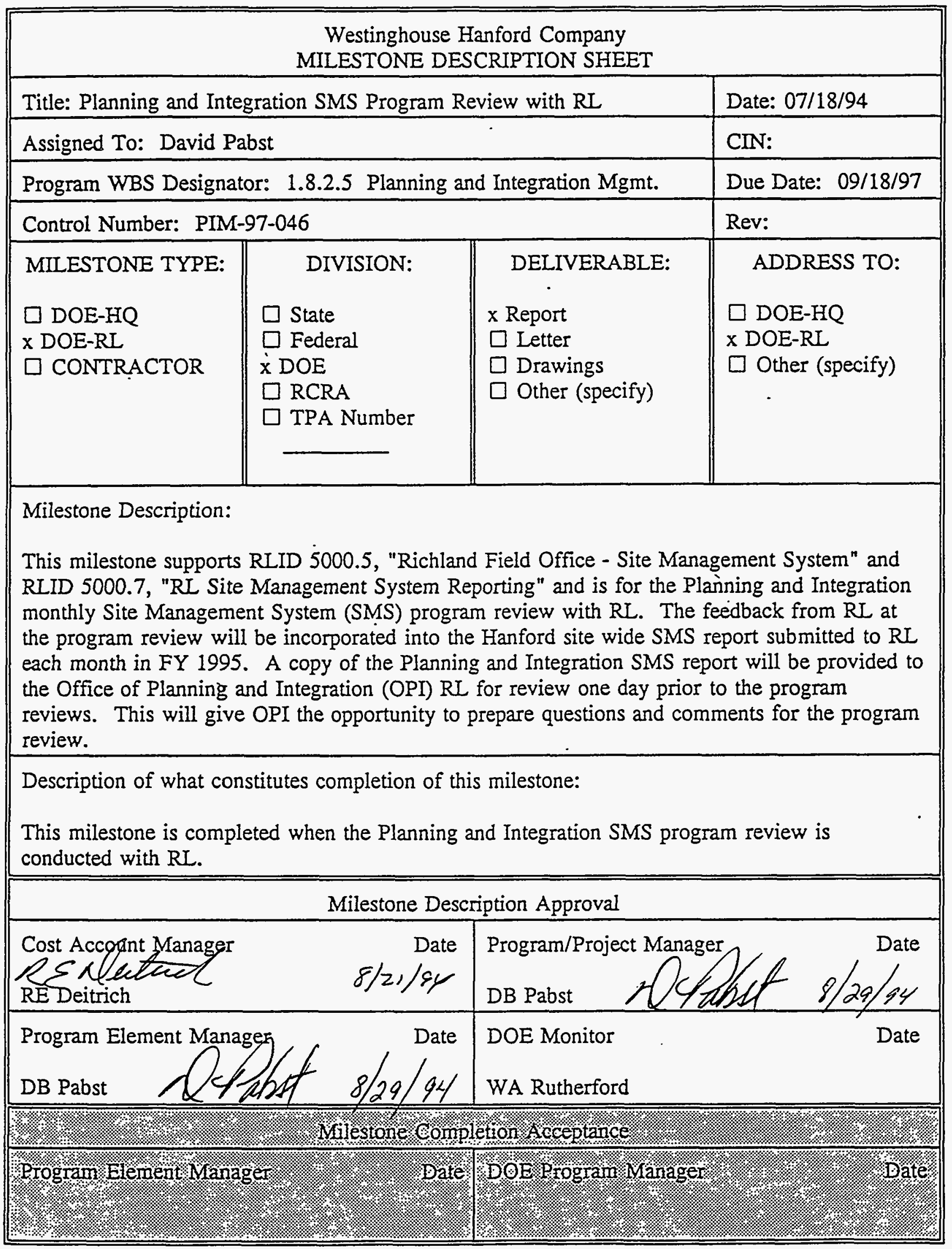




\begin{tabular}{|l||l|l||}
\hline \multicolumn{3}{|c|}{ Westinghouse Hanford Company } \\
MILESTONE DESCRIPTION SHEET
\end{tabular}




\begin{tabular}{|l||l||}
\hline \multicolumn{3}{|c|}{ Westinghouse Hanford Company } \\
MILESTONE DESCRIPTION SHEET
\end{tabular}




\begin{tabular}{|l||l||}
\hline \multicolumn{3}{|c|}{ Westinghouse Hanford Company } \\
MILESTONE DESCRIPTION SHEET
\end{tabular}




\begin{tabular}{|l||l||}
\hline \multicolumn{3}{|c|}{ Westinghouse Hanford Company } \\
MILESTONE DESCRIPTION SHEET
\end{tabular}




\begin{tabular}{||l|l||}
\hline \multicolumn{3}{|c|}{ Westinghouse Hanford Company } \\
MILESTONE DESCRIPTION SHEET
\end{tabular}




\begin{tabular}{|l||l||}
\hline \multicolumn{3}{|c|}{ Westinghouse Hanford Company } \\
MILESTONE DESCRIPTION SHEET
\end{tabular}




\begin{tabular}{|l||l||}
\hline \multicolumn{3}{|c|}{ Westinghouse Hanford Company } \\
MILESTONE DESCRIPTION SHEET
\end{tabular}




\begin{tabular}{|l|l||}
\hline \multicolumn{2}{|c|}{ Westinghouse Hanford Company } \\
MILESTONE DESCRIPTION SHEET
\end{tabular}




\begin{tabular}{|l||l|l||}
\hline \multicolumn{3}{|c|}{ Westinghouse Hanford Company } \\
MILESTONE DESCRIPTION SHEET
\end{tabular}




\begin{tabular}{|l|l|||}
\hline \multicolumn{3}{|c|}{ Westinghouse Hanford Company } \\
MILESTONE DESCRIPTION SHEET
\end{tabular}




\begin{tabular}{|l||l|||}
\hline \multicolumn{3}{|c|}{ Westinghouse Hanford Company } \\
MILESTONE DESCRIPTION SHEET
\end{tabular}




\begin{tabular}{|l||l||}
\hline \multicolumn{3}{|c|}{ Westinghouse Hanford Company } \\
MILESTONE DESCRIPTION SHEET
\end{tabular}


Planning Integration Multi-Year Program Plan

This page

intentionally left blank. 


\begin{tabular}{||l|l|||}
\hline \multicolumn{3}{|c||}{ Westinghouse Hanford Company } \\
MILESTONE DESCRIPTION SHEET
\end{tabular}




\begin{tabular}{|l||l|l||}
\hline \multicolumn{3}{|c||}{ Westinghouse Hanford Company } \\
MILESTONE DESCRIPTION SHEET
\end{tabular}




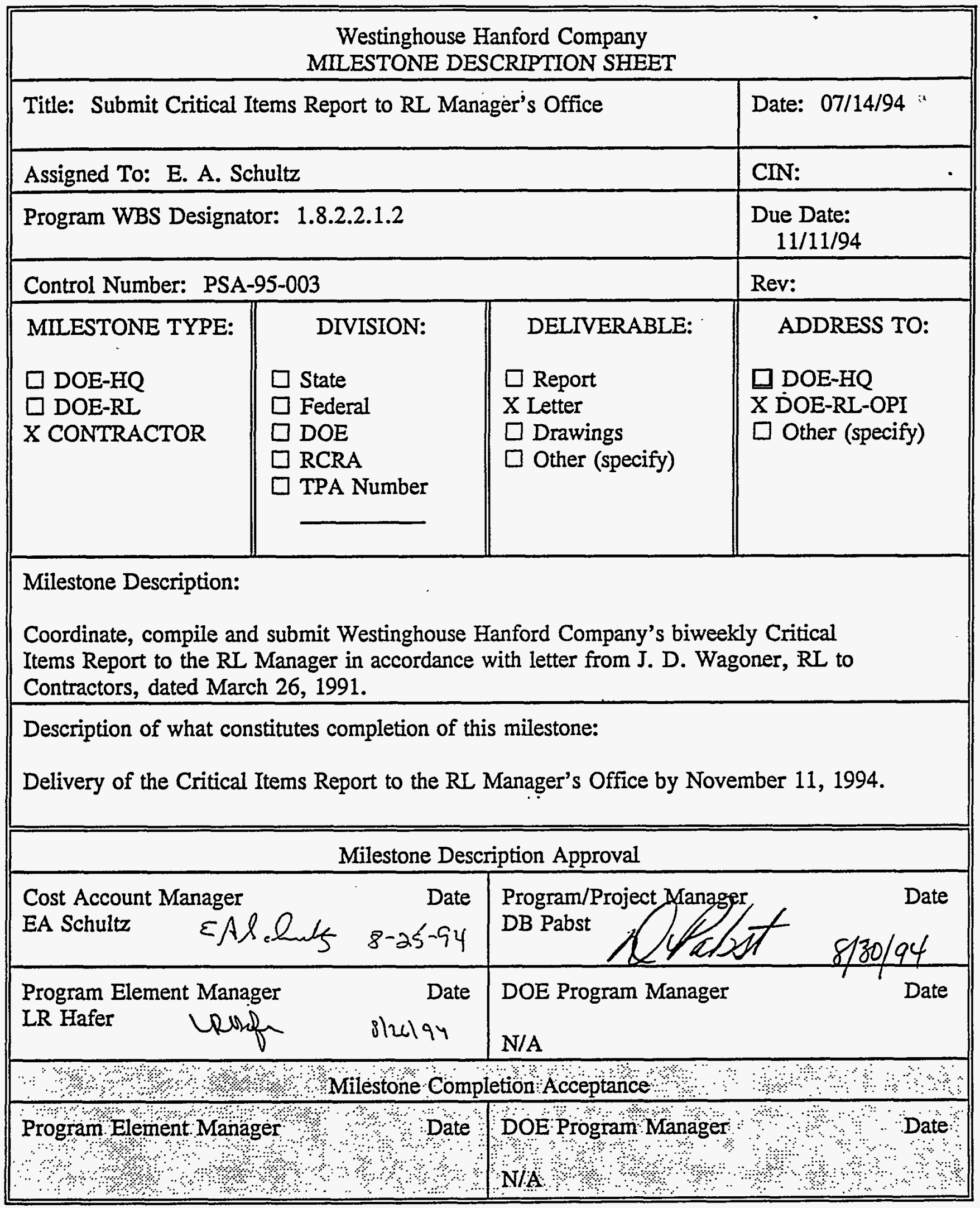




\begin{tabular}{|l||l|l||}
\hline \multicolumn{3}{|c|}{ Westinghouse Hanford Company } \\
MILESTONE DESCRIPTION SHEET
\end{tabular}




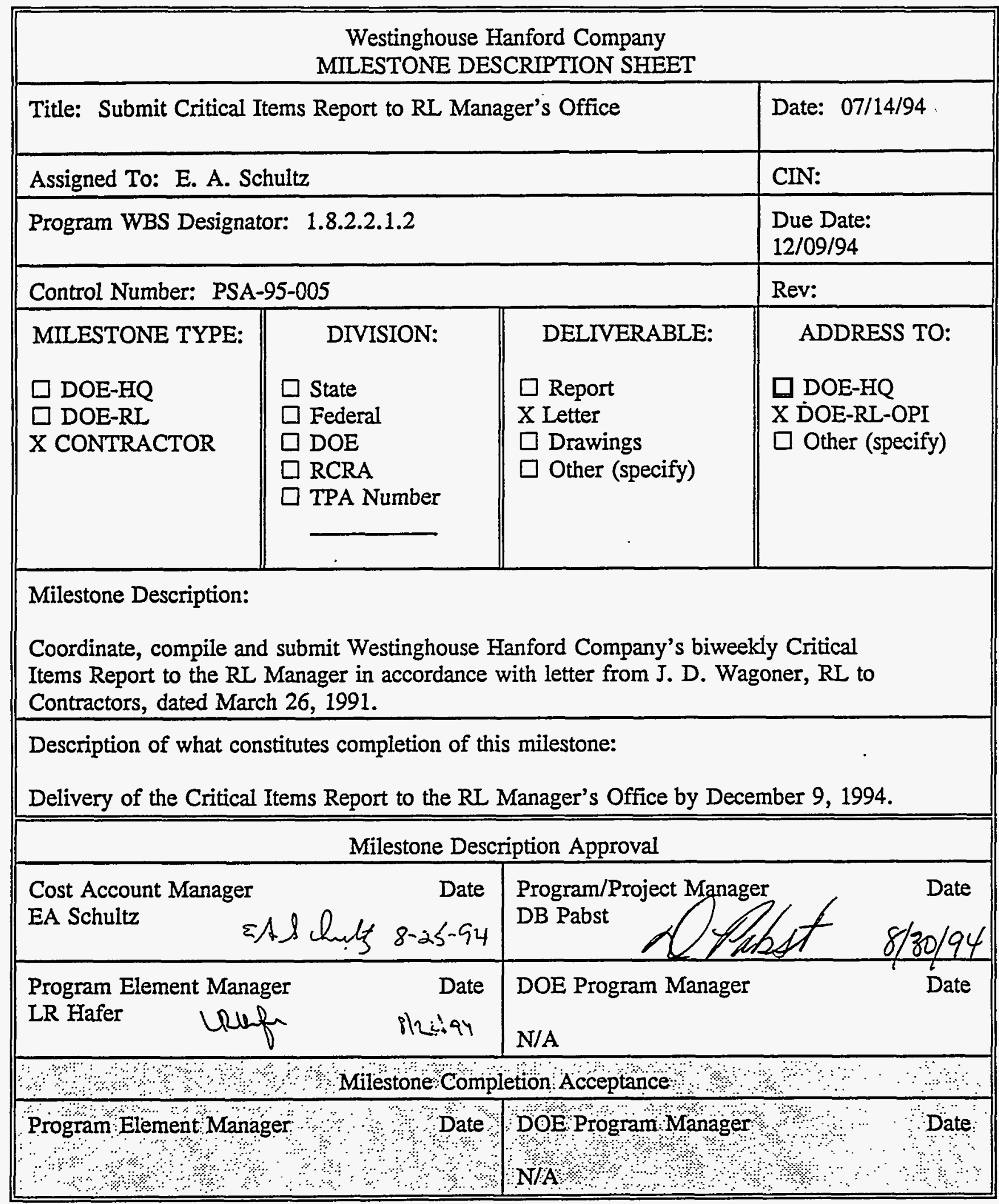




\begin{tabular}{|l||l|l||}
\hline \multicolumn{3}{|c||}{ Westinghouse Hanford Company } \\
MILESTONE DESCRIPTION SHEET
\end{tabular}




\begin{tabular}{||l|l||}
\hline \multicolumn{3}{|c|}{ Westinghouse Hanford Company } \\
MILESTONE DESCRIPTION SHEET
\end{tabular}




\begin{tabular}{|l||l|l||}
\hline \multicolumn{3}{|c|}{ Westinghouse Hanford Company } \\
MILESTONE DESCRIPTION SHEET
\end{tabular}




\begin{tabular}{|l||l|l||}
\hline \multicolumn{3}{|c|}{ Westinghouse Hanford Company } \\
MILESTONE DESCRIPTION SHEET
\end{tabular}




\begin{tabular}{|l|l|l||}
\hline \multicolumn{3}{|c||}{ Westinghouse Hanford Company } \\
MILESTONE DESCRIPTION SHEET
\end{tabular}




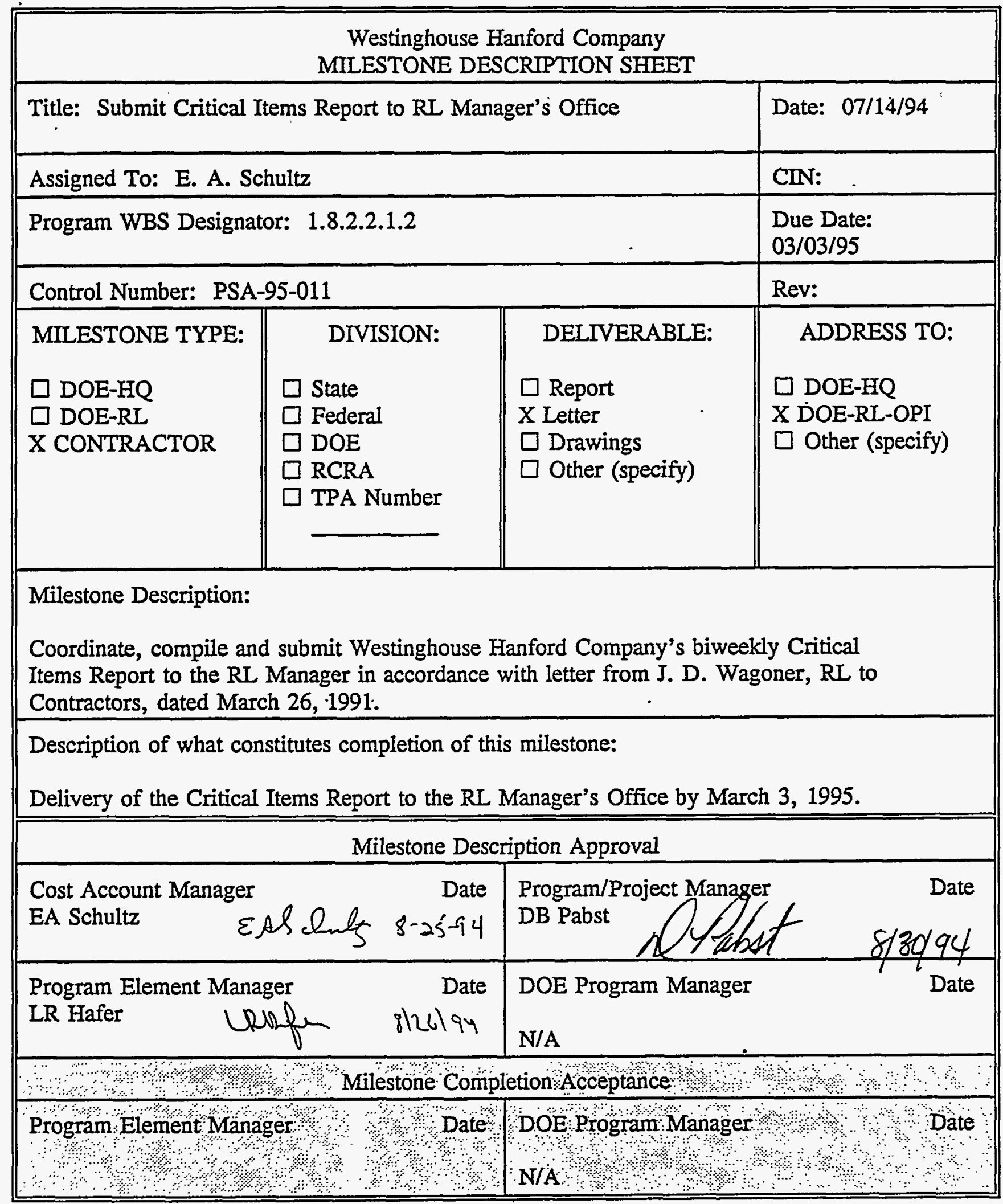




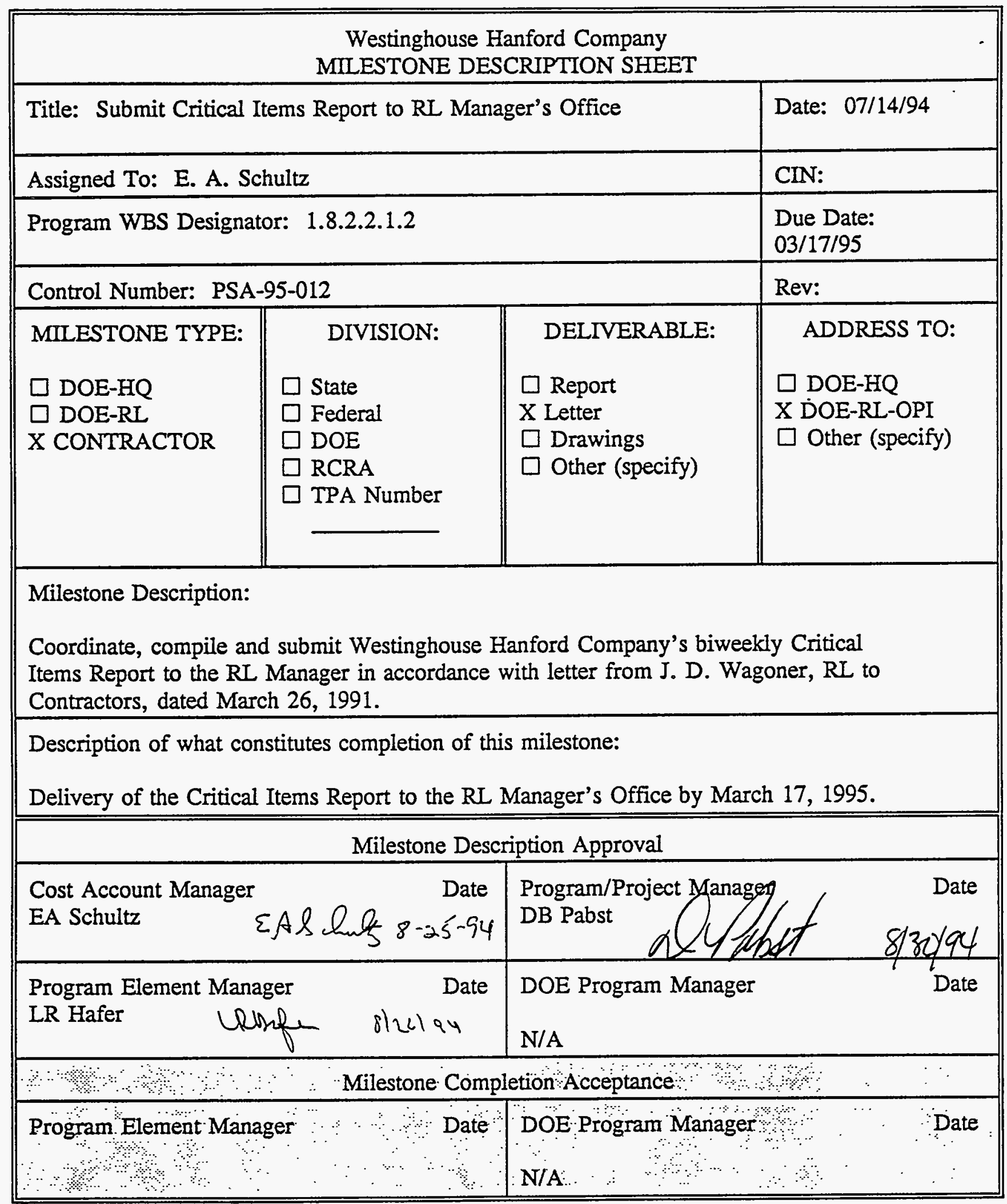




\begin{tabular}{||l||l||}
\hline \multicolumn{3}{|c|}{ Westinghouse Hanford Company } \\
MILESTONE DESCRIPTION SHEET
\end{tabular}




\begin{tabular}{|l||l|l||}
\hline \multicolumn{3}{|c||}{ Westinghouse Hanford Company } \\
MILESTONE DESCRIPTION SHEET
\end{tabular}




\begin{tabular}{|l||l|l||}
\hline \multicolumn{3}{|c|}{ Westinghouse Hanford Company } \\
MILESTONE DESCRIPTION SHEET
\end{tabular}




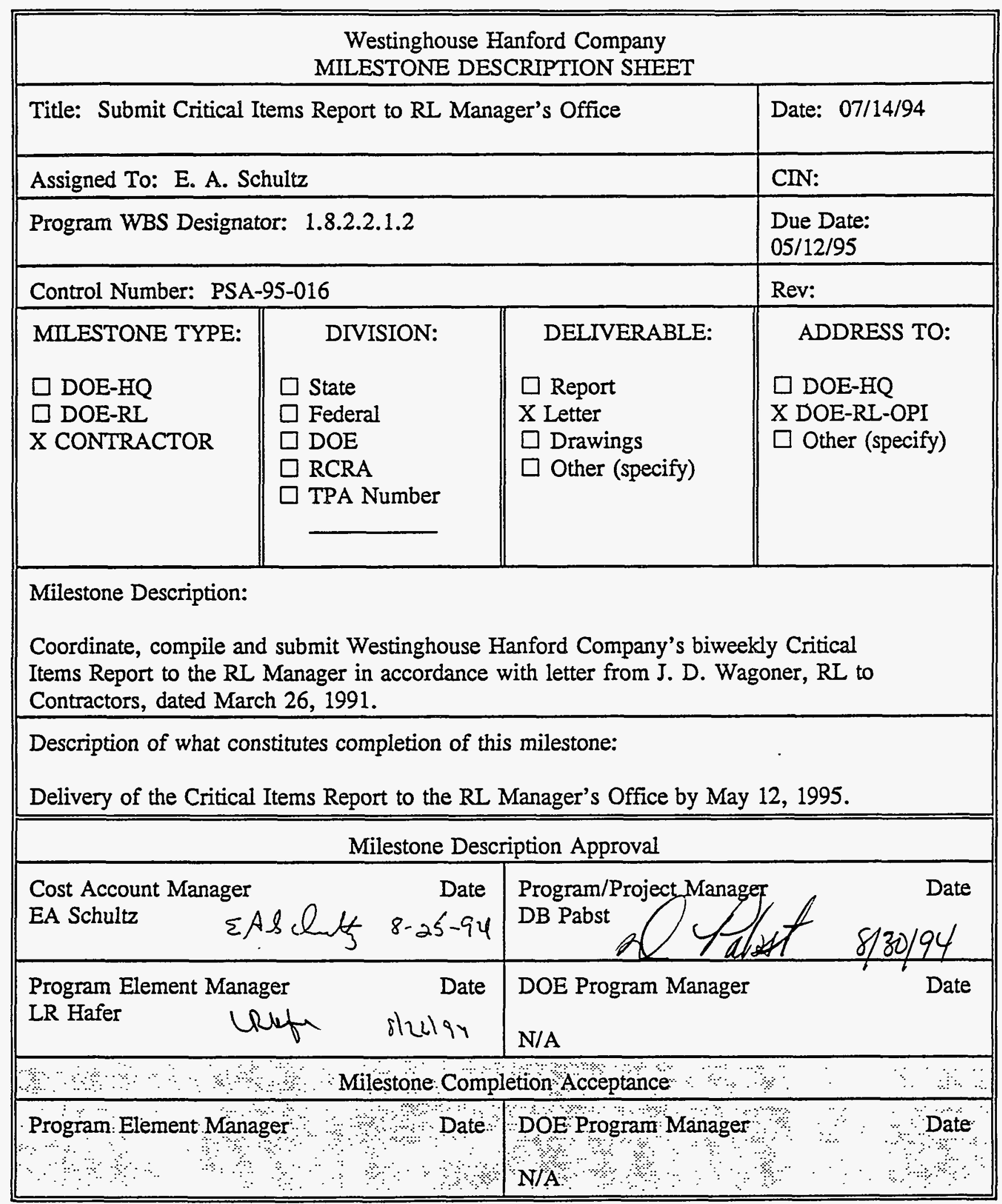




\begin{tabular}{|l||l||}
\hline \multicolumn{3}{|c|}{ Westinghouse Hanford Company } \\
MILESTONE DESCRIPTION SHEET
\end{tabular}




\begin{tabular}{|l|l|l||}
\hline \multicolumn{3}{|c|}{ Westinghouse Hanford Company } \\
MIIESTONE DESCRIPTION SHEET
\end{tabular}




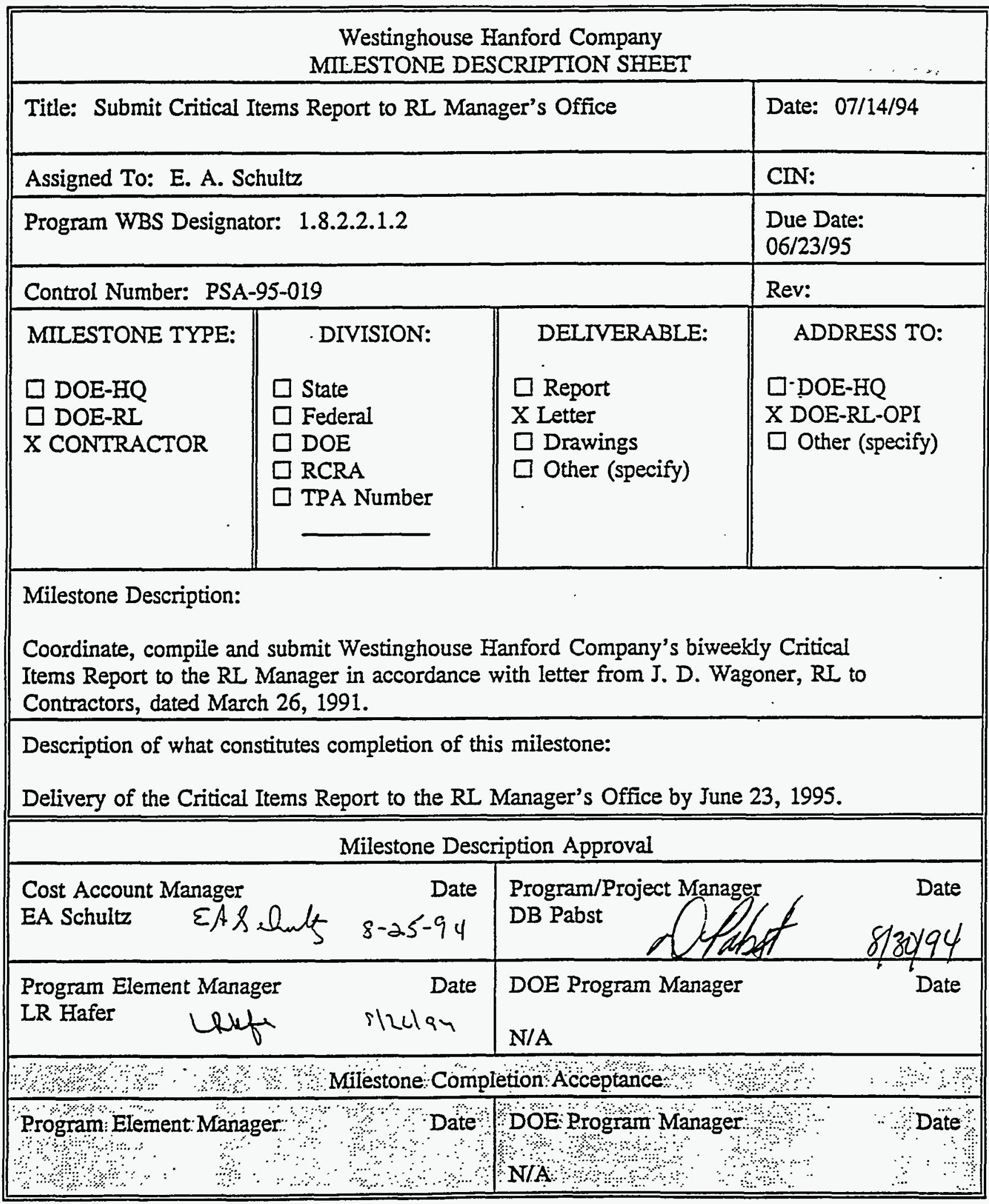




\begin{tabular}{|c|c|c|c|}
\hline \multicolumn{4}{|c|}{$\begin{array}{l}\text { Westinghouse Hanford Company } \\
\text { MILESTONE DESCRIPTION SHEET }\end{array}$} \\
\hline \multicolumn{3}{|c|}{ Title: Submit Critical Items Report to RL Manager's Office } & Date: $07 / 14 / 94$ \\
\hline \multicolumn{3}{|c|}{ Assigned To: E. A. Schultz } & CIN: \\
\hline \multicolumn{3}{|c|}{ Program WBS Designator: 1 8.2.2.1.2 } & $\begin{array}{l}\text { Due Date: } \\
07 / 07 / 95\end{array}$ \\
\hline \multicolumn{3}{|c|}{ Control Number: PSA-95-020 } & Rev: \\
\hline $\begin{array}{l}\text { MIIESTONE TYPE: } \\
\square \text { DOE-HQ } \\
\square \text { DOE-RL } \\
\text { X CONTRACTOR }\end{array}$ & $\begin{array}{l}\quad \text { DIVISION: } \\
\square \text { State } \\
\square \text { Federal } \\
\square \text { DOE } \\
\square \text { RCRA } \\
\square \text { TPA Number }\end{array}$ & $\begin{array}{l}\text { DELIVERABLE: } \\
\square \text { Report } \\
\text { X Letter } \\
\square \text { Drawings } \\
\square \text { Other (specify) }\end{array}$ & $\begin{array}{l}\text { ADDRESS TO: } \\
\square \text { DOE-HQ } \\
\text { X DOE-RL-OPI } \\
\square \text { Other (specify) }\end{array}$ \\
\hline \multicolumn{4}{|c|}{$\begin{array}{l}\text { Milestone Description: } \\
\text { Coordinate, compile and submit Westinghouse Hanford Company's biweekly Critical } \\
\text { Items Report to the RL Manager in accordance with letter from J. D. Wagoner, RL to } \\
\text { Contractors, dated March } 26,1991 \text {. }\end{array}$} \\
\hline \multicolumn{4}{|c|}{$\begin{array}{l}\text { Description of what constitutes completion of this milestone: } \\
\text { Delivery of the Critical Items Report to the RL Manager's Office by July 7, } 1995 .\end{array}$} \\
\hline \multicolumn{4}{|c|}{ Milestone Description Approval } \\
\hline \multicolumn{2}{|c|}{$\begin{array}{lcc}\text { Cost Account Manager } & \text { Date } \\
\text { EA Schultz } & \text { EAlchub5 } & 8-25-94\end{array}$} & \multicolumn{2}{|c|}{$\begin{array}{l}\text { Program/Project Manager } \\
\text { DB Pabst }\end{array}$} \\
\hline \multicolumn{2}{|c|}{$\begin{array}{l}\text { Program Element Manager } \\
\text { LR Hafer } \\
\text { LRUf }\end{array}$} & \multicolumn{2}{|c|}{$\begin{array}{l}\text { DOE Program Manager } \\
\text { N/A }\end{array}$} \\
\hline \multicolumn{4}{|c|}{ Milestone Completion Acceptance } \\
\hline \multicolumn{2}{|c|}{$\begin{array}{c}\text { Program Element Manager } \\
\because \because \\
\ddots\end{array}$} & \multicolumn{2}{|c|}{$\begin{array}{l}\text { DOE Program Manager } \\
\text { N/A }\end{array}$} \\
\hline
\end{tabular}




\begin{tabular}{||l|l|l||}
\hline \multicolumn{3}{|c|}{ Westinghouse Hanford Company } \\
MILESTONE DESCRIPTION SHEET
\end{tabular}




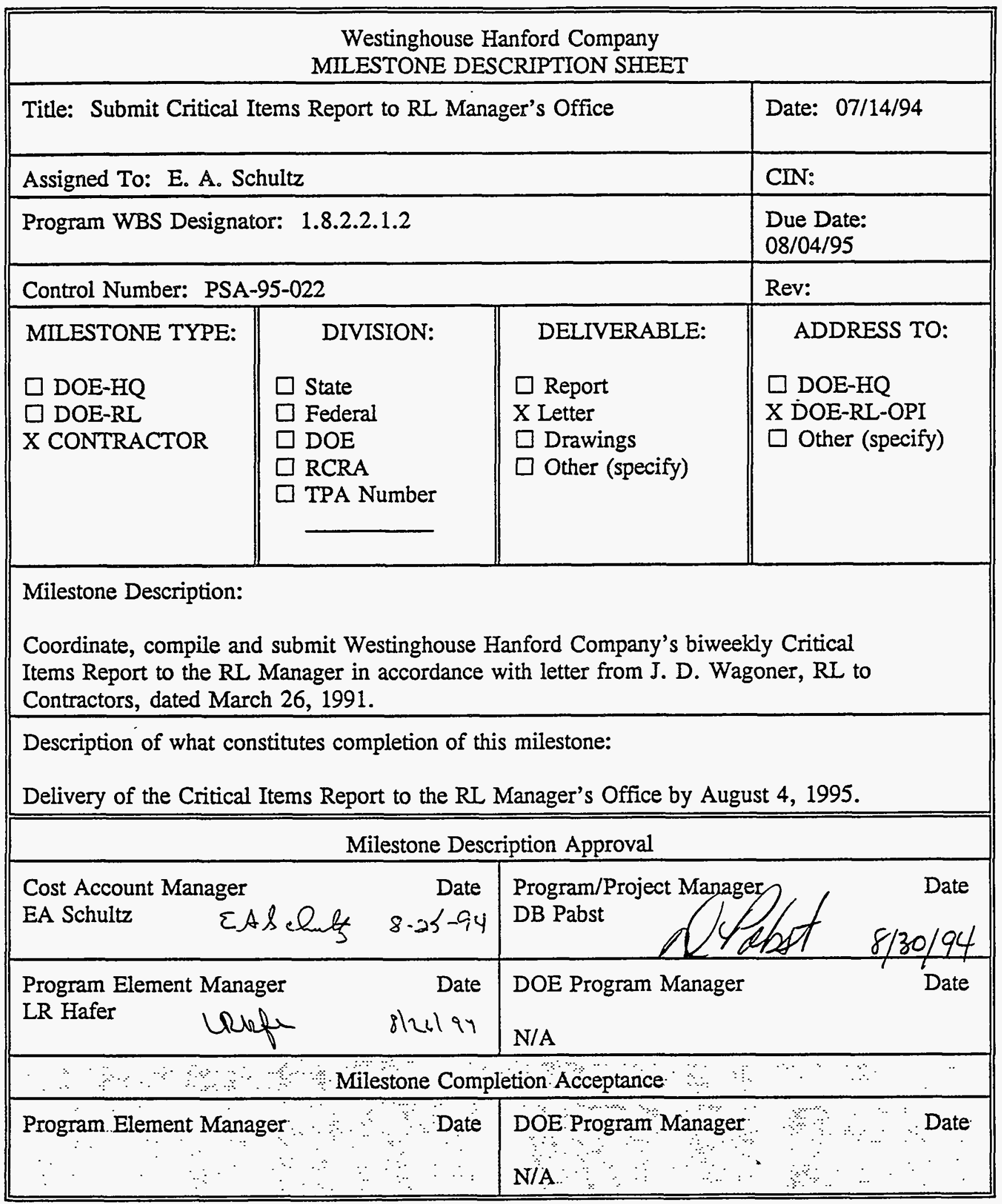




\begin{tabular}{|l||l|||}
\hline \multicolumn{3}{|c|}{ Westinghouse Hanford Company } \\
MILESTONE DESCRIPTION SHEET
\end{tabular}




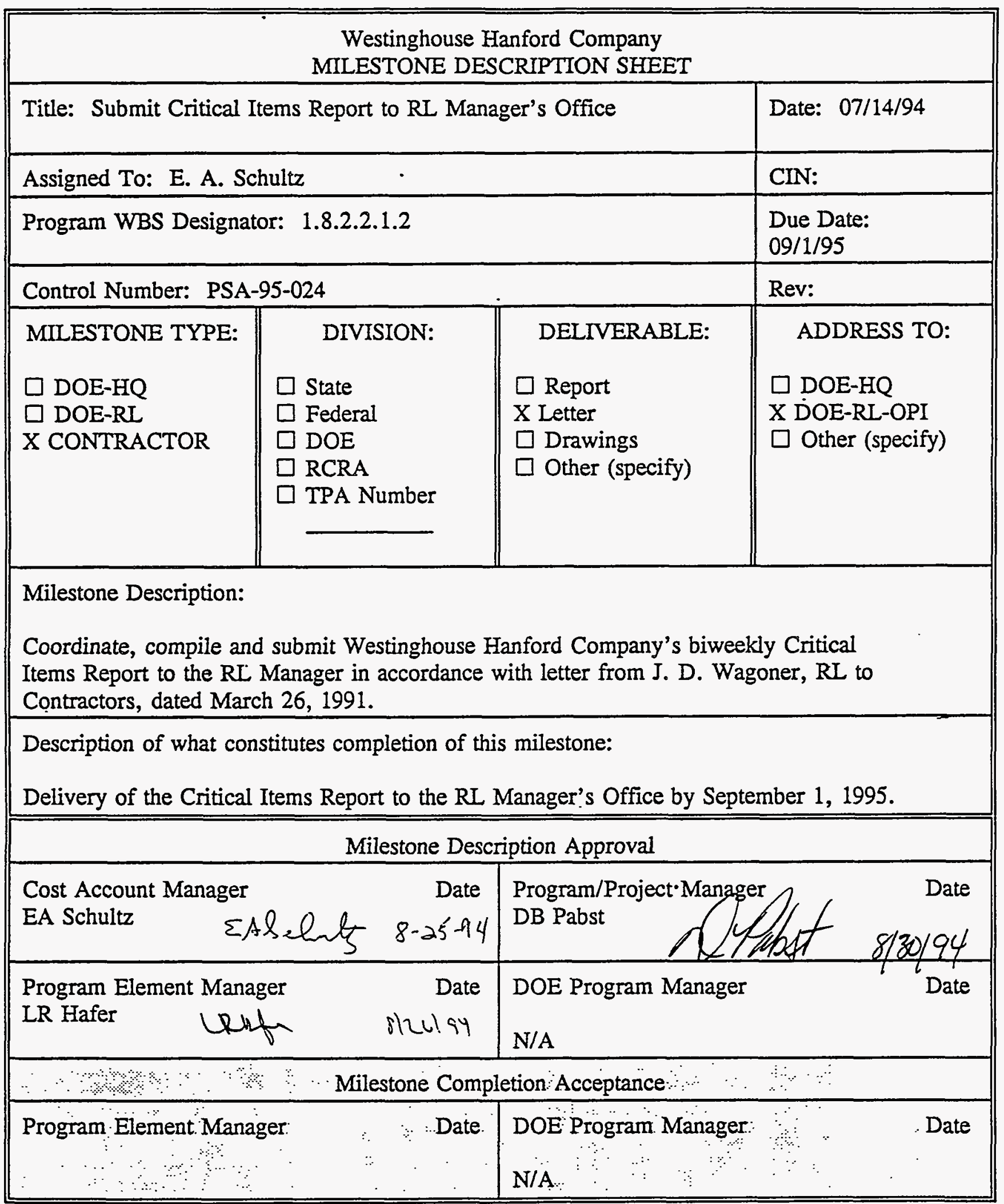




\begin{tabular}{||l||l||}
\hline \multicolumn{3}{|c||}{ Westinghouse Hanford Company } \\
MILSTONE DESCRIPTION SHEET
\end{tabular}




\begin{tabular}{|c|c|c|c|}
\hline \multicolumn{4}{|c|}{$\begin{array}{l}\text { Westinghouse Hanford Company } \\
\text { MILESTONE DESCRIPTION SHEET }\end{array}$} \\
\hline \multicolumn{3}{|c|}{ Title: Submit Critical Items Report to RL Manager's Office } & Date: $07 / 14 / 94$ \\
\hline \multicolumn{3}{|c|}{ Assigned To: E. A. Schultz } & CIN: \\
\hline \multicolumn{3}{|c|}{ Program WBS Designator: 1.8.2.2.1.2 } & $\begin{array}{l}\text { Due Date: } \\
\text { 09/29/95 }\end{array}$ \\
\hline \multicolumn{3}{|c|}{ Control Number: PSA-95-026 } & Rev: \\
\hline $\begin{array}{l}\text { MILESTONE TYPE: } \\
\square \text { DOE-HQ } \\
\square \text { DOE-RL } \\
\text { X CONTRACTOR }\end{array}$ & $\begin{array}{l}\quad \text { DIVISION: } \\
\square \text { State } \\
\square \text { Federal } \\
\square \text { DOE } \\
\square \text { RCRA } \\
\square \text { TPA Number }\end{array}$ & $\begin{array}{l}\text { DELIVERABLE: } \\
\square \text { Report } \\
\text { X Letter } \\
\square \text { Drawings } \\
\square \text { Other (specify) }\end{array}$ & $\begin{array}{l}\text { ADDRESS TO: } \\
\square \text { DOE-HQ } \\
\text { X DOE-RL-OPI } \\
\square \text { Other (specify) }\end{array}$ \\
\hline \multicolumn{4}{|c|}{$\begin{array}{l}\text { Coordinate, compile and submit Westinghouse Hanford Company's biweekly Critical } \\
\text { Items Report to the RL Manager in accordance with letter from J. D. Wagoner, RL to } \\
\text { Contractors, dated March 26, } 1991 \text {. }\end{array}$} \\
\hline \multicolumn{4}{|c|}{$\begin{array}{l}\text { Description of what constitutes completion of this milestone: } \\
\text { Delivery of the Critical Items Report to the RL Manager's Office by September 29, } 1995 .\end{array}$} \\
\hline \multicolumn{4}{|c|}{ Milestone Description Approval } \\
\hline \multicolumn{2}{|c|}{ 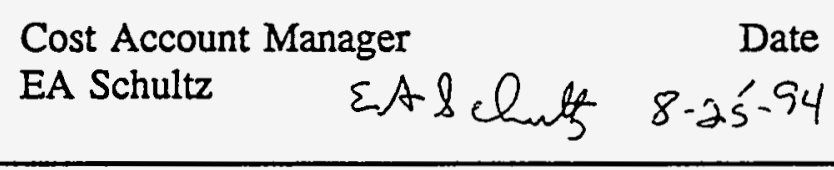 } & \multicolumn{2}{|c|}{$\begin{array}{l}\text { Program/Project Manager } \\
\text { DB Pabst }\end{array}$} \\
\hline \multicolumn{2}{|c|}{$\begin{array}{l}\text { Program Element Manager } \\
\text { LR Hafer Unify }\end{array}$} & \multirow{2}{*}{$\begin{array}{l}\text { DOE Program Manager } \\
\text { N/A } \\
\text { etion Acceptance : }\end{array}$} & Date \\
\hline 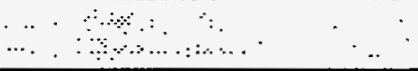 & Milestone Comp & & . \\
\hline \multicolumn{2}{|c|}{ 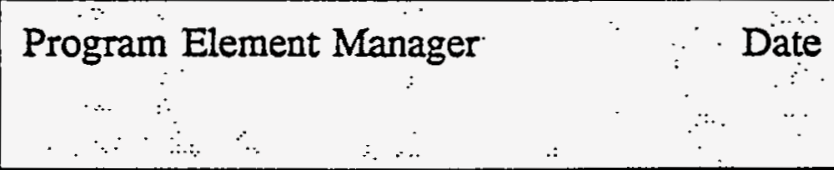 } & \multicolumn{2}{|c|}{$\begin{array}{l}\text { DOE Program Manager } \\
\text { N/A }\end{array}$} \\
\hline
\end{tabular}




\begin{tabular}{|c|c|c|c|}
\hline \multicolumn{4}{|c|}{$\begin{array}{l}\text { Westinghouse Hanford Company } \\
\text { MILESTONE DESCRIPTION SHEET }\end{array}$} \\
\hline \multicolumn{3}{|c|}{$\begin{array}{l}\text { Title: Complete Fiscal Year } 1996 \text { Microcomputer Statement of } \\
\text { Strategy (SOS) }\end{array}$} & Date: $07 / 25 / 94$ \\
\hline \multicolumn{3}{|c|}{ Assigned To: EA Schultz } & CIN: \\
\hline \multicolumn{3}{|c|}{ Program WBS Designator: 1 8.2.2.1.5 } & $\begin{array}{l}\text { Due Date: } \\
07 / 31 / 95\end{array}$ \\
\hline \multicolumn{3}{|c|}{ Control Number: PSA-95-029 } & Rev: \\
\hline $\begin{array}{l}\text { MILESTONE TYPE: } \\
\square \text { DOE-HQ } \\
\square \text { DOE-RL } \\
\text { X CONTRACTOR }\end{array}$ & $\begin{aligned} & \text { DIVISION: } \\
& \square \text { State } \\
& \square \text { Federal } \\
& \square \text { DOE } \\
& \square \text { RCRA } \\
& \text { TPA Number }\end{aligned}$ & $\begin{array}{l}\text { DELIVERABLE: } \\
\text { X Report } \\
\square \text { Letter } \\
\square \text { Drawings } \\
\square \text { Other (specify) }\end{array}$ & $\begin{array}{l}\text { ADDRESS TO: } \\
\square \text { DOE-HQ } \\
\square \text { DOE-RL-OPI } \\
\text { X Other (specify) } \\
\text { IRM }\end{array}$ \\
\hline \multicolumn{4}{|c|}{$\begin{array}{l}\text { Milestone Description: } \\
\text { The SOS is the primary WHC short-range planning document for ADPE hardware and software } \\
\text { costing less than } \$ 25,000 \text {. The Statement of Strategy is responsive to DOE Order RL-1360.1b } \\
\text { and is an acquisition plan for all ADPE included. }\end{array}$} \\
\hline \multicolumn{4}{|c|}{$\begin{array}{l}\text { Description of what constitutes completion of this milestone: } \\
\text { This milestone will be complete when prepared and submitted to IRM on July } 31,1995 .\end{array}$} \\
\hline \multicolumn{4}{|c|}{ Milestone Description Approval } \\
\hline \multicolumn{2}{|c|}{$\begin{array}{ll}\text { Cost Account Manager } \\
\text { JE Tarcza }\end{array}$} & \multicolumn{2}{|c|}{ Program/Project Manager $8 / 25 / 94$} \\
\hline \multicolumn{2}{|c|}{$\begin{array}{l}\text { Program Element Dater } \\
\text { LR Hafer LRIsf }\end{array}$} & $\begin{array}{l}\text { DOE Program Manager } \\
\text { N/A }\end{array}$ & Date \\
\hline \multicolumn{4}{|c|}{ 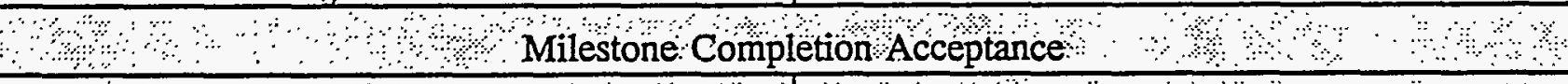 } \\
\hline \multicolumn{2}{|c|}{ Program Element Manager } & \multicolumn{2}{|c|}{ DOE Program Manager } \\
\hline
\end{tabular}




\begin{tabular}{|l||l|l||}
\hline \multicolumn{3}{|c|}{ Westinghouse Hanford Company } \\
MILESTONE DESCRIPTION SHEET
\end{tabular}




\begin{tabular}{||l|l|l||}
\hline \multicolumn{3}{|c|}{ Westinghouse Hanford Company } \\
MILESTONE DESCRIPTION SHEET
\end{tabular}




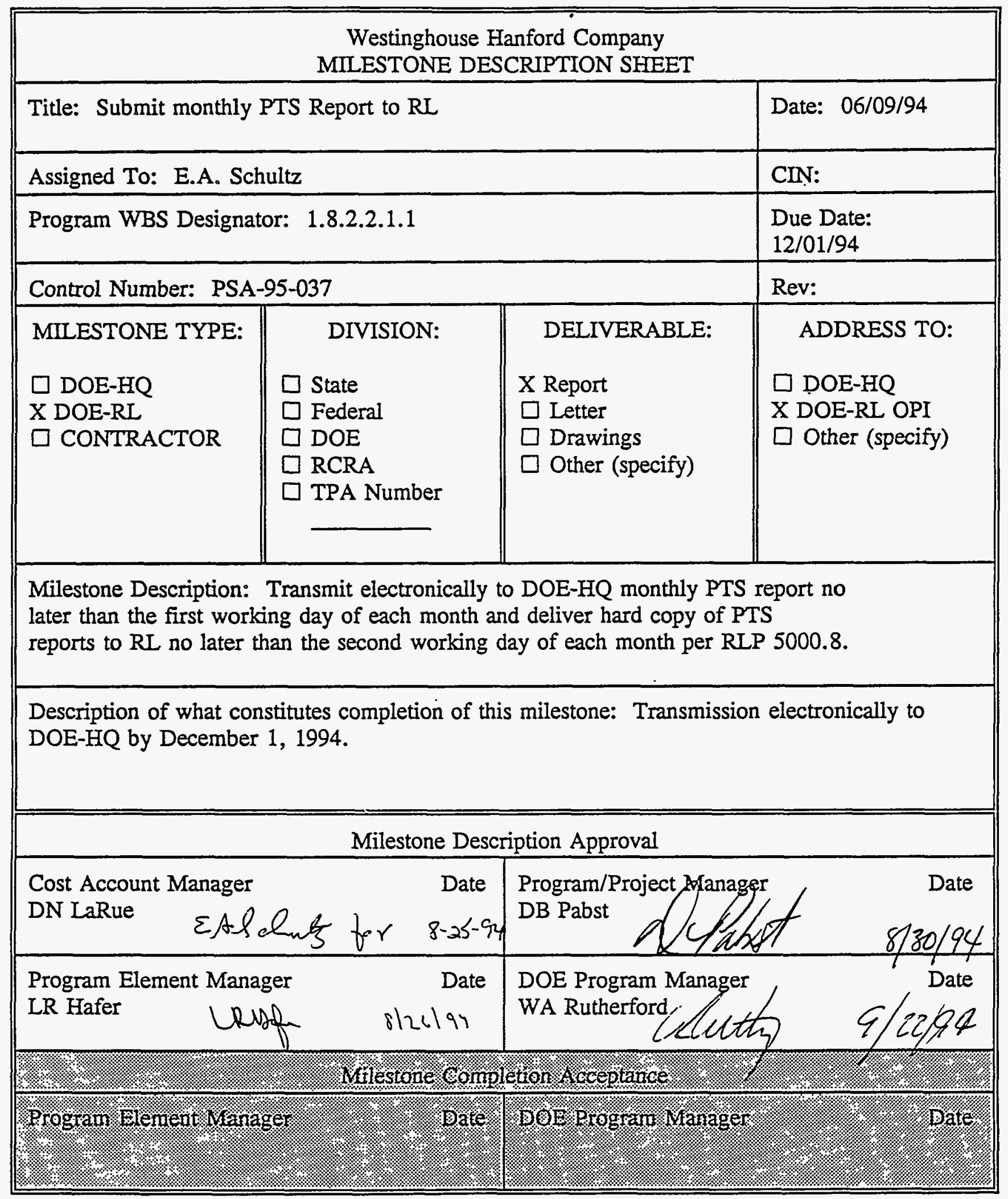




\begin{tabular}{||l||l||}
\hline \multicolumn{3}{|c|}{ Westinghouse Hanford Company } \\
MILESTONE DESCRIPTION SHEET
\end{tabular}




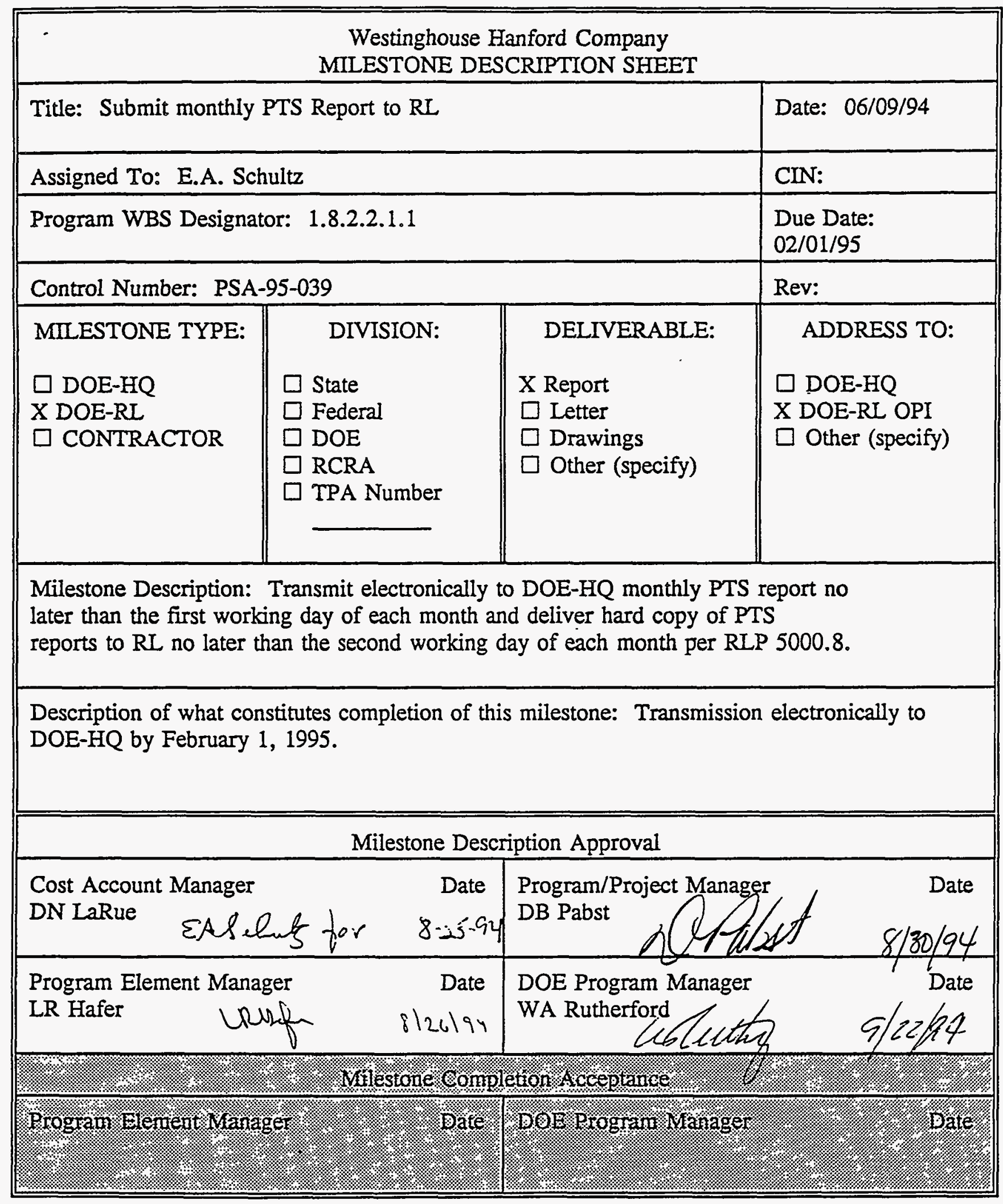




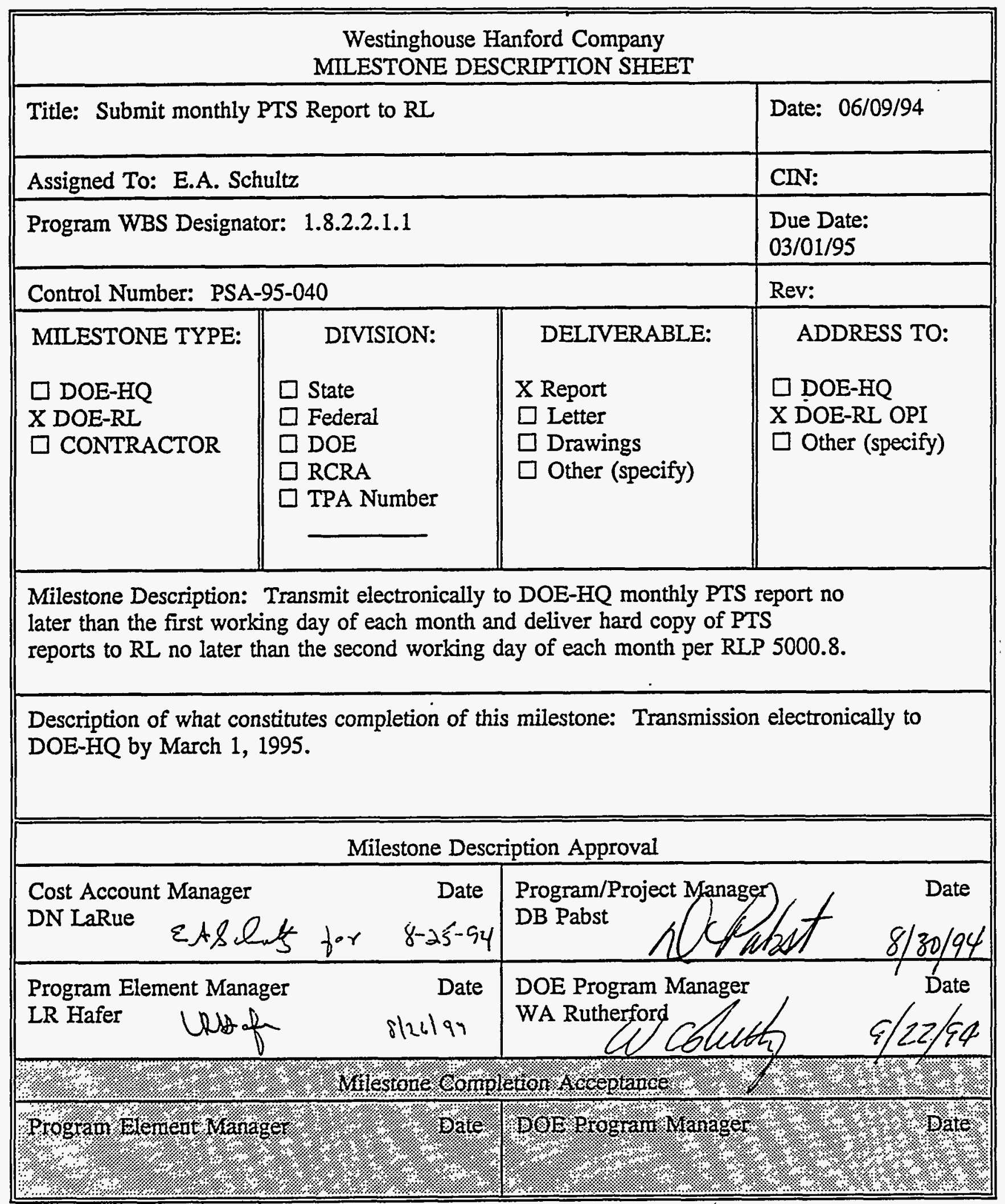




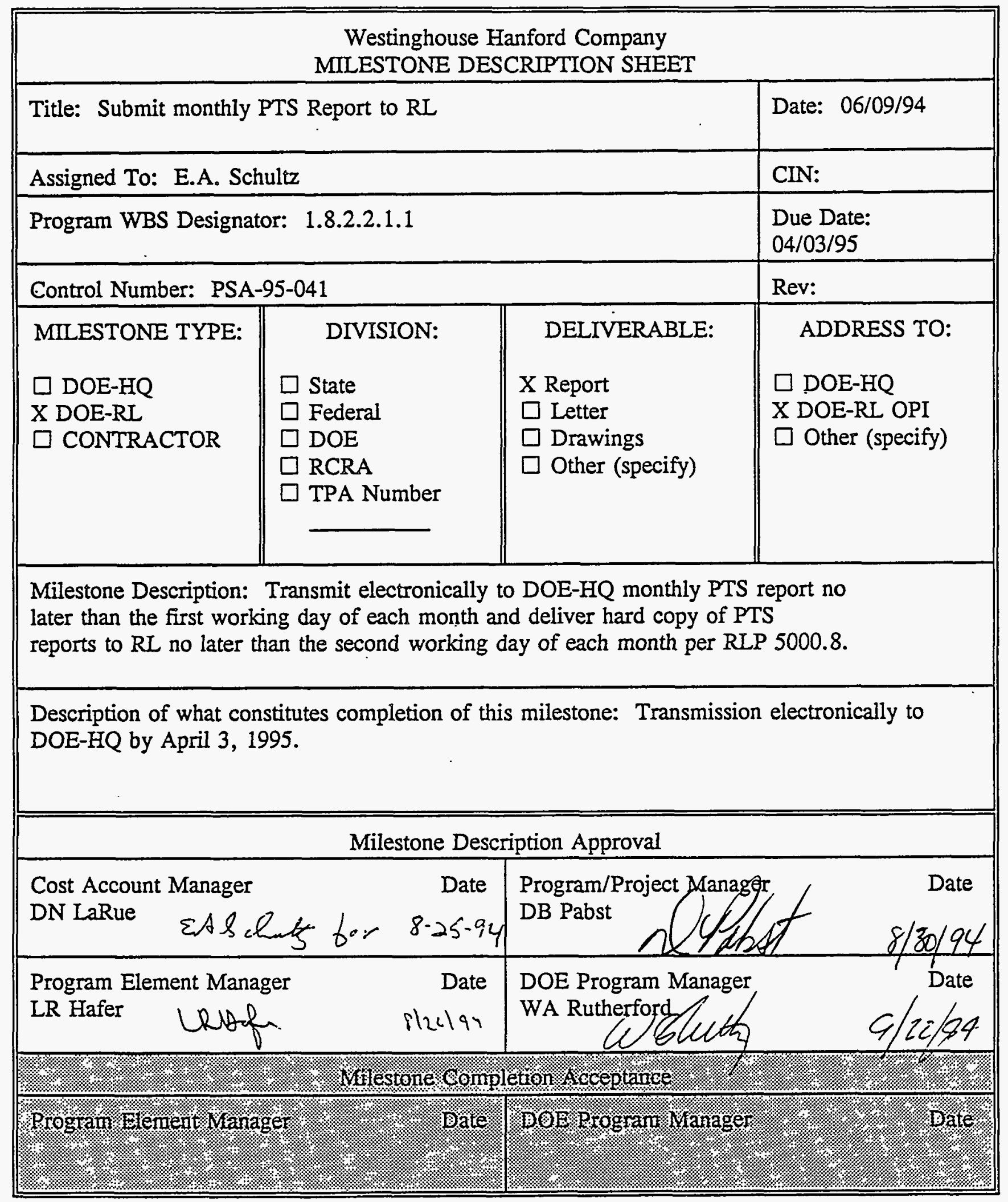




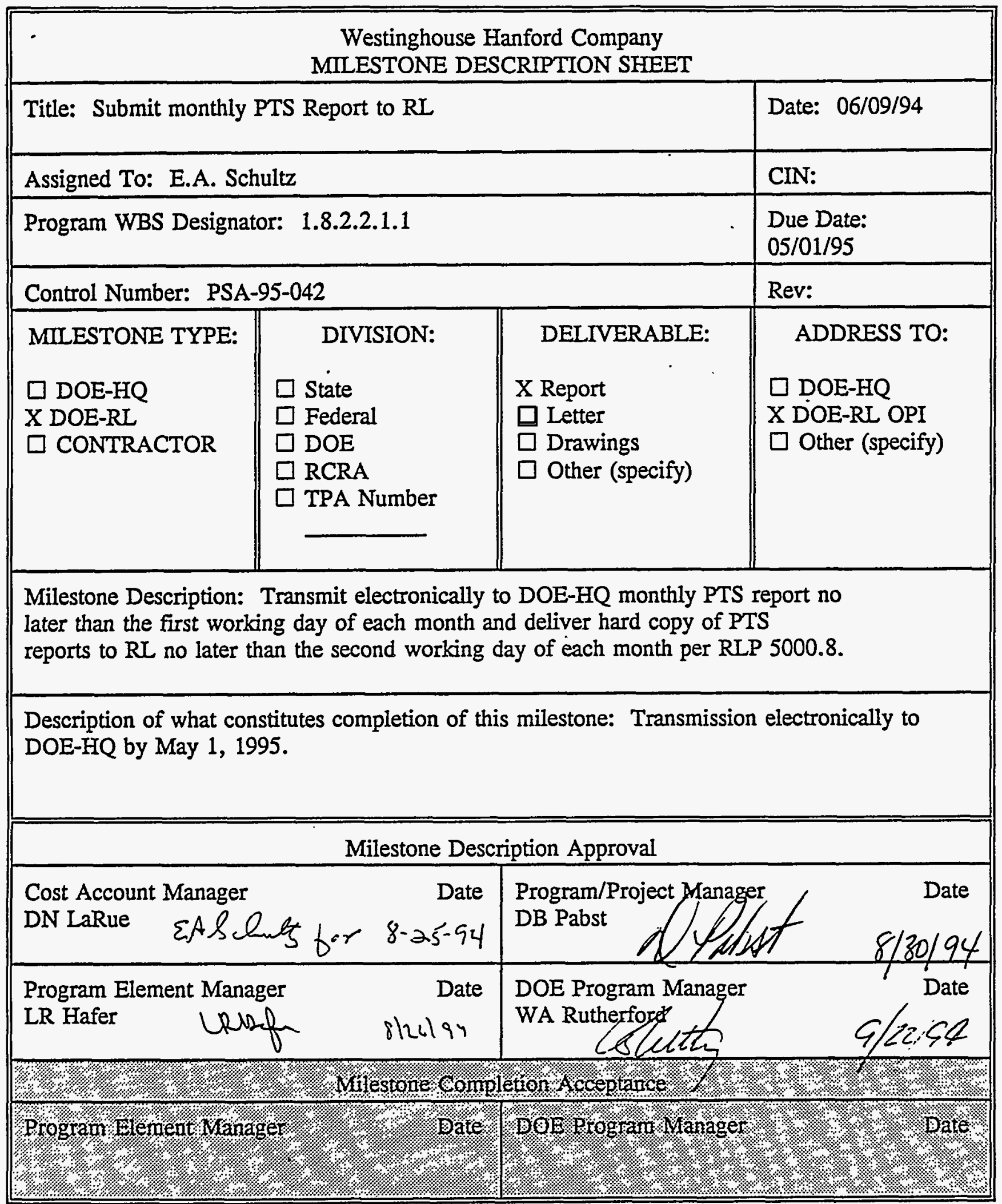




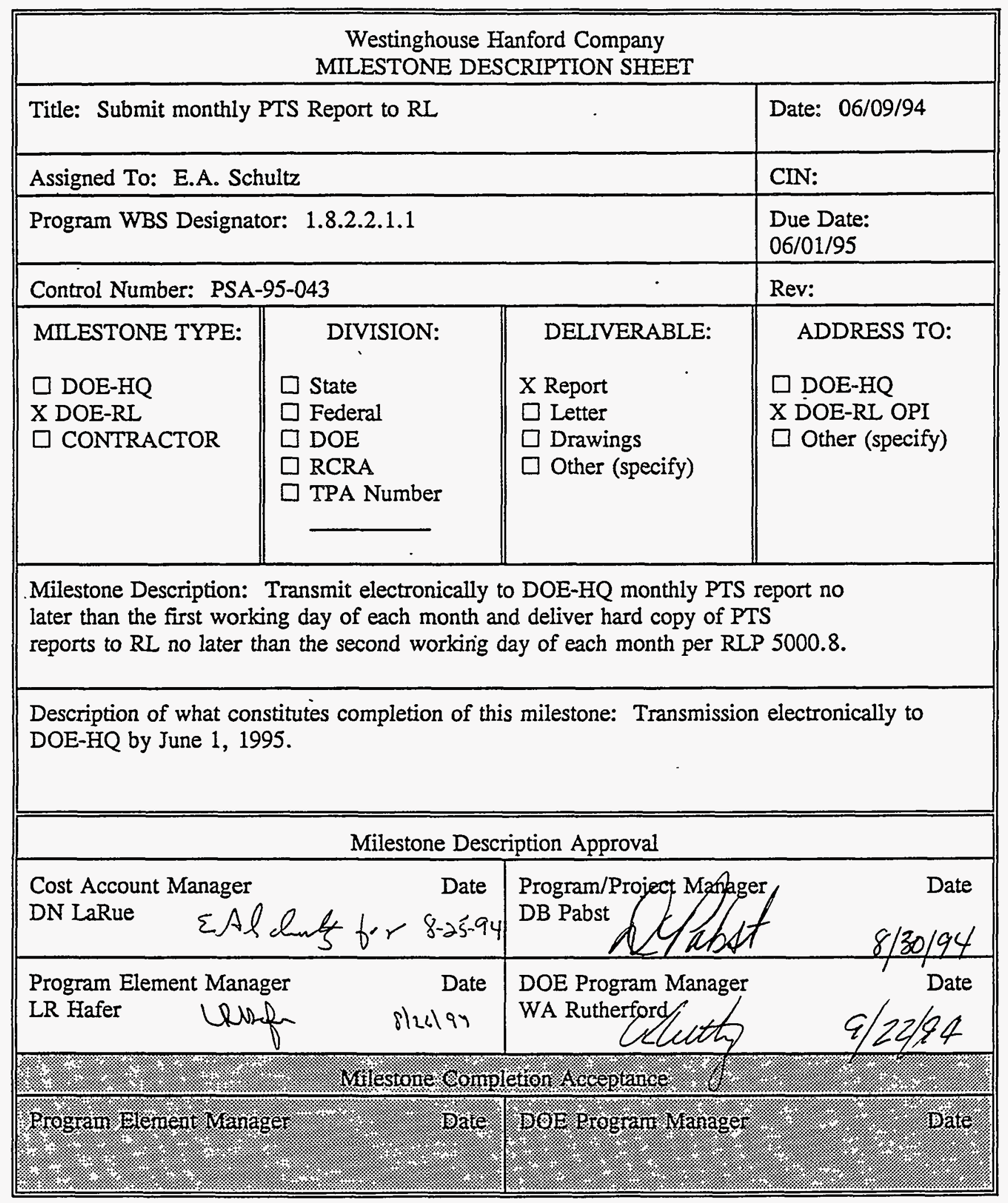




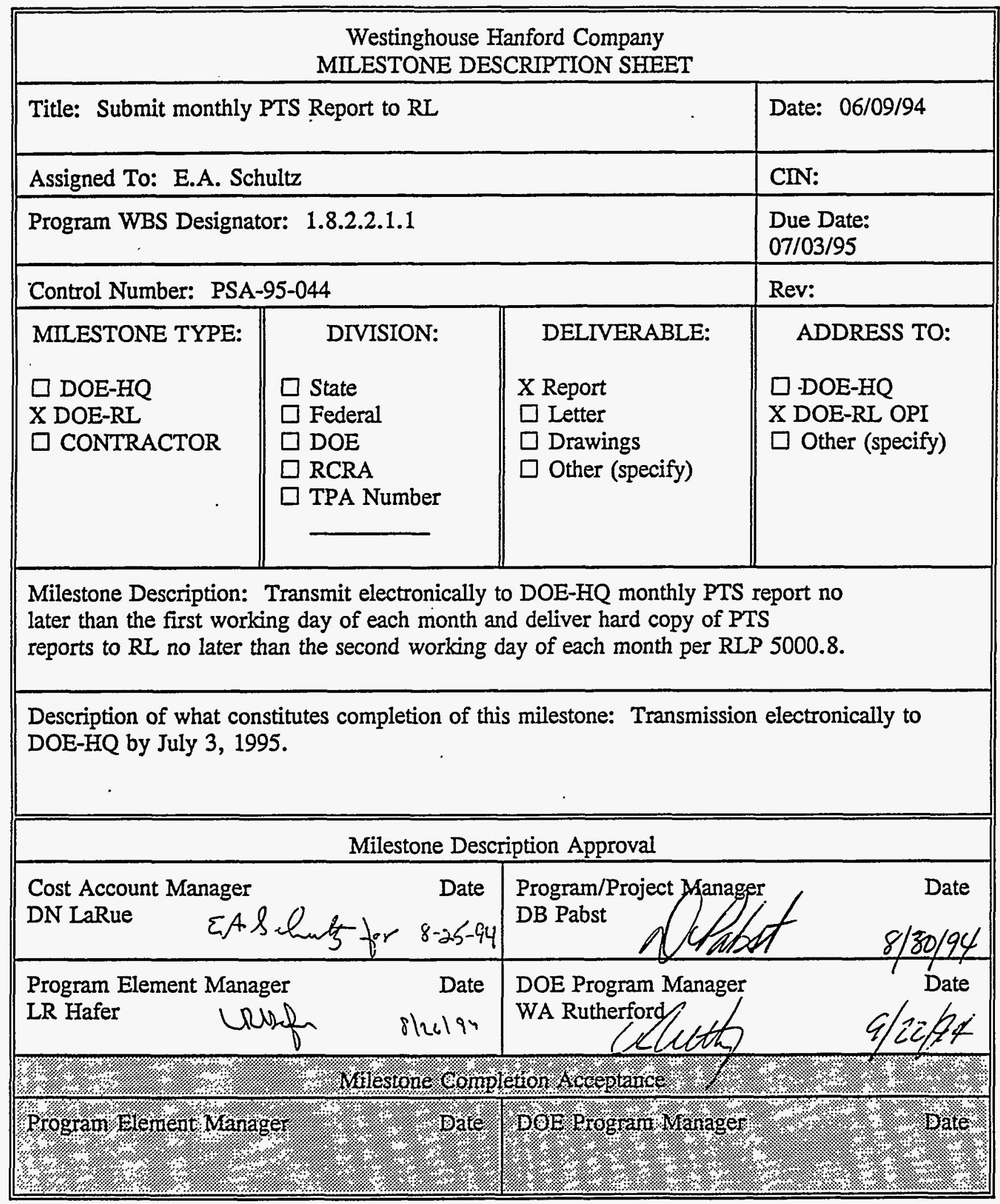




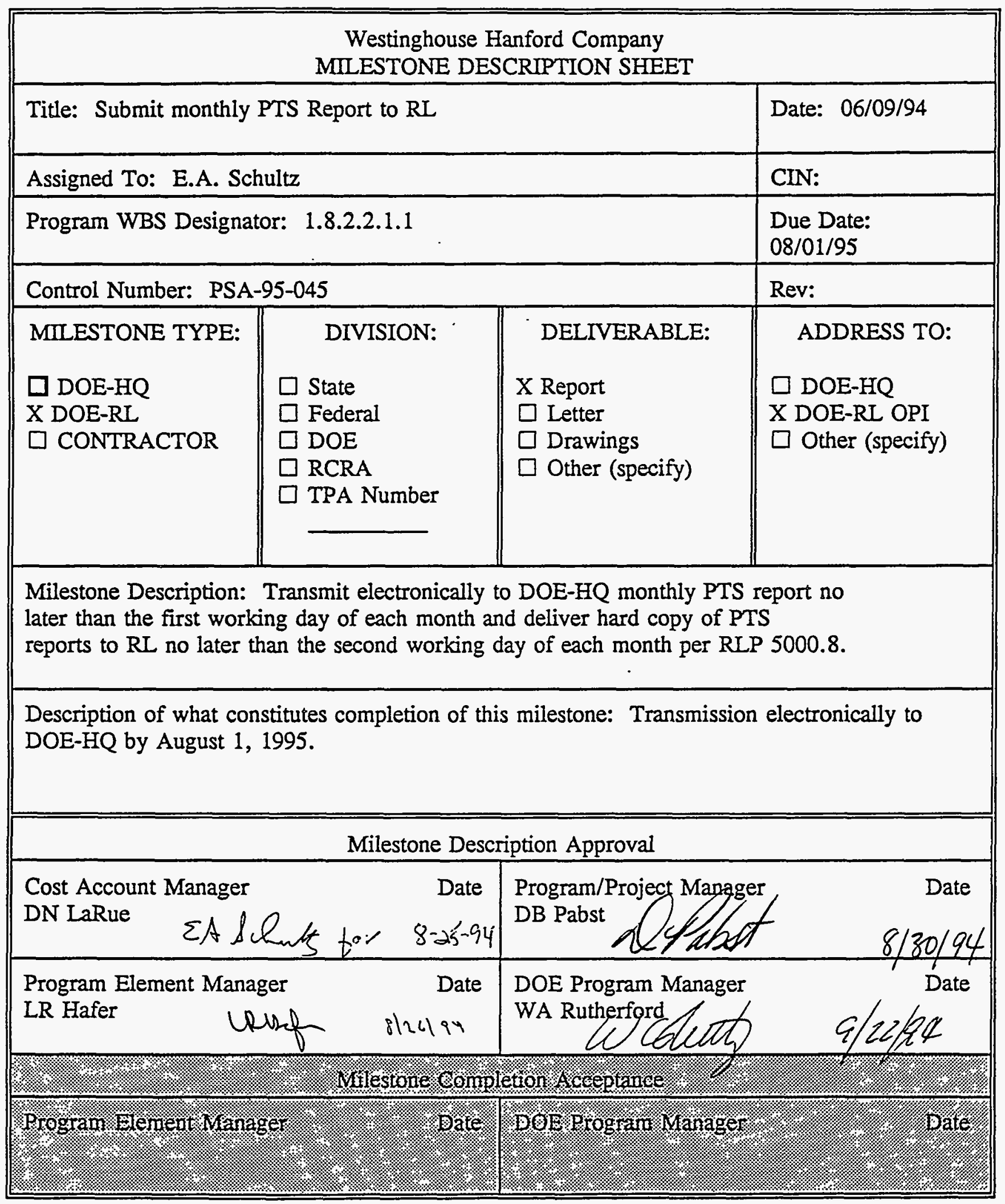




\begin{tabular}{|l|l|l||}
\hline \multicolumn{3}{|c|}{ Westinghouse Hanford Company } \\
MILESTONE DESCRIPTION SHEET
\end{tabular}




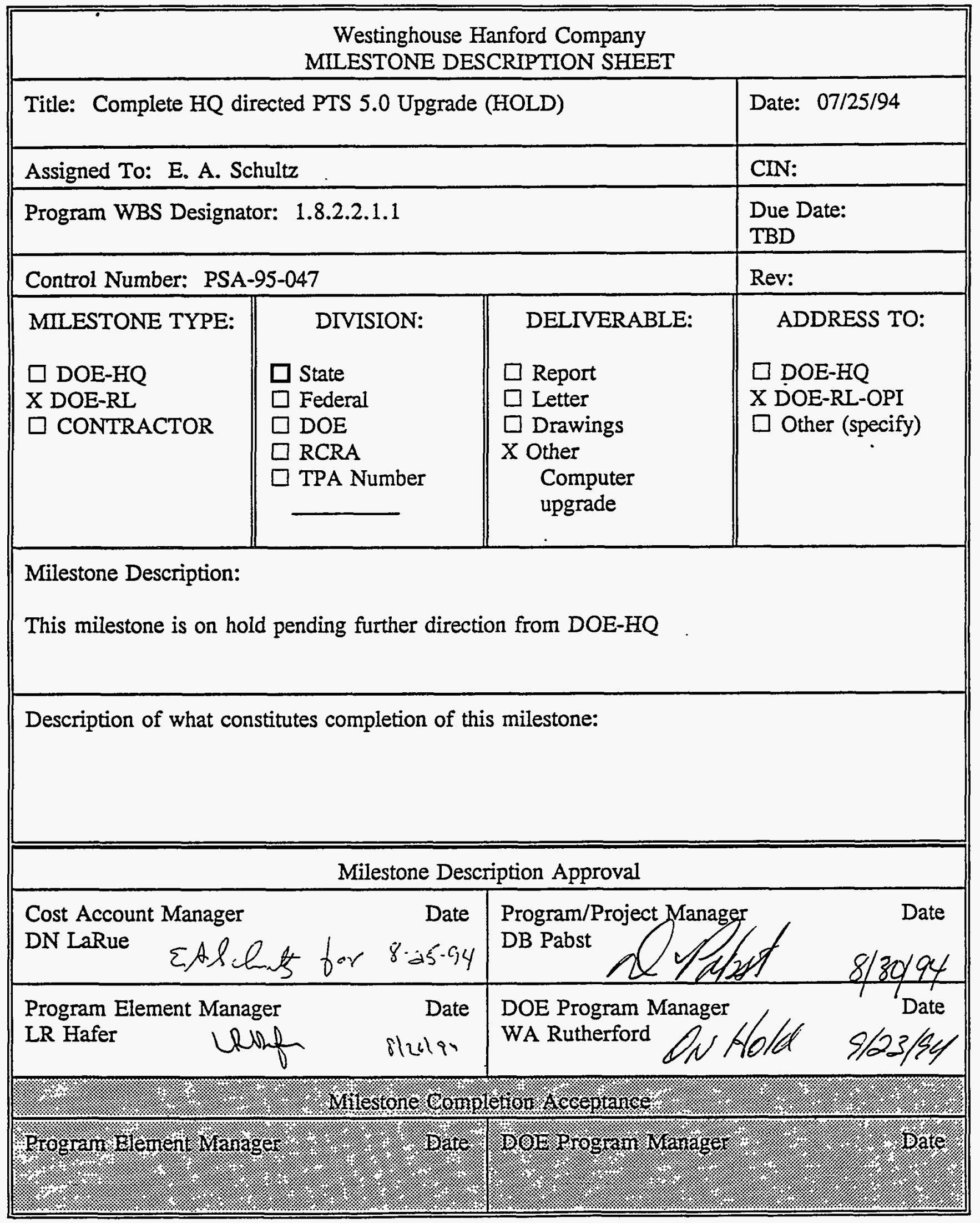




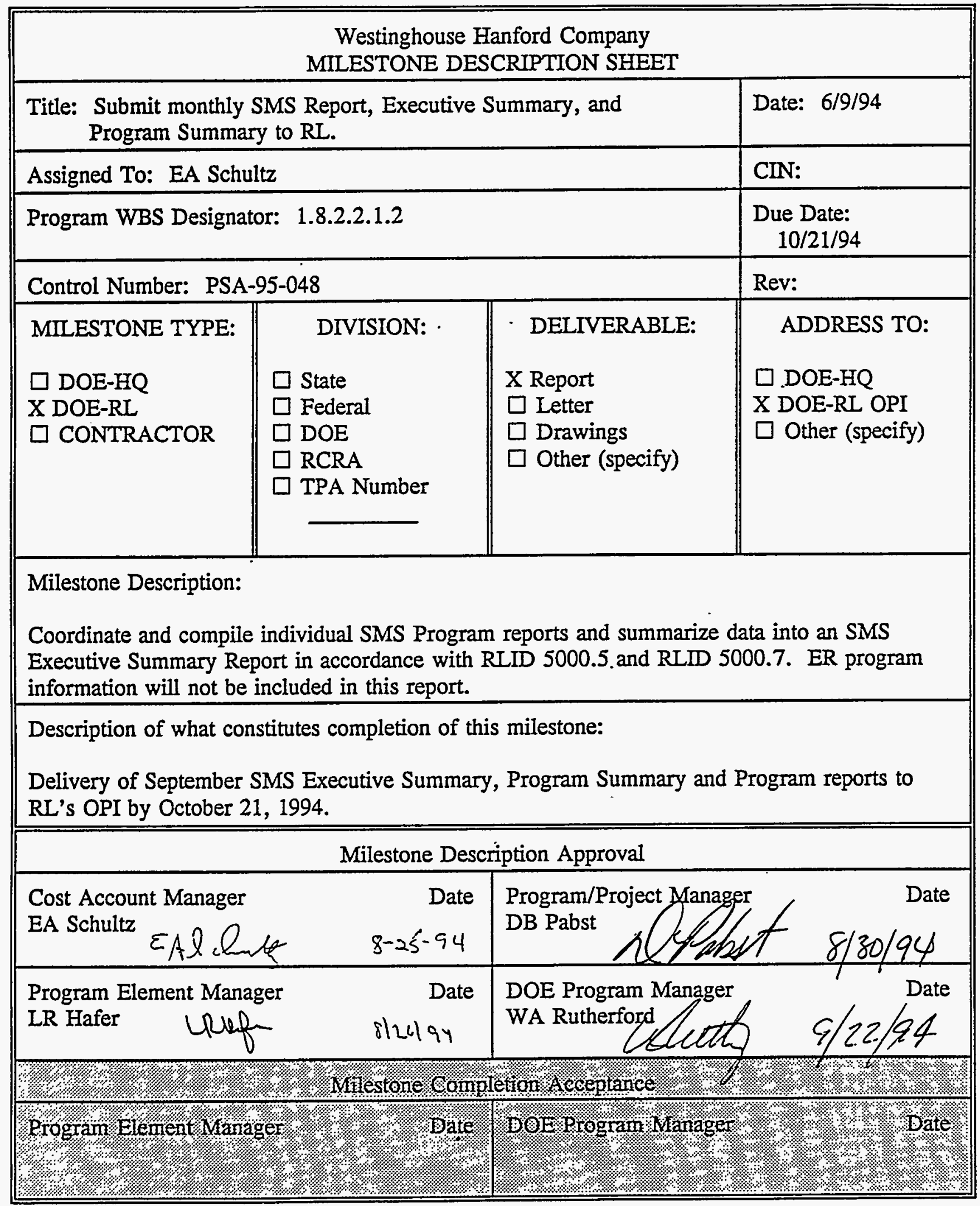




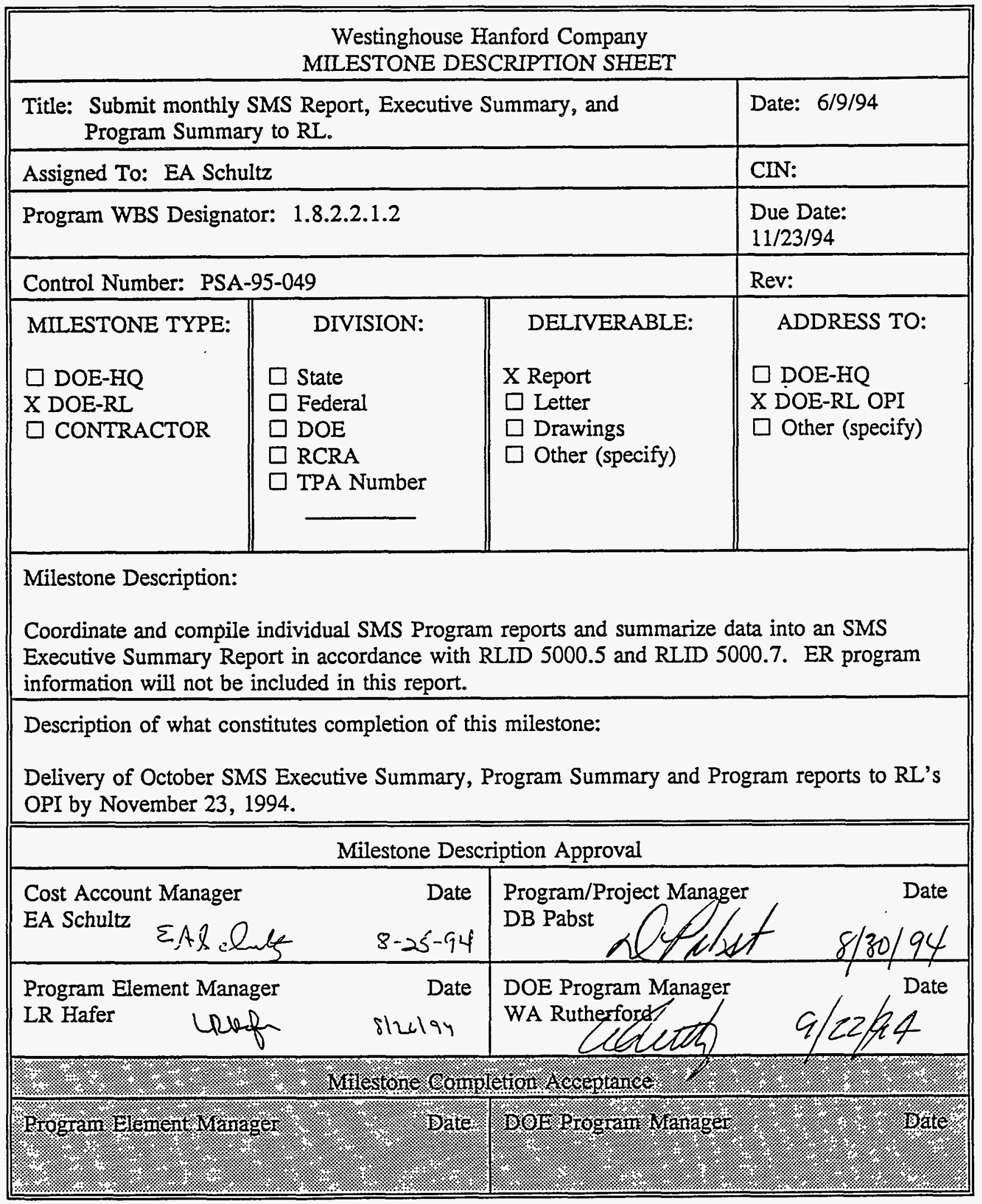




\begin{tabular}{|l||l|l||}
\hline \multicolumn{3}{|c||}{ Westinghouse Hanford Company } \\
MILESTONE DESCRIPTION SHEET
\end{tabular}




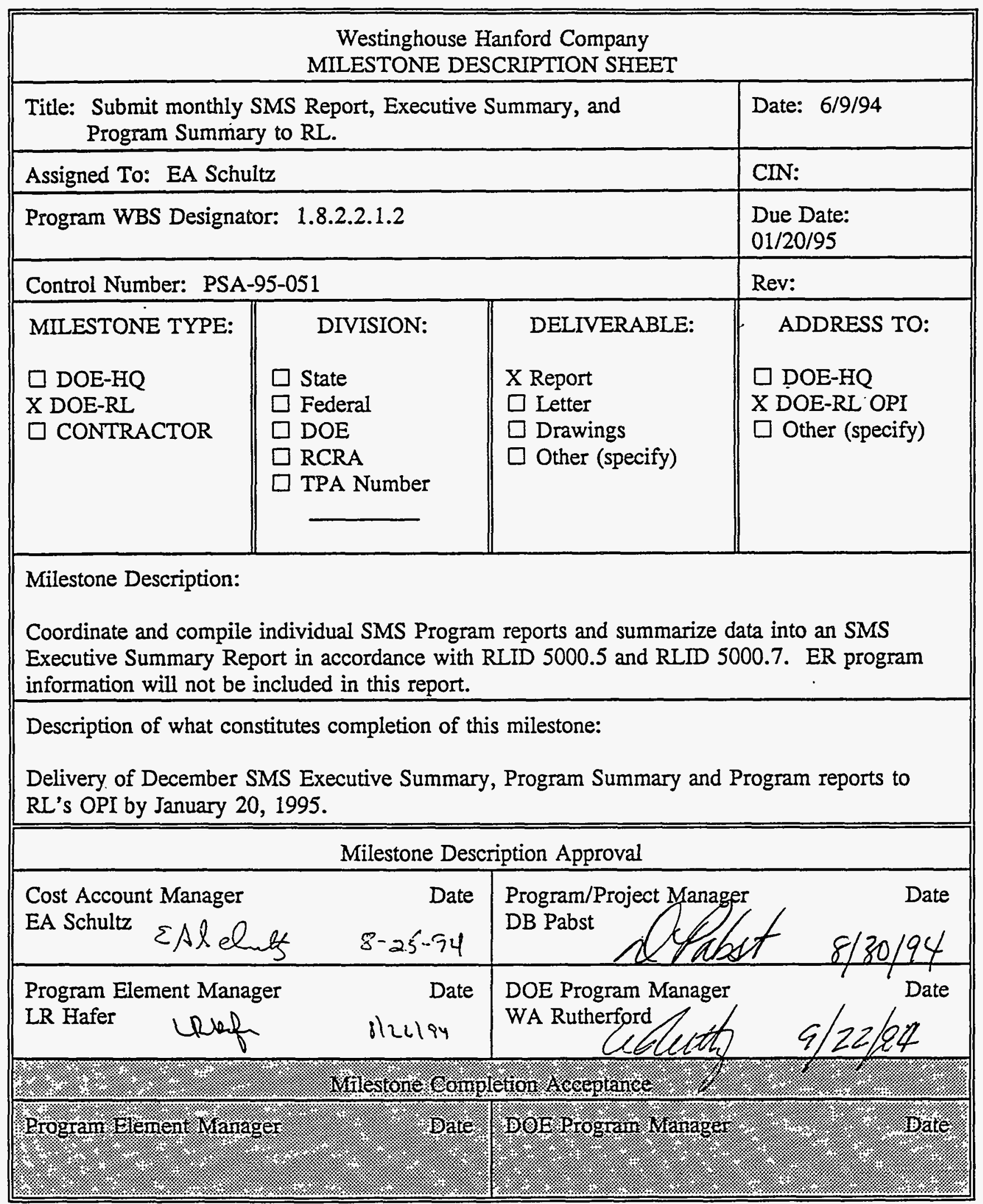




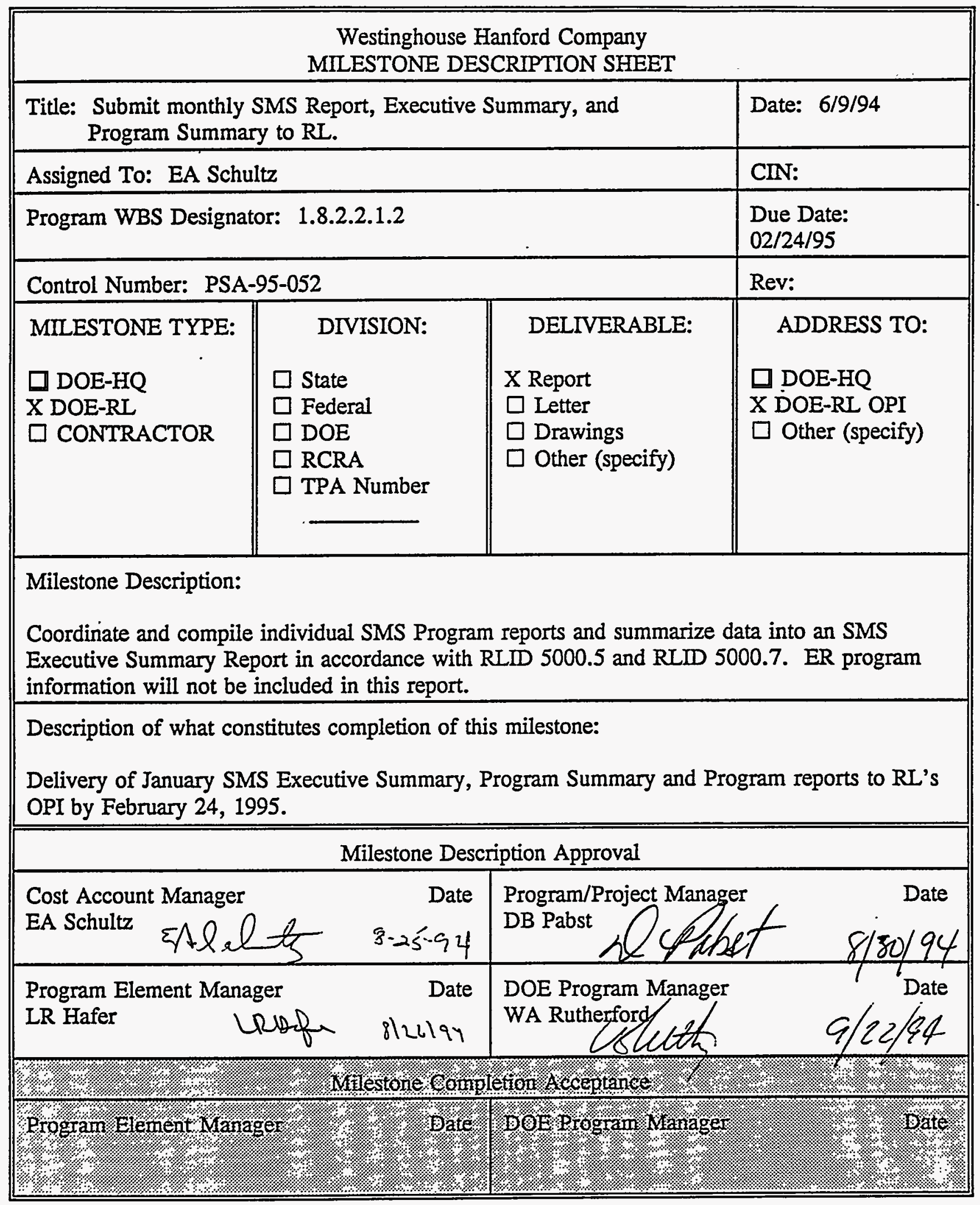




\begin{tabular}{|l|l|l||}
\hline \multicolumn{3}{|c|}{ Westinghouse Hanford Company } \\
MILSTONE DESCRIPTION SHEET
\end{tabular}




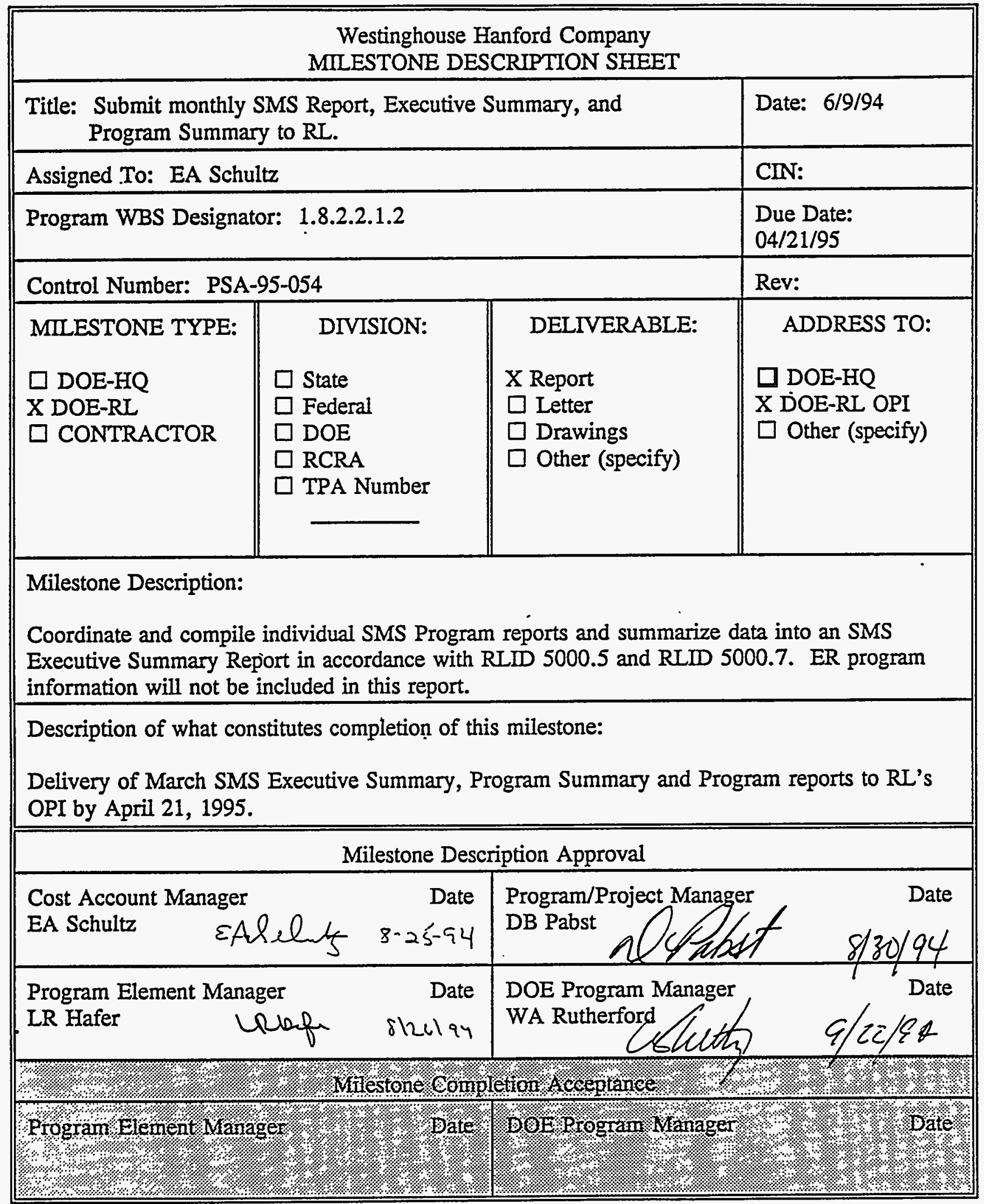




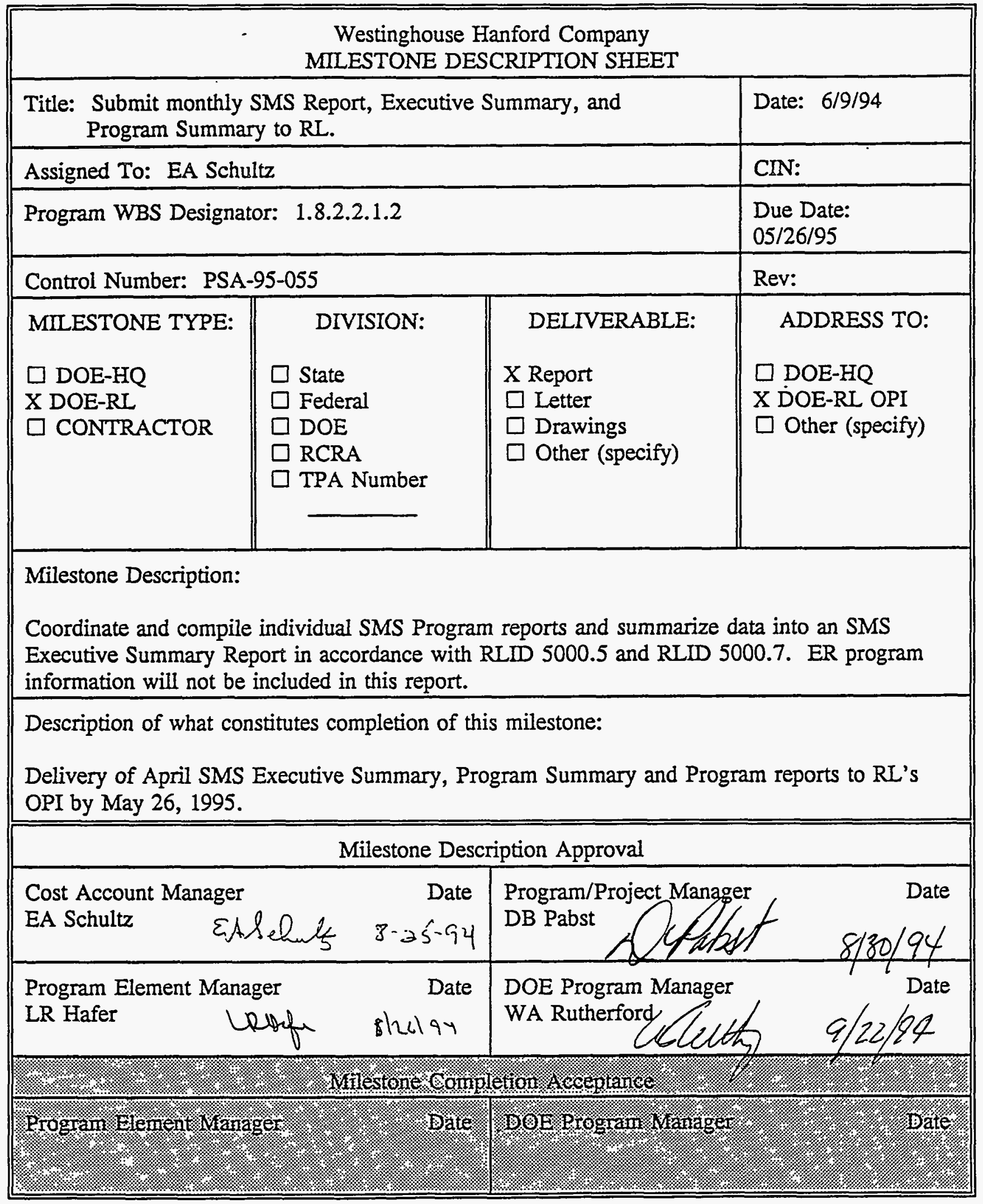




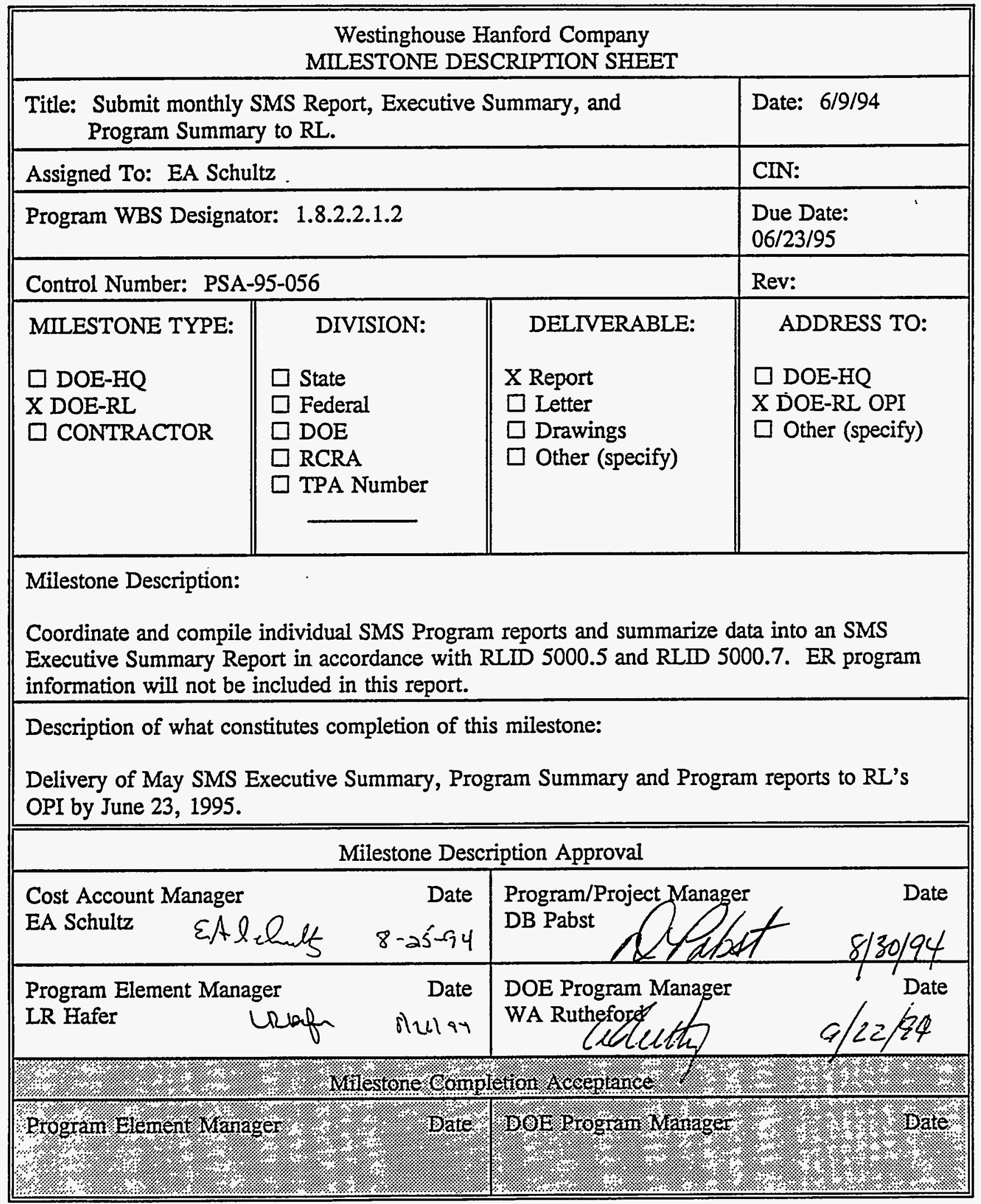




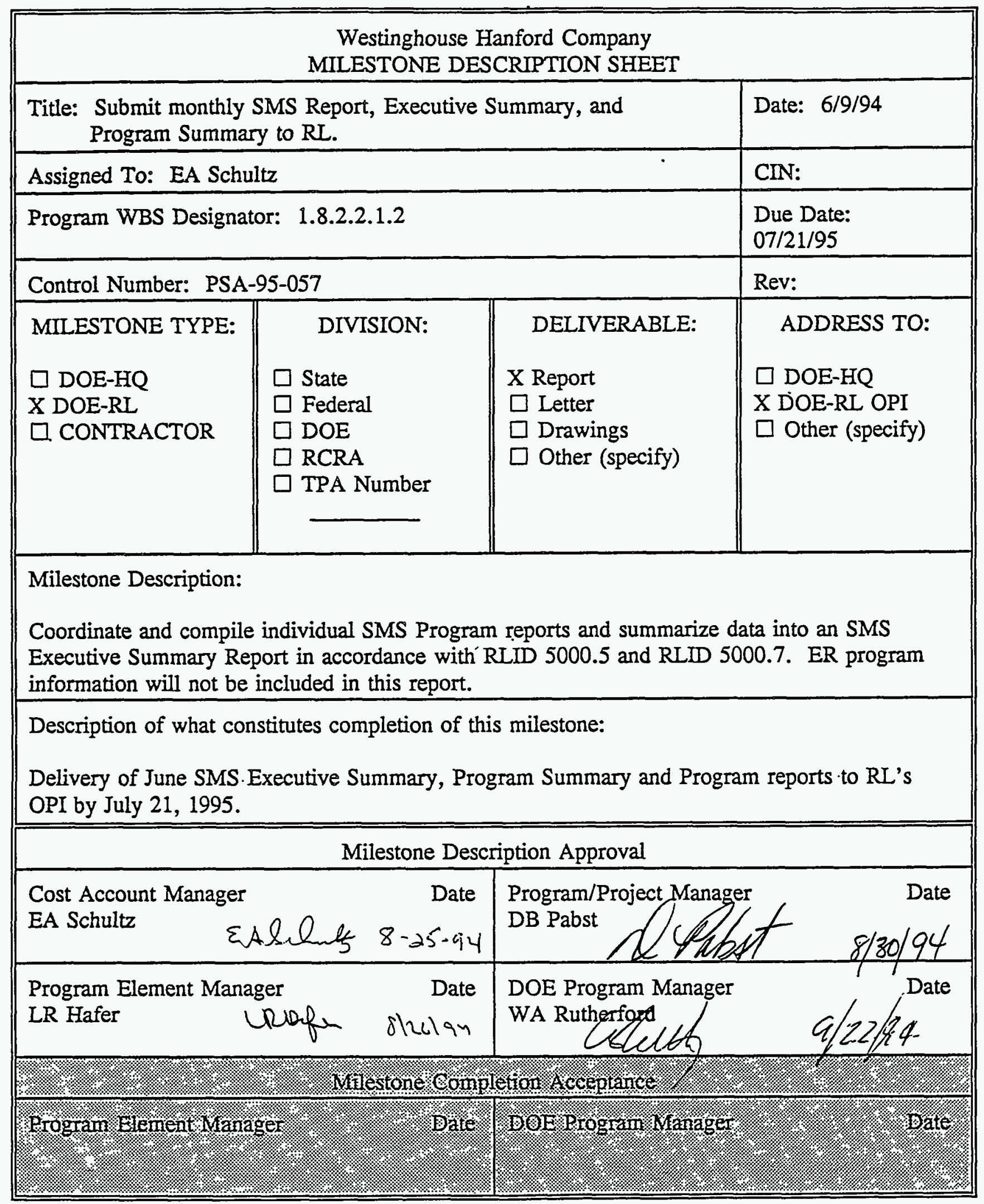




\begin{tabular}{|l||l|l||}
\hline \multicolumn{3}{|c|}{ Westinghouse Hanford Company } \\
MILSTONE DESCRIPTION SHEET
\end{tabular}




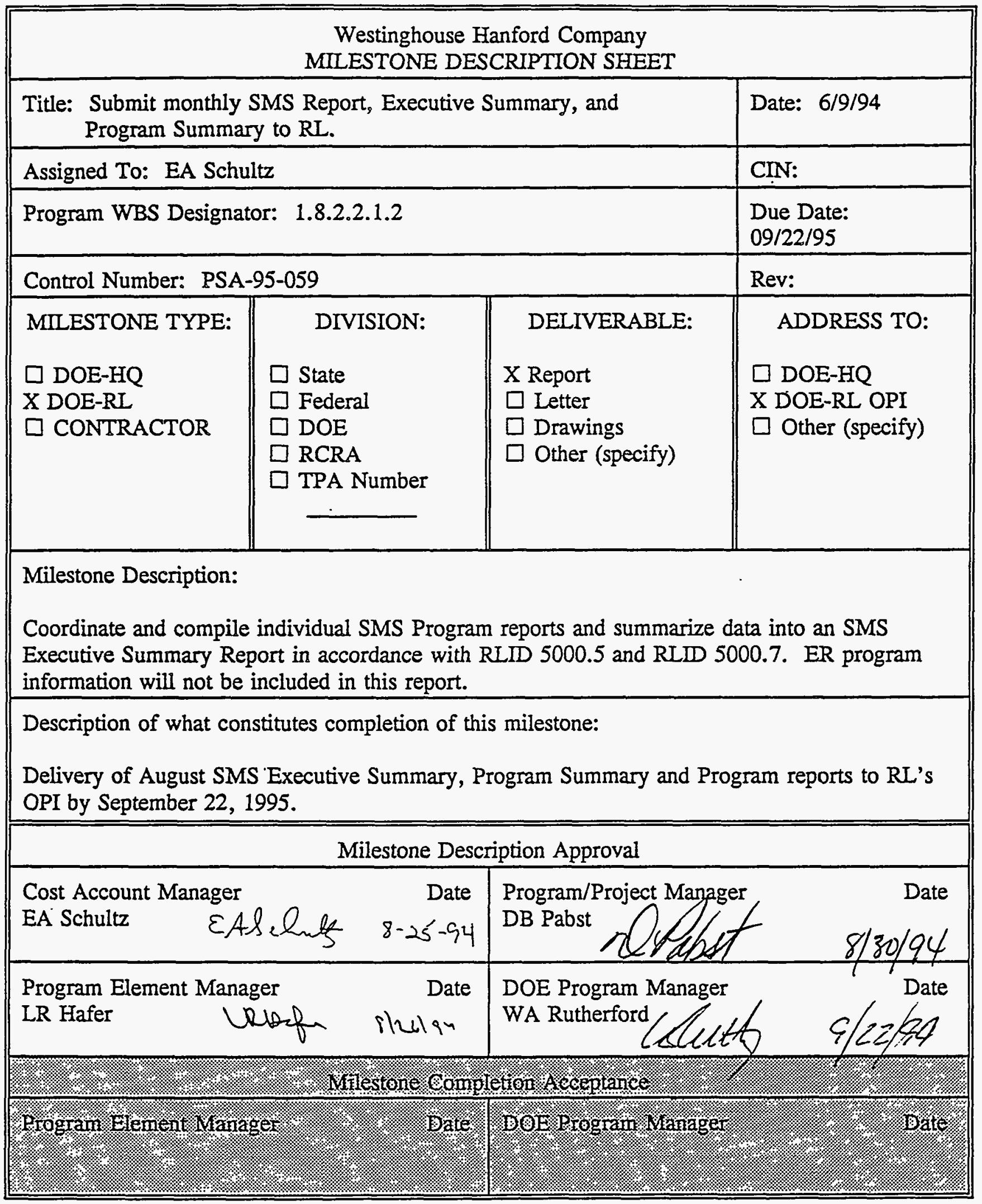




\begin{tabular}{|l||l|l||}
\hline \multicolumn{3}{|c|}{ Westinghouse Hanford Company } \\
MILESTONE DESCRIPTION SHEET
\end{tabular}




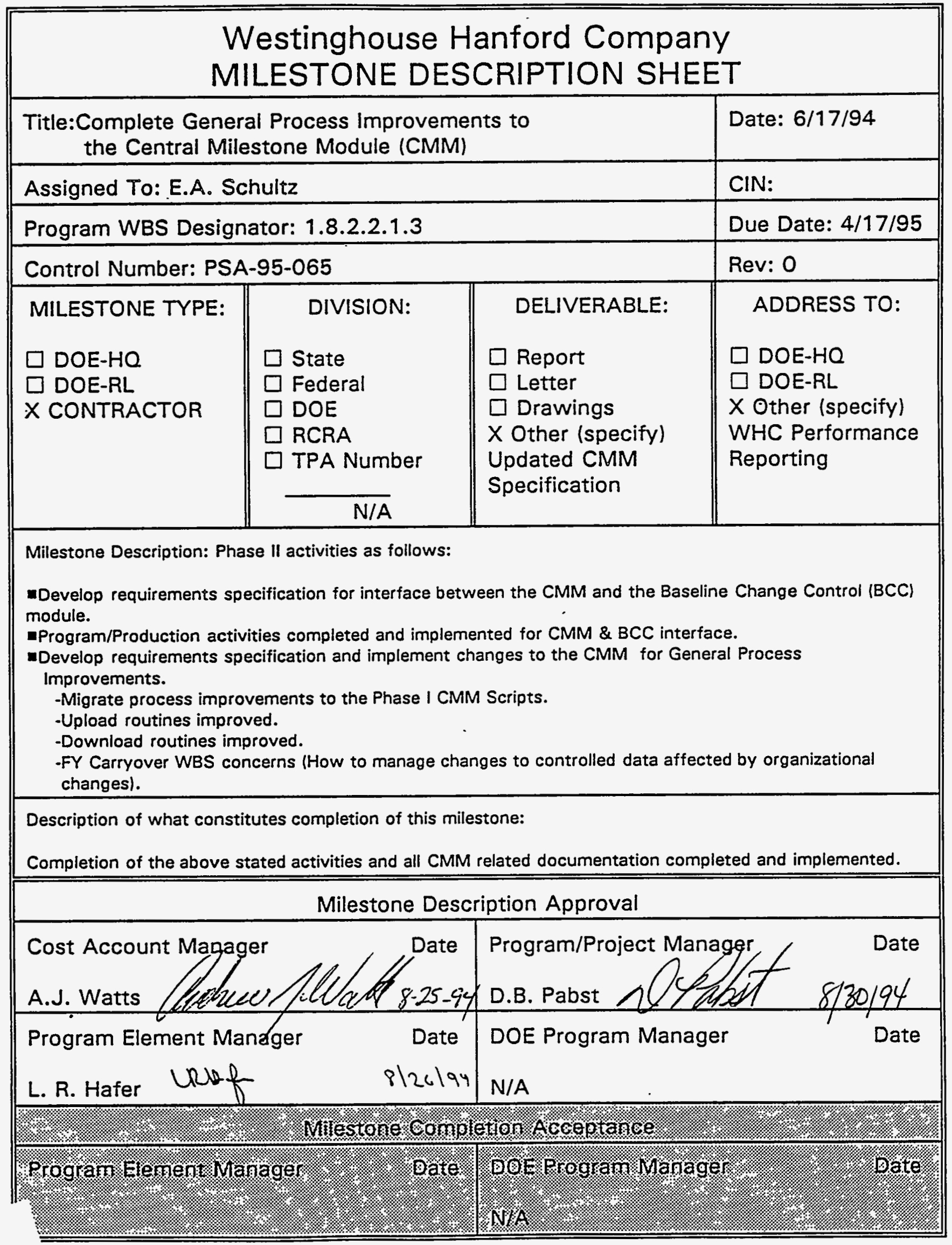




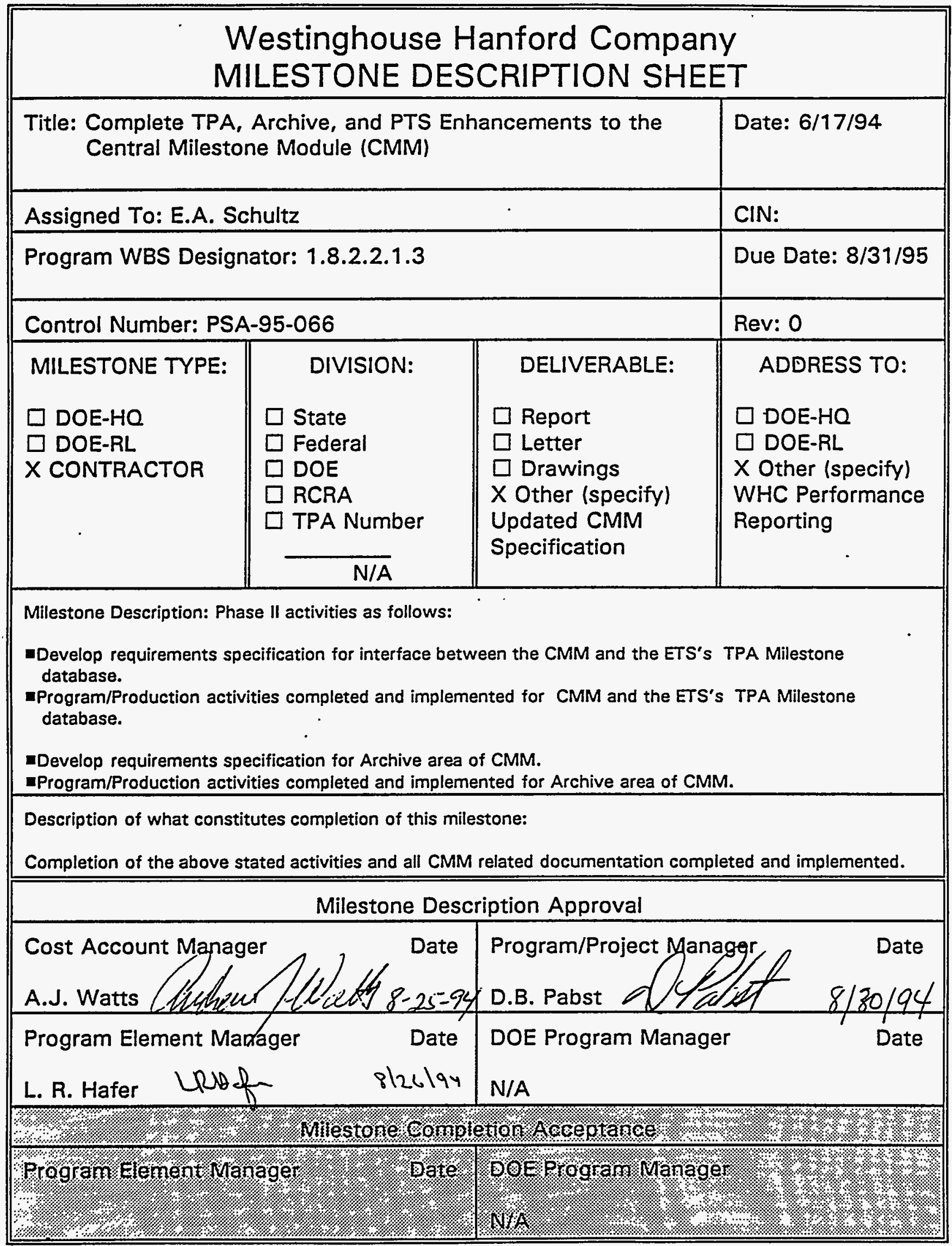




\begin{tabular}{|l||l|l||}
\hline \multicolumn{3}{|c||}{ Westinghouse Hanford Company } \\
MILESTONE DESCRIPTION SHEET
\end{tabular}




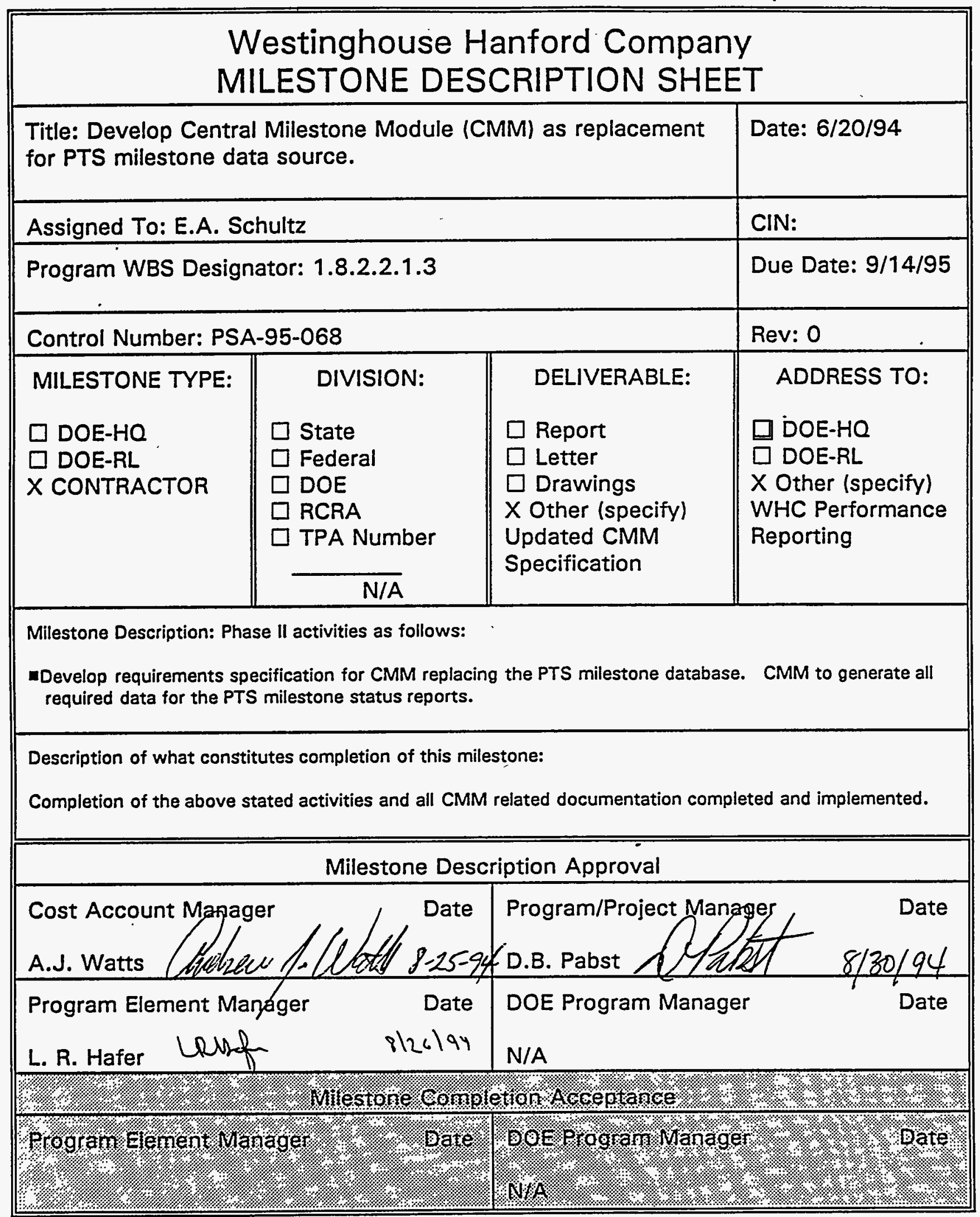




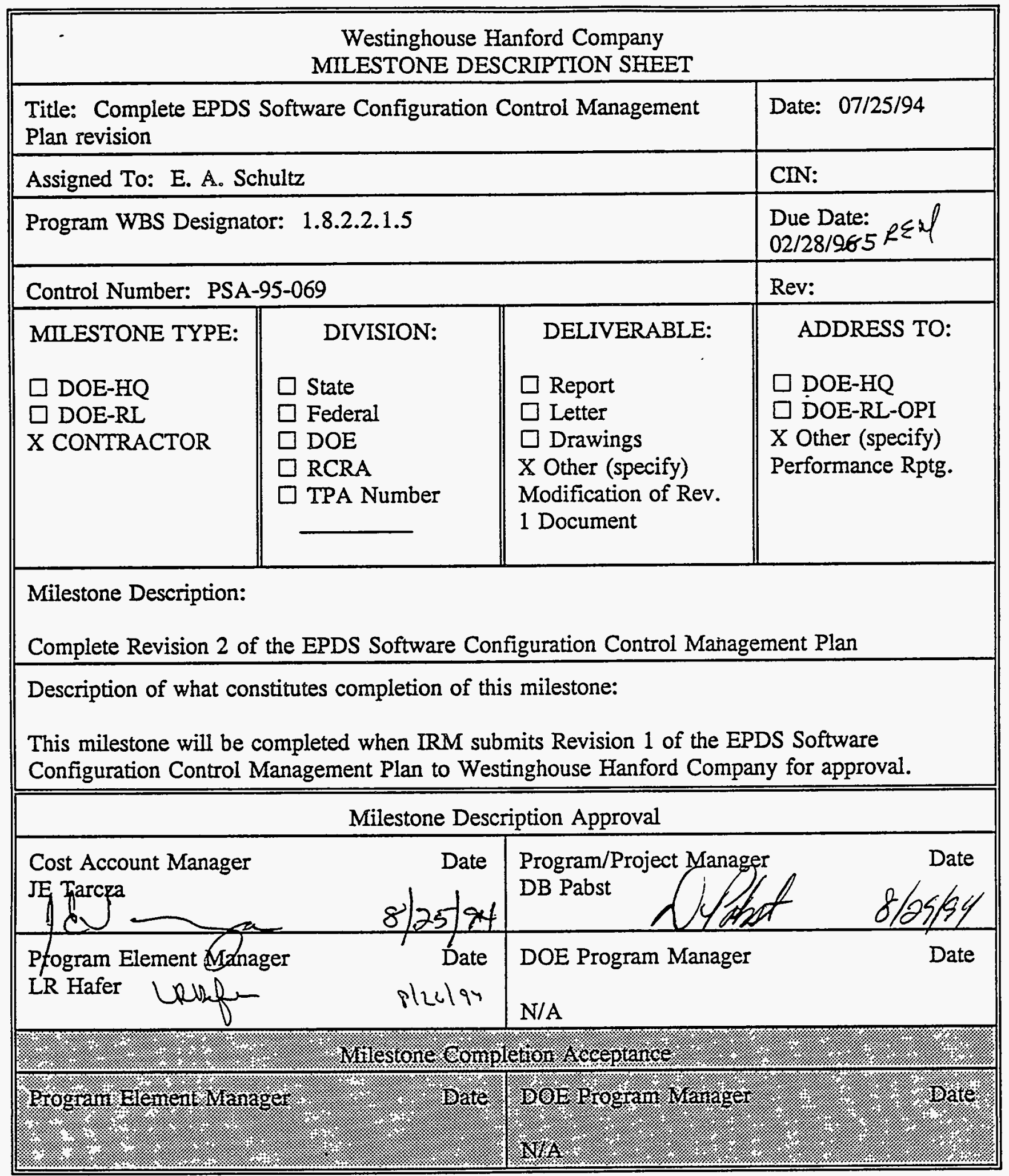




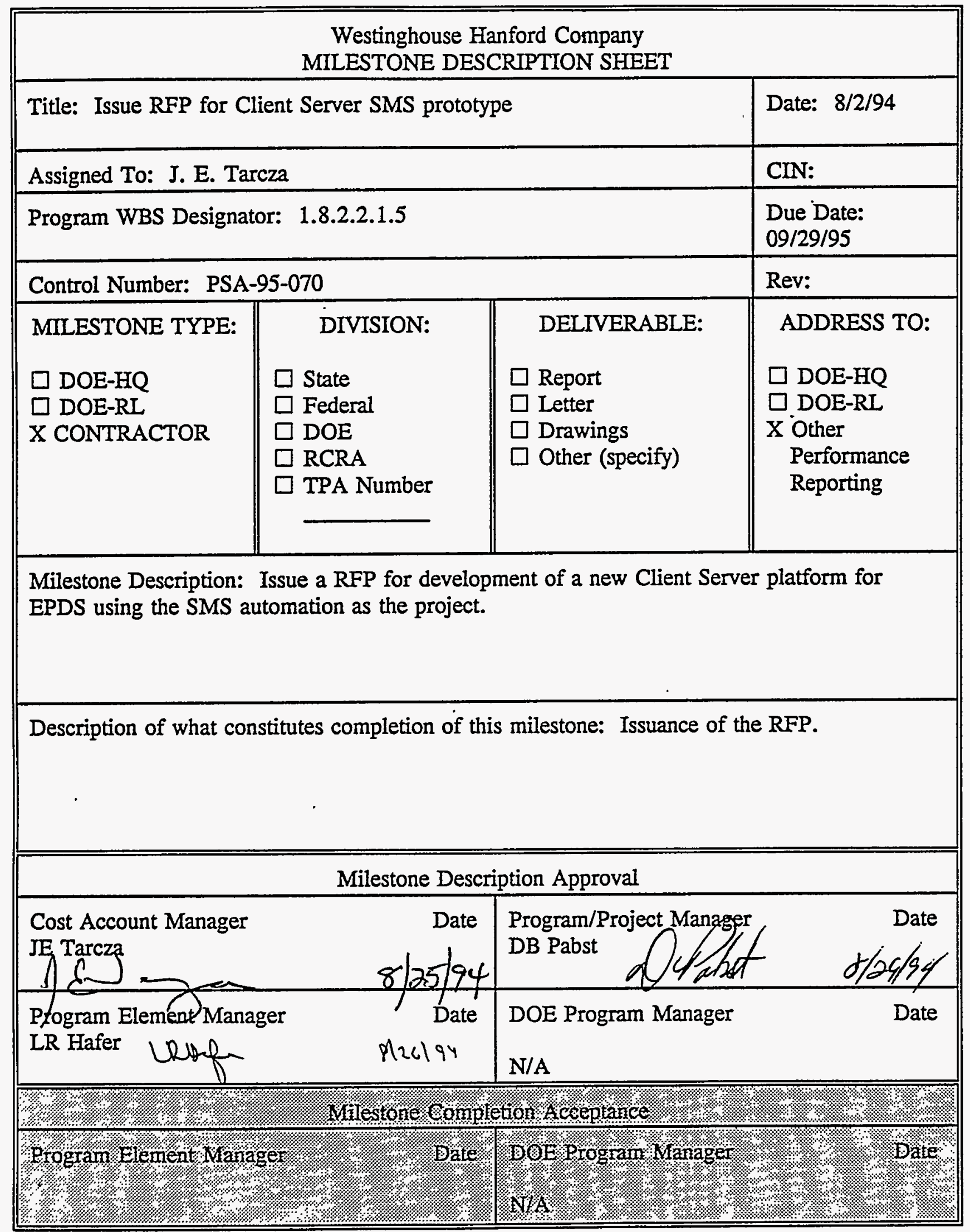




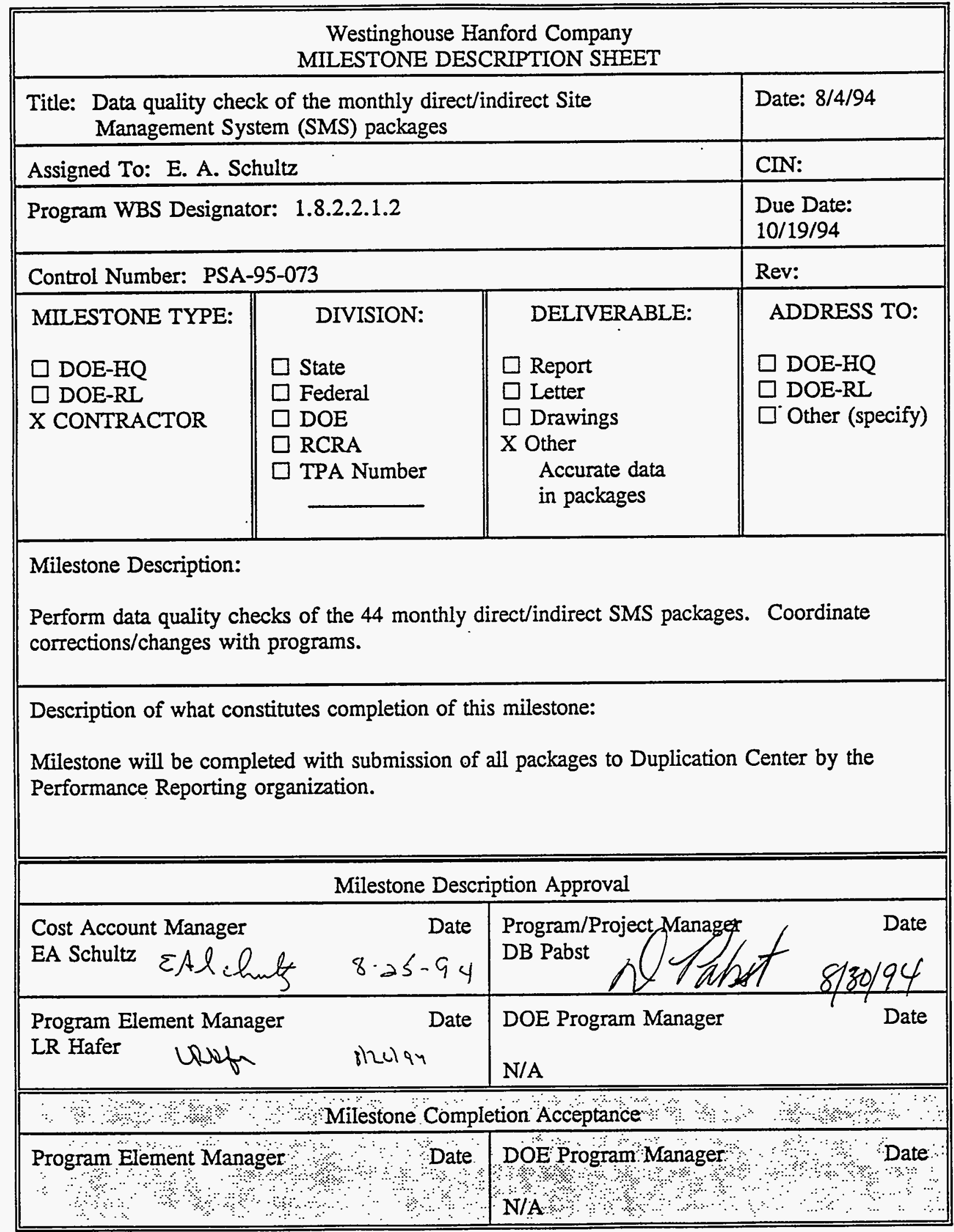




\begin{tabular}{||l|l||}
\hline \multicolumn{3}{|c|}{ Westinghouse Hanford Company } \\
MILESTONE DESCRIPTION SHEET
\end{tabular}




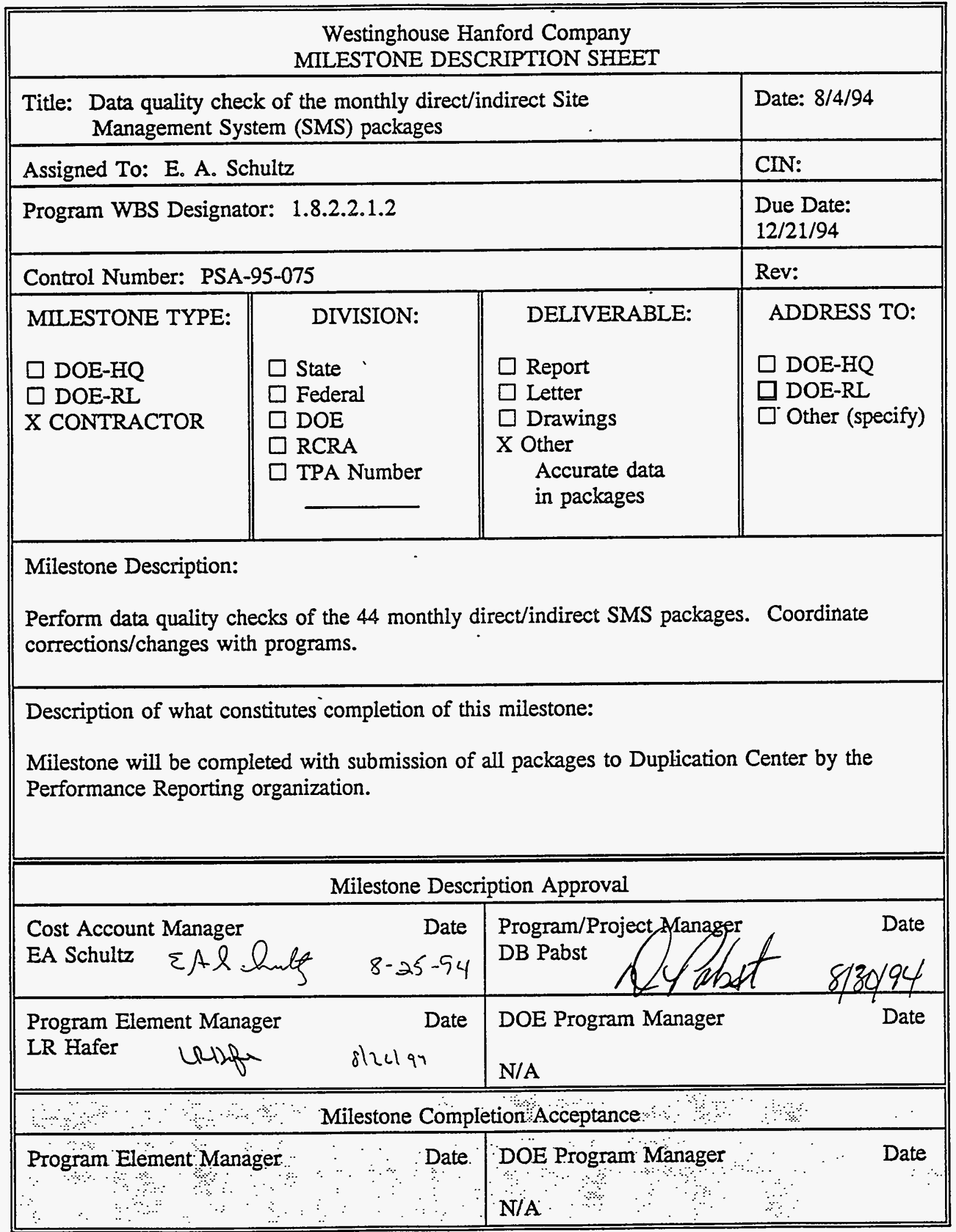




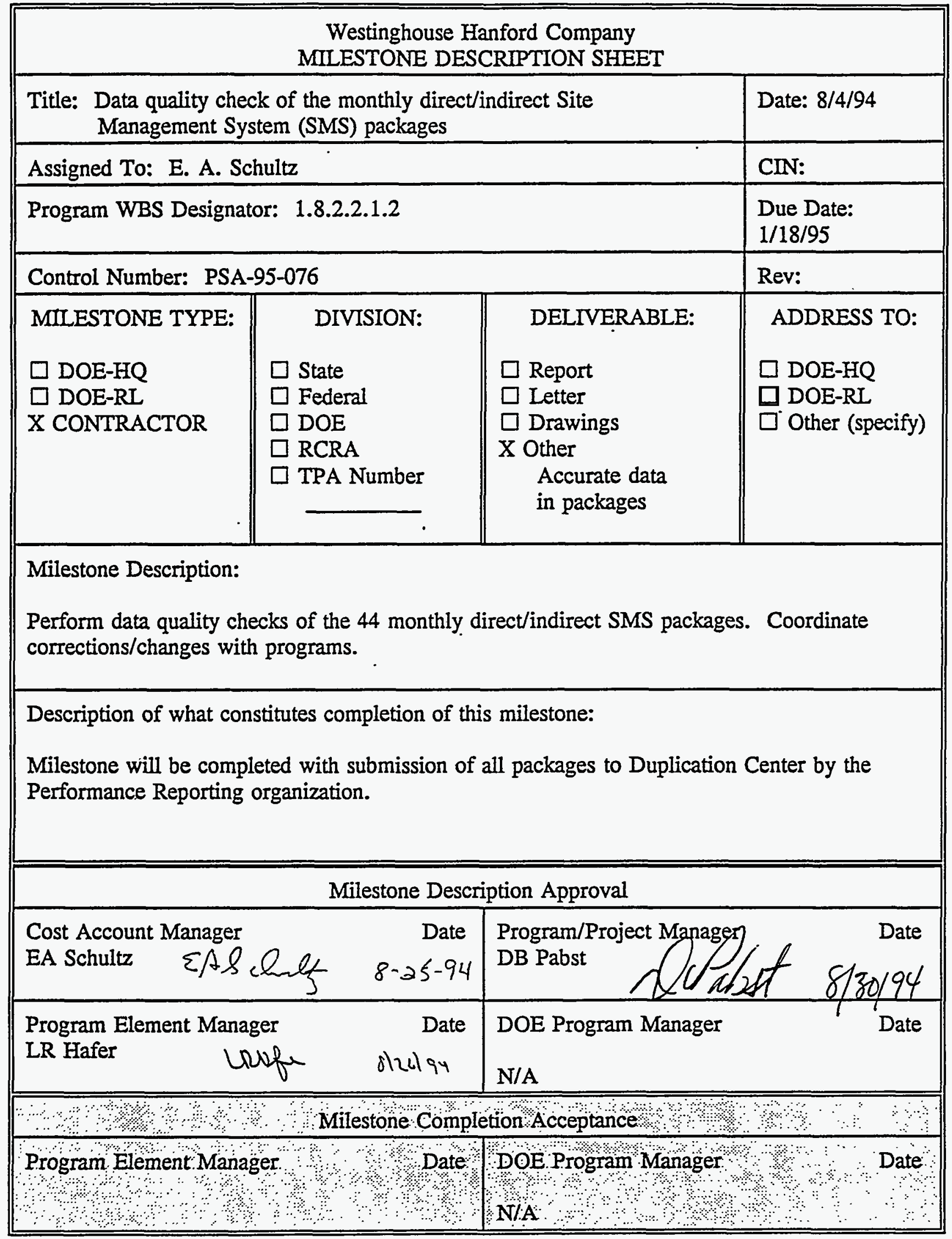




\begin{tabular}{||l||l||}
\hline \multicolumn{3}{|c|}{ Westinghouse Hanford Company } \\
MILESTONE DESCRIPTION SHEET
\end{tabular}




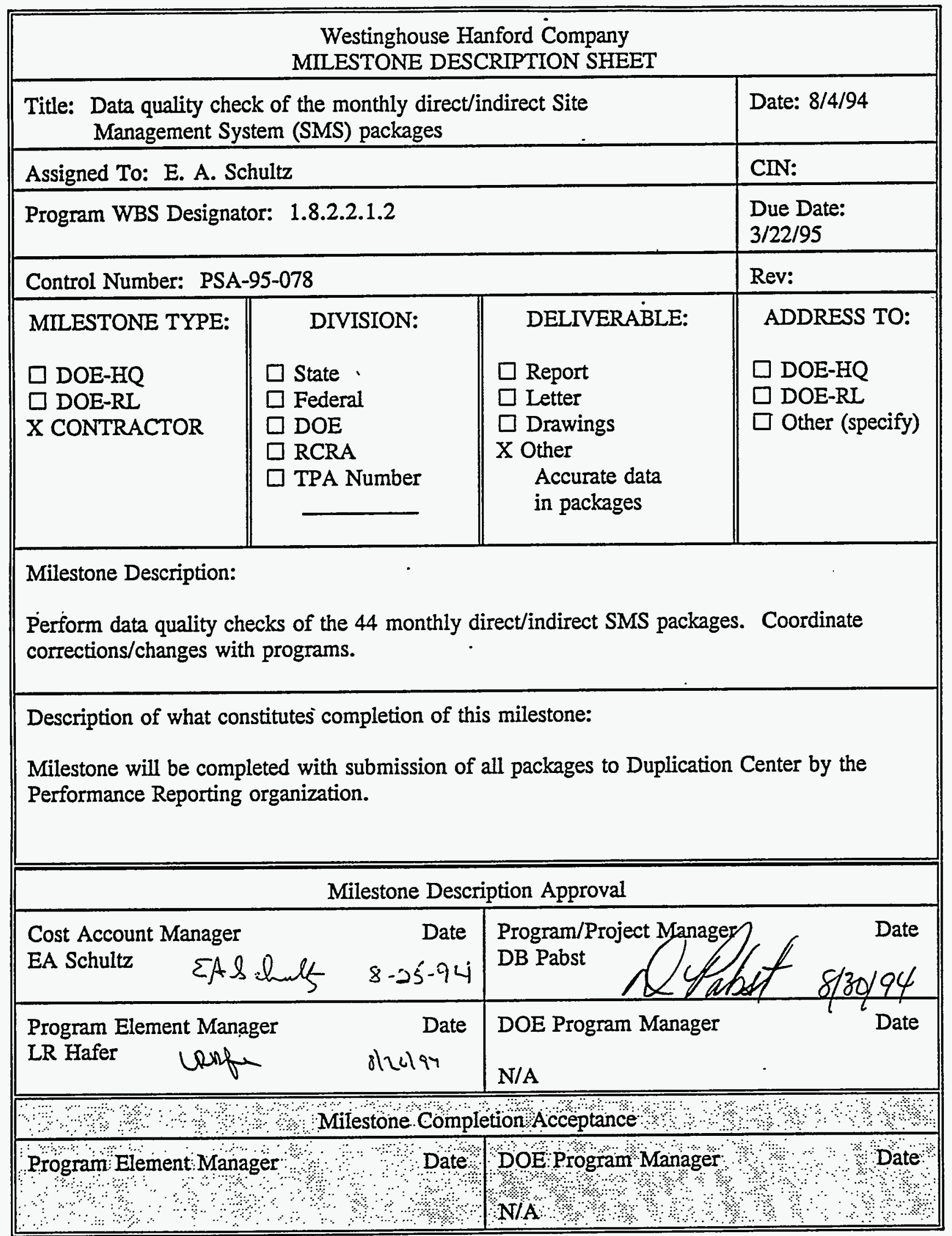




\begin{tabular}{|l|l|l||}
\hline \multicolumn{3}{|c|}{ Westinghouse Hanford Company } \\
MILESTONE DESCRIPTION SHEET
\end{tabular}




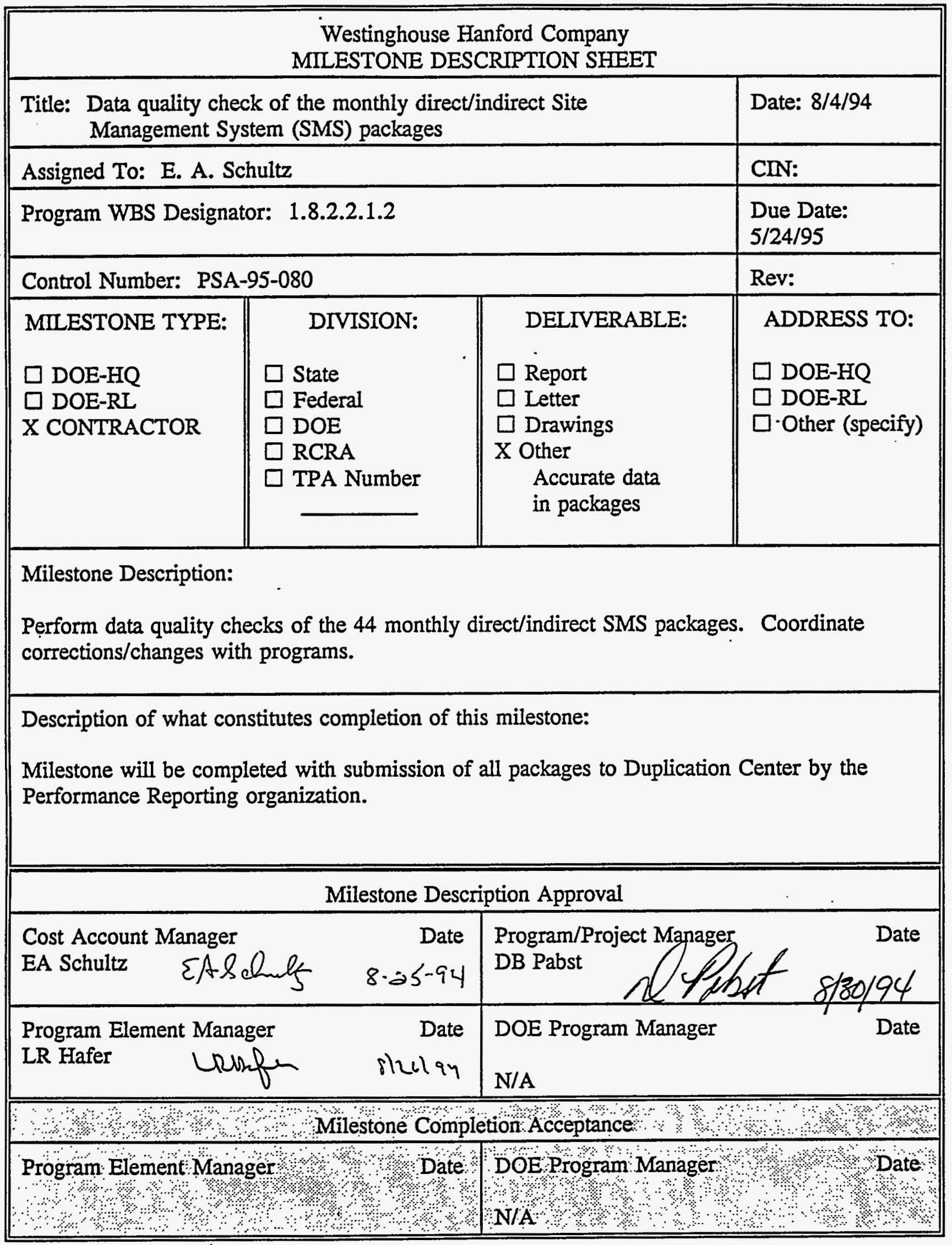




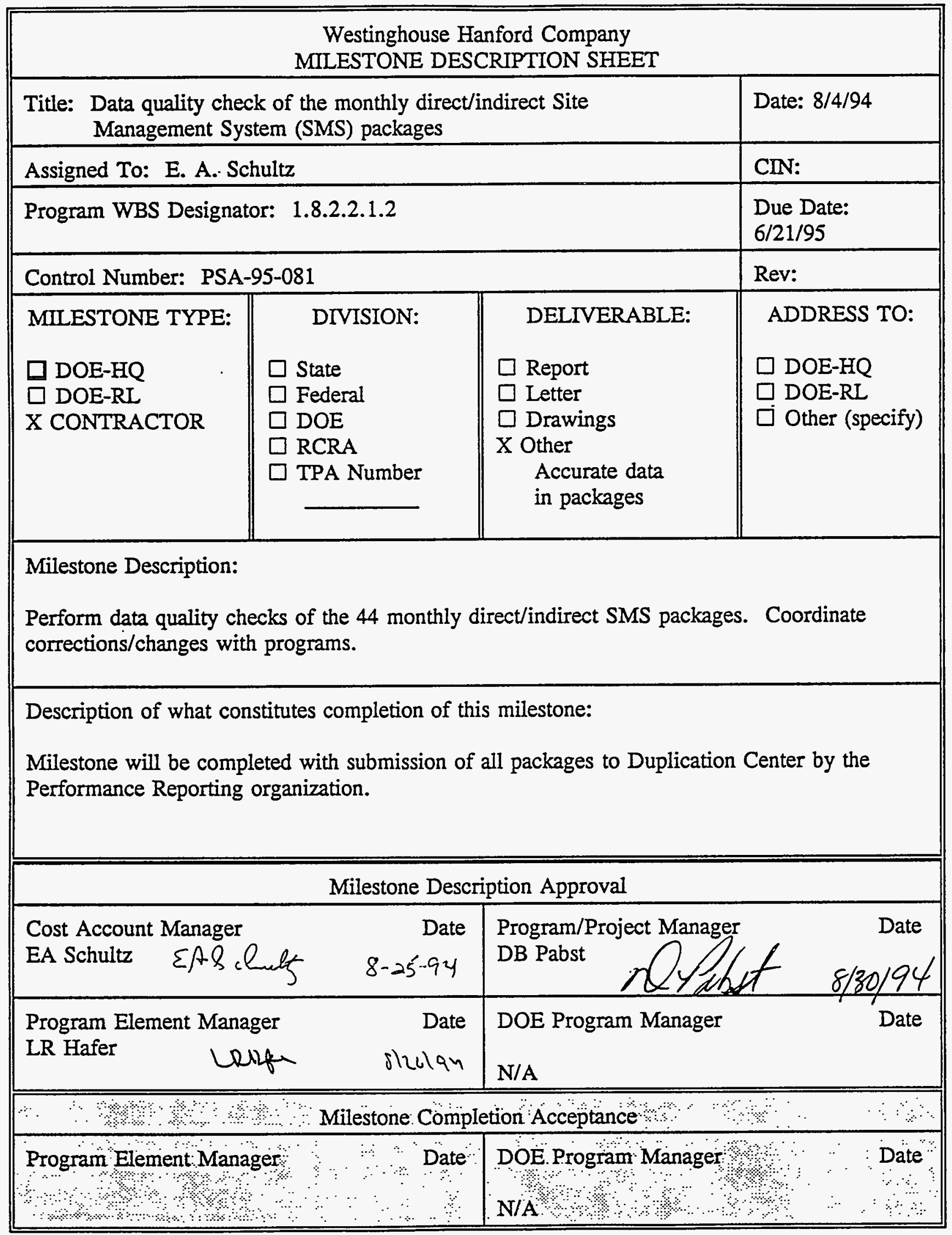




\begin{tabular}{|c|c|c|c|}
\hline \multicolumn{4}{|c|}{$\begin{array}{l}\text { Westinghouse Hanford Company } \\
\text { MILESTONE DESCRIPTION SHEET }\end{array}$} \\
\hline \multicolumn{3}{|c|}{$\begin{array}{l}\text { Title: Data quality check of the monthly direct/indirect Site } \\
\text { Management System (SMS) packages }\end{array}$} & Date: $8 / 4 / 94$ \\
\hline \multicolumn{3}{|c|}{ Assigned To: E. A. Schultz } & CIN: \\
\hline \multicolumn{3}{|c|}{ Program WBS Designator: 1.8 .2 .2 .1 .2} & $\begin{array}{l}\text { Due Date: } \\
7 / 19 / 95\end{array}$ \\
\hline \multicolumn{3}{|c|}{ Control Number: PSA-95-082 } & Rev: \\
\hline $\begin{array}{l}\text { MILESTONE TYPE: } \\
\square \text { DOE-HQ } \\
\square \text { DOE-RL } \\
\text { X CONTRACTOR }\end{array}$ & $\begin{aligned} & \text { DIVISION: } \\
& \\
\square & \text { State } \\
& \text { Federal } \\
& \square \text { DOE } \\
& \text { RCRA } \\
& \square \text { TPA Number }\end{aligned}$ & $\begin{array}{l}\text { DELIVERABLE: } \\
\square \text { Report } \\
\square \text { Letter } \\
\square \text { Drawings } \\
X \text { Other } \\
\text { Accurate data } \\
\text { in packages }\end{array}$ & $\begin{array}{l}\text { ADDRESS TO: } \\
\square \text { DOE-HQ } \\
\square \text { DOE-RL } \\
\square \text { Other (specify) }\end{array}$ \\
\hline \multicolumn{4}{|c|}{$\begin{array}{l}\text { Perform data quality checks of the } 44 \text { monthly direct/indirect SMS packages. } \\
\text { corrections/changes with programs. }\end{array}$} \\
\hline \multicolumn{4}{|c|}{$\begin{array}{l}\text { Description of what constitutes completion of this milestone: } \\
\text { Milestone will be completed with submission of all'packages to Duplication Center by the } \\
\text { Performance Reporting organization. }\end{array}$} \\
\hline \multicolumn{4}{|c|}{ Milestone Description Approval } \\
\hline \multicolumn{2}{|c|}{$\begin{array}{l}\text { Cost Account Manager } \\
\text { EA Schultz }\end{array}$} & \multicolumn{2}{|c|}{ PBogram/Projegg Manager } \\
\hline \multicolumn{2}{|c|}{$\begin{array}{l}\text { Program Element Manager } \\
\text { LR Hafer UUS }\end{array}$} & \multicolumn{2}{|c|}{$\begin{array}{l}\text { DOE Program Manager Date } \\
\text { N/A }\end{array}$} \\
\hline \multicolumn{4}{|c|}{ Milestone Completion Acceptance } \\
\hline \multicolumn{2}{|c|}{ Program Element Manager } & \multicolumn{2}{|c|}{$\begin{array}{l}\text { DOE Program Manager } \\
\text { NIA }\end{array}$} \\
\hline
\end{tabular}




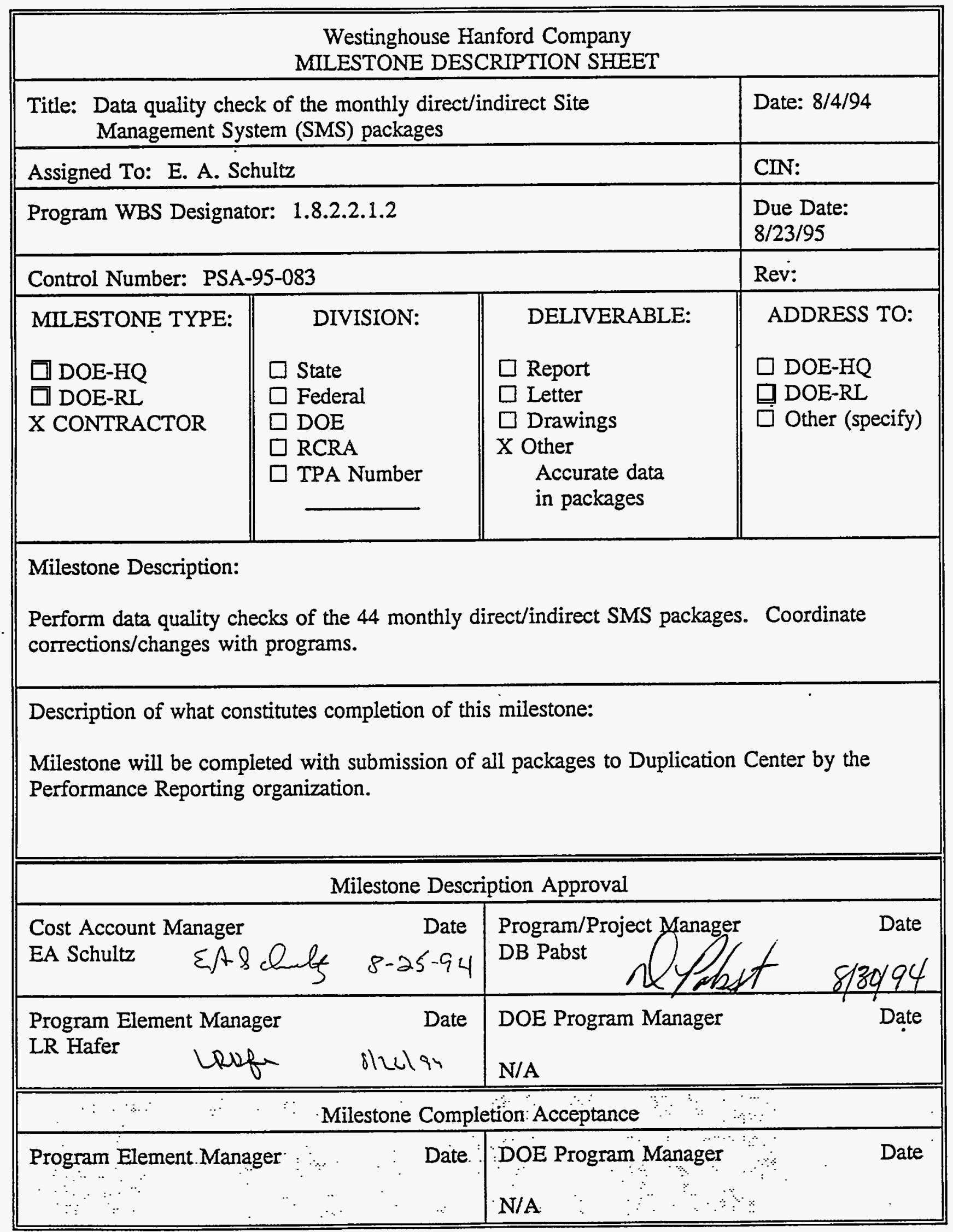




\begin{tabular}{|l|l|l||}
\hline \multicolumn{3}{|c|}{ Westinghouse Hanford Company } \\
MLESTONE DESCRIPTION SHEET
\end{tabular}




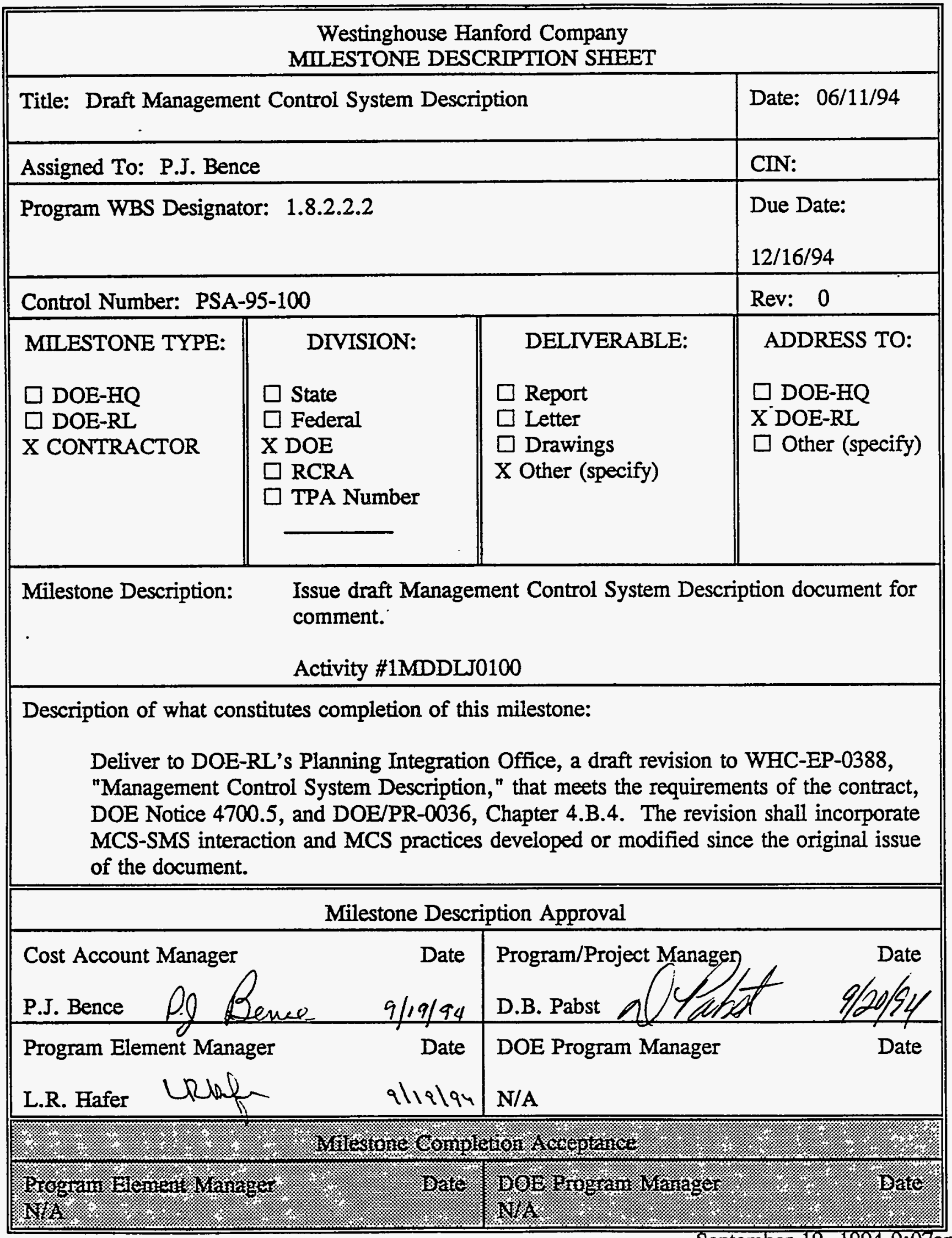




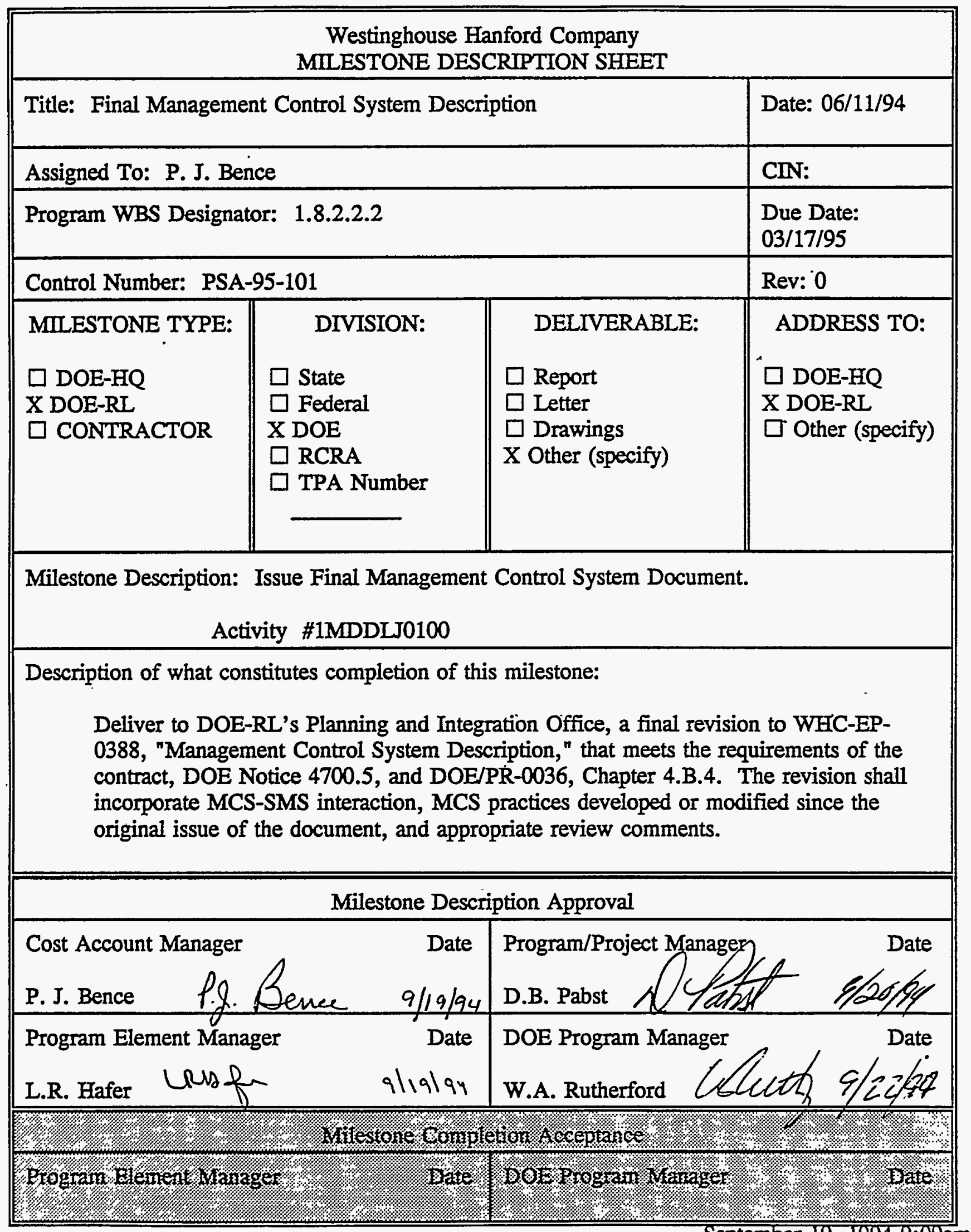




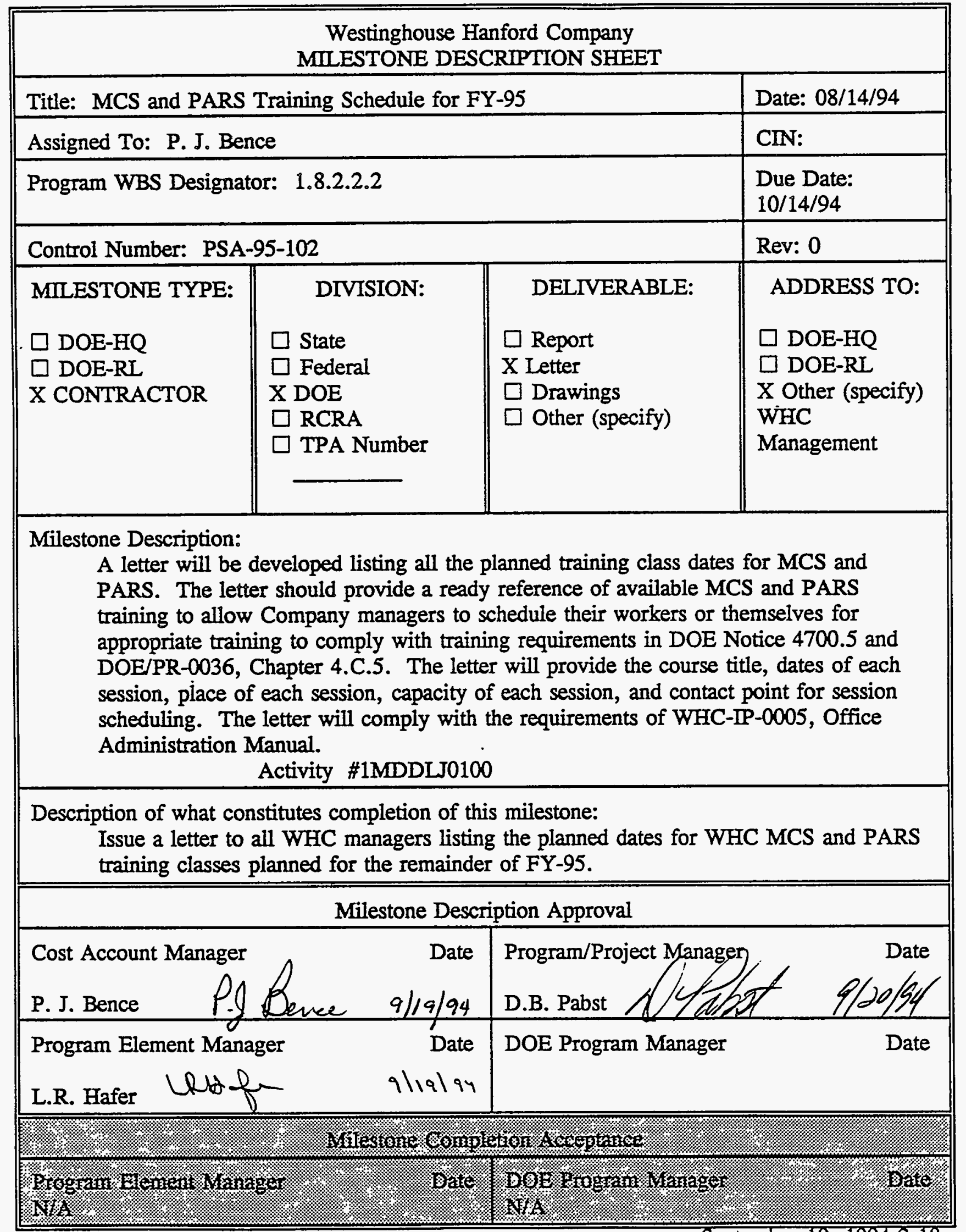




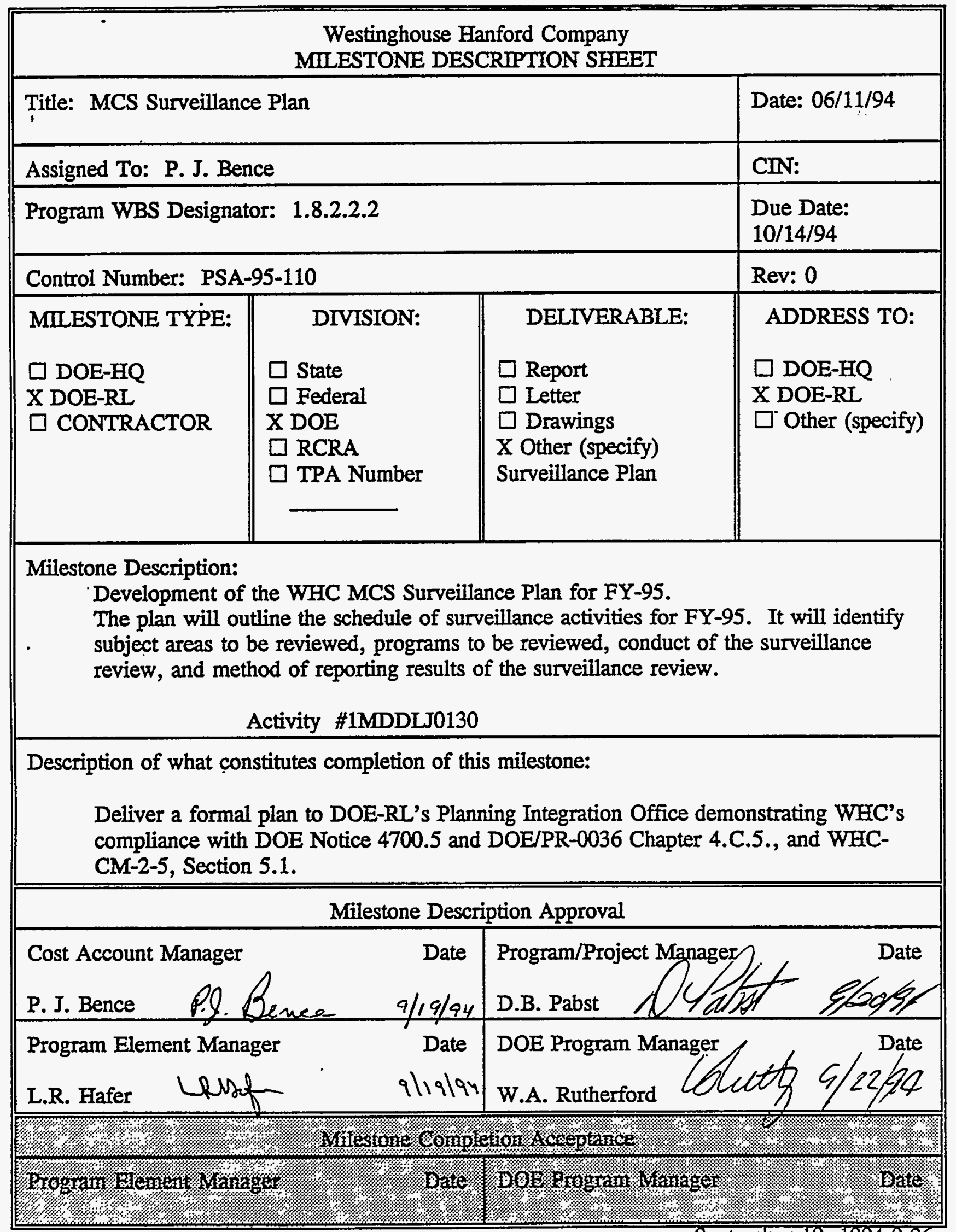




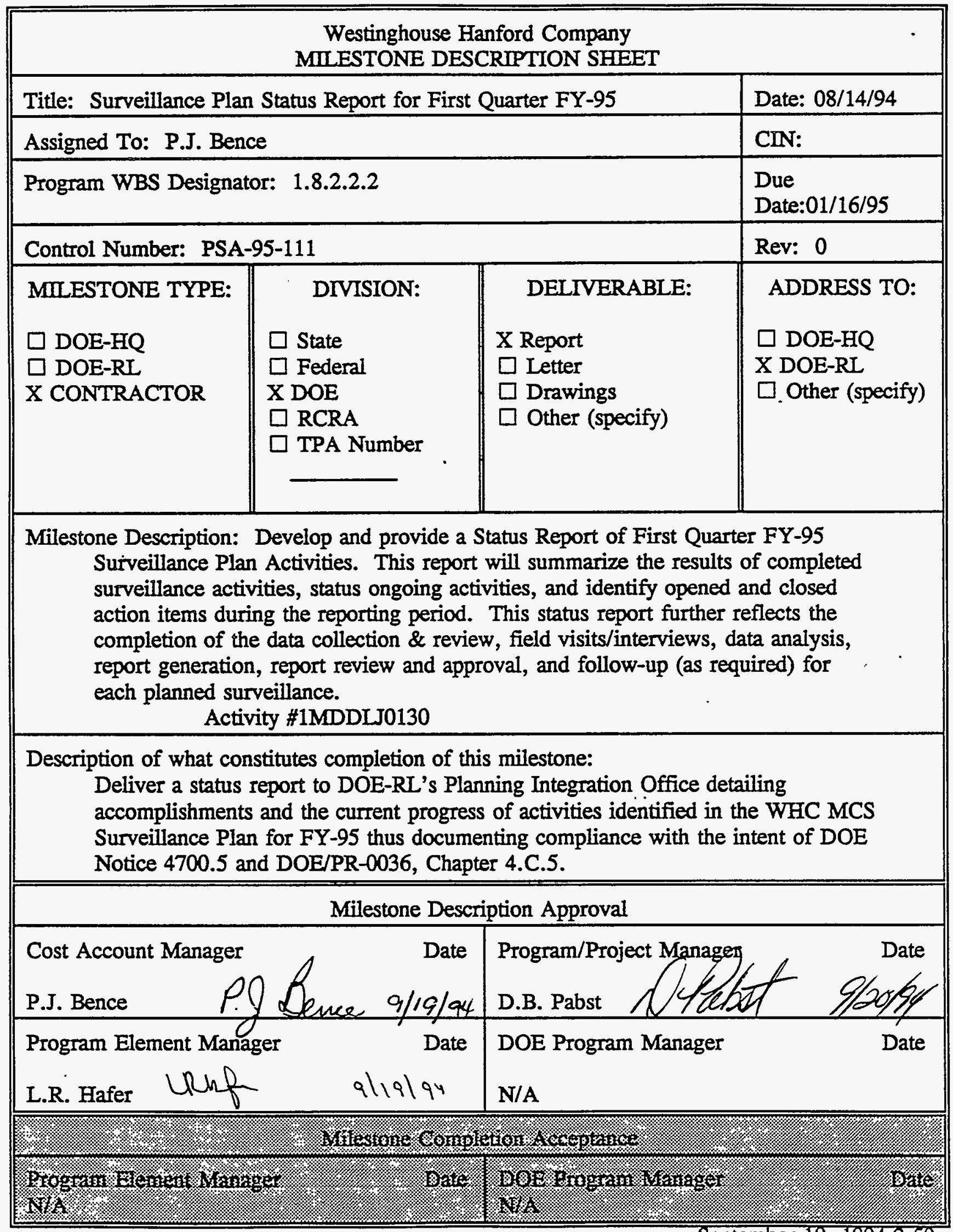




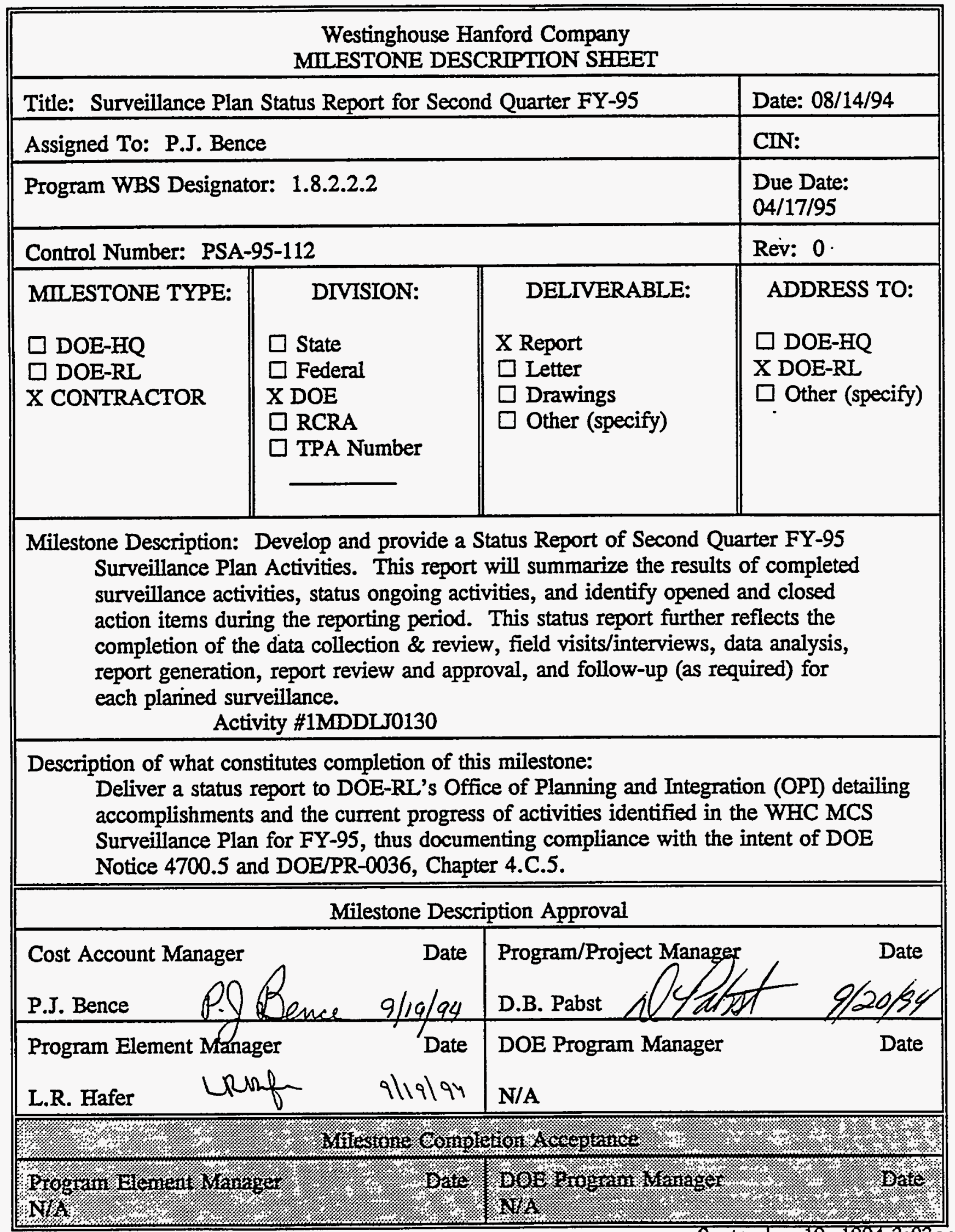




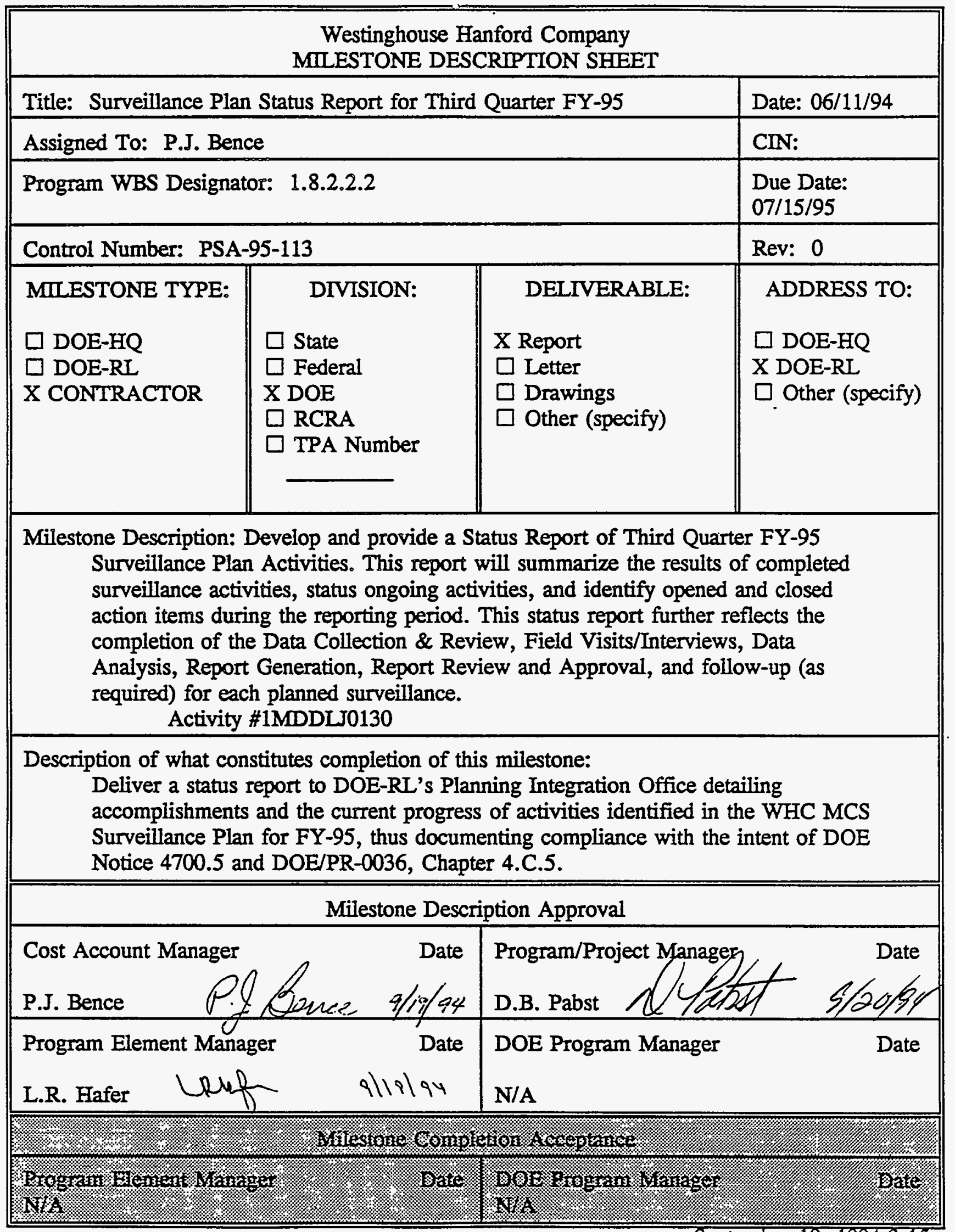




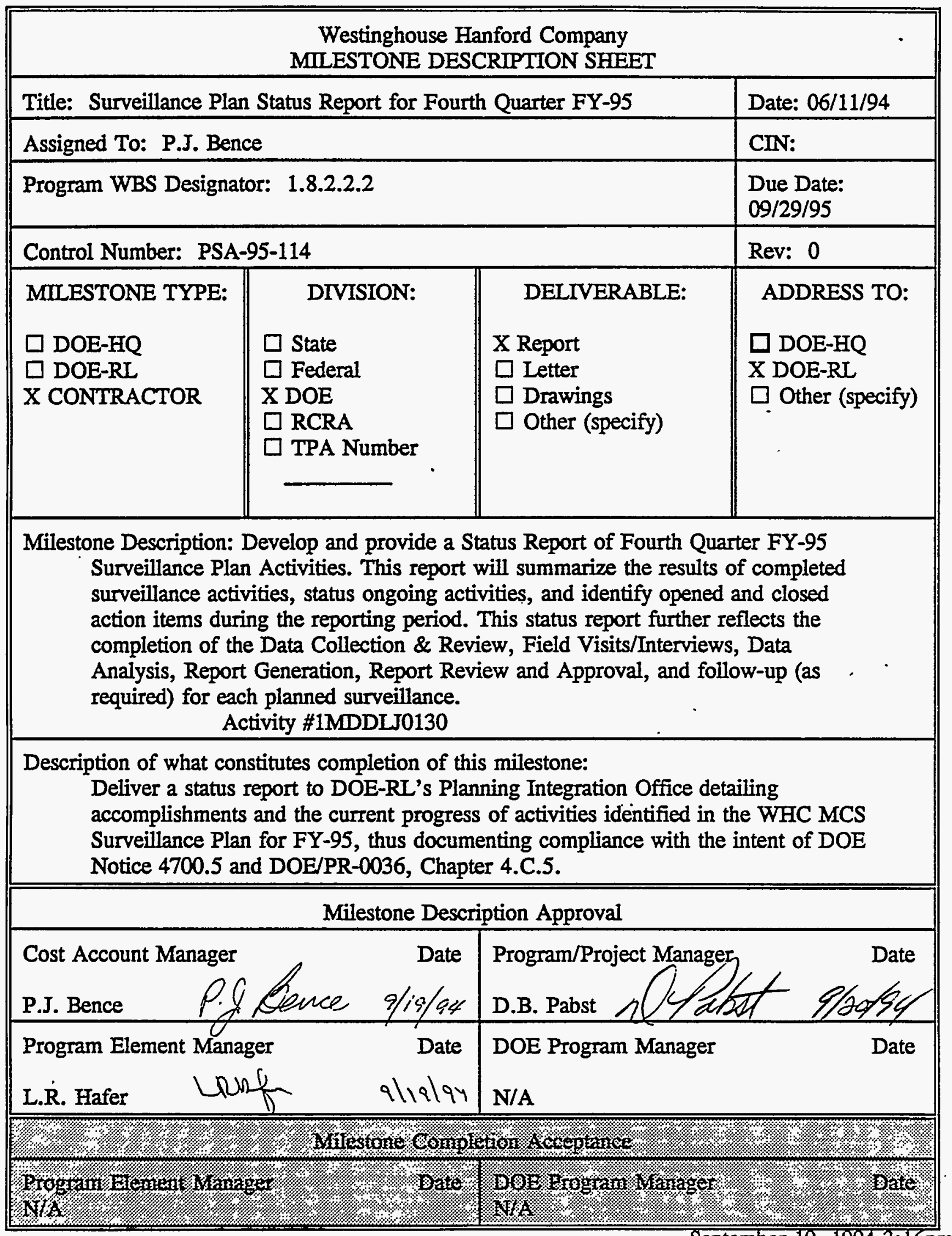




\begin{tabular}{|l||l|l||}
\hline \multicolumn{3}{|c|}{ Westinghouse Hanford Company } \\
MILESONE DESCRIPTION SHEET
\end{tabular}




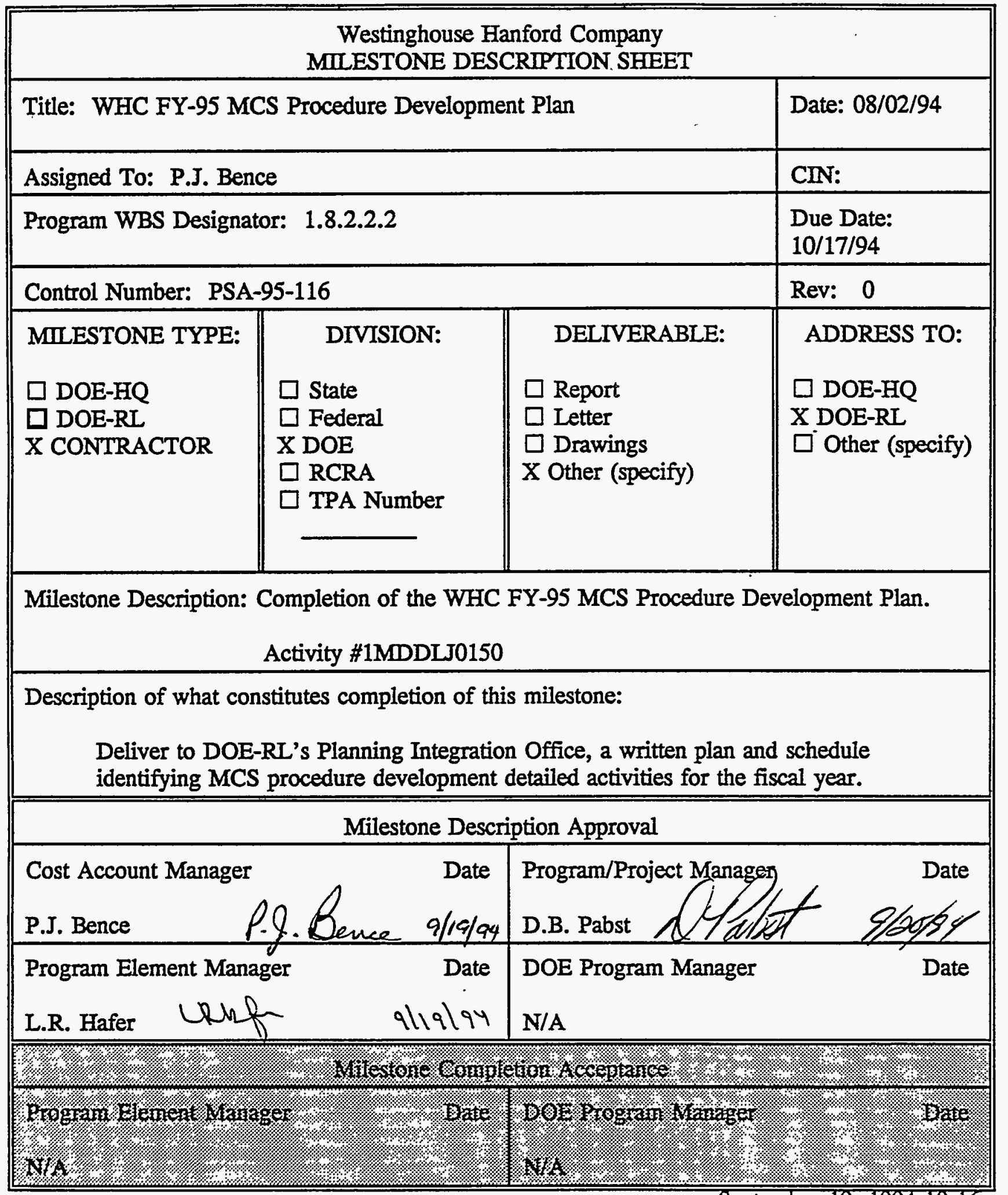




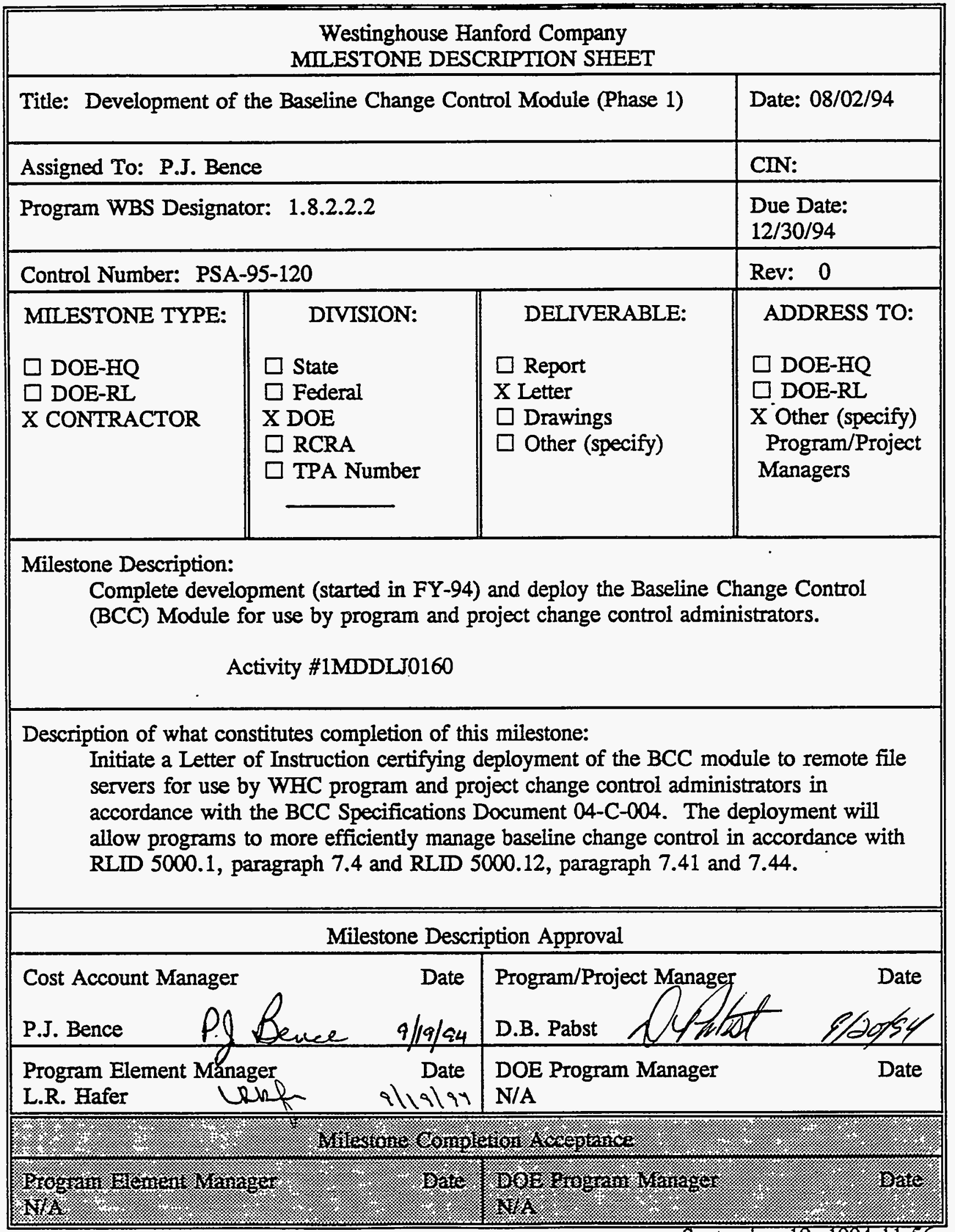




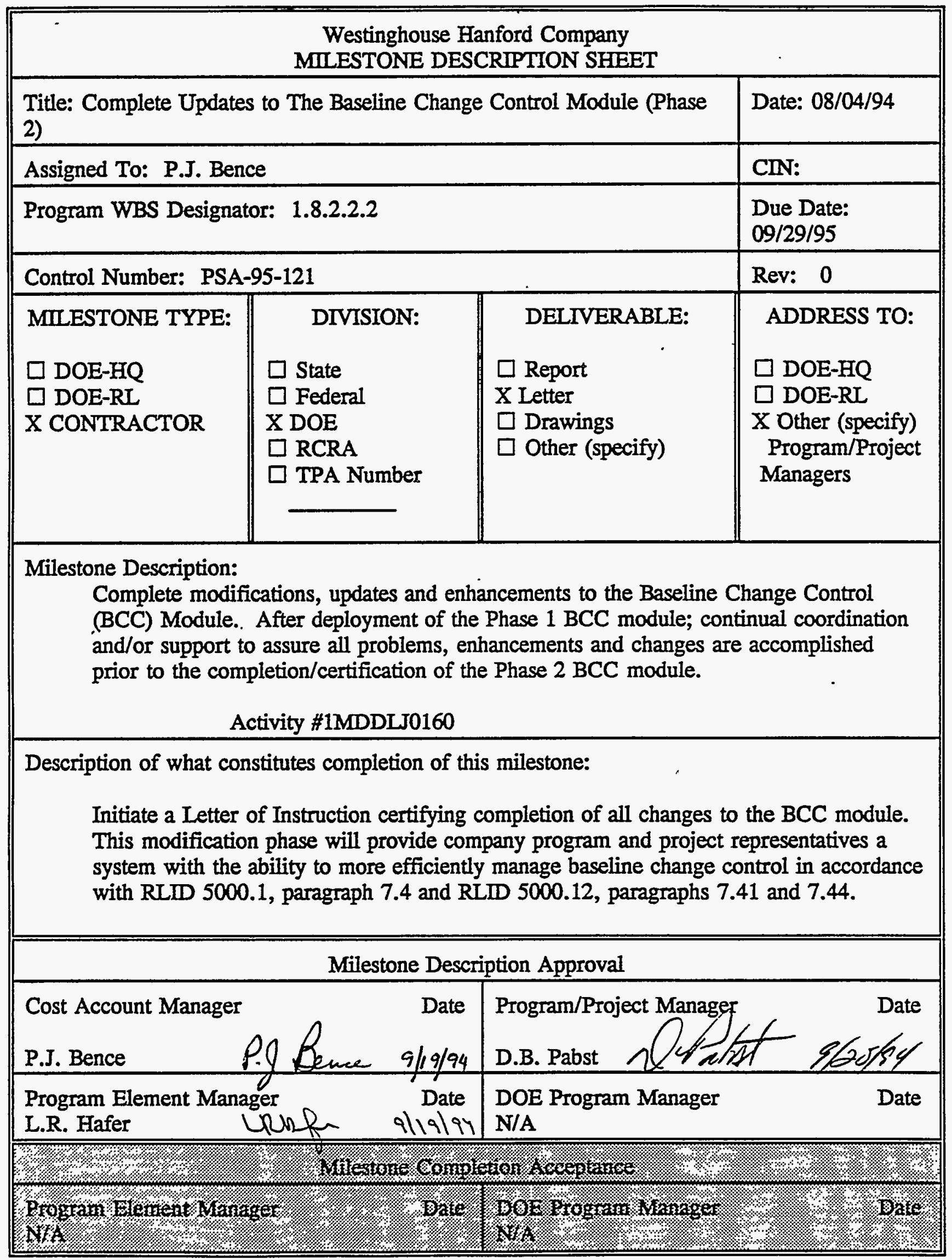




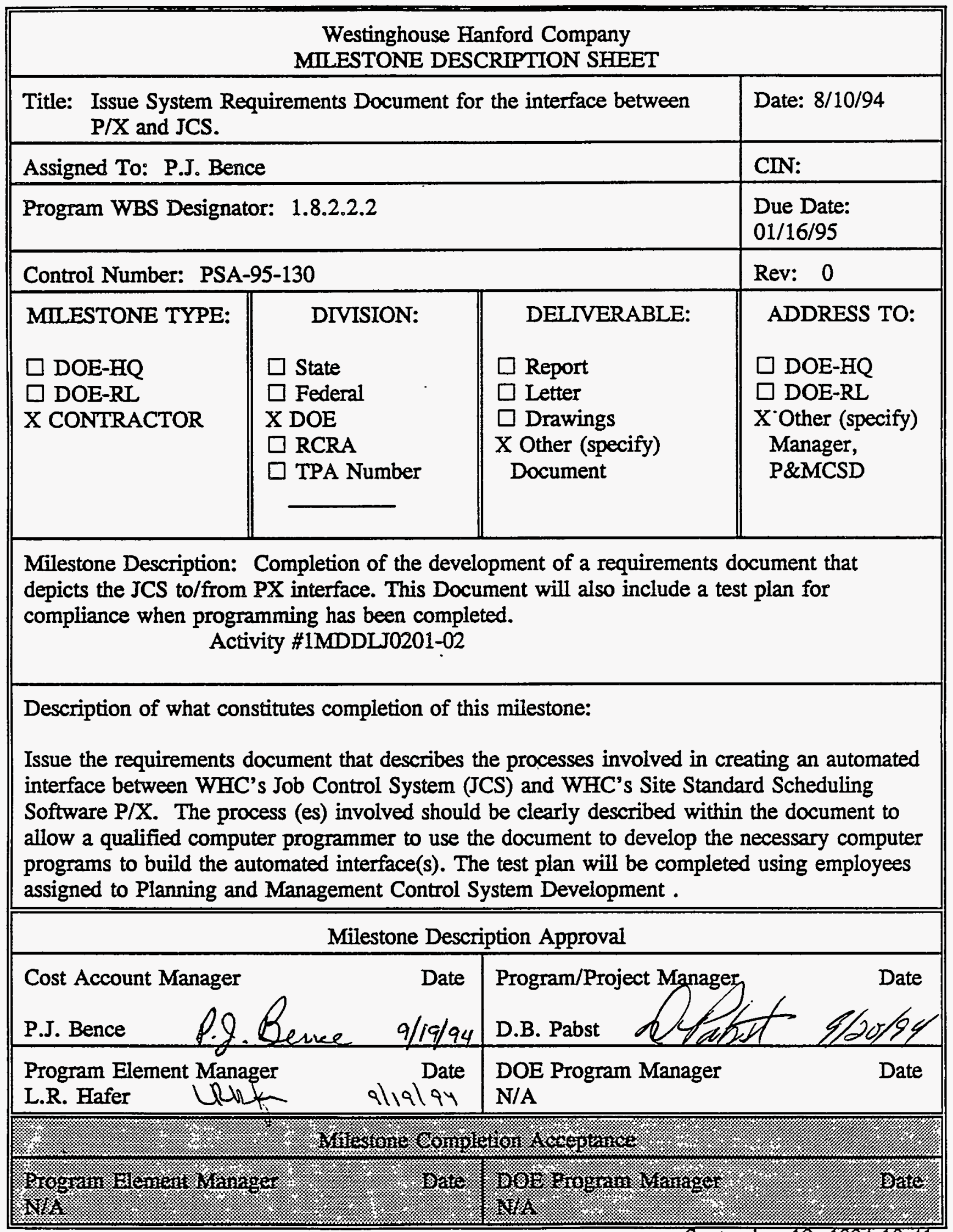




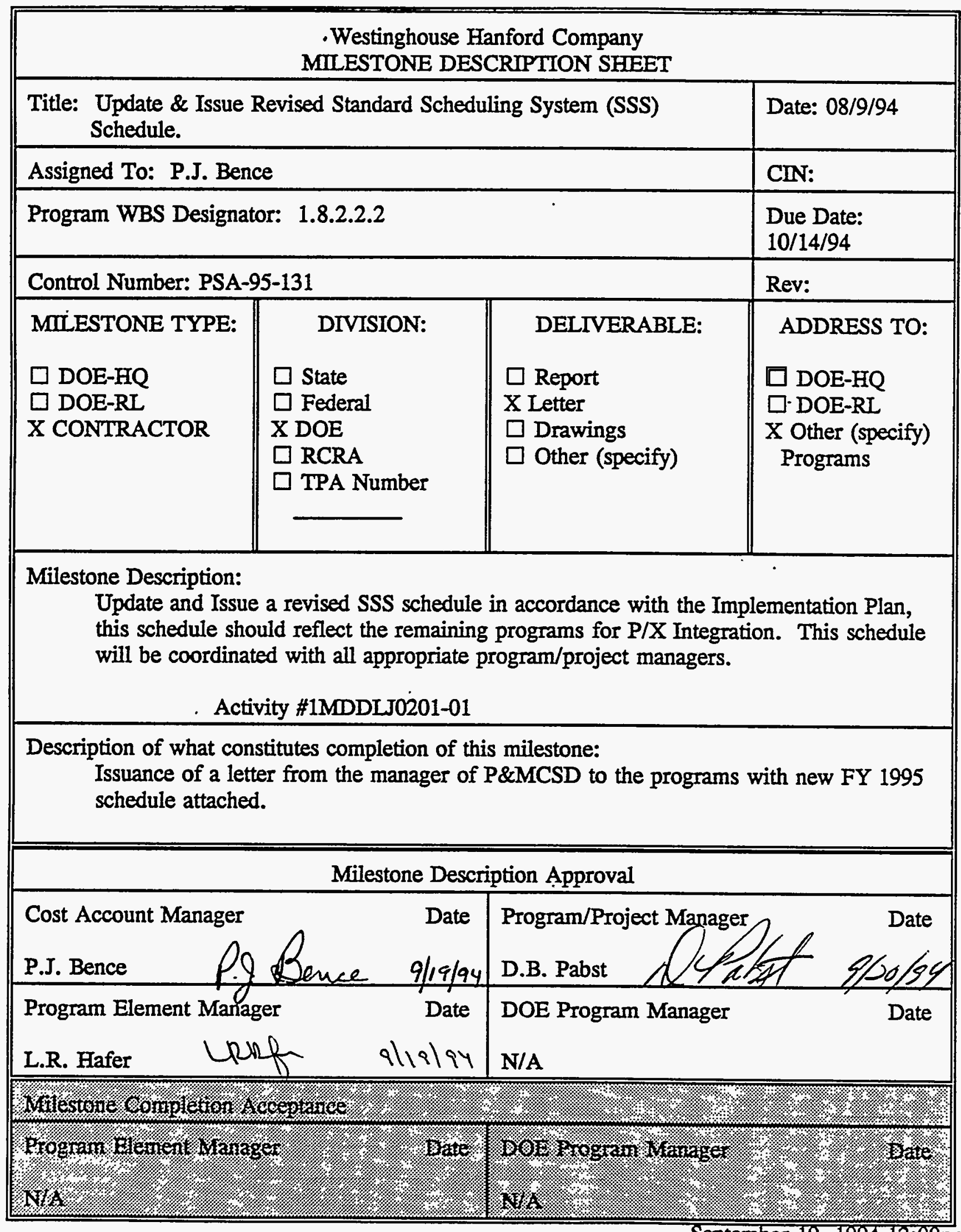




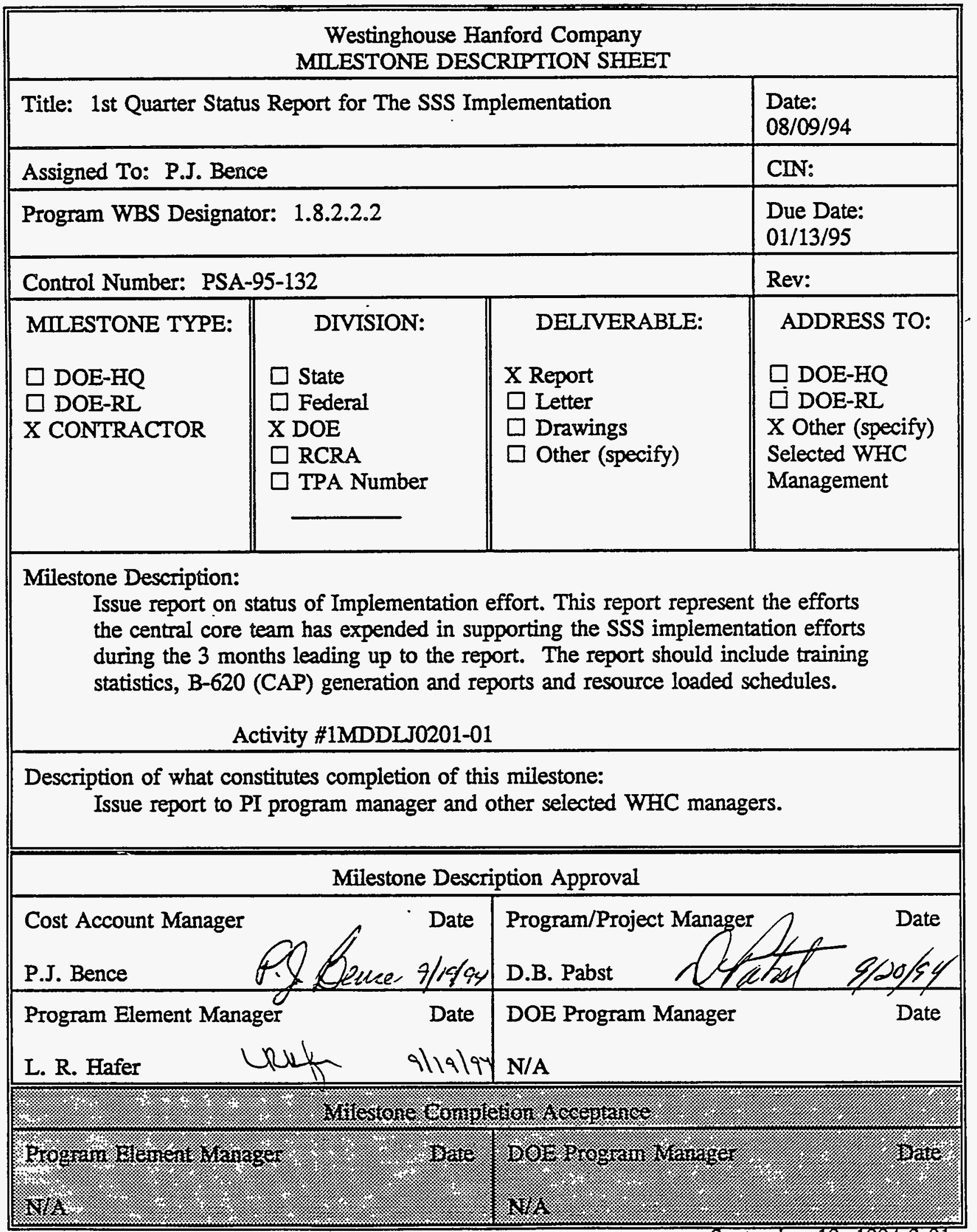




\begin{tabular}{|c|c|c|c|}
\hline \multicolumn{4}{|c|}{$\begin{array}{l}\text { Westinghouse Hanford Company } \\
\text { MILESTONE DESCRIPTION SHEET }\end{array}$} \\
\hline \multicolumn{3}{|c|}{ Title: 2nd Quarter Status Report for the SSS Implementation } & $\begin{array}{l}\text { Date: } \\
08 / 09 / 94\end{array}$ \\
\hline \multicolumn{3}{|c|}{ Assigned To: P.J. Bence } & CIN: \\
\hline \multicolumn{3}{|c|}{ Program WBS Designator: 1 8.2.2.2 } & $\begin{array}{l}\text { Due Date: } \\
04 / 14 / 95\end{array}$ \\
\hline \multicolumn{3}{|c|}{ Control Number: PSA-95-133 } & Rev: \\
\hline $\begin{array}{l}\text { MIILSTONE TYPE: } \\
\square \text { DOE-HQ } \\
\square \text { DOE-RL } \\
\text { X CONTRACTOR }\end{array}$ & $\begin{array}{l}\quad \text { DIVISION: } \\
\quad \square \text { State } \\
\square \text { Federal } \\
\text { X DOE } \\
\square \text { RCRA } \\
\square \text { TPA Number }\end{array}$ & $\begin{array}{l}\text { DELIVERABLE: } \\
\text { X Report } \\
\square \text { Letter } \\
\square \text { Drawings } \\
\square \text { Other (specify) }\end{array}$ & $\begin{array}{l}\text { ADDRESS TO: } \\
\square \text { DOE-HQ } \\
\square \text { DOE-RL } \\
\text { X'Other (specify) } \\
\text { Selected WHC } \\
\text { Management }\end{array}$ \\
\hline \multicolumn{4}{|c|}{$\begin{array}{l}\text { Milestone Description: Issue report on status of Implementation effort. This report represent } \\
\text { the efforts the central core team has expended in supporting the SSS } \\
\text { implementation efforts during the } 3 \text { months leading up to the report. The report } \\
\text { should include training statistics, B- } 620 \text { (CAP) generation and reports, and } \\
\text { resource loaded schedules. }\end{array}$} \\
\hline \multicolumn{4}{|c|}{$\begin{array}{l}\text { Description of what constitutes completion of this milestone: Issue report to PI program } \\
\text { manager and other selected WHC managers. }\end{array}$} \\
\hline \multicolumn{4}{|c|}{ Milestone Description Approval } \\
\hline \multicolumn{2}{|c|}{$\begin{array}{l}\text { Cost Account Manager } \\
\text { P.J. Bence Pate }\end{array}$} & \multicolumn{2}{|c|}{ Program/Project Manager Pabst Date } \\
\hline \multicolumn{2}{|c|}{$\begin{array}{l}\text { Program Element Manager Date } \\
\text { L. R. Hafer Leff }\end{array}$} & \multicolumn{2}{|c|}{$\begin{array}{l}\text { DOE Program Manager Date } \\
\text { N/A }\end{array}$} \\
\hline \multicolumn{4}{|c|}{ 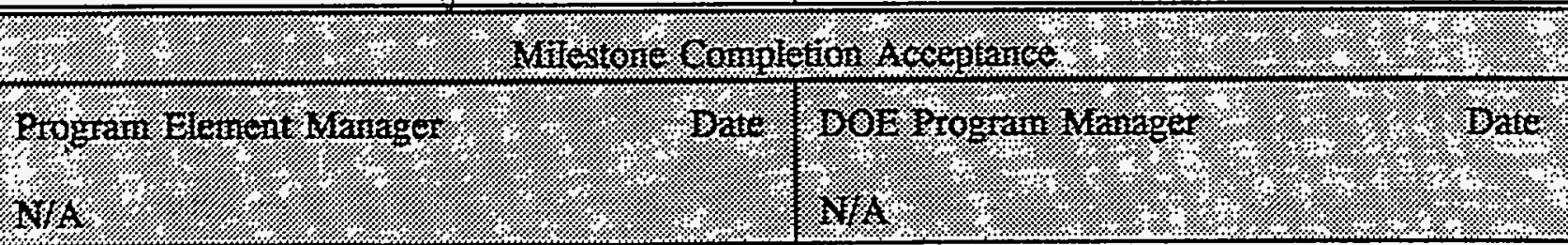 } \\
\hline
\end{tabular}




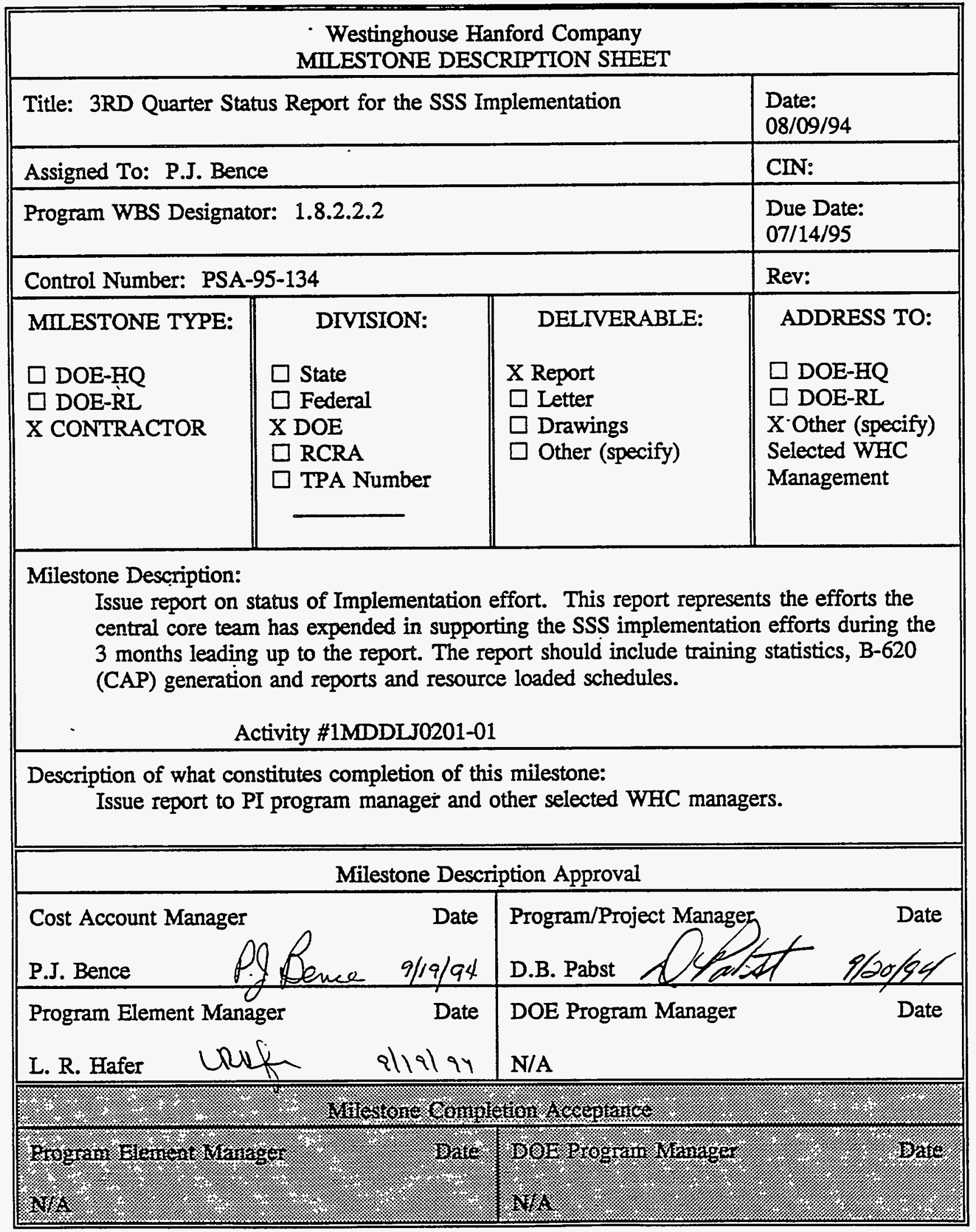




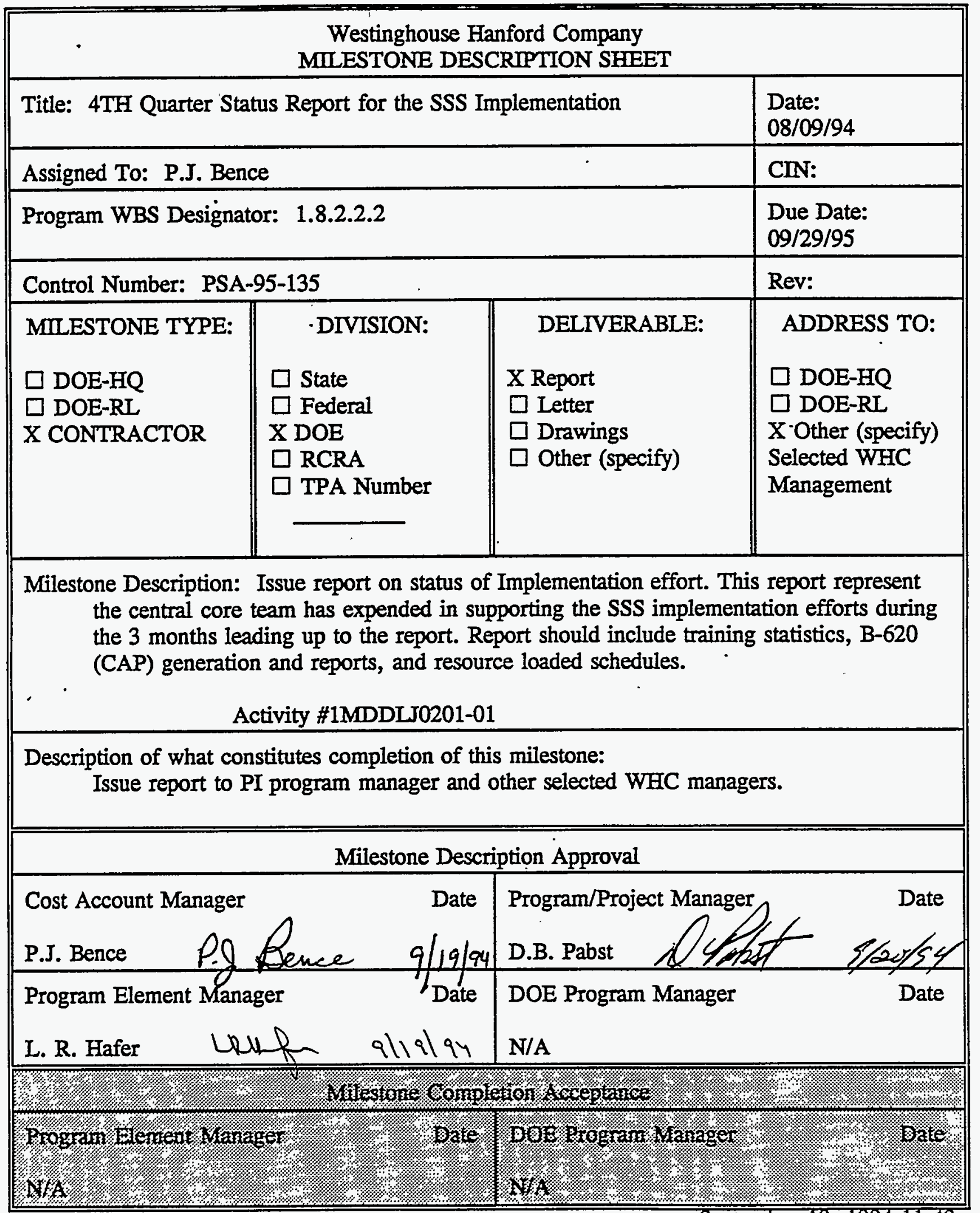

September 19, 1994 11:43am 


\begin{tabular}{|l||l|l||}
\hline \multicolumn{3}{|c|}{ Westinghouse Hanford Company } \\
MILESTONE DESCRIPTON SHEET
\end{tabular}




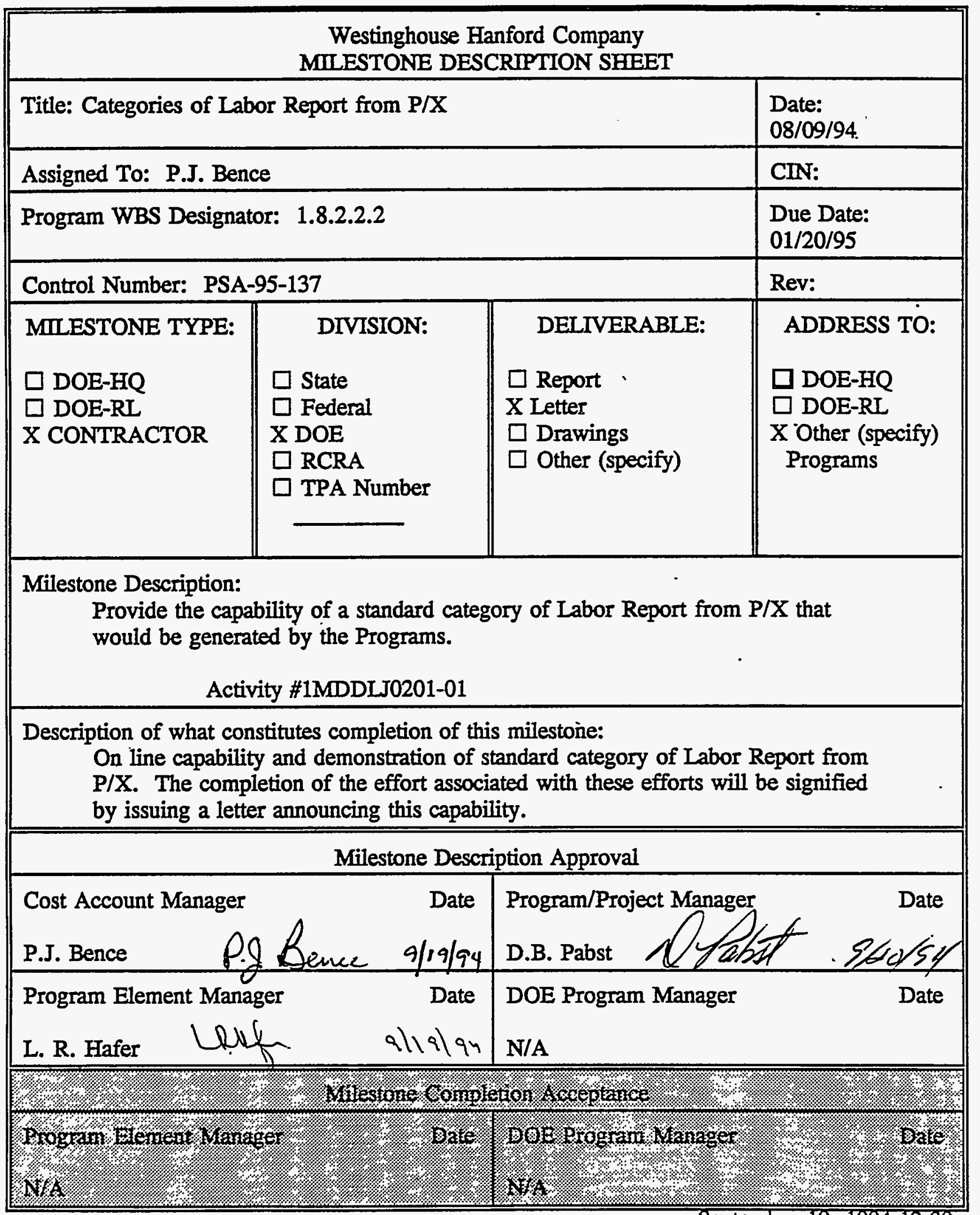




\begin{tabular}{||l|l|l||}
\hline \multicolumn{3}{|c|}{ Westinghouse Hanford Company } \\
MIESTONE DESCRIPTION SHEET
\end{tabular}

September 19,1994 12:43pm 


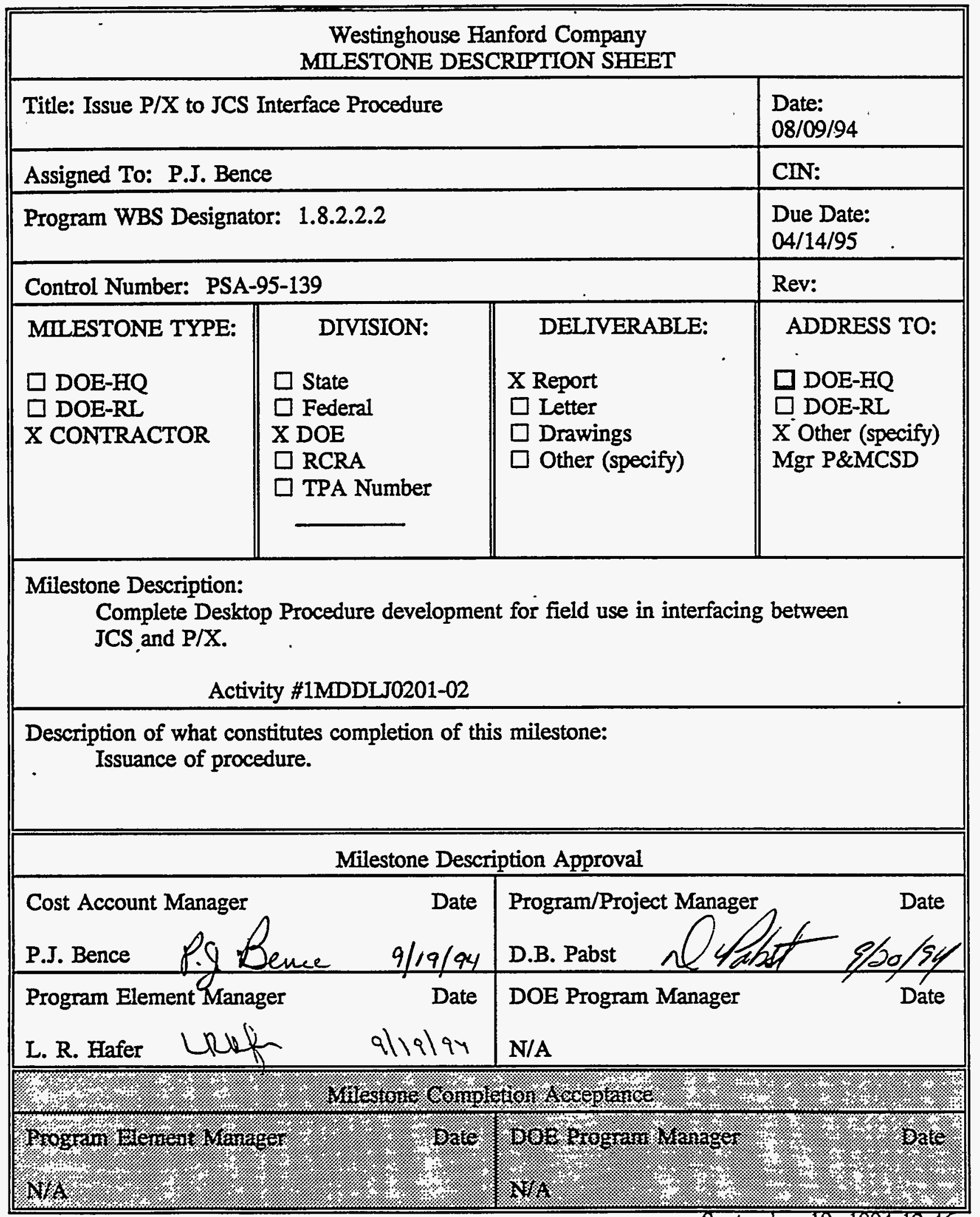




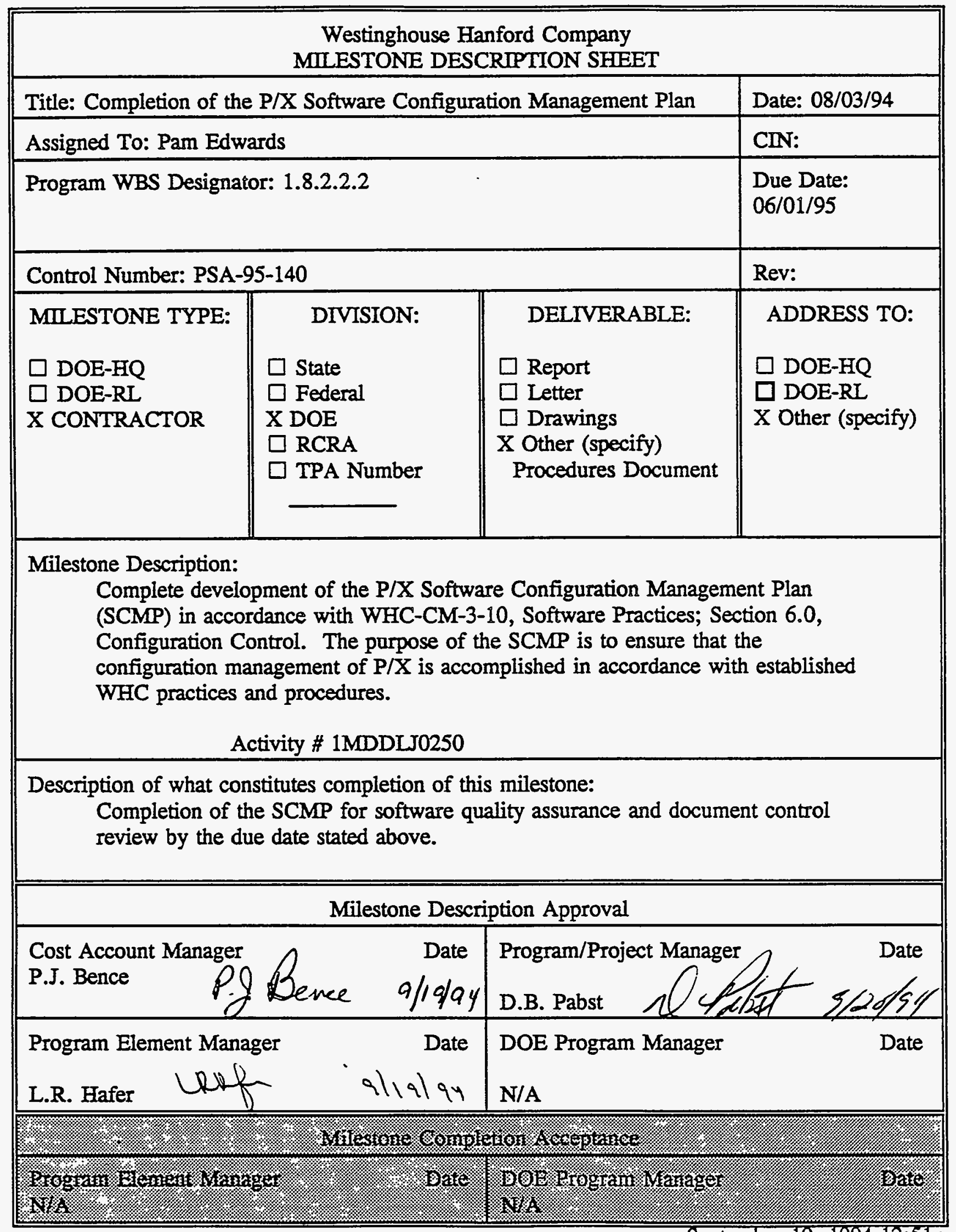




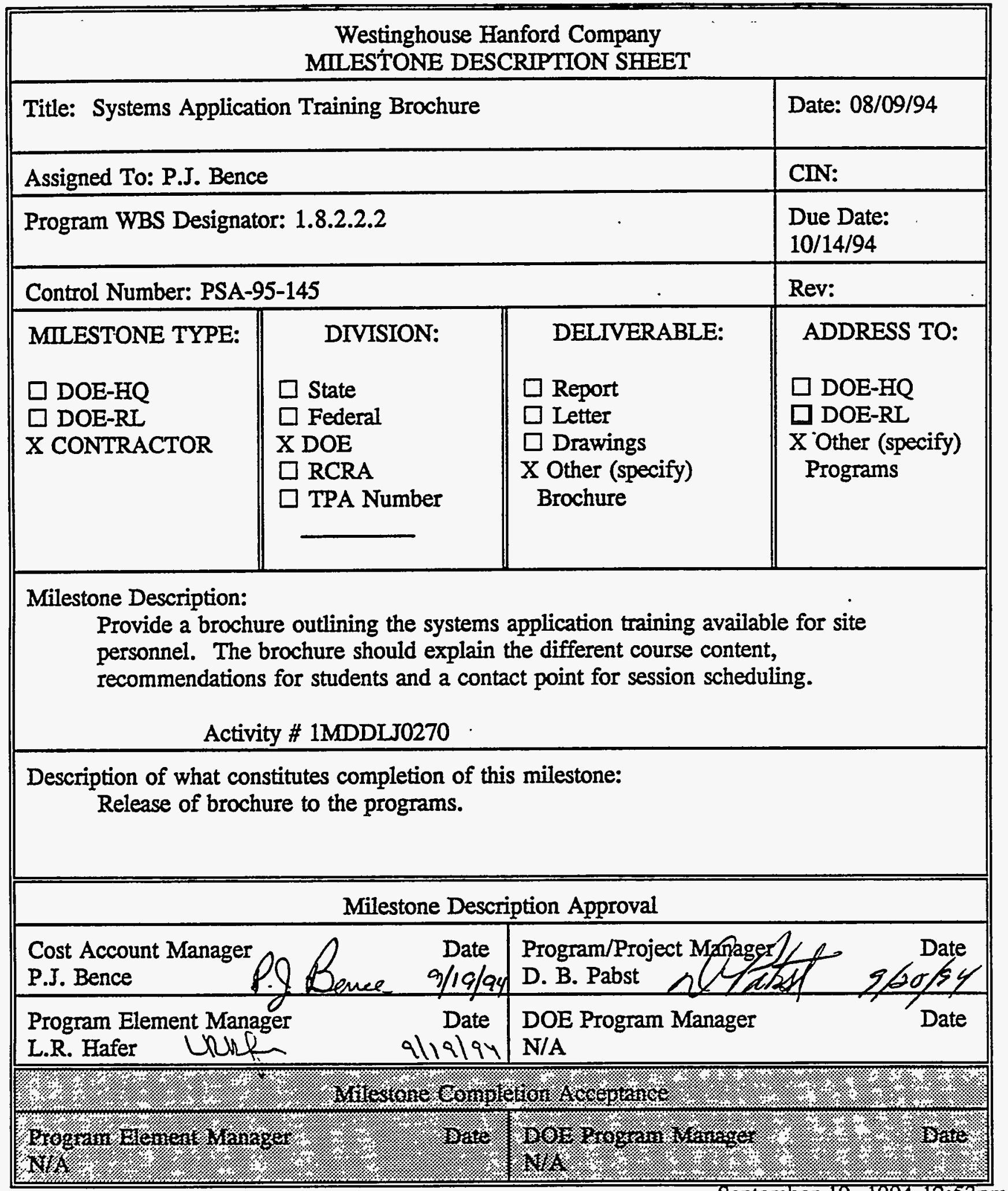




\begin{tabular}{|l||l||}
\hline \multicolumn{3}{|c||}{ Westinghouse Hanford Company } \\
MIIESTONE DESCRIPTION SHEET
\end{tabular}




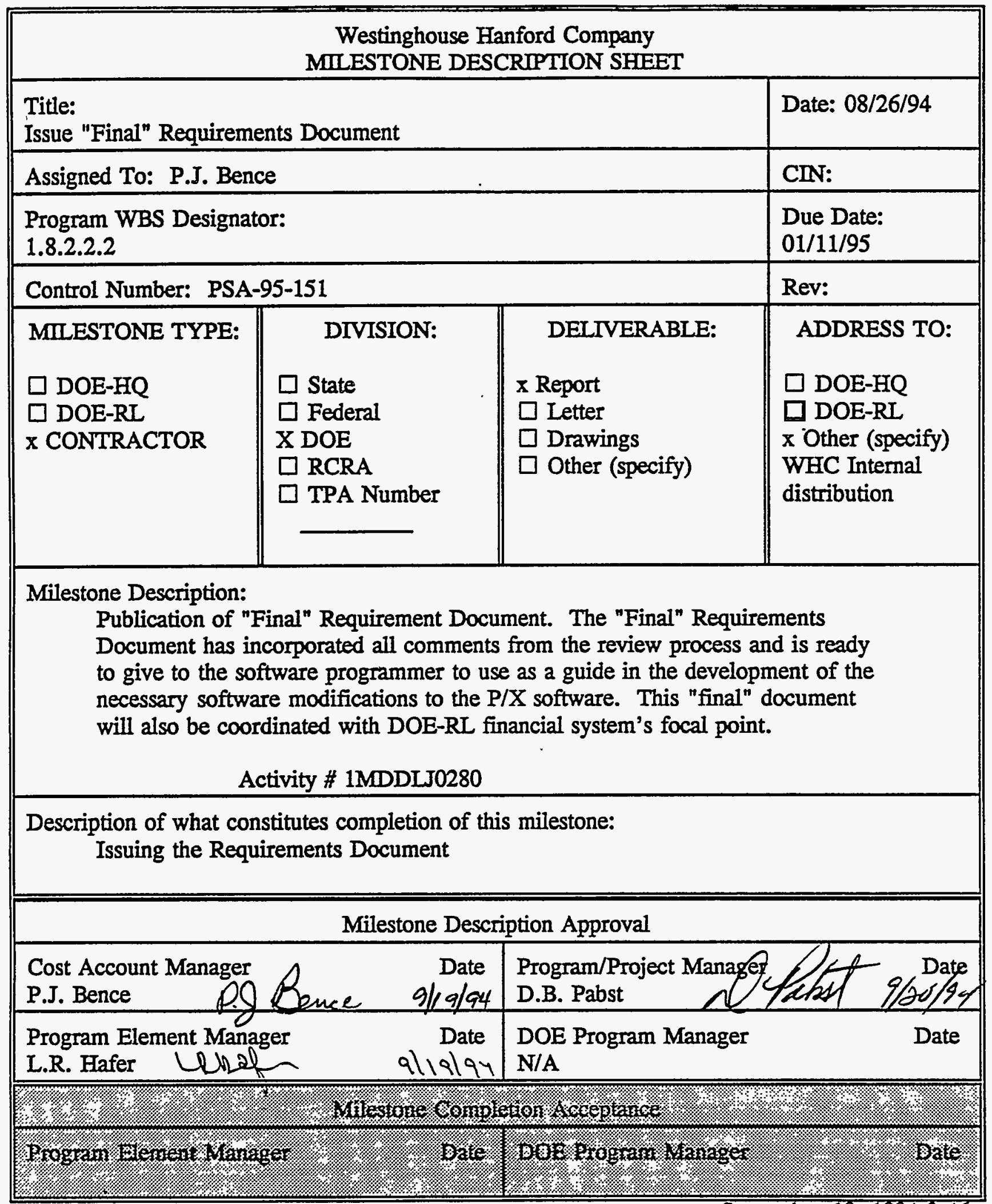




\begin{tabular}{|l|l||}
\hline \multicolumn{3}{|c||}{ Westinghouse Hanford Company } \\
MILSTONE DESCRIPTION SHEET
\end{tabular}

September 19, 1994 1:08pm 


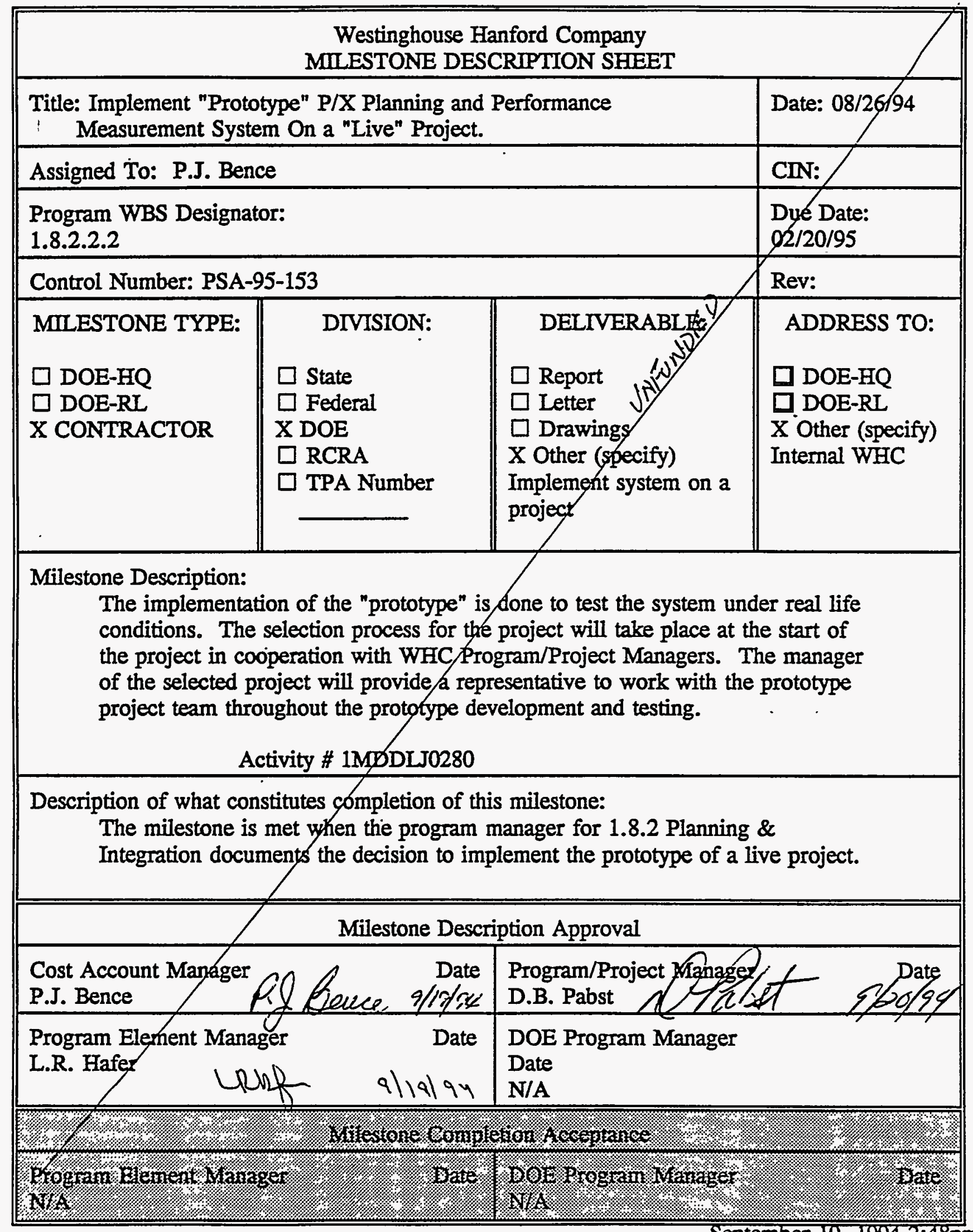




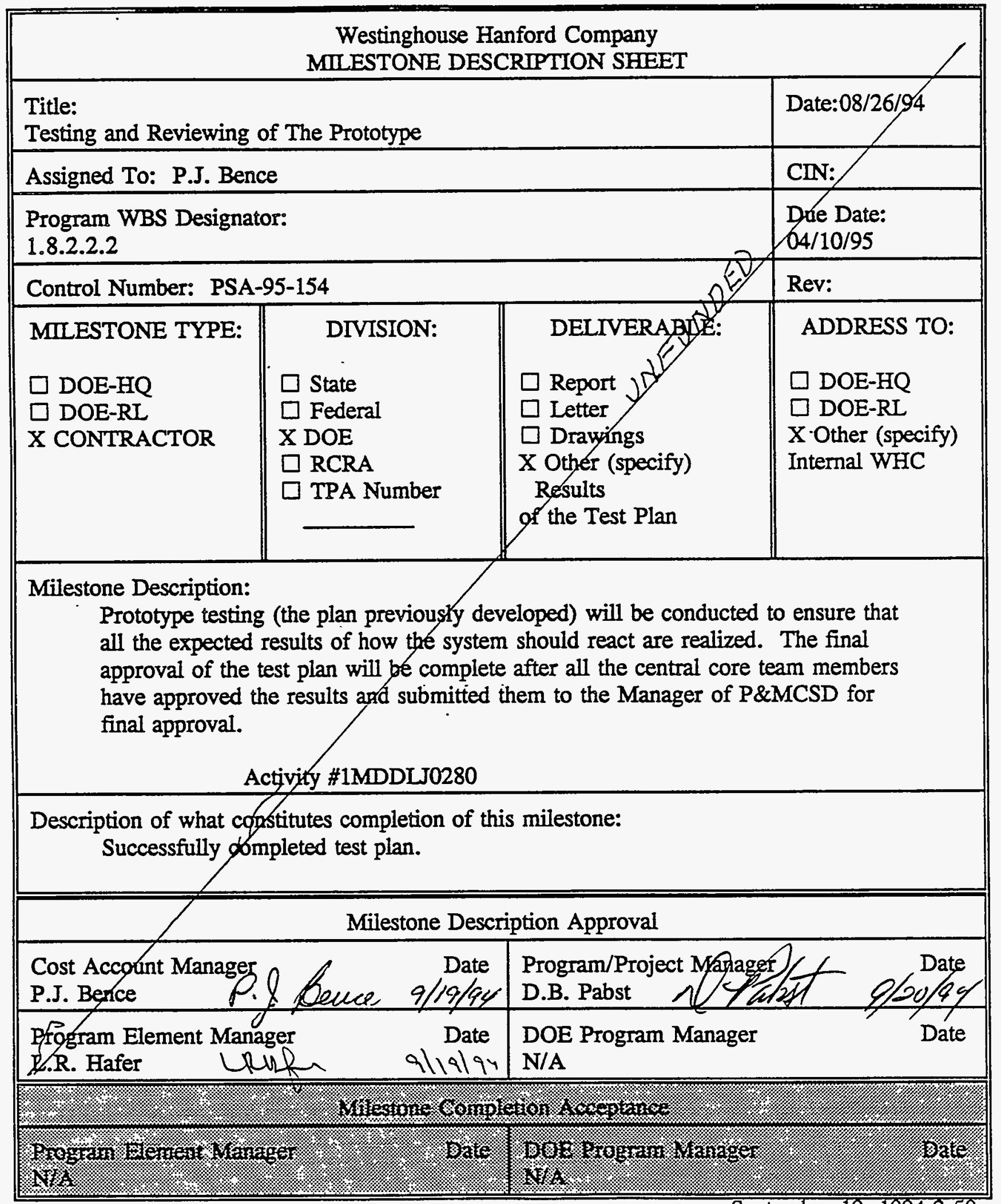




\begin{tabular}{|c|c|c|c|}
\hline \multicolumn{4}{|c|}{$\begin{array}{l}\text { Westinghouse Hanford Company } \\
\text { MILESTONE DESCRIPTION SHIEET }\end{array}$} \\
\hline \multicolumn{3}{|c|}{$\begin{array}{l}\text { Title: } \\
\text { Management Review of the Prototype.Project }\end{array}$} & Date: $08 / 26194$ \\
\hline \multicolumn{3}{|c|}{ Assigned To: P.J. Bence } & CIN: \\
\hline \multicolumn{3}{|c|}{$\begin{array}{l}\text { Program WBS Designator: } \\
\text { 1.8.2.2.2 }\end{array}$} & $\begin{array}{l}\text { Due Date: } \\
\text { 65/01/95 }\end{array}$ \\
\hline \multicolumn{3}{|c|}{ Control Number: PSA-95-155 } & Rev: \\
\hline $\begin{array}{l}\text { MILESTONE TYPE: } \\
\square \text { DOE-HQ } \\
\square \text { DOE-RL } \\
\text { X CONTRACTOR }\end{array}$ & $\begin{array}{l}\quad \text { DIVISION: } \\
\square \text { State } \\
\square \text { Federal } \\
\text { X DOE } \\
\square \text { RCRA } \\
\square \text { TPA Number }\end{array}$ & $\begin{array}{l}\text { DELIVERABCE: } \\
\square \text { Report } 15 \\
\square \text { Letter } 15 \\
\square \text { Drawings } \\
\text { X Other (specify) } \\
\text { Presentation of the } \\
\text { results of the prototype } \\
\text { to Management }\end{array}$ & $\begin{array}{l}\text { ADDRESS TO: } \\
\square \text { DOE-HQ } \\
\square \text { DOE-RL } \\
\text { X Other (specify) } \\
\text { Internally WHC }\end{array}$ \\
\hline \multicolumn{4}{|c|}{$\begin{array}{l}\text { Milestone Description: } \\
\text { A "Live" presentation will be provided to WHC upper management and } \\
\text { appropriate managers from DOE-BJ of the capabilities of the prototype. These } \\
\text { individuals will see how the system is used and what it will provide to the user } \\
\text { community. Comparisons will be presented as the pros and cons of the system } \\
\text { as it relates to the present yse of the FDS. Cost comparisons of the two systems } \\
\text { will be provided during this presentation. } \\
\text { Activity \# 1MDDLJ0280 }\end{array}$} \\
\hline \multicolumn{4}{|c|}{$\begin{array}{l}\text { Description of what constitutes completion of this milestone: } \\
\text { A formal presentation to management of the prototypes capabilities. }\end{array}$} \\
\hline \multicolumn{4}{|c|}{ Milestone Description Approval } \\
\hline \multicolumn{2}{|c|}{ 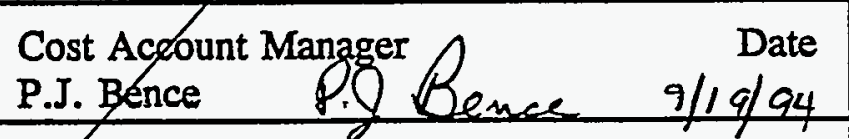 } & \multicolumn{2}{|c|}{$\begin{array}{l}\text { Program/Project Marager } \\
\text { D. B. Pabst }\end{array}$} \\
\hline \multicolumn{2}{|c|}{$\begin{array}{l}\text { Program Element Manager } \\
\text { L.R. Hafer Uate }\end{array}$} & $\begin{array}{l}\text { DOE Program Manager } \\
\text { N/A }\end{array}$ & Date \\
\hline \multicolumn{4}{|c|}{ 1. } \\
\hline \multicolumn{2}{|c|}{ 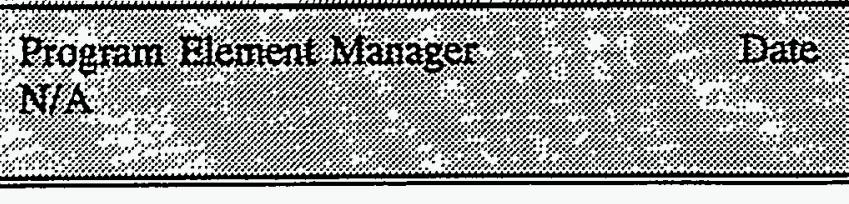 } & 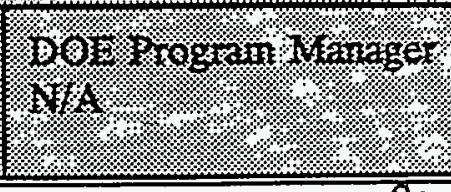 & (4. \\
\hline
\end{tabular}




\begin{tabular}{|c|c|c|c|}
\hline \multicolumn{4}{|c|}{$\begin{array}{l}\text { Westinghouse Hanford Company } \\
\text { MILESTONE DESCRIPTION SHEET }\end{array}$} \\
\hline \multicolumn{3}{|c|}{$\begin{array}{l}\text { Title: } \\
\text { GO/NO GO Decision to Implement the Prototype Sitewide }\end{array}$} & Date: $08 / 26 / 94$ \\
\hline \multicolumn{3}{|c|}{ Assigned To: P.J. Bence } & ÇIN: \\
\hline \multicolumn{3}{|c|}{ Program WBS Designator: 1 .8.2.2.2 } & $\begin{array}{l}\text { Due Date: } \\
05 / 30 / 95\end{array}$ \\
\hline \multicolumn{3}{|c|}{ Control Number: PSA-95-156 } & Rev: \\
\hline $\begin{array}{l}\text { MILESTONE TYPE: } \\
\square \text { DOE-HQ } \\
\square \text { DOE-RL } \\
\text { X CONTRACTOR }\end{array}$ & $\begin{aligned} & \text { DIVISION: } \\
& \square \text { State } \\
& \square \text { Federal } \\
& \text { X DOE } \\
& \text { RCRA } \\
& \quad \text { TPA Number }\end{aligned}$ & $\begin{array}{l}\text { DELIVERABLE: } \\
\square \text { Report } \\
\square \text { Letter } \\
\square \text { Drawings } \\
\text { X Other (specify) } \\
\text { Memo from Senior } \\
\text { Management }\end{array}$ & $\begin{array}{l}\text { ADDRESS TO: } \\
\square \text { DOE-HQ } \\
\square \text { DOE-RL } \\
\text { X Other (specify) } \\
\text { Affected } \\
\text { Programs and } \\
\text { their DOE-RL } \\
\text { counter parts }\end{array}$ \\
\hline \multicolumn{4}{|c|}{$\begin{array}{l}\text { Milestone Description: } \\
\text { The Chief Financial Officer (CFO) for WHC will issue a formal letter notifying } \\
\text { DOE-RL and affegted organizations if the implementation of the P/X Planning } \\
\text { and Performance Measuring System is a Go or a No Go. NOTE: If it is a GO, } \\
\text { the project team will have to put together a formal training class, provide the } \\
\text { training to those programs that have been selected to implement the system and } \\
\text { provide on site help. } \\
\text { Activity \# 1MDDLJ0280 }\end{array}$} \\
\hline \multicolumn{4}{|c|}{$\begin{array}{l}\text { Description of what constitutes completion of this milestone: } \\
\text { A letter issued by the WHC CFO announcing the Go/No Go decision as to the } \\
\text { implementation of a replacement system to the WHC Financial Data System. }\end{array}$} \\
\hline \multicolumn{4}{|c|}{ Milestone Description Approval } \\
\hline \multicolumn{2}{|c|}{$\begin{array}{l}\text { Cost Account Manager } \\
\text { P.J. Bence }\end{array}$} & \multicolumn{2}{|c|}{$\begin{array}{l}\text { Program/Project Manager } \\
\text { D.B. Pabst Date }\end{array}$} \\
\hline \multicolumn{2}{|c|}{$\begin{array}{l}\text { Program Element Manager } \\
\text { L.R. Hafer Late } \\
\end{array}$} & \multicolumn{2}{|c|}{$\begin{array}{l}\text { DOE Program Manager Date } \\
\text { N/A }\end{array}$} \\
\hline \multicolumn{4}{|c|}{ 1 } \\
\hline \multicolumn{2}{|c|}{ 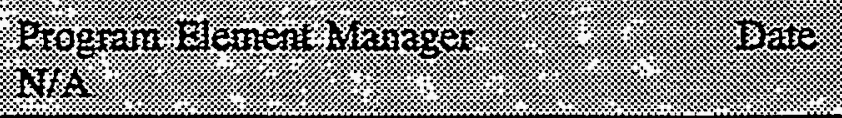 } & \multicolumn{2}{|c|}{ 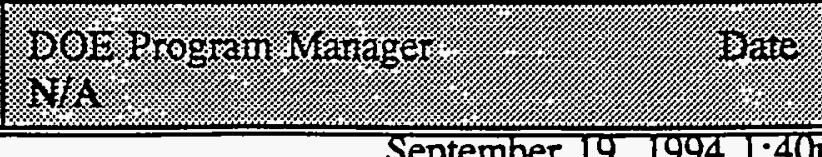 } \\
\hline
\end{tabular}




\begin{tabular}{|c|c|c|c|}
\hline \multicolumn{4}{|c|}{$\begin{array}{l}\text { Westinghouse Hanford Company } \\
\text { MILESTONE DESCRIPTION SHEET }\end{array}$} \\
\hline \multicolumn{3}{|c|}{ Title: Submit Critical Items Report to RL Manager's Office } & Date: $07 / 14 / 94$ \\
\hline \multicolumn{3}{|c|}{ Assigned To: E. A. Schultz } & CIN: \\
\hline \multicolumn{3}{|c|}{ Program WBS Designator: 1 8.2.2.1.2 } & $\begin{array}{l}\text { Due Date: } \\
10 / 13 / 95\end{array}$ \\
\hline \multicolumn{3}{|c|}{ Control Number: PSA-96-001 } & Rev: \\
\hline $\begin{array}{l}\text { MILESTONE TYPE: } \\
\square \text { DOE-HQ } \\
\square \text { DOE-RL } \\
\text { X CONTRACTOR }\end{array}$ & $\begin{array}{l}\quad \text { DIVISION: } \\
\square \text { State } \\
\square \text { Federal } \\
\square \text { DOE } \\
\square \text { RCRA } \\
\square \text { TPA Number }\end{array}$ & $\begin{array}{l}\text { DELIVERABLE: } \\
\square \text { Report } \\
\text { X Letter } \\
\square \text { Drawings } \\
\square \text { Other (specify) }\end{array}$ & $\begin{array}{l}\text { ADDRESS TO: } \\
\square \text { DOE-HQ } \\
\text { X DOE-RL-OPI } \\
\square \text { Other (specify) }\end{array}$ \\
\hline \multicolumn{4}{|c|}{$\begin{array}{l}\text { Milestone Description: } \\
\text { Coordinate, compile and submit Westinghouse Hanford Company's biweekly Critical } \\
\text { Items Report to the RL Manager in accordance with letter from J. D. Wagoner, RL to } \\
\text { Contractors, dated March } 26,1991 \text {. }\end{array}$} \\
\hline \multicolumn{4}{|c|}{$\begin{array}{l}\text { Description of what constitutes completion of this milestone: } \\
\text { Delivery of the Critical Items Report to the RL Manager's Office by October 13, } 1995 .\end{array}$} \\
\hline \multicolumn{4}{|c|}{ Milestone Description Approval } \\
\hline \multicolumn{2}{|l|}{$\begin{array}{l}\text { Cost Account Manager } \\
\text { EA Schultz }\end{array}$} & $\begin{array}{l}\text { Program/Project Manager } \\
\text { DB Pabst }\end{array}$ & Date \\
\hline \multicolumn{2}{|c|}{$\begin{array}{l}\text { Program Element Manager } \\
\text { LR Hafer }\end{array}$} & \multicolumn{2}{|c|}{$\begin{array}{l}\text { DOE Program Manager Date } \\
\text { N/A }\end{array}$} \\
\hline \multicolumn{4}{|c|}{ अ० } \\
\hline \multicolumn{3}{|c|}{ 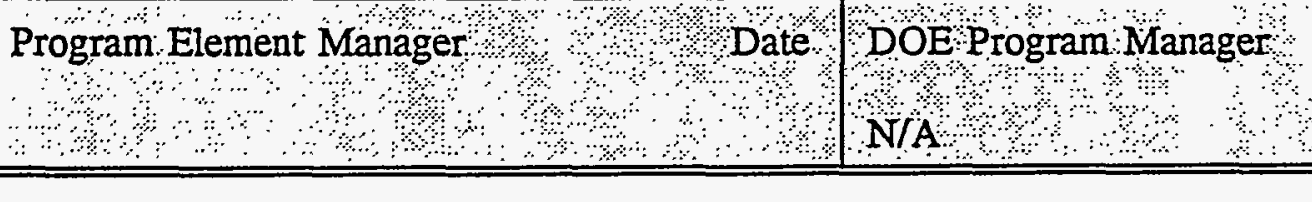 } & Date \\
\hline
\end{tabular}




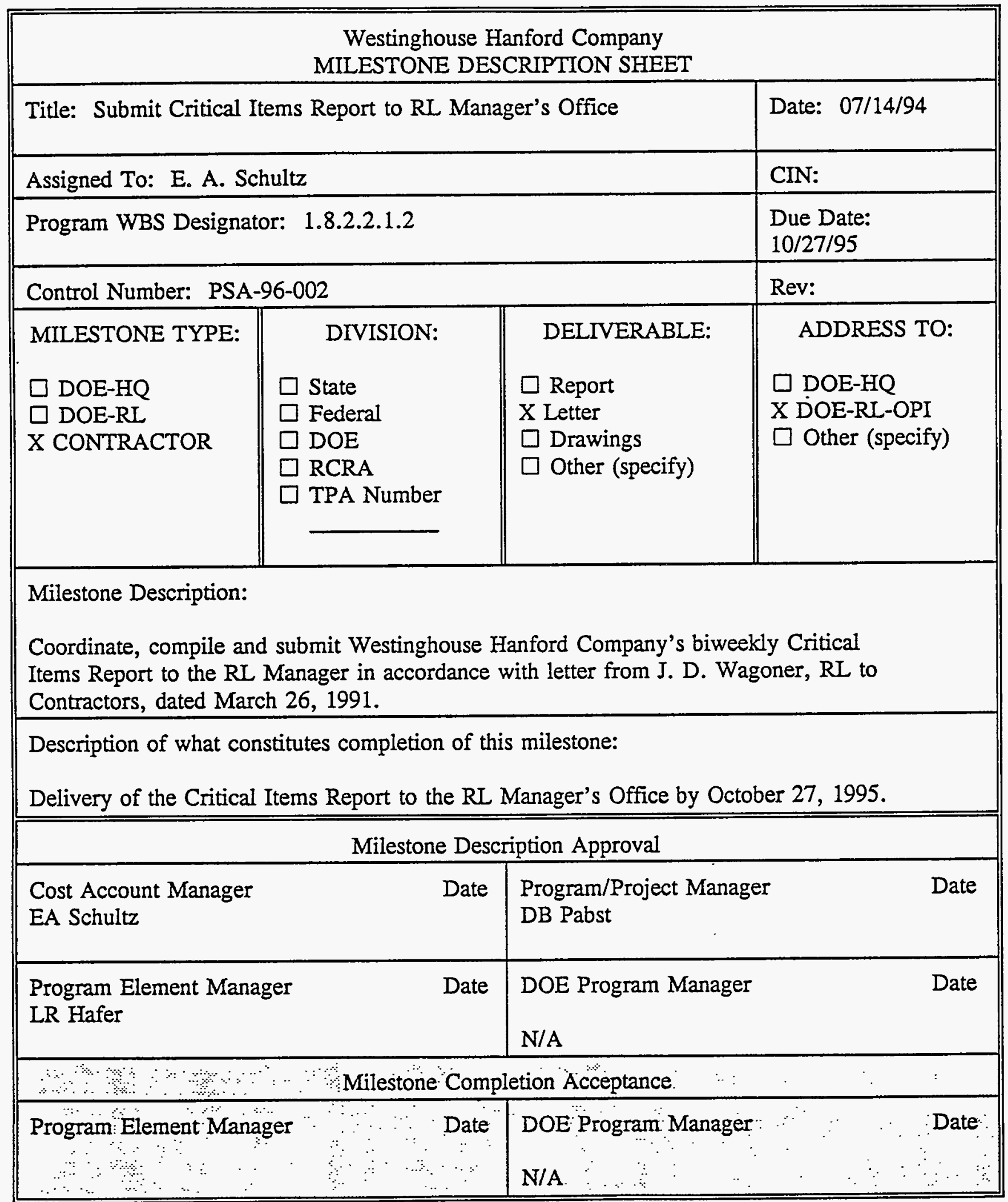




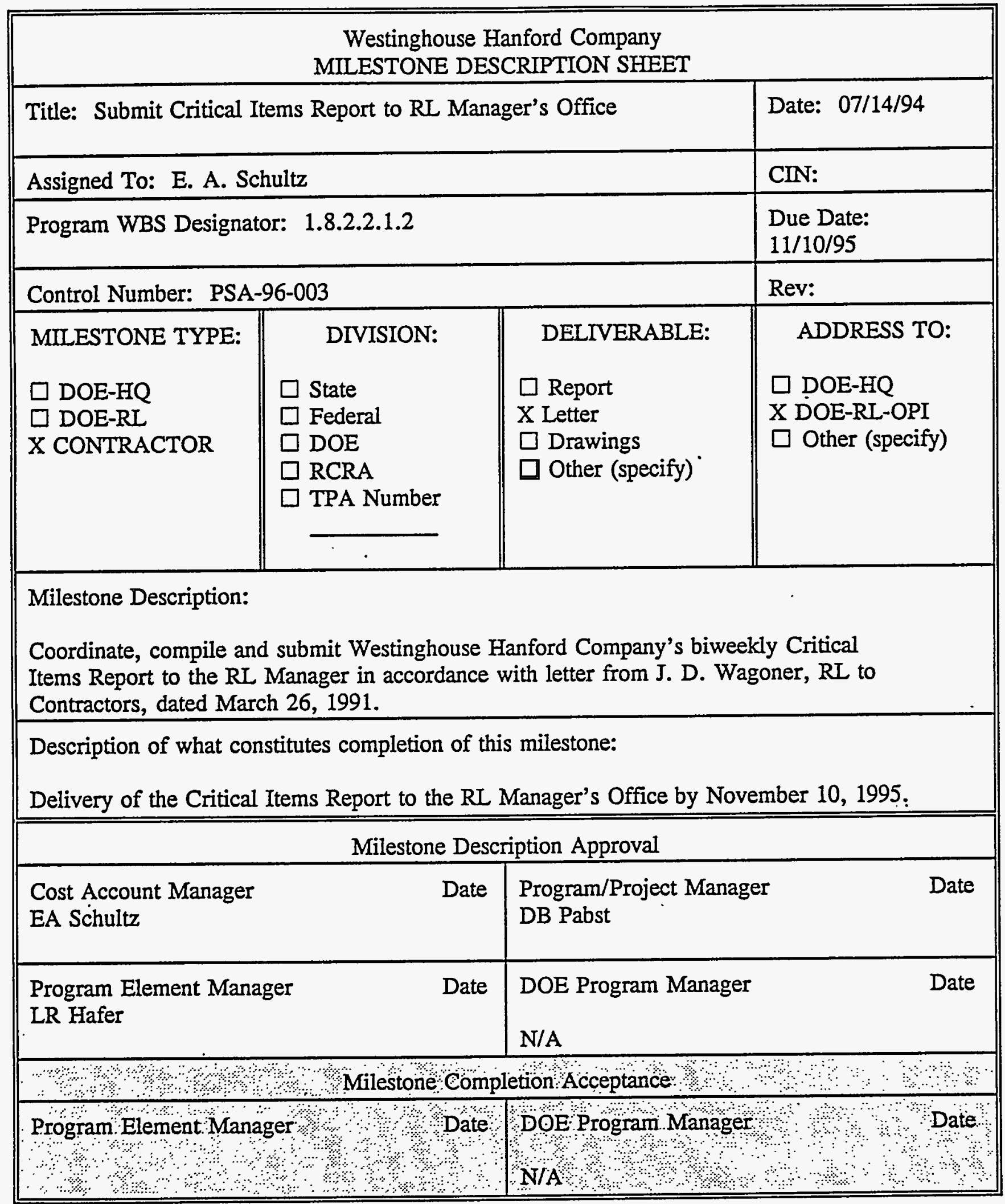




\begin{tabular}{|c|c|c|c|}
\hline \multicolumn{4}{|c|}{$\begin{array}{l}\text { Westinghouse Hanford Company } \\
\text { MILESTONE DESCRIPTION SHEET }\end{array}$} \\
\hline \multicolumn{3}{|c|}{ Title: Submit Critical Items Report to RL Manager's Office } & Date: $07 / 14 / 94$ \\
\hline \multicolumn{3}{|c|}{ Assigned To: E. A. Schultz } & $\mathrm{CIN}:$ \\
\hline \multicolumn{3}{|c|}{ Program WBS Designator: 1 8.2.2.1.2 } & $\begin{array}{l}\text { Due Date: } \\
11 / 24 / 95\end{array}$ \\
\hline \multicolumn{3}{|c|}{ Control Number: PSA-96-004 } & Rev: \\
\hline $\begin{array}{l}\text { MILESTONE TYPE: } \\
\square \text { DOE-HQ } \\
\square \text { DOE-RL } \\
\text { X CONTRACTOR }\end{array}$ & $\begin{aligned} & \text { DIVISION: } \\
& \\
& \text { State } \\
& \text { Federal } \\
& \square \text { DOE } \\
& \quad \text { RCRA } \\
& \square \text { TPA Number }\end{aligned}$ & $\begin{array}{l}\text { DÉLIVERABLE: } \\
\square \text { Report } \\
\text { X Letter } \\
\square \text { Drawings } \\
\square \text { Other (specify) }\end{array}$ & $\begin{array}{l}\text { ADDRESS TO: } \\
\square \text { DOE-HQ } \\
\text { X DOE-RL-OPI } \\
\square \text { Other (specify) }\end{array}$ \\
\hline \multicolumn{4}{|c|}{$\begin{array}{l}\text { Milestone Description: } \\
\text { Coordinate, compile and submit Westinghouse Hanford Company's biweekly Critical } \\
\text { Items Report to the RL Manager in accordance with letter from J. D. Wagoner, RL to } \\
\text { Contractors, dated March 26,. } 1991 \text {. }\end{array}$} \\
\hline \multicolumn{4}{|c|}{$\begin{array}{l}\text { Description of what constitutes completion of this milestone: } \\
\text { Delivery of the Critical Items Report to the RL Manager's Office by November 24, } 1995 .\end{array}$} \\
\hline \multicolumn{4}{|c|}{ Milestone Description Approval } \\
\hline \multicolumn{2}{|l|}{$\begin{array}{l}\text { Cost Account Manager } \\
\text { EA Schultz }\end{array}$} & $\begin{array}{l}\text { Program/Project Manager } \\
\text { DB Pabst }\end{array}$ & Date \\
\hline \multicolumn{2}{|c|}{$\begin{array}{l}\text { Program Element Manager } \\
\text { LR Hafer }\end{array}$} & \multicolumn{2}{|l|}{$\begin{array}{l}\text { DOE Program Manager } \\
\text { N/A }\end{array}$} \\
\hline \multicolumn{4}{|c|}{ : $\quad$ Milestone Completion Acceptance } \\
\hline $\begin{array}{l}\text { Program Element Mana } \\
\vdots \\
\qquad \text { : }\end{array}$ & $\begin{array}{cccc}\text { ser } & \ddots & \ddots & \text { Date } \\
\ddots & \ddots & \ddots & \\
& \ddots & \end{array}$ & $\begin{array}{l}\text { DOE Program Manager } \\
\text { N/A }\end{array}$ & 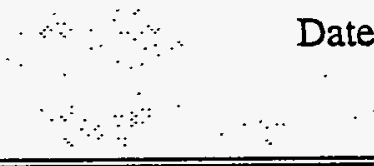 \\
\hline
\end{tabular}




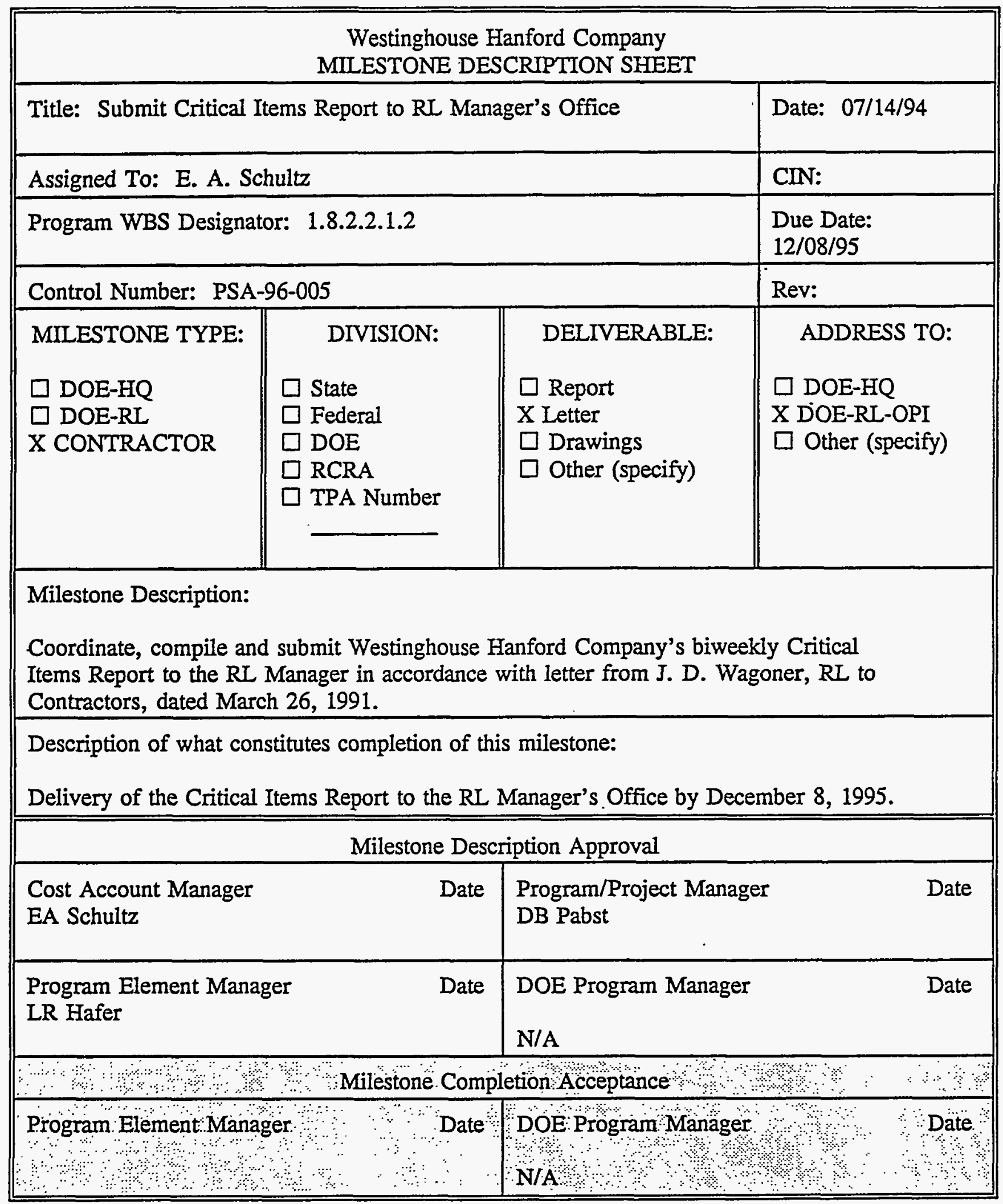




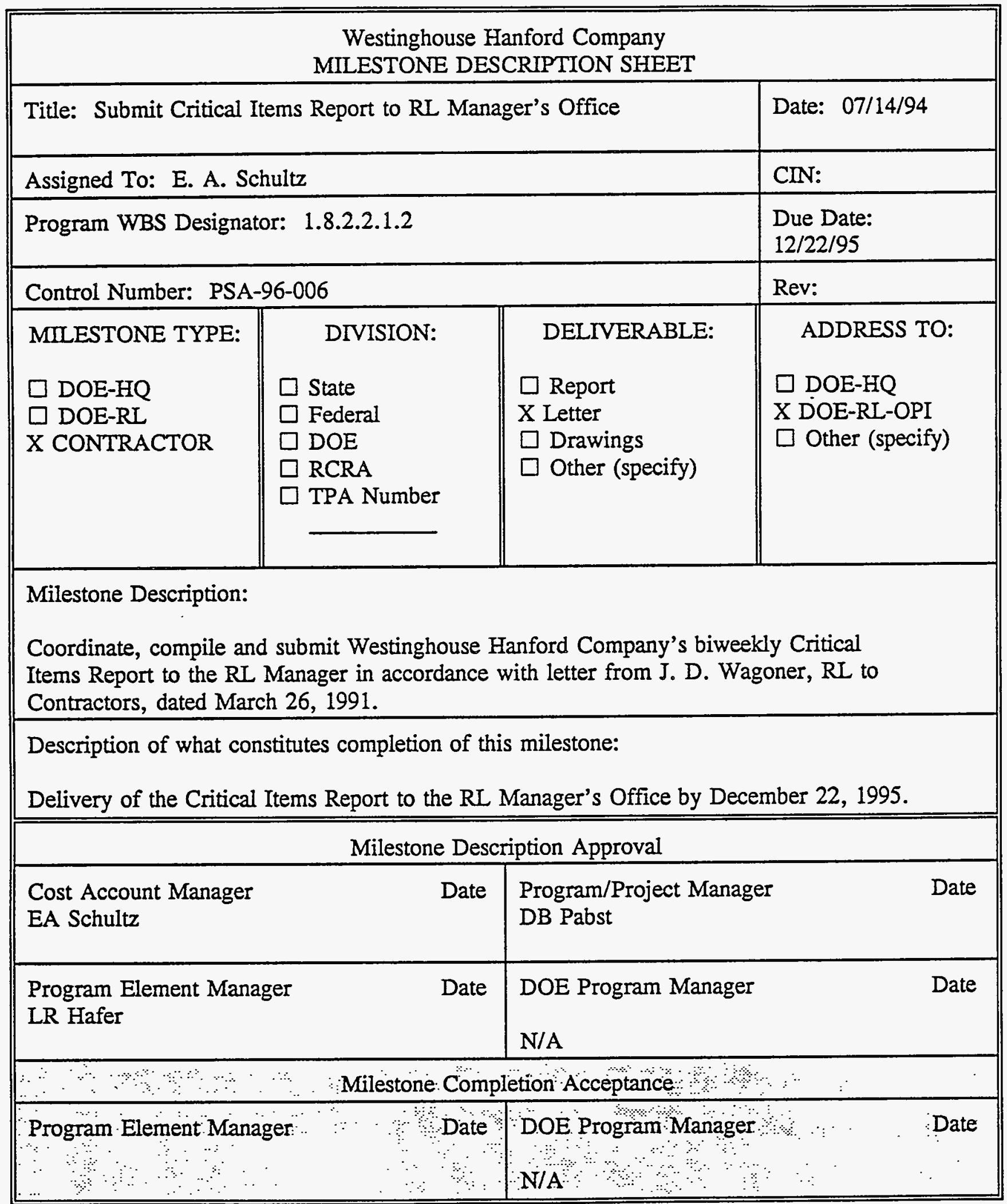




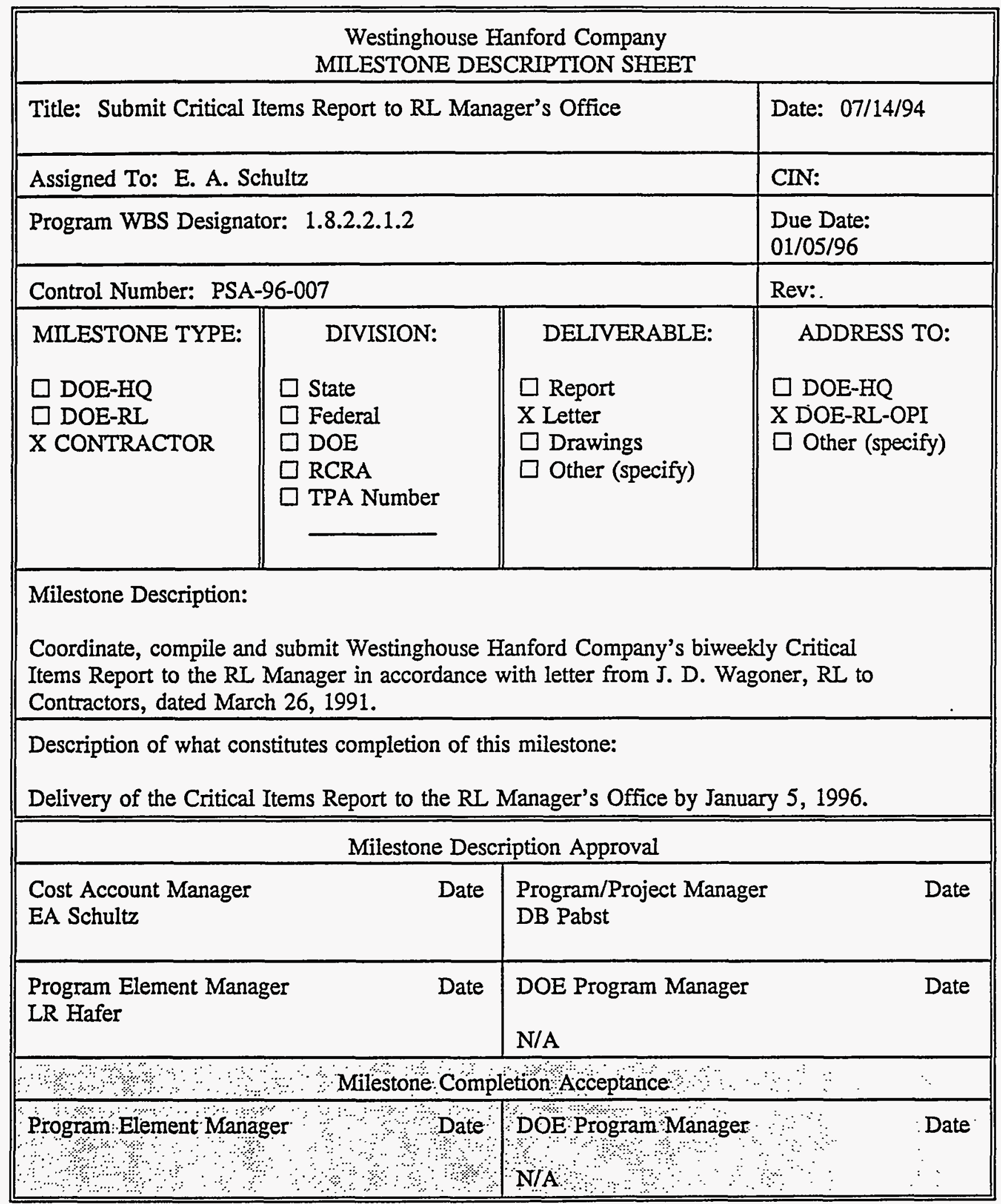




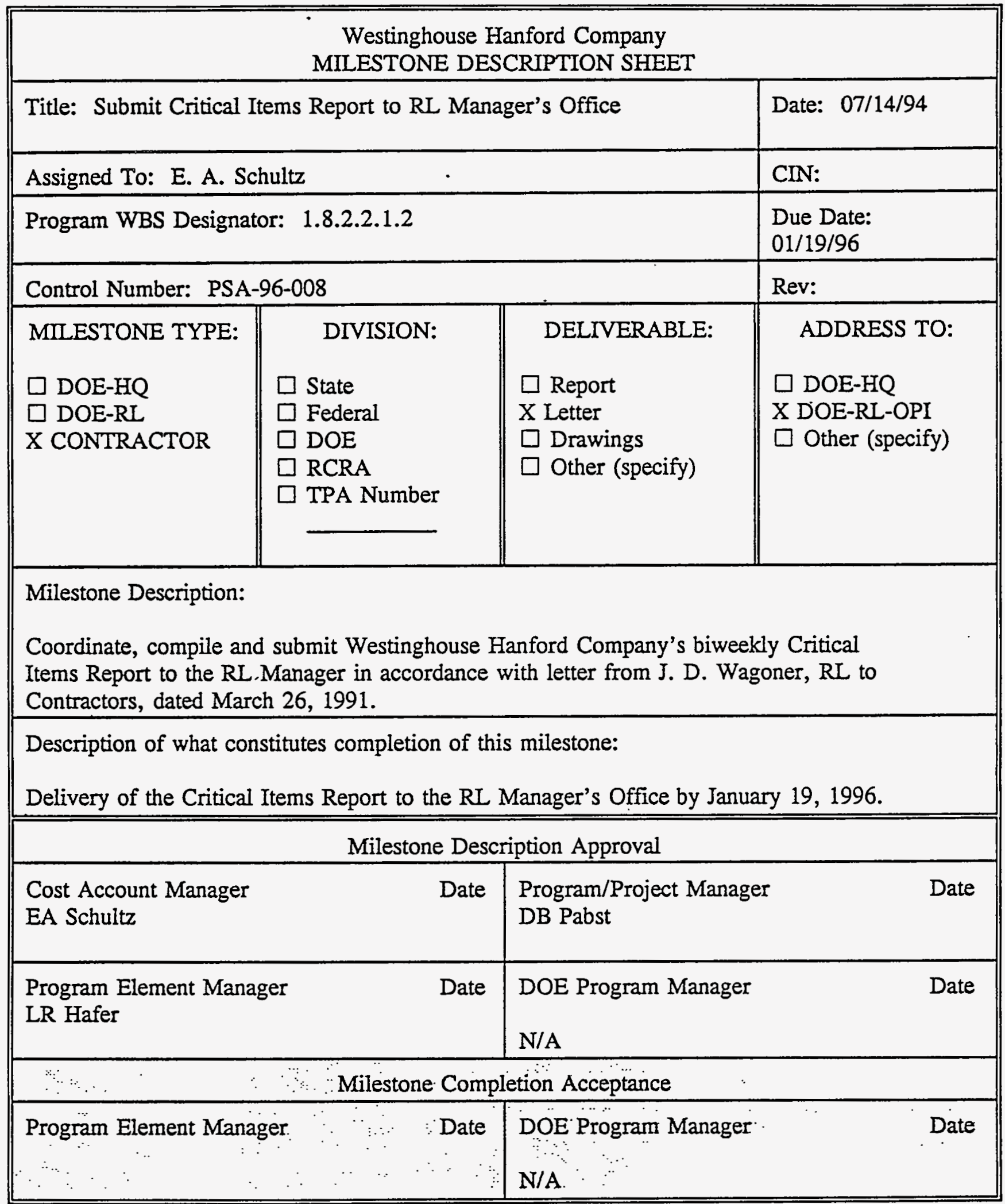




\begin{tabular}{||l|l||}
\hline \multicolumn{3}{|c|}{ Westinghouse Hanford Company } \\
MILESTONE DESCRIPTION SHEET
\end{tabular}




\begin{tabular}{|c|c|c|c|}
\hline \multicolumn{4}{|c|}{$\begin{array}{l}\text { Westinghouse Hanford Company } \\
\text { MILESTONE DESCRIPTION SHEET }\end{array}$} \\
\hline \multicolumn{3}{|c|}{ Title: Submit Critical Items Report to RL Manager's Office } & Date: $07 / 14 / 94$ \\
\hline \multicolumn{3}{|c|}{ Assigned To: E. A. Schultz } & CIN: \\
\hline \multicolumn{3}{|c|}{ Program WBS Designator: 1 8.2.2.1.2 } & $\begin{array}{l}\text { Due Date: } \\
02 / 16 / 96 \\
\end{array}$ \\
\hline \multicolumn{3}{|c|}{ Control Number: PSA-96-010 } & \multirow{2}{*}{$\begin{array}{l}\text { Rev: } \\
\text { ADDRESS TO: } \\
\square \text { DOE-HQ } \\
\text { X DOE-RL-OPI } \\
\square \text { Other (specify) }\end{array}$} \\
\hline $\begin{array}{l}\text { MILESTONE TYPE: } \\
\square \text { DOE-HQ } \\
\square \text { DOE-RL } \\
\text { X CONTRACTOR }\end{array}$ & $\begin{array}{l}\quad \text { DIVISION: } \\
\square \text { State } \\
\square \text { Federal } \\
\square \text { DOE } \\
\square \text { RCRA } \\
\square \text { TPA Number }\end{array}$ & $\begin{array}{l}\text { DELIVERABLE: } \\
\square \text { Report } \\
\text { X Letter } \\
\square \text { Drawings } \\
\square \text { Other (specify) }\end{array}$ & \\
\hline \multicolumn{4}{|c|}{$\begin{array}{l}\text { Milestone Description: } \\
\text { Coordinate, compile and submit Westinghouse Hanford Company's biweekly Critical } \\
\text { Items Report to the RL Manager in accordance with letter from J. D. Wagoner, RL to } \\
\text { Contractors, dated March 26, } 1991 .\end{array}$} \\
\hline \multicolumn{4}{|c|}{$\begin{array}{l}\text { Description of what constitutes.completion of this milestone: } \\
\text { Delivery of the Critical Items Report to the RL Manager's Office by February 16, } 1996 .\end{array}$} \\
\hline \multicolumn{4}{|c|}{ Milestone Description Approval } \\
\hline \multicolumn{2}{|l|}{$\begin{array}{l}\text { Cost Account Manager } \\
\text { EA Schultz }\end{array}$} & \multicolumn{2}{|l|}{$\begin{array}{l}\text { Program/Project Manager } \\
\text { DB Pabst }\end{array}$} \\
\hline \multicolumn{2}{|c|}{$\begin{array}{l}\text { Program Element Manager } \\
\text { LR Hafer }\end{array}$} & \multicolumn{2}{|c|}{$\begin{array}{l}\text { DOE Program Manager Date } \\
\text { N/A }\end{array}$} \\
\hline \multicolumn{4}{|c|}{\begin{tabular}{|lllll} 
& $\cdots$ & $\cdots$ & $\cdots$ & Milestone Completion Acceptance: \\
\end{tabular}} \\
\hline \multicolumn{2}{|c|}{ 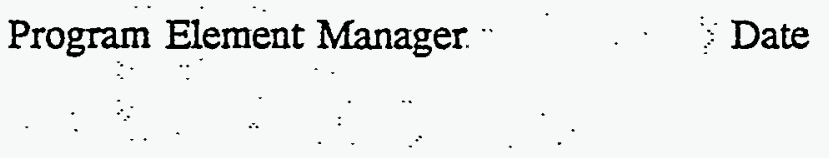 } & \multicolumn{2}{|l|}{$\begin{array}{l}\text { DOE Program Manager } \\
\text { N/A }\end{array}$} \\
\hline
\end{tabular}




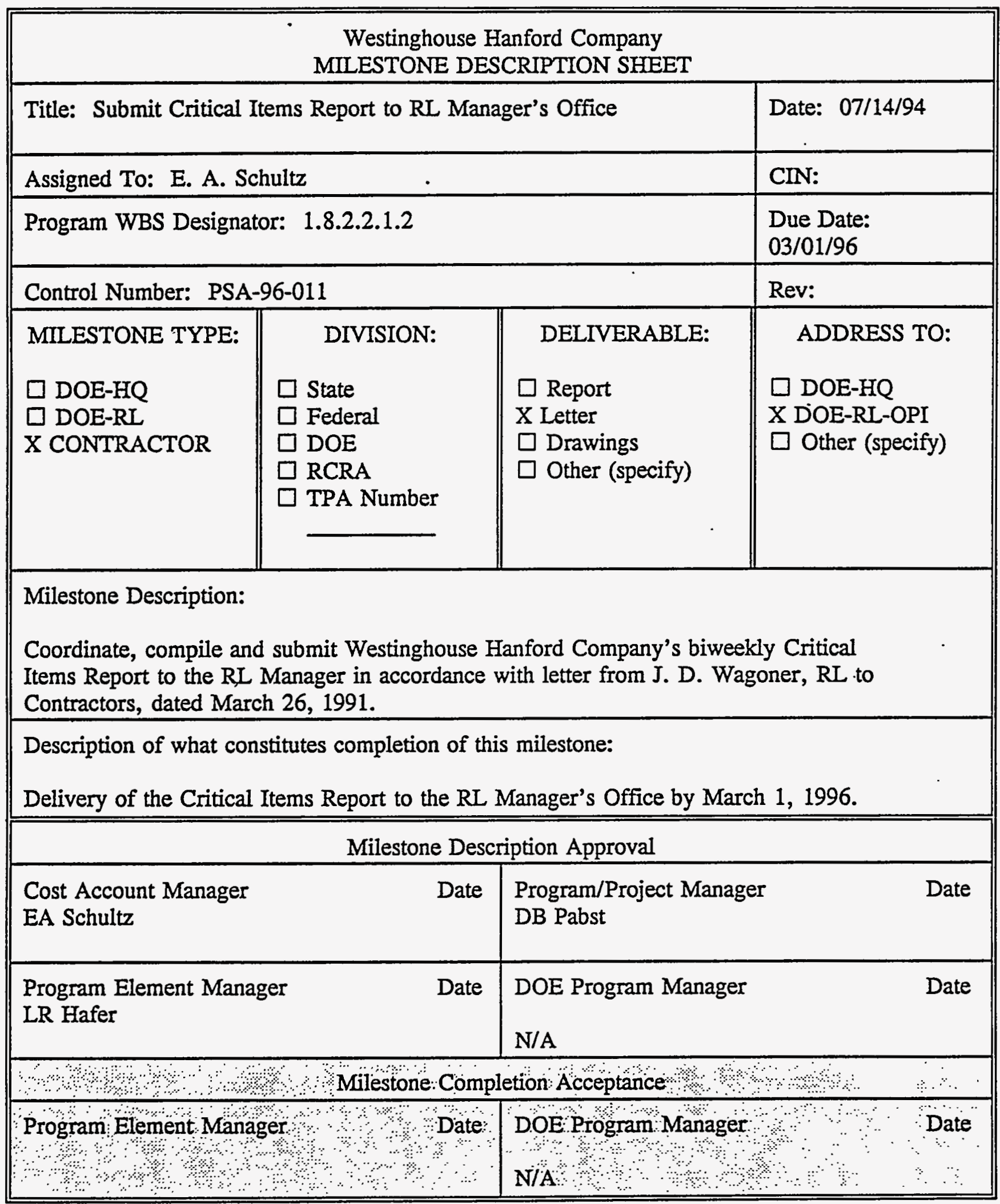




\begin{tabular}{|l||l|l||}
\hline \multicolumn{3}{|c|}{ Westinghouse Hanford Company } \\
MILESTONE DESCRIPTION SHEET
\end{tabular}




\begin{tabular}{|c|c|c|c|c|}
\hline \multicolumn{5}{|c|}{$\begin{array}{l}\text { Westinghouse Hanford Company } \\
\text { MILESTONE DESCRIPTION SHEET }\end{array}$} \\
\hline \multicolumn{3}{|c|}{ Title: Submit Critical Items Report to RL Manager's Office } & \multicolumn{2}{|c|}{ Date: $07 / 14 / 94$} \\
\hline \multicolumn{3}{|c|}{ Assigned To: E. A. Schultz } & \multicolumn{2}{|c|}{ CIN: } \\
\hline \multicolumn{3}{|c|}{ Program WBS Designator: 1 8.2.2.1.2 } & \multicolumn{2}{|c|}{$\begin{array}{l}\text { Due Date: } \\
\text { 03/29/96 }\end{array}$} \\
\hline \multicolumn{3}{|c|}{ Control Number: PSA-96-013 } & \multicolumn{2}{|c|}{ Rev: } \\
\hline $\begin{array}{l}\text { MILESTONE TYPE: } \\
\square \text { DOE-HQ } \\
\square \text { DOE-RL } \\
\text { X CONTRACTOR }\end{array}$ & $\begin{aligned} & \text { DIVISION: } \\
& \square \text { State } \\
& \square \text { Federal } \\
& \square \text { DOE } \\
& \square \text { RCRA } \\
& \square \text { TPA Number }\end{aligned}$ & $\begin{array}{l}\text { DELIVERABLE: } \\
\square \text { Report } \\
\text { X Letter } \\
\square \text { Drawings } \\
\square \text { Other (specify) }\end{array}$ & \multicolumn{2}{|c|}{$\begin{array}{l}\text { ADDRESS TO: } \\
\square \text { DOE-HQ } \\
\text { X DOE-RL-OPI } \\
\square \text { Other (specify) }\end{array}$} \\
\hline \multicolumn{5}{|c|}{$\begin{array}{l}\text { Milestone Description: } \\
\text { Coordinate, compile and submit Westinghouse Hanford Company's biweekly Critical } \\
\text { Items Report to the RL Manager in accordance with letter from J. D. Wagoner, RL to } \\
\text { Contractors, dated March } 26,1991 \text {. }\end{array}$} \\
\hline \multicolumn{5}{|c|}{$\begin{array}{l}\text { Description of what constitutes completion of this milestone: } \\
\text { Delivery of the Critical Items Report to the RL Manager's Office by March 29, } 1996 .\end{array}$} \\
\hline \multicolumn{5}{|c|}{ Milestone Description Approval } \\
\hline \multicolumn{2}{|l|}{$\begin{array}{l}\text { Cost Account Manager } \\
\text { EA Schultz }\end{array}$} & \multicolumn{2}{|c|}{$\begin{array}{l}\text { Program/Project Manager } \\
\text { DB Pabst }\end{array}$} & Date \\
\hline \multicolumn{2}{|c|}{$\begin{array}{l}\text { Program Element Manager } \\
\text { LR Hafer }\end{array}$} & \multicolumn{2}{|c|}{$\begin{array}{l}\text { DOE Program Manager } \\
\text { N/A }\end{array}$} & Date \\
\hline \multicolumn{5}{|c|}{ औ o Milestone Completton Acceptance } \\
\hline \multicolumn{2}{|c|}{ Program Element Manager } & \multicolumn{3}{|c|}{$\begin{array}{l}\mathrm{DOE} \text { Program Manager } \\
\mathrm{N} / \mathrm{A}\end{array}$} \\
\hline
\end{tabular}




\begin{tabular}{|c|c|c|c|}
\hline \multicolumn{4}{|c|}{$\begin{array}{l}\text { Westinghouse Hanford Company } \\
\text { MILESTONE DESCRIPTION SHEET }\end{array}$} \\
\hline \multicolumn{3}{|c|}{ Title: Submit Critical Items Report to RL Manager's Office } & Date: $07 / 14 / 94$ \\
\hline \multicolumn{3}{|c|}{ Assigned To: E. A. Schultz } & CIN: \\
\hline \multicolumn{3}{|c|}{ Program WBS Designator: 1 8.2.2.1.2 } & $\begin{array}{l}\text { Due Date: } \\
\text { 04/12/96 }\end{array}$ \\
\hline \multicolumn{3}{|c|}{ Control Number: PSA-96-014 } & Rev: \\
\hline $\begin{array}{l}\text { MILESTONE TYPE: } \\
\square \text { DOE-HQ } \\
\square \text { DOE-RL } \\
\text { X CONTRACTOR }\end{array}$ & $\begin{aligned} & \text { DIVISION: } \\
& \square \text { State } \\
& \square \text { Federal } \\
& \square \text { DOE } \\
& \quad \text { RCRA } \\
& \square \text { TPA Number }\end{aligned}$ & $\begin{array}{l}\text { DELIVERABLE: } \\
\square \text { Report } \\
\text { X Letter } \\
\square \text { Drawings } \\
\square \text { Other (specify) }\end{array}$ & $\begin{array}{l}\text { ADDRESS TO: } \\
\square \text { DOE-HQ } \\
\text { X DOE-RL-OPI } \\
\square \text { Other (specify) }\end{array}$ \\
\hline \multicolumn{4}{|c|}{$\begin{array}{l}\text { Milestone Description: } \\
\text { Coordinate, compile and submit Westinghouse Hanford Company's biweekly Critical } \\
\text { Items Report to the RL Manager in accordance with letter from J. D. Wagoner, RL to } \\
\text { Contractors, dated March } 26,1991 \text {. }\end{array}$} \\
\hline \multicolumn{4}{|c|}{$\begin{array}{l}\text { Description of what constitutes completion of this milestone: } \\
\text { Delivery of the Critical Items Report to the RL Manager's Office by April 12, } 1996 .\end{array}$} \\
\hline \multicolumn{4}{|c|}{ Milestone Description Approval } \\
\hline \multicolumn{2}{|l|}{$\begin{array}{l}\text { Cost Account Manager } \\
\text { EA Schultz }\end{array}$} & $\begin{array}{l}\text { Program/Project Manager } \\
\text { DB Pabst }\end{array}$ & Date \\
\hline \multicolumn{2}{|c|}{$\begin{array}{l}\text { Program Element Manager } \\
\text { LR Hafer }\end{array}$} & $\begin{array}{l}\text { DOE Program Manager } \\
\text { N/A }\end{array}$ & Date \\
\hline \multicolumn{2}{|c|}{$\begin{array}{c}\text { Program Element Manager } \\
\therefore\end{array}$} & $\begin{array}{l}\text { DOE Program Manager } \\
\text { N/A } \\
\end{array}$ & $\therefore$ \\
\hline
\end{tabular}




\begin{tabular}{||l|l||}
\hline \multicolumn{3}{|c|}{ Westinghouse Hanford Company } \\
MILESTONE DESCRIPTION SHEET
\end{tabular}




\begin{tabular}{|c|c|c|c|}
\hline \multicolumn{4}{|c|}{$\begin{array}{l}\text { Westinghouse Hanford Company } \\
\text { MILESTONE DESCRIPTION SHEET }\end{array}$} \\
\hline \multicolumn{3}{|c|}{ Title: Submit Critical Items Report to RL Manager's Office } & Date: $07 / 14 / 94$ \\
\hline \multicolumn{3}{|c|}{ Assigned To: E. A. Schultz } & CIN: \\
\hline \multicolumn{3}{|c|}{ Program WBS Designator: 1 8.2.2.1.2 } & $\begin{array}{l}\text { Due Date: } \\
05 / 10 / 96\end{array}$ \\
\hline \multicolumn{3}{|c|}{ Control Number: PSA-96-016 } & Rev: \\
\hline $\begin{array}{l}\text { MILESTONE TYPE: } \\
\square \text { DOE-HQ } \\
\square \text { DOE-RL } \\
\text { X CONTRACTOR }\end{array}$ & $\begin{array}{l}\quad \text { DIVISION: } \\
\quad \square \text { State } \\
\square \text { Federal } \\
\square \text { DOE } \\
\square \text { RCRA } \\
\square \text { TPA Number }\end{array}$ & $\begin{array}{l}\text { DELIVERABLE: } \\
\square \text { Report } \\
\text { X Letter } \\
\square \text { Drawings } \\
\square \text { Other (specify) }\end{array}$ & $\begin{array}{l}\text { ADDRESS TO: } \\
\square \text { DOE-HQ } \\
\text { X DOE-RL-OPI } \\
\square \text { Other (specify) }\end{array}$ \\
\hline \multicolumn{4}{|c|}{$\begin{array}{l}\text { Milestone Description: } \\
\text { Coordinate, compile and submit Westinghouse Hanford Company's biweekly Critical } \\
\text { Items Report to the RL Manager in accordance with letter from J. D. Wagoner, RL to } \\
\text { Contractors, dated March 26, } 1991 \text {. }\end{array}$} \\
\hline \multicolumn{4}{|c|}{$\begin{array}{l}\text { Description of what constitutes completion of this milestone: } \\
\text { Delivery of the Critical Items Report to the RL Manager's Office by May 10, } 1996 .\end{array}$} \\
\hline \multicolumn{4}{|c|}{ Milestone Description Approval } \\
\hline \multicolumn{2}{|l|}{$\begin{array}{l}\text { Cost Account Manager } \\
\text { EA Schultz }\end{array}$} & $\begin{array}{l}\text { Program/Project Manager } \\
\text { DB Pabst }\end{array}$ & Date \\
\hline \multicolumn{2}{|c|}{$\begin{array}{l}\text { Program Element Manager } \\
\text { LR Hafer }\end{array}$} & $\begin{array}{l}\text { DOE Program Manager } \\
\text { N/A }\end{array}$ & Date \\
\hline 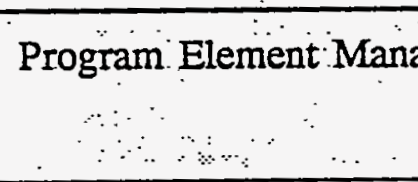 & ger: Date & $\begin{array}{l}\text { DOE Program Manager } \\
\text { N/A. }\end{array}$ & $\because$ \\
\hline
\end{tabular}




\begin{tabular}{|l||l|||}
\hline \multicolumn{3}{|c|}{ Westinghouse Hanford Company } \\
MLESTONE DESCRIPTION SHEET
\end{tabular}




\begin{tabular}{||l||l||}
\hline \multicolumn{3}{|c||}{ Westinghouse Hanford Company } \\
MILESTONE DESCRIPTION SHEET
\end{tabular}




\begin{tabular}{|c|c|c|c|}
\hline \multicolumn{4}{|c|}{$\begin{array}{l}\text { Westinghouse Hanford Company } \\
\text { MILESTONE DESCRIPTION SHEET }\end{array}$} \\
\hline \multicolumn{3}{|c|}{ Title: Submit Critical Items Report to RL Manager's Office } & Date: $07 / 14 / 94$ \\
\hline \multicolumn{3}{|c|}{ Assigned To: E. A. Schultz } & CIN: \\
\hline \multicolumn{3}{|c|}{ Program WBS Designator: 1 8.2.2.1.2 } & $\begin{array}{l}\text { Due Date: } \\
06 / 21 / 96\end{array}$ \\
\hline \multicolumn{3}{|c|}{ Control Number: PSA-96-019 } & Rev: \\
\hline $\begin{array}{l}\text { MILESTONE TYPE: } \\
\square \text { DOE-HQ } \\
\square \text { DOE-RL } \\
\text { X CONTRACTOR }\end{array}$ & $\begin{array}{l}\quad \text { DIVISION: } \\
\square \text { State } \\
\square \text { Federal } \\
\square \text { DOE } \\
\square \text { RCRA } \\
\square \text { TPA Number }\end{array}$ & $\begin{array}{l}\text { DELIVERABLE: } \\
\square \text { Report } \\
\text { X Letter } \\
\square \text { Drawings } \\
\square \text { Other (specify) }\end{array}$ & $\begin{array}{l}\text { ADDRESS TO: } \\
\square \text { DOE-HQ } \\
\text { X DOE-RL-OPI } \\
\square \text { Other (specify) }\end{array}$ \\
\hline \multicolumn{4}{|c|}{$\begin{array}{l}\text { Milestone Description: } \\
\text { Coordinate, compile and submit Westinghouse Hanford Company's biweekly Critical } \\
\text { Items Report to the RL Manager in accordance with letter from J. D. Wagoner, RL to } \\
\text { Contractors, dated March } 26,1991 \text {. }\end{array}$} \\
\hline \multicolumn{4}{|c|}{$\begin{array}{l}\text { Description of what constitutes completion of this milestone: } \\
\text { Delivery of the Critical Items Report to the RL Manager's Office by June 21, } 1996 .\end{array}$} \\
\hline \multicolumn{4}{|c|}{ Milestone Description Approval } \\
\hline \multicolumn{2}{|l|}{$\begin{array}{l}\text { Cost Account Manager } \\
\text { EA Schultz }\end{array}$} & \multicolumn{2}{|l|}{$\begin{array}{l}\text { Program/Project Manager } \\
\text { DB Pabst }\end{array}$} \\
\hline \multicolumn{2}{|c|}{$\begin{array}{l}\text { Program Element Manager } \\
\text { LR Hafer }\end{array}$} & \multicolumn{2}{|c|}{$\begin{array}{l}\text { DOE Program Manager Date } \\
\text { N/A }\end{array}$} \\
\hline \multicolumn{4}{|c|}{ u } \\
\hline Program Element Mana & Date & $\begin{array}{l}\text { DOE Program Manager } \\
\text { N/A }\end{array}$ & 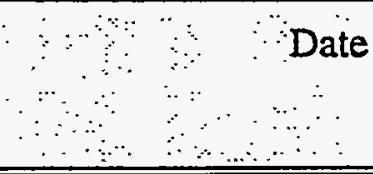 \\
\hline
\end{tabular}




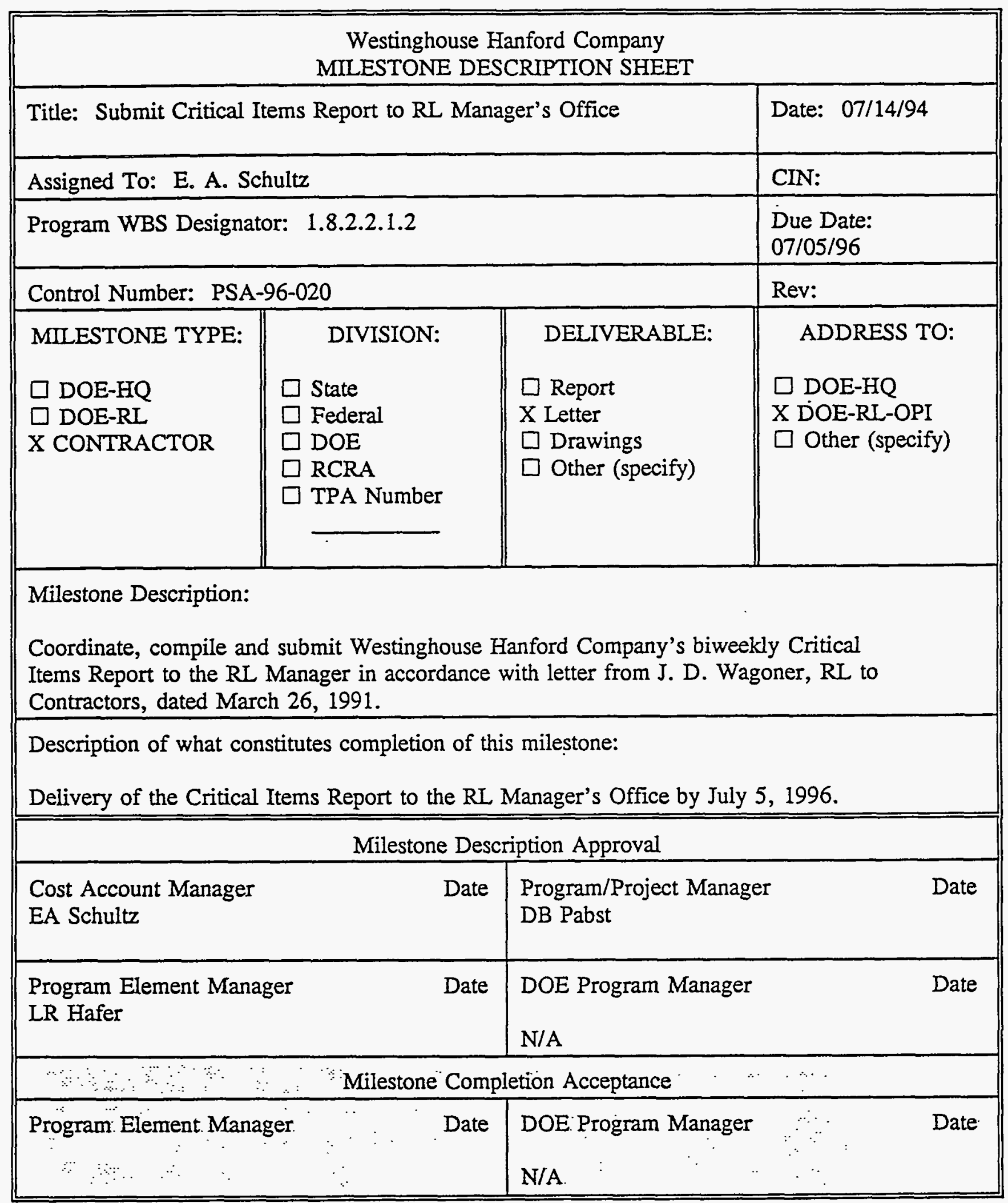




\begin{tabular}{|c|c|c|c|}
\hline \multicolumn{4}{|c|}{$\begin{array}{l}\text { Westinghouse Hanford Company } \\
\text { MILESTONE DESCRIPTION SHEET }\end{array}$} \\
\hline \multicolumn{3}{|c|}{ Title: Submit Critical Items Report to RL Manager's Office } & Date: $07 / 14 / 94$ \\
\hline \multicolumn{3}{|c|}{ Assigned To: E. A. Schultz } & CIN: \\
\hline \multicolumn{3}{|c|}{ Program WBS Designator: 1 8.2.2.1.2 } & $\begin{array}{l}\text { Due Date: } \\
07 / 19 / 96 \\
\end{array}$ \\
\hline \multicolumn{3}{|c|}{ Control Number: PSA-96-021 } & Rev: \\
\hline $\begin{array}{l}\text { MILESTONE TYPE: } \\
\square \text { DOE-HQ } \\
\square \text { DOE-RL } \\
\text { X CONTRACTOR }\end{array}$ & $\begin{array}{l}\quad \text { DIVISION: } \\
\square \text { State } \\
\square \text { Federal } \\
\square \text { DOE } \\
\square \text { RCRA } \\
\square \text { TPA Number }\end{array}$ & $\begin{array}{l}\text { DELIVERABLE: } \\
\square \text { Report } \\
\text { X Letter } \\
\square \text { Drawings } \\
\square \text { Other (specify) }\end{array}$ & $\begin{array}{l}\text { ADDRESS TO: } \\
\square \text { DOE-HQ } \\
\text { X DOE-RL-OPI } \\
\square \text { Other (specify) }\end{array}$ \\
\hline \multicolumn{4}{|c|}{$\begin{array}{l}\text { Milestone Description: } \\
\text { Coordinate, compile and submit Westinghouse Hanford Company's biweekly Critical } \\
\text { Items Report to the RL Manager in accordance with letter from J. D. Wagoner, RL to } \\
\text { Contractors, dated March 26, } 1991 \text {. }\end{array}$} \\
\hline \multicolumn{4}{|c|}{$\begin{array}{l}\text { Description of what constitutes completion of this milestone: } \\
\text { Delivery of the Critical Items Report to the RL Manager's Office by July 19, } 1996 .\end{array}$} \\
\hline \multicolumn{4}{|c|}{ Milestone Description Approval } \\
\hline \multicolumn{2}{|l|}{$\begin{array}{l}\text { Cost Account Manager } \\
\text { EA Schultz }\end{array}$} & \multicolumn{2}{|c|}{$\begin{array}{l}\text { Program/Project Manager } \\
\text { DB Pabst }\end{array}$} \\
\hline \multicolumn{2}{|c|}{$\begin{array}{l}\text { Program Element Manager } \\
\text { LR Hafer }\end{array}$} & \multicolumn{2}{|c|}{$\begin{array}{l}\text { DOE Program Manager Date } \\
\text { N/A }\end{array}$} \\
\hline \multicolumn{4}{|c|}{, Milestone Completion Acceptance } \\
\hline \multicolumn{2}{|c|}{$\begin{array}{c}\text { Programelement Manager } \\
\text { ele }\end{array}$} & \multicolumn{2}{|c|}{$\begin{array}{l}\text { DOE Program Manager } \\
\text { N } / \text { A }\end{array}$} \\
\hline
\end{tabular}




\begin{tabular}{|c|c|c|c|}
\hline \multicolumn{4}{|c|}{$\begin{array}{l}\text { Westinghouse Hanford Company } \\
\text { MILESTONE DESCRIPTION SHEET }\end{array}$} \\
\hline \multicolumn{3}{|c|}{ Title: Submit Critical Items Report to RL Manager's Office } & Date: $07 / 14 / 94$ \\
\hline \multicolumn{3}{|c|}{ Assigned To: E. A. Schultz } & CIN: \\
\hline \multicolumn{3}{|c|}{ Program WBS Designator: 1 8.2.2.1.2 } & $\begin{array}{l}\text { Due Date: } \\
08 / 02 / 96\end{array}$ \\
\hline \multicolumn{3}{|c|}{ Control Number: PSA-96-022 } & Rev: \\
\hline $\begin{array}{l}\text { MILESTONE TYPE: } \\
\square \text { DOE-HQ } \\
\square \text { DOE-RI } \\
\text { X CONTRACTOR }\end{array}$ & $\begin{aligned} & \text { DIVISION: } \\
& \\
& \text { State } \\
& \square \text { Federal } \\
& \square \text { DOE } \\
& \square \text { RCRA } \\
& \square \text { TPA Number }\end{aligned}$ & $\begin{array}{l}\text { DELIVERABLE: } \\
\square \text { Report } \\
\text { X Letter } \\
\square \text { Drawings } \\
\square \text { Other (specify) }\end{array}$ & $\begin{array}{l}\text { ADDRESS TO: } \\
\square \text { DOE-HQ } \\
\text { X DOE-RL-OPI } \\
\square \text { Other (specify) }\end{array}$ \\
\hline \multicolumn{4}{|c|}{$\begin{array}{l}\text { Milestone Description: } \\
\text { Coordinate, compile and submit Westinghouse Hanford Company's biweekly Critical } \\
\text { Items Report to the RL Manager in accordance with letter from J. D. Wagoner, RL to } \\
\text { Contractors, dated March } 26,1991 \text {. }\end{array}$} \\
\hline \multicolumn{4}{|c|}{$\begin{array}{l}\text { Description of what constitutes completion of this milestone: } \\
\text { Delivery of the Critical Items Report to the RL Manager's Office by August 2, } 1996 .\end{array}$} \\
\hline \multicolumn{4}{|c|}{ Milestone Description Approval } \\
\hline \multicolumn{2}{|l|}{$\begin{array}{l}\text { Cost Account Manager } \\
\text { EA Schultz }\end{array}$} & \multicolumn{2}{|l|}{$\begin{array}{l}\text { Program/Project Manager } \\
\text { DB Pabst }\end{array}$} \\
\hline \multicolumn{2}{|c|}{$\begin{array}{l}\text { Program Element Manager } \\
\text { LR Hafer }\end{array}$} & \multicolumn{2}{|c|}{$\begin{array}{l}\text { DOE Program Manager Date } \\
\text { N/A }\end{array}$} \\
\hline \multicolumn{4}{|c|}{ A $\quad$ Milestone Completion Acceptance } \\
\hline $\begin{array}{c}\text { Program Element Manag } \\
\qquad\end{array}$ & $\therefore$ Date & $\begin{array}{l}\text { DOE Program Manager } \\
\text { N/A }\end{array}$ & \\
\hline
\end{tabular}




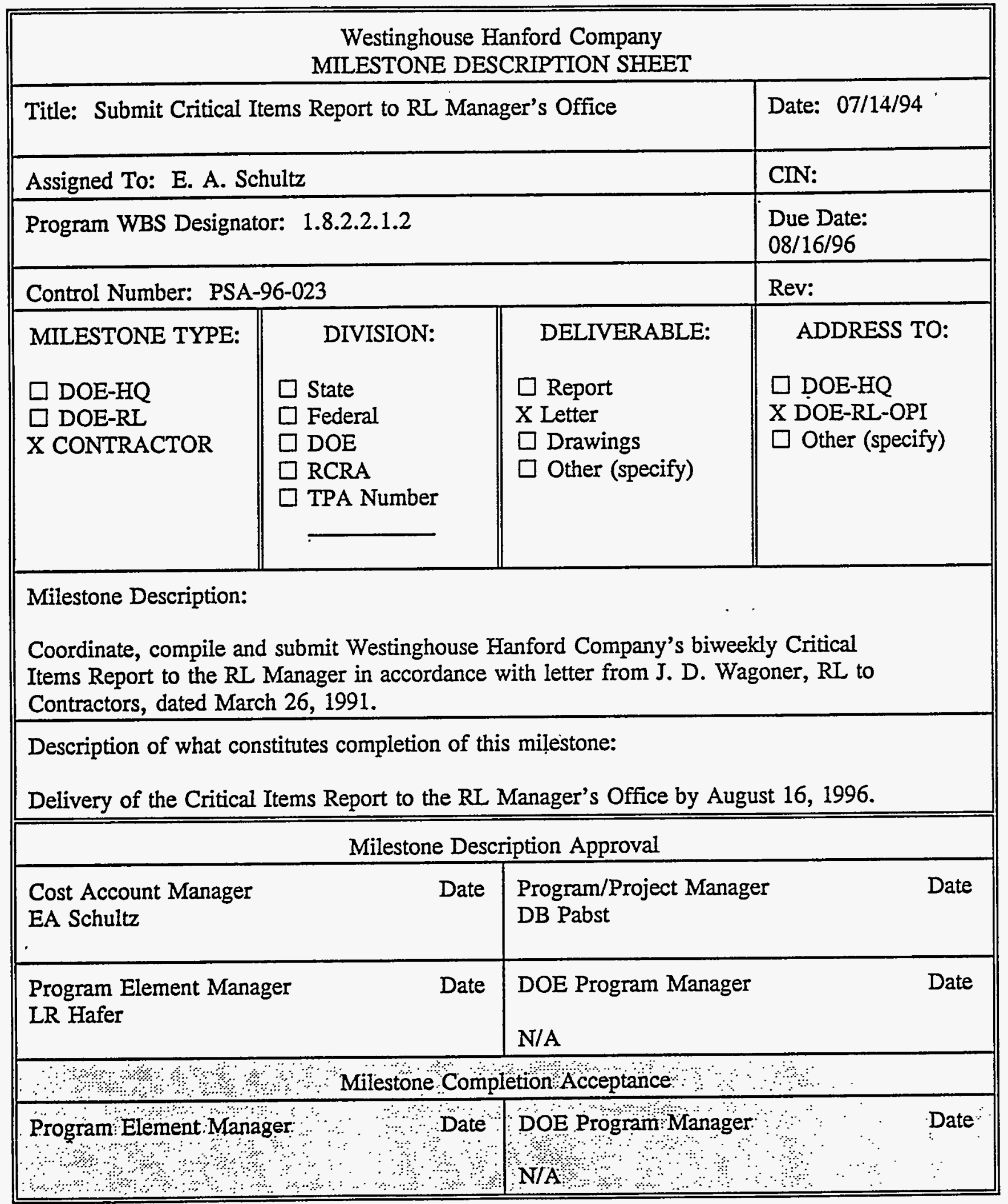




\begin{tabular}{|c|c|c|c|}
\hline \multicolumn{4}{|c|}{$\begin{array}{l}\text { Westinghouse Hanford Company } \\
\text { MILESTONE DESCRIPTION SHEET }\end{array}$} \\
\hline \multicolumn{3}{|c|}{ Title: Submit Critical Items Report to RL Manager's Office } & Date: $07 / 14 / 94$ \\
\hline \multicolumn{3}{|c|}{ Assigned To: E. A. Schultz } & CIN: \\
\hline \multicolumn{3}{|c|}{ Program WBS Designator: 1 8.2.2.1.2 } & $\begin{array}{l}\text { Due Date: } \\
\text { 08/30/96 }\end{array}$ \\
\hline \multicolumn{3}{|c|}{ Control Number: PSA-96-024 } & Rev: \\
\hline $\begin{array}{l}\text { MILESTONE TYPE: } \\
\square \text { DOE-HQ } \\
\square \text { DOE-RI } \\
\text { X CONTRACTOR }\end{array}$ & $\begin{aligned} & \text { DIVISION: } \\
& \square \text { State } \\
\square & \text { Federal } \\
& \text { DOE } \\
& \quad \text { RCRA } \\
& \quad \text { TPA Number }\end{aligned}$ & $\begin{array}{l}\text { DELIVERABLE: } \\
\square \text { Report } \\
\text { X Letter } \\
\square \text { Drawings } \\
\square \text { Other (specify) }\end{array}$ & $\begin{array}{l}\text { ADDRESS TO: } \\
\text { D DOE-HQ } \\
\text { X DOE-RL-OPI } \\
\square \text { Other (specify) }\end{array}$ \\
\hline \multicolumn{4}{|c|}{$\begin{array}{l}\text { Milestone Description: } \\
\text { Coordinate, compile and submit Westinghouse Hanford Company's biweekly Critical } \\
\text { Items Report to the RL Manager in accordance with letter from J. D. Wagoner, RL to } \\
\text { Contractors, dated March } 26,1991 \text {. }\end{array}$} \\
\hline \multicolumn{4}{|c|}{$\begin{array}{l}\text { Description of what constitutes completion of this milestone: } \\
\text { Delivery of the Critical Items Report to the RL Manager's Office by August 30, } 1996 .\end{array}$} \\
\hline \multicolumn{4}{|c|}{ Milestone Description Approval } \\
\hline \multicolumn{2}{|l|}{$\begin{array}{l}\text { Cost Account Manager } \\
\text { EA Schultz }\end{array}$} & \multicolumn{2}{|l|}{$\begin{array}{l}\text { Program/Project Manager } \\
\text { DB Pabst }\end{array}$} \\
\hline \multicolumn{2}{|c|}{$\begin{array}{l}\text { Program Element Manager } \\
\text { LR Hafer }\end{array}$} & $\begin{array}{l}\text { DOE Program Manager } \\
\text { N/A }\end{array}$ & Date \\
\hline \multicolumn{4}{|c|}{ औै $\quad \because$ Milestone Completion Acceptance } \\
\hline $\begin{array}{l}\text { Program Element Man } \\
\quad: \quad: \quad \cdots\end{array}$ & Date & $\begin{array}{l}\text { DOE Program Manager } \\
\text { N/A }\end{array}$ & Date \\
\hline
\end{tabular}




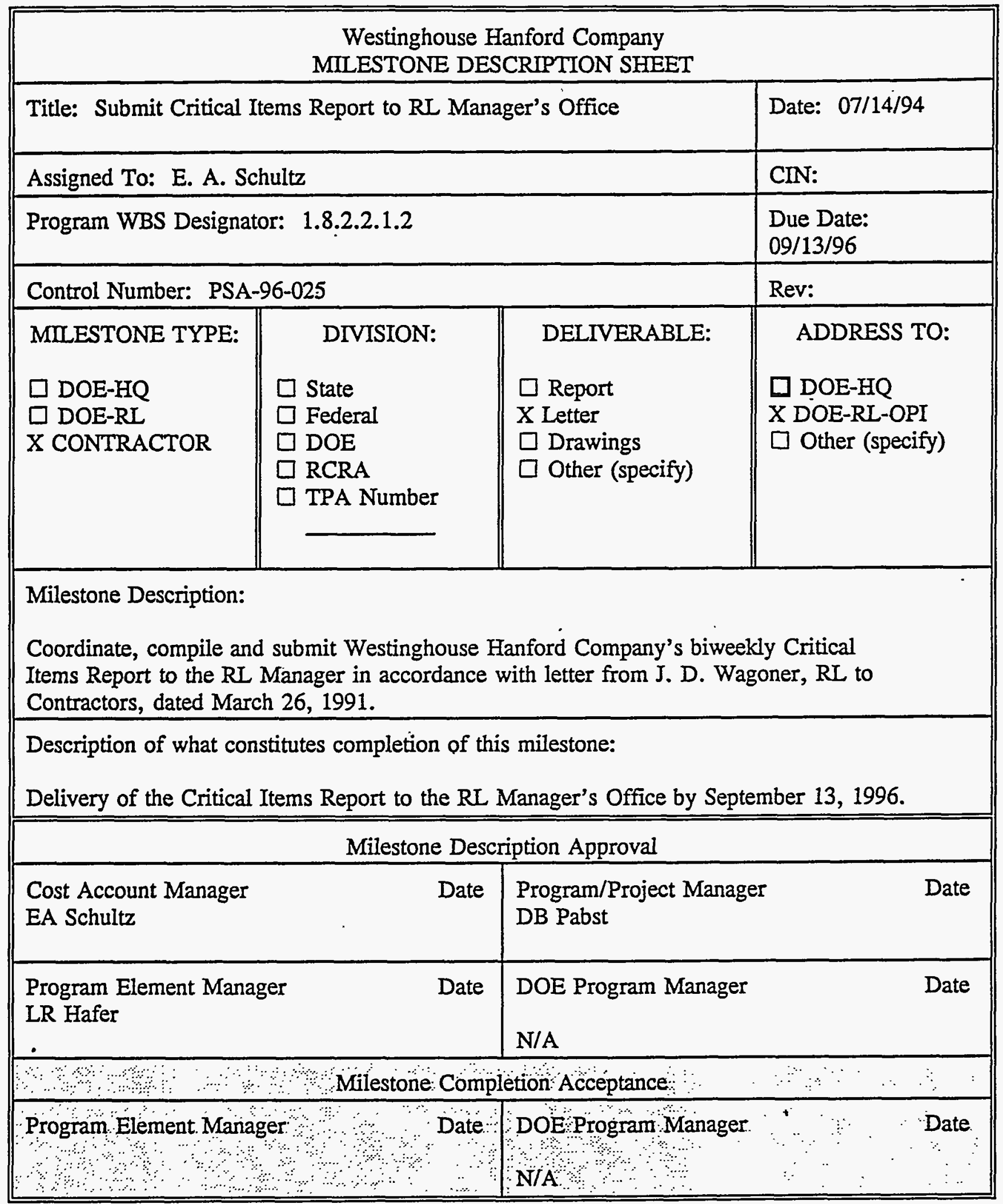




\begin{tabular}{|c|c|c|c|}
\hline \multicolumn{4}{|c|}{$\begin{array}{l}\text { Westinghouse Hanford Company } \\
\text { MILESTONE DESCRIPTION SHEET }\end{array}$} \\
\hline \multicolumn{3}{|c|}{ Title: Submit Critical Items Report to RL Manager's Office } & Date: $07 / 14 / 94$ \\
\hline \multicolumn{3}{|c|}{ Assigned To: E. A. Schultz } & CIN: \\
\hline \multicolumn{3}{|c|}{ Program WBS Designator: 1 8.2.2.1.2 } & $\begin{array}{l}\text { Due Date: } \\
09 / 27 / 96\end{array}$ \\
\hline \multicolumn{3}{|c|}{ Control Number: PSA-96-026 } & Rev: \\
\hline $\begin{array}{l}\text { MILESTONE TYPE: } \\
\square \text { DOE-HQ } \\
\square \text { DOE-RL } \\
\text { X CONTRACTOR }\end{array}$ & $\begin{aligned} & \text { DIVISION: } \\
& \square \text { State } \\
& \square \text { Federal } \\
& \square \text { DOE } \\
& \square \text { RCRA } \\
& \square \text { TPA Number }\end{aligned}$ & $\begin{array}{l}\text { DELIVERABLE: } \\
\square \text { Report } \\
\text { X Letter } \\
\square \text { Drawings } \\
\square \text { Other (specify) }\end{array}$ & $\begin{array}{l}\text { ADDRESS TO: } \\
\square \text { DOE-HQ } \\
\text { X DOE-RL-OPI } \\
\square \text { Other (specify) }\end{array}$ \\
\hline \multicolumn{4}{|c|}{$\begin{array}{l}\text { Milestone Description: } \\
\text { Coordinate, compile and submit Westinghouse Hanford Company's biweekly Critical } \\
\text { Items Report to the RL Manager in accordance with letter from J. D. Wagoner, RL to } \\
\text { Contractors, dated March 26, } 1991 \text {. }\end{array}$} \\
\hline \multicolumn{4}{|c|}{$\begin{array}{l}\text { Description of what constitutes completion of this milestone: } \\
\text { Delivery of the Critical Items Report to the RL Manager's Office by September 27, } 1996 .\end{array}$} \\
\hline \multicolumn{4}{|c|}{ Milestone Description Approval } \\
\hline \multicolumn{2}{|l|}{$\begin{array}{l}\text { Cost Account Manager } \\
\text { EA Schultz }\end{array}$} & $\begin{array}{l}\text { Program/Project Manager } \\
\text { DB Pabst }\end{array}$ & Date \\
\hline \multicolumn{2}{|c|}{$\begin{array}{l}\text { Program Element Manager } \\
\text { LR Hafer }\end{array}$} & \multirow{2}{*}{$\begin{array}{l}\text { DOE Program Manager } \\
\text { N/A } \\
\text { etion Acceptance }\end{array}$} & Date \\
\hline$\therefore$ & $\because$ Milestone Com! & & $\therefore$ \\
\hline \multicolumn{2}{|c|}{$\begin{array}{l}\text { Program Element Manager } \\
\quad\end{array}$} & $\begin{array}{l}\text { DOE Program Manager } \\
\text { N/A }\end{array}$ & Date \\
\hline
\end{tabular}




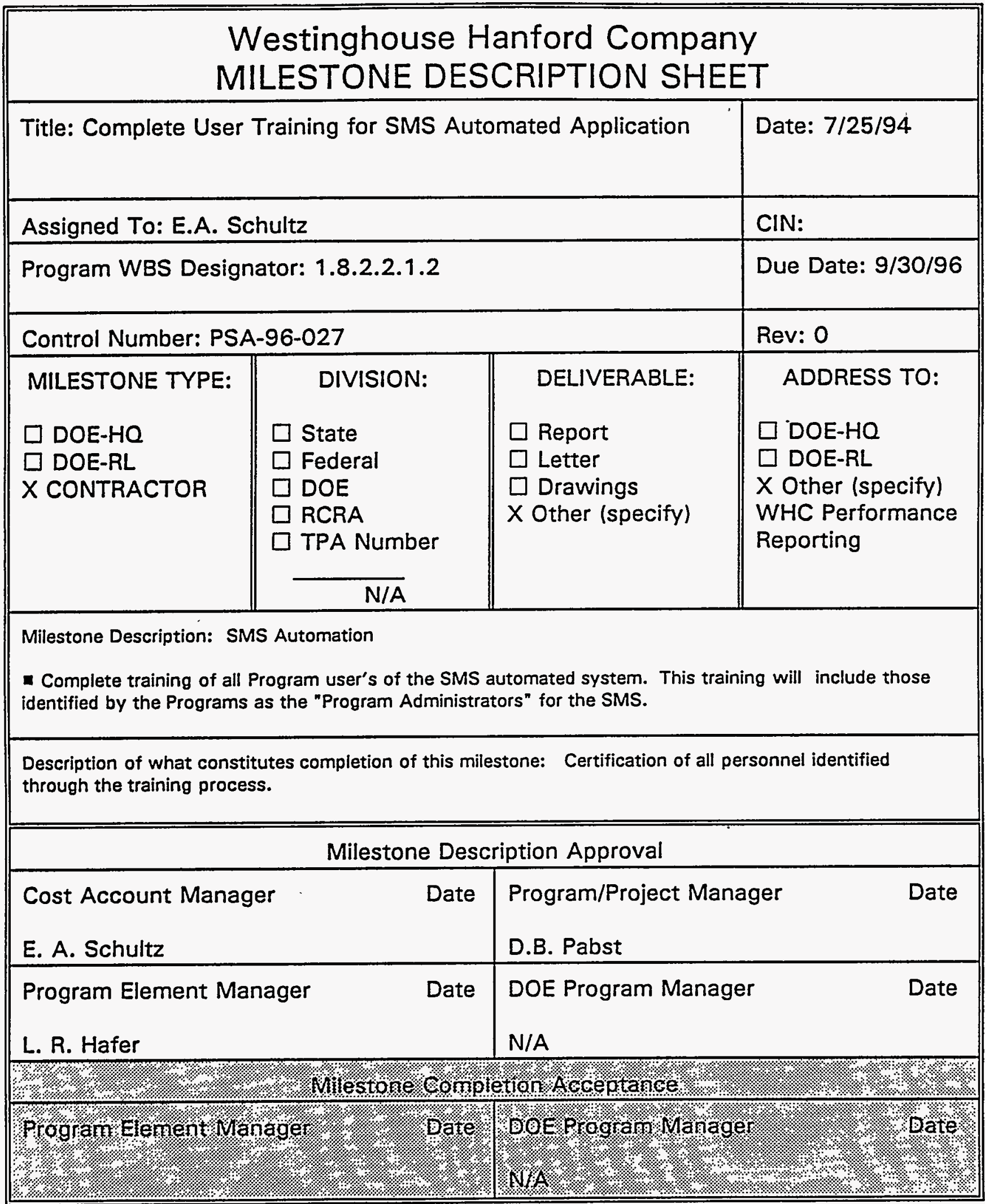




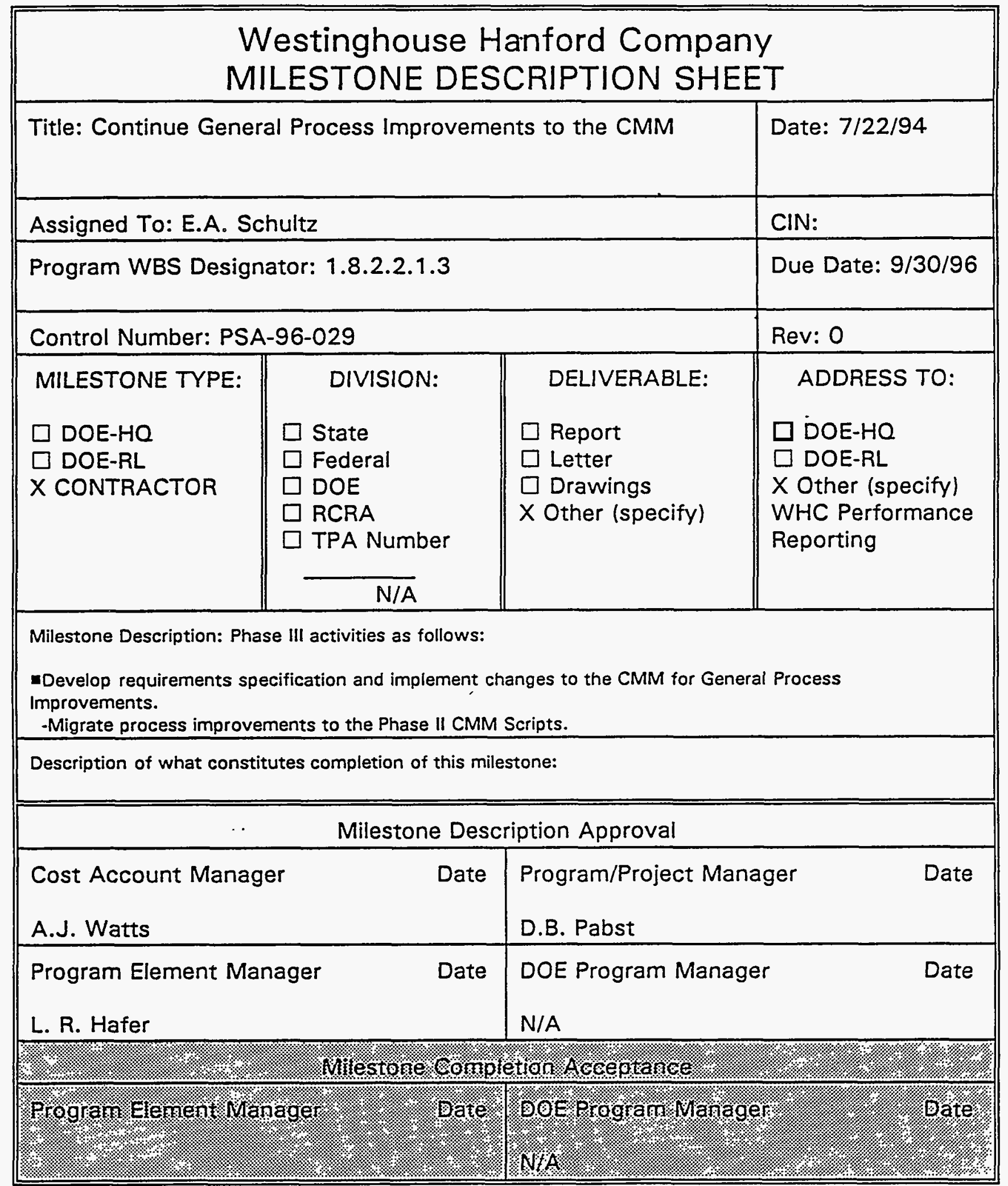




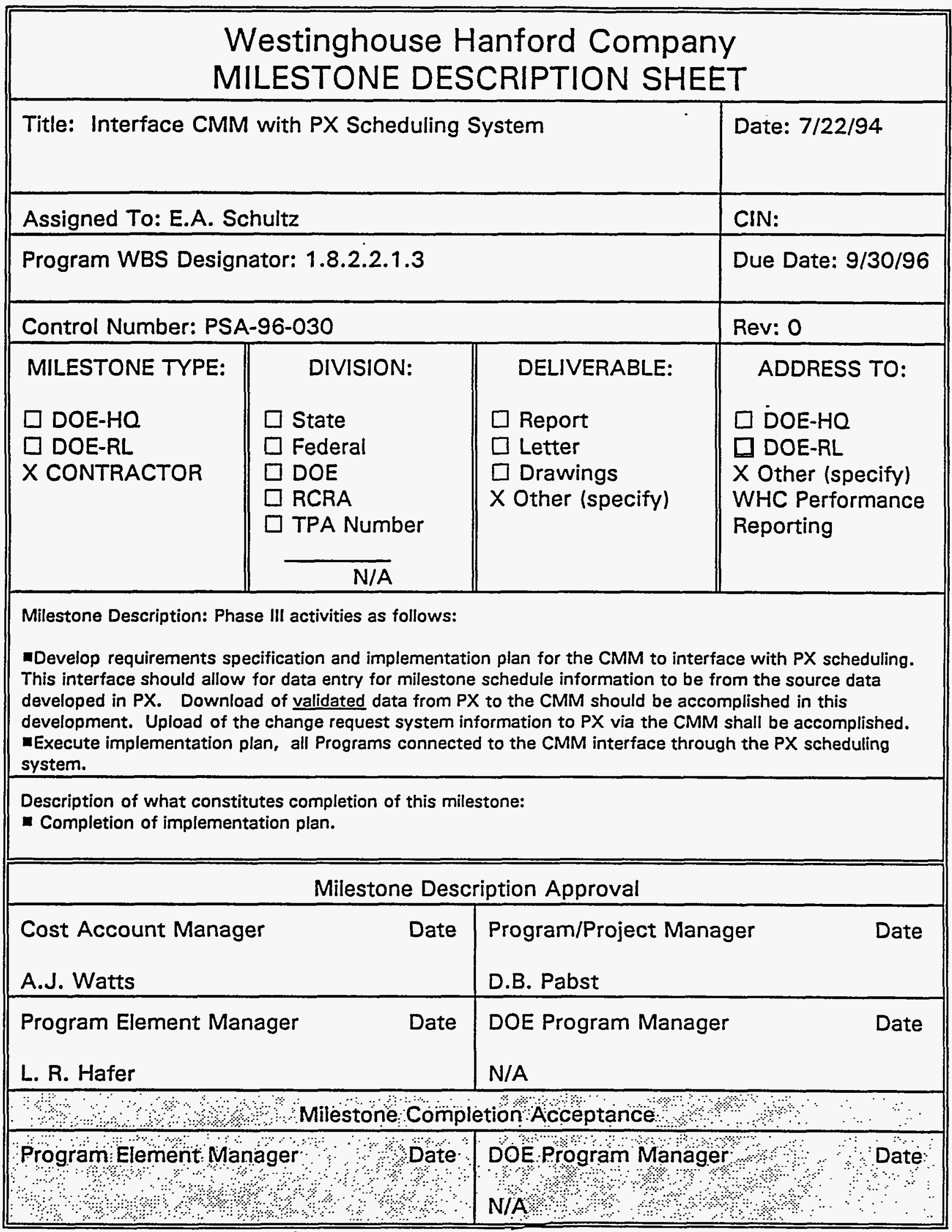




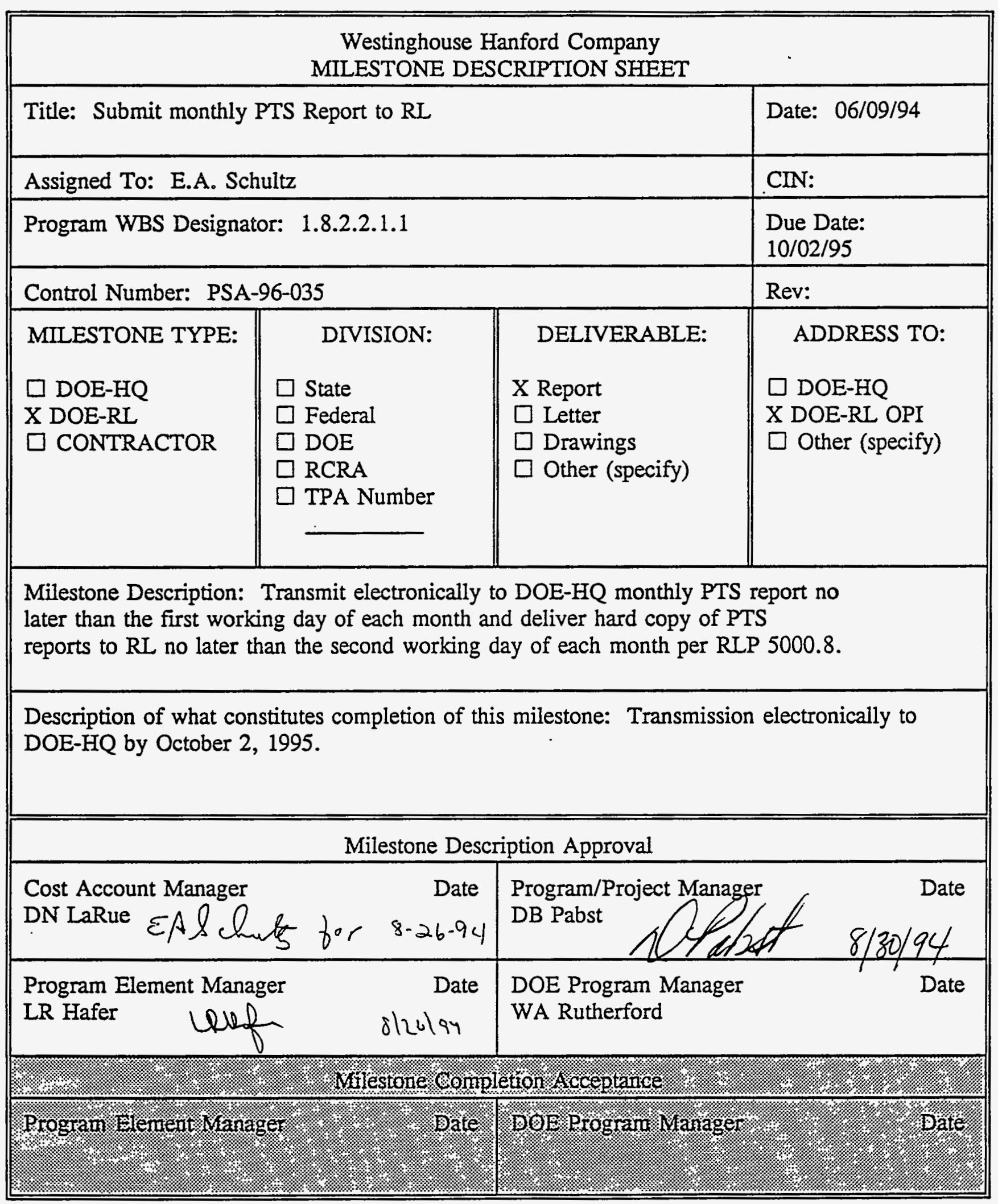




\begin{tabular}{|l||l|l||}
\hline \multicolumn{3}{|c|}{ Westinghouse Hanford Company } \\
MILESTONE DESCRIPTION SHEET
\end{tabular}




\begin{tabular}{|c|c|c|c|}
\hline \multicolumn{4}{|c|}{$\begin{array}{l}\text { Westinghouse Hanford Company } \\
\text { MILESTONE DESCRIPTION SHEET }\end{array}$} \\
\hline \multicolumn{3}{|c|}{ Title: Submit monthly PTS Report to RL } & Date: $06 / 09 / 94$ \\
\hline \multicolumn{3}{|c|}{ Assigned To: E.A. Schultz } & CIN: \\
\hline \multicolumn{3}{|c|}{ Program WBS Designator: $1.8,2.2 .1 .1$} & $\begin{array}{l}\text { Due Date: } \\
12 / 01 / 95\end{array}$ \\
\hline \multicolumn{3}{|c|}{ Control Number: PSA-96-037 } & Rev: \\
\hline $\begin{array}{l}\text { MILESTONE TYPE: } \\
\square \text { DOE-HQ } \\
\text { X DOE-RL } \\
\square \text { CONTRACTOR }\end{array}$ & $\begin{array}{l}\quad \text { DIVISION: } \\
\square \text { State } \\
\square \text { Federal } \\
\square \text { DOE } \\
\square \text { RCRA } \\
\square \text { TPA Number }\end{array}$ & $\begin{array}{l}\text { DELIVERABLE: } \\
\text { X Report } \\
\square \text { Letter } \\
\square \text { Drawings } \\
\square \text { Other (specify) }\end{array}$ & $\begin{array}{l}\text { ADDRESS TO: } \\
\square \text { DOE-HQ } \\
\text { X DOE-RL OPI } \\
\square \text { Other (specify) }\end{array}$ \\
\hline \multicolumn{4}{|c|}{$\begin{array}{l}\text { Milestone Description: Transmit electronically to DOE-HQ monthly PTS report no } \\
\text { later than the first working day of each month and deliver hard copy of PTS } \\
\text { reports to RL no later than the second working day of each month per RLP 5000.8. }\end{array}$} \\
\hline \multicolumn{4}{|c|}{$\begin{array}{l}\text { Description of what constitutes completion of this milestone: Transmission electronically to } \\
\text { DOE-HQ by December } 1,1995 \text {. }\end{array}$} \\
\hline \multicolumn{4}{|c|}{ Milestone Description Approval } \\
\hline \multicolumn{2}{|c|}{$\begin{array}{lr}\text { Cost Account Manager } & \text { Date } \\
\text { DN LaRue } & \text { EA\& habt for } 8.26 .94 \\
\end{array}$} & \multicolumn{2}{|c|}{ Program/Project Manager } \\
\hline \multicolumn{2}{|c|}{$\begin{array}{l}\text { Program Element Manager } \\
\text { LR Hafer Lefof } \\
\text {. }\end{array}$} & $\begin{array}{l}\text { DOE Program Manager } \\
\text { WA Rutherford }\end{array}$ & Date \\
\hline \multicolumn{4}{|c|}{ 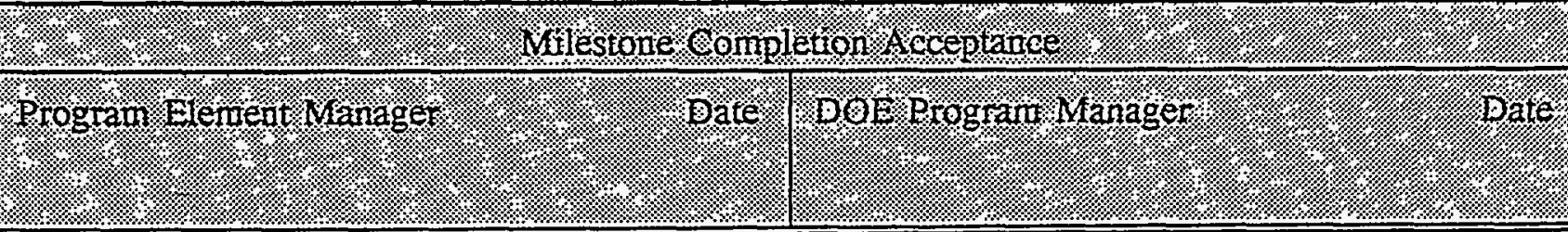 } \\
\hline
\end{tabular}




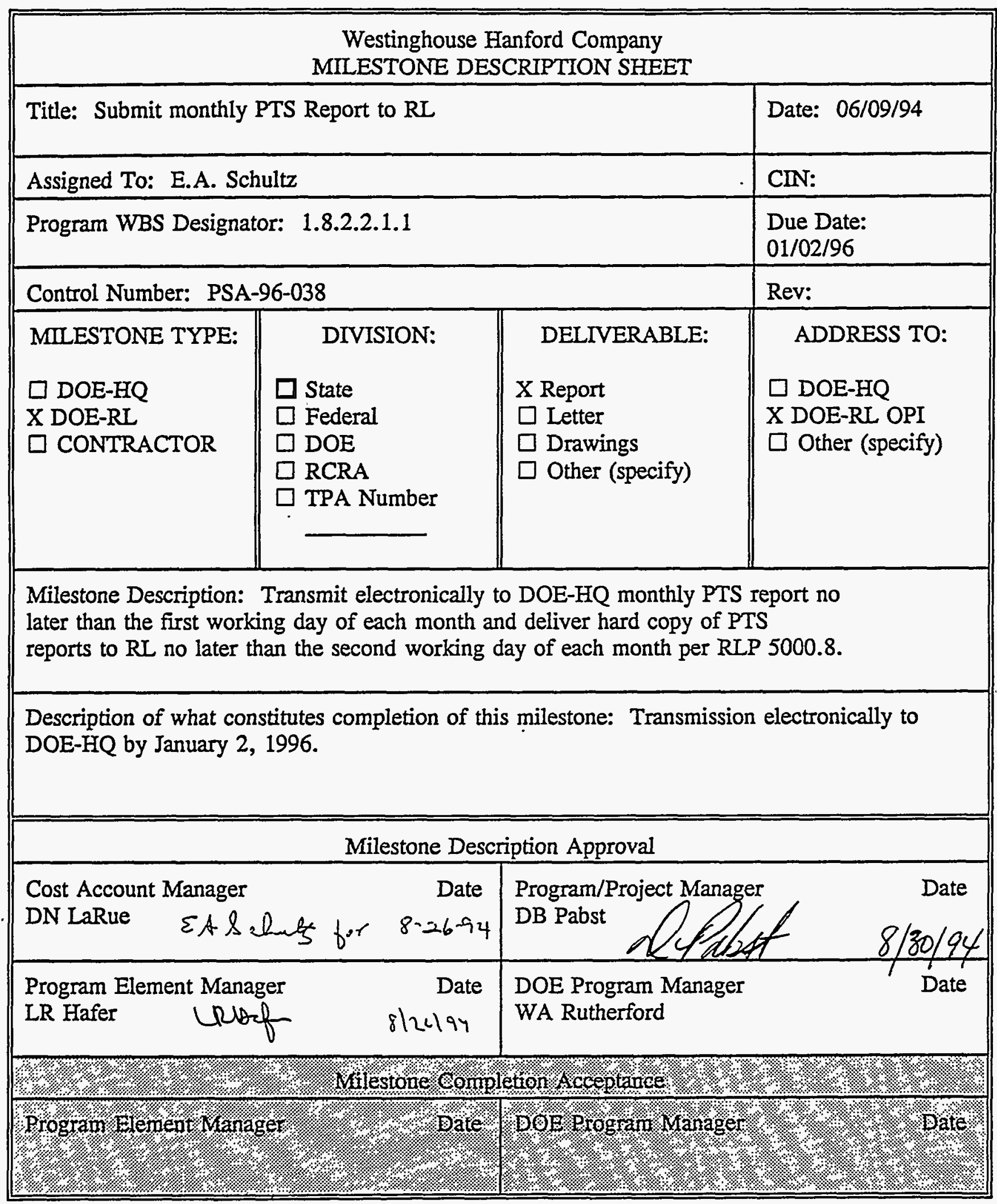




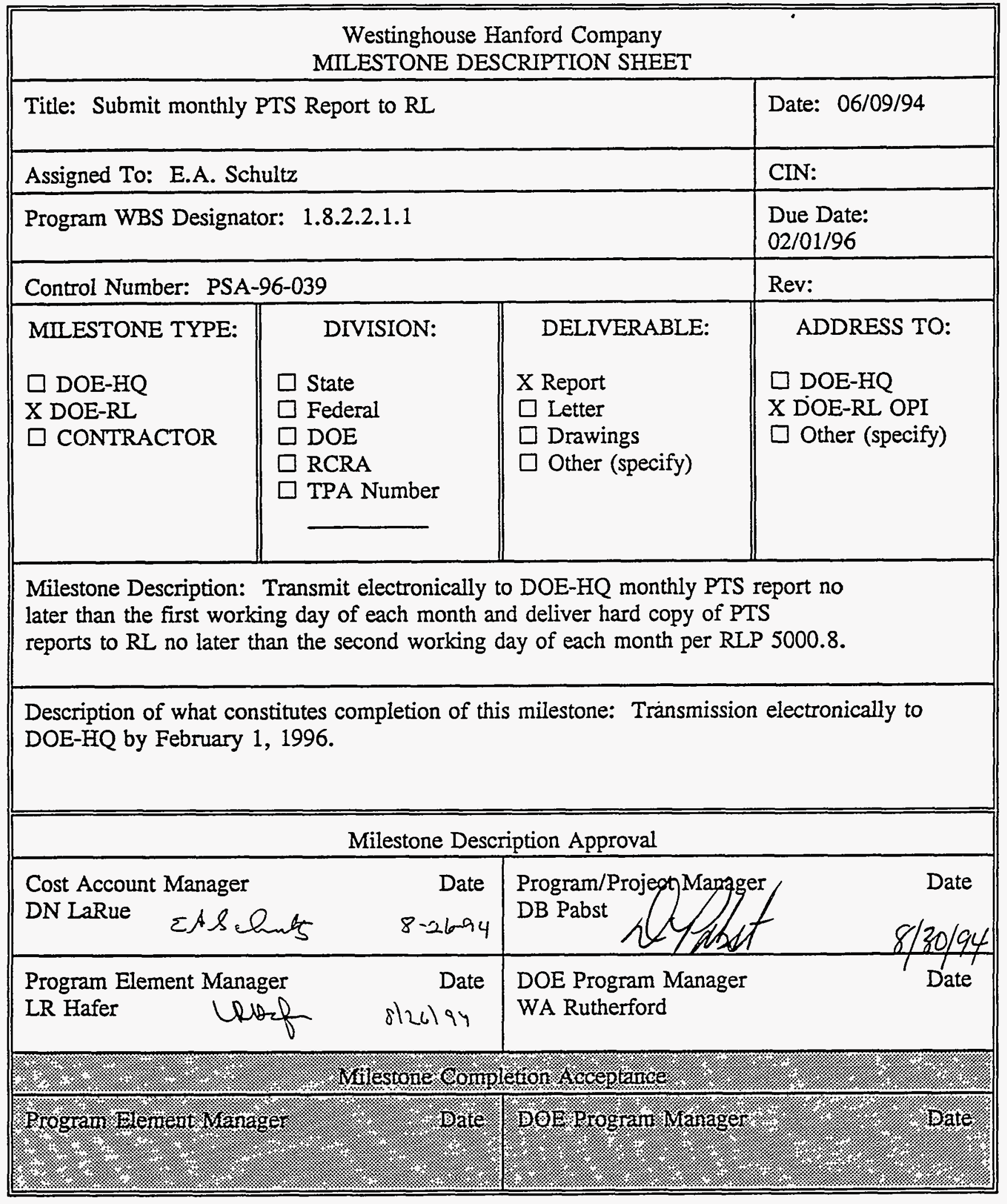




\begin{tabular}{|l||l|l||}
\hline \multicolumn{3}{|c||}{ Westinghouse Hanford Company } \\
MILESTONE DESCRIPTION SHEET
\end{tabular}




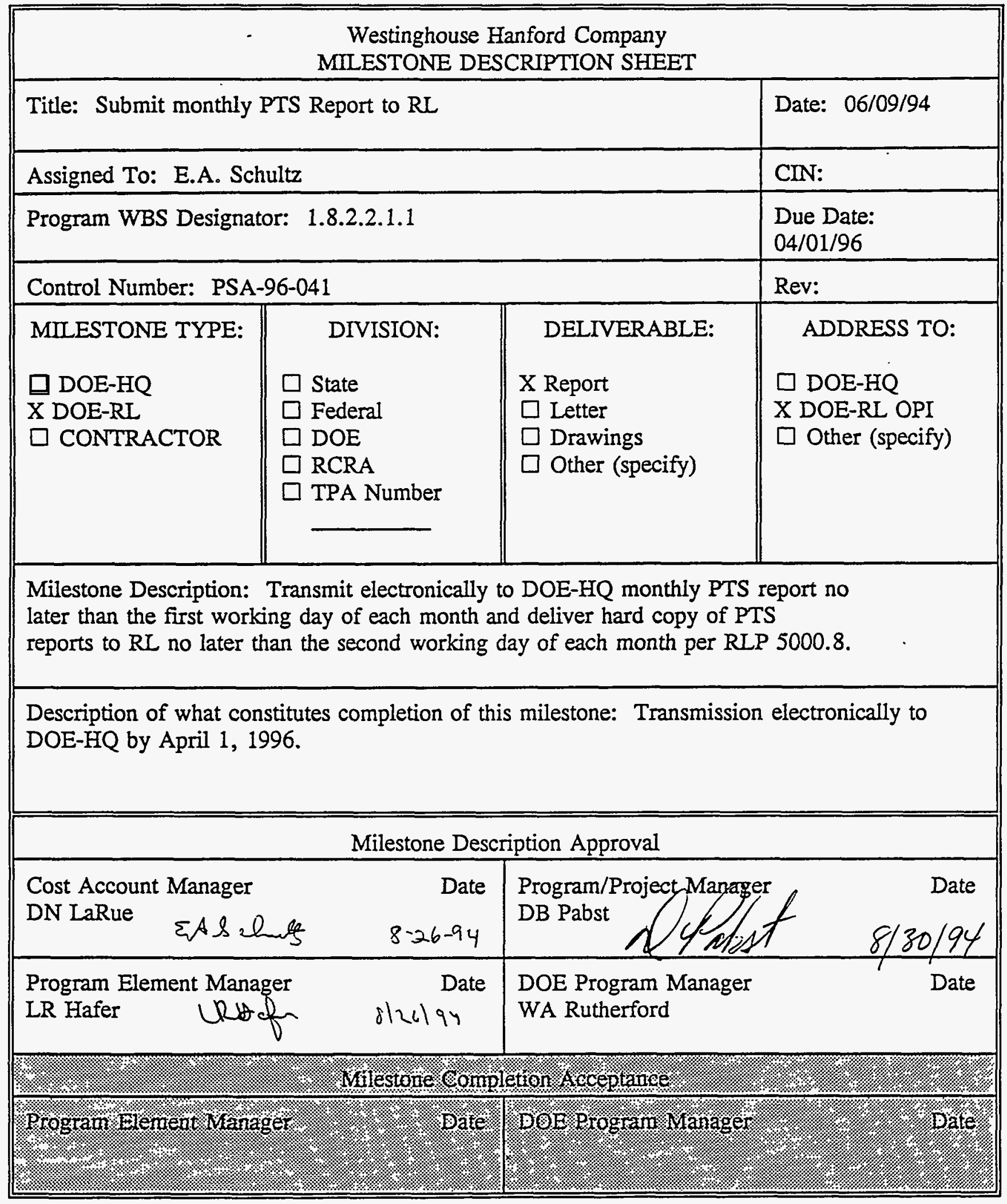




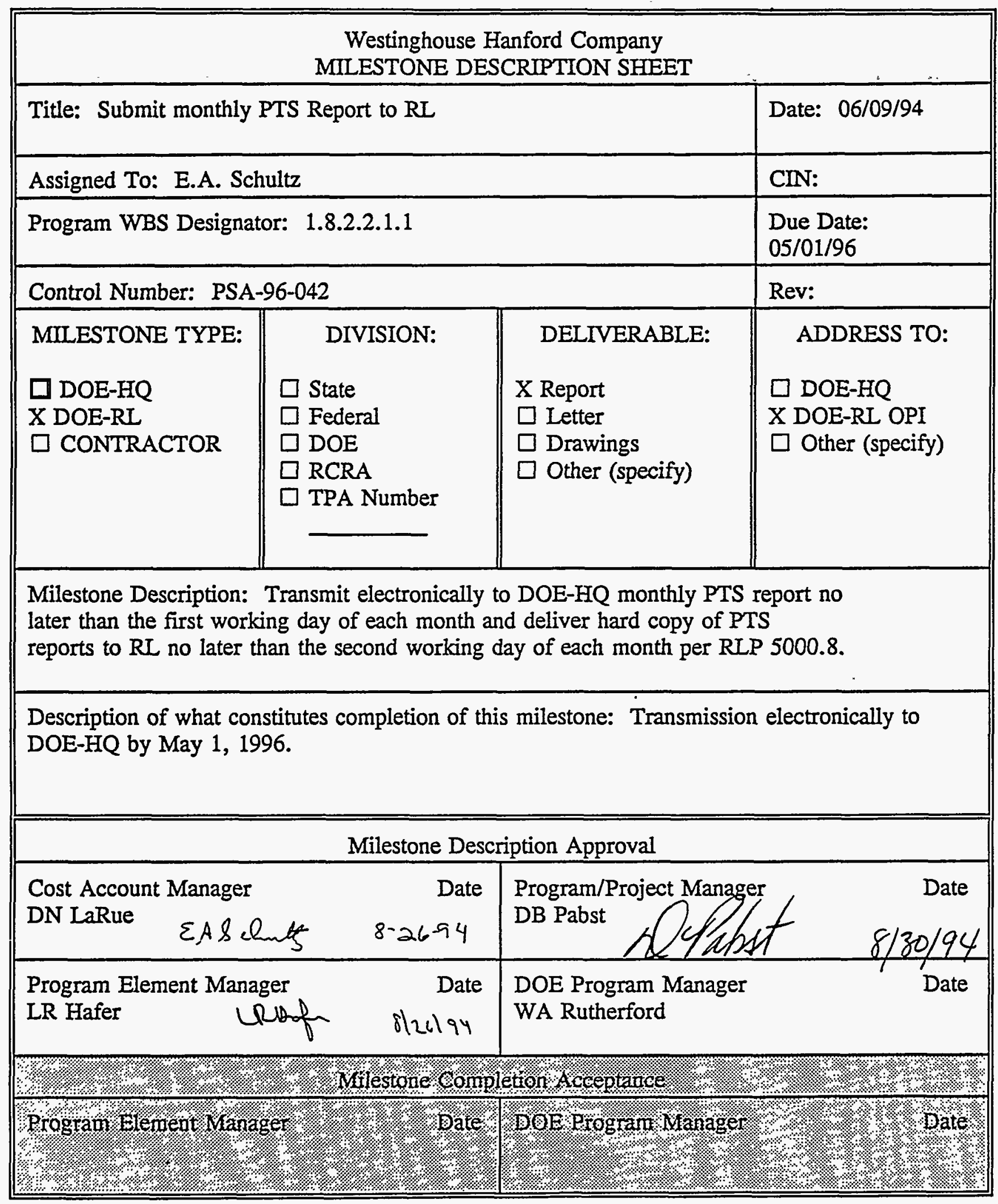




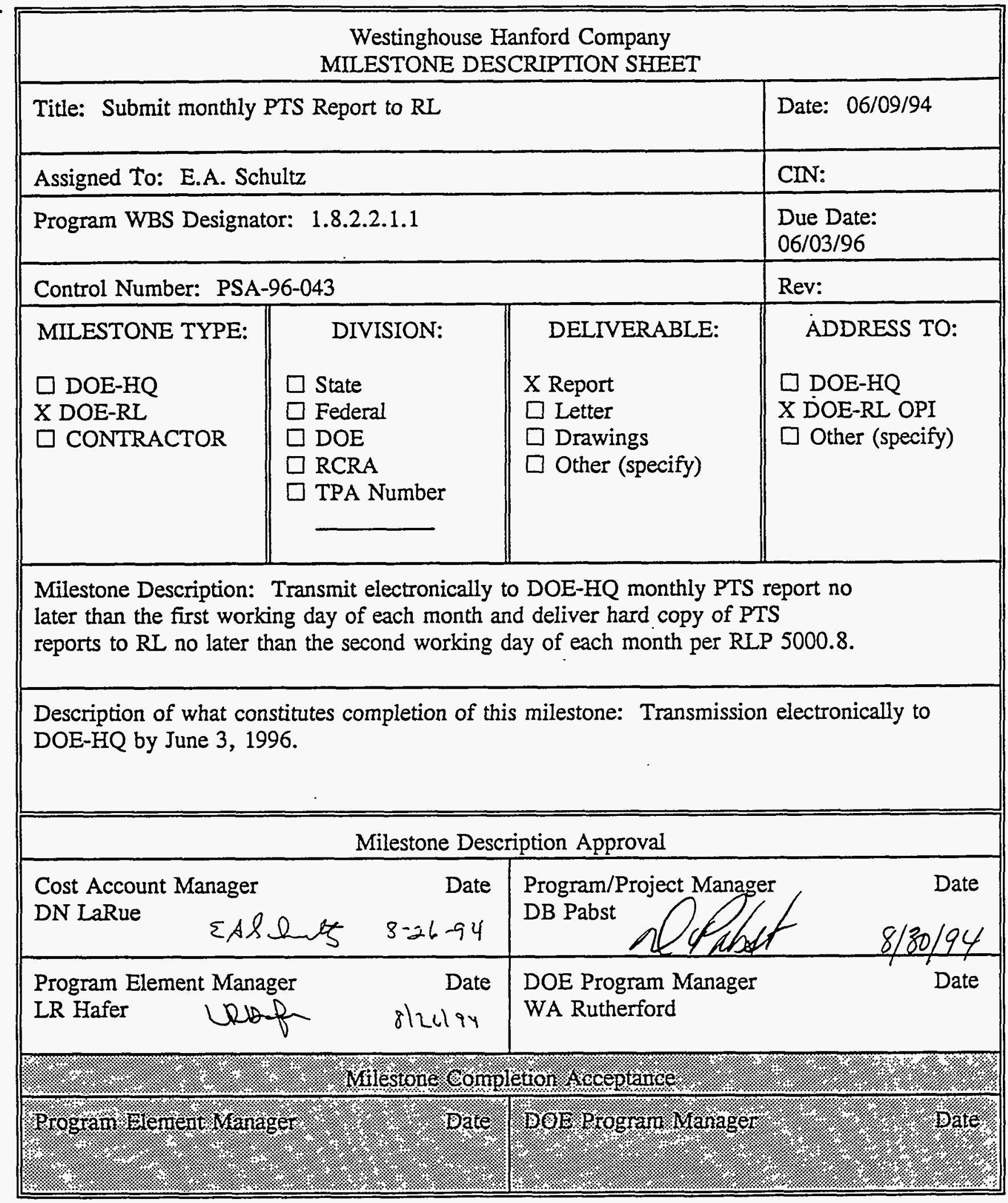




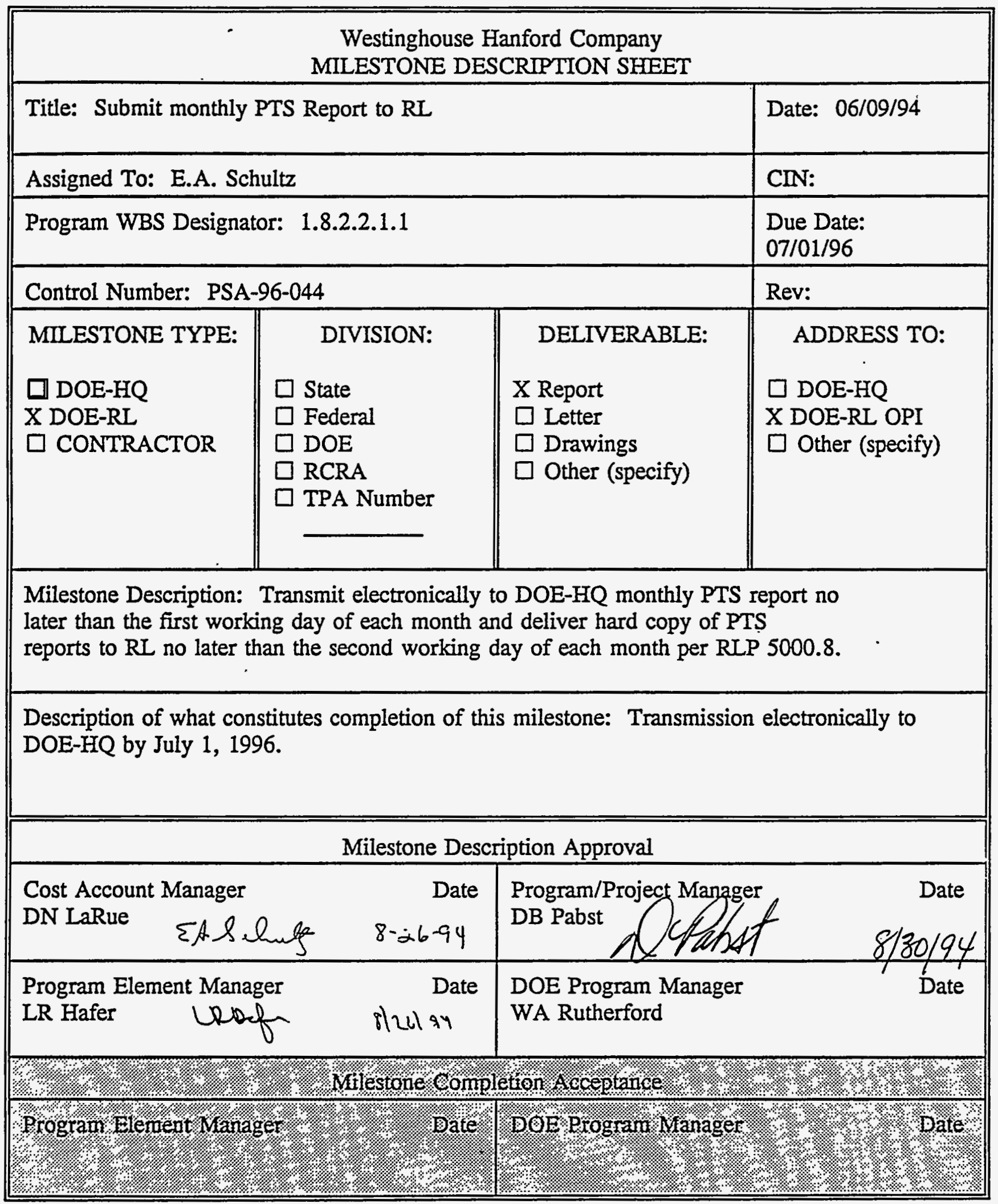




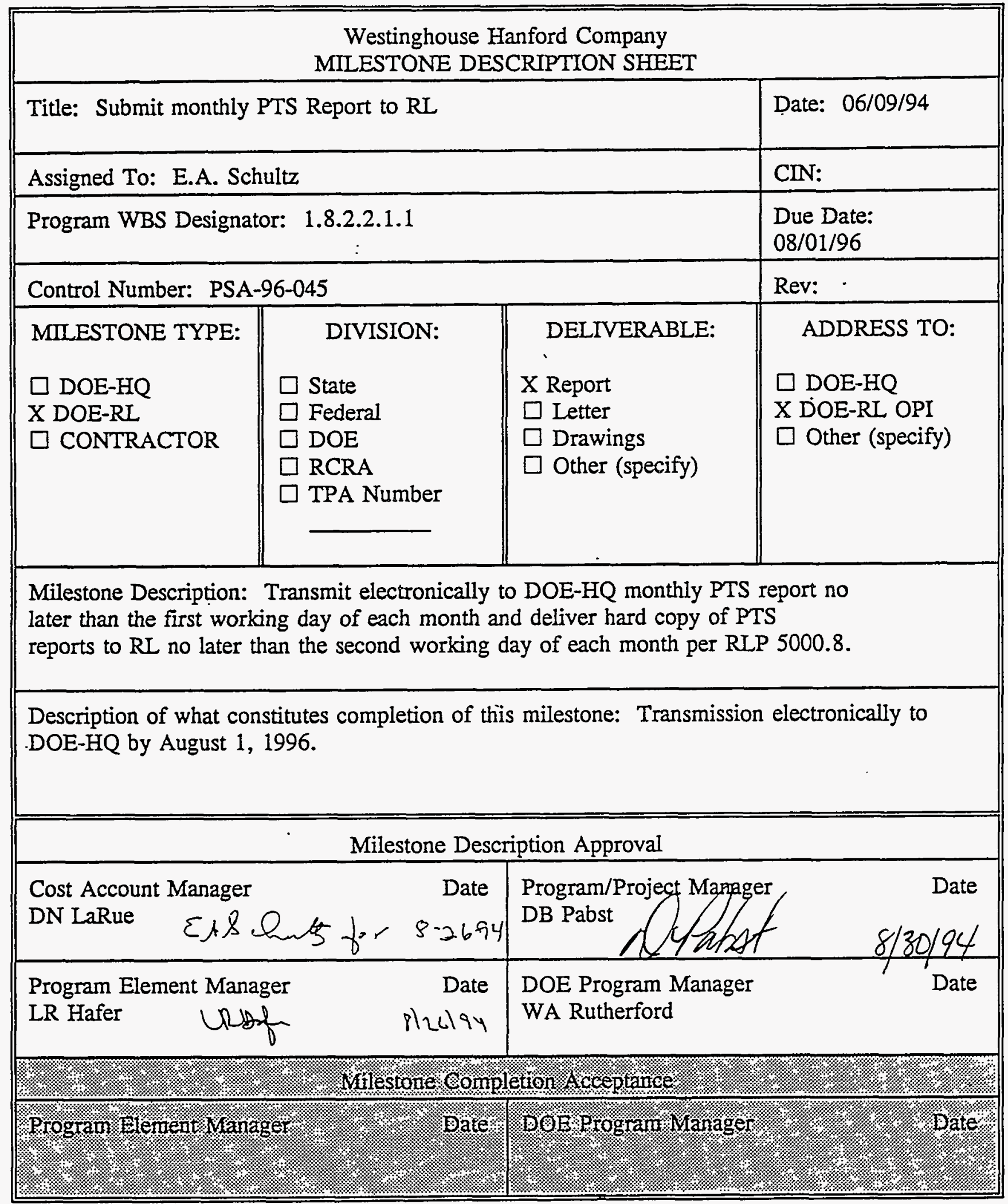




\begin{tabular}{|l|l|l||}
\hline \multicolumn{3}{|c|}{ Westinghouse Hanford Company } \\
MILESTONE DESCRIPTION SHEET
\end{tabular}




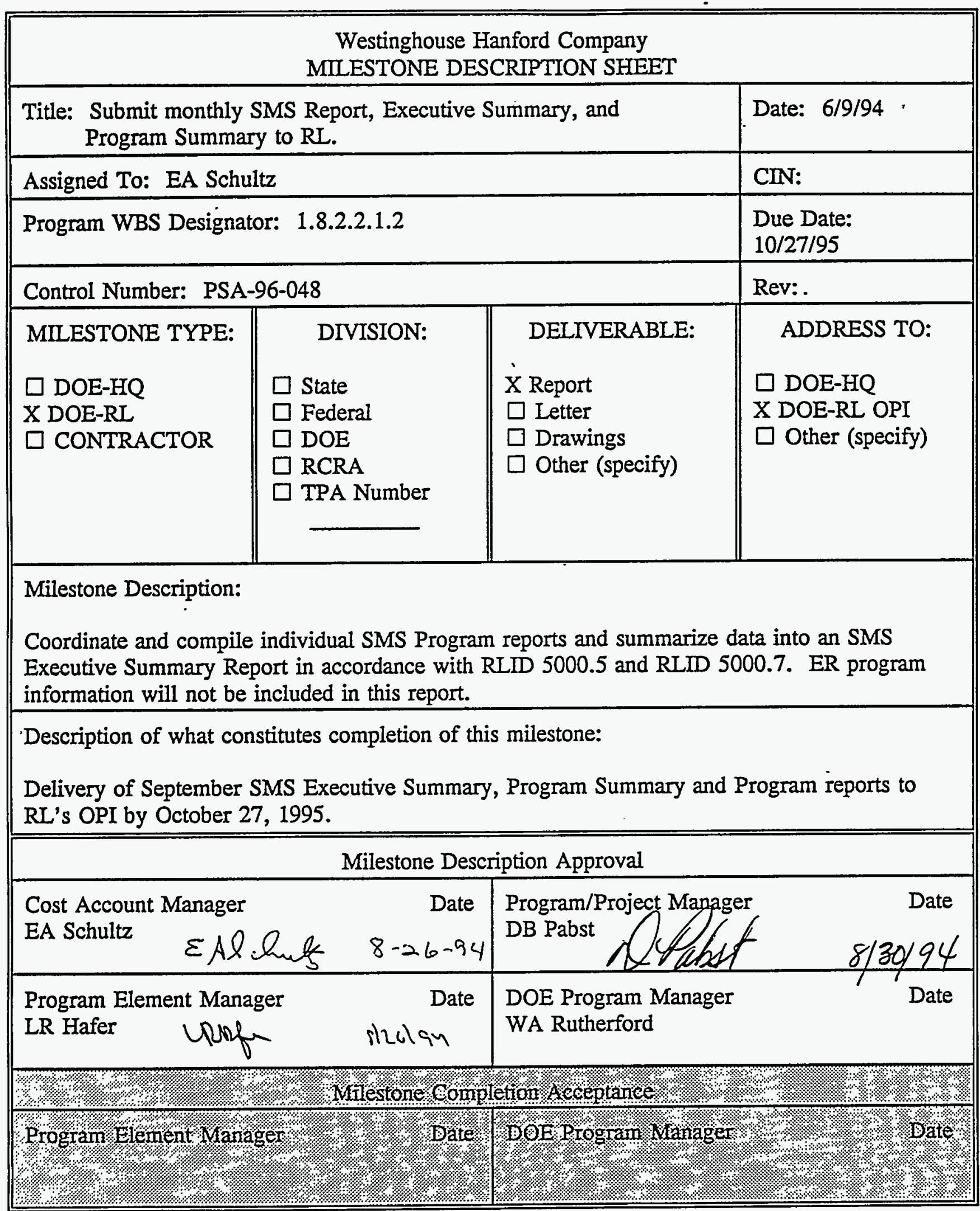




\begin{tabular}{|l||l|l||}
\hline \multicolumn{3}{|c||}{ Westinghouse Hanford Company } \\
MILESTONE DESCRIPTION SHEET
\end{tabular}




\begin{tabular}{|l|l|l||}
\hline \multicolumn{3}{|c||}{ Westinghouse Hanford Company } \\
MILESTONE DESCRIPTION SHEET
\end{tabular}




\begin{tabular}{|l|l|l||}
\hline \multicolumn{3}{|c||}{ Westinghouse Hanford Company } \\
MILESTONE DESCRIPTION SHEET
\end{tabular}




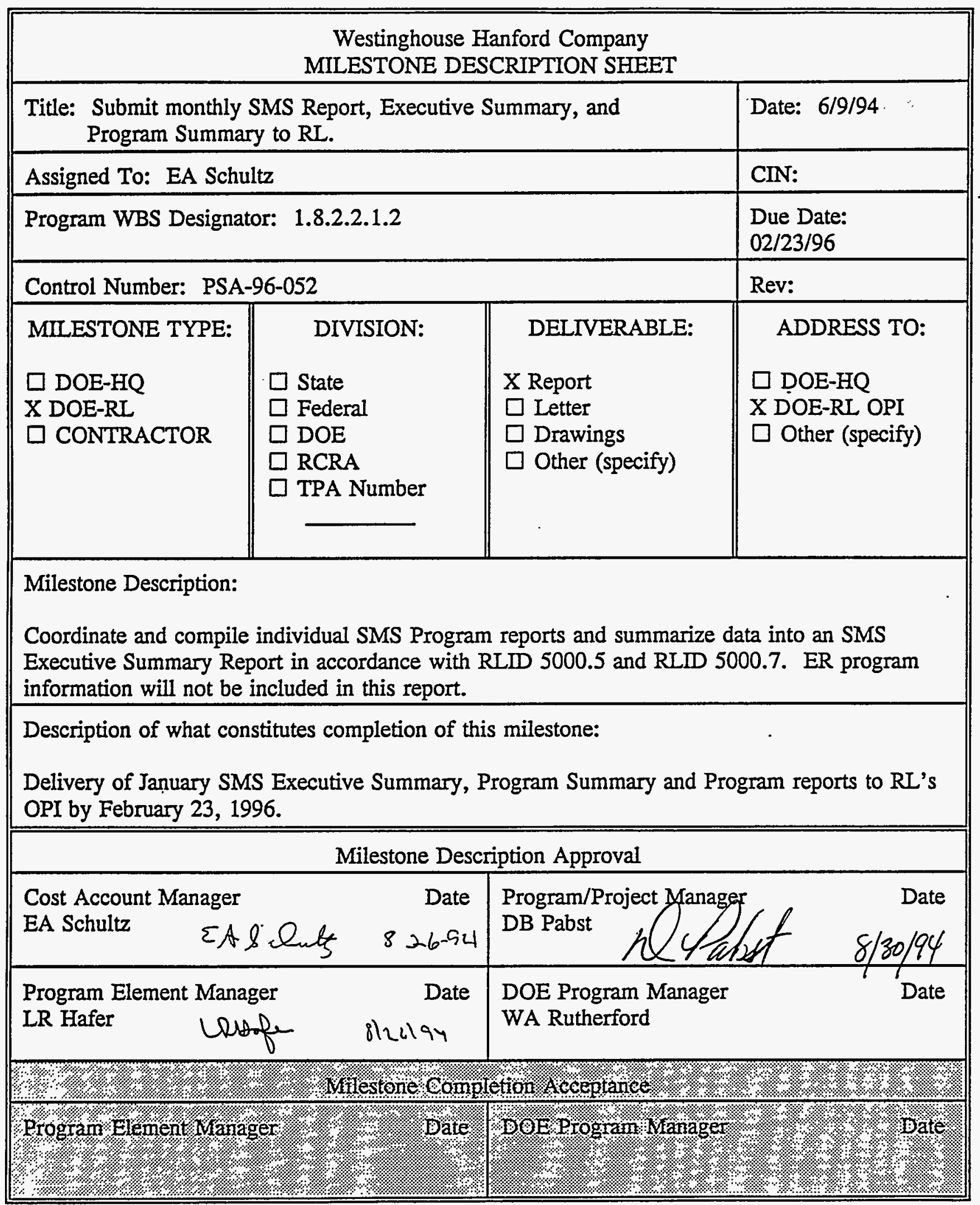




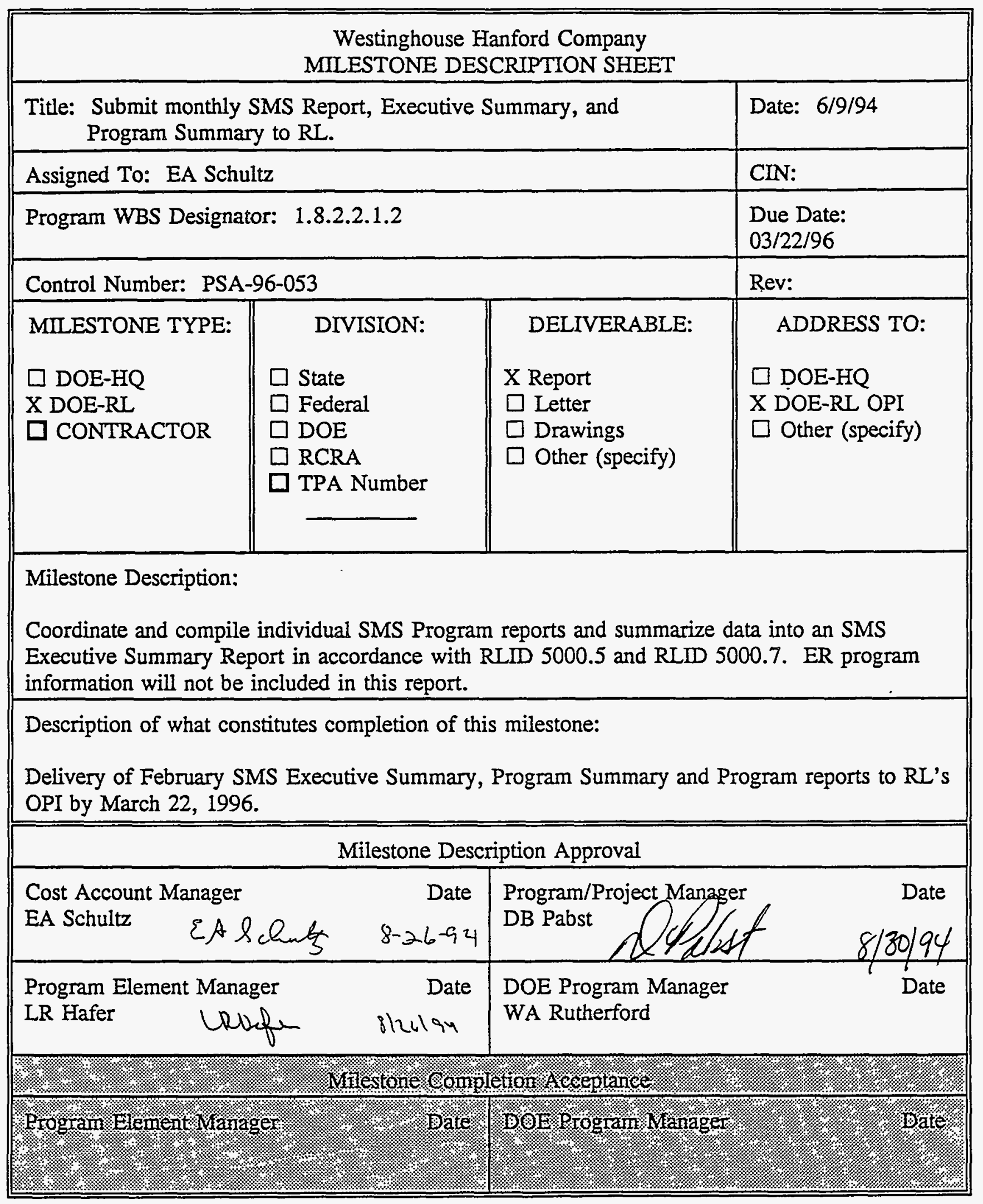




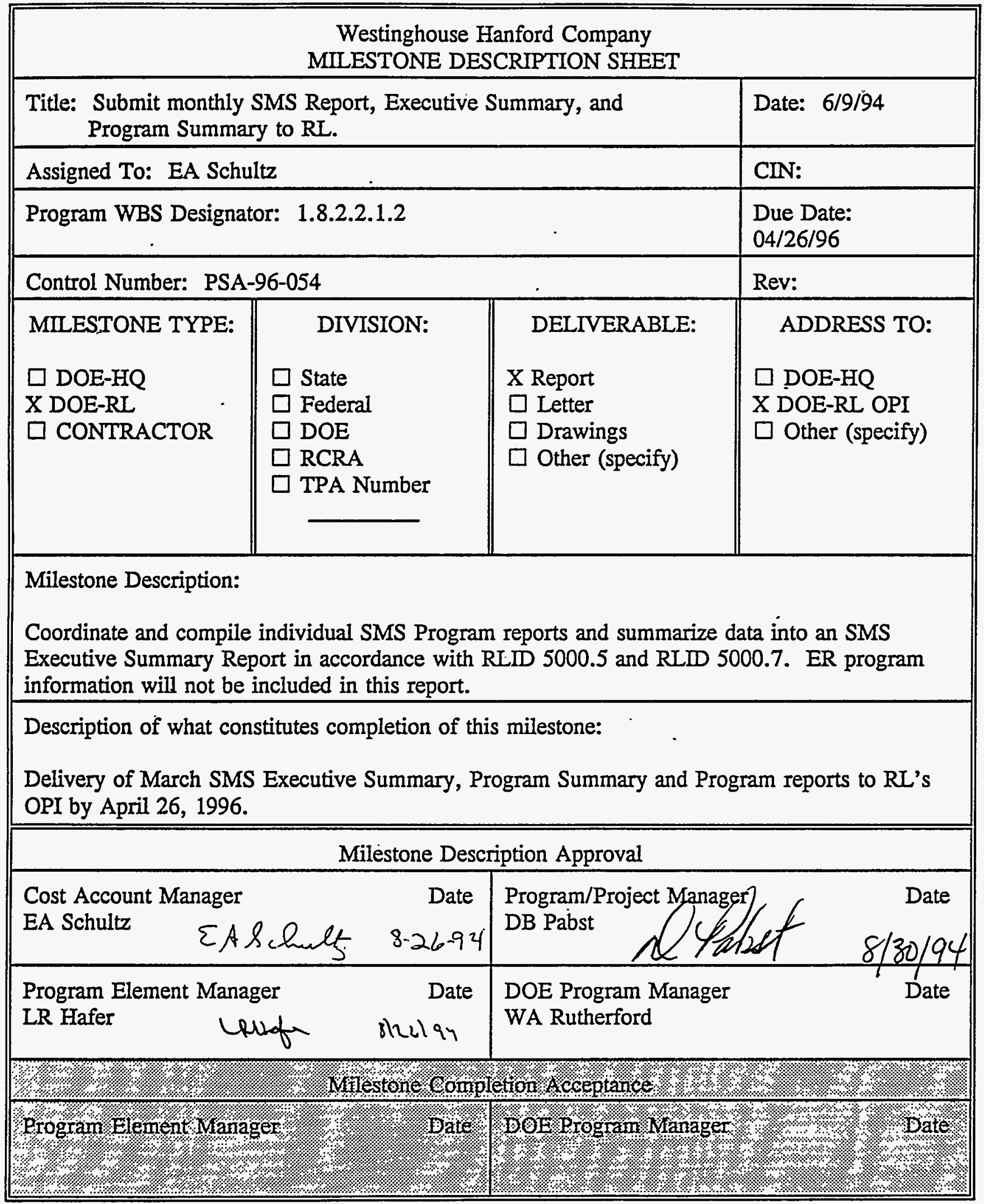




\begin{tabular}{|l||l|l||}
\hline \multicolumn{3}{|c|}{ Westinghouse Hanford Company } \\
MILESTONE DESCRIPTION SHEET
\end{tabular}




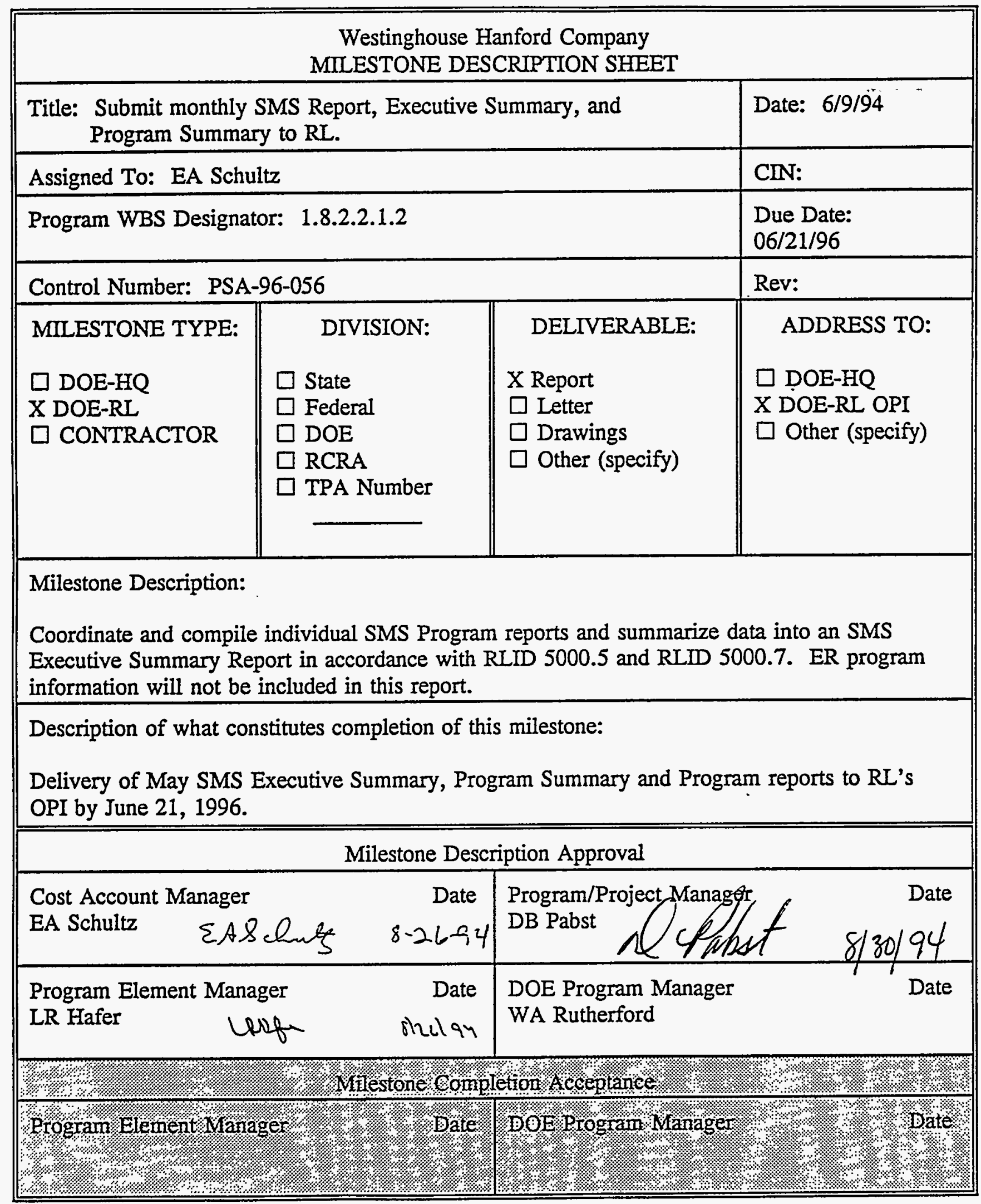




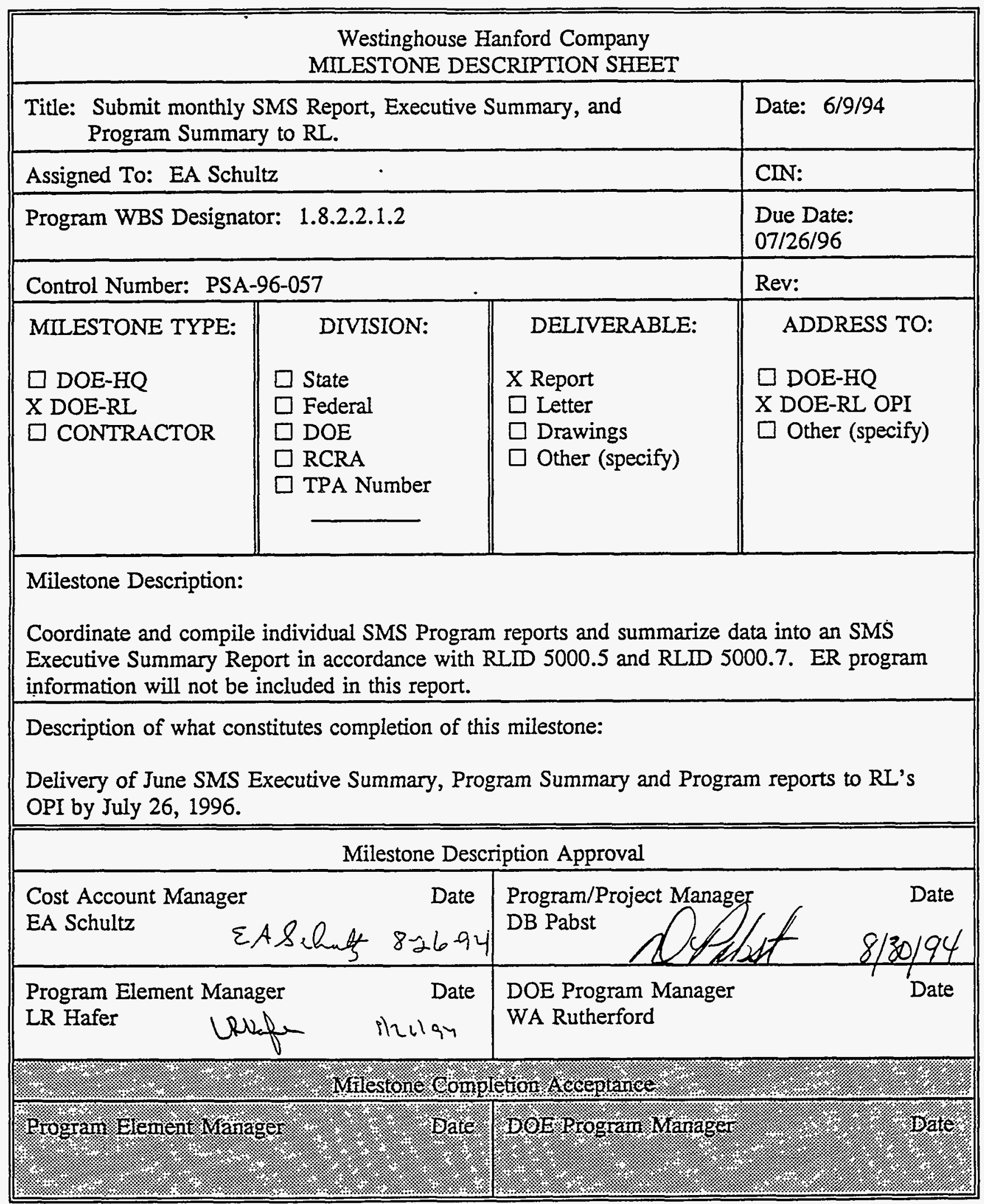




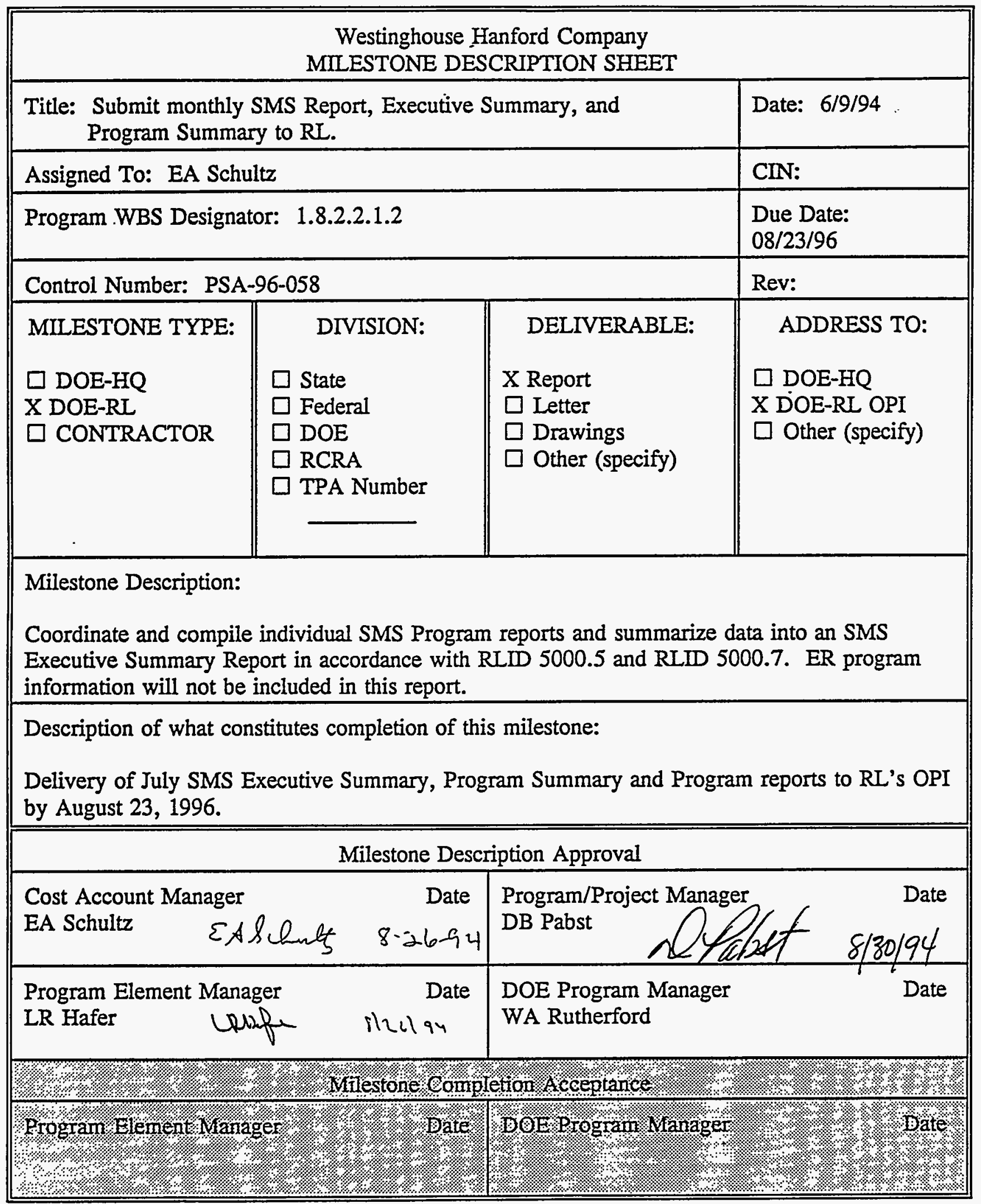




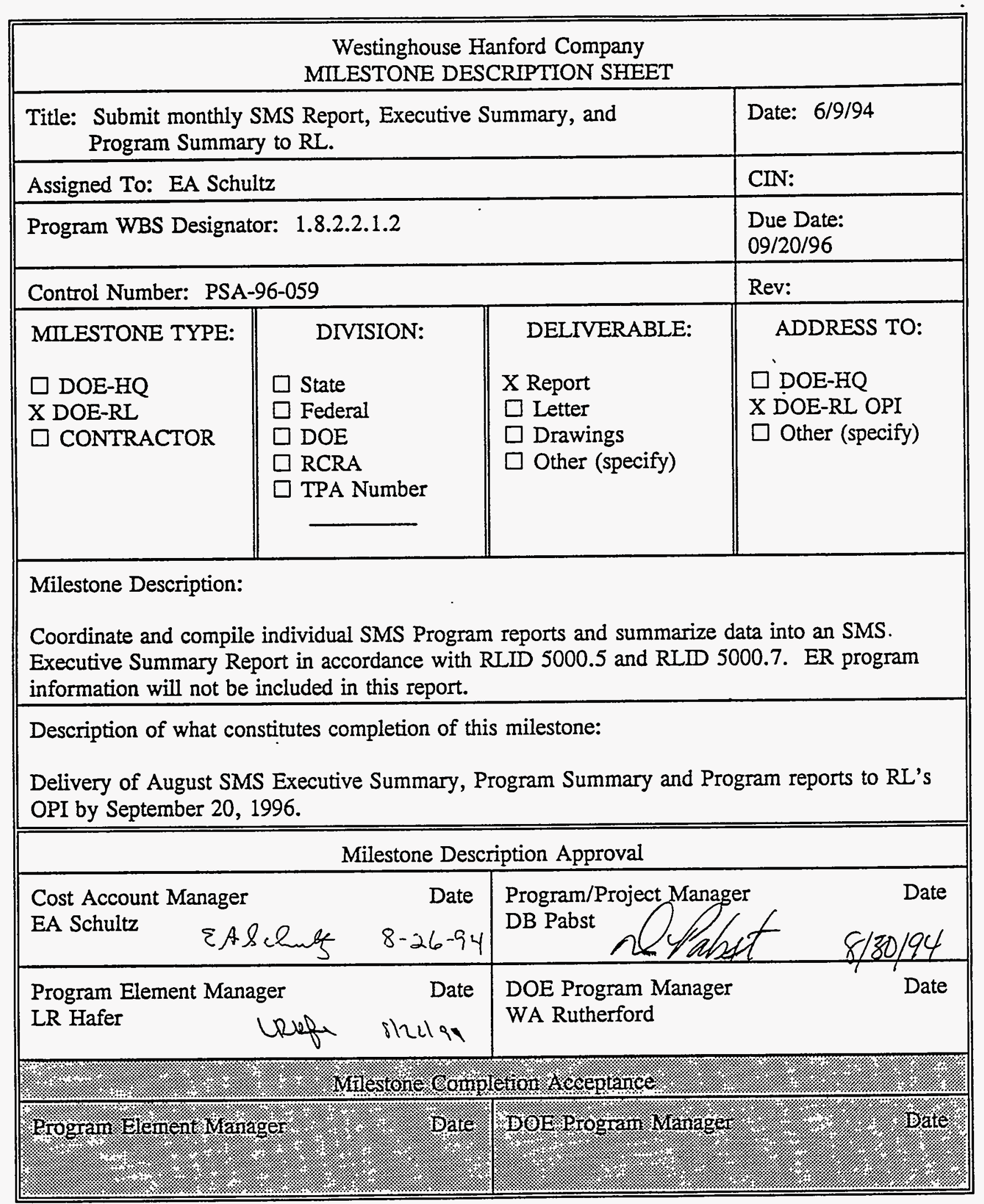




\begin{tabular}{||l||l||}
\hline \multicolumn{3}{|c|}{ Westinghouse Hanford Company } \\
MILSTONE DESCRIPTION SHEET
\end{tabular}




\begin{tabular}{|l|l|l||}
\hline \multicolumn{3}{|c||}{ Westinghouse Hanford Company } \\
MILESTONE DESCRIPTION SHEET
\end{tabular}




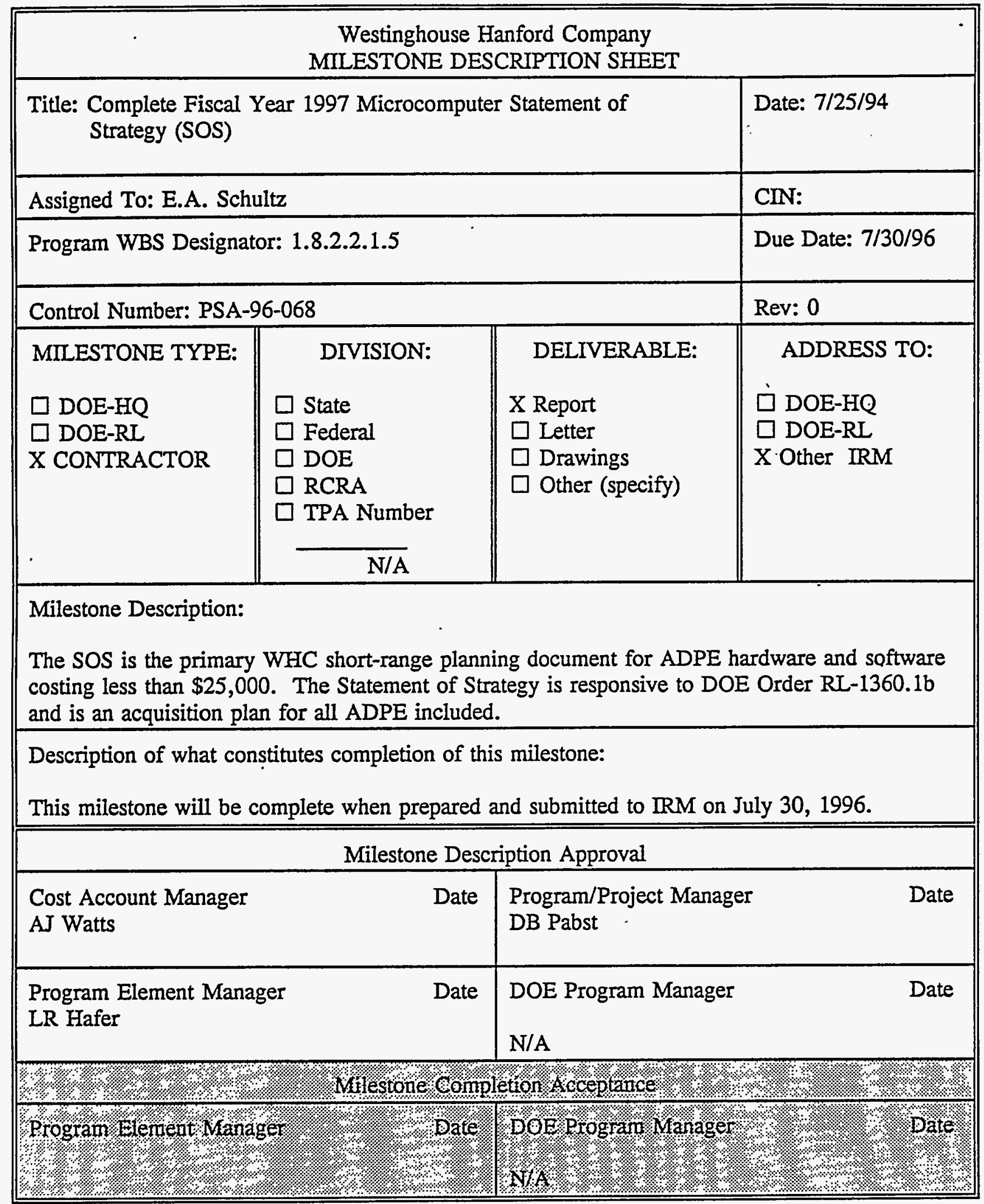




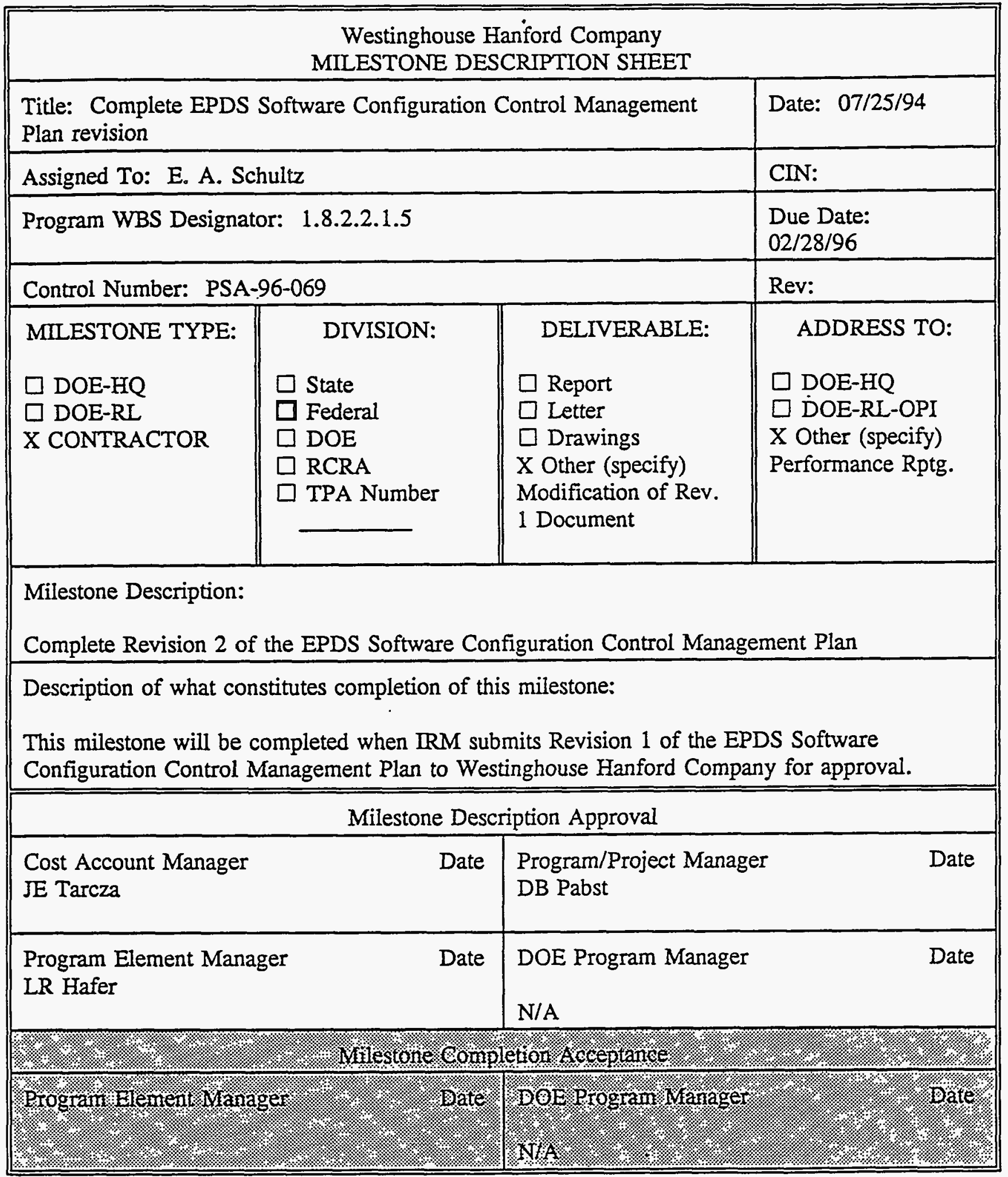




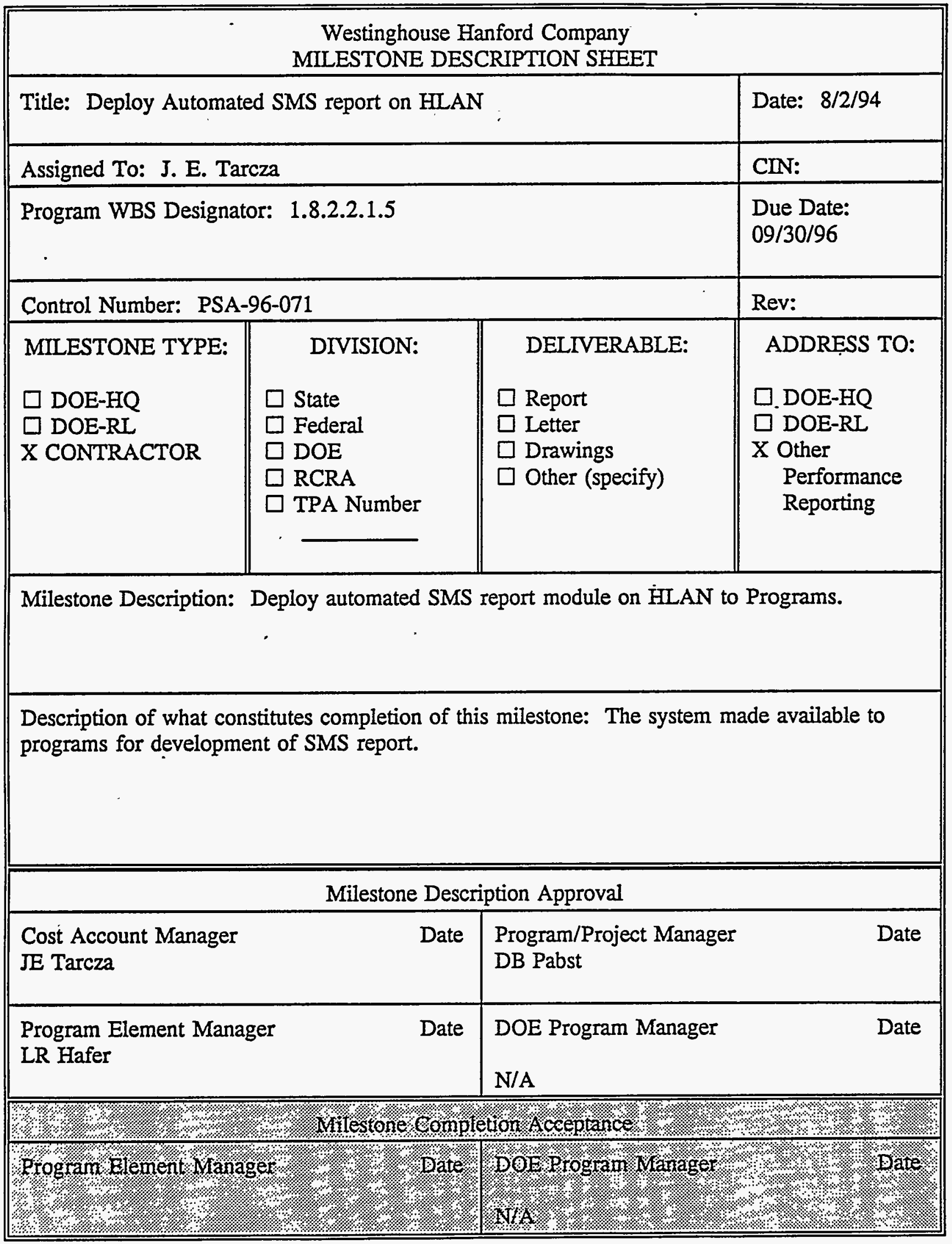




\begin{tabular}{|c|c|c|c|}
\hline \multicolumn{4}{|c|}{$\begin{array}{l}\text { Westinghouse Hanford Company } \\
\text { MILESTONE DESCRIPTION SHEET }\end{array}$} \\
\hline \multicolumn{3}{|c|}{$\begin{array}{l}\text { Title: Data quality check of the monthly direct/indirect Site } \\
\text { Management System (SMS) packages }\end{array}$} & Date: $8 / 4 / 94$ \\
\hline \multicolumn{3}{|c|}{ Assigned To: E. A. Schultz } & CIN: \\
\hline \multicolumn{3}{|c|}{ Program WBS Designator: 1.8.2.2.1.2 } & $\begin{array}{l}\text { Due Date: } \\
\text { 10/25/95 }\end{array}$ \\
\hline \multicolumn{3}{|c|}{ Control Number: PSA-96-073 } & Rev: \\
\hline MILESTONE TYPE: & DIVISION: & DELIVERABLE: & ADDRESS TO: \\
\hline $\begin{array}{l}\square \text { DOE-HQ } \\
\square \text { DOE-RL } \\
\text { X CONTRACTOR }\end{array}$ & $\begin{array}{l}\square \text { State } \\
\square \text { Federal } \\
\square \text { DOE } \\
\square \text { RCRA } \\
\square \text { TPA Number }\end{array}$ & $\begin{array}{l}\square \text { Report. } \\
\square \text { Letter } \\
\square \text { Drawings } \\
\text { X Other } \\
\text { Accurate data } \\
\text { in packages }\end{array}$ & $\begin{array}{l}\square \text { DOE-HQ } \\
\square \text { DOE-RI } \\
\square \text { Other (specify) }\end{array}$ \\
\hline \multicolumn{4}{|c|}{$\begin{array}{l}\text { Milestone Description: } \\
\text { Perform data quality checks of the } 44 \text { monthly direct/indirect SMS packages. Coordinate } \\
\text { corrections/changes with programs. }\end{array}$} \\
\hline \multicolumn{4}{|c|}{$\begin{array}{l}\text { Description of what constitutes completion of this milestone: } \\
\text { Milestone will be completed with submission of all packages to Duplication Center by the } \\
\text { Performance Reporting organization. }\end{array}$} \\
\hline \multicolumn{4}{|c|}{ Milestone Description Approval } \\
\hline \multicolumn{2}{|l|}{$\begin{array}{l}\text { Cost Account Manager } \\
\text { EA Schultz }\end{array}$} & \multicolumn{2}{|l|}{$\begin{array}{l}\text { Program/Project Manager } \\
\text { DB Pabst }\end{array}$} \\
\hline \multicolumn{2}{|c|}{$\begin{array}{l}\text { Program Element Manager } \\
\text { LR Hafer }\end{array}$} & \multicolumn{2}{|l|}{$\begin{array}{l}\text { DOE Program Manager } \\
\text { N/A }\end{array}$} \\
\hline \multicolumn{2}{|c|}{$\begin{array}{l}\text { Program Element Manager } \\
\qquad\end{array}$} & \multicolumn{2}{|c|}{$\begin{array}{l}\text { DOE Program Manager } \\
\text { N/A }\end{array}$} \\
\hline
\end{tabular}




\begin{tabular}{|c|c|c|c|}
\hline \multicolumn{4}{|c|}{$\begin{array}{l}\text { Westinghouse Hanford Company } \\
\text { MILESTONE DESCRIPTION SHEET }\end{array}$} \\
\hline \multicolumn{3}{|c|}{$\begin{array}{l}\text { Title: Data quality check of the monthly direct/indirect Site } \\
\text { Management System (SMS) packages }\end{array}$} & Date: $8 / 4 / 94$ \\
\hline \multicolumn{3}{|c|}{ Assigned To: E. A. Schultz } & CIN: \\
\hline \multicolumn{3}{|c|}{ Program WBS Designator: 1.8 .2 .2 .1 .2} & $\begin{array}{l}\text { Due Date: } \\
11 / 20 / 95\end{array}$ \\
\hline \multicolumn{3}{|c|}{ Control Number: PSA-96-074 } & Rev: \\
\hline $\begin{array}{l}\text { MULESTONE TYPE: } \\
\text { D DOE-HQ } \\
\text { ㅁ DOE-RL } \\
\text { X CONTRACTOR }\end{array}$ & $\begin{aligned} & \text { DIVISION: } \\
& \quad \text { State } \\
& \text { Federal } \\
& \square \text { DOE } \\
& \text { RCRA } \\
& \square \text { TPA Number }\end{aligned}$ & $\begin{array}{l}\text { DELIVERABLE: } \\
\square \text { Report } \\
\square \text { Letter } \\
\square \text { Drawings } \\
\text { X Other } \\
\text { Accurate data } \\
\text { in packages }\end{array}$ & $\begin{array}{l}\text { ADDRESS TO: } \\
\square \text { DOE-HQ } \\
\square \text { DOE-RL } \\
\square \text { Other (specify) }\end{array}$ \\
\hline \multicolumn{4}{|c|}{$\begin{array}{l}\text { Milestone Description: } \\
\text { Perform data quality checks of the } 44 \text { monthly direct/indirect SMS packages. Coordinate } \\
\text { corrections/changes with programs. }\end{array}$} \\
\hline \multicolumn{4}{|c|}{$\begin{array}{l}\text { Description of what constitutes completion of this milestone: } \\
\text { Milestone will be completed with submission of all packages to Duplication Center by the } \\
\text { Performance Reporting organization. }\end{array}$} \\
\hline \multicolumn{4}{|c|}{ Milestone Description Approval } \\
\hline \multicolumn{2}{|l|}{$\begin{array}{l}\text { Cost Account Manager } \\
\text { EA Schultz }\end{array}$} & \multicolumn{2}{|l|}{$\begin{array}{l}\text { Program/Project Manager } \\
\text { DB Pabst }\end{array}$} \\
\hline \multicolumn{2}{|c|}{$\begin{array}{l}\text { Program Element Manager } \\
\text { LR Hafer }\end{array}$} & $\begin{array}{l}\text { DOE Program Manager } \\
\text { N/A }\end{array}$ & Date \\
\hline \multicolumn{4}{|c|}{ ४ै।, M Milestone Completion Acceptance } \\
\hline \multicolumn{2}{|c|}{ Program Element Manager $\quad$ Date } & $\begin{array}{l}\text { DOE Program Manager } \\
\text { N/A }\end{array}$ & $\therefore$ \\
\hline
\end{tabular}




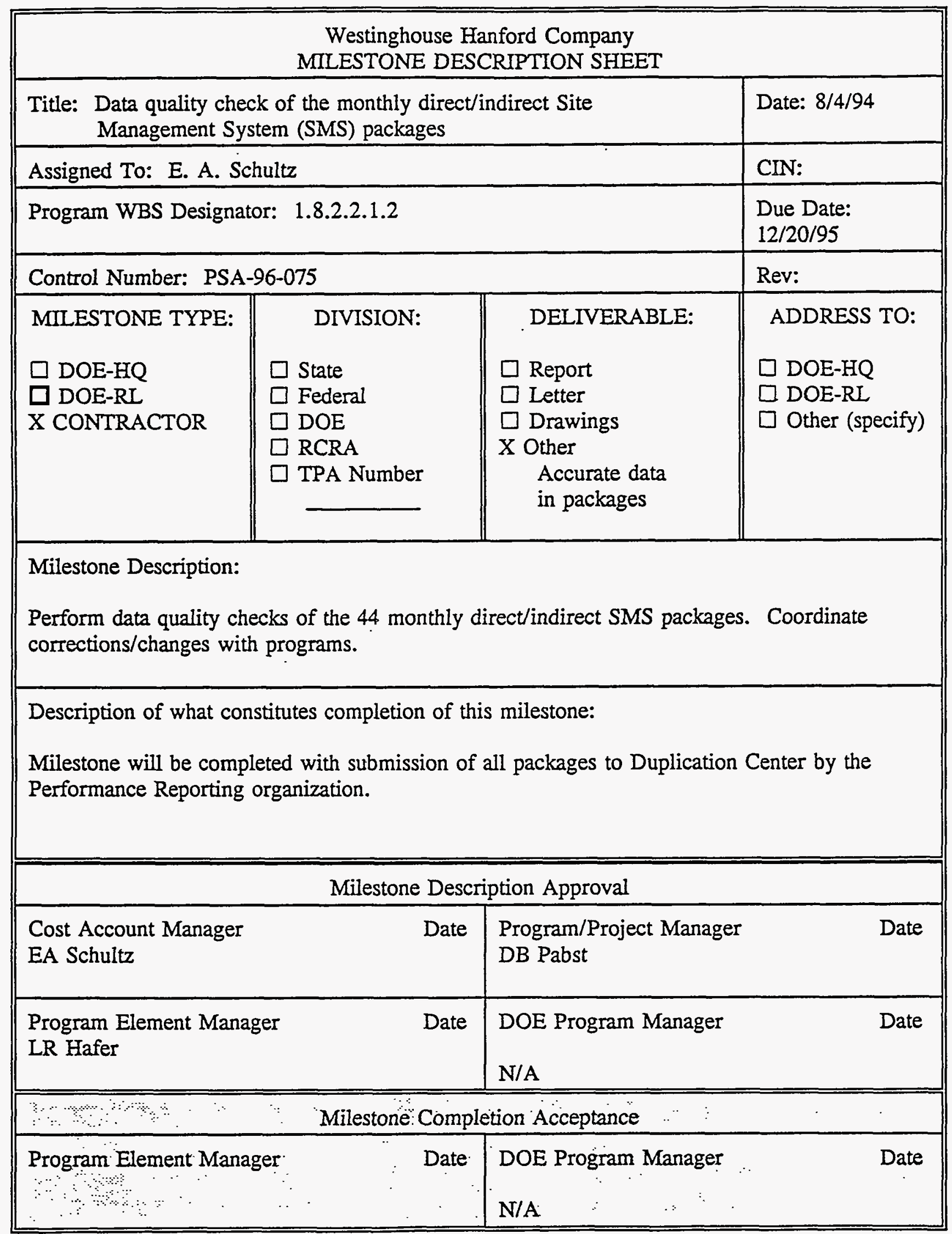




\begin{tabular}{|c|c|c|c|}
\hline \multicolumn{4}{|c|}{$\begin{array}{l}\text { Westinghouse Hanford Company } \\
\text { MILESTONE DESCRIPTION SHEET }\end{array}$} \\
\hline \multicolumn{3}{|c|}{$\begin{array}{l}\text { Title: Data quality check of the monthly direct/indirect Site } \\
\text { Management System (SMS) packages }\end{array}$} & Date: $8 / 4 / 94$ \\
\hline \multicolumn{3}{|c|}{ Assigned To: E. A. Schultz } & CIN: \\
\hline \multicolumn{3}{|c|}{ Program WBS Designator: 1.8 .2 .2 .1 .2} & $\begin{array}{l}\text { Due Date: } \\
1 / 24 / 96\end{array}$ \\
\hline \multicolumn{3}{|c|}{ Control Number: PSA-96-076 } & Rev: \\
\hline MILESTONE TYPE: & DIVISION: & DELIVERABLE: & ADDRESS TO: \\
\hline $\begin{array}{l}\text { D DOE-HQ } \\
\square \text { DOE-RL } \\
\text { X CONTRACTOR }\end{array}$ & $\begin{array}{l}\square \text { State } \\
\square \text { Federal } \\
\square \text { DOE } \\
\square \text { RCRA } \\
\square \text { TPA Number }\end{array}$ & $\begin{array}{l}\square \text { Report } \\
\square \text { Letter } \\
\square \text { Drawings } \\
\text { X Other } \\
\text { Accurate data } \\
\text { in packages }\end{array}$ & $\begin{array}{l}\square \text { DOE-HQ } \\
\square \text {-DOE-RL } \\
\square \text { Other (specify) }\end{array}$ \\
\hline \multicolumn{4}{|l|}{ Milestone Description: } \\
\hline \multicolumn{4}{|c|}{$\begin{array}{l}\text { Perform data quality checks of the } 44 \text { monthly direct/indirect SMS packages. Coordinate } \\
\text { corrections/changes with programs. }\end{array}$} \\
\hline \multicolumn{4}{|c|}{$\begin{array}{l}\text { Description of what constitutes completion of this milestone: } \\
\text { Milestone will be completed with submission of all packages to Duplication Center by the } \\
\text { Performance Reporting organization. }\end{array}$} \\
\hline \multicolumn{4}{|c|}{ Milestone Description Approval } \\
\hline \multicolumn{2}{|l|}{$\begin{array}{l}\text { Cost Account Manager } \\
\text { EA Schultz }\end{array}$} & \multicolumn{2}{|l|}{$\begin{array}{l}\text { Program/Project Manager } \\
\text { DB Pabst }\end{array}$} \\
\hline \multicolumn{2}{|c|}{$\begin{array}{l}\text { Program Element Manager } \\
\text { LR Hafer }\end{array}$} & $\begin{array}{l}\text { DOE Program Manager } \\
\text { N/A }\end{array}$ & Date \\
\hline \multicolumn{4}{|c|}{ औ। $\quad$ Milestone Completion Acceptance } \\
\hline \multicolumn{2}{|c|}{ Program Element Manager a } & \multicolumn{2}{|c|}{$\begin{array}{lll}\text { DOE Program Manger } & \text { Date } \\
\mathrm{N} / \mathrm{AP} & \end{array}$} \\
\hline
\end{tabular}




\begin{tabular}{|c|c|c|c|}
\hline \multicolumn{4}{|c|}{$\begin{array}{l}\text { Westinghouse Hanford Company } \\
\text { MILESTONE DESCRIPTION SHEET }\end{array}$} \\
\hline \multicolumn{3}{|c|}{$\begin{array}{l}\text { Title: Data quality check of the monthly direct/indirect Site } \\
\text { Management System (SMS) packages }\end{array}$} & Date: $8 / 4 / 94$ \\
\hline \multicolumn{3}{|c|}{ Assigned To: E. A. Schultz } & CIN: \\
\hline \multicolumn{3}{|c|}{ Program WBS Designator: 1 8.2.2.1.2 } & $\begin{array}{l}\text { Due Date: } \\
2 / 21 / 96\end{array}$ \\
\hline \multicolumn{3}{|c|}{ Control Number: PSA-96-077 } & \multirow{2}{*}{$\begin{array}{l}\text { Rev: } \\
\text { ADDRESS TO: } \\
\square \text { DOE-HQ } \\
\square \text { DOE-RL } \\
\square \text { Other (specify) }\end{array}$} \\
\hline $\begin{array}{l}\text { MILESTONE TYPE: } \\
\square \text { DOE-HQ } \\
\square \text { DOE-RL } \\
\text { X CONTRACTOR }\end{array}$ & $\begin{aligned} & \text { DIVISION: } \\
& \square \text { State } \\
& \text { Federal } \\
& \square \text { DOE } \\
& \square \text { RCRA } \\
& \square \text { TPA Number }\end{aligned}$ & $\begin{array}{l}\text { DELIVERABLE: } \\
\square \text { Report } \\
\square \text { Letter } \\
\square \text { Drawings } \\
X \text { Other } \\
\text { Accurate data } \\
\text { in packages }\end{array}$ & \\
\hline \multicolumn{4}{|c|}{$\begin{array}{l}\text { Milestone Description: } \\
\text { Perform data quality checks of the } 44 \text { monthly direct/indirect SMS packages. Coordinate } \\
\text { corrections/changes with programs. }\end{array}$} \\
\hline \multicolumn{4}{|c|}{$\begin{array}{l}\text { Description of what constitutes completion of this milestone: } \\
\text { Milestone will be completed with submission of all packages to Duplication Center by the } \\
\text { Performance Reporting organization. }\end{array}$} \\
\hline \multicolumn{4}{|c|}{ Milestone Description Approval } \\
\hline \multicolumn{2}{|l|}{$\begin{array}{l}\text { Cost Account Manager } \\
\text { EA Schultz }\end{array}$} & \multicolumn{2}{|l|}{$\begin{array}{l}\text { Program/Project Manager } \\
\text { DB Pabst }\end{array}$} \\
\hline \multicolumn{2}{|c|}{$\begin{array}{l}\text { Program Element Manager } \\
\text { LR Hafer }\end{array}$} & \multicolumn{2}{|c|}{$\begin{array}{l}\text { DOE Program Manager Date } \\
\text { N/A }\end{array}$} \\
\hline \multicolumn{4}{|c|}{$\quad \quad \quad \because \quad$ Milestone Completion Acceptance } \\
\hline $\begin{array}{c}\text { Program Element Mana } \\
\ddots \\
\ddots\end{array}$ & $\begin{array}{c}\text { ger } \quad \cdots \quad \text { Date } \\
\because \cdots \\
\cdots\end{array}$ & $\begin{array}{l}\text { DOE Program Manager } \\
\text { N/A }\end{array}$ & $\begin{array}{lll}\ddots & \ddots\end{array}$ Date \\
\hline
\end{tabular}




\begin{tabular}{|c|c|c|c|}
\hline \multicolumn{4}{|c|}{$\begin{array}{l}\text { Westinghouse Hanford Company } \\
\text { MILESTONE DESCRIPTION SHEET }\end{array}$} \\
\hline \multicolumn{3}{|c|}{$\begin{array}{l}\text { Title: Data quality check of the monthly direct/indirect Site } \\
\text { Management System (SMS) packages }\end{array}$} & Date: $8 / 4 / 94$ \\
\hline \multicolumn{3}{|c|}{ Assigned To: E. A. Schultz } & CIN: \\
\hline \multicolumn{3}{|c|}{ Program WBS Designator: 1 8.2.2.1.2 } & $\begin{array}{l}\text { Due Date: } \\
3 / 20 / 96\end{array}$ \\
\hline \multicolumn{3}{|c|}{ Control Number: PSA-96-078 } & Rev: \\
\hline $\begin{array}{l}\text { MILESTONE TYPE: } \\
\square \text { DOE-HQ } \\
\square \text { DOE-RL } \\
\text { X CONTRACTOR }\end{array}$ & $\begin{aligned} & \text { DIVISION: } \\
& \\
& \text { State } \\
& \text { Federal } \\
& \square \text { DOE } \\
& \text { RCRA } \\
& \text { RPA Number }\end{aligned}$ & $\begin{array}{l}\text { DELIVERABLE: } \\
\text {. Report } \\
\square \text { Letter } \\
\square \text { Drawings } \\
\text { X Other } \\
\text { Accurate data } \\
\text { in packages }\end{array}$ & $\begin{array}{l}\text { ADDRESS TO: } \\
\square \text { DOE-HQ } \\
\square \text { DOE-RL } \\
\square \text { Other (specify) }\end{array}$ \\
\hline \multicolumn{4}{|c|}{$\begin{array}{l}\text { Milestone Description: } \\
\text { Perform data quality checks of the } 44 \text { monthly direct/indirect SMS packages. Coordinate } \\
\text { corrections/changes with programs. }\end{array}$} \\
\hline \multicolumn{4}{|c|}{$\begin{array}{l}\text { Description of what constitutes completion of this milestone: } \\
\text { Milestone will be completed with submission of all packages to Duplication Center by the } \\
\text { Performance Reporting organization. }\end{array}$} \\
\hline \multicolumn{4}{|c|}{ Milestone Description Approval } \\
\hline \multicolumn{2}{|l|}{$\begin{array}{l}\text { Cost Account Manager } \\
\text { EA Schultz }\end{array}$} & $\begin{array}{l}\text { Program/Project Manager } \\
\text { DB Pabst }\end{array}$ & Date \\
\hline \multicolumn{2}{|c|}{$\begin{array}{l}\text { Program Element Manager } \\
\text { LR Hafer }\end{array}$} & \multicolumn{2}{|c|}{$\begin{array}{l}\text { DOE Program Manager Date } \\
\text { N/A }\end{array}$} \\
\hline \multicolumn{4}{|c|}{ 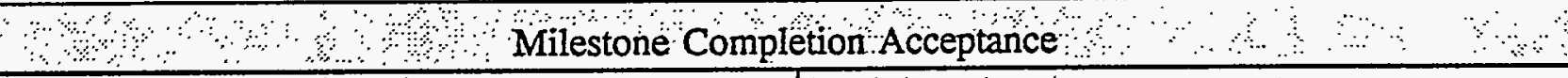 } \\
\hline Program ElementMana & pate & $\begin{array}{l}\text { DOE Program Manager } \\
\text { N/A }\end{array}$ & Date \\
\hline
\end{tabular}




\begin{tabular}{|c|c|c|c|}
\hline \multicolumn{4}{|c|}{$\begin{array}{l}\text { Westinghouse Hanford Company } \\
\text { MILESTONE DESCRIPTION SHEET }\end{array}$} \\
\hline \multicolumn{3}{|c|}{$\begin{array}{l}\text { Title: Data quality check of the monthly direct/indirect Site } \\
\text { Management System (SMS) packages }\end{array}$} & Date: $8 / 4 / 94$ \\
\hline \multicolumn{3}{|c|}{ Assigned To: E. A. Schultz } & CIN: \\
\hline \multicolumn{3}{|c|}{ Program WBS Designator: 1 8.2.2.1.2 } & $\begin{array}{l}\text { Due Date: } \\
4 / 24 / 96\end{array}$ \\
\hline \multicolumn{3}{|c|}{ Control Number: PSA-96-079 } & Rev: \\
\hline $\begin{array}{l}\text { MILESTONE TYPE: } \\
\square \text { DOE-HQ } \\
\square \text { DOE-RL } \\
\text { X CONTRACTOR }\end{array}$ & $\begin{aligned} & \text { DIVISION: } \\
& \text { State } \\
& \square \text { Federal } \\
& \text { DOE } \\
& \square \text { RCRA } \\
& \square \text { TPA Number } \\
&\end{aligned}$ & $\begin{array}{l}\text { DELIVERABLE: } \\
\square \text { Report } \\
\square \text { Letter } \\
\square \text { Drawings } \\
\text { X Other } \\
\text { Accurate data } \\
\text { in packages }\end{array}$ & $\begin{array}{l}\text { ADDRESS TO: } \\
\square \text { DOE-HQ } \\
\square \text { DOE-RL } \\
\square \text { Other (specify) }\end{array}$ \\
\hline \multicolumn{4}{|c|}{$\begin{array}{l}\text { Perform data quality checks of the } 44 \text { monthly direct/indirect SMS packages. Coordinate } \\
\text { corrections/changes with programs. }\end{array}$} \\
\hline \multicolumn{4}{|c|}{$\begin{array}{l}\text { Description of what constitutes completion of this milestone: } \\
\text { Milestone will be completed with submission of all packages to Duplication Center by the } \\
\text { Performance Reporting organization. }\end{array}$} \\
\hline \multicolumn{4}{|c|}{ Milestone Description Approval } \\
\hline \multicolumn{2}{|l|}{$\begin{array}{l}\text { Cost Account Manager } \\
\text { EA Schultz }\end{array}$} & $\begin{array}{l}\text { Program/Project Manager } \\
\text { DB Pabst }\end{array}$ & Date \\
\hline \multicolumn{2}{|c|}{$\begin{array}{l}\text { Program Element Manager } \\
\text { LR Hafer }\end{array}$} & $\begin{array}{l}\text { DOE Program Manager } \\
\text { N/A }\end{array}$ & Date \\
\hline \multicolumn{2}{|c|}{\begin{tabular}{c} 
Program Element Manager \\
\hdashline$y$ \\
\hdashline
\end{tabular}} & \multicolumn{2}{|c|}{$\begin{array}{l}\text { DOE Program Manager } \\
\text { N/A }\end{array}$} \\
\hline
\end{tabular}




\begin{tabular}{|c|c|c|c|}
\hline \multicolumn{4}{|c|}{$\begin{array}{l}\text { Westinghouse Hanford Company } \\
\text { MILESTONE DESCRIPTION SHEET }\end{array}$} \\
\hline \multicolumn{3}{|c|}{$\begin{array}{l}\text { Title: Data quality check of the monthly direct/indirect Site } \\
\text { Management Syster (SMS) packages }\end{array}$} & Date: $8 / 4 / 94$ \\
\hline \multicolumn{3}{|c|}{ Assigned To: E. A. Schultz } & CIN: \\
\hline \multicolumn{3}{|c|}{ Program WBS Designator: 1.8.2.2.1.2 } & $\begin{array}{l}\text { Due Date: } \\
5 / 22 / 96\end{array}$ \\
\hline \multicolumn{3}{|c|}{ Control Number: PSA-96-080 } & Rev: \\
\hline MILESTONE TYPE: & DIVISION: & DELIVERABLE: & ADDRESS TO: \\
\hline $\begin{array}{l}\square \text { DOE-HQ } \\
\square \text { DOE-RI } \\
\text { X CONTRACTOR }\end{array}$ & $\begin{array}{l}\square \text { State } \\
\square \text { Federal } \\
\square \text { DOE } \\
\square \text { RCRA } \\
\square \text { TPA Number }\end{array}$ & $\begin{array}{l}\square \text { Report } \\
\square \text { Letter } \\
\square \text { Drawings } \\
\text { X Other } \\
\text { Accurate data } \\
\text { in packages }\end{array}$ & $\begin{array}{l}\square \text { DOE-HQ } \\
\square \text { DOE-RI } \\
\square \text { Other (specify) }\end{array}$ \\
\hline \multicolumn{4}{|l|}{ Milestone Description: } \\
\hline \multicolumn{4}{|c|}{$\begin{array}{l}\text { Perform data quality checks of the } 44 \text { monthly direct/indirect SMS packages. Coordinate } \\
\text { corrections/changes with programs. }\end{array}$} \\
\hline \multirow{2}{*}{\multicolumn{4}{|c|}{$\begin{array}{l}\text { Description of what constitutes completion of this milestone: } \\
\text { Milestone will be completed with submission of all packages to Duplication Center by the } \\
\text { Performance Reporting organization. }\end{array}$}} \\
\hline & & & \\
\hline \multicolumn{4}{|c|}{ Milestone Description Approval } \\
\hline \multicolumn{2}{|l|}{$\begin{array}{l}\text { Cost Account Manager } \\
\text { EA Schultz }\end{array}$} & \multicolumn{2}{|l|}{$\begin{array}{l}\text { Program/Project Manager } \\
\text { DB Pabst }\end{array}$} \\
\hline \multicolumn{2}{|c|}{$\begin{array}{l}\text { Program Element Manager } \\
\text { LR Hafer }\end{array}$} & \multicolumn{2}{|c|}{$\begin{array}{l}\text { DOE Program Manager } \\
\text { N/A }\end{array}$} \\
\hline \multicolumn{4}{|c|}{ " $\quad$ Milestone Completion Acceptance } \\
\hline \multicolumn{2}{|c|}{ 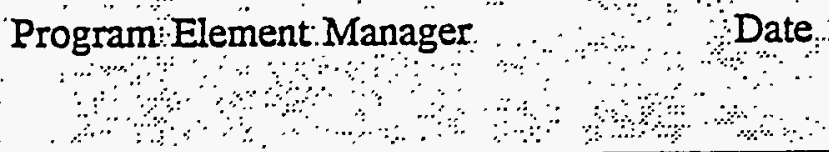 } & \multicolumn{2}{|c|}{$\begin{array}{l}\text { DOE Program Manager } \\
\text { N/A } \mathrm{Al}\end{array}$} \\
\hline
\end{tabular}




\begin{tabular}{|c|c|c|c|}
\hline \multicolumn{4}{|c|}{$\begin{array}{l}\text { Westinghouse Hanford Company } \\
\text { MILESTONE DESCRIPTION SHEET }\end{array}$} \\
\hline \multicolumn{3}{|c|}{$\begin{array}{l}\text { Title: Data quality check of the monthly direct/indirect Site } \\
\text { Management System (SMS) packages }\end{array}$} & Date: $8 / 4 / 94$ \\
\hline \multicolumn{3}{|c|}{ Assigned To: E. A. Schultz } & CIN: \\
\hline \multicolumn{3}{|c|}{ Program WBS Designator: 1.8.2.2.1.2 } & $\begin{array}{l}\text { Due Date: } \\
6 / 19 / 96\end{array}$ \\
\hline \multicolumn{3}{|c|}{ Control Number: PSA-96-081 } & Rev: \\
\hline $\begin{array}{l}\text { MILESTONE TYPE: } \\
\square \text { DOE-HQ } \\
\square \text { DOE-RL } \\
\text { X CONTRACTOR }\end{array}$ & $\begin{aligned} & \text { DIVISION: } \\
& \square \text { State } \\
& \square \text { Federal } \\
& \square \text { DOE } \\
& \square \text { RCRA } \\
& \square \text { TPA Number }\end{aligned}$ & $\begin{array}{l}\text { DELIVERABLE: } \\
\square \text { Report } \\
\square \text { Letter } \\
\square \text { Drawings } \\
X \text { Other } \\
\text { Accurate data } \\
\text { in packages }\end{array}$ & $\begin{array}{l}\text { ADDRESS TO: } \\
\square \text { DOE-HQ } \\
\square \text { DOE-RL } \\
\square \text { Other (specify) }\end{array}$ \\
\hline \multicolumn{4}{|c|}{$\begin{array}{l}\text { Perform data quality checks of the } 44 \text { monthly direct/indirect SMS packages. Coordinate } \\
\text { corrections/changes with programs. }\end{array}$} \\
\hline \multicolumn{4}{|c|}{$\begin{array}{l}\text { Description of what constitutes completion of this milestone: } \\
\text { Milestone will be completed with submission of all packages to Duplication Center by the } \\
\text { Performance Reporting organization. }\end{array}$} \\
\hline \multicolumn{4}{|c|}{ Milestone Description Approval } \\
\hline \multicolumn{2}{|l|}{$\begin{array}{l}\text { Cost Account Manager } \\
\text { EA Schultz }\end{array}$} & \multicolumn{2}{|l|}{$\begin{array}{l}\text { Program/Project Manager } \\
\text { DB Pabst }\end{array}$} \\
\hline \multicolumn{2}{|c|}{$\begin{array}{l}\text { Program Element Manager } \\
\text { LR Hafer }\end{array}$} & \multicolumn{2}{|l|}{$\begin{array}{l}\text { DOE Program Manager } \\
\text { N/A }\end{array}$} \\
\hline \multicolumn{4}{|c|}{ arestone Completion Acceptance } \\
\hline 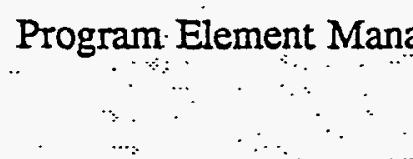 & $\therefore$ Date & $\begin{array}{l}\text { DOE Program Manager } \\
\because \\
\text { N/A }\end{array}$ & Date \\
\hline
\end{tabular}




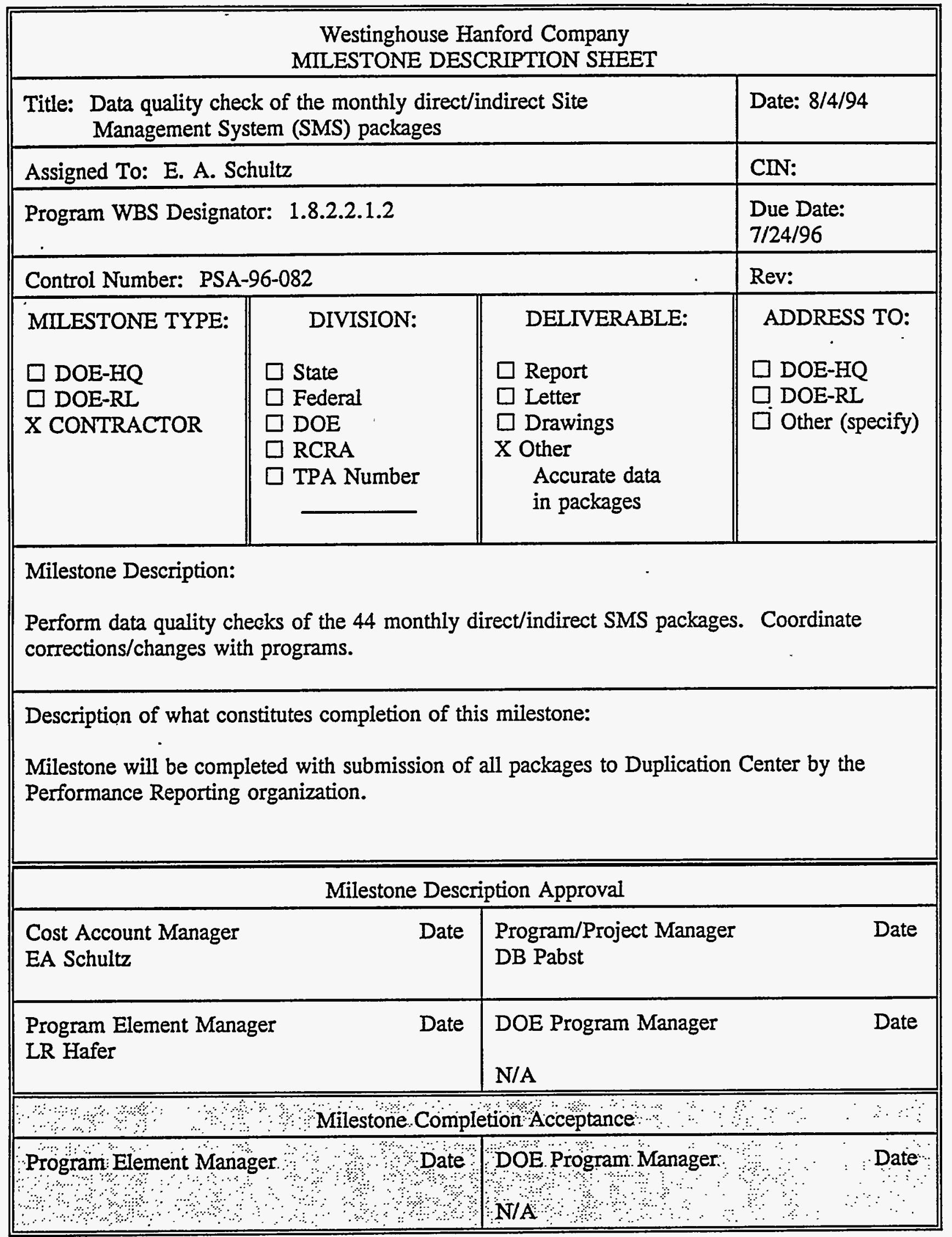




\begin{tabular}{|c|c|c|c|}
\hline \multicolumn{4}{|c|}{$\begin{array}{l}\text { Westinghouse Hanford Company } \\
\text { MILESTONE DESCRIPTION SHEET }\end{array}$} \\
\hline \multicolumn{3}{|c|}{$\begin{array}{l}\text { Title: Data quality check of the monthly direct/indirect Site } \\
\text { Management System (SMS) packages }\end{array}$} & Date: $8 / 4 / 94$ \\
\hline \multicolumn{3}{|c|}{ Assigned To: E. A. Schultz } & CIN: \\
\hline \multicolumn{3}{|c|}{ Program WBS Designator: 1 .8.2.2.1.2 } & $\begin{array}{l}\text { Due Date: } \\
8 / 21 / 96\end{array}$ \\
\hline \multicolumn{3}{|c|}{ Control Number: PSA-96-083 } & Rev: \\
\hline $\begin{array}{l}\text { MILESTONE TYPE: } \\
\square \text { DOE-HQ } \\
\square \text { DOE-RL } \\
\text { X CONTRACTOR }\end{array}$ & $\begin{aligned} & \text { DIVISION: } \\
& \\
& \text { State } \\
& \text { Federal } \\
\square & \text { DOE } \\
& \text { RCRA } \\
& \square \text { TPA Number }\end{aligned}$ & $\begin{array}{l}\text { DELIVERABLE: } \\
\square \text { Report. } \\
\square \text { Letter } \\
\square \text { Drawings } \\
\text { X Other } \\
\text { Accurate data } \\
\text { in packages }\end{array}$ & $\begin{array}{l}\text { ADDRESS TO: } \\
\square \text { DOE-HQ } \\
\square \text { DOE-RL } \\
\square \text { Other (specify) }\end{array}$ \\
\hline \multicolumn{4}{|c|}{$\begin{array}{l}\text { Milestone Description: } \\
\text { Perform data quality checks of the } 44 \text { monthly direct/indirect SMS packages. Coordinate } \\
\text { corrections/changes with programs. }\end{array}$} \\
\hline \multicolumn{4}{|c|}{$\begin{array}{l}\text { Description of what constitutes completion of this milestone: } \\
\text { Milestone will be completed with submission of all packages to Duplication Center by the } \\
\text { Performance Reporting organization. }\end{array}$} \\
\hline \multicolumn{4}{|c|}{ Milestone Description Approval } \\
\hline \multicolumn{2}{|l|}{$\begin{array}{l}\text { Cost Account Manager } \\
\text { EA Schultz }\end{array}$} & \multicolumn{2}{|l|}{$\begin{array}{l}\text { Program/Project Manager } \\
\text { DB Pabst }\end{array}$} \\
\hline \multicolumn{2}{|c|}{$\begin{array}{l}\text { Program Element Manager } \\
\text { LR Hafer }\end{array}$} & \multicolumn{2}{|c|}{$\begin{array}{l}\text { DOE Program Manager Date } \\
\text { N/A }\end{array}$} \\
\hline \multicolumn{4}{|c|}{ Milestone Completion Acceptance } \\
\hline $\begin{array}{c}\text { Program Element Mana } \\
\therefore \quad\end{array}$ & ger $\therefore \quad \because$ Date & $\begin{array}{l}\text { DOE Program Manager } \\
\text { N/A }\end{array}$ & Date \\
\hline
\end{tabular}




\begin{tabular}{|c|c|c|c|}
\hline \multicolumn{4}{|c|}{$\begin{array}{l}\text { Westinghouse Hanford Company } \\
\text { MILESTONE DESCRIPTION SHEET }\end{array}$} \\
\hline \multicolumn{3}{|c|}{$\begin{array}{l}\text { Title: Data quality check of the monthly direct/indirect Site } \\
\text { Management System (SMS) packages }\end{array}$} & Date: $8 / 4 / 94$ \\
\hline \multicolumn{3}{|c|}{ Assigned To: E. A. Schultz } & CIN: \\
\hline \multicolumn{3}{|c|}{ Program WBS Designator: 1 8.2.2.1.2 } & $\begin{array}{l}\text { Due Date: } \\
9 / 18 / 96\end{array}$ \\
\hline \multicolumn{3}{|c|}{ Control Number: PSA-96-084 } & Rev: \\
\hline $\begin{array}{l}\text { MILESTONE TYPE: } \\
\square \text { DOE-HQ } \\
\square \text { DOE-RL } \\
\text { X CONTRACTOR }\end{array}$ & $\begin{array}{l}\quad \text { DIVISION: } \\
\square \text { State } \\
\square \text { Federal } \\
\square \text { DOE } \\
\square \text { RCRA } \\
\square \text { TPA Number }\end{array}$ & $\begin{array}{l}\text { DELIVERABLE: } \\
\square \text { Report } \\
\square \text { Letter } \\
\square \text { Drawings } \\
\text { X Other } \\
\text { Accurate data } \\
\text { in packages }\end{array}$ & $\begin{array}{l}\text { ADDRESS TO: } \\
\square \text { DOE-HQ } \\
\square \text { DOE-RL } \\
\square \text { Other (specify) }\end{array}$ \\
\hline \multicolumn{4}{|c|}{$\begin{array}{l}\text { Milestone Description: } \\
\text { Perform data quality checks of the } 44 \text { monthly direct/indirect SMS packages. Coordinate } \\
\text { corrections/changes with programs. }\end{array}$} \\
\hline \multicolumn{4}{|c|}{$\begin{array}{l}\text { Description of what constitutes completion of this milestone: } \\
\text { Milestone will be completed with submission of all packages to Duplication Center by the } \\
\text { Performance Reporting organization. }\end{array}$} \\
\hline \multicolumn{4}{|c|}{ Milestone Description Approval } \\
\hline \multicolumn{2}{|l|}{$\begin{array}{l}\text { Cost Account Manager } \\
\text { EA Schultz }\end{array}$} & \multicolumn{2}{|l|}{$\begin{array}{l}\text { Program/Project Manager } \\
\text { DB Pabst }\end{array}$} \\
\hline \multicolumn{2}{|c|}{$\begin{array}{l}\text { Program Element Manager } \\
\text { LR Hafer }\end{array}$} & \multicolumn{2}{|c|}{$\begin{array}{l}\text { DOE Program Manager Date } \\
\text { N/A }\end{array}$} \\
\hline \multicolumn{4}{|c|}{ Milestone Completion Acceptance $\quad: \quad \cdots$} \\
\hline Program Element Mana & er & $\begin{array}{l}\text { DOE Program Manager } \\
\text { N/A }\end{array}$ & Date \\
\hline
\end{tabular}




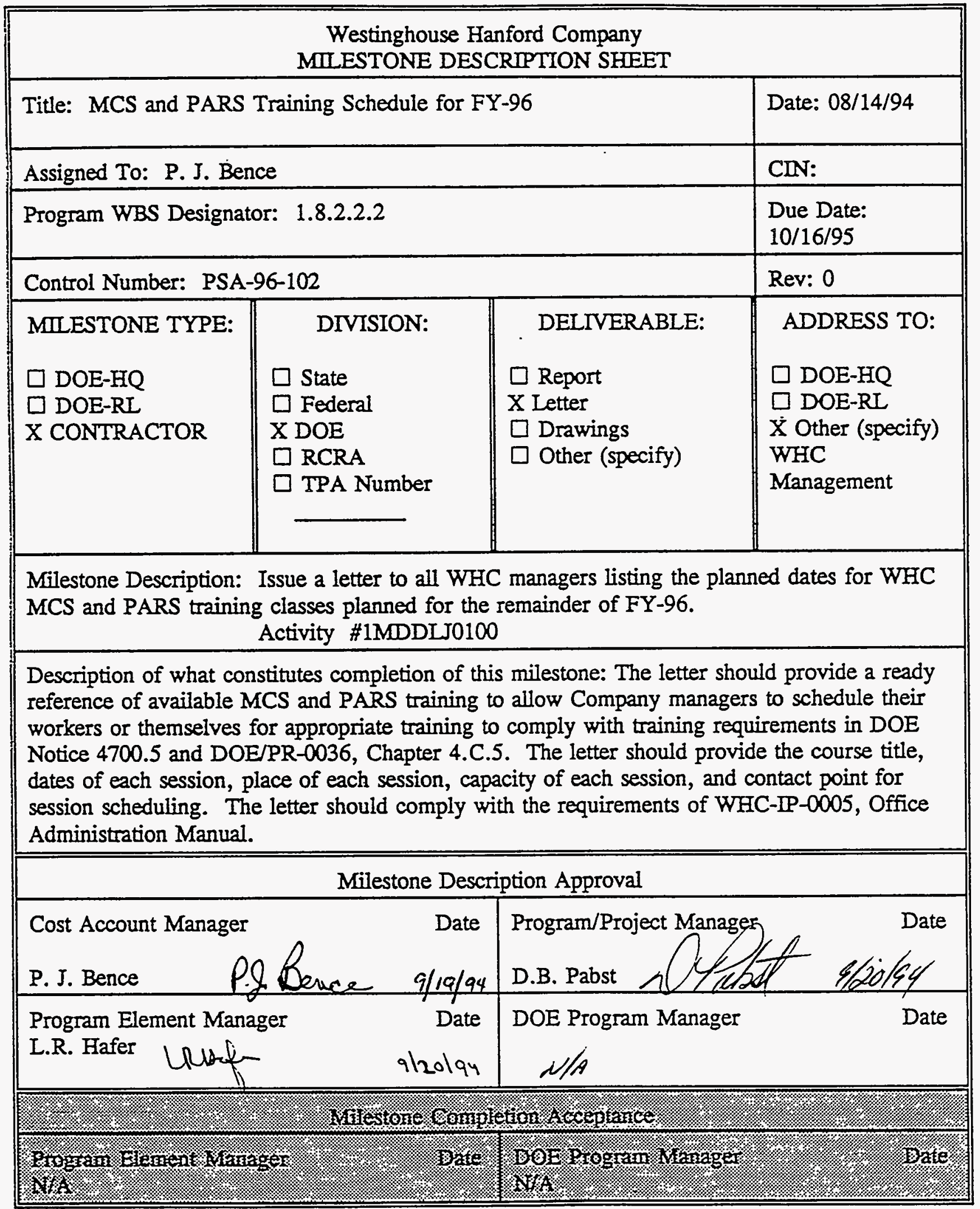




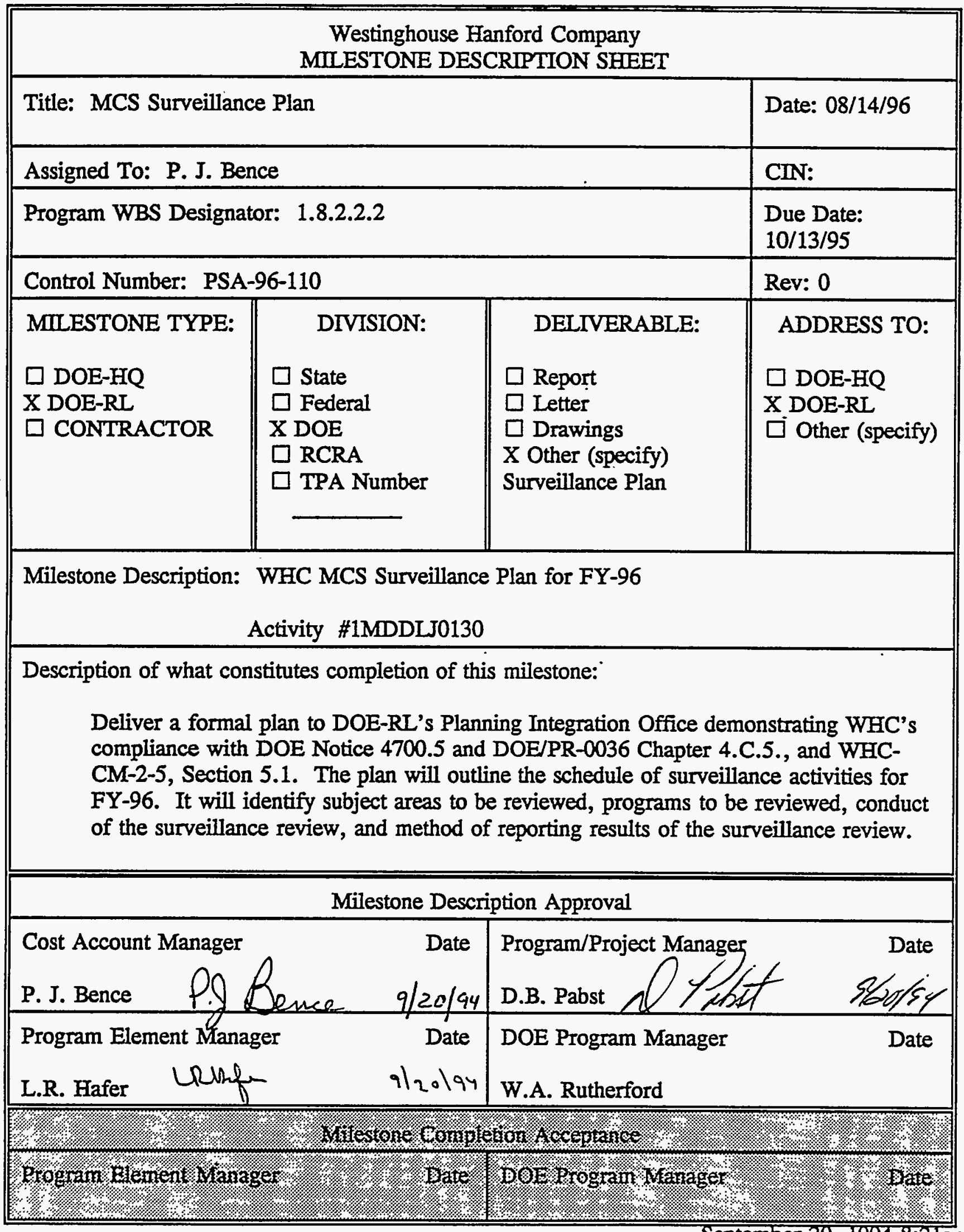




\begin{tabular}{|l||l|l||}
\hline \multicolumn{3}{|c|}{ Westinghouse Hanford Company } \\
MILSTONE DESCRIPTION SHEET
\end{tabular}




\begin{tabular}{|c|c|c|c|}
\hline \multicolumn{4}{|c|}{$\begin{array}{l}\text { Westinghouse Hanford Company } \\
\text { MIIESTONE DESCRIPTION SHEET }\end{array}$} \\
\hline \multicolumn{3}{|c|}{ Title: Surveillance Plan Status Report for Second Quarter FY-96 } & Date: $08 / 14 / 94$ \\
\hline \multicolumn{3}{|c|}{ Assigned To: P.J. Bence } & CIN: \\
\hline \multicolumn{3}{|c|}{ Program WBS Designator: 1 8.2.2.2 } & $\begin{array}{l}\text { Due Date: } \\
04 / 15 / 96\end{array}$ \\
\hline \multicolumn{3}{|c|}{ Control Number: PSA-96-112 } & Rev: 0 \\
\hline $\begin{array}{l}\text { MILESTONE TYPE: } \\
\square \text { DOE-HQ } \\
\square \text { DOE-RL } \\
\text { X CONTRACTOR }\end{array}$ & $\begin{aligned} & \text { DIVISION: } \\
& \square \text { State } \\
& \square \text { Federal } \\
\text { X DOE } & \square \text { RCRA } \\
& \square \text { TPA Number }\end{aligned}$ & $\begin{array}{l}\text { DELIVERABLE: } \\
\text { X Report } \\
\square \text { Letter } \\
\square \text { Drawings } \\
\square \text { Other (specify) }\end{array}$ & $\begin{array}{l}\text { ADDRESS TO: } \\
\square \text { DOE-HQ } \\
\text { X DOE-RL } \\
\square \text { Other (specify) }\end{array}$ \\
\hline \multirow{2}{*}{\multicolumn{4}{|c|}{$\begin{array}{l}\text { Milestone Description: Develop and provide a Status Report of First Quarter FY-96 } \\
\text { Surveillance Plan Activities. This report will summarize the results of completed surveillance } \\
\text { activities, status ongoing activities, and identify opened and closed action items during the } \\
\text { reporting period. This status report further reflects the completion of the data collection \& } \\
\text { review, field visits/interviews, data analysis, report generation, report review and approval, and } \\
\text { follow-up (as required) for each planned surveillance. }\end{array}$}} \\
\hline & & & \\
\hline \multicolumn{4}{|c|}{$\begin{array}{l}\text { Description of what constitutes completion of this milestone: Deliver a status report to DOE- } \\
\text { RL's Planning and Integration Office detailing accomplishments and the current progress of } \\
\text { activities identified in the WHC MCS Surveillance Plan for FY-96, thus documenting } \\
\text { compliance with the intent of DOE Notice } 4700.5 \text { and DOE/PR-0036, Chapter 4.C.5. }\end{array}$} \\
\hline \multicolumn{4}{|c|}{ Milestone Description Approval } \\
\hline \multicolumn{2}{|c|}{$\begin{array}{l}\text { Cost Account Manager } \\
\text { P.J. Bence Pate } \\
\text { Pence 9/20/94 }\end{array}$} & \multicolumn{2}{|c|}{ Program/Project Manager, Date } \\
\hline \multicolumn{2}{|c|}{$\begin{array}{l}\text { Program Element Manager } \quad \text { Date } \\
\text { L.R. Hafer Lefff }\end{array}$} & $\begin{array}{l}\text { DOE Program Manage } \\
\text { N/A }\end{array}$ & Date \\
\hline \multicolumn{4}{|c|}{ (1) } \\
\hline \multicolumn{4}{|c|}{ 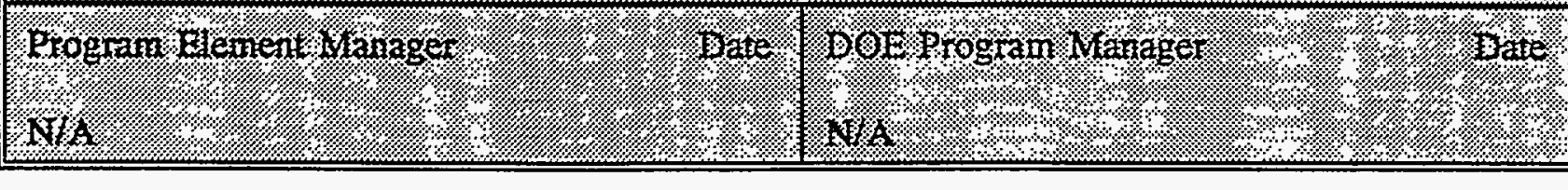 } \\
\hline
\end{tabular}




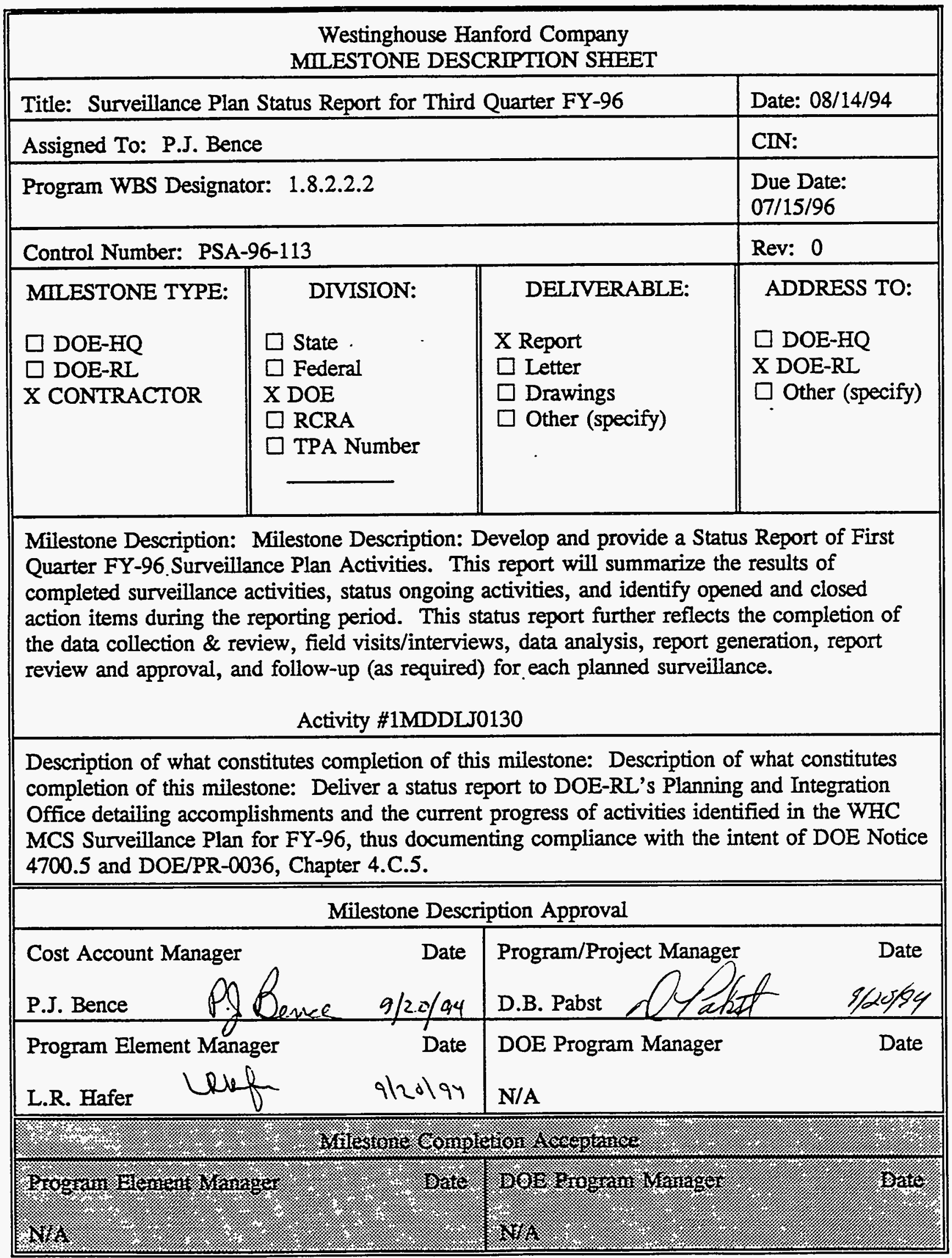




\begin{tabular}{|l|l||}
\hline \multicolumn{3}{|c|}{ Westinghouse Hanford Company } \\
MILESTONE DESCRIPTION SHEET
\end{tabular}




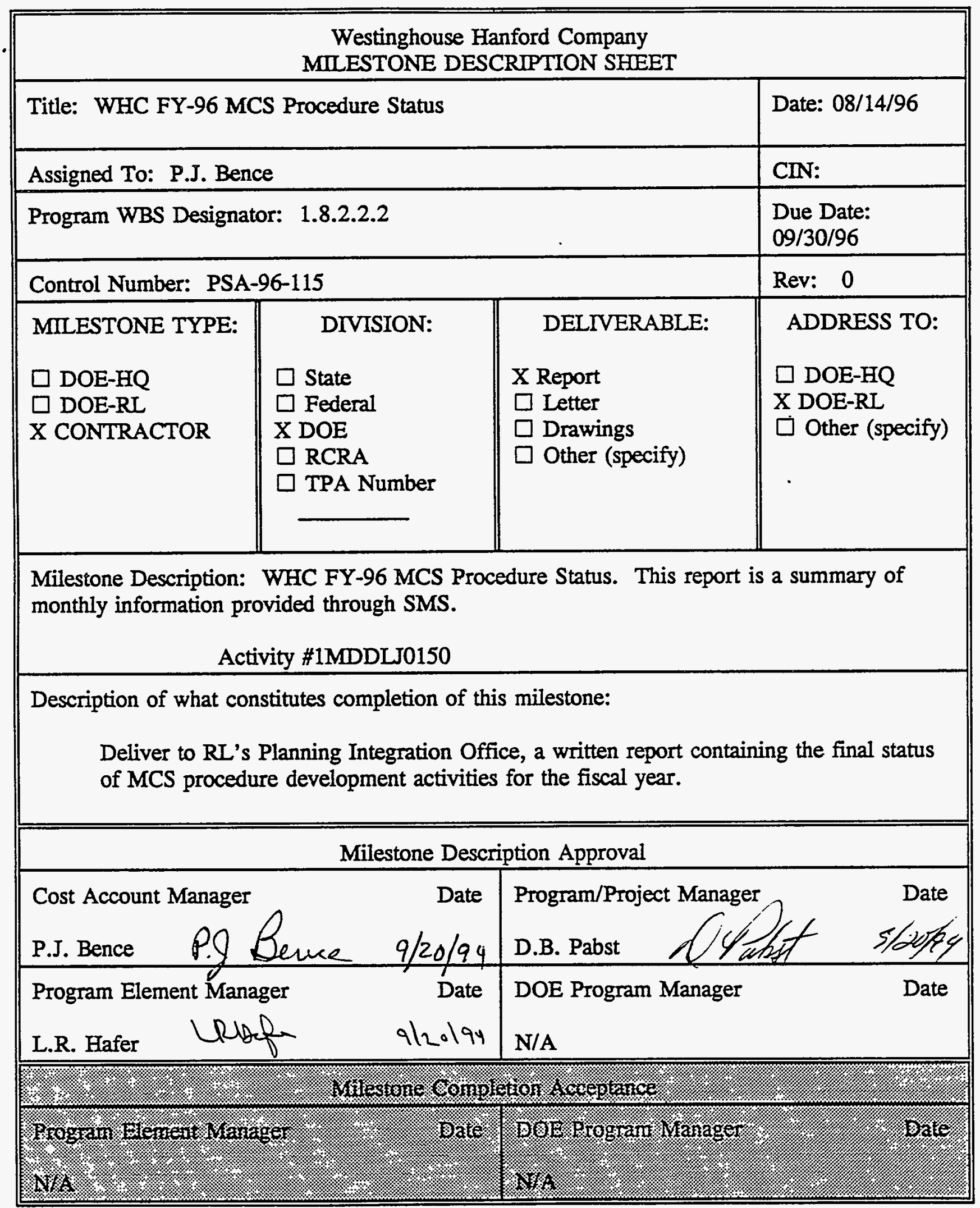




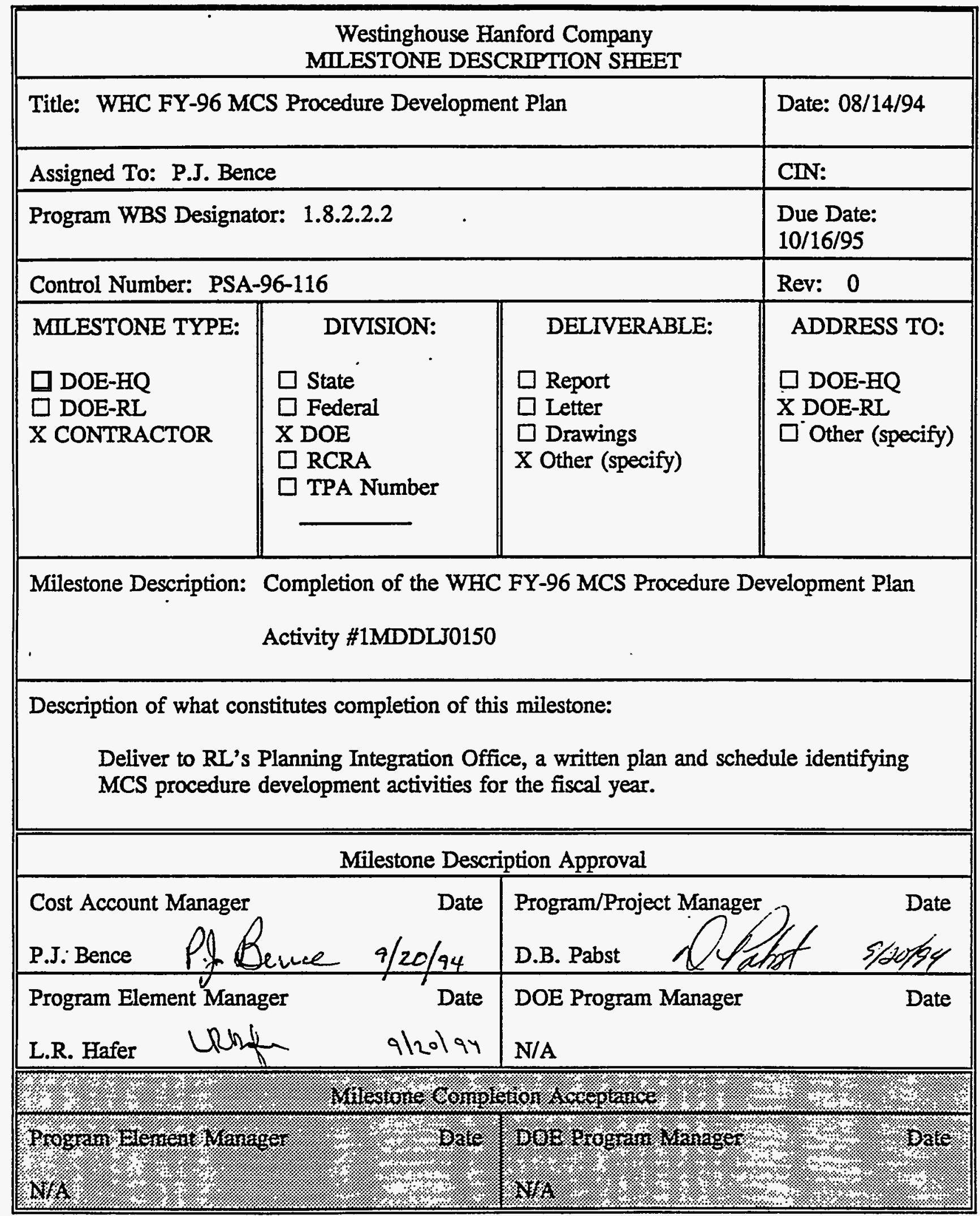




\begin{tabular}{|l|l|l||}
\hline \multicolumn{3}{|c|}{ Westinghouse Hanford Company } \\
MILSTONE DESCRIPTION SHEET
\end{tabular}




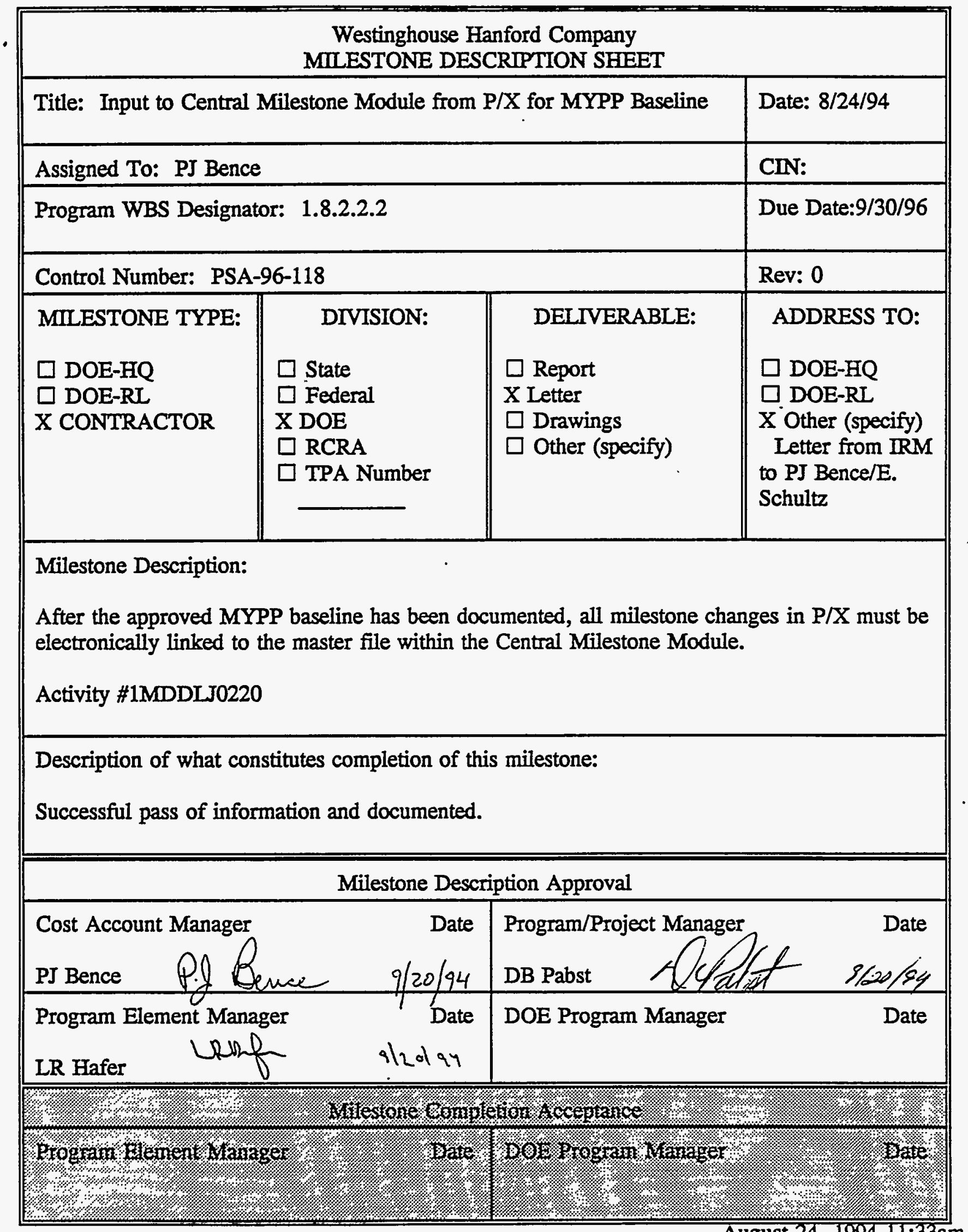




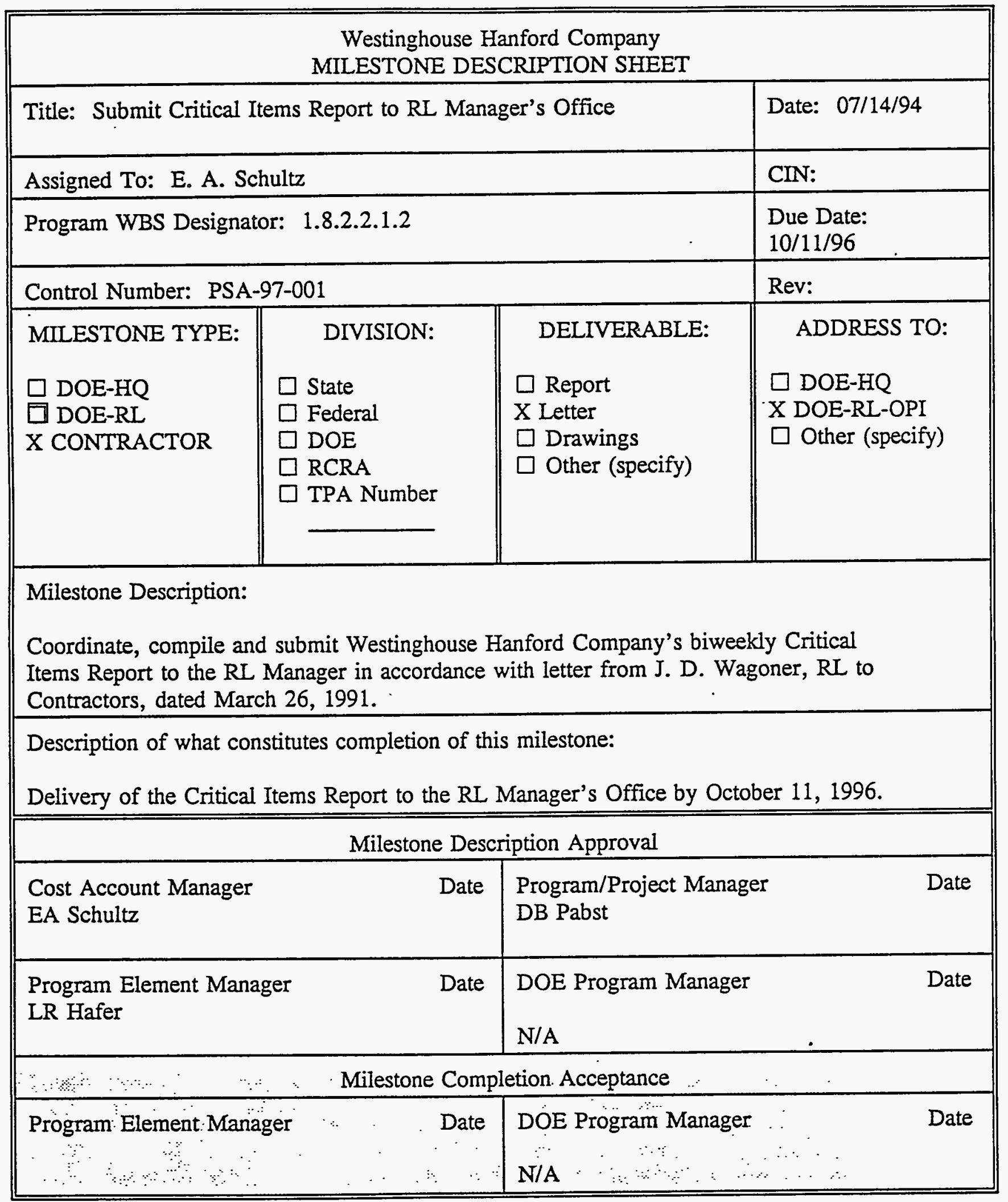




\begin{tabular}{||l|l|l||}
\hline \multicolumn{3}{|c||}{ Westinghouse Hanford Company } \\
MILESTONE DESCRIPTION SHEET
\end{tabular}




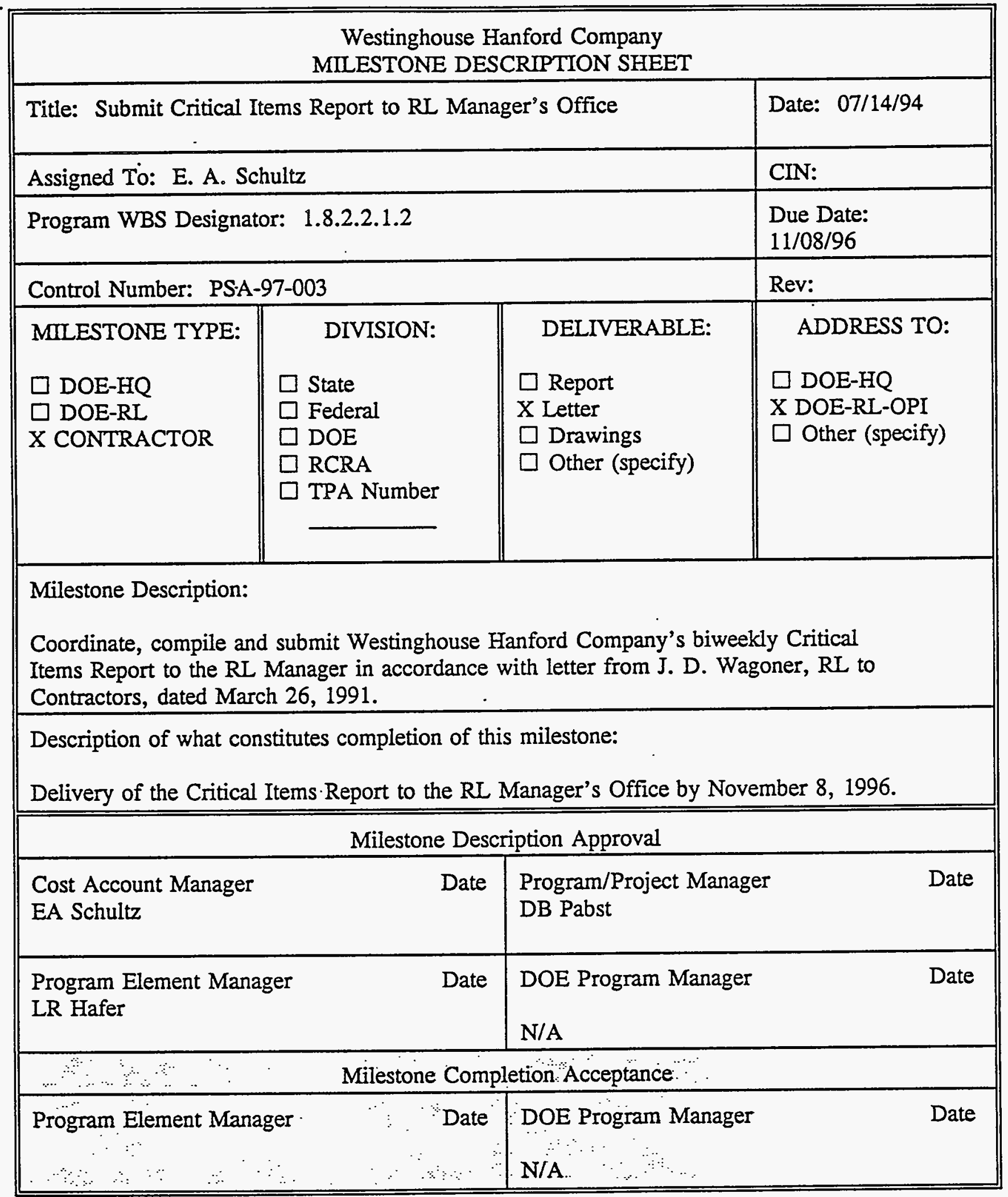




\begin{tabular}{|l||l|l||}
\hline \multicolumn{3}{|c|}{ Westinghouse Hanford Company } \\
MILESTONE DESCRIPTION SHEET
\end{tabular}




\begin{tabular}{||l|l||}
\hline \multicolumn{3}{|c||}{ Westinghouse Hanford Company } \\
MILESTONE DESCRIPTION SHEET
\end{tabular}




\begin{tabular}{|c|c|c|c|}
\hline \multicolumn{4}{|c|}{$\begin{array}{l}\text { Westinghouse Hanford Company } \\
\text { MILESTONE DESCRIPTION SHEET }\end{array}$} \\
\hline \multicolumn{3}{|c|}{ Title: Submit Critical Items Report to RL Manager's Office } & Date: $07 / 14 / 94$ \\
\hline \multicolumn{3}{|c|}{ Assigned To: E. A. Schultz } & CIN: \\
\hline \multicolumn{3}{|c|}{ Program WBS Designator: 1 8.2.2.1.2 } & $\begin{array}{l}\text { Due Date: } \\
12 / 20 / 96\end{array}$ \\
\hline \multicolumn{3}{|c|}{ Control Number: PSA-97-006 } & Rev: \\
\hline $\begin{array}{l}\text { MILESTONE TYPE: } \\
\square \text { DOE-HQ } \\
\square \text { DOE-RL } \\
\text { X CONTRACTOR }\end{array}$ & $\begin{array}{l}\quad \text { DIVISION: } \\
\square \text { State } \\
\square \text { Federal } \\
\square \text { DOE } \\
\square \text { RCRA } \\
\square \text { TPA Number }\end{array}$ & $\begin{array}{l}\text { DELIVERABLE: } \\
\square \text { Report } \\
\text { X Letter } \\
\square \text { Drawings } \\
\square \text { Other (specify) }\end{array}$ & $\begin{array}{l}\text { ADDRESS TO: } \\
\square \text { DOE-HQ } \\
\text { X DOE-RL-OPI } \\
\square \text { Other (specify) }\end{array}$ \\
\hline \multicolumn{4}{|c|}{$\begin{array}{l}\text { Milestone Description: } \\
\text { Coordinate, compile and submit Westinghouse Hanford Company's biweekly Critical } \\
\text { Items Report to the RL Manager in accordance with letter from J. D. Wagoner, RL to } \\
\text { Contractors, dated March } 26,1991 \text {. }\end{array}$} \\
\hline \multicolumn{4}{|c|}{$\begin{array}{l}\text { Description of what constitutes completion of this milestone: } \\
\text { Delivery of the Critical Items Report to the RL Manager's Office by December 20, } 1996 .\end{array}$} \\
\hline \multicolumn{4}{|c|}{ Milestone Description Approval } \\
\hline \multicolumn{2}{|l|}{$\begin{array}{l}\text { Cost Account Manager } \\
\text { EA Schultz }\end{array}$} & \multicolumn{2}{|l|}{$\begin{array}{l}\text { Program/Project Manager } \\
\text { DB Pabst }\end{array}$} \\
\hline \multicolumn{2}{|c|}{$\begin{array}{l}\text { Program Element Manager } \\
\text { LR Hafer }\end{array}$} & \multicolumn{2}{|c|}{$\begin{array}{ll}\text { DOE Program Manager } & \text { Date } \\
\text { N/A } & \\
\end{array}$} \\
\hline \multicolumn{4}{|c|}{ Milestone Completion Acceptance ${ }^{\circ}$ o } \\
\hline \multicolumn{2}{|c|}{ Program Element Manager Date } & \multicolumn{2}{|c|}{$\begin{array}{l}\text { DOE Program Manager } \\
\text { N/A }\end{array}$} \\
\hline
\end{tabular}




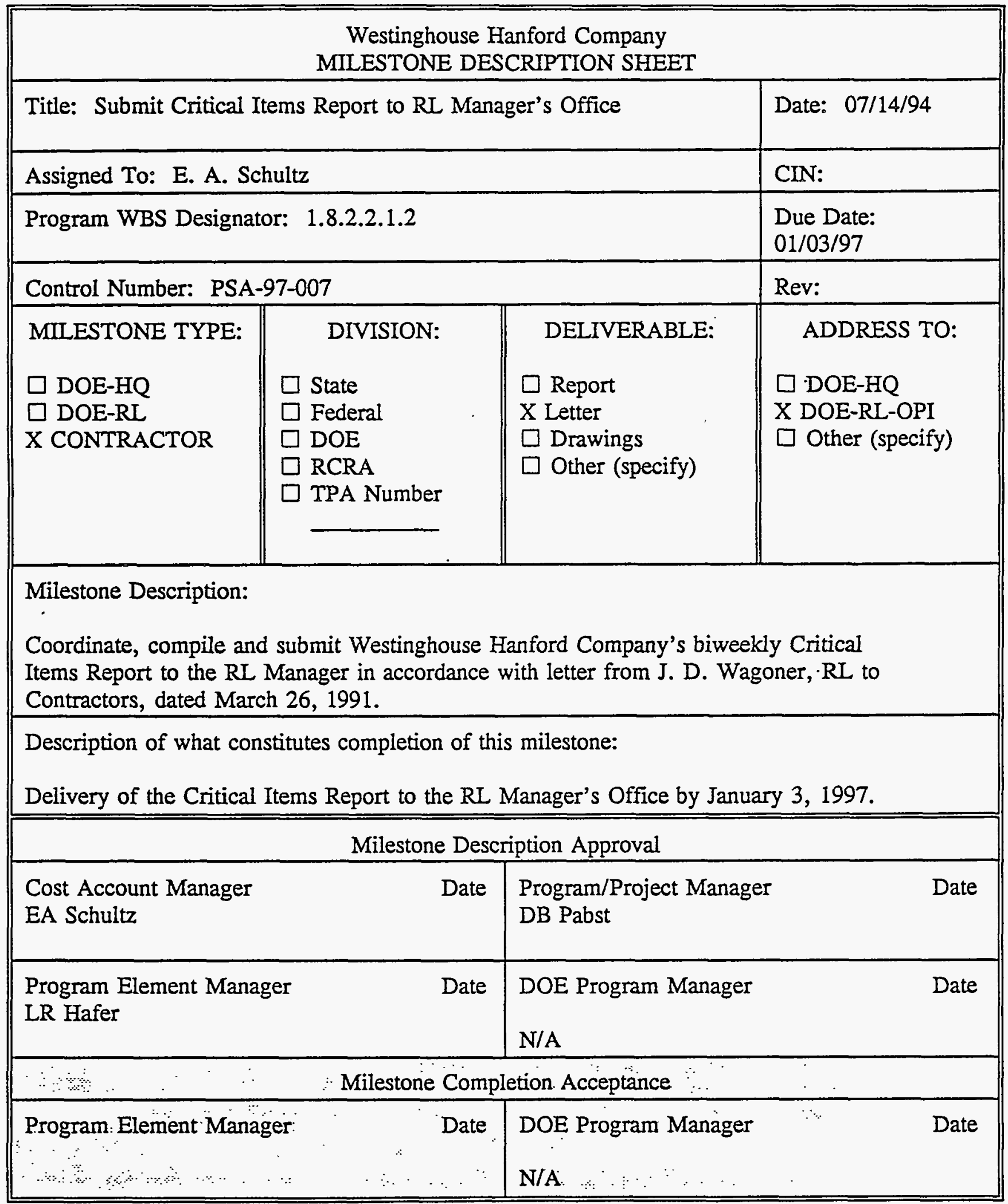




\begin{tabular}{|c|c|c|c|}
\hline \multicolumn{4}{|c|}{$\begin{array}{l}\text { Westinghouse Hanford Company } \\
\text { MILESTONE DESCRIPTION SHEET }\end{array}$} \\
\hline \multicolumn{3}{|c|}{ Title: Submit Critical Items Report to RL Manager's Office } & Date: $07 / 14 / 94$ \\
\hline \multicolumn{3}{|c|}{ Assigned To: E. A. Schultz } & CIN: \\
\hline \multicolumn{3}{|c|}{ Program WBS Designator: $1 \cdot 8 \cdot 2 \cdot 2 \cdot 1.2$} & $\begin{array}{l}\text { Due Date: } \\
01 / 17 / 97\end{array}$ \\
\hline \multicolumn{3}{|c|}{ Control Number: PSA-97-008 } & Rev: ${ }^{\circ}$ \\
\hline $\begin{array}{l}\text { MILESTONE TYPE: } \\
\square \text { DOE-HQ } \\
\square \text { DOE-RL } \\
\text { X CONTRACTOR }\end{array}$ & $\begin{aligned} & \text { DIVISION: } \\
& \\
& \square \text { State } \\
& \square \text { Federal } \\
& \square \text { DOE } \\
& \square \text { RCRA } \\
& \square \text { TPA Number }\end{aligned}$ & $\begin{array}{l}\text { DELIVERABLE: } \\
\square \text { Report } \\
\text { X Letter } \\
\square \text { Drawings } \\
\square \text { Other (specify) }\end{array}$ & $\begin{array}{l}\text { ADDRESS TO: } \\
\square \text { DOE-HQ } \\
\text { X DOE-RL-OPI } \\
\square \text { Other (specify) }\end{array}$ \\
\hline \multicolumn{4}{|c|}{$\begin{array}{l}\text { Milestone Description: } \\
\text { Coordinate, compile and submit Westinghouse Hanford Company's biweekly Critical } \\
\text { Items Report to the RL Manager in accordance with letter from J. D. Wagoner, RL to } \\
\text { Contractors, dated March } 26,1991 \text {. }\end{array}$} \\
\hline \multicolumn{4}{|c|}{$\begin{array}{l}\text { Description of what constitutes completion of this milestone: } \\
\text { Delivery of the Critical Items Report to the RL Manager's Office by January 17, } 1997 .\end{array}$} \\
\hline \multicolumn{4}{|c|}{ Milestone Description Approval } \\
\hline \multicolumn{2}{|l|}{$\begin{array}{l}\text { Cost Account Manager } \\
\text { EA Schultz }\end{array}$} & $\begin{array}{l}\text { Program/Project Manager } \\
\text { DB Pabst }\end{array}$ & Date \\
\hline \multicolumn{2}{|c|}{$\begin{array}{l}\text { Program Element Manager } \\
\text { LR Hafer }\end{array}$} & $\begin{array}{l}\text { DOE Program Manager } \\
\text { N/A }\end{array}$ & Date \\
\hline \multicolumn{4}{|c|}{$\mathrm{A}^{\circ} \mathrm{Q}$ Milestone Completion Acceptance } \\
\hline Program Element Man & 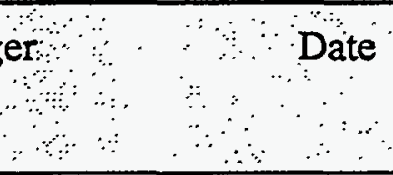 & $\begin{array}{l}\text { DOE Program Manager } \\
\text { N/A }\end{array}$ & a \\
\hline
\end{tabular}




\begin{tabular}{|l||l||}
\hline \multicolumn{3}{|c||}{ Westinghouse Hanford Company } \\
MILESTONE DESCRIPTION SHEET
\end{tabular}




\begin{tabular}{|l||l|l||}
\hline \multicolumn{3}{|c|}{ Westinghouse Hanford Company } \\
MILESTONE DESCRIPTION SHEET
\end{tabular}




\begin{tabular}{|c|c|c|c|}
\hline \multicolumn{4}{|c|}{$\begin{array}{l}\text { Westinghouse Hanford Company } \\
\text { MILESTONE DESCRIPTION SHEET }\end{array}$} \\
\hline \multicolumn{3}{|c|}{ Title: Submit Critical Items Report to RL Manager's Office } & Date: $07 / 14 / 94$ \\
\hline \multicolumn{3}{|c|}{ Assigned To: E. A. Schultz } & CIN: \\
\hline \multicolumn{3}{|c|}{ Program WBS Designator: 1 8.2.2.1.2 } & $\begin{array}{l}\text { Due Date: } \\
02 / 28 / 97 \\
\end{array}$ \\
\hline \multicolumn{3}{|c|}{ Control Number: PSA-97-011 } & Rev: \\
\hline $\begin{array}{l}\text { MILESTONE TYPE: } \\
\square \text { DOE-HQ } \\
\square \text { DOE-RL } \\
\text { X CONTRACTOR }\end{array}$ & $\begin{array}{l}\quad \text { DIVISION: } \\
\square \text { State } \\
\square \text { Federal } \\
\square \text { DOE } \\
\square \text { RCRA } \\
\square \text { TPA Number }\end{array}$ & $\begin{array}{l}\text { DELIVERABLE: } \\
\square \text { Report } \\
\text { X Letter } \\
\square \text { Drawings } \\
\square \text { Other (specify) }\end{array}$ & $\begin{array}{l}\text { ADDRESS TO: } \\
\square \text { DOE-HQ } \\
\text { X DOE-RL-OPI } \\
\square \text { Other (specify) }\end{array}$ \\
\hline \multicolumn{4}{|c|}{$\begin{array}{l}\text { Milestone Description: } \\
\text { Coordinate, compile and submit Westinghouse Hanford Company's biweekly Critical } \\
\text { Items Report to the RL Manager in accordance with letter from J. D. Wagoner, RL to } \\
\text { Contractors, dated March } 26,1991 \text {. }\end{array}$} \\
\hline \multicolumn{4}{|c|}{$\begin{array}{l}\text { Description of what constitutes completion of this milestone: } \\
\text { Delivery of the Critical Items Report to the RL Manager's Office by February 28, } 1997 .\end{array}$} \\
\hline \multicolumn{4}{|c|}{ Milestone Description Approval } \\
\hline \multicolumn{2}{|l|}{$\begin{array}{l}\text { Cost Account Manager } \\
\text { EA Schultz }\end{array}$} & \multicolumn{2}{|l|}{$\begin{array}{l}\text { Program/Project Manager } \\
\text { DB Pabst }\end{array}$} \\
\hline \multicolumn{2}{|c|}{$\begin{array}{l}\text { Program Element Manager } \\
\text { LR Hafer }\end{array}$} & \multicolumn{2}{|c|}{$\begin{array}{l}\text { DOE Program Manager Date } \\
\text { N/A }\end{array}$} \\
\hline \multicolumn{4}{|c|}{ 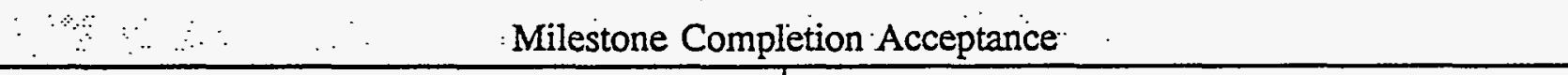 } \\
\hline \multicolumn{2}{|c|}{\begin{tabular}{c} 
Program Element Manager \\
\hdashline$\quad 1$ \\
\end{tabular}} & \multicolumn{2}{|l|}{$\begin{array}{l}\text { DOE Program Manager: } \\
\text { N/A } \\
\end{array}$} \\
\hline
\end{tabular}




\begin{tabular}{||l|l||}
\hline \multicolumn{3}{|c|}{ Westinghouse Hanford Company } \\
MILESTONE DESCRIPTION SHEET
\end{tabular}




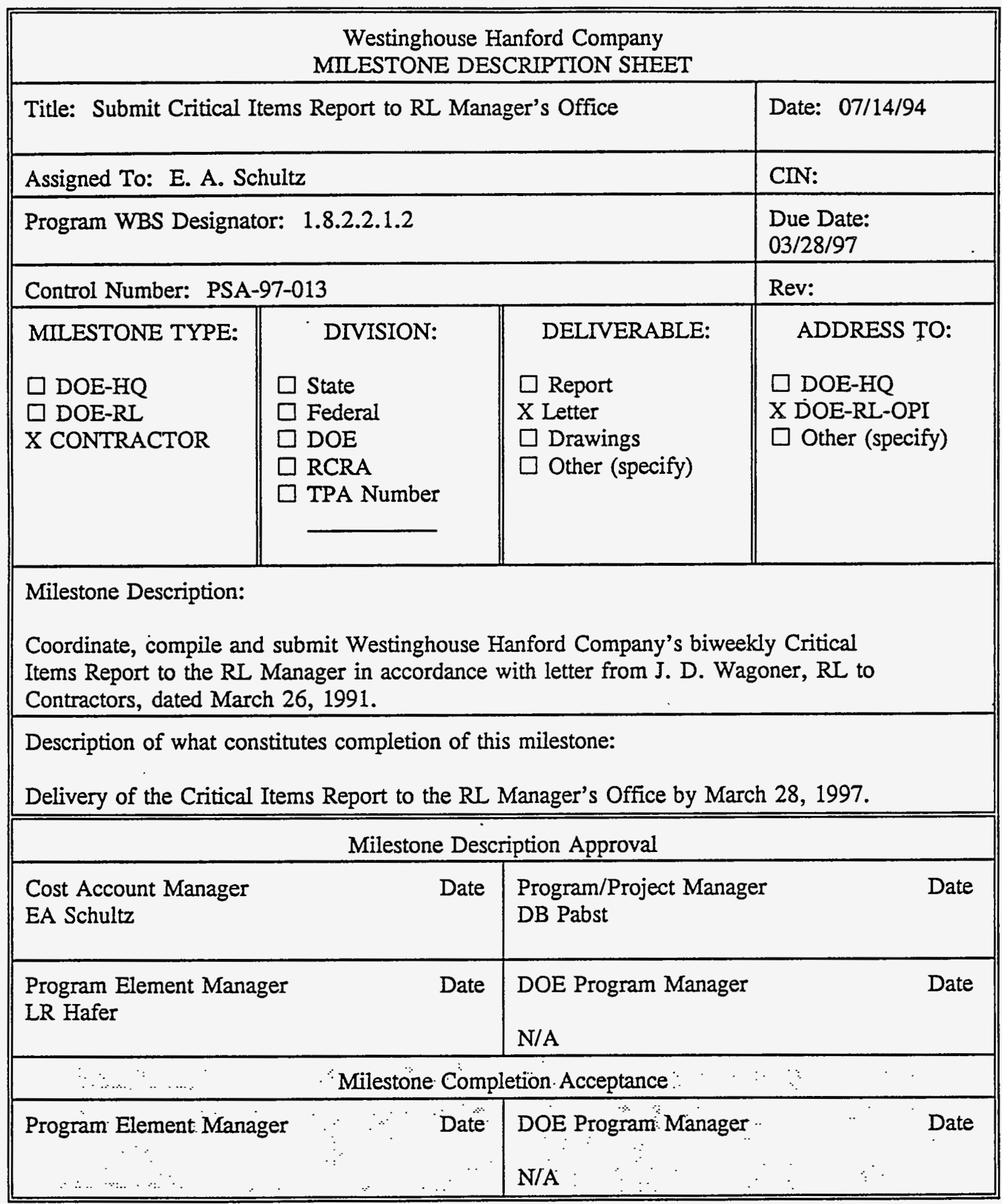




\begin{tabular}{||l|l||}
\hline \multicolumn{3}{|c||}{ Westinghouse Hanford Company } \\
MILESTONE DESCRIPTION SHEET
\end{tabular}




\begin{tabular}{|c|c|c|c|}
\hline \multicolumn{4}{|c|}{$\begin{array}{l}\text { Westinghouse Hanford Company } \\
\text { MILESTONE DESCRIPTION SHEET }\end{array}$} \\
\hline \multicolumn{3}{|c|}{ 'Title: Submit Critical Items Report to RL Manager's Office } & Date: $07 / 14 / 94$ \\
\hline \multicolumn{3}{|c|}{ Assigned To: E. A. Schultz } & CIN: \\
\hline \multicolumn{3}{|c|}{ Program WBS Designator: 1.8.2.2.1.2 } & $\begin{array}{l}\text { Due Date: } \\
04 / 25 / 97\end{array}$ \\
\hline \multicolumn{3}{|c|}{ Control Number: PSA-97-015. } & \multirow{2}{*}{$\begin{array}{l}\text { Rev: } \\
\text { ADDRESS TO: } \\
\square \text { DOE-HQ } \\
\text { X DOE-RL-OPI } \\
\square \text { Other (specify) }\end{array}$} \\
\hline $\begin{array}{l}\text { MILESTONE TYPE: } \\
\text { D DOE-HQ } \\
\square \text { DOE-RL } \\
\text { X CONTRACTOR }\end{array}$ & $\begin{array}{l}\quad \text { DIVISION: } \\
\square \text { State } \\
\square \text { Federal } \\
\square \text { DOE } \\
\square \text { RCRA } \\
\square \text { TPA Number }\end{array}$ & $\begin{array}{l}\text { DELIVERABLE: } \\
\square \text { Report } \\
\text { X Letter } \\
\square \text { Drawings } \\
\square \text { Other (specify) }\end{array}$ & \\
\hline \multicolumn{4}{|c|}{$\begin{array}{l}\text { Milestone Description: } \\
\text { Coordinate, compile and submit Westinghouse Hanford Company's biweekly Critical } \\
\text { Items Report to the RL Manager in accordance with letter from J. D. Wagoner, RL to } \\
\text { Contractors, dated March } 26,1991 \text {. }\end{array}$} \\
\hline \multicolumn{4}{|c|}{$\begin{array}{l}\text { Description of what constitutes completion of this milestone: } \\
\text { Delivery of the Critical Items Report to the RL Manager's Office by April 25, } 1997 .\end{array}$} \\
\hline \multicolumn{4}{|c|}{ Milestone Description Approval } \\
\hline \multicolumn{2}{|l|}{$\begin{array}{l}\text { Cost Account Manager } \\
\text { EA Schultz }\end{array}$} & \multicolumn{2}{|l|}{$\begin{array}{l}\text { Program/Project Manager } \\
\text { DB Pabst }\end{array}$} \\
\hline \multicolumn{2}{|c|}{$\begin{array}{l}\text { Program Element Manager } \\
\text { LR Hafer }\end{array}$} & \multicolumn{2}{|l|}{$\begin{array}{l}\text { DOE Program Manager } \\
\text { N/A }\end{array}$} \\
\hline \multicolumn{4}{|c|}{ Milestone Completion Acceptance } \\
\hline $\begin{array}{c}\text { Program Element Man } \\
\begin{array}{c}\because \\
\because\end{array}\end{array}$ & ser: $: \ldots$ & $\begin{array}{c}\text { DOE Program Manager } \\
\\
\text { N/A } \\
\text { N }\end{array}$ & Date \\
\hline
\end{tabular}




\begin{tabular}{|c|c|c|c|}
\hline \multicolumn{4}{|c|}{$\begin{array}{l}\text { Westinghouse Hanford Company } \\
\text { MILESTONE DESCRIPTION SHEET }\end{array}$} \\
\hline \multicolumn{3}{|c|}{ Title: Submit Critical Items Report to RL Manager's Office } & Date: $07 / 14 / 94$ \\
\hline \multicolumn{3}{|c|}{ Assigned To: E. A. Schultz } & CIN: \\
\hline \multicolumn{3}{|c|}{ Program WBS Designator: 1 8.2.2.1.2 } & $\begin{array}{l}\text { Due Date: } \\
\text { 05/09/97 }\end{array}$ \\
\hline \multicolumn{3}{|c|}{ Control Number: PSA-97-016 } & Rev: \\
\hline $\begin{array}{l}\text { MILESTONE TYPE: } \\
\square \text { DOE-HQ } \\
\square \text { DOE-RL } \\
\text { X CONTRACTOR }\end{array}$ & $\begin{array}{l}\quad \text { DIVISION: } \\
\square \text { State } \\
\square \text { Federal } \\
\square \text { DOE } \\
\square \text { RCRA } \\
\square \text { TPA Number }\end{array}$ & $\begin{array}{l}\text { DELIVERABLE: } \\
\square \text { Report } \\
\text { X Letter } \\
\square \text { Drawings } \\
\square \text { Other (specify) }\end{array}$ & $\begin{array}{l}\text { ADDRESS TO: } \\
\square \text { DOE-HQ } \\
\text { X DOE-RL-OPI } \\
\square \text { Other (specify) }\end{array}$ \\
\hline \multicolumn{4}{|c|}{$\begin{array}{l}\text { Milestone Description: } \\
\text { Coordinate, compile and submit Westinghouse Hanford Company's biweekly Critical } \\
\text { Items Report to the RL Manager in accordance with letter from J. D. Wagoner, RL to } \\
\text { Contractors, dated March } 26,1991 \text {. }\end{array}$} \\
\hline \multicolumn{4}{|c|}{$\begin{array}{l}\text { Description of what constitutes completion of this milestone: } \\
\text { Delivery of the Critical Items Report to the RL Manager's Office by May 9, } 1997 .\end{array}$} \\
\hline \multicolumn{4}{|c|}{ Milestone Description Approval } \\
\hline \multicolumn{2}{|l|}{$\begin{array}{l}\text { Cost Account Manager } \\
\text { EA Schultz }\end{array}$} & $\begin{array}{l}\text { Program/Project Manager } \\
\text { DB Pabst }\end{array}$ & Date \\
\hline \multicolumn{2}{|c|}{$\begin{array}{l}\text { Program Element Manager } \\
\text { LR Hafer }\end{array}$} & $\begin{array}{l}\text { DOE Program Manager } \\
\text { N/A }\end{array}$ & Date \\
\hline \multicolumn{4}{|c|}{ 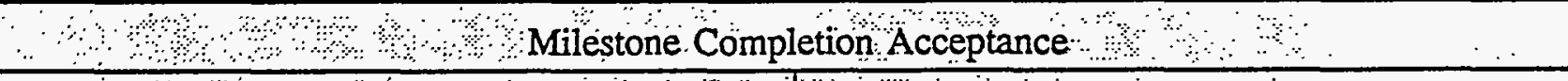 } \\
\hline \multicolumn{2}{|c|}{ Program Element Manager } & $\begin{array}{l}\mathrm{DOE} \text { Program Manager } \\
\mathrm{N} / \mathrm{A}\end{array}$ & $\because$ \\
\hline
\end{tabular}




\begin{tabular}{||l|l||}
\hline \multicolumn{3}{|c||}{ Westinghouse Hanford Company } \\
MILESTONE DESCRIPTION SHEET
\end{tabular}




\begin{tabular}{||l||l||}
\hline \multicolumn{3}{|c|}{ Westinghouse Hanford Company } \\
MILESTONE DESCRIPTION SHEET
\end{tabular}




\begin{tabular}{|c|c|c|c|c|}
\hline \multicolumn{5}{|c|}{$\begin{array}{l}\text { Westinghouse Hanford Company } \\
\text { MILESTONE DESCRIPTION SHEET }\end{array}$} \\
\hline \multicolumn{4}{|c|}{ Title: Submit Critical Items Report to RL Manager's Office } & Date: $07 / 14 / 94$ \\
\hline \multicolumn{4}{|c|}{ Assigned To: E. A. Schultz } & CIN: \\
\hline \multicolumn{4}{|c|}{ Program WBS Designator: 1 8.2.2.1.2 } & $\begin{array}{l}\text { Due Date: } \\
06 / 20 / 97\end{array}$ \\
\hline \multicolumn{4}{|c|}{ Control Number: PSA-97-019 } & Rev: \\
\hline $\begin{array}{l}\text { MILESTONE TYPE: } \\
\square \text { DOE-HQ } \\
\square \text { DOE-RL } \\
\text { X CONTRACTOR }\end{array}$ & $\begin{array}{l}\square \\
\square \\
\square 1 \\
\square 1 \\
\square 1 \\
\square \\
\square\end{array}$ & & $\begin{array}{l}\text { DELIVERABLE: } \\
\square \text { Report } \\
\text { X Letter } \\
\square \text { Drawings } \\
\square \text { Other (specify) }\end{array}$ & $\begin{array}{l}\text { ADDRESS TO: } \\
\square \text { DOE-HQ } \\
\text { X DOE-RL-OPI } \\
\square \text { Other (specify) }\end{array}$ \\
\hline \multicolumn{5}{|c|}{$\begin{array}{l}\text { Milestone Description: } \\
\text { Coordinate, compile and submit Westinghouse Hanford Company's biweekly Critical } \\
\text { Items Report to the RL Manager in accordance with letter from J. D. Wagoner, RL to } \\
\text { Contractors, dated March 26, } 1991 \text {. }\end{array}$} \\
\hline \multicolumn{5}{|c|}{$\begin{array}{l}\text { Description of what constitutes completion of this milestone: } \\
\text { Delivery of the Critical Items Report to the RL Manager's Office by June 20, } 1997 .\end{array}$} \\
\hline \multicolumn{5}{|c|}{ Milestone Description Approval } \\
\hline \multicolumn{3}{|l|}{$\begin{array}{l}\text { Cost Account Manager } \\
\text { EA Schultz }\end{array}$} & \multicolumn{2}{|c|}{$\begin{array}{l}\text { Program/Project Manager } \\
\text { DB Pabst }\end{array}$} \\
\hline \multicolumn{3}{|c|}{$\begin{array}{l}\text { Program Element Manager } \\
\text { LR Hafer }\end{array}$} & \multicolumn{2}{|c|}{$\begin{array}{l}\text { DOE Program Manager Date } \\
\text { N/A }\end{array}$} \\
\hline \multicolumn{5}{|c|}{$\cdots \quad$ Milestone Completion Acceptance: } \\
\hline \multicolumn{3}{|c|}{$\begin{array}{cccc}\text { Program Element Manager } & & & \text { Date } \\
\cdots & & & \ddots \\
\ddots & \cdots & \ddots & \ddots \\
\end{array}$} & \multicolumn{2}{|l|}{$\begin{array}{l}\text { DOE Program Manager } \\
\text { N/A. }\end{array}$} \\
\hline
\end{tabular}




\begin{tabular}{||l|l||}
\hline \multicolumn{3}{|c|}{ Westinghouse Hanford Company } \\
MILESTONE DESCRIPTION SHEET
\end{tabular}




\begin{tabular}{|c|c|c|c|}
\hline \multicolumn{4}{|c|}{$\begin{array}{l}\text { Westinghouse Hanford Company } \\
\text { MILESTONE DESCRIPTION SHEET }\end{array}$} \\
\hline \multicolumn{3}{|c|}{ Title: Submit Critical Items Report to RL Manager's Office } & Date: $07 / 14 / 94$ \\
\hline \multicolumn{3}{|c|}{ Assigned To: E. A. Schuitz } & CIN: \\
\hline \multicolumn{3}{|c|}{ Program WBS Designator: 1 8.2.2.1.2 } & $\begin{array}{l}\text { Due Date: } \\
07 / 18 / 97\end{array}$ \\
\hline \multicolumn{3}{|c|}{ Control Number: PSA-97-021 } & Rev: \\
\hline $\begin{array}{l}\text { MILESTONE TYPE: } \\
\square \text { DOE-HQ } \\
\square \text { DOE-RL } \\
\text { X CONTRACTOR }\end{array}$ & $\begin{aligned} & \text { DIVISION: } \\
& \square \text { State } \\
& \square \text { Federal } \\
& \square \text { DOE } \\
& \quad \text { RCRA } \\
& \square \text { TPA Number }\end{aligned}$ & $\begin{array}{l}\text { DELIVERABLE: } \\
\square \text { Report } \\
\text { X Letter } \\
\square \text { Drawings } \\
\square \text { Other (specify) }\end{array}$ & $\begin{array}{l}\text { ADDRESS TO: } \\
\square \text { DOE-HQ } \\
\text { X DOE-RL-OPI } \\
\square \text { Other (specify) }\end{array}$ \\
\hline \multicolumn{4}{|c|}{$\begin{array}{l}\text { Milestone Description: } \\
\text { Coordinate, compile and submit Westinghouse Hanford Company's biweekly Critical } \\
\text { Items Report to the RL Manager in accordance with letter from J. D. Wagoner, RL to } \\
\text { Contractors, dated March } 26,1991 \text {. }\end{array}$} \\
\hline \multicolumn{4}{|c|}{$\begin{array}{l}\text { Description of what constitutes completion of this milestone: } \\
\text { Delivery of the Critical Items Report to the RL Manager's Office by July } 18,1997 .\end{array}$} \\
\hline \multicolumn{4}{|c|}{ Milestone Description Approval } \\
\hline \multicolumn{2}{|l|}{$\begin{array}{l}\text { Cost Account Manager } \\
\text { EA Schultz }\end{array}$} & \multicolumn{2}{|l|}{$\begin{array}{l}\text { Program/Project Manager } \\
\text { DB Pabst }\end{array}$} \\
\hline \multicolumn{2}{|c|}{$\begin{array}{l}\text { Program Element Manager } \\
\text { LR Hafer }\end{array}$} & \multicolumn{2}{|c|}{$\begin{array}{l}\text { DOE Program Manager Date } \\
\text { N/A }\end{array}$} \\
\hline \multicolumn{4}{|c|}{ - $\quad \ldots \quad$ Milestone Completion Acceptance } \\
\hline Program Element Mana & er $\quad$ Date & $\begin{array}{l}\text { DOE Program Manager } \\
\text { N/A. }\end{array}$ & Date \\
\hline
\end{tabular}




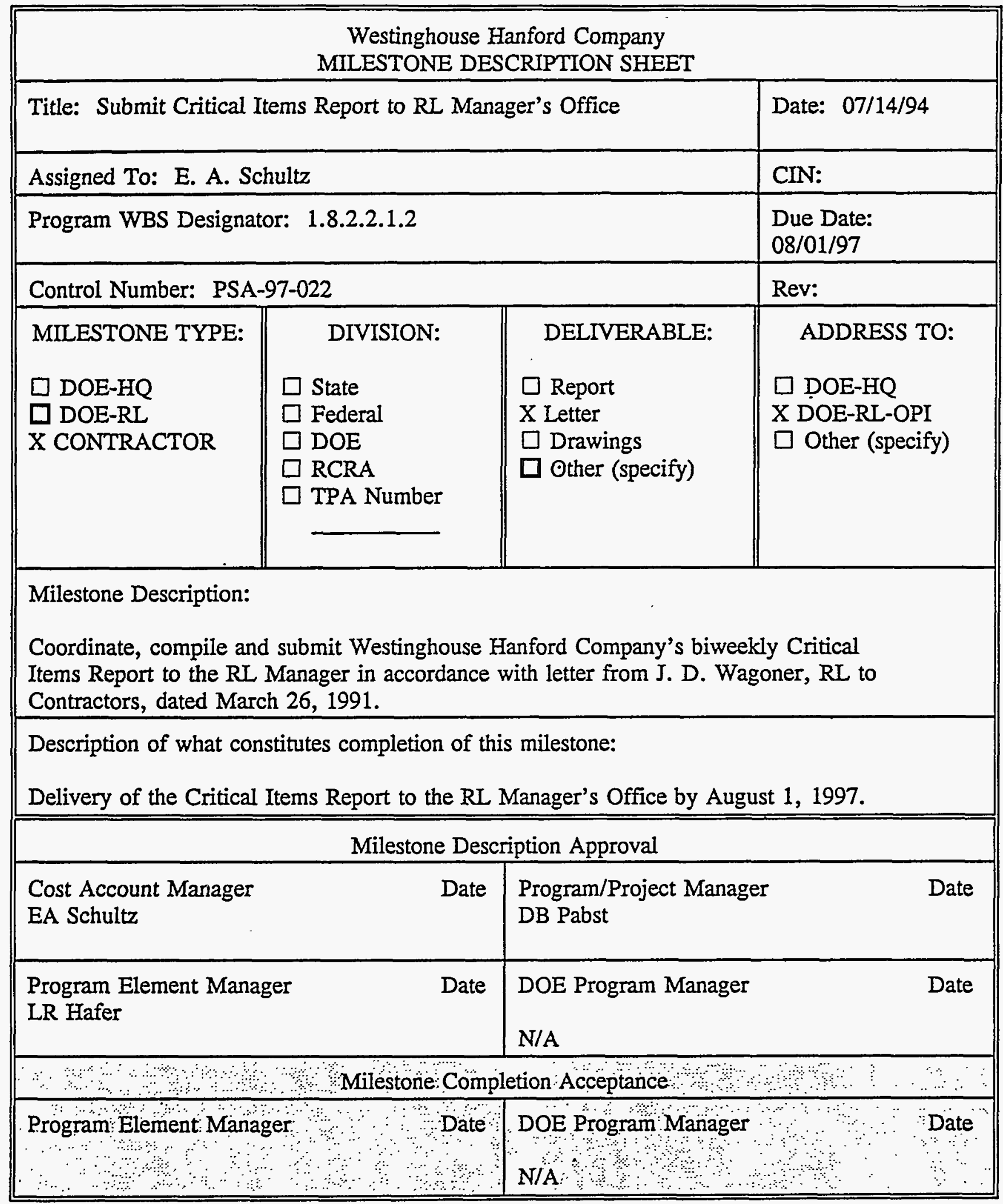




\begin{tabular}{|c|c|c|c|}
\hline \multicolumn{4}{|c|}{$\begin{array}{l}\text { Westinghouse Hanford Company } \\
\text { MILESTONE DESCRIPTION SHEET }\end{array}$} \\
\hline \multicolumn{3}{|c|}{ Title: Submit Critical Items Report to RL Manager's Office } & Date: $07 / 14 / 94$ \\
\hline \multicolumn{3}{|c|}{ Assigned To: E. A. Schultz } & CIN: \\
\hline \multicolumn{3}{|c|}{ Program WBS Designator: 1 8.2.2.1.2 } & $\begin{array}{l}\text { Due Pate: } \\
08 / 15 / 97 \\
\end{array}$ \\
\hline \multicolumn{3}{|c|}{ Control Number: PSA-97-023 } & Rev: \\
\hline $\begin{array}{l}\text { MILESTONE TYPE: } \\
\square \text { DOE-HQ } \\
\square \text { DOE-RL } \\
\text { X CONTRACTOR }\end{array}$ & $\begin{aligned} & \text { DIVISION: } \\
& \square \text { State } \\
& \square \text { Federal } \\
& \square \text { DOE } \\
& \quad \text { RCRA } \\
& \square \text { TPA Number }\end{aligned}$ & $\begin{array}{l}\text { DELIVERABLE: } \\
\square \text { Report } \\
\text { X Letter } \\
\square \text { Drawings } \\
\square \text { Other (specify) }\end{array}$ & $\begin{array}{l}\text { ADDRESS TO: } \\
\square \text { DOE-HQ } \\
\text { X DOE-RL-OPI } \\
\square \text { Other (specify) }\end{array}$ \\
\hline \multicolumn{4}{|c|}{$\begin{array}{l}\text { Milestone Description: } \\
\text { Coordinate, compile and submit Westinghouse Hanford Company's biweekly Critical } \\
\text { Items Report to the RL Manager in accordance with letter from J. D. Wagoner, RL to } \\
\text { Contractors, dated March } 26,1991 \text {. }\end{array}$} \\
\hline \multicolumn{4}{|c|}{$\begin{array}{l}\text { Description of what constitutes completion of this milestone: } \\
\text { Delivery of the Critical Items Report to the RL Manager's Office by August 15, } 1997 .\end{array}$} \\
\hline \multicolumn{4}{|c|}{ Milestone Description Approval } \\
\hline \multicolumn{2}{|l|}{$\begin{array}{l}\text { Cost Account Manager } \\
\text { EA Schultz }\end{array}$} & \multicolumn{2}{|l|}{$\begin{array}{l}\text { Program/Project Manager } \\
\text { DB Pabst }\end{array}$} \\
\hline \multicolumn{2}{|c|}{$\begin{array}{l}\text { Program Element Manager } \\
\text { LR Hafer }\end{array}$} & \multicolumn{2}{|l|}{$\begin{array}{l}\text { DOE Program Manager } \\
\text { N/A }\end{array}$} \\
\hline \multicolumn{4}{|c|}{ a $:-$ Milestone Completion Acceptance } \\
\hline 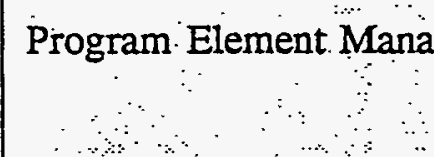 & ger Date & $\begin{array}{l}\text { DOE Program Manager } \\
\text { N/A }\end{array}$ & Date \\
\hline
\end{tabular}




\begin{tabular}{||l|l||}
\hline \multicolumn{3}{|c||}{ Westinghouse Hanford Company } \\
MILESTONE DESCRIPTION SHEET
\end{tabular}




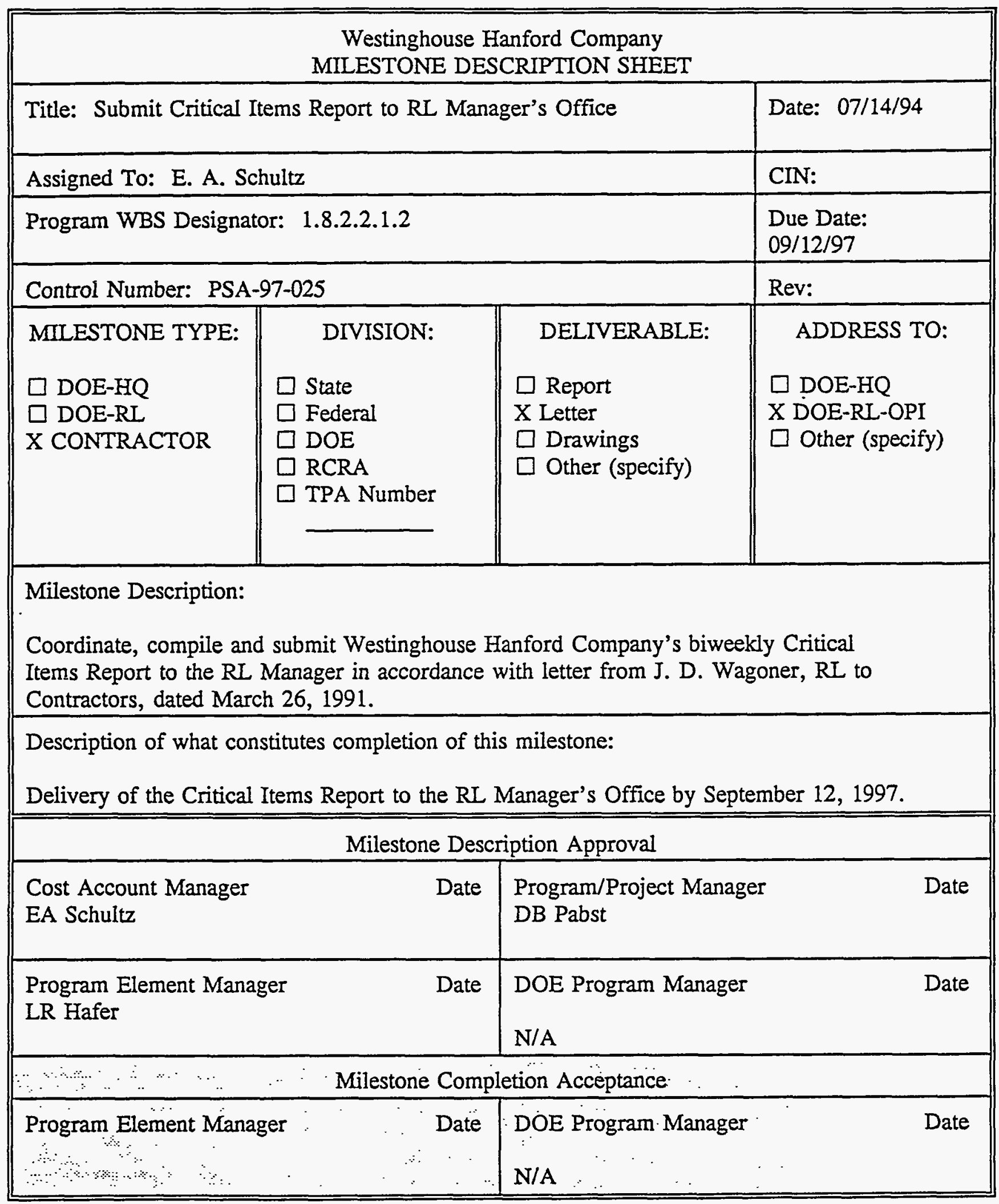




\begin{tabular}{|l|l|l||}
\hline \multicolumn{3}{|c|}{ Westinghouse Hanford Company } \\
MILESTONE DESCRIPTION SHEET
\end{tabular}




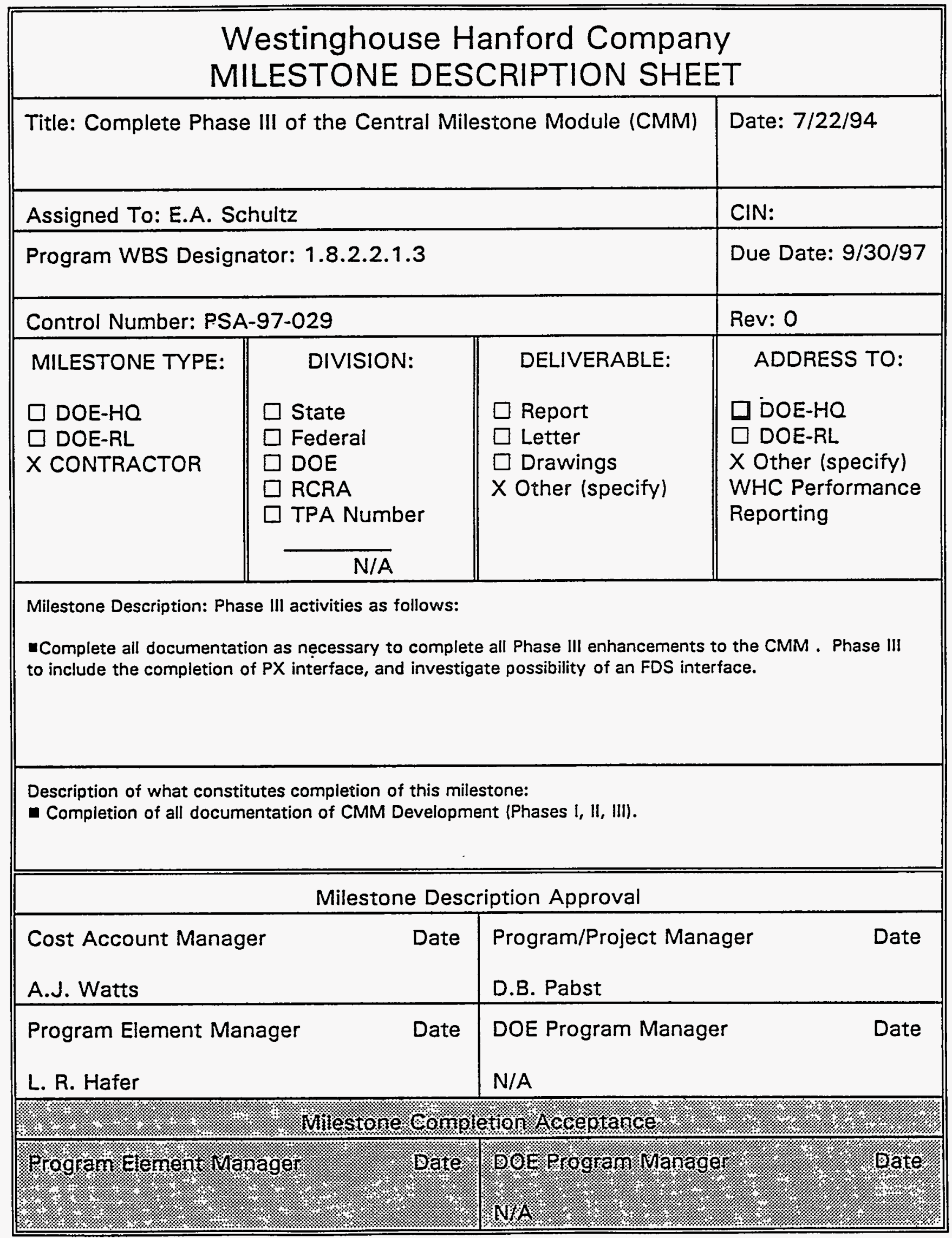




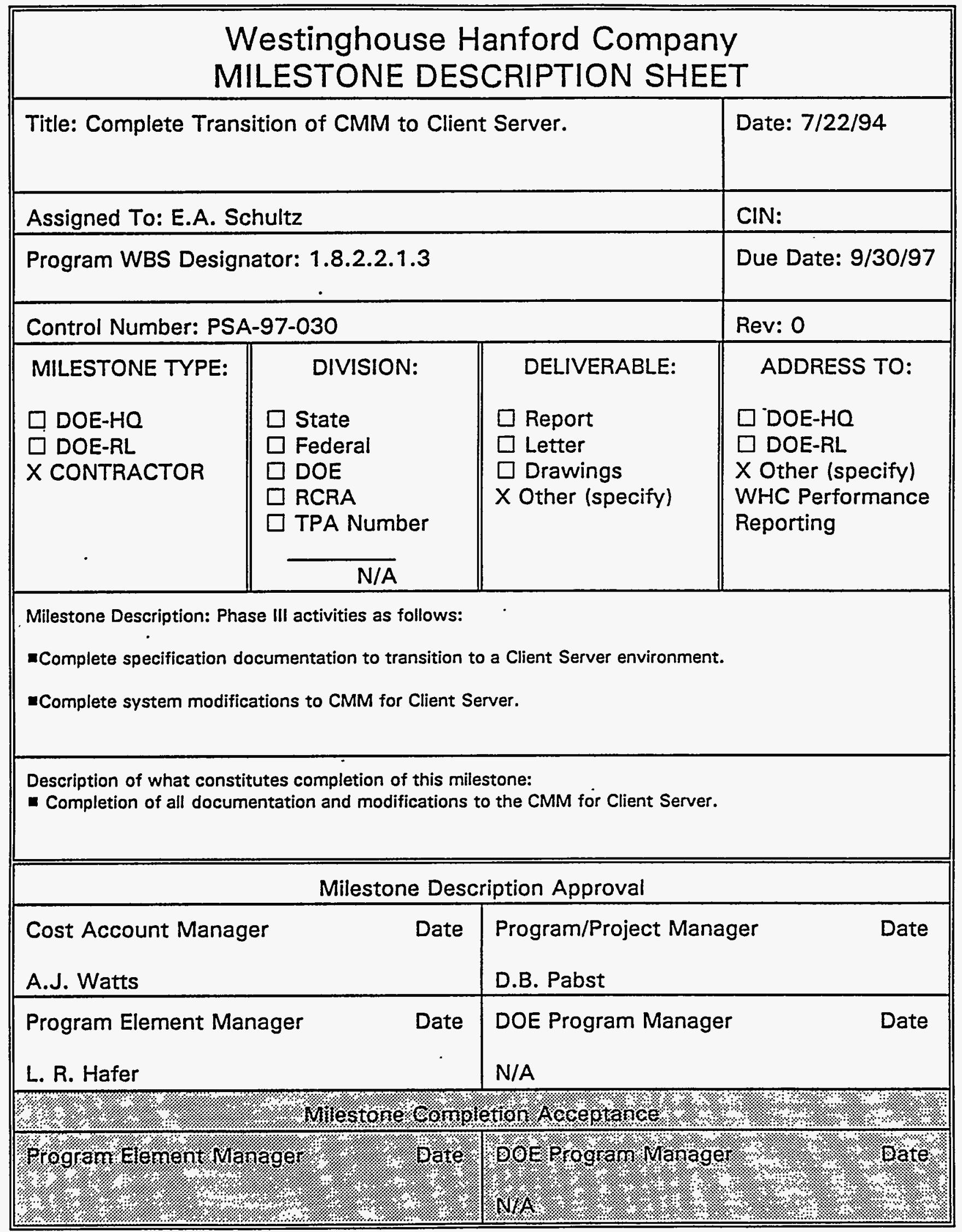




\begin{tabular}{|c|c|c|}
\hline \multicolumn{3}{|c|}{$\begin{array}{l}\text { Westinghouse Hanford Company } \\
\text { MILESTONE DESCRIPTION SHEET }\end{array}$} \\
\hline \multicolumn{2}{|c|}{ Title: Submit monthly PTS Report to RL } & e: $06 / 09 / 94$ \\
\hline \multicolumn{2}{|c|}{ Assigned To: E.A. Schultz } & \\
\hline \multicolumn{2}{|c|}{ Program WBS Designator: 1 8.2.2.1.1 } & $\begin{array}{l}\text { e Date: } \\
01 / 96\end{array}$ \\
\hline \multicolumn{2}{|c|}{ Control Number: PSA-97-035 } & \\
\hline $\begin{array}{l}\text { MILESTONE TYPE: } \\
\square \text { DOE-HQ } \\
\text { X DOE-RL } \\
\square \text { CONTRACTOR }\end{array}$ & $\begin{array}{l}\square s \\
\square I \\
\square I \\
\square I \\
\square ?\end{array}$ & $\begin{array}{l}\text { ADDRESS TO: } \\
\text { DOE-HQ } \\
\text { JOE-RL OPI } \\
\text { Other (specify) }\end{array}$ \\
\hline \multicolumn{3}{|c|}{$\begin{array}{l}\text { Milestone Description: Transmit electronically to DOE-HQ monthly PTS report no } \\
\text { later than the first working day of each month and deliver hard copy of PTS } \\
\text { reports to RL no later than the second working day of each month per RLP 5000.8. }\end{array}$} \\
\hline \multicolumn{3}{|c|}{$\begin{array}{l}\text { Description of what constitutes completion of this milestone: Transmission electronically to } \\
\text { DOE-HQ by October } 1,1996 \text {. }\end{array}$} \\
\hline \multicolumn{3}{|c|}{ Milestone Description Approval. } \\
\hline \multicolumn{2}{|c|}{ 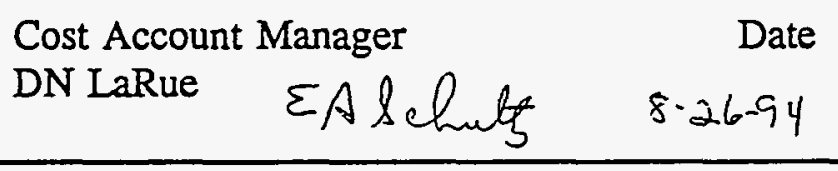 } & $\begin{array}{l}\text { Program/Project Manager } \\
\text { DB Pabst }\end{array}$ \\
\hline \multicolumn{2}{|c|}{$\begin{array}{l}\text { Program Element Manager } \\
\text { LR Hafer Lerof }\end{array}$} & Date \\
\hline \multicolumn{3}{|c|}{ 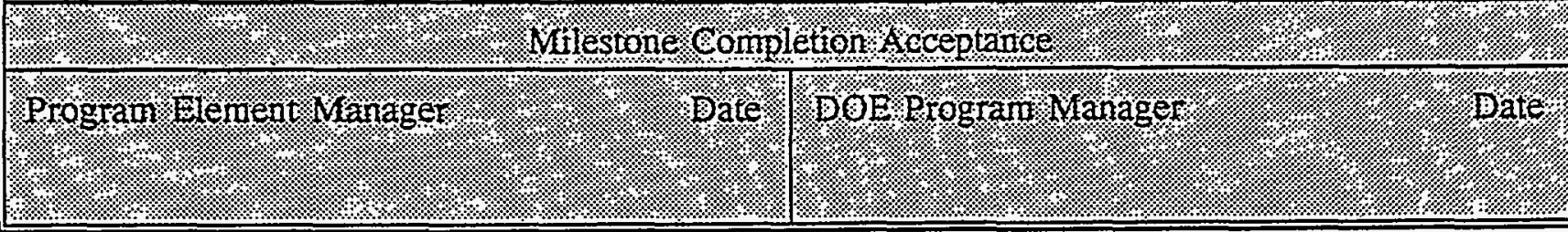 } \\
\hline
\end{tabular}




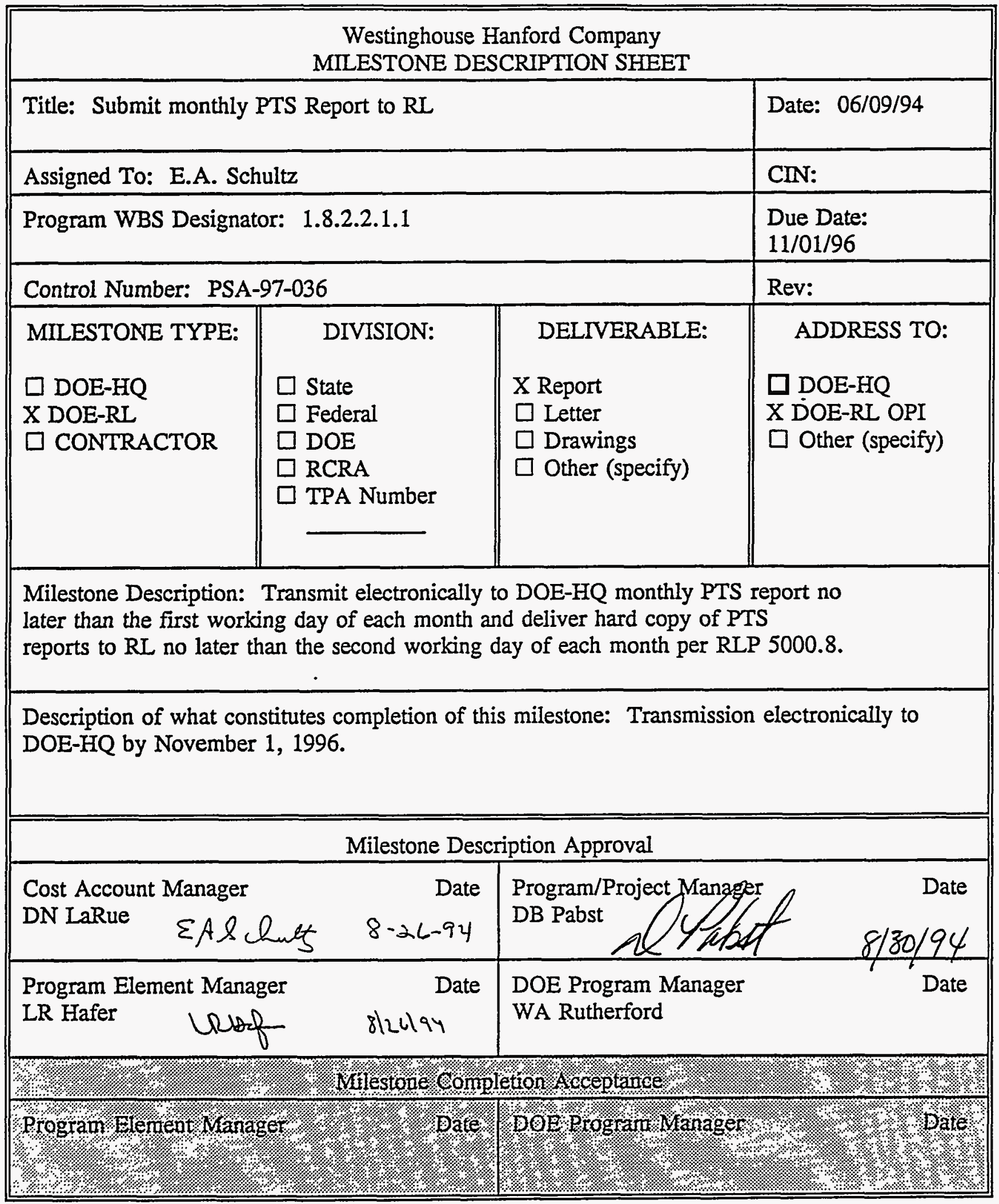




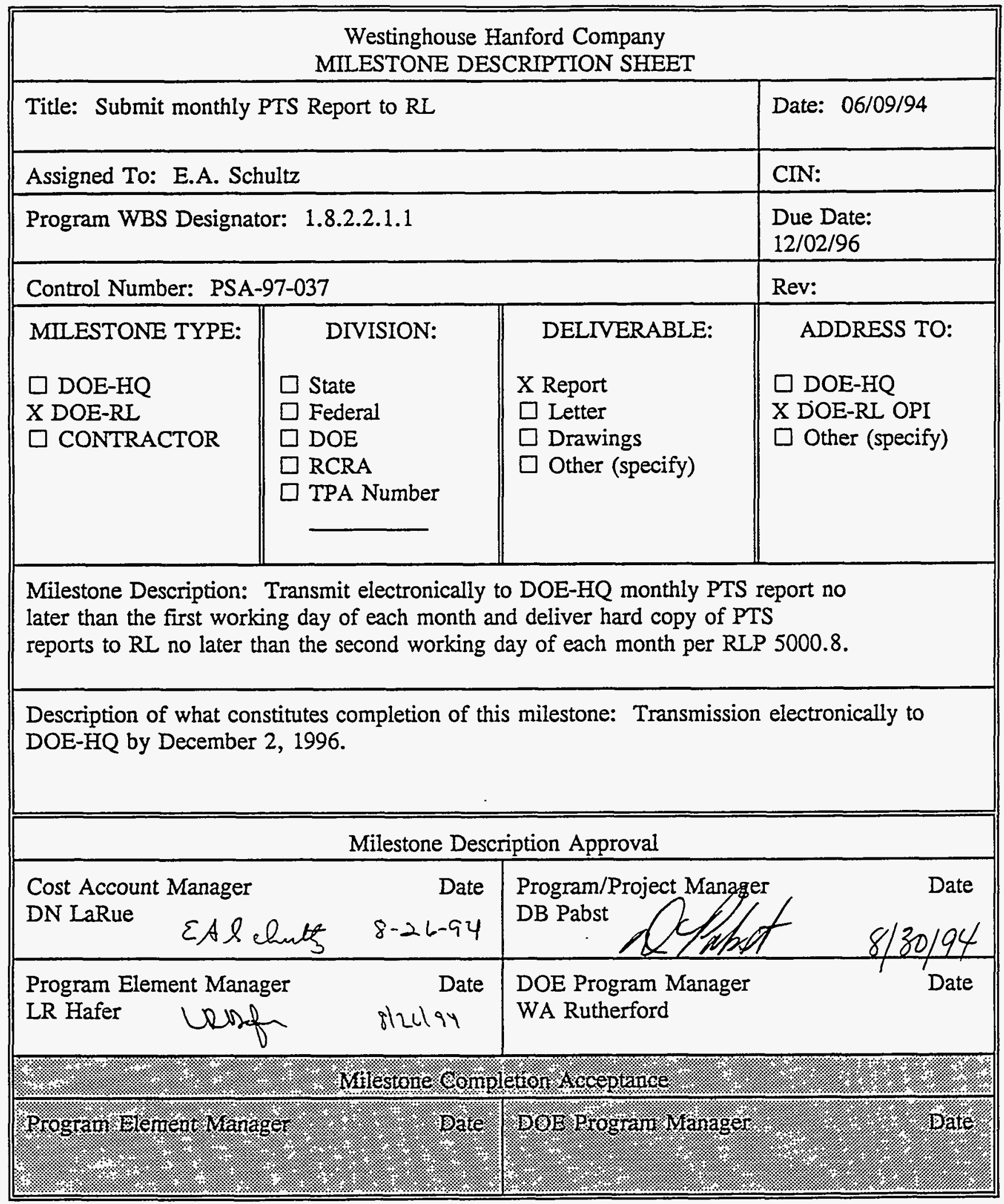




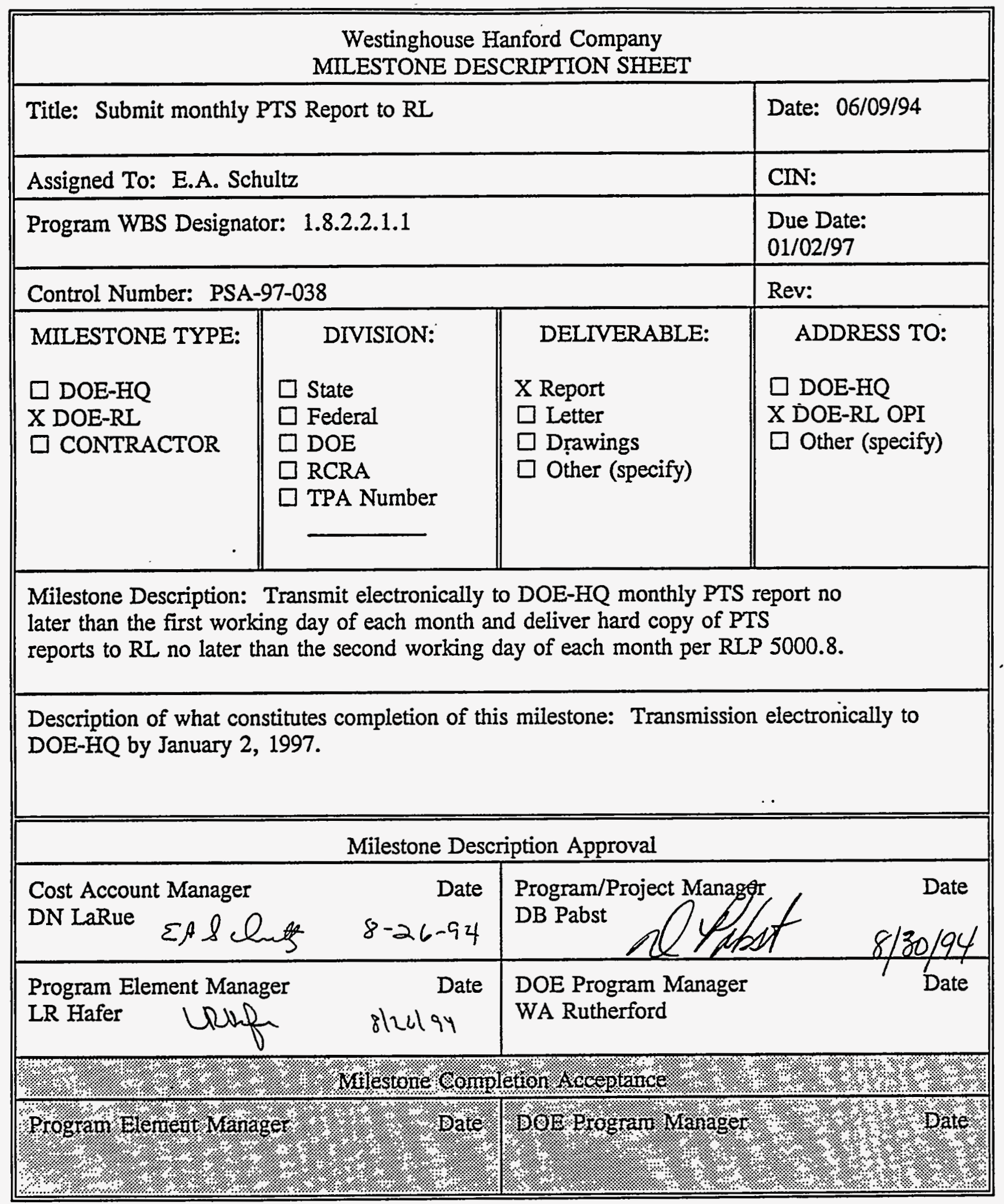




\begin{tabular}{|l|l|l||}
\hline \multicolumn{3}{|c||}{ Westinghouse Hanford Company } \\
MILESTNE DESCRIPTION SHEET
\end{tabular}




\begin{tabular}{||l|l||}
\hline \multicolumn{3}{|c|}{ Westinghouse Hanford Company } \\
MILESTONE DESCRIPTION SHEET
\end{tabular}




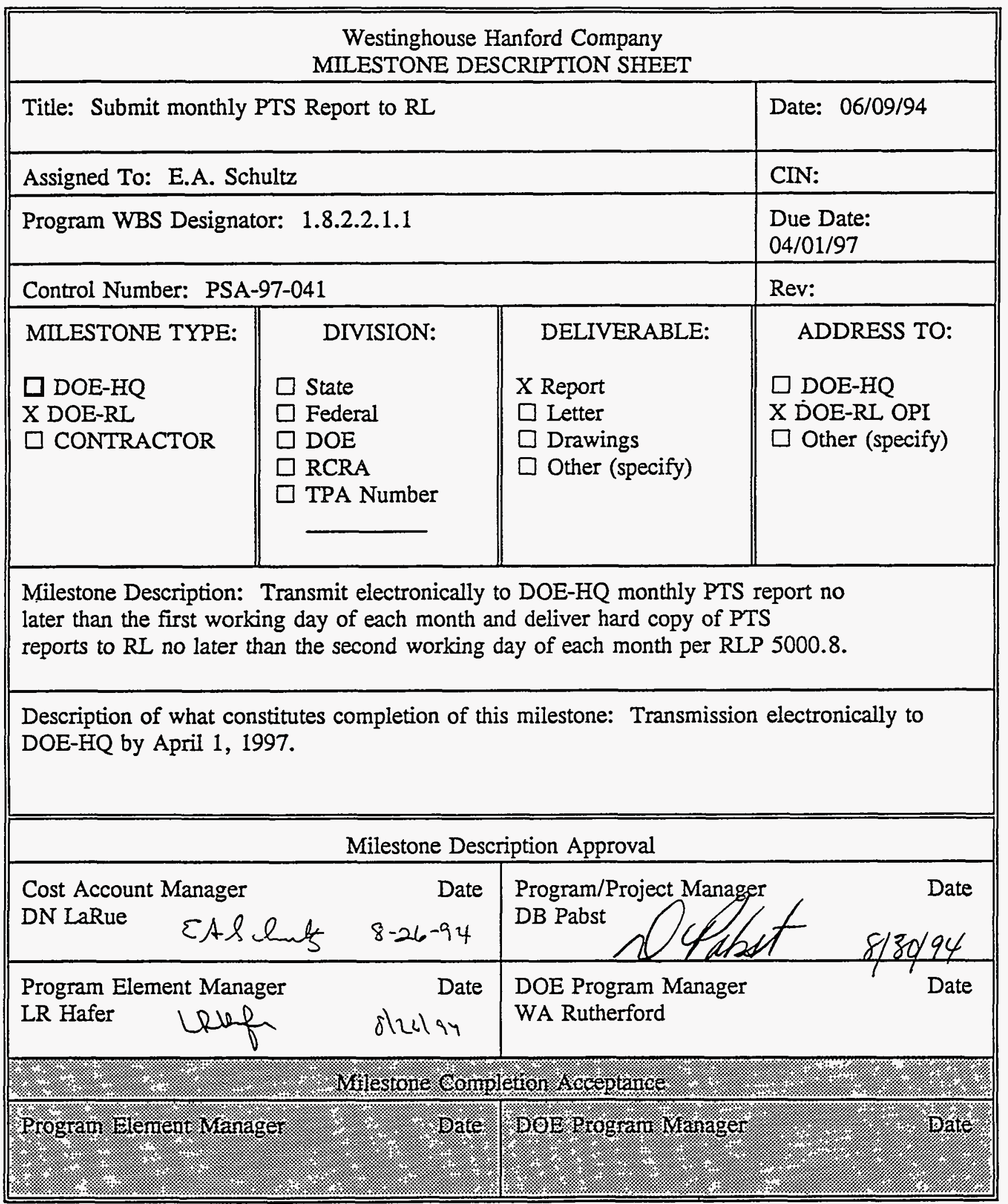




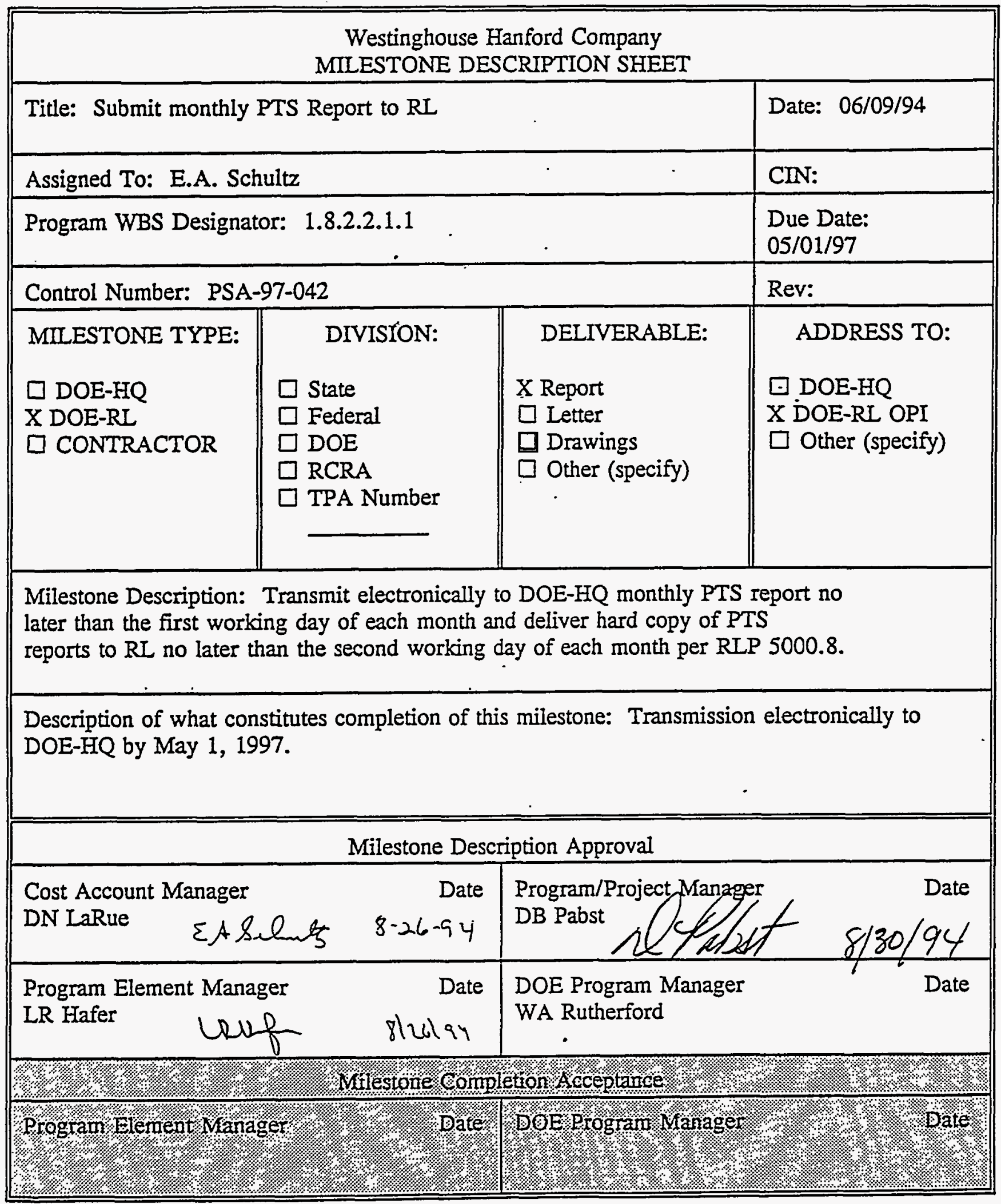




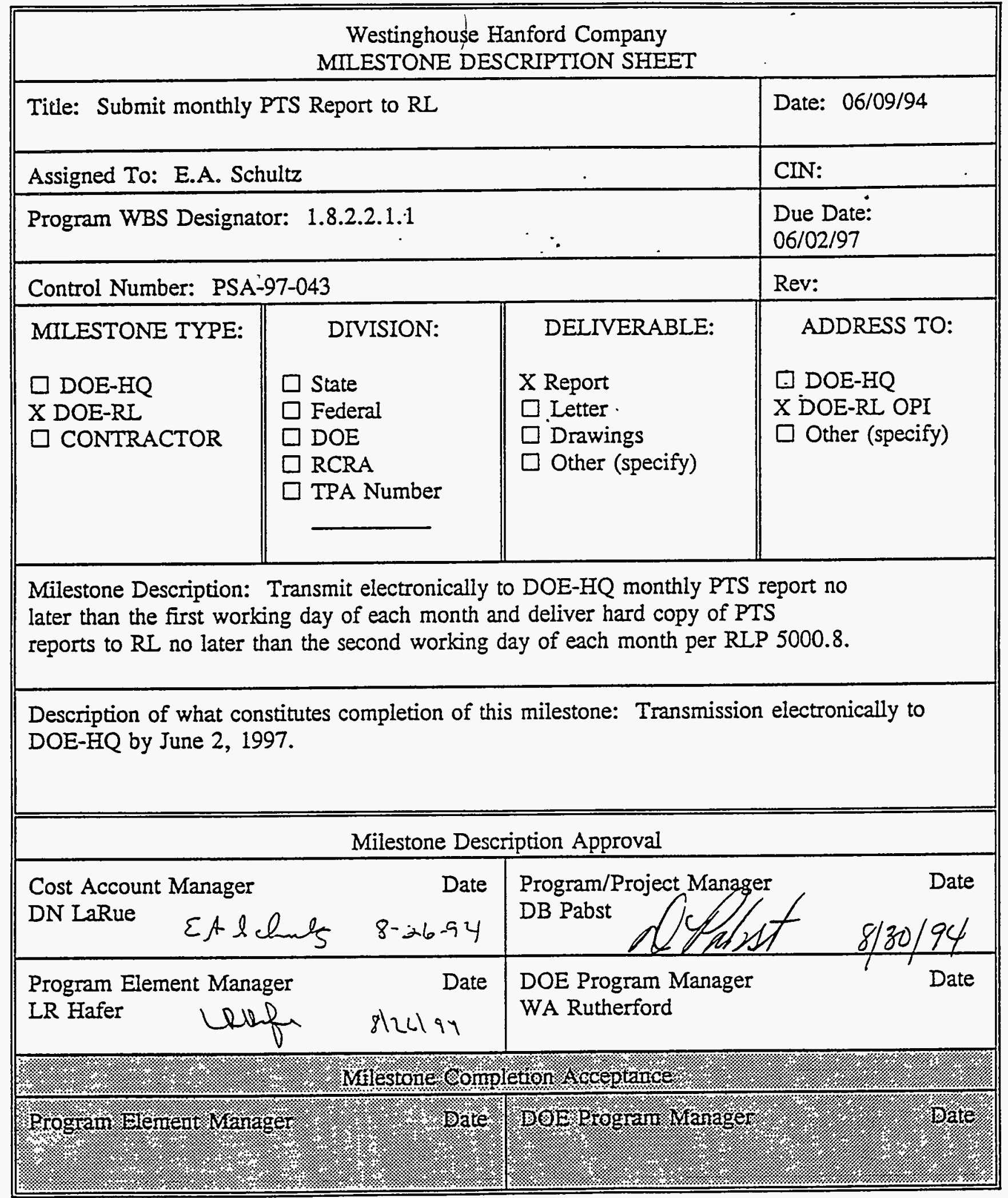




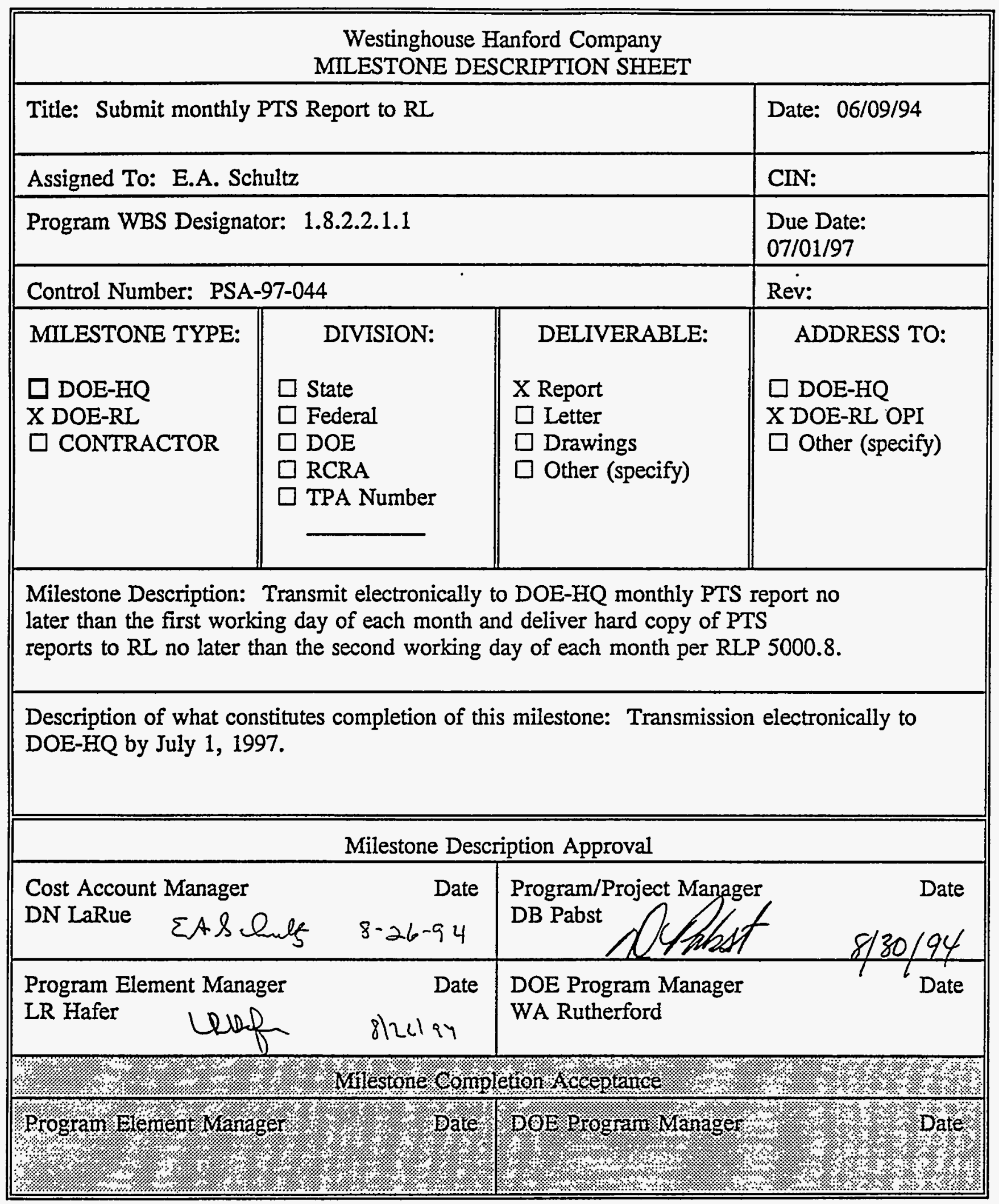




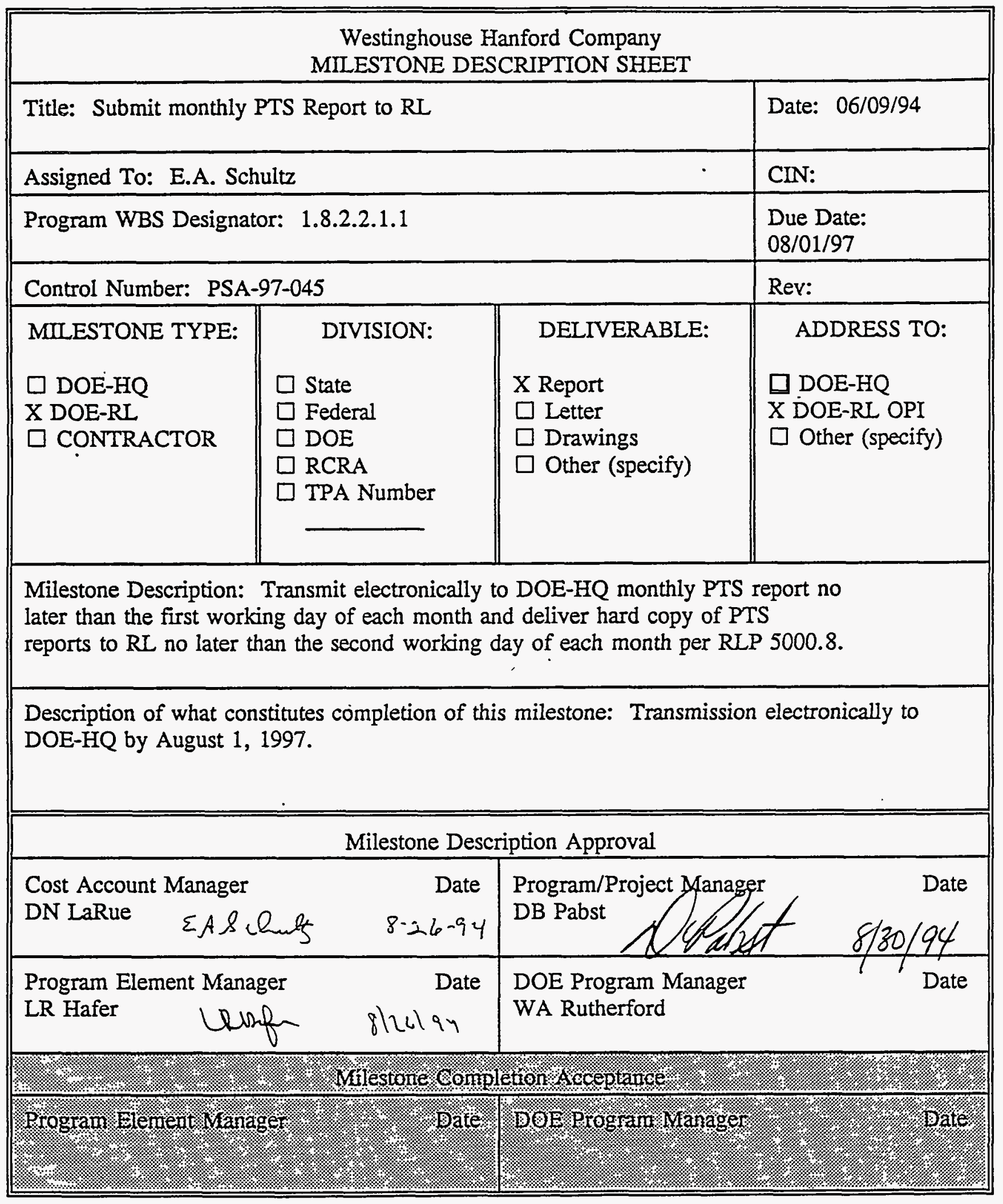




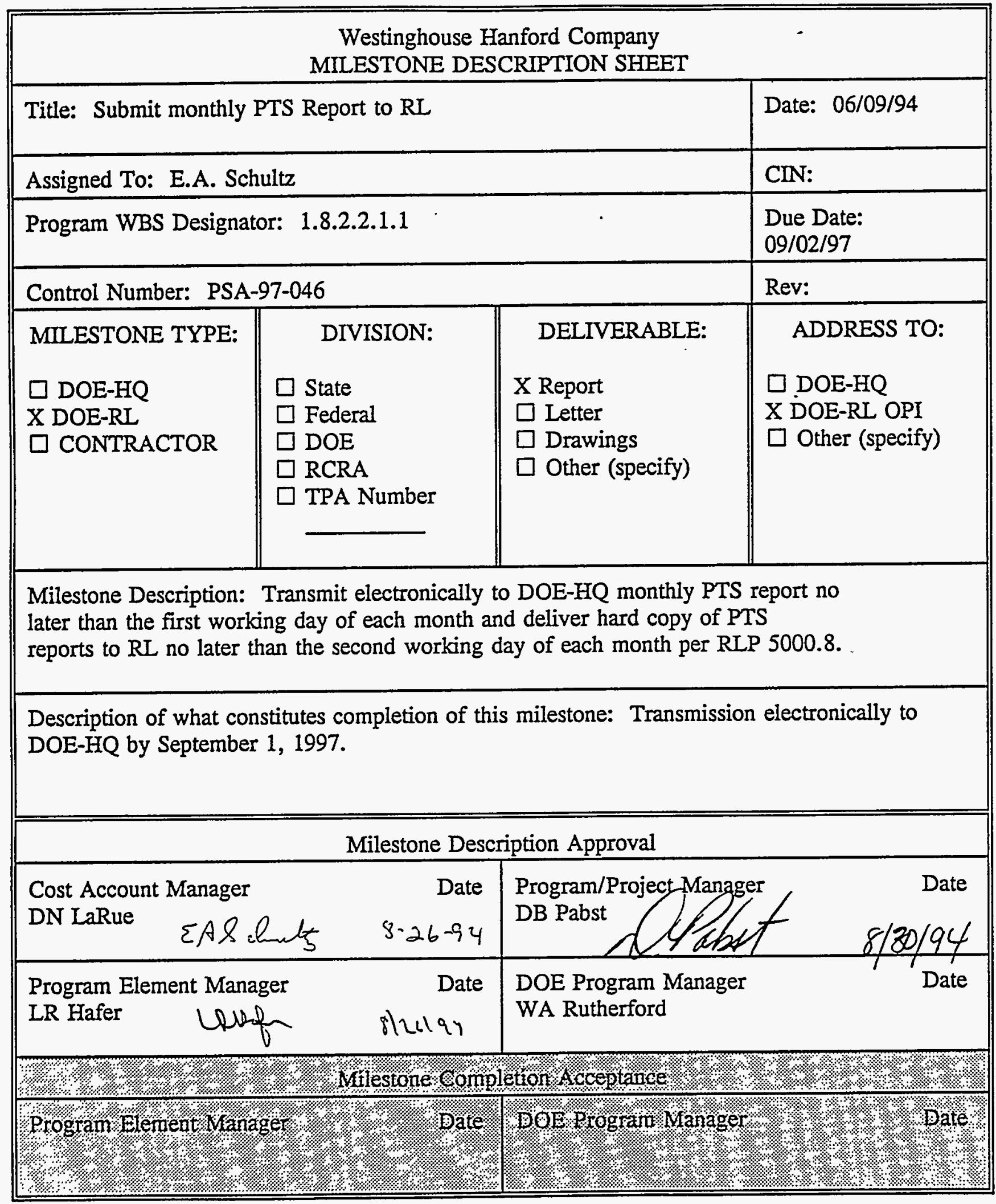




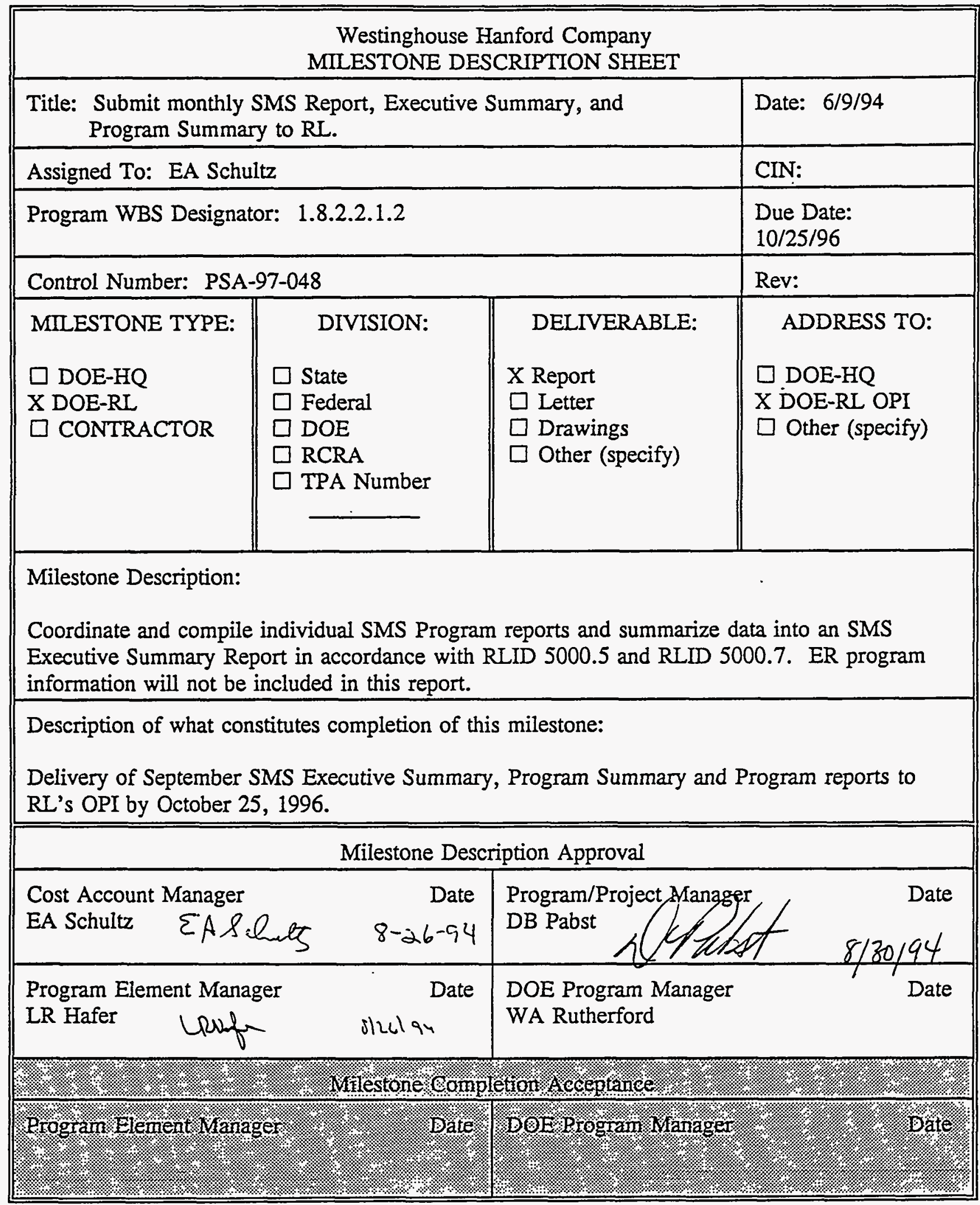




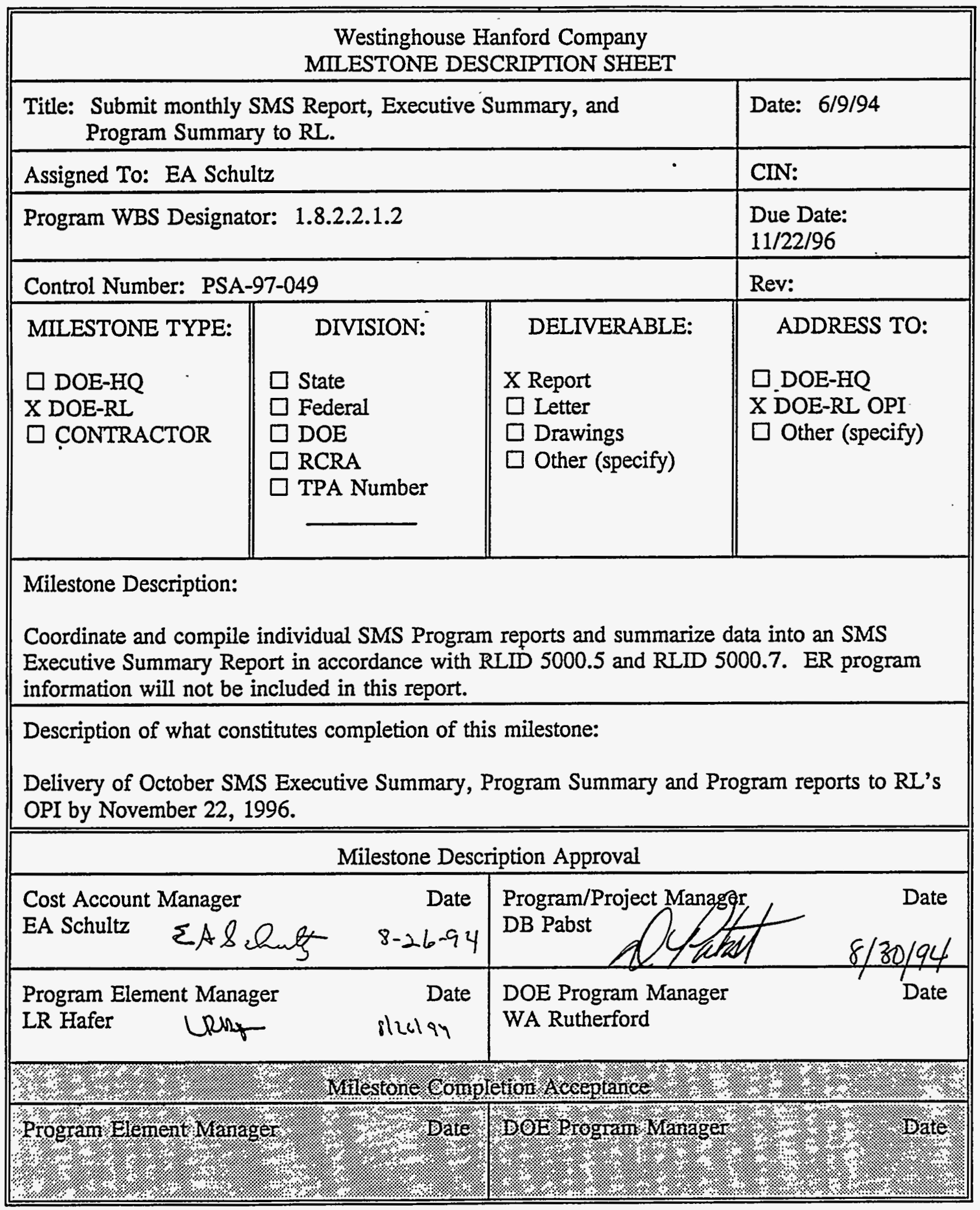




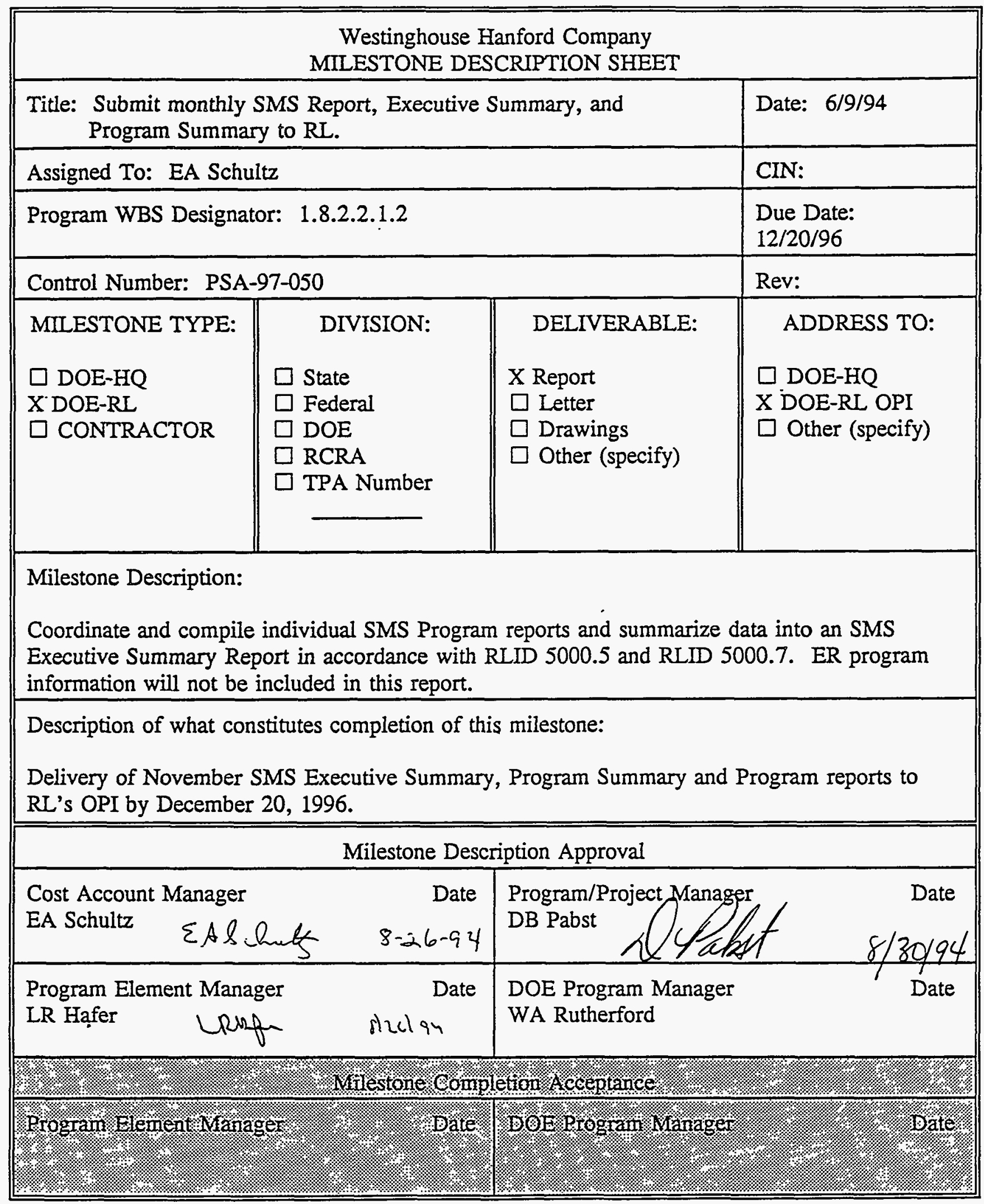




\begin{tabular}{|l|l|l||}
\hline \multicolumn{3}{|c||}{ Westinghouse Hanford Company } \\
MILESTONE DESCRIPTION SHEET
\end{tabular}




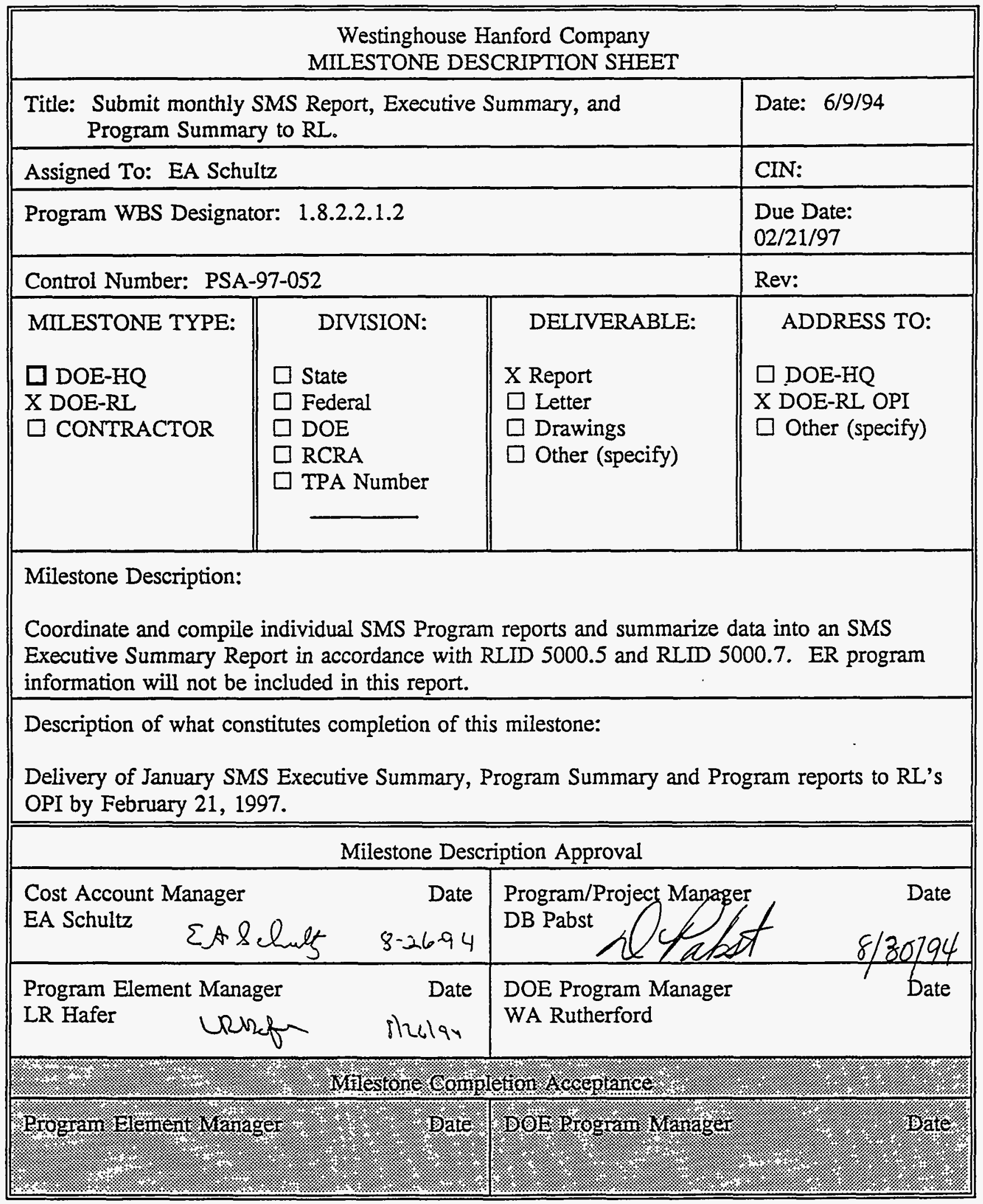




\begin{tabular}{||l|l|l||}
\hline \multicolumn{3}{|c|}{ Westinghouse Hanford Company } \\
MILESTONE DESCRIPTION SHEET
\end{tabular}




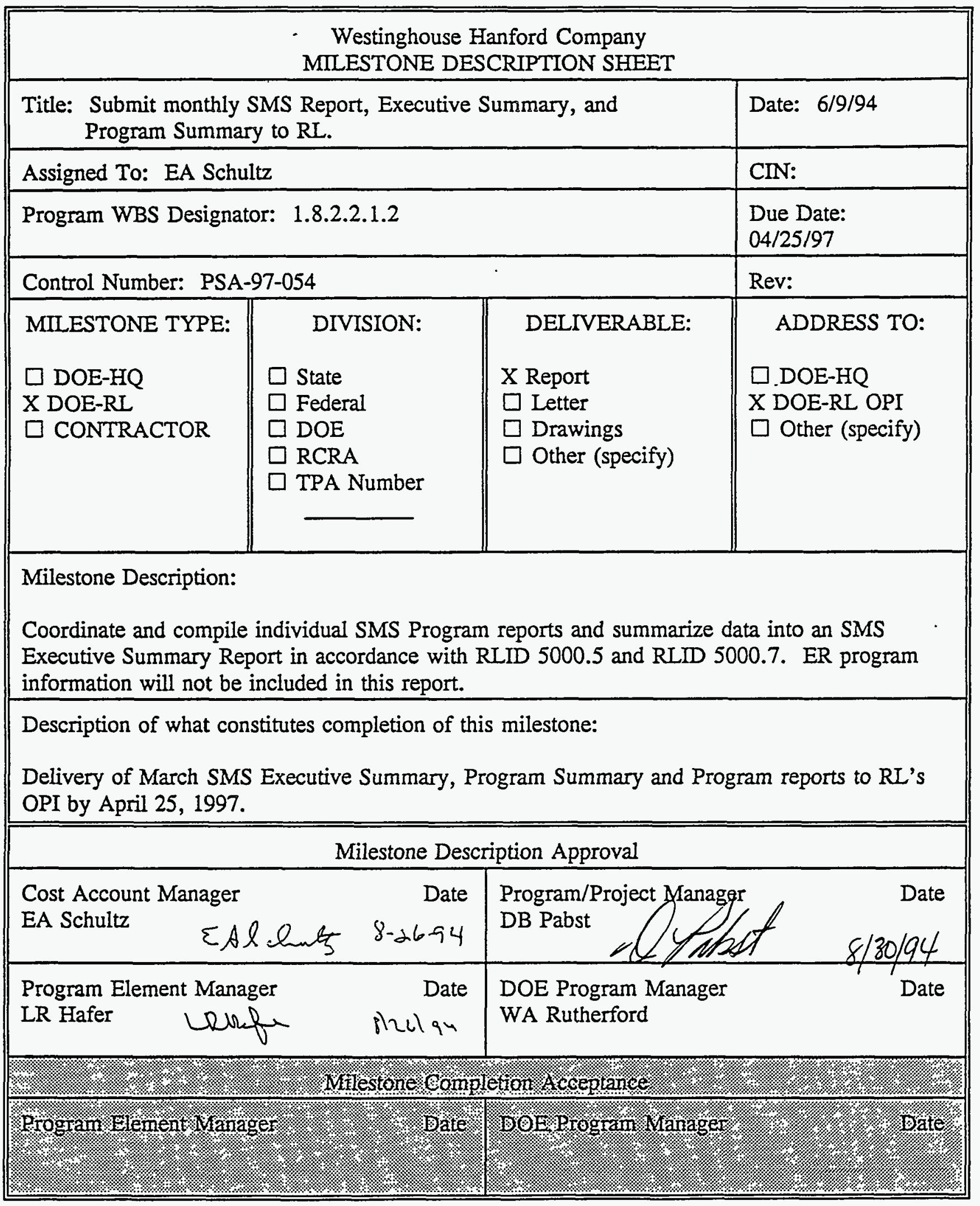




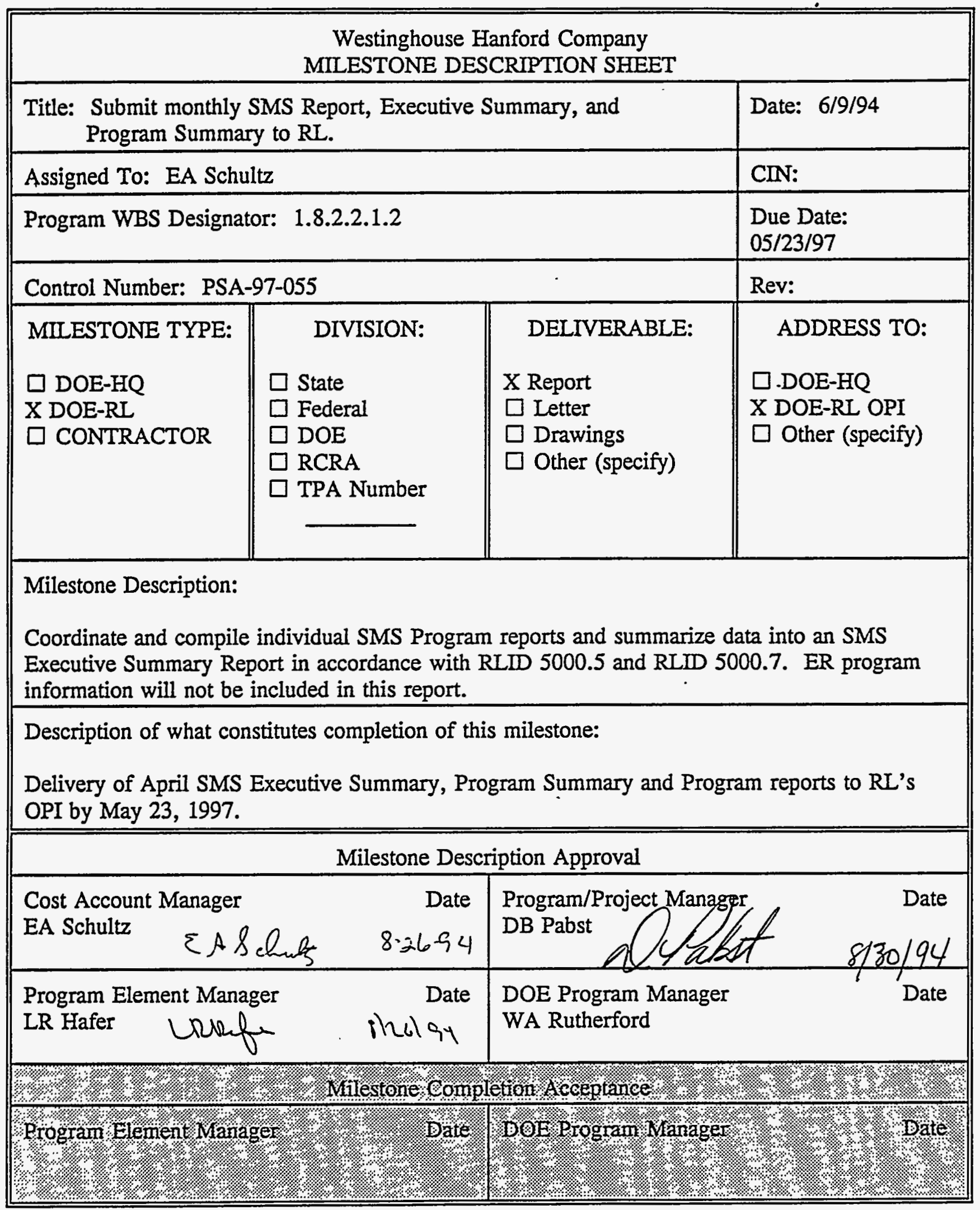




\begin{tabular}{|l||l|l||}
\hline \multicolumn{3}{|c|}{ Westinghouse Hanford Company } \\
MILESTONE DESCRIPTION SHEET
\end{tabular}




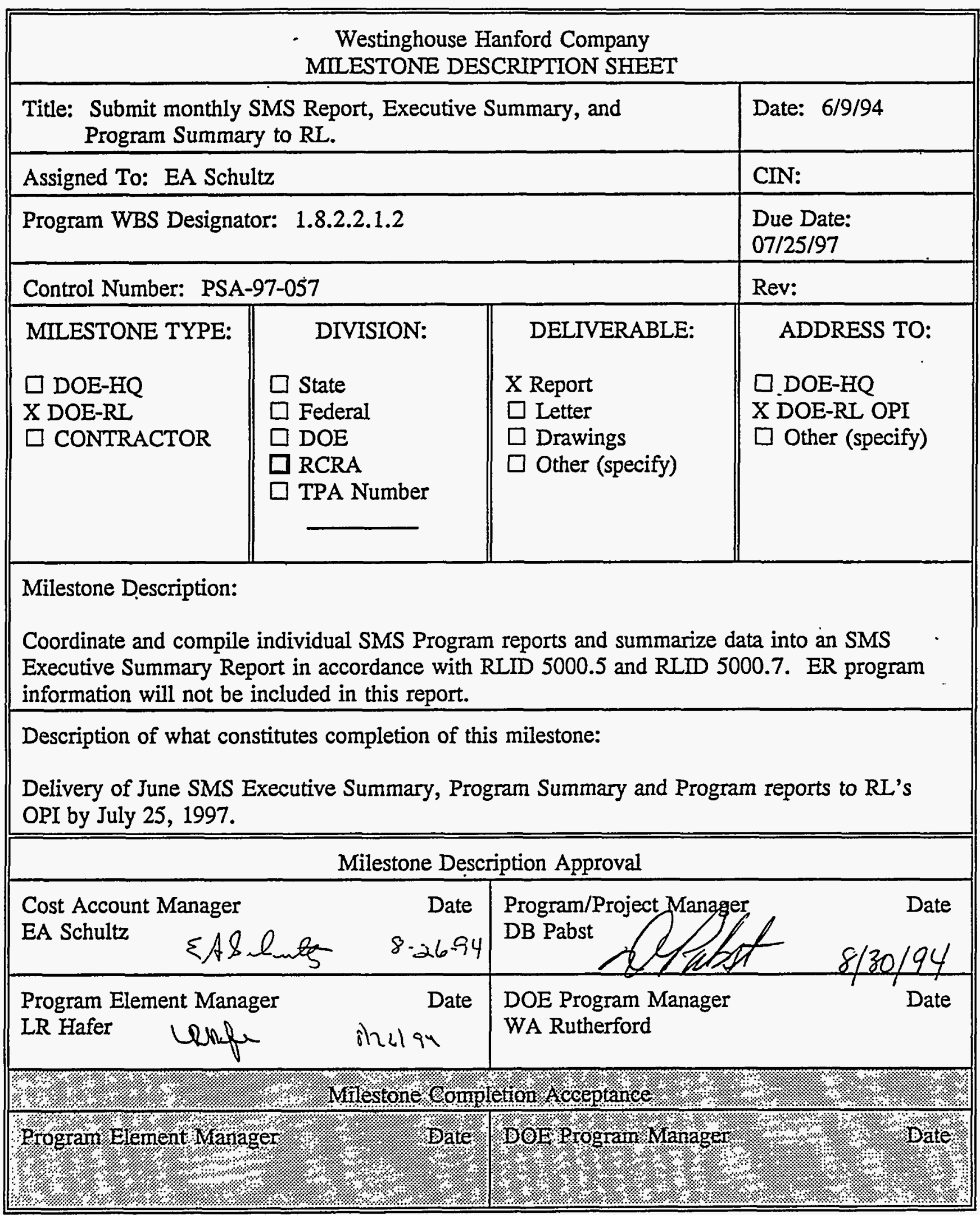




\begin{tabular}{|l||l|l||}
\hline \multicolumn{3}{|c||}{ Westinghouse Hanford Company } \\
MILESTONE DESCRIPTION SHEET
\end{tabular}




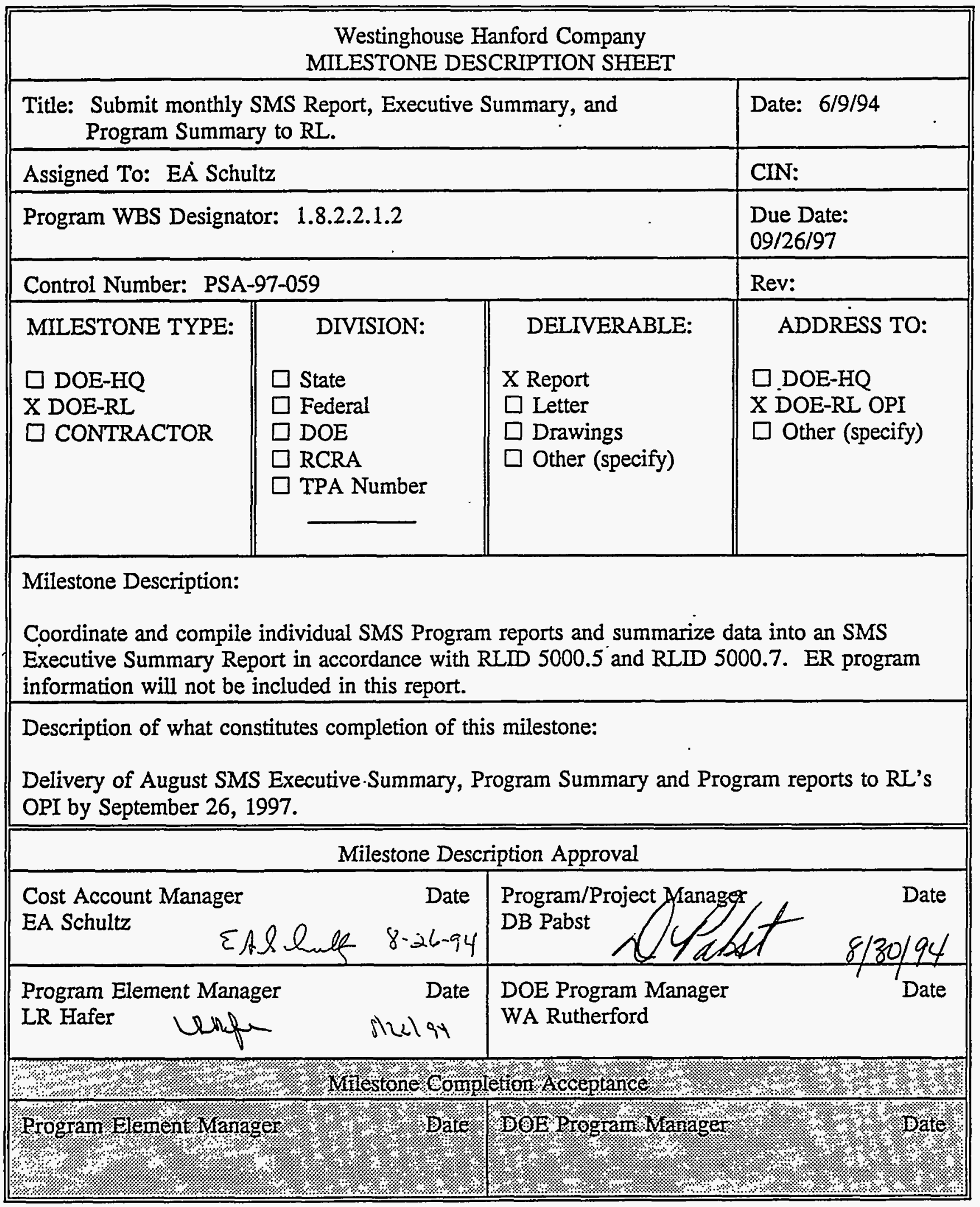




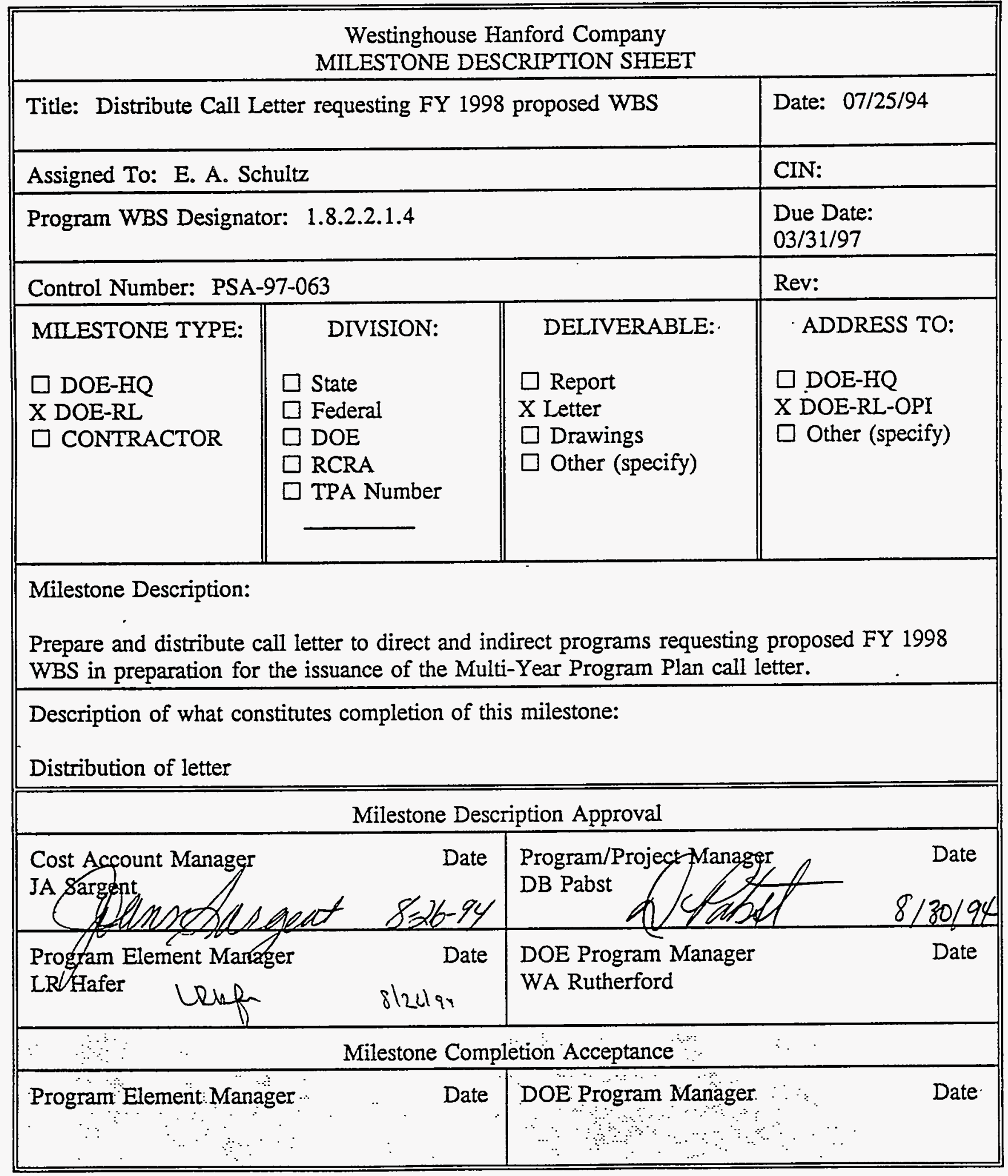




\begin{tabular}{|l||l|l||}
\hline \multicolumn{3}{|c|}{ Westinghouse Hanford Company } \\
MILESTONE DESCRIPTION SHEET
\end{tabular}




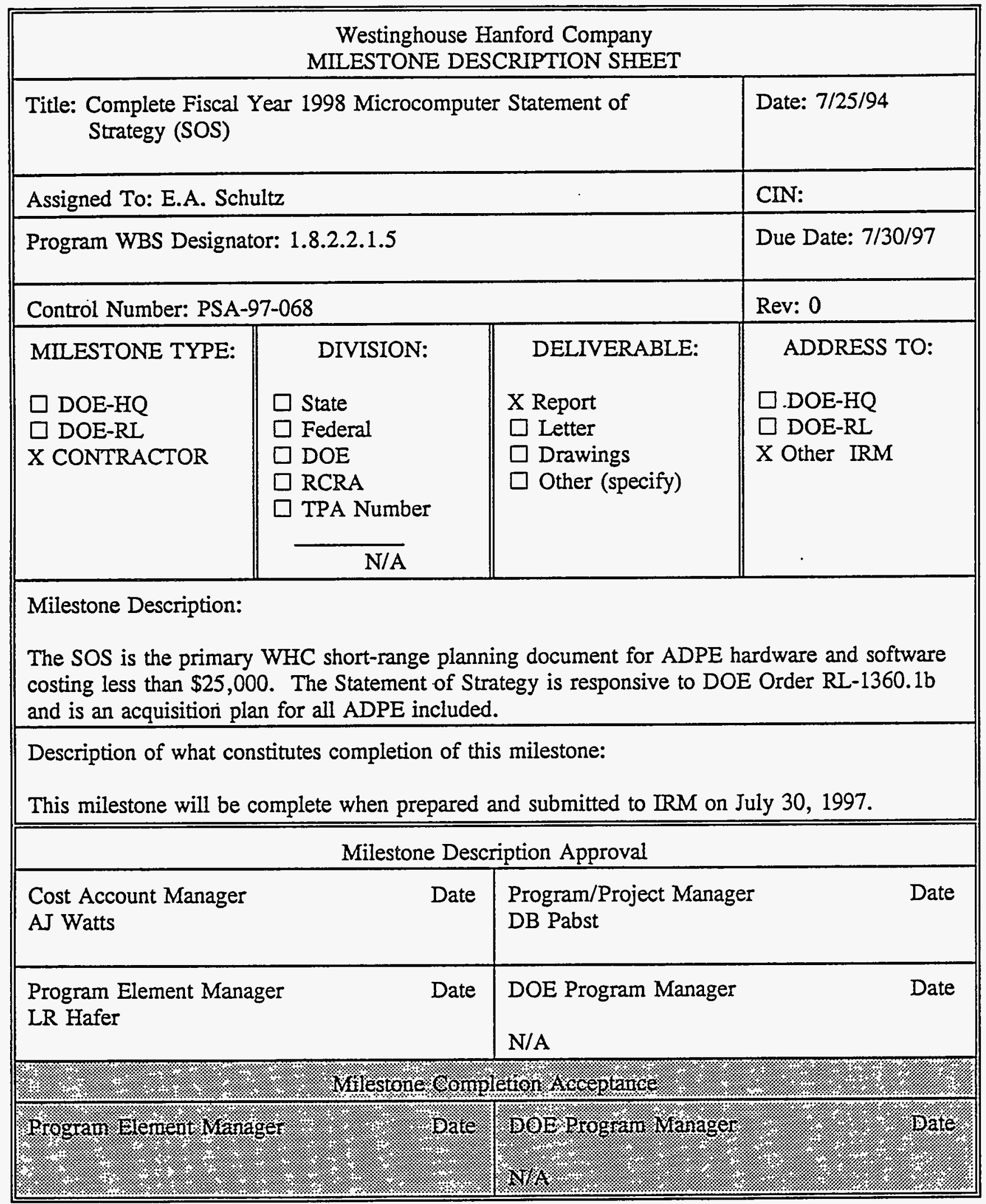




\begin{tabular}{|c|c|c|c|}
\hline \multicolumn{4}{|c|}{$\begin{array}{l}\text { Westinghouse Hanford Company } \\
\text { MILESTONE DESCRIPTION SHEET }\end{array}$} \\
\hline \multicolumn{3}{|c|}{$\begin{array}{l}\text { Title: Complete EPDS Software Configuration Control Management } \\
\text { Plan revision }\end{array}$} & Date: $07 / 25 / 94$ \\
\hline \multicolumn{3}{|c|}{ Assigned To: E. A. Schultz } & CIN: \\
\hline \multicolumn{3}{|c|}{ Program WBS Designator: 1 8.2.2.1.5 } & $\begin{array}{l}\text { Due Date: } \\
02 / 28 / 97\end{array}$ \\
\hline \multicolumn{3}{|c|}{ Control Number: PSA-97-069 } & Rev: \\
\hline $\begin{array}{l}\text { MILESTONE TYPE: } \\
\square \text { DOE-HQ } \\
\square \text { DOE-RL } \\
\text { X CONTRACTOR }\end{array}$ & $\begin{aligned} & \text { DIVISION: } \\
& \\
\square & \text { State } \\
& \square \text { Federal } \\
& \square \text { DOE } \\
& \square \text { RCRA } \\
& \square \text { TPA Number }\end{aligned}$ & $\begin{array}{l}\text { DELIVERABLE: } \\
\square \text { Report } \\
\square \text { Letter } \\
\square \text { Drawings } \\
\text { X Other (specify) } \\
\text { Modification of Rev. } \\
1 \text { Document }\end{array}$ & $\begin{array}{l}\text { ADDRESS TO: } \\
\square \text { DOE-HQ } \\
\square \text { DOE-RL-OPI } \\
\text { X Other (specify) } \\
\text { Performance Rptg. }\end{array}$ \\
\hline \multicolumn{4}{|c|}{$\begin{array}{l}\text { Milestone Description: } \\
\text { Complete Revision } 2 \text { of the EPDS Software Configuration Control Management Plan }\end{array}$} \\
\hline \multicolumn{4}{|c|}{$\begin{array}{l}\text { Description of what constitutes completion of this milestone: } \\
\text { This milestone will be completed when IRM submits Revision } 1 \text { of the EPDS Software } \\
\text { Configuration Control Management Plan to Westinghouse Hanford Company for approval. }\end{array}$} \\
\hline \multicolumn{4}{|c|}{ Milestone Description Approval } \\
\hline \multicolumn{2}{|l|}{$\begin{array}{l}\text { Cost Account Manager } \\
\text { JE Tarcza }\end{array}$} & \multicolumn{2}{|c|}{$\begin{array}{l}\text { Program/Project Manager } \\
\text { DB Pabst }\end{array}$} \\
\hline \multicolumn{2}{|c|}{$\begin{array}{l}\text { Program Element Manager } \\
\text { LR Hafer }\end{array}$} & \multicolumn{2}{|c|}{$\begin{array}{l}\text { DOE Program Manager Date } \\
\text { N/A }\end{array}$} \\
\hline \multicolumn{4}{|c|}{ 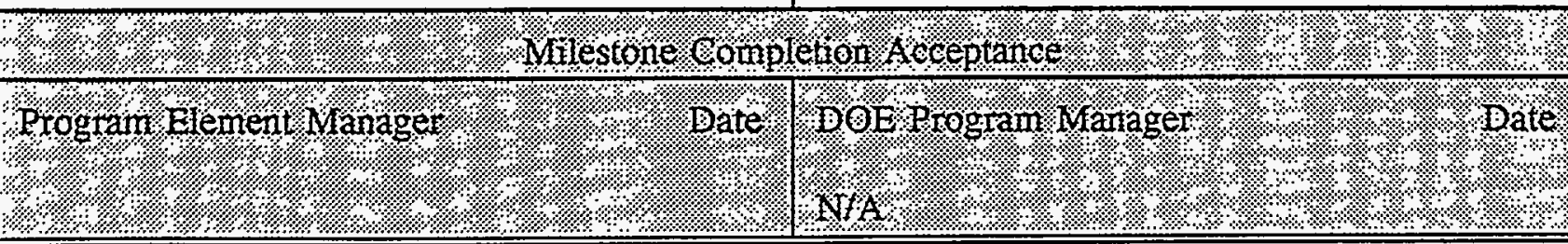 } \\
\hline
\end{tabular}




\begin{tabular}{|c|c|c|c|}
\hline \multicolumn{4}{|c|}{$\begin{array}{l}\text { Westinghouse Hanford Company } \\
\text { MILESTONE DESCRIPTION SHEET }\end{array}$} \\
\hline \multicolumn{3}{|c|}{$\begin{array}{l}\text { Title: Complete EPDS platform upgrade (PTS, CMM, ADS, BCC) } \\
\text { to Client Server environment }\end{array}$} & Date: $8 / 2 / 94$ \\
\hline \multicolumn{3}{|c|}{ Assigned To: J. E. Tarcza } & CIN: \\
\hline \multicolumn{3}{|c|}{ Program WBS Designator: 1 8.2.2.1.5 } & $\begin{array}{l}\text { Due Date: } \\
\text { 09/30/97 }\end{array}$ \\
\hline \multicolumn{3}{|c|}{ Control Number: PSA-97-072 } & Rev: \\
\hline $\begin{array}{l}\text { MILESTONE TYPE: } \\
\square \text { DOE-HQ } \\
\square \text { DOE-RL } \\
\text { X CONTRACTOR }\end{array}$ & $\begin{aligned} & \text { DIVISION: } \\
& \square \text { State } \\
& \square \text { Federal } \\
& \square \text { DOE } \\
& \square \text { RCRA } \\
& \square \text { TPA Number }\end{aligned}$ & $\begin{array}{l}\text { DELIVERABLE: } \\
\square \text { Report } \\
\square \text { Letter } \\
\square \text { Drawings } \\
\square \text { Other (specify) }\end{array}$ & $\begin{array}{l}\text { ADDRESS TO: } \\
\square \text { DOE-HQ } \\
\square \text { DOE-RI } \\
\text { X Other } \\
\text { Performance } \\
\text { Reporting }\end{array}$ \\
\hline \multicolumn{4}{|c|}{ Milestone Description: Upgrade all existing modules on EPDS to Client Server platform. } \\
\hline \multicolumn{4}{|c|}{$\begin{array}{l}\text { Description of what constitutes completion of this milestone: All existing modules moved from } \\
\text { existing Paradox platform to new Client Server operating environment. }\end{array}$} \\
\hline \multicolumn{4}{|c|}{ Milestone Description Approval } \\
\hline \multicolumn{2}{|c|}{$\begin{array}{l}\text { Cost Account Manager } \quad \text { Date } \\
\text { JE Tarcza }\end{array}$} & $\begin{array}{l}\text { Program/Project Manager } \\
\text { DB Pabst }\end{array}$ & Date \\
\hline \multicolumn{2}{|c|}{$\begin{array}{l}\text { Program Element Manager } \\
\text { LR Hafer }\end{array}$} & $\begin{array}{l}\text { DOE Program Manager } \\
\text { N/A }\end{array}$ & Date \\
\hline \multicolumn{4}{|c|}{ 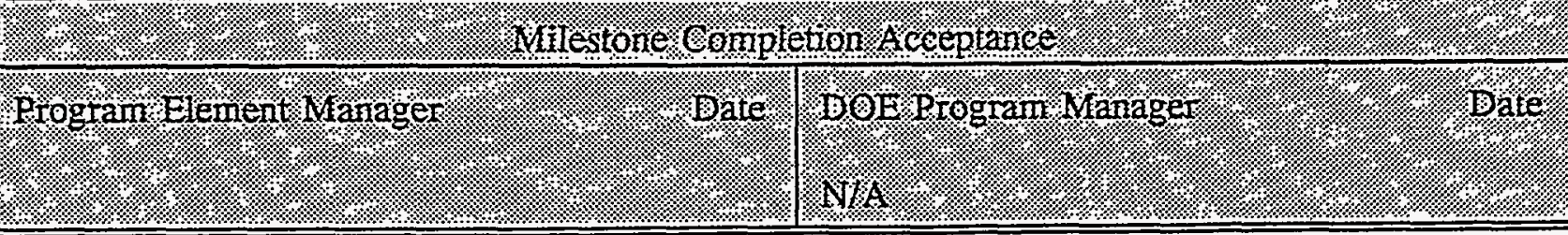 } \\
\hline
\end{tabular}




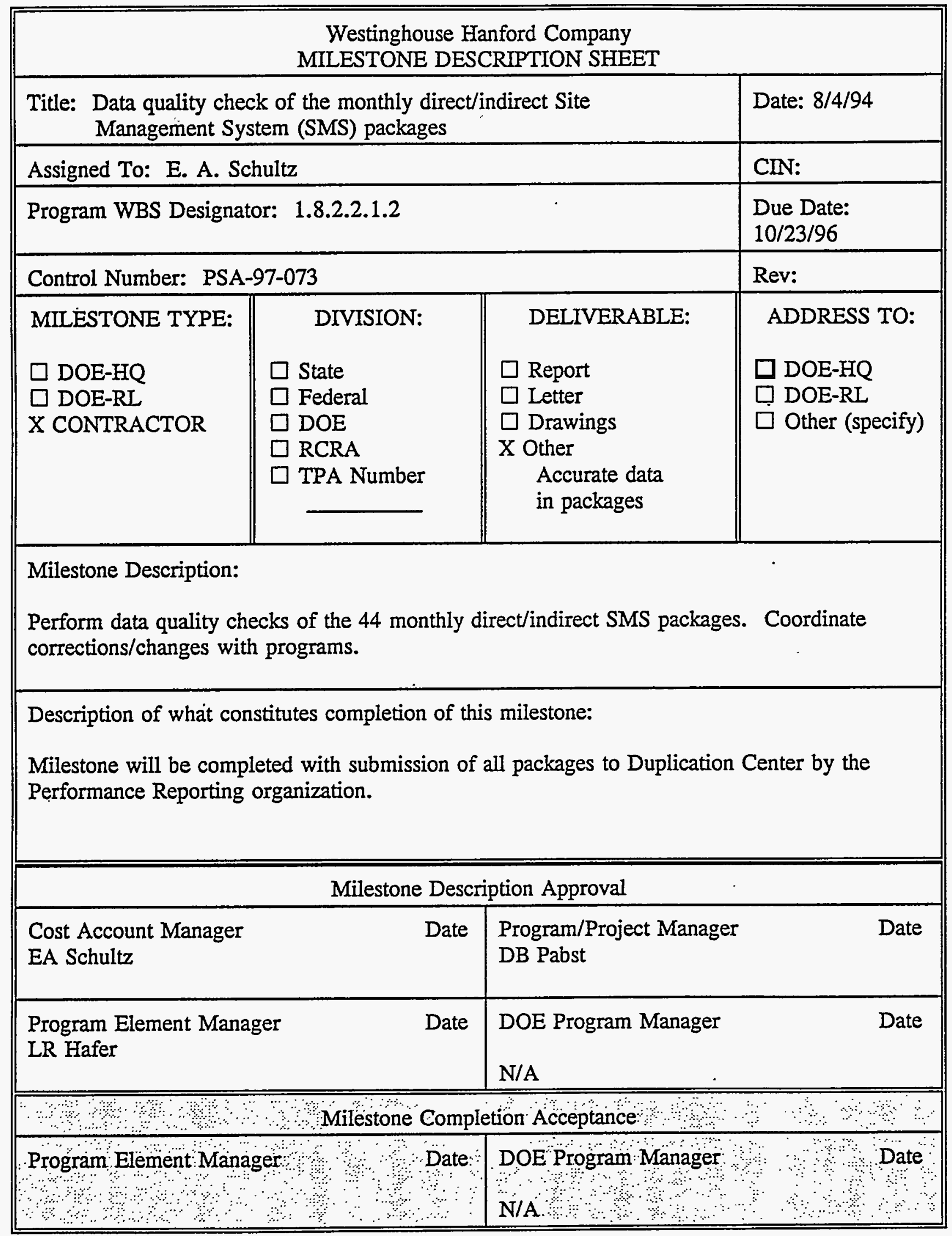




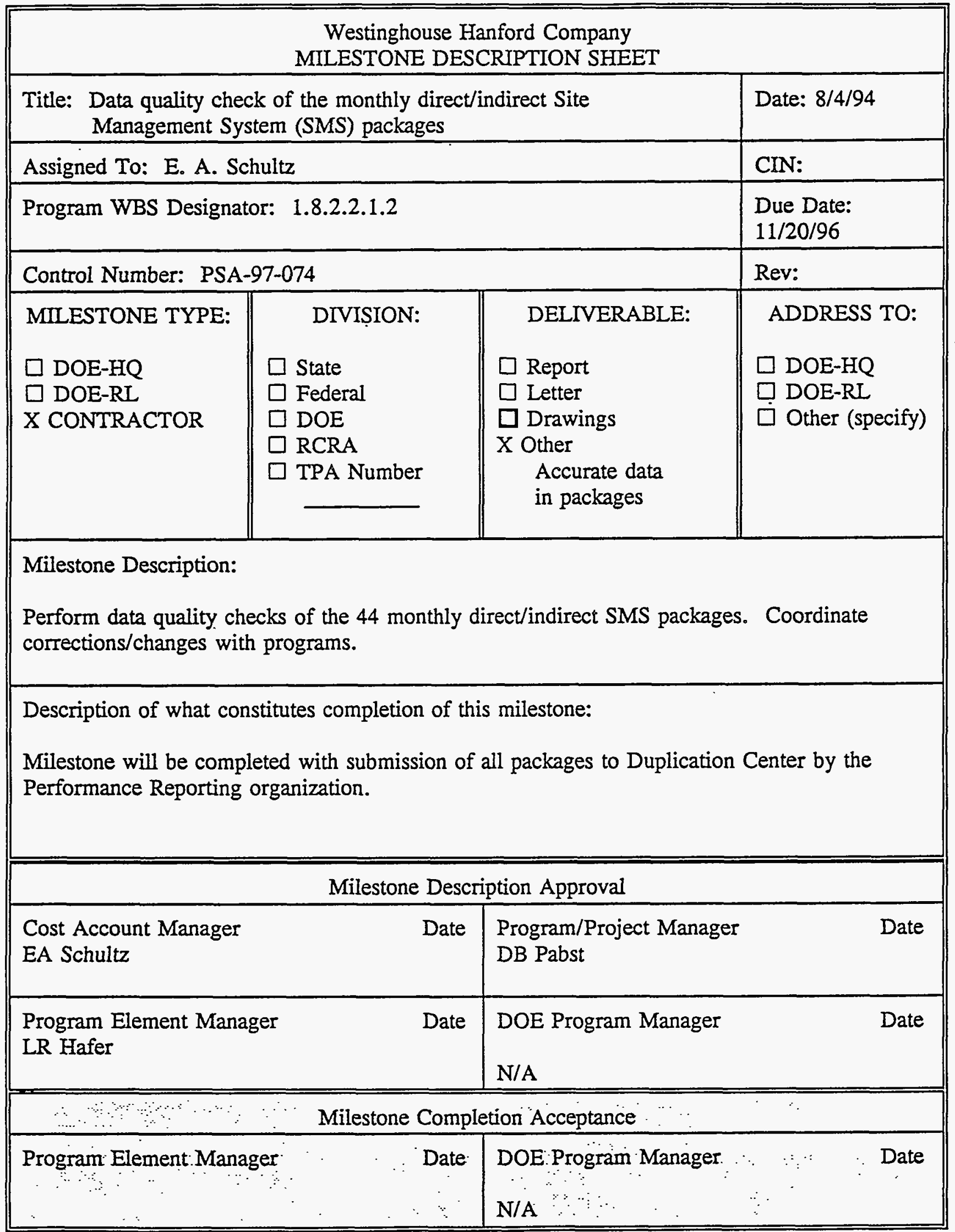




\begin{tabular}{|c|c|c|c|}
\hline \multicolumn{4}{|c|}{$\begin{array}{l}\text { Westinghouse Hanford Company } \\
\text { MILESTONE DESCRIPTION SHEET }\end{array}$} \\
\hline \multicolumn{3}{|c|}{$\begin{array}{l}\text { Title: Data quality check of the monthly direct/indirect Site } \\
\text { Management System (SMS) packages }\end{array}$} & Date: $8 / 4 / 94$ \\
\hline \multicolumn{3}{|c|}{ Assigned To: E. A. Schultz } & CIN: \\
\hline \multicolumn{3}{|c|}{ Program WBS Designator: 1 8.2.2.1.2 } & $\begin{array}{l}\text { Due Date: } \\
12 / 18 / 96 \\
\end{array}$ \\
\hline \multicolumn{3}{|c|}{ Control Number: PSA-97-075 } & Rev: \\
\hline MILESTONE TYPE: & DIVISION: & DELIVERABLE: & ADDRESS TO: \\
\hline $\begin{array}{l}\square \text { DOE-HQ } \\
\square \text { DOE-RL } \\
\text { X CONTRACTOR }\end{array}$ & $\begin{array}{l}\square \text { State } \\
\square \text { Federal } \\
\square \text { DOE } \\
\square \text { RCRA } \\
\square \text { TPA Number }\end{array}$ & $\begin{array}{l}\square \text { Report } \\
\square \text { Letter } \\
\square \text { Drawings } \\
\text { X Other } \\
\text { Accurate data } \\
\text { in packages }\end{array}$ & $\begin{array}{l}\square \text { DOE-HQ } \\
\square \text { DOE-RL } \\
\square \text { Other (specify) }\end{array}$ \\
\hline \multicolumn{4}{|c|}{$\begin{array}{l}\text { Milestone Description: } \\
\text { Perform data quality checks of the } 44 \text { monthly direct/indirect SMS packages. Coordinate } \\
\text { corrections/changes with programs. }\end{array}$} \\
\hline \multicolumn{4}{|c|}{$\begin{array}{l}\text { Description of what constitutes completion of this milestone: } \\
\text { Milestone will be completed with submission of all packages to Duplication Center by the } \\
\text { Performance Reporting organization. }\end{array}$} \\
\hline \multicolumn{4}{|c|}{ Milestone Description Approval } \\
\hline \multicolumn{2}{|l|}{$\begin{array}{l}\text { Cost Account Manager } \\
\text { EA Schultz }\end{array}$} & $\begin{array}{l}\text { Program/Project Manager } \\
\text { DB Pabst }\end{array}$ & Date \\
\hline \multicolumn{2}{|c|}{$\begin{array}{l}\text { Program Element Manager } \\
\text { LR Hafer }\end{array}$} & $\begin{array}{l}\text { DOE Program Manager } \\
\text { N/A }\end{array}$ & Date \\
\hline \multicolumn{4}{|c|}{ 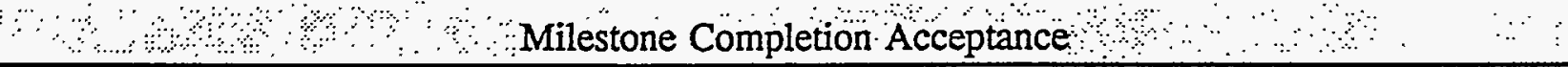 } \\
\hline Program Element Mana & मा Date & $\begin{array}{l}\text { DOE Program Manager } \\
\text { Ni }{ }^{2} \text { l }\end{array}$ & 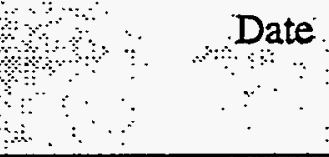 \\
\hline
\end{tabular}




\begin{tabular}{|c|c|c|c|}
\hline \multicolumn{4}{|c|}{$\begin{array}{l}\text { Westinghouse Hanford Company } \\
\text { MILESTONE DESCRIPTION SHEET }\end{array}$} \\
\hline \multicolumn{3}{|c|}{$\begin{array}{l}\text { Title: Data quality check of the monthly direct/indirect Site } \\
\text { Management System (SMS) packages }\end{array}$} & Date: $8 / 4 / 94$ \\
\hline \multicolumn{3}{|c|}{ Assigned To: E. A. Schultz } & CIN: \\
\hline \multicolumn{3}{|c|}{ Program WBS Designator: 1 8.8.2.2.1.2 } & $\begin{array}{l}\text { Due Date: } \\
1 / 22 / 97\end{array}$ \\
\hline \multicolumn{3}{|c|}{ Control Number: PSA-97-076 } & Rev: \\
\hline MILESTONE TYPE: & DIVISION: & DELIVERABLE: & ADDRESS TO: \\
\hline $\begin{array}{l}\square \text { DOE-HQ } \\
\square \text { DOE-RL } \\
\text { X CONTRACTOR }\end{array}$ & $\begin{array}{l}\square \text { State } \\
\square \text { Federal } \\
\square \text { DOE } \\
\square \text { RCRA } \\
\square \text { TPA Number }\end{array}$ & $\begin{array}{l}\square \text { Report } \\
\square \text { Letter } \\
\square \text { Drawings } \\
\text { X Other } \\
\text { Accurate data } \\
\text { in packages }\end{array}$ & $\begin{array}{l}\square \text { DOE-HQ } \\
\square \text { DOE-RL } \\
\square \text { Other (specify) }\end{array}$ \\
\hline \multirow{2}{*}{\multicolumn{4}{|c|}{$\begin{array}{l}\text { Milestone Description: } \\
\text { Perform data quality checks of the } 44 \text { monthly direct/indirect SMS packages. Coordinate } \\
\text { corrections/changes with programs. }\end{array}$}} \\
\hline & & & \\
\hline \multicolumn{4}{|c|}{$\begin{array}{l}\text { Description of what constitutes completion of this milestone: } \\
\text { Milestone will be completed with submission of all packages to Duplication Center by the } \\
\text { Performance Reporting organization. }\end{array}$} \\
\hline \multicolumn{4}{|c|}{ Milestone Description Approval } \\
\hline \multicolumn{2}{|l|}{$\begin{array}{l}\text { Cost Account Manager } \\
\text { EA Schultz }\end{array}$} & $\begin{array}{l}\text { Program/Project Manager } \\
\text { DB Pabst }\end{array}$ & Date \\
\hline \multicolumn{2}{|c|}{$\begin{array}{l}\text { Program Element Manager } \\
\text { LR Hafer }\end{array}$} & $\begin{array}{l}\text { DOE Program Manager } \\
\text { N/A }\end{array}$ & Date \\
\hline \multicolumn{2}{|c|}{$\begin{array}{c}\text { Program Element Manager } \\
\text { (n) }\end{array}$} & \multicolumn{2}{|c|}{$\begin{array}{l}\text { DOE Program Manager } \\
\text { N/A } \\
\text { N }\end{array}$} \\
\hline
\end{tabular}




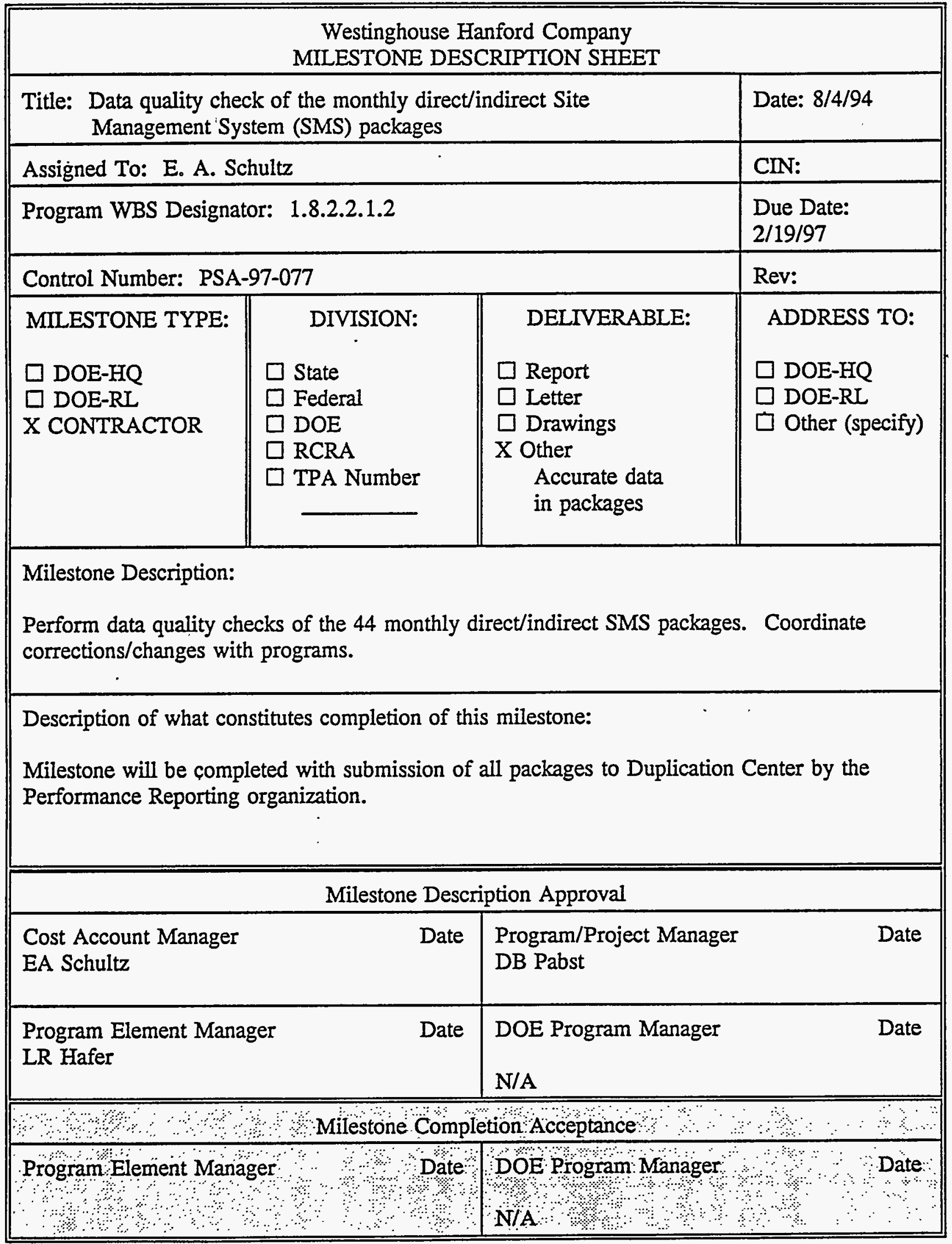




\begin{tabular}{|c|c|c|c|}
\hline \multicolumn{4}{|c|}{$\begin{array}{l}\text { Westinghouse Hanford Company } \\
\text { MILESTONE DESCRIPTION SHEET }\end{array}$} \\
\hline \multicolumn{3}{|c|}{$\begin{array}{l}\text { Title: Data quality check of the monthly direct/indirect Site } \\
\text { Management System (SMS) packages }\end{array}$} & Date: $8 / 4 / 94$ \\
\hline \multicolumn{3}{|c|}{ Assigned To: E. A. Schultz } & CIN: \\
\hline \multicolumn{3}{|c|}{ Program WBS Designator: 1 8.2.2.1.2 } & $\begin{array}{l}\text { Due Date: } \\
3 / 19 / 97\end{array}$ \\
\hline \multicolumn{3}{|c|}{ Control Number: PSA-97-078 } & Rev: \\
\hline $\begin{array}{l}\text { MILESTONE TYPE: } \\
\square \text { DOE-HQ } \\
\square \text { DOE-RL } \\
\text { X CONTRACTOR }\end{array}$ & $\begin{array}{l}\quad \text { DIVISION: } \\
\quad \text { State } \\
\square \text { Federal } \\
\square \text { DOE } \\
\square \text { RCRA } \\
\square \text { TPA Number }\end{array}$ & $\begin{array}{l}\text { DELIVERABLE: } \\
\square \text { Report } \\
\square \text { Letter } \\
\square \text { Drawings } \\
\text { X Other } \\
\text { Accurate data } \\
\text { in packages }\end{array}$ & $\begin{array}{l}\text { ADDRESS TO: } \\
\square \text { DOE-HQ } \\
\square \text { DOE-RL } \\
\square \text { Other (specify) }\end{array}$ \\
\hline \multicolumn{4}{|c|}{$\begin{array}{l}\text { Milestone Description: } \\
\text { Perform data quality checks of the } 44 \text { monthly direct/indirect SMS packages. Coordinate } \\
\text { corrections/changes with programs. }\end{array}$} \\
\hline \multicolumn{4}{|c|}{$\begin{array}{l}\text { Description of what constitutes completion of this milestone: } \\
\text { Milestone will be completed with submission of all packages to Duplication Center by the } \\
\text { Performance Reporting organization. }\end{array}$} \\
\hline \multicolumn{4}{|c|}{ Milestone Description Approval } \\
\hline \multicolumn{2}{|l|}{$\begin{array}{l}\text { Cost Account Manager } \\
\text { EA Schultz }\end{array}$} & $\begin{array}{l}\text { Program/Project Manager } \\
\text { DB Pabst }\end{array}$ & Date \\
\hline \multicolumn{2}{|c|}{$\begin{array}{l}\text { Program Element Manager } \\
\text { LR Hafer }\end{array}$} & $\begin{array}{l}\text { DOE Program Manager } \\
\text { N/A }\end{array}$ & Date \\
\hline \multicolumn{4}{|c|}{ Milestone Completion Acceptance } \\
\hline $\begin{array}{c}\text { Program Element Mana } \\
\qquad\end{array}$ & $\begin{array}{llll} & \\
\ddots & \ddots\end{array}$ & $\begin{array}{l}\text { DOE Program Manager } \\
\text { N/A }\end{array}$ & Date \\
\hline
\end{tabular}




\begin{tabular}{|l|l||}
\hline \multicolumn{3}{|c|}{ Westinghouse Hanford Company } \\
MILESTONE DESCRIPTION SHEET
\end{tabular}




\begin{tabular}{|c|c|c|c|}
\hline \multicolumn{4}{|c|}{$\begin{array}{l}\text { Westinghouse Hanford Company } \\
\text { MILESTONE DESCRIPTION SHEET }\end{array}$} \\
\hline \multicolumn{3}{|c|}{$\begin{array}{l}\text { Title: Data quality check of the monthly direct/indirect Site } \\
\text { Management System (SMS) packages }\end{array}$} & Date: $8 / 4 / 94$ \\
\hline \multicolumn{3}{|c|}{ Assigned To: E. A. Schultz } & CIN: \\
\hline \multicolumn{3}{|c|}{ Program WBS Designator: 1 8.2.2.1.2 } & $\begin{array}{l}\text { Due Date: } \\
5 / 21 / 97\end{array}$ \\
\hline \multicolumn{3}{|c|}{ Control Number: PSA-97-080 } & Rev: \\
\hline $\begin{array}{l}\text { MILESTONE TYPE: } \\
\square \text { DOE-HQ } \\
\square \text { DOE-RL } \\
\text { X CONTRACTOR }\end{array}$ & $\begin{aligned} & \text { DIVISION: } \\
& \quad \text { State } \\
& \text { Federal } \\
& \square \text { DOE } \\
& \quad \text { RCRA } \\
& \square \text { TPA Number }\end{aligned}$ & $\begin{array}{l}\text { DELIVERABLE: } \\
\square \text { Report } \\
\square \text { Letter } \\
\square \text { Drawings } \\
\text { X Other } \\
\text { Accurate data } \\
\text { in packages }\end{array}$ & $\begin{array}{l}\text { ADDRESS TO: } \\
\square \text { DOE-HQ } \\
\square \text { DOE-RL } \\
\square \text { Other (specify) }\end{array}$ \\
\hline \multicolumn{4}{|c|}{$\begin{array}{l}\text { Milestone Description: } \\
\text { Perform data quality checks of the } 44 \text { monthly direct/indirect SMS packages. Coordinate } \\
\text { corrections/changes with programs. }\end{array}$} \\
\hline \multicolumn{4}{|c|}{$\begin{array}{l}\text { Description of what constitutes completion of this milestone: } \\
\text { Milestone will be completed with submission of all packages to Duplication Center by the } \\
\text { Performance Reporting organization. }\end{array}$} \\
\hline \multicolumn{4}{|c|}{ Milestone Description Approval } \\
\hline \multicolumn{2}{|l|}{$\begin{array}{l}\text { Cost Account Manager } \\
\text { EA Schultz }\end{array}$} & $\begin{array}{l}\text { Program/Project Manager } \\
\text { DB Pabst }\end{array}$ & Date \\
\hline \multicolumn{2}{|c|}{$\begin{array}{l}\text { Program Element Manager } \\
\text { LR Hafer }\end{array}$} & $\begin{array}{l}\text { DOE Program Manager } \\
\text { N/A }\end{array}$ & Date \\
\hline \multicolumn{2}{|c|}{ 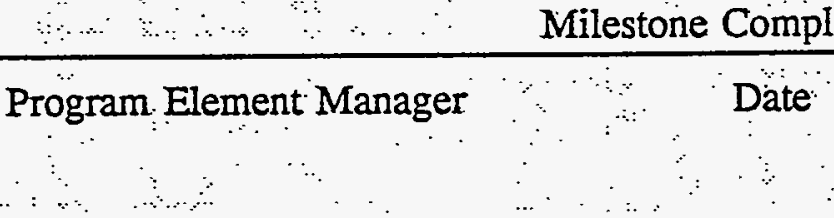 } & $\begin{array}{l}\text { DOE Program Manager } \\
\text { D/A } \\
\text { N/A }\end{array}$ & $\begin{array}{ll}\therefore & \text { Date } \\
\because & \end{array}$ \\
\hline
\end{tabular}




\begin{tabular}{|c|c|c|c|}
\hline \multicolumn{4}{|c|}{$\begin{array}{l}\text { Westinghouse Hanford Company } \\
\text { MILESTONE DESCRIPTION SHEET }\end{array}$} \\
\hline \multicolumn{3}{|c|}{$\begin{array}{l}\text { Title: Data quality check of the monthly direct/indirect Site } \\
\text { Management System (SMS) packages }\end{array}$} & Date: $8 / 4 / 94$ \\
\hline \multicolumn{3}{|c|}{ Assigned To: E. A. Schultz } & CIN: \\
\hline \multicolumn{3}{|c|}{ Program WBS Designator: 1 8.2.2.1.2 } & $\begin{array}{l}\text { Due Date: } \\
6 / 18 / 97\end{array}$ \\
\hline \multicolumn{3}{|c|}{ Control Number: PSA-97-081 } & Rev: \\
\hline MILESTONE TYPE: & DIVISION: & DELIVERABLE: & ADDRESS TO: \\
\hline $\begin{array}{l}\square \text { DOE-HQ } \\
\square \text { DOE-RL } \\
\text { X CONTRACTOR }\end{array}$ & $\begin{array}{l}\square \text { State } \\
\square \text { Federal } \\
\square \text { DOE } \\
\square \text { RCRA } \\
\square \text { TPA Number }\end{array}$ & $\begin{array}{l}\square \text { Report } \\
\square \text { Letter } \\
\square \text { Drawings } \\
\text { X Other } \\
\text { Accurate data } \\
\text { in packages }\end{array}$ & $\begin{array}{l}\square \text { DOE-HQ } \\
\square \text { DOE-RL } \\
\square \text { Other (specify) }\end{array}$ \\
\hline \multicolumn{4}{|c|}{$\begin{array}{l}\text { Milestone Description: } \\
\text { Perform data quality checks of the } 44 \text { monthly direct/indirect SMS packages. Coordinate } \\
\text { corrections/changes with programs. }\end{array}$} \\
\hline \multicolumn{4}{|c|}{$\begin{array}{l}\text { Description of what constitutes completion of this milestone: } \\
\text { Milestone will be completed with submission of all packages to Duplication Center by the } \\
\text { Performance Reporting organization. }\end{array}$} \\
\hline \multicolumn{4}{|c|}{ Milestone Description Approval } \\
\hline \multicolumn{2}{|l|}{$\begin{array}{l}\text { Cost Account Manager } \\
\text { EA Schultz }\end{array}$} & \multicolumn{2}{|l|}{$\begin{array}{l}\text { Program/Project Manager } \\
\text { DB Pabst }\end{array}$} \\
\hline \multicolumn{2}{|c|}{$\begin{array}{l}\text { Program Element Manager } \\
\text { LR Hafer }\end{array}$} & $\begin{array}{l}\text { DOE Program Manager } \\
\text { N/A }\end{array}$ & Date \\
\hline \multicolumn{4}{|c|}{ Milestone Completion Acceptance } \\
\hline \multicolumn{2}{|c|}{ Program Element:Manager } & \multicolumn{2}{|c|}{ DOE Program Manager } \\
\hline
\end{tabular}




\begin{tabular}{|c|c|c|c|}
\hline \multicolumn{4}{|c|}{$\begin{array}{l}\text { Westinghouse Hanford Company } \\
\text { MILESTONE DESCRIPTION SHEET }\end{array}$} \\
\hline \multicolumn{3}{|c|}{$\begin{array}{l}\text { Title: Data quality check of the monthly direct/indirect Site } \\
\text { Management System (SMS) packages }\end{array}$} & Date: $8 / 4 / 94$ \\
\hline \multicolumn{3}{|c|}{ Assigned To: E. A. Schultz } & CIN: \\
\hline \multicolumn{3}{|c|}{ Program WBS Designator: 1 8.2.2.1.2 } & $\begin{array}{l}\text { Due Date: } \\
7 / 23 / 97\end{array}$ \\
\hline \multicolumn{3}{|c|}{ Control Number: PSA-97-082 } & Rev: \\
\hline MILESTONE TYPE: & DIVISION: & DELIVERABLE: & ADDRESS TO: \\
\hline $\begin{array}{l}\square \text { DOE-HQ } \\
\square \text { DOE-RL } \\
\text { X CONTRACTOR }\end{array}$ & $\begin{array}{l}\square \text { State } \\
\square \text { Federal } \\
\square \text { DOE } \\
\square \text { RCRA } \\
\square \text { TPA Number }\end{array}$ & $\begin{array}{l}\square \text { Report } \\
\square \text { Letter } \\
\square \text { Drawings } \\
\text { X Other } \\
\text { Accurate data } \\
\text { in packages }\end{array}$ & $\begin{array}{l}\square \text { DOE-HQ } \\
\square \text { DOE-RL } \\
\square \text { Other (specify) }\end{array}$ \\
\hline \multicolumn{4}{|c|}{$\begin{array}{l}\text { Milestone Description: } \\
\text { Perform data quality checks of the } 44 \text { monthly direct/indirect SMS packages. Coordinate } \\
\text { corrections/changes with programs. }\end{array}$} \\
\hline \multicolumn{4}{|c|}{$\begin{array}{l}\text { Description of what constitutes completion of this milestone: } \\
\text { Milestone will be completed with submission of all packages to Duplication Center by the } \\
\text { Performance Reporting organization. }\end{array}$} \\
\hline \multicolumn{4}{|c|}{ Milestone Description Approval } \\
\hline \multicolumn{2}{|l|}{$\begin{array}{l}\text { Cost Account Manager } \\
\text { EA Schultz }\end{array}$} & \multicolumn{2}{|l|}{$\begin{array}{l}\text { Program/Project Manager } \\
\text { DB Pabst }\end{array}$} \\
\hline \multicolumn{2}{|c|}{$\begin{array}{l}\text { Program Element Manager } \\
\text { LR Hafer }\end{array}$} & \multicolumn{2}{|c|}{$\begin{array}{l}\text { DOE Program Manager Date } \\
\text { N/A }\end{array}$} \\
\hline \multicolumn{4}{|c|}{ Milestone Completion Acceptance } \\
\hline \multicolumn{2}{|c|}{$\begin{array}{c}\text { Program Element Manager } \\
\begin{array}{ccc} \\
0 & \cdots & \end{array}\end{array}$} & \multicolumn{2}{|c|}{$\begin{array}{l}\text { DOE Program Manager } \\
\text { N/A }\end{array}$} \\
\hline
\end{tabular}




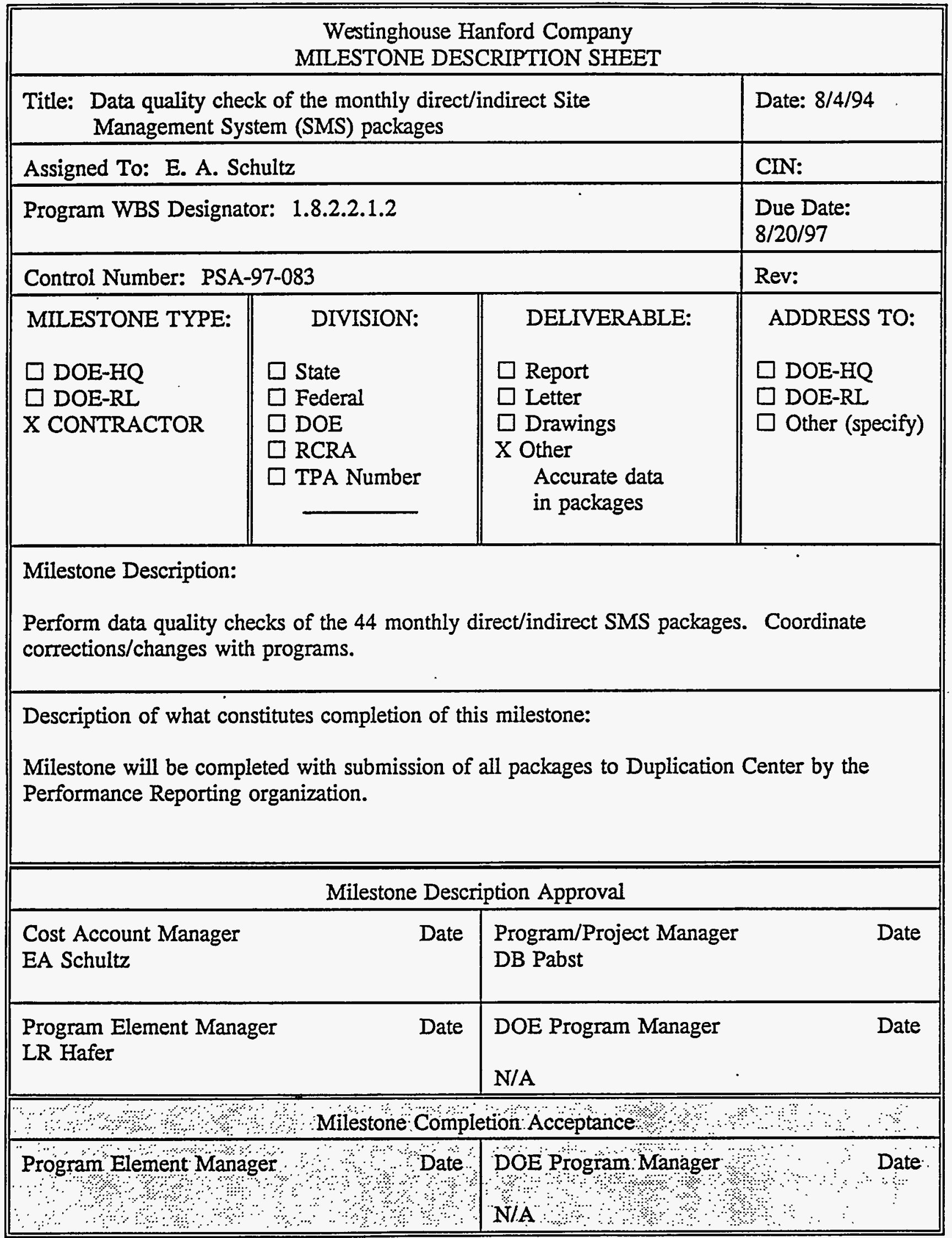




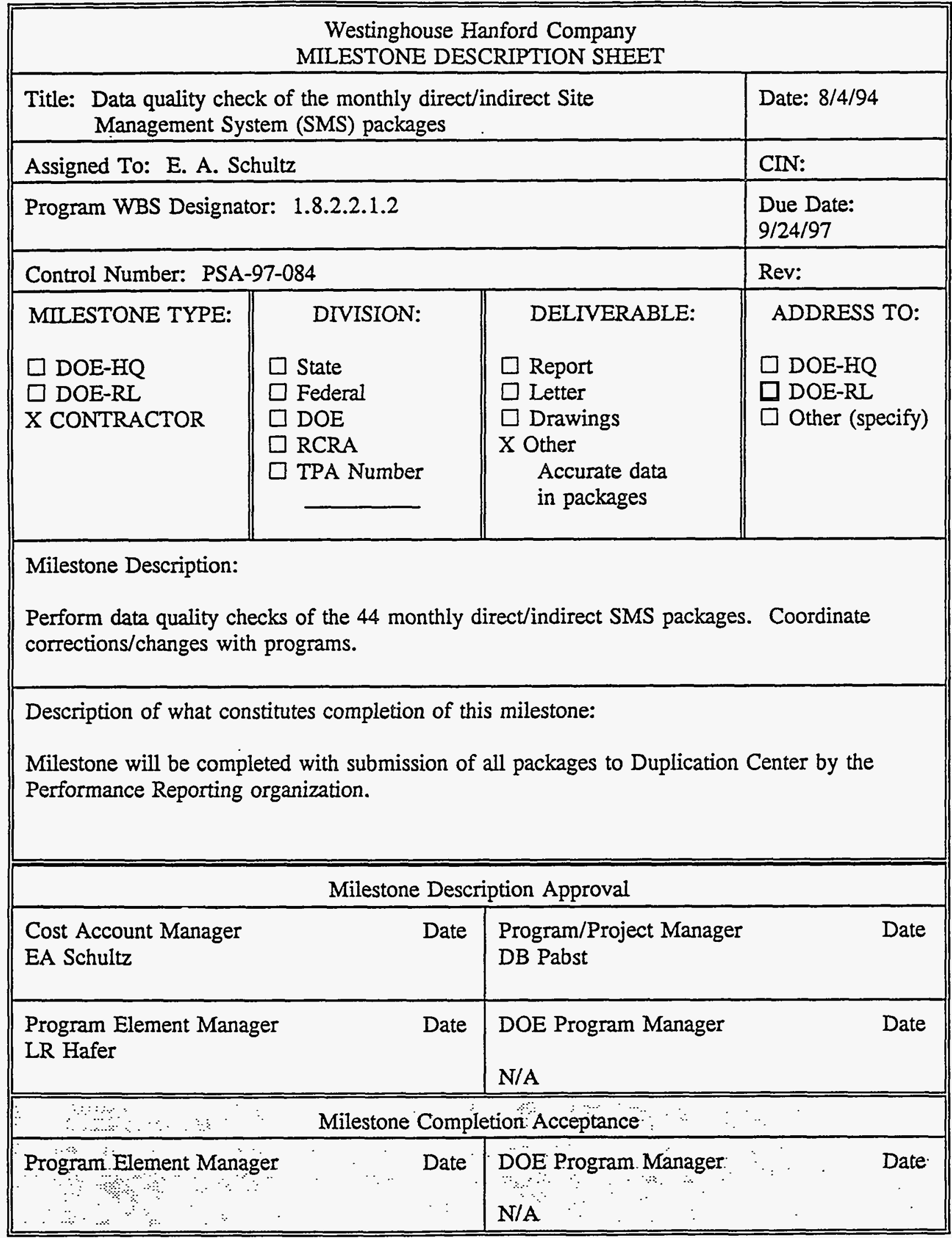




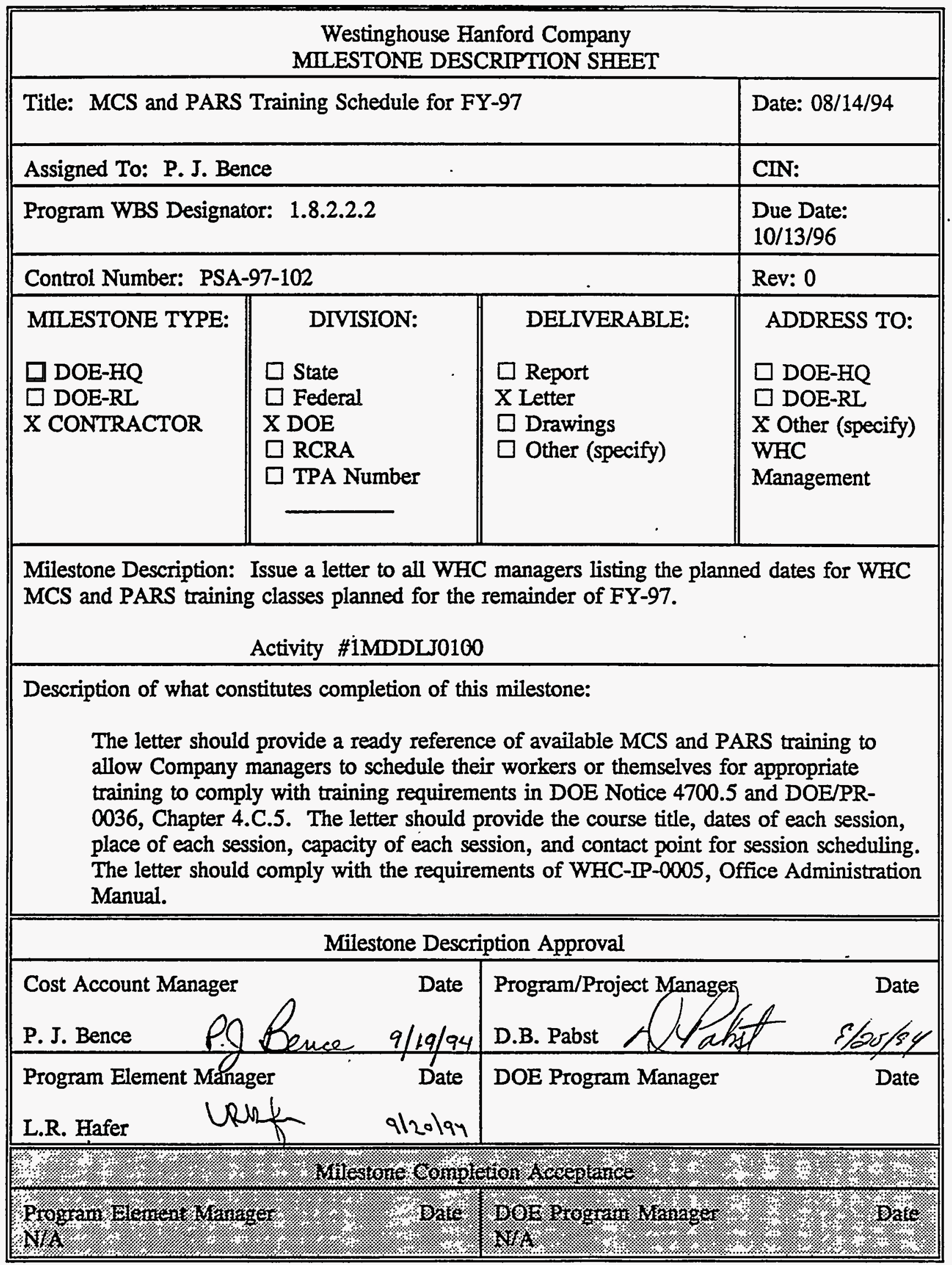




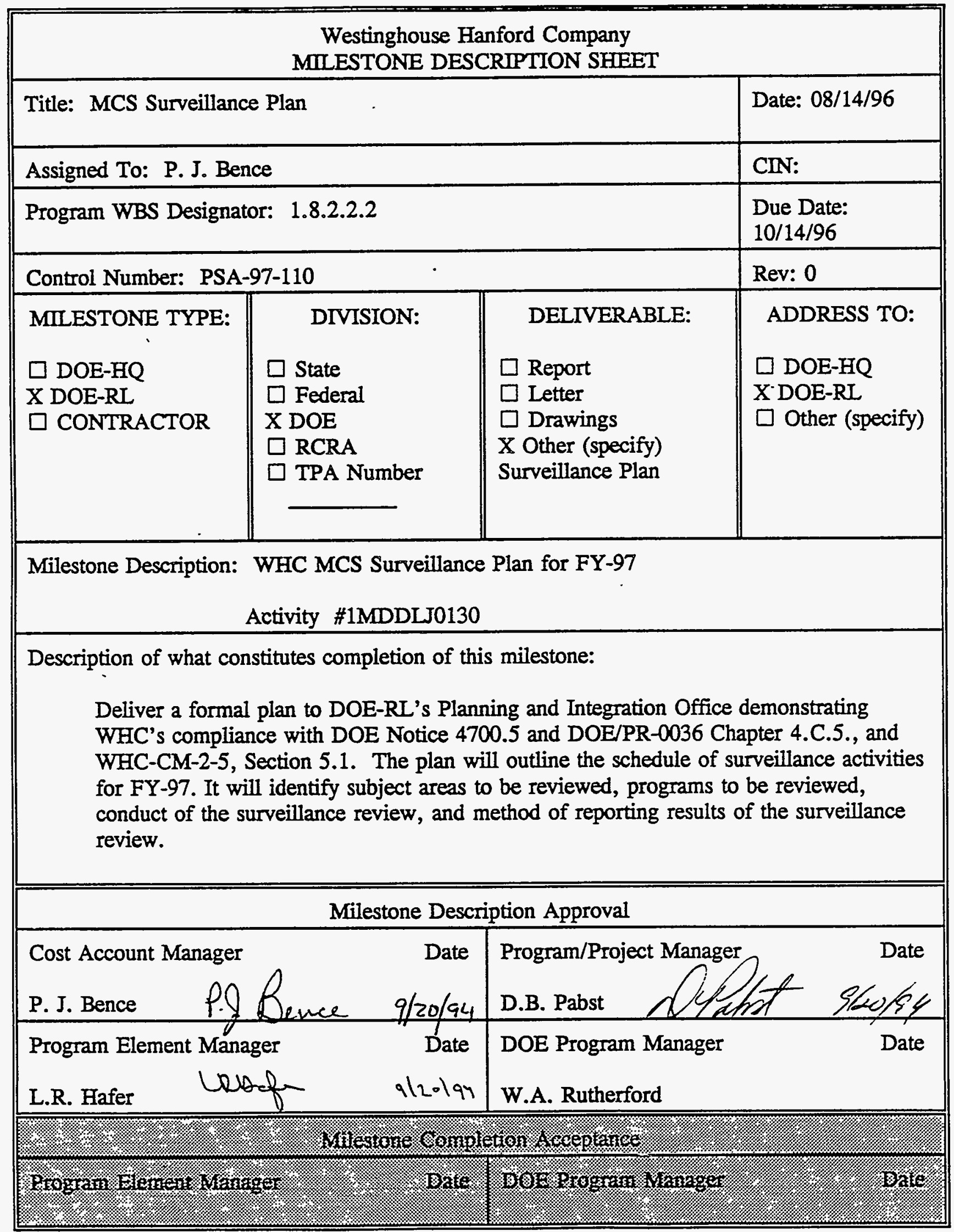




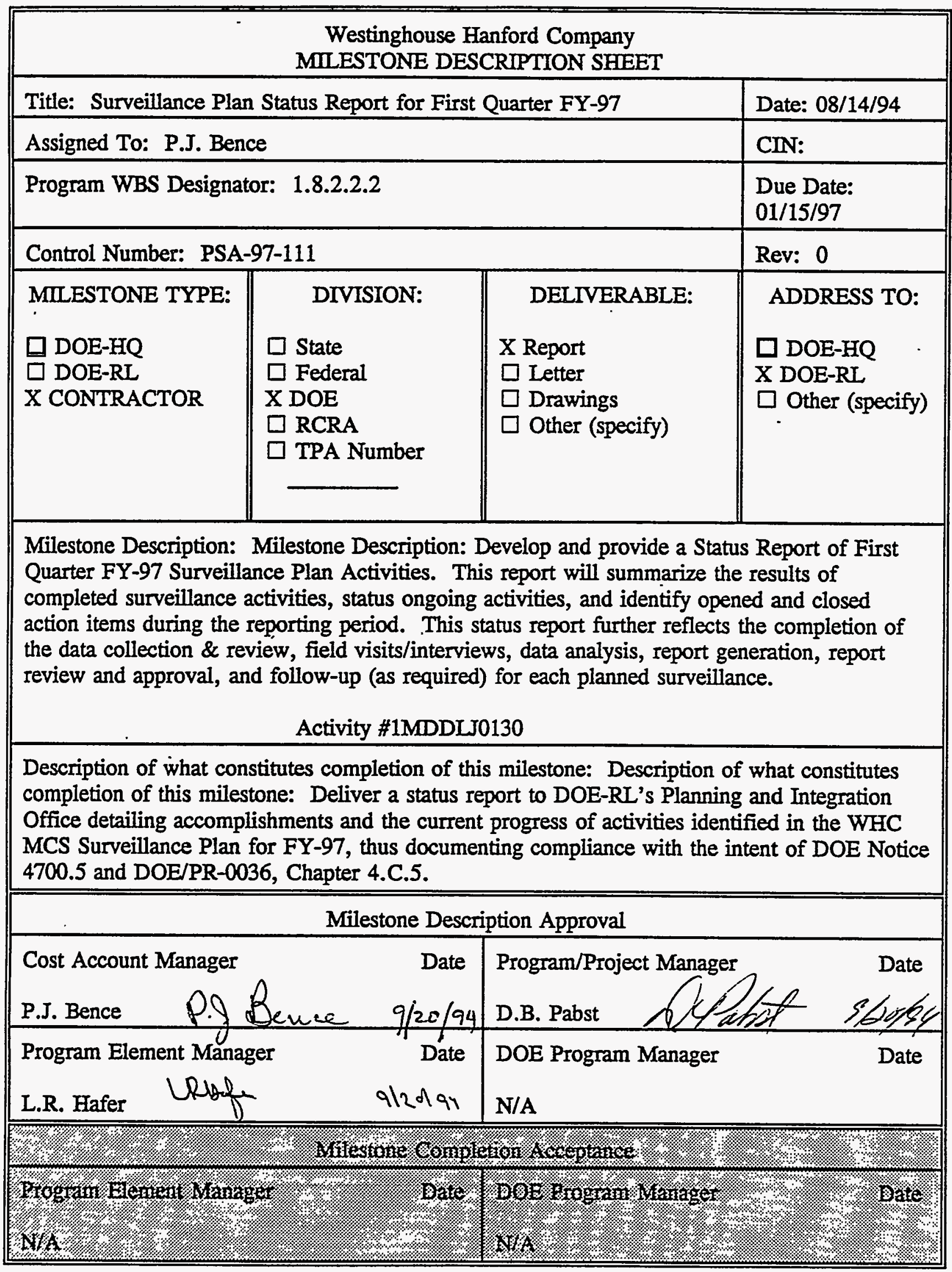




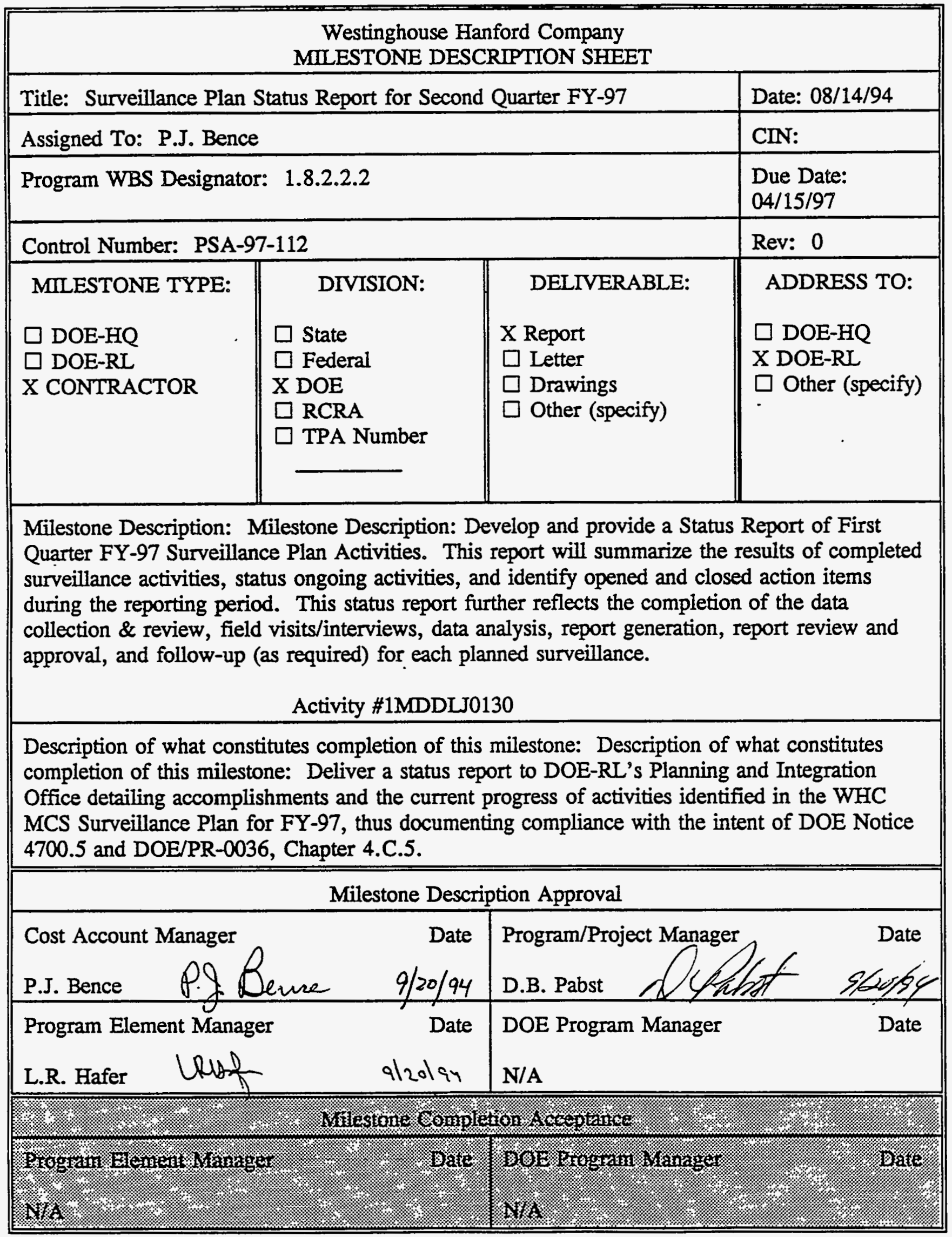




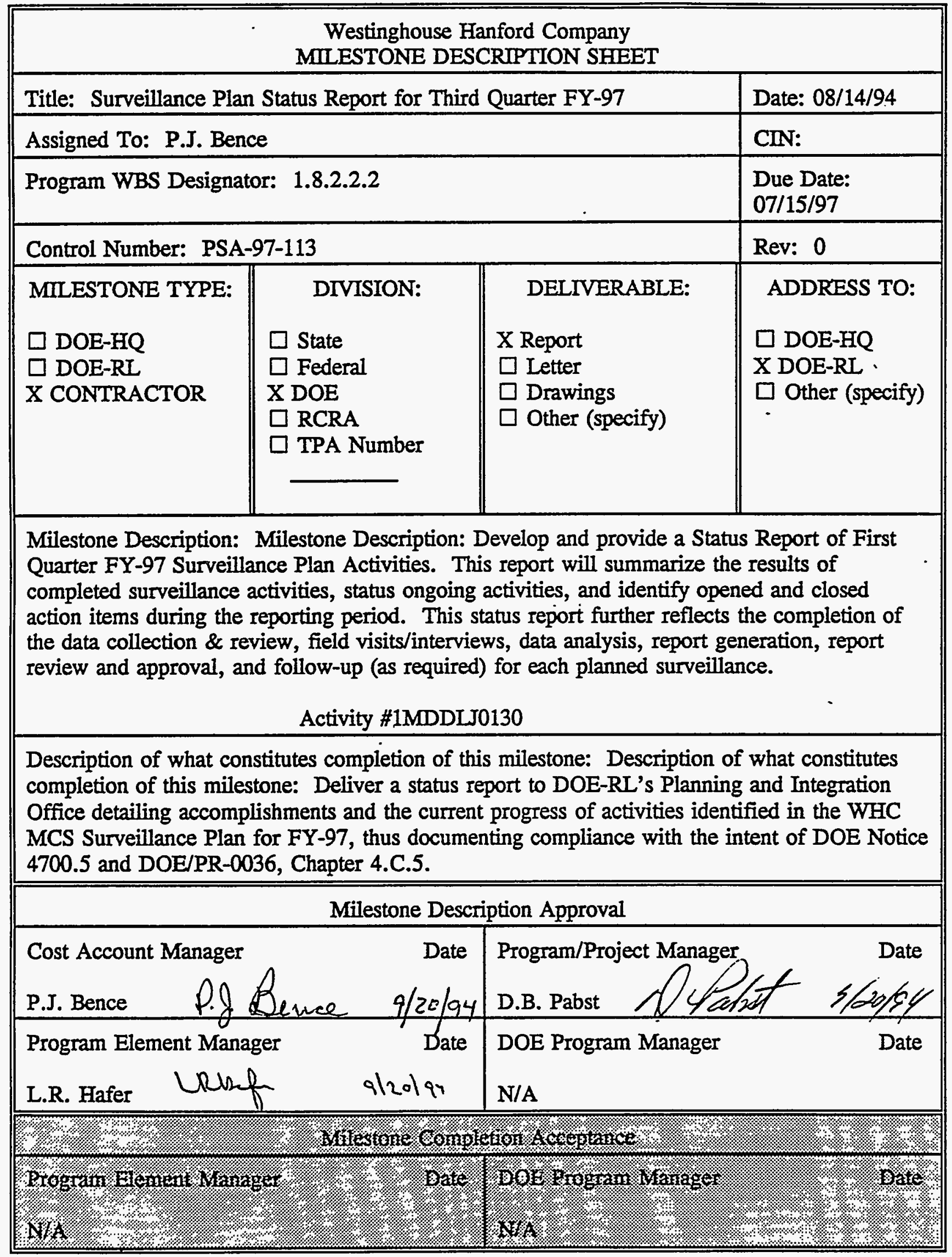




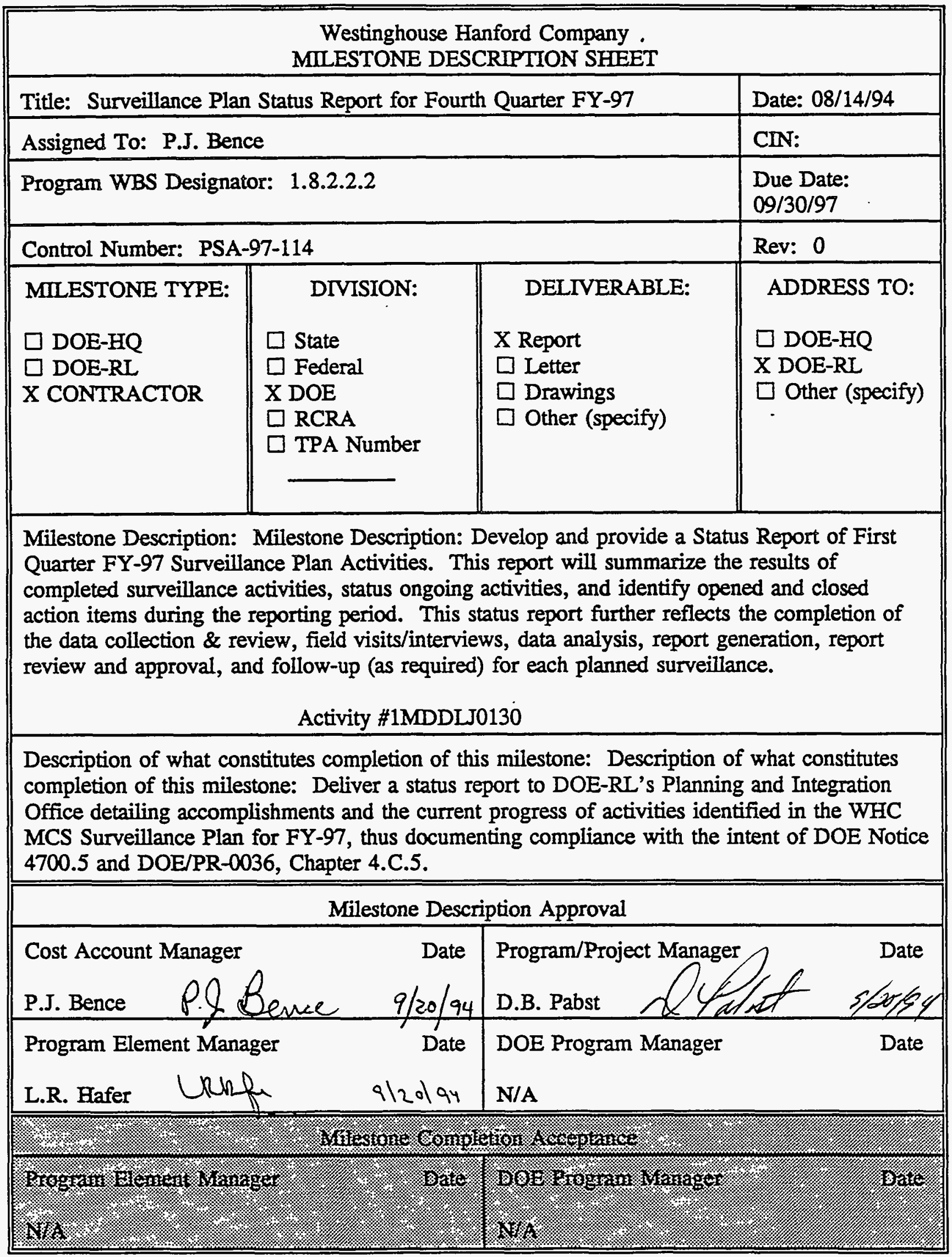




\begin{tabular}{|l|l|l||}
\hline \multicolumn{3}{|c|}{ Westinghouse Hanford Company } \\
MILESTONE DESCRIPTION SHEET
\end{tabular}




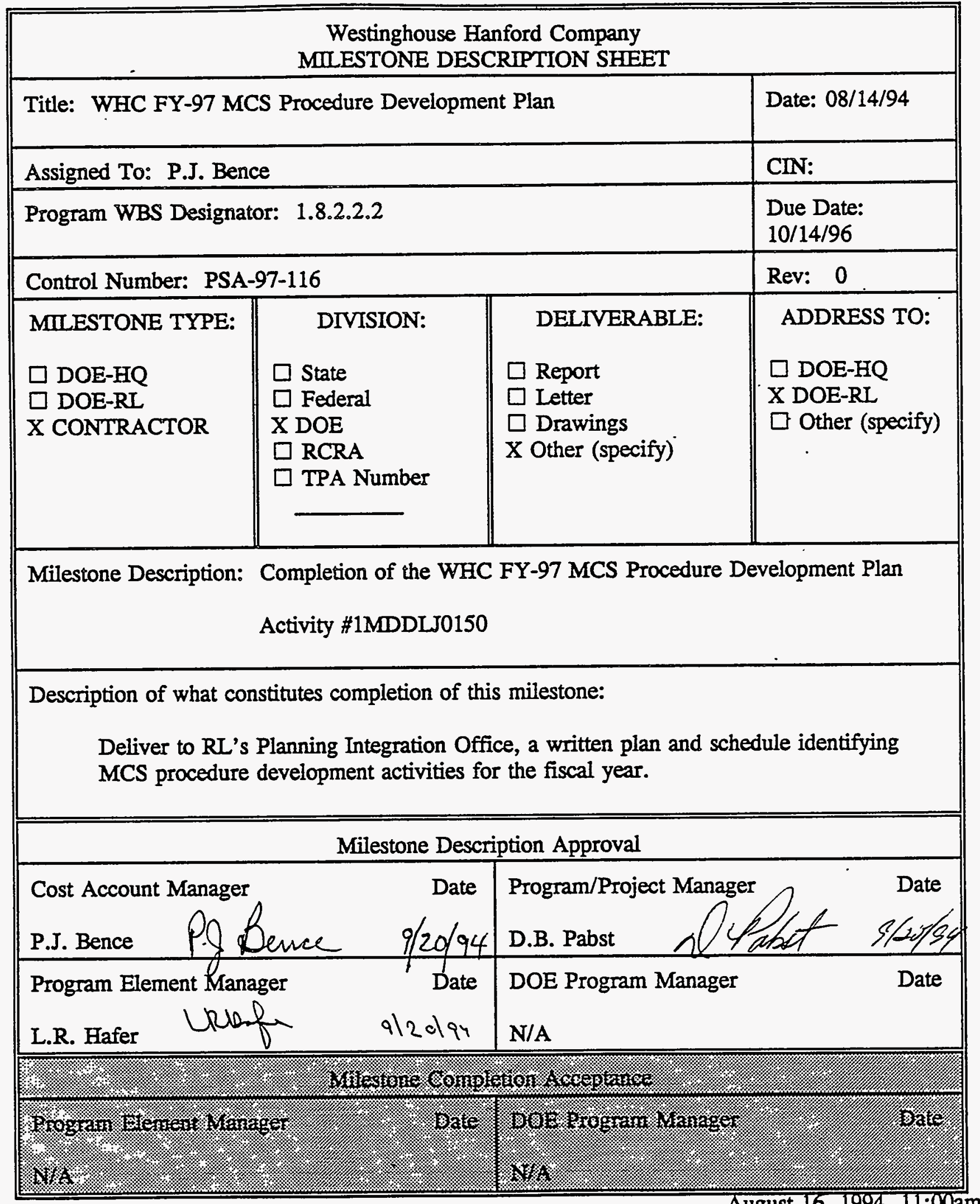


Planning Integration Multi-Year Program Plan

This page

intentionally left blank. 


\begin{tabular}{|c|c|c|c|}
\hline \multicolumn{3}{|c|}{$\begin{array}{l}\text { Westinghouse Hanford Company } \\
\text { MILESTONE DESCRIPTION SHEET }\end{array}$} & $\begin{array}{r}W H C-S P-1096 \\
09 / 23 / 94 \\
\end{array}$ \\
\hline \multicolumn{3}{|c|}{ Title: Complete Draft Hanford Strategic Plan } & Date: $9 / 20 / 94$ \\
\hline \multicolumn{3}{|c|}{ Assigned To: H. H. Yoshikawa } & CIN: \\
\hline \multicolumn{3}{|c|}{ Program WBS Designator: 1 8.2.1.1 } & $\begin{array}{l}\text { Due Date: } \\
01 / 19 / 95\end{array}$ \\
\hline \multicolumn{3}{|c|}{ Control Number: PSS-95-100 } & Rev: 0 \\
\hline $\begin{array}{l}\text { MILESTONE TYPE: } \\
\square \text { DOE-HQ } \\
\text { X DOE-RL } \\
\square \text { CONTRACTOR }\end{array}$ & $\begin{array}{l}\quad \text { DIVISION: } \\
\square \text { State } \\
\square \text { Federal } \\
\text { X DOE } \\
\square \text { RCRA } \\
\square \text { TPA Number }\end{array}$ & $\begin{array}{l}\text { DELIVERABLE: } \\
\text { X Report } \\
\square \text { Letter } \\
\square \text { Drawings } \\
\square \text { Other (specify) }\end{array}$ & $\begin{array}{l}\text { ADDRESS TO: } \\
\square \text { DOE-HQ } \\
\text { X DOE-RL } \\
\square \text { Other (specify) }\end{array}$ \\
\hline \multicolumn{4}{|c|}{$\begin{array}{l}\text { Milestone Description: } \\
\text { Submittal of the draft Fiscal Year (FY) } 1996 \text { Hanford Strategic Plan to U.S. Department of } \\
\text { Energy, Richland Operations Office (RL) Planning Integration Division (PID) in accordance } \\
\text { with Hanford Quality Council direction for review. Contents of the FY } 1996 \text { draft Hanford } \\
\text { Strategic Plan will include: }\end{array}$} \\
\hline \multicolumn{4}{|c|}{$\begin{array}{l}\text { Description of what constitutes completion of this milestone: } \\
\text { The draft FY } 1996 \text { Hanford Strategic Plan is submitted to RL-PID consistent with direction by } \\
\text { the Hanford Quality Council. The draft Hanford Strategic Plan will be submitted to RL-PID } \\
\text { for transmittal to DOE-HQ, Regulators, and Indian Nations. }\end{array}$} \\
\hline \multicolumn{4}{|c|}{$\begin{array}{l}\text { The draft Hanford Strategic Plan will comply with requirements of RLID } 5000.16 \text { provided to } \\
\text { the contractor for information September } 9,1994 \text {, or as modified by the Hanford Quality } \\
\text { Council. }\end{array}$} \\
\hline
\end{tabular}




\begin{tabular}{|c|c|c|}
\hline \multicolumn{3}{|c|}{$\begin{array}{l}\text { Westinghouse Hanford Company } \\
\text { MILESTONE DESCRIPTION SHEET }\end{array}$} \\
\hline \multicolumn{2}{|l|}{ Title: Complete Draft Hanford Strategic Plan } & Date: $9 / 20 / 94$ \\
\hline \multicolumn{2}{|l|}{ Assigned To: H. H. Yoshikawa } & CIN: \\
\hline \multicolumn{2}{|l|}{ Program WBS Designator: 1 8.2.1.1 } & $\begin{array}{l}\text { Due Date: } \\
01 / 19 / 95\end{array}$ \\
\hline \multicolumn{2}{|l|}{ Control Number: PSS-95-100 } & Rev: 0 \\
\hline $\begin{array}{l}\text { Cost Account Manager } \\
\text { Date } \\
\text { H. H. Yoshikawa }\end{array}$ & \multicolumn{2}{|c|}{ Program/Project Manager 1 Babst } \\
\hline 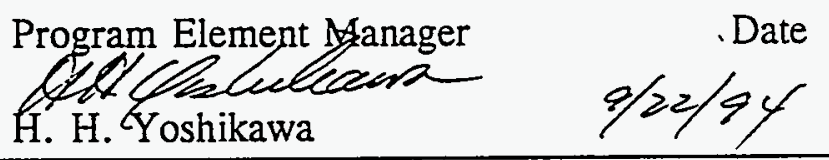 & \multicolumn{2}{|c|}{ 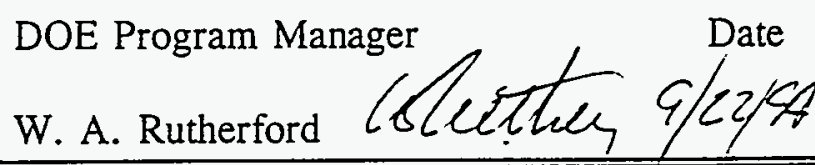 } \\
\hline \multicolumn{3}{|c|}{ 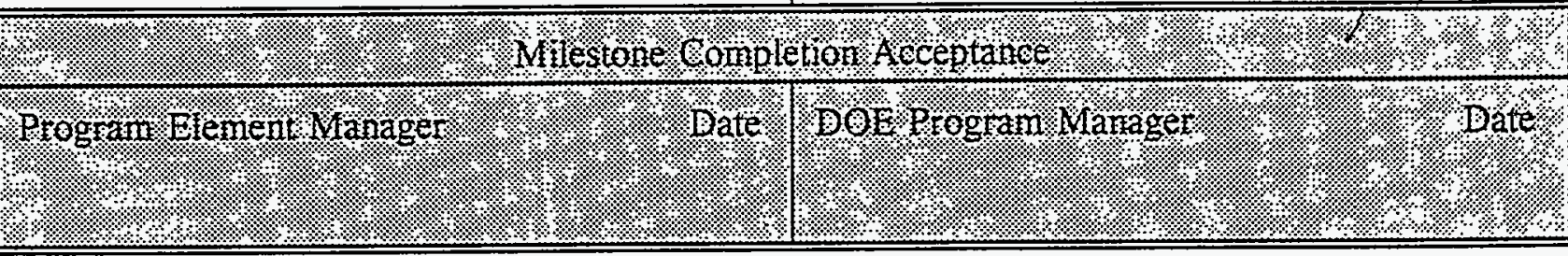 } \\
\hline
\end{tabular}




\begin{tabular}{|c|c|c|c|}
\hline \multicolumn{3}{|c|}{$\begin{array}{l}\text { Westinghouse Hanford Company } \\
\text { MILESTONE DESCRIPTION SHEET }\end{array}$} & $\begin{array}{r}\text { WHC-SP-1096 } \\
09 / 23 / 94\end{array}$ \\
\hline \multicolumn{3}{|c|}{ Title: Complete Hanford Strategic Plan } & Date: $9 / 20 / 94$ \\
\hline \multicolumn{3}{|c|}{ Assigned To: H. H. Yoshikawa } & CIN: \\
\hline \multicolumn{3}{|c|}{ Program WBS Designator: 1 8.2.1.1 } & $\begin{array}{l}\text { Due Date: } \\
.03 / 31 / 95\end{array}$ \\
\hline \multicolumn{3}{|c|}{ Control Number: PSS-95-101 } & Rev: 0 \\
\hline $\begin{array}{l}\text { MILESTONE TYPE: } \\
\square \text { DOE-HQ } \\
\text { X DOE-RL } \\
\square \text { CONTRACTOR }\end{array}$ & $\begin{array}{l}\quad \text { DIVISION: } \\
\square \text { State } \\
\square \text { Federal } \\
\text { X DOE } \\
\square \text { RCRA } \\
\square \text { TPA Number }\end{array}$ & $\begin{array}{l}\text { DELIVERABLE: } \\
\text { X Report } \\
\square \text { Letter } \\
\square \text { Drawings . } \\
\square \text { Other (specify) }\end{array}$ & $\begin{array}{l}\text { ADDRESS TO: } \\
\square \text { DOE-HQ } \\
\text { X DOE-RL } \\
\square \text { Other (specify) }\end{array}$ \\
\hline \multicolumn{4}{|c|}{$\begin{array}{l}\text { Milestone Description: } \\
\text { Delivery of the final Fiscal Year (FY) } 1996 \text { Hanford Strategic Plan to U.S. Department of } \\
\text { Energy, Richland Operations Office (RL) Planning Integration Division (PID) for processing } \\
\text { approval by Site Manager. Contents will include: } \\
\text { - Hanford Site Mission, Vision, and Values } \\
\text { - Hanford Site Goals } \\
\text { - Sinford Site Strategies and Success Indicators } \\
\text { - Hanford Planning Assumptions } \\
\text { - EM Supplement Details (e.g., Actions, Completion Dates, and Action Assignee). }\end{array}$} \\
\hline \multicolumn{4}{|c|}{$\begin{array}{l}\text { Description of what constitutes completion of this milestone: } \\
\text { The FY } 1996 \text { Hanford Strategic Plan is initiated by written guidance from the RL-PID } \\
\text { consistent with a decision by the Hanford Quality Council. The Hanford Strategic Plan is } \\
\text { compiled by WHC in support of the Hanford Quality Council. Review comments from } \\
\text { contractors, RL, Regulators, Indian Nations, and stakeholders are resolved and the document } \\
\text { content has been finalized. Copies of the Hanford Strategic Plan will be made and distributed } \\
\text { following Site managers approval. } \\
\text { The document's quality, will be reflected by solid systems thinking, well written in } \\
\text { understandable Layman's terms, professionally formatted, grammatically correct, and concise } \\
\text { and to the point. }\end{array}$} \\
\hline \multicolumn{4}{|c|}{$\begin{array}{l}\text { The Hanford Strategic Plan will comply with requirements of RLID } 5000.16 \text { provided to the } \\
\text { contractor for information September } 6,1994 \text {, or as modified by the Hanford Quality Council. }\end{array}$} \\
\hline \multicolumn{4}{|c|}{ Milestone Description Approval } \\
\hline $\begin{array}{l}\text { Cost Account Manager } \\
\text { flef Co Cuetean } \\
\text { H. H. Yoshikawa }\end{array}$ & $\begin{array}{l}\text { Date } \\
9 / 2 \geq / 9 y\end{array}$ & $\begin{array}{l}\text { Program/Project Man } \\
\text { D. B. Pabst }\end{array}$ & - Glozlsy \\
\hline
\end{tabular}




\begin{tabular}{|c|c|c|}
\hline \multicolumn{2}{|c|}{$\begin{array}{l}\text { Westinghouse Hanford Company } \\
\text { MILESTONE DESCRIPTION SHEET }\end{array}$} & $\begin{array}{r}\text { WHC-SP-1096 } \\
09 / 23 / 94\end{array}$ \\
\hline \multicolumn{2}{|l|}{ Title: Complete Hanford Strategic Plan } & Date: $9 / 20 / 94$ \\
\hline \multicolumn{2}{|l|}{ Assigned To: H. H. Yoshikawa } & CIN: \\
\hline \multicolumn{2}{|l|}{ Program WBS Designator: 1 8.2.1.1 } & $\begin{array}{l}\text { Due Date: } \\
03 / 31 / 95\end{array}$ \\
\hline \multicolumn{2}{|l|}{ Control Number: PSS-95-101 } & Rev: 0 \\
\hline $\begin{array}{l}\text { Program Element Manager } \\
\text { Off Seleclecewa } \\
\text { H. H. Yoshikawa }\end{array}$ & \multicolumn{2}{|c|}{ DOE Program Manager } \\
\hline \multicolumn{3}{|c|}{ 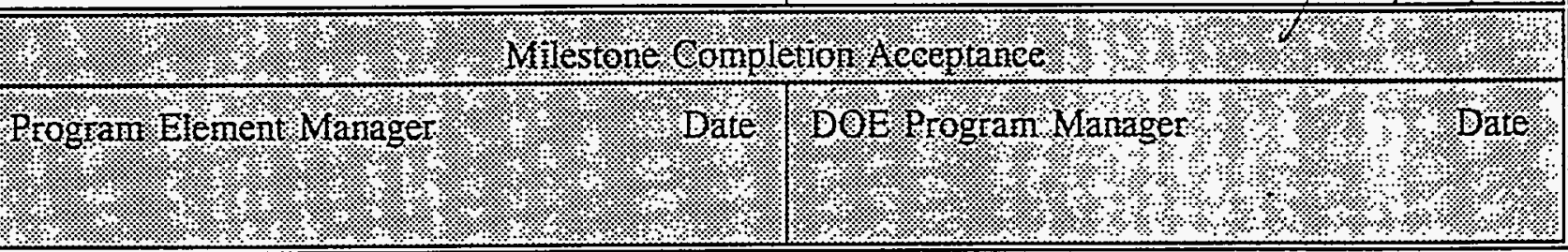 } \\
\hline
\end{tabular}




\begin{tabular}{|c|c|c|c|}
\hline \multicolumn{4}{|c|}{$\begin{array}{l}\text { Westinghouse Hanford Company } \\
\text { MILESTONE DESCRIPTION ȘHEET }\end{array}$} \\
\hline \multicolumn{3}{|c|}{$\begin{array}{l}\text { Title: Proposal for High-Level Work Breakdown Structure to Planning } \\
\text { Integration Division }\end{array}$} & Date: $9 / 20 / 94$ \\
\hline \multicolumn{3}{|c|}{ Assigned To: S. M. O'Toole } & CIN: \\
\hline \multicolumn{3}{|c|}{ Program WBS Designator: $1 \cdot 8 \cdot 2 \cdot 1.2 .1$} & $\begin{array}{l}\text { Due Date: } \\
\text { 11/29/94 }\end{array}$ \\
\hline \multicolumn{3}{|c|}{ Control Number: PSS-95-200 } & Rev: 0 \\
\hline $\begin{array}{l}\text { MILESTONE TYPE: } \\
\square \text { DOE-HQ } \\
\text { X DOE-RL } \\
\square \text { CONTRACTOR }\end{array}$ & $\begin{array}{l}\quad \text { DIVISION: } \\
\square \text { State } \\
\square \text { Federal } \\
\text { X DOE } \\
\square \text { RCRA } \\
\square \text { TPA Number }\end{array}$ & $\begin{array}{l}\text { DELIVERABLE: } \\
\square \text { Report } \\
\square \text { Letter } \\
\square \text { Drawings } \\
\text { X Other (specify) } \\
\quad \text { Presentation }\end{array}$ & $\begin{array}{l}\text { ADDRESS TO: } \\
\square \text { DOE-HQ } \\
\text { X DOE-RL } \\
\square \text { Other (specify) }\end{array}$ \\
\hline \multicolumn{4}{|c|}{$\begin{array}{l}\text { Milestone Description: } \\
\text { This milestone consists of providing to U.S. Department of Energy, Richland Operations Office } \\
\text { (RL) - Planning Integration Division (PID) an Fiscal Year } 1995 \text { High-level RL Work } \\
\text { Breakdown Structure (WBS), based on DOE guidance. RLID } 5000.2 \text { and } 5000.12 \text { define } \\
\text { Hanford Mission Plan: Site Guidance--A comprehensive definition of total Site mission and } \\
\text { issues as outlined in the Hanford Strategic Plan including a Site WBS which defines essential } \\
\text { RL programs. The final WBS will be included in the Hanford Mission Plan, Volume 1, Site } \\
\text { Guidance (refer to PSS-95-202 and 203). }\end{array}$} \\
\hline \multicolumn{4}{|c|}{$\begin{array}{l}\text { Description of what constitutes completion of this milestone: } \\
\text { This commitment is completed by presentation of the proposed High-Level WBS, to RL-PID } \\
\text { staff. The presentation will include slides and handouts of the High-Level WBS. The } \\
\text { presentation will consist of a proposal for the top } 3 \text { levels of the WBS across the Site. The } \\
\text { WBS proposal will show linkages to the Site Product Breakdown Structure. A crosswalk will } \\
\text { be included in the presentation. }\end{array}$} \\
\hline \multicolumn{4}{|c|}{ Milestone Description Approval } \\
\hline \multicolumn{4}{|c|}{ 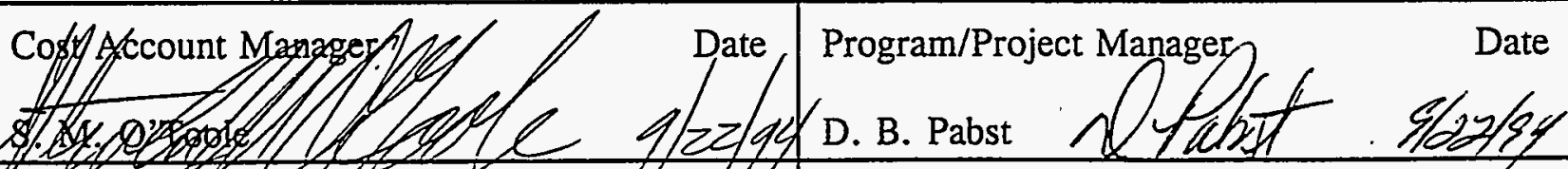 } \\
\hline \multicolumn{4}{|c|}{ 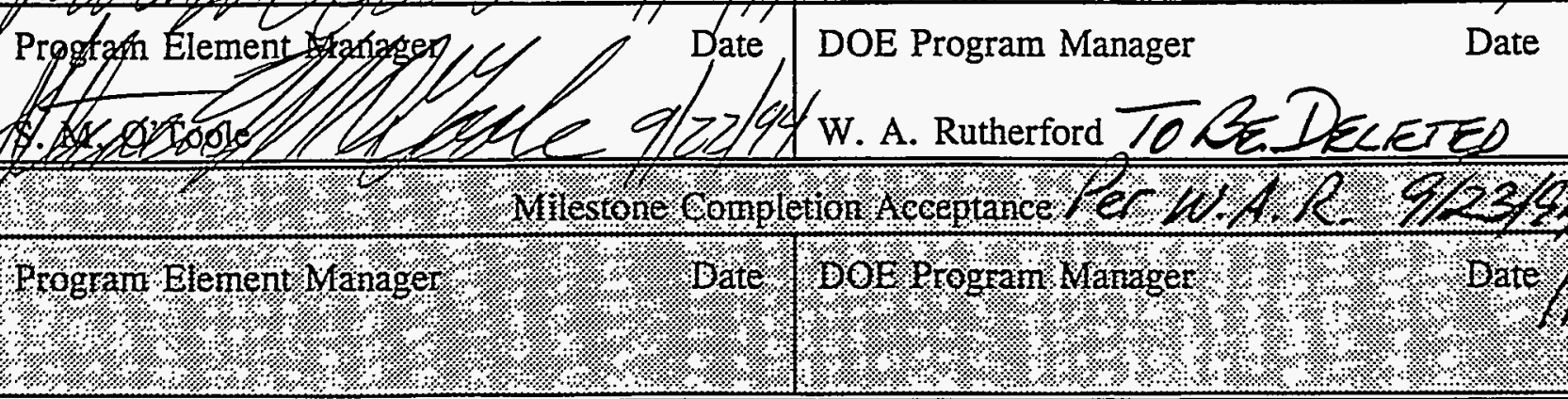 } \\
\hline
\end{tabular}




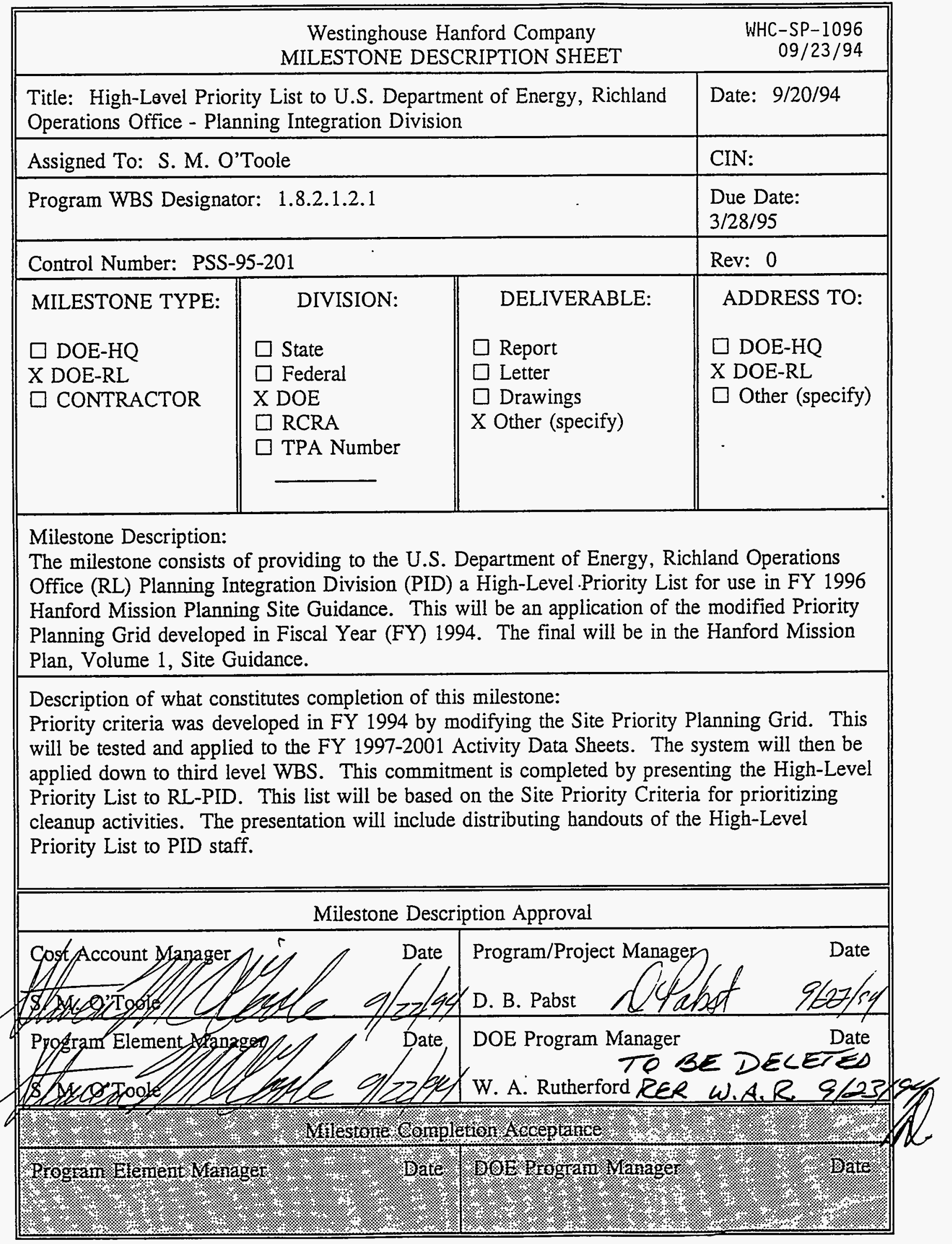




\begin{tabular}{||l|l||}
\hline \multicolumn{3}{|c|}{ Westinghouse Hanford Company } & \multicolumn{1}{|c||}{ MHC-SP-1096 } \\
$09 / 23 / 94$
\end{tabular}




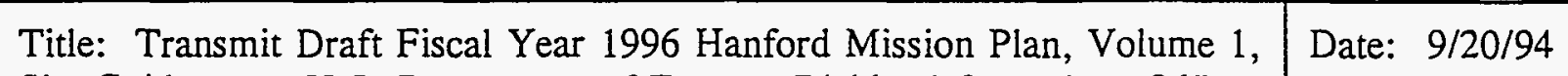
Site Guidance to U.S. Department of Energy, Richland Operations Office for Review

Assigned To: S. M. O'Toole

Program WBS Designator: 1.8.2.1.2.1

Control Number: PSS-95-202

Description of what constitutes completion of this milestone:

Prepare and transmit to RL Planning Integration Division (PID) copies of the draft FY 1996 Hanford Mission Plan (HMP), Volume 1. The FY 1996 document will be an update based on information developed since issuance of the FY 1995 HMP. It will provide the technical guidance and direction to Hanford Site programs on which to base their planning for FY 1996 and beyond. The primary sources of information include the 1994 Hanford Strategic Plan; FY 1995 HMP; systems engineering activities; program rebaselining; Tri-Party Agreement renegotiations; and public/regulator/Indian nation/stakeholder input.

The document's quality, will be reflected by and coordinated with programs, solid systems thinking, aligned and consistent with other planning documentation (e.g., the Hanford Strategic Plan), well written in understandable Layman's term, professionally formatted, grammatically correct, and concise and to the point.

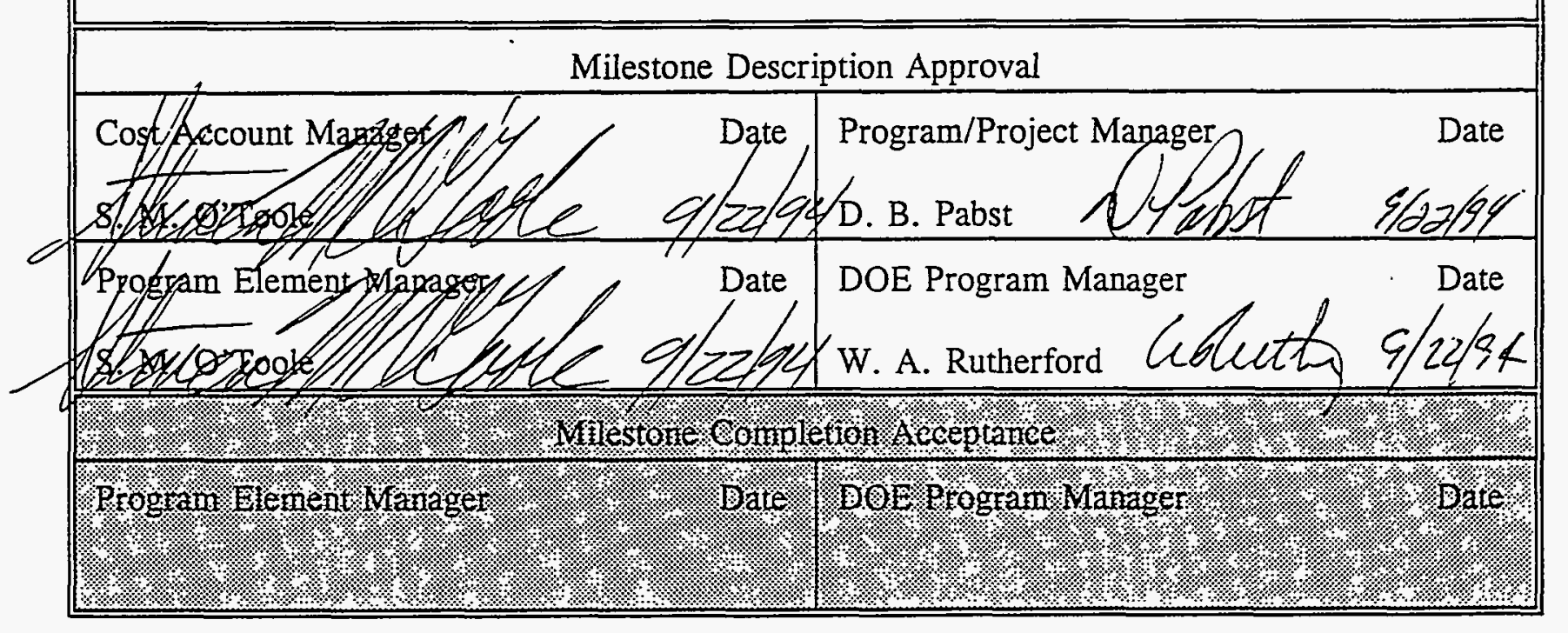




\begin{tabular}{|c|c|c|c|}
\hline & \multicolumn{2}{|c|}{$\begin{array}{l}\text { Westinghouse Hanford Company } \\
\text { MILESTONE DESCRIPTION SHEET }\end{array}$} & $\begin{array}{r}\text { WHC-SP-1096 } \\
09 / 23 / 94\end{array}$ \\
\hline \multicolumn{3}{|c|}{$\begin{array}{l}\text { Title: Complęte Fiscal Year } 1996 \text { Hanford Mission Plan, Volume 1, Site } \\
\text { Guidance }\end{array}$} & Date: $9 / 20 / 94$ \\
\hline \multicolumn{3}{|c|}{ Assigned To: S. M. O'Toole } & CIN: \\
\hline \multicolumn{3}{|c|}{ Program WBS Designator: 1 8.2.1.2.1 } & Due Date: $3 / 31 / 95$ \\
\hline \multicolumn{3}{|c|}{ Control Number: PSS-95-203 } & Rev: 0 \\
\hline \multirow{2}{*}{$\begin{array}{l}\text { MILESTONE TYPE: } \\
\square \text { DOE-HQ } \\
\text { X DOE-RL } \\
\square \text { CONTRACTOR }\end{array}$} & DIVISION: & DELIVERABLE: & ADDRESS TO: \\
\hline & $\begin{array}{l}\square \text { State } \\
\square \text { Federal } \\
\text { X DOE } \\
\square \text { RCRA } \\
\square \text { TPA Number }\end{array}$ & $\begin{array}{l}\text { X Report } \\
\square \text { Letter } \\
\square \text { Drawings } \\
\square \text { Other (specify) }\end{array}$ & $\begin{array}{l}\square \text { DOE-HQ } \\
\text { X DOE-RL } \\
\square \text { Other (specify) }\end{array}$ \\
\hline \multicolumn{4}{|c|}{$\begin{array}{l}\text { Milestone Description: } \\
\text { Transmit an U.S. Department of Energy, Richland Operations Office (RL) reviewed draft } \\
\text { Fiscal Year (FY) } 1996 \text { Hanford Mission Plan (HMP), Volume 1, Site Guidance to RL for } \\
\text { approval. Content of the document (consistent with PSS-95-202) is as follows: }\end{array}$} \\
\hline \multicolumn{4}{|c|}{$\begin{array}{l}\text { Resolve Comments Received as a Result of RL Review of Draft FY } 1996 \text { HMP, Volume 1, } \\
\text { Site Guidance. }\end{array}$} \\
\hline
\end{tabular}




\begin{tabular}{|c|c|}
\hline $\begin{array}{l}\text { Westinghouse Hanford Company } \\
\text { MILESTONE DESCRIPTION SHEET }\end{array}$ & $\begin{array}{r}W H C-S P-1096 \\
09 / 23 / 94\end{array}$ \\
\hline $\begin{array}{l}\text { Title: Complete Fiscal Year } 1996 \text { Hanford Mission Plan, Volume 1, Site } \\
\text { Guidance }\end{array}$ & Date: $9 / 20 / 94$ \\
\hline Assigned To: S. M. O'Toole & CIN: \\
\hline Program WBS Designator: 1.8 .2 .1 .2 .1 & $\begin{array}{l}\text { Due Date: } \\
7105 / 95-3 / 31 / 95\end{array}$ \\
\hline Control Number: PSS-95-203 & Rev: 0 \\
\hline \multicolumn{2}{|c|}{$\begin{array}{l}\text { Description of what constitutes completion of this milestone: } \\
\text { Provide final FY } 1996 \text { HMP, Volume } 1 \text { to RL for RL Manager's approval. All RL comments } \\
\text { received by March } 21,1995 \text {, will be dispositioned prior to delivery of this milestone. } \\
\text { The document's quality, will be reflected by and coordinated with programs, solid systems } \\
\text { thinking, aligned and consistent with other planning documentation, well written in } \\
\text { understandable Layman's terms, professionally formatted, grammatically correct, and concise } \\
\text { and to the point. }\end{array}$} \\
\hline \multicolumn{2}{|l|}{ Milestone Description Approval } \\
\hline Date ${ }_{\text {Program/Project Manag }}$ & $\begin{array}{l}\text { Date } \\
\text { S/23/94 }\end{array}$ \\
\hline Prosym Element & ith $\varepsilon / z 2 \beta p$ \\
\hline 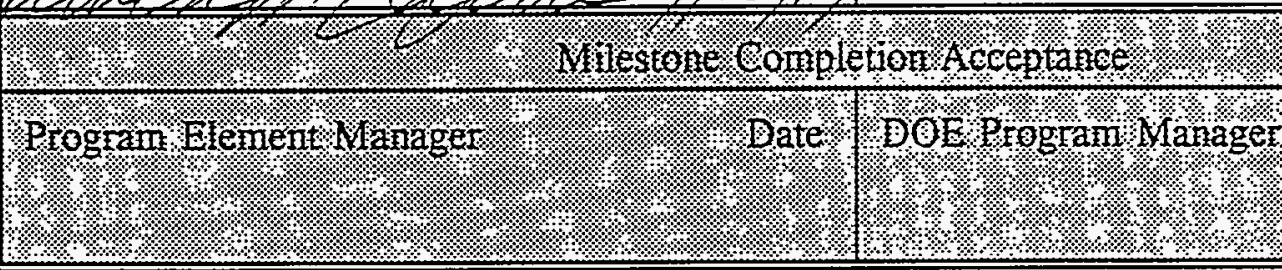 & (1.7. \\
\hline
\end{tabular}

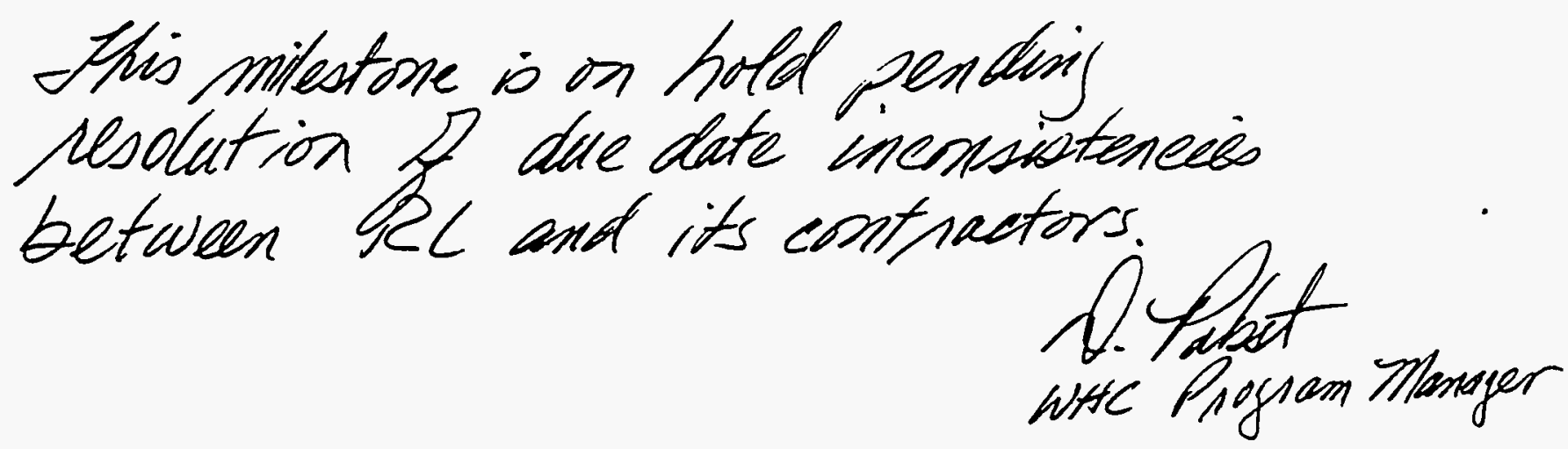




\begin{tabular}{|c|c|c|c|}
\hline & \multicolumn{2}{|c|}{$\begin{array}{l}\text { Westinghouse Hanford Company } \\
\text { MILESTONE DESCRIPTION SHEET }\end{array}$} & $\begin{array}{r}\text { WHC-SP-1096 } \\
09 / 23 / 94\end{array}$ \\
\hline \multicolumn{3}{|c|}{ Title: Complete Hanford Strategic Analysis Addendum for 1995} & Date: $9 / 20 / 94$ \\
\hline \multicolumn{3}{|c|}{ Assigned To: S. M. O'Toole } & CIN: \\
\hline \multicolumn{3}{|c|}{ Program WBS Designator: $1 \cdot 8 \cdot 2 \cdot 1 \cdot 2.1$} & $\begin{array}{l}\text { Due Date: } \\
\text { 9/30/95 }\end{array}$ \\
\hline \multicolumn{3}{|c|}{ Control Number: PSS-95-204 } & Rev: 0 \\
\hline $\begin{array}{l}\text { MILESTONE TYPE: } \\
\square \text { DOE-HQ } \\
\square \text { DOE-RL } \\
\text { X CONTRACTOR }\end{array}$ & $\begin{array}{l}\quad \text { DIVISION: } \\
\quad \square \text { State } \\
\square \text { Federal } \\
\text { X DOE } \\
\square \text { RCRA } \\
\square \text { TPA Number }\end{array}$ & $\begin{array}{l}\text { DELIVERABLE: } \\
\text { X Report } \\
\square \text { Letter } \\
\square \text { Drawings } \\
\square \text { Other (specify) }\end{array}$ & $\begin{array}{l}\text { ADDRESS TO: } \\
\square \text { DOE-HQ } \\
\square \text { DOE-RL } \\
\text { X. Other (specify) } \\
\text { Systems } \\
\text { Engineering } \\
(1.8 .2 .4)\end{array}$ \\
\hline \multicolumn{4}{|c|}{$\begin{array}{l}\text { Milestone Description: } \\
\text { Provide to WHC Systems Engineering (1.8.2.4) an update of the } 1994 \text { Hanford Strategic } \\
\text { Analysis (HSA) in the form of an addendum prepared in Fiscal Year (FY) 1995. Contents will } \\
\text { include sitewide material balance, ROM cost and schedule. }\end{array}$} \\
\hline \multicolumn{4}{|c|}{$\begin{array}{l}\text { Description of what constitutes completion of this milestone: } \\
\text { This commitment is completed by transmittal of one hard copy of the final HSA Addendum } \\
\text { document containing additional alternative sitewide material balances, ROM cost and schedule } \\
\text { to Systems Engineering. Systems Engineering will provide the necessary input (technical } \\
\text { baseline, case description) to initiate the analysis and produce the HSA Addendum. The HSA } \\
\text { addendum will then represent a technical document reflecting the current alternative for } \\
\text { material disposition at Hanford the update technical baseline and one optimization case. The } \\
\text { content and level of detail will be consistent with the Hanford Strategic Analysis Study, } \\
\text { WHC-EP-0549. This document will be suitable for release to the public. } \\
\text { The document will reflect the following: } \\
\text { - Technically Sound and Tied to Systems Engineering } \\
\text { Well Written } \\
\text { - Grammatically Correct and Professionally Formatted }\end{array}$} \\
\hline \multicolumn{4}{|c|}{ Milestone Description Approval } \\
\hline costs Accou & & Program/Project Man & If $9 / 22 / 54$ \\
\hline
\end{tabular}




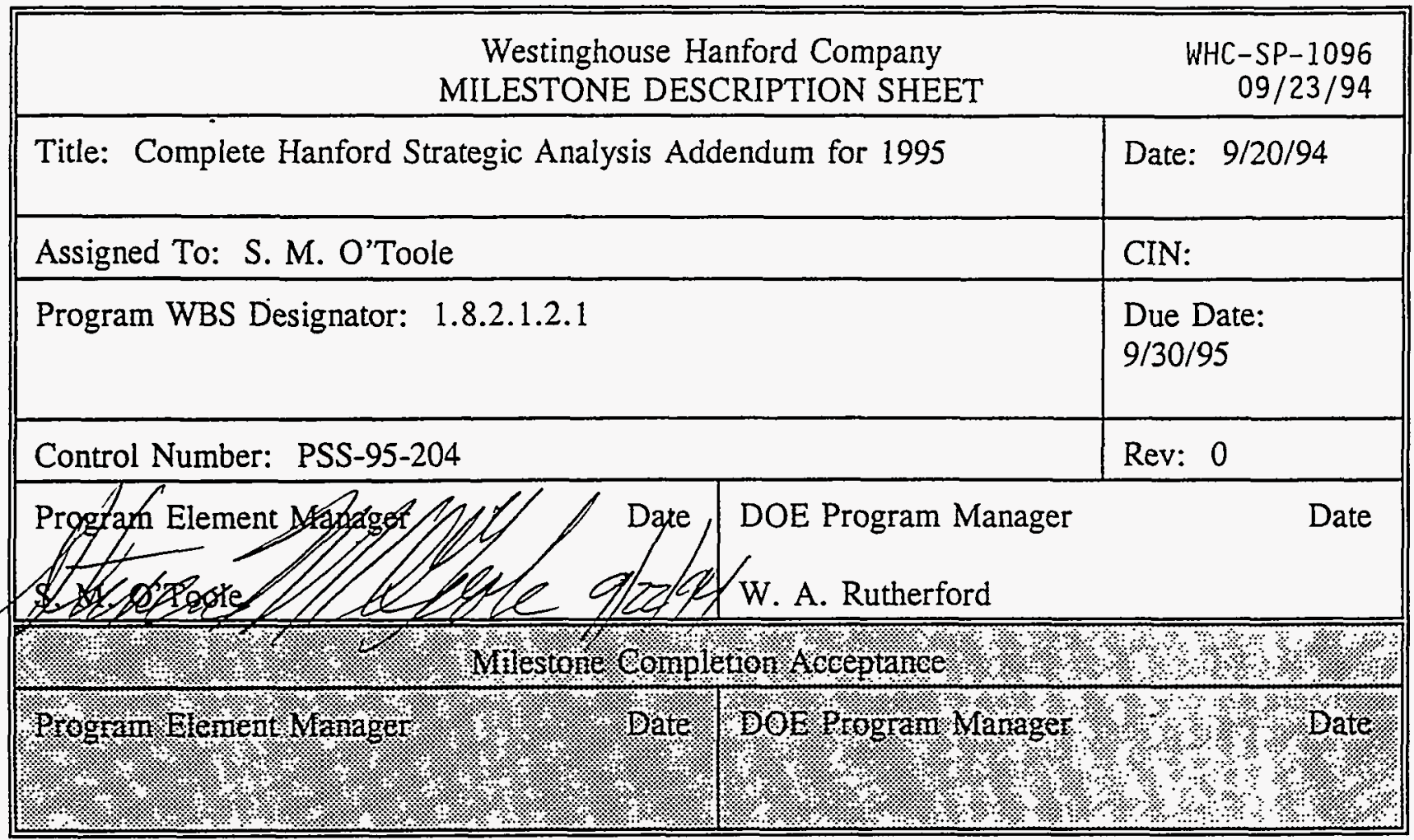




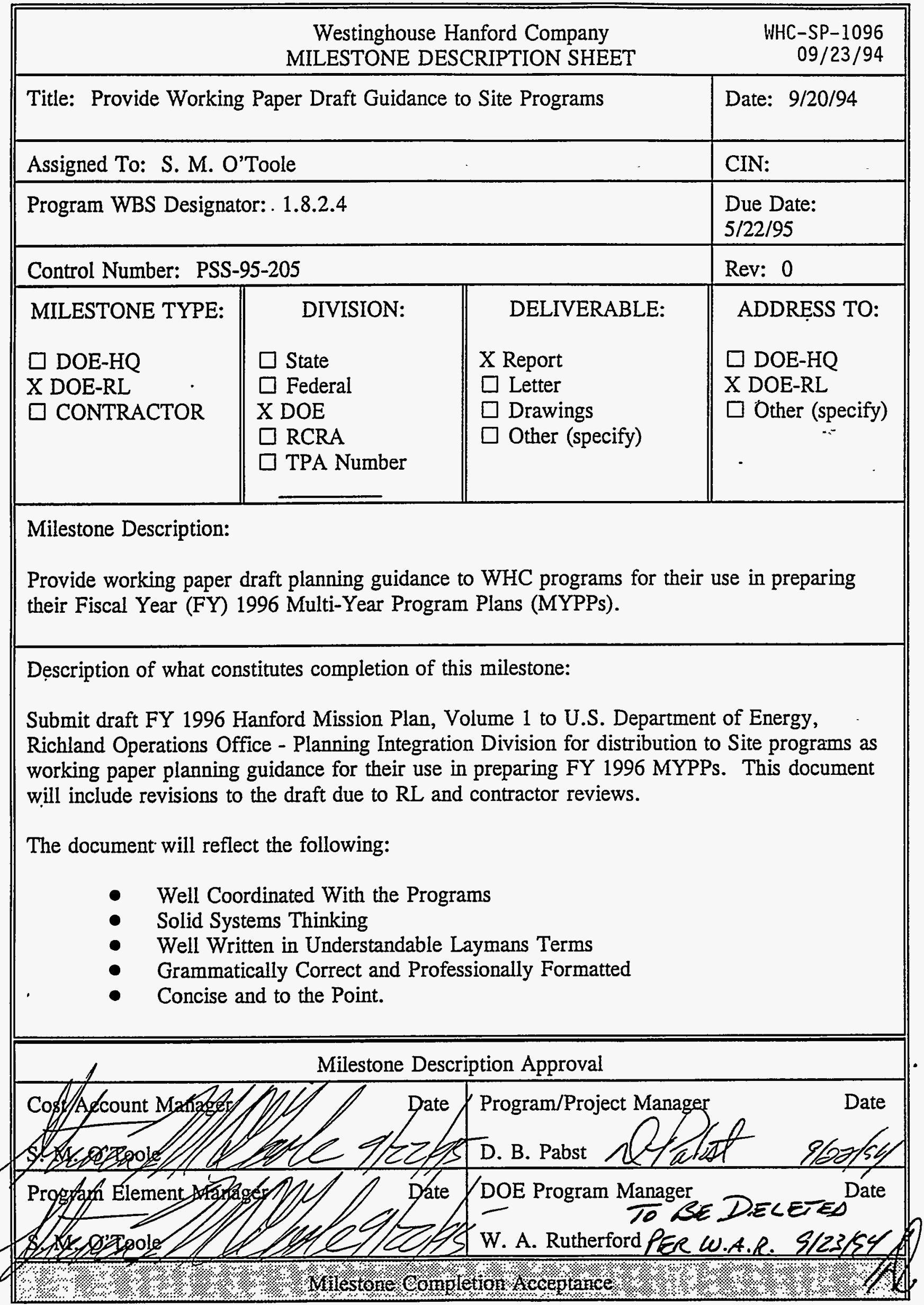




\begin{tabular}{|c|c|}
\hline $\begin{array}{l}\text { Westinghouse Hanford Company } \\
\text { MILESTONE DESCRIPTION SHEET }\end{array}$ & $\begin{array}{r}\text { WHC-SP-1096 } \\
09 / 23 / 94 \\
\end{array}$ \\
\hline Title: Provide Working Paper Draft Guidance to Site Programs & Date: $9 / 20 / 94$ \\
\hline Assigned To: S. M. O'Toole & CIN: \\
\hline Program WBS Designator: 1.8 .2 .4 & $\begin{array}{l}\text { Due Date: } \\
5 / 22 / 95\end{array}$ \\
\hline Control Number: PSS-95-205 & Rev: 0 \\
\hline \multicolumn{2}{|c|}{ Progran Elengent quanger } \\
\hline
\end{tabular}




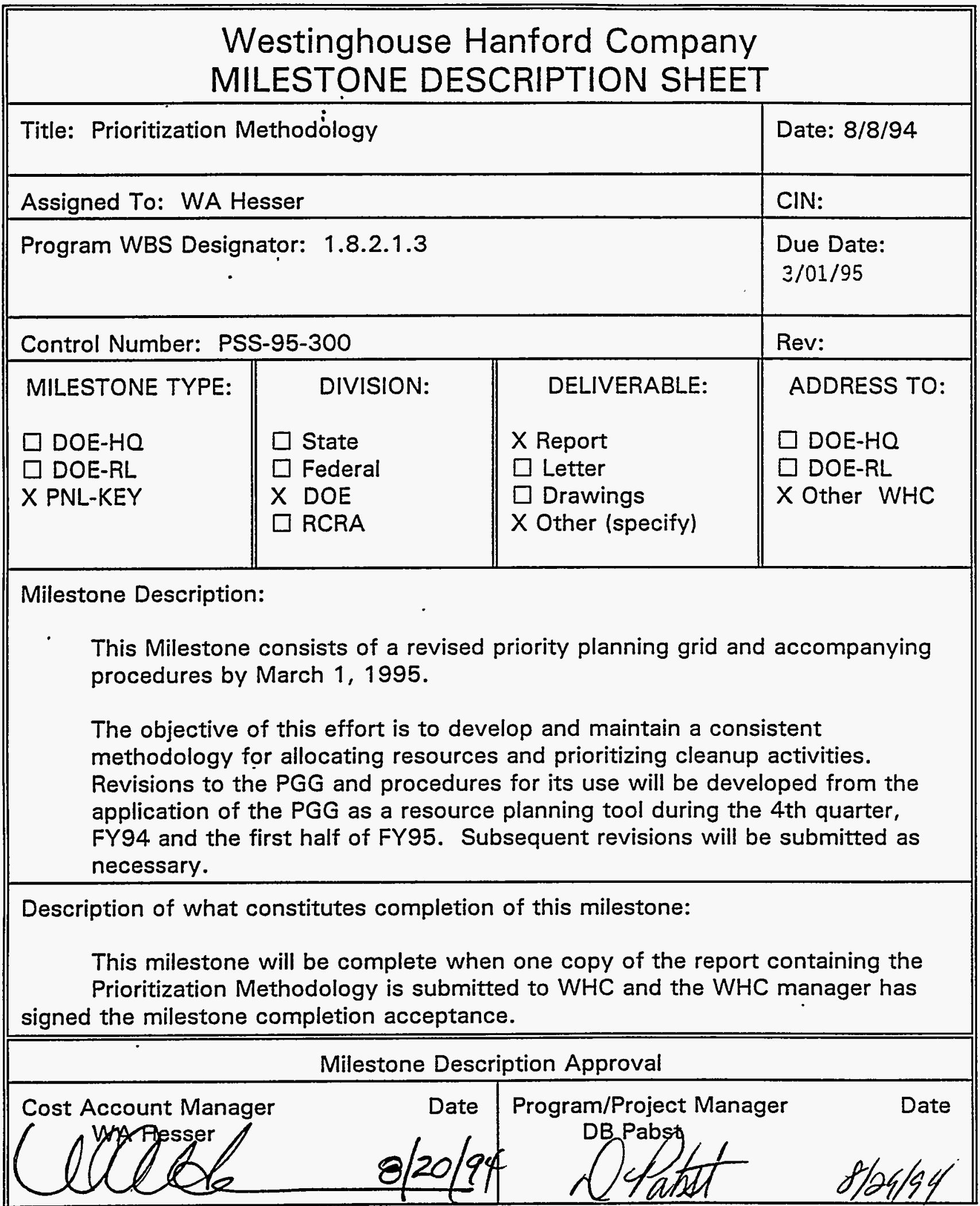




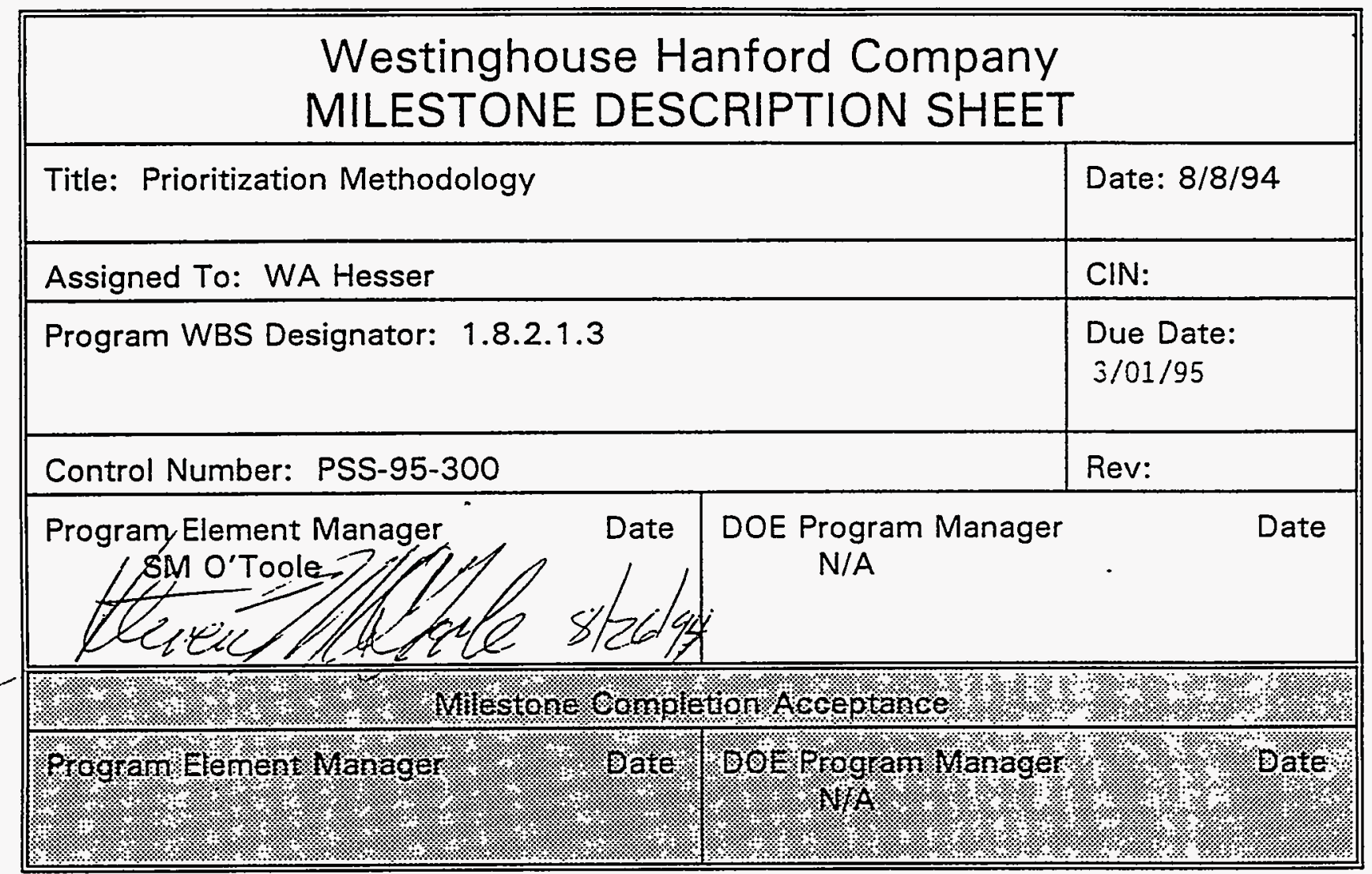




\section{Westinghouse Hanford Company MILESTONE DESCRIPTION SHEET}

\begin{tabular}{|c|c|c|c|}
\hline \multicolumn{3}{|c|}{ Title: Analysis of Risks and Costs at Hanford } & Date: $8 / 8 / 94$ \\
\hline \multicolumn{3}{|c|}{ Assigned To: WA Hesser } & CIN: \\
\hline \multicolumn{3}{|c|}{ Program WBS Designator: 1.8.2.1.3 } & $\begin{array}{l}\text { Due Date: } \\
4 / 30 / 95\end{array}$ \\
\hline \multicolumn{3}{|c|}{ Control Number: PSS-95-301 } & Rev: \\
\hline MILESTONE TYPE: & DIVISION: & DELIVERABLE: & ADDRESS TO: \\
\hline $\begin{array}{l}\square \text { DOE-HQ } \\
\text { X DOE-RL } \\
\square \text { CONTRACTOR }\end{array}$ & $\begin{array}{l}\square \text { State } \\
\square \text { Federal } \\
X \text { DOE } \\
\square \text { RCRA }\end{array}$ & $\begin{array}{l}\text { X Report } \\
\square \text { Letter } \\
\square \text { Drawings } \\
\square \text { Other (specify) }\end{array}$ & $\begin{array}{l}\square \text { DOE-HQ } \\
\text { X DOE-RL } \\
\square \text { Other } \\
\text { (specify) }\end{array}$ \\
\hline
\end{tabular}

Milestone Description:

The objective of this analysis is to review the Site cleanup strategy from a cost-risk-benefit perspective and to determine whether or not the Site is complying with $\mathrm{HQ}$ guidance to allocate its resources to those areas of greatest risk. A methodology for relating the different types of environmental, safety, and health risks will be developed, based upon work done in FY94. This analysis will consider cost and schedule baselines from FY94 and FY95 budget allocations and the technical baseline developed by systems engineering. It will also consider risk baseline information developed by IRAP and program activities as part of the risk management system. The product of the research will be a report analyzing the Site work, with recommendations for priority of effort and resource allocation.

Description of what constitutes completion of this milestone:

This milestone will be completed when one copy of the report containing the Analysis of Risks and Costs at Hanford is submitted to RL and the RL manager has signed the milestone completion acceptance.

\begin{tabular}{|c|c|c|c|}
\hline \multicolumn{4}{|c|}{ Milestone Description Approval } \\
\hline Cost Account Manager & $\begin{array}{l}\text { Date } \\
\text { ol94 }\end{array}$ & Program/Project Manager & $\begin{array}{l}\text { Date } \\
194\end{array}$ \\
\hline
\end{tabular}




\section{Westinghouse Hanford Company MILESTONE DESCRIPTION SHEET}

Title: Analysis of Risks and Costs at Hanford

Date: $8 / 8 / 94$

Assigned To: WA Hesser

Program WBS Designator: 1.8.2.1.3

CIN:

Due Date:

$4 / 30 / 95$

Control Number: PSS-95-301

Rev:

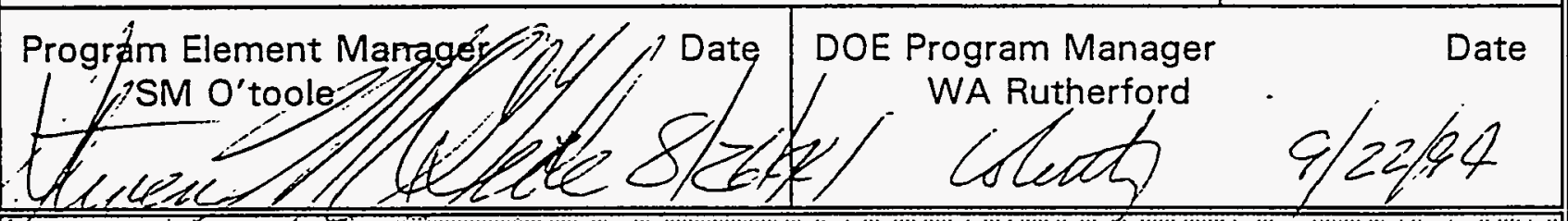

Witestone Conpletion Aceptance 


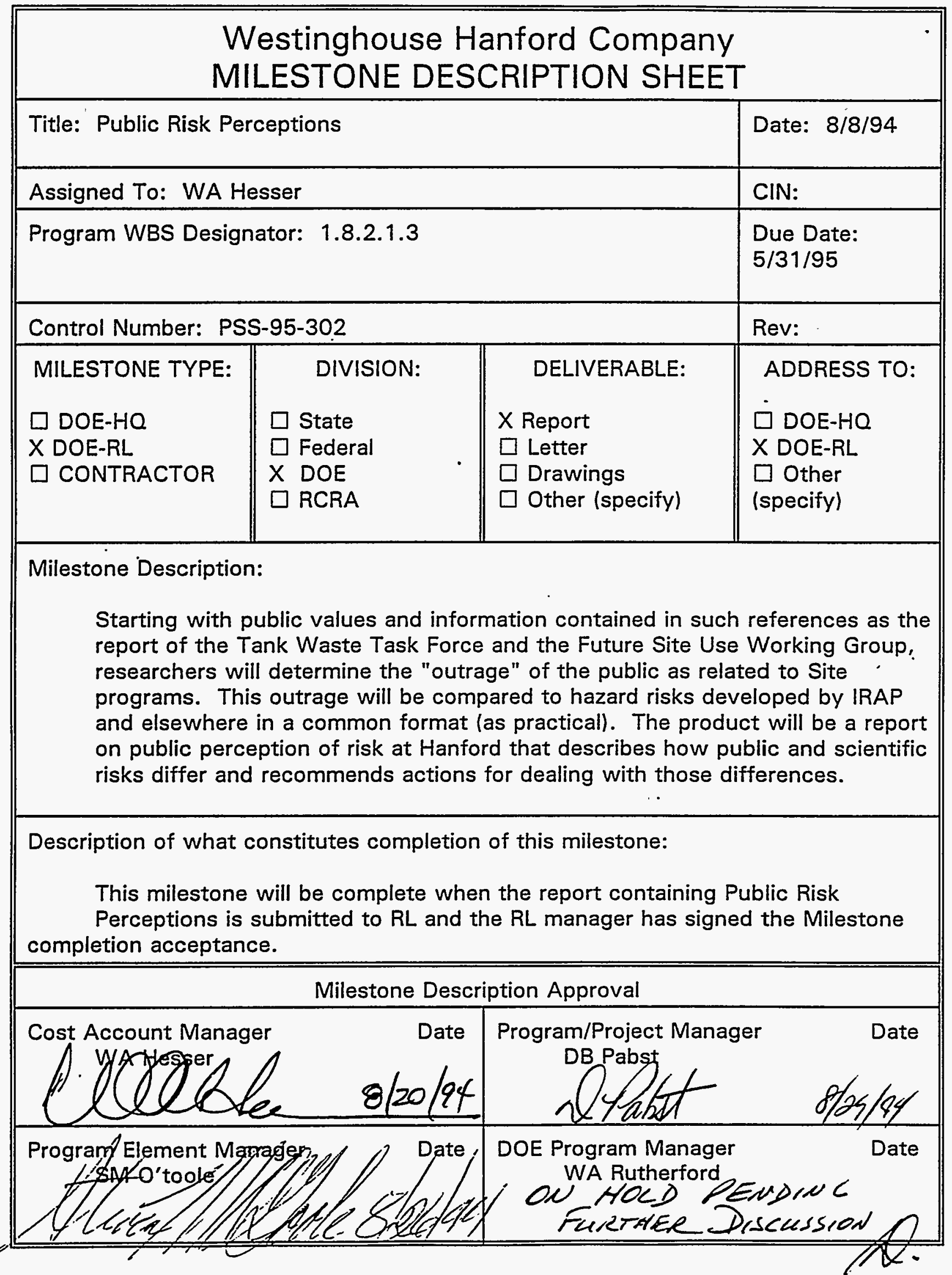




\begin{tabular}{|c|c|}
\hline \multicolumn{2}{|c|}{$\begin{array}{l}\text { Westinghouse Hanford Company } \\
\text { MILESTONE DESCRIPTION SHEET }\end{array}$} \\
\hline Title: Public Risk Perceptions & Date: $8 / 8 / 94$ \\
\hline Assigned To: WA Hesser & CIN: \\
\hline Program WBS Designator: 1.8.2.1.3 & $\begin{array}{l}\text { Due Date: } \\
5 / 31 / 95\end{array}$ \\
\hline Control Number: PSS-95-302 & Rev: \\
\hline \multicolumn{2}{|c|}{ 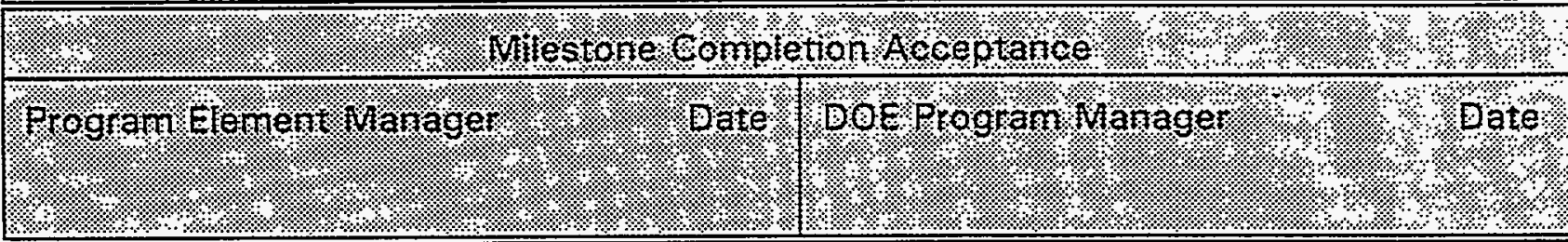 } \\
\hline
\end{tabular}




\begin{tabular}{|c|c|c|c|}
\hline \multicolumn{4}{|c|}{$\begin{array}{l}\text { Westinghouse Hanford Company } \\
\text { MILESTONE DESCRIPTION SHEET }\end{array}$} \\
\hline \multicolumn{3}{|c|}{ Risk Management in Systems Engineering } & Date:8/8/94 \\
\hline \multicolumn{3}{|c|}{ Assigned To: WA Hesser } & CIN: \\
\hline \multicolumn{3}{|c|}{ Program WBS Designator: 1.8.2.1.3 } & $\begin{array}{c}\text { Due Date: } \\
3 / 31 / 95\end{array}$ \\
\hline \multicolumn{3}{|c|}{ Control Number: PSS-95-304 } & Rev: \\
\hline $\begin{array}{l}\text { MILESTONE TYPE: } \\
\square \text { DOE-HO } \\
\square \text { DOE-RL } \\
\times \text { PNL-KEY }\end{array}$ & $\begin{array}{l}\text { DIVISION: } \\
\square \text { State } \\
\square \text { Federal } \\
\square \text { DOE } \\
\square \text { RCRA }\end{array}$ & $\begin{aligned} & \text { DELIVERABLE: } \\
& \times \text { Report } \\
& \square \text { Letter } \\
& \square \text { Drawings } \\
& \square \text { Other (specify) }\end{aligned}$ & $\begin{array}{l}\text { ADDRESS TO: } \\
\square \text { DOE-HO } \\
\square \text { DOE-RL } \\
X \text { WHC }\end{array}$ \\
\hline \multicolumn{4}{|c|}{$\begin{array}{l}\text { The risk management policy requires that alternatives to address risk be } \\
\text { developed using systems engineering (and similar) techniques. Then, preferred } \\
\text { alternatives will be chosen based upon consistent, risk-based methodology. } \\
\text { PNL researchers will assist systems engineering in this effort through the } \\
\text { application of decision analysis and risk-based decision techniques. Perceived } \\
\text { risk, stakeholder values, and socio-economic implications will be considered in } \\
\text { the decision process. }\end{array}$} \\
\hline \multicolumn{4}{|c|}{$\begin{array}{l}\text { PNL will aiso provide support in the management of risk of the preferred } \\
\text { alternative, linkage to the Integrated Risk Assessment Program, solidification of } \\
\text { public values, concerns, and priorities for guidance to programs to use in their } \\
\text { systems engineering processes, and training at the program and project level in } \\
\text { risk management techniques. }\end{array}$} \\
\hline \multicolumn{4}{|c|}{$\begin{array}{l}\text { The primary product is assistance on a continuing basis to systems } \\
\text { engineering, especially including the preparation of recommendations to } \\
\text { management and decision makers regarding technical alternatives. PNL will } \\
\text { also provide a report of the results of the year's activities describing the } \\
\text { lessons learned. }\end{array}$} \\
\hline
\end{tabular}




\begin{tabular}{|c|c|c|}
\hline \multicolumn{3}{|c|}{$\begin{array}{l}\text { Westinghouse Hanford Company } \\
\text { MILESTONE DESCRIPTION SHEET }\end{array}$} \\
\hline \multicolumn{2}{|l|}{ Title: Risk Management in Systems Engineering } & Date:8/8/94 \\
\hline \multicolumn{2}{|l|}{ Assigned To: WA Hesser } & CIN: \\
\hline \multicolumn{2}{|l|}{ Program WBS Designator: 1.8.2.1.3 } & $\begin{array}{c}\text { Due Date: } \\
3 / 31 / 95\end{array}$ \\
\hline \multicolumn{2}{|l|}{ Control Number: PSS-95-304 } & Rev: \\
\hline \multicolumn{3}{|c|}{$\begin{array}{l}\text { This milestone will be completed when one copy of the report containing Risk } \\
\text { Management in Systems Engineering is submitted to WHC and the WHC } \\
\text { manager has signed the milestone completion acceptance. }\end{array}$} \\
\hline \multicolumn{3}{|c|}{ Milestone Description Approval } \\
\hline Cost Account Manager & Program/Project Manager & $\begin{array}{l}\text { Date } \\
8 / 2 y / 54\end{array}$ \\
\hline Program Element Manager & 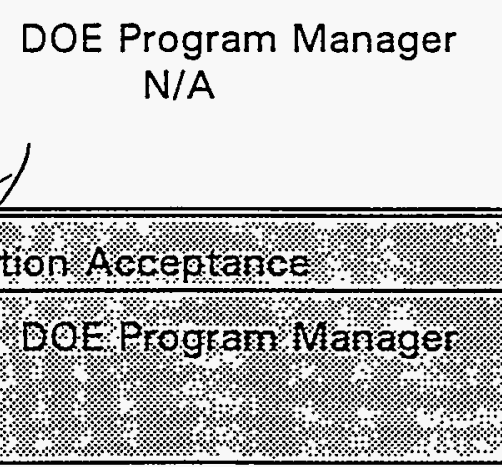 & 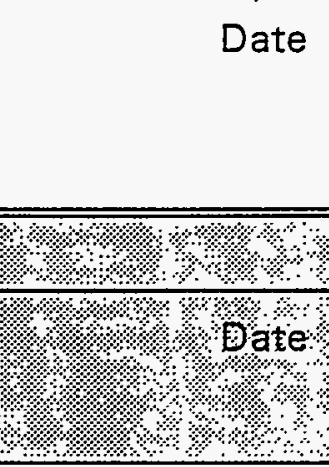 \\
\hline
\end{tabular}




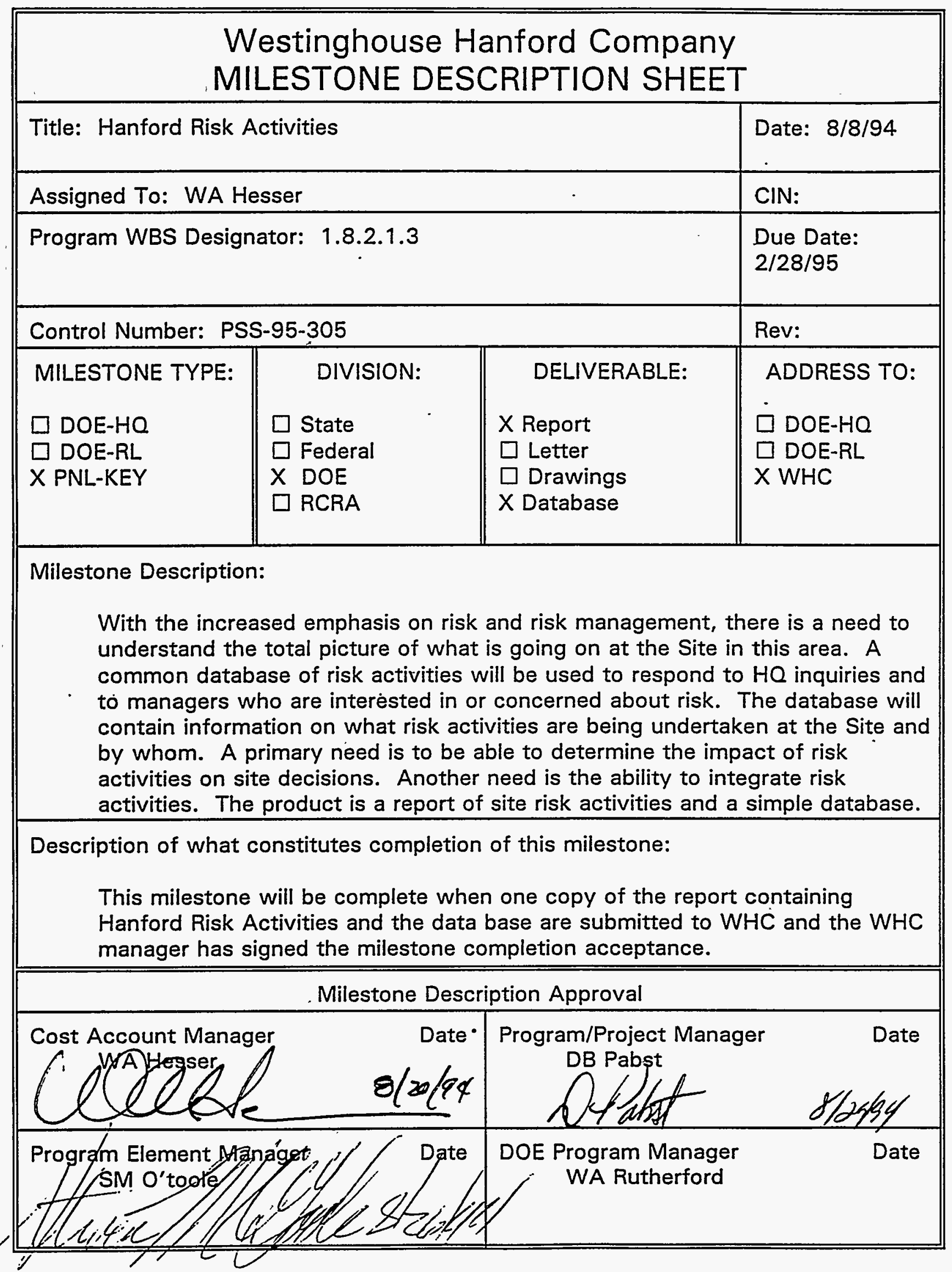




\begin{tabular}{|c|c|}
\hline \multicolumn{2}{|c|}{$\begin{array}{l}\text { Westinghouse Hanford Company } \\
\text { MILESTONE DESCRIPTION SHEET }\end{array}$} \\
\hline Title: Hanford Risk Activities & Date: $8 / 8 / 94$ \\
\hline Assigned To: WA Hesser & CIN: \\
\hline Program WBS Designator: 1.8.2.1.3 & $\begin{array}{l}\text { Due Date: } \\
2 / 28 / 95\end{array}$ \\
\hline Control Number: PSS-95-305 & Rev: \\
\hline 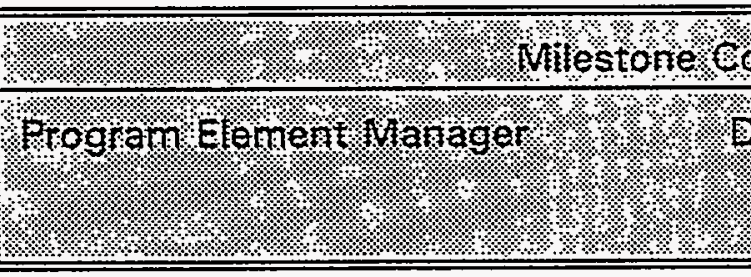 & (1) \\
\hline
\end{tabular}




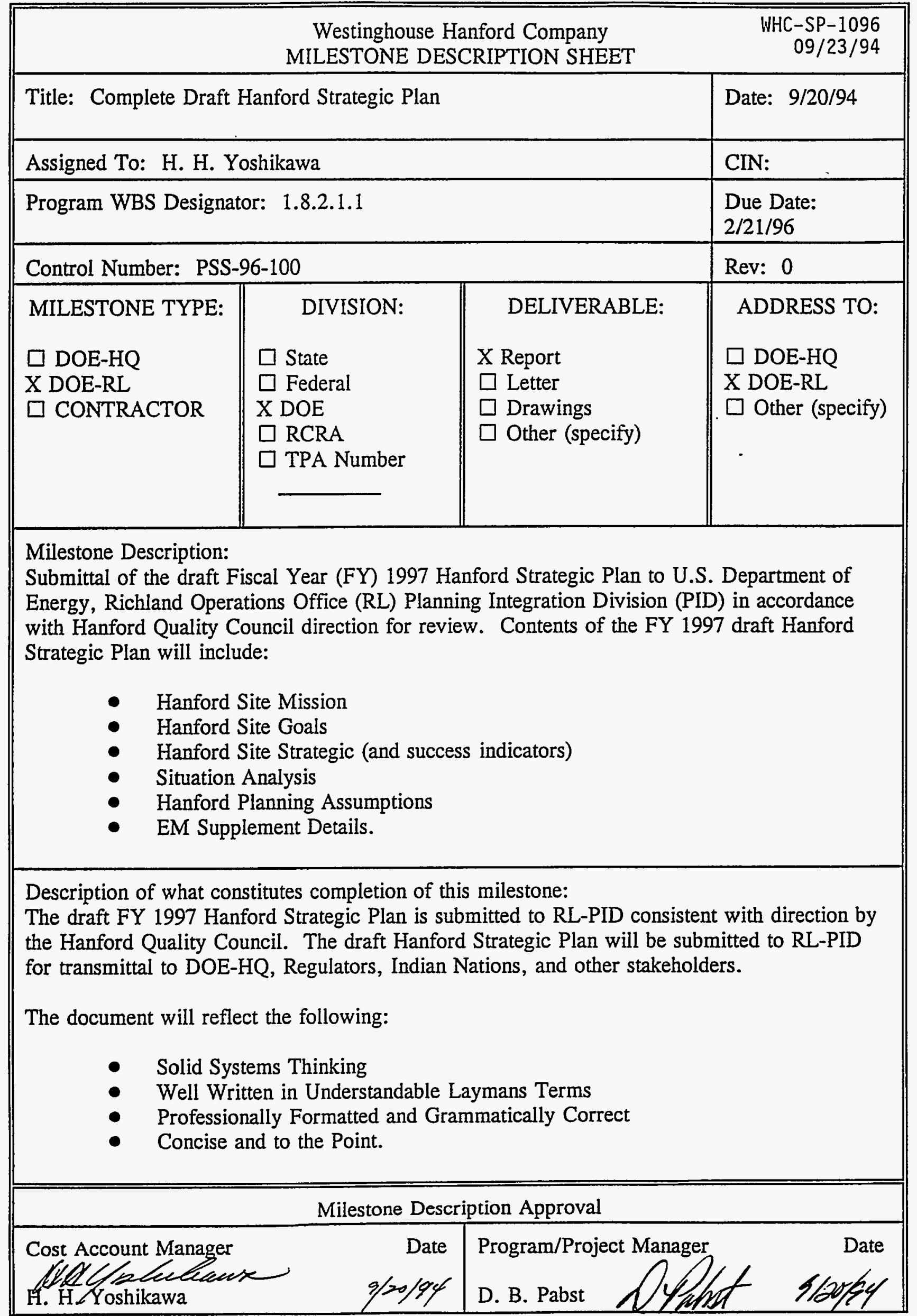




\begin{tabular}{|c|c|c|}
\hline \multicolumn{2}{|c|}{$\begin{array}{l}\text { Westinghouse Hanford Company } \\
\text { MILESTONE DESCRIPTION SHEET }\end{array}$} & $\begin{array}{r}W H C-S P-1096 \\
09 / 23 / 94\end{array}$ \\
\hline \multicolumn{2}{|l|}{ Title: Complete Draft Hanford Strategic Plan } & Date: $9 / 20 / 94$ \\
\hline \multicolumn{2}{|l|}{ Assigned To: H. H. Yoshikawa } & CIN: \\
\hline \multicolumn{2}{|l|}{ Program WBS Designator: 1 8.2.1.1 } & $\begin{array}{l}\text { Due Date: } \\
2 / 21 / 96\end{array}$ \\
\hline \multicolumn{2}{|l|}{ Control Number: PSS-96-100 } & Rev: 0 \\
\hline $\begin{array}{l}\text { Program Element Manager } \\
\text { H. H. Yoshikawa }\end{array}$ & $\begin{array}{l}\text { DOE Program Manager } \\
\text { W. A. Rutherford }\end{array}$ & Date \\
\hline \multicolumn{3}{|c|}{ 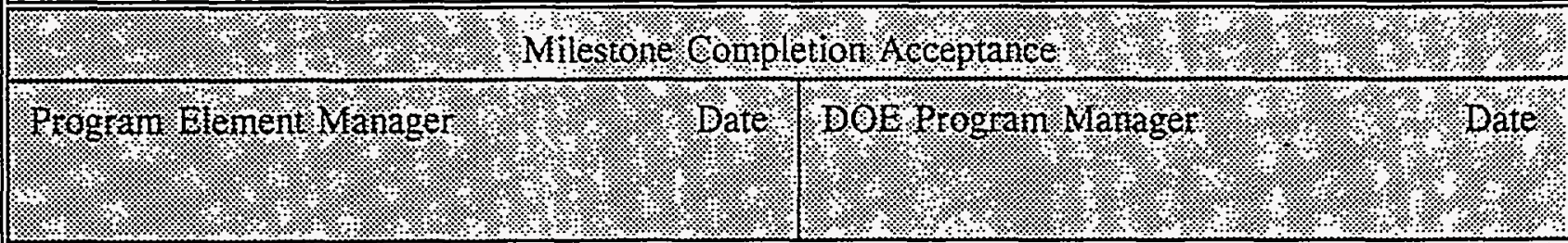 } \\
\hline
\end{tabular}




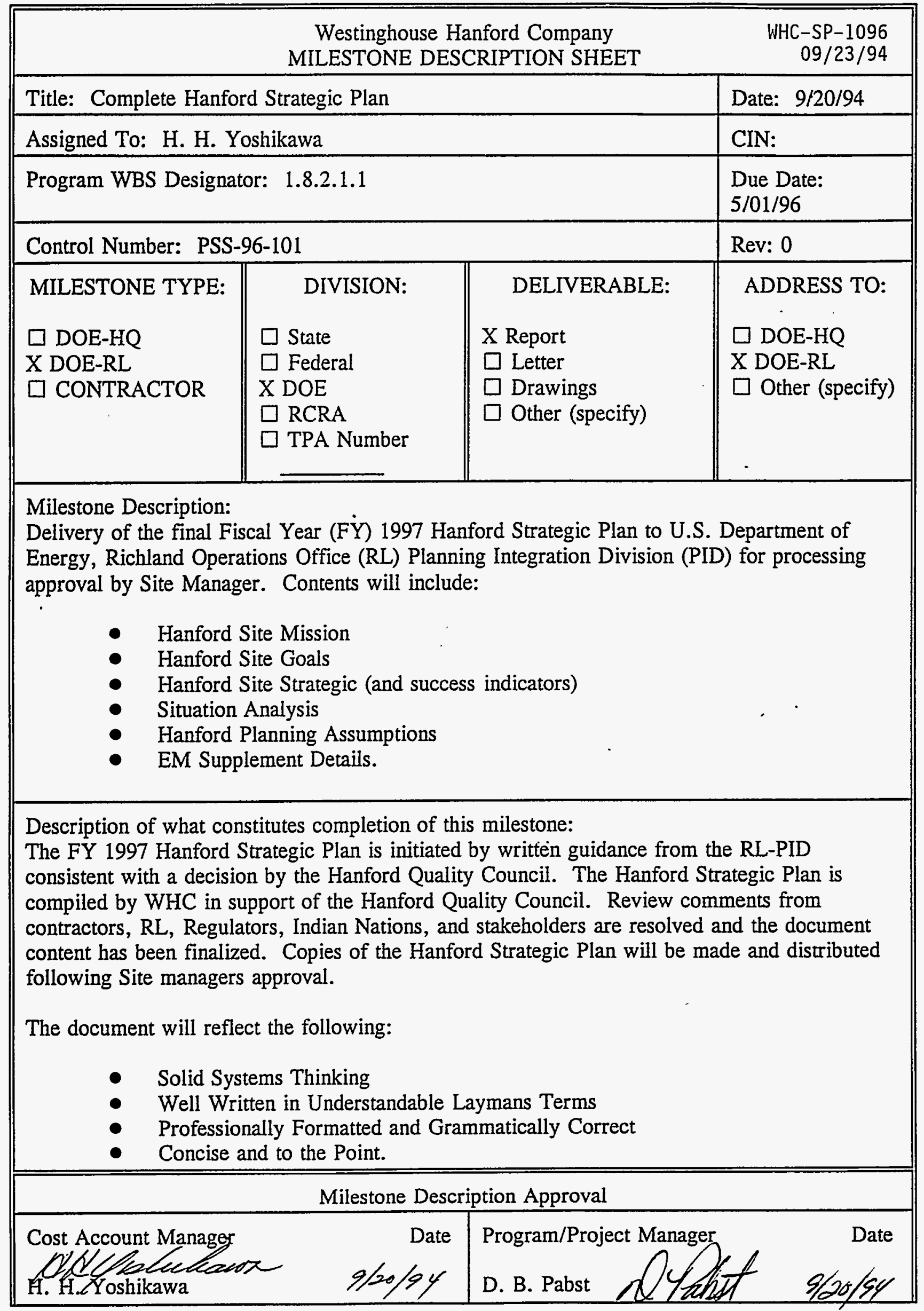




\begin{tabular}{|c|c|c|}
\hline \multicolumn{2}{|c|}{$\begin{array}{l}\text { Westinghouse Hanford Company } \\
\text { MILESTONE DESCRIPTION SHEET }\end{array}$} & $\begin{array}{r}W H C-S P-1096 \\
09 / 23 / 94\end{array}$ \\
\hline \multicolumn{2}{|l|}{ Title: Complete Hanford Strategic Plan } & Date: $9 / 20 / 94$ \\
\hline \multicolumn{2}{|l|}{ Assigned To: H. H. Yoshikawa } & CIN: \\
\hline \multicolumn{2}{|l|}{ Program WBS Designator: 1.8.2.1.1 } & $\begin{array}{l}\text { Due Date: } \\
5 / 01 / 96\end{array}$ \\
\hline \multicolumn{2}{|l|}{ Control Number: PSS-96-101 } & Rev: 0 \\
\hline $\begin{array}{l}\text { Program Element Manager } \\
\text { Date } \\
\text { H. H, Yoshikawa }\end{array}$ & $\begin{array}{l}\text { DOE Program Manager } \\
\text { W. A. Rutherford }\end{array}$ & Date \\
\hline Progran Elenent Managet & tion teceptance & (3.) \\
\hline
\end{tabular}




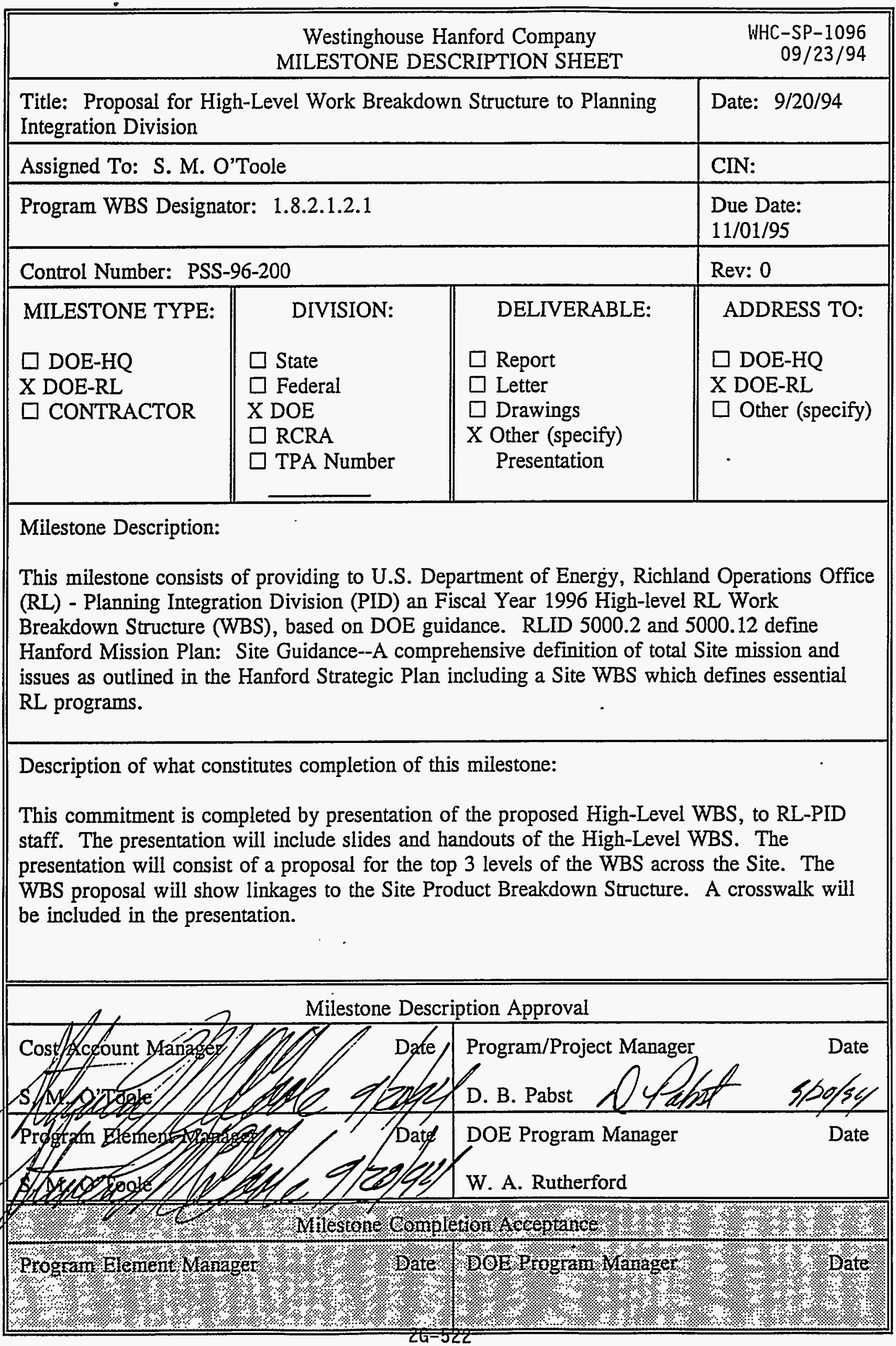




\begin{tabular}{|c|c|c|c|}
\hline \multicolumn{3}{|c|}{$\begin{array}{l}\text { Westinghouse Hanford Company } \\
\text { MILESTONE DESCRIPTION SHEET }\end{array}$} & $\begin{array}{r}\text { WHC-SP-1096 } \\
09 / 23 / 94\end{array}$ \\
\hline \multicolumn{3}{|c|}{$\begin{array}{l}\text { Title: High-Level Priority List to U.S. Department of Energy, Richland } \\
\text { Operations Office - Planning Integration Division }\end{array}$} & Date: $9 / 20 / 94$ \\
\hline \multicolumn{3}{|c|}{ Assigned To: S. M. O'Toole } & CIN: \\
\hline \multicolumn{3}{|c|}{ Program WBS Designator: 1 8.2.1.2.1 } & $\begin{array}{l}\text { Due Date: } \\
\text { 4/10/96 }\end{array}$ \\
\hline \multicolumn{3}{|c|}{ Control Number: PSS-96-201 } & Rev: 0 \\
\hline $\begin{array}{l}\text { MILESTONE TYPE: } \\
\square \text { DOE-HQ } \\
\text { X DOE-RL } \\
\square \text { CONTRACTOR }\end{array}$ & $\begin{aligned} & \text { DIVISION: } \\
& \quad \text { State } \\
& \text { Federal } \\
& \text { X DOE } \\
& \text { RCRA } \\
& \square \text { TPA Number }\end{aligned}$ & $\begin{array}{l}\text { DELIVERABLE: } \\
\square \text { Report } \\
\square \text { Letter } \\
\square \text { Drawings } \\
\text { X Other (specify) }\end{array}$ & $\begin{array}{l}\text { ADDRESS TO: } \\
\square \text { DOE-HQ } \\
\text { X DOE-RL } \\
\square \text { Other (specify) }\end{array}$ \\
\hline \multicolumn{4}{|c|}{$\begin{array}{l}\text { Milestone Description: } \\
\text { The milestone consists of providing to the U.S. Department of Energy, Richland Operations } \\
\text { Office (RL) Planning Integration Division (PID) a High-Level Priority List for use in FY } 1997 \\
\text { Hanford Mission Planning Site Guidance. This will be an application of the modified Priority } \\
\text { Planning Grid developed in Fiscal Year (FY) } 1995 \text { to High-Level Site Work Scope. }\end{array}$} \\
\hline \multicolumn{4}{|c|}{$\begin{array}{l}\text { Description of what constitutes completion of this milestone: } \\
\text { Priority criteria was developed in FY } 1995 \text { by modifying the Site Priority Planning Grid. This } \\
\text { will be tested and applied to the FY } 1998-2002 \text { Activity Data Sheets. The system will then be } \\
\text { applied to High-Level Site Work Scope. This commitment is completed by presenting the } \\
\text { High-Level Priority List to RL-PID. This list will be based on the Site Priority Criteria for } \\
\text { prioritizing cleanup activities. The presentation will include distributing handouts of the High- } \\
\text { Level Priority List to PID staff. }\end{array}$} \\
\hline \multicolumn{4}{|c|}{ Milestone Description Approval } \\
\hline \multicolumn{4}{|c|}{ 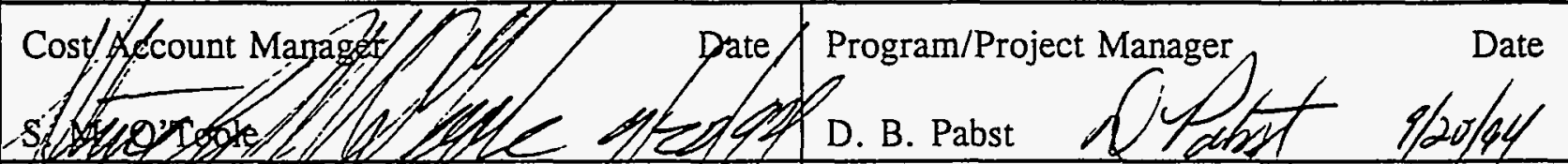 } \\
\hline \multicolumn{4}{|c|}{ 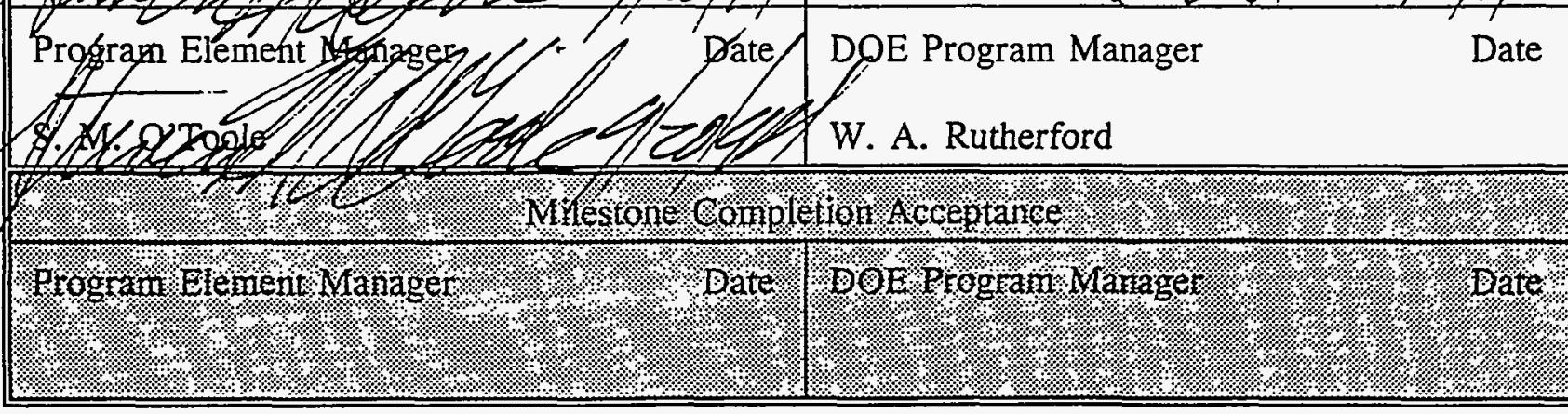 } \\
\hline
\end{tabular}




\begin{tabular}{|c|c|c|c|}
\hline \multicolumn{3}{|c|}{$\begin{array}{l}\text { Westinghouse Hanford Company } \\
\text { MILESTONE DESCRIPTION SHEET }\end{array}$} & $\begin{array}{r}\text { WHC-SP-1096 } \\
09 / 23 / 94\end{array}$ \\
\hline \multicolumn{3}{|c|}{$\begin{array}{l}\text { Title: Transmit Draft Fiscal Year } 1997 \text { Hanford Mission Plan, Volume 1, } \\
\text { Site Guidance to U.S. Department of Energy, Richland Operations Office } \\
\text { for Review }\end{array}$} & Date: $9 / 20 / 94$ \\
\hline \multicolumn{3}{|c|}{ Assigned To: S. M. O'Toole } & CIN: \\
\hline \multicolumn{3}{|c|}{ Program WBS Designator: $1.8 \cdot 2 \cdot 1 \cdot 2.1$} & $\begin{array}{l}\text { Due Date: } \\
5 / 30 / 96\end{array}$ \\
\hline \multicolumn{3}{|c|}{ Control Number: PSS-96-202 } & Rev: 0 \\
\hline $\begin{array}{l}\text { MILESTONE TYPE: } \\
\square \text { DOE-HQ } \\
\text { X DOE-RL } \\
\square \text { CONTRACTOR }\end{array}$ & $\begin{aligned} & \text { DIVISION: } \\
& \square \text { State } \\
& \quad \text { Federal } \\
\text { X DOE } & \\
& \text { RCRA } \\
& \square \text { TPA Number }\end{aligned}$ & $\begin{array}{l}\text { DELIVERABLE: } \\
\text { X Report } \\
\square \text { Letter } \\
\square \text { Drawings } \\
\square \text { Other (specify) }\end{array}$ & $\begin{array}{l}\text { ADDRESS TO: } \\
\square \text { DOE-HQ } \\
\text { X DOE-RL } \\
\square \text { Other (specify) } \\
\cdot\end{array}$ \\
\hline \multicolumn{4}{|c|}{$\begin{array}{l}\text { Milestone Description: } \\
\text { Transmit draft Fiscal Year (FY) } 1997 \text { Hanford Mission Plan (HMP), Volume 1, Site Guidance } \\
\text { to U.S. Department of Energy, Richland Operations Office (RL) for review. Contents of the } \\
\text { document is as follows: } \\
\text { - Hanford Site Mission } \\
\text { - Hanford Site Goals } \\
\text { - FY } 1997 \text { Planning Basis } \\
\text { - Hanning Assumptions } \\
\text { - High Level Priority Criteria/List } \\
\text { - Organizational Breakdown Structure (OBS) } \\
\text { - Site Systems Engineering Interface } \\
\text { - Hanford Site Integrated Schedule } \\
\text { - Crosswalk Hanford Waste Categories With Waste Types. }\end{array}$} \\
\hline
\end{tabular}




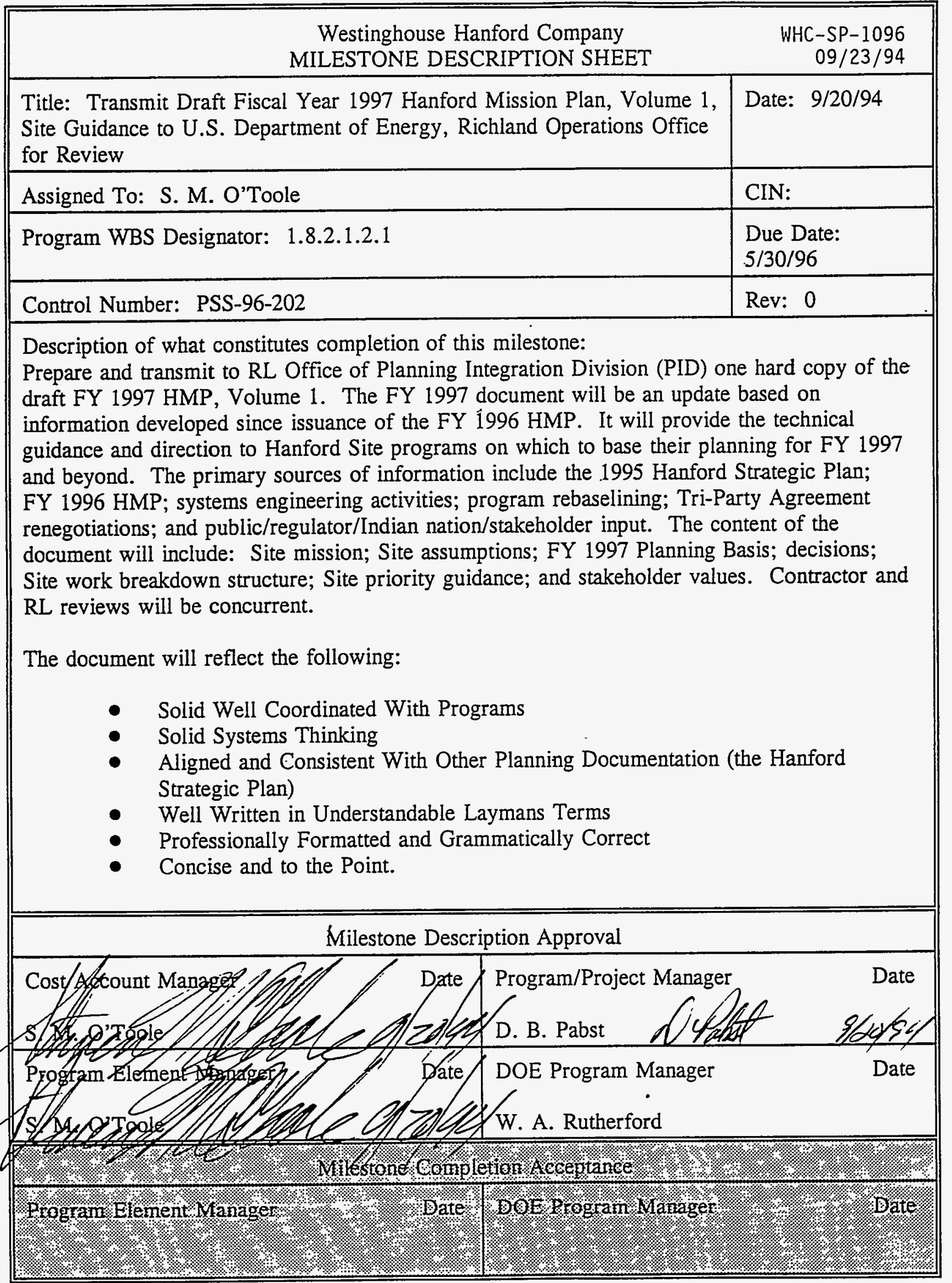




\begin{tabular}{|c|c|c|c|}
\hline \multicolumn{3}{|c|}{$\begin{array}{l}\text { Westinghouse Hanford Company } \\
\text { MILESTONE DESCRIPTION SHEET }\end{array}$} & $\begin{array}{r}\text { WHC-SP-1096 } \\
09 / 23 / 94\end{array}$ \\
\hline \multicolumn{3}{|c|}{$\begin{array}{l}\text { Title: Complete Fiscal Year } 1997 \text { Hanford Mission Plan, Volume 1, Site } \\
\text { Guidance }\end{array}$} & Date: $9 / 20 / 94$ \\
\hline \multicolumn{3}{|c|}{ Assigned To: S. M. O'Toole } & CIN: \\
\hline \multicolumn{3}{|c|}{ Program WBS Designator: 1 8.2.1.2.1 } & $\begin{array}{l}\text { Due Date: } \\
8 / 16 / 96\end{array}$ \\
\hline \multicolumn{3}{|c|}{ Control Number: PSS-96-203 } & Rev: 0 \\
\hline $\begin{array}{l}\text { MILESTONE TYPE: } \\
\square \text { DOE-HQ } \\
\text { X DOE-RL } \\
\square \text { CONTRACTOR }\end{array}$ & $\begin{array}{l}\quad \text { DIVISION: } \\
\qquad \text { State } \\
\square \text { Federal } \\
\text { X DOE } \\
\square \text { RCRA } \\
\square \text { TPA Number }\end{array}$ & $\begin{array}{l}\text { DELIVERABLE: } \\
\text { X Report } \\
\square \text { Letter } \\
\square \text { Drawings } \\
\square \text { Other (specify) }\end{array}$ & $\begin{array}{l}\text { ADDRESS TO: } \\
\square \text { DOE-HQ } \\
\text { X DOE-RL } \\
\square \text { Other (specify) }\end{array}$ \\
\hline 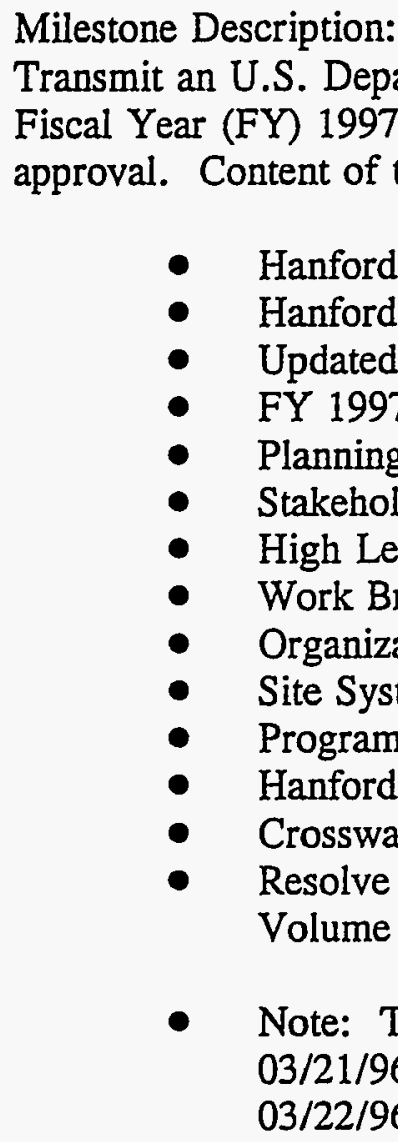 & $\begin{array}{l}\text { tment of Energy, } \\
\text { lanford Mission PI } \\
\text { document is as } \mathrm{ft} \\
\text { ite Mission } \\
\text { ite Goals } \\
\text { Material Balance } \\
\text { Planning Basis } \\
\text { Assumptions } \\
\text { er Values } \\
\text { l Priority Criteria/ } \\
\text { akdown Structure } \\
\text { onal Breakdown } S \\
\text { ms Engineering Int } \\
\text { nterfaces } \\
\text { ite Integrated Sche } \\
\text { Hanford Waste C } \\
\text { omments Receivec } \\
\text { Site Guidance } \\
\text { is milestone is con } \\
\text { and RL commento } \\
\text { and 04/11/96. It } \mathrm{r}\end{array}$ & $\begin{array}{l}\text { Md Operations Office (RL) } \\
\text { MP), Volume 1, Site Gui } \\
\text { (OBS) } \\
\text { Result of RL Review of } ~ \\
\text { t upon receiving RL revie } \\
\text { available to discuss con } \\
\text { s DOE-HQ review comm }\end{array}$ & $\begin{array}{l}\text { eviewed draft } \\
\text { nce to RL for } \\
\text { aft FY 1997 HMP, } \\
\text { comments by } \\
\text { ts by between } 05 / 096 \text {. }\end{array}$ \\
\hline
\end{tabular}




\begin{tabular}{|c|c|}
\hline $\begin{array}{l}\text { Westinghouse Hanford Company } \\
\text { MILESTONE DESCRIPTION SHEET }\end{array}$ & $\begin{array}{r}\text { WHC-SP-1096 } \\
09 / 23 / 94\end{array}$ \\
\hline $\begin{array}{l}\text { Title: Complete Fiscal Year } 1997 \text { Hanford Mission Plan, Volume 1, Site } \\
\text { Guidance }\end{array}$ & Date: $9 / 20 / 94$ \\
\hline Assigned To: S. M. O'Toole & CIN: \\
\hline Program WBS Designator: 1.8.2.1.2.1 & $\begin{array}{l}\text { Due Date: } \\
8 / 16 / 96\end{array}$ \\
\hline Control Number: PSS-96-203 & Rev: 0 \\
\hline $\begin{array}{l}\text { Description of what constitutes completion of this milestone: } \\
\text { Provide final FY } 1997 \text { HMP, Volume } 1 \text { to RL for RL Manager's approva } \\
\text { received by } 06 / 13 / 96 \text { will be dispositioned prior to delivery of this milesto } \\
\text { The document will reflect the following: } \\
\text { - Solid Well Coordinated With Programs } \\
\text { - Solid Systems Thinking } \\
\text { - Well Written in Understandable Laymans Terms } \\
\text { - Professionally Formatted and Grammatically Correct } \\
\text { - Concise and to the Point. }\end{array}$ & nts \\
\hline Milestone Description Approval & \\
\hline 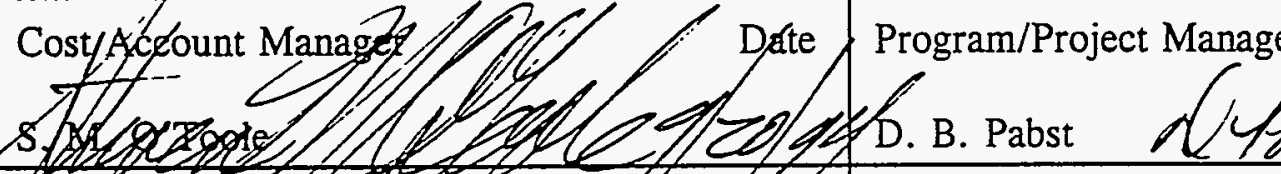 & Ot Date \\
\hline Proffar Element Minger & Date \\
\hline 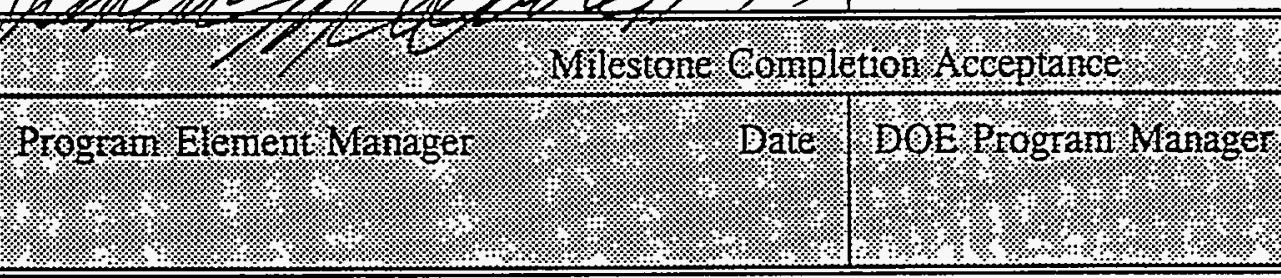 & ?: \\
\hline
\end{tabular}




\begin{tabular}{|c|c|c|c|}
\hline \multicolumn{3}{|c|}{$\begin{array}{l}\text { Westinghouse Hanford Company } \\
\text { MILESTONE DESCRIPTION SHEET }\end{array}$} & $\begin{array}{r}\text { WHC-SP-1096 } \\
09 / 23 / 94\end{array}$ \\
\hline \multicolumn{3}{|c|}{ Title: Complete Hanford Strategic Analysis Addendum for 1996} & Date: $9 / 20 / 94$ \\
\hline \multicolumn{3}{|c|}{ Assigned To: S. M. O’Toole } & CIN: \\
\hline \multicolumn{3}{|c|}{ Program WBS Designator: $1.8 \cdot 2 \cdot 1 \cdot 2.1$} & $\begin{array}{l}\text { Due Date: } \\
\text { 9/30/96 }\end{array}$ \\
\hline \multicolumn{3}{|c|}{ Control Number: PSS-96-204 } & Rev: 0 \\
\hline $\begin{array}{l}\text { MILESTONE TYPE: } \\
\square \text { DOE-HQ } \\
\square \text { DOE-RL } \\
\text { X CONTRACTOR }\end{array}$ & $\begin{array}{l}\quad \text { DIVISION: } \\
\square \text { State } \\
\square \text { Federal } \\
\text { X DOE } \\
\square \text { RCRA } \\
\square \text { TPA Number }\end{array}$ & $\begin{array}{l}\quad \text { DELIVERABLE: } \\
\text { X Report } \\
\square \text { Letter } \\
\square \text { Drawings } \\
\square \text { Other (specify) }\end{array}$ & $\begin{array}{l}\text { ADDRESS TO: } \\
\square \text { DOE-HQ } \\
\square \text { DOE-RL } \\
\text { X Other (specify) } \\
\text {-Systems } \\
\text { Engineering } \\
(1.8 .2 .4)\end{array}$ \\
\hline \multicolumn{4}{|c|}{$\begin{array}{l}\text { Milestone Description: } \\
\text { Provide to WHC Systems Engineering (1.8.2.4) an update of the } 1995 \text { Hanford Strategic } \\
\text { Analysis (HSA) in the form of an addendum prepared in Fiscal Year (FY) 1996. Contents will } \\
\text { include sitewide material balance, ROM cost and schedule. }\end{array}$} \\
\hline \multicolumn{4}{|c|}{$\begin{array}{l}\text { Description of what constitutes completion of this milestone: } \\
\text { This commitment is completed by transmittal of one hard copy of the final HSA Addendum } \\
\text { document containing additional alternative sitewide material balances, ROM cost and schedule } \\
\text { to Systems Engineering. Systems Engineering will provide the necessary input (technical } \\
\text { baseline, case description) to initiate the analysis and produce the HSA Addendum. The HSA } \\
\text { addendum will then represent a technical document reflecting the three alternatives for material } \\
\text { disposition at Hanford. The content and level of detail will be consistent with the Hanford } \\
\text { Strategic Analysis Study, WHC-EP-0549. This document will be suitable for release to the } \\
\text { public. }\end{array}$} \\
\hline \multicolumn{4}{|c|}{ Milestone Description Approval } \\
\hline 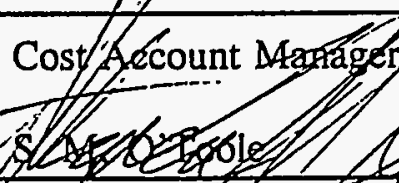 & & Program/Project Man & Date \\
\hline & & $\begin{array}{l}\text { DOE Program Manag } \\
\text { W. A. Rutherford }\end{array}$ & Date \\
\hline
\end{tabular}




\begin{tabular}{|c|c|}
\hline $\begin{array}{l}\text { Westinghouse Hanford Company } \\
\text { MILESTONE DESCRIPTION SHEET }\end{array}$ & $\begin{array}{r}\text { WHC-SP-1096 } \\
09 / 23 / 94\end{array}$ \\
\hline Title: Complete Hanford Strategic Analysis Addendum for 1996 & Date: $9 / 20 / 94$ \\
\hline Assigned To: S. M. O'Toole & CIN: \\
\hline Program WBS Designator: 1.8.2.1.2.1 & $\begin{array}{l}\text { Due Date: } \\
9 / 30 / 96\end{array}$ \\
\hline Control Number: PSS-96-204 & Rev: 0 \\
\hline \multicolumn{2}{|c|}{ 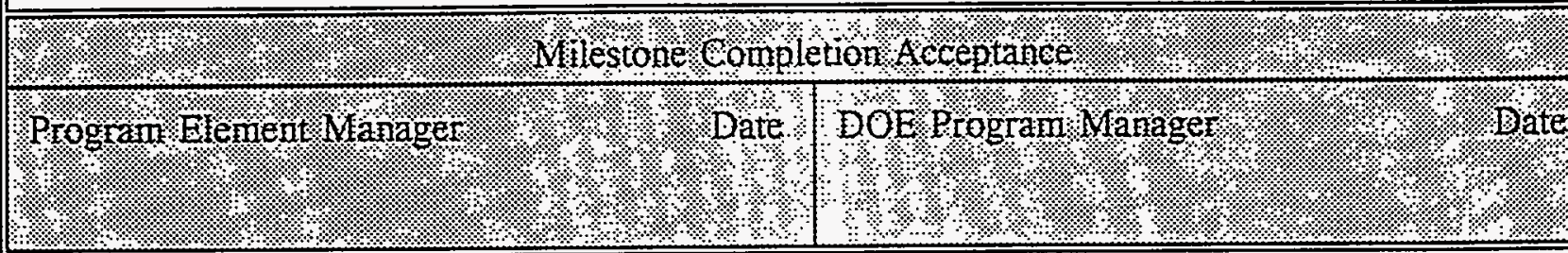 } \\
\hline
\end{tabular}




\begin{tabular}{|c|c|c|c|}
\hline \multicolumn{4}{|c|}{$\begin{array}{l}\text { Westinghouse Hanford Company } \\
\text { MILESTONE DESCRIPTION SHEET }\end{array}$} \\
\hline \multicolumn{3}{|c|}{ Title: Provide Working Paper Draft Guidance to Site Programs } & Date: $9 / 20 / 94$ \\
\hline \multicolumn{3}{|c|}{ Assigned To: S. M. O'Toole } & CIN: \\
\hline \multicolumn{3}{|c|}{ Program WBS Designator: 1.8 .2 .1 .2 .1} & $\begin{array}{l}\text { Due Date: } \\
7 / 12 / 96\end{array}$ \\
\hline \multicolumn{3}{|c|}{ Control Number: PSS-96-205 } & Rev: 0 \\
\hline $\begin{array}{l}\text { MILESTONE TYPE: } \\
\square \text { DOE-HQ } \\
\text { X DOE-RL } \\
\square \text { CONTRACTOR }\end{array}$ & $\begin{array}{l}\quad \text { DIVISION: } \\
\square \text { State } \\
\square \text { Federal } \\
\text { X DOE } \\
\square \text { RCRA } \\
\square \text { TPA Number }\end{array}$ & $\begin{array}{l}\quad \text { DELIVERABLE: } \\
\text { X Report } \\
\square \text { Letter } \\
\square \text { Drawings } \\
\square \text { Other (specify) }\end{array}$ & $\begin{array}{l}\text { ADDRESS TO: } \\
\square \text { DOE-HQ } \\
\text { X DOE-RL } \\
\square \text { Other (specify) }\end{array}$ \\
\hline \multicolumn{4}{|c|}{$\begin{array}{l}\text { Milestone Description: } \\
\text { Provide working paper draft planning guidance to WHC programs for their use in preparing } \\
\text { their Fiscal Year (FY) } 1997 \text { Multi-Year Program Plans (MYPPs). }\end{array}$} \\
\hline \multicolumn{4}{|c|}{$\begin{array}{l}\text { Description of what constitutes completion of this milestone: } \\
\text { Submit draft FY } 1997 \text { Hanford Mission Plan, Volume } 1 \text { to U.S. Department of Energy, } \\
\text { Richland Operations Office - Planning Integration Division for distribution to Site programs as } \\
\text { working paper planning guidance for their use in preparing FY } 1997 \cdot \text { MYPPs. This document } \\
\text { will include revisions to the draft due to RL and contractor reviews. } \\
\text { The document will reflect the following: } \\
\qquad \text { Well Coordinated With the Programs } \\
\text { - Solid Systems Thinking } \\
\text { - Gell Written in Understandable Laymans Terms } \\
\text { - Concise and to the Point. }\end{array}$} \\
\hline & & ption Approval & \\
\hline S S lor & $y 912-1172$ & Program/Project Mar & Date \\
\hline
\end{tabular}


WHC-SP-1096

09/23/94

\begin{tabular}{||l|l||}
\hline \multicolumn{3}{|c||}{ Westinghouse Hanford Company } \\
MILESTONE DESCRIPTION SHEET
\end{tabular}




\begin{tabular}{|c|c|c|c|}
\hline \multicolumn{4}{|c|}{$\begin{array}{l}\text { Westinghouse Hanford Company } \\
\text { MILESTONE DESCRIPTION SHEET }\end{array}$} \\
\hline \multicolumn{3}{|c|}{ Title: Analysis of Risks and Costs at Hanford } & Date: $8 / 8 / 94$ \\
\hline \multicolumn{3}{|c|}{ Assigned To: WA Hesser } & CIN: \\
\hline \multicolumn{3}{|c|}{ Program WBS Designator: 1.8.2.1.3 } & $\begin{array}{l}\text { Due Date: } \\
1 / 31 / 96\end{array}$ \\
\hline \multicolumn{3}{|c|}{ Control Number: PSS-96-300 } & Rev: \\
\hline $\begin{array}{l}\text { MILESTONE TYPE: } \\
\square \text { DOE-HQ } \\
\text { X DOE-RL } \\
\square \text { CONTRACTOR }\end{array}$ & $\begin{array}{l}\quad \text { DIVISION: } \\
\square \text { State } \\
\square \text { Federal } \\
\text { X DOE } \\
\square \text { RCRA }\end{array}$ & $\begin{array}{l}\text { DELIVERABLE: } \\
\text { X Report } \\
\square \text { Letter } \\
\square \text { Drawings } \\
\square \text { Other (specify) }\end{array}$ & $\begin{array}{l}\text { ADDRESS TO: } \\
\square \text { DOE-HQ } \\
\text { X DOE-RL } \\
\square \text { Other } \\
\text { (specify) }\end{array}$ \\
\hline \multirow{2}{*}{\multicolumn{4}{|c|}{$\begin{array}{l}\text { The most important decisions at Hanford involve the allocation of funding to } \\
\text { competing activities. This report analyzes these decisions and describes the } \\
\text { relationship between costs, risks, and risk reduction. Risks, in this case, mean } \\
\text { public health, worker safety, and environmental/ecological. Costs are those } \\
\text { associated with program activities. The overall objective of the analysis is to } \\
\text { determine whether or not the Site is complying with DOE-HQ directives to } \\
\text { allocate its resources to those areas of greatest risk, realizing, of course, that } \\
\text { there are many other inputs to the decision process. } \\
\text { This analysis builds on similar work begun in FY94 and continued in FY95. }\end{array}$}} \\
\hline & & & \\
\hline \multicolumn{4}{|c|}{$\begin{array}{l}\text { This milestone will be complete when one copy of the report containing the } \\
\text { Analysis of Risks and Costs at Hanford is submitted to } R L \text { and the } R L \text { manager } \\
\text { has signed the milestone completion acceptance. }\end{array}$} \\
\hline \multicolumn{4}{|c|}{ Milestone Description Approval } \\
\hline \multicolumn{2}{|c|}{ Cost Account Manager } & \multicolumn{2}{|c|}{ Program/Project Manager Date } \\
\hline
\end{tabular}




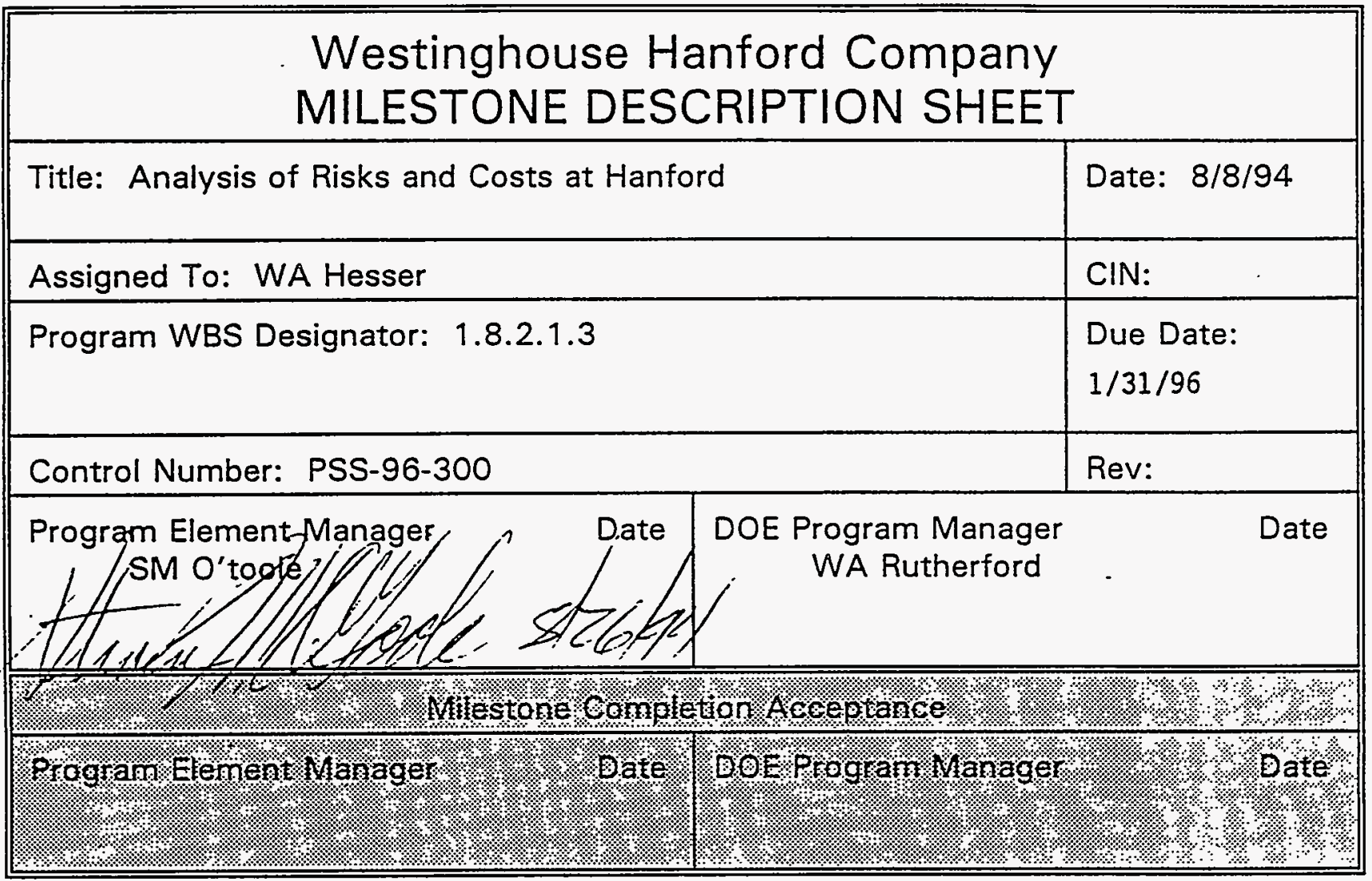




\begin{tabular}{|c|c|c|c|}
\hline \multicolumn{4}{|c|}{$\begin{array}{l}\text { Westinghouse Hanford Company } \\
\text { MILESTONE DESCRIPTION SHEET }\end{array}$} \\
\hline $\begin{array}{l}\text { Report } \\
\text { making }\end{array}$ & \multicolumn{2}{|c|}{$\begin{array}{l}\text { Report of the Application of Risk-Based decision } \\
\text { making at Hanford }\end{array}$} & Date: $8 / 8 / 94$ \\
\hline \multicolumn{3}{|c|}{ Assigned To: WA Hesser } & CIN: \\
\hline \multicolumn{3}{|c|}{ Program WBS Designator: 1.8.2.1.3 } & $\begin{array}{l}\text { Due Date: } \\
9 / 30 / 96\end{array}$ \\
\hline \multicolumn{3}{|c|}{ Control Number: PSS-96-301 } & Rev: \\
\hline MILESTONE TYPE: & DIVISION: & DELIVERABLE: & ADDRESS TO: \\
\hline $\begin{array}{l}\square \text { DOE-HQ } \\
\text { X DOE-RL } \\
\square \text { CONTRACTOR }\end{array}$ & $\begin{array}{l}\square \text { State } \\
\square \text { Federal } \\
X \text { DOE } \\
\square \text { RCRA }\end{array}$ & $\begin{array}{l}\text { X Report } \\
\square \text { Letter } \\
\square \text { Drawings } \\
\square \text { Other (specify) }\end{array}$ & $\begin{array}{l}\square \text { DOE-HQ } \\
\text { X DOE-RL } \\
\square \text { Other } \\
\text { (specify) }\end{array}$ \\
\hline
\end{tabular}




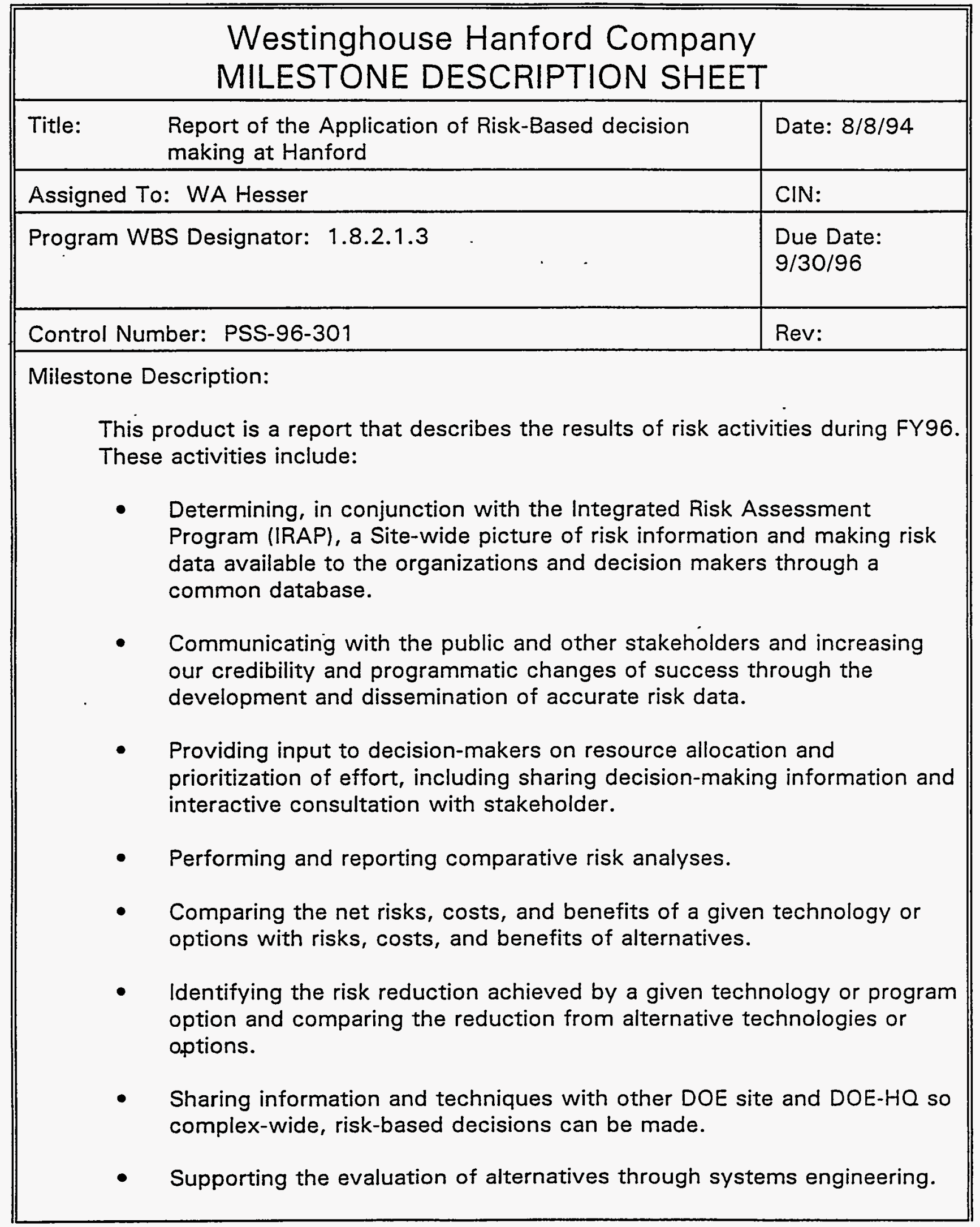




\section{Westinghouse Hanford Company MILESTONE DESCRIPTION SHEET}

\begin{tabular}{|c|c|}
\hline $\begin{array}{l}\text { Report of the Application of Risk-Based decision } \\
\text { making at Hanford }\end{array}$ & Date: $8 / 8 / 94$ \\
\hline Assigned To: WA Hesser & CIN: \\
\hline Program WBS Designator: 1.8.2.1.3 & $\begin{array}{l}\text { Due Date: } \\
\text { 9/30/96 }\end{array}$ \\
\hline Control Number: PSS-96-301 & Rev: \\
\hline
\end{tabular}

Description of what constitutes completion of this milestone:

This milestone will be complete when one copy of the report containing the Application of Risk-Based decision making at Hanford is submitted to RL and the RL manager has signed the milestone completion acceptance.

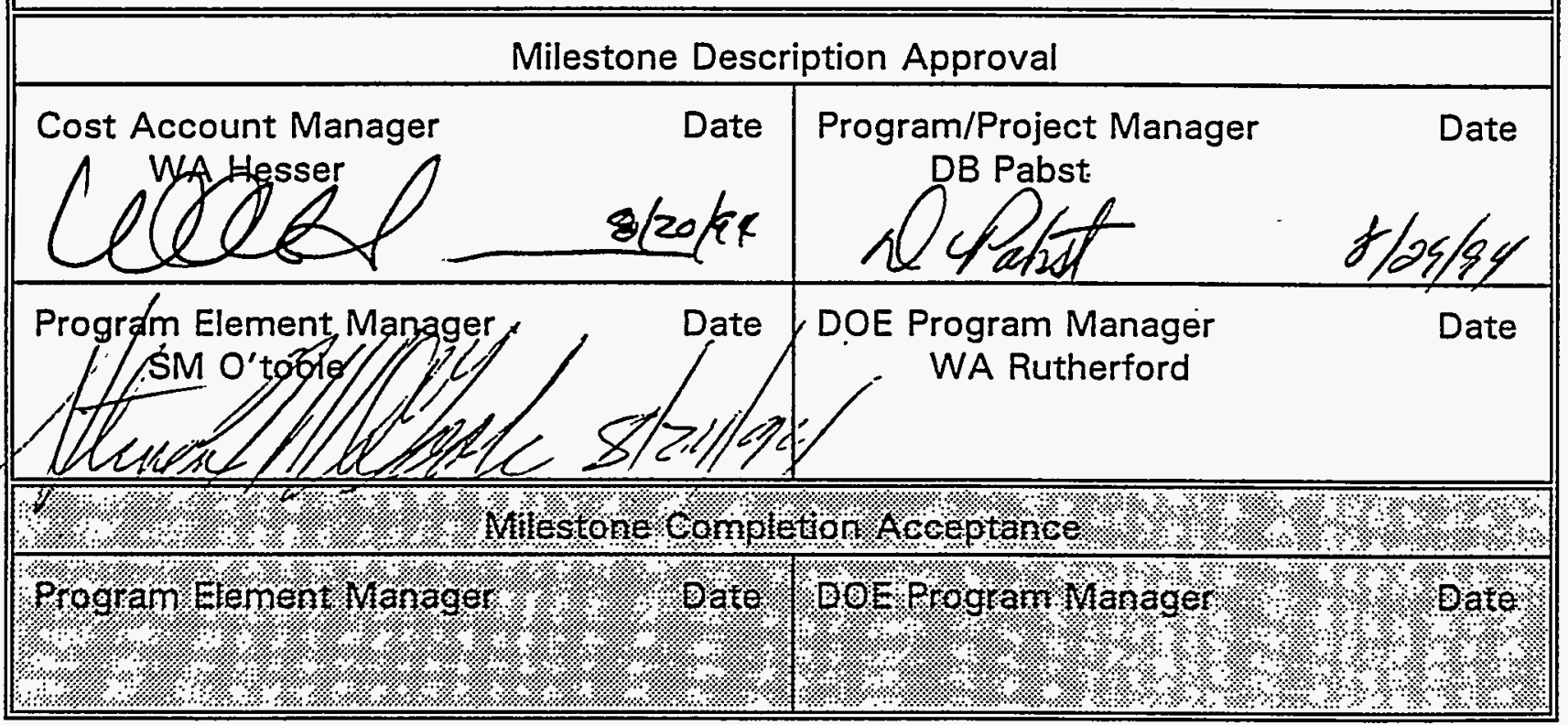




\begin{tabular}{|c|c|c|c|}
\hline \multicolumn{3}{|c|}{$\begin{array}{l}\text { Westinghouse Hanford Company } \\
\text { MILESTONE DESCRIPTION SHEET }\end{array}$} & $\begin{array}{r}\text { WHC-SP-1096 } \\
09 / 23 / 94\end{array}$ \\
\hline \multicolumn{3}{|c|}{ Title: Complete Draft Hanford Strategic Plan } & Date: $9 / 20 / 94$ • \\
\hline \multicolumn{3}{|c|}{ Assigned To: H. H. Yoshikawa } & CIN: \\
\hline \multicolumn{3}{|c|}{ Program WBS Designator: 1 8.2.1.1 } & $\begin{array}{l}\text { Due Date: } \\
2 / 21 / 97\end{array}$ \\
\hline \multicolumn{3}{|c|}{ Control Number: PSS-97-100 } & Rev: 0 \\
\hline $\begin{array}{l}\text { MILESTONE TYPE: } \\
\square \text { DOE-HQ } \\
\text { X DOE-RL } \\
\square \text { CONTRACTOR }\end{array}$ & $\begin{aligned} & \text { DIVISION: } \\
& \square \text { State } \\
& \square \text { Federal } \\
& \text { X DOE } \\
& \square \text { RCRA } \\
& \square \text { TPA Number }\end{aligned}$ & $\begin{array}{l}\text { DELIVERABLE: } \\
\text { X Report } \\
\square \text { Letter } \\
\square \text { Drawings } \\
\square \text { Other (specify) }\end{array}$ & $\begin{array}{l}\text { ADDRESS TO: } \\
\square \text { DOE-HQ } \\
\text { X DOE-RL } \\
\square \text { Other (specify) }\end{array}$ \\
\hline \multicolumn{4}{|c|}{$\begin{array}{l}\text { Milestone Description: } \\
\text { Submittal of the draft Fiscal Year (FY) } 1998 \text { Hanford Strategic Plan to U.S. Department of } \\
\text { Energy, Richland Operations Office (RL) Planning Integration Division (PID) in accordance } \\
\text { with Hanford Quality Council direction for review. Contents of the FY } 1998 \text { draft Hanford } \\
\text { Strategic Plan will include: }\end{array}$} \\
\hline \multicolumn{4}{|c|}{$\begin{array}{l}\text { Description of what constitutes completion of this milestone: } \\
\text { The draft FY } 1998 \text { Hanford Strategic Plan is submitted to RL-PID co } \\
\text { the Hanford Quality Council. The draft Hanford Strategic Plan will b } \\
\text { for transmittal to DOE-HQ, Regulators, Indian Nations, and other stal } \\
\text { The document will reflect the following: } \\
\text { - Solid Systems Thinking } \\
\text { - Well Written in Understandable Laymans Terms } \\
\text { - Concise and to the Point. }\end{array}$} \\
\hline \multicolumn{4}{|c|}{ Milestone Description Approval } \\
\hline \multicolumn{2}{|c|}{$\begin{array}{l}\text { Cost Account Manager } \\
\text { Aff Lloluleara } \\
\text { H. H. Yoshikawa }\end{array}$} & \multicolumn{2}{|c|}{ Program/Project Manager } \\
\hline
\end{tabular}




\begin{tabular}{|c|c|c|}
\hline \multicolumn{2}{|c|}{$\begin{array}{l}\text { Westinghouse Hanford Company } \\
\text { MILESTONE DESCRIPTION SHEET }\end{array}$} & $\begin{array}{r}W H C-S P-1096 \\
09 / 23 / 94\end{array}$ \\
\hline \multicolumn{2}{|l|}{ Title: Complete Draft Hanford Strategic Plan } & Date: $9 / 20 / 94$ \\
\hline \multicolumn{2}{|l|}{ Assigned To: H. H. Yoshikawa } & CIN: \\
\hline \multicolumn{2}{|l|}{ Program WBS Designator: 1 8.2.1.1 } & $\begin{array}{l}\text { Due Date: } \\
2 / 21 / 97\end{array}$ \\
\hline \multicolumn{2}{|l|}{ Control Number: PSS-97-100 } & Rev: 0 \\
\hline 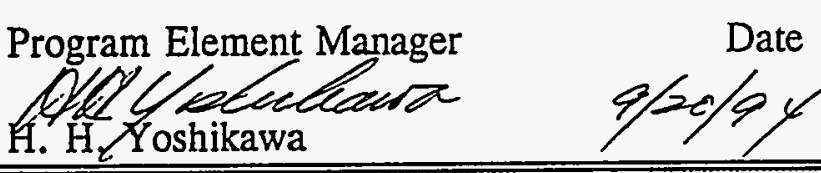 & \multicolumn{2}{|c|}{$\begin{array}{l}\text { DOE Program Manager Date } \\
\text { W. A. Rutherford }\end{array}$} \\
\hline \multicolumn{3}{|c|}{ 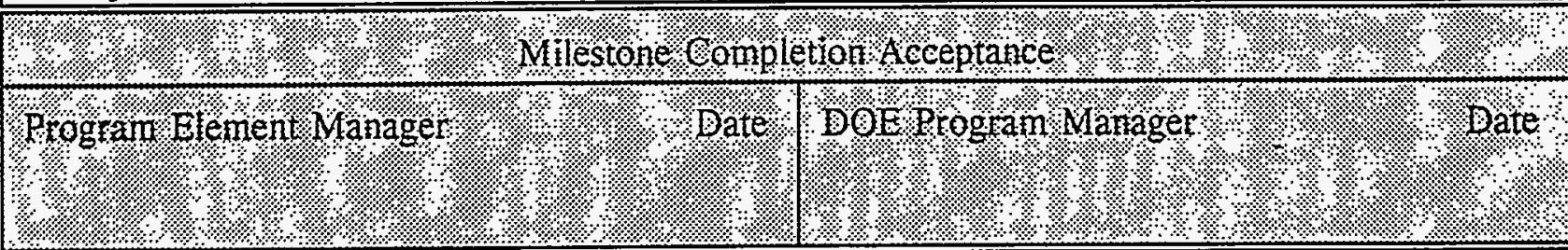 } \\
\hline
\end{tabular}




\begin{tabular}{|c|c|c|c|}
\hline & \multicolumn{2}{|c|}{$\begin{array}{l}\text { Westinghouse Hanford Company } \\
\text { MILESTONE DESCRIPTION SHEET }\end{array}$} & $\begin{array}{r}\text { WHC-SP-1096 } \\
09 / 23 / 94 \\
\end{array}$ \\
\hline \multicolumn{3}{|c|}{ Title: Complete Hanford Strategic Plan } & Date: $9 / 20 / 94$ \\
\hline \multicolumn{3}{|c|}{ Assigned To: H. H. Yoshikawa } & CIN: \\
\hline \multicolumn{3}{|c|}{ Program WBS Designator: 1.8 .2 .1 .1} & $\begin{array}{l}\text { Due Date: } \\
\text { 5/02/97 }\end{array}$ \\
\hline \multicolumn{3}{|c|}{ Control Number: PSS-97-101 } & Rev: 0 \\
\hline $\begin{array}{l}\text { MILESTONE TYPE: } \\
\square \text { DOE-HQ } \\
\text { X DOE-RL } \\
\square \text { CONTRACTOR }\end{array}$ & $\begin{array}{l}\quad \text { DIVISION: } \\
\quad \square \text { State } \\
\square \text { Federal } \\
\text { X DOE } \\
\square \text { RCRA } \\
\square \text { TPA Number }\end{array}$ & $\begin{array}{l}\text { DELIVERABLE: } \\
\text { X Report } \\
\square \text { Letter } \\
\square \text { Drawings } \\
\square \text { Other (specify) }\end{array}$ & $\begin{array}{l}\text { ADDRESS TO: } \\
\square \text { DOE-HQ } \\
\text { X DOE-RL } \\
\square \text { Other (specify) }\end{array}$ \\
\hline \multicolumn{4}{|c|}{$\begin{array}{l}\text { Milestone Description: } \\
\text { Delivery of the final Fiscal Year (FY) } 1998 \text { Hanford Strategic Plan to U.S. Department of } \\
\text { Energy, Richland Operations Office (RL) Planning Integration Division (PID) for processing } \\
\text { approval by Site Manager. Contents will include: }\end{array}$} \\
\hline \multicolumn{4}{|c|}{$\begin{array}{l}\text { Description of what constitutes completion of this milestone: } \\
\text { The FY } 1998 \text { Hanford Strategic Plan is initiated by written guidance } \\
\text { consistent with a decision by the Hanford Quality Council. The Hanf } \\
\text { compiled by WHC in support of the Hanford Quality Council. Revie } \\
\text { contractors, RL, Regulators, Indian Nations, and stakeholders are res } \\
\text { content has been finalized. Copies of the Hanford Strategic Plan will } \\
\text { following Site managers approval. } \\
\text { The document will reflect the following: } \\
\text { - Solid Systems Thinking } \\
\text { Well Written in Understandable Laymans Terms } \\
\text { - Concise and to the Point. }\end{array}$} \\
\hline \multicolumn{4}{|c|}{ Milestone Description Approval } \\
\hline $\begin{array}{l}\text { Cost Account Manage } \\
\text { H. H. yoshikawa }\end{array}$ & a $9 / 20 / 94$ & $\begin{array}{l}\text { Program/Project Man } \\
\text { D. B. Pabst }\end{array}$ & If I/zolsy \\
\hline
\end{tabular}




\begin{tabular}{|c|c|c|}
\hline \multicolumn{2}{|c|}{$\begin{array}{l}\text { Westinghouse Hanford Company } \\
\text { MILESTONE DESCRIPTION SHEET }\end{array}$} & $\begin{array}{r}\text { WHC-SP-1096 } \\
09 / 23 / 94 \\
\end{array}$ \\
\hline \multicolumn{2}{|l|}{ Title: Complete Hanford Strategic Plan } & Date: $9 / 20 / 94$ \\
\hline \multicolumn{2}{|l|}{ Assigned To: H. H. Yoshikawa } & CIN: \\
\hline \multicolumn{2}{|l|}{ Program WBS Designator: 1.8.2.1.1 } & $\begin{array}{l}\text { Due Date: } \\
5 / 02 / 97\end{array}$ \\
\hline \multicolumn{2}{|l|}{ Control Number: PSS-97-101 } & Rev: 0 \\
\hline 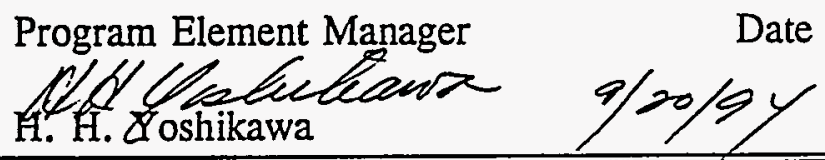 & $\begin{array}{l}\text { DOE Program Manager } \\
\text { W. A. Rutherford }\end{array}$ & Date \\
\hline \multicolumn{3}{|c|}{ 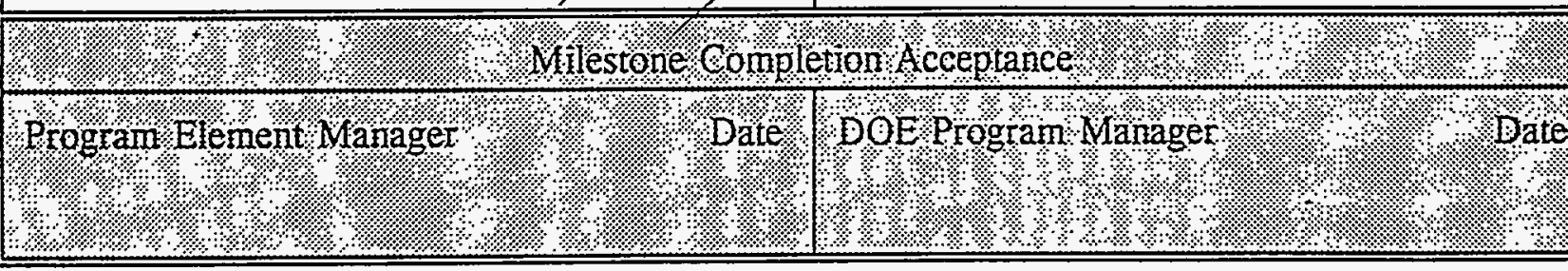 } \\
\hline
\end{tabular}




\begin{tabular}{|c|c|c|c|}
\hline \multicolumn{3}{|c|}{$\begin{array}{l}\text { Westinghouse Hanford Company } \\
\text { MILESTONE DESCRIPTION SHEET }\end{array}$} & $\begin{array}{r}\text { WHC-SP-1096 } \\
09 / 23 / 94\end{array}$ \\
\hline \multicolumn{3}{|c|}{$\begin{array}{l}\text { Title: Proposal for High-Level Work Breakdown Structure to Planning } \\
\text { Integration Division }\end{array}$} & Date: $9 / 20 / 94$ \\
\hline \multicolumn{3}{|c|}{ Assigned To: S. M. O'Toole } & CIN: \\
\hline \multicolumn{3}{|c|}{ Program WBS Designator: 1 8.2.1.2.1 } & $\begin{array}{l}\text { Due Date: } \\
\text { 11/27/96 }\end{array}$ \\
\hline \multicolumn{3}{|c|}{ Control Number: PSS-97-200 } & Rev: 0 \\
\hline $\begin{array}{l}\text { MILESTONE TYPE: } \\
\square \text { DOE-HQ } \\
\text { X DOE-RL } \\
\square \text { CONTRACTOR }\end{array}$ & $\begin{aligned} & \text { DIVISION: } \\
& \square \text { State } \\
& \text { Federal } \\
& \text { X DOE } \\
& \square \text { RCRA } \\
& \square \text { TPA Number }\end{aligned}$ & $\begin{array}{l}\text { DELIVERABLE: } \\
\square \text { Report } \\
\square \text { Letter } \\
\square \text { Drawings } \\
\text { X Other (specify) } \\
\quad \text { Presentation }\end{array}$ & $\begin{array}{l}\text { ADDRESS TO: } \\
\square \text { DOE-HQ } \\
\text { X DOE-RL } \\
\square \text { Other (specify) }\end{array}$ \\
\hline \multicolumn{4}{|c|}{$\begin{array}{l}\text { This milestone consists of providing to U.S. Department of Energy, Richland Operations Office } \\
\text { (RL) - Planning Integration Division (PID) an Fiscal Year } 1997 \text { High-level RL Work } \\
\text { Breakdown Structure (WBS), based on DOE guidance. RLID 5000.2 and } 5000.12 \text { define } \\
\text { Hanford Mission Plan: Site Guidance--A comprehensive definition of total Site mission and } \\
\text { issues as outlined in the Hanford Strategic Plan including a Site WBS which defines essential } \\
\text { RL programs. }\end{array}$} \\
\hline \multicolumn{4}{|c|}{$\begin{array}{l}\text { Description of what constitutes completion of this milestone: } \\
\text { This commitment is completed by presentation of the proposed High-Level WBS, to RL-PID } \\
\text { staff. The presentation will include slides and handouts of the High-Level WBS. The } \\
\text { presentation will consist of a proposal for the top } 3 \text { levels of the WBS across the Site. The } \\
\text { WBS proposal will show linkages to the Site Product Breakdown Structure. A crosswalk will } \\
\text { be included in the presentation. }\end{array}$} \\
\hline \multicolumn{4}{|c|}{ Milestone Description Approval } \\
\hline \multirow{2}{*}{\multicolumn{2}{|c|}{ 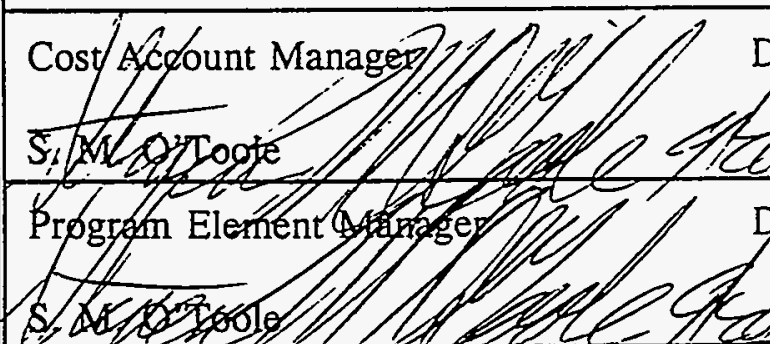 }} & Program/Project Man & $\begin{array}{l}\text { Date } \\
\text { Sborlay }\end{array}$ \\
\hline & & $\begin{array}{l}\text { DOE Program Manag } \\
\text { W. A. Rutherford }\end{array}$ & Date \\
\hline \multicolumn{4}{|c|}{ Pregram Eienent Manager } \\
\hline
\end{tabular}




\begin{tabular}{|c|c|c|c|}
\hline \multicolumn{3}{|c|}{$\begin{array}{l}\text { Westinghouse Hanford Company } \\
\text { MILESTONE DESCRIPTION SHEET }\end{array}$} & $\begin{array}{r}\text { WHC-SP-1096 } \\
09 / 23 / 94\end{array}$ \\
\hline \multicolumn{3}{|c|}{$\begin{array}{l}\text { Title: High-Level Priority List to U.S. Department of Energy, Richland } \\
\text { Operations Office - Planning Integration Division }\end{array}$} & Date: $9 / 20 / 94$ \\
\hline \multicolumn{3}{|c|}{ Assigned To: S. M. O'Toole } & CIN: \\
\hline \multicolumn{3}{|c|}{ Program WBS Designator: 1.8.2.1.2.1 } & $\begin{array}{l}\text { Due Date: } \\
4 / 10 / 97\end{array}$ \\
\hline \multicolumn{3}{|c|}{ Control Number: PSS-97-201 } & Rev: 0 \\
\hline $\begin{array}{l}\text { MILESTONE TYPE: } \\
\square \text { DOE-HQ } \\
\text { X DOE-RL } \\
\square \text { CONTRACTOR }\end{array}$ & $\begin{array}{l}\quad \text { DIVISION: } \\
\square \text { State } \\
\square \text { Federal } \\
\text { X DOE } \\
\square \text { RCRA } \\
\square \text { TPA Number }\end{array}$ & $\begin{array}{l}\text { DELIVERABLE: } \\
\square \text { Report } \\
\square \text { Letter } \\
\square \text { Drawings } \\
\text { X Other (specify) }\end{array}$ & $\begin{array}{l}\text { ADDRESS TO: } \\
\square \text { DOE-HQ } \\
\text { X DOE-RL } \\
\square \text { Other (specify) }\end{array}$ \\
\hline \multicolumn{4}{|c|}{$\begin{array}{l}\text { Milestone Description: } \\
\text { The milestone consists of providing to the U.S. Department of Energy, Richland Operations } \\
\text { Office (RL) Planning Integration Division (PID) } \text { a High-Level Priority List for use in FY } 1998 \\
\text { Hanford Mission Planning Site Guidance. This will be an application of the modified Priority } \\
\text { Planning 'Grid developed in Fiscal Year (FY) } 1994 \text { to High-Level Site Work Scope. }\end{array}$} \\
\hline \multicolumn{4}{|c|}{$\begin{array}{l}\text { Description of what constitutes completion of this milestone: } \\
\text { Priority criteria was developed in FY } 1994 \text { by modifying the Site Priority Planning Grid. This } \\
\text { will be tested and applied to the FY } 1999-2003 \text { Activity Data Sheets. The system will then be } \\
\text { applied to High-Level Site Work Scope. This commitment is completed by presenting the } \\
\text { High-Level Priority List to RL-PID. This list will be based on the Site Priority Criteria for } \\
\text { prioritizing cleanup activities. The presentation will include distributing handouts of the High- } \\
\text { Level Priority List to PID staff. }\end{array}$} \\
\hline \multicolumn{4}{|c|}{$\ldots$ Milestone Description Approval } \\
\hline \multicolumn{4}{|c|}{ Cost. Alcount pate/ Program/Project Manager } \\
\hline Prógám Element & & $\begin{array}{l}\text { DOE Program Manager } \\
\text { W. A. Rutherford }\end{array}$ & Date \\
\hline \multicolumn{4}{|c|}{ 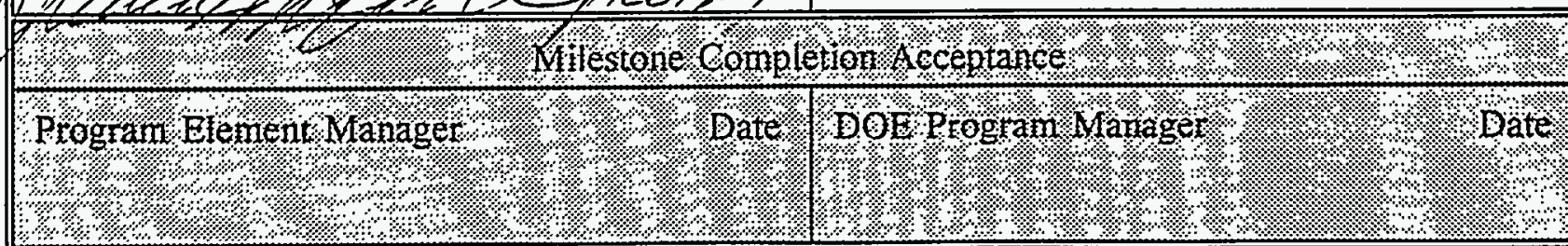 } \\
\hline
\end{tabular}




\begin{tabular}{|l|l||}
\hline \multicolumn{2}{|c|}{ Westinghouse Hanford Company } \\
\hline Title: Transmit Draft Fiscal Year 1998 Hanford Mission Plan, Volume 1, \\
Site Guidance to U.S. Department of Energy, Richland Operations Office \\
for Review
\end{tabular}




\begin{tabular}{|c|c|c|c|}
\hline \multicolumn{3}{|c|}{$\begin{array}{l}\text { Westinghouse Hanford Company } \\
\text { MILESTONE DESCRIPTION SHEET }\end{array}$} & $\begin{array}{r}\text { WHC-SP-1096 } \\
09 / 23 / 94 \\
\end{array}$ \\
\hline \multicolumn{3}{|c|}{$\begin{array}{l}\text { Title: Complete Fiscal Year } 1998 \text { Hanford Mission Plan, Volume 1, Site } \\
\text { Guidance }\end{array}$} & Date: $9 / 20 / 94$ \\
\hline \multicolumn{3}{|c|}{ Assigned To: S. M. O'Toole } & CIN: \\
\hline \multicolumn{3}{|c|}{ Program WBS Designator: 1.8.2.1.2.1 } & $\begin{array}{l}\text { Due Date: } \\
8 / 19 / 97 \\
\end{array}$ \\
\hline \multicolumn{3}{|c|}{ Control Number: PSS-97-203 } & Rev: 0 \\
\hline \multirow{2}{*}{$\begin{array}{l}\text { MILESTONE TYPE: } \\
\square \text { DOE-HQ } \\
\text { X DOE-RL } \\
\square \text { CONTRACTOR }\end{array}$} & DIVISION: & DELIVERABLE: & ADDRESS TO: \\
\hline & $\begin{array}{l}\square \text { State } \\
\square \text { Federal } \\
\text { X DOE } \\
\square \text { RCRA } \\
\square \text { TPA Number }\end{array}$ & $\begin{array}{l}\text { X Report } \\
\square \text { Letter } \\
\square \text { Drawings } \\
\square \text { Other (specify) }\end{array}$ & $\begin{array}{l}\square \text { DOE-HQ } \\
\text { X DOE-RL } \\
\square \text { Other (specify) }\end{array}$ \\
\hline \multicolumn{4}{|c|}{ 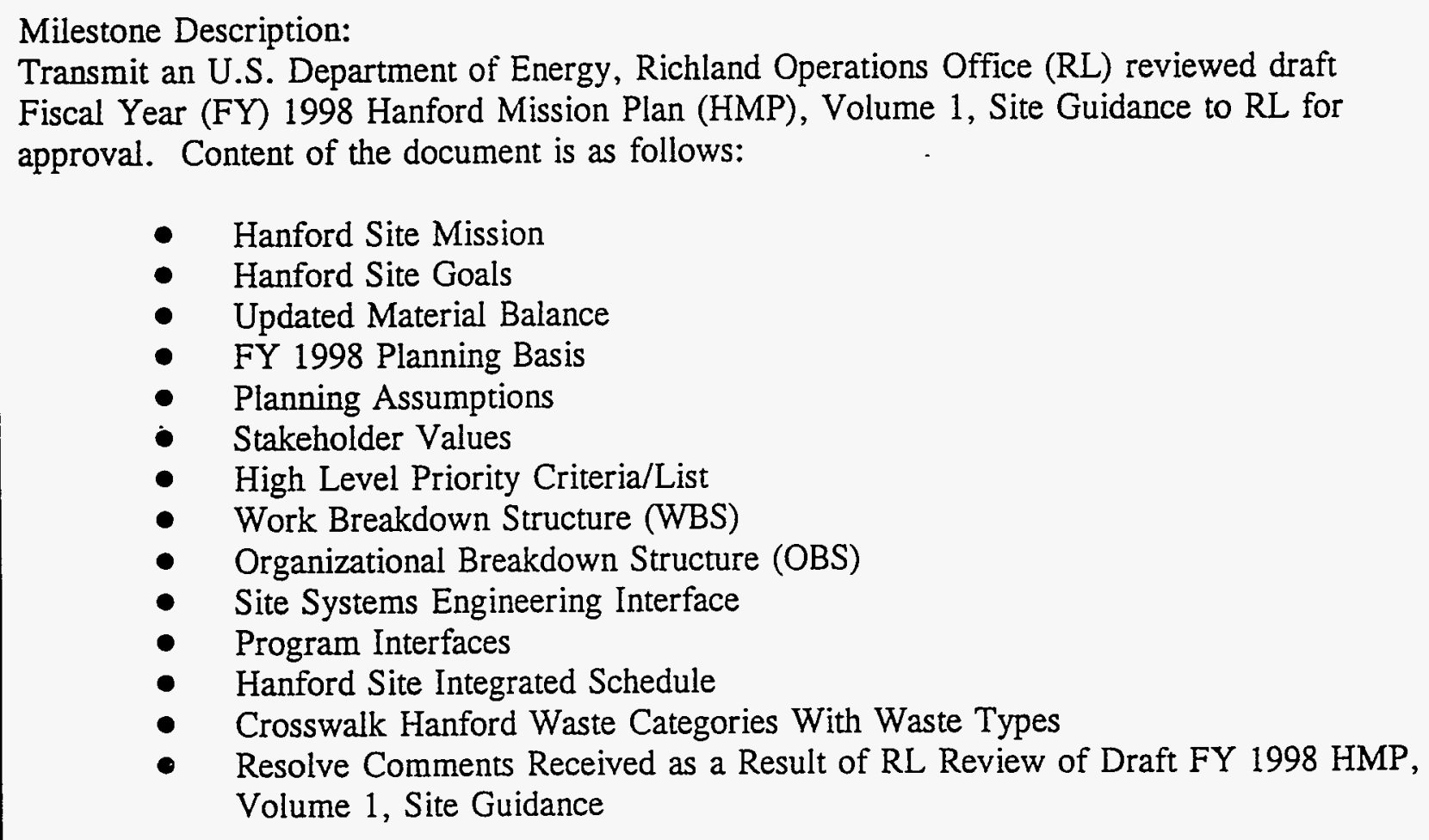 } \\
\hline
\end{tabular}




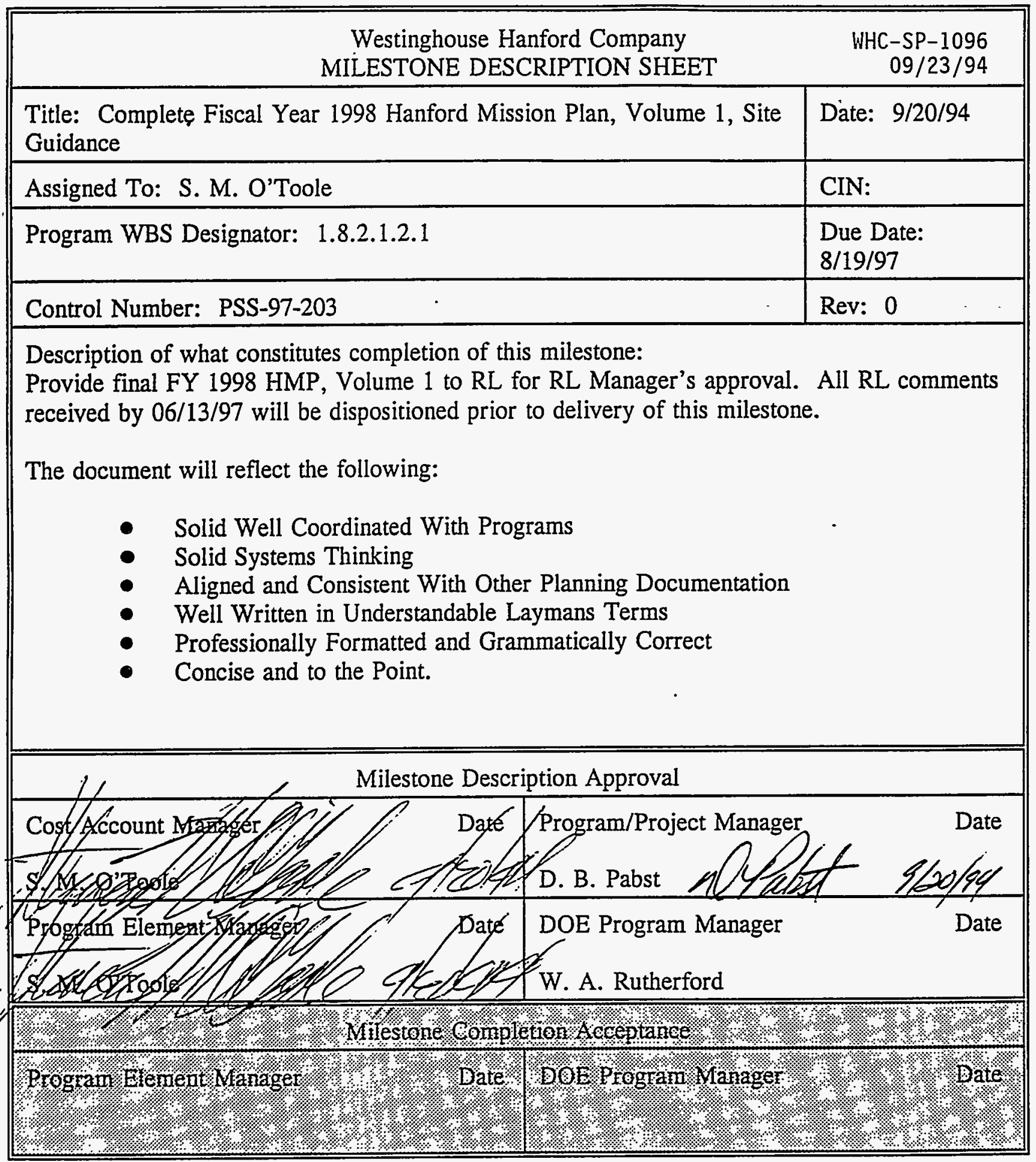




\begin{tabular}{|c|c|c|c|}
\hline & \multicolumn{2}{|c|}{$\begin{array}{l}\text { Westinghouse Hanford Company } \\
\text { MILESTONE DESCRIPTION SHEET }\end{array}$} & $\begin{array}{r}\text { WHC-SP-1096 } \\
09 / 23 / 94 \\
\end{array}$ \\
\hline \multicolumn{3}{|c|}{ Title: Complete Hanford Strategic Analysis Addendum for 1997} & Date: $9 / 20 / 94$ \\
\hline \multicolumn{3}{|c|}{ Assigned To: S. M. O'Toole } & CIN: \\
\hline \multicolumn{3}{|c|}{ Program WBS Designator: 1.8.2.1.2.1 } & $\begin{array}{l}\text { Due Date: } \\
\text { 9/30/97 }\end{array}$ \\
\hline \multicolumn{3}{|c|}{ Control Number: PSS-97-204 } & Rev: 0 \\
\hline $\begin{array}{l}\text { MILESTONE TYPE: } \\
\square \text { DOE-HQ } \\
\square \text { DOE-RL } \\
\text { X CONTRACTOR }\end{array}$ & 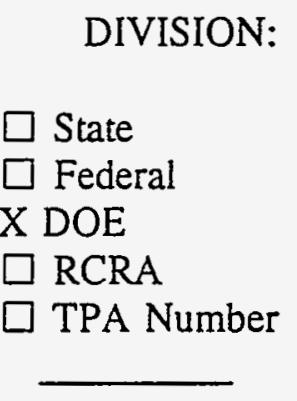 & $\begin{array}{l}\text { DELIVERABLE: } \\
\text { X Report } \\
\square \text { Letter } \\
\square \text { Drawings } \\
\square \text { Other (specify) }\end{array}$ & $\begin{array}{l}\text { ADDRESS TO: } \\
\square \text { DOE-HQ } \\
\square \text { DOE-RL } \\
\text { X Other (specify) } \\
\text { Systems } \\
\text { Engineering } \\
(1.8 .2 .4)\end{array}$ \\
\hline \multicolumn{4}{|c|}{$\begin{array}{l}\text { Milestone Description: } \\
\text { Provide to WHC Systems Engineering (1.8.2.4) an update of the } 1994 \text { Hanford Strategic } \\
\text { Analysis (HSA) in the form of an addendum prepared in Fiscal Year (FY) 1997. Contents will } \\
\text { include sitewide material balance, ROM cost and schedule. }\end{array}$} \\
\hline \multicolumn{4}{|c|}{$\begin{array}{l}\text { Description of what constitutes completion of this milestone: } \\
\text { This commitment is completed by transmittal of one hard copy of the } \\
\text { document containing additional alternative sitewide material balances, } \\
\text { to Systems Engineering. Systems Engineering will provide the neces } \\
\text { baseline, case description) to initiate the analysis and produce the HS } \\
\text { addendum will then represent a technical document reflecting three al } \\
\text { disposition at Hanford. The content and level of detail will be consis } \\
\text { Strategic Analysis Study, WHC-EP-0549. This document will be sui } \\
\text { public. } \\
\text { The document will reflect the following: } \\
\text { - Technically Sound and Tied to Systems Engineering } \\
\text { - Gell Written } \\
\text { - Concise and to the Point. }\end{array}$} \\
\hline \multicolumn{4}{|c|}{ _ Milestone Description Approval } \\
\hline \multicolumn{3}{|c|}{ Cost/fGeount Managey/fyid Date/ Program/Proje } & $\begin{array}{l}\text { Date } \\
\text { Q10194 }\end{array}$ \\
\hline \multicolumn{3}{|c|}{ Program Elementutanager } & \\
\hline
\end{tabular}




\begin{tabular}{|c|c|}
\hline $\begin{array}{l}\text { Westinghouse Hanford Company } \\
\text { MILESTONE DESCRIPTION SHEET }\end{array}$ & $\begin{array}{r}W H C-S P-1096 \\
09 / 23 / 94 \\
\end{array}$ \\
\hline Title: Complete Hanford Strategic Analysis Addendum for 1997 & Date: $9 / 20 / 94$ \\
\hline Assigned To: S. M. O'Toole & CIN: \\
\hline Program WBS Designator: 1.8 .2 .1 .2 .1 & $\begin{array}{l}\text { Due Date: } \\
\text { 9/30/97 }\end{array}$ \\
\hline Control Number: PSS-97-204 & Rev: 0 \\
\hline 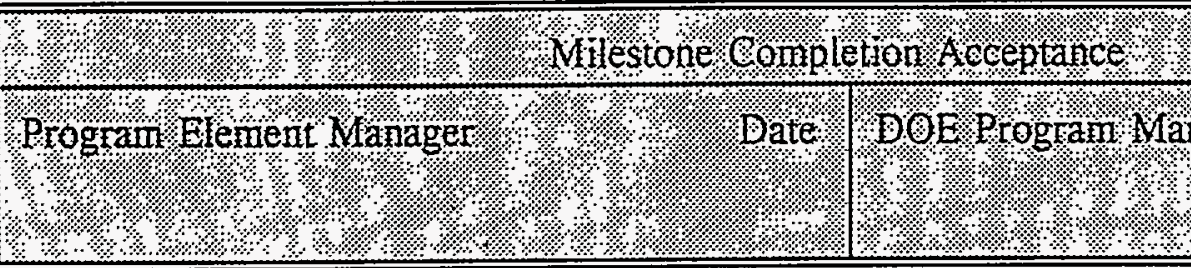 & $(4)$ \\
\hline
\end{tabular}




\begin{tabular}{|l|l||}
\hline \multicolumn{2}{|c|}{ Westinghouse Hanford Company } \\
MILESTONE DESCRIPTION SHEET
\end{tabular}




\begin{tabular}{|l|l||}
\hline \multicolumn{3}{|c||}{ Westinghouse Hanford Company } \\
MILESTONE DESCRIPTION SHEET
\end{tabular}




\begin{tabular}{|c|c|c|c|}
\hline \multicolumn{4}{|c|}{$\begin{array}{l}\text { Westinghouse Hanford Company } \\
\text { MILESTONE DESCRIPTION SHEET }\end{array}$} \\
\hline \multicolumn{3}{|c|}{ Title: Analysis of Risks and Costs at Hanford (Update) } & Date: $8 / 8 / 94$ \\
\hline \multicolumn{3}{|c|}{ Assigned To: WA Hesser } & CIN: \\
\hline \multicolumn{3}{|c|}{ Program WBS Designator: 1.8.2.1.3 } & $\begin{array}{l}\text { Due Date: } \\
1 / 31 / 97\end{array}$ \\
\hline \multicolumn{3}{|c|}{ Control Number: PSS-97-300 } & Rev: \\
\hline $\begin{array}{l}\text { MILESTONE TYPE: } \\
\square \text { DOE-HQ } \\
\text { X DOE-RL } \\
\square \text { CONTRACTOR }\end{array}$ & $\begin{array}{l}\quad \text { DIVISION: } \\
\square \text { State } \\
\square \text { Federal } \\
\text { X DOE } \\
\square \text { RCRA }\end{array}$ & $\begin{array}{l}\text { DELIVERABLE: } \\
\text { X Report } \\
\square \text { Letter } \\
\square \text { Drawings } \\
\square \text { Other (specify) }\end{array}$ & $\begin{array}{l}\text { ADDRESS TO: } \\
\text { D DOE-HO } \\
\text { X DOE-RL } \\
\square \text { Other } \\
\text { (specify) }\end{array}$ \\
\hline \multirow{2}{*}{\multicolumn{4}{|c|}{$\begin{array}{l}\text { The most important decisions at Hanford involve the allocation of funding to } \\
\text { competing activities. This report analyzes these decisions and describes the } \\
\text { relationship between costs, risks, and risk reduction. Risks, in this case, mean } \\
\text { public health, worker safety, and environmental/ecological. Costs are those } \\
\text { associated with program activities. The overall objective of the analysis is to } \\
\text { determine whether or not the Site is complying with DOE-HO directives to } \\
\text { allocate its resources to those areas of greatest risk, realizing of course, that } \\
\text { there are many other inputs to the decision process. } \\
\text { This analysis builds on similar work begun in FY94 and continued in FY95 and } \\
\text { FY96. }\end{array}$}} \\
\hline & & & \\
\hline \multicolumn{4}{|c|}{$\begin{array}{l}\text { The milestone will be complete when one copy of the report containing the } \\
\text { Analysis of Risks and Costs at Hanford is submitted to } R L \text { and the } R L \text { manager } \\
\text { has signed the milestone completion acceptance. }\end{array}$} \\
\hline \multicolumn{4}{|c|}{ Milestone Description Approval } \\
\hline \multicolumn{2}{|c|}{ Cost Account Manager } & \multicolumn{2}{|c|}{$\begin{array}{c}\text { Program/Project Manager } \\
\text { DB Pabst }\end{array}$} \\
\hline
\end{tabular}




\section{Westinghouse Hanford Company MILESTONE DESCRIPTION SHEET}

Title: Analysis of Risks and Costs at Hanford (Update)

Date: $8 / 8 / 94$

Assigned To: WA Hesser

CIN:

Program WBS Designator: 1.8.2.1.3

Due Date:

$1 / 31 / 97$

Control Number: PSS-97-300

Rev:

Prograán Element Manager

S.M O'toole

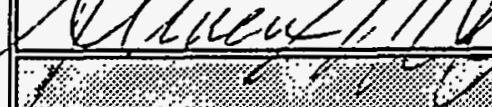

Uthestone Eompletion Aceeptance

Progran Elentent Wan ager 


\begin{tabular}{|c|c|c|c|}
\hline \multicolumn{4}{|c|}{$\begin{array}{l}\text { Westinghouse Hanford Company } \\
\text { MILESTONE DESCRIPTION SHEET }\end{array}$} \\
\hline \multicolumn{3}{|c|}{$\begin{array}{l}\text { Report of the Application of Risk-Based decision } \\
\text { making at Hanford }\end{array}$} & Date: $8 / 8 / 94$ \\
\hline \multicolumn{3}{|c|}{ Assigned To: WA Hesser } & CIN: \\
\hline \multicolumn{3}{|c|}{ Program WBS Designator: $1.8 \cdot 2.1 .3$} & $\begin{array}{l}\text { Due Date: } \\
9 / 30 / 97\end{array}$ \\
\hline \multicolumn{3}{|c|}{ Control Number: PSS-97-301 } & Rev: \\
\hline MILESTONE TYPE: & DIVISION: & DELIVERABLE: & ADDRESS TO: \\
\hline $\begin{array}{l}\square \text { DOE-HQ } \\
\times \text { DOE-RL } \\
\square \text { CONTRACTOR }\end{array}$ & $\begin{array}{l}\square \text { State } \\
\square \text { Federal } \\
X \text { DOE } \\
\square \text { RCRA }\end{array}$ & $\begin{array}{l}\text { X Report } \\
\square \text { Letter } \\
\square \text { Drawings } \\
\square \text { Other (specify) }\end{array}$ & $\begin{array}{l}\square \text { DOE-HQ } \\
X \text { DOE-RL } \\
\square \text { Other } \\
\text { (specify) }\end{array}$ \\
\hline
\end{tabular}




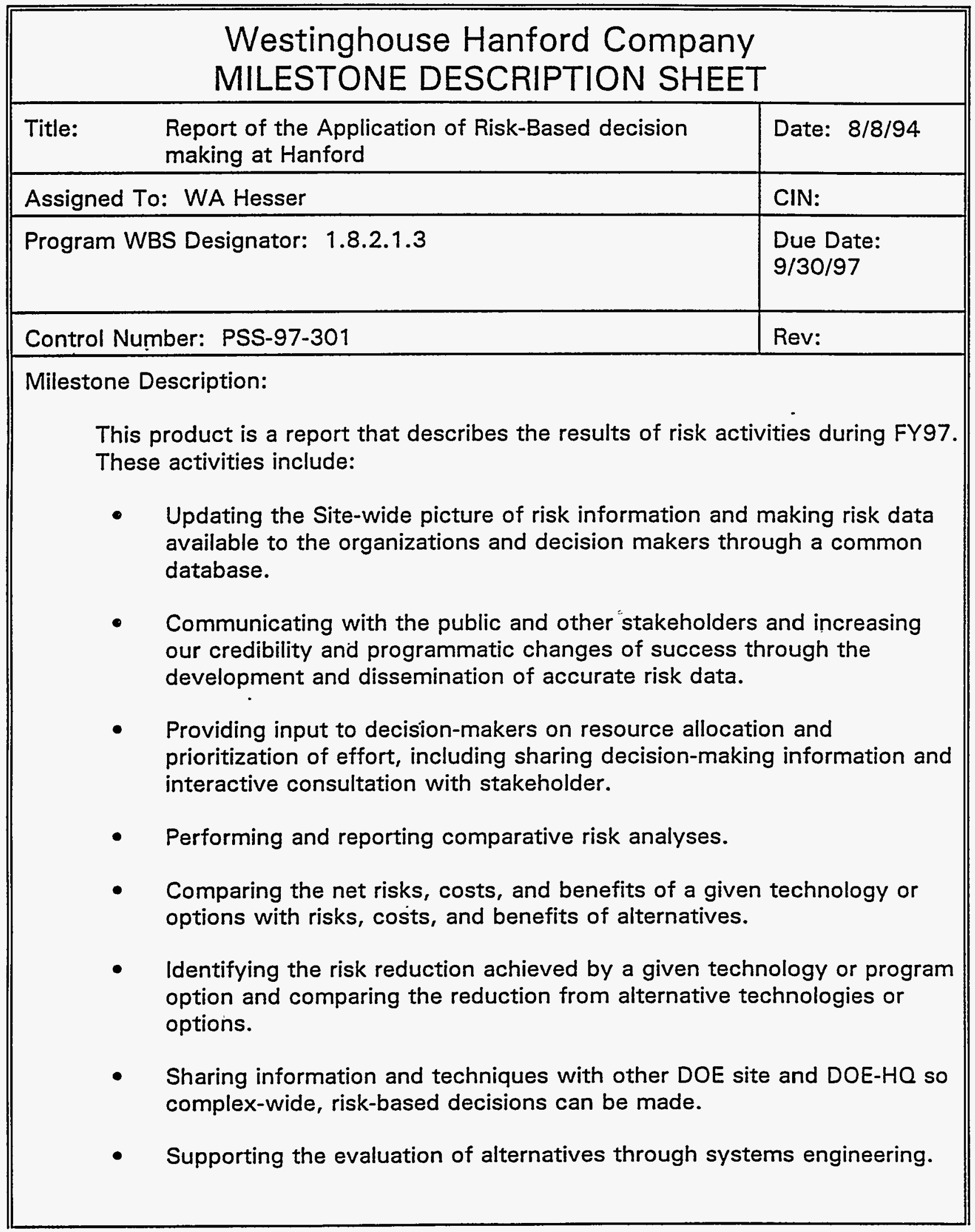




\begin{tabular}{|l|l||}
\hline \multicolumn{3}{|c|}{ Westinghouse Hanford Company } \\
MILESTONE DESCRIPTION SHEET \\
\hline Title: $\begin{array}{l}\text { Report of the Application of Risk-Based decision } \\
\text { making at Hanford }\end{array}$ & Date: $8 / 8 / 94$ \\
\hline Assigned To: WA Hesser & CIN: \\
\hline Program WBS Designator: 1.8.2.1.3 & Due Date: \\
$9 / 30 / 97$
\end{tabular}


Planning Integration Multi-Year Progran Plan

\section{This page}

intentionally left blank. 


\section{Westinghouse Hanford Company MILESTONE DESCRIPTION SHEET}

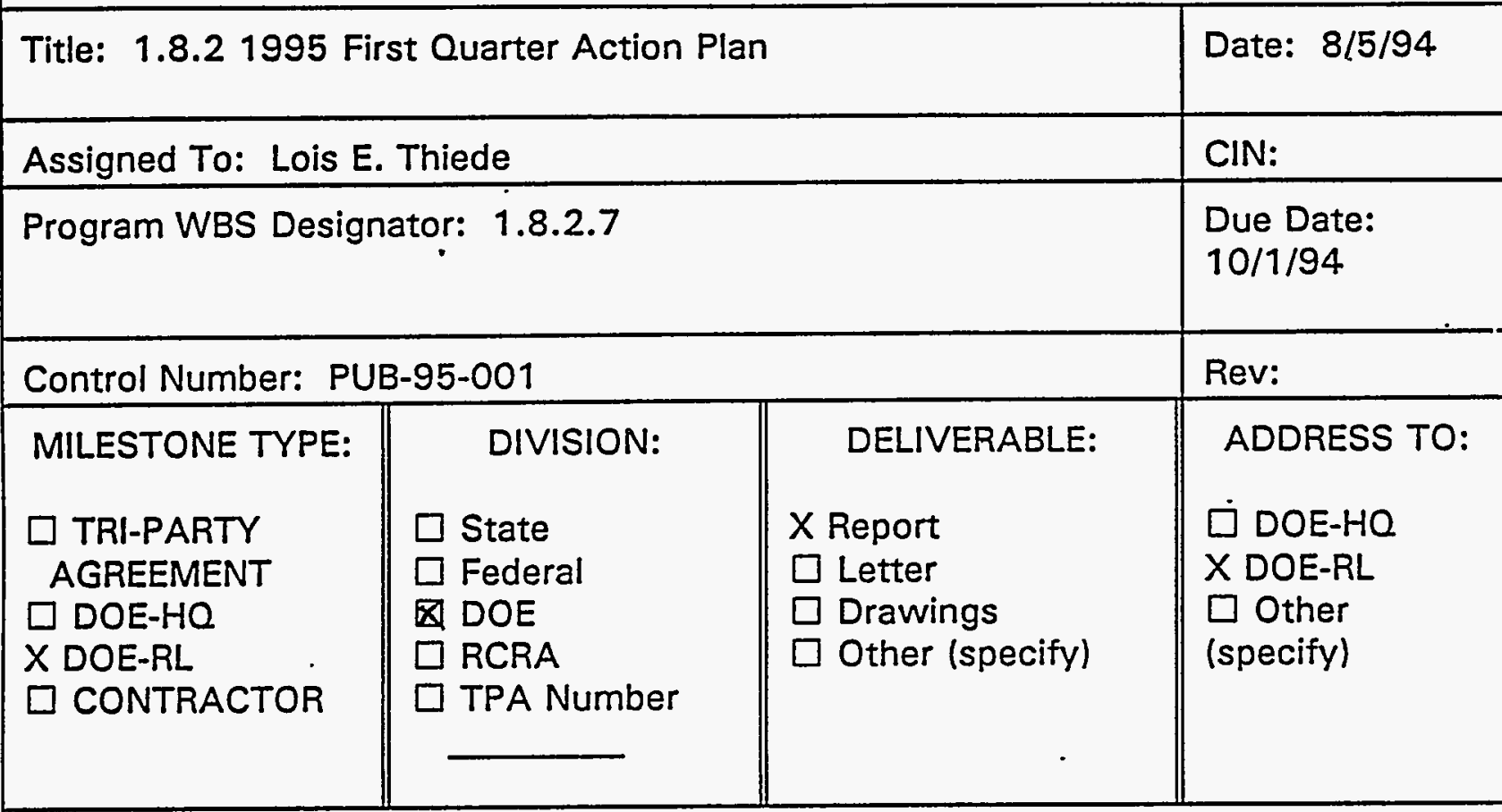

Milestone Description: Provide an Action Plan for specific public involvement actions and schedule to be completed in the First Quarter of FY 1995.

Description of what constitutes completion of this milestone: This milestone will be complete when a document entitled "1.8.2 1995 First Quarter Public Involvement Action Plan" is provided to RL for their signoff to authorize implementation.

\begin{tabular}{|c|c|}
\hline \multicolumn{2}{|c|}{ Milestone Description Approval } \\
\hline $\begin{array}{l}\text { Cost Account Manager Date } \\
\text { Lois E. Thiedteriuf the the 8-25.94t }\end{array}$ & Drogram/Project Manager \\
\hline 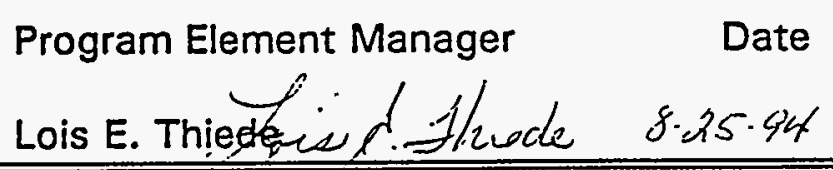 & $\begin{array}{lc}\text { DOE Program Manager } & \text { Date } \\
\text { Bill A. Rutherford on Hesd } & 9 / 23 / 44 \\
\end{array}$ \\
\hline 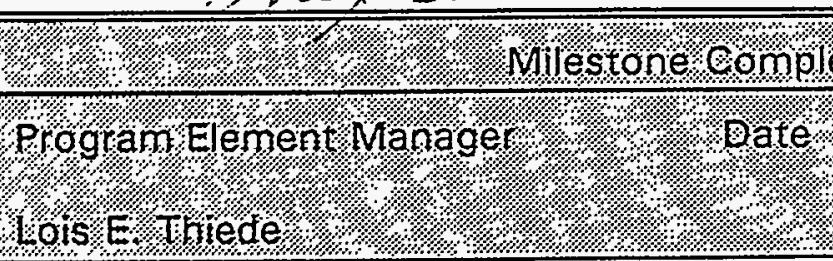 & 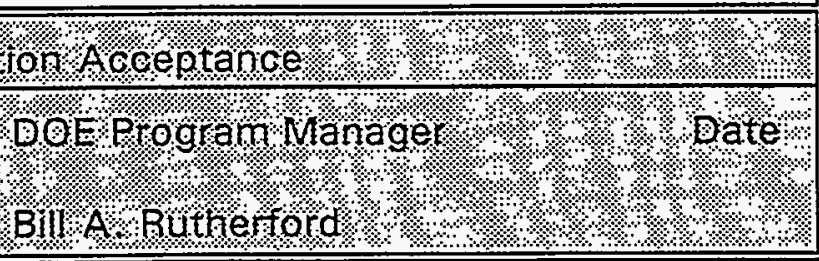 \\
\hline
\end{tabular}




\begin{tabular}{|c|c|c|c|}
\hline \multicolumn{4}{|c|}{$\begin{array}{l}\text { Westinghouse Hanford Company } \\
\text { MILESTONE DESCRIPTION SHEET }\end{array}$} \\
\hline \multicolumn{3}{|c|}{ Title: 1.8.2 1995 Second Quarter Action Plan } & Date: $8 / 5 / 94$ \\
\hline \multicolumn{3}{|c|}{ Assigned To: Lois E. Thiede } & CIN: \\
\hline \multicolumn{3}{|c|}{ Program WBS Designator: 1 8.2.7 } & $\begin{array}{l}\text { Due Date: } \\
12 / 9 / 94\end{array}$ \\
\hline \multicolumn{3}{|c|}{ Control Number: PUB-95-002 } & Rev: \\
\hline $\begin{array}{l}\text { MILESTONE TYPE: } \\
\square \text { TRI-PARTY } \\
\text { AGREEMENT } \\
\square \text { DOE-HQ } \\
\text { X DOE-RL } \\
\square \text { CONTRACTOR }\end{array}$ & $\begin{array}{l}\text { - DIVISION: } \\
\square \text { State } \\
\square \text { Federal } \\
\otimes \text { DOE } \\
\square \text { RCRA } \\
\square \text { TPA Number }\end{array}$ & $\begin{array}{l}\text { DELIVERABLE: } \\
\times \text { Report } \\
\square \text { Letter } \\
\square \text { Drawings } \\
\square \text { Other (specify) }\end{array}$ & $\begin{array}{l}\text { ADDRESS TO: } \\
\square \text { DOE-HQ } \\
\text { X DOE-RL } \\
\square \text { Other } \\
\text { (specify) }\end{array}$ \\
\hline \multicolumn{4}{|c|}{$\begin{array}{l}\text { Milestone Description: Provide an Action Plan for specific public involvement actions } \\
\text { and schedule to be completed in the Second Quarter of FY } 1995 \text {. }\end{array}$} \\
\hline \multicolumn{4}{|c|}{$\begin{array}{l}\text { Description of what constitutes completion of this milestone: This milestone will be } \\
\text { complete when a document entitled "1.8.2 } 1995 \text { Second Quarter Public Involvement } \\
\text { Action Plan" is provided to RL for their signoff to authorize implementation. }\end{array}$} \\
\hline \multicolumn{4}{|c|}{ Milestone Description Approval } \\
\hline \multicolumn{2}{|c|}{ 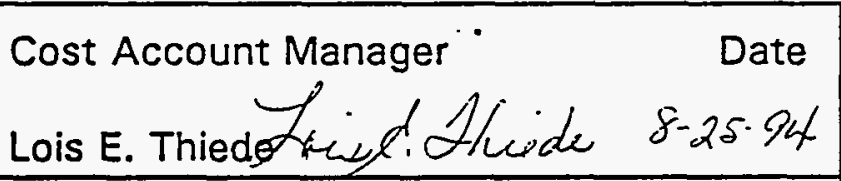 } & \multicolumn{2}{|c|}{ Program/Project Manager Bate } \\
\hline \multicolumn{2}{|c|}{ Program Element Manager } & \multicolumn{2}{|c|}{$\begin{array}{l}\text { DOE Program Manager } \\
\text { Bill A. Rutherford ON Nold } 9 / 23 / 54\end{array}$} \\
\hline \multicolumn{4}{|c|}{ 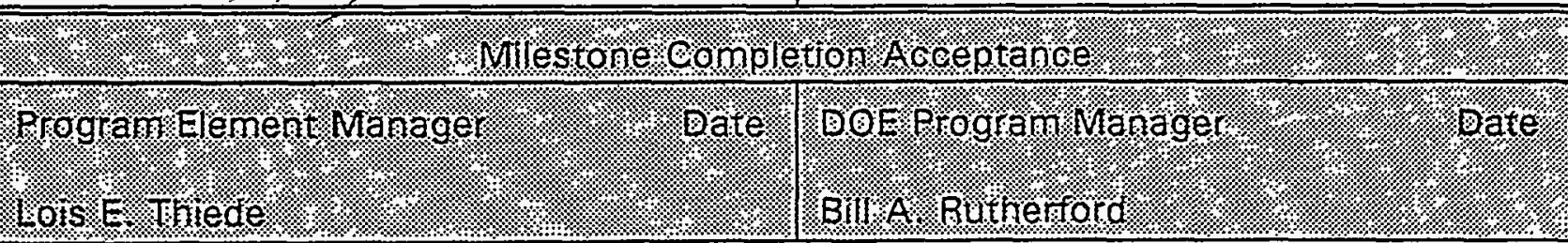 } \\
\hline
\end{tabular}




\begin{tabular}{|c|c|c|c|}
\hline \multicolumn{4}{|c|}{$\begin{array}{l}\text { Westinghouse Hanford Company } \\
\text { MILESTONE DESCRIPTION SHEET }\end{array}$} \\
\hline \multicolumn{3}{|c|}{ Title: Summary of Action Plan } & Date: $8 / 3 / 94$ \\
\hline \multicolumn{3}{|c|}{ Assigned To: Lois E. Thiede } & CIN: \\
\hline \multicolumn{3}{|c|}{ Program WBS Designator: 1.8 .2 .7} & $\begin{array}{l}\text { Due Date: } \\
1 / 13 / 95\end{array}$ \\
\hline \multicolumn{3}{|c|}{ Control Number: PUB-95-003 } & Rev: \\
\hline $\begin{array}{l}\text { MILESTONE TYPE: } \\
\square \text { TRI-PARTY } \\
\text { AGREEMENT } \\
\square \text { DOE-HQ } \\
\square \text { DOE-RL } \\
X \text { CONTRACTOR }\end{array}$ & $\begin{aligned} & \text { DIVISION: } \\
& \\
& \square \text { State } \\
& \square \text { Federal } \\
& \otimes \text { DOE } \\
& \square \text { RCRA } \\
& \square \text { TPA Number } \\
&\end{aligned}$ & $\begin{aligned} & \text { DELIVERABLE: } \\
X & \text { Report } \\
& \text { Letter } \\
& \square \text { Drawings } \\
& \text { Other (specify) }\end{aligned}$ & $\begin{array}{l}\text { ADDRESS TO: } \\
\text { म DOE-HO } \\
\square \text { DOE-RL } \\
\otimes \text { Other } \\
\text { (specify) } \\
1.8 .2 \text { Peo RAM } \\
\text { ELENENTS }\end{array}$ \\
\hline \multicolumn{4}{|c|}{$\begin{array}{l}\text { Milestone Description: Provide a summary of results from implementing the Public } \\
\text { Involvement Action Plan' for the first quarter of the fiscal year. Summary to describe } \\
\text { the process undertaken and evaluation of how well stated objectives were met. }\end{array}$} \\
\hline \multicolumn{4}{|c|}{$\begin{array}{l}\text { Description of what constitutes completion of this milestone: This milestone will be } \\
\text { complete when a document entitled "1995 First Quarter } 1.8 .2 \text { Public Involvement } \\
\text { Action Plan Summary" is provided to all requesting Program Elements. }\end{array}$} \\
\hline \multicolumn{4}{|c|}{ Milestone Description Approval } \\
\hline \multicolumn{2}{|c|}{ 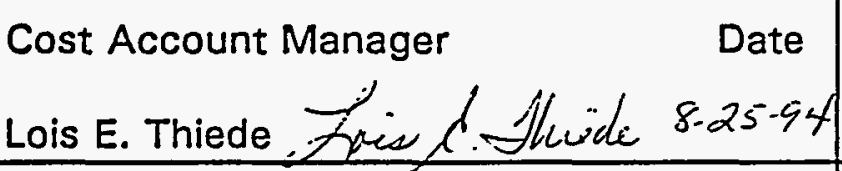 } & \multicolumn{2}{|c|}{ Dave B. Pabst Date } \\
\hline \multicolumn{2}{|c|}{$\begin{array}{l}\text { Program Element Manager } \quad \text { Date } \\
\text { Lois E. Thiede frief. H/wide } 8.2594\end{array}$} & \multicolumn{2}{|c|}{$\begin{array}{l}\text { DOE Program Manager Date } \\
\text { NA }\end{array}$} \\
\hline \multicolumn{4}{|c|}{ 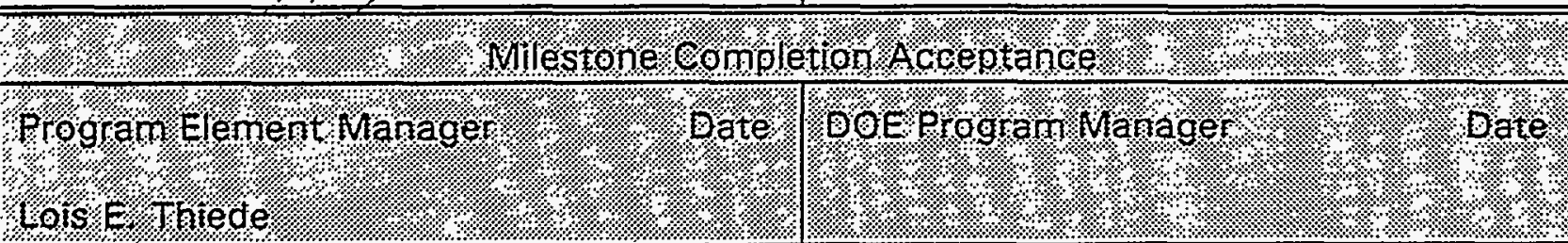 } \\
\hline
\end{tabular}




\begin{tabular}{|c|c|c|c|}
\hline \multicolumn{4}{|c|}{$\begin{array}{l}\text { Westinghouse Hanford Company } \\
\text { MILESTONE DESCRIPTION SHEET }\end{array}$} \\
\hline \multicolumn{3}{|c|}{ Title: 1.8.2 1995 Third Quarter Action Plan } & Date: $8 / 5 / 94$ \\
\hline \multicolumn{3}{|c|}{ Assigned To: Lois E. Thiede } & CIN: \\
\hline \multicolumn{3}{|c|}{ Program WBS Designator: 1.8.2.7 } & $\begin{array}{l}\text { Due Date: } \\
3 / 10 / 95\end{array}$ \\
\hline \multicolumn{3}{|c|}{ Control Number: PUB-95-004 } & Rev: \\
\hline $\begin{array}{l}\text { MILESTONE TYPE: } \\
\square \text { TRI-PARTY } \\
\text { AGREEMENT } \\
\square \text { DOE-HO } \\
\text { X DOE-RL } \\
\square \text { CONTRACTOR }\end{array}$ & $\begin{array}{l}\text { DIVISION: } \\
\square \text { State } \\
\square \text { Federal } \\
\text { DOE } \\
\square \text { RCRA } \\
\square \text { TPA Number }\end{array}$ & $\begin{array}{l}\text { DELIVERABLE: } \\
\text { X Report } \\
\square \text { Letter } \\
\square \text { Drawings } \\
\square \text { Other (specify) }\end{array}$ & $\begin{array}{l}\text { ADDRESS TO: } \\
\square \text { DOE-HO } \\
\text { X DOE-RL } \\
\square \text { Other } \\
\text { (specify) }\end{array}$ \\
\hline \multicolumn{4}{|c|}{$\begin{array}{l}\text { Milestone Description: Provide an Action Plan for specific public involvement actions } \\
\text { and schedule to be completed in the Third Quarter of FY } 1995 .\end{array}$} \\
\hline \multicolumn{4}{|c|}{$\begin{array}{l}\text { Description of what constitutes completion of this milestone: This milestone will be } \\
\text { complete when a document entitled " } 1.8 .21995 \text { Third Quarter Public Involvement } \\
\text { Action Plan" is provided to RL for their signoff to authorize implementation. }\end{array}$} \\
\hline \multicolumn{4}{|c|}{ Milestone Description Approval } \\
\hline \multicolumn{2}{|c|}{ 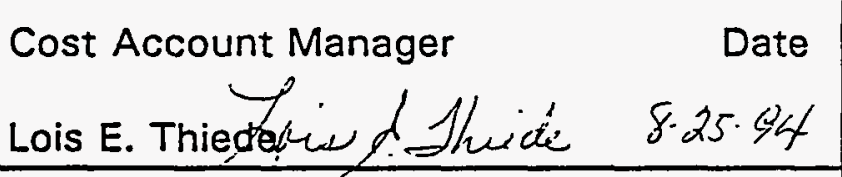 } & \multicolumn{2}{|c|}{ Drogram/Project Manager B. Pabst } \\
\hline \multicolumn{2}{|c|}{$\begin{array}{ll}\text { Program Element Manager } & \text { Date } \\
\text { Lois E. Thieforew Hevide } & 8 \cdot 2.5 \cdot 9.4\end{array}$} & \multicolumn{2}{|c|}{$\begin{array}{ll}\text { DOE Program Manager } & \text { Date } \\
\text { Bill A. Rutherford } \mathrm{ON} \text { Klold } 9 / 27 / 5 \mathrm{~S}\end{array}$} \\
\hline \multicolumn{4}{|c|}{ 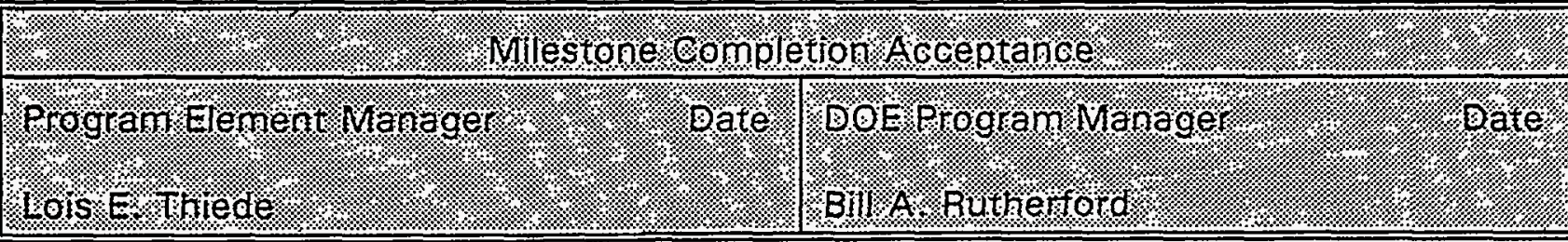 } \\
\hline
\end{tabular}




\begin{tabular}{|c|c|c|c|}
\hline \multicolumn{4}{|c|}{$\begin{array}{l}\text { Westinghouse Hanford Company } \\
\text { MILESTONE DESCRIPTION SHEET }\end{array}$} \\
\hline \multicolumn{3}{|c|}{ Title: Summary of Action Plan } & Date: $8 / 3 / 94$ \\
\hline \multicolumn{3}{|c|}{ Assigned To: Lois E. Thiede } & CIN: \\
\hline \multicolumn{3}{|c|}{ Program WBS Designator: 1 (.8.2.7 } & $\begin{array}{l}\text { Due Date: } \\
4 / 13 / 95\end{array}$ \\
\hline \multicolumn{3}{|c|}{ Control Number: PUB-95-006 } & Rev: \\
\hline $\begin{array}{l}\text { MILESTONE TYPE: } \\
\square \text { TRI-PARTY } \\
\text { AGREEMENT } \\
\square \text { DOE-HQ } \\
\square \text { DOE-RL } \\
X \text { CONTRACTOR }\end{array}$ & $\begin{aligned} & \text { DIVISION: } \\
& \square \text { State } \\
& \square \text { Federal } \\
& \text { DOE } \\
& \square \text { RCRA } \\
& \square \text { TPA Number }\end{aligned}$ & $\begin{array}{l}\text { DELIVERABLE: } \\
\times \text { Report } \\
\square \text { Letter } \\
\square \text { Drawings } \\
\square \text { Other (specify) }\end{array}$ & $\begin{array}{l}\text { ADDRESS TO: } \\
\text { 口 DOE-HO } \\
\square \text { DOE-RL } \\
\text { Q Other } \\
\text { (specify) } \\
\text { 1.8.2. PhoGram } \\
\text { ELEMErith. }\end{array}$ \\
\hline \multicolumn{4}{|c|}{$\begin{array}{l}\text { Milestone Description: Provide a summary of results from implementing the Public } \\
\text { Involvement Action Plan'for the second quarter of the fiscal year. Summary to } \\
\text { describe the process undertaken and evaluation of how well stated objectives were } \\
\text { met. }\end{array}$} \\
\hline \multicolumn{4}{|c|}{$\begin{array}{l}\text { Description of what constitutes completion of this milestone: This milestone will be } \\
\text { complete when a document entitled "1995 Second Quarter } 1.8 .2 \text { Public Involvement } \\
\text { Action Plan Summary" is provided to all requesting Program Elements. }\end{array}$} \\
\hline \multicolumn{4}{|c|}{ Milestone Description Approval } \\
\hline \multicolumn{2}{|c|}{$\begin{array}{l}\text { Cost Account Manager } \\
\text { Lois E. Thiede Late } \\
\text { Srift. Shick } \\
\end{array}$} & \multicolumn{2}{|c|}{$\begin{array}{l}\text { Program/Project Manager } \\
\text { Dave B. Pabst }\end{array}$} \\
\hline \multicolumn{2}{|c|}{ 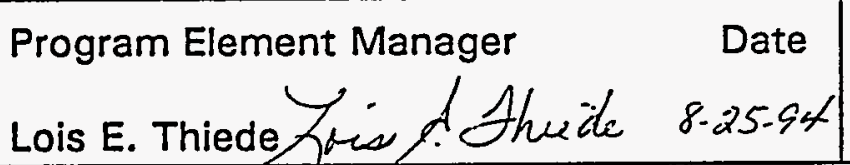 } & \multicolumn{2}{|c|}{$\begin{array}{l}\text { DOE Program Manager Date } \\
\text { NA }\end{array}$} \\
\hline \multicolumn{4}{|c|}{ 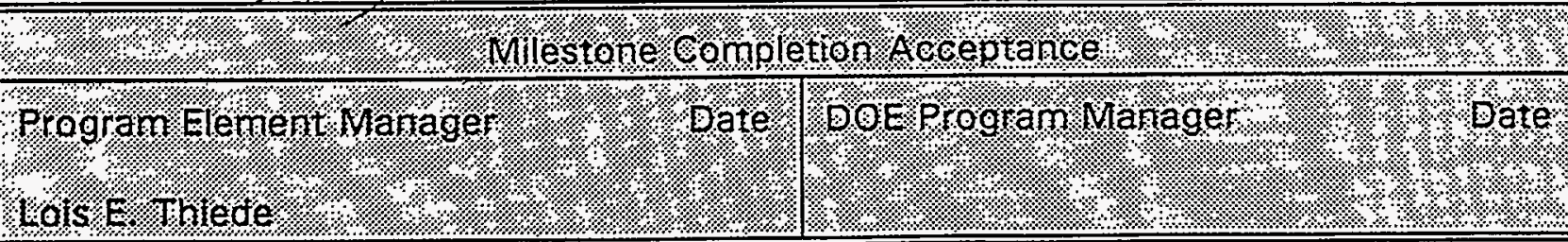 } \\
\hline
\end{tabular}




\begin{tabular}{|c|c|c|c|}
\hline \multicolumn{4}{|c|}{$\begin{array}{l}\text { Westinghouse Hanford Company } \\
\text { MILESTONE DESCRIPTION SHEET }\end{array}$} \\
\hline \multicolumn{3}{|c|}{ Title: 1.8.2 1995 Fourth Quarter Action Plan } & Date: $8 / 5 / 94$ \\
\hline \multicolumn{3}{|c|}{ Assigned To: Lois E. Thiede } & CIN: \\
\hline \multicolumn{3}{|c|}{ Program WBS Designator: 1 1.8.2.7 } & $\begin{array}{l}\text { Due Date: } \\
6 / 9 / 95\end{array}$ \\
\hline \multicolumn{3}{|c|}{ Control Number: PUB-95-007 } & Rev: \\
\hline $\begin{array}{l}\text { MILESTONE TYPE: } \\
\square \text { TRI-PARTY } \\
\text { AGREEMENT } \\
\square \text { DOE-HQ } \\
\text { X DOE-RL } \\
\square \text { CONTRACTOR }\end{array}$ & $\begin{aligned} & \text { DIVISION: } \\
& \square \text { State } \\
& \square \text { Federal } \\
& \square \text { DOE } \\
& \square \text { RCRA } \\
& \square \text { TPA Number }\end{aligned}$ & $\begin{array}{l}\text { DELIVERABLE: } \\
\times \text { Report } \\
\square \text { Letter } \\
\square \text { Drawings } \\
\square \text { Other (specify) }\end{array}$ & 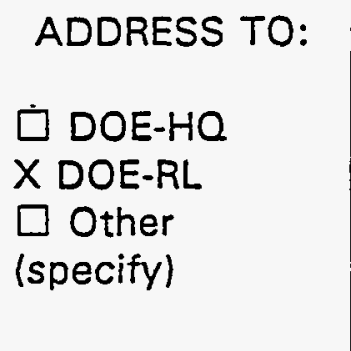 \\
\hline \multicolumn{4}{|c|}{$\begin{array}{l}\text { Milestone Description: Provide an Action Plan for specific public involvement actions } \\
\text { and schedule to be completed in the Fourth Quarter of FY } 1995 .\end{array}$} \\
\hline \multicolumn{4}{|c|}{$\begin{array}{l}\text { Description of what constitutes completion of this milestone: This milestone will be } \\
\text { complete when a document entitled "1.8.2 } 1995 \text { Fourth Quarter Public Involvement } \\
\text { Action Plan" is provided to RL for their signoff to authorize implementation. }\end{array}$} \\
\hline \multicolumn{4}{|c|}{ Milestone Description Approval } \\
\hline \multicolumn{2}{|c|}{ 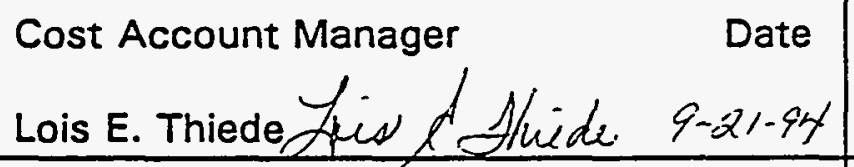 } & \multicolumn{2}{|c|}{ Dave B. Pabst Date } \\
\hline \multicolumn{2}{|c|}{$\begin{array}{l}\text { Program Element Manager } \\
\text { Lois E. Thiede Tris /. Sthide } 9-21-94\end{array}$} & \multicolumn{2}{|c|}{$\begin{array}{ll}\text { DOE Program Manager } & \text { Date } \\
\text { Bill A. Rutherford } & \text { flold } \\
\end{array}$} \\
\hline \multicolumn{4}{|c|}{ 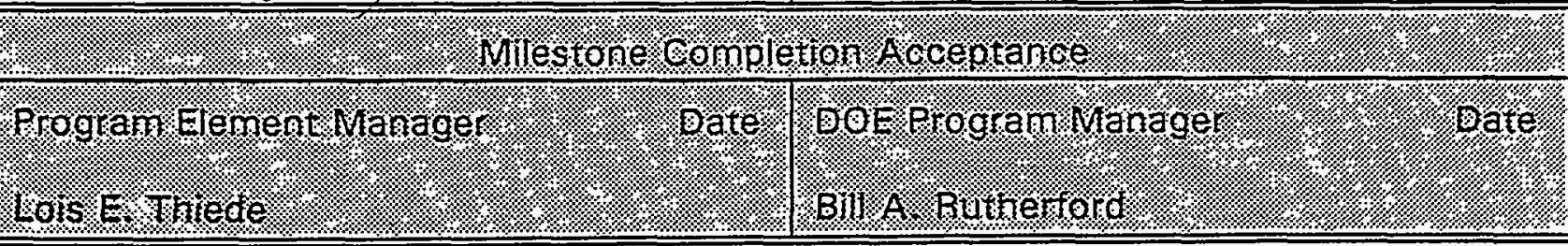 } \\
\hline
\end{tabular}




\section{Westinghouse Hanford Company MILESTONE DESCRIPTION SHEET}

\begin{tabular}{|c|c|c|c|}
\hline \multicolumn{3}{|c|}{ Title: Summary of Action Plan } & Date: $8 / 3 / 94$ \\
\hline \multicolumn{3}{|c|}{ Assigned To: Lois E. Thiede } & CIN: \\
\hline \multicolumn{3}{|c|}{ Program WBS Designator: 1.8.2.7 } & $\begin{array}{l}\text { Due Date: } \\
7 / 14 / 95\end{array}$ \\
\hline \multicolumn{3}{|c|}{ Control Number: PUB-95-008 } & Rev: \\
\hline MILESTONE TYPE: & DIVISION: & DELIVERABLE: & ADDRESS TO: \\
\hline $\begin{array}{l}\square \text { TRI-PARTY } \\
\text { AGREEMENT } \\
\square \text { DOE-HQ } \\
\square \text { DOE-RL } \\
\times \text { CONTRACTOR }\end{array}$ & $\begin{array}{l}\square \text { State } \\
\square \text { Federal } \\
\triangle \text { DOE } \\
\square \text { RCRA } \\
\square \text { TPA Number }\end{array}$ & $\begin{array}{l}X \text { Report } \\
\square \text { Letter } \\
\square \text { Drawings } \\
\square \text { Other (specify) }\end{array}$ & 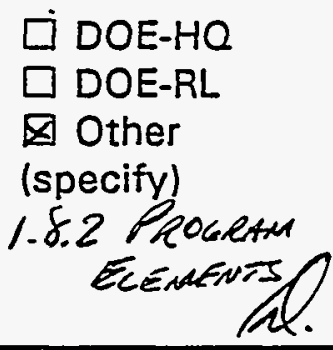 \\
\hline
\end{tabular}

Milestone Description: Provide a summary of results from implementing the Public Involvement Action Plan for the third quarter of the fiscal year. Summary to describe the process undertaken and evaluation of how well stated objectives were met.

Description of what constitutes completion of this milestone: This milestone will be complete when a document entitled "1995 Third Quarter 1.8.2 Public Involvement Action Plan Summary" is provided to all requesting Program Elements.

\begin{tabular}{|c|c|}
\hline \multicolumn{2}{|c|}{ Milestone Description Approval } \\
\hline 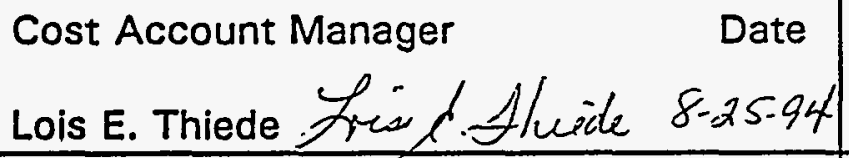 & Drogram/Project Manager B. Pabst Date \\
\hline 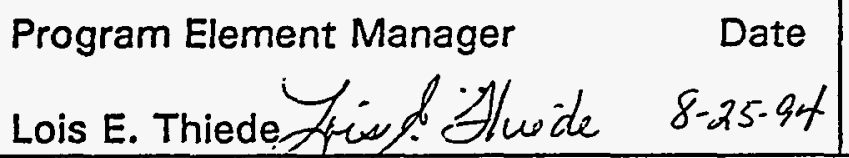 & $\begin{array}{l}\text { DOE Program Manager } \\
\text { NA }\end{array}$ \\
\hline 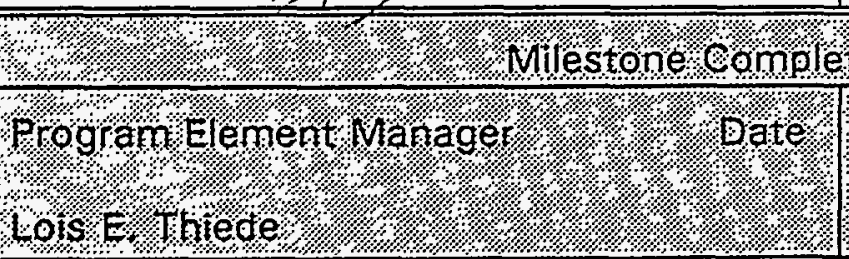 & 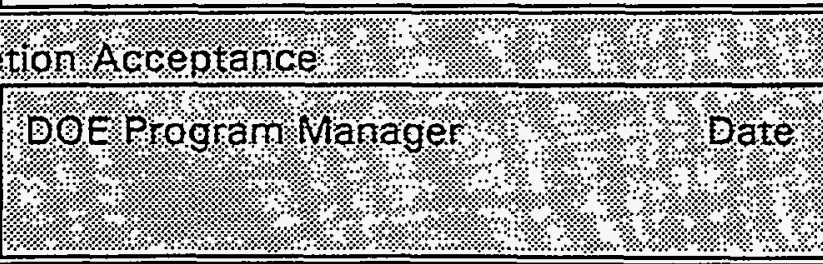 \\
\hline
\end{tabular}




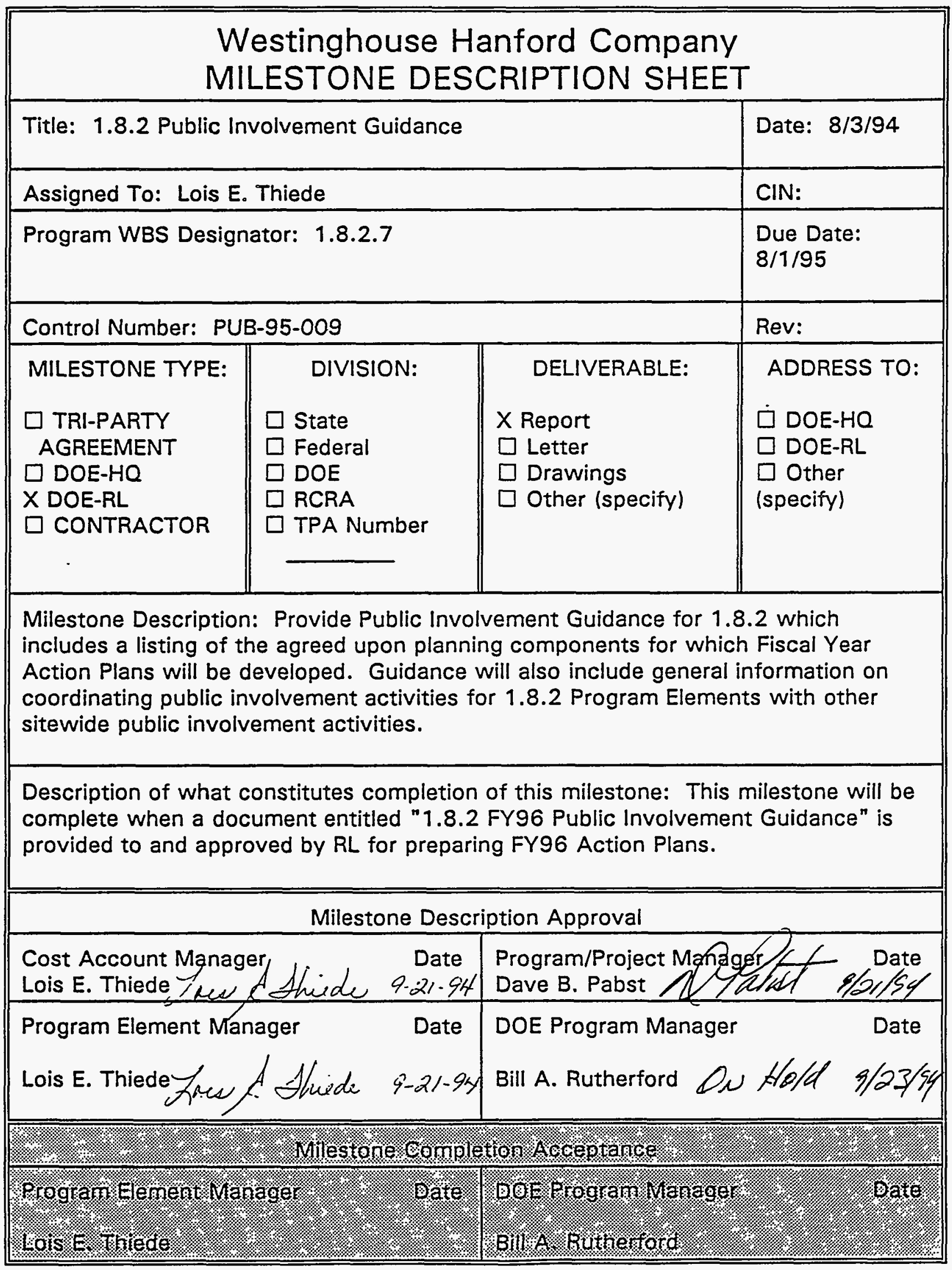




\begin{tabular}{|c|c|c|c|}
\hline \multicolumn{4}{|c|}{$\begin{array}{l}\text { Westinghouse Hanford Company } \\
\text { MILESTONE DESCRIPTION SHEET }\end{array}$} \\
\hline \multicolumn{3}{|c|}{ Title: 1.8 .21996 First Quarter Action Plan } & Date: $8 / 5 / 94$ \\
\hline \multicolumn{3}{|c|}{ Assigned To: Lois E. Thiede } & CIN: \\
\hline \multicolumn{3}{|c|}{ Program WBS Designator: 1 8.2.7 } & $\begin{array}{l}\text { Due Date: } \\
9 / 8 / 95\end{array}$ \\
\hline \multicolumn{3}{|c|}{ Control Number: PUB-95-010 } & Rev: \\
\hline $\begin{array}{l}\text { MILESTONE TYPE: } \\
\square \text { TRI-PARTY } \\
\text { AGREEMENT } \\
\square \text { DOE-HO } \\
\text { X DOE-RL } \\
\square \text { CONTRACTOR }\end{array}$ & 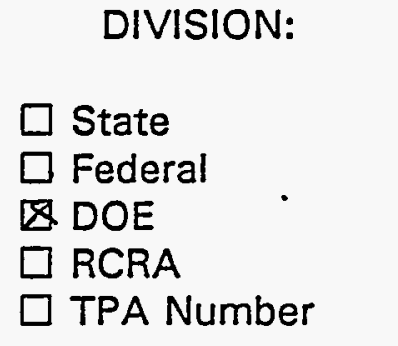 & $\begin{aligned} & \text { DELIVERABLE: } \\
& X \text { Report } \\
& \square \text { Letter } \\
& \square \text { Drawings } \\
& \square \text { Other (specify) }\end{aligned}$ & $\begin{array}{l}\text { ADDRESS TO: } \\
\square \text { DOE-HQ } \\
\text { X DOE-RL } \\
\square \text { Other } \\
\text { (specify) }\end{array}$ \\
\hline \multicolumn{4}{|c|}{$\begin{array}{l}\text { Milestone Description: Provide an Action Plan for specific public involvement actions } \\
\text { and schedule to be completed in the First Quarter of FY } 1996 .\end{array}$} \\
\hline \multicolumn{4}{|c|}{$\begin{array}{l}\text { Description of what constitutes completion of this milestone: "This milestone will be } \\
\text { complete when a document entitled "1.8.2 } 1996 \text { First Quarter Public Involvement } \\
\text { Action Plan" is provided to RL for their signoff to authorize implementation. }\end{array}$} \\
\hline \multicolumn{4}{|c|}{ Milestone Description Approval } \\
\hline \multicolumn{2}{|c|}{ 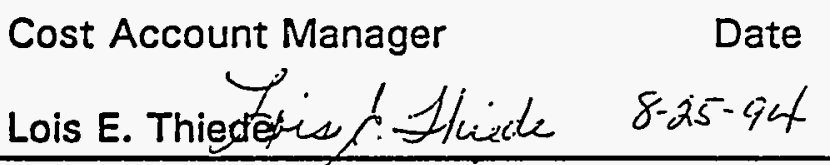 } & \multicolumn{2}{|c|}{ Program/Project Managen B. Pabst 1 Date } \\
\hline \multicolumn{2}{|c|}{ 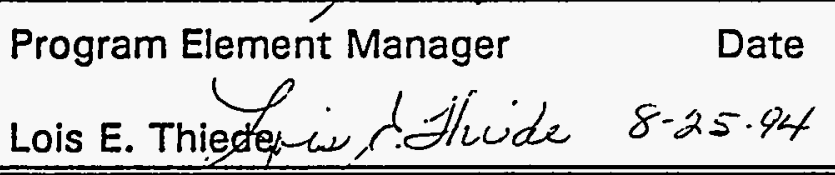 } & \multicolumn{2}{|c|}{$\begin{array}{l}\text { DOE Program Manager } \\
\text { Bill A. Rutherford DN flold } 9 / 23 / 54\end{array}$} \\
\hline \multicolumn{4}{|c|}{ 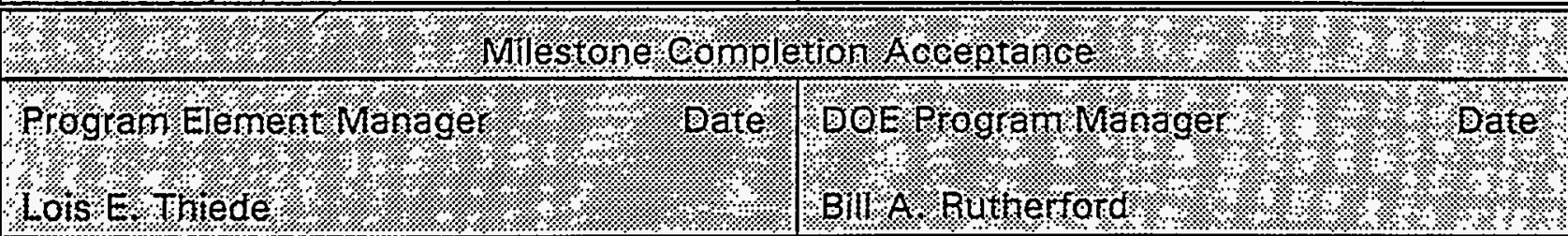 } \\
\hline
\end{tabular}




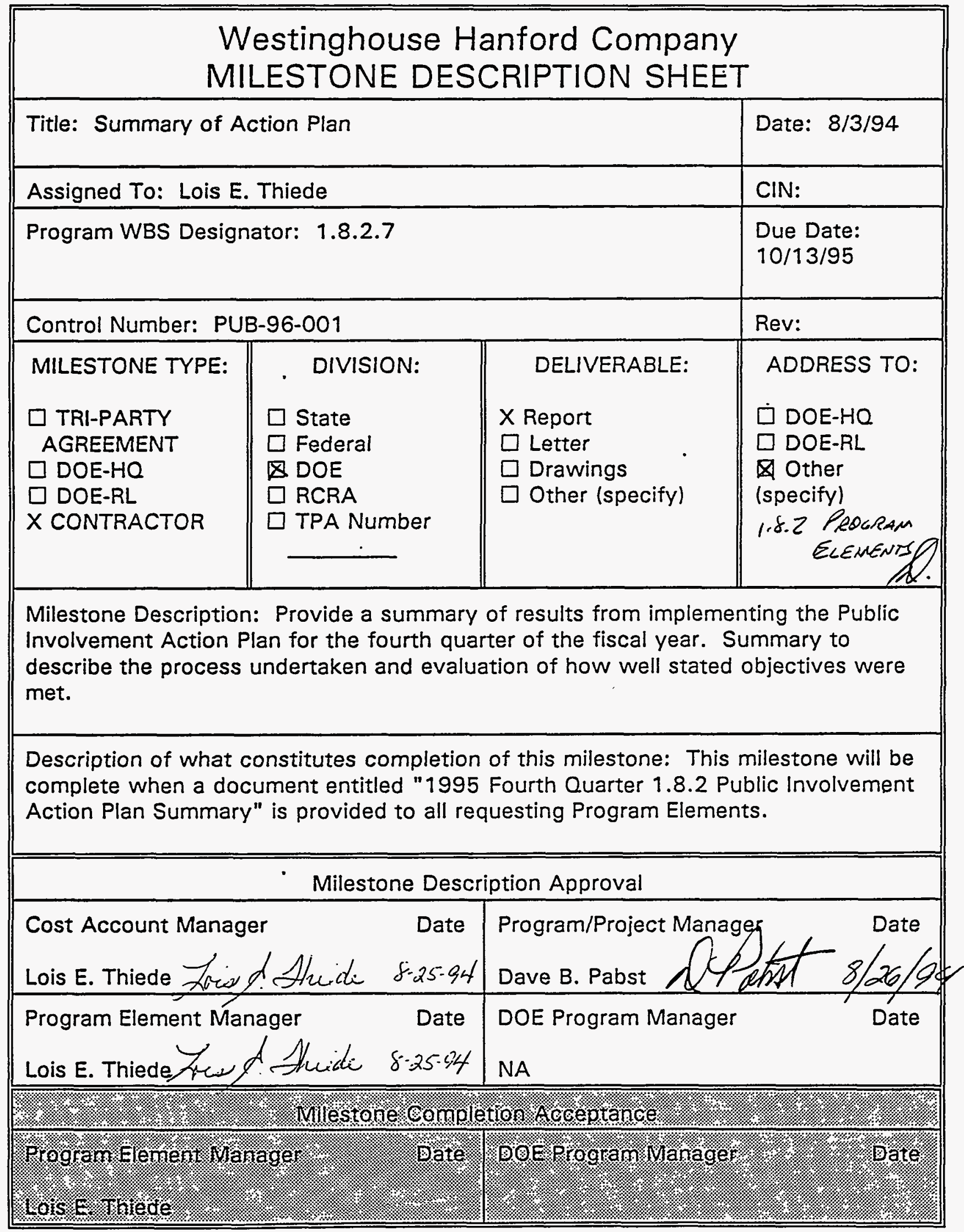




\begin{tabular}{|c|c|c|c|}
\hline \multicolumn{4}{|c|}{$\begin{array}{l}\text { Westinghouse Hanford Company } \\
\text { MILESTONE DESCRIPTION SHEET }\end{array}$} \\
\hline \multicolumn{3}{|c|}{ Title: 1.8.2 1996 Second Quarter Action Plan } & Date: $8 / 5 / 94$ \\
\hline \multicolumn{3}{|c|}{ Assigned To: Lois E. Thiede } & CIN: \\
\hline \multicolumn{3}{|c|}{ Program WBS Designator: 1 8.2.7 } & $\begin{array}{l}\text { Due Date: } \\
12 / 9 / 95\end{array}$ \\
\hline \multicolumn{3}{|c|}{ Control Number: PUB-96-002 } & Rev: \\
\hline $\begin{array}{l}\text { MILESTONE TYPE: } \\
\square \text { TRI-PARTY } \\
\text { AGREEMENT } \\
\square \text { DOE-HQ } \\
\text { X DOE-RL } \\
\square \text { CONTRACTOR }\end{array}$ & $\begin{aligned} & \text { DIVISION: } \\
& \square \text { State } \\
& \square \text { Federal } \\
& \triangle \text { DOE } \\
& \square \text { RCRA } \\
& \square \text { TPA Number }\end{aligned}$ & $\begin{aligned} & \text { DELIVERABLE: } \\
& \times \text { Report } \\
& \square \text { Letter } \\
& \square \text { Drawings } \\
& \square \text { Other (specify) }\end{aligned}$ & $\begin{array}{l}\text { ADDRESS TO: } \\
\square \text { DOE-HO } \\
\text { X DOE-RL } \\
\square \text { Other } \\
\text { (specify) }\end{array}$ \\
\hline \multicolumn{4}{|c|}{$\begin{array}{l}\text { Milestone Description: Provide an Action Plan for specific public involvement actions } \\
\text { and schedule to be completed in the Second Quarter of FY } 1996 .\end{array}$} \\
\hline \multicolumn{4}{|c|}{$\begin{array}{l}\text { Description of what constitutes completion of this milestone: This milestone will be } \\
\text { complete when a document entitled "1.8.2 } 1996 \text { Second Quarter Public Involvement } \\
\text { Action Plan" is provided to RL for their signoff to authorize implementation. }\end{array}$} \\
\hline \multicolumn{4}{|c|}{ Milestone Description Approval } \\
\hline \multicolumn{2}{|c|}{ 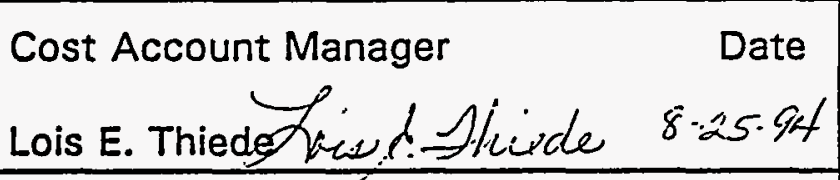 } & \multicolumn{2}{|c|}{ Dave B. Pabst } \\
\hline \multicolumn{2}{|c|}{$\begin{array}{lr}\text { Program Element Manager } & \text { Date } \\
\text { Lois E. Thiedeftin thicuide } & 8.95 .94 \\
\end{array}$} & \multicolumn{2}{|c|}{$\begin{array}{l}\text { DOE Program Manager } \\
\text { Bill A. Rutherford }\end{array}$} \\
\hline \multicolumn{4}{|c|}{ 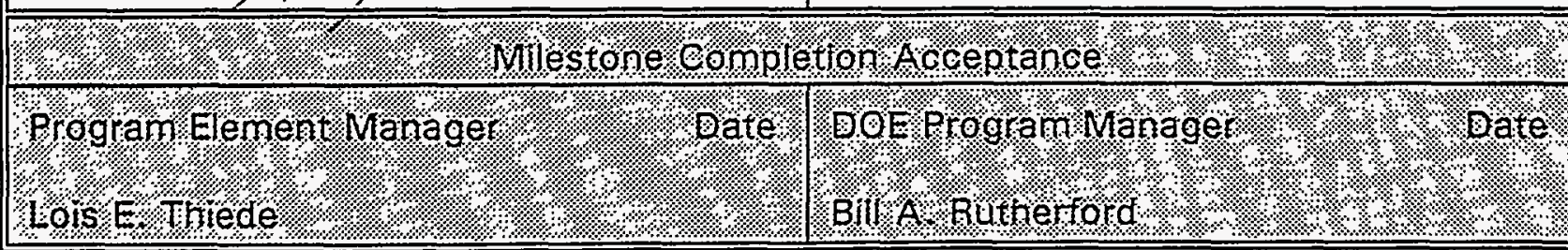 } \\
\hline
\end{tabular}




\begin{tabular}{|c|c|c|c|}
\hline \multicolumn{4}{|c|}{$\begin{array}{l}\text { Westinghouse Hanford Company } \\
\text { MILESTONE DESCRIPTION SHEET }\end{array}$} \\
\hline \multicolumn{3}{|c|}{ Title: Summary of Action Plan } & Date: $8 / 3 / 94$ \\
\hline \multicolumn{3}{|c|}{ Assigned To: Lois E. Thiede } & CIN: \\
\hline \multicolumn{3}{|c|}{ Program WBS Designator: 1 8.2.7 } & $\begin{array}{l}\text { Due Date: } \\
1 / 12 / 96\end{array}$ \\
\hline \multicolumn{3}{|c|}{ Control Number: PUB-96-003 } & Rev: \\
\hline $\begin{array}{l}\text { MILESTONE TYPE: } \\
\square \text { TRI-PARTY } \\
\text { AGREEMENT } \\
\square \text { DOE-HO } \\
\square \text { DOE-RL } \\
X \text { CONTRACTOR }\end{array}$ & $\begin{array}{l}\quad \text { DIVISION: } \\
\square \text { State } \\
\square \text { Federal } \\
\text { DOE } \\
\square \text { RCRA } \\
\square \text { TPA Number }\end{array}$ & $\begin{array}{l}\text { DELIVERABLE: } \\
\times \text { Report } \\
\square \text { Letter } \\
\square \text { Drawings } \\
\square \text { Other (specify) }\end{array}$ & $\begin{array}{l}\text { ADDRESS TO: } \\
\square \text { DOE-HO } \\
\square \text { DOE-RL } \\
\otimes \text { Other } \\
\text { (specify) } \\
1.8 .2 \text { PleGRAM } \\
\text { ELEMENTS }\end{array}$ \\
\hline \multicolumn{4}{|c|}{$\begin{array}{l}\text { Milestone Description: Provide a summary of results from implementing the Public } \\
\text { linvolvement Action Plan for the first quarter of the fiscal year. Summary to describe } \\
\text { the process undertaken and evaluation of how well stated objectives were met. }\end{array}$} \\
\hline \multicolumn{4}{|c|}{$\begin{array}{l}\text { Description of what constitutes completion of this milestone: This milestone will be } \\
\text { complete when a document entitled "1.996 First Quarter } 1.8 .2 \text { Public Involvement } \\
\text { Action Plan Summary" is provided to all requesting Program Elements. }\end{array}$} \\
\hline \multicolumn{4}{|c|}{ Milestone Description Approval } \\
\hline \multicolumn{2}{|c|}{$\begin{array}{l}\text { Cost Account Manager } \\
\text { Lois E. Thiede Zrised. Hate } \\
\end{array}$} & \multicolumn{2}{|c|}{ Drogram/Project Manager B. Pabst 1 Date } \\
\hline \multicolumn{2}{|c|}{ 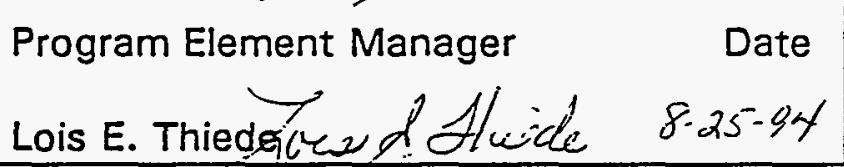 } & \multicolumn{2}{|c|}{$\begin{array}{l}\text { DOE Program Manager Date } \\
\text { NA }\end{array}$} \\
\hline \multicolumn{4}{|c|}{ 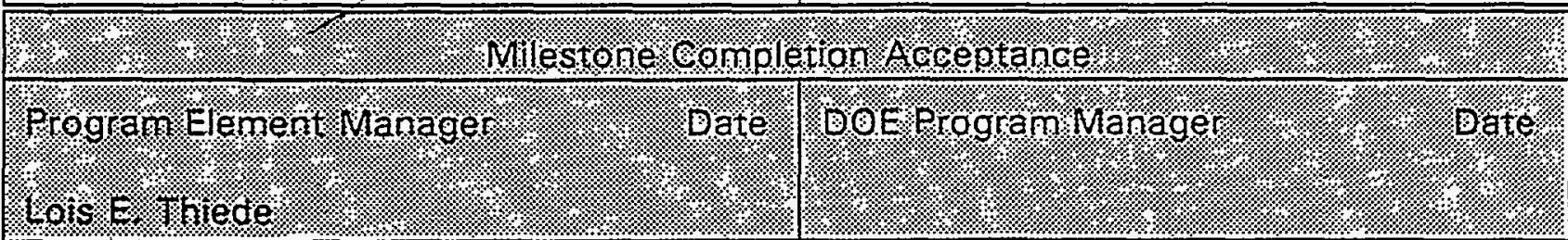 } \\
\hline
\end{tabular}




\begin{tabular}{|c|c|c|c|}
\hline \multicolumn{4}{|c|}{$\begin{array}{l}\text { Westinghouse Hanford Company } \\
\text { MILESTONE DESCRIPTION SHEET }\end{array}$} \\
\hline \multicolumn{3}{|c|}{ Title: 1.8 .21996 Third Quarter Action Plan } & Date: $8 / 5 / 94$ \\
\hline \multicolumn{3}{|c|}{ Assigned To: Lois E. Thiede } & CIN: \\
\hline \multicolumn{3}{|c|}{ Program WBS Designator: 1.8.2.7 } & $\begin{array}{l}\text { Due Date: } \\
3 / 10 / 96\end{array}$ \\
\hline \multicolumn{3}{|c|}{ Control Number: PUB-96-004 } & Rev: \\
\hline $\begin{array}{l}\text { MILESTONE TYPE: } \\
\square \text { TRI-PARTY } \\
\text { AGREEMENT } \\
\square \text { DOE-HO } \\
\text { X DOE-RL } \\
\square \text { CONTRACTOR }\end{array}$ & $\begin{array}{l}\text { DIVISION: } \\
\quad \square \text { State } . \\
\square \text { Federal } \\
\otimes \text { DOE } \\
\square \text { RCRA } \\
\square \text { TPA Number }\end{array}$ & $\begin{array}{l}\text { DELIVERABLE: } \\
\times \text { Report } \\
\square \text { Letter } \\
\square \text { Drawings } \\
\square \text { Other (specify) }\end{array}$ & $\begin{array}{l}\text { ADDRESS TO: } \\
\square \text { DOE-HQ } \\
\text { X DOE-RL } \\
\square \text { Other } \\
\text { (specify) }\end{array}$ \\
\hline \multicolumn{4}{|c|}{$\begin{array}{l}\text { Milestone Description: Provide an Action Plan for specific public involvement actions } \\
\text { and schedule to be completed in the Third Quarter of FY } 1996 .\end{array}$} \\
\hline \multicolumn{4}{|c|}{$\begin{array}{l}\text { Description of what constitutes completion of this milestone: This milestone will be } \\
\text { complete when a document entitled "1.8.2 } 1996 \text { Third Quarter Public Involvement } \\
\text { Action Plan" is provided to RL for their signoff to authorize implementation. }\end{array}$} \\
\hline \multicolumn{4}{|c|}{ Milestone Description Approval } \\
\hline \multicolumn{2}{|c|}{ 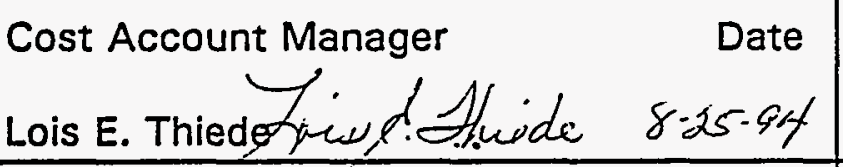 } & \multicolumn{2}{|c|}{ Dave B. Pabst Date } \\
\hline \multicolumn{2}{|c|}{ 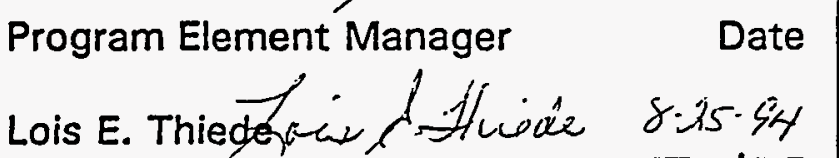 } & \multicolumn{2}{|c|}{$\begin{array}{l}\text { DOE Program Manager Date } \\
\text { Bill A. Rutherford }\end{array}$} \\
\hline \multicolumn{4}{|c|}{ 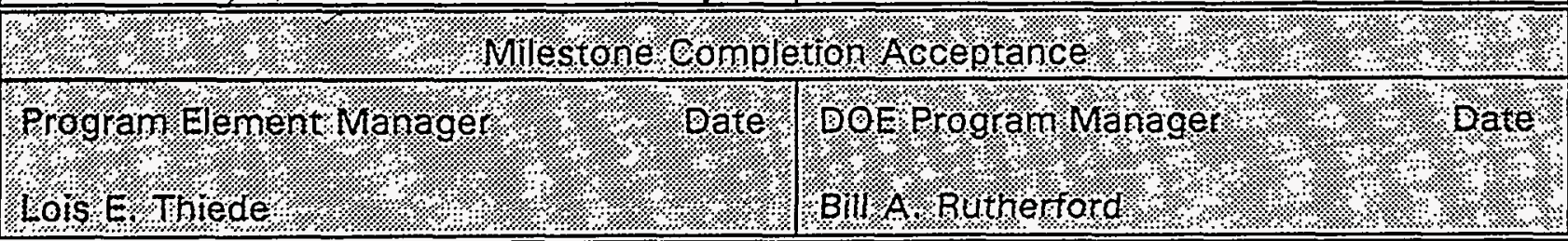 } \\
\hline
\end{tabular}




\begin{tabular}{|c|c|c|c|}
\hline \multicolumn{4}{|c|}{$\begin{array}{l}\text { Westinghouse Hanford Company } \\
\text { MILESTONE DESCRIPTION SHEET }\end{array}$} \\
\hline \multicolumn{3}{|c|}{ Title: Summary of Action Plan } & Date: $8 / 3 / 94$ \\
\hline \multicolumn{3}{|c|}{ Assigned To: Lois E. Thiede } & CIN: \\
\hline \multicolumn{3}{|c|}{ Program WBS Designator: 1 8.2.7 } & $\begin{array}{l}\text { Due Date: } \\
4 / 15 / 96\end{array}$ \\
\hline \multicolumn{3}{|c|}{ Control Number: PUB-96-005 } & Rev: \\
\hline $\begin{array}{l}\text { MILESTONE TYPE: } \\
\square \text { TRI-PARTY } \\
\text { AGREEMENT } \\
\square \text { DOE-HO } \\
\square \text { DOE-RL } \\
X \text { CONTRACTOR }\end{array}$ & $\begin{aligned} & \text { DIVISION: } \\
& \square \text { State } \\
& \square \text { Federal } \\
& \triangle \text { DOE } \\
& \square \text { RCRA } \\
& \square \text { TPA Number } \\
&\end{aligned}$ & $\begin{array}{l}\text { DELIVERABLE: } \\
\times \text { Report } \\
\square \text { Letter } \\
\square \text { Drawings } \\
\square \text { Other (specify) }\end{array}$ & $\begin{array}{l}\text { ADDRESS TO: } \\
\square \text { DOE-HO } \\
\square \text { DOE-RL } \\
\text { 囚 Other } \\
\text { (specify) } \\
1.8 .2 \text { PROGRAM } \\
\text { ELENENTS }\end{array}$ \\
\hline \multicolumn{4}{|c|}{$\begin{array}{l}\text { Milestone Description: Provide a summary of results from implementing the Public } \\
\text { Involvement Action Plan for the second quarter of the fiscal year. Summary to } \\
\text { describe the process undertaken and evaluation of how well stated objectives were } \\
\text { met. }\end{array}$} \\
\hline \multicolumn{4}{|c|}{$\begin{array}{l}\text { Description of what constitutes completion of this milestone: This milestone will be } \\
\text { complete when a document entitled "1996 Second Quarter } 1.8 .2 \text { Public Involvement } \\
\text { Action Plan Summary" is provided to all requesting Program Elements. }\end{array}$} \\
\hline \multicolumn{4}{|c|}{ Milestone Description Approval } \\
\hline \multicolumn{2}{|c|}{ 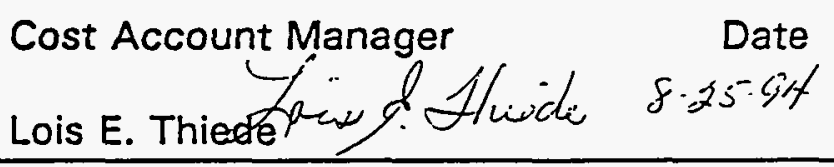 } & \multicolumn{2}{|c|}{ Program/Project Manager B. Pabst } \\
\hline \multicolumn{2}{|c|}{ 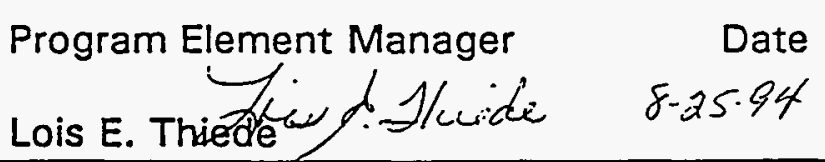 } & \multicolumn{2}{|c|}{$\begin{array}{l}\text { DOE Program Manager Date } \\
\text { NA }\end{array}$} \\
\hline \multicolumn{4}{|c|}{ 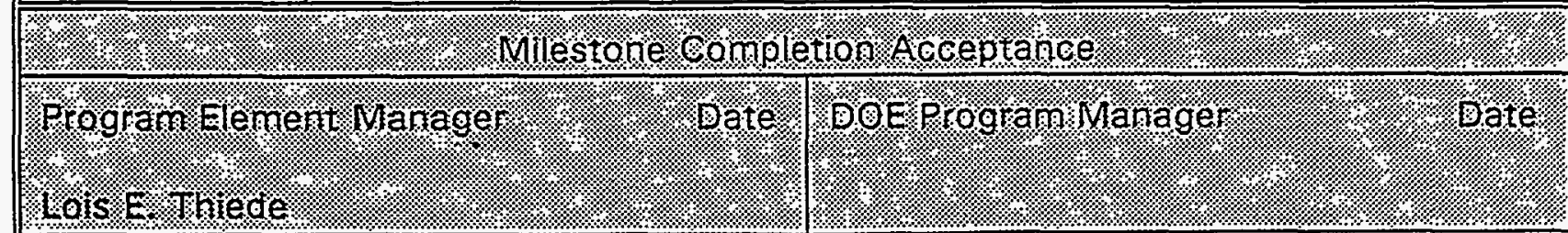 } \\
\hline
\end{tabular}




\begin{tabular}{|c|c|c|c|}
\hline \multicolumn{4}{|c|}{$\begin{array}{l}\text { Westinghouse Hanford Company } \\
\text { MILESTONE DESCRIPTION SHEET }\end{array}$} \\
\hline \multicolumn{3}{|c|}{ Title: 1.8.2 1996 Fourth Quarter Action Plan } & Date: $8 / 5 / 94$ \\
\hline \multicolumn{3}{|c|}{ Assigned To: Lois E. Thiede } & CIN: \\
\hline \multicolumn{3}{|c|}{ Program WBS Designator: 1 8.2.7 } & $\begin{array}{l}\text { Due Date: } \\
6 / 9 / 96\end{array}$ \\
\hline \multicolumn{3}{|c|}{ Control Number: PUB-96-006 } & Rev: \\
\hline $\begin{array}{l}\text { MILESTONE TYPE: } \\
\square \text { TRI-PARTY } \\
\text { AGREEMENT } \\
\square \text { DOE-HO } \\
\times \text { DOE-RL } \\
\square \text { CONTRACTOR }\end{array}$ & $\begin{array}{l}\text { DIVISION: } \\
\square \text { State } \\
\square \text { Federal } \\
\otimes \text { DOE } \\
\square \text { RCRA } \\
\square \text { TPA Number }\end{array}$ & $\begin{array}{l}\text { DELIVERABLE: } \\
\text { X Report } \\
\square \text { Letter } \\
\square \text { Drawings } \\
\square \text { Other (specify) }\end{array}$ & $\begin{array}{l}\text { ADDRESS TO: } \\
\text { } \square \text { DOE-HQ } \\
\text { X DOE-RL } \\
\square \text { Other } \\
\text { (specify) }\end{array}$ \\
\hline \multicolumn{4}{|c|}{$\begin{array}{l}\text { Milestone Description: Provide an Action Plan for specific public involvement actions } \\
\text { and schedule to be completed in the Fourth Quarter of FY } 1996 \text {. }\end{array}$} \\
\hline \multicolumn{4}{|c|}{$\begin{array}{l}\text { Description of what constitutes completion of this milestone: This milestone will be } \\
\text { complete when a document entitled "1.8.2 } 1996 \text { Fourth Quarter Public Involvement } \\
\text { Action Plan" is provided to RL for their signoff to authorize implementation. }\end{array}$} \\
\hline \multicolumn{4}{|c|}{ Milestone Description Approval } \\
\hline \multicolumn{2}{|c|}{ 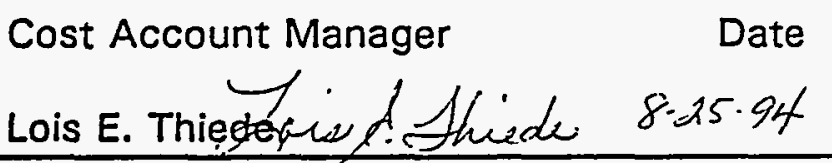 } & \multicolumn{2}{|c|}{ Program/Project Manageg/f Date } \\
\hline \multicolumn{2}{|c|}{ 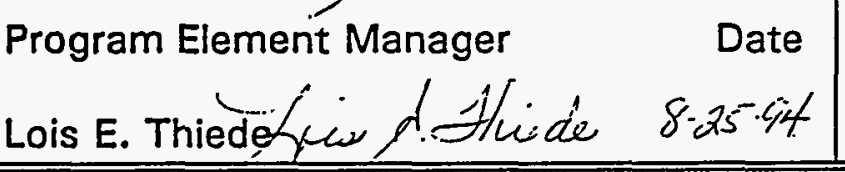 } & \multicolumn{2}{|c|}{$\begin{array}{l}\text { DOE Program Manager Date } \\
\text { Bill A. Rutherford }\end{array}$} \\
\hline \multicolumn{4}{|c|}{ 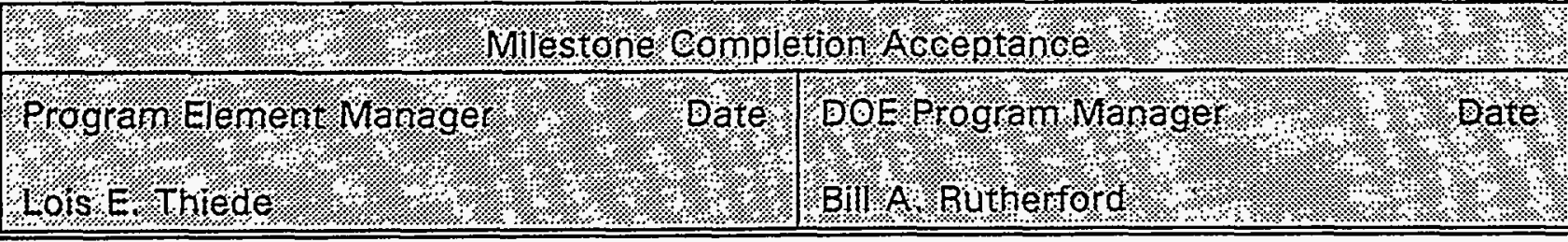 } \\
\hline
\end{tabular}




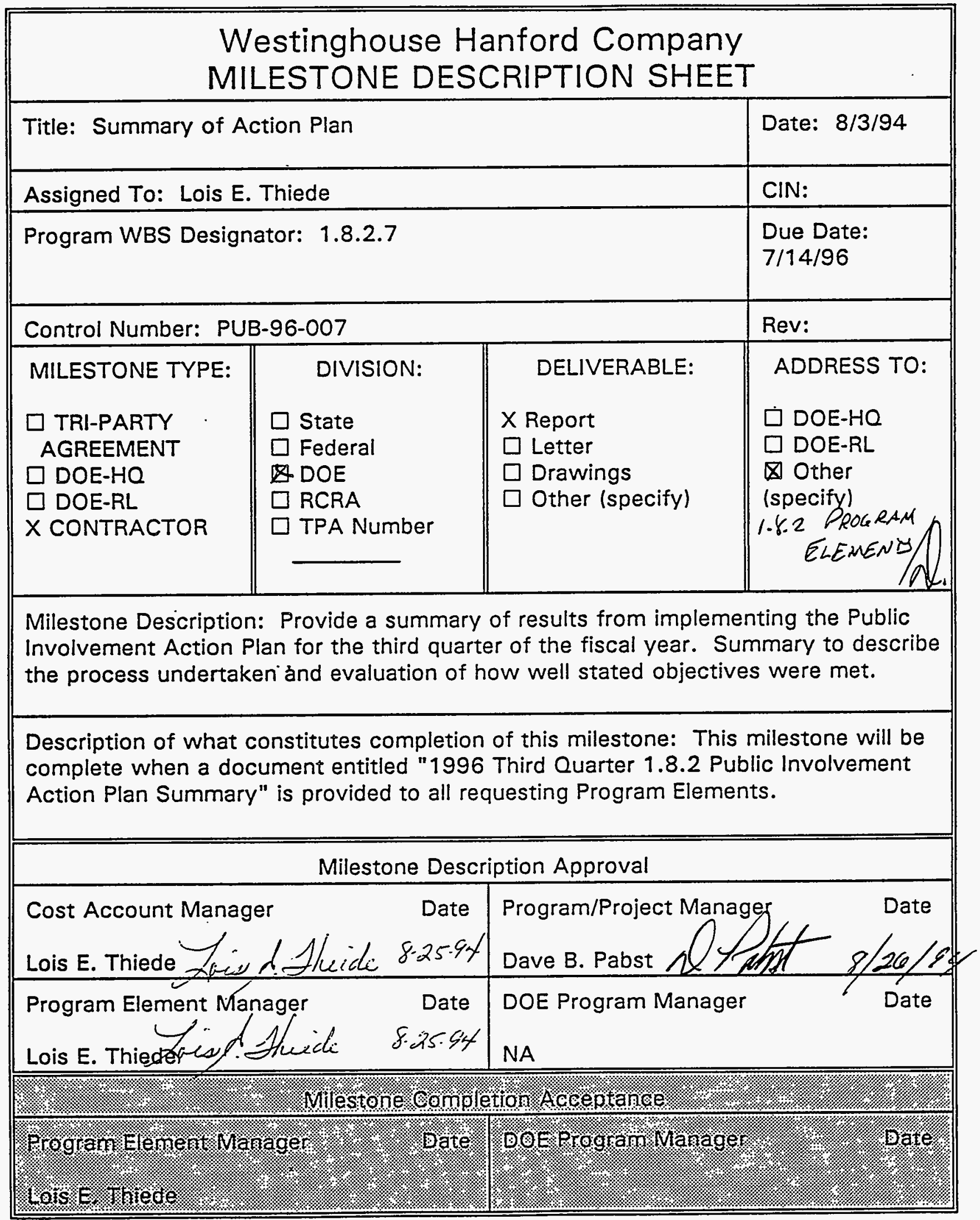




\begin{tabular}{|c|c|c|c|}
\hline \multicolumn{4}{|c|}{$\begin{array}{l}\text { Westinghouse Hanford Company } \\
\text { MILESTONE DESCRIPTION SHEET }\end{array}$} \\
\hline \multicolumn{3}{|c|}{ Title: 1.8.2 Public Involvement Guidance } & Date: $8 / 3 / 94$ \\
\hline \multicolumn{3}{|c|}{ Assigned To: Lois E. Thiede } & CIN: \\
\hline \multicolumn{3}{|c|}{ Program WBS Designator: 1.8.2.7 } & $\begin{array}{l}\text { Due Date: } \\
8 / 1 / 96\end{array}$ \\
\hline \multicolumn{3}{|c|}{ Control Number: PUB-96-008 } & Rev: \\
\hline $\begin{array}{l}\text { MILESTONE TYPE: } \\
\square \text { TRI-PARTY } \\
\text { AGREEMENT } \\
\square \text { DOE-HQ } \\
\text { X DOE-RL } \\
\square \text { CONTRACTOR }\end{array}$ & $\begin{array}{l}\quad \text { DIVISION: } \\
\square \text { State } \\
\square \text { Federal } \\
\text { DOE } \\
\square \text { RCRA } \\
\square \text { TPA Number }\end{array}$ & $\begin{array}{l}\text { DELIVERABLE: } \\
\times \text { Report } \\
\square \text { Letter } \\
\square \text { Drawings } \\
\square \text { Other (specify) }\end{array}$ & $\begin{array}{l}\text { ADDRESS TO: } \\
\dot{\square} \text { DOE-HQ } \\
\otimes \text { DOE-RL } \\
\square \text { Other } \\
\text { (specify) }\end{array}$ \\
\hline \multicolumn{4}{|c|}{$\begin{array}{l}\text { Milestone Description: Provide Public Involvement Guidance for } 1.8 .2 \text { which } \\
\text { includes a listing of the agreed upon planning components for which Fiscal Year } \\
\text { Action Plans will be developed. Guidance will also include general information on } \\
\text { coordinating public involvement activities for } 1.8 .2 \text { Program Elements with other } \\
\text { sitewide public involvement activities. }\end{array}$} \\
\hline \multicolumn{4}{|c|}{$\begin{array}{l}\text { Description of what constitutes completion of this milestone: This milestone will be } \\
\text { complete when a document entitled "1.8.2 FY97 Public Involvement Guidance" is } \\
\text { provided to and approved by RL for preparing FY97 Action Plans. }\end{array}$} \\
\hline \multicolumn{4}{|c|}{ Milestone Description Approval } \\
\hline \multicolumn{2}{|c|}{$\begin{array}{l}\text { Cost Account Manager". Date } \\
\text { Lois E. Thiede Tris d the de 8-2.5.94 }\end{array}$} & \multicolumn{2}{|c|}{ Dave B. Pabst } \\
\hline \multicolumn{2}{|c|}{ 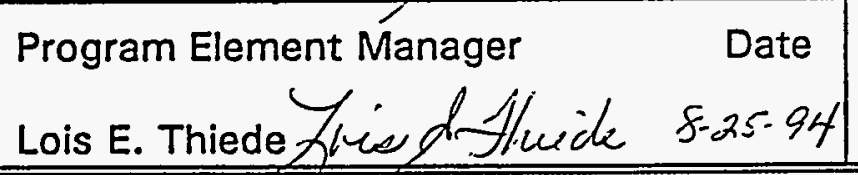 } & \multicolumn{2}{|c|}{$\begin{array}{l}\text { DOE Program Manager Date } \\
\text { Bill A. Rutherford }\end{array}$} \\
\hline \multicolumn{4}{|c|}{ 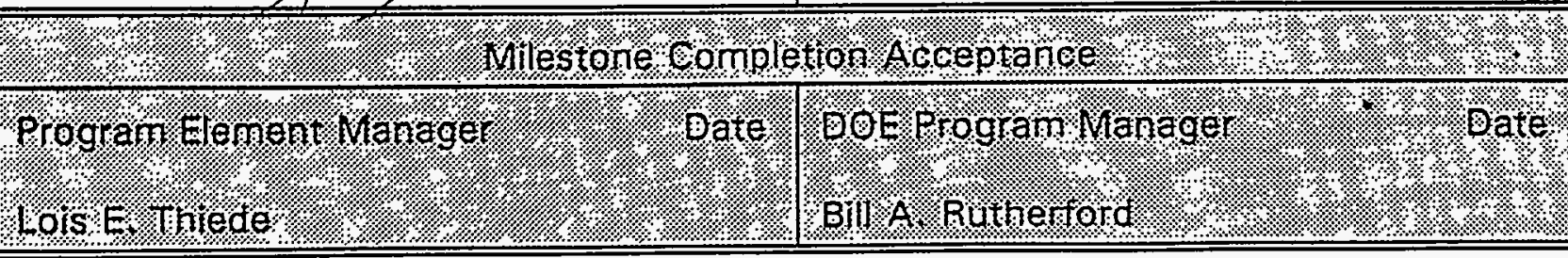 } \\
\hline
\end{tabular}




\begin{tabular}{|c|c|c|c|}
\hline \multicolumn{4}{|c|}{$\begin{array}{l}\text { Westinghouse Hanford Company } \\
\text { MILESTONE DESCRIPTION SHEET }\end{array}$} \\
\hline \multicolumn{3}{|c|}{ Title: 1.8.2 1997 First Quarter Action Plan } & Date: $8 / 5 / 94$ \\
\hline \multicolumn{3}{|c|}{ Assigned To: Lois E. Thiede } & CIN: \\
\hline \multicolumn{3}{|c|}{ Program WBS Designator: 1.8 .2 .7} & $\begin{array}{l}\text { Due Date: } \\
9 / 8 / 96\end{array}$ \\
\hline \multicolumn{3}{|c|}{ Control Number: PUB-96-009 } & Rev: \\
\hline $\begin{array}{l}\text { MILESTONE TYPE: } \\
\square \text { TRI-PARTY } \\
\text { AGREEMENT } \\
\square \text { DOE-HO } \\
\text { X DOE-RL } \\
\square \text { CONTRACTOR }\end{array}$ & $\begin{aligned} & \text { DIVISION: } \\
& \\
& \square \text { State } \\
& \square \text { Federal } \\
& \text { DOE } \\
& \square \text { RCRA } \\
& \square \text { TPA Number }\end{aligned}$ & $\begin{array}{l}\text { DELIVERABLE: } \\
\times \text { Report } \\
\square \text { Letter } \\
\square \text { Drawings } \\
\square \text { Other (specify) }\end{array}$ & $\begin{array}{l}\text { ADDRESS TO: } \\
\square \text { DOE-HO } \\
\text { X DOE-RL } \\
\square \text { Other } \\
\text { (specify) }\end{array}$ \\
\hline \multicolumn{4}{|c|}{$\begin{array}{l}\text { Milestone Description: Provide an Action Plan for specific public involvement actions } \\
\text { and schedule to be completed in the First Quarter of FY } 1997 .\end{array}$} \\
\hline \multicolumn{4}{|c|}{$\begin{array}{l}\text { Description of what constitutes completion of this milestone: This milestone will be } \\
\text { complete when a document entitled "1.8.2 } 1997 \text { First Quarter Public Involvement } \\
\text { Action Plan" is provided to RL for their signoff to authorize implementation. }\end{array}$} \\
\hline \multicolumn{4}{|c|}{ Milestone Description Approval } \\
\hline \multicolumn{2}{|c|}{$\begin{array}{l}\text { Cost Account Manager } \quad \text { Date } \\
\text { Lois E. Thiedegind. Thide } 8 \text { - } 25 \text {-9.t }\end{array}$} & \multicolumn{2}{|c|}{ Drogram/Project Manager B. Pabst } \\
\hline \multicolumn{2}{|c|}{$\begin{array}{ll}\text { Program Element Manager } & \text { Date } \\
\text { Lois E. Thiedeffeyd Huvitu } & 8.25 \cdot 94\end{array}$} & \multicolumn{2}{|c|}{$\begin{array}{l}\text { DOE Program Manager Date } \\
\text { Bill A. Rutherford }\end{array}$} \\
\hline \multicolumn{4}{|c|}{ 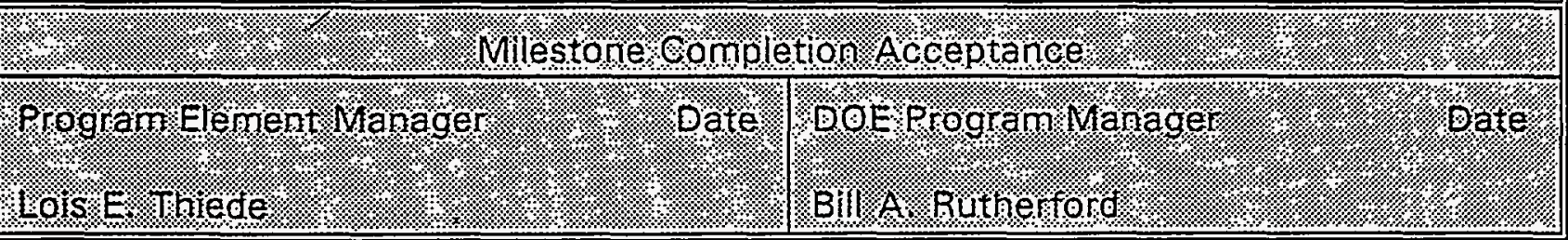 } \\
\hline
\end{tabular}




\begin{tabular}{|c|c|c|c|}
\hline \multicolumn{4}{|c|}{$\begin{array}{l}\text { Westinghouse Hanford Company } \\
\text { MILESTONE DESCRIPTION SHEET }\end{array}$} \\
\hline \multicolumn{3}{|c|}{ Title: Summary of Action Plan } & Date: $8 / 3 / 94$ \\
\hline \multicolumn{3}{|c|}{ Assigned To: Lois E. Thiede } & CIN: \\
\hline \multicolumn{3}{|c|}{ Program WBS Designator: 1 (18.2.7 } & $\begin{array}{l}\text { Due Date: } \\
\text { 10/14/96 }\end{array}$ \\
\hline \multicolumn{3}{|c|}{ Control Number: PUB-97-001 } & Rev: \\
\hline $\begin{array}{l}\text { MILESTONE TYPE: } \\
\square \text { TRI-PARTY } \\
\text { AGREEMENT } \\
\square \text { DOE-HQ } \\
\square \text { DOE-RL } \\
X \text { CONTRACTOR }\end{array}$ & $\begin{array}{l}\text { DIVISION: } \\
\square \text { State } \\
\square \text { Federal } \\
\text { D DOE } \\
\square \text { RCRA } \\
\square \text { TPA Number }\end{array}$ & $\begin{array}{l}\text { DELIVERABLE: } \\
\times \text { Report } \\
\square \text { Letter } \\
\square \text { Drawings } \\
\square \text { Other (specify) }\end{array}$ & $\begin{array}{l}\text { ADDRESS TO: } \\
\text { Dं DOE-HO } \\
\square \text { DOE-RL } \\
\text { Other } \\
\text { (specify) } \\
\text { l.YY RoOCA+Ah } \\
\text { ELEMENTS }\end{array}$ \\
\hline \multicolumn{4}{|c|}{$\begin{array}{l}\text { Milestone Description: Provide a summary of results from implementing the Public } \\
\text { Involvement Action Plan for the fourth quarter of the fiscal year. Summary to } \\
\text { describe the process undertaken and evaluation of how well stated objectives were } \\
\text { met. }\end{array}$} \\
\hline \multicolumn{4}{|c|}{$\begin{array}{l}\text { Description of what constitutes completion of this milestone: This milestone will be } \\
\text { complete when a document entitled "1996 Fourth Quarter } 1.8 .2 \text { Public Involvement } \\
\text { Action Plan Summary" is provided to all requesting Program Elements. }\end{array}$} \\
\hline \multicolumn{4}{|c|}{ Milestone Description Approval } \\
\hline \multicolumn{2}{|c|}{ 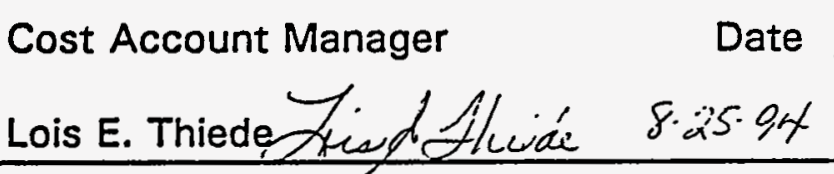 } & \multicolumn{2}{|c|}{ Dave B. Pabst } \\
\hline \multicolumn{2}{|c|}{$\begin{array}{l}\text { Program Element Manager } \quad \text { Date } \\
\text { Lois E. Thiegexis/. H/wide } \\
8.25 \cdot 94\end{array}$} & \multicolumn{2}{|c|}{$\begin{array}{l}\text { DOE Program Manager Date } \\
\text { NA }\end{array}$} \\
\hline \multicolumn{4}{|c|}{ 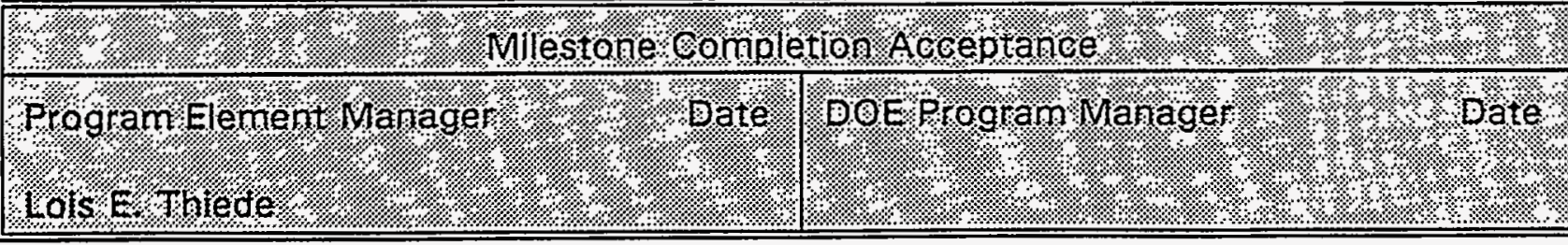 } \\
\hline
\end{tabular}




\section{Westinghouse Hanford Company MILESTONE DESCRIPTION SHEET}

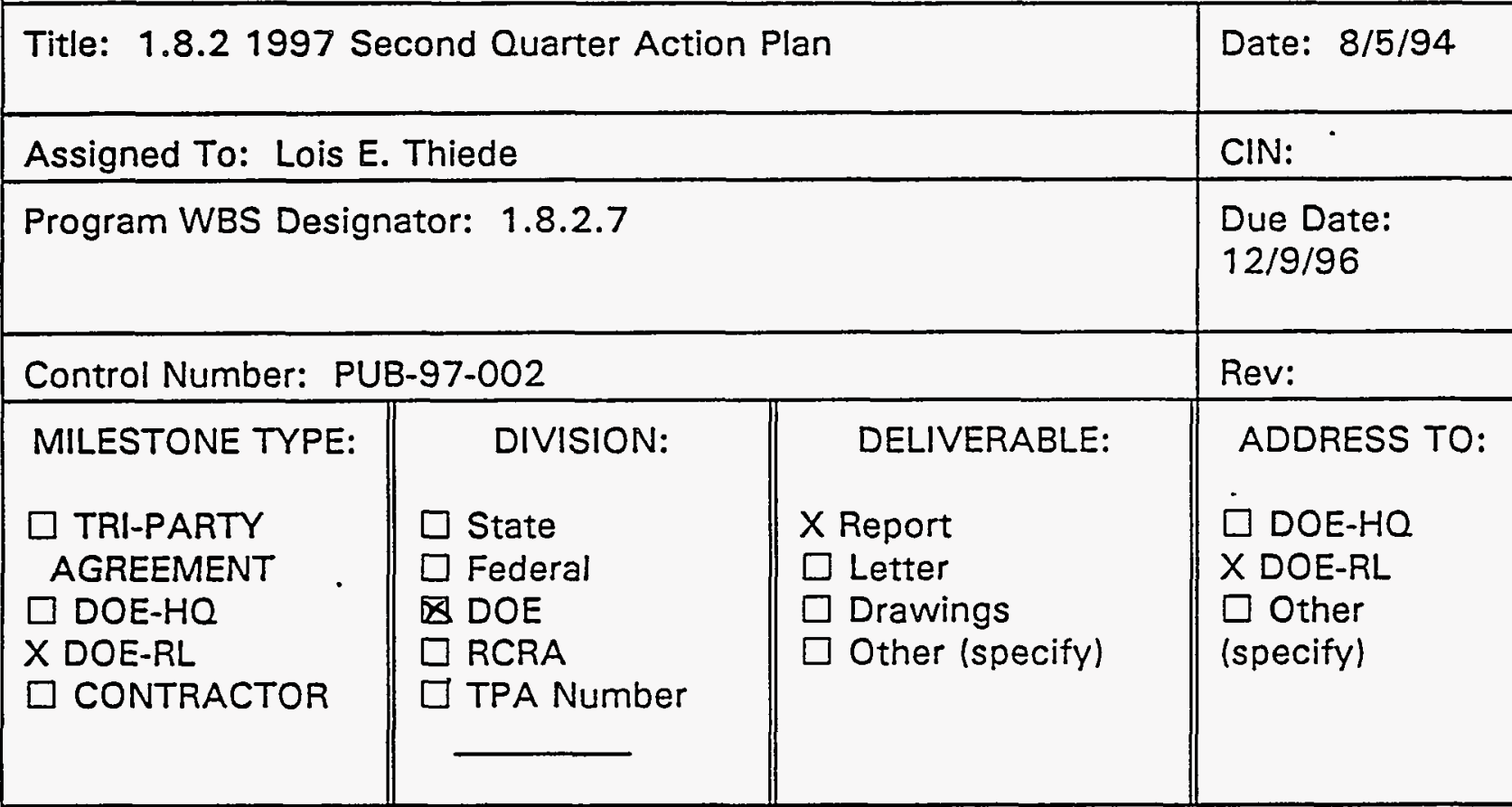

Milestone Description: Provide an Action Plan for specific public involvement actions and schedule to be completed in the Second Quarter of FY 1997.

Description of what constitutes completion of this milestone: This milestone will be complete when a document entitled "1.8.2 1997 Second Quarter Public Involvement Action Plan" is provided to RL for their signoff to authorize implementation.

\begin{tabular}{|c|c|}
\hline \multicolumn{2}{|c|}{ Milestone Description Approval } \\
\hline Lost Account Manager Date & Drogram/Project Managers \\
\hline $\begin{array}{l}\text { Program Element Manager Date } \\
\text { Lois E. Thiedetriz/. Lhide 8.25.54 }\end{array}$ & $\begin{array}{l}\text { DOE Program Manager } \\
\text { Bill A. Rutherford }\end{array}$ \\
\hline 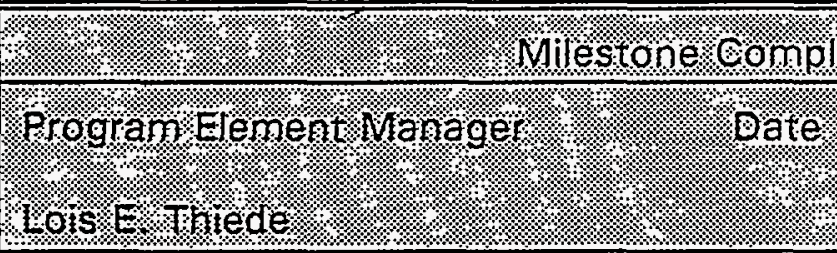 & 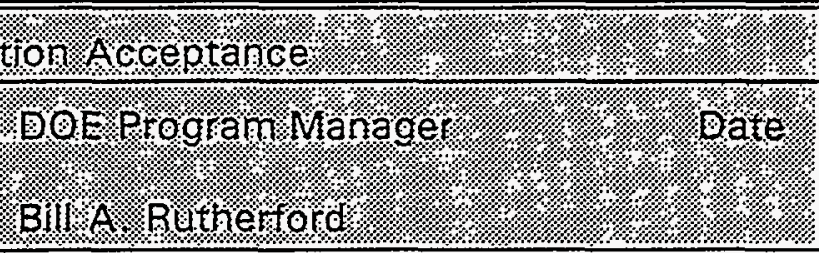 \\
\hline
\end{tabular}




\begin{tabular}{|c|c|c|c|}
\hline \multicolumn{4}{|c|}{$\begin{array}{l}\text { Westinghouse Hanford Company } \\
\text { MILESTONE DESCRIPTION SHEET }\end{array}$} \\
\hline \multicolumn{3}{|c|}{ Title: Summary of Action Plan } & Date: $8 / 3 / 94$ \\
\hline \multicolumn{3}{|c|}{ Assigned To: Lois E. Thiede } & CIN: \\
\hline \multicolumn{3}{|c|}{ Program WBS Designator: 1 8.2.7 } & $\begin{array}{l}\text { Due Date: } \\
1 / 13 / 97\end{array}$ \\
\hline \multicolumn{3}{|c|}{ Control Number: PUB-97-003 } & Rev: \\
\hline $\begin{array}{l}\text { MILESTONE TYPE: } \\
\square \text { TRI-PARTY } \\
\text { AGREEMENT } \\
\square \text { DOE-HQ } \\
\square \text { DOE-RL } \\
X \text { CONTRACTOR. }\end{array}$ & $\begin{array}{l}\quad \text { DIVISION: } \\
\square \text { State } \\
\square \text { Federal } \\
\text { D DOE } \\
\square \text { RCRA } \\
\square \text { TPA Number }\end{array}$ & $\begin{array}{l}\text { DELIVERABLE: } \\
\times \text { Report } \\
\square \text { Letter } \\
\square \text { Drawings } \\
\square \text { Other (specify) }\end{array}$ & $\begin{array}{l}\text { ADDRESS TO: } \\
\text { ' DOE-HO } \\
\square \text { DOE-RL } \\
\text { Q Other } \\
\text { (specifyl } \\
1.8 .2 \text { ProGRAM } \\
\text { ELEMENTS }\end{array}$ \\
\hline \multicolumn{4}{|c|}{$\begin{array}{l}\text { Milestone Description: Provide a summary of results from implementing the Public } \\
\text { Involvement Action Plan for the first quarter of the fiscal year. Summary to describe } \\
\text { the process undertaken and evaluation of how well stated objectives were met. }\end{array}$} \\
\hline \multicolumn{4}{|c|}{$\begin{array}{l}\text { Description of what constitutes completion of this milestone: This milestone will be } \\
\text { complete when a document entitled "1997 First Quarter } 1.8 .2 \text { Public Involvement } \\
\text { Action Plan Summary" is provided to all requesting Program Elements. }\end{array}$} \\
\hline \multicolumn{4}{|c|}{ Milestone Description Approval } \\
\hline \multicolumn{2}{|c|}{$\begin{array}{l}\text { Cost Account Manager } \\
\text { Lois E. Thiedghis \& Allude } \\
\end{array}$} & \multicolumn{2}{|c|}{ Dave B. Pabst } \\
\hline \multicolumn{2}{|c|}{ 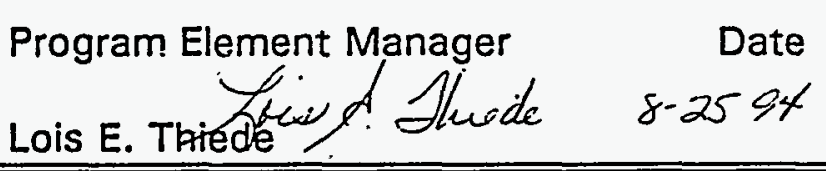 } & \multicolumn{2}{|c|}{$\begin{array}{l}\text { DOE Program Manager Date } \\
\text { NA }\end{array}$} \\
\hline \multicolumn{4}{|c|}{ 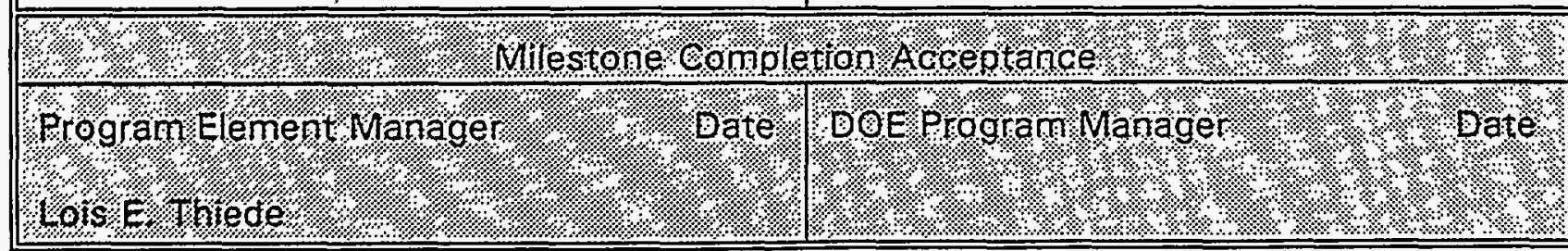 } \\
\hline
\end{tabular}




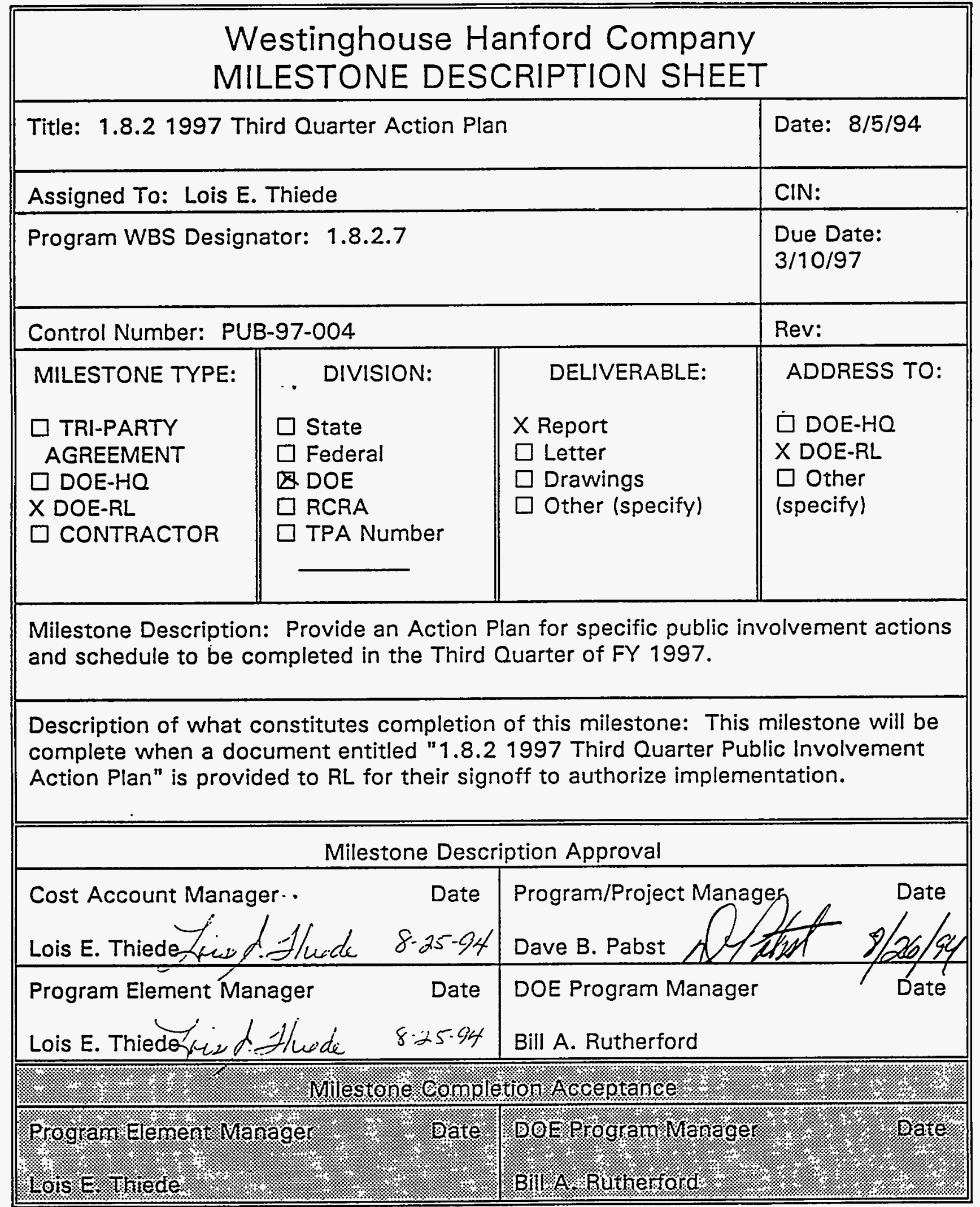




\begin{tabular}{|c|c|c|c|}
\hline \multicolumn{4}{|c|}{$\begin{array}{l}\text { Westinghouse Hanford Company } \\
\text { MILESTONE DESCRIPTION SHEET }\end{array}$} \\
\hline \multicolumn{3}{|c|}{ Title: Summary of Action Plan } & Date: $8 / 3 / 94$ \\
\hline \multicolumn{3}{|c|}{ Assigned To: Lois E. Thiede } & CIN: \\
\hline \multicolumn{3}{|c|}{ Program WBS Designator: 1.8 .2 .7} & $\begin{array}{l}\text { Due Date: } \\
4 / 14 / 97\end{array}$ \\
\hline \multicolumn{3}{|c|}{ Control Number: PUB-97-005 } & Rev: \\
\hline $\begin{array}{l}\text { MILESTONE TYPE: } \\
\square \text { TRI-PARTY } \\
\text { AGREEMENT } \\
\square \text { DOE-HO } \\
\square \text { DOE-RL } \\
X \text { CONTRACTOR }\end{array}$ & $\begin{array}{l}\quad \text { DIVISION: } \\
\square \text { State } \\
\square \text { Federal } \\
\text { DOE } \\
\square \text { RCRA } \\
\square \text { TPA Number } \\
. .\end{array}$ & $\begin{aligned} & \text { DELIVERABLE: } \\
\times & \text { Report } \\
& \text { Letter } \\
& \text { Drawings } \\
& \text { Other (specify) }\end{aligned}$ & 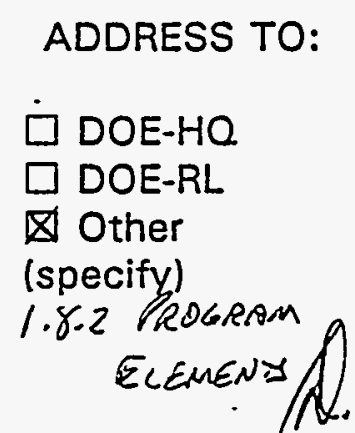 \\
\hline \multicolumn{4}{|c|}{$\begin{array}{l}\text { Milestone Description: Provide a summary.of results from implementing the Public } \\
\text { Involvement Action Plan for the second quarter of the fiscal year. Summary to } \\
\text { describe the process undertaken and evaluation of how well stated objectives were } \\
\text { met. }\end{array}$} \\
\hline \multicolumn{4}{|c|}{$\begin{array}{l}\text { Description of what constitutes completion of this milestone: This milestone will be } \\
\text { complete when a document entitled "1997 Second Quarter } 1.8 .2 \text { Public Involvement } \\
\text { Action Plan Summary" is provided to all requesting Program Elements. }\end{array}$} \\
\hline \multicolumn{4}{|c|}{ Milestone Description Approval } \\
\hline \multicolumn{2}{|c|}{ 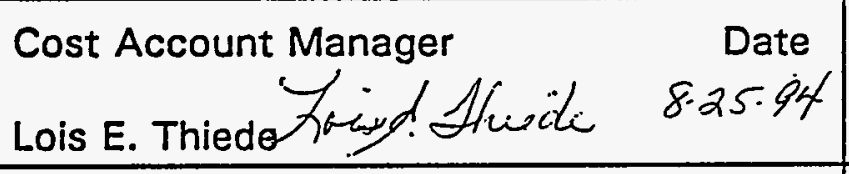 } & \multicolumn{2}{|c|}{ Dave B. Pabst Date } \\
\hline \multicolumn{2}{|c|}{ 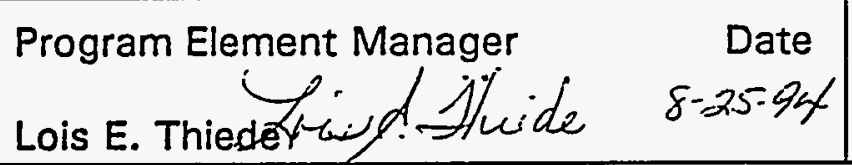 } & \multicolumn{2}{|c|}{$\begin{array}{l}\text { DOE Program Manager Date } \\
\text { NA }\end{array}$} \\
\hline \multicolumn{4}{|c|}{ 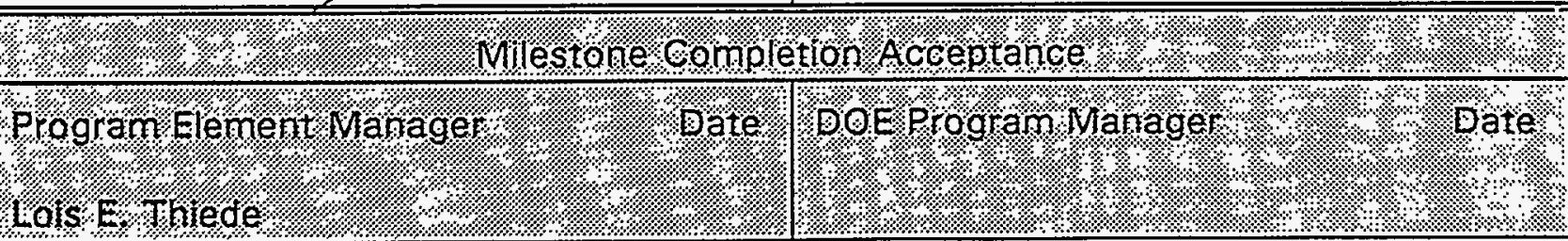 } \\
\hline
\end{tabular}




\begin{tabular}{|c|c|c|c|}
\hline \multicolumn{4}{|c|}{$\begin{array}{l}\text { Westinghouse Hanford Company } \\
\text { MILESTONE DESCRIPTION SHEET }\end{array}$} \\
\hline \multicolumn{3}{|c|}{ Title: 1.8.2 1997 Fourth Quarter Action Plan } & Date: $8 / 5 / 94$ \\
\hline \multicolumn{3}{|c|}{ Assigned To: Lois E. Thiede } & CIN: \\
\hline \multicolumn{3}{|c|}{ Program WBS Designator: 1.8 .2 .7} & $\begin{array}{l}\text { Due Date: } \\
6 / 9 / 97\end{array}$ \\
\hline \multicolumn{3}{|c|}{ Control Number: PUB-97-006 } & Rev: \\
\hline $\begin{array}{l}\text { MILESTONE TYPE: } \\
\square \text { TRI-PARTY } \\
\text { AGREEMENT } \\
\square \text { DOE-HQ } \\
\text { X DOE-RL } \\
\square \text { CONTRACTOR }\end{array}$ & $\begin{aligned} & \text { DIVISION: } \\
& \text { State } \\
\square & \text { Federal } \\
\square & \text { DOE } \\
\square & \text { RCRA } \\
& \square \text { TPA Number }\end{aligned}$ & $\begin{aligned} & \text { DELIVERABLE: } \\
\times & \text { Report } \\
& \square \text { Letter } \\
& \text { Drawings } \\
& \square \text { Other (specify) }\end{aligned}$ & $\begin{array}{l}\text { ADDRESS TO: } \\
\square \text { DOE-HO } \\
\text { X DOE-RL } \\
\square \text { Other } \\
\text { (specify) }\end{array}$ \\
\hline \multicolumn{4}{|c|}{$\begin{array}{l}\text { Milestone Description: Provide an Action Plan for specific public invalvement actions } \\
\text { and schedule to be completed in the Fourth Quarter of FY } 1997 .\end{array}$} \\
\hline \multicolumn{4}{|c|}{$\begin{array}{l}\text { Description of what constitutes completion of this milestone: This milestone will be } \\
\text { complete when a document entitled "1.8.2 } 1997 \text { Fourth Quarter Public Involvement } \\
\text { Action Plan" is provided to RL for their signoff to authorize implementation. }\end{array}$} \\
\hline \multicolumn{4}{|c|}{ Milestone Description Approval } \\
\hline \multicolumn{2}{|c|}{ 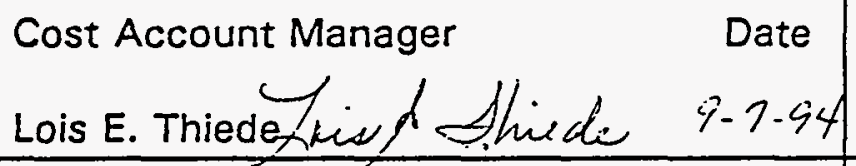 } & \multicolumn{2}{|c|}{ Program/Project Manager) Date } \\
\hline \multicolumn{2}{|c|}{ Program Element Manager $\quad$ Date } & \multicolumn{2}{|c|}{$\begin{array}{l}\text { DOE Program Manager Date } \\
\text { Bill A. Rutherford }\end{array}$} \\
\hline \multicolumn{4}{|c|}{ 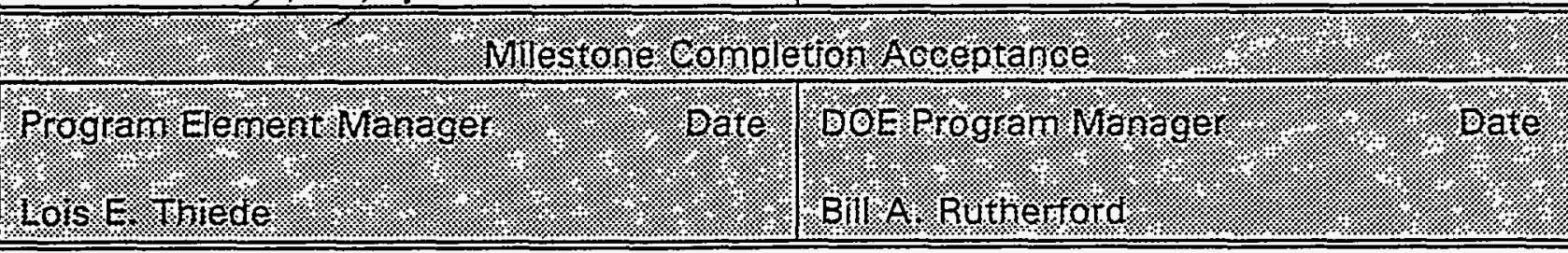 } \\
\hline
\end{tabular}




\begin{tabular}{|c|c|c|c|}
\hline \multicolumn{4}{|c|}{$\begin{array}{l}\text { Westinghouse Hanford Companiy } \\
\text { MILESTONE DESCRIPTION SHEET }\end{array}$} \\
\hline \multicolumn{3}{|c|}{ Title: Summary of Action Plan } & Date: $8 / 3 / 94$ \\
\hline \multicolumn{3}{|c|}{ Assigned To: Lois E. Thiede } & CIN: \\
\hline \multicolumn{3}{|c|}{ Program WBS Designator: 1.8.2.7 } & $\begin{array}{l}\text { Due Date: } \\
7 / 11 / 97\end{array}$ \\
\hline \multicolumn{3}{|c|}{ Control Number: PUB-97-007 } & Rev: \\
\hline $\begin{array}{l}\text { MILESTONE TYPE: } \\
\square \text { TRI-PARTY } \\
\text { AGREEMENT } \\
\square \text { DOE-HQ } \\
\square \text { DOE-RL } \\
X \text { CONTRACTOR }\end{array}$ & $\begin{array}{l}\quad \text { DIVISION: } \\
\square \text { State } \\
\square \text { Federal } \\
\square \text { DOE } \\
\square \text { RCRA } \\
\square \text { TPA Number }\end{array}$ & $\begin{array}{l}\text { DELIVERABLE: } \\
\times \text { Report } \\
\square \text { Letter } \\
\square \text { Drawings } \\
\square \text { Other (specify) }\end{array}$ & $\begin{array}{l}\text { ADDRESS TO: } \\
\dot{\square} \text { DOE-HQ } \\
\square \text { DOE-RL } \\
\square \text { Other } \\
\text { (specify) }\end{array}$ \\
\hline \multicolumn{4}{|c|}{$\begin{array}{l}\text { Milestone Description: Provide a summary of results from implementing the Public } \\
\text { Involvement Action Plan for the third quarter of the fiscal year. Summary to describe } \\
\text { the process undertaken and evaluation of how well stated objectives were met. }\end{array}$} \\
\hline \multicolumn{4}{|c|}{$\begin{array}{l}\text { Description of what constitutes completion of this milestone: This milestone will be } \\
\text { complete when a document entitled "1997 Third Quarter } 1.8 .2 \text { Public Involvement } \\
\text { Action Plan Summary" is provided to all requesting Program Elements. }\end{array}$} \\
\hline \multicolumn{4}{|c|}{ Milestone Description Approval } \\
\hline \multicolumn{2}{|c|}{$\begin{array}{l}\text { Cost Account Manager } \quad \text { Date } \\
\text { Lois E. Thiede faid the thide } 9-7-94\end{array}$} & \multicolumn{2}{|c|}{ 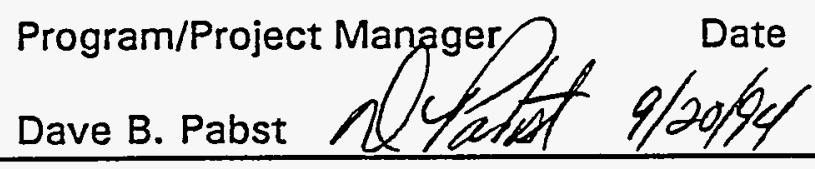 } \\
\hline \multicolumn{2}{|c|}{ 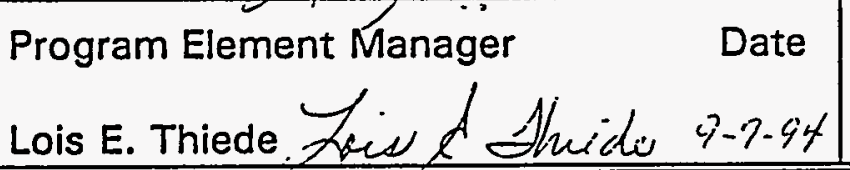 } & \multicolumn{2}{|c|}{$\begin{array}{l}\text { DOE Program Manager } \\
\text { NA }\end{array}$} \\
\hline \multicolumn{4}{|c|}{ 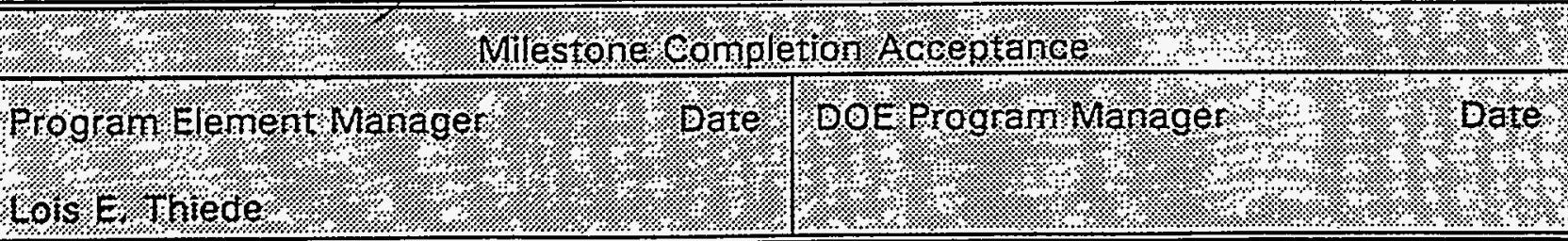 } \\
\hline
\end{tabular}




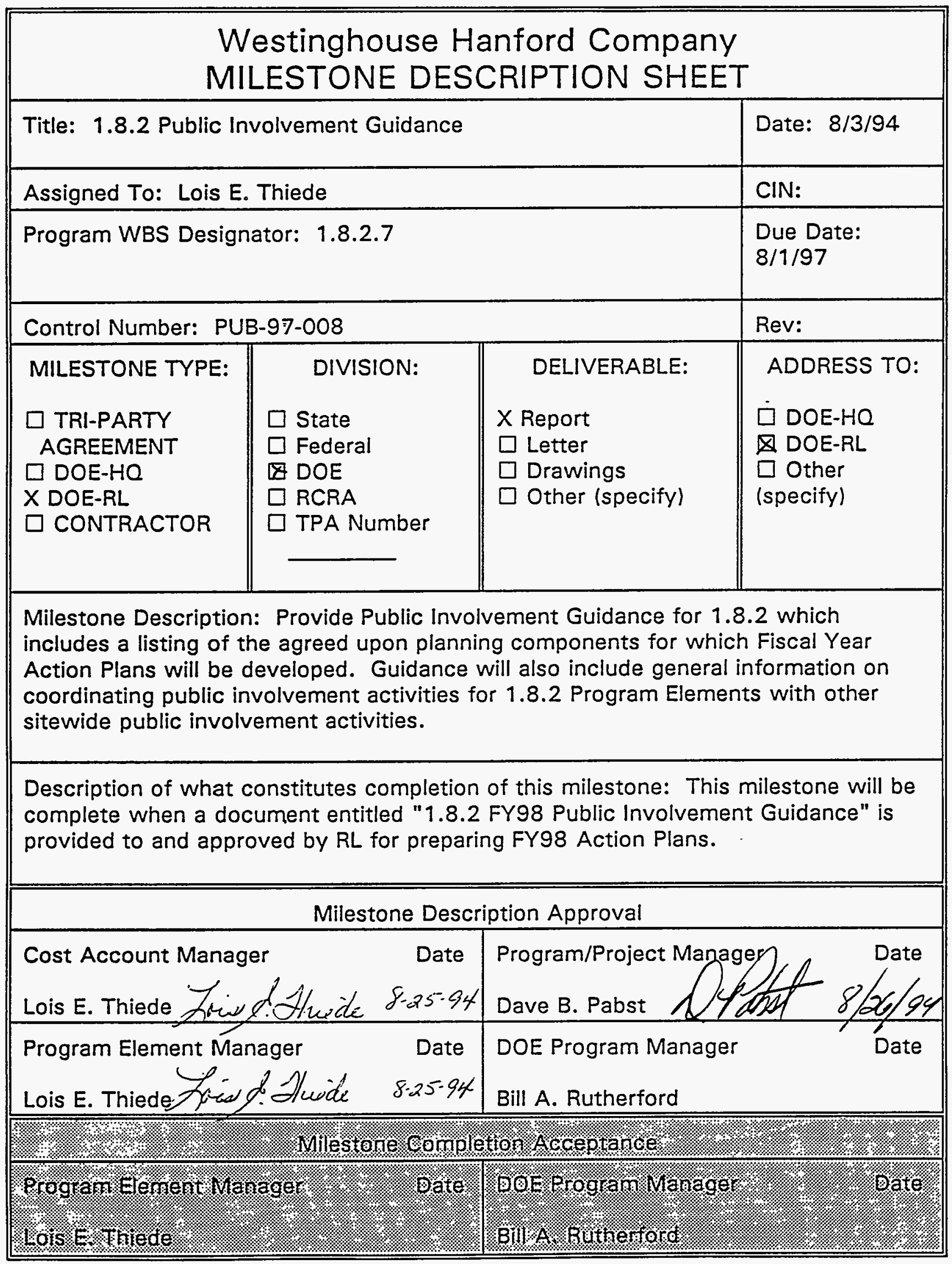




\begin{tabular}{|c|c|c|c|}
\hline \multicolumn{4}{|c|}{$\begin{array}{l}\text { Westinghouse Hanford Company } \\
\text { MILESTONE DESCRIPTION SHEET }\end{array}$} \\
\hline \multicolumn{3}{|c|}{ Title: 1.8 .21997 First Quarter Action Plan } & Date: $8 / 5 / 94$ \\
\hline \multicolumn{3}{|c|}{ Assigned To: Lois E. Thiede } & CIN: \\
\hline \multicolumn{3}{|c|}{ Program WBS Designator: 1.8.2.7 } & $\begin{array}{l}\text { Due Date: } \\
9 / 5 / 97\end{array}$ \\
\hline \multicolumn{3}{|c|}{ Control Number: PUB-97-009 } & Rev: \\
\hline $\begin{array}{l}\text { MILESTONE TYPE: } \\
\square \text { TRI-PARTY } \\
\text { AGREEMENT } \\
\square \text { DOE-HO } \\
\text { X DOE-RL } \\
\square \text { CONTRACTOR }\end{array}$ & $\begin{aligned} & \text { DIVISION: } \\
& \square \text { State } \\
& \square \text { Federal } \\
& \square \text { DOE } \\
& \square \text { RCRA } \\
& \square \text { TPA Number }\end{aligned}$ & $\begin{aligned} & \text { DELIVERABLE: } \\
& \times \text { Report } \\
& \square \text { Letter } \\
& \square \text { Drawings } \\
& \square \text { Other (specify) }\end{aligned}$ & $\begin{array}{l}\text { ADDRESS TO: } \\
\text { मं DOE-HO } \\
\text { X DOE-RL } \\
\square \text { Other } \\
\text { (specify) }\end{array}$ \\
\hline \multicolumn{4}{|c|}{$\begin{array}{l}\text { Milestone Description: Provide an Action Plan for specific public involvement actions } \\
\text { and schedule to be completed in the First Quarter of FY } 1998 .\end{array}$} \\
\hline \multicolumn{4}{|c|}{$\begin{array}{l}\text { Description of what constitutes completion of this milestone: This milestone will be } \\
\text { complete when a document entitled "1.8.2 } 1998 \text { First Quarter Public Involvement } \\
\text { Action Plan" is provided to RL for their signoff to authorize implementation. }\end{array}$} \\
\hline \multicolumn{4}{|c|}{ Milestone Description Approval } \\
\hline \multicolumn{2}{|c|}{ 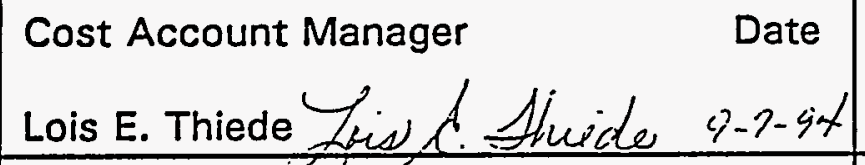 } & \multicolumn{2}{|c|}{ Dave B. Pabst Datect } \\
\hline \multicolumn{2}{|c|}{$\begin{array}{ll}\text { Program Element Manager } & \text { Date } \\
\text { Lois E. Thiede fins \&l Fluide } & 9-7-94 \\
\end{array}$} & \multicolumn{2}{|c|}{$\begin{array}{l}\text { DOE Program Manager Date } \\
\text { Bill A. Rutherford }\end{array}$} \\
\hline \multicolumn{4}{|c|}{ 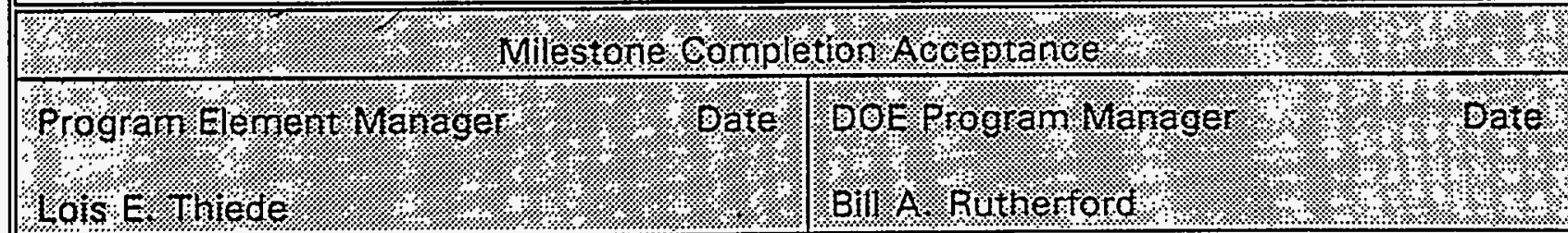 } \\
\hline
\end{tabular}


WHC-SP-1096

$09 / 23 / 94$

Planning Integration Multi-Year Program Plan

This page

intentionally left blank. 


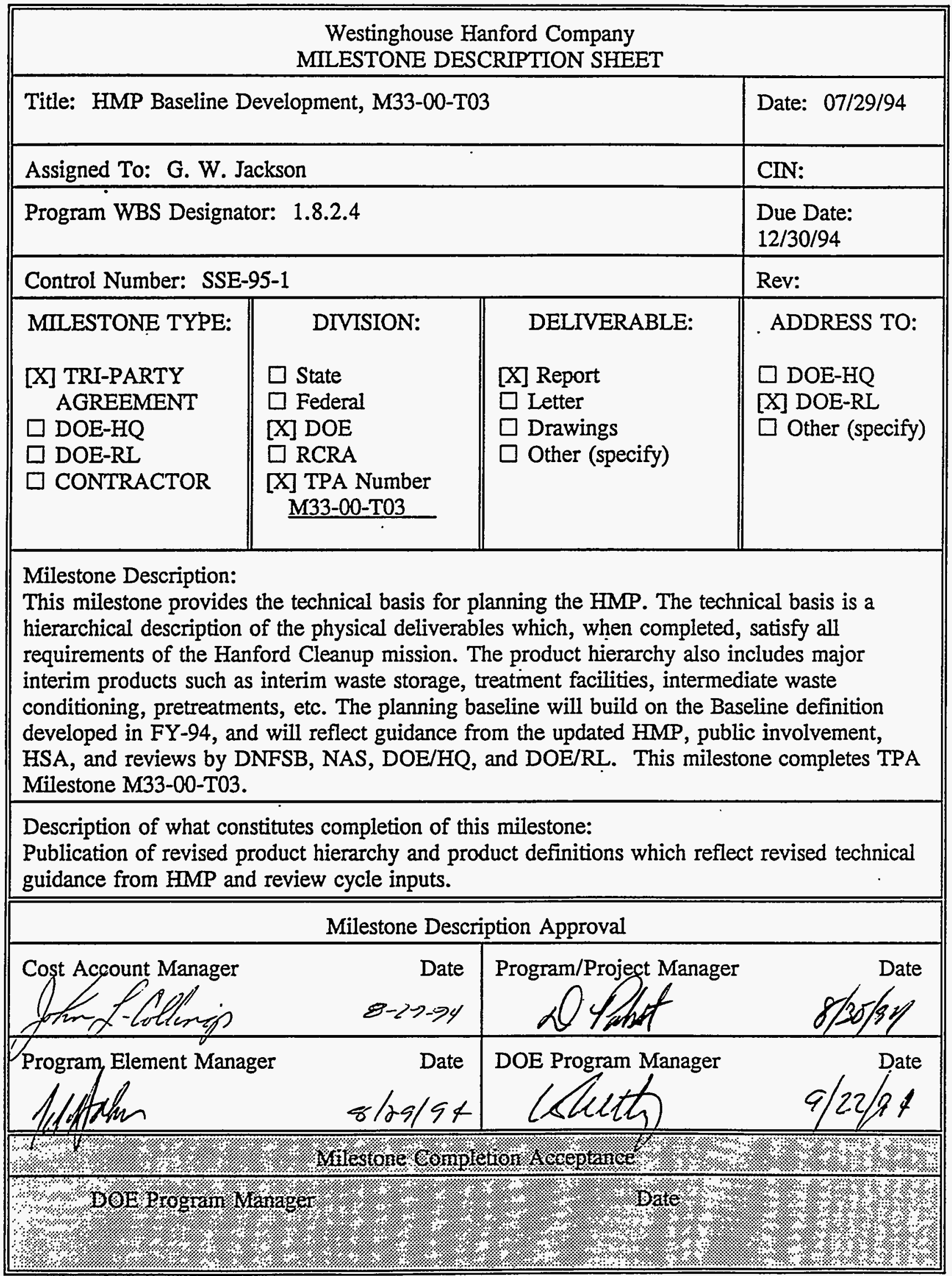




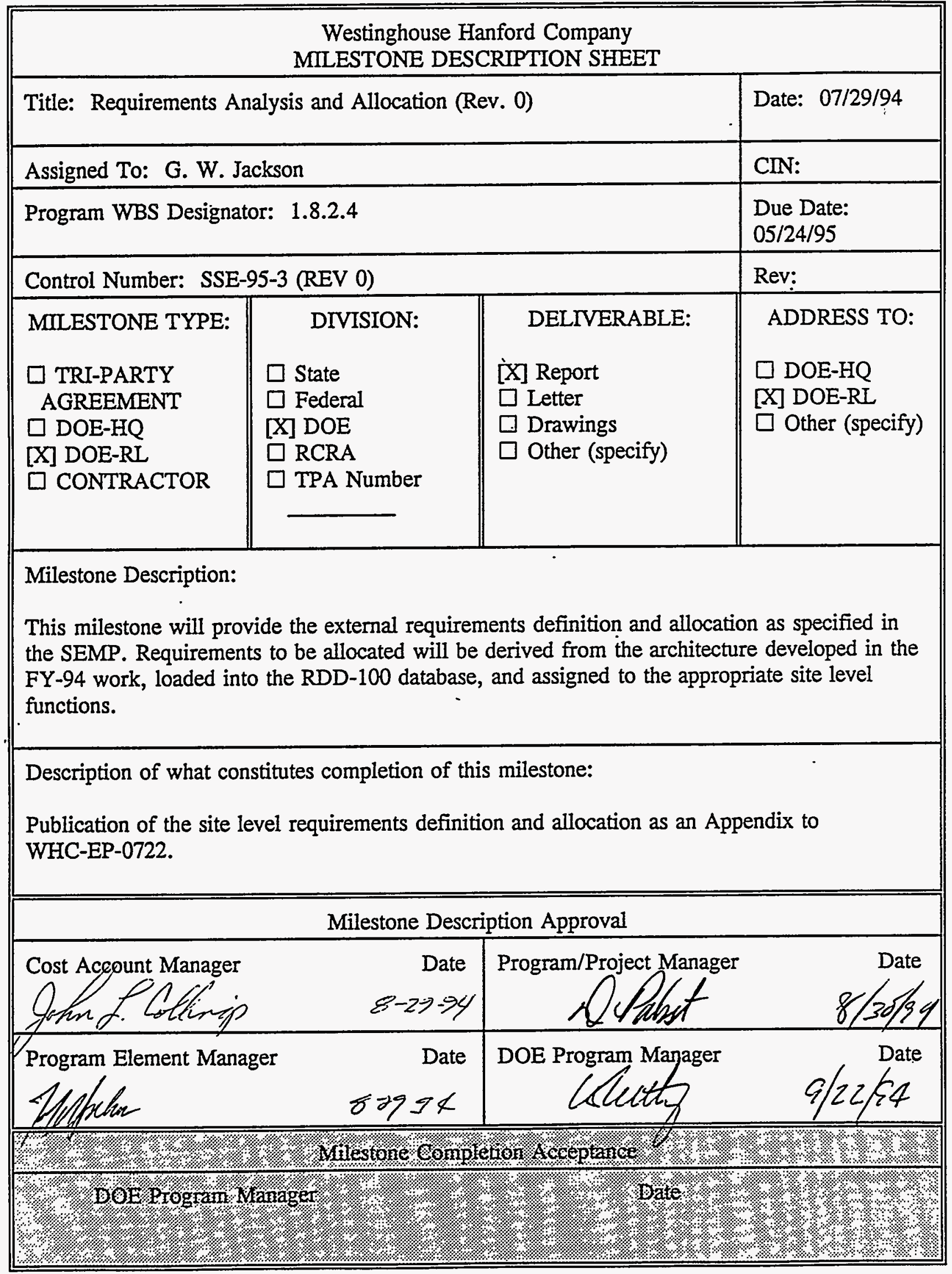




\begin{tabular}{|c|c|c|c|}
\hline \multicolumn{4}{|c|}{$\begin{array}{l}\text { Westinghouse Hanford Company } \\
\text { MILESTONE DESCRIPTION SHEET }\end{array}$} \\
\hline \multicolumn{3}{|c|}{ Title: Measures of Effectiveness Analysis (Rev. 0) } & Date: $07 / 29 / 94$ \\
\hline \multicolumn{3}{|c|}{ Assigned To: G. W. Jackson } & CIN: \\
\hline \multicolumn{3}{|c|}{ Program WBS Designator: 1.8.2.4 } & $\begin{array}{l}\text { Due Date: } \\
05 / 23 / 95\end{array}$ \\
\hline \multicolumn{3}{|c|}{ Control Number: SSE-95-4 } & Rev: \\
\hline $\begin{array}{l}\text { MILESTONE TYPE: } \\
\square \text { TRI-PARTY } \\
\text { AGREEMENT } \\
\square \text { DOE-HQ } \\
{[\mathrm{X}] \text { DOE-RL }} \\
\square \text { CONTRACTOR }\end{array}$ & $\begin{array}{l}\quad \text { DIVISION: } \\
\square \text { State } \\
\square \text { Federal } . \\
{[\mathrm{X}] \text { DOE }} \\
\square \text { RCRA } \\
\square \text { TPA Number }\end{array}$ & $\begin{array}{l}\text { DELIVERABLE: } \\
{[X] \text { Report }} \\
\square \text { Letter } \\
\square \text { Drawings } \\
\square \text { Other (specify) }\end{array}$ & $\begin{array}{l}\text { ADDRESS TO: } \\
\square \text { DOE-HQ } \\
{[X] \text { DOE-RL }} \\
\square \text { Other (specify) }\end{array}$ \\
\hline \multicolumn{4}{|c|}{$\begin{array}{l}\text { Milestone Description: } \\
\text { This milestone will develop the top level measures of effectiveness and allocate these to the } \\
\text { lower level site functions. The measures will be specific to the architecture developed in } \\
\text { WHC-EP-0779 and will follow the guidance provided in the SEMP. }\end{array}$} \\
\hline \multicolumn{4}{|c|}{$\begin{array}{l}\text { Description of what constitutes completion of this milestone: } \\
\text { Publication of the site level measures of effectiveness definitions as an Appendix to } \\
\text { WHC-EP-0722. }\end{array}$} \\
\hline \multicolumn{4}{|c|}{ Milestone Description Approval } \\
\hline \multicolumn{2}{|c|}{ Cost Account Manager $\quad$ Date } & Program/Project Manager & $\begin{array}{l}\text { Date } \\
8 / 30 / 94\end{array}$ \\
\hline \multirow{2}{*}{\multicolumn{2}{|c|}{$\begin{array}{l}\text { Program Element Manager } \\
\text { Alififalm }\end{array}$}} & \multicolumn{2}{|c|}{$\begin{array}{l}\text { DOE Program Manager } \\
\text { on Hold PER W.A. Ratherfire } \\
\text { S/23/s4 }\end{array}$} \\
\hline & & 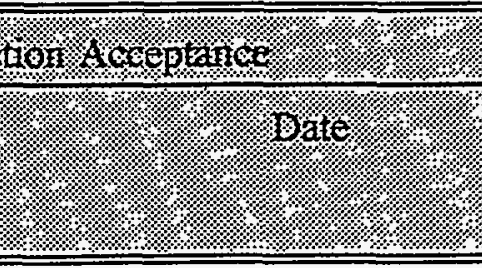 & 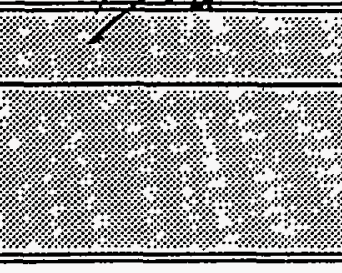 \\
\hline
\end{tabular}




\begin{tabular}{|l||l||}
\hline \multicolumn{3}{|c|}{ Westinghouse Hanford Company } \\
MILESTONE DESCRIPTION SHEET
\end{tabular}




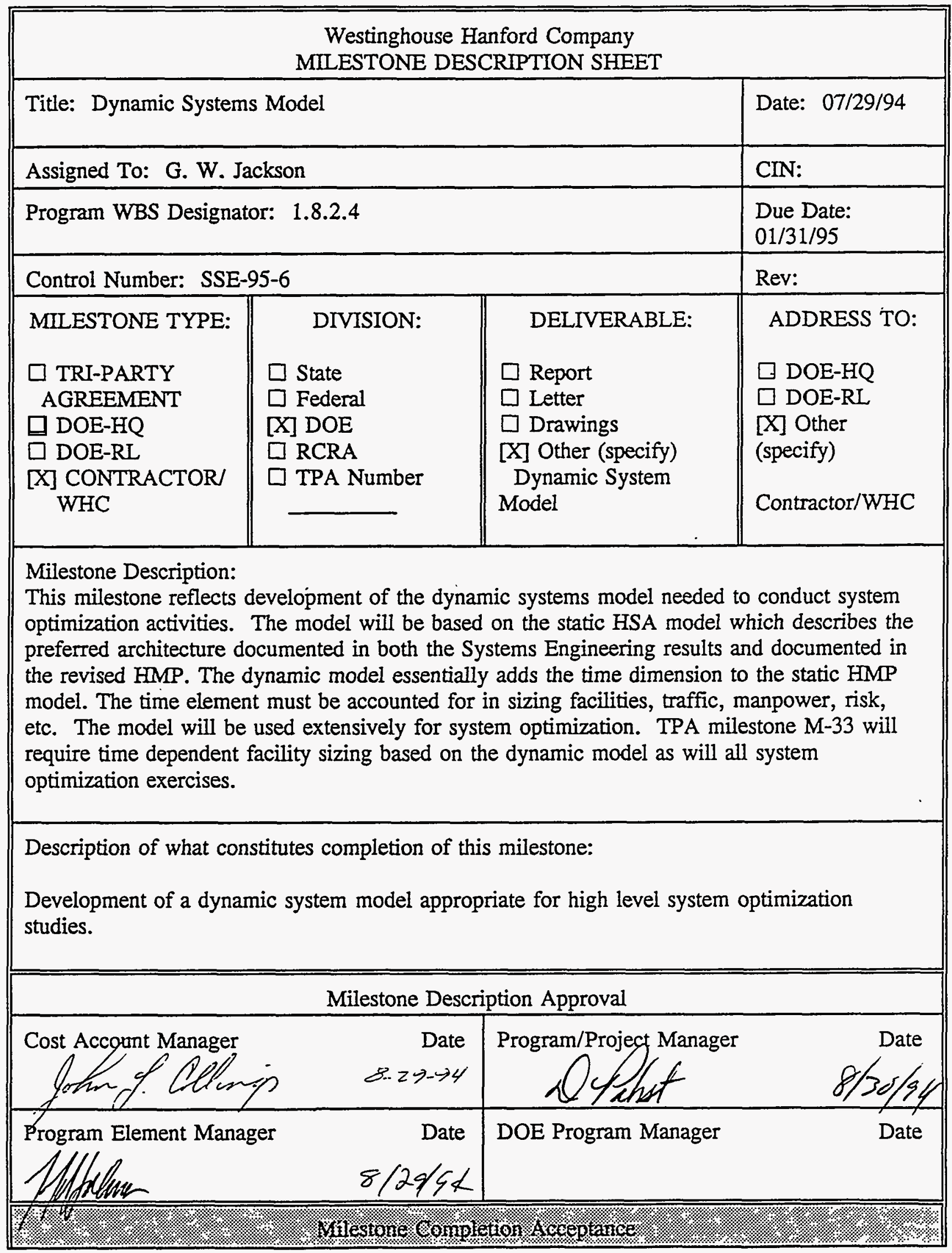




\begin{tabular}{|c|c|c|c|}
\hline \multicolumn{4}{|c|}{$\begin{array}{l}\text { Westinghouse Hanford Company } \\
\text { MILESTONE DESCRIPTION SHEET }\end{array}$} \\
\hline \multicolumn{3}{|c|}{ Title: TPA Milestone M33-00-T04 } & Date: $07 / 29 / 94$ \\
\hline \multicolumn{3}{|c|}{ Assigned To: G. W. Jackson } & CIN: \\
\hline \multicolumn{3}{|c|}{ Program WBS Designator: 1 8.2.4 } & $\begin{array}{l}\text { Due Date: } \\
\text {-08/31/95 } 6 / 30\end{array}$ \\
\hline \multicolumn{3}{|c|}{ Control Number: SSE-95-7 } & Rev: \\
\hline $\begin{array}{l}\text { MILESTONE TYPE: } \\
\text { [X] TRI-PARTY } \\
\text { AGREEMENT } \\
\square \text { DOE-HQ } \\
\square \text { DOE-RL } \\
\square \text { CONTRACTOR }\end{array}$ & $\begin{array}{l}\text { DIVISION: } \\
\square \text { State } \\
\square \text { Federal } \\
\square \text { DOE } \\
\square \text { RCRA } \\
\text { [X] TPA Number } \\
\text { M33-00-T04 }\end{array}$ & $\begin{array}{l}\text { DELIVERABLE: } \\
\square \text { Report } \\
\square \text { Letter } \\
\square \text { Drawings } \\
{[\mathrm{X}] \text { Other (specify) }} \\
\text { TPA Change } \\
\text { Package }\end{array}$ & $\begin{array}{l}\text { ADDRESS TO: } \\
\square \text { DOE-HQ } \\
{[\mathrm{X}] \text { DOE-RL }} \\
\square \text { Other (specify) }\end{array}$ \\
\hline \multicolumn{4}{|c|}{ 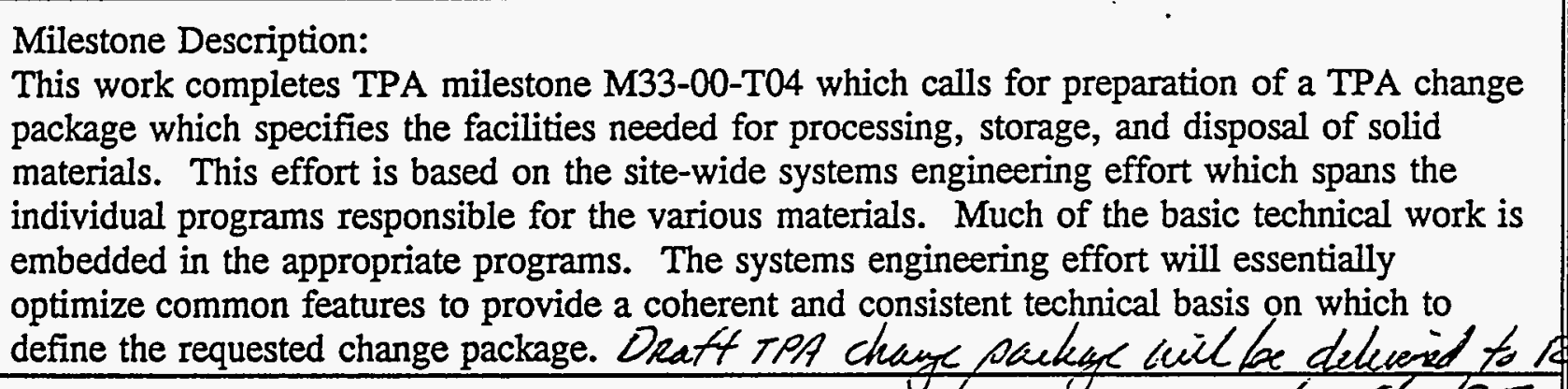 } \\
\hline \multicolumn{4}{|c|}{$\begin{array}{l}\text { Description of what constitutes completion of this milestone: } \\
\text { Prepare a TPA change package for the processing, storage and disposal of solid wastes and } \\
\text { materials. }\end{array}$} \\
\hline \multicolumn{4}{|c|}{ Milestone Description Approval } \\
\hline \multicolumn{2}{|c|}{ Cost Account Manager } & Program/Projecy Manager & $\begin{array}{l}\text { Date } \\
8 / 30 / 94\end{array}$ \\
\hline \multicolumn{2}{|c|}{ Program Element Manager } & DOE Program Manager & $8 / 22 / 94$ \\
\hline \multicolumn{4}{|c|}{ 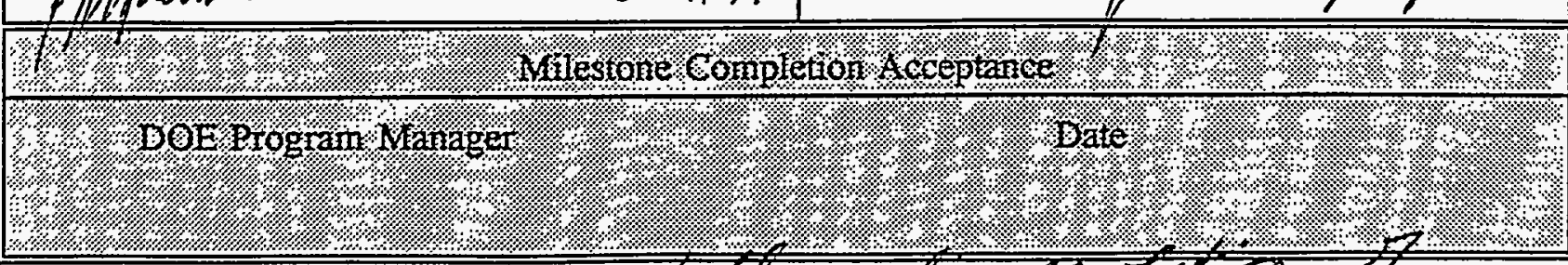 } \\
\hline
\end{tabular}




\begin{tabular}{|l|l||}
\hline \multicolumn{3}{|c|}{ Westinghouse Hanford Company } \\
MILESTONE DESCRIPTION SHEET
\end{tabular}




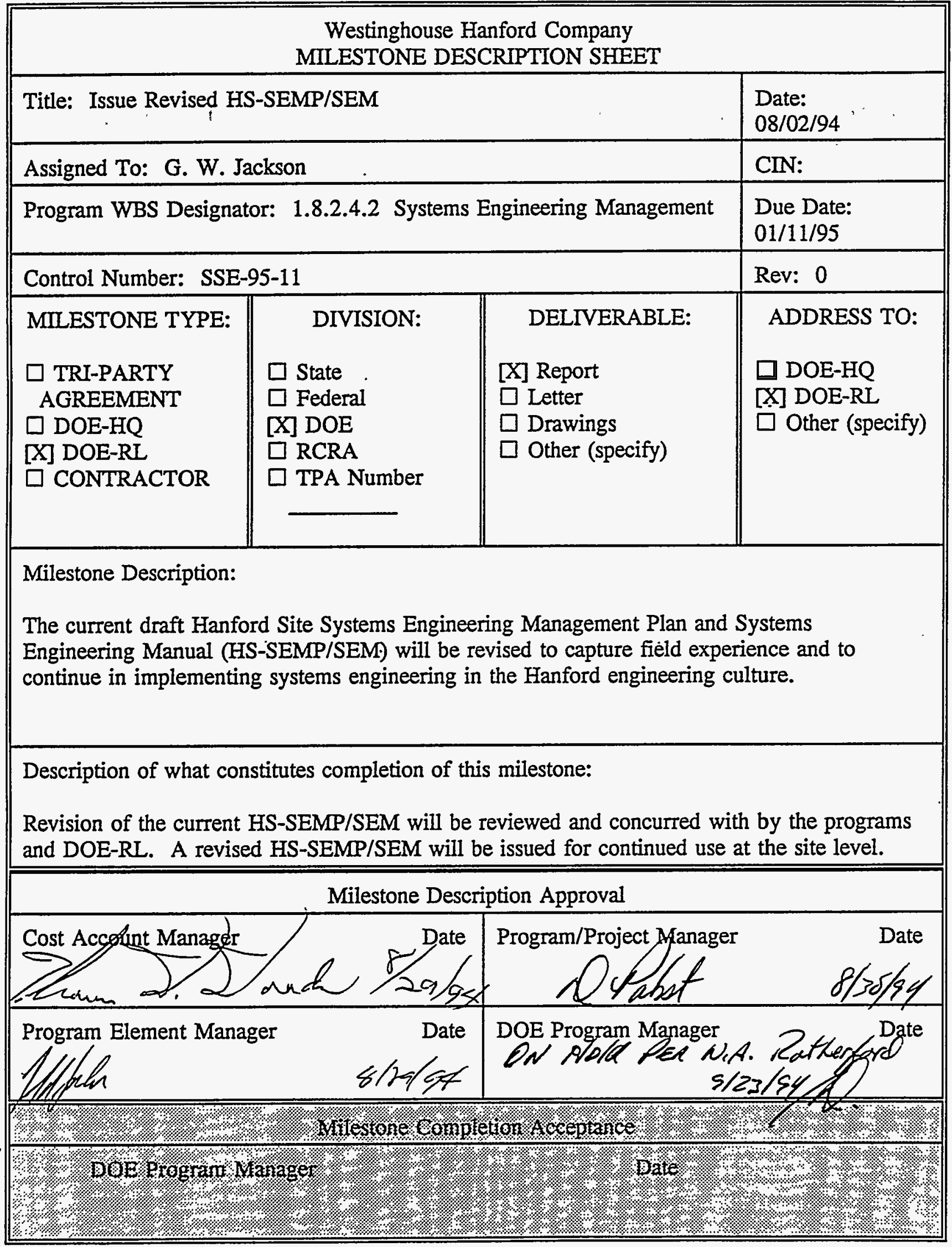




\begin{tabular}{|l|l|||}
\hline \multicolumn{3}{|c||}{ Westinghouse Hanford Company } \\
MILESTONE DESCRIPTION SHEET
\end{tabular}




\begin{tabular}{|l|l||}
\hline \multicolumn{3}{|c|}{ Westinghouse Hanford Company } \\
MILESTONE DESCRIPTION SHEET
\end{tabular}




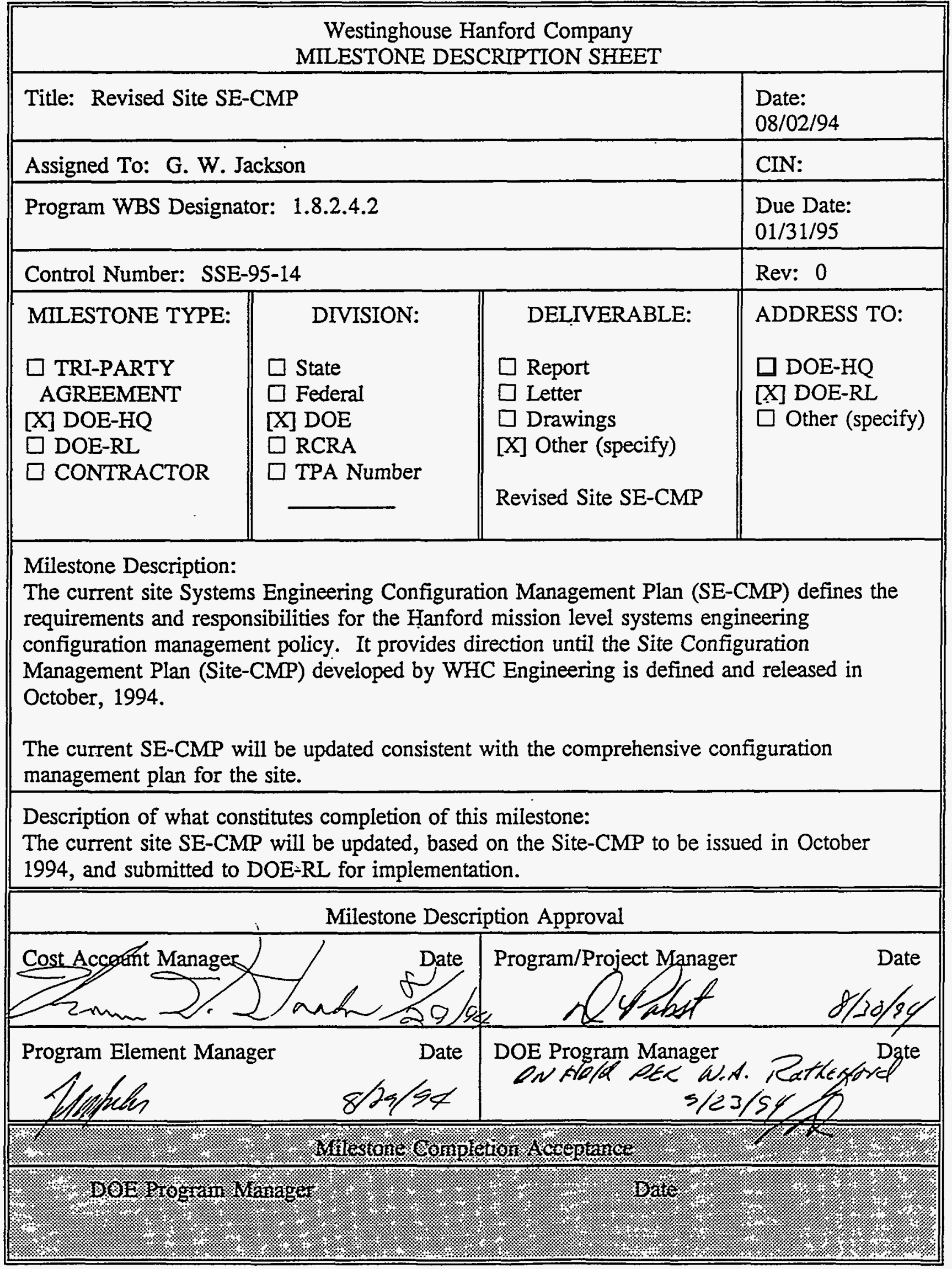




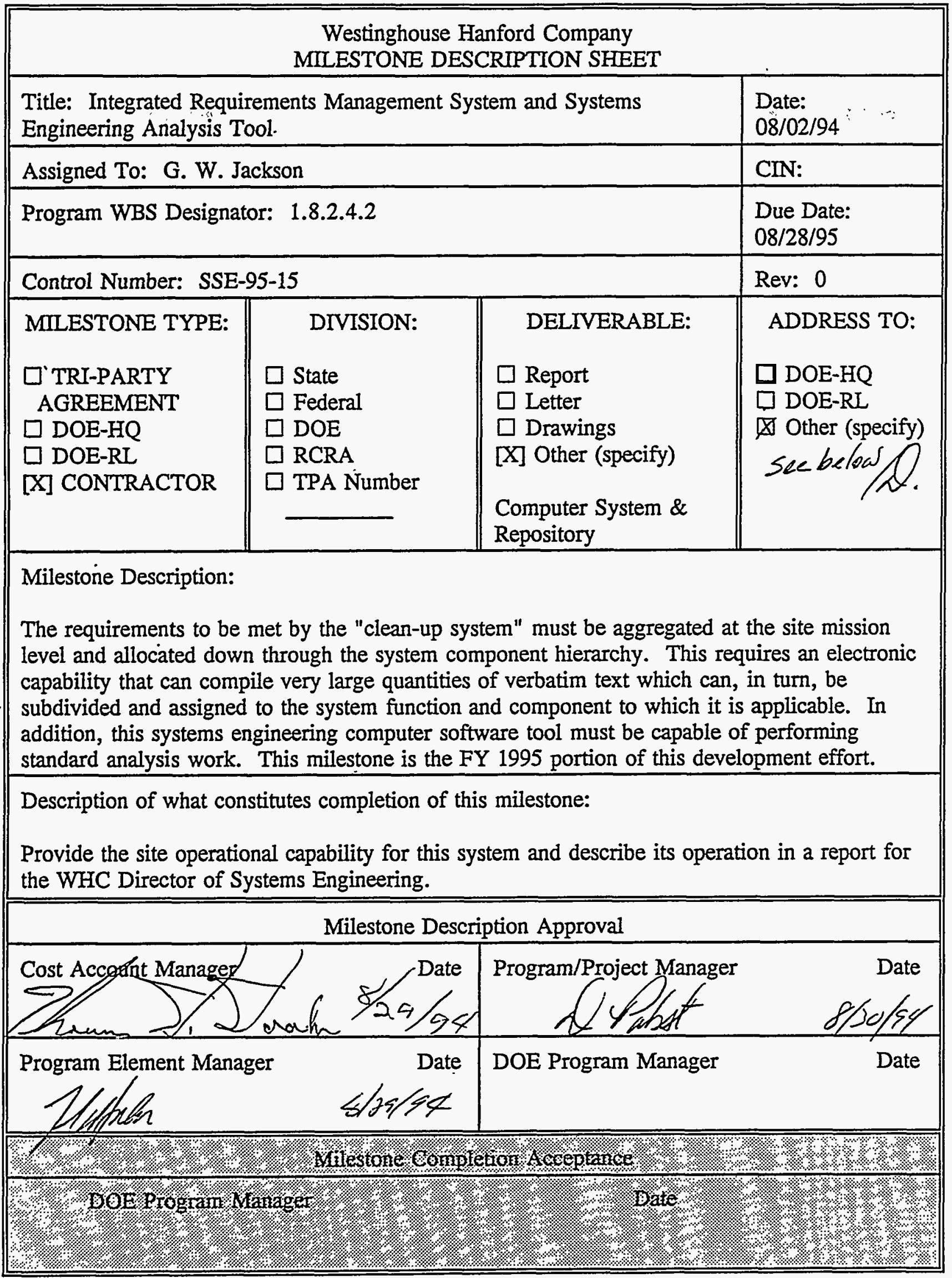




\begin{tabular}{|c|c|c|c|}
\hline \multicolumn{4}{|c|}{$\begin{array}{l}\text { Westinghouse Hanford Company } \\
\text { MILESTONE DESCRIPTION SHEET }\end{array}$} \\
\hline \multicolumn{3}{|c|}{ Title: HMP Baseline Development } & Date: $07 / 29 / 94$ \\
\hline \multicolumn{3}{|c|}{ Assigned To: G. W. Jackson } & CIN: \\
\hline \multicolumn{3}{|c|}{ Program WBS Designator: 1.8.2.4 } & $\begin{array}{l}\text { Due Date: } \\
01 / 02 / 96\end{array}$ \\
\hline \multicolumn{3}{|c|}{ Control Number: SSE-96-1 } & Rev: \\
\hline $\begin{array}{l}\text { MILESTONE TYPE: } \\
\square \text { TRI-PARTY } \\
\text { AGREEMENT } \\
\square \text { DOE-HQ } \\
{[X] \text { DOE-RL }} \\
\square \text { CONTRACTOR }\end{array}$ & $\begin{array}{l}\quad \text { DIVISION: } \\
\quad \square \text { State } \\
\square \text { Federal } \\
{[X] \text { DOE }} \\
\square \text { RCRA } \\
\square \text { TPA Number }\end{array}$ & $\begin{array}{l}\text { DELIVERABLE: } \\
{[X] \text { Report }} \\
\square \text { Letter } \\
\square \text { Drawings } \\
\square \text { Other (specify) }\end{array}$ & $\begin{array}{l}\text { ADDRESS TO: } \\
\square \text { DOE-HQ } \\
{[X] \text { DOE-RL }} \\
\square \text { Other (specify) }\end{array}$ \\
\hline \multicolumn{4}{|c|}{$\begin{array}{l}\text { Milestone Description: } \\
\text { This milestone provides the technical basis for planning the HMP. The technical basis is a } \\
\text { hierarchical description of the physical deliverables which, when completed, satisfy all } \\
\text { requirements of the Hanford Cleanup mission. The product hierarchy also includes major } \\
\text { interim products such as interim waste storage, treatment facilities, intermediate waste } \\
\text { conditioning, pretreatments, etc. The planning baseline will build on the Baseline definition } \\
\text { developed in FY-95, and will reflect guidance from the updated HMP, public involvement, } \\
\text { HSA, and reviews by DNFSB, NAS, DOE/HQ, and DOE/RL. }\end{array}$} \\
\hline \multicolumn{4}{|c|}{$\begin{array}{l}\text { Description of what constitutes completion of this milestone: } \\
\text { Publication of revised product hierarchy and product definitions which reflect revised technical } \\
\text { guidance from HMP and review cycle inputs. }\end{array}$} \\
\hline \multicolumn{4}{|c|}{ Milestone Description Approval } \\
\hline \multicolumn{2}{|c|}{ Cost Aceount Manager $\quad$ Date } & Program/Project Manager & $\begin{array}{l}\text { Date } \\
8 / 30 / 24 \\
\end{array}$ \\
\hline \multicolumn{2}{|c|}{$\begin{array}{lr}\text { Program Element Manager } & \text { Date } \\
\text { Nhllhele } & 8-29-5 \% \\
\end{array}$} & DOE Program Manager & Date \\
\hline \multicolumn{4}{|c|}{ 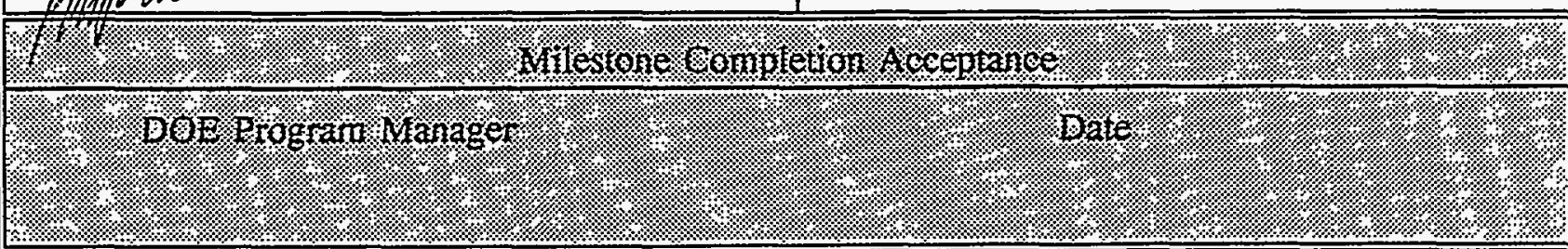 } \\
\hline
\end{tabular}




\begin{tabular}{|c|c|c|c|}
\hline \multicolumn{4}{|c|}{$\begin{array}{l}\text { Westinghouse Hanford Company } \\
\text { MILESTONE DESCRIPTION SHEET }\end{array}$} \\
\hline \multicolumn{3}{|c|}{ Title: Update Functional Analysis } & Date: $07 / 29 / 94$ \\
\hline \multicolumn{3}{|c|}{ Assigned To: G. W. Jackson } & CIN: \\
\hline \multicolumn{3}{|c|}{ Program WBS Designator: 1 1.8.2.4 } & $\begin{array}{l}\text { Due Date: } \\
03 / 27 / 96\end{array}$ \\
\hline \multicolumn{3}{|c|}{ Control Number: SSE-96-2 } & Rev: \\
\hline $\begin{array}{l}\text { MILESTONE TYPE: } \\
\square \text { TRI-PARTY } \\
\text { AGREEMENT } \\
\square \text { DOE-HQ } \\
{[X] \text { DOE-RL }} \\
\square \text { CONTRACTOR }\end{array}$ & $\begin{array}{l}\quad \text { DIVISION: } \\
\square \text { State } \\
\square \text { Federal } \\
{[\mathrm{X}] \text { DOE }} \\
\square \text { RCRA } \\
\square \text { TPA Number }\end{array}$ & $\begin{array}{l}\text { DELIVERABLE: } \\
{[\mathrm{X}] \text { Report }} \\
\square \text { Letter } \\
\square \text { Drawings } \\
\square \text { Other (specify) }\end{array}$ & $\begin{array}{l}\text { ADDRESS TO: } \\
\square \text { DOE-HQ } \\
{[X] \text { DOE-RL }} \\
\square \text { Other (specify) }\end{array}$ \\
\hline \multicolumn{4}{|c|}{$\begin{array}{l}\text { Milestone Description: } \\
\text { This milestone consists of updating the Cleanup System Functional analysis to reflect guidance } \\
\text { from the updated HMP, HSA, and DOE-RL, and appropriate reviews. }\end{array}$} \\
\hline \multicolumn{4}{|c|}{$\begin{array}{l}\text { Description of what constitutes completion of this milestone: } \\
\text { Publication of the revised functional analysis as a revision to WHC EP-0722. }\end{array}$} \\
\hline \multicolumn{4}{|c|}{ Milestone Description Approval } \\
\hline \multicolumn{2}{|c|}{ Cost Acsount Manager $\quad$ Date } & Program/Project Manager & $\begin{array}{l}\text { Date } \\
8 / 30 / 94\end{array}$ \\
\hline \multicolumn{2}{|c|}{$\begin{array}{ll}\text { Program Element Manager } & \text { Date } \\
\text { S/ffech } & 8-27.9 K\end{array}$} & DOE Program Manager & Date \\
\hline \multicolumn{4}{|c|}{ 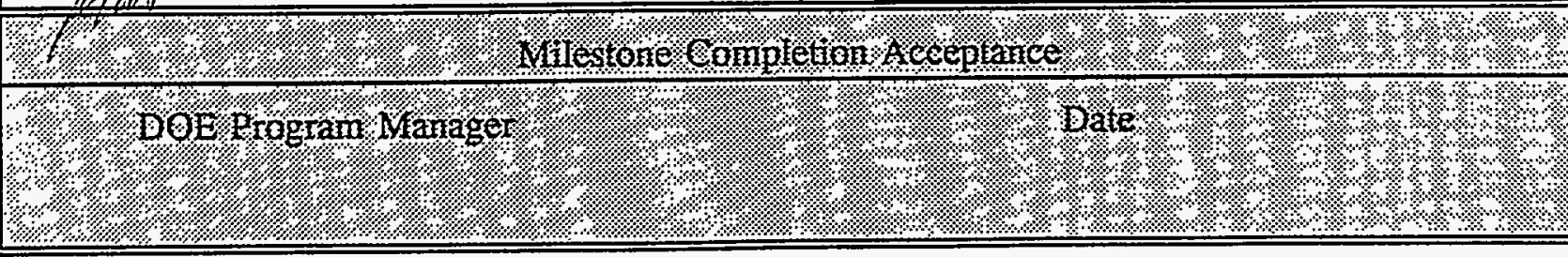 } \\
\hline
\end{tabular}


WHC-SP-1096

$09 / 23 / 94$

\begin{tabular}{|c|c|c|c|}
\hline \multicolumn{4}{|c|}{$\begin{array}{l}\text { Westinghouse Hanford Company } \\
\text { MILESTONE DESCRIPTION SHEET }\end{array}$} \\
\hline \multicolumn{3}{|c|}{ Title: Requirements Analysis and Allocation } & Date: $07 / 29 / 94$ \\
\hline \multicolumn{3}{|c|}{ Assigned To: G. W. Jackson } & CIN: \\
\hline \multicolumn{3}{|c|}{ Program WBS Designator: 1.8 .2 .4} & $\begin{array}{l}\text { Due Date: } \\
05 / 22 / 96\end{array}$ \\
\hline \multicolumn{3}{|c|}{ Control Number: SSE-96-3 } & Rev: \\
\hline $\begin{array}{l}\text { MILESTONE TYPE: } \\
\square \text { TRI-PARTY } \\
\text { AGREEMENT } \\
\square \text { DOE-HQ } \\
{[X] \text { DOE-RL }} \\
\square \text { CONTRACTOR }\end{array}$ & $\begin{array}{l}\quad \text { DIVISION: } \\
\square \text { State } \\
\square \text { Federal } \\
{[\mathrm{X}] \text { DOE }} \\
\square \text { RCRA } \\
\square \text { TPA Number }\end{array}$ & $\begin{array}{l}\text { DELIVERABLE: } \\
{[X] \text { Report }} \\
\square \text { Letter } \\
\square \text { Drawings } \\
\square \text { Other (specify) }\end{array}$ & $\begin{array}{l}\text { ADDRESS TO: } \\
\square \text { DOE-HQ } \\
{[X] \text { DOE-RL }} \\
\square \text { Other (specify) }\end{array}$ \\
\hline \multicolumn{4}{|c|}{$\begin{array}{l}\text { Milestone Description: } \\
\text { This milestone will update the external requirements definition and allocation as specified in the } \\
\text { SEMP. Requirements to be allocated will be derived from the architecture developed in the } \\
\text { FY- } 95 \text { work, loaded into the RDD- } 100 \text { database, and assigned to the appropriate site level } \\
\text { functions. }\end{array}$} \\
\hline \multicolumn{4}{|c|}{$\begin{array}{l}\text { Description of what constitutes completion of this milestone: } \\
\text { Publication of the site level requirements definition and allocation as an updated Appendix to } \\
\text { WHC-EP-0722. }\end{array}$} \\
\hline \multicolumn{4}{|c|}{ Milestone Description Approval } \\
\hline Cost Aceount Manager & $\begin{array}{r}\text { Date } \\
3-29-94\end{array}$ & Program/Project Manager & $\begin{array}{r}\text { Date } \\
8 / 30 / 54\end{array}$ \\
\hline $\begin{array}{l}\text { Program Element Mana } \\
\text { Hffleter }\end{array}$ & $\begin{array}{l}\text { Date } \\
8-29-5 \alpha\end{array}$ & DOE Program Manager & Date \\
\hline (1. & imager & tivit & 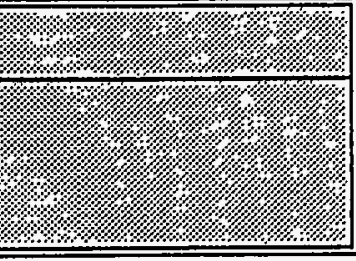 \\
\hline
\end{tabular}




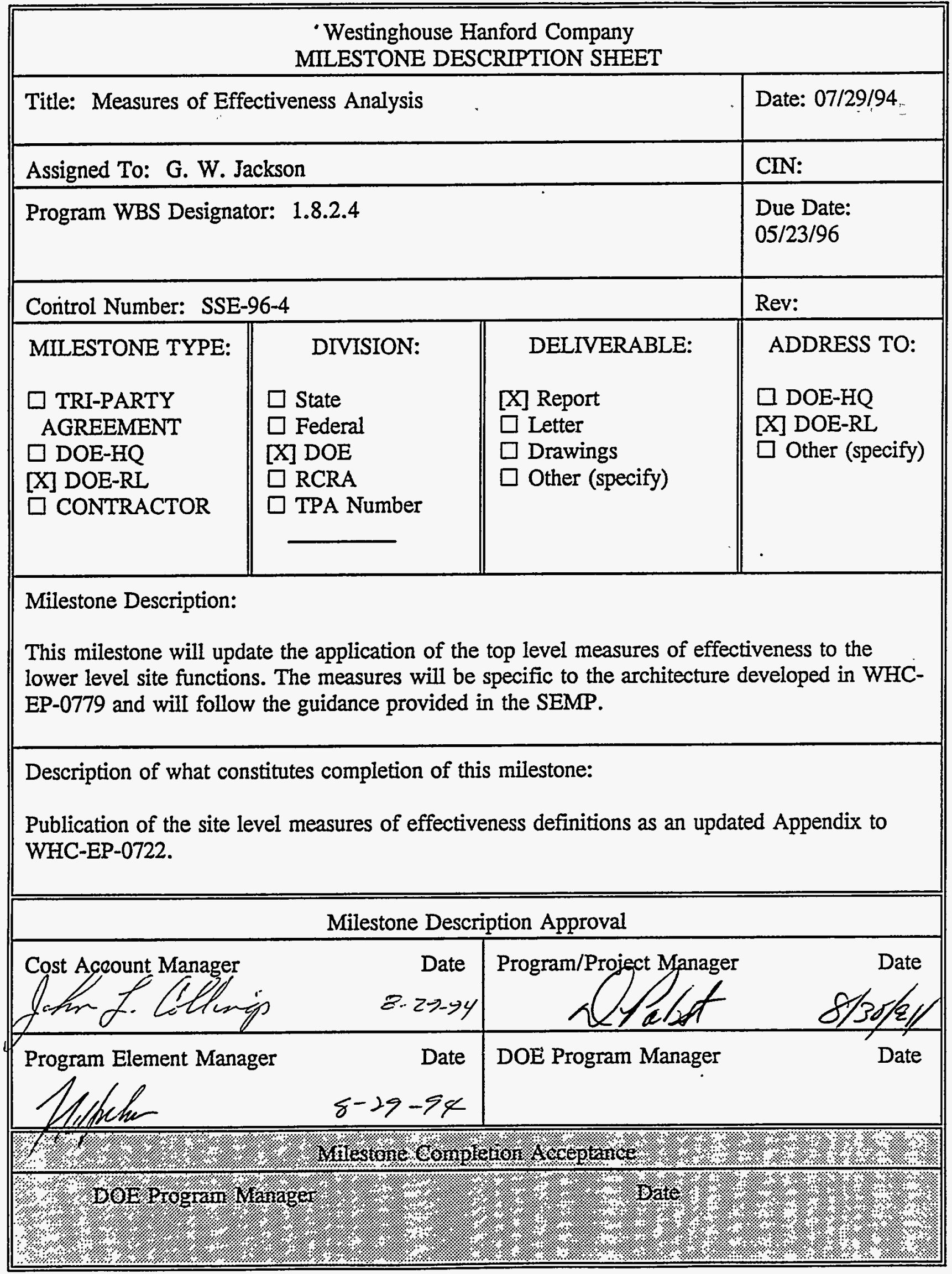




\begin{tabular}{|l|l||}
\hline \multicolumn{3}{|c|}{ Westinghouse Hanford Company } \\
MILESTONE DESCRIPTION SHEET
\end{tabular}




\begin{tabular}{|c|c|c|c|}
\hline \multicolumn{4}{|c|}{$\begin{array}{l}\text { Westinghouse Hanford Company } \\
\text { MILESTONE DESCRIPTION SHEET }\end{array}$} \\
\hline \multicolumn{3}{|c|}{ Title: System Optimization - Case 2, 200 Area Disposal Site } & Date: $07 / 29 / 94$ \\
\hline \multicolumn{3}{|c|}{ Assigned To: G. W. Jackson } & CIN: \\
\hline \multicolumn{3}{|c|}{ Program WBS Designator: 1 8.2.4 } & $\begin{array}{l}\text { Due Date: } \\
05 / 29 / 96 \\
\end{array}$ \\
\hline \multicolumn{3}{|c|}{ Control Number: SSE-96-6 } & Rev: \\
\hline $\begin{array}{l}\text { MILESTONE TYPE: } \\
\square \text { TRI-PARTY } \\
\text { AGREEMENT } \\
\square \text { DOE-HQ } \\
{[X] \text { DOE-RL. }} \\
\square \text { CONTRACTOR }\end{array}$ & $\begin{array}{l}\text { DIVISION: } \\
\square \text { State } \\
\square \text { Federal } \\
{[X] \text { DOE }} \\
\square \text { RCRA } \\
\square \text { TPA Number }\end{array}$ & $\begin{array}{l}\text { DELIVERABLE: } \\
{[\mathrm{X}] \text { Report }} \\
\square \text { Letter } \\
\square \text { Drawings } \\
\square \text { Other (specify) }\end{array}$ & $\begin{array}{l}\text { ADDRESS TO: } \\
\square \text { DOE-HQ } \\
{[X] \text { DOE-RL }} \\
\square \text { Other (specify) }\end{array}$ \\
\hline \multicolumn{4}{|c|}{$\begin{array}{l}\text { Milestone Description: } \\
\text { This milestone consists of the physical optimization of the } 200 \text { area disposal site with respect to } \\
\text { types, capacities and quantities of final disposal facilities, consistency of disposal approach, risk } \\
\text { management, physical size, cost and schedule. The optimization work will utilize the baseline } \\
\text { update and dynamic model developed within } 1.8 .2 .4 \text {. The optimization will not provide a } \\
\text { specific } 200 \text { area design, but will provide a design concept or concept set which, after } \\
\text { stakeholder, regulator and system owner review, will provide the general criteria for design. }\end{array}$} \\
\hline \multicolumn{4}{|c|}{$\begin{array}{l}\text { Description of what constitutes completion of this milestone: } \\
\text { A report documenting the optimization study will complete this milestone. The report will be } \\
\text { the vehicle used to elicit public, regulator, and DOE comment on the } 200 \text { area disposal } \\
\text { concept. }\end{array}$} \\
\hline \multicolumn{4}{|c|}{ Milestone Description Approval } \\
\hline \multicolumn{2}{|c|}{ Cost Account Manager $\quad$ Date } & Program/Project Alanager & $\begin{array}{l}\text { Date } \\
8 / 30 / 94 \\
\end{array}$ \\
\hline Program Element Manag & $\begin{array}{r}\text { Date } \\
6-29-94 \\
\end{array}$ & DOE Program Manager & Date \\
\hline \multicolumn{4}{|c|}{ 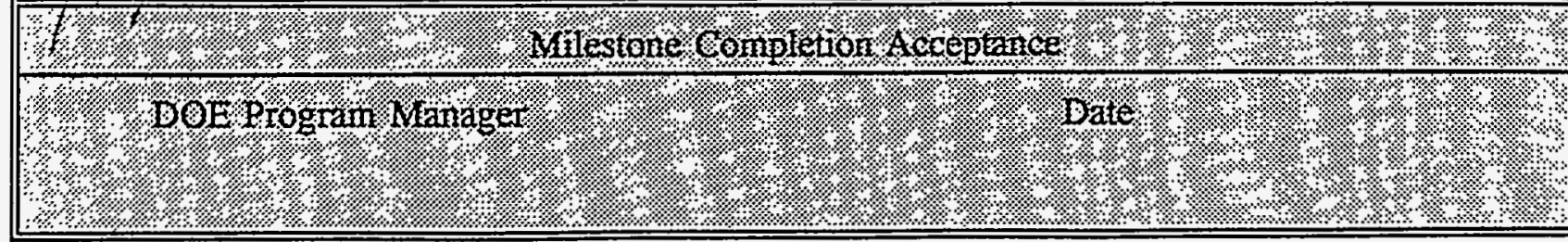 } \\
\hline
\end{tabular}




\begin{tabular}{|c|c|c|c|}
\hline \multicolumn{4}{|c|}{$\begin{array}{l}\text { Westinghouse Hanford Company } \\
\text { MLESTONE DESCRIPTION SHEET }\end{array}$} \\
\hline \multicolumn{3}{|c|}{ Title: Baseline Specification } & Date: $07 / 29 / 94$ \\
\hline \multicolumn{3}{|c|}{ Assigned To: G. W. Jackson } & CIN: \\
\hline \multicolumn{3}{|c|}{ Program WBS Designator: 1 1.8.2.4 } & $\begin{array}{l}\text { Due Date: } \\
\text { 09/30/96 }\end{array}$ \\
\hline \multicolumn{3}{|c|}{ Control Number: SSE-96-7 } & Rev: \\
\hline $\begin{array}{l}\text { MILESTONE TYPE: } \\
\square \text { TRI-PARTY } \\
\text { AGREEMENT } \\
\square \text { DOE-HQ } \\
{[X] \text { DOE-RL }} \\
\square \text { CONTRACTOR }\end{array}$ & $\begin{aligned} & \text { DIVISION: } \\
& \square \text { State } \\
& \square \text { Federal } \\
& {[X] \text { DOE } } \\
& \square \text { RCRA } \\
& \square \text { TPA Number }\end{aligned}$ & $\begin{array}{l}\text { DELIVERABLE: } \\
{[\mathrm{X}] \text { Report }} \\
\square \text { Letter } \\
\square \text { Drawings } \\
\square \text { Other (specify) }\end{array}$ & $\begin{array}{l}\text { ADDRESS TO: } \\
\square \text { DOE-HQ } \\
{[X] \text { DOE-RL }} \\
\square \text { Other (specify) }\end{array}$ \\
\hline \multicolumn{4}{|c|}{$\begin{array}{l}\text { Milestone Description: } \\
\text { This milestone provides the physical system baseline in terms of a product breakdown } \\
\text { structure, associated product definitions, functions which produce the products, and external } \\
\text { and internal requirements. The baseline specification differs from the technical baseline } \\
\text { developed in FY- } 95 \text { in terms of the added detail provided and the changes to the FY-95 } \\
\text { baseline resulting from the baseline update and system optimization work. The Baseline } \\
\text { specification will replace the MYPP planning basis used in FY-96 and become the new } \\
\text { planning basis for FY-97 MYPP activities. The cycle of Baseline Planning, baseline update, } \\
\text { and baseline specification will repeat every year until a stable site specification emerges. }\end{array}$} \\
\hline \multicolumn{4}{|c|}{$\begin{array}{l}\text { Description of what constitutes completion of this milestone: } \\
\text { A report documenting the site specification will complete this milestone. The report will be the } \\
\text { vehicle used to elicit public, regulator, and DOE comment needed to construct the planning } \\
\text { basis for FY-97. }\end{array}$} \\
\hline \multicolumn{4}{|c|}{ Milestone Description Approval } \\
\hline \multicolumn{2}{|c|}{ Cost Account Manager } & Program/Project Manager & Date \\
\hline Program Element Manag & $\begin{array}{r}\text { Date } \\
8-29-94 \\
\end{array}$ & DOE Program Manager & Date \\
\hline \multicolumn{4}{|c|}{ 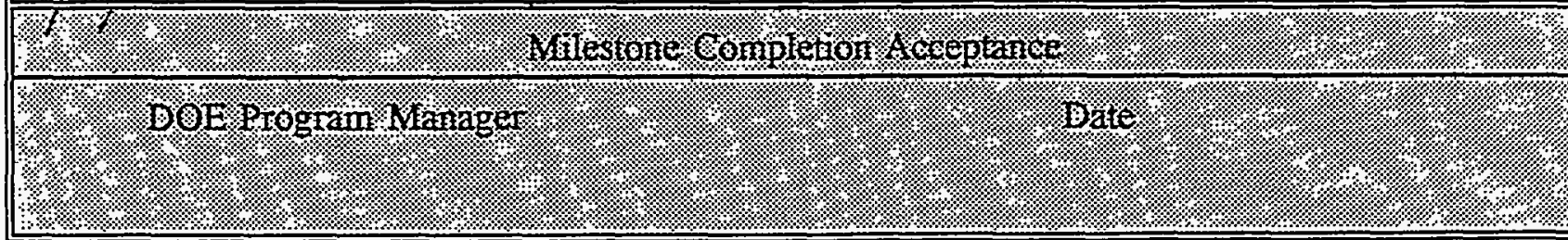 } \\
\hline
\end{tabular}




\begin{tabular}{|l|l||}
\hline \multicolumn{3}{|c|}{ Westinghouse Hanford Company } \\
MILESTONE DESCRIPTION SHEET
\end{tabular}




\begin{tabular}{|l|l|l||}
\hline \multicolumn{3}{|c|}{ Westinghouse Hanford Company } \\
MILESTONE DESCRIPTION SHEET
\end{tabular}




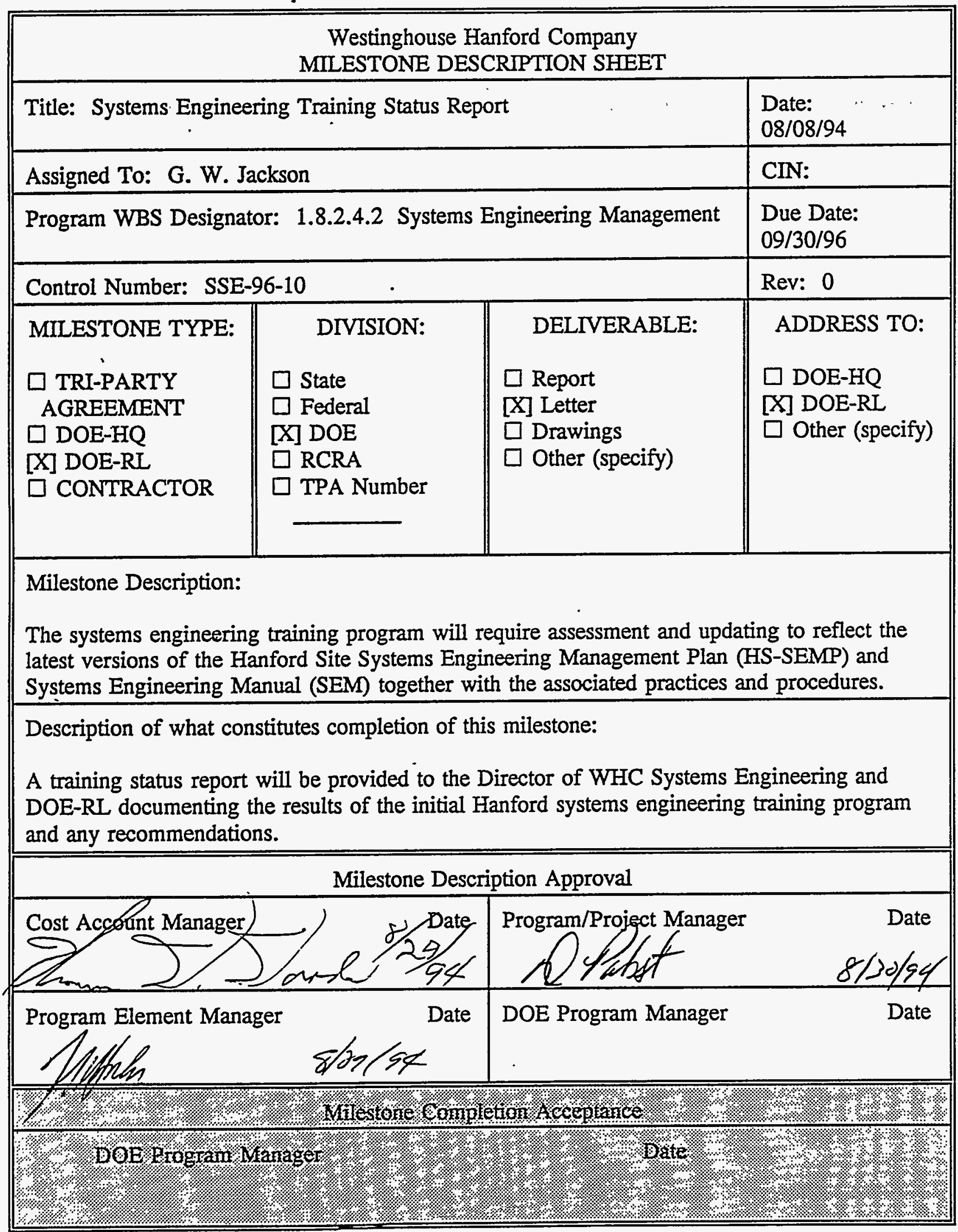




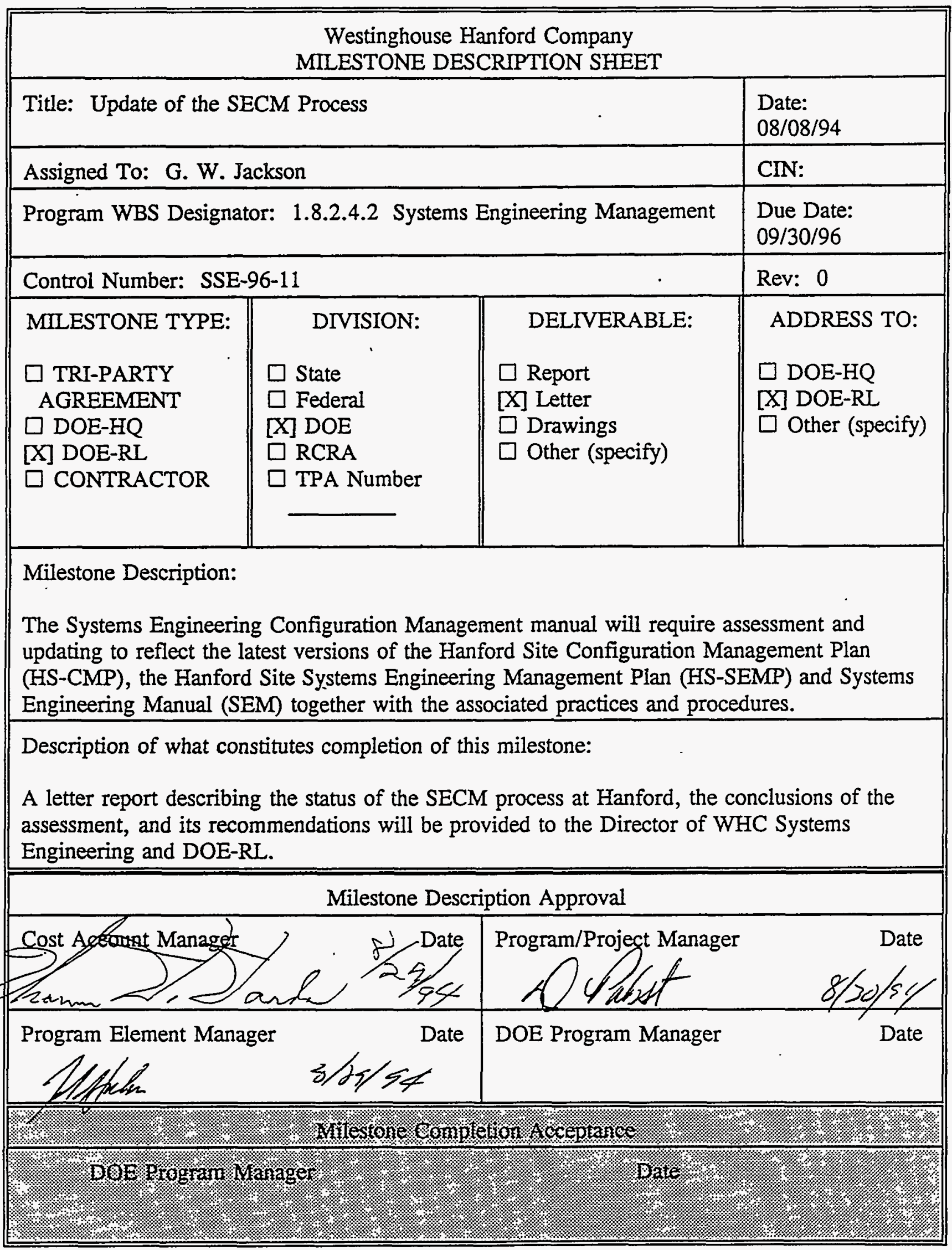




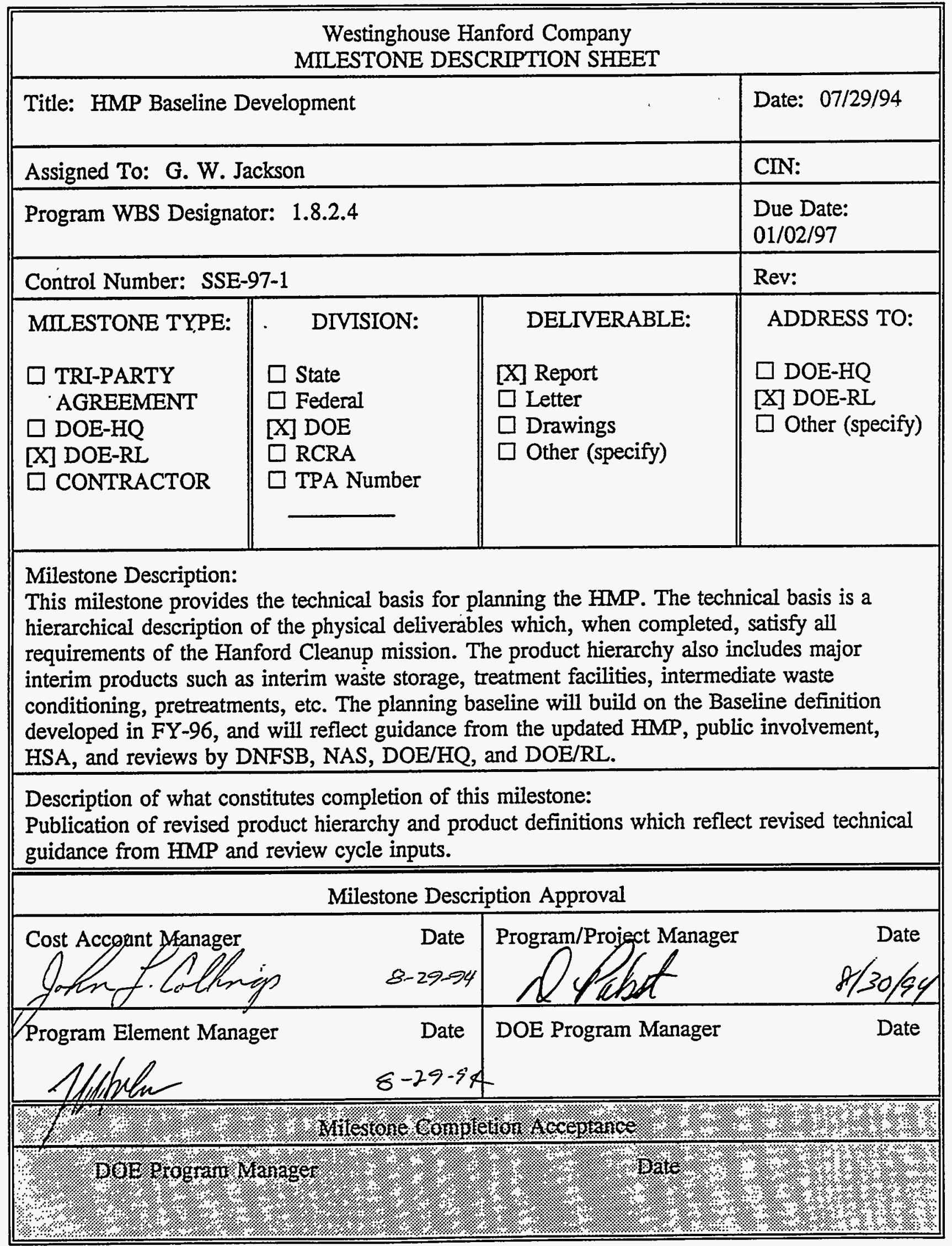




\begin{tabular}{|l|l||}
\hline \multicolumn{3}{|c||}{ Westinghouse Hanford Company } \\
MILESTONE DESCRIPTION SHEET
\end{tabular}




\begin{tabular}{|c|c|c|c|}
\hline \multicolumn{4}{|c|}{$\begin{array}{l}\text { Westinghouse Hanford Company } \\
\text { MILESTONE DESCRIPTION SHEET }\end{array}$} \\
\hline \multicolumn{3}{|c|}{ Title: Requirements Analysis and Allocation (Rev. 2) } & Date: $07 / 29 / 94$ \\
\hline \multicolumn{3}{|c|}{ Assigned To: G. W. Jackson } & CIN: \\
\hline \multicolumn{3}{|c|}{ Program WBS Designator: 1 8.2.4 } & $\begin{array}{l}\text { Due Date: } \\
\text { 05/23/97 }\end{array}$ \\
\hline \multicolumn{3}{|c|}{ Control Number: SSE-97-3 } & Rev: \\
\hline $\begin{array}{l}\text { MMLESTONE TYPE: } \\
\square \text { TRI-PARTY } \\
\text { AGREEMENT } \\
\square \text { DOE-HQ } \\
{[X] \text { DOE-RI }} \\
\square \text { CONTRACTOR }\end{array}$ & $\begin{array}{l}\quad \text { DIVISION: } \\
\square \text { State } \\
\square \text { Federal } \\
{[\mathrm{X}] \text { DOE }} \\
\square \text { RCRA } \\
\square \text { TPA Number }\end{array}$ & $\begin{array}{l}\text { DELIVERABLE: } \\
{[X] \text { Report }} \\
\square \text { Letter } \\
\square \text { Drawings } \\
\square \text { Other (specify) }\end{array}$ & $\begin{array}{l}\text { ADDRESS TO: } \\
\square \text { DOE-HQ } \\
{[X] \text { DOE-RL }} \\
\square \text { Other (specify) }\end{array}$ \\
\hline \multicolumn{4}{|c|}{$\begin{array}{l}\text { Milestone Description: } \\
\text { This milestone will revise the external requirements definition and allocation as specified in the } \\
\text { SEMP. Requirements to be allocated will be derived from the architecture developed in the } \\
\text { FY-96 work, loaded into the RDD-100 database, and assigned to the appropriate site level } \\
\text { functions. }\end{array}$} \\
\hline \multicolumn{4}{|c|}{$\begin{array}{l}\text { Description of what constitutes completion of this milestone: } \\
\text { Publication of the site level requirements definition and allocation as a revised Appendix to } \\
\text { WHC-EP-0722. }\end{array}$} \\
\hline \multicolumn{4}{|c|}{ Milestone Description Approval } \\
\hline \multicolumn{2}{|c|}{ Cost Account Manager } & Program/ProjectManager & $8 / 30 / 4$ \\
\hline \multicolumn{2}{|c|}{$\begin{array}{lr}\text { Program Element Manager } & \text { Date } \\
\text { Mffhlm } & 8^{-29-9} 4\end{array}$} & DOE Program Manager & Date \\
\hline \multicolumn{4}{|c|}{ 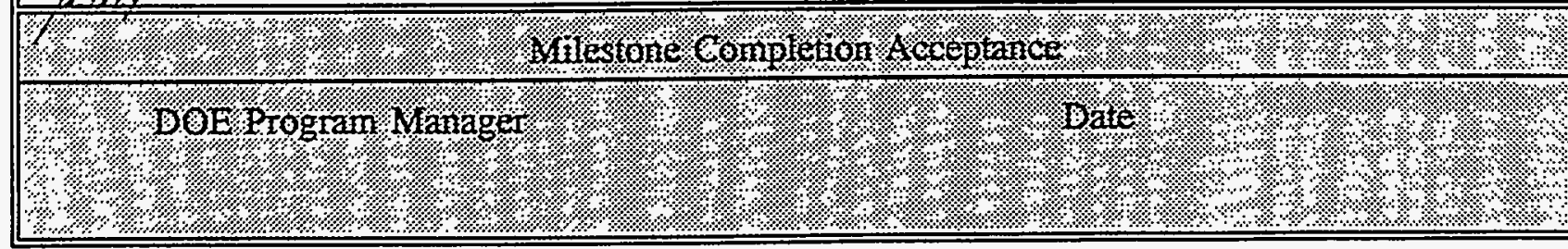 } \\
\hline
\end{tabular}




\begin{tabular}{|l|l||}
\hline \multicolumn{3}{|c||}{ Westinghouse Hanford Company } \\
MILESTONE DESCRIPTION SHEET
\end{tabular}




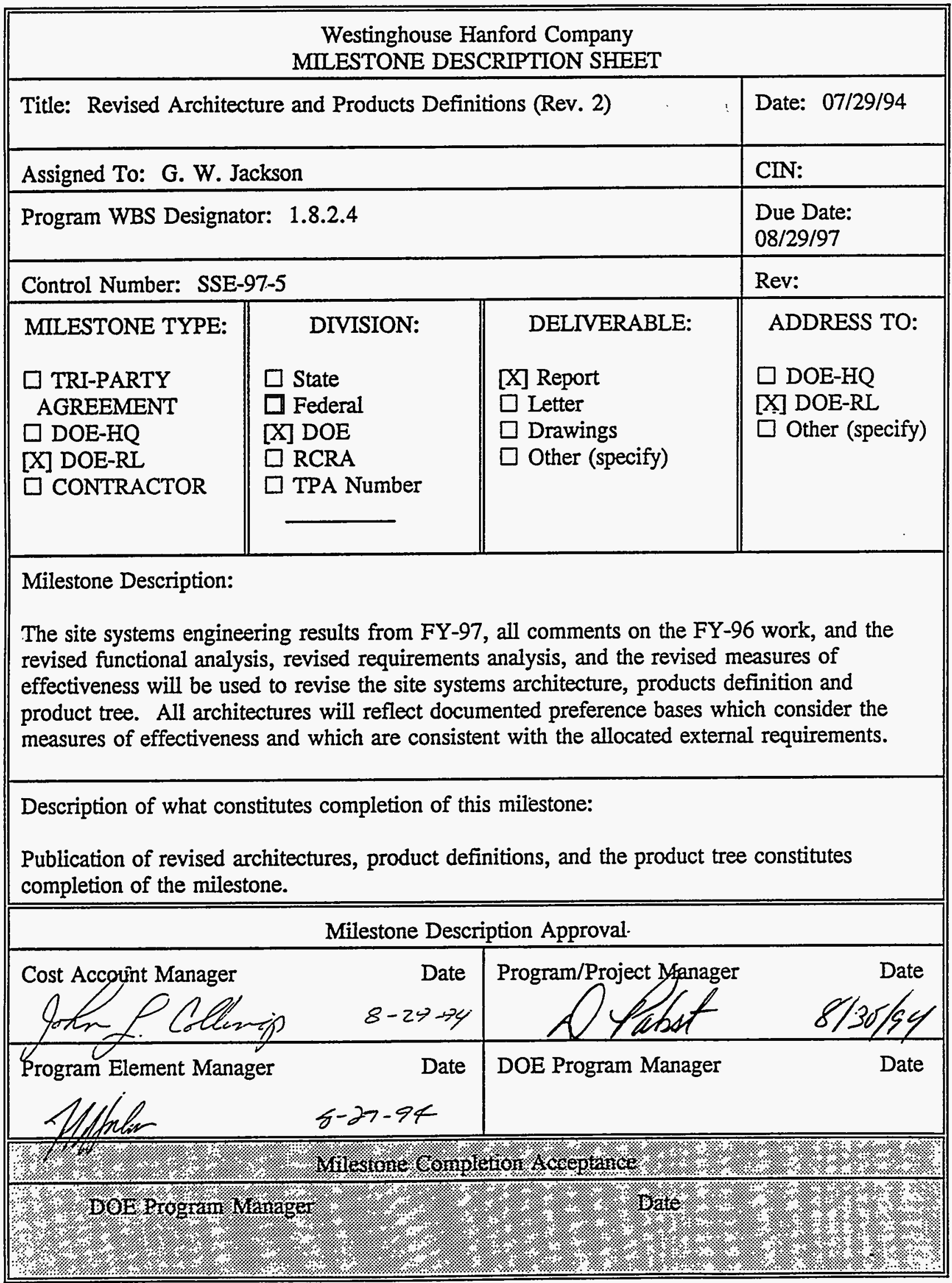




\begin{tabular}{|l|l||}
\hline \multicolumn{3}{|c||}{ Westinghouse Hanford Company } \\
MILESTONE DESCRIPTION SHEET
\end{tabular}




\begin{tabular}{|l|l||}
\hline \multicolumn{3}{|c|}{ Westinghouse Hanford Company } \\
MILESTONE DESCRIPTION SHEET
\end{tabular}




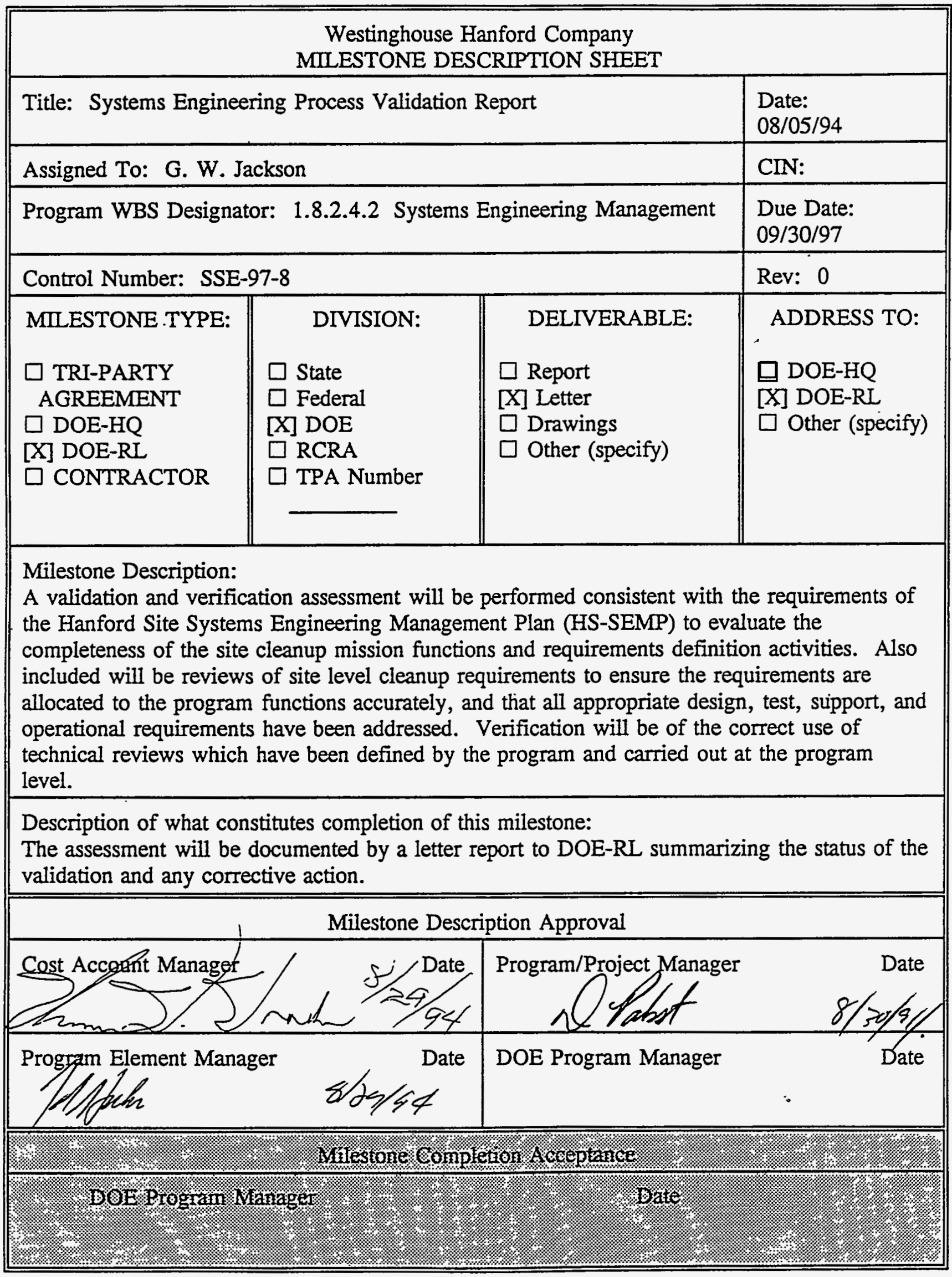




\begin{tabular}{|l|l|l||}
\hline \multicolumn{3}{|c|}{ Westinghouse Hanford Company } \\
MILESTONE DESCRIPTION SHEET
\end{tabular}




\begin{tabular}{|l|l||}
\hline \multicolumn{3}{|c|}{ Westinghouse Hanford Company } \\
MILESTONE DESCRIPTION SHEET
\end{tabular}




\begin{tabular}{|c|c|c|c|}
\hline \multicolumn{4}{|c|}{$\begin{array}{l}\text { Westinghouse Hanford Company } \\
\text { MILESTONE DESCRIPTION SHEET }\end{array}$} \\
\hline \multicolumn{3}{|c|}{$\begin{array}{l}\text { Title: Complete Integration of Systems Engineering Data Management } \\
\text { System }\end{array}$} & $\begin{array}{l}\text { Date: } \\
\text { 08/08/94 }\end{array}$ \\
\hline \multicolumn{3}{|c|}{ Assigned To: G. W. Jackson } & CIN: \\
\hline \multicolumn{3}{|c|}{ Program WBS Designator: 1 8.2.4.2 Systems Engineering Management } & $\begin{array}{l}\text { Due Date: } \\
09 / 30 / 97\end{array}$ \\
\hline \multicolumn{3}{|c|}{ Control Number: SSE-97-11 } & Rev: 0 \\
\hline $\begin{array}{l}\text { MULESTONE TYPE: } \\
\square \text { TRI-PARTY } \\
\text { AGREEMENT } \\
\square \text { DOE-HQ } \\
{[X] \text { DOE-RL }} \\
\square \text { CONTRACTOR }\end{array}$ & $\begin{array}{l}\text { DIVISION: } \\
\square \text { State } \\
\square \text { Federal } \\
{[\text { X] DOE }} \\
\square \text { RCRA } \\
\square \text { TPA Number }\end{array}$ & $\begin{array}{l}\text { DELIVERABLE: } \\
\square \text { Report } \\
{[X] \text { Letter }} \\
\square \text { Drawings } \\
\square \text { Other (specify) }\end{array}$ & $\begin{array}{l}\text { ADDRESS TO: } \\
\square \text { DOE-HQ } \\
{[X] \text { DOE-RL }} \\
\square \text { Other (specify) }\end{array}$ \\
\hline \multicolumn{4}{|c|}{$\begin{array}{l}\text { Milestone Description: Integration of the Hanford systems engineering information } \\
\text { management system will be completed for all appropriate site programs and projects. A final } \\
\text { report will be issued defining and assessing the application of the information and data system } \\
\text { to fulfill corresponding information products required by the Hanford Site Systems Engineering } \\
\text { Management Plan (HS-SEMP) and the RLID } 4900.1 \text {. Information and data requirements for } \\
\text { Hanford site programs and projects with respect to all sitewide SE products will be monitored } \\
\text { including those programs that are not part of the integration effort. This monitoring will } \\
\text { include assessment on a site perspective of the systems engineering information process and } \\
\text { include recommendations for any future revisions needed to correct deficiencies or to improve } \\
\text { overall site systems engineering information handling (e.g. consolidation of duplication between } \\
\text { programs, elimination of counter-productive data handling methods, etc.) }\end{array}$} \\
\hline \multicolumn{4}{|c|}{$\begin{array}{l}\text { Description of what constitutes completion of this milestone: A letter report describing the } \\
\text { final integration of the appropriate site programs and projects into the SE data base } \\
\text { management system will be provided to DOE-RL, and will include conclusions and } \\
\text { recommendations for data system improvements. }\end{array}$} \\
\hline \multicolumn{4}{|c|}{ Milestone Description Approval } \\
\hline \multicolumn{2}{|c|}{ Cost Acceunt Manager / Date } & Program/Project $z$ Manager & $\begin{array}{l}\text { Date } \\
8 / 3 / 94\end{array}$ \\
\hline \multicolumn{2}{|c|}{$\begin{array}{l}\text { Program Element Manager } \\
\text { Allfhel }\end{array}$} & DOE Program Manager & Date \\
\hline \multicolumn{4}{|c|}{ 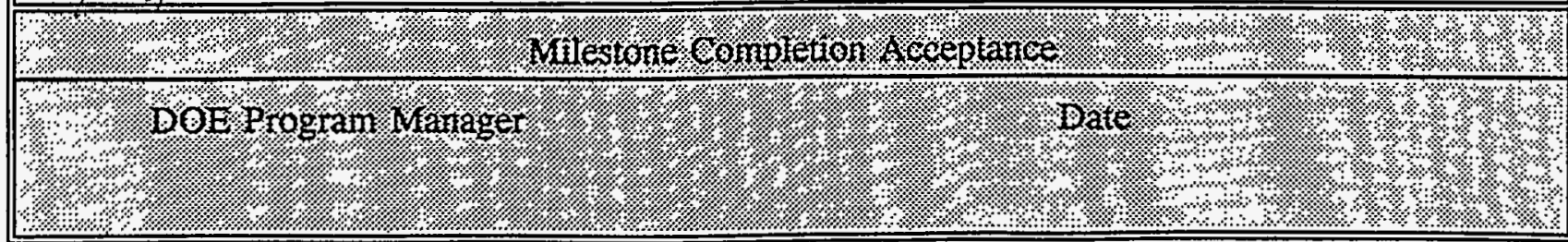 } \\
\hline
\end{tabular}


WHC-SP-1096

$09 / 23 / 94$

Planning Integration

Multi-Year Program Plan

This page intentionally left blank. 
The Planning Integration Program cost baseline was derived from the output of the time-phased, resource-loaded, logic-driven schedule network database for the first three years of the program. The following four years of the program were defined through a process which combined reviews of the FY 1996 Activity Data Sheets for ADS 7250 and 3410, and escalation of previous-year data.

The nature of the majority of the Planning Integration Program is repetitive, and will continue for the duration of activity at Hanford. The planning of the workscope that is not repetitive (e.g., Integrated Risk Assessment) has not been planned in detail beyond a seven-year window. Therefore, the Program Cost Baseline reflects the costs required to support program activities for a seven-year period.

Section 2.H.1 of this MYPP provides the costs for each program element in terms of direct and G\&A dollars over the period of FY 1995 through FY 2001. 
WHC-SP-1096

$09 / 23 / 94$

Planning Integration

Multi-Year Program Plan

This page intentionally left blank.

$2 \mathrm{H}-1 \mathrm{~A}$ 
Table 2.H-1 Cost Baseline Summary (\$ in Thousands)

Direct Funded

Program

Element/ADS

Fund Type FY 95 SMS FY 96 FY 97

FY 98

FY 99 FY $00 \quad$ FY 01 * Guidance Plan Plan Plan Plan Plan Plan

1.8 .2 .1

Work Identification

$\begin{array}{lllllll}2039 & 2100 & 2163 & 3500 & 2295 & 2364 & 2435\end{array}$

1.8.2.2

Baseline Reporting

1.8.2.3

Baseline Integration

1.8 .2 .4

$\begin{array}{lllllll}1137 & 1230 & 1214 & 1250 & 1288 & 1326 & 1366\end{array}$

Systems Engineering

1.8.2.5

Program Mgt

1.8 .2 .6

IRAP

1.8 .2 .7

Public Involvement

1.8.2.8

$\begin{array}{lllllll}1584 & 1666 & 1690 & 1741 & 1793 & 1847 & 1902\end{array}$

$\begin{array}{lllllll}1321 & 1162 & 1023 & 927 & 955 & 984 & 1013\end{array}$

$\begin{array}{lllllll}324 & 334 & 344 & 354 & 365 & 376 & 387\end{array}$

$\begin{array}{lllllll}2379 & 2134 & 4346 & 6194 & 2839 & 2924 & 3012\end{array}$

$\begin{array}{lllllll}0 & 0 & 0 & 0 & 0 & 0 & 0\end{array}$

ADS Prep/Coordination 1.8 .2

$\begin{array}{lllllll}1166 & 1375 & 1329 & 1369 & 1410 & 1452 & 1496\end{array}$

Planning Integration

$\begin{array}{lllllll}9950 & 10001 & 12109 & 15336 & 10945 & 11273 & 11611\end{array}$

G\&A Funded

Program

Element/ADS

Fund Type FY 95 SMS FY 96

$$
\text { * Guidance PIan }
$$

FY 97

Plan

FY 98

Plan

195

201

207

213

$3089-3000$

$3699 \quad 3881$

30893000

Baseline Reporting

1.8.2.3

Baseline Integration

1.8 .2 .4

Systems Engineering

1.8.2.5

Program Mgt

1.8 .2 .6

IRAP

1.8.2.7

Public Involvement

1.8.2.8

ADS Prep/Coordination

1.8 .2

Planning Integration

$\begin{array}{lllllll}0 & 0 & 0 & 0 & 0 & 0 & 0\end{array}$

$\begin{array}{lllllll}1744 & 1722 & 1774 & 1827 & 1882 & 1938 & 1996\end{array}$

$\begin{array}{lllllll}0 & 0 & 0 & 0 & 0 & 0 & 0\end{array}$

$\begin{array}{lllllll}0 & 0 & 0 & 0 & 0 & 0 & 0\end{array}$

$\begin{array}{lllllll}45 & 46 & 48 & 49 & 51 & 52 & 54\end{array}$

$\begin{array}{lllllll}0 & 0 & 0 & 0 & 0 & 0 & 0\end{array}$

$\begin{array}{lllllll}5683 & 5850 & 5118 & 5089 & 5242 & 5399 & 5561\end{array}$

* The 1.8.2 Planning Integration Program only has OP funds. 
Planning Integration Multi-Year Program Plan

This page intentionally left blank. 


\section{I BASES OF ESTIMATES}

Estimates were developed by the program elements. Each program element has developed its estimates and undergone the rigors of internal validations to assure adequacy of cost justification. A summary of the bases of estimates is provided in the following paragraphs.

\section{I.1 Work Identification Basis of Estimate}

A combination of the specific analogy and cost review and update techniques "were used in developing this estimate. The process was begun by developing the logic of work scope activities. This was carried to the lowest level possible followed by estimating duration timeframes. The technical team members then developed their estimate of this year's requirements based on Tast year's work (FY 1995 Hanford Strategic Plan support, Draft Fiscal Year 1995 HMP Volume 1, Hanford Strategic Analysis, MYPP support, etc.) and the relative complexity of each work scope item. This produced a somewhat uneven staffing levels which were iterated against resources and milestones or other commitments.

\section{I.2 BaseTine Reporting and System Development Basis of Estimate}

A combination of historical analogy, which includes cost reviews and projections, and bottoms up estimating techniques, utilizing estimating worksheets, was used in developing these cost estimates.

Specifically, each work scope area was broken down into its individual activities, or even lower, as in the case of the site standard scheduling system, due to its complexity. A network logic has been developed.

The three key performance reports within Performance Reporting are of a cyclical nature, required by DOE-HQ or RL for the past two years. Past experience on these cyclical products factored heavily into the basis of the estimates.

\section{I.3 Program Baseline Integration Basis of Estimate}

The basis of the cost estimates for the WBS 1.8.2.3 "Program Baseline Integration" was developed using the guidelines in the DOE Office of Waste Management Cost and ScheduTe Estimating Guide (EM-30), and provides a cost baseline which can be validated under guidelines presented in RLD 5700.9, " 
Planning Integration

Multi-Year Program Plan

Cost Validation." The EM-30 guide applies the techniques of Activity-Based Costing $(A B C)$ to estimating waste operations activities and provides an activity-based framework to improve the rigor, consistency, and credibility of waste management estimates. $A B C$ also provides a consistent framework for measuring and reporting program performance:

$A B C$ estimating is a method for preparing cost estimates which breaks work into discrete, quantifiable activities. The cost of each activity is then estimated in terms of requirements for 1 abor and materials on a per unit basis.

The cost estimate, maintained in department files, was developed to:

- Provide an activity-based cost estimate for all department work, under a defined WBS structure, based on resource-loaded schedules, historical actual cost records, and activity-based work scope analyses,

- Provide a valid documentation basis, either by reference or inclusion, to support the cost estimate and schedule,

- Provide definition to the methodology used in the formulation of the estimate and schedule logic,

- Develop costs allocable to department production milestones and deliverables, and

- Shift cost estimates from organizational to activity based estimating, thus improving estimate quality and providing budget independence from organizational considerations.

\section{I.4 Technical Baseline Development Basis of Estimate}

Estimates for program element 1.8.2.4 are based on the experience from FY 1994 systems engineering activities and the technical judgement of the systems engineering managers. Standards for estimating the resources needed to conduct systems engineering work on unprecedented applications do not exist.

The approach taken was to break up the work into tasks of sufficiently smal1 increment that the effort could be roughly compared to previous systems engineering work. These packages were compared to their counterparts from FY 1994 and a judgement factor applied to arrive at an estimate for FY 1995. Fiscal years 1996 and 1997 are iterations of the FY 1995 work and are resource loaded at a level similar to FY 1995. 
Planning Integration Multi-Year Program Plan

\section{I.5 Planning Integration Program Management Basis of Estimate}

The work scope for this program element was decomposed to the lowest plannable level or tasks. These tasks were placed into a logical sequence and then scheduled. Once scheduled, resource estimates were defined based upon experience with similar or same tasks in FY 1994. These resource estimates were then applied to the schedule network, and the network and resources were adjusted to form a workable profile that met milestone and interface commitments.

\section{I.6 Integrated Risk Assessment Basis of Estimate}

A combination of the specific analogy and buildup techniques were used in developing IRAP's scope, cost, and schedule estimates. FY 1995 is considered the base case, and other years are considered repetitive to a large degree.

Microsoft Project (in conjunction with internal software) is the tool that PNL uses to track planned versus actual work using resource-loaded schedules. To develop information to populate this tool, activities were defined, then Scheduling Activity Planning forms and Scheduling Activity Estimating Work Sheet forms were completed to define resource requirements and schedule information. Conflicting or inconsistent results were iteratively refined.

Activities were sequenced to produce required products. This sequence is reflected in the IRAP network schedule (logic). Products were grouped to define subtasks. Subtasks were aggregated into logical groups to define the Work Breakdown Structure. In addition, interfaces with other 1.8.2 program elements and with other organizations were also incorporated into the planning and scheduling.

Resource requirements were developed based upon work scope and personnel we intend to assign to specific tasks.

\section{I.7 Public Involvement Basis of Estimate}

The basis of the cost estimates for the WBS 1.8.2.7, "Public Involvement," was developed using prior experience in planning for the Hanford Site Future Uses Working Group, the Tank Waste Task Force, Tank Waste Remediation System/Tri-Party Agreement renegotiations, and other Hanford Site public involvement actions. Manhour needs were extrapolated from 


\section{Planning Integration Multi-Year Program Plan}

experience with similar programs. Manhour costs were estimated based on four action plans to be developed, approved, and implemented throughout the year.

This activity is fully funded in G\&A under SSPP 6.10.6.2.

2.I.8 Activity Data Sheet Preparation and Coordination Basis of Estimate

The basis of the cost estimates for the WBS 1.8.2.8 are identical to those employed to develop the cost estimates for WBS 1.8.2.3, Program Baseline Integration. 
WHC-SP-1096

$09 / 23 / 94$

Planning Integration Multi-Year Program Plan

\section{J Waste Type Data}

The Planning Integration Program neither generates, stores, nor disposes of any waste in the execution of its program. 
WHC-SP-1096

$09 / 23 / 94$

Planning Integration

Multi=Year Program Plan

This page intentionally left blank. 


\section{FY 1994 MYPP \\ PLANNING INTEGRATION PROGRAM \\ 1.8 .2}

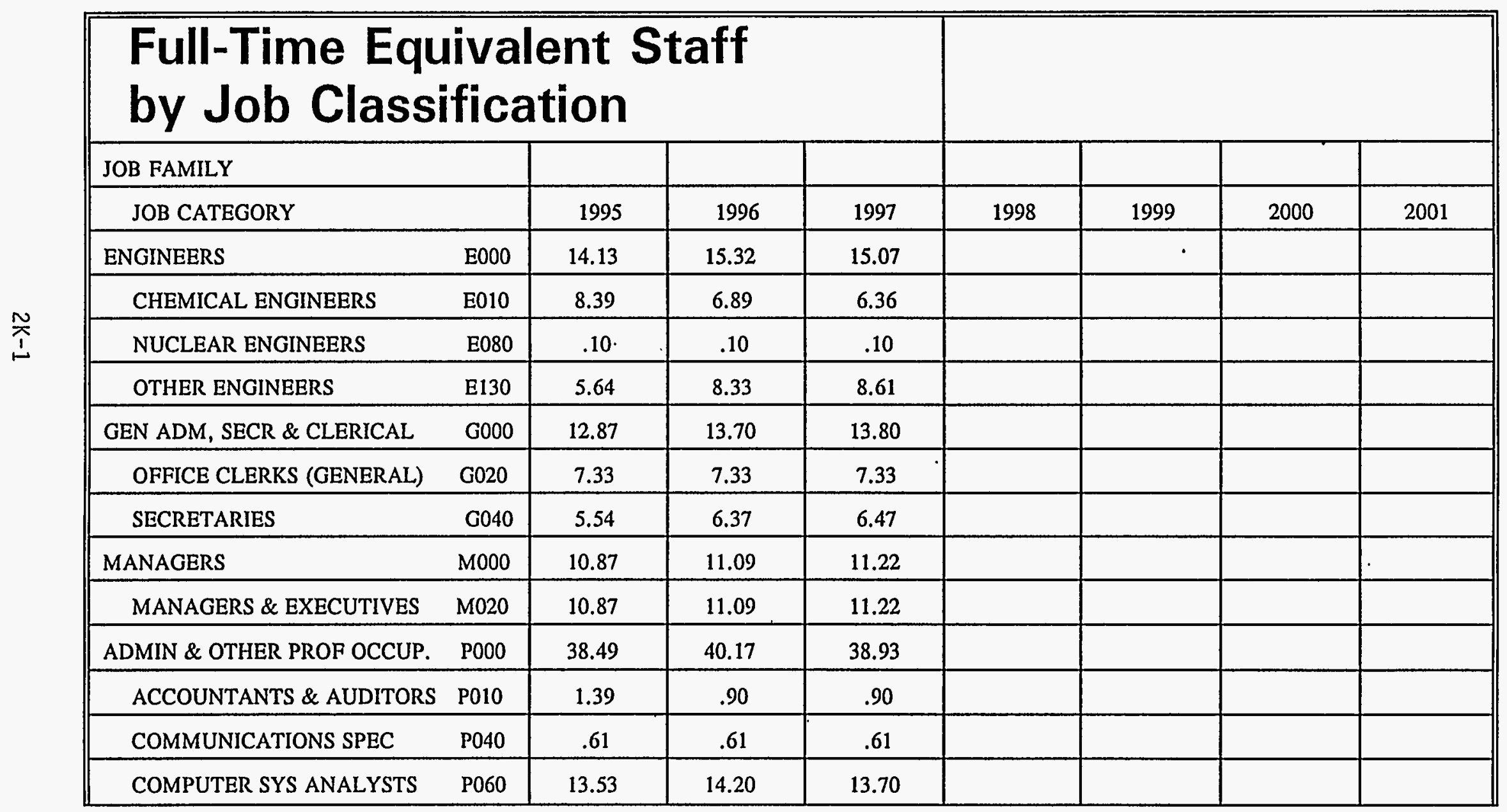




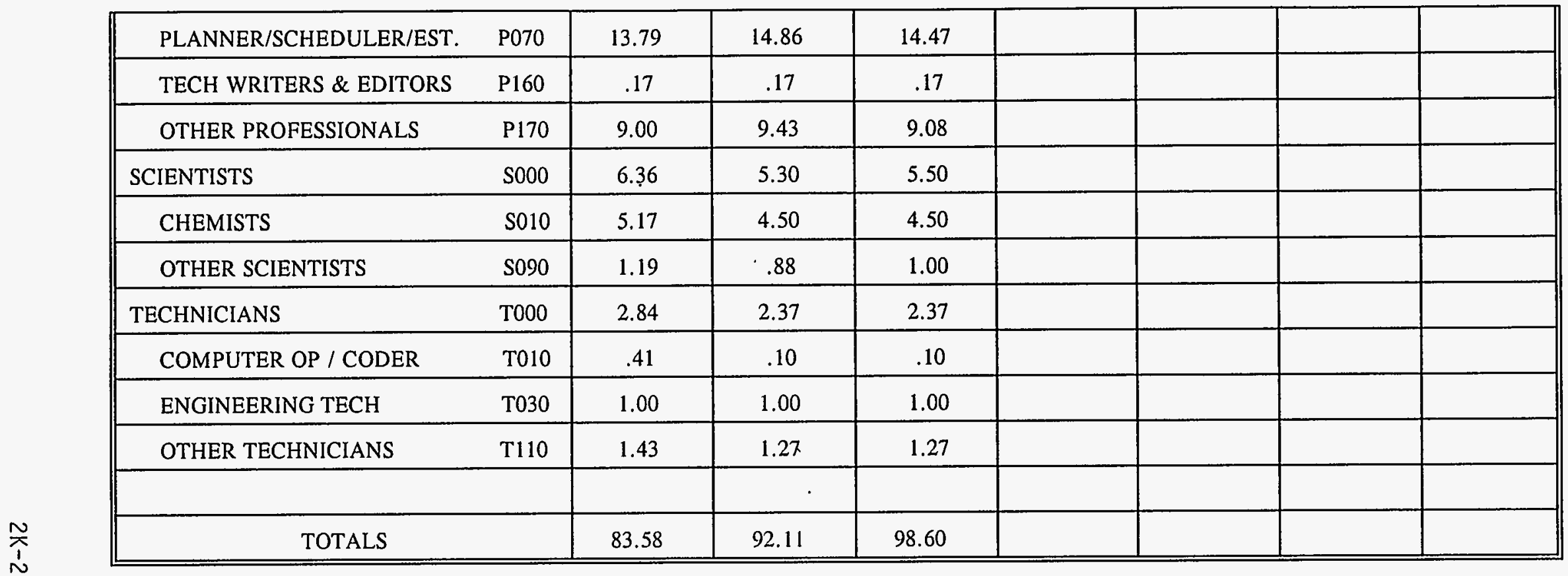


Labor Staffing Levẹls, PNL

\begin{tabular}{|c|c|c|c|c|c|}
\hline eost: & Qost Elenent Deseription? & Hours: & $\frac{189}{995}$ & 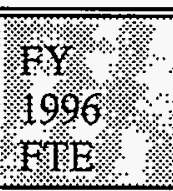 & 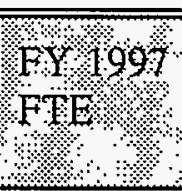 \\
\hline 0MM030 & Program Managers & 1308 & 0.73 & 0.65 & 1.56 \\
\hline OEE010 & Chemical Engineers & 785 & 0.44 & 0.39 & 0.94 \\
\hline 00E050 & Environmental Engineers & 1628 & 0.90 & 0.81 & 1.94 \\
\hline OEE080 & Nuclear Engineers & 5444 & 3.02 & 2.70 & 6.50 \\
\hline OEE110 & Quality Control Engineers & 908 & 0.50 & 0.45 & 1.08 \\
\hline OESO20 & Environmental Scientists & 1834 & 1.02 & 0.91 & 2.19 \\
\hline OES040 & Life Scientists & 2540 & 1.41 & 1.26 & 3.03 \\
\hline 0ES066 & $\begin{array}{l}\text { Mathematicians/ } \\
\text { Statisticians }\end{array}$ & 1290 & 0.72 & $0.64^{-}$ & 1.54 \\
\hline OEP040 & Communication Specialists & 320 & 0.18 & 0.16 & 0.38 \\
\hline 0EP060 & Computer System Analysts & 3100 & 1.72 & 1.54 & 3.70 \\
\hline 0ЕP080 & Health Physicists & 2100 & 1.17 & 1.04 & 2.51 \\
\hline 0EP160 & Technical Writers/Editors & 608 & 0.34 & 0.30 & 0.73 \\
\hline OEG010 & Administrative Assistants & 1288 & 0.72 & 0.64 & 1.54 \\
\hline 0NG030 & Office Clerks (special) & 1308 & 0.73 & 0.65 & 1.56 \\
\hline \multirow[t]{2}{*}{$\begin{array}{l}\text { OEG040/ } \\
\text { ONGO40 }\end{array}$} & Secretaries & 1876 & 1.04 & 0.93 & 2.24 \\
\hline & Total & & 14.64 & 13.06 & 31.48 \\
\hline
\end{tabular}

FTE $=1800$ Hours

Table 2.K-2. Program Staffing Levels, PNL 
WHC-SP-1096

$09 / 23 / 94$

Planning Integration

Multi-Year Program Plan

This page intentionally left blank.

$2 K-4$ 
Planning Integration Multi-Year Program Plan

2L-1 Building BTocks

Building Blocks were removed by direction of the U.S. Department of Energy, Richland Operations office; to be added at a later date. 
Planning Integration Multi-Year Program Plan

This page intentionaliy left blank. 


\subsection{FISCAL YEAR WORK PLAN}

This section of the MYPP contains the information for the execution year of the Planning Integration Program. It provides the details required to measure performance against the baseline plan. The cost, funding, and carryover data contained in the following sections is time phased, by month, based on scheduled work scope. These costs are documented in the Cost Account Authorizations with associated work scope and milestones identified to facilitate performance analysis. 
WHC-SP-1096

$09 / 23 / 94$

Planning Integration Multi-Year Program Plan

This page intentionally left blank. 


\section{Table 3.A-1 Cost Baseline Summary/Program Element - Operating ( $\$$ in Thousands)}

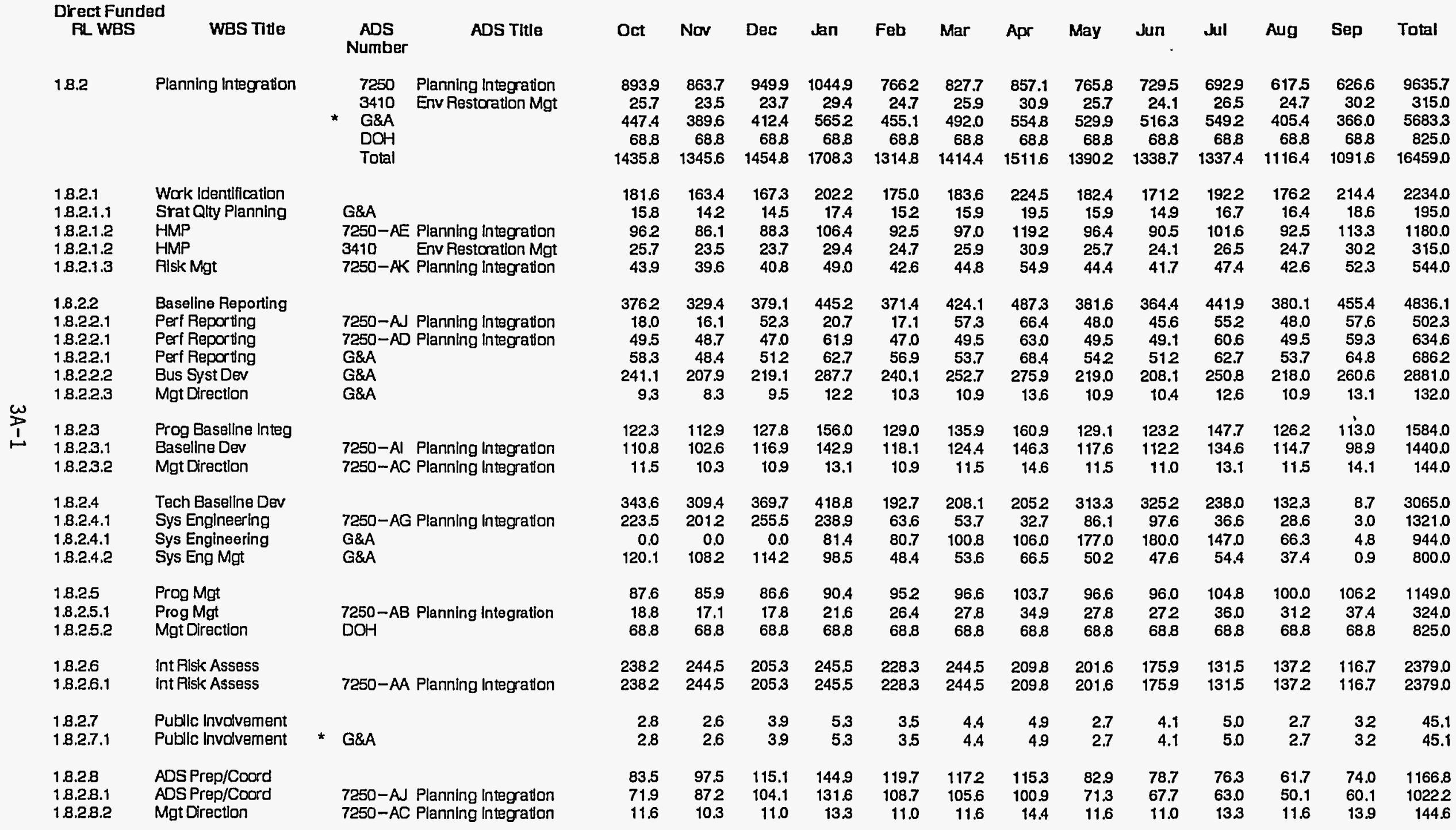

- Funded by SSPP 6.10.6.2 
WHC-SP-1096

$09 / 23 / 94$

Planning Integration Multi-Yeạr Program P1an

This page intentionally left blank. 
Planning Integration

Multi-Year Program Plan

\section{B}

PROGRAM FUNDING

ADS 3410

Within ADS 3410, The P1anning Integration Program has planned its activities to a Budgeted Cost of Work Scheduled (BCWS). For the portion of the program funded from ADS 3410, funds are required to support the BCWS.

\section{BCWS $=\$ 315 \mathrm{~K}$ Funds $=\$ 315 \mathrm{~K}$}

ADS 7250

Within ADS 7250, the Planning Integration Program has planned its activities to a Budgeted Cost of Work Scheduled (BCWS). The program is also participating in the productivity commitment described in the Cost. and Management Efficiency Initiative (State of Washington Department of . Ecology, et al, January 1994). To achieve the productivity commitment, funds are being requested that acknowledge the contractors commitments to finding and achieving these productivity gains. The following describes this approach.

\section{BCWS $=\$ 9,636 \mathrm{~K}$ \\ PC* $=(749 K)$ \\ Funds $\quad=\$ 8,887 \mathrm{~K}$}

*Productivity Commitment 
WHC-SP-1096

09/23/94

Planning Integration

Multi-Year Program Plan

This page intentionally left blank.

$3 B-2$ 
Planning Integration Multi-Year Program Plan

3.C CARRY OVER

The Planning Integration Program has identified no carryover scope from FY 1994 to FY 1995. 
Planning Integration Multi-Year Program Plan

This page intentionally left blank. 
Planning Integration Multi-Year Program Plan

3.D COST ACCOUNT AUTHORIZATIONS

$3 D-1$ 


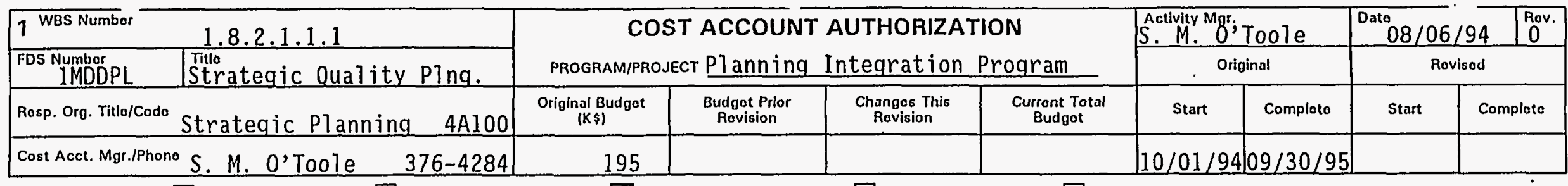

Funding Source: $\square$ O Operating Expense $\square$ CENRTC FY $\square$ Lino Item FY $\square$ GPP FY $\quad \square$ Small Projocts FY

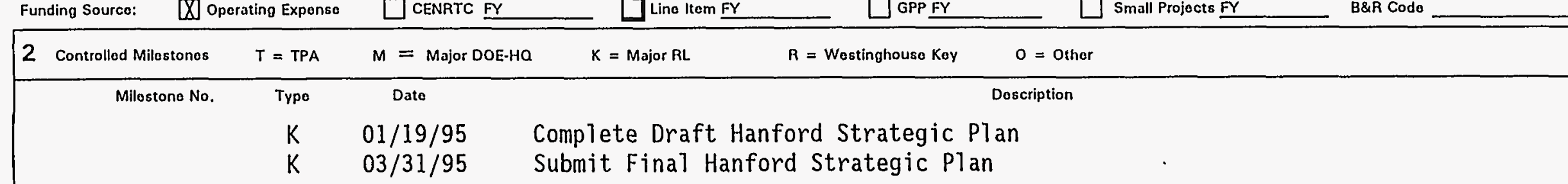

3 Doscription/Instructions (scopo, objoctives, approach, constraints, further milostono dofinition) (capital justification)

WHC supports the RL Hanford Quality Council in the preparation of the

Hanford Strategic Plan and supplement. The Hanford Strategic Plan is

revisited annually and revised at the direction of the Hanford Quality

Council. This activity compiles the input from the Hanford Quality Council

and its teams into a succinct document. After the plan is approved by RL it

is distributed by this activity. This effort assists planning and

facilitation of on-going Hanford Quality Council and Strategy/Goal Team

meetings.

\begin{tabular}{|c|c|r|}
\hline ADS Number & \multicolumn{2}{|c|}{7250 - G\&A } \\
\hline Total CAP \$000 & G \& A/CSP \$000 & Total CAA \$000 \\
\hline & & \\
& 1 & 195 \\
\hline
\end{tabular}

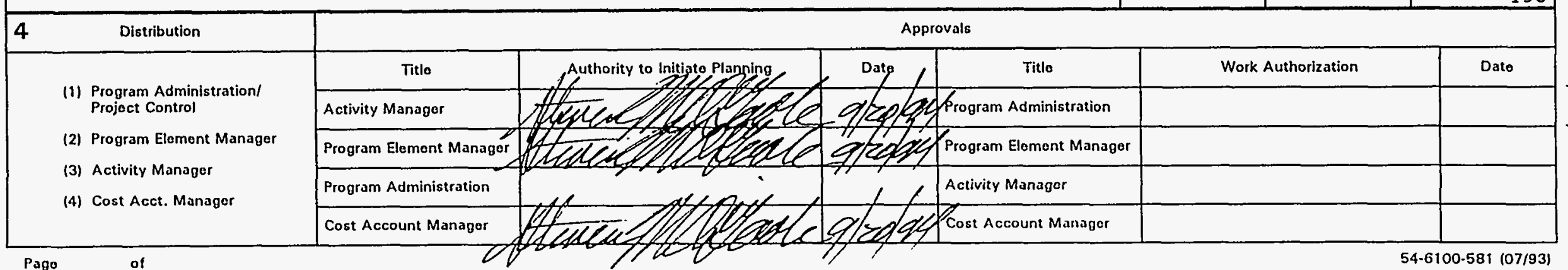




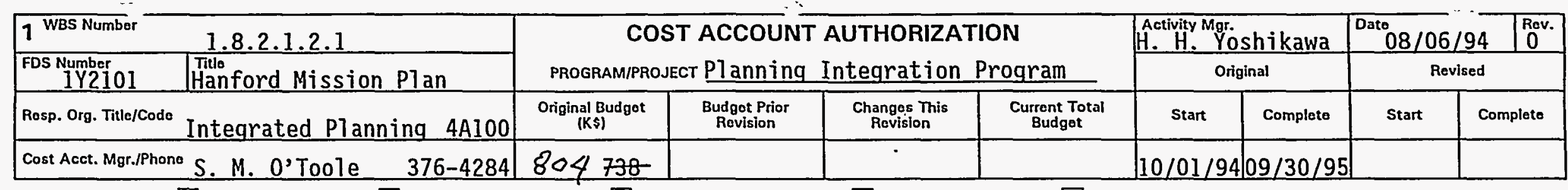

\begin{tabular}{|c|c|c|c|c|c|c|c|}
\hline \multicolumn{2}{|c|}{ Funding Source: } & {$[X]$ Operating Expense } & \multirow{2}{*}{$\frac{\square \text { CENRTC } F Y}{M=\text { Major DOE-HQ }}$} & \multirow{2}{*}{$\mathrm{K}=$ Major RL } & \multirow{2}{*}{$=\square$ GPP FY } & \multirow{2}{*}{$=\square$ Small Projects FY } & \multirow[t]{2}{*}{ B\&R Codo } \\
\hline 2 & Controlled Milostonos & $T=T P A$ & & & & & \\
\hline
\end{tabular}

Milostono No. Type Dato Description

K 11/29/94 Proposal for High level WBS to RL Planning Integration Division

$\mathrm{K} \quad 03 / 28 / 95 \quad$ Proposal for High Level Priority List to RL Planning Integration Division

K 04/17/95 Transmit Draft FY 1996 HMP Volume 1, Site Guidance to RL for Review

K 07/05/95 Complete FY 1996 HMP Volume 1, Site Guidance

R 09/30/95 Complete Hanford Strategic Analysis Addendum

3 Doscription/Instructions (scopo, objectives, approach, constraints, further millostono dofinition) (capital justification)

Develop an input to Site level technical baseline development. Develop and document Site level guidance and analysis to define Site mission, programs, strategy assumptions and issues. The guidance will include extracts from the Hanford Strategic Plan (Hanford Mission) as well as objectives, Fiscal Year 1996 Planning Basis, stakeholder values, priority list/criteria, WBS/OBS based on systems engineered PBS, interfaces and Site summary schedule based on the Site integrated schedule. The Hanford Strategic Analysis addendum is an evaluation of alternatives and states for the site examining Material Blances, ROM cost and schedule data in support of systems Engineering. The Site Guidance directs the MYPPs.

(Continued on Page 2)

\begin{tabular}{|c|r|r|}
\hline ADS Number & \multicolumn{2}{|c|}{7250} \\
\hline Total CAP $\$ 000$ & G \& AICSP \$000 & Total CAA $\$ 000$ \\
\hline 859 & 145 & 804 \\
605 & 133 & 738 \\
\hline
\end{tabular}

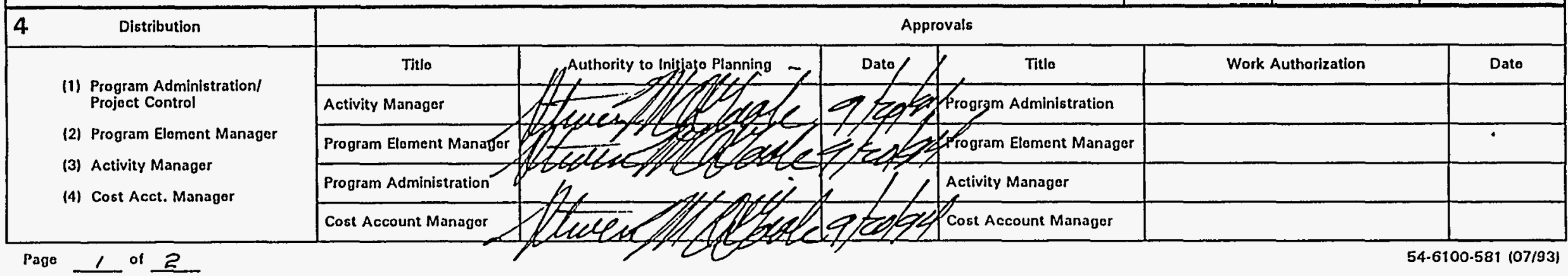




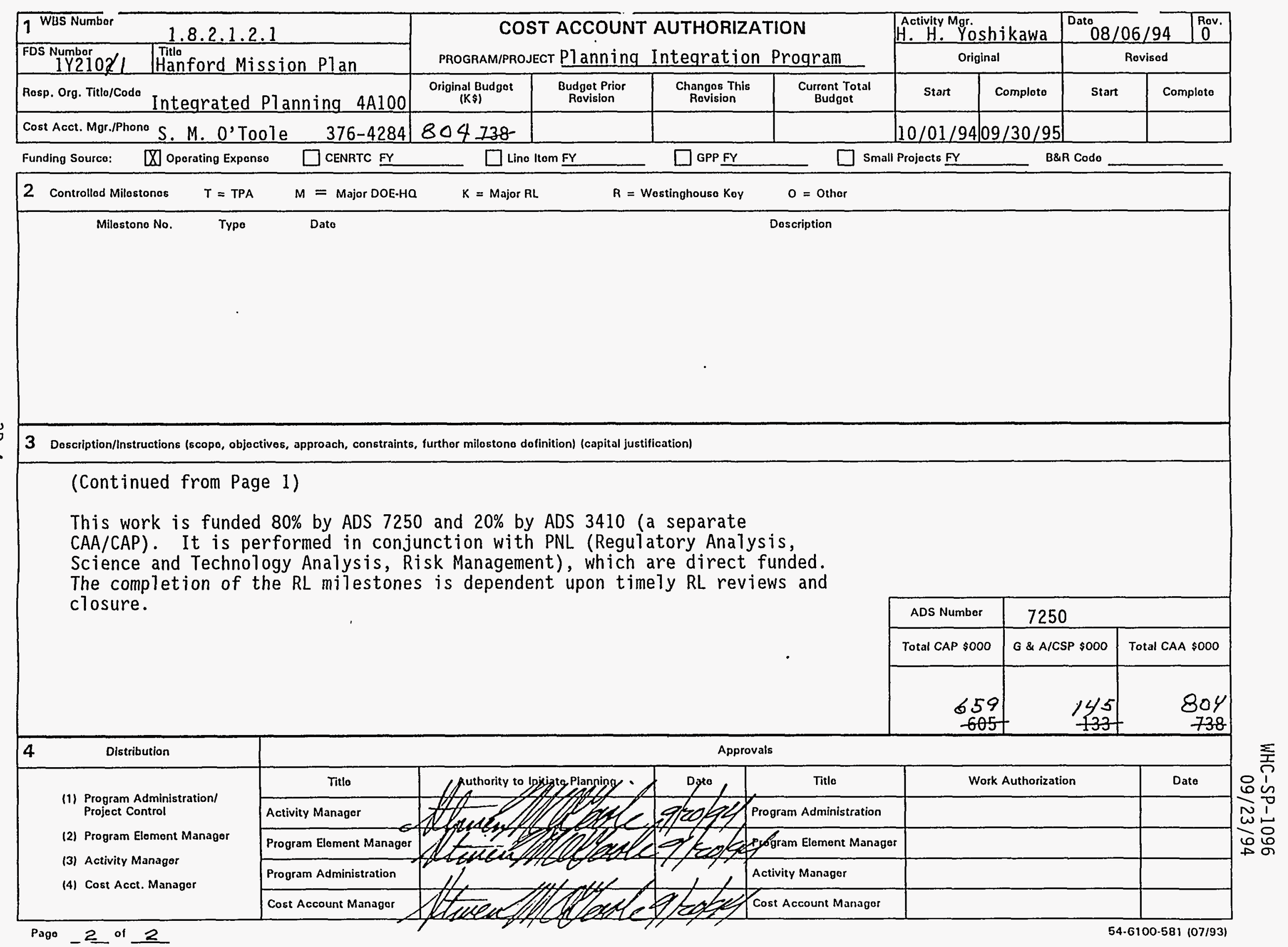




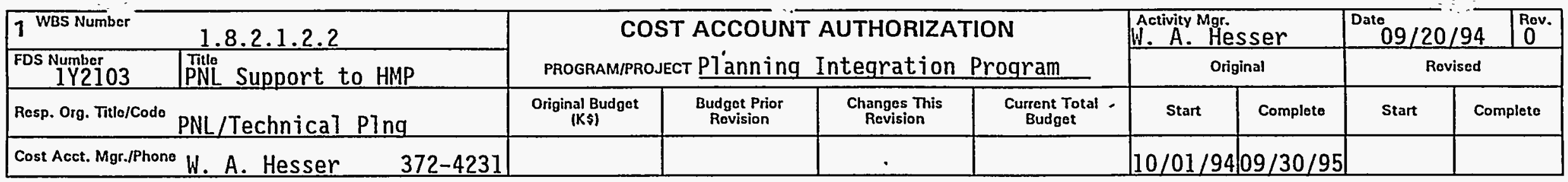

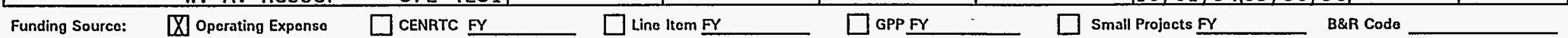

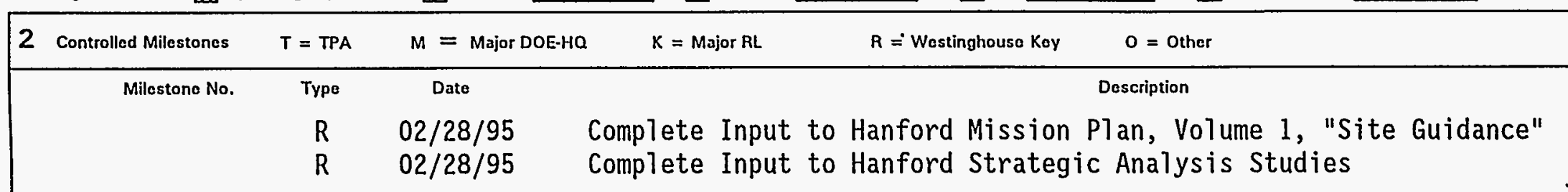

3 Doscription/Instructions (scopo, objoctives, approach, constraints, further milestone definition) (capital justification)

Support the Tong range planning process, Hanford Mission Plan (HMP). PNL

will lead the Risk Management Task (1.8.2.1.3). PNL will provide general

support to the preparation of HMP Vol. I by assisting in the development of

Site objectives and priority criteria, updating Site decisions, compiling

stakeholder values, and updating Public Involvement Policy, as well as

reviews. Provide input to Hanford Strategic Analysis (HSA) special cases

and analyze FY 1996 Multi-Year Program Plan (MYPP) assumptions and

regulatory drivers. Program P1an Analysis will identify sitewide

programmatic risk as wel1. The analysis will focus on identification of

sitewide and interface issues. This CAA represents $80 \%$ of budget (the

balance is funded through ADS 3410).

\begin{tabular}{|r|r|r|}
\hline ADS Numbor & \multicolumn{2}{|c|}{7250} \\
\hline Total CAP $\$ 000$ & G \& A/CSP \$000 & Total CAA \$000 \\
\hline & & \\
376 & & 376 \\
\hline
\end{tabular}

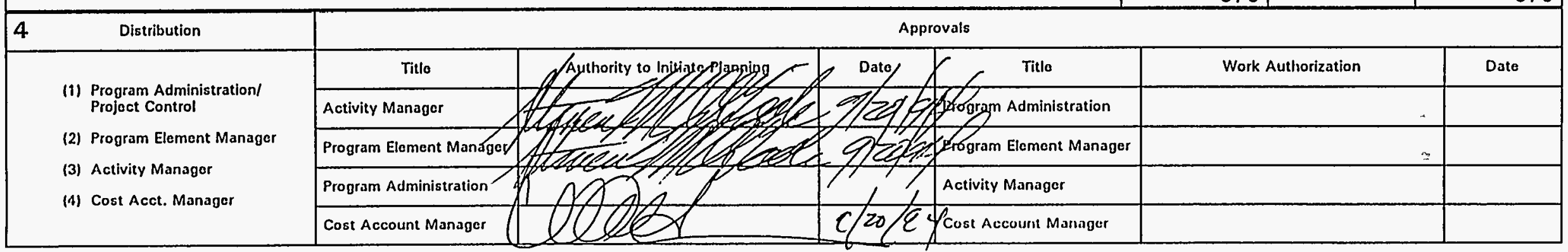




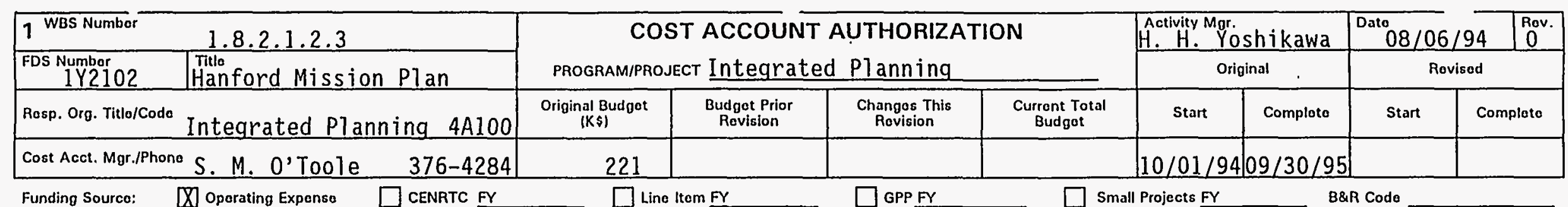

\begin{tabular}{|c|c|c|c|c|c|c|c|}
\hline & ding Sourco: & iting Expenso & $\square$ CENRTC FY & D Line Ite & $\square$ GPP FY & L] Small Projects FY & B\&A Codo \\
\hline 2 & Controllod Milostones & $T=T P A$ & $M=$ Major DOE-HO & $K=$ Major RL & $R=$ Westinghouse Key & $0=$ Other & \\
\hline
\end{tabular}

K 11/29/94 Proposal for High Level WBS to RL Planning Integration Division

$K \quad 03 / 28 / 95$ Proposal for High Level Priority List to RL Planning Integration Division

K 04/17/95 Transmit Draft FY 1996 HMP Volume 1, Site Guidance to RL

K 07/05/95 Complete FY 1996 HMP Volume 1, Site Guidance

$R \quad 09 / 30 / 95 \quad$ Complete Hanford Strategic Analysis Addendum

3 Doscription/Instructions (scope, objectives, approach, constraints, further milestone definition) (capital justification)

Develop an input to Site level technical baseline development. Develop and

document Site Tevel guidance and analysis to define Site mission, programs, strategy assumptions and issues. The guidance will include extracts from

the Hanford Strategic Plan (Hanford Mission Plan) as well as objectives,

FY 1996 Planning Basis, stakeholder values, priority list/criteria, WBS/OBS

based on systems engineered PBS, interfaces and Site summary scheduled

integrated based on the Site schedule. The Hanford Strategic Analysis

addendum is an evaluation of alternatives and states from the site examining

Material Balances, ROM cost and schedule data in support of Systems

Engineering. The Site Guidance directs the MYPPs.

(Continued on Page 2)

\begin{tabular}{|r|r|r|}
\hline \multicolumn{1}{|c|}{ ADS Number } & \multicolumn{2}{|c|}{3410} \\
\hline Total CAP $\$ 000$ & G \& A/CSP $\$ 000$ & Total CAA $\$ 000$ \\
\hline & & \\
181 & & \\
& 40 & 221 \\
\hline
\end{tabular}

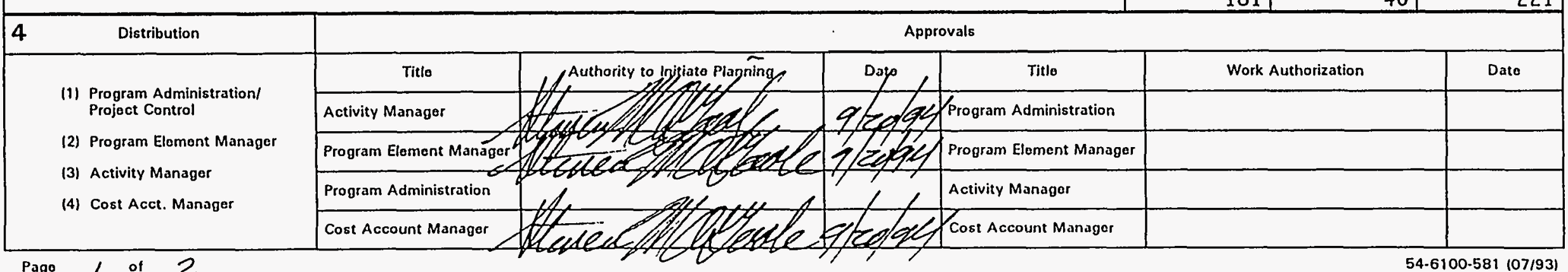




\begin{tabular}{|c|c|c|c|c|c|c|c|c|c|c|}
\hline 1 WBS Number & 1.8 .2 .1 .2 .3 & & ACCOUN & UTHORIZA & & $\begin{array}{l}\text { Activity M } \\
\text { H. H. }\end{array}$ & hikawa & $\begin{array}{r}\text { Dato } \\
08 / 0\end{array}$ & & $\begin{array}{c}\text { Rev. } \\
0\end{array}$ \\
\hline $\begin{array}{l}\text { FDS Number } \\
1 Y 2102\end{array}$ & $\begin{array}{l}\text { Tullo } \\
\text { Hanford Mission Plan }\end{array}$ & PROGRAM/PR & TPlannin & tegration & rogram & & & & & \\
\hline Resp. Org. Title/Code & Integrated Planning $4 \mathrm{Al} 100$ & $\begin{array}{c}\text { Original Budget } \\
(K \$)\end{array}$ & $\begin{array}{l}\text { Budget Prior } \\
\text { Revision }\end{array}$ & $\begin{array}{l}\text { Changos This } \\
\text { Revision }\end{array}$ & $\begin{array}{c}\text { Current Total } \\
\text { Budget }\end{array}$ & Start & Complete & Start & & plete \\
\hline Cost Acct. Mgr./Phone & S. M. O'Toole & 221 & & & & $10 / 10 /$ & $9 / 30 / 9$ & & & \\
\hline
\end{tabular}

Funding Source: $\quad$ 对 Operating Expenso $\square$ CENRTC FY $\square$ Lino Item FY $\square$ GPP FY $\quad \square$ Small Projocts FY

\begin{tabular}{|cccccc}
\hline 2 Controlled Milestones & $T=$ TPA & $M=$ Major DOE-HO & $K=$ Major RL & $R=$ Wostinghouse Koy $\quad O=$ Other \\
\hline Milestono No. & Typo & Dato & Description
\end{tabular}

3 Doscription/Instructions (scopo, objectives, approach, constraints, further milostono definition) (capital justification)

\section{(Continued from Page 1)}

This work is funded $80 \%$ by ADS 7250 and $20 \%$ by ADS 3410 (a separate

CAA/CAP). It is performed in conunction with PNL (Regulatory Analysis,

Science and Technology Analysis, Risk Management), which are direct funded.

The completion of the RL milestones is dependent upon timely RL reviews

and closure.

\begin{tabular}{|r|r|r|}
\hline ADS Numbor & \multicolumn{1}{|c|}{3410} \\
\hline Total CAP \$000 & G \& A/CSP \$000 & Total CAA \$000 \\
\hline & & \\
181 & 1 & \\
\hline
\end{tabular}

Distribution
(1) Program Administration/
Project Control
(2) Program Element Manager
(3) Activity Manager
(4) Cost Acct. Manager

Pago 2 of 2

\begin{tabular}{|l|l|l|l|}
\hline \multicolumn{1}{|c|}{ Titlo } & Work Authorization & Dato \\
\hline Activity Manager & Puthority to Initiato Planning & \\
\hline Program Element Man foer & & \\
\hline Costram Administration Account Managor & & \\
\hline
\end{tabular}




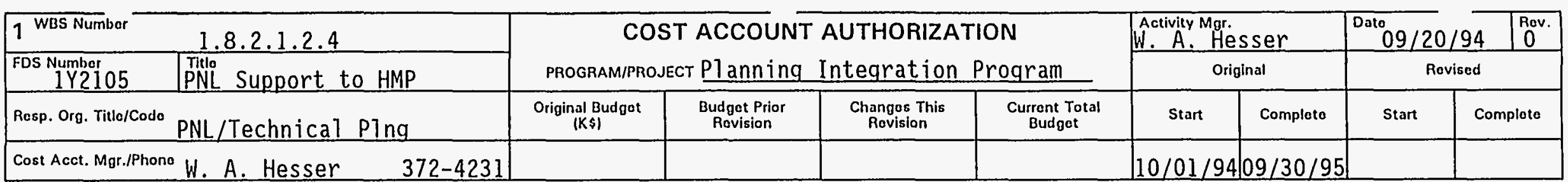

Funding Source: $[\mathrm{X}$ Operating Exponse $\square$ CENRTC FY $\square$ Line Itom FY $\square$ GPP FY $\square$ Small Projocts FY

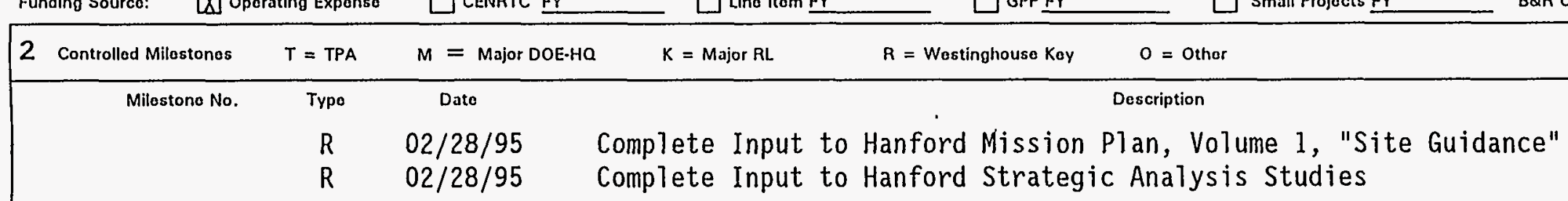

Support the long range planning process, Hanford Mission Plan (HMP). PNL

will lead the Risk Management Task (1.8.2.1.3). PNL will provide general

support to the preparation of HMP Vol. 1 by assisting in the development of

Site objectives and priority criteria, updating Site decisions, compiling

stakeholder values, and updating Public Involvement Policy, as well as

reviews. Provide input to Hanford Strategic Analysis (HSA) special cases

and analyze FY 1996 Multi-Year Program Plan (MYPP) assumptions and

regulatory drivers. Program Plan Analysis will identify sitewide

programmatic risk as wel1. The analysis will focus on identification of

sitewide and interface issues. This CAA represents $20 \%$ of budget (the

balance is funded through ADS 7250).

\begin{tabular}{|r|r|r|}
\hline ADS Number & \multicolumn{2}{|c|}{3410} \\
\hline Total CAP \$000 & G \& A/CSP \$000 & Total CAA \$000 \\
\hline & & \\
94 & & 94 \\
\hline
\end{tabular}

\begin{tabular}{|c|c|c|c|c|c|}
\hline Distribution & \multicolumn{5}{|c|}{ Approvals } \\
\hline \multirow{5}{*}{$\begin{array}{l}\text { (1) Program Administration/ } \\
\text { Project Control } \\
\text { (2) Program Elcment Manager } \\
\text { (3) Activity Manager } \\
\text { (4) Cost Acct. Manager }\end{array}$} & Titlo & optopings & Titlo & Work Authorization & Date \\
\hline & Activity Manager & 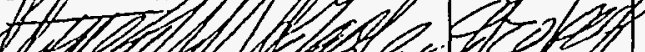 & Program Administration & & \\
\hline & Program Element Manager & $g$ & Arogram Element Manager & & \\
\hline & Program Administration & & Activity Manager & & \\
\hline & Cost Account Managdr & grofen & Cost Account Manager & & \\
\hline
\end{tabular}

Pago $-{ }^{\text {of }}$ 


\begin{tabular}{|c|c|c|c|c|c|c|c|c|c|}
\hline \multicolumn{2}{|c|}{1 WBS Number 1.8 .2 .1 .3 .1} & \multirow{2}{*}{\multicolumn{4}{|c|}{ COST ACCOUNT AUTHORIZATION }} & \multicolumn{2}{|c|}{$\begin{array}{l}\text { Activity Mgr. } \\
\text { W.A. Hesser }\end{array}$} & \begin{tabular}{|l|l|} 
Dato \\
$07 / 18$ \\
\end{tabular} & $\begin{array}{c}\text { Rev. } \\
0\end{array}$ \\
\hline $\begin{array}{l}\text { FDS Number } \\
1 Y 2104\end{array}$ & $\begin{array}{l}\text { Title } \\
\text { Risk Management }\end{array}$ & & & & & \multicolumn{2}{|c|}{ Original } & \multicolumn{2}{|c|}{ Revised } \\
\hline \multicolumn{2}{|c|}{ Resp. Org. Title/Code PNL/Technical Plng $4 \mathrm{Al} 100$} & $\begin{array}{l}\text { Original Budget } \\
(\mathrm{K} \$)\end{array}$ & $\begin{array}{l}\text { Budget Prior } \\
\text { Revision }\end{array}$ & $\begin{array}{l}\text { Changes This } \\
\text { Revision }\end{array}$ & $\begin{array}{l}\text { Current Total } \\
\text { Budget }\end{array}$ & Start & Complete & Start & Complete \\
\hline Cost Acct. Mgr./Phone & W.A. Hesser/372-4231 & & & & & $10 / 94$ & $09 / 30 / 95$ & & \\
\hline
\end{tabular}

Funding Source: $\square$ Operating Expense $\square$ CENRTC FY

$\square$ Line Item FY

$\square \mathrm{GPPFY}$

Small Projects FY B\&R Codo

2 Controlled Milestones $T=T P A \quad M=$ Major DOE-HO $\quad K=$ Major RL $\quad R=$ Westinghouse Key $O=$ Other

$\begin{array}{rlll}\text { Milestone No. Type Date } & \text { Description }\end{array}$

PSS $-95-300 \quad$ PNL-KEY 03/31/95

PSS $-95-301 \quad \mathrm{~K} \quad 04 / 30 / 95$

PSS-95-302 K $05 / 31 / 95$

PSS-95-304 PNL-KEY 03/31/95

PSS-95-305 PNL-KEY 02/28/94

Revised Priority Planning Grid and Procedures

Analysis of Hanford Costs and Risks

Public Risk Perceptions at Hanford

Lessons from Risk Management Support of Systems Engineering

Risk Activities

3 Description/Instructions (scope, objectives, approach, constraints, further milestone definition) (capital justification)

FY 1995 - PNL will: assist WHC and RL strategic planners in developing and implementing a risk-based budget prioritization process; in conjunction with IRAP, assist in the development of a total risk picture of the site; relate ES\&H risks and budgets and perform cost-benefit analysis; assist in the development and implementation of risk management policies and procedures; and assist in the implementation of risk-based decision making. PNL wilf
also identify risk perceptions of the site to reflect public values and concerns. Risk mangers will coordinate with the Integrated Risk Assessment Program and will serve as an interface between risk and planning activities. The logic of the risk management program is attached as Enclosure 1 .

\begin{tabular}{|c|c|c|}
\hline ADS Number & \multicolumn{2}{|c|}{7250} \\
\hline Total CAP \$000 & G \& A/CSP \$000 & Total CAA \$000 \\
\hline 544 & & 544 \\
\hline
\end{tabular}

\begin{tabular}{|c|c|c|c|c|c|c|}
\hline Distribution & \multicolumn{6}{|c|}{ Approvals } \\
\hline \multirow{5}{*}{$\begin{array}{l}\text { (1) Program Administration/ } \\
\text { Project Control } \\
\text { (2) Program Element Manager } \\
\text { (3) Activity Manager } \\
\text { (4) Cost Acct. Manager }\end{array}$} & Titlo & Aythority to Initiorg Plgnning & Date & Titlo & Work Authorization & Date \\
\hline & Activity Manager & & esterles & Program Administration & m & \\
\hline & Program Element Manag & Hever lll lesode & sifised & Program Element Manager & $\infty$ & \\
\hline & Program Administration & & & Activity Manager & & \\
\hline & Cost Account Managor & & $-9 / 20 / 94$ & Cost Account Manager & & \\
\hline
\end{tabular}


FY 19?

\begin{tabular}{|c|c|c|c|c|c|c|c|c|c|}
\hline 1 WBS Number & 1.8 .2 .2 .1 .1 & & Accoun & UTHORIZA & & $\begin{array}{l}\text { Activity M } \\
\text { EA Sch }\end{array}$ & & $\begin{array}{r}\text { Dato } \\
10 / 0\end{array}$ & 94 \\
\hline $\begin{array}{l}\text { FDS Numbor } \\
1 Y 2202\end{array}$ & $\begin{array}{l}\text { Trto } \\
\text { Progress Tracking System }\end{array}$ & PROGRAM/PR & Plannin & tegratior & & & & & \\
\hline Rosp. Org. Titlo/Code & Performance Rptg & $\begin{array}{l}\text { Orialnal Budgot } \\
|K \&|\end{array}$ & $\begin{array}{l}\text { Budgot Prlor } \\
\text { Aovision }\end{array}$ & $\begin{array}{l}\text { Changos This } \\
\text { Rovision }\end{array}$ & $\begin{array}{l}\text { Curront Total } \\
\text { Budgot }\end{array}$ & Start & Comploto & Start & Complete \\
\hline Cout Acct. Mor./Pho & - DN LaRue & & & & & $10 / 01 /$ & $9 / 30 / 95$ & & \\
\hline
\end{tabular}

Funding Sourco: $\square$ Oporating Exponso $\square$ CENRTC $F$

2 Controllod Milostonos $T=T P A \quad M=$ Major DOE-HO $K=$ Major AL $\quad R=$ Wostinghouso Koy

\begin{tabular}{|cccccc}
\hline 2 & & $K=$ Major $A L$ & $R=$ Wostinghouso Koy & $0=$ Othor \\
\hline Milostono No. & Typo & Dato & Description
\end{tabular}

PSA-95-035 K Monthly Submit PTS report to RL

thru 046

PSA-95-047 R TBD

Begin HQ directed PTS 5.0 Upgrade (Hold)

3 Doscription/instructions (scopo, objoctivos, approach, constrainte, further milostono dofinttion) (capital Juatification)

The PTS activity involves integration and implementation of the DOE-HQ mandated PTS reporting requirement. DOE-HQ established an Environmental Management (EM) program reporting system to track work-scope progress at the ADS level. PTS serves as the primary EM management tool, which provides a consistent EM-wide reporting format and database for responding to internal and external requests for program information. This is an ongoing activity required to meet monthly reporting submittals to DOE-HQ.

PTS reports activity status for all EM ADSs and technical task plans. Non EM ADSs are not included in this activity. PTS integrated data from the site Management System and Financial Data Systems and incorporates them into the PTS format via the Environmental Planning Data System on Site.

\begin{tabular}{|l}
\hline $4 \quad$ Distribution \\
\hline (1) Program Administration/ \\
Projact Control \\
(2) Program Elomont Managor \\
(3) Actlulty Managor \\
(4) Cost Acct. Managor
\end{tabular}

\begin{tabular}{|l|}
\hline \multicolumn{2}{|c|}{ Titlo } \\
\hline Activity Managor \\
\hline Program Elamont Managor \\
\hline Program Adminietratlon \\
\hline Coat Account Managor \\
\hline
\end{tabular}

\begin{tabular}{|c|c|}
\hline Authority to Initlato Planning & \\
\hline EA Schultz EA \& chus & 8 \\
\hline LR Hafer UHA & 81 \\
\hline JP Carandanis & \\
\hline DN LaRue $\varepsilon A S_{\text {e }}$ & 8 \\
\hline
\end{tabular}

Approvals

\begin{tabular}{|c|l|l|l|}
\hline \multicolumn{1}{|c|}{ Dato } & \multicolumn{1}{|c|}{ Mitlo } & \multicolumn{1}{|c|}{ Work Authorlzotion } & Dato \\
\hline 8.31 .94 & Program Adminletrotion & JP Carandanis & \\
\hline 8131194 & Program Elomont Managor & LR Hafer & \\
\hline & Actlvity Managor & EA Schultz & \\
\hline $8-31-0,4$ & Cost Account Managor & DN LaRue & \\
\hline
\end{tabular}

\begin{tabular}{|r|r|r|}
\hline ADS Numbor & \multicolumn{2}{|c|}{$7250-A D$} \\
\hline Total CAP 1000 & o \& A/CSP 000 & rotal CAA 1000 \\
\hline 40 & & \\
520 & 114 & 634 \\
634 & & 114 \\
\hline
\end{tabular}


FY 19.

\begin{tabular}{|c|c|c|c|c|c|c|c|c|c|}
\hline \multirow{2}{*}{\multicolumn{2}{|c|}{\begin{tabular}{|l|l|}
1 WBS Numbor & 1.8 .2 .2 .1 .2 \\
FDS Numbor & Titlo \\
\end{tabular}}} & \multicolumn{4}{|c|}{ COST ACCOUNT AUTHORIZATION } & \multicolumn{2}{|c|}{$\begin{array}{l}\text { Activity Mori } \\
\text { EA Schuitz }\end{array}$} & \multicolumn{2}{|r|}{ Rov. } \\
\hline & & \multicolumn{4}{|c|}{ PROGRAM/PROJECT Planning Integration } & & & \multicolumn{2}{|c|}{ Rovisod } \\
\hline Rosp. Oro. Titlo/Codo & Performance Rptg. & $\begin{array}{c}\text { Original Budgot } \\
(K \leqslant\})\end{array}$ & $\begin{array}{l}\text { Budget Prior } \\
\text { Rovision }\end{array}$ & $\begin{array}{l}\text { Changes This } \\
\text { Revision }\end{array}$ & $\begin{array}{l}\text { Curront Total } \\
\text { Budgot }\end{array}$ & Start & Comploto & Start & Comploto \\
\hline Cost Acct. Mgr./Phon & EA Schultz & & & & & $10 / 01$ & $99 / 30 / 9$ & & \\
\hline
\end{tabular}

Funding Source: $\square$ O Oporatine Exponso $\square$ CENRTC FY $\square$ Lino Item FY $\quad \square$ GPP FY GER Codo G\&A

2 Controllod Milostonos $T=$ TPA $\quad M=$ Major DOE-Ha $\quad K=$ Major RL $\quad R=$ Wostinghouse Koy $O=$ Othor

Milostono No. Typo Dato Doscription

PSA-95-048 K Monthiy Submit SMS report to RL

thru 059

PSA-95-001 R Biweekly Submit Critical Items Report to RL Manager's 0ffice

thru 026

PSA-95-073 R Monthly Data Quality Check of the monthly direct/indirect SMS Packages thru 084

3 Doscription/Instructions (scopo, objoctives, approach, constraints, further milostono dofinition) (capital jurtfication)

The SMS activity involves the coordination and compilation of the individual SMS program reports and summarization of this data into a SMS executive summary and program summary report. This activity is the primary vehicle used by Hanford Site organizations to manage the work necessary to accomplish the goals and objectives of the RL Mission. This an ongoing activity to meet monthly reporting submittals to $\mathrm{RL}$ as required by RLID 5000.5, "Richland Field Office-Site Management System," and RLID 5000.7, "RL Site Management System Reporting."

The SMS monthly report statuses and measures the accomplishments, progress, and expenditures against an approved baseline for all activities (direct/indirect.) The SMS report is an assimilation of data from the milestone and TPA databases, Level 0 Schedule, Change Control, and FDS into a standardized format. (Description continued on page 2)

\begin{tabular}{|r|l|r|}
\hline ADS Numbor & \multicolumn{1}{|c|}{ G\&A } \\
\hline Total CAP $\$ 000$ & G \& A/CSP \$000 & Total CAA $\$ 000$ \\
\hline & & \\
345 & $-0-$ & 345 \\
\hline
\end{tabular}

\begin{tabular}{|l|ll}
\hline 4 & Distribution & Approvals
\end{tabular}

\begin{tabular}{|c|c|c|c|c|c|c|}
\hline Distribution & \multicolumn{6}{|c|}{ Approvals } \\
\hline \multirow{2}{*}{$\begin{array}{l}\text { (1) Program Administration/ } \\
\text { Projoct Control }\end{array}$} & Titlo & Authority to Initiato Planning & Dato & Titlo & Work Authorization & Dato \\
\hline & Activity Managor & EA Schuitz $\Sigma A$ del be & $8-26-94$ & Program Administration & JP Carandanis & \\
\hline (2) Program Elomont Managor & Program Elomont Managor & LR Hafer UReffa & plzel94 & Program Elomont Manager & LR Hafer & \\
\hline (4) Cost Acct. Managor & Program Administration & JP Carandanis & & Activity Managor & EA Schultz & \\
\hline & Cost Account Managor & EA Schuitz $\Sigma A \ell_{\text {s }}$ lult & $8.26-54$ & Cost Account Managor & EA Schultz & \\
\hline
\end{tabular}




\begin{tabular}{|c|c|c|c|c|c|c|c|c|c|}
\hline \multirow{2}{*}{\multicolumn{2}{|c|}{\begin{tabular}{|l|l|}
1 WBS Numbor & $1.8 \cdot 2 \cdot 2 \cdot 1.3$ \\
$\begin{array}{c}\text { FDS Numbor } \\
1 M M Y L L M\end{array}$ & $\begin{array}{l}\text { Trtelo } \\
\text { Central Milestone Module }\end{array}$ \\
\end{tabular}}} & \multirow{2}{*}{\multicolumn{4}{|c|}{$\begin{array}{l}\text { COST ACCOUNT AUTHORIZATION } \\
\text { PROGRAM/PROJECT Planning Integration }\end{array}$}} & \multirow{2}{*}{\multicolumn{2}{|c|}{$\begin{array}{c}\text { Activity Mgr. } \\
\text { A. Schultz } \\
\text { Original }\end{array}$}} & ${ }^{\text {Dato }} 10 /$ & \multirow{2}{*}{$\frac{14}{d}$} \\
\hline & & & & & & & & Rovisod & \\
\hline Rosp. Org. Titlo/Codo & Performance Rep. $\quad 4$ A220 & $\begin{array}{c}\text { Orioinal Budgot } \\
\mid K \$\}\end{array}$ & $\begin{array}{l}\text { Budgot Prior } \\
\text { Revision }\end{array}$ & $\begin{array}{l}\text { Changos This } \\
\text { Rovision }\end{array}$ & $\begin{array}{l}\text { Curront Total } \\
\text { Budgot }\end{array}$ & Start & Comploto & Start & Comploto \\
\hline Cost Acct. Mgr./Phone & - AJ Watts & & & & & $10 / 01 /$ & $9 / 30 / 95$ & & \\
\hline
\end{tabular}
Fundino Sourco: $[\mathrm{D}]$ Operating Exponso $\square$ CENATC $\mathrm{FY}$

\begin{tabular}{rccccc}
\hline 2 Controllod Milostonos & $T=$ TPA & $M=$ Major DOE-HO & $K=$ Mojor RL & $R=$ Wostinghouso Koy $\quad O=$ Othor \\
\hline Milostono No. & Typs & Dato & Doscription
\end{tabular}

$\begin{array}{llll}\text { PSA-95-065 } & R & 04 / 17 / 95 & \text { Complete general process improvements to the Central Milestone Module } \\ \text { PSA-95-066 } & R & 08 / 31 / 95 & \text { Complete TPA, Archive, and PTS enhancements to the CMM } \\ \text { PSA-95-068 } & R & 09 / 14 / 95 & \text { Develop CMM as replacement for PTS milestone data source }\end{array}$

3 Doscription/Instructions (scopo, objoctives, approach, constraints, further mllostono dofinition) (capital Justification)

The Central Milestone Module is a database which houses all RL, HQ, TPA and DNFSB milestones for the Hanford Site. Milestones are statused within this database and fed to the Multiyear Program Plan/Fiscal Year Work Plan; Five Year Plan; PTS and SMS reports. Programs also have the ability to enter and track contractor milestones at their discretion.

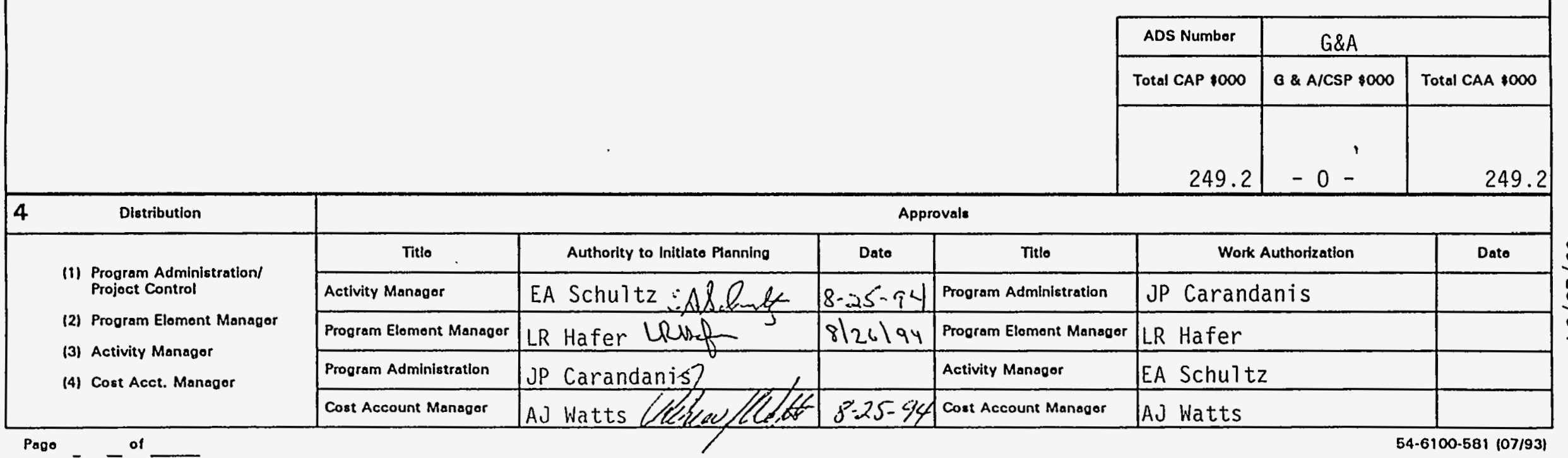


FY 15

\begin{tabular}{|c|c|c|c|c|c|c|c|c|}
\hline \begin{tabular}{|l|l} 
WBS Numbor & $1.8 .2 \cdot 2.1 .4$ \\
FDS Number & $\frac{1}{\text { Titlo }}$ \\
\end{tabular} & \multirow{2}{*}{\multicolumn{4}{|c|}{$\begin{array}{l}\text { COST ACCOUNT AUTHORIZATION } \\
\text { PROGRAM/PROJECT Planning Integration }\end{array}$}} & \multirow{2}{*}{\multicolumn{2}{|c|}{$\frac{\text { Activity Mgr }}{\text { EA Schuitz }}$}} & \multirow{2}{*}{\multicolumn{2}{|c|}{\begin{tabular}{|r|} 
Dato $10 / 01 / 94$ \\
Rovised \\
\end{tabular}}} \\
\hline \begin{tabular}{c|l} 
FDS Number \\
IMDDLN & Masto \\
Master WBS \& Valid. Tbls.
\end{tabular} & & & & & & & & \\
\hline Rosp. Org. Titlo/Codo Performance Rptg. & $\begin{array}{l}\text { Original Budgot } \\
\text { (Ks) }\end{array}$ & $\begin{array}{l}\text { Budget Prior } \\
\text { Rovision }\end{array}$ & $\begin{array}{c}\text { Changes This } \\
\text { Rovision }\end{array}$ & $\begin{array}{l}\text { Curront Total } \\
\text { Budget }\end{array}$ & Start & Complote & Start & Complote \\
\hline Cost Acct. Mgr./Phono JA Sargent $376-9575$ & & & & & $10 / 01 /$ & $9 / 30 / 95$ & & \\
\hline
\end{tabular}

Funding Sourco: $[X]$ Oporating Exponso $\square$ CENRTC FY $\square$ LLino ttom FY $\square$ GPP FY $\square$ Small Projocts FY

\begin{tabular}{|rccccc}
\hline 2 Controllod Milostonos & $T=$ TPA & $M=$ Major DOE-HO & $K=$ Major RL $\quad . \quad R=$ Wostinghouso Kay $\quad 0=$ Othor \\
\hline Milostono No. & Typo & Dato & Doscription
\end{tabular}

PSA-95-063 K 03/31/95 Distributed Cali Letter requesting FY 1996 proposed WBS

PSA-95-067 K 06/15/95 Finalize Draft FY 1996 Proposed WBS and distribute

3 Doscription/Instructions (scopo, objoctivos, approach, constrainte, furthor mllostono definition) (capital justification)

The master WBS and validation tables is a database which encompasses the U.S. Department of Energy-Headquarters (DOE-HQ) Work. Breakdown Structure (WBS) numbers/titles, U.S. Department of Energy Richland Operations Office .

(RL) WBS numbers/titles, Activity Data Sheet numbers/titles,

Contractor/DOE-HQ project numbers, and DOE-HQ/RL/Contractor Program

Managers. This database feeds ali reports mentioned above to provide

consistency and credibility between DOE-HQ and RL reports.

\begin{tabular}{|r|r|r|}
\hline ADS Numbor & \multicolumn{2}{|c|}{ G\&A } \\
\hline Total CAP 1000 & G\& A/CSP t000 & Total CAA 1000 \\
\hline & 1 & \\
92.0 & -0 & 92.0 \\
\hline
\end{tabular}

\begin{tabular}{|c|c|c|c|c|c|c|c|}
\hline 4 & Distribution & \multicolumn{6}{|c|}{ Approvale } \\
\hline \multirow{5}{*}{\multicolumn{2}{|c|}{$\begin{array}{l}\text { (1) Program Administration/ } \\
\text { Projoct Control } \\
\text { (2) Prooram Elomont Managor } \\
\text { (3) Activity Managor } \\
\text { (4) Cost Acct. Managor }\end{array}$}} & Titlo & Authority to Initlato Planning & Dato & Titlo & Work Authorization & Dato \\
\hline & & Activity Managor & EA Schultz $\varepsilon A l \ln h$ & $8.25-94$ & Program Administration & JP Carandanis & \\
\hline & & Program Eloment Managor & LR Hafer lesif & 8126199 & Program Elomont Managor & LR Hafer & \\
\hline & & Program Adminlstration & JP Carandanis & & Activity Managor & EA Schultz & \\
\hline & & Cost Account Managor & JA Sarge $1 / 0 A$ & & Cost Account Managor & JA Sargent & \\
\hline
\end{tabular}




\begin{tabular}{|c|c|c|c|c|c|c|c|c|c|}
\hline \multirow{2}{*}{\multicolumn{2}{|c|}{\begin{tabular}{|l|l|}
1 WBS Numbor & $1 \cdot 8 \cdot 2 \cdot 2 \cdot 1 \cdot 5$ \\
$\begin{array}{c}\text { FDS Numbor } \\
1 Y 2201\end{array}$ & $\begin{array}{l}\text { Tila } \\
\text { EPDS Plat form Oversight }\end{array}$ \\
\end{tabular}}} & \multirow{2}{*}{\multicolumn{4}{|c|}{$\begin{array}{l}\text { COST ACCOUNT AUTHORIZATION } \\
\text { PROGRAM/PROJECT Planning Integration }\end{array}$}} & \multirow{2}{*}{\multicolumn{2}{|c|}{\begin{tabular}{|r|} 
Activity Mor \\
Schuitz \\
Original \\
\end{tabular}}} & \multirow{2}{*}{\multicolumn{2}{|c|}{\begin{tabular}{|c|c|} 
Dato $07 / 25 / 94$ & Rov. \\
Rovisod \\
\end{tabular}}} \\
\hline & & & & & & & & & \\
\hline Rosp. Org. Titlo/Codo & Performance Rptg. $\quad 4$ A220 & $\begin{array}{l}\text { Original Budgot } \\
(K \$)\end{array}$ & $\begin{array}{l}\text { Budgot Prior } \\
\text { Rovision }\end{array}$ & $\begin{array}{l}\text { Chonges This } \\
\text { Revision }\end{array}$ & $\begin{array}{c}\text { Current Total } \\
\text { Budgot }\end{array}$ & Start & Complato & Start & Comploto \\
\hline Cost Acct. Mor./Phon & - JE Tarcza & & & & & $10 / 01 /$ & $19 / 30 / 95$ & & \\
\hline
\end{tabular}

Funding Sourco: $\square$ X] Oporating Exponso $\square$ CENRTC FY $\square$ Lino Item FY $\square$ GPP FY

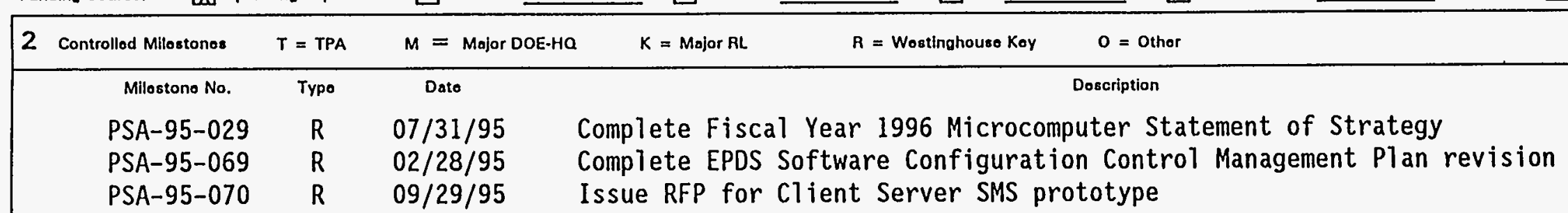

3 Doscription/Instructions (scopo, objoctivos, approsch, constraints, furthor milostono doflnition) (capital justiflcation)

EPDS is a computer platform deployed on the HLAN, used for the collection of

financial/budget planning and reporting data which is electronically fed to

the DOE-HQ PTS and ADS40 systems. This platform currently supports PTS, FYP,

RPM, CMM, and CCM database modules. Platform oversight includes the

management operation and system development activities required to maintain

compatability with DOE-HQ and other DOE-RL systems. The Client Server

Project is necessary to enhance the existing 1 imited DOS based platform to accomodate in excess of 50 concurrent users, more efficient data

validation/management capabilities, and take advantage of newer technologies such as multitasking graphical user interface and network fiber optics. CSG activities include development of the SOS for 4A000, review of company level computer strategies, and approval of all computer acquisitions. CPPM activities include the monitoring of Org $4 A 000$ computer related property.

\begin{tabular}{|r|r|r|}
\hline ADS Numbor & \multicolumn{2}{|c|}{$7250-0-A J$} \\
\hline Total CAP $\$ 000$ & G \& A/CSP $\$ 000$ & Total CAA $\$ 000$ \\
\hline &, & \\
411.7 & 90.6 & 502.3 \\
\hline
\end{tabular}

\begin{tabular}{|c|c|}
\hline 4 & Distribution \\
\hline & $\begin{array}{l}\text { Program Administration/ } \\
\text { Projoct Control }\end{array}$ \\
\hline (2) & Program Eloment Managor \\
\hline (3) & Activity Managor \\
\hline (4) & Cost Acct. Managor \\
\hline
\end{tabular}

Pago _- of

\begin{tabular}{|c|c|c|c|c|c|}
\hline \multicolumn{6}{|c|}{ Approvals } \\
\hline Titlo & Authority to Initiato Planning & Dato & Titlo & Work Authorization & Dato \\
\hline Aćtivity Managor & EA Schuitz EA & $8-.25-94$ & Program Administration & JP Carandanis & \\
\hline Program Elomont Managor & LR Hafer UNbef & 8126194 & Program Elomont Managor & LR Hafer & \\
\hline Program Administration & JP Carandanis & & Activity Managor & EA Schultz & \\
\hline Cost Account Managor & JE Tarcza & $d A$ & Cost Account Managor & JE Tarcza & \\
\hline
\end{tabular}




\begin{tabular}{|c|c|c|c|c|c|c|c|c|c|c|}
\hline \multicolumn{2}{|c|}{1 WBS Number $\quad 1.8 .2 .2 .2 .1$} & \multirow{2}{*}{\multicolumn{4}{|c|}{ COST ACCOUNT AUTHORIZATION }} & \multirow{2}{*}{\multicolumn{2}{|c|}{$\begin{array}{l}\text { Activity Mor. } \\
\text { Larry Hafer } \\
\text { Original }\end{array}$}} & \multicolumn{3}{|c|}{$\begin{array}{r}\text { Dato } \\
09 / 19 / 94 \\
\end{array}$} \\
\hline $\begin{array}{l}\text { FDS Numbor } \\
\text { IMDDLJ }\end{array}$ & $\begin{array}{l}\text { Title } \\
\text { Business Systems Devel. }\end{array}$ & & & & & & & \multicolumn{3}{|c|}{ Revised } \\
\hline Resp. Org. Title/Codo & Planning \& MCS Dev & $\begin{array}{l}\text { Original Budgot } \\
\text { (Ks) }\end{array}$ & $\begin{array}{l}\text { Budget Prior } \\
\text { Rovision }\end{array}$ & $\begin{array}{l}\text { Changes This } \\
\text { Rovision }\end{array}$ & $\begin{array}{l}\text { Current Total } \\
\text { Budget }\end{array}$ & Start & Comploto & Start & & plete \\
\hline Cost Acct. Mgr./Phon & P.J. Bence & 3,187 & & & & $10 / 01 /$ & $9 / 30 / 9$ & & & \\
\hline
\end{tabular}

Funding Source: $[X]$ Operating Expense $\square$ CENRTC FY $\square$ Lino Itom FY $\square$ GPP FY B $\quad \square$ Small Projocts FY Code

2 Controlled Milostones $T=T P A \quad M=$ Major DOE-HQ $\quad K=$ Major RL $\quad R=$ Wastinghousa Koy $O=$ Ollhor

\begin{tabular}{|cccccc}
2 Controlled Milestones & $T=$ TPA & $M=$ Major DOE-Ha & $K=$ Major RL & $R=$ Wastinghouse Koy $\quad O=$ Other \\
\hline Milestone No. & Typo & Date & Dascription
\end{tabular}

PSA-95-100 R 12/16/94 Draft Management Control System Description

PSA-95-101 K 03/17/95 Final Management Control System Description

PSA-95-102 $R \quad 10 / 14 / 94 \quad$ MCS and PARS Training Schedule for FY-95

PSA-95-110 K 10/14/94 MCS Surveillance Plan

PSA-95-111 $\quad R \quad 01 / 16 / 95 \quad$ Surveillance Plan Status Report for First Quarter FY-95

PSA-95-112 R 04/17/95 Surveillance P1an Status Report for Second Quarter FY-95

PSA-95-113 $\quad R \quad 07 / 15 / 95 \quad$ Surveillance Plan Status Report for Third Quarter FY-95

$\omega$

PSA-95-114 R $09 / 29 / 95$

Surveillance Plan Status Report for Fourth Quarter FY-95

3 Description/Instructions (scope, objectivos, approach, constraints, further milostono definition) (capital justification)

This activity supports the company's Management Control System (MCS)

procedure (including the WHC's Systen Description) update/development and

training of site personnel. It includes field support for Site Management

System (SMS) requirements, surveillance activities, change control

administration and compliance reviews.

This activity also supports the development and maintenance of the company's standard scheduling system. The activity contains the implementation process including but not limited to process development, specific scheduling software $(P / X)$ training, configuration control of the WHC site specific modifications to the $P / X$ software, field support, interface to the milestone module and JCS/Work Package linkage.

(Milestones and Descriptions continued on page 2 )

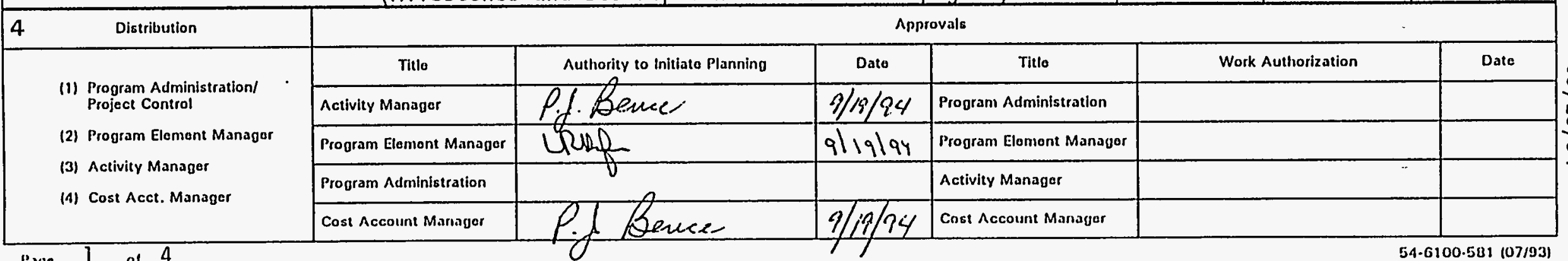

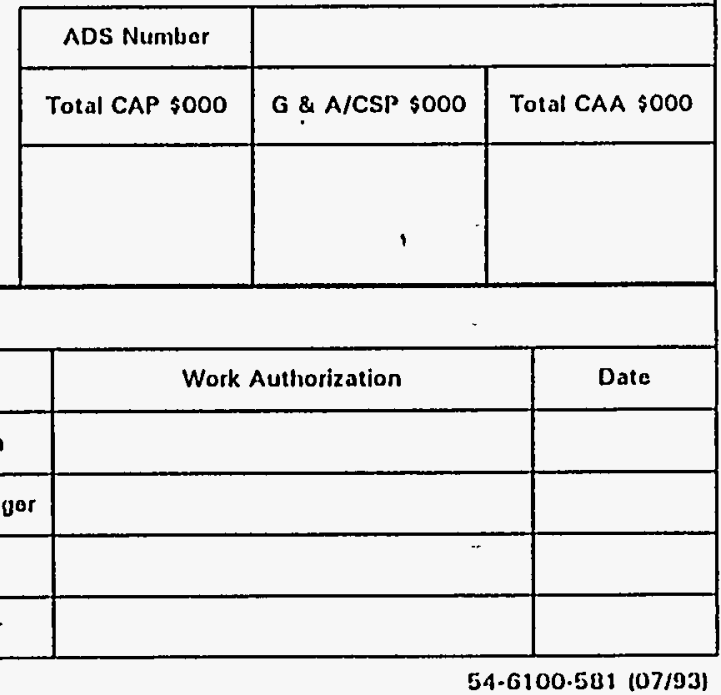

Poge 1 of 4 


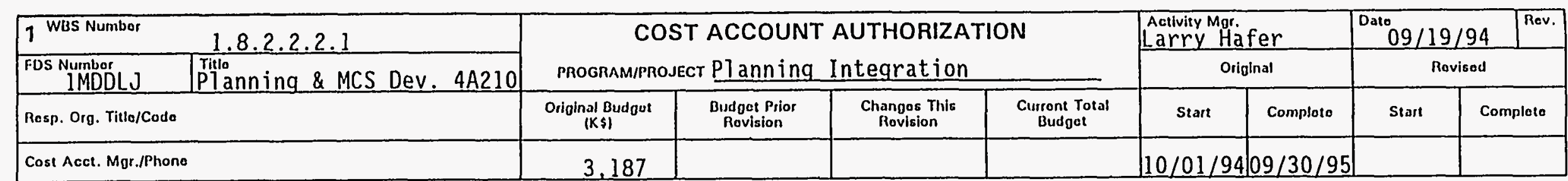

Funding Source: $[X]$ Operating Expense

$\square$ CENRTC FY

187

$\square \mathrm{GPPF}$

$\square$ Small Projects FY B\&R Coda

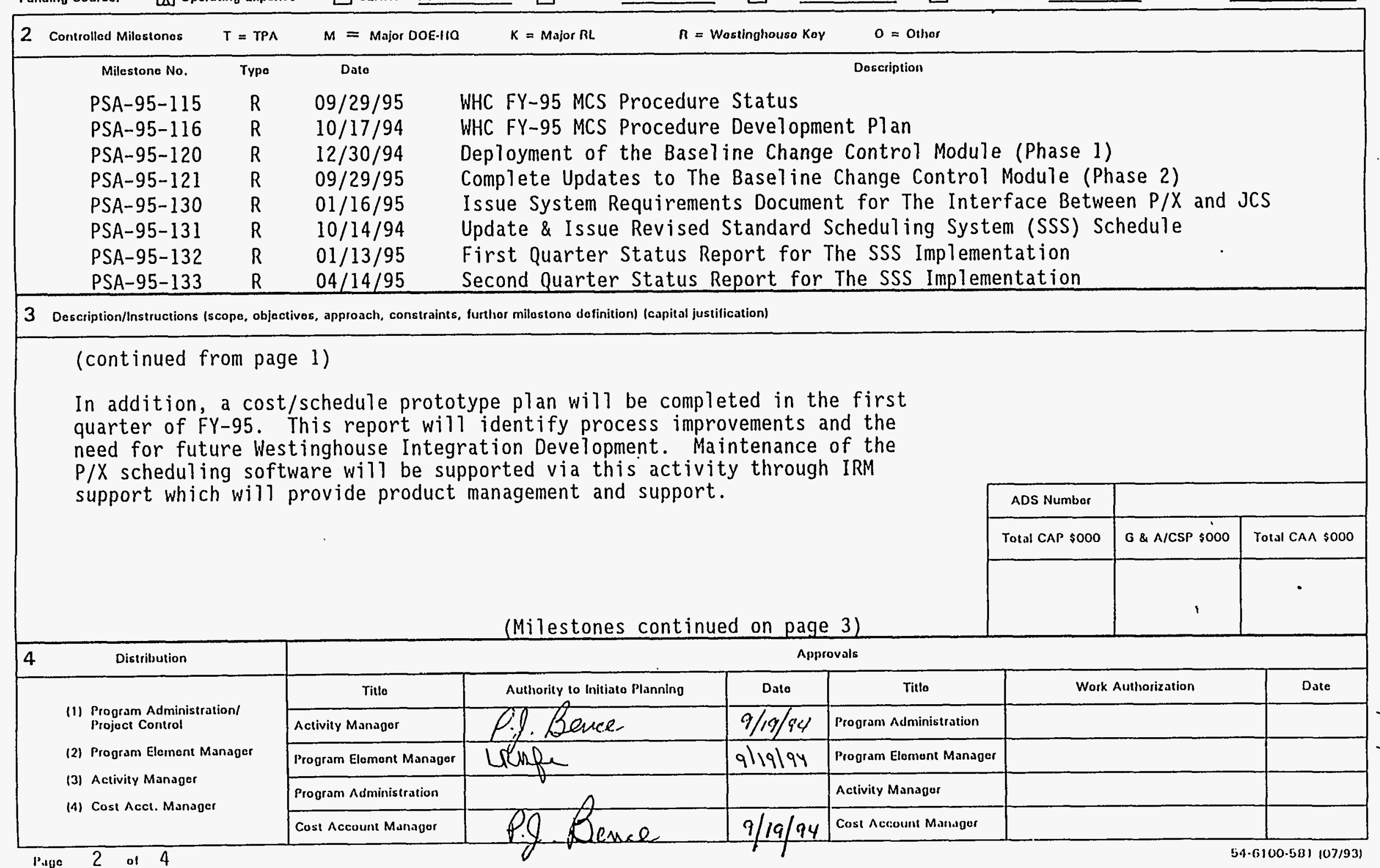

Piove 2 of 4 


\begin{tabular}{|c|c|c|c|c|c|c|c|c|c|c|}
\hline \multicolumn{2}{|l|}{1 WBS Number } & \multicolumn{4}{|c|}{ COST ACCOUNT AUTHORIZATION } & \multirow{2}{*}{\multicolumn{2}{|c|}{$\begin{array}{l}\text { Activity Moro } \\
\text { Larry Hafer } \\
\text { Original }\end{array}$}} & \multicolumn{3}{|c|}{\begin{tabular}{|l|} 
Dato \\
$09 / 19 / 94$ \\
\end{tabular}} \\
\hline $\begin{array}{l}\text { FDS Numbior } \\
\text { IMDDLJ }\end{array}$ & $\begin{array}{l}\text { Tillo } \\
\text { Business Systems Devel. }\end{array}$ & \multicolumn{4}{|c|}{ PROGRAMIPROJECT PLANNING INTEGRATION } & & & \multicolumn{3}{|c|}{ Revisod } \\
\hline Resp. Org. Titte/Codo & Planning \& MCS Dev & $\begin{array}{l}\text { Original Budgat } \\
\text { (Ks) }\end{array}$ & $\begin{array}{l}\text { Budget Prior } \\
\text { Rovision }\end{array}$ & $\begin{array}{l}\text { Changos This } \\
\text { Revision }\end{array}$ & $\begin{array}{l}\text { Current Total } \\
\text { Budgot }\end{array}$ & Start & Completo & Stant & & plete \\
\hline Cost Acct. Mgr.JPhon & P. J. Bence & 3,187 & & & & $10 / 01$ & $9 / 30 / 9$ & & & \\
\hline
\end{tabular}

Funding Source: $\square$ Operating Expense $\square$ CENRTC FY $\square$ Lino Item FY $\quad \square$ Small Projocts FY

\begin{tabular}{|rccccc}
2 Controlled Milestones & $T=T P A$ & $M=$ Major DOE-HO & $K=$ Major $\mathrm{RL}$ & $\mathrm{R}=$ Wostinghouso Koy & $O=0$ Other \\
\hline Milestono No. & Type & Dato & Description
\end{tabular}

PSA-95-134 R $\quad$ 07/14/95 Third Quarter Status Report for The SSS Implmentation

PSA-95-135 R 09/29/95 Fourth Quarter Status Report for The SSS Implementation

PSA-95-136 R 11/18/94 Complete Procedure That Describes Inputting BCWP Into PX for Schedule Status

PSA-95-137 R 01/20/95 Category of Labor Report From P/X

PSA-95-138 $R \quad 03 / 10 / 95 \quad$ Program P/X Software Changes for JCS Interface and Completion of Testing

PSA-95-139 R 04/14/95 Issue $P / X$ to JCS Interface Procedure

PSA-95-140 R 06/01/95 Completion of the P/X Software Configuration Management Plan

PSA-95-145 R 10/14/94 Systems Application Training Brochure

3 Description/Instructions (scopo, objoctivos, approach, constraints, furthor milostono dofiniltont (capital jụstification)

(continued from page 3)

(Milestones continued on page 4)

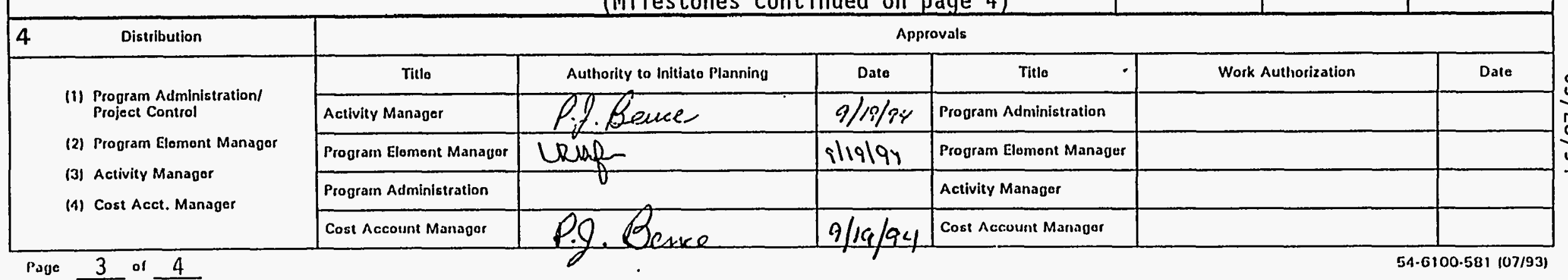




\begin{tabular}{|c|c|c|c|c|c|c|c|c|c|c|}
\hline 1 Wus Nurnbor & 1.8 .2 .2 .2 .1 & & ACCOUn & UTHORIZ & & $\begin{array}{l}\text { Activity M } \\
\text { L.R. }\end{array}$ & & \begin{tabular}{|c|} 
Dato \\
$09 /$ \\
\end{tabular} & & Rev. \\
\hline $\begin{array}{l}\text { FDS Numbor } \\
\text { IMDDLJ J }\end{array}$ & $\begin{array}{l}\text { Titlo } \\
\text { Business Systems Devel. }\end{array}$ & PROGRAM/PRC & PLANNIN & ITEGRATIOI & & & & & & \\
\hline Rosp. Org. Titlo/Codo & Planning \& MCS Dev & $\begin{array}{l}\text { Original Budgot } \\
(K s)\end{array}$ & $\begin{array}{l}\text { Budgot Priar } \\
\text { Rovision }\end{array}$ & $\begin{array}{l}\text { Changos This } \\
\text { Rovision }\end{array}$ & $\begin{array}{l}\text { Curront Total } \\
\text { Budgot }\end{array}$ & Statt & Complete & Statt & & plate \\
\hline Cost Acct. Mgr./Phon & P. J. Bence & 3,187 & & & & $10 / 01 /$ & $9 / 30 / 9$ & & & \\
\hline
\end{tabular}

Funding Source: $[X]$ Operating Expense $\square$ CENRTC FY $\square$ Line Item FY $\square$ GPP FY $\quad \square$ Small Projocts FY

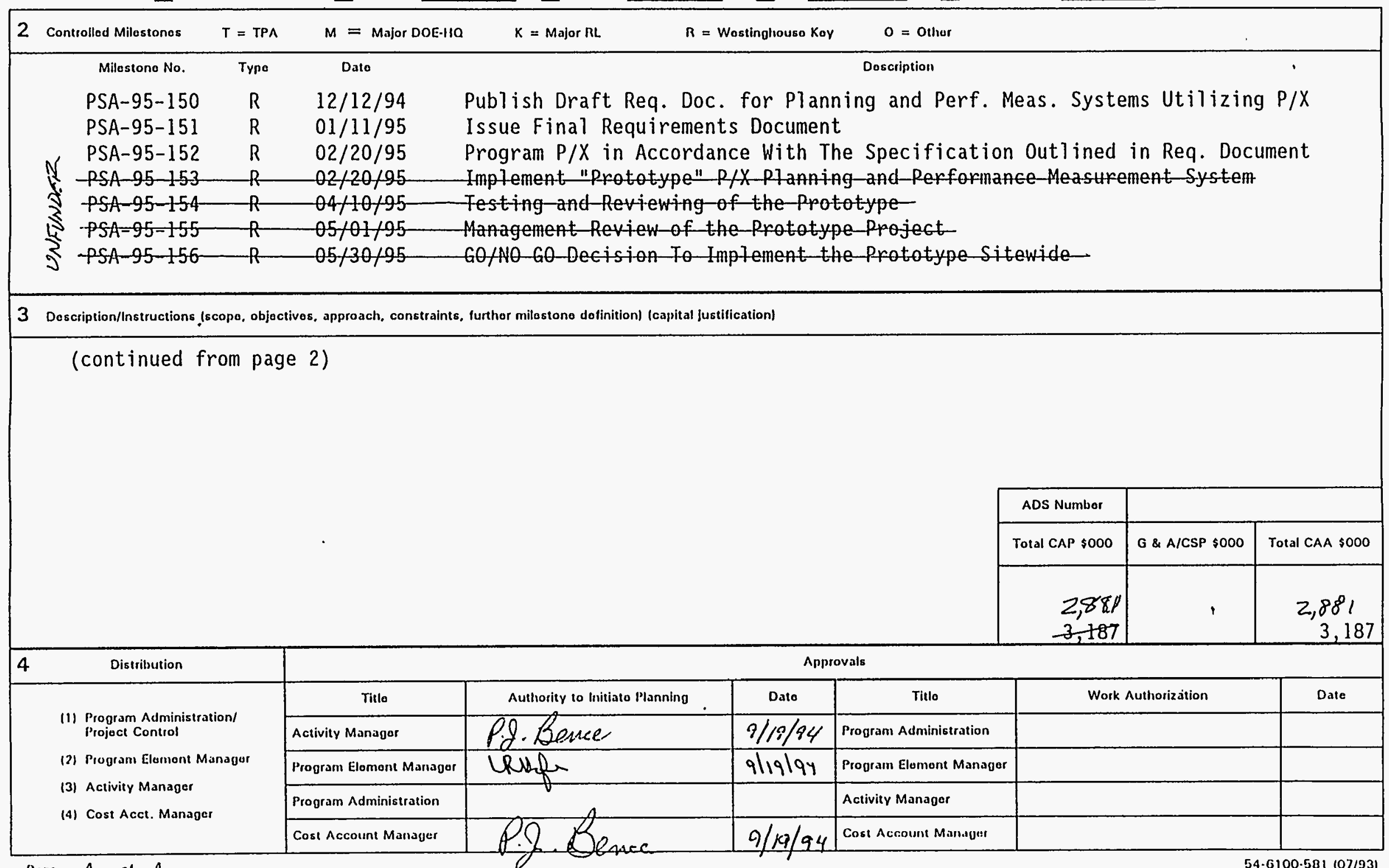




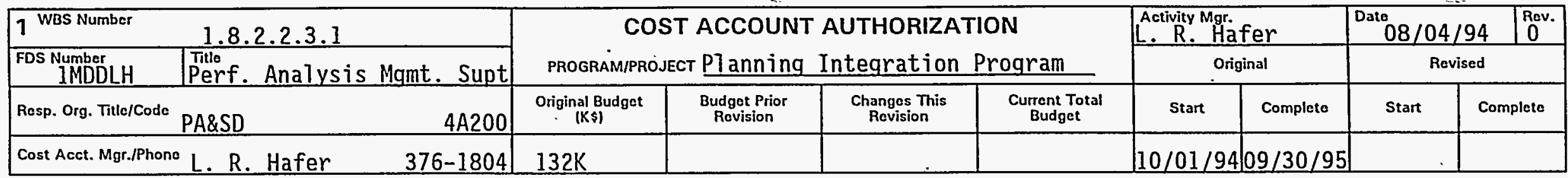

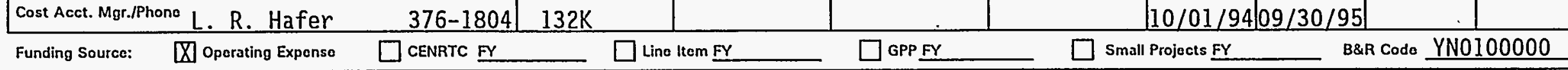

\begin{tabular}{|rccccc}
\hline 2 Controlled Milestones & $T=$ TPA & $M=$ Major DOE-HO & $K=$ Major RL & $R=$ Westinghouso Koy $\quad 0=$ Other \\
\hline Milestone No. & Typo & Dato
\end{tabular}

3 Description/Instructions (scope, objactives, approach, constraints, further milestono definition) (capital justification)

Provide program element management direction for Performance Reporting and Systems Development (1.8.2.2). Specific workscope includes reviewing the SMS Executive Summary; reviewing and approving 1.8.2.2 input to the MYPP/FYWP, SMS monthly report, and ADS submittal; attending SPI Staff meetings and conducting program element staff meetings; actively participating in business system development initiatives; monitoring and implementing Planning Integration Program Recovery Plan; attending 1.8.2 program reviews and weekly interface meetings with RL Planning Integration Division; attending financial coordination meetings and monthiy financial briefings to the Controller; acting for SPI manager as required; supporting GOCO SMS Cross-Cultivation Committee; and responding to needs of approximately 27 staff, including "management by walking around."

\begin{tabular}{|r|r|r|}
\hline ADS Number & \multicolumn{2}{|c|}{} \\
\hline Total CAP $\$ 000$ & G \& A/CSP $\$ 000$ & Total CAA $\$ 000$ \\
\hline & & \\
$132 \mathrm{~K}$ & & $132 \mathrm{~K}$ \\
\hline
\end{tabular}

\begin{tabular}{|c|c|c|c|c|c|c|c|c|}
\hline \multirow[t]{2}{*}{4} & \multirow[t]{2}{*}{ Distribution } & \multicolumn{7}{|c|}{ Approvals } \\
\hline & & Title & Authority to Initiate Planning & Date & Titla & Work Authorization & & Date \\
\hline \multirow{4}{*}{$\begin{array}{l}\text { (1) } \\
\text { (2) } \\
\text { (4) }\end{array}$} & \multirow{4}{*}{$\begin{array}{l}\text { Program Administration/ } \\
\text { Project Control } \\
\text { Program Element Manager } \\
\text { Activity Manager } \\
\text { Cost Acct. Manager }\end{array}$} & Activity Manager & L. R. Hafer URuff & 8126194 & Program Administration & J. P. Carandanis & - & \\
\hline & & Program Element Manuger & L. R. Hafer Unuf & 8126194 & Program Element Manager & L. R. Hafer & . & \\
\hline & & Program Administration & J. P. Carandanis & & Activity Manager & L. R. Hafer & & \\
\hline & & Cost Account Managor & L. R. Hafer UNuf & 8126194 & Cost Account Manager & L. R. Hafer & & \\
\hline
\end{tabular}




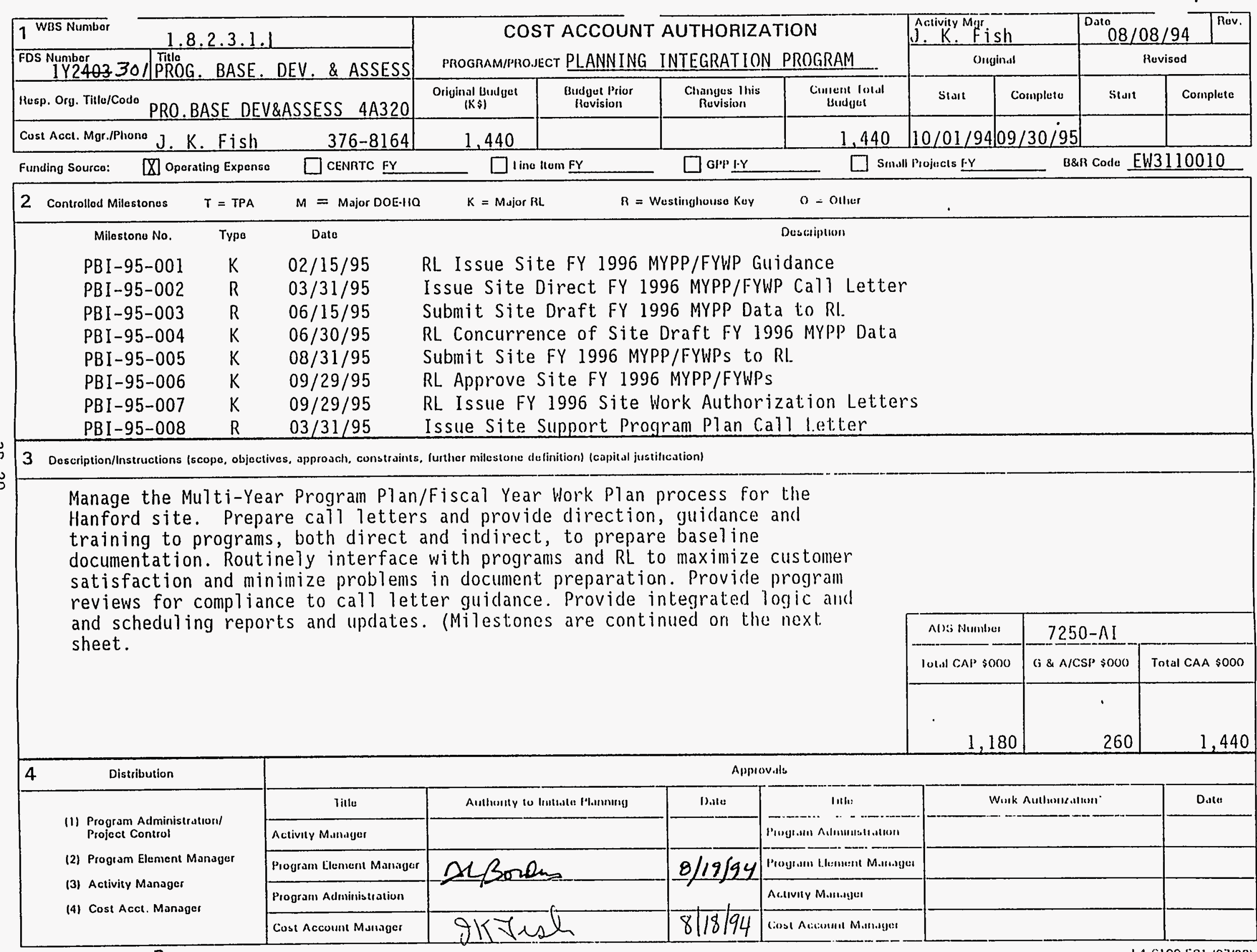




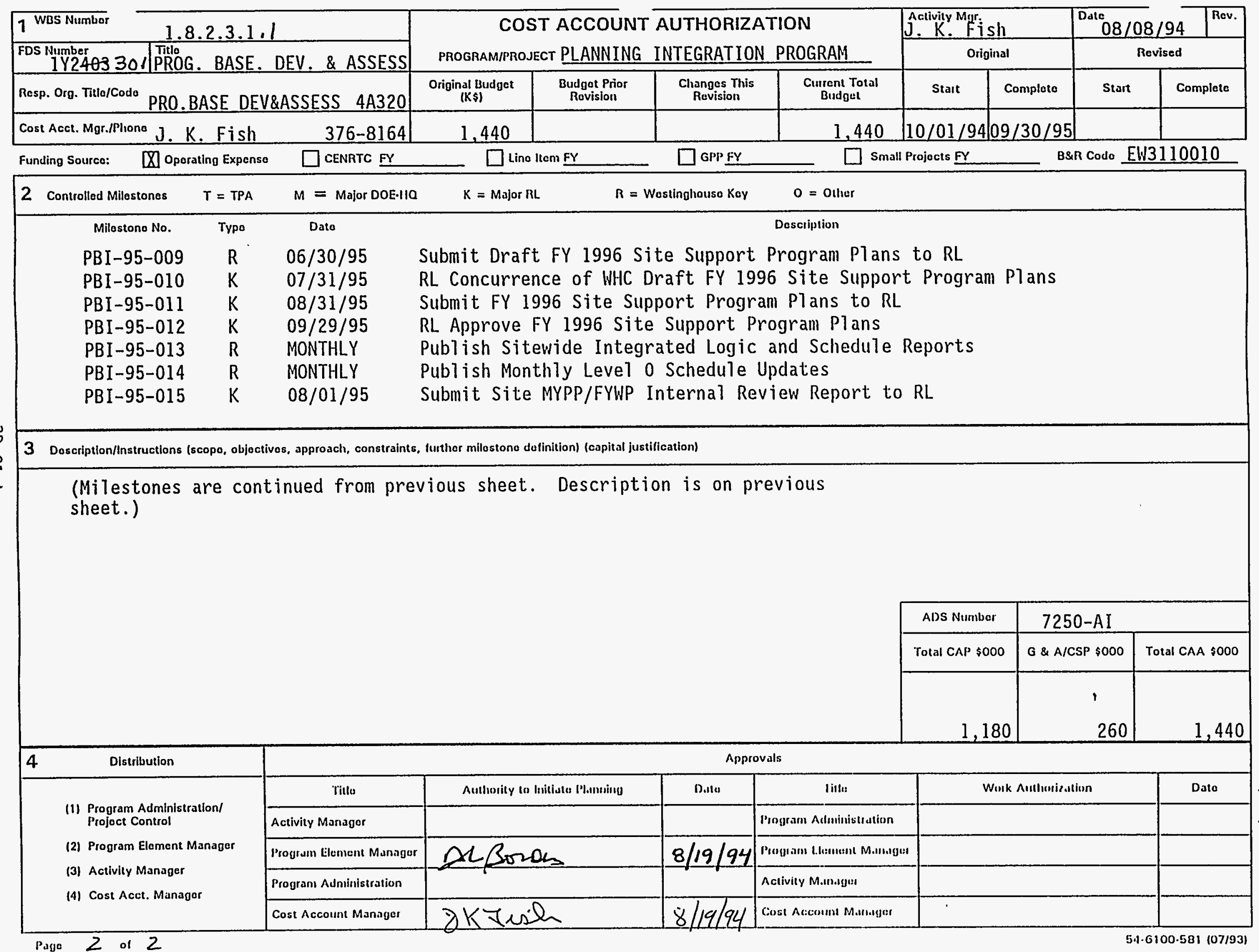




\begin{tabular}{|c|c|c|c|c|c|c|c|c|c|}
\hline 1 WBS Number $\quad 1.8 .2 .3 .2 .1$ & & ACCOUN & UTHORIZA & & $\begin{array}{l}\text { Activity M } \\
\text { D. L. }\end{array}$ & DERS & 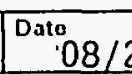 & & Rev. \\
\hline PTBI MANAGEMENT DIRECTION & PROGRAM/PR & PLANNIN & ITEGRATIOI & OGRAM & & & & & \\
\hline Rosp. Org. Titlo/Codo Prog. Baseline Int. $4 A 300$ & $\begin{array}{c}\text { Original Budgot } \\
(K \leqslant)\end{array}$ & $\begin{array}{l}\text { Budgat Prior } \\
\text { Revision }\end{array}$ & $\begin{array}{l}\text { Changes This } \\
\text { Rovision }\end{array}$ & $\begin{array}{l}\text { Curront Total } \\
\text { Budgot }\end{array}$ & Stairt & Comploto & Start & & pleto \\
\hline Cost Acct. Mgr./Phona D. L. Borders $376-9227$ & 144 & & & 144 & $10 / 01 /$ & $9 / 30 / 9$ & & & \\
\hline
\end{tabular}

Funding Source: 四 Operating Expense $\square$ CENRTC FY

$\square$ Line tem FY

$\square$ Small Projocts FY

B\&R Codo

2 Controllod Milestonos $T=$ TPA $\quad M=$ Major DOE-HQ $\quad K=$ Major RL $\quad R=$ Wastinghouse Koy $\quad O=$ Other

Milestono No. Type Date

Doscription

(A)

3 Description/Instructions (scopo, objectives, approach, constraints, further milostone dofinition) (capital justification)

Program Baseline Integration management and administration is the management

function associated with oversight of the Multi-Year Planning effort.

oversight is most significantly focused on the direction and integration of

program baseline plans and integrated logics/schedules. This activity is

associated with ADS 7250 , Subactivity AI.

The key assumption which directs the workscope associated with this activity include acknowledgement that the mission of the PBI organization is to direct, coordinate, and support effective planning by responsible line organizations. This includes the development of the planning required to guide site programs in the preparation of their multiyear baselines using integrated planning techniques and which can be validated through independent reviews and audits.

\begin{tabular}{|c|c|}
\hline 4 & Distribution \\
\hline (1) & $\begin{array}{l}\text { Program Administration/ } \\
\text { Projoct Control }\end{array}$ \\
\hline (2) & Program Element Manager \\
\hline (3) & Activity Manager \\
\hline (4) & Cost Acct. Manager \\
\hline
\end{tabular}

Page _ $\quad$ of

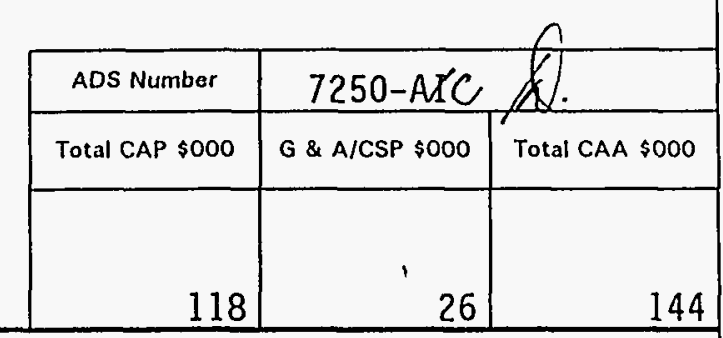

Approvals

\begin{tabular}{|c|c|c|c|c|c|}
\hline Title & Authority to Initiato Planning & Date & Titlo & Work Authorization & Dato \\
\hline Activity Manager & & & Programı Administration & & \\
\hline Program Element Manager & ALBoras & $8 / 26 / 94$ & Program Element Manager & & \\
\hline Program Administration & & & Activity Manager & & \\
\hline Cost Account Manager & AlBora & $8 / 26 / 94$ & Cost Account Manager & & \\
\hline
\end{tabular}

54-6100-581 (07/93) 


\begin{tabular}{|c|c|c|c|c|c|c|c|c|c|c|}
\hline \multicolumn{2}{|c|}{1 WBS Number $\quad 1.8 .2 .4 .1 .1$} & \multirow{2}{*}{\multicolumn{4}{|c|}{$\begin{array}{l}\text { COST ACCOUNT AUTHORIZATION } \\
\text { PROGRAM/PROJECT PLANNING INTEGRATION }\end{array}$}} & \multirow{2}{*}{\multicolumn{2}{|c|}{$\frac{\text { Activity Mgr. }}{\text { J. L. COLLINGS }}$}} & \multicolumn{3}{|c|}{ 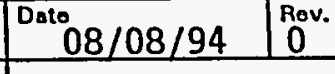 } \\
\hline $\begin{array}{l}\text { FDS Numbor } \\
1 Y 2401 \\
\end{array}$ & $\begin{array}{l}\text { Titlo } \\
\text { SYSTEMS ENGR. ARCH. DEV. }\end{array}$ & & & & & & & \multicolumn{3}{|c|}{ Rovisod } \\
\hline Resp. Org. Titlo/Codo & SYSTEMS ENGINEERING $8 F 300$ & $\begin{array}{l}\text { Original Budgot } \\
(\mathrm{K} \$)^{-}\end{array}$ & $\begin{array}{l}\text { Budgot Prior } \\
\text { Rovision }\end{array}$ & $\begin{array}{l}\text { Changos This } \\
\text { Rovision }\end{array}$ & $\begin{array}{l}\text { Curront Total } \\
\text { Budgot }\end{array}$ & Start & Complote & Start & & plato \\
\hline Cost Acct. Mor./Phono & J. L. COLLINGS & 1,321 & & & 1,321 & $10 / 01 /$ & $09 / 30 / 95$ & & & \\
\hline
\end{tabular}

Funding Sourco: $\square$ X Oporating Expenso $\square$ CENATC FY $\square$ Lino Itom FY $\square$ GPPFY

\begin{tabular}{|cccccc}
2 Controliod Milostones & $T=$ TPA & $M=$ Major DOE-HQ & $K=$ Major RL & $R=$ Wostinghouso Koy $\quad 0=$ Othor \\
\hline Milostono No. & Typo & Dato & Doscription
\end{tabular}

$\begin{array}{lccl}\text { SSE-95-1 } & \text { T } & 12 / 30 / 94 & \text { HMP BASEL INE DEVELOPMENT, } \\ \text { SSE-95-6 } & R & 01 / 31 / 95 & \text { DYNAMIC SYSTEMS MODEL } \\ \text { SSE-95-7 } & T & 08 / 31 / 95 & \text { TPA MILESTONES M33-00-TO4 } \\ \text { SSE-95-10 } & \text { K } & 09 / 29 / 95 & \text { BASELINE SPECIFICATION }\end{array}$

3 Description/Instructions (scopo, objoctivos, approach, constraints, furthor milostono definition) (capital justification)

The primary thrust of this cost account is the development of a technical specification of the Hanford cleanup mission based on systems engineering analysis. This is the first issue of a specification for the Hanford cleanup system. This element is complete when the cleanup system is fully specified with respect to end state and mission delivery system. Since the cleanup system is expected to continually evolve over mission life, the systems engineering work will continue until the cleanup mission is complete.

This work is directly funded by the 1.8 .2 program. Closely rèlated work in 1.8.2.4.1.2 is overhead funded.

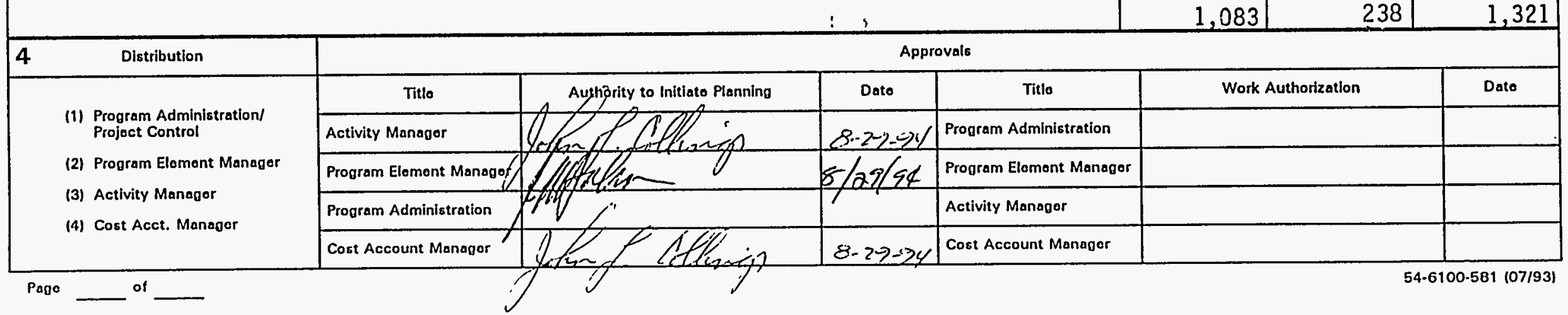




\begin{tabular}{|c|c|c|c|c|c|c|c|c|c|}
\hline \multirow{2}{*}{\begin{tabular}{|l|l|}
1 WBS Number & 1.8 .2 .4 .1 .2 \\
$\begin{array}{l}\text { FDS Numbor } \\
\text { 1MDDLA }\end{array}$ & $\begin{array}{l}\text { Titlo } \\
\text { SYSTEMS ENG INEERING }\end{array}$ \\
\end{tabular}} & \multirow{2}{*}{\multicolumn{4}{|c|}{$\begin{array}{l}\text { COST ACCOUNT AUTHORIZATION } \\
\text { PROGRAM/PROJECT PLANNING INTEGRATION }\end{array}$}} & \multirow{2}{*}{\multicolumn{2}{|c|}{\begin{tabular}{|c|c|c|} 
Activity Mgr. \\
O. L. COLiginal \\
\end{tabular}}} & \multirow{2}{*}{\multicolumn{3}{|c|}{\begin{tabular}{r|} 
Dato \\
Rovisod \\
\end{tabular}}} \\
\hline & & & & & & & & & \\
\hline Rosp. Org. ritlo/Codo SYSTEMS ENGINEERING $8 F 300$ & $\begin{array}{l}\text { Original Budgot } \\
\text { (K\$) }\end{array}$ & $\begin{array}{l}\text { Budgot Prior } \\
\text { Rovision }\end{array}$ & $\begin{array}{l}\text { Changes This } \\
\text { Aovision }\end{array}$ & $\begin{array}{l}\text { Curront Total } \\
\text { Budget }\end{array}$ & Start & Comploto & Start & & plato \\
\hline Cost Acct. Mor./Phono 3. L. COLLINGS & 944 & & & 944 & $10 / 01 /$ & $99 / 30 / 95$ & & & \\
\hline
\end{tabular}

Funding Source: $[\mathbb{X}$ Oporating Exponso $\square$ CENRTC FY

$\square$ Lino ltom Fy $\square$ GPPFY

$\square$ Small Projocts FY

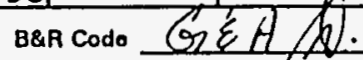

\begin{tabular}{|ccccccc}
\hline 2 Controllod Milostonos & $T=$ TPA & $M=$ Major DOE-HQ & $K=$ Major RL & $R=$ Wostinghouso Koy & $O=$ Other \\
\hline Milostono No. & Typo & Dato & Description
\end{tabular}

SSE-95-2 K $\quad$ 03/29/95 UPDATE FUNCTIONAL ANALYSIS, REV. 0

SSE-95-3 K 05/24/95 REQUIREMENTS ANALYSIS ALLOCATION, REV. 0

SSE-95-4 K $05 / 23 / 95$ UPDATE BASELINE MEASURES, REV. 0

SSE-95-5 K 08/31/95 REVISED ARCHITECTURE AND PRODUCTS DEFINITIONS, REV. 0

3 Doscription/Instructions (scope, objectives, approach, constraints, furthor milostono dofinition) (capital justification)

The primary thrust of this cost account is the development of a technical specification for the Hanford cleanup mission based on systems engineering analysis. The element is complete when the cleanup system is fully specified with respect to end state and mission delivery system. Since the cleanup system is expected to continually evolve over mission life, the systems engineering work will continue until the cleanup mission is complete.

The work is overhead funded and is closely related to element 1.8.2.4.1.1.

\begin{tabular}{|r|r|r|}
\hline ADS Numbor & \multicolumn{2}{|c|}{ G:A } \\
\hline Total CAP $\$ 000$ & G \& A/CSP $\$ 000$ & Total CAA $\$ 000$ \\
\hline & 1 & \\
944 & & 944 \\
\hline
\end{tabular}

\begin{tabular}{|c|c|c|c|c|c|c|}
\hline 4 & Distribution & \multicolumn{5}{|c|}{ Approvals } \\
\hline & \multirow{5}{*}{$\begin{array}{l}\text { (1) Program Administration/ } \\
\text { Project Control } \\
\text { (2) Program Element Manager } \\
\text { (3) Activity Managor } \\
\text { (4) Cost Acct. Managor }\end{array}$} & Aythority to Initiato Planning & Dato & Titlo & Wark Authorization & Date \\
\hline & & Activity Managor & $8-2 \rightarrow \rightarrow 4$ & Program Administration & & \\
\hline & & Program Eloment Managgr & $57-27-94$ & Program Elomont Managor & & \\
\hline & & Program Administration & & Activity Managor & & \\
\hline & & Cost Account Managor & $(3 .-70)-24$ & Cost Account Maragor & & \\
\hline
\end{tabular}




\begin{tabular}{|c|c|c|c|c|c|c|c|c|c|c|c|}
\hline \multicolumn{3}{|c|}{1 WBS Number $\quad 1.8 .2 .4 .2 .1$} & \multirow{2}{*}{\multicolumn{4}{|c|}{$\begin{array}{l}\text { COST ACCOUNT AUTHORIZATIQN } \\
\text { PROGRAM/PROJECT PLANNING INTEGRATION }\end{array}$}} & \multirow{2}{*}{\multicolumn{2}{|c|}{\begin{tabular}{|l|} 
Activity Mar. WOOODS \\
Original \\
\end{tabular}}} & \multirow{2}{*}{\multicolumn{3}{|c|}{\begin{tabular}{r|} 
Dato $08 / 08 / 94$ \\
Rovisod \\
\end{tabular}}} \\
\hline \multirow{2}{*}{$\frac{\text { FDS Numbor }}{\text { IMDDLB }}$} & \multicolumn{2}{|c|}{\begin{tabular}{|l} 
Titlo \\
SYSTEMS ENGINEERING MGMT.
\end{tabular}} & & & & & & & & & \\
\hline & SE MANAGEMENT & $8 F 200$ & $\begin{array}{l}\text { Original Budgot } \\
\text { (K\$) }\end{array}$ & $\begin{array}{l}\text { Budget Prior } \\
\text { Rovision }\end{array}$ & $\begin{array}{c}\text { Changes This } \\
\text { Rovision }\end{array}$ & $\begin{array}{l}\text { Current Total } \\
\text { Budgot }\end{array}$ & Start & Comploto & Start & & ploto \\
\hline Cost Acct. Mgr./Phone & T. W. WOODS & $376-1375$ & 800 & & & 800 & $10 / 01$ & $09 / 30 / 95$ & & & \\
\hline
\end{tabular}

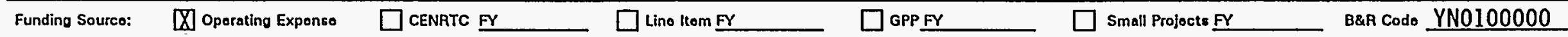

\begin{tabular}{|c|c|c|c|c|c|}
\hline 2 & Controllod Milestones & $T=T P A$ & $M=$ Major DOE-Ha & $R=$ Wostinghouso Koy & $0=$ Other \\
\hline & Milestone No. & Typo & Dato & & Description \\
\hline & SSE-95-11 & K & $01 / 11 / 95$ & ISSUE REVISED HS-SEMP/SEM BASED & FIELD EXPERIENCE (REV 1) \\
\hline & SSE-95-12 & $\mathbf{R}$ & $03 / 31 / 95$ & SE ENGINEERING PRACTICES AND PROC & DURES STATUS REPORT \\
\hline & SSE $-95-13$ & $R$ & $07 / 20 / 95$ & SE TRAINING STATUS REPORT & \\
\hline & SSE-95-14 & K & $01 / 31 / 95$ & ISSUE REVISED SITE SE-CMP & \\
\hline & SSE-95-15 & $\mathrm{R}$ & $08 / 28 / 95$ & COMPLETE DEFINITION INFORMATION & RUCTURE AND CONTROL SYSTEM \\
\hline
\end{tabular}

3 Description/lnstructione (scopo, objoctivos, approach, conetraints, furthor milostono dofinition) (capital justification)

Manage and control formal site systems engineering baselines to include mission/functional definition, requirements, alternatives, technical performance, and design configuration. This cost account will also provide development and integration of program and project SEMPs, SEMs, and other implementation standards and procedures. Policies, processes and specifications necessary to support systems engineering performance at Hanford will be provided and maintained. Information management procedures for maintaining the systems engineering baselines will be established to support an effective configuration management program and to provide linkage to the Site Management System (SMS).

\begin{tabular}{|r|r|r|}
\hline ADS Number & \multicolumn{1}{|c|}{ N/A } \\
\hline Total CAP $\$ 000$ & O \& A/CSP $\$ 000$ & Total CAA $\$ 000$ \\
\hline & & \\
800 & & 800 \\
\hline
\end{tabular}

\begin{tabular}{|c|c|c|c|c|c|c|c|}
\hline 4 & Distribution & & & & ovals & & \\
\hline & & Titlo & Authosity to Initiage Planning & Dato & Titlo & Work Authorization & Dato \\
\hline & Project Control & Activity Managor & Tes & $8 / 34 / \pi$ & Program Administration & & \\
\hline & Program Elomont Manager & Program Eloment Managar & & $F=-29 / 5$ & Program Eloment Managor & & \\
\hline & I) Activity managor & Program Administration & & & Activity Manager & & \\
\hline & & Cost Account Manager & & & Cost Account Manager & & \\
\hline
\end{tabular}




\begin{tabular}{|c|c|c|c|c|c|c|c|c|c|}
\hline $1.8 .2 .5 .1,1$ & & ACCOUN & UTHORIZ & & Activity N & & Dato $06 /$ & & $\begin{array}{l}\text { Rov. } \\
0\end{array}$ \\
\hline \begin{tabular}{c|l} 
FDS Numbor \\
$1 Y 2205501$ & Tito \\
Program Mamt. Support
\end{tabular} & PROGRAM/PR & Plannin & tegratio & & & & & & \\
\hline Resp. Org. Titlo/Codo Plng Integ Prog Mgm $4 \mathrm{~A} 800$ & $\begin{array}{l}\text { Original Budgat } \\
(K \$)\end{array}$ & $\begin{array}{l}\text { Budgot Prior } \\
\text { Revision }\end{array}$ & $\begin{array}{l}\text { Changes This } \\
\text { Rovision }\end{array}$ & $\begin{array}{l}\text { Current Total } \\
\text { Budgot }\end{array}$ & Start & Comploto & Start & & pletc \\
\hline Cost Aact. Mgr./Phono R. E. Dejtrich & & & & & $10 / 01$ & $9 / 30 / 5$ & & & \\
\hline
\end{tabular}

D

$\square$ Lino Item FY

] GPP FY B\&R Code EW311001

2 Controlled Milostones $T=T P A \quad M=$ Major DOE-HQ $\quad K=$ Major RL $\quad R=$ Wostinghouse Kay $O=O$ other

Milestono No. Type Date Description

$\begin{array}{llll}\text { PIM-95-001 } & R & 11 / 14 / 94 & \text { ADS } 7250 \text { Kick-0ff Meeting } \\ \text { PIM-95-002 } & R & 01 / 16 / 95 & \text { ADS 7250 Preparation in Prod }\end{array}$

PIM-95-002 $R$ 01/16/95 ADS 7250 Preparation in Process Review

PIM-95-003 R $01 / 20 / 95$

PIM-95-004 K $01 / 30 / 95$

PIM-95-005 R $04 / 07 / 95$

PIM-95-006 R 04/14/95

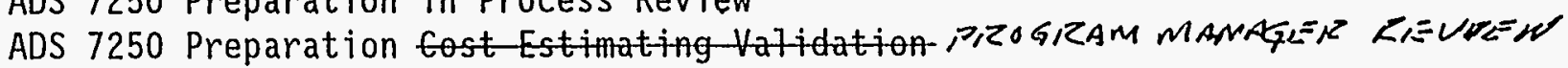

Submit Draft ADS 7250 to RL for Review and Comment

PIM-95-007 R $\quad$ 06/19/95

Complete Development of ADS 7250

FY 1996 MYPP/FYWP Kick-0ff Meeting

PIM-95-008 R $07 / 14 / 95$

MYPP/FYWP in Process Reviews

3 Description/Instructions (scope, objectives, approach, constraints, further milestono definition) (capital justification)

Provide program management support for the Planning Integration Program.

This scope includes support of the planning process, development of the

Planning Integration MYPP/FYWP, development of the ADS for Planning

Integration. Also includes interfacing with the program element teams to

gather information on program activities for preparation of monthly SMS and

PTS reports as requested by RL and DOE-HQ, respectively, as well as other

financial and budgetary reports as required for program. Also includes

coordination and administration of proper change control upon approval of

baseline. This program element will be the focal point for RL inquiries pertaining to the 1.8.2 Planning Integration Program: There is a program coordination effort which requires attendance to all program elements staff meetings, RL interface meetings and presentation of program review data. The performance analys is of each 1.8.2 Planning Integration program element

\begin{tabular}{l}
\hline 4 Distribution \\
\hline (1) Program Administration/ \\
Projact Control \\
(2) Program Element Manager \\
(3) Activity Manager \\
(4) Cost Acct. Manager
\end{tabular}

Page 1 of 2

\begin{tabular}{|c|c|c|c|c|c|}
\hline \multicolumn{6}{|c|}{ Approvals } \\
\hline Titlo & Authority to Initiato Planning & Dato & Titlo & Work Authorization & Date \\
\hline Activity Manager & D. B. Pabst & $8 / 14 / 44$ & Program Administration & J.P. Cauratalis s & $8 / 19 / 9$ \\
\hline Program Element Manager & D. B. Pabst 4 Muts & 1/ & Program Element Manager & D. B. Pabst & $-8 / 19 / 94$ \\
\hline Program Administration & J. P. Carantanto L & $8 / 14 / 94$ & Activity Managor & D. B. Pabst $\mathbb{N}$ MI & $Y^{1}$ \\
\hline Cost Account Manager & R. E. Dertistiptillent & $8 / 14 / 84$ & Cost Account Manager & R. Eferethobolstrel & $8 / 19 / 84$ \\
\hline
\end{tabular}

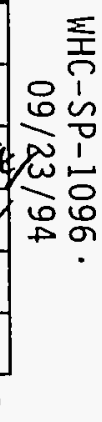




\begin{tabular}{|c|c|c|c|c|c|c|c|c|}
\hline \multirow{2}{*}{ 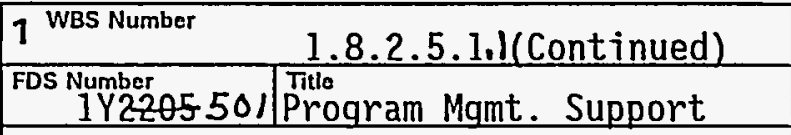 } & \multirow{2}{*}{\multicolumn{4}{|c|}{$\begin{array}{l}\text { COST ACCOUNT AUTHORIZATION } \\
\text { PROGRAM/PROJECT Planning Integration }\end{array}$}} & \multirow{2}{*}{\multicolumn{2}{|c|}{\begin{tabular}{|c|} 
Activity Mgr. B. Pabst \\
Original \\
\end{tabular}}} & \multirow{2}{*}{\multicolumn{2}{|c|}{ 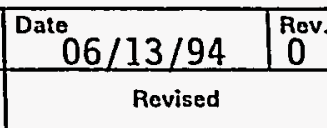 }} \\
\hline & & & & & & & & \\
\hline 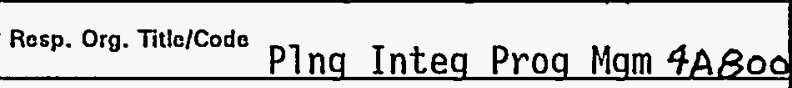 & $\begin{array}{c}\text { Original Budget } \\
\text { (Ks) }\end{array}$ & $\begin{array}{l}\text { Budget Prior } \\
\text { Revision }\end{array}$ & $\begin{array}{l}\text { Changes This } \\
\text { Revision }\end{array}$ & $\begin{array}{c}\text { Curront Total } \\
\text { Budget }\end{array}$ & Start & Complete & Start & Complete \\
\hline Cost Acct. Mgr./Phone R. E. Deitrich & & & & & $10 / 01 /$ & $09 / 30 / 95$ & & \\
\hline
\end{tabular}

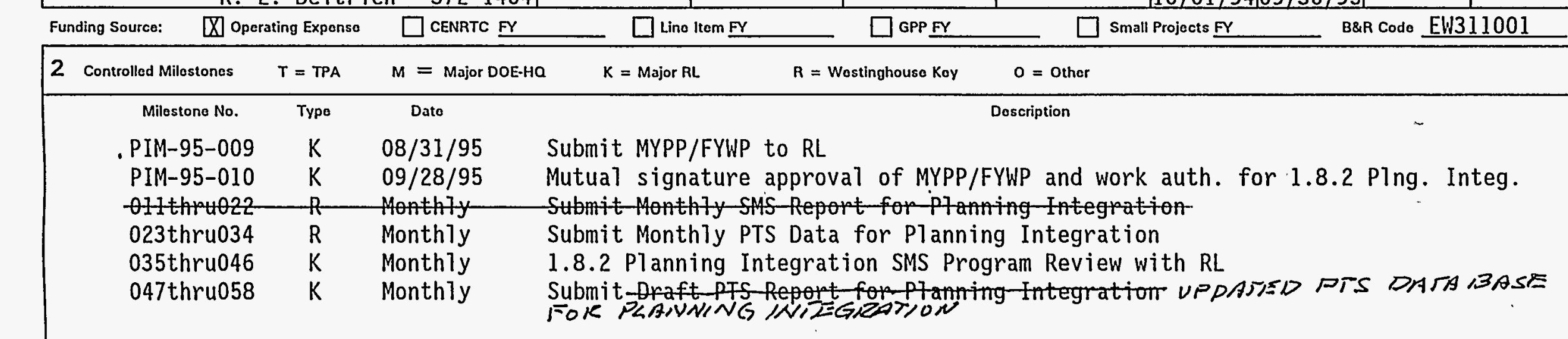

3 Description/Instructions (scopo, objoctives, approach, constraints, furthor milestono dofinition) (capital justification)

(Continued)

will be the responsibility of this organization.

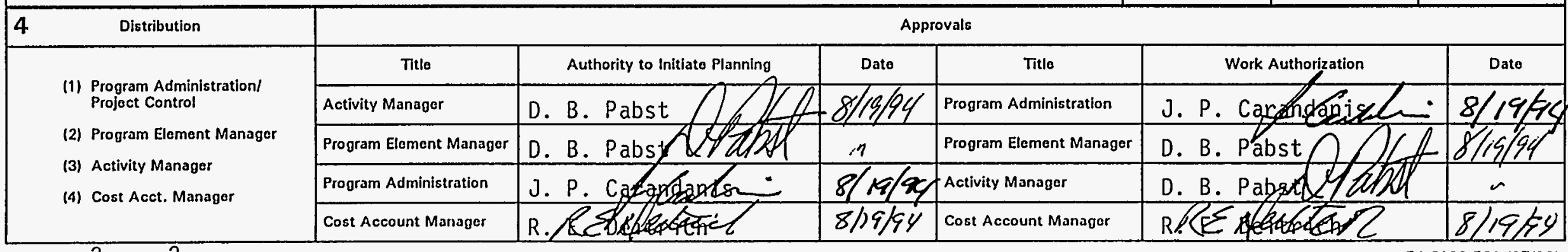

Pago 2 of 2

\begin{tabular}{|c|c|c|}
\hline ADS Numbor & \multicolumn{2}{|c|}{} \\
\hline Total CAP \$000 & G \& A/CSP \$000 & Total CAA \$000 \\
\hline & $\cdot$ & \\
& & \\
\hline
\end{tabular}

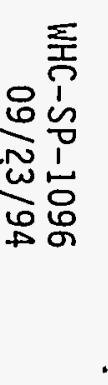




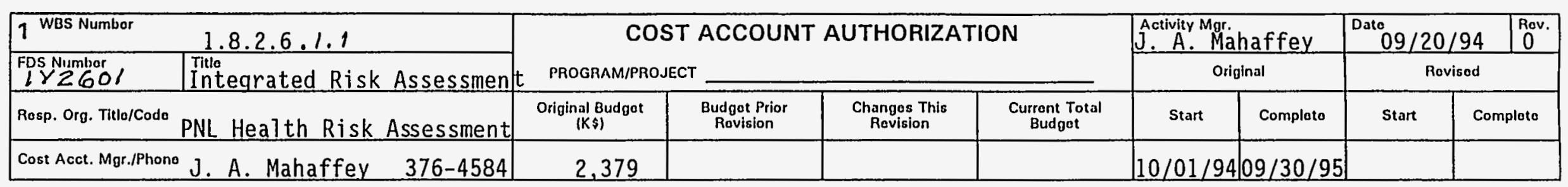

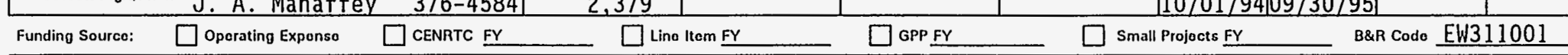

\begin{tabular}{|cccccc}
\hline 2 Controlled Milestones & $T=T P A$ & $M=$ Major DOE-HO & $K=$ Major RL & $R=$ Westinghouse Koy & $O=$ Other \\
\hline Milestone No. & Type & Dato & Description
\end{tabular}

$\begin{array}{llll}\text { IRA-95-001 } & K & 05 / 30 / 95 & \text { Guidance to programs as part of FY } 1996 \text { MYPP guidance from } 1.8 .2 \\ \text { IRA-95-002 } & K & 09 / 30 / 95 & \begin{array}{l}\text { Draft report on public health risk validation and quantification } \\ \text { IRA-95-003 }\end{array} \\ \text { K } & 09 / 30 / 95 & \begin{array}{l}\text { Draft report on qualitative evaluation of public, worker, and ecological } \\ \text { baseline risks }\end{array}\end{array}$

3 Description/Instructions (scope, objectives, approach, constraints, further milestono definition) (capital justification)

The technical scope of the Integrated Risk Assessment Program (IRAP) includes providing risks at the current tịme, during environmental restoration and waste management activities, and when the final site endstate is achieved. Receptors for which these risks will be produced are the general public including those with reserved rights, workers, the ecosystem, and natural resources. If these two (receptors by time) are considered as a matrix, there are 15 cells for which risk results are needed, and some cells will require more than one set of assumptions be considered. During FY 1995 the public health baseline risk assessment product from FY 1994 will be validated and a similar scoring system will be developed for worker and ecological risks. Guidance will be developed for Hanford programs regarding FY 1996 inputs to IRAP. IRAP products, risk assessment results (and combinations in various ways to provide information to other Hanford and DOE activities), will be documented in the form of reports. Accomplishment of the matrix of IRAP risk assessments will take several years for initial completion. Updates will occur subsequently.

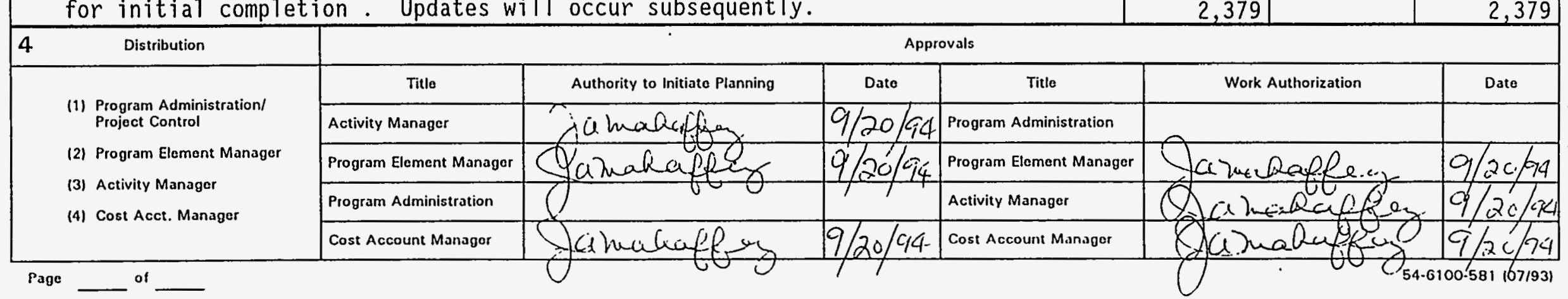




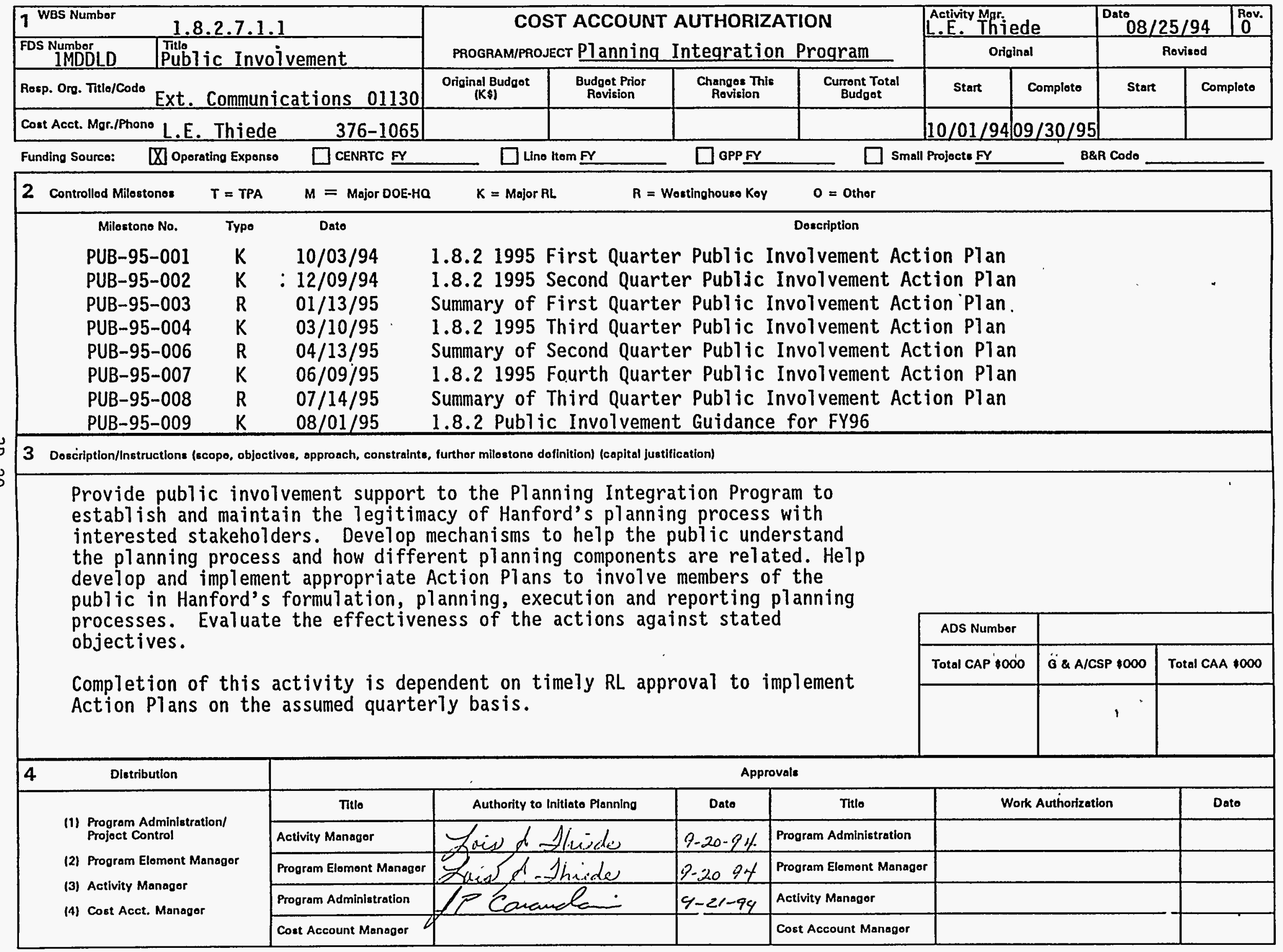




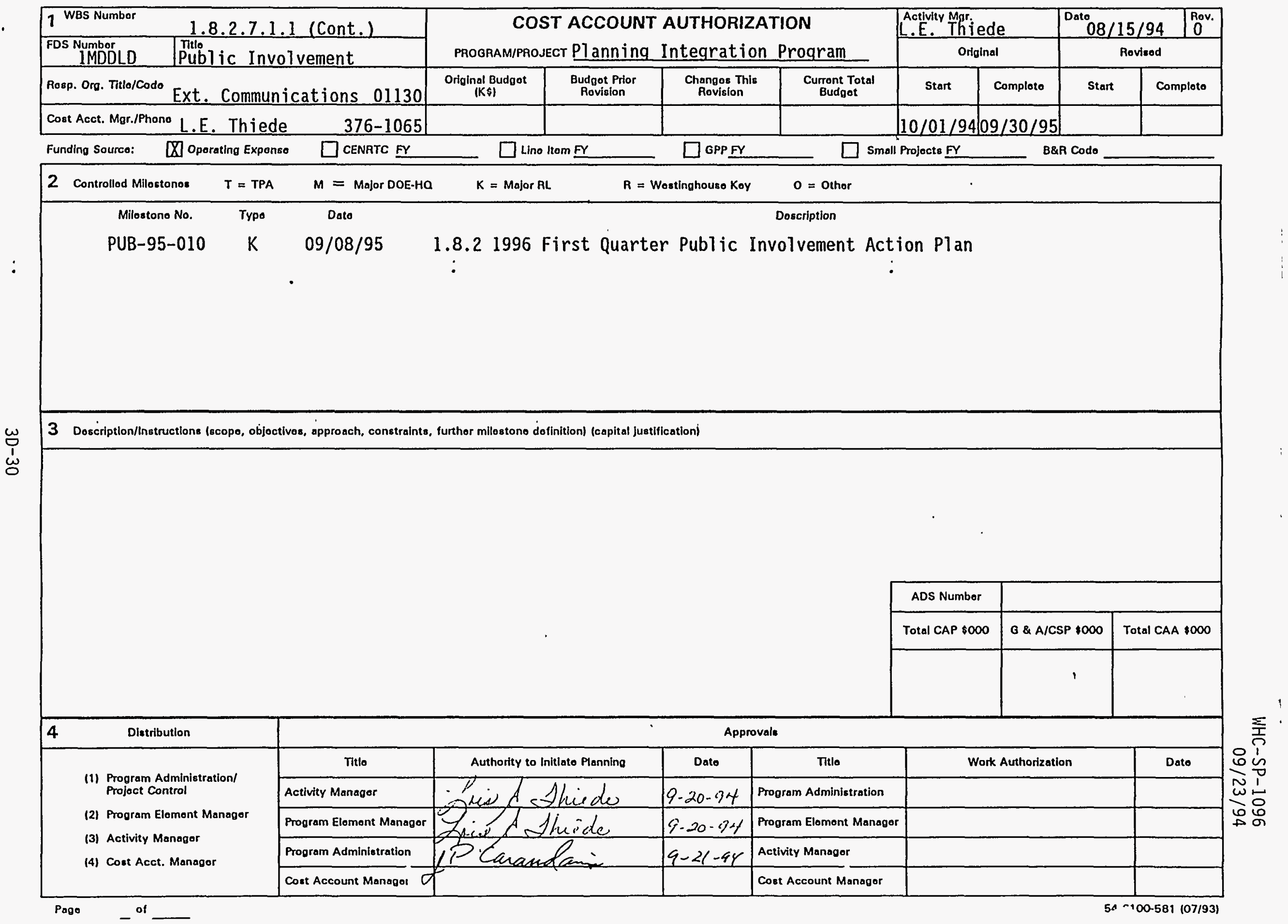




\begin{tabular}{|c|c|c|c|c|c|c|c|c|c|}
\hline \multirow{2}{*}{ 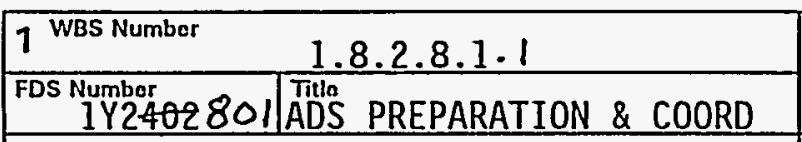 } & \multirow{2}{*}{\multicolumn{4}{|c|}{$\begin{array}{l}\text { COST ACCOUNT AUTHORIZATION } \\
\text { PROGRAM/PROJECT PLANNING INTEGRATION PROGRAM }\end{array}$}} & \multirow{2}{*}{\multicolumn{2}{|c|}{$\begin{array}{r}\text { Activity Mar. } \\
\text { EDWÁRS } \\
\text { Original } \\
\end{array}$}} & \multirow{2}{*}{\multicolumn{3}{|c|}{\begin{tabular}{|r|}
$08 / 22 / 94$ \\
Revised \\
\end{tabular}}} \\
\hline & & & & & & & & & \\
\hline Resp. Org. Titto/Codo ADS PREP. \& COORD & $\begin{array}{c}\text { Original Budget } \\
(K \$)\end{array}$ & $\begin{array}{l}\text { Budgot Prior } \\
\text { Revision }\end{array}$ & $\begin{array}{l}\text { Changes This } \\
\text { Rovision }\end{array}$ & $\begin{array}{l}\text { Current Total } \\
\text { Budget }\end{array}$ & Start & Complete & Start & & plete \\
\hline Cost Acct. Mgr./Phona C. EDWARDS & 1,022 & & & 1,022 & $10 / 01 /$ & $9 / 30 / 95$ & & & \\
\hline
\end{tabular}

Funding Source: $\quad \mathbb{X}$ Operating Expenso $\square$ CENRTC FY $\square$ Line ltem FY $\square$ GPP FY $\square$ Small Projects FY

\begin{tabular}{|c|c|c|c|c|c|c|}
\hline 2 & Controlled Milestones & $T=T P A$ & $M=$ Major DOE-Ha & $K=$ Major RL. & $\mathrm{R}=$ Wostinghouse Key & $0=$ other \\
\hline & Milestono No. & Typo & Date & & & escription \\
\hline
\end{tabular}

$\begin{array}{llll}\text { APC-95-001 } & K & 11 / 01 / 94 & \text { Receive FY 1997-2001 ADS Guidance from RL } \\ \text { APC-95-002 } & R & 11 / 11 / 94 & \text { Issue ADS Guidance Letter to Programs } \\ \text { APC-95-003 } & K & 01 / 06 / 95 & \text { Submit Draft FY 1996-1997 Building Blocks to HQ } \\ \text { APC-95-004 } & M & 02 / 17 / 95 & \text { Submit Draft FY 1997-2001 ADSs and FY 1996-1997 Bldg Blocks } \\ \text { PC-95-005 } & K & 03 / 17 / 95 & \text { Submit Internal ADS Review Report to RL } \\ \text { APC-95-006 } & K & 04 / 14 / 95 & \text { Submit Final FY 1997-2001 ADSs and Building Blocks to RL } \\ \text { APC-95-007 } & M & 04 / 14 / 95 & \text { Submit Final FY 1997-2001 ADSs and Bujlding Blocks to HQ } \\ \text { APC-95-008 } & K & 11 / 01 / 94 & \text { Publish Hanford Site Focus }\end{array}$

3 Description/Instructions (scope, objoctives, approach, constraints, further milestone definition) (capital justification)

The out-year planning activity provides for the coordination, integration, development, review, submittal, distribution and maintenance of the Hanford Site Environmental Restoration (ER) and Waste Management (WM) ADSs and

Building Blocks. This provides a summary of Hanford's Waste Management and ER status and planning. Interface with the public is included. The overall focus of these activities is the development of the Hanford Site budget request for EM/WR workscope.

Also included is the maintaining of the Environmental Planning Data Base System (EPDS), a local database system which tracks all aspects of the ADS planning process and support to the coordination of external cost estimate and budget development reviews. Included in the EPDS activity is the support for maintenance and further system enhancements by WHC and IRM

Distribution
(1) Program Administration/
Project Control
(2) Program Eloment Manager
(3) Activity Manager
(4) Cost Acct. Manager

1 of 2

\begin{tabular}{|c|c|c|c|c|c|}
\hline \multicolumn{6}{|c|}{ Approvals } \\
\hline Titlo & Authority to Initiate Planning & Dato & Titlo & Work Authorization & Date \\
\hline Activity Managor & K $\quad B \quad b$ & $f / 2 y / 9 x$ & Program Administration & & \\
\hline Program Eloment Manager & Dx/sorom & $8 / 25 / 94$ & Program Element Manager & & \\
\hline Program Administration & & & Activity Manager & & \\
\hline Cost Account Manager & & & Cost Account Managor & & \\
\hline
\end{tabular}

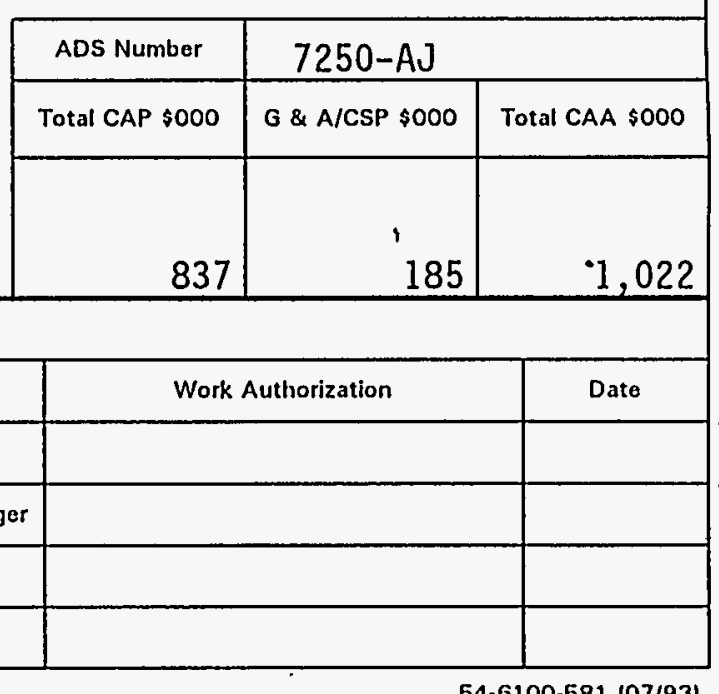

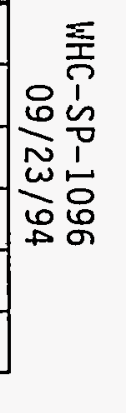




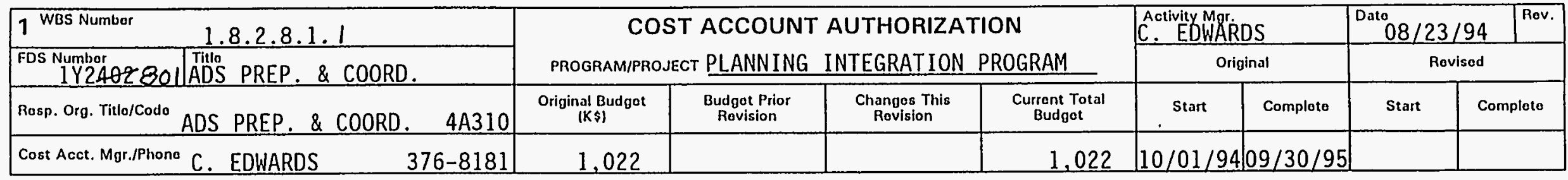

Funding Source: $\quad \mathbb{X}$ Oporating Expense $\square$ CENRTC FY

$\square$ Line Itom FY

$\square$ Small Projocts FY

B\&R Code

\begin{tabular}{|rccccc}
\hline 2 Controlled Milostones & $T=$ TPA & $M=$ Major DOE-HO & $K=$ Major RL & $. R=$ Westinghouse Key $\quad O=$ Other \\
\hline Milostono No. & Typo & Date & & Description \\
APC-95-009 & $K$ & $01 / 27 / 95$ & Publish Supplement to Hanford Site Focus
\end{tabular}

3 Description/Instructions (scopo, objoctives, approach, constraints, further milostono definition) (capital justification)

(Milestones are continued from previous sheet. Description is on previous sheet.)

\begin{tabular}{|c|c|c|c|c|c|c|c|}
\hline & & & & & \multirow{2}{*}{$\begin{array}{c}\text { ADS Number } \\
\text { Total CAP } \$ 000\end{array}$} & \multicolumn{2}{|l|}{$7250-\mathrm{AJ}$} \\
\hline & & & & & & G \& A/CSP $\$ 000$ & Total CAA $\$ 000$ \\
\hline & & & & & 837 & 185 & 1,022 \\
\hline Distribution & \multicolumn{7}{|c|}{ Approvals } \\
\hline \multirow{5}{*}{$\begin{array}{l}\text { (1) Program Administration/ } \\
\text { Project Control } \\
\text { (2) Program Element Managar } \\
\text { (3) Activity Manager } \\
\text { (4) Cost Acct. Manager }\end{array}$} & Title & Authority to Initiato Planning & Dato & Title & \multicolumn{2}{|c|}{ Work Authorization } & Date \\
\hline & Activity Manager & 5 & 8123194 & Program Administration & & & \\
\hline & Program Eloment Manager & ArBongen & $8 / 25 / 94$ & Program Eloment Manager & & & \\
\hline & Program Administration & & & Activity Manager & & & \\
\hline & Cost Account Managor & & & Cost Account Manager & & & \\
\hline
\end{tabular}




\begin{tabular}{|c|c|c|c|c|c|c|c|c|c|}
\hline \multirow{2}{*}{\multicolumn{2}{|c|}{\begin{tabular}{|l|l|}
1 WBS Number & 1.8 .2 .8 .2 .1 \\
$\begin{array}{c}\text { FDS Numbor } \\
1 Y 2802\end{array}$ & $\begin{array}{l}\text { Titlo } \\
\text { ADS MANAGEMENT DIRECTION }\end{array}$ \\
\end{tabular}}} & \multirow{2}{*}{\multicolumn{4}{|c|}{$\begin{array}{l}\text { COST ACCOUNT AUTHORIZATION } \\
\text { PROGRAM/PROJECT PLANNING INTEGRATION PROGRAM }\end{array}$}} & \multirow{2}{*}{\multicolumn{2}{|c|}{\begin{tabular}{|c|} 
Activity Mgr. \\
D.L. BÖRERS \\
Original \\
\end{tabular}}} & \multirow{2}{*}{\multicolumn{2}{|c|}{\begin{tabular}{|r|} 
Date \\
$08 / 26 / 94$ \\
Revised \\
\end{tabular}}} \\
\hline & & & & & & & & & \\
\hline \multicolumn{2}{|c|}{ Resp. Org. Tille/Code PROG. BASELINE INT. 4A300 } & $\begin{array}{c}\text { Original Budget } \\
(K \$)\end{array}$ & $\begin{array}{c}\text { Budget Prior } \\
\text { Rovision }\end{array}$ & $\begin{array}{c}\text { Changes This } \\
\text { Rovision }\end{array}$ & $\begin{array}{c}\text { Current Total } \\
\text { Budget }\end{array}$ & Start & Completo & Start & Complete \\
\hline \multicolumn{2}{|c|}{ Cost Acct. Mgr./Phono D. L. BORDERS 376-9227 } & 144 & & & 144 & $10 / 01 / 9$ & $09 / 30 / 95$ & & \\
\hline
\end{tabular}

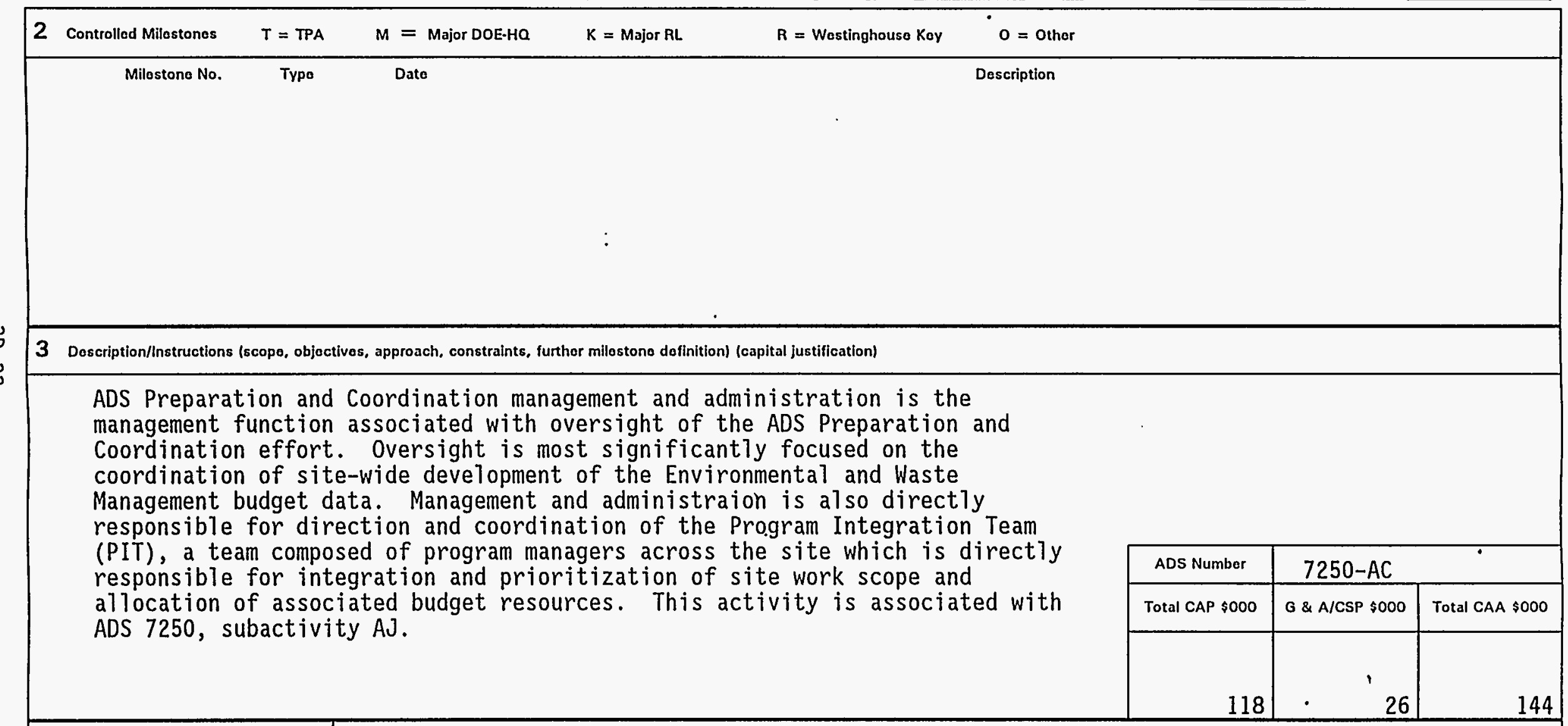

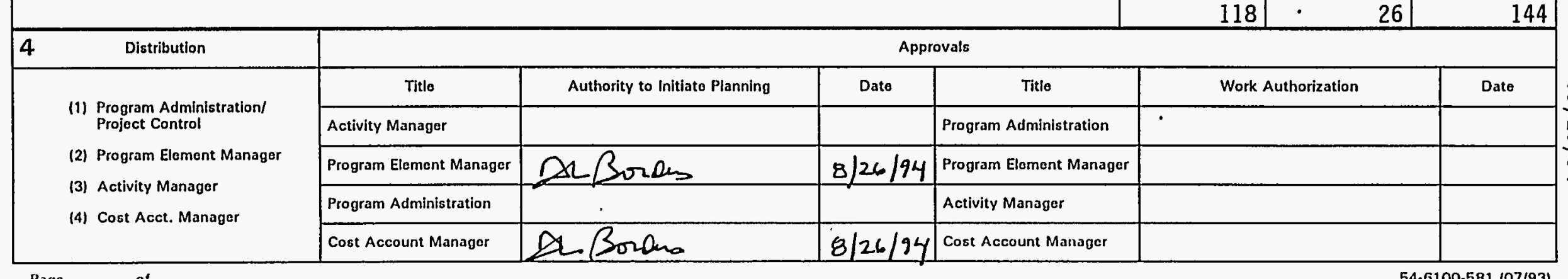

